Summary of Shock Initiation Data for TATB-based Explosives

K. S. Vandersall

April 7, 2010 
This document was prepared as an account of work sponsored by an agency of the United States government. Neither the United States government nor Lawrence Livermore National Security, LLC, nor any of their employees makes any warranty, expressed or implied, or assumes any legal liability or responsibility for the accuracy, completeness, or usefulness of any information, apparatus, product, or process disclosed, or represents that its use would not infringe privately owned rights. Reference herein to any specific commercial product, process, or service by trade name, trademark, manufacturer, or otherwise does not necessarily constitute or imply its endorsement, recommendation, or favoring by the United States government or Lawrence Livermore National Security, LLC. The views and opinions of authors expressed herein do not necessarily state or reflect those of the United States government or Lawrence Livermore National Security, LLC, and shall not be used for advertising or product endorsement purposes.

This work performed under the auspices of the U.S. Department of Energy by Lawrence Livermore National Laboratory under Contract DE-AC52-07NA27344. 


\section{Summary of Shock Initiation Data for TATB-based Explosives}

This short summary of previously published data [1,2] was compiled to provide the actual in-situ gauge data to allow modeling of these experiments. Although the purpose here is to fulfill a deliverable for a JOWOG 9 Focused Exchange (09-006), it is just as applicable to other exchanges as well. The TATB materials described here are Ultra Fine (UF) TATB and LX-17 (92.5\% TATB and 7.5\% Kel-F by weight), with the details of the experiments provided in the prior publications [1,2]. The data is provided in the appendices of the document, but will be provided electronically as text files due to being amenable to importing into the code in that manner for comparison.

\section{Ambient Temperature Ultra Fine (UF) TATB Experiments}

Table 1 provides a description of the ambient temperature Ultra Fine (UF) TATB experiments 4191, 4534, and 4535 [1]. All of these experiments used multiple manganin gauge elements on a 24 degree wedge sample. Experiment 4191 used a $15 \mathrm{~mm}$ thick AD998 aluminum oxide flyer and $3 \mathrm{~mm}$ thick Teflon impact plate. The other experiments, 4534 and 4535 used nominally $12 \mathrm{~mm}$ thick flyer plates and $5 \mathrm{~mm}$ thick impact plates. Figures 1-3 provide the gauge record plots with the gauge locations described in the figure or in the figure caption. The data from these records is included in the appendix.

Table 1. Summary of UF-TATB experiments at ambient temperature.

\begin{tabular}{||l|l|c|c|c|c|c|c|c||}
\hline SHOT & MAT'L & TEMP. & $\begin{array}{c}\text { SHOT } \\
\text { DATE }\end{array}$ & $\begin{array}{c}\text { IMPACT } \\
\text { VELOCITY }\end{array}$ & $\begin{array}{c}\text { FLYER } \\
\text { PLATE }\end{array}$ & $\begin{array}{c}\text { IMPACT } \\
\text { PLATE }\end{array}$ & $\begin{array}{c}\text { INPUT } \\
\text { PRESSURE }\end{array}$ & $\begin{array}{c}\text { RUN TO } \\
\text { DET }\end{array}$ \\
\hline 4191 & UF-TATB & $25^{\circ} \mathrm{C}$ & $11 / 21 / 88$ & $1.509 \mathrm{~km} / \mathrm{s}$ & AD-998 & Teflon & $11 \mathrm{GPa}$ & $5 \mathrm{~mm}$ \\
\hline 4534 & UF-TATB & $25^{\circ} \mathrm{C}$ & $4 / 3 / 01$ & $1.436 \mathrm{~km} / \mathrm{s}$ & $\mathrm{Al}-6061$ & $\mathrm{Al}-6061$ & $7.7 \mathrm{GPa}$ & $>35.7 \mathrm{~mm}$ \\
\hline 4535 & UF-TATB & $25^{\circ} \mathrm{C}$ & $4 / 4 / 01$ & $1.688 \mathrm{~km} / \mathrm{s}$ & $\mathrm{Al}-6061$ & $\mathrm{Al}-6061$ & $9.2 \mathrm{GPa}$ & $9.5 \mathrm{~mm}$ \\
\hline
\end{tabular}

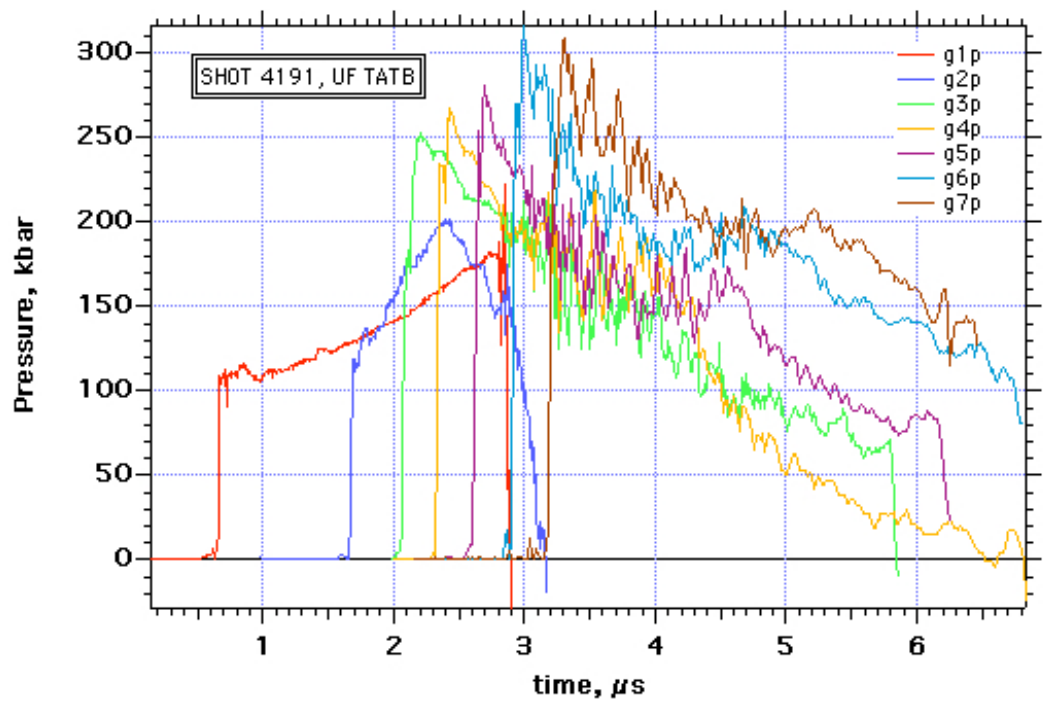

Figure 1. Manganin gauge record for experiment 4191. Gauges were located nominally at $0,4,6,8,10,12$ and $14 \mathrm{~mm}$. 


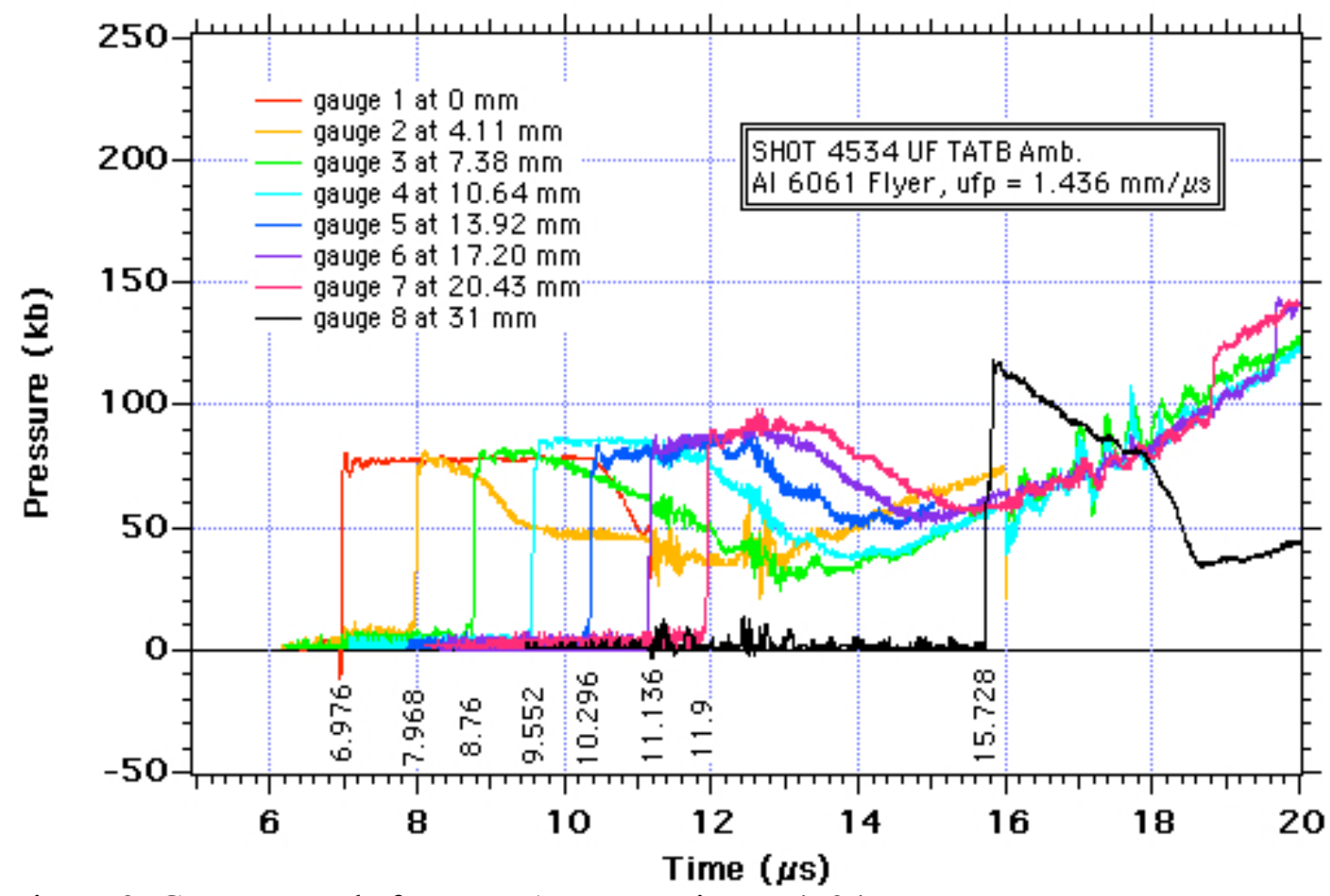

Figure 2. Gauge records for UF-TATB experiment 4534.

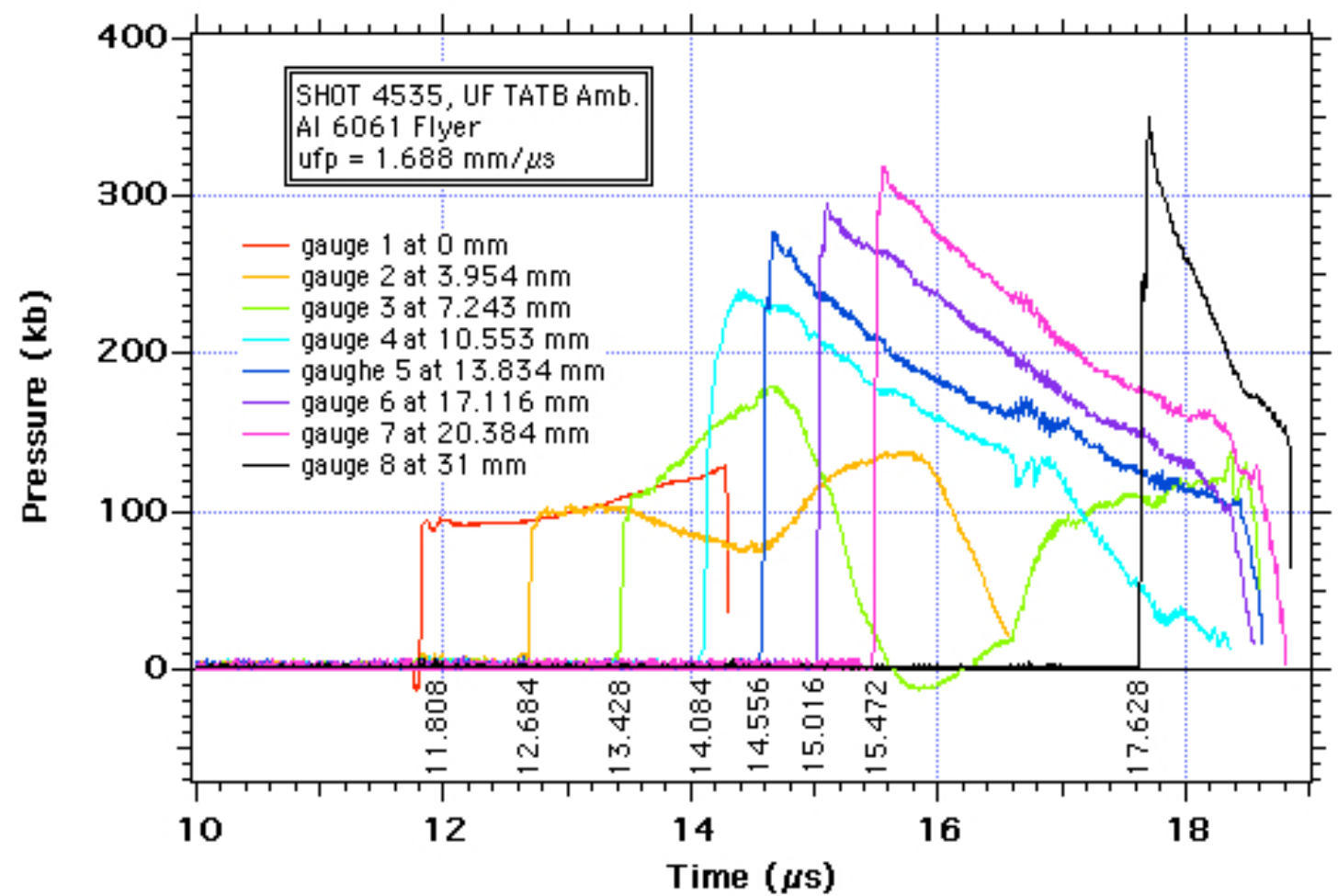

Figure 3. Gauge records for UF-TATB experiment 4535. 


\section{Ambient Temperature LX-17 Experiments}

Table 2 provides a summary of Experiments $2 \mathrm{~S}-47,2 \mathrm{~S}-50,2 \mathrm{~S}-54,2 \mathrm{~S}-64$, and $2 \mathrm{~S}-68$ that were performed at Los Alamos National Laboratory by Rick Gustavsen in collaboration with Lawrence Livermore National Laboratory researchers [2]. Figures 4-8 provide the electromagnetic velocity (EMV) gauge records with the gauge locations provided in the figure caption.

Table 2. Summary of LX-17 shock initiation experiments performed at LANL in collaboration with LLNL [2].

\begin{tabular}{||c|c|c|c|c|c|c|c|c||}
\hline SHOT & MAT'L & TEMP. & $\begin{array}{c}\text { SHOT } \\
\text { DATE }\end{array}$ & $\begin{array}{c}\text { IMPACT } \\
\text { VELOCITY }\end{array}$ & $\begin{array}{c}\text { FLYER } \\
\text { PLATE }\end{array}$ & $\begin{array}{c}\text { IMPACT } \\
\text { PLATE }\end{array}$ & $\begin{array}{c}\text { INPUT } \\
\text { PRESSURE }\end{array}$ & $\begin{array}{c}\text { RUN TO } \\
\text { DET }\end{array}$ \\
\hline 2S-47 & LX-17 & $25^{\circ} \mathrm{C}$ & $10 / 12 / 00$ & $2.951 \mathrm{~km} / \mathrm{s}$ & Kel-F & - & $15.0 \mathrm{GPa}$ & $5.53 \mathrm{~mm}$ \\
\hline $2 \mathrm{~S}-50$ & $\mathrm{LX}-17$ & $25^{\circ} \mathrm{C}$ & $15 / 15 / 00$ & $2.619 \mathrm{~km} / \mathrm{s}$ & Kel-F & - & $12.50 \mathrm{GPa}$ & $9.83 \mathrm{~mm}$ \\
\hline $2 \mathrm{~S}-54$ & $\mathrm{LX}-17$ & $25^{\circ} \mathrm{C}$ & $2 / 14 / 01$ & $2.599 \mathrm{~km} / \mathrm{s}$ & Kel-F & - & $12.36 \mathrm{GPa}$ & $10.1 \mathrm{~mm}$ \\
\hline $2 \mathrm{~S}-64$ & $\mathrm{LX}-17$ & $25^{\circ} \mathrm{C}$ & $11 / 15 / 01$ & $3.131 \mathrm{~km} / \mathrm{s}$ & Kel-F & - & $16.38 \mathrm{GPa}$ & $3.9 \mathrm{~mm}$ \\
\hline $2 \mathrm{~S}-68$ & LX-17 & $25^{\circ} \mathrm{C}$ & $19 / 19 / 01$ & $2.474 \mathrm{~km} / \mathrm{s}$ & Kel-F & - & $11.48 \mathrm{GPa}$ & $11.0 \mathrm{~mm}$ \\
\hline \hline
\end{tabular}

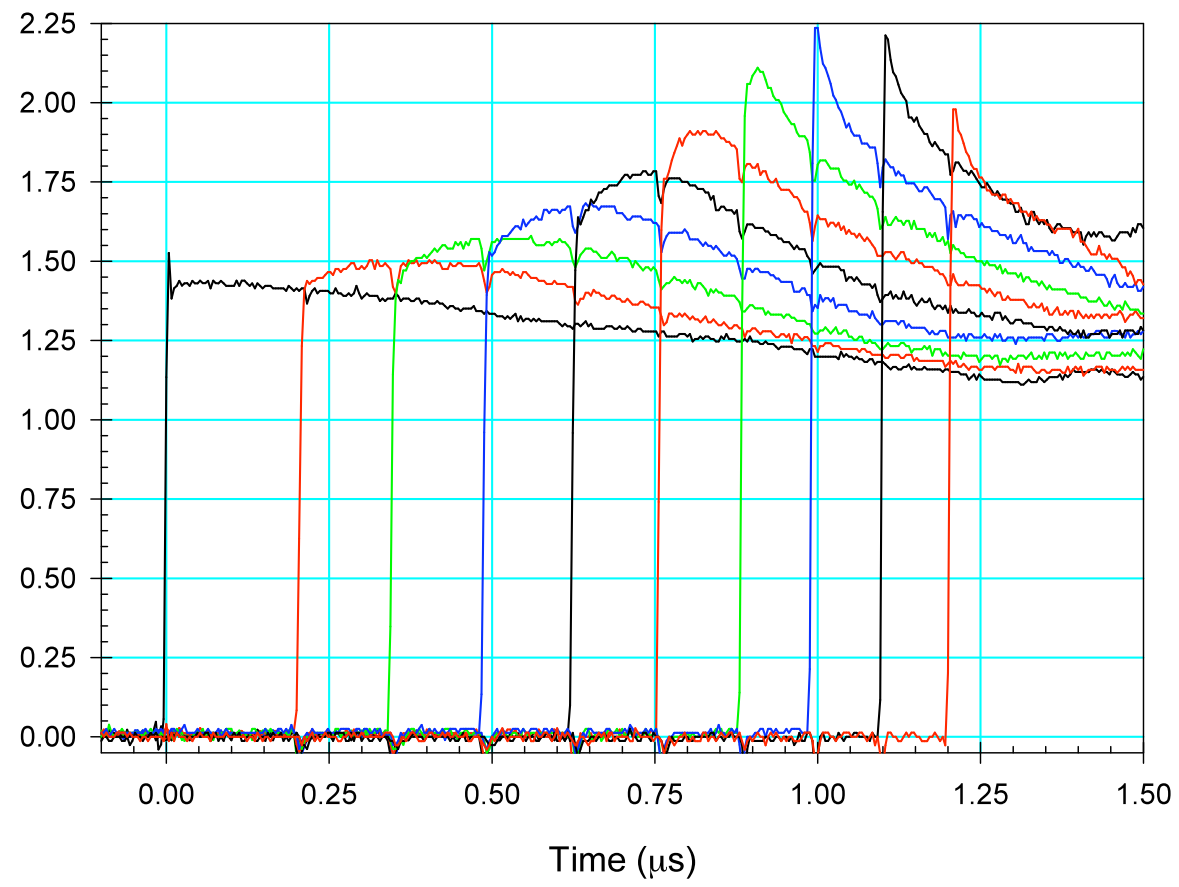

Figure 3. Gauge records for experiment $2 \mathrm{~S}-47$ with particle velocity gauges are located at depths of $0.00,1.19,1.98,2.77,3.55,4.34,5.13,5.92,6.71$, and $7.50 \mathrm{~mm}$. 


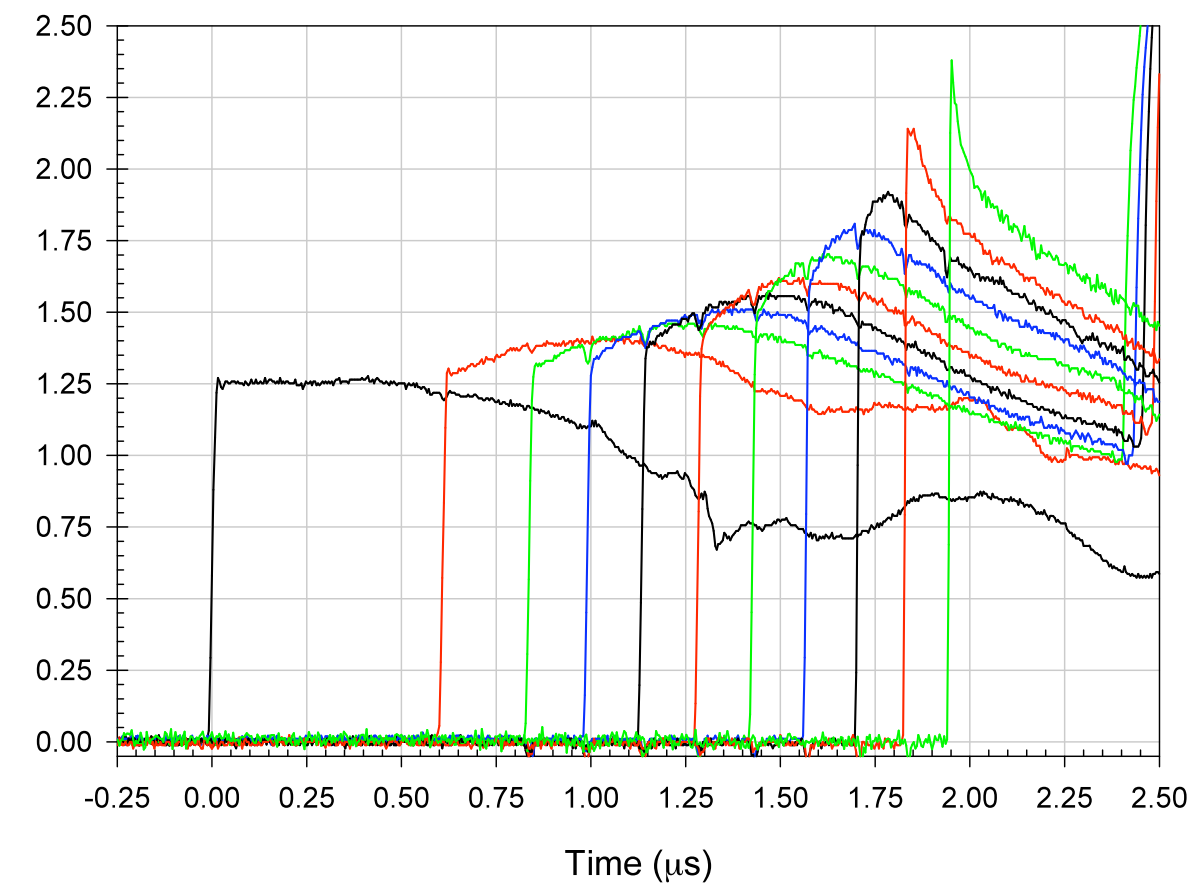

Figure 4. Gauge records for experiment $2 \mathrm{~S}-50$ with particle velocity gauges are located at depths of $0.00,3.10,4.32,5.11,5.89,6.68,7.47,8.26,9.04,9.83$, and $10.62 \mathrm{~mm}$.

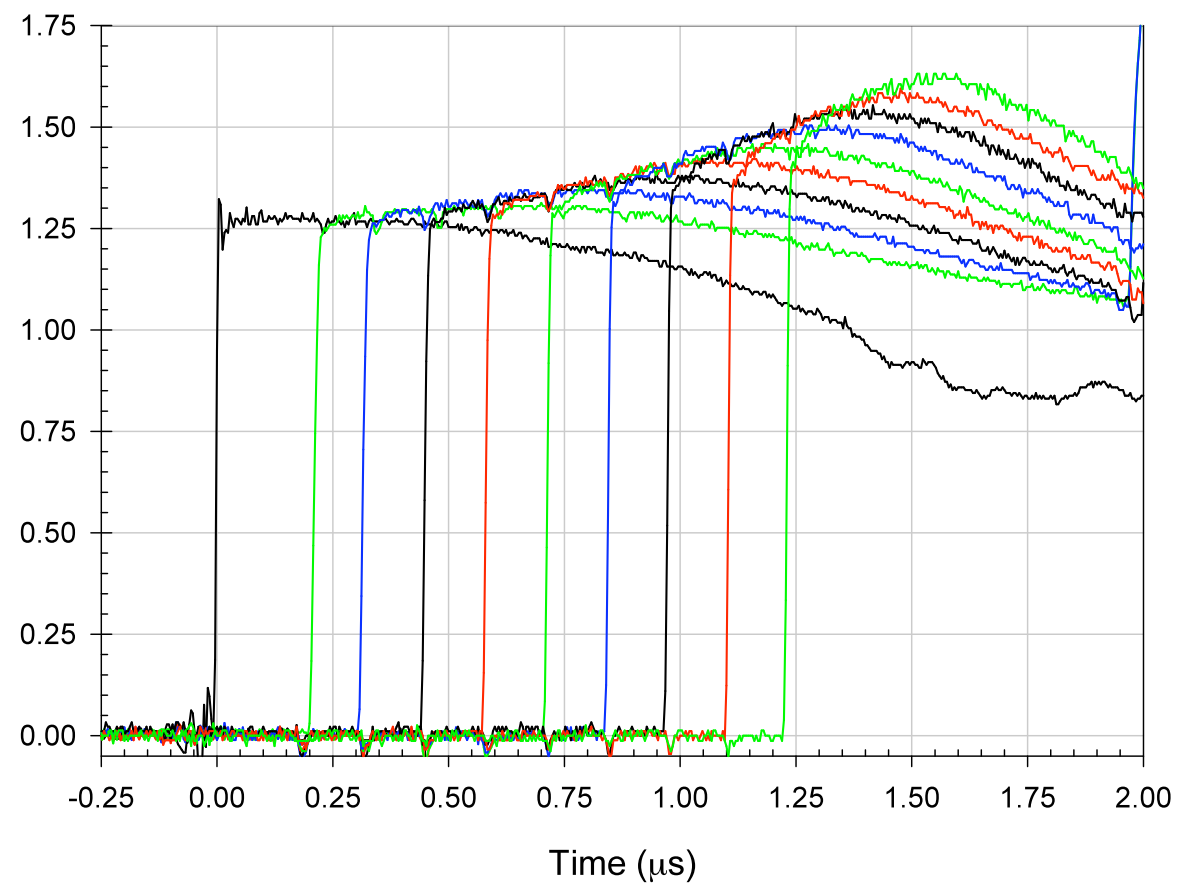

Figure 5. Gauge records for experiment $2 \mathrm{~S}-54$ with particle velocity gauges are located at depths of $0.00,1.19,1.86,2.54,3.20,3.88,4.55,5.22,5.89$, and $6.56 \mathrm{~mm}$. 


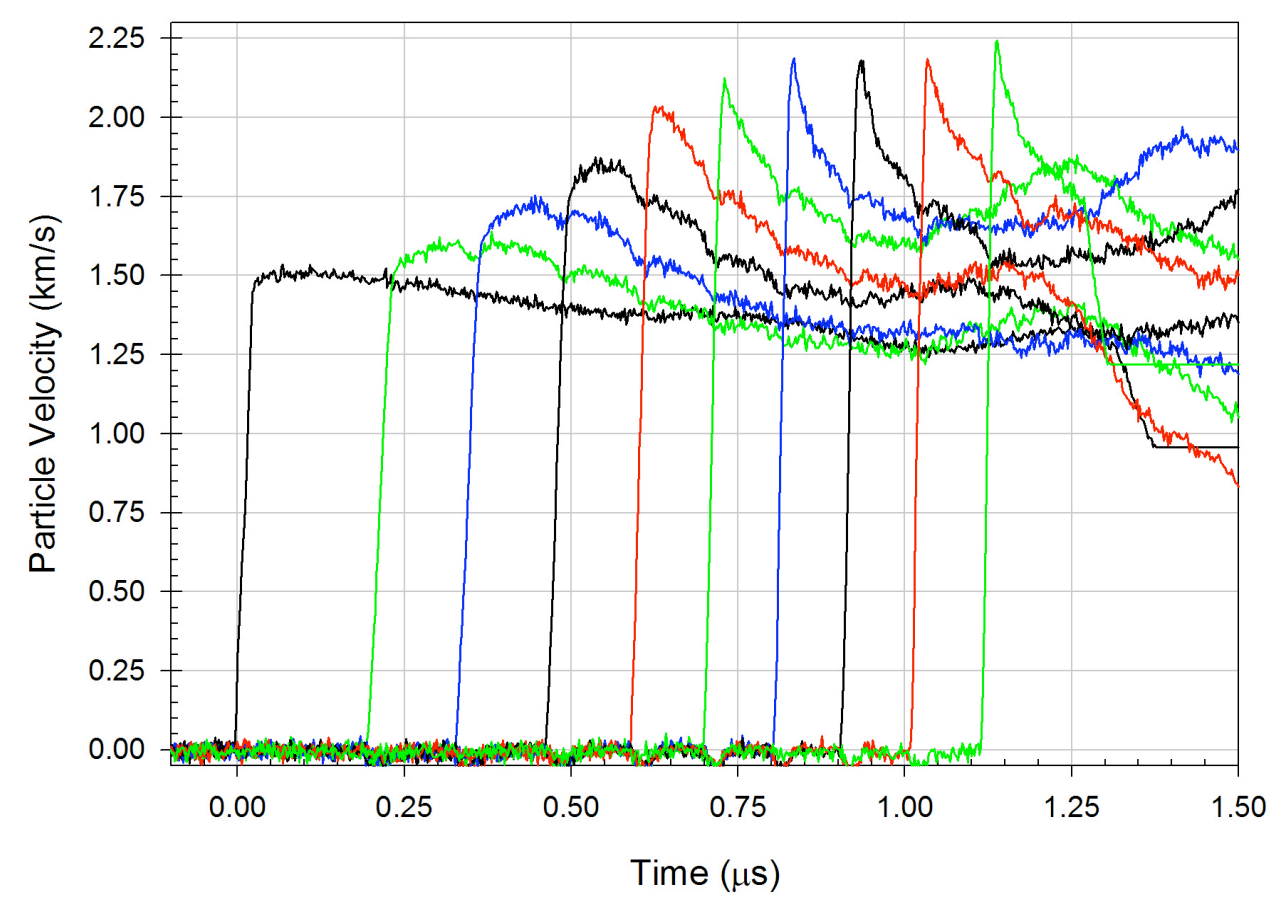

Figure 6. Gauge records for experiment $2 \mathrm{~S}-64$ with particle velocity gauges are located at depths of $0.00,1.15,1.94,2.73,3.52,4.31,5.09,5.89,6.68$, and $7.47 \mathrm{~mm}$.

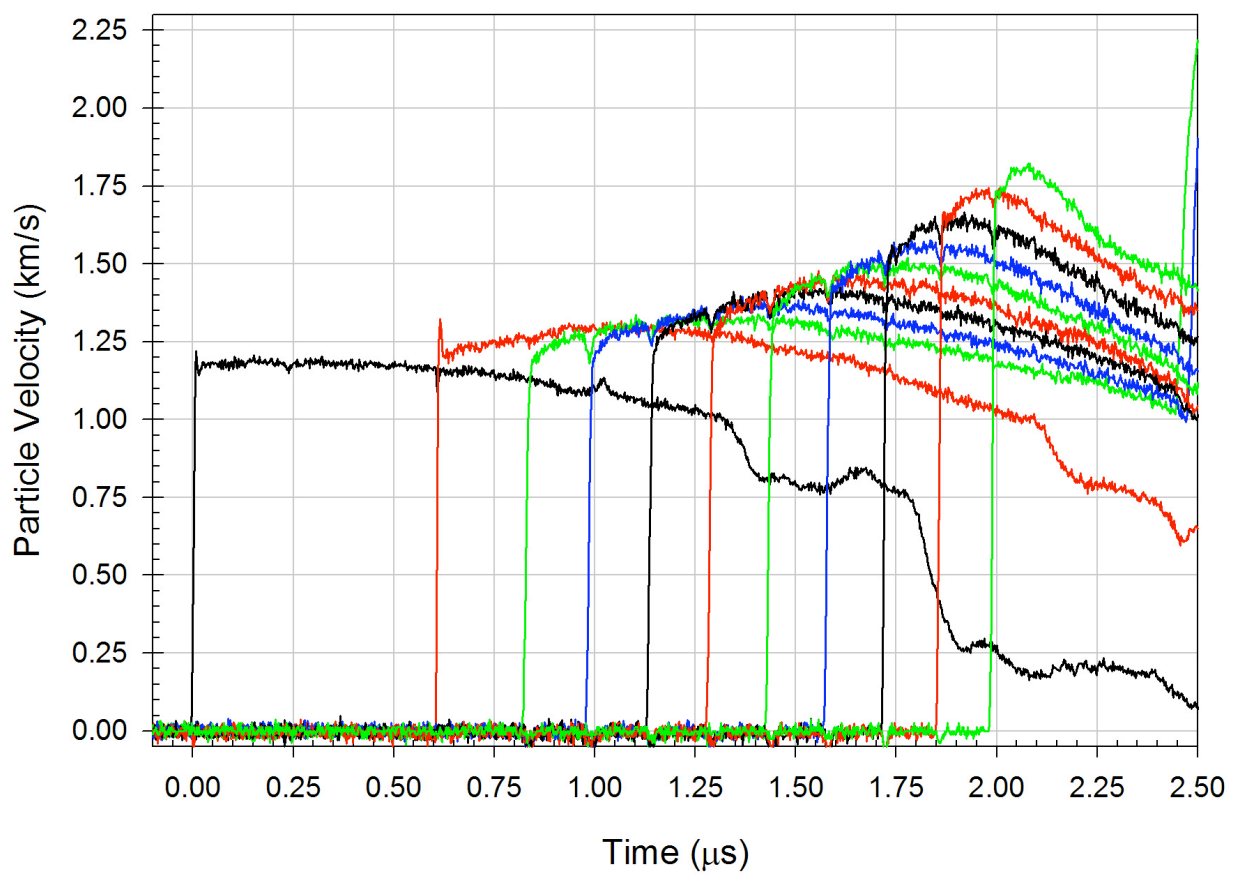

Figure 7. Gauge records for experiment $2 \mathrm{~S}-68$ with particle velocity gauges are located at depths of $0.00,3.04,4.13,4.91,5.70,6.48,7.28,8.07,8.86,9.65$, and $10.44 \mathrm{~mm}$. 


\section{References}

1. Paul A. Urtiew, Jerry W. Forbes, Frank Garcia, and Craig M. Tarver, "Shock Initiation of UF-TATB at $250^{\circ} \mathrm{C}$," Shock Compression of Condensed Matter 2001 edited by M. D. Furnish, N. N. Thadhani, and Y. Horie, 2002, pp. 10391042.

2. R. L. Gustavsen, S. A. Sheffield, R. R. Alcon, J. W. Forbes, C. M. Tarver, and F. Garcia, "Embedded Electromagnetic Gauge Measurements and Modeling of Shock Initiations in the TATB Based Explosives LX-17 and PBX-9502.

\section{Appendices to follow:}

Appendix A - Gauge data for experiment 4191. Appendix B - Gauge data for experiment 4534. Appendix C - Gauge data for experiment 4535. Appendix D - Gauge data for experiment 2S-47. Appendix E - Gauge data for experiment 2S-50. Appendix F - Gauge data for experiment 2S-54. Appendix $\mathrm{G}-$ Gauge data for experiment 2S-64. Appendix $\mathrm{H}-$ Gauge data for experiment 2S-68. 


\section{Appendix A - Gauge data for experiment 4191.}

\begin{tabular}{|c|c|c|c|c|c|c|c|c|c|c|c|}
\hline g1t & $\begin{array}{l}\text { g1p } \\
\text { g7t }\end{array}$ & $\begin{array}{l}\text { g2t } \\
\text { g7p }\end{array}$ & $\mathrm{g} 2 \mathrm{p}$ & g3t & $\mathrm{g} 3 \mathrm{p}$ & $\mathrm{g} 4 \mathrm{t}$ & $\mathrm{g} 4 \mathrm{p}$ & g5t & g5p & g6t & g6 \\
\hline 0.145 & $\begin{array}{l}0.673987 \\
2.155\end{array}$ & $\begin{array}{l}0.985 \\
0\end{array}$ & 1.68832 & 1.99 & 0 & 2 & 0 & 2.25 & 0 & 2.155 & 0 \\
\hline 0.155 & $\begin{array}{l}0 \\
2.17\end{array}$ & $\begin{array}{l}0.995 \\
0\end{array}$ & 0 & 2 & 1.83243 & 2.015 & 0 & 2.265 & 0 & 2.17 & 0 \\
\hline 0.16 & $\begin{array}{l}0 \\
2.185\end{array}$ & $\begin{array}{l}1 \\
0\end{array}$ & 0 & 2.005 & 1.82212 & 2.03 & 0 & 2.28 & 0 & 2.185 & 0 \\
\hline 0.17 & $\begin{array}{l}0 \\
2.2\end{array}$ & $\begin{array}{l}1.01 \\
0\end{array}$ & 0 & 2.015 & 1.82212 & 2.045 & 0 & 2.295 & 0 & 2.2 & 0 \\
\hline 0.18 & $\begin{array}{l}0 \\
2.215\end{array}$ & $\begin{array}{l}1.015 \\
0\end{array}$ & 0 & 2.02 & 3.67164 & 2.06 & 0 & 2.31 & 0 & 2.215 & 0 \\
\hline 0.185 & $\begin{array}{l}0 \\
2.23\end{array}$ & $\begin{array}{l}1.025 \\
0\end{array}$ & 0 & 2.03 & 3.65094 & 2.075 & 0 & 2.325 & 0 & 2.23 & 0 \\
\hline 0.195 & $\begin{array}{l}0 \\
2.25\end{array}$ & $\begin{array}{l}1.03 \\
0\end{array}$ & 0 & 2.035 & 3.65094 & 2.09 & 0 & 2.34 & 0 & 2.25 & 0 \\
\hline 0.2 & $\begin{array}{l}0 \\
2.265\end{array}$ & $\begin{array}{l}1.04 \\
0\end{array}$ & 0 & 2.045 & 5.48642 & 2.105 & 0 & 2.355 & 0 & 2.265 & 0 \\
\hline 0.21 & $\begin{array}{l}0 \\
2.28\end{array}$ & $\begin{array}{l}1.05 \\
0\end{array}$ & 0 & 2.055 & 7.32848 & 2.125 & 0 & 2.375 & 0 & 2.28 & 0 \\
\hline 0.215 & $\begin{array}{l}0 \\
2.295\end{array}$ & $\begin{array}{l}1.055 \\
0\end{array}$ & 0 & 2.06 & 14.7616 & 2.14 & 0 & 2.39 & 0 & 2.295 & 0 \\
\hline 0.225 & $\begin{array}{l}0 \\
2.31\end{array}$ & $\begin{array}{l}1.065 \\
1.72165\end{array}$ & 0 & 2.07 & 75.5157 & 2.155 & 0 & 2.405 & 1.78966 & 2.31 & 0 \\
\hline 0.23 & $\begin{array}{l}0 \\
2.325\end{array}$ & $\begin{array}{l}1.07 \\
0\end{array}$ & 0 & 2.075 & 134.203 & 2.17 & 0 & 2.42 & 1.78966 & 2.325 & 0 \\
\hline 0.24 & $\begin{array}{l}0 \\
2.34\end{array}$ & $\begin{array}{l}1.08 \\
0\end{array}$ & 0 & 2.085 & 151.454 & 2.185 & 0 & 2.435 & 0 & 2.34 & 0 \\
\hline 0.25 & $\begin{array}{l}0 \\
2.355\end{array}$ & $\begin{array}{l}1.085 \\
0\end{array}$ & 0 & 2.09 & 166.461 & 2.2 & 0 & 2.45 & 1.78966 & 2.355 & 0 \\
\hline 0.255 & $\begin{array}{l}0 \\
2.375\end{array}$ & $\begin{array}{l}1.095 \\
0\end{array}$ & 0 & 2.1 & 174.793 & 2.215 & 0 & 2.465 & 0 & 2.375 & 0 \\
\hline 0.265 & $\begin{array}{l}0 \\
2.39\end{array}$ & $\begin{array}{l}1.1 \\
1.72165\end{array}$ & 0 & 2.105 & 183.335 & 2.23 & 0 & 2.48 & 0 & 2.39 & 0 \\
\hline 0.27 & $\begin{array}{l}0 \\
2.405\end{array}$ & $\begin{array}{l}1.11 \\
0\end{array}$ & 0 & 2.115 & 174.793 & 2.25 & 0 & 2.495 & 0 & 2.405 & 0 \\
\hline 0.28 & $\begin{array}{l}0 \\
2.42\end{array}$ & $\begin{array}{l}1.12 \\
0\end{array}$ & 0 & 2.125 & 169.95 & 2.265 & 0 & 2.515 & 0 & 2.42 & 0 \\
\hline 0.285 & $\begin{array}{l}0 \\
2.435\end{array}$ & $\begin{array}{l}1.125 \\
0\end{array}$ & 0 & 2.13 & 179.646 & 2.28 & 0 & 2.53 & 0 & 2.435 & 0 \\
\hline 0.295 & $\begin{array}{l}0 \\
2.45\end{array}$ & $\begin{array}{l}1.135 \\
0\end{array}$ & 0 & 2.14 & 221.279 & 2.295 & 3.62518 & 2.545 & 0 & 2.45 & 0 \\
\hline 0.3 & $\begin{array}{l}0 \\
2.465\end{array}$ & $\begin{array}{l}1.14 \\
0\end{array}$ & 0 & 2.145 & 226.213 & 2.31 & 5.44764 & 2.56 & 3.58579 & 2.465 & 0 \\
\hline 0.31 & $\begin{array}{l}0 \\
2.48\end{array}$ & $\begin{array}{l}1.15 \\
0\end{array}$ & 0 & 2.155 & 230.142 & 2.325 & 1.80929 & 2.575 & 5.38834 & 2.48 & 0 \\
\hline 0.32 & $\begin{array}{l}0 \\
2.495\end{array}$ & $\begin{array}{l}1.155 \\
0\end{array}$ & 0 & 2.16 & 233.625 & 2.34 & 79.0136 & 2.59 & 9.06414 & 2.495 & 0 \\
\hline 0.325 & $\begin{array}{l}0 \\
2.515\end{array}$ & $\begin{array}{l}1.165 \\
0\end{array}$ & 0 & 2.17 & 238.572 & 2.355 & 234.598 & 2.605 & 9.01248 & 2.515 & 0 \\
\hline 0.335 & $\begin{array}{l}0 \\
2.53\end{array}$ & $\begin{array}{l}1.175 \\
0\end{array}$ & 0 & 2.18 & 247.564 & 2.375 & 233.638 & 2.62 & 82.1381 & 2.53 & 0 \\
\hline 0.34 & $\begin{array}{l}0 \\
2.545\end{array}$ & $\begin{array}{l}1.18 \\
0\end{array}$ & 0 & 2.185 & 250.057 & 2.39 & 211.601 & 2.64 & 239.613 & 2.545 & 0 \\
\hline 0.35 & $\begin{array}{l}0 \\
2.56\end{array}$ & $\begin{array}{l}1.19 \\
0\end{array}$ & 0 & 2.195 & 247.564 & 2.405 & 255.775 & 2.655 & 254.301 & 2.56 & 0 \\
\hline 0.355 & $\begin{array}{l}0 \\
2.575\end{array}$ & $\begin{array}{l}1.195 \\
1.72165\end{array}$ & 0 & 2.2 & 252.55 & 2.42 & 267.319 & 2.67 & 215.225 & 2.575 & 0 \\
\hline 0.365 & $\begin{array}{l}0 \\
2.59\end{array}$ & $\begin{array}{l}1.205 \\
0\end{array}$ & 0 & 2.21 & 250.057 & 2.435 & 259.881 & 2.685 & 266.561 & 2.59 & 0 \\
\hline 0.375 & $\begin{array}{l}0 \\
2.605\end{array}$ & $\begin{array}{l}1.21 \\
1.73107\end{array}$ & 0 & 2.215 & 247.564 & 2.45 & 264.839 & 2.7 & 281.285 & 2.605 & 0 \\
\hline 0.38 & $\begin{array}{l}0 \\
2.62\end{array}$ & $\begin{array}{l}1.22 \\
0\end{array}$ & 0 & 2.225 & 250.057 & 2.465 & 259.881 & 2.715 & 269.015 & 2.62 & 0 \\
\hline 0.39 & $\begin{array}{l}0 \\
2.64\end{array}$ & $\begin{array}{l}1.225 \\
1.72165\end{array}$ & 0 & 2.23 & 247.564 & 2.48 & 252.449 & 2.73 & 270.749 & 2.64 & 0 \\
\hline
\end{tabular}




\begin{tabular}{|c|c|c|c|c|c|c|c|c|c|c|c|}
\hline 0.395 & $\begin{array}{l}0 \\
2.655\end{array}$ & $\begin{array}{l}1.235 \\
0\end{array}$ & 0 & 2.24 & 249.145 & 2.495 & 251.577 & 2.745 & 264.108 & 2.655 & 0 \\
\hline 0.405 & $\begin{array}{l}0 \\
2.67\end{array}$ & $\begin{array}{l}1.245 \\
1.72165\end{array}$ & 0 & 2.25 & 247.564 & 2.515 & 244.11 & 2.765 & 255.949 & 2.67 & 0 \\
\hline 0.41 & $\begin{array}{l}0 \\
2.685\end{array}$ & $\begin{array}{l}1.25 \\
0\end{array}$ & 0 & 2.255 & 245.072 & 2.53 & 244.11 & 2.78 & 249.402 & 2.685 & 0 \\
\hline 0.42 & $\begin{array}{l}0 \\
2.7\end{array}$ & $\begin{array}{l}1.26 \\
0\end{array}$ & 0 & 2.265 & 245.072 & 2.545 & 239.137 & 2.795 & 243.634 & 2.7 & 0 \\
\hline 0.425 & $\begin{array}{l}0 \\
2.715\end{array}$ & $\begin{array}{l}1.265 \\
0\end{array}$ & 0 & 2.27 & 244.132 & 2.56 & 241.623 & 2.81 & 246.953 & 2.715 & 0 \\
\hline 0.435 & $\begin{array}{l}0 \\
2.73\end{array}$ & $\begin{array}{l}1.275 \\
0\end{array}$ & 0 & 2.28 & 241.627 & 2.575 & 241.623 & 2.825 & 239.613 & 2.73 & 0 \\
\hline 0.445 & $\begin{array}{l}0 \\
2.745\end{array}$ & $\begin{array}{l}1.28 \\
0\end{array}$ & 0 & 2.285 & 242.581 & 2.59 & 239.137 & 2.84 & 249.402 & 2.745 & 0 \\
\hline 0.45 & $\begin{array}{l}0 \\
2.765\end{array}$ & $\begin{array}{l}1.29 \\
0\end{array}$ & 0 & 2.295 & 237.602 & 2.605 & 241.623 & 2.855 & 237.169 & 2.765 & 0 \\
\hline 0.46 & $\begin{array}{l}0 \\
2.78\end{array}$ & $\begin{array}{l}1.295 \\
1.73107\end{array}$ & 0 & 2.3 & 241.627 & 2.62 & 236.652 & 2.87 & 234.725 & 2.78 & 0 \\
\hline 0.465 & $\begin{array}{l}0 \\
2.795\end{array}$ & $\begin{array}{l}1.305 \\
0\end{array}$ & 0 & 2.31 & 240.091 & 2.64 & 231.686 & 2.885 & 235.641 & 2.795 & 0 \\
\hline 0.475 & $\begin{array}{l}0 \\
2.81\end{array}$ & $\begin{array}{l}1.315 \\
0\end{array}$ & 0 & 2.32 & 241.627 & 2.655 & 226.725 & 2.905 & 228.358 & 2.81 & 0 \\
\hline 0.48 & $\begin{array}{l}0 \\
2.825\end{array}$ & $\begin{array}{l}1.32 \\
0\end{array}$ & 0 & 2.325 & 240.091 & 2.67 & 224.246 & 2.92 & 227.402 & 2.825 & 0 \\
\hline 0.49 & $\begin{array}{l}0 \\
2.84\end{array}$ & $\begin{array}{l}1.33 \\
0\end{array}$ & 0 & 2.335 & 242.581 & 2.685 & 224.246 & 2.935 & 232.283 & 2.84 & 0 \\
\hline 0.495 & $\begin{array}{l}0 \\
2.855\end{array}$ & $\begin{array}{l}1.335 \\
0\end{array}$ & 0 & 2.34 & 242.581 & 2.7 & 221.769 & 2.95 & 229.842 & 2.855 & 12.2521 \\
\hline 0.505 & $\begin{array}{l}0 \\
2.87\end{array}$ & $\begin{array}{l}1.345 \\
1.73107\end{array}$ & 0 & 2.35 & 242.581 & 2.715 & 221.769 & 2.965 & 233.213 & 2.87 & 17.5906 \\
\hline 0.515 & $\begin{array}{l}0 \\
2.885\end{array}$ & $\begin{array}{l}1.35 \\
5.18287\end{array}$ & 0 & 2.355 & 242.581 & 2.73 & 221.769 & 2.98 & 228.358 & 2.885 & 0 \\
\hline 0.52 & $\begin{array}{l}0.689229 \\
2.905\end{array}$ & $\begin{array}{l}1.36 \\
0\end{array}$ & 0 & 2.365 & 242.581 & 2.745 & 219.294 & 2.995 & 227.402 & 2.905 & 10.4841 \\
\hline 0.53 & $\begin{array}{l}0.685354 \\
2.92\end{array}$ & $\begin{array}{l}1.37 \\
0\end{array}$ & 0 & 2.375 & 240.091 & 2.765 & 216.82 & 3.01 & 211.414 & 2.92 & 180.755 \\
\hline 0.535 & $\begin{array}{l}1.37942 \\
2.935\end{array}$ & $\begin{array}{l}1.375 \\
0\end{array}$ & 0 & 2.38 & 241.627 & 2.78 & 214.348 & 3.03 & 218.667 & 2.935 & 269.827 \\
\hline 0.545 & $\begin{array}{l}1.37942 \\
2.95\end{array}$ & $\begin{array}{l}1.385 \\
1.72165\end{array}$ & 0 & 2.39 & 240.091 & 2.795 & 211.878 & 3.045 & 199.363 & 2.95 & 269.827 \\
\hline 0.55 & $\begin{array}{l}2.05893 \\
2.965\end{array}$ & $\begin{array}{l}1.39 \\
1.72165\end{array}$ & 0 & 2.395 & 237.602 & 2.81 & 210.511 & 3.06 & 233.213 & 2.965 & 240.88 \\
\hline 0.56 & $\begin{array}{l}2.05893 \\
2.98\end{array}$ & $\begin{array}{l}1.4 \\
1.73107\end{array}$ & 0 & 2.405 & 237.602 & 2.825 & 195.821 & 3.075 & 194.556 & 2.98 & 300.36 \\
\hline 0.57 & $\begin{array}{l}2.05893 \\
2.995\end{array}$ & $\begin{array}{l}1.405 \\
0\end{array}$ & 0 & 2.41 & 237.602 & 2.84 & 210.511 & 3.09 & 196.958 & 2.995 & 316.396 \\
\hline 0.575 & $\begin{array}{l}3.45578 \\
3.01\end{array}$ & $\begin{array}{l}1.415 \\
0\end{array}$ & 0 & 2.42 & 235.114 & 2.855 & 195.821 & 3.105 & 212.451 & 3.01 & 299.885 \\
\hline 0.585 & $\begin{array}{l}3.4363 \\
3.03\end{array}$ & $\begin{array}{l}1.42 \\
0\end{array}$ & 0 & 2.425 & 235.114 & 2.87 & 193.379 & 3.12 & 204.177 & 3.03 & 299.885 \\
\hline 0.59 & $\begin{array}{l}3.41704 \\
3.045\end{array}$ & $\begin{array}{l}1.43 \\
12.1757\end{array}$ & 0 & 2.435 & 235.114 & 2.885 & 193.379 & 3.135 & 213.83 & 3.045 & 278.028 \\
\hline 0.6 & $\begin{array}{l}3.4363 \\
3.06\end{array}$ & $\begin{array}{l}1.44 \\
1.72165\end{array}$ & 0 & 2.445 & 235.114 & 2.905 & 186.07 & 3.155 & 213.83 & 3.06 & 271.542 \\
\hline 0.605 & $\begin{array}{l}3.4363 \\
3.075\end{array}$ & $\begin{array}{l}1.445 \\
0\end{array}$ & 0 & 2.45 & 233.625 & 2.92 & 200.709 & 3.17 & 184.969 & 3.075 & 262.098 \\
\hline 0.615 & $\begin{array}{l}4.1264 \\
3.09\end{array}$ & $\begin{array}{l}1.455 \\
6.96044\end{array}$ & 0 & 2.46 & 230.142 & 2.935 & 193.379 & 3.185 & 202.857 & 3.09 & 292.801 \\
\hline 0.62 & $\begin{array}{l}6.85741 \\
3.105\end{array}$ & $\begin{array}{l}1.46 \\
3.44929\end{array}$ & 0 & 2.465 & 232.627 & 2.95 & 190.941 & 3.2 & 157.777 & 3.105 & 280.403 \\
\hline 0.63 & $\begin{array}{l}0.677734 \\
3.12\end{array}$ & $\begin{array}{l}1.47 \\
1.73107\end{array}$ & 0 & 2.475 & 228.682 & 2.965 & 195.821 & 3.215 & 228.358 & 3.12 & 283.353 \\
\hline 0.64 & $\begin{array}{l}3.41704 \\
3.135\end{array}$ & $\begin{array}{l}1.475 \\
0\end{array}$ & 0 & 2.48 & 227.658 & 2.98 & 201.85 & 3.23 & 193.295 & 3.135 & 278.628 \\
\hline 0.645 & $\begin{array}{l}4.79041 \\
3.155\end{array}$ & $\begin{array}{l}1.485 \\
0\end{array}$ & 0 & 2.49 & 226.213 & 2.995 & 188.504 & 3.245 & 198.072 & 3.155 & 292.801 \\
\hline 0.655 & $\begin{array}{l}4.79041 \\
3.17\end{array}$ & $\begin{array}{l}1.49 \\
8.66768\end{array}$ & 0 & 2.495 & 223.746 & 3.01 & 195.821 & 3.26 & 190.909 & 3.17 & 280.991 \\
\hline 0.66 & $\begin{array}{l}17.4137 \\
3.185\end{array}$ & $\begin{array}{l}1.5 \\
17.4804\end{array}$ & 0 & 2.505 & 223.746 & 3.03 & 184.868 & 3.275 & 154.1 & 3.185 & 278.628 \\
\hline
\end{tabular}




\begin{tabular}{|c|c|c|c|c|c|c|c|c|c|c|c|}
\hline 0.67 & $\begin{array}{l}109.633 \\
3.2\end{array}$ & $\begin{array}{l}1.51 \\
169.208\end{array}$ & 0 & 2.515 & 221.279 & 3.045 & 180.037 & 3.295 & 210.05 & 3.2 & 239.342 \\
\hline 0.675 & $\begin{array}{l}108.097 \\
3.215\end{array}$ & $\begin{array}{l}1.515 \\
201.546\end{array}$ & 0 & 2.52 & 221.279 & 3.06 & 192.132 & 3.31 & 174.278 & 3.215 & 278.628 \\
\hline 0.685 & $\begin{array}{l}104.645 \\
3.23\end{array}$ & $\begin{array}{l}1.525 \\
235.987\end{array}$ & 0 & 2.53 & 218.815 & 3.075 & 160.823 & 3.325 & 170.663 & 3.23 & 229.124 \\
\hline 0.69 & $\begin{array}{l}108.962 \\
3.245\end{array}$ & $\begin{array}{l}1.53 \\
232.388\end{array}$ & 0 & 2.535 & 213.891 & 3.09 & 196.987 & 3.34 & 190.909 & 3.245 & 210.38 \\
\hline 0.7 & $\begin{array}{l}108.097 \\
3.26\end{array}$ & $\begin{array}{l}1.54 \\
266.56\end{array}$ & 0 & 2.545 & 213.891 & 3.105 & 187.287 & 3.355 & 213.83 & 3.26 & 231.473 \\
\hline 0.71 & $\begin{array}{l}112.426 \\
3.275\end{array}$ & $\begin{array}{l}1.545 \\
280.719\end{array}$ & 0 & 2.55 & 213.891 & 3.12 & 199.418 & 3.37 & 194.556 & 3.275 & 248.71 \\
\hline 0.715 & $\begin{array}{l}113.294 \\
3.295\end{array}$ & $\begin{array}{l}1.555 \\
306.671\end{array}$ & 0 & 2.56 & 213.891 & 3.135 & 199.418 & 3.385 & 174.278 & 3.295 & 257.378 \\
\hline 0.725 & $\begin{array}{l}90.943 \\
3.31\end{array}$ & $\begin{array}{l}1.565 \\
309.028\end{array}$ & 0 & 2.57 & 216.352 & 3.155 & 199.418 & 3.4 & 164.831 & 3.31 & 251.054 \\
\hline 0.73 & $\begin{array}{l}108.962 \\
3.325\end{array}$ & $\begin{array}{l}1.57 \\
290.159\end{array}$ & 0 & 2.575 & 213.891 & 3.17 & 189.709 & 3.42 & 205.253 & 3.325 & 232.326 \\
\hline 0.74 & $\begin{array}{l}110.693 \\
3.34\end{array}$ & $\begin{array}{l}1.58 \\
299.597\end{array}$ & 1.67897 & 2.585 & 213.891 & 3.185 & 217.539 & 3.435 & 180.19 & 3.34 & 255.744 \\
\hline 0.745 & $\begin{array}{l}108.763 \\
3.355\end{array}$ & $\begin{array}{l}1.585 \\
275.998\end{array}$ & 1.68832 & 2.59 & 213.891 & 3.2 & 159.782 & 3.45 & 151.746 & 3.355 & 264.458 \\
\hline 0.755 & $\begin{array}{l}111.374 \\
3.37\end{array}$ & $\begin{array}{l}1.595 \\
293.074\end{array}$ & 3.3824 & 2.6 & 213.891 & 3.215 & 178.878 & 3.465 & 146.082 & 3.37 & 225.32 \\
\hline 0.765 & $\begin{array}{l}108.097 \\
3.385\end{array}$ & $\begin{array}{l}1.6 \\
262.564\end{array}$ & 3.3824 & 2.605 & 212.536 & 3.23 & 190.9 & 3.48 & 173.041 & 3.385 & 232.326 \\
\hline 0.77 & $\begin{array}{l}110.503 \\
3.4\end{array}$ & $\begin{array}{l}1.61 \\
268.919\end{array}$ & 3.3824 & 2.615 & 212.536 & 3.245 & 198.141 & 3.495 & 170.663 & 3.4 & 222.988 \\
\hline 0.78 & $\begin{array}{l}110.503 \\
3.42\end{array}$ & $\begin{array}{l}1.615 \\
245.349\end{array}$ & 3.40136 & 2.62 & 213.891 & 3.26 & 134.734 & 3.51 & 149.396 & 3.42 & 225.32 \\
\hline 0.785 & $\begin{array}{l}108.763 \\
3.435\end{array}$ & $\begin{array}{l}1.625 \\
239.14\end{array}$ & 1.68832 & 2.63 & 212.536 & 3.275 & 160.823 & 3.525 & 163.547 & 3.435 & 215.998 \\
\hline 0.795 & $\begin{array}{l}111.374 \\
3.45\end{array}$ & $\begin{array}{l}1.635 \\
264.201\end{array}$ & 1.69776 & 2.64 & 212.536 & 3.295 & 201.85 & 3.545 & 204.177 & 3.45 & 197.43 \\
\hline 0.8 & $\begin{array}{l}108.763 \\
3.465\end{array}$ & $\begin{array}{l}1.64 \\
264.91\end{array}$ & 0 & 2.645 & 212.536 & 3.31 & 178.878 & 3.56 & 189.758 & 3.465 & 199.745 \\
\hline 0.81 & $\begin{array}{l}107.233 \\
3.48\end{array}$ & $\begin{array}{l}1.65 \\
280.719\end{array}$ & 0 & 2.655 & 212.536 & 3.325 & 171.696 & 3.575 & 170.663 & 3.48 & 227.654 \\
\hline 0.815 & $\begin{array}{l}115.74 \\
3.495\end{array}$ & $\begin{array}{l}1.655 \\
271.949\end{array}$ & 0 & 2.66 & 212.536 & 3.34 & 199.418 & 3.59 & 194.556 & 3.495 & 210.38 \\
\hline 0.825 & $\begin{array}{l}113.119 \\
3.51\end{array}$ & $\begin{array}{l}1.665 \\
297.238\end{array}$ & 25.9094 & 2.67 & 212.536 & 3.355 & 211.601 & 3.605 & 192.156 & 3.51 & 211.346 \\
\hline 0.835 & $\begin{array}{l}114.865 \\
3.525\end{array}$ & $\begin{array}{l}1.67 \\
285.439\end{array}$ & 47.4758 & 2.675 & 210.092 & 3.37 & 182.451 & 3.62 & 169.549 & 3.525 & 225.32 \\
\hline 0.84 & $\begin{array}{l}113.992 \\
3.545\end{array}$ & $\begin{array}{l}1.68 \\
246.16\end{array}$ & 118.373 & 2.685 & 210.092 & 3.385 & 170.407 & 3.635 & 149.396 & 3.545 & 229.99 \\
\hline 0.85 & $\begin{array}{l}115.901 \\
3.56\end{array}$ & $\begin{array}{l}1.685 \\
250.843\end{array}$ & 99.732 & 2.69 & 212.536 & 3.4 & 158.435 & 3.65 & 154.1 & 3.56 & 218.327 \\
\hline 0.855 & $\begin{array}{l}113.992 \\
3.575\end{array}$ & $\begin{array}{l}1.695 \\
239.14\end{array}$ & 104.01 & 2.7 & 210.092 & 3.42 & 158.435 & 3.665 & 180.19 & 3.575 & 213.671 \\
\hline 0.865 & $\begin{array}{l}115.74 \\
3.59\end{array}$ & $\begin{array}{l}1.705 \\
239.14\end{array}$ & 116.94 & 2.71 & 210.092 & 3.435 & 144.172 & 3.685 & 180.19 & 3.59 & 215.998 \\
\hline 0.87 & $\begin{array}{l}113.294 \\
3.605\end{array}$ & $\begin{array}{l}1.71 \\
239.14\end{array}$ & 118.373 & 2.715 & 210.092 & 3.45 & 141.808 & 3.7 & 163.547 & 3.605 & 213.671 \\
\hline 0.88 & $\begin{array}{l}110.503 \\
3.62\end{array}$ & $\begin{array}{l}1.72 \\
245.349\end{array}$ & 114.067 & 2.725 & 212.536 & 3.465 & 163.215 & 3.715 & 161.181 & 3.62 & 195.117 \\
\hline 0.885 & $\begin{array}{l}112.246 \\
3.635\end{array}$ & $\begin{array}{l}1.725 \\
255.53\end{array}$ & 116.94 & 2.73 & 210.092 & 3.48 & 172.811 & 3.73 & 158.817 & 3.635 & 185.887 \\
\hline 0.895 & $\begin{array}{l}110.503 \\
3.65\end{array}$ & $\begin{array}{l}1.735 \\
257.874\end{array}$ & 114.776 & 2.74 & 210.092 & 3.495 & 182.451 & 3.745 & 157.777 & 3.65 & 206.7 \\
\hline 0.905 & $\begin{array}{l}110.503 \\
3.665\end{array}$ & $\begin{array}{l}1.74 \\
243.819\end{array}$ & 118.373 & 2.745 & 207.649 & 3.51 & 180.037 & 3.76 & 165.916 & 3.665 & 233.823 \\
\hline 0.91 & $\begin{array}{l}109.633 \\
3.685\end{array}$ & $\begin{array}{l}1.75 \\
242.306\end{array}$ & 129.202 & 2.755 & 207.649 & 3.525 & 218.933 & 3.775 & 170.663 & 3.685 & 206.7 \\
\hline 0.92 & $\begin{array}{l}108.763 \\
3.7\end{array}$ & $\begin{array}{l}1.76 \\
271.949\end{array}$ & 129.202 & 2.765 & 207.649 & 3.545 & 218.933 & 3.79 & 175.421 & 3.7 & 206.7 \\
\hline 0.925 & $\begin{array}{l}108.763 \\
3.715\end{array}$ & $\begin{array}{l}1.765 \\
278.99\end{array}$ & 132.193 & 2.77 & 205.208 & 3.56 & 182.451 & 3.81 & 175.421 & 3.715 & 215.057 \\
\hline 0.935 & $\begin{array}{l}107.025 \\
3.73\end{array}$ & $\begin{array}{l}1.775 \\
264.91\end{array}$ & 137.435 & 2.78 & 205.208 & 3.575 & 160.823 & 3.825 & 160.125 & 3.73 & 215.998 \\
\hline
\end{tabular}




\begin{tabular}{|c|c|c|c|c|c|c|c|c|c|c|c|}
\hline 0.94 & $\begin{array}{l}108.561 \\
3.745\end{array}$ & $\begin{array}{l}1.78 \\
257.874\end{array}$ & 135.74 & 2.785 & 205.208 & 3.59 & 141.808 & 3.84 & 147.049 & 3.745 & 208.044 \\
\hline 0.95 & $\begin{array}{l}107.025 \\
3.76\end{array}$ & $\begin{array}{l}1.79 \\
243.819\end{array}$ & 134.386 & 2.795 & 205.208 & 3.605 & 146.541 & 3.855 & 130.717 & 3.76 & 199.745 \\
\hline 0.96 & $\begin{array}{l}106.814 \\
3.775\end{array}$ & $\begin{array}{l}1.795 \\
225.13\end{array}$ & 136.582 & 2.8 & 205.208 & 3.62 & 165.609 & 3.87 & 130.717 & 3.775 & 212.717 \\
\hline 0.965 & $\begin{array}{l}107.025 \\
3.79\end{array}$ & $\begin{array}{l}1.805 \\
206.524\end{array}$ & 132.193 & 2.81 & 205.208 & 3.635 & 160.823 & 3.885 & 151.746 & 3.79 & 211.346 \\
\hline 0.975 & $\begin{array}{l}106.814 \\
3.81\end{array}$ & $\begin{array}{l}1.81 \\
223.724\end{array}$ & 129.202 & 2.815 & 205.208 & 3.65 & 172.811 & 3.9 & 157.495 & 3.81 & 199.745 \\
\hline 0.98 & $\begin{array}{l}105.289 \\
3.825\end{array}$ & $\begin{array}{l}1.82 \\
244.633\end{array}$ & 127.029 & 2.825 & 197.897 & 3.665 & 177.626 & 3.915 & 152.755 & 3.825 & 204.38 \\
\hline 0.99 & $\begin{array}{l}106.157 \\
3.84\end{array}$ & $\begin{array}{l}1.83 \\
233.006\end{array}$ & 129.202 & 2.835 & 199.066 & 3.685 & 180.037 & 3.935 & 143.314 & 3.84 & 204.38 \\
\hline 0.995 & $\begin{array}{l}109.436 \\
3.855\end{array}$ & $\begin{array}{l}1.835 \\
246.16\end{array}$ & 130.004 & 2.84 & 210.092 & 3.7 & 170.407 & 3.95 & 147.049 & 3.855 & 202.062 \\
\hline 1.005 & $\begin{array}{l}109.633 \\
3.87\end{array}$ & $\begin{array}{l}1.845 \\
250.843\end{array}$ & 132.193 & 2.85 & 188.179 & 3.715 & 180.037 & 3.965 & 147.049 & 3.87 & 185.887 \\
\hline 1.01 & $\begin{array}{l}109.633 \\
3.885\end{array}$ & $\begin{array}{l}1.85 \\
227.462\end{array}$ & 132.193 & 2.855 & 197.897 & 3.73 & 196.987 & 3.98 & 145.669 & 3.885 & 181.285 \\
\hline 1.02 & $\begin{array}{l}111.187 \\
3.9\end{array}$ & $\begin{array}{l}1.86 \\
218.141\end{array}$ & 131.378 & 2.865 & 188.179 & 3.745 & 184.868 & 3.995 & 167.014 & 3.9 & 195.117 \\
\hline 1.03 & $\begin{array}{l}111.187 \\
3.915\end{array}$ & $\begin{array}{l}1.865 \\
232.13\end{array}$ & 132.193 & 2.87 & 205.208 & 3.76 & 180.037 & 4.01 & 184.969 & 3.915 & 196.391 \\
\hline 1.035 & $\begin{array}{l}110.503 \\
3.935\end{array}$ & $\begin{array}{l}1.875 \\
243.819\end{array}$ & 136.582 & 2.88 & 173.676 & 3.775 & 160.823 & 4.025 & 179.017 & 3.935 & 183.585 \\
\hline 1.045 & $\begin{array}{l}111.187 \\
3.95\end{array}$ & $\begin{array}{l}1.88 \\
229.795\end{array}$ & 136.582 & 2.885 & 179.768 & 3.79 & 146.541 & 4.04 & 161.181 & 3.95 & 178.987 \\
\hline 1.05 & $\begin{array}{l}112.246 \\
3.965\end{array}$ & $\begin{array}{l}1.89 \\
215.815\end{array}$ & 135.74 & 2.895 & 205.208 & 3.81 & 160.823 & 4.055 & 151.746 & 3.965 & 185.887 \\
\hline 1.06 & $\begin{array}{l}112.064 \\
3.98\end{array}$ & $\begin{array}{l}1.9 \\
208.845\end{array}$ & 137.926 & 2.905 & 195.464 & 3.825 & 158.435 & 4.075 & 142.365 & 3.98 & 192.807 \\
\hline 1.065 & $\begin{array}{l}112.246 \\
3.995\end{array}$ & $\begin{array}{l}1.905 \\
208.845\end{array}$ & 138.782 & 2.91 & 195.464 & 3.84 & 161.877 & 4.09 & 144.705 & 3.995 & 183.585 \\
\hline 1.075 & $\begin{array}{l}112.246 \\
4.01\end{array}$ & $\begin{array}{l}1.915 \\
204.206\end{array}$ & 138.782 & 2.92 & 195.464 & 3.855 & 186.07 & 4.105 & 156.457 & 4.01 & 181.285 \\
\hline 1.08 & $\begin{array}{l}107.687 \\
4.025\end{array}$ & $\begin{array}{l}1.92 \\
214.463\end{array}$ & 138.782 & 2.925 & 195.464 & 3.87 & 190.941 & 4.12 & 170.663 & 4.025 & 188.191 \\
\hline 1.09 & $\begin{array}{l}112.246 \\
4.04\end{array}$ & $\begin{array}{l}1.93 \\
229.795\end{array}$ & 138.782 & 2.935 & 188.179 & 3.885 & 178.783 & 4.135 & 175.421 & 4.04 & 188.191 \\
\hline 1.1 & $\begin{array}{l}110.503 \\
4.055\end{array}$ & $\begin{array}{l}1.935 \\
227.462\end{array}$ & 143.19 & 2.94 & 193.033 & 3.9 & 171.52 & 4.15 & 161.181 & 4.055 & 187.104 \\
\hline 1.105 & $\begin{array}{l}112.064 \\
4.075\end{array}$ & $\begin{array}{l}1.945 \\
213.49\end{array}$ & 147.611 & 2.95 & 188.179 & 3.915 & 184.868 & 4.165 & 147.049 & 4.075 & 180.162 \\
\hline 1.115 & $\begin{array}{l}112.941 \\
4.09\end{array}$ & $\begin{array}{l}1.955 \\
206.524\end{array}$ & 152.044 & 2.96 & 200.332 & 3.935 & 190.941 & 4.18 & 140.028 & 4.09 & 180.162 \\
\hline 1.12 & $\begin{array}{l}112.941 \\
4.105\end{array}$ & $\begin{array}{l}1.96 \\
220.47\end{array}$ & 149.826 & 2.965 & 193.033 & 3.95 & 176.36 & 4.2 & 156.457 & 4.105 & 177.852 \\
\hline 1.13 & $\begin{array}{l}111.374 \\
4.12\end{array}$ & $\begin{array}{l}1.97 \\
214.844\end{array}$ & 151.107 & 2.975 & 195.464 & 3.965 & 157.073 & 4.215 & 177.804 & 4.12 & 174.399 \\
\hline 1.135 & $\begin{array}{l}112.941 \\
4.135\end{array}$ & $\begin{array}{l}1.975 \\
214.844\end{array}$ & 154.265 & 2.98 & 193.033 & 3.98 & 161.877 & 4.23 & 184.969 & 4.135 & 182.473 \\
\hline 1.145 & $\begin{array}{l}112.064 \\
4.15\end{array}$ & $\begin{array}{l}1.985 \\
226.552\end{array}$ & 154.265 & 2.99 & 212.536 & 3.995 & 159.473 & 4.245 & 175.421 & 4.15 & 195.117 \\
\hline 1.155 & $\begin{array}{l}112.064 \\
4.165\end{array}$ & $\begin{array}{l}1.99 \\
221.864\end{array}$ & 153.314 & 2.995 & 185.756 & 4.01 & 159.473 & 4.26 & 162.248 & 4.165 & 194.066 \\
\hline 1.16 & $\begin{array}{l}112.064 \\
4.18\end{array}$ & $\begin{array}{l}2 \\
220.47\end{array}$ & 153.314 & 3.005 & 180.916 & 4.025 & 164.283 & 4.275 & 148.027 & 4.18 & 181.285 \\
\hline 1.17 & $\begin{array}{l}112.246 \\
4.2\end{array}$ & $\begin{array}{l}2.005 \\
213.49\end{array}$ & 156.488 & 3.01 & 197.897 & 4.04 & 181.21 & 4.29 & 133.039 & 4.2 & 189.422 \\
\hline 1.175 & $\begin{array}{l}112.941 \\
4.215\end{array}$ & $\begin{array}{l}2.015 \\
213.49\end{array}$ & 156.488 & 3.02 & 202.769 & 4.055 & 176.36 & 4.305 & 128.398 & 4.215 & 196.391 \\
\hline 1.185 & $\begin{array}{l}113.119 \\
4.23\end{array}$ & $\begin{array}{l}2.025 \\
201.889\end{array}$ & 158.715 & 3.03 & 188.179 & 4.075 & 161.877 & 4.325 & 149.396 & 4.23 & 190.498 \\
\hline 1.19 & $\begin{array}{l}113.119 \\
4.245\end{array}$ & $\begin{array}{l}2.03 \\
201.889\end{array}$ & 157.737 & 3.035 & 185.756 & 4.09 & 166.692 & 4.34 & 154.1 & 4.245 & 191.743 \\
\hline 1.2 & $\begin{array}{l}113.119 \\
4.26\end{array}$ & $\begin{array}{l}2.04 \\
203.175\end{array}$ & 159.953 & 3.045 & 190.605 & 4.105 & 169.105 & 4.355 & 149.396 & 4.26 & 195.117 \\
\hline 1.205 & $\begin{array}{l}113.819 \\
4.275\end{array}$ & $\begin{array}{l}2.045 \\
204.206\end{array}$ & 163.176 & 3.05 & 180.916 & 4.12 & 176.36 & 4.37 & 144.705 & 4.275 & 196.391 \\
\hline
\end{tabular}




\begin{tabular}{|c|c|c|c|c|c|c|c|c|c|c|c|}
\hline 1.215 & $\begin{array}{l}114.697 \\
4.29\end{array}$ & $\begin{array}{l}2.055 \\
204.206\end{array}$ & 167.648 & 3.06 & 196.715 & 4.135 & 159.473 & 4.385 & 142.365 & 4.29 & 182.473 \\
\hline 1.225 & $\begin{array}{l}114.697 \\
4.305\end{array}$ & $\begin{array}{l}2.06 \\
201.889\end{array}$ & 164.392 & 3.065 & 159.273 & 4.15 & 157.073 & 4.4 & 144.705 & 4.305 & 170.939 \\
\hline 1.23 & $\begin{array}{l}113.294 \\
4.325\end{array}$ & $\begin{array}{l}2.07 \\
201.889\end{array}$ & 166.616 & 3.075 & 173.676 & 4.165 & 157.073 & 4.415 & 152.755 & 4.325 & 175.545 \\
\hline 1.24 & $\begin{array}{l}114.865 \\
4.34\end{array}$ & $\begin{array}{l}2.075 \\
197.262\end{array}$ & 169.887 & 3.08 & 189.385 & 4.18 & 149.892 & 4.43 & 165.916 & 4.34 & 174.399 \\
\hline 1.245 & $\begin{array}{l}116.615 \\
4.355\end{array}$ & $\begin{array}{l}2.085 \\
194.951\end{array}$ & 172.13 & 3.09 & 184.511 & 4.2 & 146.083 & 4.445 & 173.041 & 4.355 & 170.939 \\
\hline 1.255 & $\begin{array}{l}115.74 \\
4.37\end{array}$ & $\begin{array}{l}2.095 \\
201.889\end{array}$ & 174.374 & 3.1 & 174.793 & 4.215 & 146.083 & 4.465 & 170.663 & 4.37 & 177.852 \\
\hline 1.26 & $\begin{array}{l}116.456 \\
4.385\end{array}$ & $\begin{array}{l}2.1 \\
208.845\end{array}$ & 173.301 & 3.105 & 171.268 & 4.23 & 153.289 & 4.48 & 158.817 & 4.385 & 180.162 \\
\hline 1.27 & $\begin{array}{l}116.615 \\
4.4\end{array}$ & $\begin{array}{l}2.11 \\
207.837\end{array}$ & 173.301 & 3.115 & 188.179 & 4.245 & 153.289 & 4.495 & 152.755 & 4.4 & 178.987 \\
\hline 1.275 & $\begin{array}{l}116.615 \\
4.415\end{array}$ & $\begin{array}{l}2.115 \\
199.575\end{array}$ & 174.374 & 3.12 & 184.511 & 4.26 & 153.289 & 4.51 & 158.817 & 4.415 & 177.852 \\
\hline 1.285 & $\begin{array}{l}117.49 \\
4.43\end{array}$ & $\begin{array}{l}2.125 \\
199.575\end{array}$ & 174.374 & 3.13 & 172.37 & 4.275 & 153.289 & 4.525 & 156.457 & 4.43 & 180.162 \\
\hline 1.295 & $\begin{array}{l}116.615 \\
4.445\end{array}$ & $\begin{array}{l}2.13 \\
192.642\end{array}$ & 173.301 & 3.135 & 174.793 & 4.29 & 155.697 & 4.54 & 164.63 & 4.445 & 189.422 \\
\hline 1.3 & $\begin{array}{l}117.49 \\
4.465\end{array}$ & $\begin{array}{l}2.14 \\
181.129\end{array}$ & 173.301 & 3.145 & 174.793 & 4.305 & 146.083 & 4.555 & 174.183 & 4.465 & 196.391 \\
\hline 1.31 & $\begin{array}{l}116.771 \\
4.48\end{array}$ & $\begin{array}{l}2.15 \\
178.833\end{array}$ & 173.301 & 3.155 & 164.062 & 4.325 & 134.147 & 4.57 & 171.79 & 4.48 & 191.743 \\
\hline 1.315 & $\begin{array}{l}118.218 \\
4.495\end{array}$ & $\begin{array}{l}2.155 \\
191.551\end{array}$ & 173.301 & 3.16 & 189.385 & 4.34 & 119.952 & 4.59 & 164.63 & 4.495 & 194.066 \\
\hline 1.325 & $\begin{array}{l}117.49 \\
4.51\end{array}$ & $\begin{array}{l}2.165 \\
192.642\end{array}$ & 173.301 & 3.17 & 186.947 & 4.355 & 119.952 & 4.605 & 158.817 & 4.51 & 205.709 \\
\hline 1.33 & $\begin{array}{l}119.099 \\
4.525\end{array}$ & $\begin{array}{l}2.17 \\
191.551\end{array}$ & 173.301 & 3.175 & 212.536 & 4.37 & 124.667 & 4.62 & 161.181 & 4.525 & 173.241 \\
\hline 1.34 & $\begin{array}{l}119.099 \\
4.54\end{array}$ & $\begin{array}{l}2.18 \\
203.175\end{array}$ & 180.009 & 3.185 & 211.431 & 4.385 & 122.307 & 4.635 & 162.248 & 4.54 & 185.887 \\
\hline 1.35 & $\begin{array}{l}117.642 \\
4.555\end{array}$ & $\begin{array}{l}2.185 \\
198.519\end{array}$ & 181.122 & 3.19 & 191.826 & 4.4 & 112.911 & 4.65 & 158.817 & 4.555 & 191.743 \\
\hline 1.355 & $\begin{array}{l}118.218 \\
4.57\end{array}$ & $\begin{array}{l}2.195 \\
192.642\end{array}$ & 180.009 & 3.2 & 210.314 & 4.415 & 110.573 & 4.665 & 161.181 & 4.57 & 190.498 \\
\hline 1.365 & $\begin{array}{l}119.243 \\
4.59\end{array}$ & $\begin{array}{l}2.2 \\
192.642\end{array}$ & 177.771 & 3.205 & 174.793 & 4.43 & 110.573 & 4.68 & 156.457 & 4.59 & 189.422 \\
\hline 1.37 & $\begin{array}{l}119.243 \\
4.605\end{array}$ & $\begin{array}{l}2.21 \\
197.262\end{array}$ & 180.009 & 3.215 & 226.213 & 4.445 & 105.911 & 4.695 & 155.123 & 4.605 & 187.104 \\
\hline 1.38 & $\begin{array}{l}119.243 \\
4.62\end{array}$ & $\begin{array}{l}2.22 \\
203.175\end{array}$ & 180.009 & 3.225 & 143.537 & 4.465 & 103.587 & 4.715 & 152.755 & 4.62 & 187.104 \\
\hline 1.385 & $\begin{array}{l}119.243 \\
4.635\end{array}$ & $\begin{array}{l}2.225 \\
197.262\end{array}$ & 183.376 & 3.23 & 163.762 & 4.48 & 110.573 & 4.73 & 147.049 & 4.635 & 183.585 \\
\hline 1.395 & $\begin{array}{l}119.243 \\
4.65\end{array}$ & $\begin{array}{l}2.235 \\
190.335\end{array}$ & 183.376 & 3.24 & 172.37 & 4.495 & 110.573 & 4.745 & 137.695 & 4.65 & 195.117 \\
\hline 1.4 & $\begin{array}{l}119.243 \\
4.665\end{array}$ & $\begin{array}{l}2.24 \\
213.49\end{array}$ & 182.249 & 3.245 & 142.614 & 4.51 & 98.9526 & 4.76 & 137.695 & 4.665 & 196.391 \\
\hline 1.41 & $\begin{array}{l}119.243 \\
4.68\end{array}$ & $\begin{array}{l}2.25 \\
189.233\end{array}$ & 182.249 & 3.255 & 159.273 & 4.525 & 87.4537 & 4.775 & 133.929 & 4.68 & 209.022 \\
\hline 1.42 & $\begin{array}{l}123.635 \\
4.695\end{array}$ & $\begin{array}{l}2.255 \\
171.961\end{array}$ & 185.632 & 3.26 & 124.597 & 4.54 & 94.338 & 4.79 & 133.929 & 4.695 & 208.044 \\
\hline 1.425 & $\begin{array}{l}125.287 \\
4.715\end{array}$ & $\begin{array}{l}2.265 \\
190.335\end{array}$ & 187.891 & 3.27 & 145.921 & 4.555 & 94.338 & 4.805 & 126.084 & 4.715 & 201.046 \\
\hline 1.435 & $\begin{array}{l}125.287 \\
4.73\end{array}$ & $\begin{array}{l}2.27 \\
197.262\end{array}$ & 188.983 & 3.275 & 150.701 & 4.57 & 85.7561 & 4.82 & 138.614 & 4.73 & 198.717 \\
\hline 1.44 & $\begin{array}{l}124.516 \\
4.745\end{array}$ & $\begin{array}{l}2.28 \\
201.889\end{array}$ & 188.983 & 3.285 & 161.666 & 4.59 & 88.055 & 4.835 & 133.929 & 4.745 & 196.391 \\
\hline 1.45 & $\begin{array}{l}125.397 \\
4.76\end{array}$ & $\begin{array}{l}2.29 \\
203.175\end{array}$ & 192.413 & 3.295 & 179.646 & 4.605 & 97.3023 & 4.855 & 133.929 & 4.76 & 195.117 \\
\hline 1.455 & $\begin{array}{l}125.397 \\
4.775\end{array}$ & $\begin{array}{l}2.295 \\
207.837\end{array}$ & 192.413 & 3.3 & 189.385 & 4.62 & 88.055 & 4.87 & 121.467 & 4.775 & 196.391 \\
\hline 1.465 & $\begin{array}{l}123.753 \\
4.79\end{array}$ & $\begin{array}{l}2.305 \\
201.889\end{array}$ & 192.413 & 3.31 & 182.077 & 4.635 & 83.4625 & 4.885 & 124.605 & 4.79 & 188.191 \\
\hline 1.47 & $\begin{array}{l}125.287 \\
4.805\end{array}$ & $\begin{array}{l}2.31 \\
190.335\end{array}$ & 193.482 & 3.315 & 153.096 & 4.65 & 76.614 & 4.9 & 124.605 & 4.805 & 184.787 \\
\hline 1.48 & $\begin{array}{l}124.516 \\
4.82\end{array}$ & $\begin{array}{l}2.32 \\
193.872\end{array}$ & 193.482 & 3.325 & 134.036 & 4.665 & 90.3592 & 4.915 & 126.93 & 4.82 & 195.117 \\
\hline
\end{tabular}




\begin{tabular}{|c|c|c|c|c|c|c|c|c|c|c|c|}
\hline 1.49 & $\begin{array}{l}124.516 \\
4.835\end{array}$ & $\begin{array}{l}2.325 \\
188.03\end{array}$ & 194.678 & 3.33 & 141.156 & 4.68 & 90.3592 & 4.93 & 124.605 & 4.835 & 180.162 \\
\hline 1.495 & $\begin{array}{l}123.635 \\
4.855\end{array}$ & $\begin{array}{l}2.335 \\
190.335\end{array}$ & 196.944 & 3.34 & 127.781 & 4.695 & 88.055 & 4.945 & 126.084 & 4.855 & 184.787 \\
\hline 1.505 & $\begin{array}{l}123.635 \\
4.87\end{array}$ & $\begin{array}{l}2.345 \\
186.917\end{array}$ & 195.735 & 3.35 & 171.05 & 4.715 & 83.4625 & 4.96 & 124.605 & 4.87 & 191.743 \\
\hline 1.51 & $\begin{array}{l}123.635 \\
4.885\end{array}$ & $\begin{array}{l}2.35 \\
182.291\end{array}$ & 196.944 & 3.355 & 186.947 & 4.73 & 83.4625 & 4.98 & 119.164 & 4.885 & 195.117 \\
\hline 1.52 & $\begin{array}{l}121.877 \\
4.9\end{array}$ & $\begin{array}{l}2.36 \\
179.981\end{array}$ & 197.99 & 3.365 & 174.793 & 4.745 & 72.0761 & 4.995 & 115.345 & 4.9 & 192.807 \\
\hline 1.525 & $\begin{array}{l}122.756 \\
4.915\end{array}$ & $\begin{array}{l}2.365 \\
182.291\end{array}$ & 199.212 & 3.37 & 172.37 & 4.76 & 65.312 & 5.01 & 115.345 & 4.915 & 194.066 \\
\hline 1.535 & $\begin{array}{l}123.516 \\
4.93\end{array}$ & $\begin{array}{l}2.375 \\
184.602\end{array}$ & 199.212 & 3.38 & 168.617 & 4.775 & 73.0971 & 5.025 & 119.164 & 4.93 & 185.887 \\
\hline 1.545 & $\begin{array}{l}123.635 \\
4.945\end{array}$ & $\begin{array}{l}2.38 \\
188.03\end{array}$ & 197.99 & 3.385 & 167.533 & 4.79 & 73.0971 & 5.04 & 119.966 & 4.945 & 191.743 \\
\hline 1.55 & $\begin{array}{l}123.635 \\
4.96\end{array}$ & $\begin{array}{l}2.39 \\
193.872\end{array}$ & 201.482 & 3.395 & 172.37 & 4.805 & 70.8079 & 5.055 & 119.966 & 4.96 & 185.887 \\
\hline 1.56 & $\begin{array}{l}123.635 \\
4.98\end{array}$ & $\begin{array}{l}2.395 \\
194.951\end{array}$ & 199.212 & 3.4 & 168.617 & 4.82 & 75.3921 & 5.07 & 119.966 & 4.98 & 187.104 \\
\hline 1.565 & $\begin{array}{l}124.516 \\
4.995\end{array}$ & $\begin{array}{l}2.405 \\
197.262\end{array}$ & 199.212 & 3.41 & 150.701 & 4.835 & 63.9758 & 5.085 & 113.04 & 4.995 & 187.104 \\
\hline 1.575 & $\begin{array}{l}126.173 \\
5.01\end{array}$ & $\begin{array}{l}2.415 \\
196.195\end{array}$ & 199.212 & 3.42 & 158.92 & 4.855 & 68.5246 & 5.105 & 103.866 & 5.01 & 187.104 \\
\hline 1.58 & $\begin{array}{l}125.397 \\
5.025\end{array}$ & $\begin{array}{l}2.42 \\
191.551\end{array}$ & 197.99 & 3.425 & 153.096 & 4.87 & 75.3921 & 5.12 & 108.444 & 5.025 & 182.473 \\
\hline 1.59 & $\begin{array}{l}126.278 \\
5.04\end{array}$ & $\begin{array}{l}2.43 \\
193.872\end{array}$ & 197.99 & 3.435 & 124.597 & 4.885 & 73.0971 & 5.135 & 110.74 & 5.04 & 182.473 \\
\hline 1.595 & $\begin{array}{l}126.278 \\
5.055\end{array}$ & $\begin{array}{l}2.435 \\
196.195\end{array}$ & 201.482 & 3.44 & 132.53 & 4.9 & 70.8079 & 5.15 & 115.345 & 5.055 & 180.162 \\
\hline 1.605 & $\begin{array}{l}126.278 \\
5.07\end{array}$ & $\begin{array}{l}2.445 \\
197.262\end{array}$ & 196.944 & 3.45 & 134.911 & 4.915 & 70.8079 & 5.165 & 113.04 & 5.07 & 183.585 \\
\hline 1.615 & $\begin{array}{l}127.16 \\
5.085\end{array}$ & $\begin{array}{l}2.45 \\
196.195\end{array}$ & 194.678 & 3.455 & 138.779 & 4.93 & 63.9758 & 5.18 & 108.444 & 5.085 & 180.162 \\
\hline 1.62 & $\begin{array}{l}127.16 \\
5.105\end{array}$ & $\begin{array}{l}2.46 \\
197.262\end{array}$ & 193.482 & 3.465 & 154.09 & 4.945 & 61.7105 & 5.195 & 103.866 & 5.105 & 180.162 \\
\hline 1.63 & $\begin{array}{l}128.042 \\
5.12\end{array}$ & $\begin{array}{l}2.465 \\
198.519\end{array}$ & 192.413 & 3.47 & 158.92 & 4.96 & 52.7111 & 5.21 & 101.584 & 5.12 & 178.987 \\
\hline 1.635 & $\begin{array}{l}127.16 \\
5.135\end{array}$ & $\begin{array}{l}2.475 \\
194.951\end{array}$ & 192.413 & 3.48 & 163.762 & 4.98 & 53.1014 & 5.225 & 101.584 & 5.135 & 175.545 \\
\hline 1.645 & $\begin{array}{l}128.042 \\
5.15\end{array}$ & $\begin{array}{l}2.485 \\
199.575\end{array}$ & 190.151 & 3.49 & 168.617 & 4.995 & 50.8533 & 5.245 & 106.153 & 5.15 & 180.162 \\
\hline 1.65 & $\begin{array}{l}127.258 \\
5.165\end{array}$ & $\begin{array}{l}2.49 \\
203.175\end{array}$ & 190.151 & 3.495 & 149.274 & 5.01 & 50.8533 & 5.26 & 108.444 & 5.165 & 180.162 \\
\hline 1.66 & $\begin{array}{l}128.042 \\
5.18\end{array}$ & $\begin{array}{l}2.5 \\
205.505\end{array}$ & 187.891 & 3.505 & 124.597 & 5.025 & 55.356 & 5.275 & 104.575 & 5.18 & 180.162 \\
\hline 1.665 & $\begin{array}{l}128.926 \\
5.195\end{array}$ & $\begin{array}{l}2.505 \\
205.505\end{array}$ & 189.059 & 3.51 & 136.405 & 5.04 & 57.617 & 5.29 & 102.279 & 5.195 & 177.852 \\
\hline 1.675 & $\begin{array}{l}128.042 \\
5.21\end{array}$ & $\begin{array}{l}2.515 \\
207.837\end{array}$ & 190.151 & 3.52 & 134.911 & 5.055 & 62.1578 & 5.305 & 101.584 & 5.21 & 175.545 \\
\hline 1.685 & $\begin{array}{l}129.724 \\
5.225\end{array}$ & $\begin{array}{l}2.52 \\
206.524\end{array}$ & 186.736 & 3.525 & 143.537 & 5.07 & 62.1578 & 5.32 & 97.699 & 5.225 & 170.939 \\
\hline 1.69 & $\begin{array}{l}128.926 \\
5.245\end{array}$ & $\begin{array}{l}2.53 \\
204.206\end{array}$ & 187.891 & 3.535 & 132.53 & 5.085 & 58.0408 & 5.335 & 95.4164 & 5.245 & 166.343 \\
\hline 1.7 & $\begin{array}{l}129.724 \\
5.26\end{array}$ & $\begin{array}{l}2.54 \\
198.519\end{array}$ & 185.632 & 3.545 & 154.09 & 5.105 & 53.4965 & 5.35 & 102.279 & 5.26 & 160.697 \\
\hline 1.705 & $\begin{array}{l}129.809 \\
5.275\end{array}$ & $\begin{array}{l}2.545 \\
194.951\end{array}$ & 186.786 & 3.55 & 154.09 & 5.12 & 53.4965 & 5.37 & 104.575 & 5.275 & 164.049 \\
\hline 1.715 & $\begin{array}{l}129.809 \\
5.29\end{array}$ & $\begin{array}{l}2.555 \\
197.262\end{array}$ & 183.376 & 3.56 & 181.98 & 5.135 & 53.4965 & 5.385 & 101.584 & 5.29 & 166.343 \\
\hline 1.72 & $\begin{array}{l}128.926 \\
5.305\end{array}$ & $\begin{array}{l}2.56 \\
194.951\end{array}$ & 181.122 & 3.565 & 175.922 & 5.15 & 53.4965 & 5.4 & 97.699 & 5.305 & 164.049 \\
\hline 1.73 & $\begin{array}{l}130.693 \\
5.32\end{array}$ & $\begin{array}{l}2.57 \\
196.195\end{array}$ & 178.871 & 3.575 & 174.793 & 5.165 & 48.9784 & 5.415 & 93.1387 & 5.32 & 158.423 \\
\hline 1.74 & $\begin{array}{l}130.693 \\
5.335\end{array}$ & $\begin{array}{l}2.575 \\
198.519\end{array}$ & 178.871 & 3.58 & 180.806 & 5.18 & 51.6196 & 5.43 & 93.1387 & 5.335 & 157.184 \\
\hline 1.745 & $\begin{array}{l}131.578 \\
5.35\end{array}$ & $\begin{array}{l}2.585 \\
193.872\end{array}$ & 174.374 & 3.59 & 183.252 & 5.195 & 49.3494 & 5.445 & 95.4164 & 5.35 & 154.902 \\
\hline 1.755 & $\begin{array}{l}132.394 \\
5.37\end{array}$ & $\begin{array}{l}2.59 \\
188.03\end{array}$ & 174.374 & 3.595 & 175.922 & 5.21 & 42.5796 & 5.46 & 97.699 & 5.37 & 151.617 \\
\hline
\end{tabular}




\begin{tabular}{|c|c|c|c|c|c|c|c|c|c|c|c|}
\hline 1.76 & $\begin{array}{l}131.578 \\
5.385\end{array}$ & $\begin{array}{l}2.6 \\
185.728\end{array}$ & 174.374 & 3.605 & 162.708 & 5.225 & 44.8294 & 5.475 & 93.1387 & 5.385 & 151.617 \\
\hline 1.77 & $\begin{array}{l}133.35 \\
5.4\end{array}$ & $\begin{array}{l}2.61 \\
185.728\end{array}$ & 173.301 & 3.615 & 146.871 & 5.245 & 49.3494 & 5.495 & 89.2136 & 5.4 & 157.184 \\
\hline 1.775 & $\begin{array}{l}132.394 \\
5.415\end{array}$ & $\begin{array}{l}2.615 \\
183.427\end{array}$ & 175.46 & 3.62 & 137.295 & 5.26 & 47.086 & 5.51 & 88.598 & 5.415 & 154.902 \\
\hline 1.785 & $\begin{array}{l}133.35 \\
5.43\end{array}$ & $\begin{array}{l}2.625 \\
184.602\end{array}$ & 174.374 & 3.63 & 137.295 & 5.275 & 44.8294 & 5.525 & 88.598 & 5.43 & 152.622 \\
\hline 1.79 & $\begin{array}{l}133.35 \\
5.445\end{array}$ & $\begin{array}{l}2.63 \\
189.233\end{array}$ & 174.374 & 3.635 & 136.405 & 5.29 & 44.8294 & 5.54 & 88.598 & 5.445 & 150.346 \\
\hline 1.8 & $\begin{array}{l}134.176 \\
5.46\end{array}$ & $\begin{array}{l}2.64 \\
186.917\end{array}$ & 174.374 & 3.645 & 141.156 & 5.305 & 47.086 & 5.555 & 89.2136 & 5.46 & 149.355 \\
\hline 1.81 & $\begin{array}{l}134.236 \\
5.475\end{array}$ & $\begin{array}{l}2.645 \\
184.602\end{array}$ & 174.374 & 3.65 & 154.09 & 5.32 & 47.086 & 5.57 & 91.4959 & 5.475 & 150.346 \\
\hline 1.815 & $\begin{array}{l}133.35 \\
5.495\end{array}$ & $\begin{array}{l}2.655 \\
183.427\end{array}$ & 174.374 & 3.66 & 158.92 & 5.335 & 47.086 & 5.585 & 90.8658 & 5.495 & 150.346 \\
\hline 1.825 & $\begin{array}{l}135.96 \\
5.51\end{array}$ & $\begin{array}{l}2.66 \\
189.233\end{array}$ & 172.13 & 3.665 & 150.701 & 5.35 & 47.086 & 5.6 & 89.2136 & 5.51 & 152.622 \\
\hline 1.83 & $\begin{array}{l}133.414 \\
5.525\end{array}$ & $\begin{array}{l}2.67 \\
188.03\end{array}$ & 174.374 & 3.675 & 145.921 & 5.37 & 44.8294 & 5.615 & 86.9364 & 5.525 & 154.902 \\
\hline 1.84 & $\begin{array}{l}135.123 \\
5.54\end{array}$ & $\begin{array}{l}2.68 \\
189.233\end{array}$ & 174.374 & 3.685 & 142.614 & 5.385 & 42.5796 & 5.635 & 86.9364 & 5.54 & 152.622 \\
\hline 1.845 & $\begin{array}{l}135.123 \\
5.555\end{array}$ & $\begin{array}{l}2.685 \\
188.03\end{array}$ & 174.374 & 3.69 & 139.683 & 5.4 & 38.1011 & 5.65 & 89.2136 & 5.555 & 150.346 \\
\hline 1.855 & $\begin{array}{l}136.853 \\
5.57\end{array}$ & $\begin{array}{l}2.695 \\
189.233\end{array}$ & 172.13 & 3.7 & 146.871 & 5.415 & 38.1011 & 5.665 & 86.9364 & 5.57 & 152.622 \\
\hline 1.86 & $\begin{array}{l}136.898 \\
5.585\end{array}$ & $\begin{array}{l}2.7 \\
186.917\end{array}$ & 167.648 & 3.705 & 126.95 & 5.43 & 38.1011 & 5.68 & 84.6643 & 5.585 & 154.902 \\
\hline 1.87 & $\begin{array}{l}137.747 \\
5.6\end{array}$ & $\begin{array}{l}2.71 \\
184.602\end{array}$ & 167.648 & 3.715 & 145.921 & 5.445 & 38.405 & 5.695 & 84.6643 & 5.6 & 154.902 \\
\hline 1.88 & $\begin{array}{l}137.747 \\
5.615\end{array}$ & $\begin{array}{l}2.715 \\
183.427\end{array}$ & 165.41 & 3.72 & 165.119 & 5.46 & 33.9266 & 5.71 & 82.3974 & 5.615 & 152.622 \\
\hline 1.885 & $\begin{array}{l}137.787 \\
5.635\end{array}$ & $\begin{array}{l}2.725 \\
182.291\end{array}$ & 158.715 & 3.73 & 141.156 & 5.475 & 33.6509 & 5.725 & 82.3974 & 5.635 & 152.622 \\
\hline 1.895 & $\begin{array}{l}137.747 \\
5.65\end{array}$ & $\begin{array}{l}2.735 \\
181.129\end{array}$ & 156.488 & 3.74 & 153.096 & 5.495 & 36.1622 & 5.74 & 82.3974 & 5.65 & 150.346 \\
\hline 1.9 & $\begin{array}{l}137.787 \\
5.665\end{array}$ & $\begin{array}{l}2.74 \\
178.833\end{array}$ & 155.524 & 3.745 & 155.494 & 5.51 & 36.1622 & 5.76 & 82.3974 & 5.665 & 145.802 \\
\hline 1.91 & $\begin{array}{l}138.676 \\
5.68\end{array}$ & $\begin{array}{l}2.75 \\
176.54\end{array}$ & 152.044 & 3.755 & 150.701 & 5.525 & 33.9266 & 5.775 & 85.2582 & 5.68 & 145.802 \\
\hline 1.915 & $\begin{array}{l}137.787 \\
5.695\end{array}$ & $\begin{array}{l}2.755 \\
177.674\end{array}$ & 147.611 & 3.76 & 143.537 & 5.54 & 31.6983 & 5.79 & 80.1358 & 5.695 & 143.535 \\
\hline 1.925 & $\begin{array}{l}138.676 \\
5.71\end{array}$ & $\begin{array}{l}2.765 \\
171.961\end{array}$ & 147.611 & 3.77 & 155.494 & 5.555 & 31.9632 & 5.805 & 77.8795 & 5.71 & 143.535 \\
\hline 1.935 & $\begin{array}{l}139.536 \\
5.725\end{array}$ & $\begin{array}{l}2.77 \\
169.675\end{array}$ & 145.399 & 3.775 & 148.309 & 5.57 & 29.7283 & 5.82 & 78.43 & 5.725 & 143.535 \\
\hline 1.94 & $\begin{array}{l}139.566 \\
5.74\end{array}$ & $\begin{array}{l}2.78 \\
170.767\end{array}$ & 143.19 & 3.785 & 148.309 & 5.585 & 27.5009 & 5.835 & 76.1647 & 5.74 & 143.535 \\
\hline 1.95 & $\begin{array}{l}139.566 \\
5.76\end{array}$ & $\begin{array}{l}2.785 \\
170.767\end{array}$ & 146.306 & 3.79 & 157.895 & 5.6 & 27.5009 & 5.85 & 76.1647 & 5.76 & 140.337 \\
\hline 1.955 & $\begin{array}{l}139.566 \\
5.775\end{array}$ & $\begin{array}{l}2.795 \\
170.767\end{array}$ & 148.532 & 3.8 & 163.762 & 5.615 & 27.5009 & 5.865 & 73.9049 & 5.775 & 139.011 \\
\hline 1.965 & $\begin{array}{l}140.456 \\
5.79\end{array}$ & $\begin{array}{l}2.805 \\
170.767\end{array}$ & 147.611 & 3.81 & 161.339 & 5.635 & 27.5009 & 5.885 & 76.1647 & 5.79 & 139.011 \\
\hline 1.97 & $\begin{array}{l}140.456 \\
5.805\end{array}$ & $\begin{array}{l}2.81 \\
169.675\end{array}$ & 149.826 & 3.815 & 167.533 & 5.65 & 20.8646 & 5.9 & 76.1647 & 5.805 & 139.011 \\
\hline 1.98 & $\begin{array}{l}140.456 \\
5.82\end{array}$ & $\begin{array}{l}2.82 \\
168.47\end{array}$ & 146.701 & 3.825 & 165.119 & 5.665 & 18.6682 & 5.915 & 76.7073 & 5.82 & 140.337 \\
\hline 1.985 & $\begin{array}{l}141.347 \\
5.835\end{array}$ & $\begin{array}{l}2.825 \\
165.111\end{array}$ & 145.399 & 3.83 & 158.92 & 5.68 & 18.6682 & 5.93 & 78.9872 & 5.835 & 141.272 \\
\hline 1.995 & $\begin{array}{l}140.456 \\
5.85\end{array}$ & $\begin{array}{l}2.835 \\
167.391\end{array}$ & 158.715 & 3.84 & 158.92 & 5.695 & 23.069 & 5.945 & 80.7007 & 5.85 & 141.272 \\
\hline 2.005 & $\begin{array}{l}142.223 \\
5.865\end{array}$ & $\begin{array}{l}2.84 \\
165.111\end{array}$ & 153.314 & 3.845 & 157.895 & 5.71 & 23.069 & 5.96 & 85.2582 & 5.865 & 141.272 \\
\hline 2.01 & $\begin{array}{l}142.238 \\
5.885\end{array}$ & $\begin{array}{l}2.85 \\
163.884\end{array}$ & 156.488 & 3.855 & 150.701 & 5.725 & 27.5009 & 5.975 & 85.2582 & 5.885 & 143.535 \\
\hline 2.02 & $\begin{array}{l}142.238 \\
5.9\end{array}$ & $\begin{array}{l}2.855 \\
166.176\end{array}$ & 133.018 & 3.86 & 146.871 & 5.74 & 27.5009 & 5.99 & 85.8595 & 5.9 & 142.586 \\
\hline 2.025 & $\begin{array}{l}142.223 \\
5.915\end{array}$ & $\begin{array}{l}2.865 \\
163.884\end{array}$ & 172.13 & 3.87 & 146.871 & 5.76 & 27.7406 & 6.005 & 85.8595 & 5.915 & 143.535 \\
\hline
\end{tabular}




\begin{tabular}{|c|c|c|c|c|c|c|c|c|c|c|c|}
\hline 2.035 & $\begin{array}{l}143.119 \\
5.93\end{array}$ & $\begin{array}{l}2.875 \\
161.595\end{array}$ & 174.374 & 3.88 & 123.048 & 5.775 & 25.5067 & 6.025 & 83.5633 & 5.93 & 143.535 \\
\hline 2.04 & $\begin{array}{l}142.238 \\
5.945\end{array}$ & $\begin{array}{l}2.88 \\
160.558\end{array}$ & 144.503 & 3.885 & 119.902 & 5.79 & 27.7406 & 6.04 & 83.5633 & 5.945 & 143.535 \\
\hline 2.05 & $\begin{array}{l}144.017 \\
5.96\end{array}$ & $\begin{array}{l}2.89 \\
160.558\end{array}$ & 160.944 & 3.895 & 129.308 & 5.805 & 27.7406 & 6.055 & 83.5633 & 5.96 & 141.272 \\
\hline 2.055 & $\begin{array}{l}143.129 \\
5.975\end{array}$ & $\begin{array}{l}2.895 \\
160.558\end{array}$ & 138.782 & 3.9 & 137.295 & 5.82 & 27.9833 & 6.07 & 83.5633 & 5.975 & 141.272 \\
\hline 2.065 & $\begin{array}{l}143.129 \\
5.99\end{array}$ & $\begin{array}{l}2.905 \\
161.595\end{array}$ & 139.648 & 3.91 & 156.503 & 5.835 & 25.7351 & 6.085 & 88.1609 & 5.99 & 140.337 \\
\hline 2.075 & $\begin{array}{l}143.129 \\
6.005\end{array}$ & $\begin{array}{l}2.91 \\
160.558\end{array}$ & 153.314 & 3.915 & 157.895 & 5.85 & 21.2624 & 6.1 & 85.8595 & 6.005 & 141.272 \\
\hline 2.08 & $\begin{array}{l}144.022 \\
6.025\end{array}$ & $\begin{array}{l}2.92 \\
156.016\end{array}$ & 119.109 & 3.925 & 158.92 & 5.865 & 21.2624 & 6.115 & 85.8595 & 6.025 & 141.272 \\
\hline 2.09 & $\begin{array}{l}144.022 \\
6.04\end{array}$ & $\begin{array}{l}2.93 \\
157.026\end{array}$ & 127.818 & 3.935 & 146.871 & 5.885 & 25.7351 & 6.13 & 83.5633 & 6.04 & 140.337 \\
\hline 2.095 & $\begin{array}{l}144.022 \\
6.055\end{array}$ & $\begin{array}{l}2.935 \\
152.468\end{array}$ & 138.782 & 3.94 & 137.295 & 5.9 & 25.7351 & 6.15 & 83.5633 & 6.055 & 139.011 \\
\hline 2.105 & $\begin{array}{l}144.914 \\
6.07\end{array}$ & $\begin{array}{l}2.945 \\
150.194\end{array}$ & 112.615 & 3.95 & 131.67 & 5.915 & 30.2392 & 6.165 & 83.5633 & 6.07 & 138.09 \\
\hline 2.11 & $\begin{array}{l}144.914 \\
6.085\end{array}$ & $\begin{array}{l}2.95 \\
145.654\end{array}$ & 114.067 & 3.955 & 129.308 & 5.93 & 25.7351 & 6.18 & 76.7073 & 6.085 & 139.011 \\
\hline 2.12 & $\begin{array}{l}144.914 \\
6.1\end{array}$ & $\begin{array}{l}2.96 \\
143.389\end{array}$ & 114.776 & 3.965 & 137.295 & 5.945 & 25.7351 & 6.195 & 68.135 & 6.1 & 135.847 \\
\hline 2.13 & $\begin{array}{l}145.808 \\
6.115\end{array}$ & $\begin{array}{l}2.965 \\
140.214\end{array}$ & 123.456 & 3.97 & 143.537 & 5.96 & 21.2624 & 6.21 & 52.0108 & 6.115 & 134.501 \\
\hline 2.135 & $\begin{array}{l}148.51 \\
6.13\end{array}$ & $\begin{array}{l}2.975 \\
140.214\end{array}$ & 104.661 & 3.98 & 149.274 & 5.975 & 21.2624 & 6.225 & 41.0314 & 6.13 & 132.25 \\
\hline 2.145 & $\begin{array}{l}147.595 \\
6.15\end{array}$ & $\begin{array}{l}2.98 \\
141.128\end{array}$ & 101.239 & 3.985 & 143.537 & 5.99 & 21.4651 & 6.24 & 30.2175 & 6.15 & 126.91 \\
\hline 2.15 & $\begin{array}{l}147.595 \\
6.165\end{array}$ & $\begin{array}{l}2.99 \\
144.713\end{array}$ & 104.01 & 3.995 & 145.921 & 6.005 & 19.2265 & 6.255 & 23.8121 & 6.165 & 125.521 \\
\hline 2.16 & $\begin{array}{l}147.595 \\
6.18\end{array}$ & $\begin{array}{l}3 \\
147.922\end{array}$ & 92.7679 & 4.005 & 145.921 & 6.025 & 19.2265 & & & 6.18 & 122.462 \\
\hline 2.165 & $\begin{array}{l}148.49 \\
6.195\end{array}$ & $\begin{array}{l}3.005 \\
157.026\end{array}$ & 99.732 & 4.01 & 145.921 & 6.04 & 16.9962 & & & 6.195 & 123.286 \\
\hline 2.175 & $\begin{array}{l}149.385 \\
6.21\end{array}$ & $\begin{array}{l}3.015 \\
160.558\end{array}$ & 89.1092 & 4.02 & 150.701 & 6.055 & 14.7741 & & & 6.21 & 122.462 \\
\hline 2.18 & $\begin{array}{l}149.385 \\
6.225\end{array}$ & $\begin{array}{l}3.02 \\
160.558\end{array}$ & 88.5574 & 4.025 & 155.494 & 6.07 & 14.7741 & & & 6.225 & 123.286 \\
\hline 2.19 & $\begin{array}{l}150.281 \\
6.24\end{array}$ & $\begin{array}{l}3.03 \\
145.654\end{array}$ & 88.5574 & 4.035 & 155.494 & 6.085 & 14.7741 & & & 6.24 & 122.462 \\
\hline 2.2 & $\begin{array}{l}150.281 \\
6.255\end{array}$ & $\begin{array}{l}3.035 \\
115.717\end{array}$ & 80.6894 & 4.04 & 150.701 & 6.1 & 14.7741 & & & 6.255 & 130.004 \\
\hline 2.205 & $\begin{array}{l}151.177 \\
6.275\end{array}$ & $\begin{array}{l}3.045 \\
126.798\end{array}$ & 66.1329 & 4.05 & 136.405 & 6.115 & 14.7741 & & & 6.275 & 123.286 \\
\hline 2.215 & $\begin{array}{l}153.016 \\
6.29\end{array}$ & $\begin{array}{l}3.05 \\
135.728\end{array}$ & 70.268 & 4.055 & 126.95 & 6.13 & 14.7741 & & & 6.29 & 121.054 \\
\hline 2.22 & $\begin{array}{l}152.074 \\
6.305\end{array}$ & $\begin{array}{l}3.06 \\
140.214\end{array}$ & 69.8306 & 4.065 & 119.902 & 6.15 & 14.7741 & & & 6.305 & 120.244 \\
\hline 2.23 & $\begin{array}{l}153.919 \\
6.32\end{array}$ & $\begin{array}{l}3.07 \\
140.214\end{array}$ & 45.4713 & 4.075 & 119.125 & 6.165 & 14.7741 & & & 6.32 & 121.054 \\
\hline 2.235 & $\begin{array}{l}152.074 \\
6.335\end{array}$ & $\begin{array}{l}3.075 \\
140.214\end{array}$ & 66.1329 & 4.08 & 120.687 & 6.18 & 16.9962 & & & 6.335 & 121.054 \\
\hline 2.245 & $\begin{array}{l}154.822 \\
6.35\end{array}$ & $\begin{array}{l}3.085 \\
140.214\end{array}$ & 35.777 & 4.09 & 119.902 & 6.195 & 19.2265 & & & 6.35 & 122.462 \\
\hline 2.25 & $\begin{array}{l}153.868 \\
6.365\end{array}$ & $\begin{array}{l}3.09 \\
140.214\end{array}$ & 23.8215 & 4.095 & 129.308 & 6.21 & 21.4651 & & & 6.365 & 123.286 \\
\hline 2.26 & $\begin{array}{l}153.868 \\
6.38\end{array}$ & $\begin{array}{l}3.1 \\
144.713\end{array}$ & 16.1236 & 4.105 & 122.247 & 6.225 & 21.4651 & & & 6.38 & 122.462 \\
\hline 2.27 & $\begin{array}{l}154.766 \\
6.395\end{array}$ & $\begin{array}{l}3.105 \\
140.214\end{array}$ & 18.1676 & 4.11 & 124.597 & 6.24 & 21.4651 & & & 6.395 & 126.91 \\
\hline 2.275 & $\begin{array}{l}156.63 \\
6.415\end{array}$ & $\begin{array}{l}3.115 \\
140.214\end{array}$ & 6.70262 & 4.12 & 119.902 & 6.255 & 23.7117 & & & 6.415 & 127.761 \\
\hline 2.285 & $\begin{array}{l}156.564 \\
6.43\end{array}$ & $\begin{array}{l}3.125 \\
142.462\end{array}$ & 21.888 & 4.13 & 122.247 & 6.275 & 21.4651 & & & 6.43 & 127.761 \\
\hline 2.29 & $\begin{array}{l}156.564 \\
6.445\end{array}$ & $\begin{array}{l}3.13 \\
140.214\end{array}$ & 16.1236 & 4.135 & 117.56 & 6.29 & 19.2265 & & & 6.445 & 120.244 \\
\hline 2.3 & $\begin{array}{l}157.463 \\
6.46\end{array}$ & $\begin{array}{l}3.14 \\
126.798\end{array}$ & 12.3112 & 4.145 & 117.56 & 6.305 & 19.2265 & & & 6.46 & 124.684 \\
\hline
\end{tabular}




\begin{tabular}{|c|c|c|c|c|c|c|c|c|c|}
\hline 2.305 & 158.439 & 3.145 & 18.1676 & 4.15 & 112.891 & 6.32 & 16.9962 & 6.475 & 127.761 \\
\hline 2.315 & 158.363 & 3.155 & 8.5976 & 4.16 & 108.952 & 6.335 & 19.4173 & 6.49 & 128.284 \\
\hline 2.325 & 158.363 & 3.16 & -4.52986 & 4.165 & 108.239 & 6.35 & 16.9962 & 6.505 & 124.684 \\
\hline 2.33 & 158.363 & 3.17 & -17.2558 & 4.175 & 110.563 & 6.365 & 17.1727 & 6.52 & 124.684 \\
\hline 2.34 & 159.263 & 3.175 & -19.2066 & 4.18 & 111.29 & 6.38 & 17.1727 & 6.54 & 120.244 \\
\hline 2.345 & 160.164 & & & 4.19 & 120.687 & 6.395 & 14.9364 & 6.555 & 115.819 \\
\hline 2.355 & 160.164 & & & 4.2 & 134.911 & 6.415 & 12.7085 & 6.57 & 111.41 \\
\hline 2.36 & 161.157 & & & 4.205 & 136.405 & 6.43 & 10.4892 & 6.585 & 112.165 \\
\hline 2.37 & 161.967 & & & 4.215 & 134.036 & 6.445 & 8.27851 & 6.6 & 107.017 \\
\hline 2.375 & 162.971 & & & 4.22 & 134.911 & 6.46 & 6.07647 & 6.615 & 104.827 \\
\hline 2.385 & 159.263 & & & 4.23 & 134.911 & 6.475 & 3.88318 & 6.63 & 109.212 \\
\hline 2.395 & 161.967 & & & 4.235 & 132.53 & 6.49 & 3.97719 & 6.645 & 112.165 \\
\hline 2.4 & 162.869 & & & 4.245 & 132.53 & 6.505 & 3.97719 & 6.665 & 114.381 \\
\hline 2.41 & 162.869 & & & 4.25 & 130.153 & 6.52 & -0.410188 & 6.68 & 111.41 \\
\hline 2.415 & 163.879 & & & 4.26 & 121.483 & 6.54 & -0.410188 & 6.695 & 109.212 \\
\hline 2.425 & 163.879 & & & 4.27 & 113.632 & 6.555 & -0.410188 & 6.71 & 107.017 \\
\hline 2.43 & 163.772 & & & 4.275 & 111.29 & 6.57 & -0.410188 & 6.725 & 107.744 \\
\hline 2.44 & 165.695 & & & 4.285 & 111.29 & 6.585 & -2.59026 & 6.74 & 104.827 \\
\hline 2.445 & 164.675 & & & 4.29 & 115.98 & 6.6 & -4.76115 & 6.755 & 98.953 \\
\hline 2.455 & 165.578 & & & 4.3 & 121.483 & 6.615 & -0.410188 & 6.77 & 88.0627 \\
\hline 2.465 & 166.482 & & & 4.305 & 123.048 & 6.63 & 3.97719 & 6.785 & 81.5836 \\
\hline 2.47 & 166.604 & & & 4.315 & 123.048 & 6.645 & 3.97719 & 6.805 & 81.5836 \\
\hline 2.48 & 167.514 & & & 4.325 & 115.98 & 6.665 & 3.97719 & & \\
\hline 2.485 & 168.423 & & & 4.33 & 108.952 & 6.68 & 6.18434 & & \\
\hline 2.495 & 168.423 & & & 4.34 & 100.307 & 6.695 & 15.2672 & & \\
\hline 2.5 & 169.334 & & & 4.345 & 97.3306 & 6.71 & 17.3514 & & \\
\hline 2.51 & 168.291 & & & 4.355 & 97.9782 & 6.725 & 17.3514 & & \\
\hline 2.52 & 171.155 & & & 4.36 & 97.9782 & 6.74 & 17.3514 & & \\
\hline 2.525 & 171.155 & & & 4.37 & 100.307 & 6.755 & 15.1007 & & \\
\hline 2.535 & 171.155 & & & 4.375 & 99.6452 & 6.77 & 13.0106 & & \\
\hline 2.54 & 172.067 & & & 4.385 & 104.289 & 6.785 & 8.52372 & & \\
\hline 2.55 & 172.979 & & & 4.395 & 101.965 & 6.805 & 4.07238 & & \\
\hline 2.555 & 171.155 & & & 4.4 & 104.98 & 6.82 & -0.342757 & & \\
\hline 2.565 & 172.979 & & & 4.41 & 113.632 & 6.835 & -23.9493 & & \\
\hline 2.57 & 171.913 & & & 4.415 & 115.98 & & & & \\
\hline
\end{tabular}




\begin{tabular}{|c|c|c|c|}
\hline 2.58 & 172.979 & 4.425 & 115.98 \\
\hline 2.59 & 173.891 & 4.43 & 116.745 \\
\hline 2.595 & 172.979 & 4.44 & 114.383 \\
\hline 2.605 & 173.891 & 4.445 & 113.632 \\
\hline 2.61 & 174.804 & 4.455 & 111.29 \\
\hline 2.62 & 172.979 & 4.465 & 109.673 \\
\hline 2.625 & 173.891 & 4.47 & 112.026 \\
\hline 2.635 & 174.057 & 4.48 & 114.383 \\
\hline 2.64 & 174.057 & 4.485 & 114.383 \\
\hline 2.65 & 173.891 & 4.495 & 128.621 \\
\hline 2.66 & 174.975 & 4.5 & 97.9782 \\
\hline 2.665 & 175.893 & 4.51 & 109.673 \\
\hline 2.675 & 176.812 & 4.52 & 108.039 \\
\hline 2.68 & 175.893 & 4.525 & 99.6452 \\
\hline 2.69 & 175.717 & 4.535 & 93.3347 \\
\hline 2.695 & 179.77 & 4.54 & 100.307 \\
\hline 2.705 & 178.65 & 4.55 & 93.3347 \\
\hline 2.715 & 179.77 & 4.555 & 84.1094 \\
\hline 2.72 & 180.696 & 4.565 & 86.4079 \\
\hline 2.73 & 182.548 & 4.57 & 89.3083 \\
\hline 2.735 & 182.548 & 4.58 & 93.9607 \\
\hline 2.745 & 182.766 & 4.59 & 93.3347 \\
\hline 2.75 & 179.57 & 4.595 & 93.3347 \\
\hline 2.76 & 180.903 & 4.605 & 97.9782 \\
\hline 2.765 & 179.77 & 4.61 & 93.9607 \\
\hline 2.775 & 180.903 & 4.62 & 93.9607 \\
\hline 2.785 & 179.041 & 4.625 & 88.7116 \\
\hline 2.79 & 179.24 & 4.635 & 84.6769 \\
\hline 2.8 & 182.05 & 4.64 & 91.6319 \\
\hline 2.805 & 188.875 & 4.65 & 84.6769 \\
\hline 2.815 & 127.457 & 4.66 & 100.978 \\
\hline 2.82 & 153.301 & 4.665 & 108.039 \\
\hline 2.83 & 203.877 & 4.675 & 103.327 \\
\hline 2.835 & 169.841 & 4.68 & 96.2946 \\
\hline 2.845 & 206.583 & 4.69 & 98.6336 \\
\hline
\end{tabular}




\begin{tabular}{|c|c|c|c|}
\hline 2.855 & 158.128 & 4.695 & 110.402 \\
\hline 2.86 & 222.772 & 4.705 & 98.6336 \\
\hline 2.87 & 66.3619 & 4.715 & 100.978 \\
\hline 2.875 & 42.137 & 4.72 & 101.656 \\
\hline 2.885 & 52.8784 & 4.73 & 98.6336 \\
\hline 2.89 & 36.287 & 4.735 & 91.6319 \\
\hline \multirow[t]{29}{*}{2.9} & -28.7701 & 4.745 & 91.6319 \\
\hline & & 4.75 & 89.9122 \\
\hline & & 4.76 & 94.5943 \\
\hline & & 4.765 & 100.978 \\
\hline & & 4.775 & 93.9607 \\
\hline & & 4.785 & 99.297 \\
\hline & & 4.79 & 94.5943 \\
\hline & & 4.8 & 89.9122 \\
\hline & & 4.805 & 92.2506 \\
\hline & & 4.815 & 96.9431 \\
\hline & & 4.82 & 96.9431 \\
\hline & & 4.83 & 89.9122 \\
\hline & & 4.835 & 96.9431 \\
\hline & & 4.845 & 96.9431 \\
\hline & & 4.855 & 94.5943 \\
\hline & & 4.86 & 96.9431 \\
\hline & & 4.87 & 101.656 \\
\hline & & 4.875 & 104.02 \\
\hline & & 4.885 & 99.297 \\
\hline & & 4.89 & 96.9431 \\
\hline & & 4.9 & 95.2356 \\
\hline & & 4.91 & 92.2506 \\
\hline & & 4.915 & 89.9122 \\
\hline & & 4.925 & 92.2506 \\
\hline & & 4.93 & 95.2356 \\
\hline & & 4.94 & 95.2356 \\
\hline & & 4.945 & 99.297 \\
\hline & & 4.955 & 92.2506 \\
\hline & & 4.96 & 86.4213 \\
\hline
\end{tabular}




\begin{tabular}{|c|c|}
\hline 4.97 & 90.5235 \\
\hline 4.98 & 86.4213 \\
\hline 4.985 & 84.0692 \\
\hline 4.995 & 83.4956 \\
\hline 5 & 81.7226 \\
\hline 5.01 & 77.0465 \\
\hline 5.015 & 76.5179 \\
\hline 5.025 & 81.7226 \\
\hline 5.03 & 81.7226 \\
\hline 5.04 & 84.0692 \\
\hline 5.05 & 88.7791 \\
\hline 5.055 & 88.7791 \\
\hline 5.065 & 91.1423 \\
\hline 5.07 & 91.1423 \\
\hline 5.08 & 87.0172 \\
\hline 5.085 & 86.4213 \\
\hline 5.095 & 86.4213 \\
\hline 5.105 & 86.4213 \\
\hline 5.11 & 84.6498 \\
\hline 5.12 & 84.6498 \\
\hline 5.125 & 84.0692 \\
\hline 5.135 & 84.0692 \\
\hline 5.14 & 85.2376 \\
\hline 5.15 & 89.3902 \\
\hline 5.155 & 89.3902 \\
\hline 5.165 & 91.7687 \\
\hline 5.175 & 89.3902 \\
\hline 5.18 & 90.0089 \\
\hline 5.19 & 87.0172 \\
\hline 5.195 & 82.288 \\
\hline 5.205 & 84.6498 \\
\hline 5.21 & 87.0172 \\
\hline 5.22 & 85.2376 \\
\hline 5.225 & 85.2376 \\
\hline 5.235 & 79.93 \\
\hline
\end{tabular}




\begin{tabular}{|c|c|}
\hline 5.245 & 77.5817 \\
\hline 5.25 & 78.1236 \\
\hline 5.26 & 76.2972 \\
\hline 5.265 & 75.2374 \\
\hline 5.275 & 75.764 \\
\hline 5.28 & 76.2972 \\
\hline 5.29 & 73.4104 \\
\hline 5.3 & 73.9282 \\
\hline 5.305 & 73.4104 \\
\hline 5.315 & 73.4104 \\
\hline 5.32 & 76.2972 \\
\hline 5.33 & 80.4891 \\
\hline 5.335 & 81.0532 \\
\hline 5.345 & 81.0532 \\
\hline 5.35 & 81.0532 \\
\hline 5.36 & 81.0532 \\
\hline 5.37 & 81.0532 \\
\hline 5.375 & 81.0532 \\
\hline 5.385 & 81.0532 \\
\hline 5.39 & 83.4401 \\
\hline 5.4 & 83.4401 \\
\hline 5.405 & 83.4401 \\
\hline 5.415 & 83.4401 \\
\hline 5.42 & 84.027 \\
\hline 5.43 & 85.8328 \\
\hline 5.44 & 89.4761 \\
\hline 5.445 & 86.4354 \\
\hline 5.455 & 86.4354 \\
\hline 5.46 & 84.027 \\
\hline 5.47 & 77.3838 \\
\hline 5.475 & 73.9282 \\
\hline 5.485 & 74.4526 \\
\hline 5.495 & 69.7022 \\
\hline 5.5 & 72.5895 \\
\hline 5.51 & 74.4526 \\
\hline
\end{tabular}




\begin{tabular}{|c|c|}
\hline 5.515 & 72.0742 \\
\hline 5.525 & 67.8205 \\
\hline 5.53 & 67.3365 \\
\hline 5.54 & 67.8205 \\
\hline 5.545 & 67.8205 \\
\hline 5.555 & 66.8585 \\
\hline 5.565 & 67.8205 \\
\hline 5.57 & 65.4457 \\
\hline 5.58 & 63.5362 \\
\hline 5.585 & 60.7158 \\
\hline 5.595 & 60.7158 \\
\hline 5.6 & 61.159 \\
\hline 5.61 & 63.5362 \\
\hline 5.615 & 63.0774 \\
\hline 5.625 & 65.9201 \\
\hline 5.635 & 65.4457 \\
\hline 5.64 & 65.9201 \\
\hline 5.65 & 63.0774 \\
\hline 5.655 & 63.5362 \\
\hline 5.665 & 63.5362 \\
\hline 5.67 & 63.5362 \\
\hline 5.68 & 63.5362 \\
\hline 5.69 & 65.9201 \\
\hline 5.695 & 63.5362 \\
\hline 5.705 & 63.5362 \\
\hline 5.71 & 63.5362 \\
\hline 5.72 & 65.9201 \\
\hline 5.725 & 65.9201 \\
\hline 5.735 & 65.9201 \\
\hline 5.74 & 66.4006 \\
\hline 5.75 & 65.9201 \\
\hline 5.76 & 68.8071 \\
\hline 5.765 & 68.3107 \\
\hline 5.775 & 68.8071 \\
\hline 5.78 & 68.8071 \\
\hline
\end{tabular}




$\begin{array}{ll}5.79 & 71.2202 \\ 5.795 & 68.8071 \\ 5.805 & 56.425 \\ 5.81 & 47.3963 \\ 5.82 & 42.7169 \\ 5.83 & 24.3051 \\ 5.835 & 17.3693 \\ 5.845 & 4.13595 \\ 5.85 & -4.51238 \\ 5.86 & -8.78248\end{array}$




\section{Appendix B - Gauge data for experiment 4534.}

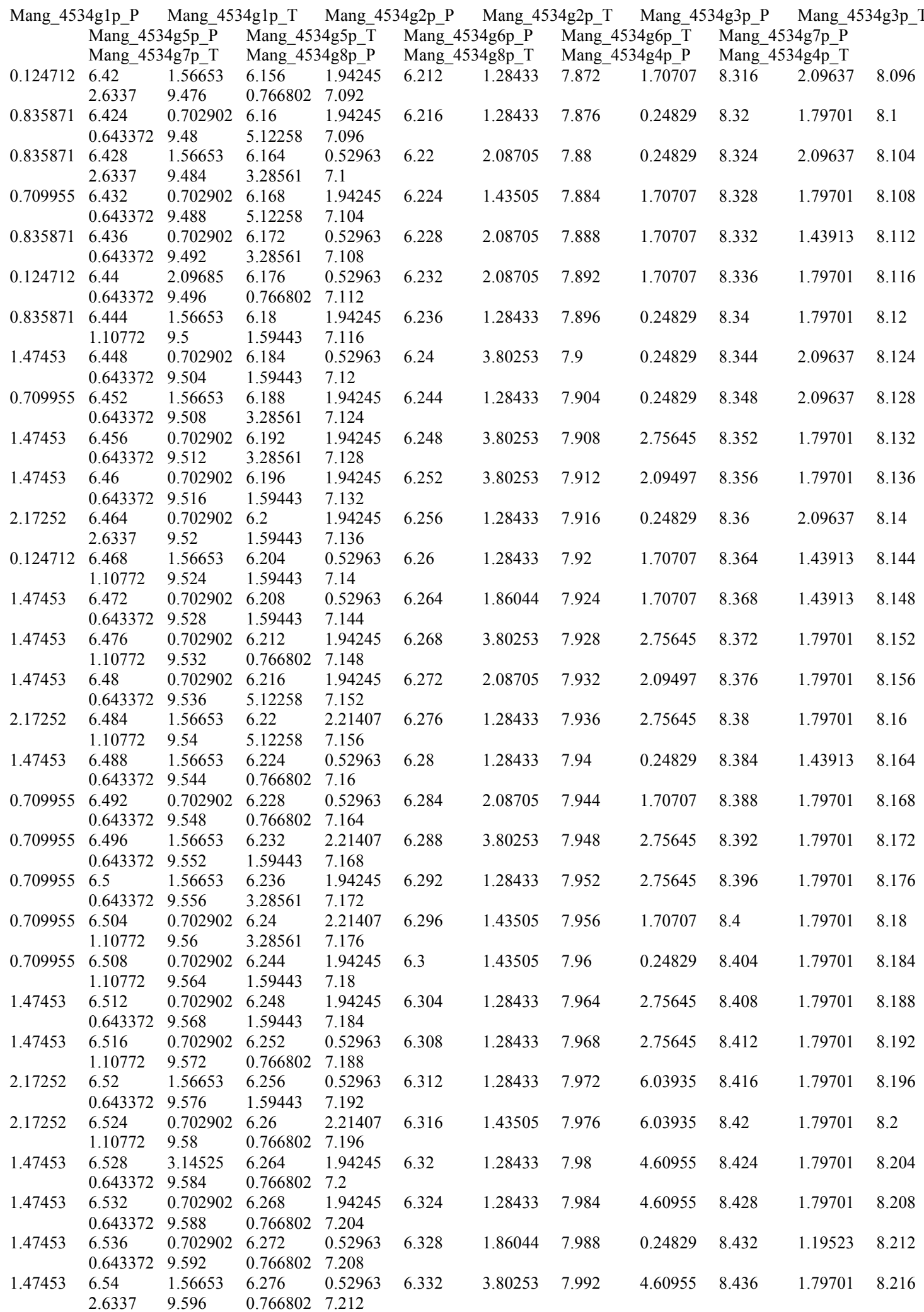




\begin{tabular}{|c|c|c|c|c|c|c|c|c|c|c|c|}
\hline 1.47453 & $\begin{array}{l}6.544 \\
1.10772\end{array}$ & $\begin{array}{l}2.09685 \\
9.6\end{array}$ & $\begin{array}{l}6.28 \\
1.59443\end{array}$ & $\begin{array}{l}0.52963 \\
7.216\end{array}$ & 6.336 & 1.28433 & 7.996 & 1.70707 & 8.44 & 1.79701 & 8.22 \\
\hline 2.17252 & $\begin{array}{l}6.548 \\
0.643372\end{array}$ & $\begin{array}{l}1.56653 \\
9.604\end{array}$ & $\begin{array}{l}6.284 \\
1.59443\end{array}$ & $\begin{array}{l}0.52963 \\
7.22\end{array}$ & 6.34 & 3.80253 & 8 & 0.24829 & 8.444 & 2.09637 & 8.224 \\
\hline 0.709955 & $\begin{array}{l}6.552 \\
2.6337\end{array}$ & $\begin{array}{l}0.702902 \\
9.608\end{array}$ & $\begin{array}{l}6.288 \\
1.59443\end{array}$ & $\begin{array}{l}2.21407 \\
7.224\end{array}$ & 6.344 & 3.80253 & 8.004 & 2.75645 & 8.448 & 1.43913 & 8.228 \\
\hline 2.17252 & $\begin{array}{l}6.556 \\
0.643372\end{array}$ & $\begin{array}{l}1.56653 \\
9.612\end{array}$ & $\begin{array}{l}6.292 \\
3.28561\end{array}$ & $\begin{array}{l}0.52963 \\
7.228\end{array}$ & 6.348 & 1.43505 & 8.008 & 0.24829 & 8.452 & 1.43913 & 8.232 \\
\hline 0.709955 & $\begin{array}{l}6.56 \\
1.10772\end{array}$ & $\begin{array}{l}1.56653 \\
9.616\end{array}$ & $\begin{array}{l}6.296 \\
0.766802\end{array}$ & $\begin{array}{l}0.52963 \\
7.232\end{array}$ & 6.352 & 1.43505 & 8.012 & 4.60955 & 8.456 & 1.43913 & 8.236 \\
\hline 1.47453 & $\begin{array}{l}6.564 \\
0.643372\end{array}$ & $\begin{array}{l}0.702902 \\
9.62\end{array}$ & $\begin{array}{l}6.3 \\
5.12258\end{array}$ & $\begin{array}{l}2.21407 \\
7.236\end{array}$ & 6.356 & 3.80253 & 8.016 & 4.60955 & 8.46 & 2.09637 & 8.24 \\
\hline 1.47453 & $\begin{array}{l}6.568 \\
1.66854\end{array}$ & $\begin{array}{l}3.14525 \\
9.624\end{array}$ & $\begin{array}{l}6.304 \\
3.28561\end{array}$ & $\begin{array}{l}0.52963 \\
7.24\end{array}$ & 6.36 & 2.08705 & 8.02 & 0.24829 & 8.464 & 2.09637 & 8.244 \\
\hline 2.17252 & $\begin{array}{l}6.572 \\
0.643372\end{array}$ & $\begin{array}{l}0.702902 \\
9.628\end{array}$ & $\begin{array}{l}6.308 \\
3.28561\end{array}$ & $\begin{array}{l}1.94245 \\
7.244\end{array}$ & 6.364 & 1.43505 & 8.024 & 1.70707 & 8.468 & 2.09637 & 8.248 \\
\hline 2.17252 & $\begin{array}{l}6.576 \\
0.643372\end{array}$ & $\begin{array}{l}1.56653 \\
9.632\end{array}$ & $\begin{array}{l}6.312 \\
3.28561\end{array}$ & $\begin{array}{l}0.52963 \\
7.248\end{array}$ & 6.368 & 2.08705 & 8.028 & 2.75645 & 8.472 & 2.09637 & 8.252 \\
\hline 2.81045 & $\begin{array}{l}6.58 \\
0.643372\end{array}$ & $\begin{array}{l}0.702902 \\
9.636\end{array}$ & $\begin{array}{l}6.316 \\
0.766802\end{array}$ & $\begin{array}{l}0.52963 \\
7.252\end{array}$ & 6.372 & .28433 & 8.032 & .75645 & 8.476 & 2.09637 & 8.256 \\
\hline 1.47453 & $\begin{array}{l}6.584 \\
0.643372\end{array}$ & $\begin{array}{l}1.56653 \\
9.64\end{array}$ & $\begin{array}{l}6.32 \\
0.766802\end{array}$ & $\begin{array}{l}0.52963 \\
7.256\end{array}$ & 6.376 & 1.43505 & 8.036 & 1.70707 & 8.48 & 1.19523 & 8.26 \\
\hline 1.47453 & $\begin{array}{l}6.588 \\
1.10772\end{array}$ & $\begin{array}{l}0.702902 \\
9.644\end{array}$ & $\begin{array}{l}6.324 \\
3.28561\end{array}$ & $\begin{array}{l}0.52963 \\
7.26\end{array}$ & 6.38 & 1.43505 & 8.04 & 1.70707 & 8.484 & 1.19523 & 8.264 \\
\hline 3.39511 & $\begin{array}{l}6.592 \\
0.643372\end{array}$ & $\begin{array}{l}0.702902 \\
9.648\end{array}$ & $\begin{array}{l}6.328 \\
5.12258\end{array}$ & $\begin{array}{l}4.26166 \\
7.264\end{array}$ & 6.384 & 3.80253 & 8.044 & 2.75645 & 8.488 & 3.65701 & 8.268 \\
\hline 1.47453 & $\begin{array}{l}6.596 \\
0.643372\end{array}$ & $\begin{array}{l}0.702902 \\
9.652\end{array}$ & $\begin{array}{l}6.332 \\
3.28561\end{array}$ & $\begin{array}{l}1.94245 \\
7.268\end{array}$ & 6.388 & 08705 & 8.048 & .75645 & 8.492 & 09637 & 3.272 \\
\hline 2.17252 & $\begin{array}{l}6.6 \\
0.643372\end{array}$ & $\begin{array}{l}0.702902 \\
9.656\end{array}$ & $\begin{array}{l}6.336 \\
1.59443\end{array}$ & $\begin{array}{l}2.21407 \\
7.272\end{array}$ & 6.392 & 1.43505 & 8.052 & 4.60955 & 8.496 & 1.79701 & 8.276 \\
\hline 2.17252 & $\begin{array}{l}6.604 \\
0.643372\end{array}$ & $\begin{array}{l}0.702902 \\
9.66\end{array}$ & $\begin{array}{l}6.34 \\
0.766802\end{array}$ & $\begin{array}{l}0.52963 \\
7.276\end{array}$ & 6.396 & 1.28433 & 8.056 & 2.75645 & 8.5 & 2.09637 & 8.28 \\
\hline 2.81045 & $\begin{array}{l}6.608 \\
1.10772\end{array}$ & $\begin{array}{l}1.56653 \\
9.664\end{array}$ & $\begin{array}{l}6.344 \\
0.766802\end{array}$ & $\begin{array}{l}0.52963 \\
7.28\end{array}$ & 6.4 & 2.08705 & 8.06 & 2.75645 & 8.504 & 2.09637 & 8.284 \\
\hline 2.81045 & $\begin{array}{l}6.612 \\
0.643372\end{array}$ & $\begin{array}{l}0.702902 \\
9.668\end{array}$ & $\begin{array}{l}6.348 \\
1.59443\end{array}$ & $\begin{array}{l}0.52963 \\
7.284\end{array}$ & 6.404 & 1.28433 & 8.064 & 2.75645 & 8.508 & 1.79701 & 8.288 \\
\hline 2.17252 & $\begin{array}{l}6.616 \\
0.643372\end{array}$ & $\begin{array}{l}0.702902 \\
9.672\end{array}$ & $\begin{array}{l}6.352 \\
5.12258\end{array}$ & $\begin{array}{l}0.52963 \\
7.288\end{array}$ & 6.408 & 1.43505 & 8.068 & 4.60955 & 8.512 & 2.09637 & 8.292 \\
\hline 2.81045 & $\begin{array}{l}6.62 \\
0.643372\end{array}$ & $\begin{array}{l}1.56653 \\
9.676\end{array}$ & $\begin{array}{l}6.356 \\
3.28561\end{array}$ & $\begin{array}{l}0.52963 \\
7.292\end{array}$ & 6.412 & 1.28433 & 8.072 & 2.75645 & 8.516 & 2.09637 & 8.296 \\
\hline 3.39511 & $\begin{array}{l}6.624 \\
1.10772\end{array}$ & $\begin{array}{l}0.702902 \\
9.68\end{array}$ & $\begin{array}{l}6.36 \\
0.766802\end{array}$ & $\begin{array}{l}0.52963 \\
7.296\end{array}$ & 6.416 & 3.80253 & 8.076 & 2.75645 & 8.52 & .09637 & 8.3 \\
\hline 3.39511 & $\begin{array}{l}6.628 \\
1.10772\end{array}$ & $\begin{array}{l}1.56653 \\
9.684\end{array}$ & $\begin{array}{l}6.364 \\
3.28561\end{array}$ & $\begin{array}{l}0.52963 \\
7.3\end{array}$ & 6.42 & 3.80253 & 8.08 & 4.60955 & 8.524 & 2.09637 & 8.304 \\
\hline 3.39511 & $\begin{array}{l}6.632 \\
1.10772\end{array}$ & $\begin{array}{l}0.702902 \\
9.688\end{array}$ & $\begin{array}{l}6.368 \\
0.766802\end{array}$ & $\begin{array}{l}0.52963 \\
7.304\end{array}$ & 6.424 & 2.08705 & 8.084 & 0.24829 & 8.528 & 2.09637 & 8.308 \\
\hline 3.39511 & $\begin{array}{l}6.636 \\
1.10772\end{array}$ & $\begin{array}{l}0.702902 \\
9.692\end{array}$ & $\begin{array}{l}6.372 \\
1.59443\end{array}$ & $\begin{array}{l}0.52963 \\
7.308\end{array}$ & 6.428 & 1.28433 & 8.088 & 1.70707 & 8.532 & 1.43913 & 8.312 \\
\hline 3.39511 & $\begin{array}{l}6.64 \\
0.643372\end{array}$ & $\begin{array}{l}1.56653 \\
9.696\end{array}$ & $\begin{array}{l}6.376 \\
0.766802\end{array}$ & $\begin{array}{l}4.26166 \\
7.312\end{array}$ & 6.432 & 1.28433 & 8.092 & 1.70707 & 8.536 & 1.43913 & 8.316 \\
\hline 3.93252 & $\begin{array}{l}6.644 \\
1.10772\end{array}$ & $\begin{array}{l}0.702902 \\
9.7\end{array}$ & $\begin{array}{l}6.38 \\
0.766802\end{array}$ & $\begin{array}{l}1.94245 \\
7.316\end{array}$ & 6.436 & 1.28433 & 8.096 & 2.75645 & 8.54 & 1.79701 & 8.32 \\
\hline 3.39511 & $\begin{array}{l}6.648 \\
1.10772\end{array}$ & $\begin{array}{l}0.702902 \\
9.704\end{array}$ & $\begin{array}{l}6.384 \\
0.766802\end{array}$ & $\begin{array}{l}0.52963 \\
7.32\end{array}$ & 6.44 & 1.43505 & 8.1 & 6.03935 & 8.544 & 2.09637 & 8.324 \\
\hline 3.39511 & $\begin{array}{l}6.652 \\
1.10772\end{array}$ & $\begin{array}{l}0.702902 \\
9.708\end{array}$ & $\begin{array}{l}6.388 \\
3.28561\end{array}$ & $\begin{array}{l}0.52963 \\
7.324\end{array}$ & 6.444 & 1.28433 & 8.104 & 0.24829 & 8.548 & 1.79701 & 8.328 \\
\hline 3.93252 & $\begin{array}{l}6.656 \\
0.643372\end{array}$ & $\begin{array}{l}0.702902 \\
9.712\end{array}$ & $\begin{array}{l}6.392 \\
3.28561\end{array}$ & $\begin{array}{l}1.94245 \\
7.328\end{array}$ & 6.448 & 3.80253 & 8.108 & 0.24829 & 8.552 & 1.43913 & 8.332 \\
\hline 2.81045 & $\begin{array}{l}6.66 \\
0.643372\end{array}$ & $\begin{array}{l}0.702902 \\
9.716\end{array}$ & $\begin{array}{l}6.396 \\
3.28561\end{array}$ & $\begin{array}{l}0.52963 \\
7.332\end{array}$ & 6.452 & 8433 & 112 & 707 & 556 & 43913 & 336 \\
\hline 2.17252 & $\begin{array}{l}6.664 \\
0.643372\end{array}$ & $\begin{array}{l}3.14525 \\
9.72\end{array}$ & $\begin{array}{l}6.4 \\
0.766802\end{array}$ & $\begin{array}{l}0.52963 \\
7.336\end{array}$ & 6.456 & 1.43505 & 8.116 & 1.70707 & 8.56 & 2.09637 & 8.34 \\
\hline 3.39511 & $\begin{array}{l}6.668 \\
0.643372\end{array}$ & $\begin{array}{l}0.702902 \\
9.724\end{array}$ & $\begin{array}{l}6.404 \\
3.28561\end{array}$ & $\begin{array}{l}1.94245 \\
7.34\end{array}$ & 6.46 & 3.80253 & 8.12 & 2.75645 & 8.564 & 1.79701 & 8.344 \\
\hline 3.39511 & $\begin{array}{l}6.672 \\
1.10772\end{array}$ & $\begin{array}{l}0.702902 \\
9.728\end{array}$ & $\begin{array}{l}6.408 \\
3.28561\end{array}$ & $\begin{array}{l}1.94245 \\
7.344\end{array}$ & 6.464 & 5.54023 & 8.124 & 1.70707 & 8.568 & 1.79701 & 8.348 \\
\hline 2. & $\begin{array}{l}6.676 \\
0.643372\end{array}$ & $\begin{array}{l}0.702902 \\
9.732\end{array}$ & $\begin{array}{l}6.412 \\
1.59443\end{array}$ & $\begin{array}{l}0.52963 \\
7.348\end{array}$ & 6 & 3505 & 8.128 & 9 & 2 & 13 & 52 \\
\hline 2.81045 & $\begin{array}{l}6.68 \\
0.643372\end{array}$ & $\begin{array}{l}0.702902 \\
9.736\end{array}$ & $\begin{array}{l}6.416 \\
1.59443\end{array}$ & $\begin{array}{l}1.94245 \\
7.352\end{array}$ & 6.472 & 3.80253 & 8.132 & 2.75645 & 8.576 & 1.79701 & 8.356 \\
\hline
\end{tabular}




\begin{tabular}{|c|c|c|c|c|c|c|c|c|c|c|c|}
\hline 2.81045 & $\begin{array}{l}6.684 \\
1.10772\end{array}$ & $\begin{array}{l}0.702902 \\
9.74\end{array}$ & $\begin{array}{l}6.42 \\
3.28561\end{array}$ & $\begin{array}{l}2.21407 \\
7.356\end{array}$ & 6.476 & 3.80253 & 8.136 & 2.75645 & 8.58 & 2.09637 & 8.36 \\
\hline 3.93252 & 6.688 & 0.702902 & $\begin{array}{l}6.424 \\
159443\end{array}$ & 4.26166 & 6.48 & 1.28433 & 8.14 & 2.75645 & 8.584 & 2.09637 & 8.364 \\
\hline 2.81045 & 6.696 & 0.702902 & $\begin{array}{l}6.432 \\
5.12258\end{array}$ & 2.21407 & 6.488 & 3.80253 & 8.148 & 4.60955 & 8.592 & 2.09637 & 8.372 \\
\hline 2.81045 & $\begin{array}{l}1.10772 \\
6.7 \\
0.643372\end{array}$ & $\begin{array}{l}9.752 \\
0.702902 \\
9.756\end{array}$ & $\begin{array}{l}5.12258 \\
6.436 \\
3.28561\end{array}$ & $\begin{array}{l}7.368 \\
4.26166 \\
7.372\end{array}$ & 6.492 & 3.80253 & 8.152 & 75645 & 6 & 2.09637 & 3.376 \\
\hline 2.17252 & $\begin{array}{l}6.704 \\
1.10772\end{array}$ & $\begin{array}{l}0.702902 \\
9.76\end{array}$ & $\begin{array}{l}6.44 \\
0.766802\end{array}$ & $\begin{array}{l}2.21407 \\
7.376\end{array}$ & 6.496 & 1.28433 & 8.156 & 2.75645 & 8.6 & 1.19523 & 8.38 \\
\hline 2.81045 & $\begin{array}{l}6.708 \\
0.643372\end{array}$ & $\begin{array}{l}1.56653 \\
9.764\end{array}$ & $\begin{array}{l}6.444 \\
1.59443\end{array}$ & $\begin{array}{l}2.21407 \\
7.38\end{array}$ & 6.5 & 1.43505 & 8.16 & 2.75645 & 8.604 & 2.09637 & 8.384 \\
\hline 3.39511 & $\begin{array}{l}6.712 \\
0.643372\end{array}$ & $\begin{array}{l}3.14525 \\
9.768\end{array}$ & $\begin{array}{l}6.448 \\
0.766802\end{array}$ & $\begin{array}{l}4.26166 \\
7.384\end{array}$ & 6.504 & 2.08705 & 8.164 & 2.75645 & 8.608 & 1.79701 & 8.388 \\
\hline 3.39511 & $\begin{array}{l}6.716 \\
0.643372\end{array}$ & $\begin{array}{l}0.702902 \\
9.772\end{array}$ & $\begin{array}{l}6.452 \\
0.766802\end{array}$ & $\begin{array}{l}0.52963 \\
7.388\end{array}$ & 6.508 & 5.54023 & 8.168 & 2.75645 & 8.612 & 1.79701 & 8.392 \\
\hline 2.81045 & $\begin{array}{l}6.72 \\
1.10772\end{array}$ & $\begin{array}{l}0.702902 \\
9.776\end{array}$ & $\begin{array}{l}6.456 \\
3.28561\end{array}$ & $\begin{array}{l}0.52963 \\
7.392\end{array}$ & 6.512 & 3.80253 & 8.172 & 0.24829 & 8.616 & 2.09637 & .396 \\
\hline 2.17252 & $\begin{array}{l}6.724 \\
1.10772\end{array}$ & $\begin{array}{l}0.702902 \\
9.78\end{array}$ & $\begin{array}{l}6.46 \\
0.766802\end{array}$ & $\begin{array}{l}2.21407 \\
7.396\end{array}$ & 6.516 & 2.08705 & 8.176 & 4.60955 & 8.62 & 2.09637 & 8.4 \\
\hline 2.81045 & $\begin{array}{l}6.728 \\
0.643372\end{array}$ & $\begin{array}{l}0.702902 \\
9.784\end{array}$ & $\begin{array}{l}6.464 \\
3.28561\end{array}$ & $\begin{array}{l}2.21407 \\
7.4\end{array}$ & 6.52 & 1.28433 & 8.18 & 2.75645 & 8.624 & 1.19523 & 8.404 \\
\hline 2.17252 & $\begin{array}{l}6.732 \\
1.10772\end{array}$ & $\begin{array}{l}0.702902 \\
9.788\end{array}$ & $\begin{array}{l}6.468 \\
0.766802\end{array}$ & $\begin{array}{l}2.21407 \\
7.404\end{array}$ & 6.524 & 1.28433 & 8.184 & 0.24829 & 8.628 & 1.79701 & 8.408 \\
\hline 3.93252 & $\begin{array}{l}6.736 \\
0.643372\end{array}$ & $\begin{array}{l}0.702902 \\
9.792\end{array}$ & $\begin{array}{l}6.472 \\
3.28561\end{array}$ & $\begin{array}{l}2.21407 \\
7.408\end{array}$ & 6.528 & .28433 & 8.188 & .75645 & 8.632 & 09637 & .412 \\
\hline 3.39511 & $\begin{array}{l}6.74 \\
0.643372\end{array}$ & $\begin{array}{l}3.14525 \\
9.796\end{array}$ & $\begin{array}{l}6.476 \\
3.28561\end{array}$ & $\begin{array}{l}0.52963 \\
7.412\end{array}$ & 6.532 & 1.28433 & 8.192 & 0.24829 & 8.636 & 1.19523 & 8.416 \\
\hline 2.81045 & $\begin{array}{l}6.744 \\
0.643372\end{array}$ & $\begin{array}{l}0.702902 \\
9.8\end{array}$ & $\begin{array}{l}6.48 \\
3.28561\end{array}$ & $\begin{array}{l}0.52963 \\
7.416\end{array}$ & 6.536 & 1.28433 & 8.196 & 0.24829 & 8.64 & 1.79701 & 8.42 \\
\hline 3.39511 & $\begin{array}{l}6.748 \\
0.643372\end{array}$ & $\begin{array}{l}3.14525 \\
9.804\end{array}$ & $\begin{array}{l}6.484 \\
0.766802\end{array}$ & $\begin{array}{l}0.52963 \\
7.42\end{array}$ & 6.54 & 1.43505 & 8.2 & 1.70707 & 8.644 & 1.79701 & 8.424 \\
\hline 3.39511 & $\begin{array}{l}6.752 \\
0.643372\end{array}$ & $\begin{array}{l}4.94625 \\
9.808\end{array}$ & $\begin{array}{l}6.488 \\
1.59443\end{array}$ & $\begin{array}{l}0.52963 \\
7.424\end{array}$ & 6.544 & 1.28433 & 8.204 & 0.24829 & 8.648 & 2.09637 & 8.428 \\
\hline 3.39511 & $\begin{array}{l}6.756 \\
0.643372\end{array}$ & $\begin{array}{l}0.702902 \\
9.812\end{array}$ & $\begin{array}{l}6.492 \\
0.766802\end{array}$ & $\begin{array}{l}1.94245 \\
7.428\end{array}$ & 6.548 & 2.08705 & 8.208 & 2.75645 & 8.652 & 1.43913 & 8.432 \\
\hline 3.39511 & $\begin{array}{l}6.76 \\
0.643372\end{array}$ & $\begin{array}{l}3.14525 \\
9.816\end{array}$ & $\begin{array}{l}6.496 \\
1.59443\end{array}$ & $\begin{array}{l}0.52963 \\
7.432\end{array}$ & 6.552 & 1.43505 & 8.212 & 2.75645 & 8.656 & 1.43913 & 8.436 \\
\hline 3.93252 & $\begin{array}{l}6.764 \\
0.643372\end{array}$ & $\begin{array}{l}0.702902 \\
9.82\end{array}$ & $\begin{array}{l}6.5 \\
3.28561\end{array}$ & $\begin{array}{l}2.21407 \\
7.436\end{array}$ & 6.556 & 80253 & 8.216 & 24829 & 8.66 & 2.09637 & 8.44 \\
\hline 3.93252 & $\begin{array}{l}6.768 \\
1.10772\end{array}$ & $\begin{array}{l}0.702902 \\
9.824\end{array}$ & $\begin{array}{l}6.504 \\
3.28561\end{array}$ & $\begin{array}{l}2.21407 \\
7.44\end{array}$ & 6.56 & 1.43505 & 8.22 & 0.24829 & 8.664 & 2.09637 & 8.444 \\
\hline 3.93252 & $\begin{array}{l}6.772 \\
1.10772\end{array}$ & $\begin{array}{l}0.702902 \\
9.828\end{array}$ & $\begin{array}{l}6.508 \\
3.28561\end{array}$ & $\begin{array}{l}2.21407 \\
7.444\end{array}$ & 6.564 & 1.28433 & 8.224 & 0.24829 & 8.668 & 2.09637 & 8.448 \\
\hline 3.93252 & $\begin{array}{l}6.776 \\
0.643372\end{array}$ & $\begin{array}{l}0.702902 \\
9.832\end{array}$ & $\begin{array}{l}6.512 \\
5.12258\end{array}$ & $\begin{array}{l}0.52963 \\
7.448\end{array}$ & 6.568 & 1.43505 & 8.228 & 0.24829 & 8.672 & 1.43913 & 8.452 \\
\hline 3.93252 & $\begin{array}{l}6.78 \\
0.643372\end{array}$ & $\begin{array}{l}1.56653 \\
9.836\end{array}$ & $\begin{array}{l}6.516 \\
1.59443\end{array}$ & $\begin{array}{l}1.94245 \\
7.452\end{array}$ & 6.572 & 1.28433 & 8.232 & 0.24829 & 8.676 & 1.19523 & 8.456 \\
\hline 3.93252 & $\begin{array}{l}6.784 \\
0.643372\end{array}$ & $\begin{array}{l}0.702902 \\
9.84\end{array}$ & $\begin{array}{l}6.52 \\
1.59443\end{array}$ & $\begin{array}{l}0.52963 \\
7.456\end{array}$ & 6.576 & 1.43505 & 8.236 & 2.75645 & 8.68 & 2.09637 & 8.46 \\
\hline 3.93252 & $\begin{array}{l}6.788 \\
0.643372\end{array}$ & $\begin{array}{l}3.14525 \\
9.844\end{array}$ & $\begin{array}{l}6.524 \\
1.59443\end{array}$ & $\begin{array}{l}1.94245 \\
7.46\end{array}$ & 6.58 & 3.80253 & 8.24 & 2.75645 & 8.684 & 1.43913 & 8.464 \\
\hline 3.93252 & $\begin{array}{l}6.792 \\
1.10772\end{array}$ & $\begin{array}{l}0.702902 \\
9.848\end{array}$ & $\begin{array}{l}6.528 \\
1.59443\end{array}$ & $\begin{array}{l}0.52963 \\
7.464\end{array}$ & 6.584 & 1.43505 & 8.244 & 4.60955 & 8.688 & 1.79701 & 8.468 \\
\hline 3.93252 & $\begin{array}{l}6.796 \\
0.643372\end{array}$ & $\begin{array}{l}3.14525 \\
9.852\end{array}$ & $\begin{array}{l}6.532 \\
1.59443\end{array}$ & $\begin{array}{l}1.94245 \\
7.468\end{array}$ & 6.588 & 1.43505 & 8.248 & 2.75645 & 8.692 & 1.79701 & 8.472 \\
\hline 3.93252 & $\begin{array}{l}6.8 \\
0.643372\end{array}$ & $\begin{array}{l}0.702902 \\
9.856\end{array}$ & $\begin{array}{l}6.536 \\
3.28561\end{array}$ & $\begin{array}{l}0.52963 \\
7.472\end{array}$ & 6.592 & 54023 & 8.252 & .09497 & 8.696 & .79701 & .476 \\
\hline 3.39511 & $\begin{array}{l}6.804 \\
0.643372\end{array}$ & $\begin{array}{l}0.702902 \\
9.86\end{array}$ & $\begin{array}{l}6.54 \\
3.28561\end{array}$ & $\begin{array}{l}0.52963 \\
7.476\end{array}$ & 6.596 & 1.43505 & 8.256 & 1.70707 & 8.7 & 2.09637 & 8.48 \\
\hline 3.39511 & $\begin{array}{l}6.808 \\
0.643372\end{array}$ & $\begin{array}{l}0.702902 \\
9.864\end{array}$ & $\begin{array}{l}6.544 \\
3.28561\end{array}$ & $\begin{array}{l}2.21407 \\
7.48\end{array}$ & 6.6 & 1.28433 & 8.26 & 1.70707 & 8.704 & 1.79701 & 8.484 \\
\hline 3.93252 & $\begin{array}{l}6.812 \\
0.643372\end{array}$ & $\begin{array}{l}0.702902 \\
9.868\end{array}$ & $\begin{array}{l}6.548 \\
1.59443\end{array}$ & $\begin{array}{l}0.52963 \\
7.484\end{array}$ & 6.604 & 2.08705 & 8.264 & 0.24829 & 8.708 & 1.79701 & 8.488 \\
\hline 3. & $\begin{array}{l}6.816 \\
0.643372\end{array}$ & $\begin{array}{l}0.702902 \\
9.872\end{array}$ & $\begin{array}{l}6.552 \\
0.766802\end{array}$ & $\begin{array}{l}1.94245 \\
7.488\end{array}$ & 6 & 8705 & 8.268 & 9 & 2 & 9701 & .492 \\
\hline 4.42834 & $\begin{array}{l}6.82 \\
0.643372\end{array}$ & $\begin{array}{l}0.702902 \\
9.876\end{array}$ & $\begin{array}{l}6.556 \\
0.766802\end{array}$ & $\begin{array}{l}1.94245 \\
7.492\end{array}$ & 6.612 & 2.08705 & 8.272 & 2.75645 & 8.716 & 2.09637 & 8.496 \\
\hline
\end{tabular}




\begin{tabular}{|c|c|c|c|c|c|c|c|c|c|c|c|}
\hline 3.93252 & $\begin{array}{l}6.824 \\
1.66854\end{array}$ & $\begin{array}{l}0.702902 \\
9.88\end{array}$ & $\begin{array}{l}6.56 \\
0.766802\end{array}$ & $\begin{array}{l}0.52963 \\
7.496\end{array}$ & 6.616 & 1.28433 & 8.276 & 0.24829 & 8.72 & 2.09637 & 8.5 \\
\hline 3.93252 & $\begin{array}{l}6.828 \\
0.643372\end{array}$ & $\begin{array}{l}0.702902 \\
9884\end{array}$ & $\begin{array}{l}6.564 \\
159443\end{array}$ & $\begin{array}{l}2.21407 \\
7.5\end{array}$ & 6.62 & 1.28433 & 8.28 & 2.75645 & 8.724 & 2.09637 & 8.504 \\
\hline 3.93252 & $\begin{array}{l}6.832 \\
0.643372\end{array}$ & $\begin{array}{l}0.702902 \\
9.888\end{array}$ & $\begin{array}{l}6.568 \\
3.28561\end{array}$ & $\begin{array}{l}2.21407 \\
7.504\end{array}$ & 6.624 & 1.43505 & 8.284 & 0.24829 & 8.728 & 2.09637 & 8.508 \\
\hline 4.42834 & $\begin{array}{l}6.836 \\
0.643372\end{array}$ & $\begin{array}{l}0.702902 \\
9.892\end{array}$ & $\begin{array}{l}6.572 \\
1.59443\end{array}$ & $\begin{array}{l}0.52963 \\
7.508\end{array}$ & 6.628 & 1.28433 & 8.288 & 0.24829 & 8.732 & 2.09637 & 8.512 \\
\hline 3.93252 & $\begin{array}{l}6.84 \\
0.643372\end{array}$ & $\begin{array}{l}0.702902 \\
9.896\end{array}$ & $\begin{array}{l}6.576 \\
3.28561\end{array}$ & $\begin{array}{l}0.52963 \\
7.512\end{array}$ & 6.632 & 1.43505 & 8.292 & 0.24829 & 8.736 & 1.43913 & 8.516 \\
\hline 3.93252 & $\begin{array}{l}6.844 \\
1.10772\end{array}$ & $\begin{array}{l}0.702902 \\
9.9\end{array}$ & $\begin{array}{l}6.58 \\
5.12258\end{array}$ & $\begin{array}{l}0.52963 \\
7.516\end{array}$ & 6.636 & 1.28433 & 8.296 & 0.24829 & 8.74 & 2.09637 & 8.52 \\
\hline 2.81045 & $\begin{array}{l}6.848 \\
0.643372\end{array}$ & $\begin{array}{l}1.56653 \\
9.904\end{array}$ & $\begin{array}{l}6.584 \\
1.59443\end{array}$ & $\begin{array}{l}1.94245 \\
7.52\end{array}$ & 6.64 & 1.43505 & 8.3 & 1.70707 & 8.744 & 2.09637 & 8.524 \\
\hline 3.93252 & $\begin{array}{l}6.852 \\
0.643372\end{array}$ & $\begin{array}{l}0.702902 \\
9.908\end{array}$ & $\begin{array}{l}6.588 \\
1.59443\end{array}$ & $\begin{array}{l}2.21407 \\
7.524\end{array}$ & 6.644 & 1.28433 & 8.304 & 1.70707 & 8.748 & 1.19523 & 8.528 \\
\hline 4.88769 & $\begin{array}{l}6.856 \\
1.10772\end{array}$ & $\begin{array}{l}2.09685 \\
9.912\end{array}$ & $\begin{array}{l}6.592 \\
1.59443\end{array}$ & $\begin{array}{l}1.94245 \\
7.528\end{array}$ & 6.648 & 2.08705 & 8.308 & 1.70707 & 8.752 & 1.79701 & 8.532 \\
\hline 3.93252 & $\begin{array}{l}6.86 \\
0.643372\end{array}$ & $\begin{array}{l}0.702902 \\
9.916\end{array}$ & $\begin{array}{l}6.596 \\
0.766802\end{array}$ & $\begin{array}{l}1.94245 \\
7.532\end{array}$ & 6.652 & 1.28433 & 8.312 & 2.75645 & 8.756 & 1.43913 & 8.536 \\
\hline 3.39511 & $\begin{array}{l}6.864 \\
1.10772\end{array}$ & $\begin{array}{l}0.702902 \\
9.92\end{array}$ & $\begin{array}{l}6.6 \\
0.766802\end{array}$ & $\begin{array}{l}0.52963 \\
7.536\end{array}$ & 6.65 & 1.43505 & 316 & .75645 & 8.76 & 2.09637 & 8.54 \\
\hline 3.93252 & $\begin{array}{l}6.868 \\
0.643372\end{array}$ & $\begin{array}{l}3.14525 \\
9.924\end{array}$ & $\begin{array}{l}6.604 \\
0.766802\end{array}$ & $\begin{array}{l}0.52963 \\
7.54\end{array}$ & 6.66 & 3.80253 & 8.32 & 0.24829 & 8.764 & 2.09637 & 8.544 \\
\hline 3.93252 & $\begin{array}{l}6.872 \\
0.643372\end{array}$ & $\begin{array}{l}0.702902 \\
9.928\end{array}$ & $\begin{array}{l}6.608 \\
3.28561\end{array}$ & $\begin{array}{l}1.94245 \\
7.544\end{array}$ & 6.664 & 1.28433 & 8.324 & 2.75645 & 8.768 & 2.09637 & 8.548 \\
\hline 3.93252 & $\begin{array}{l}6.876 \\
1.10772\end{array}$ & $\begin{array}{l}4.94625 \\
9.932\end{array}$ & $\begin{array}{l}6.612 \\
0.766802\end{array}$ & $\begin{array}{l}1.94245 \\
7.548\end{array}$ & 6.668 & 1.28433 & 8.328 & 0.24829 & 8.772 & 1.43913 & 8.552 \\
\hline 3.93252 & $\begin{array}{l}6.88 \\
0.643372\end{array}$ & $\begin{array}{l}0.702902 \\
9.936\end{array}$ & $\begin{array}{l}6.616 \\
0.766802\end{array}$ & $\begin{array}{l}2.21407 \\
7.552\end{array}$ & 6.672 & .28433 & 3.332 & 24829 & 8.776 & 3.65701 & .556 \\
\hline 2.81045 & $\begin{array}{l}6.884 \\
1.10772\end{array}$ & $\begin{array}{l}0.702902 \\
9.94\end{array}$ & $\begin{array}{l}6.62 \\
5.12258\end{array}$ & $\begin{array}{l}0.52963 \\
7.556\end{array}$ & 6.676 & 5.54023 & 8.336 & 0.24829 & 8.78 & 1.79701 & 8.56 \\
\hline 3.93252 & $\begin{array}{l}6.888 \\
0.643372\end{array}$ & $\begin{array}{l}0.702902 \\
9.944\end{array}$ & $\begin{array}{l}6.624 \\
3.28561\end{array}$ & $\begin{array}{l}5.81014 \\
7.56\end{array}$ & 6.68 & 1.43505 & 8.34 & 2.09497 & 8.784 & 1.43913 & 8.564 \\
\hline 3.93252 & $\begin{array}{l}6.892 \\
2.6337\end{array}$ & $\begin{array}{l}3.14525 \\
9.948\end{array}$ & $\begin{array}{l}6.628 \\
0.766802\end{array}$ & $\begin{array}{l}0.52963 \\
7.564\end{array}$ & 6.684 & .80253 & 8.344 & 2.75645 & 8.788 & 1.43913 & .568 \\
\hline 3.93252 & $\begin{array}{l}6.896 \\
0.643372\end{array}$ & $\begin{array}{l}0.702902 \\
9.952\end{array}$ & $\begin{array}{l}6.632 \\
1.59443\end{array}$ & $\begin{array}{l}0.52963 \\
7.568\end{array}$ & 6.688 & 1.43505 & 8.348 & 0.24829 & 8.792 & 1.79701 & 8.572 \\
\hline 3.93252 & $\begin{array}{l}6.9 \\
0.643372\end{array}$ & $\begin{array}{l}0.702902 \\
9.956\end{array}$ & $\begin{array}{l}6.636 \\
0.766802\end{array}$ & & 6.692 & 3.80253 & 8.352 & 2.75645 & 8.796 & 1.43913 & 8.576 \\
\hline 3.93252 & $\begin{array}{l}6.904 \\
0.643372\end{array}$ & $\begin{array}{l}0.702902 \\
9.96\end{array}$ & $\begin{array}{l}6.64 \\
0.766802\end{array}$ & $\begin{array}{l}1.94245 \\
7.576\end{array}$ & 6.696 & .28433 & 8.356 & 2.75645 & 8.8 & 1.43913 & 8.58 \\
\hline 3.93252 & $\begin{array}{l}6.908 \\
1.10772\end{array}$ & $\begin{array}{l}0.702902 \\
9.964\end{array}$ & $\begin{array}{l}6.644 \\
0.766802\end{array}$ & $\begin{array}{l}0.52963 \\
7.58\end{array}$ & 6.7 & 08705 & 8.36 & 0.24829 & 8.804 & 1.43913 & 3.584 \\
\hline 3.93252 & $\begin{array}{l}6.912 \\
2.6337\end{array}$ & $\begin{array}{l}0.702902 \\
9.968\end{array}$ & $\begin{array}{l}6.648 \\
0.766802\end{array}$ & $\begin{array}{l}0.52963 \\
7.584\end{array}$ & 6.704 & 1.28433 & 8.364 & 1.70707 & 8.808 & 1.19523 & 8.588 \\
\hline 2.17252 & $\begin{array}{l}6.916 \\
0.643372\end{array}$ & $\begin{array}{l}0.702902 \\
9.972\end{array}$ & $\begin{array}{l}6.652 \\
3.28561\end{array}$ & $\begin{array}{l}1.94245 \\
7.588\end{array}$ & 6.708 & .08705 & 8.368 & 1.70707 & 8.812 & 1.79701 & 3.592 \\
\hline 1.34595 & $\begin{array}{l}6.92 \\
0.643372\end{array}$ & $\begin{array}{l}0.702902 \\
9.976\end{array}$ & $\begin{array}{l}6.656 \\
1.59443\end{array}$ & $\begin{array}{l}2.04072 \\
7.592\end{array}$ & 6.712 & .08705 & 8.372 & 0.24829 & 8.816 & 1.43913 & 8.596 \\
\hline 1.63451 & $\begin{array}{l}6.924 \\
1.10772\end{array}$ & $\begin{array}{l}4.94625 \\
9.98\end{array}$ & $\begin{array}{l}6.66 \\
0.766802\end{array}$ & $\begin{array}{l}0.52963 \\
7.596\end{array}$ & 6.716 & 1.43505 & 8.376 & 0.24829 & 8.82 & 2.09637 & 8.6 \\
\hline-1.11722 & $\begin{array}{l}6.928 \\
0.643372\end{array}$ & $\begin{array}{l}3.14525 \\
9.984\end{array}$ & $\begin{array}{l}6.664 \\
1.59443\end{array}$ & $\begin{array}{l}1.94245 \\
7.6\end{array}$ & 6.72 & 1.43505 & 8.38 & 4.60955 & 8.824 & 1.43913 & 8.604 \\
\hline-4.97331 & $\begin{array}{l}6.932 \\
0.643372\end{array}$ & $\begin{array}{l}0.702902 \\
9.988\end{array}$ & $\begin{array}{l}6.668 \\
0.766802\end{array}$ & $\begin{array}{l}1.94245 \\
7.604\end{array}$ & 6.724 & .43505 & 3.384 & 2.75645 & 8.828 & 1.19523 & 8.608 \\
\hline-8.11628 & $\begin{array}{l}6.936 \\
0.643372\end{array}$ & $\begin{array}{l}0.702902 \\
9.992\end{array}$ & $\begin{array}{l}6.672 \\
1.59443\end{array}$ & $\begin{array}{l}1.94245 \\
7.608\end{array}$ & 6.728 & 1.43505 & 8.388 & 2.75645 & 8.832 & 1.79701 & 8.612 \\
\hline-10.7894 & $\begin{array}{l}6.94 \\
0.643372\end{array}$ & $\begin{array}{l}3.14525 \\
9.996\end{array}$ & $\begin{array}{l}6.676 \\
3.28561\end{array}$ & $\begin{array}{l}0.52963 \\
7.612\end{array}$ & 6.732 & 1.28433 & 8.392 & 0.24829 & 8.836 & 1.43913 & 8.616 \\
\hline-11.6764 & $\begin{array}{l}6.944 \\
1.10772\end{array}$ & $\begin{array}{l}3.14525 \\
10\end{array}$ & $\begin{array}{l}6.68 \\
0.766802\end{array}$ & $\begin{array}{l}1.94245 \\
7.616\end{array}$ & 6 & 05 & 5 & 5 & 8 & 37 & .62 \\
\hline-12.3402 & $\begin{array}{l}6.948 \\
0.643372\end{array}$ & $\begin{array}{l}0.702902 \\
10.004\end{array}$ & $\begin{array}{l}6.684 \\
1.59443\end{array}$ & $\begin{array}{l}2.21407 \\
7.62\end{array}$ & 6.74 & 1.28433 & 8.4 & 2.75645 & 8.844 & 1.43913 & 8.624 \\
\hline-11.8978 & $\begin{array}{l}6.952 \\
1.10772\end{array}$ & $\begin{array}{l}0.702902 \\
10.008\end{array}$ & $\begin{array}{l}6.688 \\
1.59443\end{array}$ & $\begin{array}{l}1.94245 \\
7.624\end{array}$ & 6.744 & 2.08705 & 8.404 & 4.60955 & 8.848 & 1.79701 & 8.628 \\
\hline-10.7894 & $\begin{array}{l}6.956 \\
1.10772\end{array}$ & $\begin{array}{l}0.702902 \\
10.012\end{array}$ & $\begin{array}{l}6.692 \\
3.28561\end{array}$ & $\begin{array}{l}1.94245 \\
7.628\end{array}$ & 6.748 & 28433 & 8 & 0.24 & 8.852 & 1.43913 & 632 \\
\hline 36 & $\begin{array}{l}6.96 \\
0.643372\end{array}$ & $\begin{array}{l}0.702902 \\
10.016\end{array}$ & $\begin{array}{l}6.696 \\
1.59443\end{array}$ & $\begin{array}{l}1.94245 \\
7.632\end{array}$ & 6.752 & 43505 & .412 & 0.24829 & 8.856 & 1.43913 & .636 \\
\hline
\end{tabular}




\begin{tabular}{|c|c|c|c|c|c|c|c|c|c|c|c|}
\hline-8.11628 & $\begin{array}{l}6.964 \\
1.10772\end{array}$ & $\begin{array}{l}1.56653 \\
10.02\end{array}$ & $\begin{array}{l}6.7 \\
0.766802\end{array}$ & $\begin{array}{l}0.52963 \\
7.636\end{array}$ & 6.756 & 1.43505 & 8.416 & 0.24829 & 8.86 & 1.19523 & 8.64 \\
\hline-6.77264 & $\begin{array}{l}6.968 \\
0.643372\end{array}$ & $\begin{array}{l}0.702902 \\
10.024\end{array}$ & $\begin{array}{l}6.704 \\
0.766802\end{array}$ & $\begin{array}{l}0.52963 \\
7.64\end{array}$ & 6.76 & 1.28433 & 8.42 & 1.70707 & 8.864 & 1.43913 & 8.644 \\
\hline 0.0113371 & $\begin{array}{l}6.972 \\
0.643372\end{array}$ & $\begin{array}{l}3.14525 \\
10.028\end{array}$ & $\begin{array}{l}6.708 \\
0.766802\end{array}$ & $\begin{array}{l}1.94245 \\
7.644\end{array}$ & 6.764 & 1.28433 & 8.424 & 0.24829 & 8.868 & 2.09637 & 8.648 \\
\hline 13.1977 & $\begin{array}{l}6.976 \\
0.643372\end{array}$ & $\begin{array}{l}0.702902 \\
10.032\end{array}$ & $\begin{array}{l}6.712 \\
0.766802\end{array}$ & $\begin{array}{l}1.94245 \\
7.648\end{array}$ & 6.768 & 1.28433 & 8.428 & 0.24829 & 8.872 & 2.09637 & 8.652 \\
\hline 29.9262 & $\begin{array}{l}6.98 \\
1.10772\end{array}$ & $\begin{array}{l}0.702902 \\
10.036\end{array}$ & $\begin{array}{l}6.716 \\
0.766802\end{array}$ & $\begin{array}{l}0.52963 \\
7.652\end{array}$ & 6.772 & 1.28433 & 8.432 & 6.03935 & 8.876 & 1.43913 & 8.656 \\
\hline 45.9336 & $\begin{array}{l}6.984 \\
1.10772\end{array}$ & $\begin{array}{l}1.56653 \\
10.04\end{array}$ & $\begin{array}{l}6.72 \\
0.766802\end{array}$ & $\begin{array}{l}0.52963 \\
7.656\end{array}$ & 6.776 & 2.08705 & 8.436 & 2.75645 & 8.88 & 1.19523 & 8.66 \\
\hline 59.8177 & $\begin{array}{l}6.988 \\
0.643372\end{array}$ & $\begin{array}{l}0.702902 \\
10.044\end{array}$ & $\begin{array}{l}6.724 \\
1.59443\end{array}$ & $\begin{array}{l}0.52963 \\
7.66\end{array}$ & 6.78 & 5.54023 & 8.44 & 1.70707 & 8.884 & 1.43913 & 8.664 \\
\hline 69.8407 & $\begin{array}{l}6.992 \\
0.643372\end{array}$ & $\begin{array}{l}0.702902 \\
10.048\end{array}$ & $\begin{array}{l}6.728 \\
0.766802\end{array}$ & $\begin{array}{l}2.21407 \\
7.664\end{array}$ & 6.784 & 1.43505 & 8.444 & 0.24829 & 8.888 & 1.43913 & 8.668 \\
\hline 77.1237 & $\begin{array}{l}6.996 \\
0.643372\end{array}$ & $\begin{array}{l}0.702902 \\
10.052\end{array}$ & $\begin{array}{l}6.732 \\
1.59443\end{array}$ & $\begin{array}{l}0.52963 \\
7.668\end{array}$ & 6.788 & 1.43505 & 8.448 & 4.60955 & 8.892 & 1.43913 & 8.672 \\
\hline 80.5291 & $\begin{array}{l}7 \\
0.643372\end{array}$ & $\begin{array}{l}1.56653 \\
10.056\end{array}$ & $\begin{array}{l}6.736 \\
0.766802\end{array}$ & $\begin{array}{l}0.52963 \\
7.672\end{array}$ & 6.792 & 43505 & 8.452 & .24829 & 8.896 & 1.19523 & 8.676 \\
\hline 80.5291 & $\begin{array}{l}7.004 \\
1.10772\end{array}$ & $\begin{array}{l}0.702902 \\
10.06\end{array}$ & $\begin{array}{l}6.74 \\
1.59443\end{array}$ & $\begin{array}{l}0.52963 \\
7.676\end{array}$ & 6.796 & 1.28433 & 8.456 & 0.24829 & 8.9 & 1.43913 & 8.68 \\
\hline 78.9555 & $\begin{array}{l}7.008 \\
1.10772\end{array}$ & $\begin{array}{l}0.702902 \\
10.064\end{array}$ & $\begin{array}{l}6.744 \\
5.12258\end{array}$ & $\begin{array}{l}0.52963 \\
7.68\end{array}$ & 6.8 & 1.28433 & 8.46 & 1.70707 & 8.904 & 1.79701 & 8.684 \\
\hline 75.818 & $\begin{array}{l}7.012 \\
2.6337\end{array}$ & & $\begin{array}{l}6.748 \\
0.766802\end{array}$ & $\begin{array}{l}2.21407 \\
7.684\end{array}$ & 6.804 & 1.43505 & 8.464 & 0.24829 & 8.908 & 3.65701 & 8.688 \\
\hline 73.9937 & $\begin{array}{l}7.016 \\
0.643372\end{array}$ & $\begin{array}{l}3.14525 \\
10.072\end{array}$ & $\begin{array}{l}6.752 \\
1.59443\end{array}$ & $\begin{array}{l}4.26166 \\
7.688\end{array}$ & 6.808 & .08705 & 8.468 & 24829 & 8.912 & 43913 & 8.692 \\
\hline 74.2541 & $\begin{array}{l}7.02 \\
1.10772\end{array}$ & $\begin{array}{l}3.14525 \\
10.076\end{array}$ & $\begin{array}{l}6.756 \\
3.28561\end{array}$ & $\begin{array}{l}0.52963 \\
7.692\end{array}$ & 6.812 & 1.43505 & 8.472 & 4.60955 & 8.916 & 3.65701 & 8.696 \\
\hline 77.1237 & $\begin{array}{l}7.024 \\
0.643372\end{array}$ & $\begin{array}{l}0.702902 \\
10.08\end{array}$ & $\begin{array}{l}6.76 \\
0.766802\end{array}$ & $\begin{array}{l}1.94245 \\
7.696\end{array}$ & 6.816 & 1.28433 & 8.476 & 2.75645 & 8.92 & 1.43913 & 8.7 \\
\hline 79.4797 & $\begin{array}{l}7.028 \\
0.643372\end{array}$ & $\begin{array}{l}0.702902 \\
10.084\end{array}$ & $\begin{array}{l}6.764 \\
0.766802\end{array}$ & $\begin{array}{l}1.94245 \\
7.7\end{array}$ & 6.82 & 3.80253 & 8.48 & 0.24829 & 8.924 & 1.43913 & 8.704 \\
\hline 80.5291 & $\begin{array}{l}7.032 \\
0.643372\end{array}$ & $\begin{array}{l}3.14525 \\
10.088\end{array}$ & $\begin{array}{l}6.768 \\
0.766802\end{array}$ & $\begin{array}{l}1.94245 \\
7.704\end{array}$ & 6.824 & 2.08705 & 8.484 & 2.75645 & 8.928 & 1.19523 & 8.708 \\
\hline 79.7419 & $\begin{array}{l}7.036 \\
0.643372\end{array}$ & $\begin{array}{l}3.14525 \\
10.092\end{array}$ & $\begin{array}{l}6.772 \\
0.766802\end{array}$ & $\begin{array}{l}1.94245 \\
7.708\end{array}$ & 6.828 & 2.08705 & 8.488 & 0.24829 & 8.932 & 1.43913 & 8.712 \\
\hline 78.1699 & $\begin{array}{l}7.04 \\
0.643372\end{array}$ & $\begin{array}{l}0.702902 \\
10.096\end{array}$ & $\begin{array}{l}6.776 \\
3.28561\end{array}$ & $\begin{array}{l}0.52963 \\
7.712\end{array}$ & 6.832 & 1.28433 & 8.492 & 1.70707 & 8.936 & 2.09637 & 8.716 \\
\hline 75.2963 & $\begin{array}{l}7.044 \\
0.643372\end{array}$ & $\begin{array}{l}0.702902 \\
10.1\end{array}$ & $\begin{array}{l}6.78 \\
0.766802\end{array}$ & $\begin{array}{l}0.52963 \\
7.716\end{array}$ & 6.836 & 28433 & 8.496 & 2.75645 & 8.94 & .65701 & 8.72 \\
\hline 72.9533 & $\begin{array}{l}7.048 \\
0.643372\end{array}$ & $\begin{array}{l}1.56653 \\
10.104\end{array}$ & $\begin{array}{l}6.784 \\
3.28561\end{array}$ & $\begin{array}{l}1.94245 \\
7.72\end{array}$ & 6.84 & 1.28433 & 8.5 & 2.75645 & 8.944 & 1.43913 & 8.724 \\
\hline 72.6934 & $\begin{array}{l}7.052 \\
2.6337\end{array}$ & $\begin{array}{l}3.14525 \\
10.108\end{array}$ & $\begin{array}{l}6.788 \\
1.59443\end{array}$ & $\begin{array}{l}0.52963 \\
7.724\end{array}$ & 6.844 & 2.08705 & 8.504 & 0.24829 & 8.948 & 2.09637 & 8.728 \\
\hline 73.4733 & $\begin{array}{l}7.056 \\
0.643372\end{array}$ & $\begin{array}{l}3.14525 \\
10.112\end{array}$ & $\begin{array}{l}6.792 \\
0.766802\end{array}$ & $\begin{array}{l}0.52963 \\
7.728\end{array}$ & 6.848 & .08705 & 8.508 & 0.24829 & 8.952 & 1.79701 & 8.732 \\
\hline 73.7335 & $\begin{array}{l}7.06 \\
0.643372\end{array}$ & $\begin{array}{l}0.702902 \\
10.116\end{array}$ & $\begin{array}{l}6.796 \\
1.59443\end{array}$ & $\begin{array}{l}1.94245 \\
7.732\end{array}$ & 6.852 & 2.08705 & 8.512 & 4.60955 & 8.956 & 2.09637 & 8.736 \\
\hline 73.7335 & $\begin{array}{l}7.064 \\
0.643372\end{array}$ & $\begin{array}{l}0.702902 \\
10.12\end{array}$ & $\begin{array}{l}6.8 \\
0.766802\end{array}$ & $\begin{array}{l}0.52963 \\
7.736\end{array}$ & 6.856 & 1.43505 & 8.516 & 1.70707 & 8.96 & 2.09637 & 8.74 \\
\hline 72.9533 & $\begin{array}{l}7.068 \\
0.643372\end{array}$ & $\begin{array}{l}0.702902 \\
10.124\end{array}$ & $\begin{array}{l}6.804 \\
3.28561\end{array}$ & $\begin{array}{l}0.52963 \\
7.74\end{array}$ & 6.86 & 43505 & 8.52 & 0.24829 & 8.964 & 1.43913 & 8.744 \\
\hline 72.1739 & $\begin{array}{l}7.072 \\
1.66854\end{array}$ & $\begin{array}{l}3.14525 \\
10.128\end{array}$ & $\begin{array}{l}6.808 \\
0.766802\end{array}$ & $\begin{array}{l}2.21407 \\
7.744\end{array}$ & 6.864 & 1.43505 & 8.524 & 2.75645 & 8.968 & 1.19523 & 8.748 \\
\hline 70.8768 & $\begin{array}{l}7.076 \\
1.10772\end{array}$ & $\begin{array}{l}3.14525 \\
10.132\end{array}$ & $\begin{array}{l}6.812 \\
0.766802\end{array}$ & $\begin{array}{l}0.52963 \\
7.748\end{array}$ & 6.868 & 1.28433 & 8.528 & 2.75645 & 8.972 & 2.09637 & 8.752 \\
\hline 71.136 & $\begin{array}{l}7.08 \\
0.643372\end{array}$ & $\begin{array}{l}0.702902 \\
10.136\end{array}$ & $\begin{array}{l}6.816 \\
5.12258\end{array}$ & $\begin{array}{l}1.94245 \\
7.752\end{array}$ & 6.872 & 8433 & 532 & 24829 & 976 & 19523 & 8.756 \\
\hline 71.6548 & $\begin{array}{l}7.084 \\
1.10772\end{array}$ & $\begin{array}{l}0.702902 \\
10.14\end{array}$ & $\begin{array}{l}6.82 \\
3.28561\end{array}$ & $\begin{array}{l}1.94245 \\
7.756\end{array}$ & 6.876 & 1.28433 & 8.536 & 2.75645 & 8.98 & 3.65701 & 8.76 \\
\hline 72.6934 & $\begin{array}{l}7.088 \\
2.6337\end{array}$ & $\begin{array}{l}3.14525 \\
10.144\end{array}$ & $\begin{array}{l}6.824 \\
0.766802\end{array}$ & $\begin{array}{l}1.94245 \\
7.76\end{array}$ & 6.88 & 2.08705 & 8.54 & 4.60955 & 8.984 & 1.79701 & 8.764 \\
\hline 72.9533 & $\begin{array}{l}7.092 \\
0.643372\end{array}$ & $\begin{array}{l}0.702902 \\
10.148\end{array}$ & $\begin{array}{l}6.828 \\
0.766802\end{array}$ & $\begin{array}{l}1.94245 \\
7.764\end{array}$ & 6.884 & 1.43505 & 8.544 & 2.75645 & 8.988 & 3.65701 & 8.768 \\
\hline 72.6 & $\begin{array}{l}7.096 \\
0.643372\end{array}$ & $\begin{array}{l}4.94625 \\
10.152\end{array}$ & $\begin{array}{l}6.832 \\
0.766802\end{array}$ & $\begin{array}{l}1.94245 \\
7.768\end{array}$ & 6 & 3 & 8.548 & 9 & 8.992 & 23 & 72 \\
\hline 72.6934 & $\begin{array}{l}7.1 \\
1.10772\end{array}$ & $\begin{array}{l}0.702902 \\
10.156\end{array}$ & $\begin{array}{l}6.836 \\
3.28561\end{array}$ & $\begin{array}{l}2.21407 \\
7.772\end{array}$ & 6.892 & 2.08705 & 8.552 & 4.60955 & 8.996 & 1.79701 & 8.776 \\
\hline
\end{tabular}




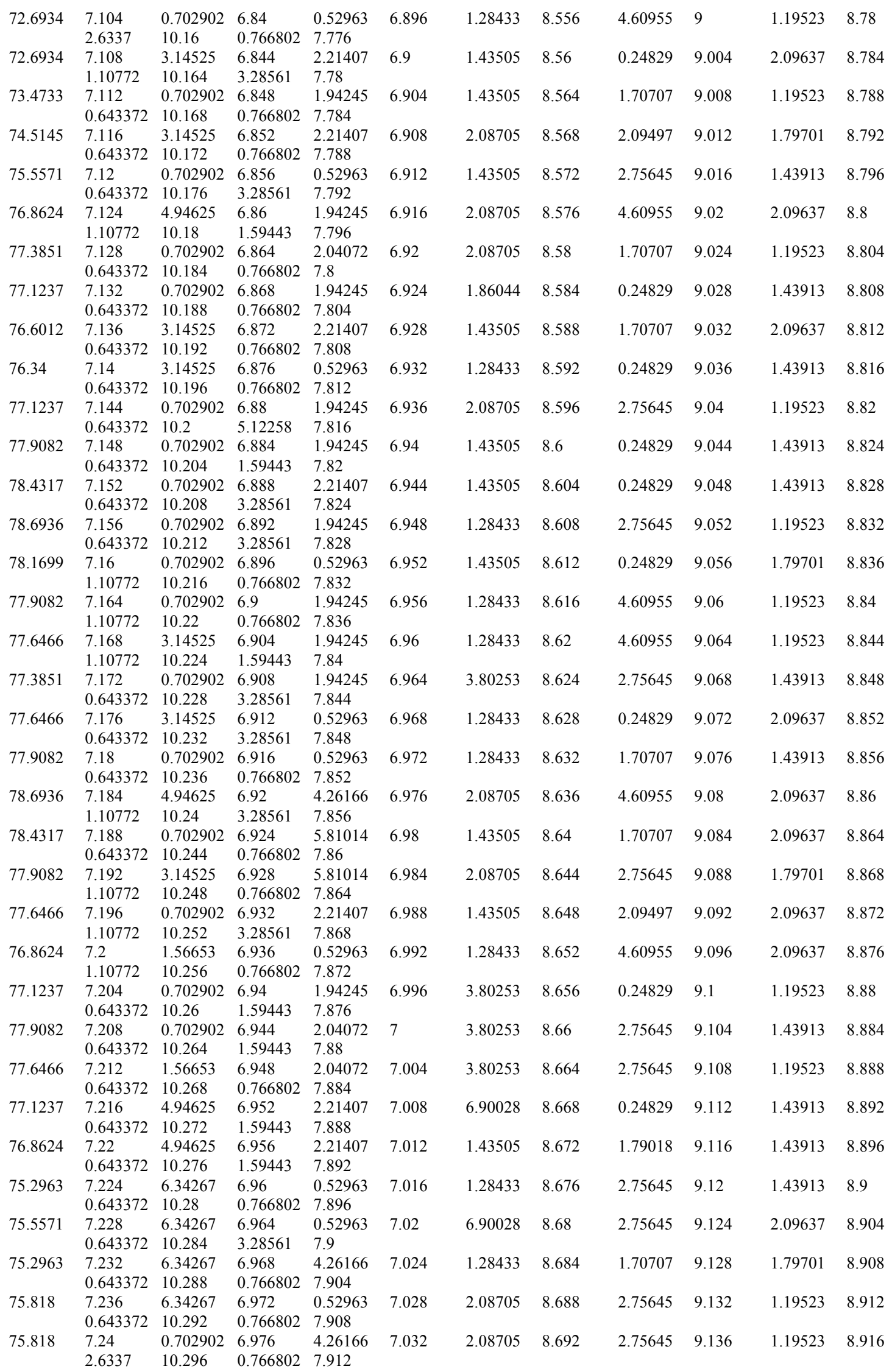




\begin{tabular}{|c|c|c|c|c|c|c|c|c|c|c|c|}
\hline 76.0789 & $\begin{array}{l}7.244 \\
0.643372\end{array}$ & $\begin{array}{l}0.702902 \\
10.3\end{array}$ & $\begin{array}{l}6.98 \\
1.59443\end{array}$ & $\begin{array}{l}2.21407 \\
7.916\end{array}$ & 7.036 & 1.43505 & 8.696 & 0.24829 & 9.14 & 2.09637 & 8.92 \\
\hline 75.5571 & $\begin{array}{l}7.248 \\
0.643372\end{array}$ & $\begin{array}{l}1.56653 \\
10.304\end{array}$ & $\begin{array}{l}6.984 \\
1.59443\end{array}$ & $\begin{array}{l}2.21407 \\
7.92\end{array}$ & 7.04 & 1.28433 & 8.7 & 0.24829 & 9.144 & 1.79701 & 8.924 \\
\hline 75.2963 & $\begin{array}{l}7.252 \\
0.643372\end{array}$ & $\begin{array}{l}1.56653 \\
10.308\end{array}$ & $\begin{array}{l}6.988 \\
0.766802\end{array}$ & $\begin{array}{l}7.05424 \\
7.924\end{array}$ & 7.044 & 1.43505 & 8.704 & 4.60955 & 9.148 & 1.79701 & 8.928 \\
\hline 74.5145 & $\begin{array}{l}7.256 \\
0.643372\end{array}$ & $\begin{array}{l}2.09685 \\
10.312\end{array}$ & $\begin{array}{l}6.992 \\
0.766802\end{array}$ & $\begin{array}{l}4.26166 \\
7.928\end{array}$ & 7.048 & 3.80253 & 8.708 & 2.75645 & 9.152 & 1.19523 & 8.932 \\
\hline 73.9937 & $\begin{array}{l}7.26 \\
0.643372\end{array}$ & $\begin{array}{l}0.702902 \\
10.316\end{array}$ & $\begin{array}{l}6.996 \\
0.766802\end{array}$ & $\begin{array}{l}2.21407 \\
7.932\end{array}$ & 7.052 & 1.43505 & 8.712 & 2.75645 & 9.156 & 1.43913 & 8.936 \\
\hline 74.5145 & $\begin{array}{l}7.264 \\
1.10772\end{array}$ & $\begin{array}{l}0.702902 \\
10.32\end{array}$ & $\begin{array}{l}7 \\
3.28561\end{array}$ & $\begin{array}{l}0.52963 \\
7.936\end{array}$ & 7.056 & 1.43505 & 8.716 & 2.75645 & 9.16 & 1.43913 & 8.94 \\
\hline 74.5145 & $\begin{array}{l}7.268 \\
0.643372\end{array}$ & $\begin{array}{l}6.34267 \\
10.324\end{array}$ & $\begin{array}{l}7.004 \\
0.766802\end{array}$ & $\begin{array}{l}1.94245 \\
7.94\end{array}$ & 7.06 & 1.28433 & 8.72 & 2.75645 & 9.164 & 1.19523 & 8.944 \\
\hline 75.0356 & $\begin{array}{l}7.272 \\
0.643372\end{array}$ & $\begin{array}{l}9.44018 \\
10.328\end{array}$ & $\begin{array}{l}7.008 \\
0.766802\end{array}$ & $\begin{array}{l}0.52963 \\
7.944\end{array}$ & 7.064 & 1.28433 & 8.724 & 0.24829 & 9.168 & 2.09637 & 8.948 \\
\hline 75.5571 & $\begin{array}{l}7.276 \\
0.643372\end{array}$ & $\begin{array}{l}9.44018 \\
10.332\end{array}$ & $\begin{array}{l}7.012 \\
0.766802\end{array}$ & $\begin{array}{l}0.52963 \\
7.948\end{array}$ & 7.068 & 2.08705 & 8.728 & 1.70707 & 9.172 & 3.65701 & 8.952 \\
\hline 75.0356 & $\begin{array}{l}7.28 \\
0.643372\end{array}$ & $\begin{array}{l}7.49597 \\
10.336\end{array}$ & $\begin{array}{l}7.016 \\
0.766802\end{array}$ & $\begin{array}{l}5.81014 \\
7.952\end{array}$ & 7.072 & 3.80253 & 8.732 & 0.24829 & 9.176 & .09637 & 8.956 \\
\hline 75.2963 & $\begin{array}{l}7.284 \\
0.643372\end{array}$ & $\begin{array}{l}4.94625 \\
10.34\end{array}$ & $\begin{array}{l}7.02 \\
3.28561\end{array}$ & $\begin{array}{l}4.26166 \\
7.956\end{array}$ & 7.076 & 1.43505 & 8.736 & 0.24829 & 9.18 & 1.79701 & 8.96 \\
\hline 74.775 & $\begin{array}{l}7.288 \\
0.643372\end{array}$ & $\begin{array}{l}3.14525 \\
10.344\end{array}$ & $\begin{array}{l}7.024 \\
0.766802\end{array}$ & $\begin{array}{l}5.81014 \\
7.96\end{array}$ & 7.08 & 1.28433 & 8.74 & 0.24829 & 9.184 & 2.09637 & 8.964 \\
\hline 75.2963 & $\begin{array}{l}7.292 \\
0.643372\end{array}$ & $\begin{array}{l}3.14525 \\
10.348\end{array}$ & $\begin{array}{l}7.028 \\
5.12258\end{array}$ & $\begin{array}{l}2.21407 \\
7.964\end{array}$ & 7.084 & 1.86044 & 8.744 & 0.24829 & 9.188 & 1.19523 & 8.968 \\
\hline 75.5571 & $\begin{array}{l}7.296 \\
0.643372\end{array}$ & $\begin{array}{l}3.14525 \\
10.352\end{array}$ & $\begin{array}{l}7.032 \\
3.28561\end{array}$ & $\begin{array}{l}2.21407 \\
7.968\end{array}$ & 7.088 & 1.28433 & 8.748 & 0.24829 & 9.192 & 1.43913 & 8.972 \\
\hline 76.34 & $\begin{array}{l}7.3 \\
0.643372\end{array}$ & $\begin{array}{l}4.94625 \\
10.356\end{array}$ & $\begin{array}{l}7.036 \\
3.28561\end{array}$ & $\begin{array}{l}2.21407 \\
7.972\end{array}$ & 7.092 & 1.28433 & 8.752 & 1.70707 & 9.196 & 3.65701 & 8.976 \\
\hline 77.1237 & $\begin{array}{l}7.304 \\
0.643372\end{array}$ & $\begin{array}{l}4.94625 \\
10.36\end{array}$ & $\begin{array}{l}7.04 \\
1.59443\end{array}$ & $\begin{array}{l}0.52963 \\
7.976\end{array}$ & 7.096 & 2.08705 & 8.756 & 0.24829 & 9.2 & 1.43913 & 8.98 \\
\hline 76.8624 & $\begin{array}{l}7.308 \\
0.643372\end{array}$ & $\begin{array}{l}8.50802 \\
10.364\end{array}$ & $\begin{array}{l}7.044 \\
1.59443\end{array}$ & $\begin{array}{l}2.21407 \\
7.98\end{array}$ & 7.1 & 1.28433 & 8.76 & 2.75645 & 9.204 & 3.65701 & 8.984 \\
\hline 76.0789 & $\begin{array}{l}7.312 \\
1.10772\end{array}$ & $\begin{array}{l}7.49597 \\
10.368\end{array}$ & $\begin{array}{l}7.048 \\
0.766802\end{array}$ & $\begin{array}{l}2.21407 \\
7.984\end{array}$ & 7.104 & .28433 & 8.764 & 0.24829 & 9.208 & 3.65701 & 8.988 \\
\hline 75.818 & $\begin{array}{l}7.316 \\
1.10772\end{array}$ & $\begin{array}{l}7.49597 \\
10.372\end{array}$ & $\begin{array}{l}7.052 \\
0.766802\end{array}$ & $\begin{array}{l}2.21407 \\
7.988\end{array}$ & 7.108 & 1.28433 & 8.768 & 4.60955 & 9.212 & 1.43913 & 8.992 \\
\hline 75.818 & $\begin{array}{l}7.32 \\
3.89069\end{array}$ & $\begin{array}{l}7.49597 \\
10.376\end{array}$ & $\begin{array}{l}7.056 \\
0.766802\end{array}$ & $\begin{array}{l}2.21407 \\
7.992\end{array}$ & 7.112 & 1.28433 & 8.772 & 4.60955 & 9.216 & 3.65701 & 8.996 \\
\hline 75.5571 & $\begin{array}{l}7.324 \\
1.10772\end{array}$ & $\begin{array}{l}4.94625 \\
10.38\end{array}$ & $\begin{array}{l}7.06 \\
0.766802\end{array}$ & $\begin{array}{l}1.94245 \\
7.996\end{array}$ & 7.116 & 3.80253 & 8.776 & 2.75645 & 9.22 & 1.43913 & 9 \\
\hline 76.0789 & $\begin{array}{l}7.328 \\
0.643372\end{array}$ & $\begin{array}{l}3.14525 \\
10.384\end{array}$ & $\begin{array}{l}7.064 \\
0.766802\end{array}$ & $\begin{array}{l}1.94245 \\
8\end{array}$ & 7.12 & 1.28433 & 8.78 & 0.24829 & 9.224 & 2.09637 & 9.004 \\
\hline 76.34 & $\begin{array}{l}7.332 \\
1.10772\end{array}$ & $\begin{array}{l}3.14525 \\
10.388\end{array}$ & $\begin{array}{l}7.068 \\
3.28561\end{array}$ & $\begin{array}{l}1.94245 \\
8.004\end{array}$ & 7.124 & 1.28433 & 8.784 & 1.70707 & 9.228 & 1.43913 & 9.008 \\
\hline 77.3851 & $\begin{array}{l}7.336 \\
0.643372\end{array}$ & $\begin{array}{l}3.14525 \\
10.392\end{array}$ & $\begin{array}{l}7.072 \\
0.766802\end{array}$ & $\begin{array}{l}1.94245 \\
8.008\end{array}$ & 7.128 & 1.43505 & 8.788 & 1.70707 & 9.232 & 3.65701 & 9.012 \\
\hline 76.8624 & $\begin{array}{l}7.34 \\
2.6337\end{array}$ & $\begin{array}{l}3.14525 \\
10.396\end{array}$ & $\begin{array}{l}7.076 \\
5.12258\end{array}$ & $\begin{array}{l}2.21407 \\
8.012\end{array}$ & 7.132 & 3.80253 & 8.792 & 2.09497 & 9.236 & 3.65701 & 9.016 \\
\hline 76.6012 & $\begin{array}{l}7.344 \\
0.643372\end{array}$ & $\begin{array}{l}3.14525 \\
10.4\end{array}$ & $\begin{array}{l}7.08 \\
3.28561\end{array}$ & $\begin{array}{l}5.81014 \\
8.016\end{array}$ & 7.136 & 1.28433 & 8.796 & 2.09497 & 9.24 & 1.19523 & 9.02 \\
\hline 76.0789 & $\begin{array}{l}7.348 \\
1.10772\end{array}$ & $\begin{array}{l}4.94625 \\
10.404\end{array}$ & $\begin{array}{l}7.084 \\
3.28561\end{array}$ & $\begin{array}{l}5.81014 \\
8.02\end{array}$ & 7.14 & .28433 & 8.8 & 1.70707 & 9.244 & .65701 & 9.024 \\
\hline 76.0789 & $\begin{array}{l}7.352 \\
0.643372\end{array}$ & $\begin{array}{l}4.94625 \\
10.408\end{array}$ & $\begin{array}{l}7.088 \\
5.12258\end{array}$ & $\begin{array}{l}5.81014 \\
8.024\end{array}$ & 7.144 & 3.80253 & 8.804 & 2.75645 & 9.248 & 1.19523 & 9.028 \\
\hline 76.34 & $\begin{array}{l}7.356 \\
0.643372\end{array}$ & $\begin{array}{l}7.49597 \\
10.412\end{array}$ & $\begin{array}{l}7.092 \\
3.28561\end{array}$ & $\begin{array}{l}7.05424 \\
8.028\end{array}$ & 7.148 & 1.28433 & 8.808 & 0.24829 & 9.252 & 3.65701 & 9.032 \\
\hline 77.6466 & $\begin{array}{l}7.36 \\
0.643372\end{array}$ & $\begin{array}{l}7.49597 \\
10.416\end{array}$ & $\begin{array}{l}7.096 \\
5.12258\end{array}$ & $\begin{array}{l}5.81014 \\
8.032\end{array}$ & 2 & 1.43505 & 2 & .24829 & 256 & 1.19523 & 036 \\
\hline 77.9082 & $\begin{array}{l}7.364 \\
0.643372\end{array}$ & $\begin{array}{l}7.49597 \\
10.42\end{array}$ & $\begin{array}{l}7.1 \\
0.766802\end{array}$ & $\begin{array}{l}4.26166 \\
8.036\end{array}$ & 7.156 & 1.43505 & 8.816 & 0.24829 & 9.26 & 3.65701 & 9.04 \\
\hline 77.9082 & $\begin{array}{l}7.368 \\
1.10772\end{array}$ & $\begin{array}{l}8.50802 \\
10.424\end{array}$ & $\begin{array}{l}7.104 \\
6.54045\end{array}$ & $\begin{array}{l}5.81014 \\
8.04\end{array}$ & 7.16 & 1.28433 & 8.82 & 0.24829 & 9.264 & 3.65701 & 9.044 \\
\hline 77.6466 & $\begin{array}{l}7.372 \\
1.10772\end{array}$ & $\begin{array}{l}7.49597 \\
10.428\end{array}$ & $\begin{array}{l}7.108 \\
3.28561\end{array}$ & $\begin{array}{l}4.26166 \\
8.044\end{array}$ & 7.164 & 2.08705 & 8.824 & 0.24829 & 9.268 & 3.65701 & 9.048 \\
\hline 77.3851 & $\begin{array}{l}7.376 \\
1.10772\end{array}$ & $\begin{array}{l}7.49597 \\
10.432\end{array}$ & $\begin{array}{l}7.112 \\
5.12258\end{array}$ & $\begin{array}{l}4.26166 \\
8.048\end{array}$ & 7 & 5 & 8.828 & 7 & 9.272 & 1 & 52 \\
\hline 76.6012 & $\begin{array}{l}7.38 \\
1.10772\end{array}$ & $\begin{array}{l}8.50802 \\
10.436\end{array}$ & $\begin{array}{l}7.116 \\
3.28561\end{array}$ & $\begin{array}{l}2.21407 \\
8.052\end{array}$ & 7.172 & 1.28433 & 8.832 & 2.75645 & 9.276 & 3.65701 & 9.056 \\
\hline
\end{tabular}




\begin{tabular}{|c|c|c|c|c|c|c|c|c|c|c|c|}
\hline 76.8624 & $\begin{array}{l}7.384 \\
0.643372\end{array}$ & $\begin{array}{l}4.94625 \\
10.44\end{array}$ & $\begin{array}{l}7.12 \\
1.59443\end{array}$ & $\begin{array}{l}2.21407 \\
8.056\end{array}$ & 7.176 & 1.28433 & 8.836 & 0.24829 & 9.28 & 1.79701 & 9.06 \\
\hline 77.3851 & $\begin{array}{l}7.388 \\
1.10772\end{array}$ & $\begin{array}{l}0.702902 \\
10.444\end{array}$ & $\begin{array}{l}7.124 \\
0.766802\end{array}$ & $\begin{array}{l}1.94245 \\
8.06\end{array}$ & 7.18 & 3.80253 & 8.84 & 0.24829 & 9.284 & 1.43913 & 9.064 \\
\hline 77.9082 & $\begin{array}{l}7.392 \\
0.643372\end{array}$ & $\begin{array}{l}0.702902 \\
10.448\end{array}$ & $\begin{array}{l}7.128 \\
0.766802\end{array}$ & $\begin{array}{l}0.52963 \\
8.064\end{array}$ & 7.184 & 2.08705 & 8.844 & 1.70707 & 9.288 & 3.65701 & 9.068 \\
\hline 77.9082 & $\begin{array}{l}7.396 \\
0.643372\end{array}$ & $\begin{array}{l}1.56653 \\
10.452\end{array}$ & $\begin{array}{l}7.132 \\
3.28561\end{array}$ & $\begin{array}{l}5.81014 \\
8.068\end{array}$ & 7.188 & 1.43505 & 8.848 & 0.24829 & 9.292 & 3.65701 & 9.072 \\
\hline 77.9082 & $\begin{array}{l}7.4 \\
0.643372\end{array}$ & $\begin{array}{l}6.34267 \\
10.456\end{array}$ & $\begin{array}{l}7.136 \\
0.766802\end{array}$ & $\begin{array}{l}7.05424 \\
8.072\end{array}$ & 7.192 & 1.28433 & 8.852 & 2.75645 & 9.296 & 3.65701 & 9.076 \\
\hline 77.1237 & $\begin{array}{l}7.404 \\
0.643372\end{array}$ & $\begin{array}{l}4.94625 \\
10.46\end{array}$ & $\begin{array}{l}7.14 \\
0.766802\end{array}$ & $\begin{array}{l}4.26166 \\
8.076\end{array}$ & 7.196 & 1.28433 & 8.856 & 0.24829 & 9.3 & 3.65701 & 9.08 \\
\hline 76.8624 & $\begin{array}{l}7.408 \\
1.10772\end{array}$ & $\begin{array}{l}8.50802 \\
10.464\end{array}$ & $\begin{array}{l}7.144 \\
1.59443\end{array}$ & $\begin{array}{l}7.05424 \\
8.08\end{array}$ & 7.2 & 1.28433 & 8.86 & 2.09497 & 9.304 & 3.65701 & 9.084 \\
\hline 76.8624 & $\begin{array}{l}7.412 \\
0.643372\end{array}$ & $\begin{array}{l}9.44018 \\
10.468\end{array}$ & $\begin{array}{l}7.148 \\
3.28561\end{array}$ & $\begin{array}{l}4.26166 \\
8.084\end{array}$ & 7.204 & 3.80253 & 8.864 & 0.24829 & 9.308 & 1.19523 & 9.088 \\
\hline 76.8624 & $\begin{array}{l}7.416 \\
1.10772\end{array}$ & $\begin{array}{l}11.1925 \\
10.472\end{array}$ & $\begin{array}{l}7.152 \\
0.766802\end{array}$ & $\begin{array}{l}2.21407 \\
8.088\end{array}$ & 7.208 & 1.43505 & 8.868 & 2.75645 & 9.312 & 1.43913 & 9.092 \\
\hline 76.8624 & $\begin{array}{l}7.42 \\
0.643372\end{array}$ & $\begin{array}{l}9.44018 \\
10.476\end{array}$ & $\begin{array}{l}7.156 \\
1.59443\end{array}$ & $\begin{array}{l}5.81014 \\
8.092\end{array}$ & 7.212 & 1.43505 & 8.872 & 2.75645 & 9.316 & 1.19523 & 9.096 \\
\hline 77.1237 & $\begin{array}{l}7.424 \\
0.643372\end{array}$ & $\begin{array}{l}8.50802 \\
10.48\end{array}$ & $\begin{array}{l}7.16 \\
5.12258\end{array}$ & $\begin{array}{l}5.81014 \\
8.096\end{array}$ & 7.216 & 1.43505 & 8.876 & 0.24829 & 9.32 & 1.19523 & 9.1 \\
\hline 77.6466 & $\begin{array}{l}7.428 \\
0.643372\end{array}$ & $\begin{array}{l}9.44018 \\
10.484\end{array}$ & $\begin{array}{l}7.164 \\
5.12258\end{array}$ & $\begin{array}{l}7.05424 \\
8.1\end{array}$ & 7.22 & 2.08705 & 8.88 & 6.03935 & 9.324 & 1.19523 & 9.104 \\
\hline 77.6466 & $\begin{array}{l}7.432 \\
0.643372\end{array}$ & $\begin{array}{l}7.49597 \\
10.488\end{array}$ & $\begin{array}{l}7.168 \\
0.766802\end{array}$ & $\begin{array}{l}5.81014 \\
8.104\end{array}$ & 7.224 & 1.28433 & 8.884 & 2.75645 & 9.328 & 1.43913 & 9.108 \\
\hline 76.8624 & $\begin{array}{l}7.436 \\
3.89069\end{array}$ & $\begin{array}{l}6.34267 \\
10.492\end{array}$ & $\begin{array}{l}7.172 \\
3.28561\end{array}$ & $\begin{array}{l}4.26166 \\
8.108\end{array}$ & 7.228 & 1.28433 & 8.888 & 1.70707 & 9.332 & 3.65701 & 9.112 \\
\hline 76.6012 & $\begin{array}{l}7.44 \\
1.10772\end{array}$ & $\begin{array}{l}7.49597 \\
10.496\end{array}$ & $\begin{array}{l}7.176 \\
3.28561\end{array}$ & $\begin{array}{l}5.81014 \\
8.112\end{array}$ & 7.232 & 2.08705 & 8.892 & 1.70707 & 9.336 & 5.45001 & 9.116 \\
\hline 76.6012 & $\begin{array}{l}7.444 \\
0.643372\end{array}$ & $\begin{array}{l}4.94625 \\
10.5\end{array}$ & $\begin{array}{l}7.18 \\
1.59443\end{array}$ & $\begin{array}{l}2.21407 \\
8.116\end{array}$ & 7.236 & 1.28433 & 8.896 & 0.24829 & 9.34 & 1.43913 & 9.12 \\
\hline 76.6012 & $\begin{array}{l}7.448 \\
1.10772\end{array}$ & $\begin{array}{l}4.94625 \\
10.504\end{array}$ & $\begin{array}{l}7.184 \\
1.59443\end{array}$ & $\begin{array}{l}4.26166 \\
8.12\end{array}$ & 7.24 & 1.28433 & 8.9 & 0.24829 & 9.344 & 1.19523 & 9.124 \\
\hline 77.3851 & $\begin{array}{l}7.452 \\
0.643372\end{array}$ & $\begin{array}{l}0.702902 \\
10.508\end{array}$ & $\begin{array}{l}7.188 \\
1.59443\end{array}$ & $\begin{array}{l}2.21407 \\
8.124\end{array}$ & 7.244 & 1.28433 & 8.904 & 2.75645 & 9.348 & 1.19523 & 9.128 \\
\hline 77.3851 & $\begin{array}{l}7.456 \\
1.10772\end{array}$ & $\begin{array}{l}4.94625 \\
10.512\end{array}$ & $\begin{array}{l}7.192 \\
0.766802\end{array}$ & $\begin{array}{l}0.52963 \\
8.128\end{array}$ & 7.248 & 1.28433 & 8.908 & 0.24829 & 9.352 & 3.65701 & 9.132 \\
\hline 77.9082 & $\begin{array}{l}7.46 \\
0.643372\end{array}$ & $\begin{array}{l}6.34267 \\
10.516\end{array}$ & $\begin{array}{l}7.196 \\
3.28561\end{array}$ & $\begin{array}{l}4.26166 \\
8.132\end{array}$ & 7.252 & 1.28433 & 8.912 & 2.75645 & 9.356 & 3.65701 & 9.136 \\
\hline 77.9082 & $\begin{array}{l}7.464 \\
2.6337\end{array}$ & $\begin{array}{l}8.50802 \\
10.52\end{array}$ & $\begin{array}{l}7.2 \\
3.28561\end{array}$ & $\begin{array}{l}5.81014 \\
8.136\end{array}$ & 7.256 & 1.28433 & 8.916 & 0.24829 & 9.36 & 3.65701 & 9.14 \\
\hline 77.6466 & $\begin{array}{l}7.468 \\
1.10772\end{array}$ & $\begin{array}{l}8.50802 \\
10.524\end{array}$ & $\begin{array}{l}7.204 \\
3.28561\end{array}$ & $\begin{array}{l}4.26166 \\
8.14\end{array}$ & 7.26 & 3.80253 & 8.92 & 2.75645 & 9.364 & 3.65701 & 9.144 \\
\hline 77.3851 & $\begin{array}{l}7.472 \\
1.10772\end{array}$ & $\begin{array}{l}7.49597 \\
10.528\end{array}$ & $\begin{array}{l}7.208 \\
3.28561\end{array}$ & $\begin{array}{l}4.26166 \\
8.144\end{array}$ & 7.264 & 2.08705 & 8.924 & 4.60955 & 9.368 & 5.45001 & 9.148 \\
\hline 77.6466 & $\begin{array}{l}7.476 \\
0.643372\end{array}$ & $\begin{array}{l}6.34267 \\
10.532\end{array}$ & $\begin{array}{l}7.212 \\
0.766802\end{array}$ & $\begin{array}{l}5.81014 \\
8.148\end{array}$ & 7.268 & 5.54023 & 8.928 & 0.24829 & 9.372 & 2.09637 & 9.152 \\
\hline 77.6466 & $\begin{array}{l}7.48 \\
2.6337\end{array}$ & $\begin{array}{l}4.94625 \\
10.536\end{array}$ & $\begin{array}{l}7.216 \\
0.766802\end{array}$ & $\begin{array}{l}4.26166 \\
8.152\end{array}$ & 7.272 & 1.28433 & 8.932 & 4.60955 & 9.376 & 2.09637 & 9.156 \\
\hline 77.9082 & $\begin{array}{l}7.484 \\
0.643372\end{array}$ & $\begin{array}{l}6.34267 \\
10.54\end{array}$ & $\begin{array}{l}7.22 \\
3.28561\end{array}$ & $\begin{array}{l}7.05424 \\
8.156\end{array}$ & 7.276 & 2.08705 & 8.936 & 2.75645 & 9.38 & 1.19523 & 9.16 \\
\hline 77.9082 & $\begin{array}{l}7.488 \\
0.643372\end{array}$ & $\begin{array}{l}7.49597 \\
10.544\end{array}$ & $\begin{array}{l}7.224 \\
0.766802\end{array}$ & $\begin{array}{l}5.81014 \\
8.16\end{array}$ & 7.28 & 1.43505 & 8.94 & 2.75645 & 9.384 & 1.19523 & 9.164 \\
\hline 77.6466 & $\begin{array}{l}7.492 \\
0.643372\end{array}$ & $\begin{array}{l}7.49597 \\
10.548\end{array}$ & $\begin{array}{l}7.228 \\
3.28561\end{array}$ & $\begin{array}{l}4.26166 \\
8.164\end{array}$ & 7.284 & 2.08705 & 8.944 & 0.24829 & 9.388 & 2.09637 & 9.168 \\
\hline 77.6466 & $\begin{array}{l}7.496 \\
0.643372\end{array}$ & $\begin{array}{l}7.49597 \\
10.552\end{array}$ & $\begin{array}{l}7.232 \\
0.766802\end{array}$ & $\begin{array}{l}4.26166 \\
8.168\end{array}$ & 7.288 & 3.80253 & 8.948 & 0.24829 & 9.392 & 3.65701 & 9.172 \\
\hline 76.8624 & $\begin{array}{l}7.5 \\
1.10772\end{array}$ & $\begin{array}{l}6.34267 \\
10.556\end{array}$ & $\begin{array}{l}7.236 \\
1.59443\end{array}$ & $\begin{array}{l}5.81014 \\
8.172\end{array}$ & 7.292 & 5.54023 & 8.952 & 0.24829 & 9.396 & 3.65701 & 9.176 \\
\hline 76.34 & $\begin{array}{l}7.504 \\
3.89069\end{array}$ & $\begin{array}{l}7.49597 \\
10.56\end{array}$ & $\begin{array}{l}7.24 \\
1.59443\end{array}$ & $\begin{array}{l}4.26166 \\
8.176\end{array}$ & 7.296 & 1.43505 & 8.956 & 4.60955 & 9.4 & 2.09637 & 9.18 \\
\hline 76.34 & $\begin{array}{l}7.508 \\
0.643372\end{array}$ & $\begin{array}{l}6.34267 \\
10.564\end{array}$ & $\begin{array}{l}7.244 \\
5.12258\end{array}$ & $\begin{array}{l}2.21407 \\
8.18\end{array}$ & 7.3 & 1.28433 & 8.96 & 1.70707 & 9.404 & 3.65701 & 9.184 \\
\hline 77.1237 & $\begin{array}{l}7.512 \\
0.643372\end{array}$ & $\begin{array}{l}7.49597 \\
10.568\end{array}$ & $\begin{array}{l}7.248 \\
0.766802\end{array}$ & $\begin{array}{l}5.81014 \\
8.184\end{array}$ & 7.304 & 1.28433 & 8.964 & 0.24829 & 9.408 & 1.43913 & 9.188 \\
\hline 77.1237 & $\begin{array}{l}7.516 \\
0.643372\end{array}$ & $\begin{array}{l}6.34267 \\
10.572\end{array}$ & $\begin{array}{l}7.252 \\
3.28561\end{array}$ & $\begin{array}{l}4.26166 \\
8.188\end{array}$ & 7.308 & 3.80253 & 8.968 & 2.09497 & 9.412 & 1.19523 & 9.192 \\
\hline 77.1237 & $\begin{array}{l}7.52 \\
1.10772\end{array}$ & $\begin{array}{l}7.49597 \\
10.576\end{array}$ & $\begin{array}{l}7.256 \\
0.766802\end{array}$ & $\begin{array}{l}4.26166 \\
8.192\end{array}$ & 7.312 & 1.28433 & 8.972 & 6.03935 & 9.416 & 1.19523 & 9.196 \\
\hline
\end{tabular}




\begin{tabular}{|c|c|c|c|c|c|c|c|c|c|c|c|}
\hline 76.8624 & $\begin{array}{l}7.524 \\
0.643372\end{array}$ & $\begin{array}{l}3.14525 \\
10.58\end{array}$ & $\begin{array}{l}7.26 \\
3.28561\end{array}$ & $\begin{array}{l}4.26166 \\
8.196\end{array}$ & 7.316 & 1.28433 & 8.976 & 0.24829 & 9.42 & 1.19523 & 9.2 \\
\hline 76.8624 & $\begin{array}{l}7.528 \\
1.10772\end{array}$ & $\begin{array}{l}6.34267 \\
10.584\end{array}$ & $\begin{array}{l}7.264 \\
3.28561\end{array}$ & $\begin{array}{l}4.26166 \\
8.2\end{array}$ & 7.32 & 2.08705 & 8.98 & 2.75645 & 9.424 & 1.19523 & 9.204 \\
\hline 76.34 & $\begin{array}{l}7.532 \\
1.10772\end{array}$ & $\begin{array}{l}7.49597 \\
10.588\end{array}$ & $\begin{array}{l}7.268 \\
0.766802\end{array}$ & $\begin{array}{l}2.21407 \\
8.204\end{array}$ & 7.324 & 1.43505 & 8.984 & 2.75645 & 9.428 & 1.19523 & 9.208 \\
\hline 75.818 & $\begin{array}{l}7.536 \\
0.643372\end{array}$ & $\begin{array}{l}7.49597 \\
10.592\end{array}$ & $\begin{array}{l}7.272 \\
3.28561\end{array}$ & $\begin{array}{l}7.05424 \\
8.208\end{array}$ & 7.328 & 2.08705 & 8.988 & 0.24829 & 9.432 & 1.19523 & 9.212 \\
\hline 77.1237 & $\begin{array}{l}7.54 \\
1.10772\end{array}$ & $\begin{array}{l}8.50802 \\
10.596\end{array}$ & $\begin{array}{l}7.276 \\
3.28561\end{array}$ & $\begin{array}{l}4.26166 \\
8.212\end{array}$ & 7.332 & 5.54023 & 8.992 & 1.79018 & 9.436 & 1.19523 & 9.216 \\
\hline 76.6012 & $\begin{array}{l}7.544 \\
0.643372\end{array}$ & $\begin{array}{l}8.50802 \\
10.6\end{array}$ & $\begin{array}{l}7.28 \\
5.12258\end{array}$ & $\begin{array}{l}5.81014 \\
8.216\end{array}$ & 7.336 & 1.43505 & 8.996 & 2.75645 & 9.44 & 1.19523 & 9.22 \\
\hline 76.8624 & $\begin{array}{l}7.548 \\
0.643372\end{array}$ & $\begin{array}{l}6.34267 \\
10.604\end{array}$ & $\begin{array}{l}7.284 \\
5.12258\end{array}$ & $\begin{array}{l}5.81014 \\
8.22\end{array}$ & 7.34 & 3.80253 & 9 & 4.60955 & 9.444 & 3.65701 & 9.224 \\
\hline 76.8624 & $\begin{array}{l}7.552 \\
1.10772\end{array}$ & $\begin{array}{l}7.49597 \\
10.608\end{array}$ & $\begin{array}{l}7.288 \\
0.766802\end{array}$ & $\begin{array}{l}2.21407 \\
8.224\end{array}$ & 7.344 & 5.54023 & 9.004 & 2.75645 & 9.448 & 1.19523 & 9.228 \\
\hline 76.8624 & $\begin{array}{l}7.556 \\
0.643372\end{array}$ & $\begin{array}{l}7.49597 \\
10.612\end{array}$ & $\begin{array}{l}7.292 \\
3.28561\end{array}$ & $\begin{array}{l}4.26166 \\
8.228\end{array}$ & 7.348 & 3.80253 & 9.008 & 0.24829 & 9.452 & 3.65701 & 9.232 \\
\hline 76.34 & $\begin{array}{l}7.56 \\
0.643372\end{array}$ & $\begin{array}{l}7.49597 \\
10.616\end{array}$ & $\begin{array}{l}7.296 \\
0.766802\end{array}$ & $\begin{array}{l}4.26166 \\
8.232\end{array}$ & 7.352 & 1.43505 & 9.012 & 2.75645 & 9.456 & 1.43913 & 9.236 \\
\hline 76.6012 & $\begin{array}{l}7.564 \\
0.643372\end{array}$ & $\begin{array}{l}7.49597 \\
10.62\end{array}$ & $\begin{array}{l}7.3 \\
0.766802\end{array}$ & $\begin{array}{l}4.26166 \\
8.236\end{array}$ & 7.356 & 3.80253 & 9.016 & 2.75645 & 9.46 & 1.43913 & 9.24 \\
\hline 76.34 & $\begin{array}{l}7.568 \\
1.10772\end{array}$ & $\begin{array}{l}6.34267 \\
10.624\end{array}$ & $\begin{array}{l}7.304 \\
0.766802\end{array}$ & $\begin{array}{l}5.81014 \\
8.24\end{array}$ & 7.36 & 1.28433 & 9.02 & 2.75645 & 9.464 & 3.65701 & 9.244 \\
\hline 76.8624 & $\begin{array}{l}7.572 \\
0.643372\end{array}$ & $\begin{array}{l}7.49597 \\
10.628\end{array}$ & $\begin{array}{l}7.308 \\
0.766802\end{array}$ & $\begin{array}{l}4.26166 \\
8.244\end{array}$ & 7.364 & 2.08705 & 9.024 & 0.24829 & 9.468 & 1.43913 & 9.248 \\
\hline 76.6012 & $\begin{array}{l}7.576 \\
0.643372\end{array}$ & $\begin{array}{l}6.34267 \\
10.632\end{array}$ & $\begin{array}{l}7.312 \\
0.766802\end{array}$ & $\begin{array}{l}2.21407 \\
8.248\end{array}$ & 7.368 & 2.08705 & 9.028 & 4.60955 & 9.472 & 3.65701 & 9.252 \\
\hline 77.3851 & $\begin{array}{l}7.58 \\
0.643372\end{array}$ & $\begin{array}{l}8.50802 \\
10.636\end{array}$ & $\begin{array}{l}7.316 \\
3.28561\end{array}$ & $\begin{array}{l}2.21407 \\
8.252\end{array}$ & 7.372 & 1.43505 & 9.032 & 4.60955 & 9.476 & 1.43913 & 9.256 \\
\hline 77.6466 & $\begin{array}{l}7.584 \\
1.10772\end{array}$ & $\begin{array}{l}7.49597 \\
10.64\end{array}$ & $\begin{array}{l}7.32 \\
3.28561\end{array}$ & $\begin{array}{l}2.21407 \\
8.256\end{array}$ & 7.376 & 1.28433 & 9.036 & 7.20896 & 9.48 & 1.19523 & 9.26 \\
\hline 77.9082 & $\begin{array}{l}7.588 \\
1.10772\end{array}$ & $\begin{array}{l}8.50802 \\
10.644\end{array}$ & $\begin{array}{l}7.324 \\
1.59443\end{array}$ & $\begin{array}{l}0.52963 \\
8.26\end{array}$ & 7.38 & 3.80253 & 9.04 & 2.75645 & 9.484 & 2.09637 & 9.264 \\
\hline 77.3851 & $\begin{array}{l}7.592 \\
0.643372\end{array}$ & $\begin{array}{l}9.44018 \\
10.648\end{array}$ & $\begin{array}{l}7.328 \\
3.28561\end{array}$ & $\begin{array}{l}7.05424 \\
8.264\end{array}$ & 7.384 & 1.28433 & 9.044 & 0.24829 & 9.488 & 1.43913 & 9.268 \\
\hline 76.8624 & $\begin{array}{l}7.596 \\
0.643372\end{array}$ & $\begin{array}{l}6.34267 \\
10.652\end{array}$ & $\begin{array}{l}7.332 \\
0.766802\end{array}$ & $\begin{array}{l}4.26166 \\
8.268\end{array}$ & 7.388 & 1.43505 & 9.048 & 4.60955 & 9.492 & 1.19523 & 9.272 \\
\hline 76.34 & $\begin{array}{l}7.6 \\
0.643372\end{array}$ & $\begin{array}{l}8.50802 \\
10.656\end{array}$ & $\begin{array}{l}7.336 \\
5.12258\end{array}$ & $\begin{array}{l}0.52963 \\
8.272\end{array}$ & 7.392 & 1.28433 & 9.052 & 2.75645 & 9.496 & 3.65701 & 9.276 \\
\hline 76.8624 & $\begin{array}{l}7.604 \\
1.10772\end{array}$ & $\begin{array}{l}7.49597 \\
10.66\end{array}$ & $\begin{array}{l}7.34 \\
0.766802\end{array}$ & $\begin{array}{l}2.21407 \\
8.276\end{array}$ & 7.396 & 1.43505 & 9.056 & 6.03935 & 9.5 & 3.65701 & 9.28 \\
\hline 77.3851 & $\begin{array}{l}7.608 \\
1.10772\end{array}$ & $\begin{array}{l}7.49597 \\
10.664\end{array}$ & $\begin{array}{l}7.344 \\
3.28561\end{array}$ & $\begin{array}{l}2.21407 \\
8.28\end{array}$ & 7.4 & 1.43505 & 9.06 & 4.60955 & 9.504 & 1.19523 & 9.284 \\
\hline 77.6466 & $\begin{array}{l}7.612 \\
1.10772\end{array}$ & $\begin{array}{l}7.49597 \\
10.668\end{array}$ & $\begin{array}{l}7.348 \\
1.59443\end{array}$ & $\begin{array}{l}4.26166 \\
8.284\end{array}$ & 7.404 & 3.80253 & 9.064 & 4.60955 & 9.508 & 1.19523 & 9.288 \\
\hline 77.6466 & $\begin{array}{l}7.616 \\
0.643372\end{array}$ & $\begin{array}{l}10.3282 \\
10.672\end{array}$ & $\begin{array}{l}7.352 \\
3.28561\end{array}$ & $\begin{array}{l}5.81014 \\
8.288\end{array}$ & 7.408 & 1.43505 & 9.068 & 2.75645 & 9.512 & 1.19523 & 9.292 \\
\hline 77.1237 & $\begin{array}{l}7.62 \\
1.10772\end{array}$ & $\begin{array}{l}8.50802 \\
10.676\end{array}$ & $\begin{array}{l}7.356 \\
3.28561\end{array}$ & $\begin{array}{l}2.21407 \\
8.292\end{array}$ & 7.412 & 1.43505 & 9.072 & 0.24829 & 9.516 & 5.45001 & 9.296 \\
\hline 77.3851 & $\begin{array}{l}7.624 \\
1.10772\end{array}$ & $\begin{array}{l}4.94625 \\
10.68\end{array}$ & $\begin{array}{l}7.36 \\
0.766802\end{array}$ & $\begin{array}{l}5.81014 \\
8.296\end{array}$ & 7.416 & 3.80253 & 9.076 & 4.60955 & 9.52 & 1.19523 & 9.3 \\
\hline 77.1237 & $\begin{array}{l}7.628 \\
0.643372\end{array}$ & $\begin{array}{l}4.94625 \\
10.684\end{array}$ & $\begin{array}{l}7.364 \\
3.28561\end{array}$ & $\begin{array}{l}4.26166 \\
8.3\end{array}$ & 7.42 & 1.28433 & 9.08 & 7.20896 & 9.524 & 1.43913 & 9.304 \\
\hline 77.3851 & $\begin{array}{l}7.632 \\
1.10772\end{array}$ & $\begin{array}{l}3.14525 \\
10.688\end{array}$ & $\begin{array}{l}7.368 \\
1.59443\end{array}$ & $\begin{array}{l}4.26166 \\
8.304\end{array}$ & 7.424 & 2.08705 & 9.084 & 2.75645 & 9.528 & 3.65701 & 9.308 \\
\hline 77.3851 & $\begin{array}{l}7.636 \\
1.10772\end{array}$ & $\begin{array}{l}4.94625 \\
10.692\end{array}$ & $\begin{array}{l}7.372 \\
1.59443\end{array}$ & $\begin{array}{l}4.26166 \\
8.308\end{array}$ & 7.428 & 1.28433 & 9.088 & 4.60955 & 9.532 & 2.09637 & 9.312 \\
\hline 77.6466 & $\begin{array}{l}7.64 \\
2.6337\end{array}$ & $\begin{array}{l}3.14525 \\
10.696\end{array}$ & $\begin{array}{l}7.376 \\
0.766802\end{array}$ & $\begin{array}{l}4.26166 \\
8.312\end{array}$ & 7.432 & 1.28433 & 9.092 & 0.24829 & 9.536 & 2.09637 & 9.316 \\
\hline 77.9082 & $\begin{array}{l}7.644 \\
1.10772\end{array}$ & $\begin{array}{l}7.49597 \\
10.7\end{array}$ & $\begin{array}{l}7.38 \\
3.28561\end{array}$ & $\begin{array}{l}2.21407 \\
8.316\end{array}$ & 7.436 & 1.43505 & 9.096 & 0.24829 & 9.54 & 5.45001 & 9.32 \\
\hline 77.9082 & $\begin{array}{l}7.648 \\
0.643372\end{array}$ & $\begin{array}{l}6.34267 \\
10.704\end{array}$ & $\begin{array}{l}7.384 \\
1.59443\end{array}$ & $\begin{array}{l}4.26166 \\
8.32\end{array}$ & 7.44 & 3.80253 & 9.1 & 0.24829 & 9.544 & 5.45001 & 9.324 \\
\hline 77.9082 & $\begin{array}{l}7.652 \\
0.643372\end{array}$ & $\begin{array}{l}7.49597 \\
10.708\end{array}$ & $\begin{array}{l}7.388 \\
3.28561\end{array}$ & $\begin{array}{l}5.81014 \\
8.324\end{array}$ & 7.444 & 1.28433 & 9.104 & 4.60955 & 9.548 & 3.65701 & 9.328 \\
\hline 77.9082 & $\begin{array}{l}7.656 \\
0.643372\end{array}$ & $\begin{array}{l}7.49597 \\
10.712\end{array}$ & $\begin{array}{l}7.392 \\
0.766802\end{array}$ & $\begin{array}{l}5.81014 \\
8.328\end{array}$ & 7.448 & 3.80253 & 9.108 & 0.24829 & 9.552 & 3.65701 & 9.332 \\
\hline 77.9082 & $\begin{array}{l}7.66 \\
0.643372\end{array}$ & $\begin{array}{l}6.34267 \\
10.716\end{array}$ & $\begin{array}{l}7.396 \\
3.28561\end{array}$ & $\begin{array}{l}7.05424 \\
8.332\end{array}$ & 7.452 & 3.80253 & 9.112 & 1.70707 & 9.556 & 3.65701 & 9.336 \\
\hline
\end{tabular}




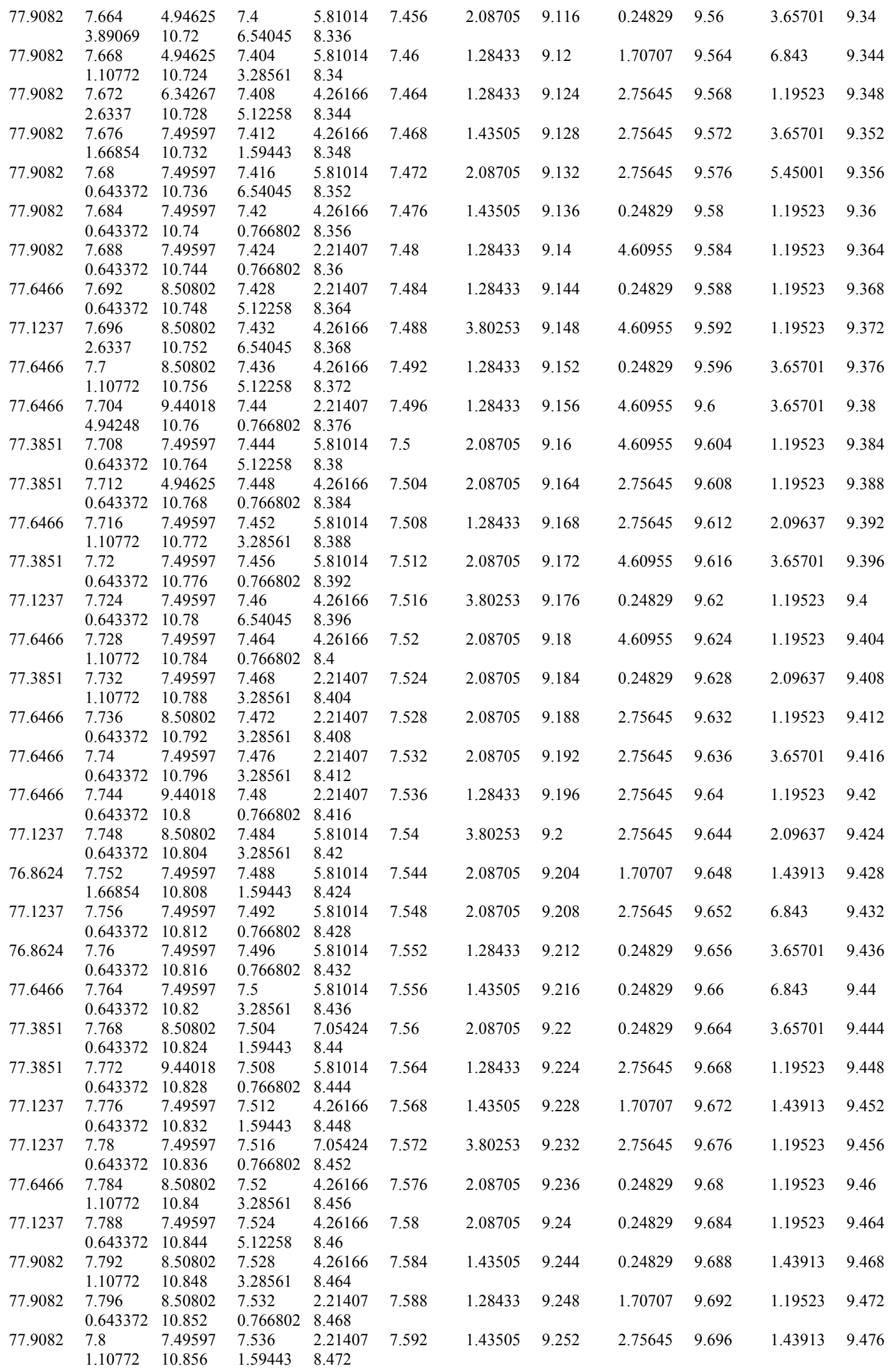




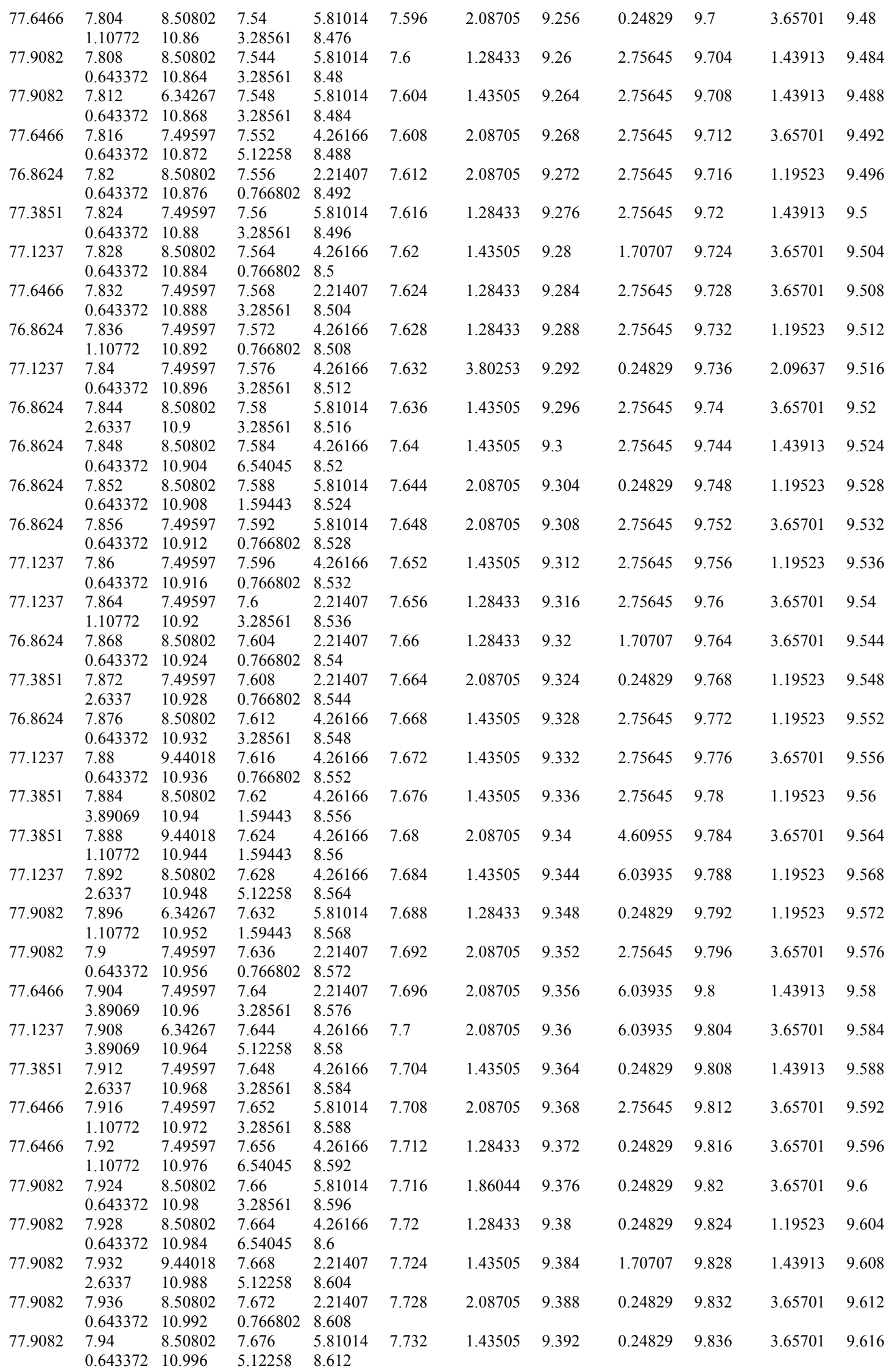




\begin{tabular}{|c|c|c|c|c|c|c|c|c|c|c|c|}
\hline 77.9082 & $\begin{array}{l}7.944 \\
0.643372\end{array}$ & $\begin{array}{l}9.44018 \\
11\end{array}$ & $\begin{array}{l}7.68 \\
3.28561\end{array}$ & $\begin{array}{l}0.52963 \\
8.616\end{array}$ & 7.736 & 3.80253 & 9.396 & 0.24829 & 9.84 & 3.65701 & 9.62 \\
\hline \multirow[t]{2}{*}{77.9082} & 7.948 & 8.50802 & 7.684 & 5.81014 & 7.74 & 3.80253 & 9.4 & 6.03935 & 9.844 & 3.65701 & 9.624 \\
\hline & 0.643372 & 11.004 & 3.28561 & 8.62 & & & & & & & \\
\hline \multirow[t]{2}{*}{77.9082} & 7.952 & 7.49597 & 7.688 & 5.81014 & 7.744 & 3.80253 & 9.404 & 0.24829 & 9.848 & 3.65701 & 9.628 \\
\hline & 1.10772 & 11.008 & 1.59443 & 8.624 & & & & & & & \\
\hline \multirow[t]{2}{*}{77.9082} & 7.956 & 7.49597 & 7.692 & 4.26166 & 7.748 & 2.08705 & 9.408 & 4.60955 & 9.852 & 3.65701 & 9.632 \\
\hline & 1.10772 & 11.012 & 0.766802 & 8.628 & & & & & & & \\
\hline \multirow[t]{2}{*}{77.9082} & 7.96 & 7.49597 & 7.696 & 0.52963 & 7.752 & 2.08705 & 9.412 & 0.24829 & 9.856 & 3.65701 & 9.636 \\
\hline & 0.643372 & 11.016 & 3.28561 & 8.632 & & & & & & & \\
\hline \multirow[t]{2}{*}{77.6466} & 7.964 & 7.49597 & 7.7 & 4.26166 & 7.756 & 1.28433 & 9.416 & 0.24829 & 9.86 & 3.65701 & 9.64 \\
\hline & 0.643372 & 11.02 & 5.12258 & 8.636 & & & & & & & \\
\hline \multirow[t]{2}{*}{77.9082} & 7.968 & 7.49597 & 7.704 & 5.81014 & 7.76 & 1.28433 & 9.42 & 0.24829 & 9.864 & 3.65701 & 9.644 \\
\hline & 3.89069 & 11.024 & 5.12258 & 8.64 & & & & & & & \\
\hline \multirow[t]{2}{*}{77.6466} & 7.972 & 6.34267 & 7.708 & 2.21407 & 7.764 & 3.80253 & 9.424 & 4.60955 & 9.868 & 5.45001 & 9.648 \\
\hline & 0.643372 & 11.0 & 5.12258 & 8.644 & & & & & & & \\
\hline \multirow[t]{2}{*}{77.9082} & 7.976 & 7.49597 & 7.712 & 5.81014 & 7.768 & 3.80253 & 9.428 & 2.75645 & 9.872 & 1.19523 & 9.652 \\
\hline & 0.643372 & 11.032 & 3.28561 & 8.648 & & & & & & & \\
\hline 77.6466 & 7.98 & 4.94625 & 7.716 & 4.26166 & 7.772 & 1.28433 & 9.432 & 0.24829 & 9.876 & 6.843 & 9.656 \\
\hline & 3.89069 & 11.036 & 5.12258 & 8.652 & & & & & & & \\
\hline 77.9082 & 7.984 & 4.94625 & 7.72 & 2.21407 & 7.776 & 1.28433 & 9.436 & 2.75645 & 9.88 & 3.65701 & 9.66 \\
\hline & 2.6337 & 11.04 & 1.59443 & 8.656 & & & & & & & \\
\hline 77.9082 & 7.988 & 6.34 & 7.724 & 4.26166 & 7.78 & 2.08705 & 9.44 & 0.24829 & 9.884 & 1.19523 & 9.664 \\
\hline & 1.10772 & 11.0 & 3.28 & 8.66 & & & & & & & \\
\hline 77.9082 & 7.992 & 6.34267 & 7.728 & 4.26166 & 7.784 & 1.28433 & 9.444 & 0.24829 & 9.888 & 3.65701 & 9.668 \\
\hline & 2.6337 & 11.048 & 5.12258 & 8.664 & & & & & & & \\
\hline 77.9082 & 7.996 & 4.94625 & 7.732 & 4.26166 & 7.788 & 1.28433 & 9.448 & 1.79018 & 9.892 & 3.65701 & 9.672 \\
\hline & 0.643372 & 11.052 & 1.59443 & 8.668 & & & & & & & \\
\hline 77.9082 & 8 & 4.94625 & 7.736 & 5.81014 & 7.792 & 1.43505 & 9.452 & 1.70707 & 9.896 & 3.65701 & 9.676 \\
\hline & 1.10772 & 11. & 3.28561 & 8.6 & & & & & & & \\
\hline 77.6466 & 8.004 & 7.4 & 7.74 & 2.21407 & 7.796 & 3.80253 & 9.456 & 4.60955 & 9.9 & 3.65701 & 9.68 \\
\hline & 1.10772 & 11.06 & 0.766802 & 8.676 & & & & & & & \\
\hline 77.6466 & 8.008 & 7.49597 & 7.744 & 4.26166 & 7.8 & 3.80253 & 9.46 & 2.75645 & 9.904 & 3.65701 & 9.684 \\
\hline & 0.643372 & 11.0 & 0.766802 & 8.68 & & & & & & & \\
\hline 77.9082 & 8.012 & 7.49597 & 7.748 & 5.81014 & 7.804 & 1.28433 & 9.464 & 4.60955 & 9.908 & 1.19523 & 9.688 \\
\hline & 0.643372 & 11.0 & 3.28 & 8.6 & & & & & & & \\
\hline 77.9082 & 8.016 & 6.34 & 7.752 & 2.21407 & 7.808 & 3.80253 & 9.468 & 0.24829 & 9.912 & 1.19523 & 9.692 \\
\hline & 0.643372 & 11.0 & 3.28561 & 8.688 & & & & & & & \\
\hline 77.9082 & 8.02 & 7.49597 & 7.756 & 2.21407 & 7.812 & 1.28433 & 9.472 & 2.75645 & 9.916 & 3.65701 & 9.696 \\
\hline & 0.643372 & 11.0 & 5.12 & 8.6 & & & & & & & \\
\hline 77.3851 & 8.024 & 4.94625 & 7.76 & 4.26166 & 7.816 & 3.80253 & 9.476 & 0.24829 & 9.92 & 3.65701 & 9.7 \\
\hline & 0.643372 & 11.08 & 0.766802 & 8.696 & & & & & & & \\
\hline 77.9082 & 8.028 & 8.50802 & 7.764 & 4.26166 & 7.82 & 1.43505 & 9.48 & 0.24829 & 9.924 & 1.43913 & 9.704 \\
\hline & 0.643372 & 1 & 3.2 & 8. & & & & & & & \\
\hline 77.6466 & 8.032 & 7.49597 & 7.768 & 5.81014 & 7.824 & 3.80253 & 9.484 & 2.75645 & 9.928 & 3.65701 & 9.708 \\
\hline & 0.643372 & 11.088 & 5.12258 & 8.7 & & & & & & & \\
\hline 77.9082 & 8.036 & 7.49597 & 7.772 & 5.81014 & 7.828 & 3.80253 & 9.488 & 0.24829 & 9.932 & 3.65701 & 9.712 \\
\hline & 0.643372 & 11.092 & 5.12258 & 8.708 & & & & & & & \\
\hline 77.9082 & 8.04 & 6.34267 & 7.776 & 4.26166 & 7.832 & 1.28433 & 9.492 & 0.24829 & 9.936 & 5.45001 & 9.716 \\
\hline & 0.643372 & 1 & 61 & 8. & & & & & & & \\
\hline 77.9082 & 8.044 & 6.34267 & 7.7 & 5.81014 & 7.836 & 1.43505 & 9.496 & 2.75645 & 9.94 & 5.45001 & 9.72 \\
\hline & 0.643372 & 1 & & & & & & & & & \\
\hline 77.6466 & 8.048 & 6.34267 & 7.784 & 5.81014 & 7.84 & 2.08705 & 9.5 & 0.24829 & 9.944 & 3.65701 & 9.724 \\
\hline & 0.643372 & 11.10 & 0.766802 & 8.72 & & & & & & & \\
\hline 77.9082 & 8.052 & 6.34267 & 7.788 & 5.81014 & 7.844 & 3.80253 & 9.504 & 4.60955 & 9.948 & 5.45001 & 9.728 \\
\hline & 0.643372 & 11.1 & 3.28561 & 8.72 & & & & & & & \\
\hline 77.3851 & 8.056 & 4.94625 & 7.79 & 4.26166 & 7.848 & 1.43505 & 9.508 & 2.75645 & 9.952 & 3.65701 & 9.732 \\
\hline & 0.643372 & 11. & & & & & & & & & \\
\hline 77.9082 & 8.06 & 7.49597 & 7.796 & 5.81014 & 7.852 & 1.28433 & 9.512 & 0.24829 & 9.956 & 6.843 & 9.736 \\
\hline & 1.10772 & 11. & 3.28 & 8.7 & & & & & & & \\
\hline 77.9082 & 8.064 & 6.34267 & 7.8 & 7.05424 & 7.856 & 1.43505 & 9.516 & 0.24829 & 9.96 & 3.65701 & 9.74 \\
\hline & 0.643372 & 11.12 & 3.28561 & 8.736 & & & & & & & \\
\hline 77.9082 & 8.068 & 4.94625 & 7.804 & 5.81014 & 7.86 & 1.43505 & 9.52 & 0.24829 & 9.964 & 3.65701 & 9.744 \\
\hline & 0.643372 & & & 8.7 & & & & & & & \\
\hline 77.9082 & 8.072 & 3.14525 & 7.808 & 5.81014 & 7.864 & 1.28433 & 9.524 & 2.75645 & 9.968 & 1.19523 & 9.748 \\
\hline & 0.64 & & & & & & & & & & \\
\hline 77.9082 & 8.076 & 7.49597 & 7.812 & 4.26166 & 7.868 & 1.28433 & 9.528 & 4.60955 & 9.972 & 1.19523 & 9.752 \\
\hline & 0.643372 & 11.132 & 5.12258 & 8.748 & & & & & & & \\
\hline 77.3851 & 8.08 & 4.94625 & 7.816 & 4.26166 & 7.872 & 1.28433 & 9.532 & 1.70707 & 9.976 & 3.65701 & 9.756 \\
\hline & 0.643372 & 11.136 & 5.12258 & 8.75 & & & & & & & \\
\hline
\end{tabular}




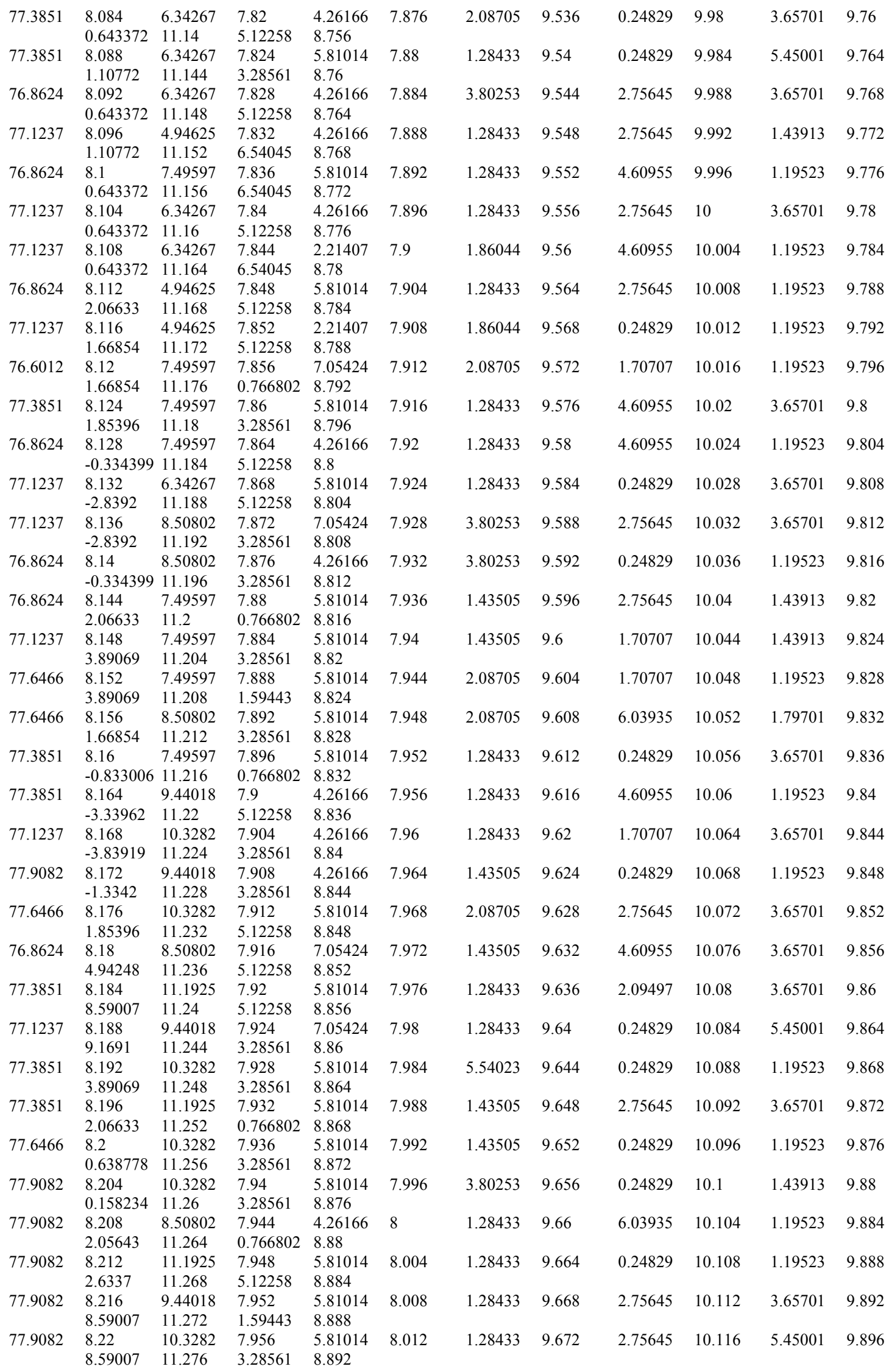




\begin{tabular}{|c|c|c|c|c|c|c|c|c|c|c|c|}
\hline 77.9082 & $\begin{array}{l}8.224 \\
3.89069\end{array}$ & $\begin{array}{l}9.44018 \\
11.28\end{array}$ & $\begin{array}{l}7.96 \\
5.12258\end{array}$ & $\begin{array}{l}8.11986 \\
8.896\end{array}$ & 8.016 & 1.28433 & 9.676 & 0.24829 & 10.12 & 1.19523 & 9.9 \\
\hline 77.9082 & $\begin{array}{l}8.228 \\
1.66854\end{array}$ & $\begin{array}{l}11.1925 \\
11.284\end{array}$ & $\begin{array}{l}7.964 \\
3.28561\end{array}$ & $\begin{array}{l}5.81014 \\
8.9\end{array}$ & 8.02 & 1.28433 & 9.68 & 0.24829 & 10.124 & 1.19523 & 9.904 \\
\hline 77.9082 & $\begin{array}{l}8.232 \\
0.158234\end{array}$ & $\begin{array}{l}12.0445 \\
11.288\end{array}$ & $\begin{array}{l}7.968 \\
3.28561\end{array}$ & $\begin{array}{l}5.81014 \\
8.904\end{array}$ & 8.024 & 2.08705 & 9.684 & 2.75645 & 10.128 & 2.09637 & 9.908 \\
\hline 77.9082 & $\begin{array}{l}8.236 \\
-0.334399\end{array}$ & $\begin{array}{l}16.2638 \\
11.292\end{array}$ & $\begin{array}{l}7.972 \\
3.28561\end{array}$ & $\begin{array}{l}5.81014 \\
8.908\end{array}$ & 8.028 & 1.43505 & 9.688 & 1.70707 & 10.132 & 1.19523 & 9.912 \\
\hline 78.1699 & $\begin{array}{l}8.24 \\
0.638778\end{array}$ & $\begin{array}{l}20.5032 \\
11.296\end{array}$ & $\begin{array}{l}7.976 \\
3.28561\end{array}$ & $\begin{array}{l}4.26166 \\
8.912\end{array}$ & 8.032 & 1.28433 & 9.692 & 0.24829 & 10.136 & 3.65701 & 9.916 \\
\hline 77.9082 & $\begin{array}{l}8.244 \\
2.6337\end{array}$ & $\begin{array}{l}27.3601 \\
11.3\end{array}$ & $\begin{array}{l}7.98 \\
0.766802\end{array}$ & $\begin{array}{l}5.81014 \\
8.916\end{array}$ & 8.036 & 1.43505 & 9.696 & 0.24829 & 10.14 & 1.19523 & 9.92 \\
\hline 77.9082 & $\begin{array}{l}8.248 \\
7.3342\end{array}$ & $\begin{array}{l}36.0579 \\
11.304\end{array}$ & $\begin{array}{l}7.984 \\
3.28561\end{array}$ & $\begin{array}{l}4.26166 \\
8.92\end{array}$ & 8.04 & 1.28433 & 9.7 & 0.24829 & 10.144 & 3.65701 & 9.924 \\
\hline 77.9082 & $\begin{array}{l}8.252 \\
10.274\end{array}$ & & $\begin{array}{l}7.988 \\
1.59443\end{array}$ & $\begin{array}{l}5.81014 \\
8.924\end{array}$ & 8.044 & 1.43505 & 9.704 & 1.70707 & 10.148 & 3.65701 & 9.928 \\
\hline 77.9082 & $\begin{array}{l}8.256 \\
9.72836\end{array}$ & & $\begin{array}{l}7.992 \\
3.28561\end{array}$ & $\begin{array}{l}5.81014 \\
8.928\end{array}$ & 8.048 & .08705 & 9.708 & .75645 & 10.152 & 6.843 & 9.932 \\
\hline 77.9082 & $\begin{array}{l}8.26 \\
7.98251\end{array}$ & $\begin{array}{l}65.6995 \\
11.316\end{array}$ & $\begin{array}{l}7.996 \\
3.28561\end{array}$ & $\begin{array}{l}5.81014 \\
8.932\end{array}$ & 8.052 & 1.43505 & 9.712 & 2.75645 & 10.156 & 3.65701 & 9.936 \\
\hline 77.9082 & $\begin{array}{l}8.264 \\
1.10772\end{array}$ & $\begin{array}{l}70.3089 \\
11.32\end{array}$ & $\begin{array}{l}8 \\
3.28561\end{array}$ & & 8.056 & 1.43505 & 9.716 & 0.24829 & 10.16 & 3.65701 & 9.94 \\
\hline 77.9082 & $\begin{array}{l}8.268 \\
1.66854\end{array}$ & & & $\begin{array}{l}5.81014 \\
8.94\end{array}$ & 8.06 & 3.80253 & 9.72 & 24829 & 10.164 & 65701 & 9.944 \\
\hline 77.9082 & $\begin{array}{l}8.272 \\
1.66854\end{array}$ & $\begin{array}{l}74.9 \\
11.3\end{array}$ & & $\begin{array}{l}4.26166 \\
8.944\end{array}$ & 8.064 & .80253 & 9.724 & 4.60955 & 10.168 & 3.65701 & 9.948 \\
\hline 77.9082 & $\begin{array}{l}8.276 \\
1.10772\end{array}$ & & & $\begin{array}{l}5.81014 \\
8.948\end{array}$ & 8.068 & 1.43505 & 9.728 & 0.24829 & 10.172 & 3.65701 & 9.952 \\
\hline 78.1699 & $\begin{array}{l}8.28 \\
7.3342\end{array}$ & & & & 8.072 & 1.43505 & 9.732 & 2.75645 & 10.176 & 3.65701 & 9.956 \\
\hline 78.1699 & $\begin{array}{l}8.284 \\
10.8104\end{array}$ & $\begin{array}{l}74 . \\
11 .\end{array}$ & $\begin{array}{l}8.02 \\
6.54045\end{array}$ & 614 & 8.076 & 28433 & 9.736 & 75645 & 10.18 & 19523 & 9.96 \\
\hline 78.1699 & $\begin{array}{l}8.288 \\
12.9123\end{array}$ & $\begin{array}{l}72.1 \\
11.3\end{array}$ & $\begin{array}{l}8.024 \\
0.766802\end{array}$ & $\begin{array}{l}4.26166 \\
8.96\end{array}$ & 8.08 & 1.43505 & 9.74 & 2.09497 & 10.184 & .43913 & 9.964 \\
\hline 78.4317 & $\begin{array}{l}8.292 \\
12.3903\end{array}$ & & & & 8.084 & 1.28433 & 9.744 & 0.24829 & 10.188 & 3.65701 & 9.968 \\
\hline 77.9082 & $\begin{array}{l}8.296 \\
11.3407\end{array}$ & & & 07 & 8.088 & 1.28433 & 9.748 & 2.75645 & 10.192 & 3.65701 & 9.972 \\
\hline 77.9082 & $\begin{array}{l}8.3 \\
8.59007\end{array}$ & $\begin{array}{l}74.0176 \\
11.356\end{array}$ & $\begin{array}{l}8.036 \\
0.766802\end{array}$ & $\begin{array}{l}5.81014 \\
8.972\end{array}$ & 8.092 & .28433 & 9.752 & 0.24829 & 10.196 & 3.65701 & 9.976 \\
\hline 78.6936 & $\begin{array}{l}8.304 \\
5.84133\end{array}$ & $\begin{array}{l}74.9477 \\
11.36\end{array}$ & $\begin{array}{l}8.04 \\
5.12258\end{array}$ & $\begin{array}{l}5.81014 \\
8.976\end{array}$ & 8.096 & 1.43505 & 9.756 & 4.60955 & 10.2 & 65701 & 9.98 \\
\hline 77.9082 & $\begin{array}{l}8.308 \\
5.84133\end{array}$ & & & $\begin{array}{l}5.81014 \\
8.98\end{array}$ & 8.1 & 1.43505 & 9.76 & 0.24829 & 10.204 & 3.65701 & 9.984 \\
\hline 78.1699 & $\begin{array}{l}8.312 \\
7.3342\end{array}$ & $\begin{array}{l}75.8 \\
11.3\end{array}$ & & $\begin{array}{l}2.21407 \\
8.984\end{array}$ & 8.104 & 1.43505 & 9.764 & 2.75645 & 10.208 & 3.65701 & 9.988 \\
\hline 78.9555 & $\begin{array}{l}8.316 \\
9.1691\end{array}$ & $\begin{array}{l}75.8 \\
11.3\end{array}$ & $\begin{array}{l}8.052 \\
5.12258\end{array}$ & $\begin{array}{l}5.81014 \\
8.988\end{array}$ & 8.108 & 3.80253 & 9.768 & 4.60955 & 10.212 & 3.65701 & 9.992 \\
\hline 79.2176 & $\begin{array}{l}8.32 \\
10.274\end{array}$ & & & $\begin{array}{l}5.81014 \\
8.992\end{array}$ & 8.112 & 3.80253 & 9.772 & 2.75645 & 10.216 & 3.65701 & 9.996 \\
\hline 78.9555 & $\begin{array}{l}8.324 \\
10.8104\end{array}$ & & $\begin{array}{l}8.06 \\
6.54045\end{array}$ & & 8.116 & 43505 & 9.776 & .75645 & 10.22 & .65701 & 10 \\
\hline 78.6936 & $\begin{array}{l}8.328 \\
9.72836\end{array}$ & $\begin{array}{l}77.7448 \\
11.384\end{array}$ & $\begin{array}{l}8.064 \\
6.54045\end{array}$ & $\begin{array}{l}4.26166 \\
9\end{array}$ & 8.12 & 2.08705 & 9.78 & 2.75645 & 10.224 & 3.65701 & 10.004 \\
\hline 78.9555 & $\begin{array}{l}8.332 \\
7.3342\end{array}$ & $\begin{array}{l}77.7448 \\
11.388\end{array}$ & $\begin{array}{l}8.068 \\
3.28561\end{array}$ & $\begin{array}{l}4.26166 \\
9.004\end{array}$ & 8.124 & 1.28433 & 9.784 & 4.60955 & 10.228 & 5.45001 & 10.008 \\
\hline 78.9555 & $\begin{array}{l}8.336 \\
6.62819\end{array}$ & & & & 8128 & 2.08705 & .788 & .75645 & 10.232 & 1.19523 & 10.012 \\
\hline 78.9555 & $\begin{array}{l}8.34 \\
3.89069\end{array}$ & $\begin{array}{l}78.6795 \\
11.396\end{array}$ & $\begin{array}{l}8.076 \\
5.1225\end{array}$ & $\begin{array}{l}2.21407 \\
9.012\end{array}$ & 8.132 & 1.43505 & 9.792 & 1.70707 & 10.236 & 3.65701 & 10.016 \\
\hline 78.9555 & $\begin{array}{l}8.344 \\
0.643372\end{array}$ & $\begin{array}{l}79.6153 \\
11.4\end{array}$ & $\begin{array}{l}8.08 \\
5.12258\end{array}$ & $\begin{array}{l}4.26166 \\
9.016\end{array}$ & 8.136 & 1.43505 & 9.796 & 0.24829 & 10.24 & 3.65701 & 10.02 \\
\hline 78.9555 & $\begin{array}{l}8.348 \\
4.94248\end{array}$ & $\begin{array}{l}80.5522 \\
11.404\end{array}$ & $\begin{array}{l}8.084 \\
3.28561\end{array}$ & $\begin{array}{l}5.81014 \\
9.02\end{array}$ & 8.14 & 05 & 8 & 5 & 4 & 23 & 24 \\
\hline 77.9082 & $\begin{array}{l}8.352 \\
5.84133\end{array}$ & & & & 8.144 & 3.80253 & 9.804 & 24829 & 10.248 & 19523 & 0.028 \\
\hline 78.1699 & $\begin{array}{l}8.356 \\
4.94248\end{array}$ & $\begin{array}{l}79.6153 \\
11.412\end{array}$ & $\begin{array}{l}8.092 \\
5.12258\end{array}$ & $\begin{array}{l}5.81014 \\
9.028\end{array}$ & 8.148 & 1.28433 & 9.808 & 1.70707 & 10.252 & 1.19523 & 10.032 \\
\hline 78.4317 & $\begin{array}{l}8.36 \\
3.89069\end{array}$ & $\begin{array}{l}81.4902 \\
11.416\end{array}$ & $\begin{array}{l}8.096 \\
5.12258\end{array}$ & $\begin{array}{l}4.26166 \\
9.032\end{array}$ & 8.152 & 3.80253 & 9.812 & 0.24829 & 10.256 & 3.65701 & 10.036 \\
\hline
\end{tabular}




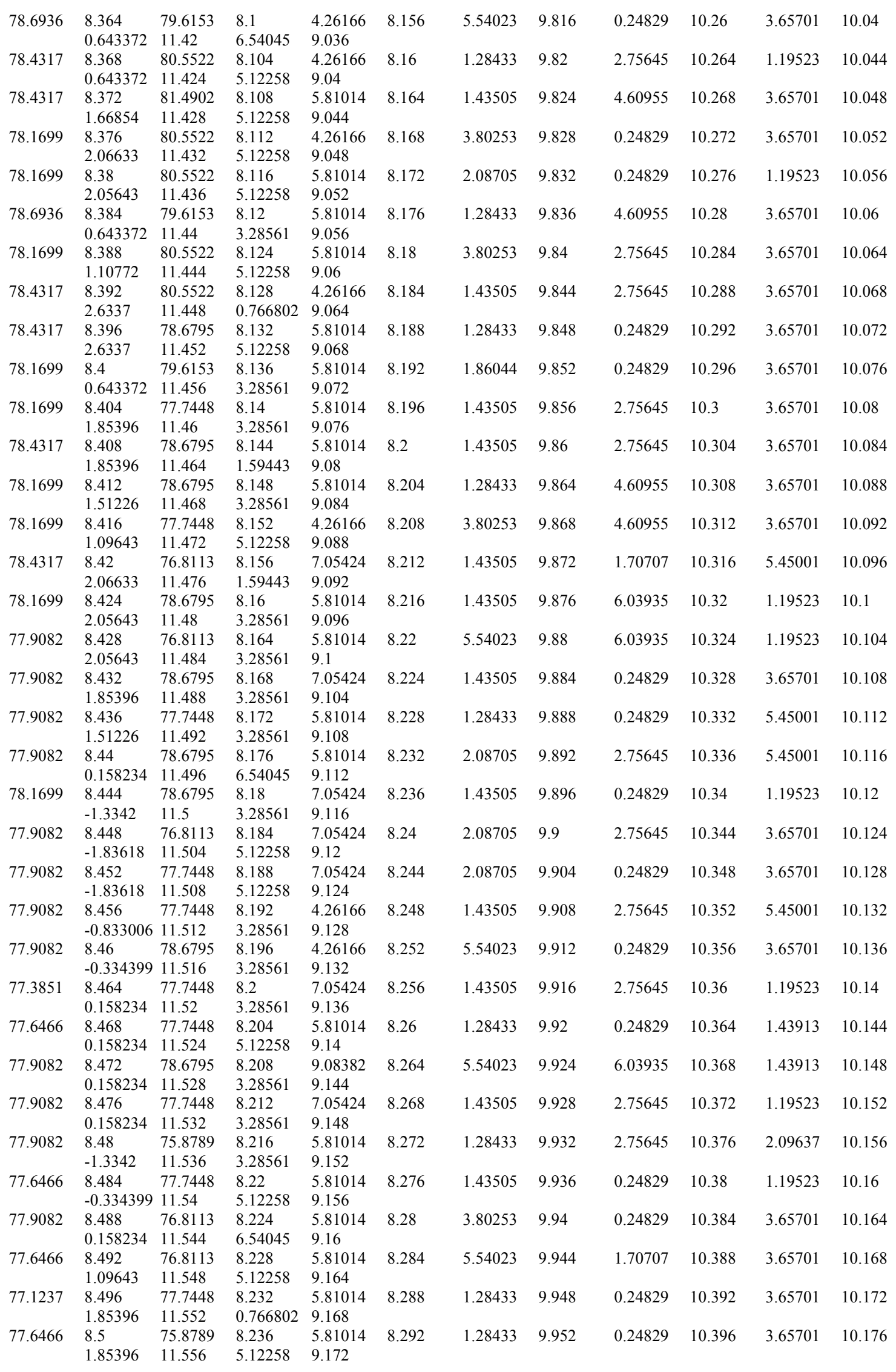




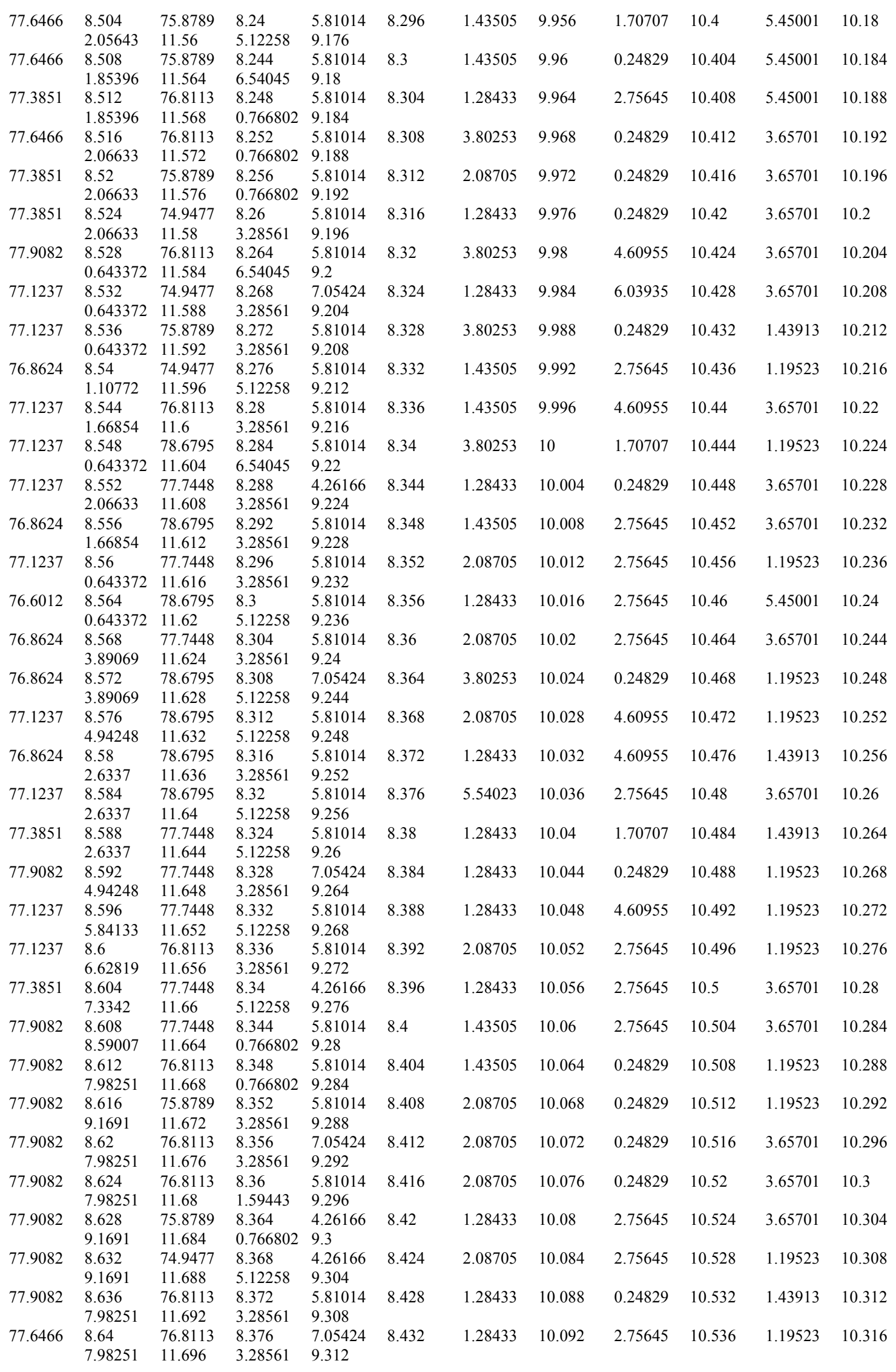




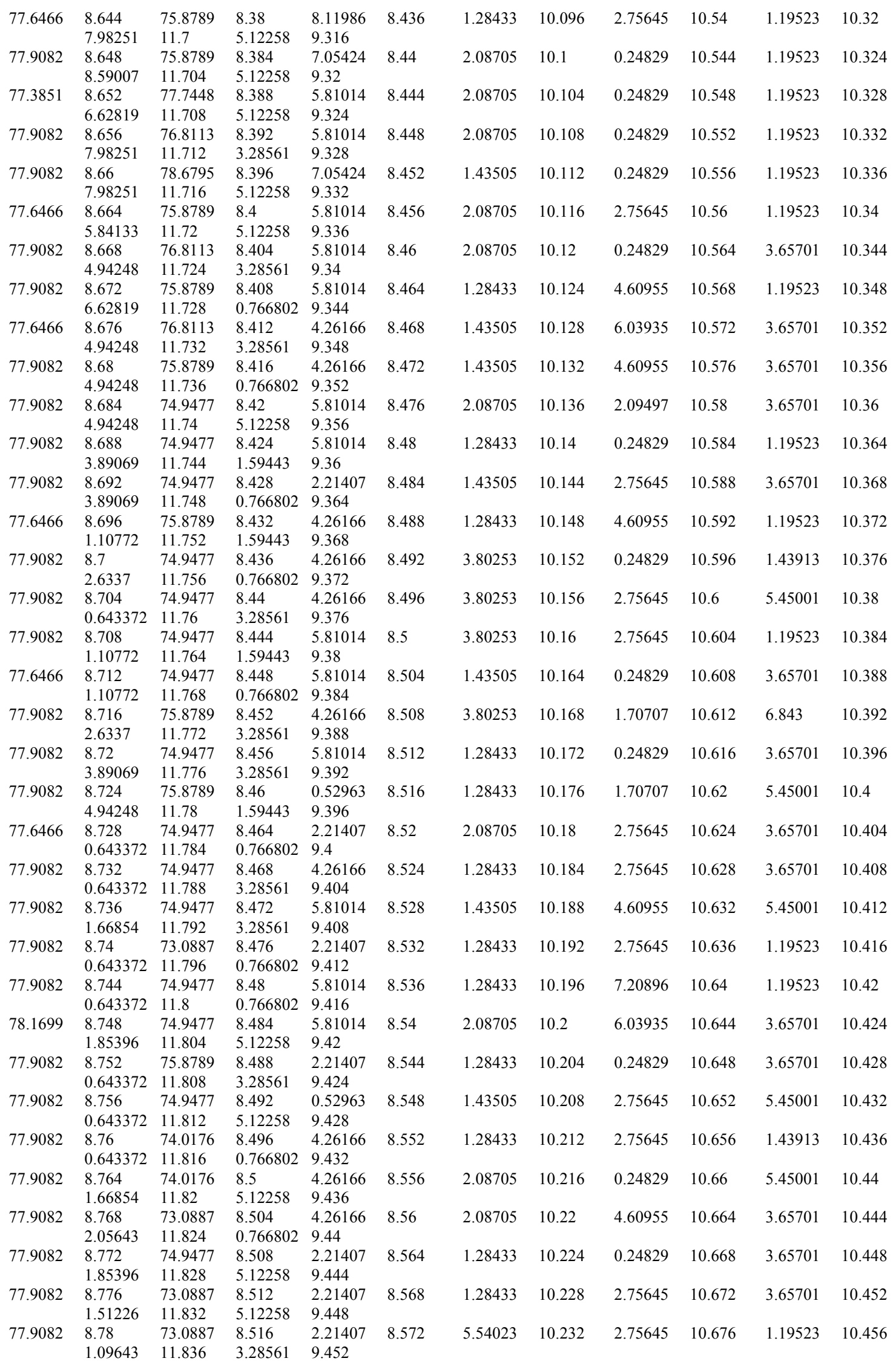




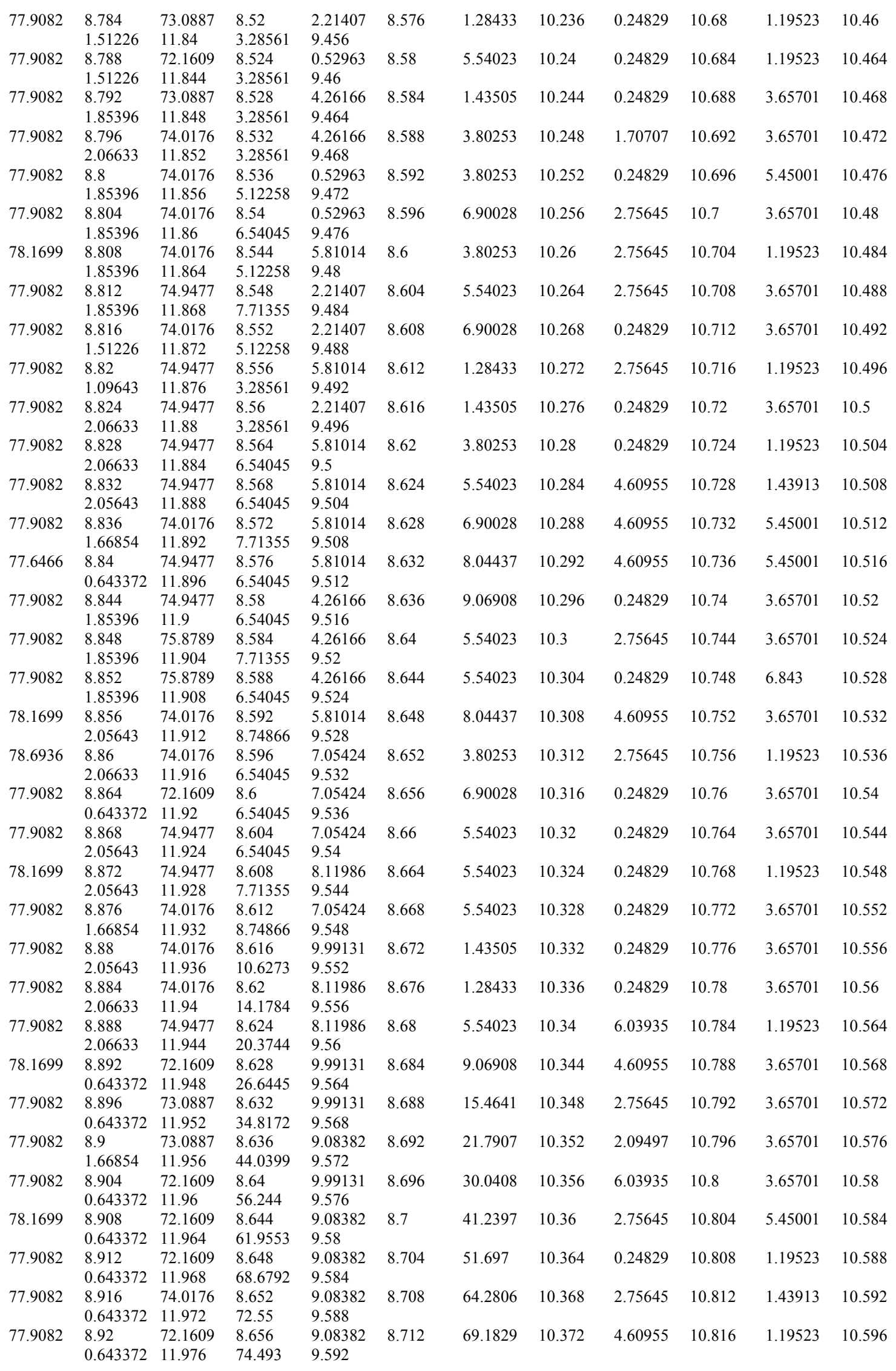




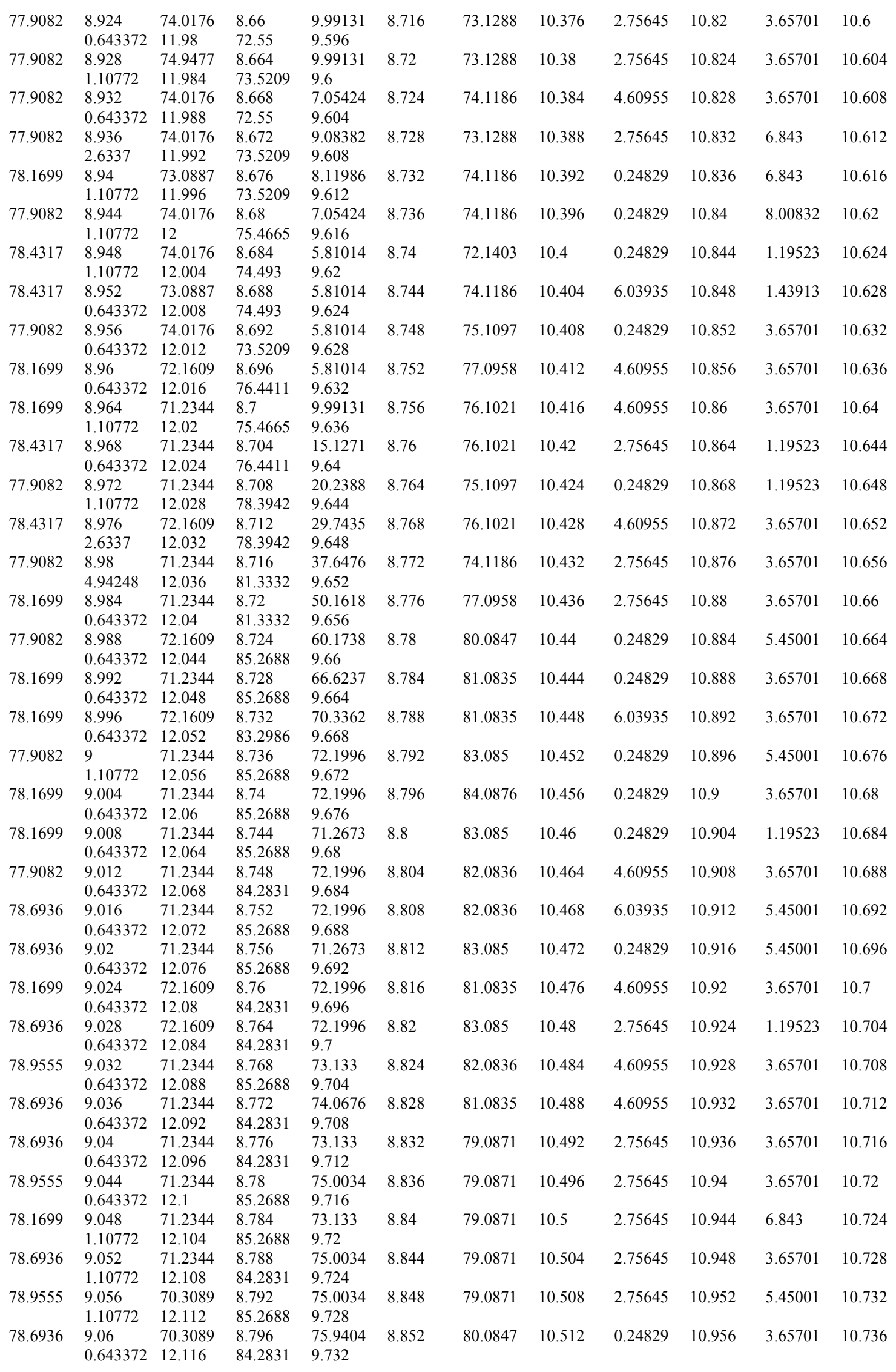




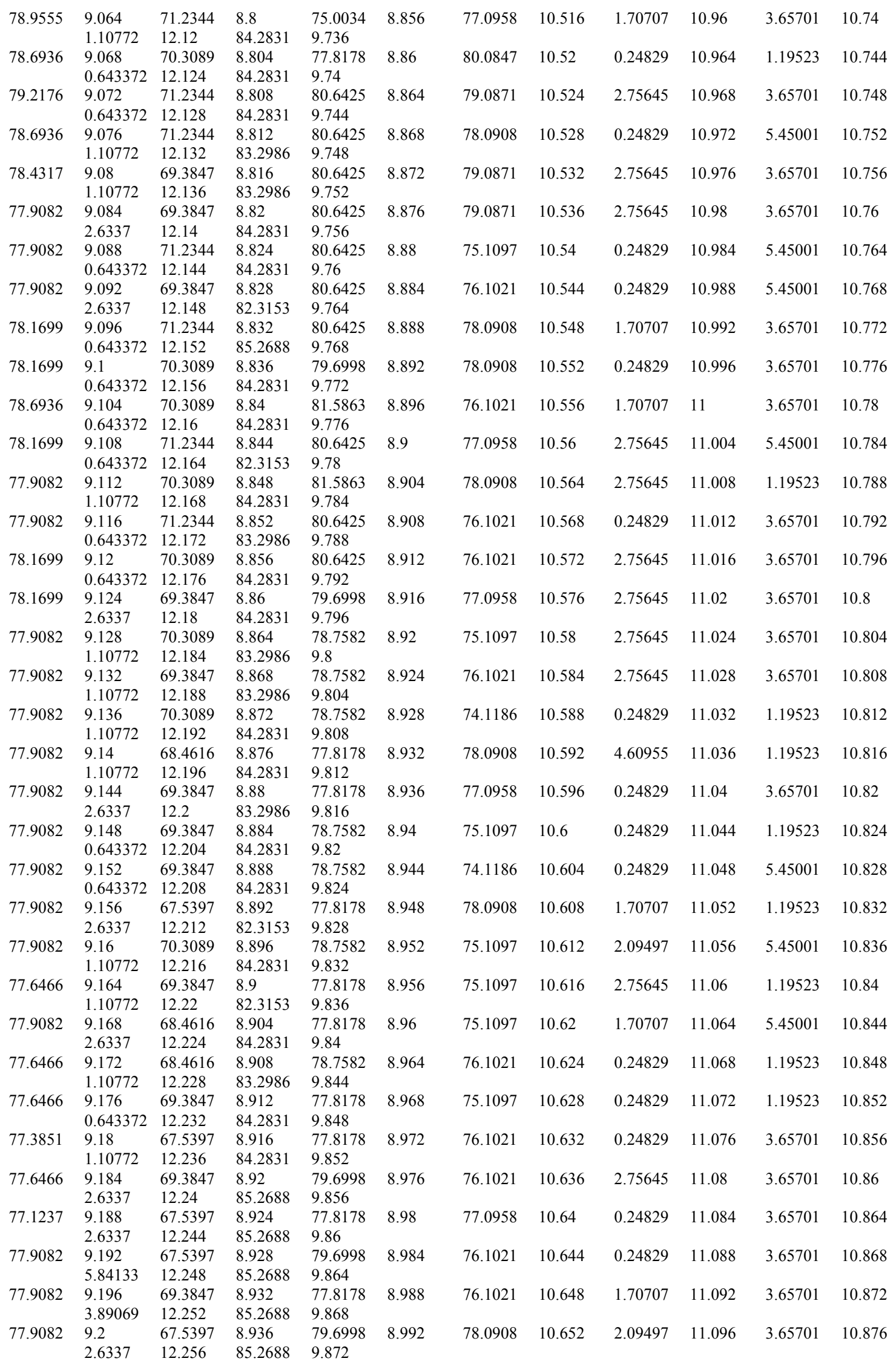




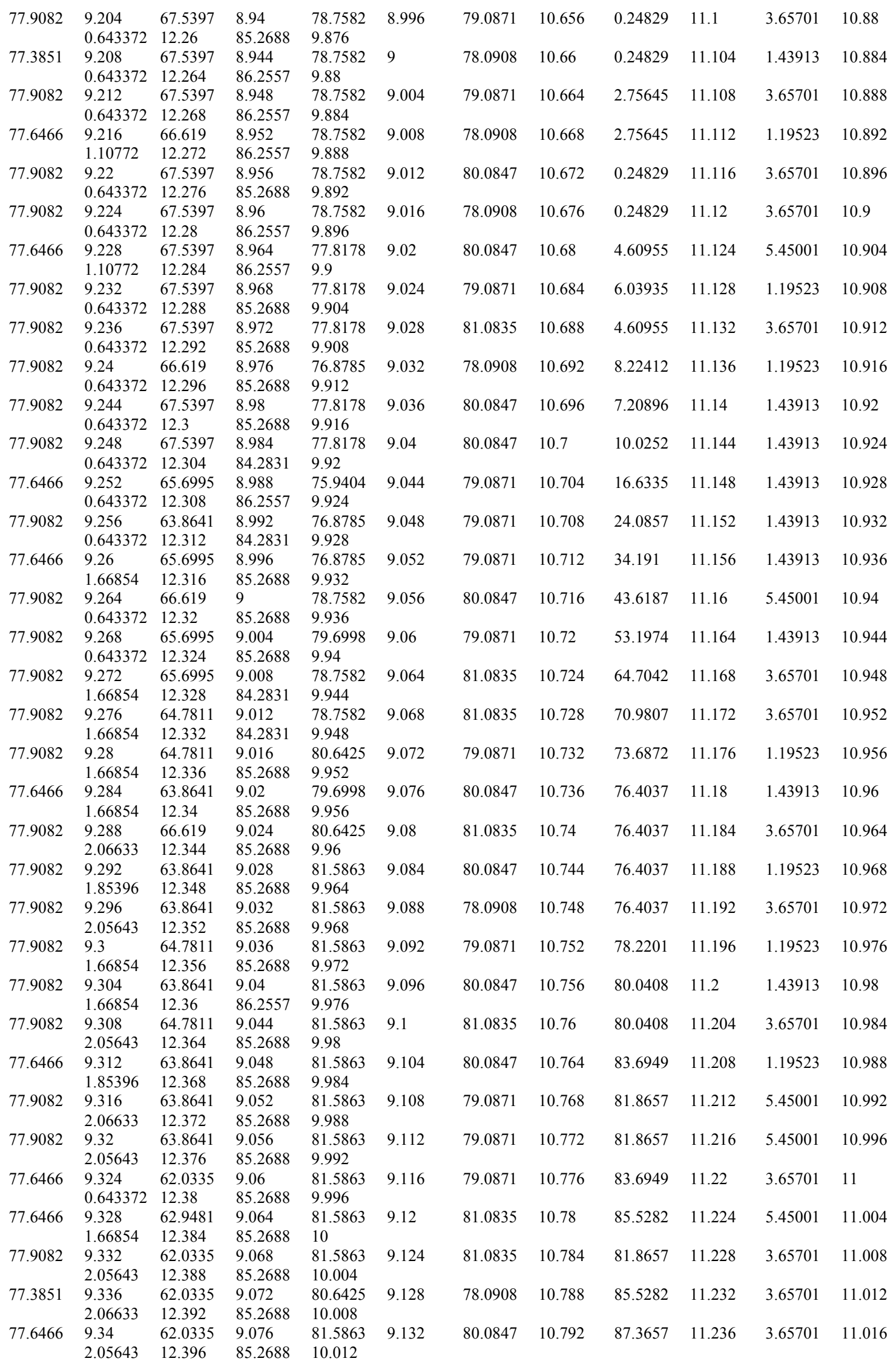




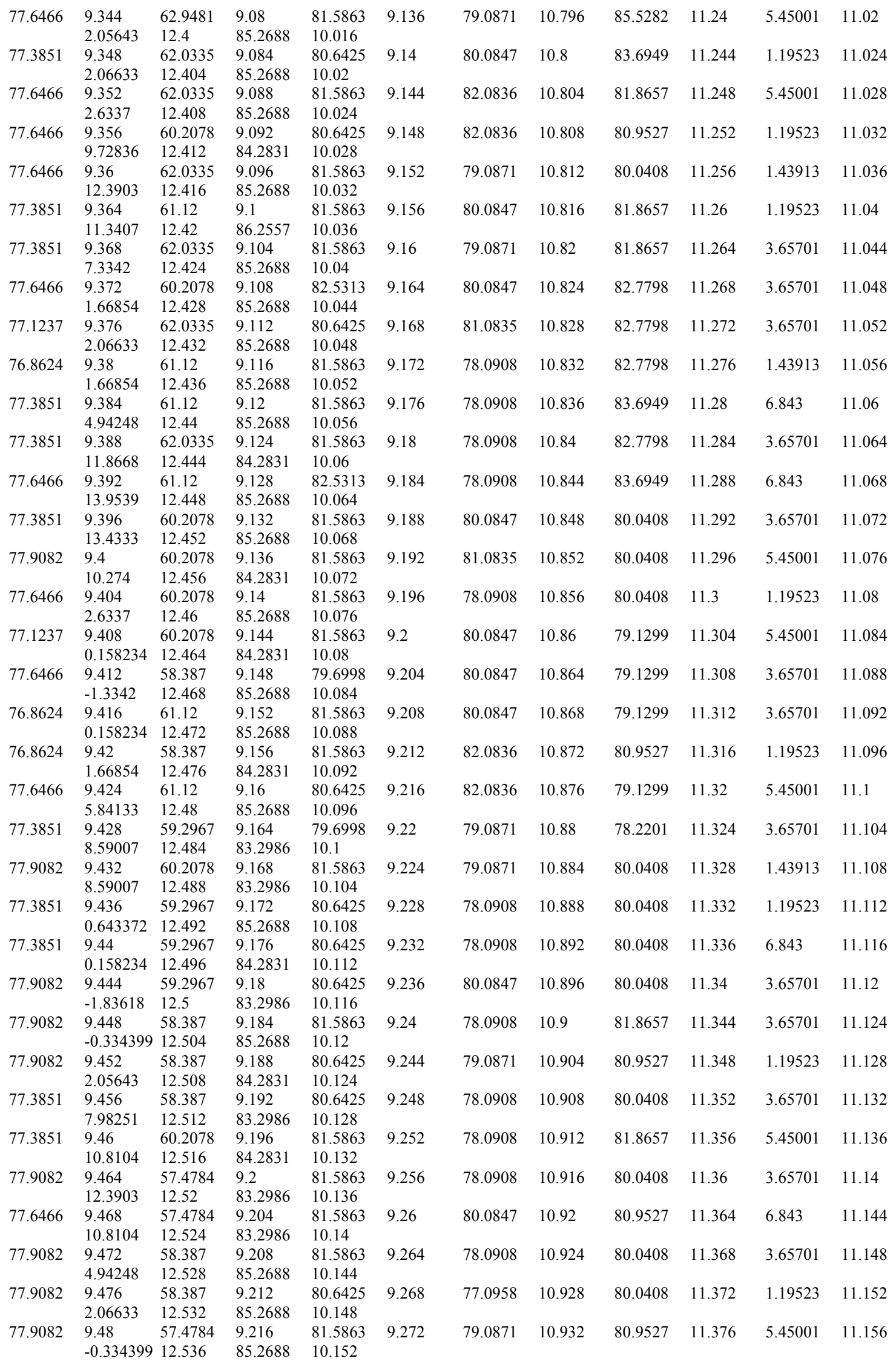




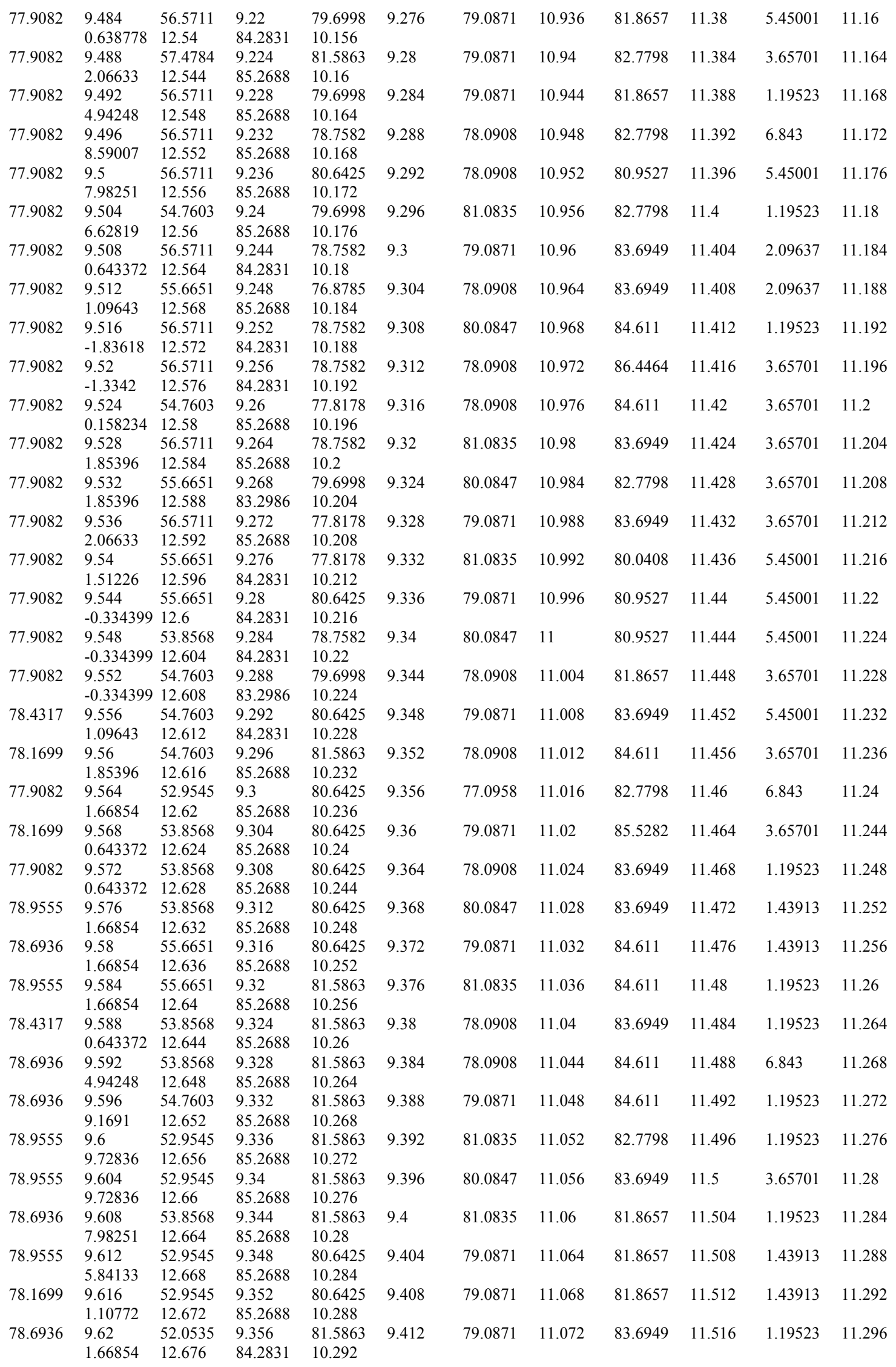




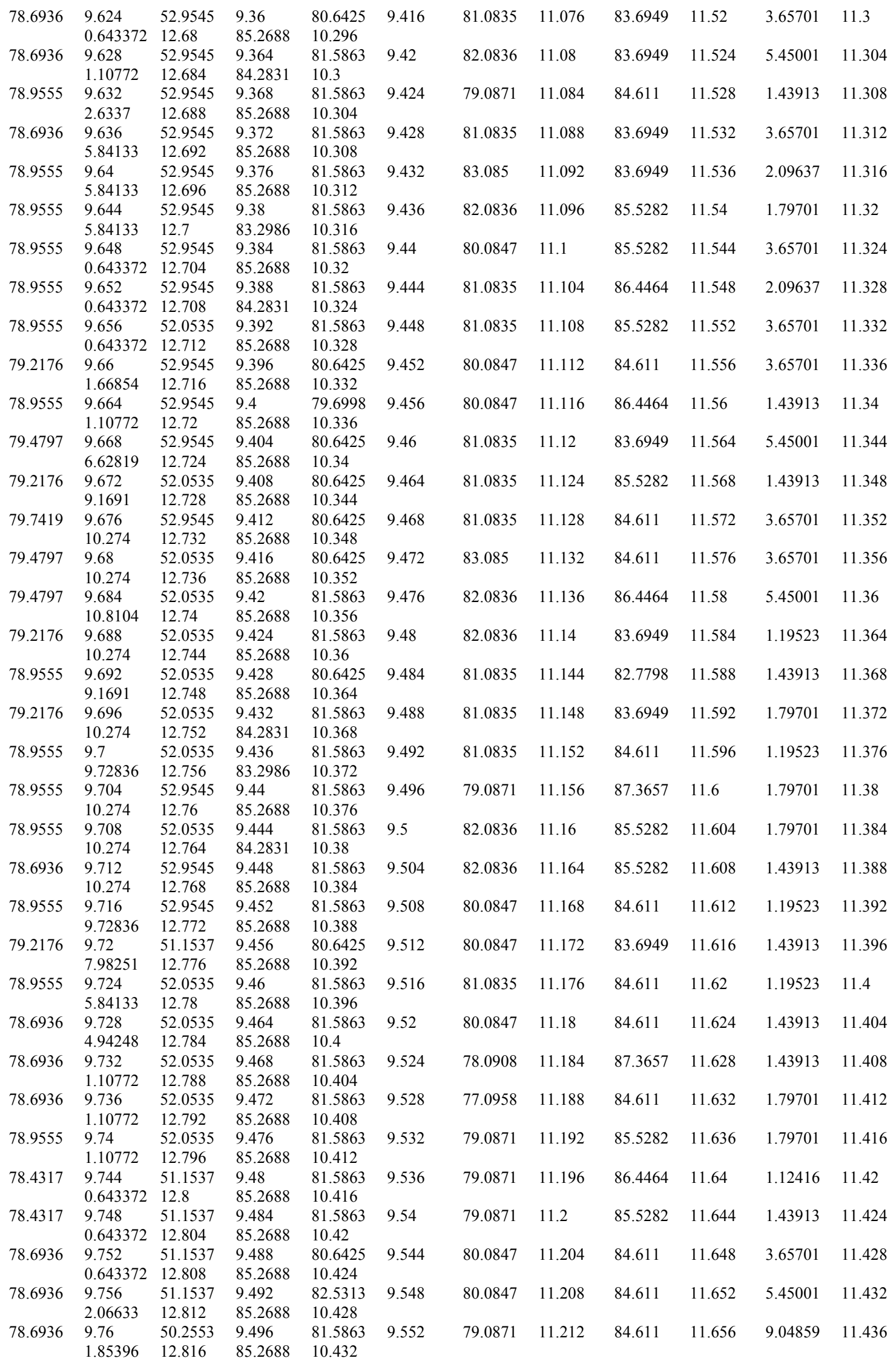




\begin{tabular}{|c|c|c|c|c|c|c|c|c|c|c|c|}
\hline \multirow[t]{2}{*}{78.6936} & 9.764 & 50.2553 & 9.5 & 80.6425 & 9.556 & 82.0836 & 11.216 & 87.3657 & 11.66 & 1.19523 & 11.44 \\
\hline & 0.638778 & 12.82 & 85.2688 & 10.436 & & & & & & & \\
\hline \multirow[t]{2}{*}{78.4317} & 9.768 & 49.3581 & 9.504 & 80.6425 & 9.56 & 78.0908 & 11.22 & 84.611 & 11.664 & 3.65701 & 11.444 \\
\hline & 1.85396 & 12.824 & 85.2688 & 10.44 & & & & & & & \\
\hline \multirow[t]{2}{*}{78.1699} & 9.772 & 52.0535 & 9.508 & 81.5863 & 9.564 & 79.0871 & 11.224 & 88.286 & 11.668 & 1.43913 & 11.448 \\
\hline & 1.85396 & 12.828 & 85.2688 & 10.444 & & & & & & & \\
\hline \multirow[t]{2}{*}{78.4317} & 9.776 & 50.2553 & 9.512 & 81.5863 & 9.568 & 80.0847 & 11.228 & 87.3657 & 11.672 & 1.79701 & 11.452 \\
\hline & 0.643372 & 12.832 & 85.2688 & 10.448 & & & & & & & \\
\hline \multirow[t]{2}{*}{78.1699} & 9.78 & 50.2553 & 9.516 & 81.5863 & 9.572 & 83.085 & 11.232 & 86.4464 & 11.676 & 1.79701 & 11.456 \\
\hline & 1.10772 & 12.836 & 85.2688 & 10.452 & & & & & & & \\
\hline \multirow[t]{2}{*}{78.1699} & 9.784 & 52.0535 & 9.52 & 81.5863 & 9.576 & 81.0835 & 11.236 & 85.5282 & 11.68 & 1.79701 & 11.46 \\
\hline & 2.6337 & 12.84 & 85.2688 & 10.456 & & & & & & & \\
\hline \multirow[t]{2}{*}{77.9082} & 9.788 & 51.1537 & 9.524 & 81.5863 & 9.58 & 81.0835 & 11.24 & 86.4464 & 11.684 & 2.09637 & 11.464 \\
\hline & 1.10772 & 12.844 & 85.2688 & 10.46 & & & & & & & \\
\hline \multirow[t]{2}{*}{77.9082} & 9.792 & 50.2553 & 9.528 & 79.6998 & 9.584 & 80.0847 & 11.244 & 86.4464 & 11.688 & 1.43913 & 11.468 \\
\hline & 0.643372 & 12.848 & 85.2688 & 10.464 & & & & & & & \\
\hline 78.1699 & 9.796 & 50.2553 & 9.532 & 81.5863 & 9.588 & 80.0847 & 11.248 & 84.611 & 11.692 & 5.45001 & 11.472 \\
\hline & 0.643372 & 12.852 & 84.2831 & 10.468 & & & & & & & \\
\hline 77.9082 & 9.8 & 50.2553 & 9.536 & 81.5863 & 9.592 & 80.0847 & 11.252 & 85.5282 & 11.696 & 5.45001 & 11.476 \\
\hline & 1.66854 & 12.856 & 85.2688 & 10.472 & & & & & & & \\
\hline 77.9082 & 9.804 & 51.1537 & 9.54 & 80.6425 & 9.596 & 81.0835 & 11.256 & 85.5282 & 11.7 & 8.00832 & 11.48 \\
\hline & 0.643372 & 12.86 & 86.2557 & 10.476 & & & & & & & \\
\hline 77.9082 & 9.808 & 51.1537 & 9.544 & 81.5863 & 9.6 & 80.0847 & 11.26 & 84.611 & 11.704 & 5.45001 & 11.484 \\
\hline & 1.66854 & 12.864 & 85.2688 & 10.48 & & & & & & & \\
\hline 77.9082 & 9.812 & 50.2553 & 9.548 & 81.5863 & 9.604 & 80.0847 & 11.264 & 82.7798 & 11.708 & 1.19523 & 11.488 \\
\hline & 0.643372 & 12.868 & 85.2688 & 10.484 & & & & & & & \\
\hline 77.9082 & 9.816 & 50.2553 & 9.552 & 80.6425 & 9.608 & 79.0871 & 11.268 & 84.611 & 11.712 & 1.43913 & 11.492 \\
\hline & 0.643372 & 12.872 & 85.2688 & 10.488 & & & & & & & \\
\hline 77.9082 & 9.82 & 50.2553 & 9.556 & 80.6425 & 9.612 & 79.0871 & 11.272 & 86.4464 & 11.716 & 1.79701 & 11.496 \\
\hline & 1.66854 & 12.876 & 85.2688 & 10.492 & & & & & & & \\
\hline 77.9082 & 9.824 & 51.1537 & 9.56 & 78.7582 & 9.616 & 78.0908 & 11.276 & 85.5282 & 11.72 & 2.09637 & 11.5 \\
\hline & 1.85396 & 12.88 & 85.2688 & 10.496 & & & & & & & \\
\hline 77.9082 & 9.828 & 49.3581 & 9.564 & 80.6425 & 9.62 & 81.0835 & 11.28 & 83.6949 & 11.724 & 1.43913 & 11.504 \\
\hline & 1.85396 & 12.884 & 85.2688 & 10.5 & & & & & & & \\
\hline 77.9082 & 9.832 & 49.3581 & 9.568 & 79.6998 & 9.624 & 80.0847 & 11.284 & 84.611 & 11.728 & 5.45001 & 11.508 \\
\hline & 0.158234 & 12.888 & 85.2688 & 10.504 & & & & & & & \\
\hline 77.9082 & 9.836 & 50.2553 & 9.572 & 78.7582 & 9.628 & 79.0871 & 11.288 & 84.611 & 11.732 & 5.45001 & 11.512 \\
\hline & 0.638778 & 12.892 & 85.2688 & 10.508 & & & & & & & \\
\hline 77.9082 & 9.84 & 51.1537 & 9.576 & 79.6998 & 9.632 & 80.0847 & 11.292 & 85.5282 & 11.736 & 5.45001 & 11.516 \\
\hline & 0.158234 & 12.896 & 85.2688 & 10.512 & & & & & & & \\
\hline 77.9082 & 9.844 & 49.3581 & 9.58 & 78.7582 & 9.636 & 80.0847 & 11.296 & 86.4464 & 11.74 & 3.65701 & 11.52 \\
\hline & 0.158234 & 12.9 & 85.2688 & 10.516 & & & & & & & \\
\hline 77.9082 & 9.848 & 50.2553 & 9.584 & 79.6998 & 9.64 & 78.0908 & 11.3 & 83.6949 & 11.744 & 5.45001 & 11.524 \\
\hline & 0.158234 & 12.904 & 85.2688 & 10.52 & & & & & & & \\
\hline 77.9082 & 9.852 & 50.2553 & 9.588 & 78.7582 & 9.644 & 77.0958 & 11.304 & 84.611 & 11.748 & 1.19523 & 11.528 \\
\hline & 0.158234 & 12.908 & 85.2688 & 10.524 & & & & & & & \\
\hline 77.9082 & 9.856 & 49.3581 & 9.592 & 79.6998 & 9.648 & 78.0908 & 11.308 & 85.5282 & 11.752 & 1.19523 & 11.532 \\
\hline & 0.158234 & 12.912 & 85.2688 & 10.528 & & & & & & & \\
\hline 77.9082 & 9.86 & 50.2553 & 9.596 & 79.6998 & 9.652 & 80.0847 & 11.312 & 85.5282 & 11.756 & 1.19523 & 11.536 \\
\hline & -0.334399 & 12.916 & 85.2688 & 10.532 & & & & & & & \\
\hline 77.9082 & 9.864 & 49.3581 & 9.6 & 78.7582 & 9.656 & 80.0847 & 11.316 & 83.6949 & 11.76 & 5.45001 & 11.54 \\
\hline & 0.158234 & 12.92 & 85.2688 & 10.536 & & & & & & & \\
\hline 77.9082 & 9.868 & 49.3581 & 9.604 & 77.8178 & 9.66 & 81.0835 & 11.32 & 82.7798 & 11.764 & 6.843 & 11.544 \\
\hline & 0.158234 & 12.924 & 85.2688 & 10.54 & & & & & & & \\
\hline 78.1699 & 9.872 & 49.3581 & 9.608 & 80.6425 & 9.664 & 78.0908 & 11.324 & 87.3657 & 11.768 & 5.45001 & 11.548 \\
\hline & -0.334399 & 12.928 & 85.2688 & 10.544 & & & & & & & \\
\hline 77.9082 & 9.876 & 50.2553 & 9.612 & 77.8178 & 9.668 & 77.0958 & 11.328 & 85.5282 & 11.772 & 9.04859 & 11.552 \\
\hline & 0.158234 & 12.932 & 85.2688 & 10.548 & & & & & & & \\
\hline 78.1699 & 9.88 & 50.2553 & 9.616 & 79.6998 & 9.672 & 77.0958 & 11.332 & 86.4464 & 11.776 & 8.00832 & 11.556 \\
\hline & 1.51226 & 12.936 & 85.2688 & 10.552 & & & & & & & \\
\hline 77.9082 & 9.884 & 49.3581 & 9.62 & 77.8178 & 9.676 & 75.1097 & 11.336 & 84.611 & 11.78 & 8.00832 & 11.56 \\
\hline & 1.85396 & 12.94 & 85.2688 & 10.556 & & & & & & & \\
\hline 78.1699 & 9.888 & 49.3581 & 9.624 & 76.8785 & 9.68 & 78.0908 & 11.34 & 85.5282 & 11.784 & 9.04859 & 11.564 \\
\hline & 2.05643 & 12.944 & 85.2688 & 10.56 & & & & & & & \\
\hline 78.6936 & 9.892 & 50.2553 & 9.628 & 77.8178 & 9.684 & 81.0835 & 11.344 & 85.5282 & 11.788 & 6.843 & 11.568 \\
\hline & 1.66854 & 12.948 & 85.2688 & 10.564 & & & & & & & \\
\hline 77.9082 & 9.896 & 50.2553 & 9.632 & 77.8178 & 9.688 & 83.085 & 11.348 & 86.4464 & 11.792 & 8.00832 & 11.572 \\
\hline & 0.643372 & 12.952 & 86.2557 & 10.568 & & & & & & & \\
\hline 77.9082 & 9.9 & 49.3581 & 9.636 & 77.8178 & 9.692 & 82.0836 & 11.352 & 84.611 & 11.796 & 8.00832 & 11.576 \\
\hline & 1.66854 & 12.956 & 85.2688 & 10.572 & & & & & & & \\
\hline
\end{tabular}




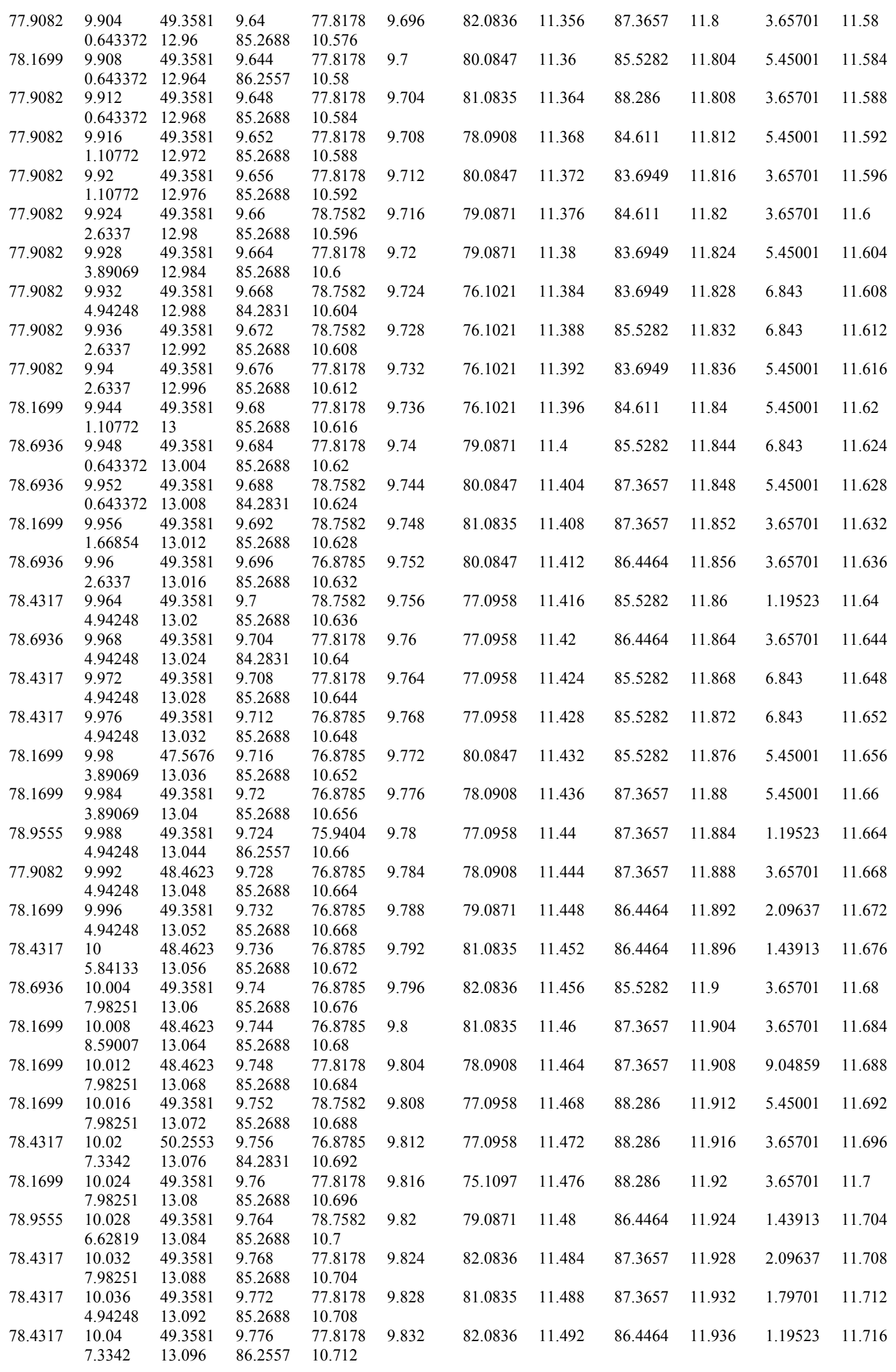




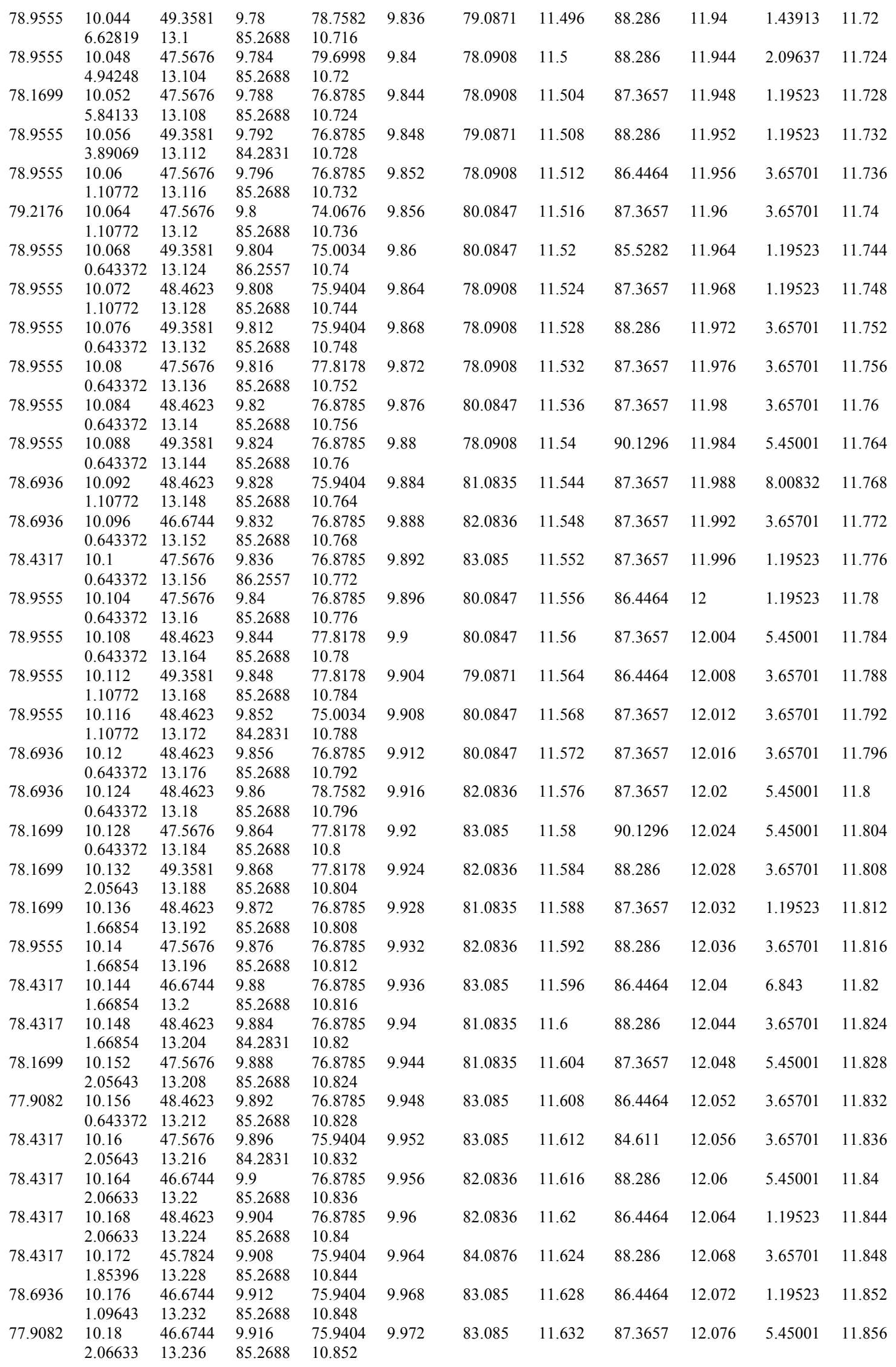




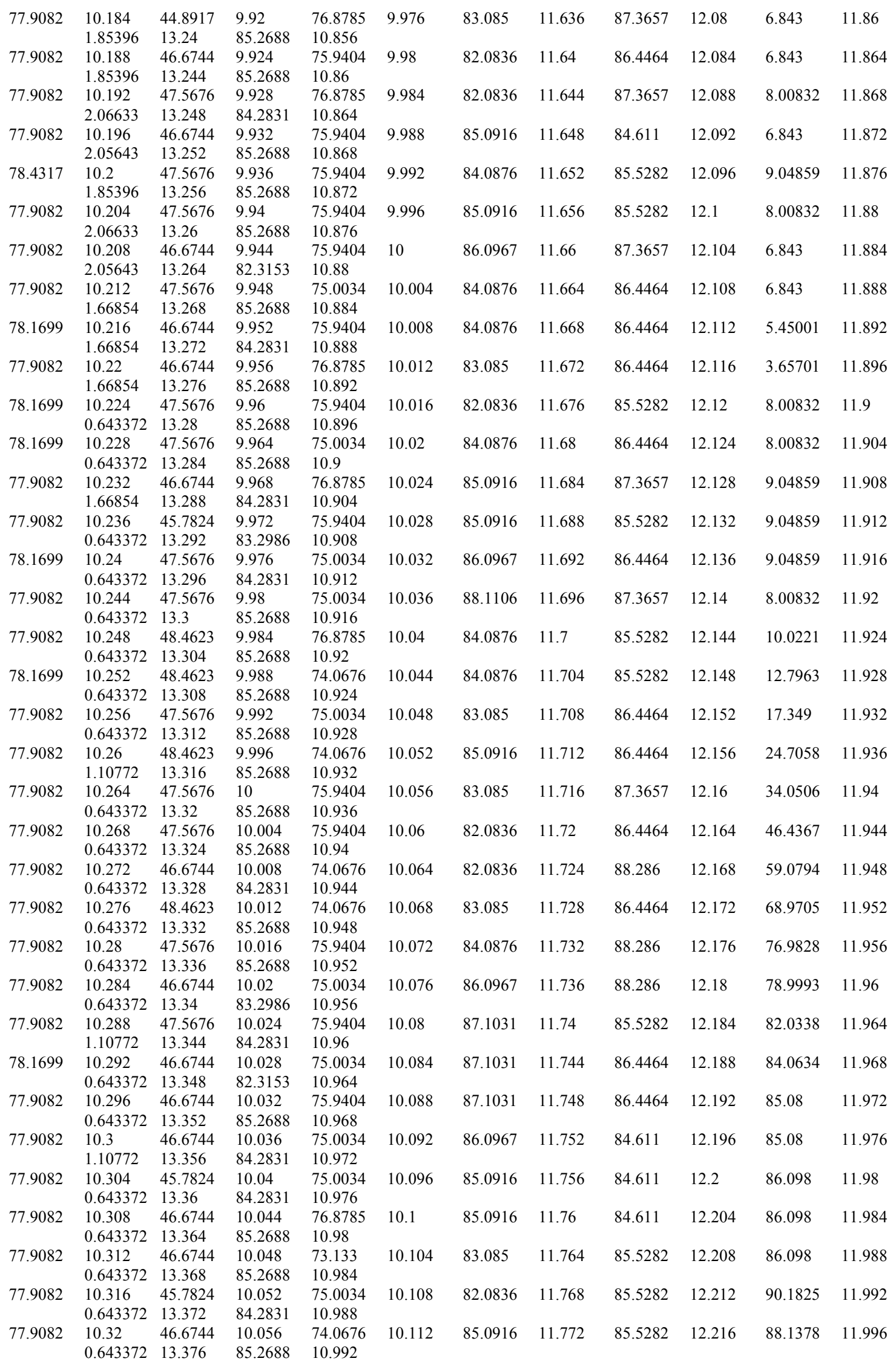




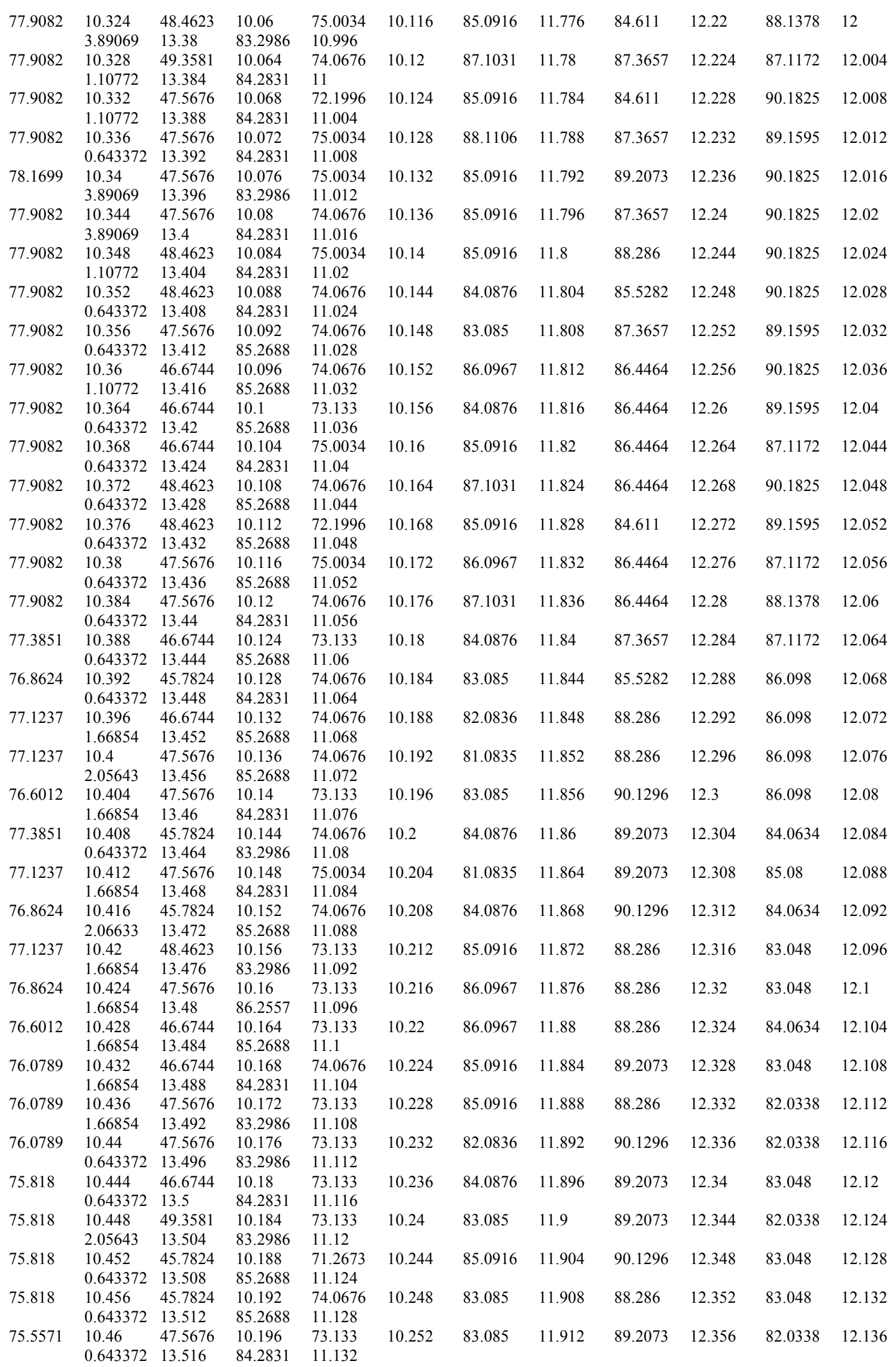




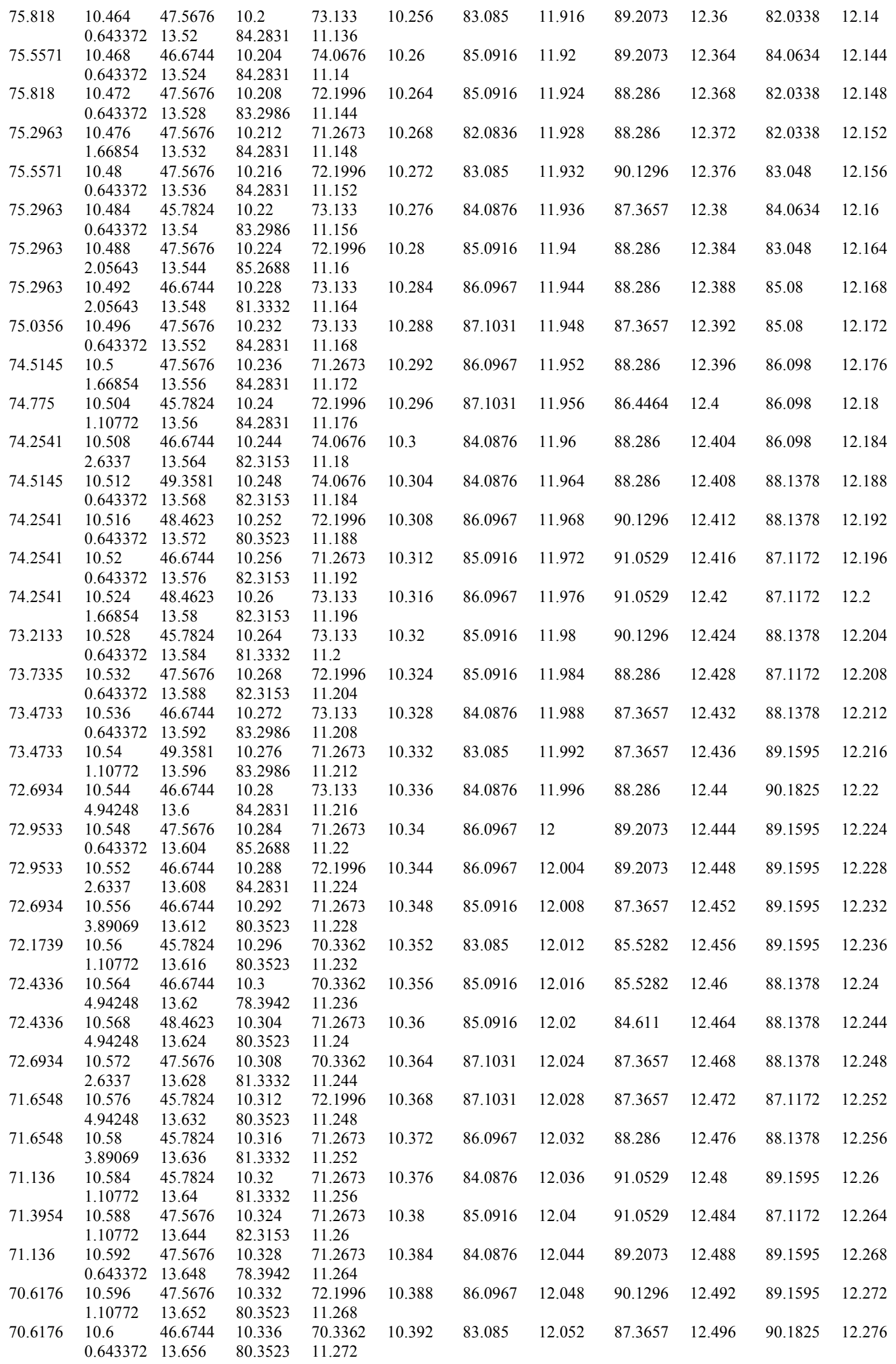




\begin{tabular}{|c|c|c|c|c|c|c|c|c|c|c|c|}
\hline 69.8407 & $\begin{array}{l}10.604 \\
3.89069\end{array}$ & $\begin{array}{l}45.7824 \\
13.66\end{array}$ & $\begin{array}{l}10.34 \\
82.3153\end{array}$ & $\begin{array}{l}70.3362 \\
11.276\end{array}$ & 10.396 & 86.0967 & 12.056 & 86.4464 & 12.5 & 90.1825 & 12.28 \\
\hline \multirow[t]{2}{*}{69.5819} & 10.608 & 48.4623 & 10.344 & 70.3362 & 10.4 & 85.0916 & 12.06 & 87.3657 & 12.504 & 86.098 & 12.284 \\
\hline & 1.10772 & 13.664 & 83.2986 & 11.28 & & & & & & & \\
\hline \multirow[t]{2}{*}{70.0996} & 10.612 & 47.5676 & 10.348 & 71.2673 & 10.404 & 85.0916 & 12.064 & 88.286 & 12.508 & 87.1172 & 12.288 \\
\hline & 0.643372 & 13.668 & 80.3523 & 11.284 & & & & & & & \\
\hline \multirow[t]{2}{*}{69.8407} & 10.616 & 48.4623 & 10.352 & 71.2673 & 10.408 & 87.1031 & 12.068 & 88.286 & 12.512 & 88.1378 & 12.292 \\
\hline & 0.643372 & 13.672 & 78.3942 & 11.288 & & & & & & & \\
\hline \multirow[t]{2}{*}{69.5819} & 10.62 & 49.3581 & 10.356 & 70.3362 & 10.412 & 86.0967 & 12.072 & 89.2073 & 12.516 & 88.1378 & 12.296 \\
\hline & 2.6337 & 13.676 & 76.4411 & 11.292 & & & & & & & \\
\hline \multirow[t]{2}{*}{69.5819} & 10.624 & 48.4623 & 10.36 & 71.2673 & 10.416 & 86.0967 & 12.076 & 89.2073 & 12.52 & 89.1595 & 12.3 \\
\hline & 0.643372 & 13.68 & 76.4411 & 11.296 & & & & & & & \\
\hline \multirow[t]{2}{*}{69.5819} & 10.628 & 47.5676 & 10.364 & 71.2673 & 10.42 & 86.0967 & 12.08 & 88.286 & 12.524 & 87.1172 & 12.304 \\
\hline & 0.643372 & 13.684 & 76.4411 & 11.3 & & & & & & & \\
\hline \multirow[t]{2}{*}{69.3232} & 10.632 & 46.6744 & 10.368 & 69.4063 & 10.424 & 86.0967 & 12.084 & 90.1296 & 12.528 & 88.1378 & 12.308 \\
\hline & 1.10772 & 13.688 & 77.4171 & 11.304 & & & & & & & \\
\hline \multirow[t]{2}{*}{68.5477} & 10.636 & 47.5676 & 10.372 & 70.3362 & 10.428 & 85.0916 & 12.088 & 92.9026 & 12.532 & 88.1378 & 12.312 \\
\hline & 2.6337 & 13.692 & 78.3942 & 11.308 & & & & & & & \\
\hline 68.5477 & 10.64 & 48.4623 & 10.376 & 71.2673 & 10.432 & 85.0916 & 12.092 & 91.9773 & 12.536 & 89.1595 & 12.316 \\
\hline & 0.643372 & 13.696 & 78.3942 & 11.312 & & & & & & & \\
\hline 68.2894 & 10.644 & 45.7824 & 10.38 & 70.3362 & 10.436 & 84.0876 & 12.096 & 93.829 & 12.54 & 90.1825 & 12.32 \\
\hline & 0.643372 & 13.7 & 77.4171 & 11.316 & & & & & & & \\
\hline 67.7731 & 10.648 & 47.5676 & 10.384 & 70.3362 & 10.44 & 83.085 & 12.1 & 91.9773 & 12.544 & 90.1825 & 12.324 \\
\hline & 0.643372 & 13.704 & 77. & 11.32 & & & & & & & \\
\hline 67.7731 & 10.652 & 48.4623 & 10.388 & 70.3362 & 10.444 & 83.085 & 12.104 & 92.9026 & 12.548 & 90.1825 & 12.328 \\
\hline & 0.643372 & 13.708 & 77.4171 & 11.324 & & & & & & & \\
\hline 67.515 & 10.656 & 47.5676 & 10.392 & 71.2673 & 10.448 & 83.085 & 12.108 & 91.0529 & 12.552 & 90.1825 & 12.332 \\
\hline & 1.10772 & 13.712 & 80.3523 & 11.328 & & & & & & & \\
\hline 67.515 & 10.66 & 48.4623 & 10.396 & 70.3362 & 10.452 & 85.0916 & 12.112 & 90.1296 & 12.556 & 88.1378 & 12.336 \\
\hline & 0.643372 & 13. & 79. & 11. & & & & & & & \\
\hline 66.9992 & 10.664 & 47.5676 & 10.4 & 71.2673 & 10.456 & 84.0876 & 12.116 & 89.2073 & 12.56 & 89.1595 & 12.34 \\
\hline & 0.643372 & 13.72 & 84.2831 & 11. & & & & & & & \\
\hline 66.9992 & 10.668 & 48.4623 & 10.404 & 68.4776 & 10.46 & 85.0916 & 12.12 & 88.286 & 12.564 & 90.1825 & 12.344 \\
\hline & 0.643372 & 13.724 & 85.2688 & 11.34 & & & & & & & \\
\hline 66.7414 & 10.672 & 48.4623 & 10.408 & 68.4776 & 10.464 & 84.0876 & 12.124 & 89.2073 & 12.568 & 90.1825 & 12.348 \\
\hline & 0.643372 & 13. & 82 & 11.344 & & & & & & & \\
\hline 66.7414 & 10.676 & 46.6744 & 10. & 69.4063 & 10.468 & 84.0876 & 12.128 & 86.4464 & 12.572 & 89.1595 & 12.352 \\
\hline & 0.643372 & 13.732 & 81.3332 & 11.348 & & & & & & & \\
\hline 66.4838 & 10.68 & 48.4623 & 10.416 & 69.4063 & 10.472 & 83.085 & 12.132 & 86.4464 & 12.576 & 90.1825 & 12.356 \\
\hline & 0.643372 & 13.736 & 77.4171 & 11.352 & & & & & & & \\
\hline 66.4838 & 10.684 & 48.4623 & 10.42 & 68.4776 & 10.476 & 83.085 & 12.136 & 86.4464 & 12.58 & 89.1595 & 12.36 \\
\hline & 0.643372 & 13.74 & 76.4411 & 11.356 & & & & & & & \\
\hline 66.9992 & 10.688 & 45.7824 & 10.424 & 70.3362 & 10.48 & 82.0836 & 12.14 & 86.4464 & 12.584 & 90.1825 & 12.364 \\
\hline & 0.6 & 13. & & 11 & & & & & & & \\
\hline 66.7414 & 10.692 & 47.5676 & 10.4 & 69.4063 & 10.484 & 82.0836 & 12.144 & 88.286 & 12.588 & 90.1825 & 12.368 \\
\hline & 1.66854 & 13.7 & 2 & & & & & & & & \\
\hline 66.4838 & 10.696 & 47.5676 & 10.432 & 70.3362 & 10.488 & 84.0876 & 12.148 & 90.1296 & 12.592 & 90.1825 & 12.372 \\
\hline & 1.66854 & 13.752 & 81.3332 & 11.368 & & & & & & & \\
\hline 65.9687 & 10.7 & 46.6744 & 10.436 & 70.3362 & 10.492 & 83.085 & 12.152 & 91.9773 & 12.596 & 89.1595 & 12.376 \\
\hline & 2.0 & 13 & & & & & & & & & \\
\hline 65.7113 & 10.704 & 46.6744 & 10.44 & 68.4776 & 10.496 & 83.085 & 12.156 & 91.9773 & 12.6 & 88.1378 & 12.38 \\
\hline & 2.06 & 13. & & & & & & & & & \\
\hline 65.1969 & 10.708 & 47.5676 & 10.444 & 69.4063 & 10.5 & 82.0836 & 12.16 & 90.1296 & 12.604 & 89.1595 & 12.384 \\
\hline & 1.85396 & 13.76 & 77.4171 & 11.38 & & & & & & & \\
\hline 64.9398 & 10.712 & 48.4623 & 10.448 & 69.4063 & 10.504 & 80.0847 & 12.164 & 91.0529 & 12.608 & 90.1825 & 12.388 \\
\hline & 2.06633 & 13.768 & 76.4411 & 11.384 & & & & & & & \\
\hline 64.6828 & 10.716 & 48.4623 & 10.452 & 70.3362 & 10.508 & 83.085 & 12.168 & 91.9773 & 12.612 & 89.1595 & 12.392 \\
\hline & 1.66854 & 13.7 & & & & & & & & & \\
\hline 64.4258 & 10.72 & 47.5676 & 10.456 & 68.4776 & 10.512 & 81.0835 & 12.172 & 93.829 & 12.616 & 90.1825 & 12.396 \\
\hline & 1.51226 & 13.7 & 77.4171 & 11.3 & & & & & & & \\
\hline 64.9398 & 10.724 & 48.4623 & 10.46 & 70.3362 & 10.516 & 82.0836 & 12.176 & 94.7563 & 12.62 & 87.1172 & 12.4 \\
\hline & 1.85396 & 13.78 & 81.3332 & 11.396 & & & & & & & \\
\hline 64.9398 & 10.728 & 49.3581 & 10.464 & 69.4063 & 10.52 & 82.0836 & 12.18 & 94.7563 & 12.624 & 89.1595 & 12.404 \\
\hline & 2.06633 & & & 11. & & & & & & & \\
\hline 64.9398 & 10.732 & 47.5676 & 10.468 & 69.4063 & 10.524 & 81.0835 & 12.184 & 93.829 & 12.628 & 90.1825 & 12.408 \\
\hline & 2.06 & & 0.3523 & & & & & & & & \\
\hline 64.9398 & 10.736 & 47.5676 & 10.472 & 70.3362 & 10.528 & 81.0835 & 12.188 & 93.829 & 12.632 & 91.2068 & 12.412 \\
\hline & 2.06633 & 13.792 & 79.3727 & 11.408 & & & & & & & \\
\hline 64.169 & 10.74 & 46.6744 & 10.476 & 70.3362 & 10.532 & 81.0835 & 12.192 & 93.829 & 12.636 & 90.1825 & 12.416 \\
\hline & 1.85396 & 13.796 & 79.3727 & 11.412 & & & & & & & \\
\hline
\end{tabular}




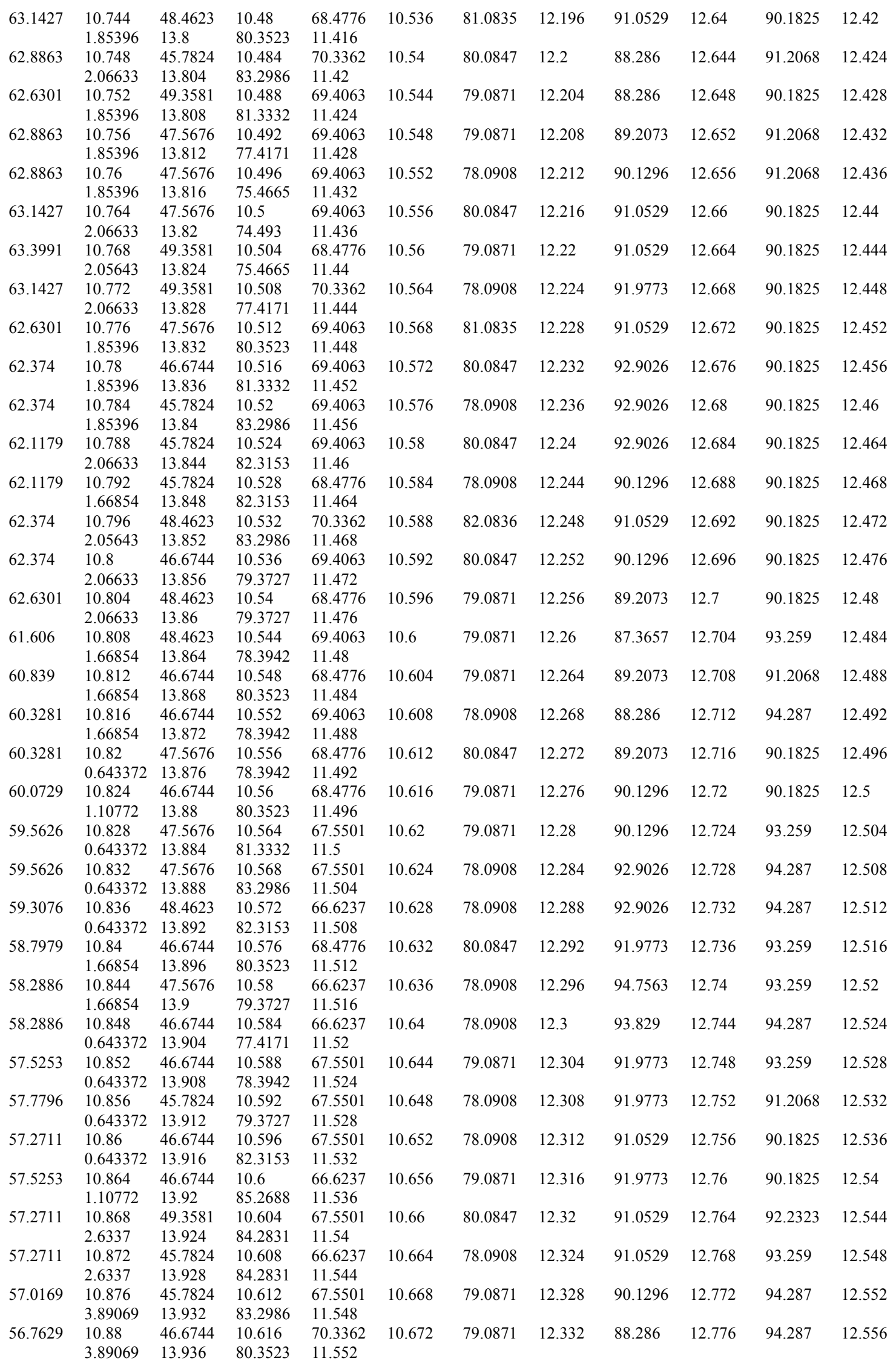




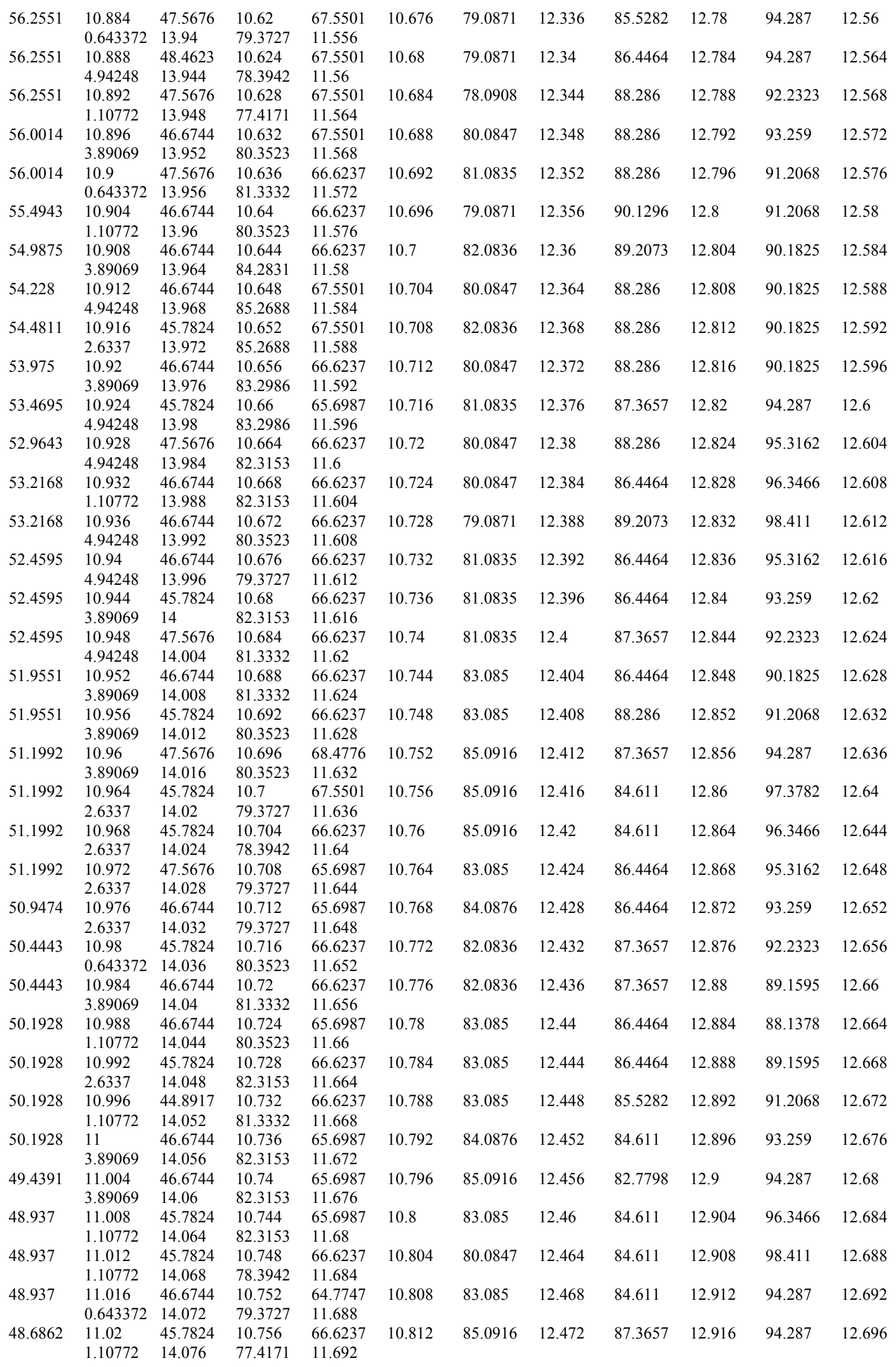




\begin{tabular}{|c|c|c|c|c|c|c|c|c|c|c|c|}
\hline 48.6862 & $\begin{array}{l}11.024 \\
0.643372\end{array}$ & $\begin{array}{l}45.7824 \\
14.08\end{array}$ & $\begin{array}{l}10.76 \\
79.3727\end{array}$ & $\begin{array}{l}65.6987 \\
11.696\end{array}$ & 10.816 & 85.0916 & 12.476 & 89.2073 & 12.92 & 90.1825 & 12.7 \\
\hline \multirow[t]{2}{*}{48.1848} & 11.028 & 45.7824 & 10.764 & 65.6987 & 10.82 & 3.085 & 12.48 & 87.3657 & 12.924 & 89.1595 & 12.704 \\
\hline & 1.66854 & 14.084 & 79.3727 & 11.7 & & & & & & & \\
\hline \multirow[t]{2}{*}{48.1848} & 11.032 & 45.7824 & 10.768 & 65.6987 & 10.824 & 87.1031 & 12.484 & 86.4464 & 12.928 & 88.1378 & 12.708 \\
\hline & 0.643372 & 14.088 & 81.3332 & 11.704 & & & & & & & \\
\hline \multirow[t]{2}{*}{48.1848} & 11.036 & 44.8917 & 10.772 & 65.6987 & 10.828 & 86.0967 & 12.488 & 86.4464 & 12.932 & 90.1825 & 12.712 \\
\hline & 1.10772 & 14.092 & 80.3523 & 11.708 & & & & & & & \\
\hline \multirow[t]{2}{*}{47.6838} & 11.04 & 45.7824 & 0.776 & 65.6987 & 10.832 & 83.085 & 12.492 & 88.286 & 12.936 & 91.2068 & 12.716 \\
\hline & 0.643372 & 14.096 & 82.3153 & 11.712 & & & & & & & \\
\hline \multirow[t]{2}{*}{47.9343} & 11.044 & 47.5676 & 10.78 & 66.6237 & 10.836 & 84.0876 & 12.496 & 87.3657 & 12.94 & 92.2323 & 12.72 \\
\hline & 0.643372 & 14.1 & 81.3332 & 11.716 & & & & & & & \\
\hline \multirow[t]{2}{*}{47.4335} & 11.048 & 46.6744 & 10.784 & 65.6987 & 10.84 & 84.0876 & 12.5 & 86.4464 & 12.944 & 94.287 & 12.724 \\
\hline & 0.64 & 4.104 & 523 & 11.72 & & & & & & & \\
\hline \multirow[t]{2}{*}{47.6838} & 11.052 & 46.6744 & 10.788 & 65.6987 & 10.844 & 84.0876 & 12.504 & 84.611 & 12.948 & 3.259 & 12.728 \\
\hline & 2.05643 & 14.108 & 78.3942 & 11.724 & & & & & & & \\
\hline \multirow[t]{2}{*}{47.9343} & 11.056 & 46.6744 & 10.792 & 66.6237 & 10.848 & 85.0916 & 12.508 & 87.3657 & 12.952 & 91.2068 & 12.732 \\
\hline & 1.66854 & 14.112 & 78.3942 & 11.728 & & & & & & & \\
\hline 47.4335 & 11.06 & 45.7824 & 10.796 & 63.8522 & 10.852 & 83.085 & 12.512 & 88.286 & 12.956 & 92.2323 & 12.736 \\
\hline & 0.643372 & 14.116 & 78.3942 & 11.732 & & & & & & & \\
\hline 47.4335 & 11.0 & 44.8917 & 10.8 & & 10.856 & 84.0876 & 12.516 & 87.3657 & 12.96 & 90.1825 & 12.74 \\
\hline & 1.6 & 14.12 & 171 & 11. & & & & & & & \\
\hline 47.4335 & 11.068 & 45.7824 & 10.804 & 64.7747 & 10.86 & 34.0876 & 12.52 & 86.4464 & 12.964 & 1.2068 & 12.744 \\
\hline & 1.66 & 14.124 & 11 & 11.74 & & & & & & & \\
\hline 47.6838 & 11.07 & 45.7824 & 10.808 & 65.6987 & 10.864 & 85.0916 & 12.524 & 87.3657 & 12.968 & 90.1825 & 12.748 \\
\hline & 1.66 & 14.128 & 77.4171 & 11.744 & & & & & & & \\
\hline 47.6838 & $11 .($ & 45.7 & 10.8 & 65.6 & 10.868 & 86.0967 & 12.528 & 84.611 & 12.972 & 92.2323 & 12.752 \\
\hline & & & & & & & & & & & \\
\hline 47.1833 & 11.08 & 45.7824 & 10.8 & 63.8 & 10.872 & 90.1295 & 12.532 & 87.3657 & 12.976 & 93.259 & 12.756 \\
\hline & 0.6 & 14.136 & & & & & & & & & \\
\hline 47.4335 & 11.08 & 45.7824 & 1 & 65.6987 & 10.876 & 89.1194 & 12.536 & 87.3657 & 12.98 & 94.287 & 12.76 \\
\hline & 0.64 & 1414 & 71 & 11756 & & & & & & & \\
\hline 47.4335 & 11.088 & .7824 & 10.824 & 64.7747 & 10.88 & 86.0967 & 12.54 & 87.3657 & 12.984 & 94.287 & 12.764 \\
\hline & & & & & & & & & & & \\
\hline 47.1833 & 11.0 & 24 & 1 & 64. & 10.884 & 81.0835 & 12.544 & 87.3657 & 12.98 & 94.287 & 12.76 \\
\hline & & & & & & & & & & & \\
\hline 47.1833 & $11.0 \mathrm{~s}$ & 46.6 & 1 & 64.7747 & 10.888 & 81.0835 & 12.548 & 85.5282 & 12. & 1.2068 & 12.772 \\
\hline & 0.643372 & 14.152 & 42 & 11768 & & & & & & & \\
\hline 47.1833 & 11.1 & 46.6744 & 1 & 522 & 10.892 & 81.0835 & 12.552 & 86.4464 & 12.996 & 91.2068 & 12.776 \\
\hline & 0. & 1 & & 11.7 & & & & & & & \\
\hline 47.6838 & 11. & 24 & 1 & 62.9307 & 10.896 & 85.0916 & 12.556 & 85.5282 & 13 & 0.1825 & 12.78 \\
\hline & & & & & & & & & & & \\
\hline 47.6838 & 11.10 & 46.6744 & 1 & 62.9307 & 10.9 & 87.1031 & 12.56 & 85.5282 & 13.004 & 90.1825 & 12.784 \\
\hline & & & & 11.7 & & & & & & & \\
\hline 47.1833 & 11.11 & 46.6744 & 1 & 64.7747 & 10.904 & 86.0967 & 12.564 & 87.3657 & 13.008 & 90.1825 & 12.788 \\
\hline & & & & 11784 & & & & & & & \\
\hline 47.9343 & 11.11 & 76 & 1 & 63.8522 & 10.908 & 85.0916 & 12.568 & 87.3657 & 13.012 & 90.1825 & 12.792 \\
\hline & & & & & & & & & & & \\
\hline 48.4355 & 11.12 & 47.5 & 1 & 65. & 10.912 & 82.0836 & 12.572 & 86.4464 & 13.016 & 90.1825 & 12.796 \\
\hline & & & & & & & & & & & \\
\hline 48.6862 & 11. & 824 & & 64.7747 & 10.916 & 79.0871 & 12.576 & 87.3657 & 13.02 & 90.1825 & 12.8 \\
\hline & & & & & & & & & & & \\
\hline 49.6902 & 11.12 & 45.7824 & 10.864 & 64.7747 & 10.92 & 80.0847 & 12.58 & 87.3657 & 13.024 & 90.1825 & 12.804 \\
\hline & & & & & & & & & & & \\
\hline 50.1928 & 11.13 & 24 & 1 & 64.7747 & 10.924 & 78.0908 & 12.584 & 85.5282 & 13.02 & 90.1825 & 12.808 \\
\hline & & & & & & & & & & & \\
\hline 50.4443 & 11.136 & 44.8917 & 1 & 63.8522 & 10.928 & 80.0847 & 12.588 & 84.611 & 13.032 & 90.1825 & 12.812 \\
\hline & 0. & & & & & & & & & & \\
\hline 49.4391 & 11.14 & 45.7824 & 10.876 & 62.9307 & 10.932 & 79.0871 & 12.592 & 85.5282 & 13.036 & 90.1825 & 12.816 \\
\hline & & & & & & & & & & & \\
\hline 48.937 & 11.144 & 45. & 10.88 & 64.7747 & 10.936 & 81.0835 & 12.59 & 7 & 13. & 1.2068 & 12.82 \\
\hline & & & & & & & & & & & \\
\hline 48.1848 & 11.14 & 744 & 10.884 & 62.9307 & 10.94 & 80.0847 & 12.6 & 88.286 & 13.044 & 91.2068 & 12.824 \\
\hline & & & & & & & & & & & \\
\hline 48.4355 & 11.152 & 45.7824 & 10.888 & 64.7747 & 10.944 & 79.0871 & 12.604 & 86.4464 & 13.048 & 92.2323 & 12.828 \\
\hline & & & & 11.8 & & & & & & & \\
\hline 48.937 & 11.156 & 45.7824 & 10.892 & 64.7747 & 10.948 & 82.0836 & 12.608 & 87.3657 & 13.052 & 1.2068 & 12.832 \\
\hline & & & & & & & & & & & \\
\hline 49.188 & & 45.7824 & 10.896 & 64.7747 & 10.952 & 84.0876 & 12.612 & 85.5282 & 13.056 & 90.1825 & 12.836 \\
\hline & 2.06633 & 14.216 & 77.4171 & 11.832 & & & & & & & \\
\hline
\end{tabular}




\begin{tabular}{|c|c|c|c|c|c|c|c|c|c|c|c|}
\hline \multirow[t]{2}{*}{49.188} & 11.164 & 45.7824 & 10.9 & 63.8522 & 10.956 & 87.1031 & 12.616 & 88.286 & 13.06 & 90.1825 & 12.84 \\
\hline & 0.643372 & 14.22 & 77.4171 & 11.836 & & & & & & & \\
\hline \multirow[t]{2}{*}{49.188} & 11.168 & 44.8917 & 10.904 & 62.9307 & 10.96 & 87.1031 & 12.62 & 87.3657 & 13.064 & 90.1825 & 12.844 \\
\hline & 1.66854 & 14.224 & 79.3727 & 11.84 & & & & & & & \\
\hline \multirow[t]{2}{*}{49.9415} & 11.172 & 45.7824 & 10.908 & 63.8522 & 10.964 & 86.0967 & 12.624 & 85.5282 & 13.068 & 91.2068 & 12.848 \\
\hline & 0.643372 & 14.228 & 80.3523 & 11.844 & & & & & & & \\
\hline \multirow[t]{2}{*}{47.6838} & 11.176 & 45.7824 & 10.912 & 62.9307 & 10.968 & 81.0835 & 12.628 & 85.5282 & 13.072 & 90.1825 & 12.852 \\
\hline & 0.643372 & 14.232 & 79.3727 & 11.848 & & & & & & & \\
\hline \multirow[t]{62}{*}{29.1983} & 11.18 & 45.7824 & 10.916 & 64.7747 & 10.972 & 77.0958 & 12.632 & 84.611 & 13.076 & 91.2068 & 12.856 \\
\hline & 0.643372 & 14.236 & 78.3942 & 11.852 & & & & & & & \\
\hline & & 45.7824 & 10.92 & 62.9307 & 10.976 & 76.1021 & 12.636 & 85.5282 & 13.08 & 93.259 & 12.86 \\
\hline & 1.66854 & 14.24 & 77.4171 & 11.856 & & & & & & & \\
\hline & & 46.6744 & 10.924 & 62.9307 & 10.98 & 75.1097 & 12.64 & 86.4464 & 13.084 & 94.287 & 12.864 \\
\hline & 0.643372 & 14.244 & 79.3727 & 11.86 & & & & & & & \\
\hline & & 44.8917 & 10.928 & 62.9307 & 10.984 & 75.1097 & 12.644 & 83.6949 & 13.088 & 94.287 & 12.868 \\
\hline & 1.66854 & 14.248 & 77.4171 & 11.864 & & & & & & & \\
\hline & & 44.8917 & 10.932 & 62.9307 & 10.988 & 79.0871 & 12.648 & 85.5282 & 13.092 & 93.259 & 12.872 \\
\hline & 1.66854 & 14.252 & 79.3727 & 11.868 & & & & & & & \\
\hline & & 45.7824 & 10.936 & 63.8522 & 10.992 & 79.0871 & 12.652 & 84.611 & 13.096 & 93.259 & 12.876 \\
\hline & 0.643372 & 14.256 & 78.3942 & 11.872 & & & & & & & \\
\hline & & 45.7824 & 10.94 & 62.9307 & 10.996 & 84.0876 & 12.656 & 87.3657 & 13.1 & 90.1825 & 12.88 \\
\hline & 0.643372 & 14.26 & 78.3942 & 11.876 & & & & & & & \\
\hline & & 44.8917 & 10.944 & 63.8522 & 11 & 82.0836 & 12.66 & 86.4464 & 13.104 & 90.1825 & 12.884 \\
\hline & 1.66854 & 14.264 & 77.4171 & 11.88 & & & & & & & \\
\hline & & 45.7824 & 10.948 & 62.0105 & 11.004 & 81.0835 & 12.664 & 84.611 & 13.108 & 90.1825 & 12.888 \\
\hline & 0.643372 & 14.268 & 77.4171 & 11.884 & & & & & & & \\
\hline & & 45.7824 & 10.952 & 62.0105 & 11.008 & 80.0847 & 12.668 & 83.6949 & 13.112 & 90.1825 & 12.892 \\
\hline & 1.10772 & 14.272 & 78.3942 & 11.888 & & & & & & & \\
\hline & & 44.8917 & 10.956 & 63.8522 & 11.012 & 82.0836 & 12.672 & 86.4464 & 13.116 & 92.2323 & 12.896 \\
\hline & 0.643372 & 14.276 & 78.3942 & 11.892 & & & & & & & \\
\hline & & 45.7824 & 10.96 & 62.0105 & 11.016 & 77.0958 & 12.676 & 84.611 & 13.12 & 94.287 & 12.9 \\
\hline & 0.643372 & 14.28 & 79.3727 & 11.896 & & & & & & & \\
\hline & & 45.7824 & 10.964 & 63.8522 & 11.02 & 78.0908 & 12.68 & 83.6949 & 13.124 & 92.2323 & 12.904 \\
\hline & 2.6337 & 14.284 & 79.3727 & 11.9 & & & & & & & \\
\hline & & 44.8917 & 10.968 & 63.8522 & 11.024 & 78.0908 & 12.684 & 83.6949 & 13.128 & 92.2323 & 12.908 \\
\hline & 2.6337 & 14.288 & 78.3942 & 11.904 & & & & & & & \\
\hline & & 46.6744 & 10.972 & 63.8522 & 11.028 & 78.0908 & 12.688 & 83.6949 & 13.132 & 92.2323 & 12.912 \\
\hline & 0.643372 & 14.292 & 81.3332 & 11.908 & & & & & & & \\
\hline & & 45.7824 & 10.976 & 62.9307 & 11.032 & 78.0908 & 12.692 & 82.7798 & 13.136 & 91.2068 & 12.916 \\
\hline & 1.10772 & 14.296 & 79.3727 & 11.912 & & & & & & & \\
\hline & & 45.7824 & 10.98 & 62.0105 & 11.036 & 78.0908 & 12.696 & 83.6949 & 13.14 & 91.2068 & 12.92 \\
\hline & 1.10772 & 14.3 & 80.3523 & 11.916 & & & & & & & \\
\hline & & 45.7824 & 10.984 & 63.8522 & 11.04 & 80.0847 & 12.7 & 83.6949 & 13.144 & 90.1825 & 12.924 \\
\hline & 1.10772 & 14.304 & 78.3942 & 11.92 & & & & & & & \\
\hline & & 44.8917 & 10.988 & 63.8522 & 11.044 & 81.0835 & 12.704 & 83.6949 & 13.148 & 91.2068 & 12.928 \\
\hline & 0.643372 & 14.308 & 78.3942 & 11.924 & & & & & & & \\
\hline & & 45.7824 & 10.992 & 62.9307 & 11.048 & 80.0847 & 12.708 & 82.7798 & 13.152 & 91.2068 & 12.932 \\
\hline & 2.6337 & 14.312 & 78.3942 & 11.928 & & & & & & & \\
\hline & & 45.7824 & 10.996 & 62.9307 & 11.052 & 77.0958 & 12.712 & 82.7798 & 13.156 & 93.259 & 12.936 \\
\hline & 1.10772 & 14.316 & 78.3942 & 11.932 & & & & & & & \\
\hline & & 45.7824 & 11 & 63.8522 & 11.056 & 73.1288 & 12.716 & 83.6949 & 13.16 & 94.287 & 12.94 \\
\hline & 1.10772 & 14.32 & 78.3942 & 11.936 & & & & & & & \\
\hline & & 44.8917 & 11.004 & 63.8522 & 11.06 & 74.1186 & 12.72 & 81.8657 & 13.164 & 95.3162 & 12.944 \\
\hline & 1.10772 & 14.324 & 76.4411 & 11.94 & & & & & & & \\
\hline & & 45.7824 & 11.008 & 62.9307 & 11.064 & 75.1097 & 12.724 & 81.8657 & 13.168 & 94.287 & 12.948 \\
\hline & 1.10772 & 14.328 & 75.4665 & 11.944 & & & & & & & \\
\hline & & 45.7824 & 11.012 & 63.8522 & 11.068 & 77.0958 & 12.728 & 83.6949 & 13.172 & 93.259 & 12.952 \\
\hline & 0.643372 & 14.332 & 77.4171 & 11.948 & & & & & & & \\
\hline & & 44.8917 & 11.016 & 63.8522 & 11.072 & 77.0958 & 12.732 & 84.611 & 13.176 & 93.259 & 12.956 \\
\hline & 0.643372 & 14.336 & 76.4411 & 11.952 & & & & & & & \\
\hline & & 45.7824 & 11.02 & 62.9307 & 11.076 & 78.0908 & 12.736 & 86.4464 & 13.18 & 93.259 & 12.96 \\
\hline & 0.643372 & 14.34 & 75.4665 & 11.956 & & & & & & & \\
\hline & & 44.8917 & 11.024 & 62.9307 & 11.08 & 76.1021 & 12.74 & 83.6949 & 13.184 & 94.287 & 12.964 \\
\hline & 0.643372 & 14.344 & 76.4411 & 11.96 & & & & & & & \\
\hline & & 44.8917 & 11.028 & 62.0105 & 11.084 & 78.0908 & 12.744 & 85.5282 & 13.188 & 92.2323 & 12.968 \\
\hline & 1.10772 & 14.348 & 77.4171 & 11.964 & & & & & & & \\
\hline & & 46.6744 & 11.032 & 62.0105 & 11.088 & 77.0958 & 12.748 & 85.5282 & 13.192 & 93.259 & 12.972 \\
\hline & 3.89069 & 14.352 & 75.4665 & 11.968 & & & & & & & \\
\hline & & 47.5676 & 11.036 & 62.0105 & 11.092 & 76.1021 & 12.752 & 84.611 & 13.196 & 92.2323 & 12.976 \\
\hline & 1.10772 & 14.356 & 76.4411 & 11.972 & & & & & & & \\
\hline
\end{tabular}




\begin{tabular}{|c|c|c|c|c|c|c|c|c|c|c|}
\hline \multirow{3}{*}{1.10772} & 45.7824 & 11.04 & 61.0915 & 11.096 & 77.0958 & 12.756 & 82.7798 & 13.2 & 93.259 & 12.98 \\
\hline & 14.36 & 76.4411 & 11.976 & & & & & & & \\
\hline & 45.7824 & 11.044 & 62.9307 & 11.1 & 79.0871 & 12.76 & 82.7798 & 13.204 & 93.259 & 12.984 \\
\hline \multirow[t]{2}{*}{0.643372} & 14.364 & 76.4411 & 11.98 & & & & & & & \\
\hline & 45.7824 & 11.048 & 62.0105 & 11.104 & 80.0847 & 12.764 & 82.7798 & 13.208 & 91.2068 & 12.988 \\
\hline \multirow[t]{2}{*}{1.10772} & 14.368 & 77.4171 & 11.984 & & & & & & & \\
\hline & 46.6744 & 11.052 & 61.0915 & 11.108 & 76.1021 & 12.768 & 81.8657 & 13.212 & 92.2323 & 12.992 \\
\hline \multirow[t]{2}{*}{2.6337} & 14.372 & 74.493 & 11.988 & & & & & & & \\
\hline & 45.7824 & 11.056 & 62.0105 & 11.112 & 75.1097 & 12.772 & 82.7798 & 13.216 & 92.2323 & 12.996 \\
\hline \multirow[t]{2}{*}{0.643372} & 14.376 & 76.4411 & 11.992 & & & & & & & \\
\hline & 44.8917 & 11.06 & 63.8522 & 11.116 & 73.1288 & 12.776 & 84.611 & 13.22 & 91.2068 & 13 \\
\hline \multirow[t]{2}{*}{2.6337} & 14.38 & 75.4665 & 11.996 & & & & & & & \\
\hline & 45.7824 & 11.064 & 62.0105 & 11.12 & 73.1288 & 12.78 & 83.6949 & 13.224 & 93.259 & 13.004 \\
\hline \multirow[t]{2}{*}{1.10772} & 14.384 & 77.4171 & 12 & & & & & & & \\
\hline & 44.8917 & 11.068 & 61.0915 & 11.124 & 73.1288 & 12.784 & 82.7798 & 13.228 & 92.2323 & 13.008 \\
\hline \multirow[t]{2}{*}{0.643372} & 14.388 & 76.4411 & 12.004 & & & & & & & \\
\hline & 45.7824 & 11.072 & 63.8522 & 11.128 & 76.1021 & 12.788 & 83.6949 & 13.232 & 93.259 & 13.012 \\
\hline \multirow[t]{2}{*}{2.6337} & 14.392 & 75.4665 & 12.008 & & & & & & & \\
\hline & 46.6744 & 11.076 & 62.0105 & 11.132 & 76.1021 & 12.792 & 84.611 & 13.236 & 90.1825 & 13.016 \\
\hline 1.10772 & 14.396 & 75.4665 & 12.012 & & & & & & & \\
\hline & 46.6744 & 11.08 & 62.0105 & 11.136 & 74.1186 & 12.796 & 82.7798 & 13.24 & 91.2068 & 13.02 \\
\hline 0.643372 & 14.4 & 75.4665 & 12.016 & & & & & & & \\
\hline & 46.6744 & 11.084 & 62.0105 & 11.14 & 77.0958 & 12.8 & 83.6949 & 13.244 & 92.2323 & 13.024 \\
\hline 0.643372 & 14.404 & 74.493 & 12.02 & & & & & & & \\
\hline & 46.6744 & 11.088 & 62.9307 & 11.144 & 76.1021 & 12.804 & 83.6949 & 13.248 & 91.2068 & 13.028 \\
\hline 0.643372 & 14.408 & 74.493 & 12.024 & & & & & & & \\
\hline & 46.6744 & 11.092 & 61.0915 & 11.148 & 73.1288 & 12.808 & 83.6949 & 13.252 & 91.2068 & 13.032 \\
\hline 1.10772 & 14.412 & 73.5209 & 12.028 & & & & & & & \\
\hline & 45.7824 & 11.096 & 61.0915 & 11.152 & 71.1532 & 12.812 & 83.6949 & 13.256 & 90.1825 & 13.036 \\
\hline 4.94248 & 14.416 & 73.5209 & 12.032 & & & & & & & \\
\hline & 44.8917 & 11.1 & 62.0105 & 11.156 & 74.1186 & 12.816 & 82.7798 & 13.26 & 90.1825 & 13.04 \\
\hline 2.6337 & 14.42 & 73.5209 & 12.036 & & & & & & & \\
\hline & 44.8917 & 11.104 & 62.0105 & 11.16 & 77.0958 & 12.82 & 83.6949 & 13.264 & 90.1825 & 13.044 \\
\hline 1.10772 & 14.424 & 74.493 & 12.04 & & & & & & & \\
\hline & 45.7824 & 11.108 & 61.0915 & 11.164 & 78.0908 & 12.824 & 83.6949 & 13.268 & 90.1825 & 13.048 \\
\hline 1.10772 & 14.428 & 74.493 & 12.044 & & & & & & & \\
\hline & 45.7824 & 11.112 & 60.1738 & 11.168 & 75.1097 & 12.828 & 84.611 & 13.272 & 90.1825 & 13.052 \\
\hline 0.643372 & 14.432 & 74.493 & 12.048 & & & & & & & \\
\hline & 46.6744 & 11.116 & 61.0915 & 11.172 & 76.1021 & 12.832 & 83.6949 & 13.276 & 90.1825 & 13.056 \\
\hline 0.643372 & 14.436 & 73.5209 & 12.052 & & & & & & & \\
\hline & 44.0023 & 11.12 & 61.0915 & 11.176 & 71.1532 & 12.836 & 82.7798 & 13.28 & 90.1825 & 13.06 \\
\hline 0.643372 & 14.44 & 73.5209 & 12.056 & & & & & & & \\
\hline & 44.0023 & 11.124 & 58.342 & 11.18 & 73.1288 & 12.84 & 84.611 & 13.284 & 90.1825 & 13.064 \\
\hline 0.643372 & 14.444 & 72.55 & 12.06 & & & & & & & \\
\hline & 44.0023 & 11.128 & 59.2573 & 11.184 & 70.1673 & 12.844 & 84.611 & 13.288 & 92.2323 & 13.068 \\
\hline 1.10772 & 14.448 & 73.5209 & 12.064 & & & & & & & \\
\hline & 44.0023 & 11.132 & 56.5153 & 11.188 & 72.1403 & 12.848 & 81.8657 & 13.292 & 91.2068 & 13.072 \\
\hline 0.643372 & 14.452 & 72.55 & 12.068 & & & & & & & \\
\hline & 44.8917 & 11.136 & 57.428 & 11.192 & 72.1403 & 12.852 & 85.5282 & 13.296 & 92.2323 & 13.076 \\
\hline 0.643372 & 14.456 & 72.55 & 12.072 & & & & & & & \\
\hline & 44.8917 & 11.14 & 57.428 & 11.196 & 73.1288 & 12.856 & 83.6949 & 13.3 & 91.2068 & 13.08 \\
\hline 1.10772 & 14.46 & 71.5804 & 12.076 & & & & & & & \\
\hline & 44.8917 & 11.144 & 57.428 & 11.2 & 74.1186 & 12.86 & 82.7798 & 13.304 & 90.1825 & 13.084 \\
\hline 0.643372 & 14.464 & 73.5209 & 12.08 & & & & & & & \\
\hline & 44.8917 & 11.148 & 58.342 & 11.204 & 74.1186 & 12.864 & 84.611 & 13.308 & 90.1825 & 13.088 \\
\hline 0.643372 & 14.468 & 71.5804 & 12.084 & & & & & & & \\
\hline & 44.8917 & 11.152 & 58.342 & 11.208 & 74.1186 & 12.868 & 82.7798 & 13.312 & 90.1825 & 13.092 \\
\hline 1.10772 & 14.472 & 72.55 & 12.088 & & & & & & & \\
\hline & 44.0023 & 11.156 & 57.428 & 11.212 & 74.1186 & 12.872 & 84.611 & 13.316 & 90.1825 & 13.096 \\
\hline 1.10772 & 14.476 & 72.55 & 12.092 & & & & & & & \\
\hline & 44.8917 & 11.16 & 56.5153 & 11.216 & 74.1186 & 12.876 & 84.611 & 13.32 & 89.1595 & 13.1 \\
\hline 1.10772 & 14.48 & 71.5804 & 12.096 & & & & & & & \\
\hline & 44.8917 & 11.164 & 59.2573 & 11.22 & 72.1403 & 12.88 & 84.611 & 13.324 & 90.1825 & 13.104 \\
\hline 1.10772 & 14.484 & 71.5804 & 12.1 & & & & & & & \\
\hline & 44.0023 & 11.168 & 58.342 & 11.224 & 69.1829 & 12.884 & 81.8657 & 13.328 & 90.1825 & 13.108 \\
\hline 1.10772 & 14.488 & 72.55 & 12.104 & & & & & & & \\
\hline & 44.8917 & 11.172 & 59.2573 & 11.228 & 69.1829 & 12.888 & 82.7798 & 13.332 & 91.2068 & 13.112 \\
\hline 0.643372 & 14.492 & 70.612 & 12.108 & & & & & & & \\
\hline & 44.0023 & 11.176 & 59.2573 & 11.232 & 70.1673 & 12.892 & 81.8657 & 13.336 & 90.1825 & 13.116 \\
\hline 0.643372 & 14.496 & 73.5209 & 12.112 & & & & & & & \\
\hline
\end{tabular}




\begin{tabular}{|c|c|c|c|c|c|c|c|c|c|c|}
\hline \multirow{3}{*}{1.10772} & 42.2275 & 11.18 & 60.1738 & 11.236 & 70.1673 & 12.896 & 81.8657 & 13.34 & 92.2323 & 13.12 \\
\hline & 14.5 & 71.5804 & 12.116 & & & & & & & \\
\hline & 40.458 & 11.184 & 60.1738 & 11.24 & 71.1532 & 12.9 & 81.8657 & 13.344 & 91.2068 & 13.124 \\
\hline \multirow[t]{2}{*}{0.643372} & 14.504 & 73.5209 & 12.12 & & & & & & & \\
\hline & 39.5753 & 11.188 & 59.2573 & 11.244 & 72.1403 & 12.904 & 83.6949 & 13.348 & 90.1825 & 13.128 \\
\hline \multirow[t]{2}{*}{1.10772} & 14.508 & 70.612 & 12.124 & & & & & & & \\
\hline & 38.6939 & 11.192 & 56.5153 & 11.248 & 71.1532 & 12.908 & 83.6949 & 13.352 & 89.1595 & 13.132 \\
\hline \multirow[t]{2}{*}{1.66854} & 14.512 & 70.612 & 12.128 & & & & & & & \\
\hline & 40.458 & 11.196 & 55.6038 & 11.252 & 70.1673 & 12.912 & 82.7798 & 13.356 & 89.1595 & 13.136 \\
\hline \multirow[t]{2}{*}{0.643372} & 14.516 & 71.5804 & 12.132 & & & & & & & \\
\hline & 38.6939 & 11.2 & 54.6936 & 11.256 & 70.1673 & 12.916 & 84.611 & 13.36 & 89.1595 & 13.14 \\
\hline \multirow[t]{2}{*}{0.643372} & 14.52 & 70.612 & 12.136 & & & & & & & \\
\hline & 38.6939 & 11.204 & 58.342 & 11.26 & 68.1997 & 12.92 & 84.611 & 13.364 & 90.1825 & 13.144 \\
\hline \multirow[t]{2}{*}{0.643372} & 14.524 & 69.645 & 12.14 & & & & & & & \\
\hline & 37.8139 & 11.208 & 59.2573 & 11.264 & 67.2179 & 12.924 & 81.8657 & 13.368 & 90.1825 & 13.148 \\
\hline \multirow[t]{2}{*}{0.643372} & 14.528 & 68.6792 & 12.144 & & & & & & & \\
\hline & 34.3073 & 11.212 & 59.2573 & 11.268 & 65.2584 & 12.928 & 82.7798 & 13.372 & 90.1825 & 13.152 \\
\hline \multirow[t]{2}{*}{0.643372} & 14.532 & 69.645 & 12.148 & & & & & & & \\
\hline & 32.5621 & 11.216 & 60.1738 & 11.272 & 64.2806 & 12.932 & 82.7798 & 13.376 & 91.2068 & 13.156 \\
\hline 0.643372 & 14.536 & 68.6792 & 12.152 & & & & & & & \\
\hline & 36.0579 & 11.22 & 59.2573 & 11.276 & 67.2179 & 12.936 & 83.6949 & 13.38 & 90.1825 & 13.16 \\
\hline 0.643372 & 14.54 & 69.645 & 12.156 & & & & & & & \\
\hline & 43.1143 & 11.224 & 57.428 & 11.28 & 68.1997 & 12.94 & 81.8657 & 13.384 & 90.1825 & 13.164 \\
\hline 0.643372 & 14.544 & 68.6792 & 12.16 & & & & & & & \\
\hline & 47.5676 & 11.228 & 56.5153 & 11.284 & 70.1673 & 12.944 & 82.7798 & 13.388 & 90.1825 & 13.168 \\
\hline 0.643372 & 14.548 & 70.612 & 12.164 & & & & & & & \\
\hline & 49.3581 & 11.232 & 54.6936 & 11.288 & 71.1532 & 12.948 & 82.7798 & 13.392 & 90.1825 & 13.172 \\
\hline 0.643372 & 14.552 & 69.645 & 12.168 & & & & & & & \\
\hline & 44.0023 & 11.236 & 55.6038 & 11.292 & 72.1403 & 12.952 & 81.8657 & 13.396 & 90.1825 & 13.176 \\
\hline 0.643372 & 14.556 & 69.645 & 12.172 & & & & & & & \\
\hline & 37.8139 & 11.24 & 54.6936 & 11.296 & 72.1403 & 12.956 & 82.7798 & 13.4 & 90.1825 & 13.18 \\
\hline 0.643372 & 14.56 & 69.645 & 12.176 & & & & & & & \\
\hline & 33.434 & 11.244 & 54.6936 & 11.3 & 71.1532 & 12.96 & 82.7798 & 13.404 & 90.1825 & 13.184 \\
\hline 0.643372 & 14.564 & 67.7147 & 12.18 & & & & & & & \\
\hline & 30.8225 & 11.248 & 54.6936 & 11.304 & 68.1997 & 12.964 & 81.8657 & 13.408 & 91.2068 & 13.188 \\
\hline 0.643372 & 14.568 & 69.645 & 12.184 & & & & & & & \\
\hline & 30.8225 & 11.252 & 53.7847 & 11.308 & 67.2179 & 12.968 & 83.6949 & 13.412 & 90.1825 & 13.192 \\
\hline 0.643372 & 14.572 & 69.645 & 12.188 & & & & & & & \\
\hline & 35.1818 & 11.256 & 54.6936 & 11.312 & 63.3043 & 12.972 & 82.7798 & 13.416 & 90.1825 & 13.196 \\
\hline 0.643372 & 14.576 & 69.645 & 12.192 & & & & & & & \\
\hline & 44.0023 & 11.26 & 55.6038 & 11.316 & 65.2584 & 12.976 & 80.9527 & 13.42 & 90.1825 & 13.2 \\
\hline 0.643372 & 14.58 & 68.6792 & 12.196 & & & & & & & \\
\hline & 44.8917 & 11.264 & 58.342 & 11.32 & 64.2806 & 12.98 & 81.8657 & 13.424 & 90.1825 & 13.204 \\
\hline 0.643372 & 14.584 & 68.6792 & 12.2 & & & & & & & \\
\hline & 44.8917 & 11.268 & 61.0915 & 11.324 & 65.2584 & 12.984 & 81.8657 & 13.428 & 90.1825 & 13.208 \\
\hline 0.643372 & 14.588 & 69.645 & 12.204 & & & & & & & \\
\hline & 41.3422 & 11.272 & 62.0105 & 11.328 & 66.2374 & 12.988 & 81.8657 & 13.432 & 90.1825 & 13.212 \\
\hline 0.643372 & 14.592 & 67.7147 & 12.208 & & & & & & & \\
\hline & 35.1818 & 11.276 & 59.2573 & 11.332 & 65.2584 & 12.992 & 81.8657 & 13.436 & 90.1825 & 13.216 \\
\hline 1.10772 & 14.596 & 68.6792 & 12.212 & & & & & & & \\
\hline & 29.0885 & 11.28 & 56.5153 & 11.336 & 67.2179 & 12.996 & 80.9527 & 13.44 & 90.1825 & 13.22 \\
\hline 0.643372 & 14.6 & 68.6792 & 12.216 & & & & & & & \\
\hline & 26.498 & 11.284 & 54.6936 & 11.34 & 67.2179 & 13 & 81.8657 & 13.444 & 90.1825 & 13.224 \\
\hline 1.10772 & 14.604 & 68.6792 & 12.22 & & & & & & & \\
\hline & 28.2236 & 11.288 & 54.6936 & 11.344 & 65.2584 & 13.004 & 81.8657 & 13.448 & 90.1825 & 13.228 \\
\hline 0.643372 & 14.608 & 69.645 & 12.224 & & & & & & & \\
\hline & 33.434 & 11.292 & 53.7847 & 11.348 & 67.2179 & 13.008 & 79.1299 & 13.452 & 90.1825 & 13.232 \\
\hline 0.643372 & 14.612 & 67.7147 & 12.228 & & & & & & & \\
\hline & 38.6939 & 11.296 & 56.5153 & 11.352 & 63.3043 & 13.012 & 80.9527 & 13.456 & 90.1825 & 13.236 \\
\hline 1.10772 & 14.616 & 67.7147 & 12.232 & & & & & & & \\
\hline & 40.458 & 11.3 & 59.2573 & 11.356 & 64.2806 & 13.016 & 80.9527 & 13.46 & 90.1825 & 13.24 \\
\hline 0.643372 & 14.62 & 68.6792 & 12.236 & & & & & & & \\
\hline & 43.1143 & 11.304 & 61.0915 & 11.36 & 64.2806 & 13.02 & 80.0408 & 13.464 & 90.1825 & 13.244 \\
\hline 0.643372 & 14.624 & 68.6792 & 12.24 & & & & & & & \\
\hline & 40.458 & 11.308 & 62.9307 & 11.364 & 63.3043 & 13.024 & 80.0408 & 13.468 & 90.1825 & 13.248 \\
\hline 0.643372 & 14.628 & 68.6792 & 12.244 & & & & & & & \\
\hline & 38.6939 & 11.312 & 62.0105 & 11.368 & 66.2374 & 13.028 & 79.1299 & 13.472 & 90.1825 & 13.252 \\
\hline 0.643372 & 14.632 & 68.6792 & 12.248 & & & & & & & \\
\hline & 36.0579 & 11.316 & 58.342 & 11.372 & 65.2584 & 13.032 & 80.0408 & 13.476 & 91.2068 & 13.256 \\
\hline 0.643372 & 14.636 & 66.7515 & 12.252 & & & & & & & \\
\hline
\end{tabular}




\begin{tabular}{|c|c|c|c|c|c|c|c|c|c|c|}
\hline & 36.0579 & 11.32 & 55.6038 & 11.376 & 68.1997 & 13.036 & 80.0408 & 13.48 & 91.2068 & 13.26 \\
\hline \multirow[t]{2}{*}{0.643372} & 14.64 & 67.7147 & 12.256 & & & & & & & \\
\hline & 39.5753 & 11.324 & 52.877 & 11.38 & 67.2179 & 13.04 & 80.0408 & 13.484 & 90.1825 & 13.264 \\
\hline \multirow[t]{2}{*}{1.10772} & 14.644 & 66.7515 & 12.26 & & & & & & & \\
\hline & 41.3422 & 11.328 & 52.877 & 11.384 & 69.1829 & 13.044 & 80.9527 & 13.488 & 90.1825 & 13.268 \\
\hline \multirow[t]{2}{*}{0.643372} & 14.648 & 68.6792 & 12.264 & & & & & & & \\
\hline & 42.2275 & 11.332 & 51.9707 & 11.388 & 68.1997 & 13.048 & 78.2201 & 13.492 & 91.2068 & 13.272 \\
\hline \multirow[t]{2}{*}{0.643372} & 14.652 & 65.7897 & 12.268 & & & & & & & \\
\hline & 43.1143 & 11.336 & 56.5153 & 11.392 & 66.2374 & 13.052 & 80.0408 & 13.496 & 91.2068 & 13.276 \\
\hline \multirow[t]{2}{*}{0.643372} & 14.656 & 68.6792 & 12.272 & & & & & & & \\
\hline & 42.2275 & 11.34 & 57.428 & 11.396 & 66.2374 & 13.056 & 77.3114 & 13.5 & 91.2068 & 13.28 \\
\hline \multirow[t]{2}{*}{0.643372} & 14.66 & 66.7515 & 12.276 & & & & & & & \\
\hline & 42.2275 & 11.344 & 60.1738 & 11.4 & 64.2806 & 13.06 & 79.1299 & 13.504 & 90.1825 & 13.284 \\
\hline \multirow[t]{2}{*}{0.643372} & 14.664 & 65.7897 & 12.28 & & & & & & & \\
\hline & 44.0023 & 11.348 & 61.0915 & 11.404 & 63.3043 & 13.064 & 80.0408 & 13.508 & 93.259 & 13.288 \\
\hline \multirow[t]{2}{*}{0.643372} & 14.668 & 65.7897 & 12.284 & & & & & & & \\
\hline & 43.1143 & 11.352 & 60.1738 & 11.408 & 64.2806 & 13.068 & 79.1299 & 13.512 & 92.2323 & 13.292 \\
\hline \multirow[t]{2}{*}{0.643372} & 14.672 & 66.7515 & 12.288 & & & & & & & \\
\hline & 43.1143 & 11.356 & 56.5153 & 11.412 & 64.2806 & 13.072 & 79.1299 & 13.516 & 91.2068 & 13.296 \\
\hline 0.643372 & 14.676 & 65.7897 & 12.292 & & & & & & & \\
\hline & 41.3422 & 11.36 & 51.9707 & 11.416 & 67.2179 & 13.076 & 78.2201 & 13.52 & 93.259 & 13.3 \\
\hline 0.643372 & 14.68 & 65.7897 & 12.296 & & & & & & & \\
\hline & 41.3422 & 11.364 & 50.1618 & 11.42 & 64.2806 & 13.08 & 77.3114 & 13.524 & 93.259 & 13.304 \\
\hline 0.643372 & 14.684 & 65.7897 & 12.3 & & & & & & & \\
\hline & 38.6939 & 11.368 & 51.9707 & 11.424 & 67.2179 & 13.084 & 77.3114 & 13.528 & 92.2323 & 13.308 \\
\hline 0.643372 & 14.688 & 67.7147 & 12.304 & & & & & & & \\
\hline & 38.6939 & 11.372 & 53.7847 & 11.428 & 65.2584 & 13.088 & 77.3114 & 13.532 & 93.259 & 13.312 \\
\hline 0.643372 & 14.692 & 65.7897 & 12.308 & & & & & & & \\
\hline & 35.1818 & 11.376 & 59.2573 & 11.432 & 65.2584 & 13.092 & 76.4037 & 13.536 & 90.1825 & 13.316 \\
\hline 0.643372 & 14.696 & 66.7515 & 12.312 & & & & & & & \\
\hline & 38.6939 & 11.38 & 59.2573 & 11.436 & 63.3043 & 13.096 & 77.3114 & 13.54 & 91.2068 & 13.32 \\
\hline 2.6337 & 14.7 & 65.7897 & 12.316 & & & & & & & \\
\hline & 40.458 & 11.384 & 62.0105 & 11.44 & 64.2806 & 13.1 & 77.3114 & 13.544 & 91.2068 & 13.324 \\
\hline 0.643372 & 14.704 & 65.7897 & 12.32 & & & & & & & \\
\hline & 42.2275 & 11.388 & 62.0105 & 11.444 & 62.3293 & 13.104 & 78.2201 & 13.548 & 90.1825 & 13.328 \\
\hline 0.643372 & 14.708 & 66.7515 & 12.324 & & & & & & & \\
\hline & 44.0023 & 11.392 & 62.0105 & 11.448 & 62.3293 & 13.108 & 77.3114 & 13.552 & 90.1825 & 13.332 \\
\hline 0.643372 & 14.712 & 67.7147 & 12.328 & & & & & & & \\
\hline & 44.0023 & 11.396 & 58.342 & 11.452 & 62.3293 & 13.112 & 77.3114 & 13.556 & 91.2068 & 13.336 \\
\hline 0.643372 & 14.716 & 66.7515 & 12.332 & & & & & & & \\
\hline & 42.2275 & 11.4 & 57.428 & 11.456 & 63.3043 & 13.116 & 76.4037 & 13.56 & 91.2068 & 13.34 \\
\hline 0.643372 & 14.72 & 65.7897 & 12.336 & & & & & & & \\
\hline & 42.2275 & 11.404 & 56.5153 & 11.46 & 65.2584 & 13.12 & 80.0408 & 13.564 & 92.2323 & 13.344 \\
\hline 0.643372 & 14.724 & 68.6792 & 12.34 & & & & & & & \\
\hline & 41.3422 & 11.408 & 57.428 & 11.464 & 62.3293 & 13.124 & 77.3114 & 13.568 & 91.2068 & 13.348 \\
\hline 0.643372 & 14.728 & 65.7897 & 12.344 & & & & & & & \\
\hline & 39.5753 & 11.412 & 56.5153 & 11.468 & 64.2806 & 13.128 & 77.3114 & 13.572 & 93.259 & 13.352 \\
\hline 1.10772 & 14.732 & 65.7897 & 12.348 & & & & & & & \\
\hline & 38.6939 & 11.416 & 57.428 & 11.472 & 64.2806 & 13.132 & 77.3114 & 13.576 & 91.2068 & 13.356 \\
\hline 0.643372 & 14.736 & 66.7515 & 12.352 & & & & & & & \\
\hline & 36.0579 & 11.42 & 56.5153 & 11.476 & 64.2806 & 13.136 & 76.4037 & 13.58 & 91.2068 & 13.36 \\
\hline 0.643372 & 14.74 & 65.7897 & 12.356 & & & & & & & \\
\hline & 37.8139 & 11.424 & 55.6038 & 11.48 & 65.2584 & 13.14 & 75.4971 & 13.584 & 90.1825 & 13.364 \\
\hline 0.643372 & 14.744 & 65.7897 & 12.36 & & & & & & & \\
\hline & 37.8139 & 11.428 & 55.6038 & 11.484 & 63.3043 & 13.144 & 78.2201 & 13.588 & 90.1825 & 13.368 \\
\hline 0.643372 & 14.748 & 64.8291 & 12.364 & & & & & & & \\
\hline & 42.2275 & 11.432 & 53.7847 & 11.488 & 63.3043 & 13.148 & 77.3114 & 13.592 & 90.1825 & 13.372 \\
\hline 0.643372 & 14.752 & 64.8291 & 12.368 & & & & & & & \\
\hline & 46.6744 & 11.436 & 52.877 & 11.492 & 64.2806 & 13.152 & 77.3114 & 13.596 & 91.2068 & 13.376 \\
\hline 0.643372 & 14.756 & 63.8699 & 12.372 & & & & & & & \\
\hline & 49.3581 & 11.44 & 52.877 & 11.496 & 66.2374 & 13.156 & 76.4037 & 13.6 & 92.2323 & 13.38 \\
\hline 0.643372 & 14.76 & 63.8699 & 12.376 & & & & & & & \\
\hline & 49.3581 & 11.444 & 51.9707 & 11.5 & 63.3043 & 13.16 & 76.4037 & 13.604 & 91.2068 & 13.384 \\
\hline 0.643372 & 14.764 & 63.8699 & 12.38 & & & & & & & \\
\hline & 48.4623 & 11.448 & 51.9707 & 11.504 & 65.2584 & 13.164 & 76.4037 & 13.608 & 90.1825 & 13.388 \\
\hline 0.643372 & 14.768 & 64.8291 & 12.384 & & & & & & & \\
\hline & 44.8917 & 11.452 & 54.6936 & 11.508 & 64.2806 & 13.168 & 76.4037 & 13.612 & 90.1825 & 13.392 \\
\hline 2.6337 & 14.772 & 64.8291 & 12.388 & & & & & & & \\
\hline & 38.6939 & 11.456 & 54.6936 & 11.512 & 63.3043 & 13.172 & 74.5917 & 13.616 & 91.2068 & 13.396 \\
\hline 0.643372 & 14.776 & 63.8699 & 12.392 & & & & & & & \\
\hline
\end{tabular}




\begin{tabular}{|c|c|c|c|c|c|c|c|c|c|c|}
\hline & 36.9352 & 11.46 & 56.5153 & 11.516 & 65.2584 & 13.176 & 74.5917 & 13.62 & 90.1825 & 13.4 \\
\hline \multirow[t]{2}{*}{1.10772} & 14.78 & 63.8699 & 12.396 & & & & & & & \\
\hline & 36.9352 & 11.464 & 57.428 & 11.52 & 63.3043 & 13.18 & 75.4971 & 13.624 & 92.2323 & 13.404 \\
\hline \multirow[t]{2}{*}{2.6337} & 14.784 & 64.8291 & 12.4 & & & & & & & \\
\hline & 35.1818 & 11.468 & 55.6038 & 11.524 & 62.3293 & 13.184 & 74.5917 & 13.628 & 90.1825 & 13.408 \\
\hline \multirow[t]{2}{*}{2.6337} & 14.788 & 65.7897 & 12.404 & & & & & & & \\
\hline & 37.8139 & 11.472 & 53.7847 & 11.528 & 62.3293 & 13.188 & 76.4037 & 13.632 & 90.1825 & 13.412 \\
\hline \multirow[t]{2}{*}{1.10772} & 14.792 & 64.8291 & 12.408 & & & & & & & \\
\hline & 38.6939 & 11.476 & 53.7847 & 11.532 & 62.3293 & 13.192 & 75.4971 & 13.636 & 90.1825 & 13.416 \\
\hline \multirow[t]{2}{*}{0.643372} & 14.796 & 67.7147 & 12.412 & & & & & & & \\
\hline & 39.5753 & 11.48 & 52.877 & 11.536 & 64.2806 & 13.196 & 76.4037 & 13.64 & 91.2068 & 13.42 \\
\hline \multirow[t]{2}{*}{1.10772} & 14.8 & 65.7897 & 12.416 & & & & & & & \\
\hline & 42.2275 & 11.484 & 53.7847 & 11.54 & 63.3043 & 13.2 & 75.4971 & 13.644 & 90.1825 & 13.424 \\
\hline \multirow[t]{2}{*}{2.6337} & 14.804 & 66.7515 & 12.42 & & & & & & & \\
\hline & 40.458 & 11.488 & 56.5153 & 11.544 & 62.3293 & 13.204 & 77.3114 & 13.648 & 90.1825 & 13.428 \\
\hline \multirow[t]{2}{*}{1.10772} & 14.808 & 64.8291 & 12.424 & & & & & & & \\
\hline & 39.5753 & 11.492 & 55.6038 & 11.548 & 65.2584 & 13.208 & 73.6872 & 13.652 & 90.1825 & 13.432 \\
\hline \multirow[t]{2}{*}{2.6337} & 14.812 & 64.8291 & 12.428 & & & & & & & \\
\hline & 38.6939 & 11.496 & 56.5153 & 11.552 & 64.2806 & 13.212 & 75.4971 & 13.656 & 90.1825 & 13.436 \\
\hline 4.94248 & 14.816 & 64.8291 & 12.432 & & & & & & & \\
\hline & 36.9352 & 11.5 & 56.5153 & 11.556 & 65.2584 & 13.216 & 73.6872 & 13.66 & 90.1825 & 13.44 \\
\hline 1.10772 & 14.82 & 63.8699 & 12.436 & & & & & & & \\
\hline & 35.1818 & 11.504 & 56.5153 & 11.56 & 64.2806 & 13.22 & 75.4971 & 13.664 & 90.1825 & 13.444 \\
\hline 0.643372 & 14.824 & 63.8699 & 12.44 & & & & & & & \\
\hline & 32.5621 & 11.508 & 57.428 & 11.564 & 62.3293 & 13.224 & 75.4971 & 13.668 & 90.1825 & 13.448 \\
\hline 1.10772 & 14.828 & 61.9553 & 12.444 & & & & & & & \\
\hline & 31.6916 & 11.512 & 58.342 & 11.568 & 62.3293 & 13.228 & 76.4037 & 13.672 & 90.1825 & 13.452 \\
\hline 2.6337 & 14.832 & 62.9119 & 12.448 & & & & & & & \\
\hline & 34.3073 & 11.516 & 57.428 & 11.572 & 61.3557 & 13.232 & 75.4971 & 13.676 & 90.1825 & 13.456 \\
\hline 1.10772 & 14.836 & 61.9553 & 12.452 & & & & & & & \\
\hline & 37.8139 & 11.52 & 57.428 & 11.576 & 62.3293 & 13.236 & 74.5917 & 13.68 & 90.1825 & 13.46 \\
\hline 2.6337 & 14.84 & 63.8699 & 12.456 & & & & & & & \\
\hline & 42.2275 & 11.524 & 55.6038 & 11.58 & 63.3043 & 13.24 & 75.4971 & 13.684 & 90.1825 & 13.464 \\
\hline 0.643372 & 14.844 & 64.8291 & 12.46 & & & & & & & \\
\hline & 44.8917 & 11.528 & 54.6936 & 11.584 & 64.2806 & 13.244 & 76.4037 & 13.688 & 90.1825 & 13.468 \\
\hline 1.10772 & 14.848 & 65.7897 & 12.464 & & & & & & & \\
\hline & 46.6744 & 11.532 & 53.7847 & 11.588 & 63.3043 & 13.248 & 73.6872 & 13.692 & 90.1825 & 13.472 \\
\hline 4.94248 & 14.852 & 66.7515 & 12.468 & & & & & & & \\
\hline & 44.0023 & 11.536 & 53.7847 & 11.592 & 63.3043 & 13.252 & 73.6872 & 13.696 & 90.1825 & 13.476 \\
\hline 2.6337 & 14.856 & 67.7147 & 12.472 & & & & & & & \\
\hline & 38.6939 & 11.54 & 53.7847 & 11.596 & 63.3043 & 13.256 & 75.4971 & 13.7 & 90.1825 & 13.48 \\
\hline 3.89069 & 14.86 & 66.7515 & 12.476 & & & & & & & \\
\hline & 35.1818 & 11.544 & 56.5153 & 11.6 & 65.2584 & 13.26 & 75.4971 & 13.704 & 90.1825 & 13.484 \\
\hline 1.10772 & 14.864 & 62.9119 & 12.48 & & & & & & & \\
\hline & 31.6916 & 11.548 & 56.5153 & 11.604 & 62.3293 & 13.264 & 72.7839 & 13.708 & 91.2068 & 13.488 \\
\hline 3.89069 & 14.868 & 61.9553 & 12.484 & & & & & & & \\
\hline & 30.8225 & 11.552 & 55.6038 & 11.608 & 62.3293 & 13.268 & 72.7839 & 13.712 & 90.1825 & 13.492 \\
\hline 2.6337 & 14.872 & 61.9553 & 12.488 & & & & & & & \\
\hline & 32.5621 & 11.556 & 55.6038 & 11.612 & 64.2806 & 13.272 & 74.5917 & 13.716 & 90.1825 & 13.496 \\
\hline 1.10772 & 14.876 & 63.8699 & 12.492 & & & & & & & \\
\hline & 36.0579 & 11.56 & 52.877 & 11.616 & 64.2806 & 13.276 & 72.7839 & 13.72 & 90.1825 & 13.5 \\
\hline 2.6337 & 14.88 & 65.7897 & 12.496 & & & & & & & \\
\hline & 37.8139 & 11.564 & 52.877 & 11.62 & 63.3043 & 13.28 & 73.6872 & 13.724 & 90.1825 & 13.504 \\
\hline 0.643372 & 14.884 & 63.8699 & 12.5 & & & & & & & \\
\hline & 39.5753 & 11.568 & 50.1618 & 11.624 & 65.2584 & 13.284 & 73.6872 & 13.728 & 90.1825 & 13.508 \\
\hline 3.89069 & 14.888 & 61.0001 & 12.504 & & & & & & & \\
\hline & 43.1143 & 11.572 & 51.9707 & 11.628 & 64.2806 & 13.288 & 72.7839 & 13.732 & 89.1595 & 13.512 \\
\hline 3.89069 & 14.892 & 60.0462 & 12.508 & & & & & & & \\
\hline & 42.2275 & 11.576 & 53.7847 & 11.632 & 65.2584 & 13.292 & 73.6872 & 13.736 & 90.1825 & 13.516 \\
\hline 3.89069 & 14.896 & 61.0001 & 12.512 & & & & & & & \\
\hline & 38.6939 & 11.58 & 55.6038 & 11.636 & 63.3043 & 13.296 & 74.5917 & 13.74 & 90.1825 & 13.52 \\
\hline 1.10772 & 14.9 & 63.8699 & 12.516 & & & & & & & \\
\hline & 36.9352 & 11.584 & 57.428 & 11.64 & 63.3043 & 13.3 & 74.5917 & 13.744 & 92.2323 & 13.524 \\
\hline 0.643372 & 14.904 & 64.8291 & 12.52 & & & & & & & \\
\hline & 33.434 & 11.588 & 58.342 & 11.644 & 63.3043 & 13.304 & 72.7839 & 13.748 & 90.1825 & 13.528 \\
\hline 0.643372 & 14.908 & 65.7897 & 12.524 & & & & & & & \\
\hline & 31.6916 & 11.592 & 59.2573 & 11.648 & 61.3557 & 13.308 & 72.7839 & 13.752 & 90.1825 & 13.532 \\
\hline 1.10772 & 14.912 & 67.7147 & 12.528 & & & & & & & \\
\hline & 31.6916 & 11.596 & 59.2573 & 11.652 & 62.3293 & 13.312 & 70.9807 & 13.756 & 90.1825 & 13.536 \\
\hline 0.643372 & 14.916 & 67.7147 & 12.532 & & & & & & & \\
\hline
\end{tabular}




\begin{tabular}{|c|c|c|c|c|c|c|c|c|c|c|}
\hline \multirow{2}{*}{2.6337} & 33.434 & $\begin{array}{l}11.6 \\
657807\end{array}$ & $\begin{array}{l}56.5153 \\
12536\end{array}$ & 11.656 & 63.3043 & 13.316 & 72.7839 & 13.76 & 90.1825 & 13.54 \\
\hline & $\begin{array}{l}14.92 \\
36.0579\end{array}$ & $\begin{array}{l}65.7897 \\
11.604\end{array}$ & $\begin{array}{l}12.536 \\
55.6038\end{array}$ & 11.66 & 2806 & 13,32 & 7 & $1.3+2$ & & 13.544 \\
\hline \multirow[t]{2}{*}{4.94248} & 14.924 & 65.7897 & 12.54 & & & 15.52 & ו & 15.1 & 30.10 & \\
\hline & 37.8139 & 11.608 & 55.6038 & 11.664 & 64.2806 & 13.324 & 71.8818 & 13.768 & 90.1825 & 13.548 \\
\hline \multirow[t]{2}{*}{1.10772} & 14.928 & 66.7515 & 12.544 & & & & & & & \\
\hline & 36.9352 & 11.612 & 55.6038 & 11.668 & 64.2806 & 13.328 & 70.0806 & 13.772 & 89.1595 & 13.552 \\
\hline \multirow[t]{2}{*}{2.6337} & 14.932 & 66.7515 & 12.548 & & & & & & & \\
\hline & 36.0579 & 11.616 & 55.6038 & 11.672 & 66.2374 & 13.332 & 71.8818 & 13.776 & 90.1825 & 13.556 \\
\hline \multirow[t]{2}{*}{2.6337} & 14.936 & 66.7515 & 12.552 & & & & & & & \\
\hline & 36.0579 & 11.62 & 54.6936 & 11.676 & 66.2374 & 13.336 & 70.9807 & 13.78 & 90.1825 & 13.56 \\
\hline \multirow[t]{2}{*}{0.643372} & 14.94 & 63.8699 & 12.556 & & & & & & & \\
\hline & 35.1818 & 11.624 & 54.6936 & 11.68 & 64.2806 & 13.34 & 69.1818 & 13.784 & 90.1825 & 13.564 \\
\hline \multirow[t]{2}{*}{1.10772} & 14.944 & 61.0001 & 12.56 & & & & & & & \\
\hline & 36.0579 & 11.628 & 55.6038 & 11.684 & 64.2806 & 13.344 & 70.0806 & 13.788 & 90.1825 & 13.568 \\
\hline \multirow[t]{2}{*}{0.643372} & 14.948 & 58.1424 & 12.564 & & & & & & & \\
\hline & 35.1818 & 11.632 & 55.6038 & 11.688 & 65.2584 & 13.348 & 70.0806 & 13.792 & 89.1595 & 13.572 \\
\hline \multirow[t]{2}{*}{0.643372} & 14.952 & 57.1925 & 12.568 & & & & & & & \\
\hline & 38.6939 & 11.636 & 56.5153 & 11.692 & 64.2806 & 13.352 & 70.0806 & 13.796 & 90.1825 & 13.576 \\
\hline 0.643372 & 14.956 & 58.1424 & 12.572 & & & & & & & \\
\hline & 38.6939 & 11.64 & 56.5153 & 11.696 & 67.2179 & 13.356 & 70.9807 & 13.8 & 90.1825 & 13.58 \\
\hline 2.6337 & 14.96 & 61.9553 & 12.576 & & & & & & & \\
\hline & 37.8139 & 11.644 & 57.428 & 11.7 & 64.2806 & 13.36 & 70.0806 & 13.804 & 89.1595 & 13.584 \\
\hline 2.6337 & 14.964 & 65.7897 & 12.58 & & & & & & & \\
\hline & 37.8139 & 11.648 & 55.6038 & 11.704 & 64.2806 & 13.364 & 70.9807 & 13.808 & 90.1825 & 13.588 \\
\hline 0.643372 & 14.968 & 65.7897 & 12.584 & & & & & & & \\
\hline & 38.6939 & 11.652 & 55.6038 & 11.708 & 61.3557 & 13.368 & 70.9807 & 13.812 & 90.1825 & 13.592 \\
\hline 0.643372 & 14.972 & 62.9119 & 12.588 & & & & & & & \\
\hline & 37.8139 & 11.656 & 55.6038 & 11.712 & 65.2584 & 13.372 & 70.0806 & 13.816 & 90.1825 & 13.596 \\
\hline 0.643372 & 14.976 & 60.0462 & 12.592 & & & & & & & \\
\hline & 36.9352 & 11.66 & 51.0656 & 11.716 & 62.3293 & 13.376 & 71.8818 & 13.82 & 89.1595 & 13.6 \\
\hline 0.643372 & 14.98 & 58.1424 & 12.596 & & & & & & & \\
\hline & 37.8139 & 11.664 & 52.877 & 11.72 & 65.2584 & 13.38 & 70.0806 & 13.824 & 88.1378 & 13.604 \\
\hline 2.6337 & 14.984 & 58.1 & 12.6 & & & & & & & \\
\hline & 39.5753 & 11.668 & 51.9707 & 11.724 & 62.3293 & 13.384 & 70.9807 & 13.828 & 89.1595 & 13.608 \\
\hline 1.10772 & 14.988 & 61.0001 & 12.604 & & & & & & & \\
\hline & 39.5753 & 11.672 & 51.9707 & 11.728 & 65.2584 & 13.388 & 69.1818 & 13.832 & 90.1825 & 13.612 \\
\hline 1.10772 & 14.992 & 64.8291 & 12.608 & & & & & & & \\
\hline & 39.5753 & 11.676 & 52.877 & 11.732 & 61.3557 & 13.392 & 69.1818 & 13.836 & 89.1595 & 13.616 \\
\hline 0.643372 & 14.996 & 65.7897 & 12.612 & & & & & & & \\
\hline & 40.458 & 11.68 & 56.5153 & 11.736 & 64.2806 & 13.396 & 69.1818 & 13.84 & 90.1825 & 13.62 \\
\hline 1.10772 & 15 & 64.8291 & 12.616 & & & & & & & \\
\hline & 37.8139 & 11.684 & 55.6038 & 11.74 & 64.2806 & 13.4 & 68.284 & 13.844 & 89.1595 & 13.624 \\
\hline 0.643372 & 15.004 & 63.8699 & 12.62 & & & & & & & \\
\hline & 37.8139 & 11.688 & 54.6936 & 11.744 & 64.2806 & 13.404 & 67.3874 & 13.848 & 87.1172 & 13.628 \\
\hline 0.643372 & 15.008 & 62.9119 & 12.624 & & & & & & & \\
\hline & 37.8139 & 11.692 & 55.6038 & 11.748 & 63.3043 & 13.408 & 70.0806 & 13.852 & 90.1825 & 13.632 \\
\hline 0.643372 & 15.012 & 61. & 12.628 & & & & & & & \\
\hline & 36.9352 & 11.696 & 55.6038 & 11.752 & 64.2806 & 13.412 & 68.284 & 13.856 & 89.1595 & 13.636 \\
\hline 0.643372 & 15.016 & 61.0001 & 12.632 & & & & & & & \\
\hline & 38.6939 & 11.7 & 52.877 & 11.756 & 63.3043 & 13.416 & 70.9807 & 13.86 & 89.1595 & 13.64 \\
\hline 0.643372 & 15.02 & 59.0936 & 12.636 & & & & & & & \\
\hline & 37.8139 & 11.704 & 52.877 & 11.76 & 66.2374 & 13.42 & 69.1818 & 13.864 & 89.1595 & 13.644 \\
\hline 0.643372 & 15.024 & 61.0001 & 12.64 & & & & & & & \\
\hline & 38.6939 & 11.708 & 51.9707 & 11.764 & 67.2179 & 13.424 & 70.0806 & 13.868 & 89.1595 & 13.648 \\
\hline 0.643372 & 15.028 & 58.1424 & 12.644 & & & & & & & \\
\hline & 38.6939 & 11.712 & 53.7847 & 11.768 & 63.3043 & 13.428 & 70.0806 & 13.872 & 89.1595 & 13.652 \\
\hline 0.643372 & 15.032 & 56.244 & 12.648 & & & & & & & \\
\hline & 38.6939 & 11.716 & 53.7847 & 11.772 & 63.3043 & 13.432 & 68.284 & 13.876 & 88.1378 & 13.656 \\
\hline 0.643372 & 15.036 & 58.1424 & 12.652 & & & & & & & \\
\hline & 39.5753 & 11.72 & 53.7847 & 11.776 & 64.2806 & 13.436 & 67.3874 & 13.88 & 88.1378 & 13.66 \\
\hline 0.643372 & 15.04 & 60.0462 & 12.656 & & & & & & & \\
\hline & 38.6939 & 11.724 & 54.6936 & 11.78 & 65.2584 & 13.44 & 68.284 & 13.884 & 86.098 & 13.664 \\
\hline 0.643372 & 15.044 & 64.8291 & 12.66 & & & & & & & \\
\hline & 38.6939 & 11.728 & 51.9707 & 11.784 & 65.2584 & 13.444 & 70.0806 & 13.888 & 87.1172 & 13.668 \\
\hline 0.643372 & 15.048 & 66.7515 & 12.664 & & & & & & & \\
\hline & 40.458 & 11.732 & 52.877 & 11.788 & 62.3293 & 13.448 & 67.3874 & 13.892 & 89.1595 & 13.672 \\
\hline 0.643372 & 15.052 & 65.7897 & 12.668 & & & & & & & \\
\hline & 39.5753 & 11.736 & 51.9707 & 11.792 & 65.2584 & 13.452 & 67.3874 & 13.896 & 88.1378 & 13.676 \\
\hline 0.643372 & 15.056 & 65.7897 & 12.672 & & & & & & & \\
\hline
\end{tabular}




\begin{tabular}{|c|c|c|c|c|c|c|c|c|c|c|}
\hline & 39.5753 & 11.74 & 51.9707 & 11.796 & 65.2584 & 13.456 & 67.3874 & 13.9 & 86.098 & 13.68 \\
\hline \multirow[t]{2}{*}{0.643372} & 15.06 & 61.9553 & 12.676 & & & & & & & \\
\hline & 39.5753 & 11.744 & 48.3582 & 11.8 & 63.3043 & 13.46 & 67.3874 & 13.904 & 88.1378 & 13.684 \\
\hline \multirow[t]{2}{*}{0.643372} & 15.064 & 58.1424 & 12.68 & & & & & & & \\
\hline & 37.8139 & 11.748 & 51.0656 & 11.804 & 64.2806 & 13.464 & 69.1818 & 13.908 & 87.1172 & 13.688 \\
\hline \multirow[t]{2}{*}{0.643372} & 15.068 & 54.3511 & 12.684 & & & & & & & \\
\hline & 37.8139 & 11.752 & 49.2593 & 11.808 & 62.3293 & 13.468 & 67.3874 & 13.912 & 89.1595 & 13.692 \\
\hline \multirow[t]{2}{*}{1.66854} & 15.072 & 53.4067 & 12.688 & & & & & & & \\
\hline & 38.6939 & 11.756 & 49.2593 & 11.812 & 63.3043 & 13.472 & 66.4919 & 13.916 & 86.098 & 13.696 \\
\hline \multirow[t]{2}{*}{0.643372} & 15.076 & 51.5221 & 12.692 & & & & & & & \\
\hline & 38.6939 & 11.76 & 48.3582 & 11.816 & 62.3293 & 13.476 & 68.284 & 13.92 & 87.1172 & 13.7 \\
\hline \multirow[t]{2}{*}{0.643372} & 15.08 & 54.3511 & 12.696 & & & & & & & \\
\hline & 40.458 & 11.764 & 48.3582 & 11.82 & 62.3293 & 13.48 & 69.1818 & 13.924 & 86.098 & 13.704 \\
\hline \multirow[t]{2}{*}{0.643372} & 15.084 & 54.3511 & 12.7 & & & & & & & \\
\hline & 41.3422 & 11.768 & 51.0656 & 11.824 & 62.3293 & 13.484 & 67.3874 & 13.928 & 86.098 & 13.708 \\
\hline \multirow[t]{2}{*}{0.643372} & 15.088 & 56.244 & 12.704 & & & & & & & \\
\hline & 41.3422 & 11.772 & 51.0656 & 11.828 & 62.3293 & 13.488 & 69.1818 & 13.932 & 84.0634 & 13.712 \\
\hline \multirow[t]{2}{*}{1.66854} & 15.092 & 60.0462 & 12.708 & & & & & & & \\
\hline & 40.458 & 11.776 & 51.9707 & 11.832 & 62.3293 & 13.492 & 69.1818 & 13.936 & 86.098 & 13.716 \\
\hline 0.643372 & 15.096 & 59.0936 & 12.712 & & & & & & & \\
\hline & 38.6939 & 11.78 & 51.0656 & 11.836 & 62.3293 & 13.496 & 69.1818 & 13.94 & 87.1172 & 13.72 \\
\hline 0.643372 & 15.1 & 59.0936 & 12.716 & & & & & & & \\
\hline & 37.8139 & 11.784 & 50.1618 & 11.84 & 62.3293 & 13.5 & 66.4919 & 13.944 & 86.098 & 13.724 \\
\hline 0.643372 & 15.104 & 58.1424 & 12.72 & & & & & & & \\
\hline & 38.6939 & 11.788 & 50.1618 & 11.844 & 62.3293 & 13.504 & 69.1818 & 13.948 & 85.08 & 13.728 \\
\hline 0.643372 & 15.108 & 58.1424 & 12.724 & & & & & & & \\
\hline & 36.0579 & 11.792 & 50.1618 & 11.848 & 61.3557 & 13.508 & 67.3874 & 13.952 & 86.098 & 13.732 \\
\hline 0.643372 & 15.112 & 54.3511 & 12.728 & & & & & & & \\
\hline & 36.9352 & 11.796 & 50.1618 & 11.852 & 62.3293 & 13.512 & 69.1818 & 13.956 & 83.048 & 13.736 \\
\hline 1.66854 & 15.116 & 54.3511 & 12.732 & & & & & & & \\
\hline & 36.9352 & 11.8 & 49.2593 & 11.856 & 61.3557 & 13.516 & 66.4919 & 13.96 & 84.0634 & 13.74 \\
\hline 0.643372 & 15.12 & 53.4067 & 12.736 & & & & & & & \\
\hline & 38.6939 & 11.804 & 49.2593 & 11.86 & 61.3557 & 13.52 & 68.284 & 13.964 & 82.0338 & 13.744 \\
\hline 0.643372 & 15.124 & 56.244 & 12.74 & & & & & & & \\
\hline & 38.6939 & 11.808 & 50.1618 & 11.864 & 61.3557 & 13.524 & 67.3874 & 13.968 & 84.0634 & 13.748 \\
\hline 0.643372 & 15.128 & 57.1925 & 12.744 & & & & & & & \\
\hline & 39.5753 & 11.812 & 50.1618 & 11.868 & 60.3834 & 13.528 & 69.1818 & 13.972 & 86.098 & 13.752 \\
\hline 0.643372 & 15.132 & 59.0936 & 12.748 & & & & & & & \\
\hline & 38.6939 & 11.816 & 48.3582 & 11.872 & 62.3293 & 13.532 & 65.5975 & 13.976 & 85.08 & 13.756 \\
\hline 0.643372 & 15.136 & 61.0001 & 12.752 & & & & & & & \\
\hline & 38.6939 & 11.82 & 49.2593 & 11.876 & 62.3293 & 13.536 & 67.3874 & 13.98 & 84.0634 & 13.76 \\
\hline 0.643372 & 15.14 & 59.0936 & 12.756 & & & & & & & \\
\hline & 37.8139 & 11.824 & 49.2593 & 11.88 & 58.4433 & 13.54 & 66.4919 & 13.984 & 84.0634 & 13.764 \\
\hline 1.10772 & 15.144 & 56.244 & 12.76 & & & & & & & \\
\hline & 36.0579 & 11.828 & 50.1618 & 11.884 & 60.3834 & 13.544 & 67.3874 & 13.988 & 84.0634 & 13.768 \\
\hline 0.643372 & 15.148 & 54.3511 & 12.764 & & & & & & & \\
\hline & 36.9352 & 11.832 & 49.2593 & 11.888 & 60.3834 & 13.548 & 67.3874 & 13.992 & 83.048 & 13.772 \\
\hline 0.643372 & 15.152 & 51.5221 & 12.768 & & & & & & & \\
\hline & 37.8139 & 11.836 & 51.0656 & 11.892 & 60.3834 & 13.552 & 68.284 & 13.996 & 83.048 & 13.776 \\
\hline 0.643372 & 15.156 & 50.5819 & 12.772 & & & & & & & \\
\hline & 36.9352 & 11.84 & 51.0656 & 11.896 & 59.4127 & 13.556 & 67.3874 & 14 & 82.0338 & 13.78 \\
\hline 0.643372 & 15.16 & 50.5819 & 12.776 & & & & & & & \\
\hline & 37.8139 & 11.844 & 50.1618 & 11.9 & 60.3834 & 13.56 & 66.4919 & 14.004 & 84.0634 & 13.784 \\
\hline 1.66854 & 15.164 & 52.4637 & 12.78 & & & & & & & \\
\hline & 38.6939 & 11.848 & 50.1618 & 11.904 & 59.4127 & 13.564 & 66.4919 & 14.008 & 85.08 & 13.788 \\
\hline 0.643372 & 15.168 & 55.2969 & 12.784 & & & & & & & \\
\hline & 38.6939 & 11.852 & 49.2593 & 11.908 & 59.4127 & 13.568 & 65.5975 & 14.012 & 83.048 & 13.792 \\
\hline 0.643372 & 15.172 & 57.1925 & 12.788 & & & & & & & \\
\hline & 37.8139 & 11.856 & 48.3582 & 11.912 & 59.4127 & 13.572 & 65.5975 & 14.016 & 82.0338 & 13.796 \\
\hline 0.643372 & 15.176 & 58.1424 & 12.792 & & & & & & & \\
\hline & 37.8139 & 11.86 & 50.1618 & 11.916 & 59.4127 & 13.576 & 66.4919 & 14.02 & 83.048 & 13.8 \\
\hline 0.643372 & 15.18 & 56.244 & 12.796 & & & & & & & \\
\hline & 38.6939 & 11.864 & 49.2593 & 11.92 & 58.4433 & 13.58 & 66.4919 & 14.024 & 82.0338 & 13.804 \\
\hline 0.643372 & 15.184 & 54.3511 & 12.8 & & & & & & & \\
\hline & 37.8139 & 11.868 & 50.1618 & 11.924 & 59.4127 & 13.584 & 66.4919 & 14.028 & 82.0338 & 13.808 \\
\hline 0.643372 & 15.188 & 52.4637 & 12.804 & & & & & & & \\
\hline & 36.9352 & 11.872 & 51.0656 & 11.928 & 60.3834 & 13.588 & 66.4919 & 14.032 & 82.0338 & 13.812 \\
\hline 1.10772 & 15.192 & 52.4637 & 12.808 & & & & & & & \\
\hline & 37.8139 & 11.876 & 51.0656 & 11.932 & 59.4127 & 13.592 & 67.3874 & 14.036 & 82.0338 & 13.816 \\
\hline 0.643372 & 15.196 & 50.5819 & 12.812 & & & & & & & \\
\hline
\end{tabular}




\begin{tabular}{|c|c|c|c|c|c|c|c|c|c|c|}
\hline & 36.9352 & 11.88 & 51.9707 & 11.936 & 59.4127 & 13.596 & 66.4919 & 14.04 & 82.0338 & 13.82 \\
\hline \multirow[t]{2}{*}{0.643372} & 15.2 & 49.6431 & 12.816 & & & & & & & \\
\hline & 36.0579 & 11.884 & 51.0656 & 11.94 & 60.3834 & 13.6 & 67.3874 & 14.044 & 82.0338 & 13.824 \\
\hline \multirow[t]{2}{*}{0.643372} & 15.204 & 48.7056 & 12.82 & & & & & & & \\
\hline & 35.1818 & 11.888 & 50.1618 & 11.944 & 57.4752 & 13.604 & 66.4919 & 14.048 & 82.0338 & 13.828 \\
\hline \multirow[t]{2}{*}{1.10772} & 15.208 & 51.5221 & 12.824 & & & & & & & \\
\hline & 36.0579 & 11.892 & 48.3582 & 11.948 & 54.5797 & 13.608 & 65.5975 & 14.052 & 82.0338 & 13.832 \\
\hline \multirow[t]{2}{*}{0.643372} & 15.212 & 50.5819 & 12.828 & & & & & & & \\
\hline & 35.1818 & 11.896 & 49.2593 & 11.952 & 56.5086 & 13.612 & 65.5975 & 14.056 & 82.0338 & 13.836 \\
\hline \multirow[t]{2}{*}{1.10772} & 15.216 & 53.4067 & 12.832 & & & & & & & \\
\hline & 35.1818 & 11.9 & 51.0656 & 11.956 & 56.5086 & 13.616 & 66.4919 & 14.06 & 82.0338 & 13.84 \\
\hline \multirow[t]{2}{*}{1.10772} & 15.22 & 54.3511 & 12.836 & & & & & & & \\
\hline & 35.1818 & 11.904 & 50.1618 & 11.96 & 56.5086 & 13.62 & 65.5975 & 14.064 & 80.0095 & 13.844 \\
\hline \multirow[t]{2}{*}{0.643372} & 15.224 & 52.4637 & 12.84 & & & & & & & \\
\hline & 35.1818 & 11.908 & 50.1618 & 11.964 & 55.5434 & 13.624 & 65.5975 & 14.068 & 82.0338 & 13.848 \\
\hline \multirow[t]{2}{*}{2.6337} & 15.228 & 54.3511 & 12.844 & & & & & & & \\
\hline & 35.1818 & 11.912 & 50.1618 & 11.968 & 55.5434 & 13.628 & 66.4919 & 14.072 & 82.0338 & 13.852 \\
\hline \multirow[t]{2}{*}{2.6337} & 15.232 & 53.4067 & 12.848 & & & & & & & \\
\hline & 36.9352 & 11.916 & 50.1618 & 11.972 & 56.5086 & 13.632 & 65.5975 & 14.076 & 82.0338 & 13.856 \\
\hline 2.6337 & 15.236 & 52.4637 & 12.852 & & & & & & & \\
\hline & 36.0579 & 11.92 & 51.9707 & 11.976 & 56.5086 & 13.636 & 65.5975 & 14.08 & 82.0338 & 13.86 \\
\hline 4.94248 & 15.24 & 51.5221 & 12.856 & & & & & & & \\
\hline & 35.1818 & 11.924 & 51.0656 & 11.98 & 56.5086 & 13.64 & 65.5975 & 14.084 & 82.0338 & 13.864 \\
\hline 2.6337 & 15.244 & 50.5819 & 12.86 & & & & & & & \\
\hline & 35.1818 & 11.928 & 50.1618 & 11.984 & 55.5434 & 13.644 & 66.4919 & 14.088 & 83.048 & 13.868 \\
\hline 3.89069 & 15.248 & 51.5221 & 12.864 & & & & & & & \\
\hline & 35.1818 & 11.932 & 51.0656 & 11.988 & 54.5797 & 13.648 & 65.5975 & 14.092 & 82.0338 & 13.872 \\
\hline 3.89069 & 15.252 & 52.4637 & 12.868 & & & & & & & \\
\hline & 35.1818 & 11.936 & 49.2593 & 11.992 & 57.4752 & 13.652 & 64.7042 & 14.096 & 82.0338 & 13.876 \\
\hline 4.94248 & 15.256 & 51.5221 & 12.872 & & & & & & & \\
\hline & 36.0579 & 11.94 & 49.2593 & 11.996 & 55.5434 & 13.656 & 66.4919 & 14.1 & 82.0338 & 13.88 \\
\hline 2.6337 & 15.26 & 50.5819 & 12.876 & & & & & & & \\
\hline & 36.9352 & 11.944 & 49.2593 & 12 & 54.5797 & 13.66 & 66.4919 & 14.104 & 82.0338 & 13.884 \\
\hline 1.10772 & 15.264 & 51.5221 & 12.88 & & & & & & & \\
\hline & 37.8139 & 11.948 & 49.2593 & 12.004 & 56.5086 & 13.664 & 64.7042 & 14.108 & 81.021 & 13.888 \\
\hline 0.643372 & 15.268 & 51.5221 & 12.884 & & & & & & & \\
\hline & 37.8139 & 11.952 & 50.1618 & 12.008 & 56.5086 & 13.668 & 63.8121 & 14.112 & 81.021 & 13.892 \\
\hline 1.10772 & 15.272 & 53.4067 & 12.888 & & & & & & & \\
\hline & 38.6939 & 11.956 & 48.3582 & 12.012 & 55.5434 & 13.672 & 65.5975 & 14.116 & 81.021 & 13.896 \\
\hline 2.6337 & 15.276 & 52.4637 & 12.892 & & & & & & & \\
\hline & 37.8139 & 11.96 & 49.2593 & 12.016 & 54.5797 & 13.676 & 66.4919 & 14.12 & 80.0095 & 13.9 \\
\hline 0.643372 & 15.28 & 50.5819 & 12.896 & & & & & & & \\
\hline & 38.6939 & 11.964 & 48.3582 & 12.02 & 55.5434 & 13.68 & 65.5975 & 14.124 & 81.021 & 13.904 \\
\hline 0.643372 & 15.284 & 49.6431 & 12.9 & & & & & & & \\
\hline & 36.9352 & 11.968 & 49.2593 & 12.024 & 53.6173 & 13.684 & 64.7042 & 14.128 & 81.021 & 13.908 \\
\hline 1.10772 & 15.288 & 47.7697 & 12.904 & & & & & & & \\
\hline & 36.9352 & 11.972 & 50.1618 & 12.028 & 54.5797 & 13.688 & 63.8121 & 14.132 & 81.021 & 13.912 \\
\hline 1.10772 & 15.292 & 47.7697 & 12.908 & & & & & & & \\
\hline & 35.1818 & 11.976 & 49.2593 & 12.032 & 52.6564 & 13.692 & 65.5975 & 14.136 & 82.0338 & 13.916 \\
\hline 0.643372 & 15.296 & 48.7056 & 12.912 & & & & & & & \\
\hline & 35.1818 & 11.98 & 49.2593 & 12.036 & 52.6564 & 13.696 & 62.9211 & 14.14 & 81.021 & 13.92 \\
\hline 1.10772 & 15.3 & 50.5819 & 12.916 & & & & & & & \\
\hline & 35.1818 & 11.984 & 47.4583 & 12.04 & 54.5797 & 13.7 & 64.7042 & 14.144 & 78.9993 & 13.924 \\
\hline 0.643372 & 15.304 & 50.5819 & 12.92 & & & & & & & \\
\hline & 36.0579 & 11.988 & 48.3582 & 12.044 & 55.5434 & 13.704 & 66.4919 & 14.148 & 81.021 & 13.928 \\
\hline 0.643372 & 15.308 & 50.5819 & 12.924 & & & & & & & \\
\hline & 36.0579 & 11.992 & 48.3582 & 12.048 & 53.6173 & 13.708 & 65.5975 & 14.152 & 77.9904 & 13.932 \\
\hline 1.10772 & 15.312 & 51.5221 & 12.928 & & & & & & & \\
\hline & 37.8139 & 11.996 & 48.3582 & 12.052 & 54.5797 & 13.712 & 65.5975 & 14.156 & 78.9993 & 13.936 \\
\hline 1.10772 & 15.316 & 51.5221 & 12.932 & & & & & & & \\
\hline & 38.6939 & 12 & 47.4583 & 12.056 & 55.5434 & 13.716 & 66.4919 & 14.16 & 78.9993 & 13.94 \\
\hline 3.89069 & 15.32 & 50.5819 & 12.936 & & & & & & & \\
\hline & 36.9352 & 12.004 & 47.4583 & 12.06 & 54.5797 & 13.72 & 62.9211 & 14.164 & 77.9904 & 13.944 \\
\hline 0.643372 & 15.324 & 47.7697 & 12.94 & & & & & & & \\
\hline & 35.1818 & 12.008 & 48.3582 & 12.064 & 54.5797 & 13.724 & 62.9211 & 14.168 & 78.9993 & 13.948 \\
\hline 2.6337 & 15.328 & 46.8351 & 12.944 & & & & & & & \\
\hline & 36.9352 & 12.012 & 47.4583 & 12.068 & 52.6564 & 13.728 & 63.8121 & 14.172 & 77.9904 & 13.952 \\
\hline 0.643372 & 15.332 & 47.7697 & 12.948 & & & & & & & \\
\hline & 36.9352 & 12.016 & 46.5598 & 12.072 & 54.5797 & 13.732 & 62.0313 & 14.176 & 78.9993 & 13.956 \\
\hline 0.643372 & 15.336 & 49.6431 & 12.952 & & & & & & & \\
\hline
\end{tabular}




\begin{tabular}{|c|c|c|c|c|c|c|c|c|c|c|}
\hline & 36.0579 & 12.02 & 46.5598 & 12.076 & 53.6173 & 13.736 & 63.8121 & 14.18 & 77.9904 & 13.96 \\
\hline \multirow[t]{2}{*}{1.66854} & 15.34 & 48.7056 & 12.956 & & & & & & & \\
\hline & 35.1818 & 12.024 & 45.6625 & 12.08 & 51.697 & 13.74 & 64.7042 & 14.184 & 78.9993 & 13.964 \\
\hline \multirow[t]{2}{*}{0.643372} & 15.344 & 50.5819 & 12.96 & & & & & & & \\
\hline & 36.0579 & 12.028 & 44.7666 & 12.084 & 52.6564 & 13.744 & 63.8121 & 14.188 & 78.9993 & 13.968 \\
\hline \multirow[t]{2}{*}{1.10772} & 15.348 & 51.5221 & 12.964 & & & & & & & \\
\hline & 36.0579 & 12.032 & 43.872 & 12.088 & 53.6173 & 13.748 & 62.0313 & 14.192 & 78.9993 & 13.972 \\
\hline \multirow[t]{2}{*}{0.643372} & 15.352 & 49.6431 & 12.968 & & & & & & & \\
\hline & 35.1818 & 12.036 & 43.872 & 12.092 & 52.6564 & 13.752 & 63.8121 & 14.196 & 78.9993 & 13.976 \\
\hline \multirow[t]{2}{*}{1.66854} & 15.356 & 51.5221 & 12.972 & & & & & & & \\
\hline & 38.6939 & 12.04 & 43.872 & 12.096 & 52.6564 & 13.756 & 63.8121 & 14.2 & 77.9904 & 13.98 \\
\hline \multirow[t]{2}{*}{0.643372} & 15.36 & 50.5819 & 12.976 & & & & & & & \\
\hline & 38.6939 & 12.044 & 42.9788 & 12.1 & 53.6173 & 13.76 & 65.5975 & 14.204 & 77.9904 & 13.984 \\
\hline \multirow[t]{2}{*}{0.643372} & 15.364 & 49.6431 & 12.98 & & & & & & & \\
\hline & 36.0579 & 12.048 & 44.7666 & 12.104 & 52.6564 & 13.764 & 64.7042 & 14.208 & 77.9904 & 13.988 \\
\hline \multirow[t]{2}{*}{0.643372} & 15.368 & 49.6431 & 12.984 & & & & & & & \\
\hline & 36.9352 & 12.052 & 44.7666 & 12.108 & 54.5797 & 13.768 & 62.9211 & 14.212 & 77.9904 & 13.992 \\
\hline \multirow[t]{2}{*}{0.643372} & 15.372 & 48.7056 & 12.988 & & & & & & & \\
\hline & 36.9352 & 12.056 & 43.872 & 12.112 & 53.6173 & 13.772 & 63.8121 & 14.216 & 78.9993 & 13.996 \\
\hline 0.643372 & 15.376 & 47.7697 & 12.992 & & & & & & & \\
\hline & 37.8139 & 12.06 & 43.872 & 12.116 & 53.6173 & 13.776 & 63.8121 & 14.22 & 77.9904 & 14 \\
\hline 0.643372 & 15.38 & 48.7056 & 12.996 & & & & & & & \\
\hline & 36.9352 & 12.064 & 42.0869 & 12.12 & 53.6173 & 13.78 & 63.8121 & 14.224 & 77.9904 & 14.004 \\
\hline 0.643372 & 15.384 & 46.8351 & 13 & & & & & & & \\
\hline & 35.1818 & 12.068 & 42.0869 & 12.124 & 51.697 & 13.784 & 62.9211 & 14.228 & 77.9904 & 14.008 \\
\hline 1.66854 & 15.388 & 46.8351 & 13.004 & & & & & & & \\
\hline & 36.9352 & 12.072 & 41.1963 & 12.128 & 52.6564 & 13.788 & 62.9211 & 14.232 & 78.9993 & 14.012 \\
\hline 0.643372 & 15.392 & 46.8351 & 13.008 & & & & & & & \\
\hline & 36.0579 & 12.076 & 42.0869 & 12.132 & 53.6173 & 13.792 & 63.8121 & 14.236 & 77.9904 & 14.016 \\
\hline 0.643372 & 15.396 & 47.7697 & 13.012 & & & & & & & \\
\hline & 36.9352 & 12.08 & 41.1963 & 12.136 & 55.5434 & 13.796 & 62.0313 & 14.24 & 77.9904 & 14.02 \\
\hline 0.643372 & 15.4 & 44.0399 & 13.016 & & & & & & & \\
\hline & 36.0579 & 12.084 & 41.1963 & 12.14 & 53.6173 & 13.8 & 61.1426 & 14.244 & 77.9904 & 14.024 \\
\hline 0.643372 & 15.404 & 44.9702 & 13.02 & & & & & & & \\
\hline & 36.0579 & 12.088 & 41.1963 & 12.144 & 53.6173 & 13.804 & 62.0313 & 14.248 & 77.9904 & 14.028 \\
\hline 0.643372 & 15.408 & 44.9702 & 13.024 & & & & & & & \\
\hline & 37.8139 & 12.092 & 41.1963 & 12.148 & 53.6173 & 13.808 & 62.9211 & 14.252 & 77.9904 & 14.032 \\
\hline 0.643372 & 15.412 & 43.1111 & 13.028 & & & & & & & \\
\hline & 36.0579 & 12.096 & 41.1963 & 12.152 & 53.6173 & 13.812 & 62.0313 & 14.256 & 77.9904 & 14.036 \\
\hline 0.643372 & 15.416 & 44.9702 & 13.032 & & & & & & & \\
\hline & 36.9352 & 12.1 & 42.0869 & 12.156 & 54.5797 & 13.816 & 64.7042 & 14.26 & 78.9993 & 14.04 \\
\hline 0.643372 & 15.42 & 44.9702 & 13.036 & & & & & & & \\
\hline & 38.6939 & 12.104 & 42.0869 & 12.16 & 52.6564 & 13.82 & 62.9211 & 14.264 & 77.9904 & 14.044 \\
\hline 0.643372 & 15.424 & 45.9019 & 13.04 & & & & & & & \\
\hline & 35.1818 & 12.108 & 41.1963 & 12.164 & 52.6564 & 13.824 & 63.8121 & 14.268 & 77.9904 & 14.048 \\
\hline 1.66854 & 15.428 & 45.9019 & 13.044 & & & & & & & \\
\hline & 35.1818 & 12.112 & 41.1963 & 12.168 & 52.6564 & 13.828 & 62.9211 & 14.272 & 77.9904 & 14.052 \\
\hline 1.66854 & 15.432 & 47.7697 & 13.048 & & & & & & & \\
\hline & 35.1818 & 12.116 & 41.1963 & 12.172 & 53.6173 & 13.832 & 61.1426 & 14.276 & 78.9993 & 14.056 \\
\hline 0.643372 & 15.436 & 46.8351 & 13.052 & & & & & & & \\
\hline & 35.1818 & 12.12 & 41.1963 & 12.176 & 52.6564 & 13.836 & 63.8121 & 14.28 & 77.9904 & 14.06 \\
\hline 0.643372 & 15.44 & 45.9019 & 13.056 & & & & & & & \\
\hline & 34.3073 & 12.124 & 40.3071 & 12.18 & 52.6564 & 13.84 & 62.9211 & 14.284 & 78.9993 & 14.064 \\
\hline 1.66854 & 15.444 & 46.8351 & 13.06 & & & & & & & \\
\hline & 36.0579 & 12.128 & 41.1963 & 12.184 & 52.6564 & 13.844 & 62.0313 & 14.288 & 77.9904 & 14.068 \\
\hline 0.643372 & 15.448 & 46.8351 & 13.064 & & & & & & & \\
\hline & 34.3073 & 12.132 & 41.1963 & 12.188 & 54.5797 & 13.848 & 62.9211 & 14.292 & 77.9904 & 14.072 \\
\hline 0.643372 & 15.452 & 45.9019 & 13.068 & & & & & & & \\
\hline & 35.1818 & 12.136 & 39.4192 & 12.192 & 55.5434 & 13.852 & 62.9211 & 14.296 & 78.9993 & 14.076 \\
\hline 0.643372 & 15.456 & 46.8351 & 13.072 & & & & & & & \\
\hline & 36.0579 & 12.14 & 41.1963 & 12.196 & 52.6564 & 13.856 & 61.1426 & 14.3 & 77.9904 & 14.08 \\
\hline 0.643372 & 15.46 & 47.7697 & 13.076 & & & & & & & \\
\hline & 36.9352 & 12.144 & 41.1963 & 12.2 & 53.6173 & 13.86 & 61.1426 & 14.304 & 77.9904 & 14.084 \\
\hline 0.643372 & 15.464 & 47.7697 & 13.08 & & & & & & & \\
\hline & 36.0579 & 12.148 & 41.1963 & 12.204 & 52.6564 & 13.864 & 61.1426 & 14.308 & 76.9828 & 14.088 \\
\hline 0.643372 & 15.468 & 46.8351 & 13.084 & & & & & & & \\
\hline & 36.9352 & 12.152 & 41.1963 & 12.208 & 52.6564 & 13.868 & 62.0313 & 14.312 & 78.9993 & 14.092 \\
\hline 1.66854 & 15.472 & 46.8351 & 13.088 & & & & & & & \\
\hline & 34.3073 & 12.156 & 40.3071 & 12.212 & 52.6564 & 13.872 & 60.2552 & 14.316 & 77.9904 & 14.096 \\
\hline 0.643372 & 15.476 & 45.9019 & 13.092 & & & & & & & \\
\hline
\end{tabular}




\begin{tabular}{|c|c|c|c|c|c|c|c|c|c|c|}
\hline & 35.1818 & 12.16 & 41.1963 & 12.216 & 52.6564 & 13.876 & 61.1426 & 14.32 & 78.9993 & 14.1 \\
\hline \multirow[t]{2}{*}{1.66854} & 15.48 & 45.9019 & 13.096 & & & & & & & \\
\hline & 34.3073 & 12.164 & 42.0869 & 12.22 & 53.6173 & 13.88 & 62.0313 & 14.324 & 77.9904 & 14.104 \\
\hline \multirow[t]{2}{*}{0.643372} & 15.484 & 44.9702 & 13.1 & & & & & & & \\
\hline & 35.1818 & 12.168 & 41.1963 & 12.224 & 49.7824 & 13.884 & 62.0313 & 14.328 & 77.9904 & 14.108 \\
\hline \multirow[t]{2}{*}{0.643372} & 15.488 & 44.9702 & 13.104 & & & & & & & \\
\hline & 35.1818 & 12.172 & 41.1963 & 12.228 & 54.5797 & 13.888 & 61.1426 & 14.332 & 77.9904 & 14.112 \\
\hline \multirow[t]{2}{*}{1.10772} & 15.492 & 44.9702 & 13.108 & & & & & & & \\
\hline & 35.1818 & 12.176 & 41.1963 & 12.232 & 54.5797 & 13.892 & 62.0313 & 14.336 & 77.9904 & 14.116 \\
\hline \multirow[t]{2}{*}{0.643372} & 15.496 & 44.9702 & 13.112 & & & & & & & \\
\hline & 35.1818 & 12.18 & 40.3071 & 12.236 & 53.6173 & 13.896 & 62.0313 & 14.34 & 77.9904 & 14.12 \\
\hline \multirow[t]{2}{*}{0.643372} & 15.5 & 44.9702 & 13.116 & & & & & & & \\
\hline & 34.3073 & 12.184 & 41.1963 & 12.24 & 53.6173 & 13.9 & 60.2552 & 14.344 & 77.9904 & 14.124 \\
\hline \multirow[t]{2}{*}{0.643372} & 15.504 & 45.9019 & 13.12 & & & & & & & \\
\hline & 35.1818 & 12.188 & 41.1963 & 12.244 & 52.6564 & 13.904 & 60.2552 & 14.348 & 77.9904 & 14.128 \\
\hline \multirow[t]{2}{*}{0.643372} & 15.508 & 45.9019 & 13.124 & & & & & & & \\
\hline & 34.3073 & 12.192 & 41.1963 & 12.248 & 51.697 & 13.908 & 61.1426 & 14.352 & 77.9904 & 14.132 \\
\hline \multirow[t]{2}{*}{0.643372} & 15.512 & 44.9702 & 13.128 & & & & & & & \\
\hline & 34.3073 & 12.196 & 41.1963 & 12.252 & 52.6564 & 13.912 & 62.0313 & 14.356 & 77.9904 & 14.136 \\
\hline 0.643372 & 15.516 & 44.9702 & 13.132 & & & & & & & \\
\hline & 35.1818 & 12.2 & 41.1963 & 12.256 & 54.5797 & 13.916 & 60.2552 & 14.36 & 78.9993 & 14.14 \\
\hline 0.643372 & 15.52 & 43.1111 & 13.136 & & & & & & & \\
\hline & 35.1818 & 12.204 & 40.3071 & 12.26 & 53.6173 & 13.92 & 59.3688 & 14.364 & 77.9904 & 14.144 \\
\hline 0.643372 & 15.524 & 42.1837 & 13.14 & & & & & & & \\
\hline & 35.1818 & 12.208 & 41.1963 & 12.264 & 53.6173 & 13.924 & 59.3688 & 14.368 & 77.9904 & 14.148 \\
\hline 0.643372 & 15.528 & 43.1111 & 13.144 & & & & & & & \\
\hline & 34.3073 & 12.212 & 41.1963 & 12.268 & 53.6173 & 13.928 & 59.3688 & 14.372 & 77.9904 & 14.152 \\
\hline 0.643372 & 15.532 & 42.1837 & 13.148 & & & & & & & \\
\hline & 35.1818 & 12.216 & 41.1963 & 12.272 & 52.6564 & 13.932 & 61.1426 & 14.376 & 77.9904 & 14.156 \\
\hline 1.10772 & 15.536 & 43.1111 & 13.152 & & & & & & & \\
\hline & 34.3073 & 12.22 & 41.1963 & 12.276 & 52.6564 & 13.936 & 59.3688 & 14.38 & 77.9904 & 14.16 \\
\hline 0.643372 & 15.54 & 43.1111 & 13.156 & & & & & & & \\
\hline & 35.1818 & 12.224 & 41.1963 & 12.28 & 50.739 & 13.94 & 62.0313 & 14.384 & 77.9904 & 14.164 \\
\hline 0.643372 & 15.544 & 43.1111 & 13.16 & & & & & & & \\
\hline & 36.9352 & 12.228 & 41.1963 & 12.284 & 51.697 & 13.944 & 60.2552 & 14.388 & 78.9993 & 14.168 \\
\hline 0.643372 & 15.548 & 44.0399 & 13.164 & & & & & & & \\
\hline & 36.9352 & 12.232 & 41.1963 & 12.288 & 52.6564 & 13.948 & 59.3688 & 14.392 & 77.9904 & 14.172 \\
\hline 1.10772 & 15.552 & 45.9019 & 13.168 & & & & & & & \\
\hline & 36.0579 & 12.236 & 42.0869 & 12.292 & 51.697 & 13.952 & 61.1426 & 14.396 & 77.9904 & 14.176 \\
\hline 0.643372 & 15.556 & 43.1111 & 13.172 & & & & & & & \\
\hline & 35.1818 & 12.24 & 40.3071 & 12.296 & 53.6173 & 13.956 & 60.2552 & 14.4 & 77.9904 & 14.18 \\
\hline 0.643372 & 15.56 & 45.9019 & 13.176 & & & & & & & \\
\hline & 36.0579 & 12.244 & 42.0869 & 12.3 & 52.6564 & 13.96 & 59.3688 & 14.404 & 78.9993 & 14.184 \\
\hline 0.643372 & 15.564 & 44.9702 & 13.18 & & & & & & & \\
\hline & 35.1818 & 12.248 & 40.3071 & 12.304 & 51.697 & 13.964 & 62.0313 & 14.408 & 77.9904 & 14.188 \\
\hline 0.643372 & 15.568 & 45.9019 & 13.184 & & & & & & & \\
\hline & 35.1818 & 12.252 & 41.1963 & 12.308 & 51.697 & 13.968 & 59.3688 & 14.412 & 77.9904 & 14.192 \\
\hline 0.643372 & 15.572 & 45.9019 & 13.188 & & & & & & & \\
\hline & 35.1818 & 12.256 & 42.0869 & 12.312 & 52.6564 & 13.972 & 59.3688 & 14.416 & 78.9993 & 14.196 \\
\hline 0.643372 & 15.576 & 44.9702 & 13.192 & & & & & & & \\
\hline & 36.9352 & 12.26 & 41.1963 & 12.316 & 54.5797 & 13.976 & 60.2552 & 14.42 & 77.9904 & 14.2 \\
\hline 1.10772 & 15.58 & 44.9702 & 13.196 & & & & & & & \\
\hline & 36.0579 & 12.264 & 42.0869 & 12.32 & 54.5797 & 13.98 & 62.0313 & 14.424 & 77.9904 & 14.204 \\
\hline 0.643372 & 15.584 & 44.9702 & 13.2 & & & & & & & \\
\hline & 36.0579 & 12.268 & 41.1963 & 12.324 & 52.6564 & 13.984 & 59.3688 & 14.428 & 77.9904 & 14.208 \\
\hline 2.6337 & 15.588 & 46.8351 & 13.204 & & & & & & & \\
\hline & 36.9352 & 12.272 & 41.1963 & 12.328 & 53.6173 & 13.988 & 58.4837 & 14.432 & 77.9904 & 14.212 \\
\hline 1.10772 & 15.592 & 45.9019 & 13.208 & & & & & & & \\
\hline & 36.0579 & 12.276 & 42.0869 & 12.332 & 53.6173 & 13.992 & 59.3688 & 14.436 & 77.9904 & 14.216 \\
\hline 0.643372 & 15.596 & 44.9702 & 13.212 & & & & & & & \\
\hline & 36.0579 & 12.28 & 41.1963 & 12.336 & 51.697 & 13.996 & 56.7168 & 14.44 & 77.9904 & 14.22 \\
\hline 2.6337 & 15.6 & 44.0399 & 13.216 & & & & & & & \\
\hline & 35.1818 & 12.284 & 41.1963 & 12.34 & 54.5797 & 14 & 59.3688 & 14.444 & 77.9904 & 14.224 \\
\hline 0.643372 & 15.604 & 44.9702 & 13.22 & & & & & & & \\
\hline & 36.0579 & 12.288 & 41.1963 & 12.344 & 50.739 & 14.004 & 58.4837 & 14.448 & 76.9828 & 14.228 \\
\hline 0.643372 & 15.608 & 44.9702 & 13.224 & & & & & & & \\
\hline & 36.9352 & 12.292 & 41.1963 & 12.348 & 51.697 & 14.008 & 58.4837 & 14.452 & 76.9828 & 14.232 \\
\hline 0.643372 & 15.612 & 44.9702 & 13.228 & & & & & & & \\
\hline & 36.9352 & 12.296 & 42.0869 & 12.352 & 51.697 & 14.012 & 59.3688 & 14.456 & 77.9904 & 14.236 \\
\hline 2.6337 & 15.616 & 44.0399 & 13.232 & & & & & & & \\
\hline
\end{tabular}




\begin{tabular}{|c|c|c|c|c|c|c|c|c|c|c|}
\hline & 36.0579 & 12.3 & 42.0869 & 12.356 & 50.739 & 14.016 & 57.5996 & 14.46 & 77.9904 & 14.24 \\
\hline \multirow[t]{2}{*}{2.6337} & 15.62 & 44.9702 & 13.236 & & & & & & & \\
\hline & 36.9352 & 12.304 & 42.0869 & 12.36 & 52.6564 & 14.02 & 59.3688 & 14.464 & 77.9904 & 14.244 \\
\hline \multirow[t]{2}{*}{2.6337} & 15.624 & 44.0399 & 13.24 & & & & & & & \\
\hline & 36.9352 & 12.308 & 42.0869 & 12.364 & 53.6173 & 14.024 & 58.4837 & 14.468 & 77.9904 & 14.248 \\
\hline \multirow[t]{2}{*}{2.6337} & 15.628 & 44.0399 & 13.244 & & & & & & & \\
\hline & 35.1818 & 12.312 & 41.1963 & 12.368 & 53.6173 & 14.028 & 56.7168 & 14.472 & 76.9828 & 14.252 \\
\hline \multirow[t]{2}{*}{0.643372} & 15.632 & 44.0399 & 13.248 & & & & & & & \\
\hline & 35.1818 & 12.316 & 42.0869 & 12.372 & 51.697 & 14.032 & 59.3688 & 14.476 & 76.9828 & 14.256 \\
\hline \multirow[t]{2}{*}{0.643372} & 15.636 & 44.0399 & 13.252 & & & & & & & \\
\hline & 35.1818 & 12.32 & 41.1963 & 12.376 & 50.739 & 14.036 & 58.4837 & 14.48 & 74.9717 & 14.26 \\
\hline \multirow[t]{2}{*}{1.10772} & 15.64 & 44.9702 & 13.256 & & & & & & & \\
\hline & 36.9352 & 12.324 & 42.0869 & 12.38 & 52.6564 & 14.04 & 58.4837 & 14.484 & 76.9828 & 14.264 \\
\hline \multirow[t]{2}{*}{0.643372} & 15.644 & 44.0399 & 13.26 & & & & & & & \\
\hline & 35.1818 & 12.328 & 41.1963 & 12.384 & 51.697 & 14.044 & 57.5996 & 14.488 & 74.9717 & 14.268 \\
\hline \multirow[t]{2}{*}{0.643372} & 15.648 & 44.0399 & 13.264 & & & & & & & \\
\hline & 36.9352 & 12.332 & 41.1963 & 12.388 & 52.6564 & 14.048 & 58.4837 & 14.492 & 74.9717 & 14.272 \\
\hline \multirow[t]{2}{*}{0.643372} & 15.652 & 44.9702 & 13.268 & & & & & & & \\
\hline & 36.9352 & 12.336 & 41.1963 & 12.392 & 50.739 & 14.052 & 57.5996 & 14.496 & 75.9766 & 14.276 \\
\hline 1.10772 & 15.656 & 45.9019 & 13.272 & & & & & & & \\
\hline & 38.6939 & 12.34 & 41.1963 & 12.396 & 52.6564 & 14.056 & 56.7168 & 14.5 & 76.9828 & 14.28 \\
\hline 2.6337 & 15.66 & 44.9702 & 13.276 & & & & & & & \\
\hline & 37.8139 & 12.344 & 41.1963 & 12.4 & 51.697 & 14.06 & 56.7168 & 14.504 & 74.9717 & 14.284 \\
\hline 0.643372 & 15.664 & 44.9702 & 13.28 & & & & & & & \\
\hline & 37.8139 & 12.348 & 41.1963 & 12.404 & 53.6173 & 14.064 & 55.8352 & 14.508 & 74.9717 & 14.288 \\
\hline 1.10772 & 15.668 & 46.8351 & 13.284 & & & & & & & \\
\hline & 37.8139 & 12.352 & 43.872 & 12.408 & 52.6564 & 14.068 & 57.5996 & 14.512 & 74.9717 & 14.292 \\
\hline 1.10772 & 15.672 & 46.8351 & 13.288 & & & & & & & \\
\hline & 36.93 & 12.356 & 43.8 & 12.412 & 52.6564 & 14.072 & 58.4837 & 14.516 & 73.9681 & 14.296 \\
\hline 0.643372 & 15.676 & 47.7697 & 13.292 & & & & & & & \\
\hline & 36.9352 & 12.36 & 44.7666 & 12.416 & 51.697 & 14.076 & 56.7168 & 14.52 & 73.9681 & 14.3 \\
\hline 1.10772 & 15.68 & 47.7697 & 13.296 & & & & & & & \\
\hline & 36.9352 & 12.364 & 43.872 & 12.42 & 54.5797 & 14.08 & 55.8352 & 14.524 & 73.9681 & 14.304 \\
\hline 1.10772 & 15.684 & 46.8351 & 13.3 & & & & & & & \\
\hline & 37.8139 & 12.368 & 42.0869 & 12.424 & 51.697 & 14.084 & 58.4837 & 14.528 & 73.9681 & 14.308 \\
\hline 1.10772 & 15.688 & 44.9702 & 13.304 & & & & & & & \\
\hline & 38.6939 & 12.372 & 41.1963 & 12.428 & 50.739 & 14.088 & 55.8352 & 14.532 & 74.9717 & 14.312 \\
\hline 1.10772 & 15.692 & 47.7697 & 13.308 & & & & & & & \\
\hline & 38.6939 & 12.376 & 41.1963 & 12.432 & 52.6564 & 14.092 & 54.9548 & 14.536 & 74.9717 & 14.316 \\
\hline 2.6337 & 15.696 & 44.9702 & 13.312 & & & & & & & \\
\hline & 38.6939 & 12.38 & 42.9788 & 12.436 & 52.6564 & 14.096 & 56.7168 & 14.54 & 73.9681 & 14.32 \\
\hline 0.643372 & 15.7 & 46.8351 & & & & & & & & \\
\hline & 38.6939 & 12.384 & 44.7666 & 12.44 & 51.697 & 14.1 & 57.5996 & 14.544 & 75.9766 & 14.324 \\
\hline 1.10772 & 15.704 & 46.8351 & 13.32 & & & & & & & \\
\hline & 37.8139 & 12.388 & 42.9788 & 12.444 & 53.6173 & 14.104 & 54.9548 & 14.548 & 73.9681 & 14.328 \\
\hline 1.10772 & 15.708 & 47.7697 & 13.324 & & & & & & & \\
\hline & 37.8139 & 12.392 & 42.0869 & 12.448 & 52.6564 & 14.108 & 56.7168 & 14.552 & 73.9681 & 14.332 \\
\hline 0.643372 & 15.712 & 46.8351 & 13.328 & & & & & & & \\
\hline & 38.6939 & 12.396 & 42.0869 & 12.452 & 52.6564 & 14.112 & 54.9548 & 14.556 & 72.9659 & 14.336 \\
\hline 1.10772 & 15.716 & 44.0399 & 13.332 & & & & & & & \\
\hline & 38.6939 & 12.4 & 42.0869 & 12.456 & 53.6173 & 14.116 & 54.9548 & 14.56 & 73.9681 & 14.34 \\
\hline 1.10772 & 15.72 & 44.9702 & 13.336 & & & & & & & \\
\hline & 38.6939 & 12.404 & 40.3071 & 12.46 & 52.6564 & 14.12 & 55.8352 & 14.564 & 73.9681 & 14.344 \\
\hline 0.643372 & 15.724 & 45.9019 & 13.34 & & & & & & & \\
\hline & 39.5753 & 12.408 & 40.3071 & 12.464 & 54.5797 & 14.124 & 56.7168 & 14.568 & 73.9681 & 14.348 \\
\hline 8.59007 & 15.728 & 44.0399 & 13.344 & & & & & & & \\
\hline & 41.3422 & 12.412 & 41.1963 & 12.468 & 53.6173 & 14.128 & 54.9548 & 14.572 & 72.9659 & 14.352 \\
\hline 17.080 & 15.732 & 44.9702 & 13.348 & & & & & & & \\
\hline & 44.0023 & 12.416 & 41.1963 & 12.472 & 49.7824 & 14.132 & 58.4837 & 14.576 & 72.9659 & 14.356 \\
\hline 31.9159 & 15.736 & 44.0399 & 13.352 & & & & & & & \\
\hline & 44.0023 & 12.42 & 42.0869 & 12.476 & 52.6564 & 14.136 & 55.8352 & 14.58 & 73.9681 & 14.36 \\
\hline 45.512 & 15.74 & 44.0399 & 13.356 & & & & & & & \\
\hline & 43.1143 & 12.424 & 44.7666 & 12.48 & 52.6564 & 14.14 & 57.5996 & 14.584 & 72.9659 & 14.364 \\
\hline 57.1746 & 15.744 & 45.9019 & 13.36 & & & & & & & \\
\hline & 42.2275 & 12.428 & 43.872 & 12.484 & 54.5797 & 14.144 & 57.5996 & 14.588 & 72.9659 & 14.368 \\
\hline 64.4999 & 15.748 & 45.9019 & 13.364 & & & & & & & \\
\hline & 45.7824 & 12.432 & 42.0869 & 12.488 & 52.6564 & 14.148 & 57.5996 & 14.592 & 72.9659 & 14.372 \\
\hline 67.9072 & 15.752 & 45.9019 & 13.368 & & & & & & & \\
\hline & 51.1537 & 12.436 & 41.1963 & 12.492 & 51.697 & 14.152 & 55.8352 & 14.596 & 71.965 & 14.376 \\
\hline 68.4767 & 15.756 & 46.8351 & 13.372 & & & & & & & \\
\hline
\end{tabular}




\begin{tabular}{|c|c|c|c|c|c|c|c|c|c|c|}
\hline & 52.9545 & 12.44 & 41.1963 & 12.496 & 52.6564 & 14.156 & 57.5996 & 14.6 & 72.9659 & 14.38 \\
\hline \multirow[t]{2}{*}{69.0466} & 15.76 & 45.9019 & 13.376 & & & & & & & \\
\hline & 51.1537 & 12.444 & 38.5327 & 12.5 & 53.6173 & 14.16 & 54.9548 & 14.604 & 72.9659 & 14.384 \\
\hline \multirow[t]{2}{*}{67.3382} & 15.764 & 47.7697 & 13.38 & & & & & & & \\
\hline & 44.8917 & 12.448 & 38.5327 & 12.504 & 54.5797 & 14.164 & 54.9548 & 14.608 & 71.965 & 14.388 \\
\hline \multirow[t]{2}{*}{66.7696} & 15.768 & 45.9019 & 13.384 & & & & & & & \\
\hline & 36.9352 & 12.452 & 42.0869 & 12.508 & 53.6173 & 14.168 & 55.8352 & 14.612 & 72.9659 & 14.392 \\
\hline \multirow[t]{2}{*}{66.7696} & 15.772 & 45.9019 & 13.388 & & & & & & & \\
\hline & 33.434 & 12.456 & 41.1963 & 12.512 & 53.6173 & 14.172 & 55.8352 & 14.616 & 71.965 & 14.396 \\
\hline \multirow[t]{2}{*}{65.0667} & 15.776 & 45.9019 & 13.392 & & & & & & & \\
\hline & 34.3073 & 12.46 & 42.9788 & 12.516 & 53.6173 & 14.176 & 54.9548 & 14.62 & 73.9681 & 14.4 \\
\hline \multirow[t]{2}{*}{65.6338} & 15.78 & 44.9702 & 13.396 & & & & & & & \\
\hline & 40.458 & 12.464 & 42.0869 & 12.52 & 54.5797 & 14.18 & 55.8352 & 14.624 & 72.9659 & 14.404 \\
\hline \multirow[t]{2}{*}{65.0667} & 15.784 & 44.9702 & 13.4 & & & & & & & \\
\hline & 46.6744 & 12.468 & 42.0869 & 12.524 & 55.5434 & 14.184 & 54.9548 & 14.628 & 73.9681 & 14.408 \\
\hline \multirow[t]{2}{*}{66.7696} & 15.788 & 45.9019 & 13.404 & & & & & & & \\
\hline & 51.1537 & 12.472 & 44.7666 & 12.528 & 56.5086 & 14.188 & 54.9548 & 14.632 & 72.9659 & 14.412 \\
\hline \multirow[t]{2}{*}{66.7696} & 15.792 & 45.9019 & 13.408 & & & & & & & \\
\hline & 54.7603 & 12.476 & 44.7666 & 12.532 & 54.5797 & 14.192 & 56.7168 & 14.636 & 72.9659 & 14.416 \\
\hline 69.0466 & 15.796 & 45.9019 & 13.412 & & & & & & & \\
\hline & 52.9545 & 12.48 & 47.4583 & 12.536 & 54.5797 & 14.196 & 54.0755 & 14.64 & 73.9681 & 14.42 \\
\hline 68.4767 & 15.8 & 44.0399 & 13. & & & & & & & \\
\hline & 47.5676 & 12.484 & 47.4583 & 12.54 & 53.6173 & 14.2 & 54.0755 & 14.644 & 72.9659 & 14.424 \\
\hline 67.9072 & 15.804 & 44.0399 & 13.42 & & & & & & & \\
\hline & 39.5753 & 12.488 & 43.872 & 12.544 & 54.5797 & 14.204 & 52.3205 & 14.648 & 71.965 & 14.428 \\
\hline 71.9029 & 15.808 & 43.1111 & 13.424 & & & & & & & \\
\hline & 35.1818 & 12.492 & 40.3071 & 12.548 & 56.5086 & 14.208 & 54.0755 & 14.652 & 72.9659 & 14.432 \\
\hline 77.0723 & 15.812 & 44.9702 & 13.428 & & & & & & & \\
\hline & 36.0579 & 12.496 & 35. & 12.552 & 54.5797 & 14.212 & 55.8352 & 14.656 & 71.965 & 14.436 \\
\hline 85.1832 & 15.816 & 44.0399 & 13.432 & & & & & & & \\
\hline & 38.6939 & 12.5 & 34.1207 & 12.556 & 55.5434 & 14.216 & 56.7168 & 14.66 & 70.9654 & 14.44 \\
\hline 95.142 & 15.82 & 43.1111 & 13.436 & & & & & & & \\
\hline & 45.7824 & 12.504 & 35.8814 & 12.56 & 52.6564 & 14.22 & 54.9548 & 14.664 & 71.965 & 14.444 \\
\hline 103.43 & 15.824 & 44.0399 & 13.44 & & & & & & & \\
\hline & 52.9545 & 12.508 & 39.4192 & 12.564 & 56.5086 & 14.224 & 55.8352 & 14.668 & 72.9659 & 14.448 \\
\hline 109.995 & 15.828 & 44.0399 & 13.444 & & & & & & & \\
\hline & 59.2967 & 12.512 & 41.1963 & 12.568 & 53.6173 & 14.228 & 56.7168 & 14.672 & 73.9681 & 14.452 \\
\hline 113.595 & 15.832 & 43.1111 & 13.448 & & & & & & & \\
\hline & 57.4784 & 12.516 & 44.7666 & 12.572 & 55.5434 & 14.232 & 55.8352 & 14.676 & 71.965 & 14.456 \\
\hline 116.605 & 15.836 & 43.1111 & 13.452 & & & & & & & \\
\hline & 54.7603 & 12.52 & 42.0869 & 12.576 & 55.5434 & 14.236 & 55.8352 & 14.68 & 72.9659 & 14.46 \\
\hline 118.415 & 15.84 & 43.1111 & & & & & & & & \\
\hline & 48.4623 & 12.524 & 40.3071 & 12.58 & 55.5434 & 14.24 & 54.9548 & 14.684 & 73.9681 & 14.464 \\
\hline 117.811 & 15.844 & 43.1111 & 13.46 & & & & & & & \\
\hline & 40.458 & 12.528 & 35.0003 & 12.584 & 55.5434 & 14.244 & 54.9548 & 14.688 & 71.965 & 14.468 \\
\hline 117.811 & 15.848 & 43.1111 & 13.464 & & & & & & & \\
\hline & 38.6939 & 12.532 & 33.2424 & 12.588 & 55.5434 & 14.248 & 57.5996 & 14.692 & 70.9654 & 14.472 \\
\hline 117.208 & 15.852 & 42.1837 & 13.468 & & & & & & & \\
\hline & 39.5753 & 12.536 & 29.7435 & 12.592 & 53.6173 & 14.252 & 54.9548 & 14.696 & 70.9654 & 14.476 \\
\hline 116.002 & 15.856 & 42.1837 & 13.472 & & & & & & & \\
\hline & 43.1143 & 12.54 & 32.3656 & 12.596 & 55.5434 & 14.256 & 54.9548 & 14.7 & 71.965 & 14.48 \\
\hline 116.002 & 15.86 & 43.1111 & 13.476 & & & & & & & \\
\hline & 44.8917 & 12.544 & 35.8814 & 12.6 & 55.5434 & 14.26 & 54.9548 & 14.704 & 71.965 & 14.484 \\
\hline 116.002 & 15.864 & 41.2577 & 13.48 & & & & & & & \\
\hline & 44.0023 & 12.548 & 39.4192 & 12.604 & 56.5086 & 14.264 & 54.9548 & 14.708 & 71.965 & 14.488 \\
\hline 115.4 & 15.868 & 43.1111 & 13.484 & & & & & & & \\
\hline & 41.3422 & 12.552 & 42.0869 & 12.608 & 54.5797 & 14.268 & 55.8352 & 14.712 & 71.965 & 14.492 \\
\hline 116.002 & 15.872 & 41.2577 & 13.488 & & & & & & & \\
\hline & 39.5753 & 12.556 & 42.0869 & 12.612 & 55.5434 & 14.272 & 54.9548 & 14.716 & 71.965 & 14.496 \\
\hline 116.002 & 15.876 & 43.1111 & 13.492 & & & & & & & \\
\hline & 38.6939 & 12.56 & 43.872 & 12.616 & 56.5086 & 14.276 & 55.8352 & 14.72 & 70.9654 & 14.5 \\
\hline 113.595 & 15.88 & 43.1111 & 13.496 & & & & & & & \\
\hline & 38.6939 & 12.564 & 41.1963 & 12.62 & 55.5434 & 14.28 & 55.8352 & 14.724 & 70.9654 & 14.504 \\
\hline 114.798 & 15.884 & 43.1111 & 13.5 & & & & & & & \\
\hline & 38.6939 & 12.568 & 39.4192 & 12.624 & 55.5434 & 14.284 & 55.8352 & 14.728 & 70.9654 & 14.508 \\
\hline 116.002 & 15.888 & 43.1111 & 13.504 & & & & & & & \\
\hline & 38.6939 & 12.572 & 36.7638 & 12.628 & 56.5086 & 14.288 & 54.0755 & 14.732 & 69.9673 & 14.512 \\
\hline 116.605 & 15.892 & 43.1111 & 13.508 & & & & & & & \\
\hline & 36.0579 & 12.576 & 34.1207 & 12.632 & 58.4433 & 14.292 & 55.8352 & 14.736 & 71.965 & 14.516 \\
\hline 117.208 & 15.896 & 42.1837 & 13.512 & & & & & & & \\
\hline
\end{tabular}




\begin{tabular}{|c|c|c|c|c|c|c|c|c|c|c|}
\hline & 34.3073 & 12.58 & 35.0003 & 12.636 & 56.5086 & 14.296 & 54.0755 & 14.74 & 70.9654 & 14.52 \\
\hline \multirow[t]{2}{*}{117.208} & 15.9 & 42.1837 & 13.516 & & & & & & & \\
\hline & 33.434 & 12.584 & 39.4192 & 12.64 & 52.6564 & 14.3 & 54.9548 & 14.744 & 70.9654 & 14.524 \\
\hline \multirow[t]{2}{*}{117.208} & 15.904 & 43.1111 & 13.52 & & & & & & & \\
\hline & 33.434 & 12.588 & 44.7666 & 12.644 & 54.5797 & 14.304 & 55.8352 & 14.748 & 69.9673 & 14.528 \\
\hline \multirow[t]{2}{*}{116.605} & 15.908 & 43.1111 & 13.524 & & & & & & & \\
\hline & 35.1818 & 12.592 & 45.6625 & 12.648 & 53.6173 & 14.308 & 54.9548 & 14.752 & 69.9673 & 14.532 \\
\hline \multirow[t]{2}{*}{117.811} & 15.912 & 43.1111 & 13.528 & & & & & & & \\
\hline & 39.5753 & 12.596 & 47.4583 & 12.652 & 55.5434 & 14.312 & 54.0755 & 14.756 & 69.9673 & 14.536 \\
\hline \multirow[t]{2}{*}{116.002} & 15.916 & 42.1837 & 13.532 & & & & & & & \\
\hline & 43.1143 & 12.6 & 43.872 & 12.656 & 53.6173 & 14.316 & 54.9548 & 14.76 & 69.9673 & 14.54 \\
\hline \multirow[t]{2}{*}{116.002} & 15.92 & 41.2577 & 13.536 & & & & & & & \\
\hline & 45.7824 & 12.604 & 42.0869 & 12.66 & 53.6173 & 14.32 & 54.0755 & 14.764 & 69.9673 & 14.544 \\
\hline \multirow[t]{2}{*}{116.002} & 15.924 & 42.1837 & 13.54 & & & & & & & \\
\hline & 44.8917 & 12.608 & 37.6476 & 12.664 & 55.5434 & 14.324 & 53.1974 & 14.768 & 69.9673 & 14.548 \\
\hline \multirow[t]{2}{*}{114.196} & 15.928 & 40.3332 & 13.544 & & & & & & & \\
\hline & 43.1143 & 12.612 & 35.8814 & 12.668 & 54.5797 & 14.328 & 54.0755 & 14.772 & 69.9673 & 14.552 \\
\hline \multirow[t]{2}{*}{116.002} & 15.932 & 39.4102 & 13.548 & & & & & & & \\
\hline & 40.458 & 12.616 & 35.0003 & 12.672 & 53.6173 & 14.332 & 55.8352 & 14.776 & 68.9705 & 14.556 \\
\hline 117.208 & 15.936 & 41.2577 & 13.552 & & & & & & & \\
\hline & 36.9352 & 12.62 & 35.8814 & 12.676 & 54.5797 & 14.336 & 54.0755 & 14.78 & 68.9705 & 14.56 \\
\hline 116.605 & 15.94 & 41.2577 & 13.556 & & & & & & & \\
\hline & 35.1818 & 12.624 & 36.7638 & 12.68 & 54.5797 & 14.34 & 54.0755 & 14.784 & 69.9673 & 14.564 \\
\hline 116.002 & 15.944 & 40.3332 & 13.56 & & & & & & & \\
\hline & 35.1818 & 12.628 & 36.7638 & 12.684 & 53.6173 & 14.344 & 54.0755 & 14.788 & 67.9751 & 14.568 \\
\hline 115.4 & 15.948 & 40.3332 & 13.564 & & & & & & & \\
\hline & 35.1818 & 12.632 & 36.7638 & 12.688 & 51.697 & 14.348 & 54.9548 & 14.792 & 68.9705 & 14.572 \\
\hline 115.4 & 15.952 & 41.2577 & 13.568 & & & & & & & \\
\hline & 36.0579 & 12.636 & 35.0003 & 12.692 & 52.6564 & 14.352 & 54.9548 & 14.796 & 69.9673 & 14.576 \\
\hline 114.798 & 15.956 & 40.3332 & 13.572 & & & & & & & \\
\hline & 37.8139 & 12.64 & 35.0003 & 12.696 & 53.6173 & 14.356 & 54.0755 & 14.8 & 68.9705 & 14.58 \\
\hline 114.798 & 15.96 & 41.2577 & 13.576 & & & & & & & \\
\hline & 43.1143 & 12.644 & 35.8814 & 12.7 & 53.6173 & 14.36 & 54.9548 & 14.804 & 69.9673 & 14.584 \\
\hline 113.595 & 15.964 & 40.3332 & 13.58 & & & & & & & \\
\hline & 45.7824 & 12.648 & 37.6476 & 12.704 & 54.5797 & 14.364 & 54.9548 & 14.808 & 69.9673 & 14.588 \\
\hline 114.196 & 15.968 & 40.3332 & 13.584 & & & & & & & \\
\hline & 48.4623 & 12.652 & 37.6476 & 12.708 & 52.6564 & 14.368 & 55.8352 & 14.812 & 69.9673 & 14.592 \\
\hline 113.595 & 15.972 & 40.3332 & 13.588 & & & & & & & \\
\hline & 46.6744 & 12.656 & 39.4192 & 12.712 & 54.5797 & 14.372 & 54.0755 & 14.816 & 68.9705 & 14.596 \\
\hline 113.595 & 15.976 & 41.2577 & 13.592 & & & & & & & \\
\hline & 41.3422 & 12.66 & 41.1963 & 12.716 & 54.5797 & 14.376 & 54.0755 & 14.82 & 68.9705 & 14.6 \\
\hline 113.595 & 15.98 & 40.3332 & 13.596 & & & & & & & \\
\hline & 36.0579 & 12.664 & 41.1963 & 12.72 & 53.6173 & 14.38 & 53.1974 & 14.824 & 69.9673 & 14.604 \\
\hline 114.196 & 15.984 & 40.3332 & 13.6 & & & & & & & \\
\hline & 27.3601 & 12.668 & 41.1963 & 12.724 & 53.6173 & 14.384 & 54.9548 & 14.828 & 69.9673 & 14.608 \\
\hline 112.994 & 15.988 & 39.4102 & 13.604 & & & & & & & \\
\hline & 23.0638 & 12.672 & 42.0869 & 12.728 & 52.6564 & 14.388 & 54.0755 & 14.832 & 67.9751 & 14.612 \\
\hline 112.393 & 15.992 & 40.3332 & 13.608 & & & & & & & \\
\hline & 21.3552 & 12.676 & 40.3071 & 12.732 & 52.6564 & 14.392 & 54.0755 & 14.836 & 69.9673 & 14.616 \\
\hline 112.994 & 15.996 & 40.3332 & 13.612 & & & & & & & \\
\hline & 23.9202 & 12.68 & 39.4192 & 12.736 & 52.6564 & 14.396 & 52.3205 & 14.84 & 67.9751 & 14.62 \\
\hline 113.595 & 16 & 40.3332 & 13.616 & & & & & & & \\
\hline & 29.9548 & 12.684 & 40.3071 & 12.74 & 52.6564 & 14.4 & 54.0755 & 14.844 & 66.9811 & 14.624 \\
\hline 112.994 & 16.004 & 40.3332 & 13.62 & & & & & & & \\
\hline & 36.0579 & 12.688 & 37.6476 & 12.744 & 52.6564 & 14.404 & 53.1974 & 14.848 & 67.9751 & 14.628 \\
\hline 113.595 & 16.008 & 40.3332 & 13.624 & & & & & & & \\
\hline & 40.458 & 12.692 & 37.6476 & 12.748 & 53.6173 & 14.408 & 50.5704 & 14.852 & 68.9705 & 14.632 \\
\hline 112.994 & 16.012 & 40.3332 & 13.628 & & & & & & & \\
\hline & 42.2275 & 12.696 & 37.6476 & 12.752 & 52.6564 & 14.412 & 53.1974 & 14.856 & 69.9673 & 14.636 \\
\hline 112.994 & 16.016 & 40.3332 & 13.632 & & & & & & & \\
\hline & 44.8917 & 12.7 & 39.4192 & 12.756 & 53.6173 & 14.416 & 51.4448 & 14.86 & 66.9811 & 14.64 \\
\hline 113.595 & 16.02 & 40.3332 & 13.636 & & & & & & & \\
\hline & 43.1143 & 12.704 & 40.3071 & 12.76 & 53.6173 & 14.42 & 54.0755 & 14.864 & 66.9811 & 14.644 \\
\hline 112.393 & 16.024 & 41.2577 & 13.64 & & & & & & & \\
\hline & 41.3422 & 12.708 & 38.5327 & 12.764 & 51.697 & 14.424 & 53.1974 & 14.868 & 68.9705 & 14.648 \\
\hline 111.193 & 16.028 & 41.2577 & 13.644 & & & & & & & \\
\hline & 38.6939 & 12.712 & 40.3071 & 12.768 & 52.6564 & 14.428 & 54.0755 & 14.872 & 69.9673 & 14.652 \\
\hline 112.393 & 16.032 & 41.2577 & 13.648 & & & & & & & \\
\hline & 35.1818 & 12.716 & 37.6476 & 12.772 & 52.6564 & 14.432 & 52.3205 & 14.876 & 69.9673 & 14.656 \\
\hline 11.193 & 16.036 & 39.4102 & 13.652 & & & & & & & \\
\hline
\end{tabular}




\begin{tabular}{|c|c|c|c|c|c|c|c|c|c|c|}
\hline & 35.1818 & 12.72 & 37.6476 & 12.776 & 52.6564 & 14.436 & 54.0755 & 14.88 & 67.9751 & 14.66 \\
\hline \multirow[t]{2}{*}{111.793} & 16.04 & 41.2577 & 13.656 & & & & & & & \\
\hline & 36.9352 & 12.724 & 36.7638 & 12.78 & 51.697 & 14.44 & 54.0755 & 14.884 & 67.9751 & 14.664 \\
\hline \multirow[t]{2}{*}{112.393} & 16.044 & 40.3332 & 13.66 & & & & & & & \\
\hline & 41.3422 & 12.728 & 35.8814 & 12.784 & 52.6564 & 14.444 & 56.7168 & 14.888 & 66.9811 & 14.668 \\
\hline \multirow[t]{2}{*}{111.793} & 16.048 & 39.4102 & 13.664 & & & & & & & \\
\hline & 45.7824 & 12.732 & 34.1207 & 12.788 & 53.6173 & 14.448 & 54.0755 & 14.892 & 66.9811 & 14.672 \\
\hline \multirow[t]{2}{*}{111.193} & 16.052 & 39.4102 & 13.668 & & & & & & & \\
\hline & 50.2553 & 12.736 & 30.6161 & 12.792 & 52.6564 & 14.452 & 53.1974 & 14.896 & 66.9811 & 14.676 \\
\hline \multirow[t]{2}{*}{111.193} & 16.056 & 39.4102 & 13.672 & & & & & & & \\
\hline & 53.8568 & 12.74 & 31.4902 & 12.796 & 51.697 & 14.456 & 54.9548 & 14.9 & 65.9884 & 14.68 \\
\hline \multirow[t]{2}{*}{111.793} & 16.06 & 39.4102 & 13.676 & & & & & & & \\
\hline & 51.1537 & 12.744 & 30.6161 & 12.8 & 51.697 & 14.46 & 53.1974 & 14.904 & 64.9972 & 14.684 \\
\hline \multirow[t]{2}{*}{111.793} & 16.064 & 40.3332 & 13.68 & & & & & & & \\
\hline & 48.4623 & 12.748 & 32.3656 & 12.804 & 52.6564 & 14.464 & 54.0755 & 14.908 & 66.9811 & 14.688 \\
\hline \multirow[t]{2}{*}{112.994} & 16.068 & 40.3332 & 13.684 & & & & & & & \\
\hline & 41.3422 & 12.752 & 32.3656 & 12.808 & 51.697 & 14.468 & 55.8352 & 14.912 & 65.9884 & 14.692 \\
\hline \multirow[t]{2}{*}{112.393} & 16.072 & 38.4886 & 13.688 & & & & & & & \\
\hline & 36.9352 & 12.756 & 34.1207 & 12.812 & 51.697 & 14.472 & 53.1974 & 14.916 & 66.9811 & 14.696 \\
\hline 112.994 & 16.076 & 39.4102 & 13.692 & & & & & & & \\
\hline & 31.6916 & 12.76 & 36.7638 & 12.816 & 51.697 & 14.476 & 54.0755 & 14.92 & 65.9884 & 14.7 \\
\hline 112.393 & 16.08 & 39.4102 & 13.696 & & & & & & & \\
\hline & 30.8225 & 12.764 & 35.0003 & 12.82 & 50.739 & 14.48 & 53.1974 & 14.924 & 65.9884 & 14.704 \\
\hline 111.793 & 16.084 & 39.4102 & 13.7 & & & & & & & \\
\hline & 36.0579 & 12.768 & 36.7638 & 12.824 & 50.739 & 14.484 & 54.0755 & 14.928 & 65.9884 & 14.708 \\
\hline 111.193 & 16.088 & 39.4102 & 13.704 & & & & & & & \\
\hline & 41.3422 & 12.772 & 33.2424 & 12.828 & 49.7824 & 14.488 & 54.9548 & 14.932 & 66.9811 & 14.712 \\
\hline 111.193 & 16.092 & 40.3332 & 13.708 & & & & & & & \\
\hline & 47.5676 & 12.776 & 30.6161 & 12.832 & 49.7824 & 14.492 & 54.9548 & 14.936 & 64.9972 & 14.716 \\
\hline 112.393 & 16.096 & 39.4102 & 13.712 & & & & & & & \\
\hline & 49.3581 & 12.78 & 30.6161 & 12.836 & 49.7824 & 14.496 & 54.9548 & 14.94 & 65.9884 & 14.72 \\
\hline 111.793 & 16.1 & 38.4886 & 13.716 & & & & & & & \\
\hline & 49.3581 & 12.784 & 32.3656 & 12.84 & 50.739 & 14.5 & 55.8352 & 14.944 & 64.0074 & 14.724 \\
\hline 111.793 & 16.104 & 39.4102 & 13.72 & & & & & & & \\
\hline & 47.5676 & 12.788 & 34.1207 & 12.844 & 51.697 & 14.504 & 55.8352 & 14.948 & 65.9884 & 14.728 \\
\hline 111.793 & 16.108 & 38.4886 & 13.724 & & & & & & & \\
\hline & 41.3422 & 12.792 & 37.6476 & 12.848 & 52.6564 & 14.508 & 54.0755 & 14.952 & 64.9972 & 14.732 \\
\hline 111.793 & 16.112 & 39.4102 & 13.728 & & & & & & & \\
\hline & 37.8139 & 12.796 & 38.5327 & 12.852 & 49.7824 & 14.512 & 55.8352 & 14.956 & 64.9972 & 14.736 \\
\hline 111.193 & 16.116 & 38.4886 & 13.732 & & & & & & & \\
\hline & 33.434 & 12.8 & 39.4192 & 12.856 & 51.697 & 14.516 & 54.0755 & 14.96 & 64.0074 & 14.74 \\
\hline 111.193 & 16.12 & 39.4102 & 13.736 & & & & & & & \\
\hline & 34.3073 & 12.804 & 39.4192 & 12.86 & 50.739 & 14.52 & 54.9548 & 14.964 & 64.9972 & 14.744 \\
\hline 111.193 & 16.124 & 38.4886 & 13.74 & & & & & & & \\
\hline & 36.0579 & 12.808 & 35.8814 & 12.864 & 51.697 & 14.524 & 55.8352 & 14.968 & 64.9972 & 14.748 \\
\hline 111.193 & 16.128 & 39.4102 & 13.744 & & & & & & & \\
\hline & 40.458 & 12.812 & 33.2424 & 12.868 & 50.739 & 14.528 & 54.0755 & 14.972 & 65.9884 & 14.752 \\
\hline 111.193 & 16.132 & 39.4102 & 13.748 & & & & & & & \\
\hline & 42.2275 & 12.816 & 30.6161 & 12.872 & 51.697 & 14.532 & 52.3205 & 14.976 & 64.9972 & 14.756 \\
\hline 111.793 & 16.136 & 38.4886 & 13.752 & & & & & & & \\
\hline & 45.7824 & 12.82 & 30.6161 & 12.876 & 52.6564 & 14.536 & 53.1974 & 14.98 & 64.0074 & 14.76 \\
\hline 111.793 & 16.14 & 39.4102 & 13.756 & & & & & & & \\
\hline & 45.7824 & 12.824 & 28.0024 & 12.88 & 51.697 & 14.54 & 54.0755 & 14.984 & 65.9884 & 14.764 \\
\hline 111.793 & 16.144 & 39.4102 & 13.76 & & & & & & & \\
\hline & 45.7824 & 12.828 & 28.0024 & 12.884 & 50.739 & 14.544 & 53.1974 & 14.988 & 64.9972 & 14.768 \\
\hline 111.793 & 16.148 & 39.4102 & 13.764 & & & & & & & \\
\hline & 44.0023 & 12.832 & 27.1341 & 12.888 & 50.739 & 14.548 & 54.9548 & 14.992 & 64.9972 & 14.772 \\
\hline 111.193 & 16.152 & 37.5685 & 13.768 & & & & & & & \\
\hline & 45.7824 & 12.836 & 27.1341 & 12.892 & 52.6564 & 14.552 & 55.8352 & 14.996 & 64.9972 & 14.776 \\
\hline 111.193 & 16.156 & 39.4102 & 13.772 & & & & & & & \\
\hline & 43.1143 & 12.84 & 28.8723 & 12.896 & 49.7824 & 14.556 & 54.0755 & 15 & 64.9972 & 14.78 \\
\hline 111.193 & 16.16 & 38.4886 & 13.776 & & & & & & & \\
\hline & 44.8917 & 12.844 & 29.7435 & 12.9 & 50.739 & 14.56 & 54.0755 & 15.004 & 65.9884 & 14.784 \\
\hline 110.594 & 16.164 & 37.5685 & 13.78 & & & & & & & \\
\hline & 43.1143 & 12.848 & 28.0024 & 12.904 & 50.739 & 14.564 & 55.8352 & 15.008 & 64.0074 & 14.788 \\
\hline 111.193 & 16.168 & 38.4886 & 13.784 & & & & & & & \\
\hline & 43.1143 & 12.852 & 30.6161 & 12.908 & 50.739 & 14.568 & 54.9548 & 15.012 & 65.9884 & 14.792 \\
\hline 110.594 & 16.172 & 39.4102 & 13.788 & & & & & & & \\
\hline & 44.0023 & 12.856 & 30.6161 & 12.912 & 51.697 & 14.572 & 54.9548 & 15.016 & 64.9972 & 14.796 \\
\hline 1.193 & 16.176 & 37.5685 & 13.792 & & & & & & & \\
\hline
\end{tabular}




\begin{tabular}{|c|c|c|c|c|c|c|c|c|c|c|}
\hline & 41.3422 & 12.86 & 29.7435 & 12.916 & 51.697 & 14.576 & 53.1974 & 15.02 & 65.9884 & 14.8 \\
\hline \multirow[t]{2}{*}{111.193} & 16.18 & 39.4102 & 13.796 & & & & & & & \\
\hline & 40.458 & 12.864 & 28.0024 & 12.92 & 51.697 & 14.58 & 54.0755 & 15.024 & 64.0074 & 14.804 \\
\hline \multirow[t]{2}{*}{111.193} & 16.184 & 37.5685 & 13.8 & & & & & & & \\
\hline & 39.5753 & 12.868 & 25.4016 & 12.924 & 50.739 & 14.584 & 53.1974 & 15.028 & 64.9972 & 14.808 \\
\hline \multirow[t]{2}{*}{111.193} & 16.188 & 38.4886 & 13.804 & & & & & & & \\
\hline & 39.5753 & 12.872 & 24.5374 & 12.928 & 51.697 & 14.588 & 54.0755 & 15.032 & 64.0074 & 14.812 \\
\hline \multirow[t]{2}{*}{111.193} & 16.192 & 37.5685 & 13.808 & & & & & & & \\
\hline & 39.5753 & 12.876 & 26.2671 & 12.932 & 50.739 & 14.592 & 55.8352 & 15.036 & 64.0074 & 14.816 \\
\hline \multirow[t]{2}{*}{109.995} & 16.196 & 38.4886 & 13.812 & & & & & & & \\
\hline & 38.6939 & 12.88 & 28.0024 & 12.936 & 50.739 & 14.596 & 54.9548 & 15.04 & 63.0189 & 14.82 \\
\hline \multirow[t]{2}{*}{108.798} & 16.2 & 37.5685 & 13.816 & & & & & & & \\
\hline & 38.6939 & 12.884 & 29.7435 & 12.94 & 52.6564 & 14.6 & 54.9548 & 15.044 & 63.0189 & 14.824 \\
\hline \multirow[t]{2}{*}{109.396} & 16.204 & 39.4102 & 13.82 & & & & & & & \\
\hline & 37.8139 & 12.888 & 32.3656 & 12.944 & 51.697 & 14.604 & 54.9548 & 15.048 & 62.0319 & 14.828 \\
\hline \multirow[t]{2}{*}{109.995} & 16.208 & 39.4102 & 13.824 & & & & & & & \\
\hline & 36.0579 & 12.892 & 33.2424 & 12.948 & 51.697 & 14.608 & 53.1974 & 15.052 & 63.0189 & 14.832 \\
\hline \multirow[t]{2}{*}{108.798} & 16.212 & 37.5685 & 13.828 & & & & & & & \\
\hline & 35.1818 & 12.896 & 34.1207 & 12.952 & 50.739 & 14.612 & 53.1974 & 15.056 & 63.0189 & 14.836 \\
\hline 110.594 & 16.216 & 39.4102 & 13.832 & & & & & & & \\
\hline & 35.1818 & 12.9 & 34.1207 & 12.956 & 51.697 & 14.616 & 52.3205 & 15.06 & 63.0189 & 14.84 \\
\hline 109.396 & 16.22 & 38.4886 & 13.836 & & & & & & & \\
\hline & 34.3073 & 12.904 & 33.2424 & 12.96 & 52.6564 & 14.62 & 54.0755 & 15.064 & 64.0074 & 14.844 \\
\hline 108.798 & 16.224 & 39.4102 & 13.84 & & & & & & & \\
\hline & 35.1818 & 12.908 & 31.4902 & 12.964 & 52.6564 & 14.624 & 51.4448 & 15.068 & 64.0074 & 14.848 \\
\hline 110.594 & 16.228 & 38.4886 & 13.844 & & & & & & & \\
\hline & 36.0579 & 12.912 & 29.7435 & 12.968 & 51.697 & 14.628 & 55.8352 & 15.072 & 64.9972 & 14.852 \\
\hline 109.995 & 16.232 & 37.5685 & 13.848 & & & & & & & \\
\hline & 37.8139 & 12.916 & 28.0024 & 12.972 & 53.6173 & 14.632 & 55.8352 & 15.076 & 64.9972 & 14.856 \\
\hline 109.396 & 16.236 & 37.5685 & 13.852 & & & & & & & \\
\hline & 38.6939 & 12.92 & 28.0024 & 12.976 & 51.697 & 14.636 & 54.0755 & 15.08 & 64.9972 & 14.86 \\
\hline 108.798 & 16.24 & 37.5685 & 13.856 & & & & & & & \\
\hline & 39.5753 & 12.924 & 29.7435 & 12.98 & 51.697 & 14.64 & 54.9548 & 15.084 & 65.9884 & 14.864 \\
\hline 109.995 & 16.244 & 38.4886 & 13.86 & & & & & & & \\
\hline & 38.6939 & 12.928 & 30.6161 & 12.984 & 53.6173 & 14.644 & 56.7168 & 15.088 & 63.0189 & 14.868 \\
\hline 109.995 & 16.248 & 37.5685 & 13.864 & & & & & & & \\
\hline & 38.6939 & 12.932 & 31.4902 & 12.988 & 53.6173 & 14.648 & 55.8352 & 15.092 & 63.0189 & 14.872 \\
\hline 111.193 & 16.252 & 37.5685 & 13.868 & & & & & & & \\
\hline & 38.6939 & 12.936 & 31.4902 & 12.992 & 51.697 & 14.652 & 54.9548 & 15.096 & 62.0319 & 14.876 \\
\hline 108.798 & 16.256 & 38.4886 & 13.872 & & & & & & & \\
\hline & 38.6939 & 12.94 & 31.4902 & 12.996 & 53.6173 & 14.656 & 54.0755 & 15.1 & 63.0189 & 14.88 \\
\hline 109.396 & 16.26 & 37.5685 & 13.876 & & & & & & & \\
\hline & 36.9352 & 12.944 & 28.8723 & 13 & 53.6173 & 14.66 & 56.7168 & 15.104 & 64.0074 & 14.884 \\
\hline 109.396 & 16.264 & 38.4886 & 13.88 & & & & & & & \\
\hline & 35.1818 & 12.948 & 28.8723 & 13.004 & 53.6173 & 14.664 & 54.9548 & 15.108 & 64.0074 & 14.888 \\
\hline 108.798 & 16.268 & 37.5685 & 13.884 & & & & & & & \\
\hline & 36.9352 & 12.952 & 28.0024 & 13.008 & 52.6564 & 14.668 & 54.9548 & 15.112 & 63.0189 & 14.892 \\
\hline 108.798 & 16.272 & 38.4886 & 13.888 & & & & & & & \\
\hline & 35.1818 & 12.956 & 28.0024 & 13.012 & 52.6564 & 14.672 & 54.9548 & 15.116 & 64.0074 & 14.896 \\
\hline 108.798 & 16.276 & 36.6499 & 13.892 & & & & & & & \\
\hline & 36.0579 & 12.96 & 28.8723 & 13.016 & 53.6173 & 14.676 & 54.9548 & 15.12 & 64.0074 & 14.9 \\
\hline 108.798 & 16.28 & 37.5685 & 13.896 & & & & & & & \\
\hline & 34.3073 & 12.964 & 29.7435 & 13.02 & 51.697 & 14.68 & 54.0755 & 15.124 & 64.0074 & 14.904 \\
\hline 108.2 & 16.284 & 39.4102 & 13.9 & & & & & & & \\
\hline & 35.1818 & 12.968 & 29.7435 & 13.024 & 53.6173 & 14.684 & 54.0755 & 15.128 & 62.0319 & 14.908 \\
\hline 107.005 & 16.288 & 37.5685 & 13.904 & & & & & & & \\
\hline & 35.1818 & 12.972 & 31.4902 & 13.028 & 54.5797 & 14.688 & 54.0755 & 15.132 & 63.0189 & 14.912 \\
\hline 107.603 & 16.292 & 38.4886 & 13.908 & & & & & & & \\
\hline & 36.0579 & 12.976 & 30.6161 & 13.032 & 53.6173 & 14.692 & 54.0755 & 15.136 & 62.0319 & 14.916 \\
\hline 108.798 & 16.296 & 38.4886 & 13.912 & & & & & & & \\
\hline & 38.6939 & 12.98 & 30.6161 & 13.036 & 52.6564 & 14.696 & 53.1974 & 15.14 & 64.9972 & 14.92 \\
\hline 108.798 & 16.3 & 38.4886 & 13.916 & & & & & & & \\
\hline & 37.8139 & 12.984 & 33.2424 & 13.04 & 54.5797 & 14.7 & 54.0755 & 15.144 & 62.0319 & 14.924 \\
\hline 108.798 & 16.304 & 39.4102 & 13.92 & & & & & & & \\
\hline & 36.9352 & 12.988 & 34.1207 & 13.044 & 53.6173 & 14.704 & 54.0755 & 15.148 & 63.0189 & 14.928 \\
\hline 107.603 & 16.308 & 39.4102 & 13.924 & & & & & & & \\
\hline & 36.0579 & 12.992 & 33.2424 & 13.048 & 55.5434 & 14.708 & 54.0755 & 15.152 & 63.0189 & 14.932 \\
\hline 107.603 & 16.312 & 36.6499 & 13.928 & & & & & & & \\
\hline & 32.5621 & 12.996 & 33.2424 & 13.052 & 54.5797 & 14.712 & 56.7168 & 15.156 & 64.0074 & 14.936 \\
\hline 106.408 & 16.316 & 38.4886 & 13.932 & & & & & & & \\
\hline
\end{tabular}




\begin{tabular}{|c|c|c|c|c|c|c|c|c|c|c|}
\hline & 32.5621 & 13 & 34.1207 & 13.056 & 54.5797 & 14.716 & 53.1974 & 15.16 & 62.0319 & 14.94 \\
\hline \multirow[t]{2}{*}{105.812} & 16.32 & 37.5685 & 13.936 & & & & & & & \\
\hline & 32.5621 & 13.004 & 32.3656 & 13.06 & 53.6173 & 14.72 & 54.0755 & 15.164 & 63.0189 & 14.944 \\
\hline \multirow[t]{2}{*}{105.812} & 16.324 & 38.4886 & 13.94 & & & & & & & \\
\hline & 35.1818 & 13.008 & 32.3656 & 13.064 & 54.5797 & 14.724 & 54.0755 & 15.168 & 63.0189 & 14.948 \\
\hline \multirow[t]{2}{*}{105.812} & 16.328 & 39.4102 & 13.944 & & & & & & & \\
\hline & 35.1818 & 13.012 & 32.3656 & 13.068 & 54.5797 & 14.728 & 54.0755 & 15.172 & 64.0074 & 14.952 \\
\hline \multirow[t]{2}{*}{106.408} & 16.332 & 39.4102 & 13.948 & & & & & & & \\
\hline & 37.8139 & 13.016 & 30.6161 & 13.072 & 53.6173 & 14.732 & 53.1974 & 15.176 & 63.0189 & 14.956 \\
\hline \multirow[t]{2}{*}{106.408} & 16.336 & 38.4886 & 13.952 & & & & & & & \\
\hline & 38.6939 & 13.02 & 30.6161 & 13.076 & 53.6173 & 14.736 & 53.1974 & 15.18 & 63.0189 & 14.96 \\
\hline \multirow[t]{2}{*}{107.005} & 16.34 & 39.4102 & 13.956 & & & & & & & \\
\hline & 38.6939 & 13.024 & 33.2424 & 13.08 & 56.5086 & 14.74 & 55.8352 & 15.184 & 61.0463 & 14.964 \\
\hline \multirow[t]{2}{*}{107.603} & 16.344 & 39.4102 & 13.96 & & & & & & & \\
\hline & 39.5753 & 13.028 & 33.2424 & 13.084 & 54.5797 & 14.744 & 54.0755 & 15.188 & 62.0319 & 14.968 \\
\hline \multirow[t]{2}{*}{106.408} & 16.348 & 39.4102 & 13.964 & & & & & & & \\
\hline & 38.6939 & 13.032 & 32.3656 & 13.088 & 55.5434 & 14.748 & 55.8352 & 15.192 & 64.0074 & 14.972 \\
\hline \multirow[t]{2}{*}{105.216} & 16.352 & 40.3332 & 13.968 & & & & & & & \\
\hline & 38.6939 & 13.036 & 32.3656 & 13.092 & 55.5434 & 14.752 & 54.9548 & 15.196 & 64.0074 & 14.976 \\
\hline 106.408 & 16.356 & 39.4102 & 13.972 & & & & & & & \\
\hline & 37.8139 & 13.04 & 32.3656 & 13.096 & 55.5434 & 14.756 & 54.9548 & 15.2 & 63.0189 & 14.98 \\
\hline 105.812 & 16.36 & 38.4886 & 13.976 & & & & & & & \\
\hline & 36.0579 & 13.044 & 31.4902 & 13.1 & 52.6564 & 14.76 & 54.0755 & 15.204 & 64.0074 & 14.984 \\
\hline 104.025 & 16.364 & 39.4102 & 13.98 & & & & & & & \\
\hline & 39.5753 & 13.048 & 31.4902 & 13.104 & 52.6564 & 14.764 & 54.0755 & 15.208 & 64.0074 & 14.988 \\
\hline 105.216 & 16.368 & 40.3332 & 13.984 & & & & & & & \\
\hline & 37.8139 & 13.052 & 30.6161 & 13.108 & 54.5797 & 14.768 & 54.9548 & 15.212 & 65.9884 & 14.992 \\
\hline 104.025 & 16.372 & 40.3332 & 13.988 & & & & & & & \\
\hline & 38.6939 & 13.056 & 30.6161 & 13.112 & 54.5797 & 14.772 & 54.9548 & 15.216 & 64.0074 & 14.996 \\
\hline 106.408 & 16.376 & 39.4102 & 13.992 & & & & & & & \\
\hline & 38.6939 & 13.06 & 30.6161 & 13.116 & 54.5797 & 14.776 & 52.3205 & 15.22 & 63.0189 & 15 \\
\hline 106.408 & 16.38 & 39.4102 & 13.996 & & & & & & & \\
\hline & 40.458 & 13.064 & 30.6161 & 13.12 & 53.6173 & 14.78 & 55.8352 & 15.224 & 63.0189 & 15.004 \\
\hline 105.812 & 16.384 & 38.4886 & 14 & & & & & & & \\
\hline & 39.5753 & 13.068 & 30.6161 & 13.124 & 52.6564 & 14.784 & 53.1974 & 15.228 & 62.0319 & 15.008 \\
\hline 105.216 & 16.388 & 39.4102 & 14.004 & & & & & & & \\
\hline & 40.458 & 13.072 & 31.4902 & 13.128 & 54.5797 & 14.788 & 54.9548 & 15.232 & 62.0319 & 15.012 \\
\hline 105.216 & 16.392 & 38.4886 & 14.008 & & & & & & & \\
\hline & 41.3422 & 13.076 & 30.6161 & 13.132 & 54.5797 & 14.792 & 54.9548 & 15.236 & 63.0189 & 15.016 \\
\hline 104.025 & 16.396 & 39.4102 & 14.012 & & & & & & & \\
\hline & 40.458 & 13.08 & 32.3656 & 13.136 & 53.6173 & 14.796 & 55.8352 & 15.24 & 62.0319 & 15.02 \\
\hline 104.025 & 16.4 & 38.4886 & 14.016 & & & & & & & \\
\hline & 39.5753 & 13.084 & 33.2424 & 13.14 & 56.5086 & 14.8 & 54.9548 & 15.244 & 62.0319 & 15.024 \\
\hline 104.025 & 16.404 & 39.4102 & 14.02 & & & & & & & \\
\hline & 38.6939 & 13.088 & 33.2424 & 13.144 & 56.5086 & 14.804 & 56.7168 & 15.248 & 62.0319 & 15.028 \\
\hline 104.025 & 16.408 & 39.4102 & 14.024 & & & & & & & \\
\hline & 37.8139 & 13.092 & 33.2424 & 13.148 & 54.5797 & 14.808 & 55.8352 & 15.252 & 62.0319 & 15.032 \\
\hline 104.025 & 16.412 & 39.4102 & 14.028 & & & & & & & \\
\hline & 37.8139 & 13.096 & 32.3656 & 13.152 & 56.5086 & 14.812 & 54.9548 & 15.256 & 63.0189 & 15.036 \\
\hline 103.43 & 16.416 & 38.4886 & 14.032 & & & & & & & \\
\hline & 37.8139 & 13.1 & 30.6161 & 13.156 & 57.4752 & 14.816 & 55.8352 & 15.26 & 62.0319 & 15.04 \\
\hline 104.025 & 16.42 & 39.4102 & 14.036 & & & & & & & \\
\hline & 38.6939 & 13.104 & 29.7435 & 13.16 & 56.5086 & 14.82 & 54.0755 & 15.264 & 62.0319 & 15.044 \\
\hline 104.025 & 16.424 & 39.4102 & 14.04 & & & & & & & \\
\hline & 39.5753 & 13.108 & 30.6161 & 13.164 & 57.4752 & 14.824 & 54.9548 & 15.268 & 62.0319 & 15.048 \\
\hline 104.025 & 16.428 & 39.4102 & 14.044 & & & & & & & \\
\hline & 42.2275 & 13.112 & 29.7435 & 13.168 & 57.4752 & 14.828 & 54.9548 & 15.272 & 61.0463 & 15.052 \\
\hline 104.025 & 16.432 & 39.4102 & 14.048 & & & & & & & \\
\hline & 43.1143 & 13.116 & 30.6161 & 13.172 & 57.4752 & 14.832 & 54.9548 & 15.276 & 62.0319 & 15.056 \\
\hline 103.43 & 16.436 & 39.4102 & 14.052 & & & & & & & \\
\hline & 44.8917 & 13.12 & 33.2424 & 13.176 & 54.5797 & 14.836 & 54.9548 & 15.28 & 62.0319 & 15.06 \\
\hline 104.025 & 16.44 & 39.4102 & 14.056 & & & & & & & \\
\hline & 44.0023 & 13.124 & 33.2424 & 13.18 & 57.4752 & 14.84 & 55.8352 & 15.284 & 60.0621 & 15.064 \\
\hline 103.43 & 16.444 & 39.4102 & 14.06 & & & & & & & \\
\hline & 44.0023 & 13.128 & 31.4902 & 13.184 & 54.5797 & 14.844 & 55.8352 & 15.288 & 61.0463 & 15.068 \\
\hline 103.43 & 16.448 & 39.4102 & 14.064 & & & & & & & \\
\hline & 42.2275 & 13.132 & 31.4902 & 13.188 & 55.5434 & 14.848 & 57.5996 & 15.292 & 60.0621 & 15.072 \\
\hline 104.025 & 16.452 & 39.4102 & 14.068 & & & & & & & \\
\hline & 42.2275 & 13.136 & 30.6161 & 13.192 & 56.5086 & 14.852 & 55.8352 & 15.296 & 60.0621 & 15.076 \\
\hline 104.025 & 16.456 & 39.4102 & 14.072 & & & & & & & \\
\hline
\end{tabular}




\begin{tabular}{|c|c|c|c|c|c|c|c|c|c|c|}
\hline & 41.3422 & 13.14 & 30.6161 & 13.196 & 56.5086 & 14.856 & 56.7168 & 15.3 & 60.0621 & 15.08 \\
\hline \multirow[t]{2}{*}{102.836} & 16.46 & 39.4102 & 14.076 & & & & & & & \\
\hline & 42.2275 & 13.144 & 29.7435 & 13.2 & 59.4127 & 14.86 & 56.7168 & 15.304 & 61.0463 & 15.084 \\
\hline \multirow[t]{2}{*}{103.43} & 16.464 & 40.3332 & 14.08 & & & & & & & \\
\hline & 43.1143 & 13.148 & 28.0024 & 13.204 & 59.4127 & 14.864 & 55.8352 & 15.308 & 59.0794 & 15.088 \\
\hline \multirow[t]{2}{*}{102.836} & 16.468 & 40.3332 & 14.084 & & & & & & & \\
\hline & 44.0023 & 13.152 & 29.7435 & 13.208 & 56.5086 & 14.868 & 55.8352 & 15.312 & 61.0463 & 15.092 \\
\hline \multirow[t]{2}{*}{103.43} & 16.472 & 40.3332 & 14.088 & & & & & & & \\
\hline & 44.0023 & 13.156 & 29.7435 & 13.212 & 59.4127 & 14.872 & 55.8352 & 15.316 & 61.0463 & 15.096 \\
\hline \multirow[t]{2}{*}{101.648} & 16.476 & 39.4102 & 14.092 & & & & & & & \\
\hline & 44.8917 & 13.16 & 31.4902 & 13.216 & 57.4752 & 14.876 & 54.9548 & 15.32 & 62.0319 & 15.1 \\
\hline \multirow[t]{2}{*}{101.648} & 16.48 & 39.4102 & 14.096 & & & & & & & \\
\hline & 44.8917 & 13.164 & 30.6161 & 13.22 & 58.4433 & 14.88 & 55.8352 & 15.324 & 61.0463 & 15.104 \\
\hline \multirow[t]{2}{*}{101.648} & 16.484 & 39.4102 & 14.1 & & & & & & & \\
\hline & 45.7824 & 13.168 & 33.2424 & 13.224 & 57.4752 & 14.884 & 55.8352 & 15.328 & 61.0463 & 15.108 \\
\hline \multirow[t]{2}{*}{104.025} & 16.488 & 38.4886 & 14.104 & & & & & & & \\
\hline & 44.0023 & 13.172 & 31.4902 & 13.228 & 56.5086 & 14.888 & 56.7168 & 15.332 & 61.0463 & 15.112 \\
\hline \multirow[t]{2}{*}{102.836} & 16.492 & 40.3332 & 14.108 & & & & & & & \\
\hline & 42.2275 & 13.176 & 31.4902 & 13.232 & 56.5086 & 14.892 & 54.9548 & 15.336 & 61.0463 & 15.116 \\
\hline 102.836 & 16.496 & 41.2577 & 14.112 & & & & & & & \\
\hline & 40.458 & 13.18 & 32.3656 & 13.236 & 57.4752 & 14.896 & 54.9548 & 15.34 & 61.0463 & 15.12 \\
\hline 103.43 & 16.5 & 40.3332 & 14.116 & & & & & & & \\
\hline & 40.458 & 13.184 & 30.6161 & 13.24 & 57.4752 & 14.9 & 56.7168 & 15.344 & 63.0189 & 15.124 \\
\hline 102.241 & 16.504 & 40.3332 & 14.12 & & & & & & & \\
\hline & 39.5753 & 13.188 & 30.6161 & 13.244 & 55.5434 & 14.904 & 55.8352 & 15.348 & 60.0621 & 15.128 \\
\hline 101.648 & 16.508 & 39.4102 & 14.124 & & & & & & & \\
\hline & 38.6939 & 13.192 & 29.7435 & 13.248 & 56.5086 & 14.908 & 56.7168 & 15.352 & 61.0463 & 15.132 \\
\hline 101.648 & 16.512 & 39.4102 & 14.128 & & & & & & & \\
\hline & 41.3422 & 13.196 & 30.6161 & 13.252 & 58.4433 & 14.912 & 54.9548 & 15.356 & 61.0463 & 15.136 \\
\hline 101.648 & 16.516 & 39.4102 & 14.132 & & & & & & & \\
\hline & 42.2275 & 13.2 & 30.6161 & 13.256 & 58.4433 & 14.916 & 57.5996 & 15.36 & 59.0794 & 15.14 \\
\hline 101.648 & 16.52 & 41.2577 & 14.136 & & & & & & & \\
\hline & 42.2275 & 13.204 & 30.6161 & 13.26 & 58.4433 & 14.92 & 56.7168 & 15.364 & 59.0794 & 15.144 \\
\hline 101.648 & 16.524 & 39.4102 & 14.14 & & & & & & & \\
\hline & 43.1143 & 13.208 & 32.3656 & 13.264 & 56.5086 & 14.924 & 56.7168 & 15.368 & 60.0621 & 15.148 \\
\hline 102.836 & 16.528 & 40.3332 & 14.144 & & & & & & & \\
\hline & 44.8917 & 13.212 & 32.3656 & 13.268 & 56.5086 & 14.928 & 55.8352 & 15.372 & 61.0463 & 15.152 \\
\hline 102.241 & 16.532 & 41.2577 & 14.148 & & & & & & & \\
\hline & 43.1143 & 13.216 & 32.3656 & 13.272 & 58.4433 & 14.932 & 55.8352 & 15.376 & 60.0621 & 15.156 \\
\hline 101.054 & 16.536 & 40.3332 & 14.152 & & & & & & & \\
\hline & 43.1143 & 13.22 & 32.3656 & 13.276 & 56.5086 & 14.936 & 56.7168 & 15.38 & 61.0463 & 15.16 \\
\hline 101.648 & 16.54 & 41.2577 & 14.156 & & & & & & & \\
\hline & 42.2275 & 13.224 & 33.2424 & 13.28 & 56.5086 & 14.94 & 56.7168 & 15.384 & 61.0463 & 15.164 \\
\hline 101.648 & 16.544 & 40.3332 & 14.16 & & & & & & & \\
\hline & 42.2275 & 13.228 & 34.1207 & 13.284 & 56.5086 & 14.944 & 56.7168 & 15.388 & 61.0463 & 15.168 \\
\hline 100.461 & 16.548 & 40.3332 & 14.164 & & & & & & & \\
\hline & 42.2275 & 13.232 & 32.3656 & 13.288 & 57.4752 & 14.948 & 57.5996 & 15.392 & 60.0621 & 15.172 \\
\hline 101.648 & 16.552 & 39.4102 & 14.168 & & & & & & & \\
\hline & 43.1143 & 13.236 & 31.4902 & 13.292 & 57.4752 & 14.952 & 56.7168 & 15.396 & 58.0981 & 15.176 \\
\hline 101.648 & 16.556 & 39.4102 & 14.172 & & & & & & & \\
\hline & 44.8917 & 13.24 & 34.1207 & 13.296 & 59.4127 & 14.956 & 56.7168 & 15.4 & 61.0463 & 15.18 \\
\hline 101.648 & 16.56 & 40.3332 & 14.176 & & & & & & & \\
\hline & 43.1143 & 13.244 & 30.6161 & 13.3 & 58.4433 & 14.96 & 55.8352 & 15.404 & 61.0463 & 15.184 \\
\hline 101.648 & 16.564 & 39.4102 & 14.18 & & & & & & & \\
\hline & 45.7824 & 13.248 & 34.1207 & 13.304 & 59.4127 & 14.964 & 55.8352 & 15.408 & 59.0794 & 15.188 \\
\hline 101.648 & 16.568 & 42.1837 & 14.184 & & & & & & & \\
\hline & 44.8917 & 13.252 & 33.2424 & 13.308 & 58.4433 & 14.968 & 56.7168 & 15.412 & 61.0463 & 15.192 \\
\hline 101.054 & 16.572 & 40.3332 & 14.188 & & & & & & & \\
\hline & 44.8917 & 13.256 & 34.1207 & 13.312 & 58.4433 & 14.972 & 56.7168 & 15.416 & 60.0621 & 15.196 \\
\hline 101.054 & 16.576 & 41.2577 & 14.192 & & & & & & & \\
\hline & 43.1143 & 13.26 & 34.1207 & 13.316 & 59.4127 & 14.976 & 56.7168 & 15.42 & 59.0794 & 15.2 \\
\hline 101.054 & 16.58 & 41.2577 & 14.196 & & & & & & & \\
\hline & 45.7824 & 13.264 & 34.1207 & 13.32 & 59.4127 & 14.98 & 56.7168 & 15.424 & 59.0794 & 15.204 \\
\hline 100.461 & 16.584 & 42.1837 & 14.2 & & & & & & & \\
\hline & 44.0023 & 13.268 & 34.1207 & 13.324 & 59.4127 & 14.984 & 57.5996 & 15.428 & 59.0794 & 15.208 \\
\hline 100.461 & 16.588 & 39.4102 & 14.204 & & & & & & & \\
\hline & 43.1143 & 13.272 & 35.0003 & 13.328 & 59.4127 & 14.988 & 56.7168 & 15.432 & 58.0981 & 15.212 \\
\hline 101.648 & 16.592 & 41.2577 & 14.208 & & & & & & & \\
\hline & 43.1143 & 13.276 & 34.1207 & 13.332 & 58.4433 & 14.992 & 56.7168 & 15.436 & 60.0621 & 15.216 \\
\hline 101.054 & 16.596 & 41.2577 & 14.212 & & & & & & & \\
\hline
\end{tabular}




\begin{tabular}{|c|c|c|c|c|c|c|c|c|c|c|}
\hline \multirow{3}{*}{101.054} & 42.2275 & 13.28 & 35.0003 & 13.336 & 60.3834 & 14.996 & 57.5996 & 15.44 & 58.0981 & 15.22 \\
\hline & 16.6 & 40.3332 & 14.216 & & & & & & & \\
\hline & 42.2275 & 13.284 & 35.8814 & 13.34 & 60.3834 & 15 & 57.5996 & 15.444 & 58.0981 & 15.224 \\
\hline \multirow[t]{2}{*}{101.648} & 16.604 & 40.3332 & 14.22 & & & & & & & \\
\hline & 42.2275 & 13.288 & 35.0003 & 13.344 & 59.4127 & 15.004 & 57.5996 & 15.448 & 58.0981 & 15.228 \\
\hline \multirow[t]{2}{*}{101.648} & 16.608 & 40.3332 & 14.224 & & & & & & & \\
\hline & 41.3422 & 13.292 & 35.0003 & 13.348 & 58.4433 & 15.008 & 55.8352 & 15.452 & 58.0981 & 15.232 \\
\hline \multirow[t]{2}{*}{101.054} & 16.612 & 41.2577 & 14.228 & & & & & & & \\
\hline & 42.2275 & 13.296 & 35.8814 & 13.352 & 60.3834 & 15.012 & 57.5996 & 15.456 & 58.0981 & 15.236 \\
\hline \multirow[t]{2}{*}{101.054} & 16.616 & 40.3332 & 14.232 & & & & & & & \\
\hline & 43.1143 & 13.3 & 36.7638 & 13.356 & 58.4433 & 15.016 & 57.5996 & 15.46 & 58.0981 & 15.24 \\
\hline \multirow[t]{2}{*}{100.461} & 16.62 & 41.2577 & 14.236 & & & & & & & \\
\hline & 44.0023 & 13.304 & 36.7638 & 13.36 & 58.4433 & 15.02 & 57.5996 & 15.464 & 59.0794 & 15.244 \\
\hline \multirow[t]{2}{*}{99.2765} & 16.624 & 40.3332 & 14.24 & & & & & & & \\
\hline & 44.0023 & 13.308 & 36.7638 & 13.364 & & & 58.4837 & 15.468 & 58.0981 & 15.248 \\
\hline \multirow[t]{2}{*}{99.2765} & 16.628 & 41.2577 & 14.244 & & & & & & & \\
\hline & 44.8917 & 13.312 & 37.6476 & 13.368 & & & 57.5996 & 15.472 & 58.0981 & 15.252 \\
\hline \multirow[t]{2}{*}{99.2765} & 16.632 & 40.3332 & 14.248 & & & & & & & \\
\hline & 44.0023 & 13.316 & 35.8814 & 13.372 & & & 57.5996 & 15.476 & 58.0981 & 15.256 \\
\hline 101.054 & 16.636 & 41.2577 & 14.252 & & & & & & & \\
\hline & 43.1143 & 13.32 & 36.7638 & 13.376 & & & 57.5996 & 15.48 & 60.0621 & 15.26 \\
\hline 100.461 & 16.64 & 39.4102 & 14.256 & & & & & & & \\
\hline & 43.1143 & 13.324 & 35.8814 & 13.38 & & & 57.5996 & 15.484 & 59.0794 & 15.264 \\
\hline 101.648 & 16.644 & 41.2577 & 14.26 & & & & & & & \\
\hline & 42.2275 & 13.328 & 36.7638 & 13.384 & & & 57.5996 & 15.488 & 58.0981 & 15.268 \\
\hline 100.461 & 16.648 & 39.4102 & 14.264 & & & & & & & \\
\hline & 42.2275 & 13.332 & 34.1207 & 13.388 & & & 57.5996 & 15.492 & 58.0981 & 15.272 \\
\hline 99.2765 & 16.652 & 41.2577 & 14.268 & & & & & & & \\
\hline & 43.1143 & 13.336 & 37.6476 & 13.392 & & & 57.5996 & 15.496 & 58.0981 & 15.276 \\
\hline 99.2765 & 16.656 & 41.2577 & 14.272 & & & & & & & \\
\hline & 43.1143 & 13.34 & 36.7638 & 13.396 & & & 56.7168 & 15.5 & 58.0981 & 15.28 \\
\hline 99.2765 & 16.66 & 41.2577 & 14.276 & & & & & & & \\
\hline & 45.7824 & 13.344 & 35.8814 & 13.4 & & & 57.5996 & 15.504 & 58.0981 & 15.284 \\
\hline 99.2765 & 16.664 & 41.2577 & 14.28 & & & & & & & \\
\hline & 46.6744 & 13.348 & 36.7638 & 13.404 & & & 56.7168 & 15.508 & 58.0981 & 15.288 \\
\hline 99.8687 & 16.668 & 41.2577 & 14.284 & & & & & & & \\
\hline & 49.3581 & 13.352 & 37.6476 & 13.408 & & & 57.5996 & 15.512 & 58.0981 & 15.292 \\
\hline 99.8687 & 16.672 & 41.2577 & 14.288 & & & & & & & \\
\hline & 47.5676 & 13.356 & 37.6476 & 13.412 & & & 58.4837 & 15.516 & 58.0981 & 15.296 \\
\hline 99.2765 & 16.676 & 41.2577 & 14.292 & & & & & & & \\
\hline & 46.6744 & 13.36 & 37.6476 & 13.416 & & & 58.4837 & 15.52 & 59.0794 & 15.3 \\
\hline 99.2765 & 16.68 & 40.3332 & 14.296 & & & & & & & \\
\hline & 46.6744 & 13.364 & 37.6476 & 13.42 & & & 59.3688 & 15.524 & 57.1182 & 15.304 \\
\hline 99.2765 & 16.684 & 41.2577 & 14.3 & & & & & & & \\
\hline & 44.0023 & 13.368 & 37.6476 & 13.424 & & & 57.5996 & 15.528 & 58.0981 & 15.308 \\
\hline 99.2765 & 16.688 & 41.2577 & 14.304 & & & & & & & \\
\hline & 44.8917 & 13.372 & 38.5327 & 13.428 & & & 57.5996 & 15.532 & 58.0981 & 15.312 \\
\hline 99.2765 & 16.692 & 40.3332 & 14.308 & & & & & & & \\
\hline & 44.0023 & 13.376 & 37.6476 & 13.432 & & & 58.4837 & 15.536 & 59.0794 & 15.316 \\
\hline 98.6847 & 16.696 & 41.2577 & 14.312 & & & & & & & \\
\hline & 44.8917 & 13.38 & 36.7638 & 13.436 & & & 58.4837 & 15.54 & 58.0981 & 15.32 \\
\hline 99.2765 & 16.7 & 41.2577 & 14.316 & & & & & & & \\
\hline & 45.7824 & 13.384 & 36.7638 & 13.44 & & & 57.5996 & 15.544 & 58.0981 & 15.324 \\
\hline 99.2765 & 16.704 & 40.3332 & 14.32 & & & & & & & \\
\hline & 45.7824 & 13.388 & 37.6476 & 13.444 & & & 56.7168 & 15.548 & 58.0981 & 15.328 \\
\hline 99.2765 & 16.708 & 41.2577 & 14.324 & & & & & & & \\
\hline & 45.7824 & 13.392 & 37.6476 & 13.448 & & & 57.5996 & 15.552 & 58.0981 & 15.332 \\
\hline 98.0932 & 16.712 & 39.4102 & 14.328 & & & & & & & \\
\hline & 45.7824 & 13.396 & 35.0003 & 13.452 & & & 59.3688 & 15.556 & 58.0981 & 15.336 \\
\hline 98.6847 & 16.716 & 39.4102 & 14.332 & & & & & & & \\
\hline & 45.7824 & 13.4 & 37.6476 & 13.456 & & & 58.4837 & 15.56 & 58.0981 & 15.34 \\
\hline 99.2765 & 16.72 & 40.3332 & 14.336 & & & & & & & \\
\hline & 46.6744 & 13.404 & 35.8814 & 13.46 & & & 59.3688 & 15.564 & 58.0981 & 15.344 \\
\hline 98.0932 & 16.724 & 40.3332 & 14.34 & & & & & & & \\
\hline & 45.7824 & 13.408 & 36.7638 & 13.464 & & & 58.4837 & 15.568 & 58.0981 & 15.348 \\
\hline 98.0932 & 16.728 & 39.4102 & 14.344 & & & & & & & \\
\hline & 45.7824 & 13.412 & 35.8814 & 13.468 & & & 59.3688 & 15.572 & 58.0981 & 15.352 \\
\hline 98.0932 & 16.732 & 40.3332 & 14.348 & & & & & & & \\
\hline & 44.8917 & 13.416 & 35.0003 & 13.472 & & & 58.4837 & 15.576 & 58.0981 & 15.356 \\
\hline 96.9115 & 16.736 & 40.3332 & 14.352 & & & & & & & \\
\hline
\end{tabular}




\begin{tabular}{|c|c|c|c|c|}
\hline & 45.7824 & 13.42 & 36.7638 & 13.476 \\
\hline \multirow[t]{2}{*}{98.0932} & 16.74 & 40.3332 & 14.356 & \\
\hline & 45.7824 & 13.424 & 35.0003 & 13.48 \\
\hline \multirow[t]{2}{*}{97.5022} & 16.744 & 41.2577 & 14.36 & \\
\hline & 45.7824 & 13.428 & 35.8814 & 13.484 \\
\hline \multirow[t]{2}{*}{96.9115} & 16.748 & 41.2577 & 14.364 & \\
\hline & 47.5676 & 13.432 & 35.0003 & 3.488 \\
\hline \multirow[t]{2}{*}{98.6847} & 16.752 & 41.2577 & 14.368 & \\
\hline & 47.5676 & 13.436 & 33.2424 & 13.492 \\
\hline \multirow[t]{2}{*}{96.9115} & 16.756 & 41.2577 & 14.372 & \\
\hline & 48.4623 & 13.44 & 35.0003 & 3.496 \\
\hline \multirow[t]{2}{*}{96.9115} & 16.76 & 40.3332 & 14.376 & \\
\hline & 49.3581 & 13.444 & 33.2424 & 13.5 \\
\hline \multirow[t]{2}{*}{96.9115} & 16.764 & 41.2577 & 14.38 & \\
\hline & 48.4623 & 13.448 & 34.1207 & 13.504 \\
\hline \multirow[t]{2}{*}{95.142} & 16.768 & 40.3332 & 14.384 & \\
\hline & 48.4623 & 13.452 & 207 & 13.508 \\
\hline \multirow[t]{2}{*}{96.3213} & 16.772 & 41.2577 & 14.388 & \\
\hline & 48.4623 & 13.456 & 35.0003 & 13.512 \\
\hline \multirow[t]{2}{*}{95.7315} & 16.776 & 41.2577 & 14.392 & \\
\hline & & & 207 & 13.516 \\
\hline \multirow[t]{2}{*}{96.9115} & 16.78 & 40.3332 & 14.396 & \\
\hline & 49.3 & & 207 & 13.52 \\
\hline \multirow[t]{2}{*}{96.9115} & 16.784 & 39.4102 & 14. & \\
\hline & 47.5 & 13. & 207 & 13.524 \\
\hline 96.9115 & 16.7 & 40.3332 & & \\
\hline & 48.4 & 13.472 & 24 & 13.528 \\
\hline 96.9115 & 16.792 & & & \\
\hline & 49.3 & 13. & 207 & 13.532 \\
\hline 97.5022 & 16.796 & & & \\
\hline & 48.4 & 13 & 207 & 3.536 \\
\hline 95.142 & 16.8 & & & \\
\hline & 49.3 & 13.4 & 33.2424 & 13.54 \\
\hline 95.142 & & 40.3 & 14.42 & \\
\hline & 47.5 & 13.4 & 34.1207 & 13.544 \\
\hline 96.3213 & & $41 ?$ & 14.424 & \\
\hline & 46.6 & 13.492 & 33.2424 & 13.548 \\
\hline 42 & 16.812 & 40.3 & 14.428 & \\
\hline & & & & 13.552 \\
\hline 95.142 & 16.8 & 39.4102 & 14. & \\
\hline & & 13.5 & & 13.556 \\
\hline 95.142 & 16.82 & 39.4102 & 14. & \\
\hline & & & & 13.56 \\
\hline 96.3213 & 16.8 & 41.2 & 14.44 & \\
\hline & 48.4 & 13.508 & 33.2424 & 13.564 \\
\hline 4.553 & 16.828 & 40.3332 & 14.444 & \\
\hline & 46.6 & 13.512 & 31.4902 & 13.568 \\
\hline 94.553 & 16.832 & 40.3332 & 14.448 & \\
\hline & 47.5 & 13. & 33.2424 & 13.572 \\
\hline 93.9643 & 16.836 & 39.4102 & 14.452 & \\
\hline & 48.4623 & 13.5 & 32.3656 & 13.576 \\
\hline 95.142 & 16.84 & 40.3332 & 14.456 & \\
\hline & 46.6744 & 13.524 & 33.2424 & 13.58 \\
\hline 94.553 & & 39.4102 & & \\
\hline & 45.7824 & 13.528 & 31.4902 & 13.584 \\
\hline 94.553 & 16.848 & 38.4886 & & \\
\hline & 46.6744 & 13.532 & 30.6161 & 13.588 \\
\hline 93.376 & 16.852 & 38.4886 & 14.4 & \\
\hline & & & 31.4902 & 3.592 \\
\hline 94.553 & 16.856 & 39.4102 & 14.472 & \\
\hline & & & 31.4902 & 13.596 \\
\hline 94.553 & 16.86 & 40.3332 & 14.476 & \\
\hline & 46.6744 & & 32.3656 & 13.6 \\
\hline 94.553 & 16.864 & 40.3332 & 14.48 & \\
\hline & 48.4623 & & 33.2424 & 13.60 \\
\hline 93.9643 & 16.868 & 39.4102 & 14.484 & \\
\hline & 46.6744 & 13.552 & 34.1207 & 13.608 \\
\hline 94.553 & 16.872 & 40.3332 & 14.488 & \\
\hline & 46.6744 & 13.556 & 31.4902 & 13.612 \\
\hline 4.553 & 16.876 & 39.4102 & 14.492 & \\
\hline
\end{tabular}

\begin{tabular}{|c|c|c|c|}
\hline 58.4837 & 15.58 & 58.0981 & 15.36 \\
\hline 57.5996 & 15.584 & 57.1182 & 15.364 \\
\hline 58.4837 & 15.588 & 59.0794 & 15.368 \\
\hline 59.3688 & 15.592 & 58.0981 & 15.372 \\
\hline 59.3688 & 15.596 & 59.0794 & 15.376 \\
\hline 58.4837 & 15.6 & 58.0981 & 15.38 \\
\hline 59.3688 & 15.604 & 58.0981 & 15.384 \\
\hline 59.3688 & 15.608 & 58.0981 & 15.388 \\
\hline 59.3688 & 15.612 & 58.0981 & 15.392 \\
\hline 61.1426 & 15.616 & 58.0981 & 15.396 \\
\hline 57.5996 & 15.62 & 58.0981 & 15.4 \\
\hline 59.3688 & 15.624 & 58.0981 & 15.404 \\
\hline 59.3688 & 15.628 & 58.0981 & 15.408 \\
\hline 58.4837 & 15.632 & 57.1182 & 15.412 \\
\hline 59.3688 & 15.636 & 58.0981 & 15.416 \\
\hline 60.2552 & 15.64 & 57.1182 & 15.42 \\
\hline 58.4837 & 15.644 & 58.0981 & 15.424 \\
\hline 58.4837 & 15.648 & 58.0981 & 15.428 \\
\hline 60.2552 & 15.652 & 58.0981 & 15.432 \\
\hline 60.2552 & 15.656 & 58.0981 & 15.436 \\
\hline 60.2552 & 15.66 & 58.0981 & 15.44 \\
\hline 59.3688 & 15.664 & 58.0981 & 15.444 \\
\hline 59.3688 & 15.668 & 57.1182 & 15.448 \\
\hline 59.3688 & 15.672 & 58.0981 & 15.452 \\
\hline 61.1426 & 15.676 & 58.0981 & 15.456 \\
\hline 60.2552 & 15.68 & 58.0981 & 15.46 \\
\hline 60.2552 & 15.684 & 58.0981 & 15.464 \\
\hline 60.2552 & 15.688 & 58.0981 & 15.468 \\
\hline 61.1426 & 15.692 & 58.0981 & 15.472 \\
\hline 61.1426 & 15.696 & 58.0981 & 15.476 \\
\hline 62.0313 & 15.7 & 58.0981 & 15.48 \\
\hline 60.2552 & 15.704 & 58.0981 & 15.484 \\
\hline 60.2552 & 15.708 & 58.0981 & 15.488 \\
\hline 62.0313 & 15.712 & 57.1182 & 15.492 \\
\hline 61.1426 & 15.716 & 58.0981 & 15.496 \\
\hline
\end{tabular}




\begin{tabular}{|c|c|c|c|c|}
\hline & 48.4623 & 13.56 & 32.3656 & 13.616 \\
\hline \multirow[t]{2}{*}{93.9643} & 16.88 & 39.4102 & 14.496 & \\
\hline & 46.6744 & 13.564 & 32.3656 & 13.62 \\
\hline \multirow[t]{2}{*}{93.9643} & 16.884 & 40.3332 & 14.5 & \\
\hline & 47.5676 & 13.568 & 30.6161 & 13.624 \\
\hline \multirow[t]{2}{*}{93.9643} & 16.888 & 39.4102 & 14.504 & \\
\hline & 46.6744 & 13.572 & 31.4902 & 13.628 \\
\hline \multirow[t]{2}{*}{92.2007} & 16.892 & 40.3332 & 14.508 & \\
\hline & 46.6744 & 13.576 & 32.3656 & 13.632 \\
\hline \multirow[t]{2}{*}{93.9643} & 16.896 & 40.3332 & 14.512 & \\
\hline & 47.5676 & 13.58 & 33.2424 & 3.636 \\
\hline \multirow[t]{2}{*}{94.553} & 16.9 & 39.4102 & 14.516 & \\
\hline & 46.6744 & 13.584 & 31.4902 & 13.64 \\
\hline \multirow[t]{2}{*}{94.553} & 16.904 & 39.4102 & 14.52 & \\
\hline & 47.5 & 13.588 & 32.3656 & 13.644 \\
\hline \multirow[t]{2}{*}{93.9643} & 16.908 & 41.2577 & 14.524 & \\
\hline & 48.4 & & 33.2424 & 13.648 \\
\hline \multirow[t]{2}{*}{93.9643} & 16.912 & 40.3332 & 14.528 & \\
\hline & 46.6 & & 31.4902 & 13.652 \\
\hline \multirow[t]{2}{*}{93.376} & 16.916 & 40.3332 & 14.532 & \\
\hline & & & 33.2424 & 13.656 \\
\hline \multirow[t]{2}{*}{92.7882} & 16.92 & 39.4102 & 14.536 & \\
\hline & & & 02 & 13.66 \\
\hline \multirow[t]{2}{*}{92.7882} & 16.924 & 40.3332 & 14.54 & \\
\hline & & & 32.3 & 13.664 \\
\hline 93.376 & 16.928 & 40.3 & 14.544 & \\
\hline & 46. & & 33.2424 & 13.668 \\
\hline 93.376 & & & 14.548 & \\
\hline & 46. & & 902 & 13.672 \\
\hline 93.376 & & & & \\
\hline & 45. & 13 & 02 & 13.676 \\
\hline 92.2007 & & & & \\
\hline & 47.5 & & 32.3656 & 13.68 \\
\hline .2007 & & & 14.56 & \\
\hline & 46.6 & 13. & 33.2424 & 13.684 \\
\hline 93.376 & 16.948 & & 14.5 & \\
\hline & 46.6 & & 32.3656 & 13.688 \\
\hline 882 & 16.952 & 41 & 14.568 & \\
\hline & & & & 13.692 \\
\hline 9643 & 16 & 40. & 14 & \\
\hline & & & & 13.696 \\
\hline 92.2007 & 16.96 & 40. & 14.576 & \\
\hline & & & & 13.7 \\
\hline 92.7882 & 16.9 & 41. & 14.58 & \\
\hline & & 13. & & 13.704 \\
\hline 376 & 16.968 & 40.3 & 14.584 & \\
\hline & 45 & 13. & 56 & 13.708 \\
\hline 93.376 & 16.972 & 41.2 & 14.588 & \\
\hline & 4 & & 34.1207 & 13.712 \\
\hline 92.2007 & 16.976 & 40.3 & 14.592 & \\
\hline & 45.7 & 13. & 32.3656 & 13.716 \\
\hline 92.2007 & 16.98 & $40 . ?$ & 14.596 & \\
\hline & 45.7 & 13. & 33.2424 & 13.72 \\
\hline 92.7882 & 16.984 & 41.2577 & & \\
\hline & 44.8917 & 13. & 34.1207 & 13.724 \\
\hline 92.2007 & & 41.2577 & & \\
\hline & 47.5676 & & 34.1207 & 13.728 \\
\hline 92.7882 & & & & \\
\hline & & & 33.2424 & 3.73 \\
\hline 92.2007 & 16.996 & 41.2577 & 14.612 & \\
\hline & 45.7824 & & 34.1207 & 13.73 \\
\hline 93.376 & 17 & 41.2577 & 14.616 & \\
\hline & & & 32.3656 & 13.74 \\
\hline 92.2007 & 17.004 & 41.2577 & 14.62 & \\
\hline & & & 34.1207 & 13.74 \\
\hline 92.2007 & 17.008 & 42.1837 & 14.624 & \\
\hline & 44.8917 & 13.692 & 34.1207 & 13.74 \\
\hline 2001 & 17.012 & 42.1837 & 14.628 & \\
\hline & 44.8917 & 13.696 & 34.1207 & 13.752 \\
\hline .2007 & 17.016 & 41.2577 & 14.632 & \\
\hline
\end{tabular}

\begin{tabular}{|c|c|c|c|}
\hline 61.1426 & 15.72 & 58.0981 & 15.5 \\
\hline 61.1426 & 15.724 & 57.1182 & 15.504 \\
\hline 59.3688 & 15.728 & 58.0981 & 15.508 \\
\hline 62.0313 & 15.732 & 58.0981 & 15.512 \\
\hline 61.1426 & 15.736 & 57.1182 & 15.516 \\
\hline 61.1426 & 15.74 & 58.0981 & 15.52 \\
\hline 62.0313 & 15.744 & 58.0981 & 15.524 \\
\hline 62.0313 & 15.748 & 58.0981 & 15.528 \\
\hline 59.3688 & 15.752 & 57.1182 & 15.532 \\
\hline 62.0313 & 15.756 & 56.1398 & 15.536 \\
\hline 62.0313 & 15.76 & 57.1182 & 15.54 \\
\hline 58.4837 & 15.764 & 57.1182 & 15.544 \\
\hline 62.0313 & 15.768 & 58.0981 & 15.548 \\
\hline 62.0313 & 15.772 & 57.1182 & 15.552 \\
\hline 62.0313 & 15.776 & 57.1182 & 15.556 \\
\hline 61.1426 & 15.78 & 58.0981 & 15.56 \\
\hline 61.1426 & 15.784 & 58.0981 & 15.564 \\
\hline 60.2552 & 15.788 & 57.1182 & 15.568 \\
\hline 61.1426 & 15.792 & 58.0981 & 15.572 \\
\hline 62.0313 & 15.796 & 58.0981 & 15.576 \\
\hline 62.9211 & 15.8 & 58.0981 & 15.58 \\
\hline 62.9211 & 15.804 & 57.1182 & 15.584 \\
\hline 62.0313 & 15.808 & 55.1628 & 15.588 \\
\hline 61.1426 & 15.812 & 56.1398 & 15.592 \\
\hline 62.0313 & 15.816 & 58.0981 & 15.596 \\
\hline 62.0313 & 15.82 & 58.0981 & 15.6 \\
\hline 62.0313 & 15.824 & 58.0981 & 15.604 \\
\hline 62.0313 & 15.828 & 58.0981 & 15.608 \\
\hline 62.0313 & 15.832 & 57.1182 & 15.612 \\
\hline 62.0313 & 15.836 & 58.0981 & 15.616 \\
\hline 62.9211 & 15.84 & 57.1182 & 15.62 \\
\hline 62.0313 & 15.844 & 58.0981 & 15.624 \\
\hline 62.0313 & 15.848 & 58.0981 & 15.628 \\
\hline 64.7042 & 15.852 & 57.1182 & 15.632 \\
\hline 60.2552 & 15.856 & 57.1182 & 15.636 \\
\hline
\end{tabular}




\begin{tabular}{|c|c|c|c|c|}
\hline & 45.7824 & 13.7 & 33.2424 & 13.756 \\
\hline \multirow[t]{2}{*}{92.7882} & 17.02 & 41.2577 & 14.636 & \\
\hline & 44.0023 & 13.704 & 34.1207 & 13.76 \\
\hline \multirow[t]{2}{*}{92.2007} & 17.024 & 42.1837 & 14.64 & \\
\hline & 44.8917 & 13.708 & 34.1207 & 13.764 \\
\hline \multirow[t]{2}{*}{92.2007} & 17.028 & 40.3332 & 14.644 & \\
\hline & 46.6744 & 13.712 & 34.1207 & 13.768 \\
\hline \multirow[t]{2}{*}{92.2007} & 17.032 & 42.1837 & 14.648 & \\
\hline & 45.7824 & 13.716 & 33.2424 & 13.772 \\
\hline \multirow[t]{2}{*}{92.2007} & 17.036 & 41.2577 & 14.652 & \\
\hline & 48.4623 & 13.72 & 33.2424 & 3.776 \\
\hline \multirow[t]{2}{*}{92.2007} & 17.04 & 43.1111 & 14.656 & \\
\hline & 47.5676 & 13.724 & 33.2424 & 13.78 \\
\hline \multirow[t]{2}{*}{92.2007} & 17.044 & 41.2577 & 14.66 & \\
\hline & 48.4623 & 13.728 & 32.3656 & 13.784 \\
\hline \multirow[t]{2}{*}{91.6137} & 17.048 & 40.3332 & 14.664 & \\
\hline & 47.5 & 13.732 & 33.2424 & 13.788 \\
\hline \multirow[t]{2}{*}{92.2007} & 17.052 & 42.1837 & 14.668 & \\
\hline & 47.5676 & 3.736 & 34.1207 & 13.792 \\
\hline \multirow[t]{2}{*}{91.6137} & 17.056 & 41.2577 & 14.672 & \\
\hline & & & 656 & 13.796 \\
\hline \multirow[t]{2}{*}{92.2007} & 17.06 & 42.1837 & 14.676 & \\
\hline & 45 ? & & 207 & 13.8 \\
\hline \multirow[t]{2}{*}{92.2007} & 17.064 & 42.1837 & 14.68 & \\
\hline & 45. & 13.748 & 34.1207 & 13.804 \\
\hline 92.2007 & 17.0 & 43.1111 & 14.684 & \\
\hline & 45.7 & 13.752 & 24 & 13.808 \\
\hline 92.2007 & 17.072 & 43.1111 & 14.6 & \\
\hline & 45.7 & 6 & 556 & 13.812 \\
\hline 92.2007 & 17.076 & & & \\
\hline & 46. & 13.76 & 33. & 13.816 \\
\hline 92.2007 & & & & \\
\hline & 47.5676 & 13.764 & 33.2424 & 13.82 \\
\hline 91.6137 & 17.0 & 304 & 14.7 & \\
\hline & 48.4623 & 13.768 & 32.3656 & 13.824 \\
\hline 6137 & 17.088 & 41.2577 & 14.704 & \\
\hline & 48.4623 & 13.772 & 33.2424 & 13.828 \\
\hline 90.4408 & 17.092 & 43.1 & 14.708 & \\
\hline & & & & 13.832 \\
\hline 92.2007 & 17.096 & 43.1111 & 14.712 & \\
\hline & & & & 13.8 \\
\hline 91.0271 & 17.1 & 41.2577 & 14.716 & \\
\hline & & & & 13.84 \\
\hline 91.6137 & 17.1 & 43.1111 & 14.72 & \\
\hline & 46.6 & 13.7 & 33.2424 & 13.844 \\
\hline 91.6137 & 17.108 & 42.1837 & 14.724 & \\
\hline & 48.4 & 13.792 & 33.2424 & 13.848 \\
\hline 91.6137 & 17.112 & 42.1837 & 14.728 & \\
\hline & 46. & 13.7 & 34.1207 & 13.852 \\
\hline 92.2007 & 17.116 & 43.1111 & 14.732 & \\
\hline & 45.7 & 13.8 & 33.2424 & 13.856 \\
\hline 91.6137 & & 43.11 & 14.736 & \\
\hline & 45.7 & 13.8 & 32.3656 & 13.86 \\
\hline 91.0271 & & & & \\
\hline & 47.5676 & 13.8 & 32.3656 & 13.86 \\
\hline 90.4408 & & 43.1111 & & \\
\hline & 47.5676 & & 33.2424 & 13.868 \\
\hline 89.855 & & & & \\
\hline & 46.6744 & & 34.1207 & 3.8 \\
\hline 89.2695 & 17.136 & 43.1111 & 14.752 & \\
\hline & 49.3581 & & 34.1207 & 13.8 \\
\hline 89.855 & 17.14 & 43.1111 & 14.756 & \\
\hline & 46.6744 & & 34.1207 & 13.8 \\
\hline 91.0271 & 17.144 & 43.1111 & 14.76 & \\
\hline & 47.5676 & & 33.2424 & 13.8 \\
\hline 91.0271 & 17.148 & 42.1837 & 14.764 & \\
\hline & 46.6744 & 13.8 & 33.2424 & 13.88 \\
\hline 89.855 & 17.152 & 43.1111 & 14.768 & \\
\hline & 48.4623 & 13.836 & 33.2424 & 13.8 \\
\hline 5 & 17.156 & 43.1111 & 14.772 & \\
\hline
\end{tabular}

\begin{tabular}{|c|c|c|c|}
\hline 62.9211 & 15.86 & 58.0981 & 15.64 \\
\hline 60.2552 & 15.864 & 56.1398 & 15.644 \\
\hline 62.0313 & 15.868 & 57.1182 & 15.648 \\
\hline 62.9211 & 15.872 & 57.1182 & 15.652 \\
\hline 63.8121 & 15.876 & 56.1398 & 15.656 \\
\hline 62.0313 & 15.88 & 58.0981 & 15.66 \\
\hline 62.0313 & 15.884 & 58.0981 & 15.664 \\
\hline 62.9211 & 15.888 & 57.1182 & 15.668 \\
\hline 63.8121 & 15.892 & 58.0981 & 15.672 \\
\hline 62.9211 & 15.896 & 58.0981 & 15.676 \\
\hline 62.9211 & 15.9 & 58.0981 & 15.68 \\
\hline 65.5975 & 15.904 & 58.0981 & 15.684 \\
\hline 63.8121 & 15.908 & 58.0981 & 15.688 \\
\hline 63.8121 & 15.912 & 59.0794 & 15.692 \\
\hline 62.9211 & 15.916 & 58.0981 & 15.696 \\
\hline 64.7042 & 15.92 & 57.1182 & 15.7 \\
\hline 64.7042 & 15.924 & 58.0981 & 15.704 \\
\hline 63.8121 & 15.928 & 58.0981 & 15.708 \\
\hline 63.8121 & 15.932 & 58.0981 & 15.712 \\
\hline 62.9211 & 15.936 & 58.0981 & 15.716 \\
\hline 63.8121 & 15.94 & 58.0981 & 15.72 \\
\hline 62.0313 & 15.944 & 58.0981 & 15.724 \\
\hline 62.9211 & 15.948 & 58.0981 & 15.728 \\
\hline 62.0313 & 15.952 & 58.0981 & 15.732 \\
\hline 62.9211 & 15.956 & 58.0981 & 15.736 \\
\hline 62.9211 & 15.96 & 58.0981 & 15.74 \\
\hline 62.9211 & 15.964 & 58.0981 & 15.744 \\
\hline 64.7042 & 15.968 & 58.0981 & 15.748 \\
\hline 63.8121 & 15.972 & 58.0981 & 15.752 \\
\hline 64.7042 & 15.976 & 58.0981 & 15.756 \\
\hline 62.9211 & 15.98 & 58.0981 & 15.76 \\
\hline 63.8121 & 15.984 & 59.0794 & 15.764 \\
\hline 63.8121 & 15.988 & 58.0981 & 15.768 \\
\hline 63.8121 & 15.992 & 58.0981 & 15.772 \\
\hline 63.8121 & 15.996 & 59.0794 & 15.776 \\
\hline
\end{tabular}




\begin{tabular}{|c|c|c|c|c|}
\hline & 48.4623 & 13.84 & 33.2424 & 13.896 \\
\hline \multirow[t]{2}{*}{89.855} & 17.16 & 43.1111 & 14.776 & \\
\hline & 49.3581 & 13.844 & 32.3656 & 13.9 \\
\hline \multirow[t]{2}{*}{88.0999} & 17.164 & 43.1111 & 14.78 & \\
\hline & 49.3581 & 13.848 & 32.3656 & 13.904 \\
\hline \multirow[t]{2}{*}{88.6845} & 17.168 & 43.1111 & 14.784 & \\
\hline & 49.3581 & 13.852 & 32.3656 & 3.908 \\
\hline \multirow[t]{2}{*}{87.5158} & 17.172 & 44.0399 & 14.788 & \\
\hline & 48.4623 & 13.8 & 33.2424 & 13.912 \\
\hline \multirow[t]{2}{*}{87.5158} & 17.176 & 43.1111 & 14.792 & \\
\hline & 49.3581 & 13.86 & 32.3656 & 13.916 \\
\hline \multirow[t]{2}{*}{87.5158} & 17.18 & 43.1111 & 14.796 & \\
\hline & 49.3581 & 4 & 32.3656 & 13.92 \\
\hline \multirow[t]{2}{*}{88.0999} & 17.184 & 43.1111 & 14.8 & \\
\hline & 49.3581 & & 31.4902 & 13.924 \\
\hline \multirow[t]{2}{*}{87.5158} & 17.188 & 44.0 & 14.804 & \\
\hline & 49.3 & & 34. & 13.928 \\
\hline \multirow[t]{2}{*}{87.5158} & 17.192 & 43. & 14.808 & \\
\hline & & & 24 & 13.932 \\
\hline \multirow[t]{2}{*}{88.0999} & 17.196 & 43. & 14.812 & \\
\hline & 49.3 & & 207 & .936 \\
\hline \multirow[t]{2}{*}{87.5158} & 17.2 & & & \\
\hline & 48.46 & & 656 & 13.94 \\
\hline \multirow[t]{2}{*}{87.5158} & 17.204 & & & \\
\hline & 48.4623 & & 33.2424 & 13.944 \\
\hline 87.5158 & 17.208 & 43. & 14.824 & \\
\hline & 49.3581 & & 34.1207 & 13.948 \\
\hline 86.932 & 17.212 & & 14.828 & \\
\hline & 49.3581 & & 34.1207 & 13.952 \\
\hline 86.932 & 17.216 & & & \\
\hline & 49.3581 & 13 & 207 & 13.956 \\
\hline 8 & 17.22 & & 14. & \\
\hline & 49.3581 & & 207 & 13.96 \\
\hline 932 & 17.224 & & 14 & \\
\hline & & & & 13.964 \\
\hline 375158 & 17.228 & & 14 & \\
\hline & & & & 3.968 \\
\hline 86.932 & 17.232 & 2 & 14 & \\
\hline & 48.4623 & & 207 & 13.972 \\
\hline 86.932 & 17.236 & 43. & 14 & \\
\hline & 48.4623 & & 35.0003 & 13.976 \\
\hline 86.932 & 17.24 & 02 & 14.856 & \\
\hline & 49.3 & & 814 & 13.98 \\
\hline 87.5158 & 17.244 & 99 & 14.86 & \\
\hline & 48.4623 & & 003 & 13.984 \\
\hline 86.932 & 17.248 & 44. & & \\
\hline & 49.3581 & & 207 & 13.988 \\
\hline 85.1832 & & & & \\
\hline & 50.2553 & & 34.1207 & 13.992 \\
\hline 85.7657 & 17.256 & & & \\
\hline & 48.4623 & & 207 & .996 \\
\hline 86.932 & 17.26 & & & \\
\hline & 49.3581 & & 207 & 14 \\
\hline 85.7657 & 17.264 & & & \\
\hline & 49.3581 & & 35.0003 & 14.004 \\
\hline 85.7657 & 17.268 & 02 & 14.884 & \\
\hline & 50.2553 & & 35.8814 & 14.008 \\
\hline 86.3486 & 17.272 & 43. & 14.888 & \\
\hline & 49.3581 & & 34.1207 & 14.012 \\
\hline 86.932 & 17.276 & 44. & 14.892 & \\
\hline & 49.3581 & & 34.1207 & 14.016 \\
\hline 86.3486 & 17.28 & 46.8351 & 14.896 & \\
\hline & 49.3581 & 13.964 & 35.0003 & 14.02 \\
\hline 85.7657 & 17.284 & 44.0399 & 14.9 & \\
\hline & 49.3581 & & & 14.024 \\
\hline 1832 & 17.288 & & & \\
\hline & 49.3581 & 13. & 35.0003 & 4.028 \\
\hline 1832 & 17.292 & 46.8351 & 14.908 & \\
\hline & 49.3581 & 13.976 & 35.0003 & 14.03 \\
\hline .1832 & 17.296 & 46.8351 & 14.912 & \\
\hline
\end{tabular}

\begin{tabular}{|c|c|c|c|}
\hline 64.7042 & 16 & 58.0981 & 15.78 \\
\hline 62.0313 & 16.004 & 58.0981 & 15.784 \\
\hline 62.9211 & 16.008 & 58.0981 & 15.788 \\
\hline 60.2552 & 16.012 & 58.0981 & 15.792 \\
\hline 60.2552 & 16.016 & 58.0981 & 15.796 \\
\hline 59.3688 & 16.02 & 58.0981 & 15.8 \\
\hline 62.0313 & 16.024 & 58.0981 & 15.804 \\
\hline 63.8121 & 16.028 & 59.0794 & 15.808 \\
\hline 65.5975 & 16.032 & 58.0981 & 15.812 \\
\hline 62.9211 & 16.036 & 58.0981 & 15.816 \\
\hline 62.9211 & 16.04 & 58.0981 & 15.82 \\
\hline 62.0313 & 16.044 & 58.0981 & 15.824 \\
\hline 60.2552 & 16.048 & 59.0794 & 15.828 \\
\hline 60.2552 & 16.052 & 59.0794 & 15.832 \\
\hline 60.2552 & 16.056 & 59.0794 & 15.836 \\
\hline 62.0313 & 16.06 & 58.0981 & 15.84 \\
\hline 60.2552 & 16.064 & 58.0981 & 15.844 \\
\hline 59.3688 & 16.068 & 58.0981 & 15.848 \\
\hline 59.3688 & 16.072 & 58.0981 & 15.852 \\
\hline 62.0313 & 16.076 & 58.0981 & 15.856 \\
\hline 62.0313 & 16.08 & 58.0981 & 15.86 \\
\hline 60.2552 & 16.084 & 58.0981 & 15.864 \\
\hline 59.3688 & 16.088 & 59.0794 & 15.868 \\
\hline 57.5996 & 16.092 & 58.0981 & 15.872 \\
\hline 60.2552 & 16.096 & 58.0981 & 15.876 \\
\hline 60.2552 & 16.1 & 59.0794 & 15.88 \\
\hline 60.2552 & 16.104 & 58.0981 & 15.884 \\
\hline 61.1426 & 16.108 & 58.0981 & 15.888 \\
\hline 61.1426 & 16.112 & 59.0794 & 15.892 \\
\hline 62.0313 & 16.116 & 58.0981 & 15.896 \\
\hline 61.1426 & 16.12 & 59.0794 & 15.9 \\
\hline 60.2552 & 16.124 & 58.0981 & 15.904 \\
\hline 60.2552 & 16.128 & 58.0981 & 15.908 \\
\hline 62.0313 & 16.132 & 58.0981 & 15.912 \\
\hline 61.1426 & 16.136 & 59.0794 & 15.916 \\
\hline
\end{tabular}




\begin{tabular}{|c|c|c|c|c|}
\hline & 50.2553 & 13.98 & 34.1207 & 14.036 \\
\hline \multirow[t]{2}{*}{85.1832} & 17.3 & 45.9019 & 14.916 & \\
\hline & 50.2553 & 13.984 & 35.8814 & 14.04 \\
\hline \multirow[t]{2}{*}{85.7657} & 17.304 & 44.0399 & 14.92 & \\
\hline & 50.2553 & 13.988 & 35.8814 & 14.044 \\
\hline \multirow[t]{2}{*}{86.3486} & 17.308 & 44.9702 & 14.924 & \\
\hline & 50.2553 & 13.992 & 37.6476 & 14.048 \\
\hline \multirow[t]{2}{*}{85.1832} & 17.312 & 45.9019 & 14.928 & \\
\hline & 50.2553 & 13.996 & 35.8814 & 14.052 \\
\hline \multirow[t]{2}{*}{85.1832} & 17.316 & 45.9019 & 14.932 & \\
\hline & 48.4623 & 14 & 36.7638 & 14.056 \\
\hline \multirow[t]{2}{*}{85.7657} & 17.32 & 46.8351 & 14.936 & \\
\hline & 49.3581 & 14.004 & 36.7638 & 14.06 \\
\hline \multirow[t]{2}{*}{85.1832} & 17.324 & 46.8351 & 14.94 & \\
\hline & 50.2553 & 14.008 & 37.6476 & 14.064 \\
\hline \multirow[t]{2}{*}{84.6011} & 17.328 & 45.9019 & 14.944 & \\
\hline & 49.3581 & 14.012 & 36.7638 & 14.068 \\
\hline \multirow[t]{2}{*}{85.1832} & 17.332 & 44.9702 & 14.948 & \\
\hline & 50.2553 & 14.016 & 36.7638 & 14.072 \\
\hline \multirow[t]{2}{*}{86.3486} & 17.336 & 45.9019 & 14.952 & \\
\hline & 50.2553 & 14.02 & 37.6476 & 14.076 \\
\hline \multirow{2}{*}{85.1832} & 17.34 & 46.8351 & 14.956 & \\
\hline & 49.3581 & 14.024 & 36.7638 & 14.08 \\
\hline \multirow[t]{2}{*}{86.3486} & 17.344 & 46.8351 & 14.96 & \\
\hline & 50.2553 & 14.028 & 36.7638 & 14.084 \\
\hline 86.3486 & 17.348 & 46.8351 & 14.964 & \\
\hline & 51.1537 & 14.032 & 36.7638 & 14.088 \\
\hline 86.932 & 17.352 & 46.8351 & 14.968 & \\
\hline & 50.2553 & 14.036 & 36.7638 & 14.092 \\
\hline 86.932 & 17.356 & 46.8351 & 14.972 & \\
\hline & 50.2553 & 14.04 & 37.6476 & 14.096 \\
\hline 85.7657 & 17.36 & 45.9019 & 14.976 & \\
\hline & 52.0535 & 14.044 & 37.6476 & 14.1 \\
\hline 85.7657 & 17.364 & 47.7697 & 14.98 & \\
\hline & 52.9545 & 14.048 & 37.6476 & 14.104 \\
\hline 85.7657 & 17.368 & 47.7697 & 14.984 & \\
\hline & 52.0535 & 14.052 & 37.6476 & 14.108 \\
\hline 85.7657 & 17.372 & 47.7697 & 14.988 & \\
\hline & 50.2553 & 14.056 & 37.6476 & 14.112 \\
\hline 86.3486 & 17.376 & 46.8351 & 14.992 & \\
\hline & 52.0535 & 14.06 & 39.4192 & 14.116 \\
\hline 87.5158 & 17.38 & 47.7697 & 14.996 & \\
\hline & 51.1537 & 14.064 & 38.5327 & 14.12 \\
\hline 87.5158 & 17.384 & 49.6431 & 15 & \\
\hline & 52.9545 & 14.068 & 37.6476 & 14.124 \\
\hline 87.5158 & 17.388 & 46.8351 & 15.004 & \\
\hline & 52.9545 & 14.072 & 35.8814 & 14.128 \\
\hline 87.5158 & 17.392 & 47.7697 & 15.008 & \\
\hline & 52.9545 & 14.076 & 37.6476 & 14.132 \\
\hline 86.932 & 17.396 & 47.7697 & 15.012 & \\
\hline & 51.1537 & 14.08 & 37.6476 & 14.136 \\
\hline 86.3486 & 17.4 & 47.7697 & 15.016 & \\
\hline & 52.0535 & 14.084 & 39.4192 & 14.14 \\
\hline 85.1832 & 17.404 & 48.7056 & 15.02 & \\
\hline & 52.0535 & 14.088 & 38.5327 & 14.144 \\
\hline 85.7657 & 17.408 & 47.7697 & 15.024 & \\
\hline & 52.0535 & 14.092 & 38.5327 & 14.148 \\
\hline 85.1832 & 17.412 & 47.7697 & 15.028 & \\
\hline & 52.9545 & 14.096 & 38.5327 & 14.152 \\
\hline 87.5158 & 17.416 & 50.5819 & 15.032 & \\
\hline & 51.1537 & 14.1 & 37.6476 & 14.156 \\
\hline 87.5158 & 17.42 & 49.6431 & 15.036 & \\
\hline & 51.1537 & 14.104 & 36.7638 & 14.16 \\
\hline 88.0999 & 17.424 & 47.7697 & 15.04 & \\
\hline & 52.9545 & 14.108 & 37.6476 & 14.164 \\
\hline 86.932 & 17.428 & 46.8351 & 15.044 & \\
\hline & 52.9545 & 14.112 & 37.6476 & 14.168 \\
\hline 86.3486 & 17.432 & 49.6431 & 15.048 & \\
\hline & 52.9545 & 14.116 & 38.5327 & 14.172 \\
\hline 86.3486 & 17.436 & 49.6431 & 15.052 & \\
\hline
\end{tabular}

\begin{tabular}{|c|c|c|c|}
\hline 62.9211 & 16.14 & 58.0981 & 15.92 \\
\hline 65.5975 & 16.144 & 59.0794 & 15.924 \\
\hline 64.7042 & 16.148 & 60.0621 & 15.928 \\
\hline 62.9211 & 16.152 & 58.0981 & 15.932 \\
\hline 61.1426 & 16.156 & 58.0981 & 15.936 \\
\hline 62.0313 & 16.16 & 58.0981 & 15.94 \\
\hline 62.9211 & 16.164 & 59.0794 & 15.944 \\
\hline 62.0313 & 16.168 & 57.1182 & 15.948 \\
\hline 62.0313 & 16.172 & 59.0794 & 15.952 \\
\hline 65.5975 & 16.176 & 58.0981 & 15.956 \\
\hline 66.4919 & 16.18 & 58.0981 & 15.96 \\
\hline 66.4919 & 16.184 & 59.0794 & 15.964 \\
\hline 64.7042 & 16.188 & 60.0621 & 15.968 \\
\hline 65.5975 & 16.192 & 59.0794 & 15.972 \\
\hline 63.8121 & 16.196 & 59.0794 & 15.976 \\
\hline 62.0313 & 16.2 & 58.0981 & 15.98 \\
\hline 60.2552 & 16.204 & 59.0794 & 15.984 \\
\hline 63.8121 & 16.208 & 58.0981 & 15.988 \\
\hline 64.7042 & 16.212 & 59.0794 & 15.992 \\
\hline 64.7042 & 16.216 & 58.0981 & 15.996 \\
\hline 64.7042 & 16.22 & 59.0794 & 16 \\
\hline 62.9211 & 16.224 & 58.0981 & 16.004 \\
\hline 67.3874 & 16.228 & 58.0981 & 16.008 \\
\hline 64.7042 & 16.232 & 58.0981 & 16.012 \\
\hline 64.7042 & 16.236 & 58.0981 & 16.016 \\
\hline 65.5975 & 16.24 & 57.1182 & 16.02 \\
\hline 65.5975 & 16.244 & 58.0981 & 16.024 \\
\hline 63.8121 & 16.248 & 61.0463 & 16.028 \\
\hline 63.8121 & 16.252 & 62.0319 & 16.032 \\
\hline 66.4919 & 16.256 & 62.0319 & 16.036 \\
\hline 64.7042 & 16.26 & 62.0319 & 16.04 \\
\hline 64.7042 & 16.264 & 59.0794 & 16.044 \\
\hline 65.5975 & 16.268 & 59.0794 & 16.048 \\
\hline 66.4919 & 16.272 & 58.0981 & 16.052 \\
\hline 65.5975 & 16.276 & 58.0981 & 16.056 \\
\hline
\end{tabular}




\begin{tabular}{|c|c|c|c|c|}
\hline & 2.9545 & 14.12 & 39.4192 & 14.176 \\
\hline \multirow[t]{2}{*}{86.3486} & 17.44 & 47.7697 & 15.056 & \\
\hline & 52.9545 & 14.124 & 39.4192 & 14.18 \\
\hline \multirow[t]{2}{*}{86.3486} & 17.444 & 47.7697 & 15.06 & \\
\hline & 52.9545 & 14.128 & 39.4192 & 14.184 \\
\hline \multirow[t]{2}{*}{86.932} & 17.448 & 48.7056 & 15.064 & \\
\hline & 53.8568 & 14.132 & 38.5327 & 14.188 \\
\hline \multirow[t]{2}{*}{86.3486} & 17.452 & 49.6431 & 15.068 & \\
\hline & 53.8568 & 14.136 & 39.4192 & 14.192 \\
\hline \multirow[t]{2}{*}{85.7657} & 17.456 & 49.6431 & 15.072 & \\
\hline & 53.8568 & 14.14 & 40.3071 & 14.196 \\
\hline \multirow[t]{2}{*}{86.932} & 17.46 & 48.7056 & 15.076 & \\
\hline & 52.9545 & 14.144 & 39.4192 & 14.2 \\
\hline \multirow[t]{2}{*}{86.3486} & 17.464 & 48.7056 & 15.08 & \\
\hline & 53.8568 & & 327 & 14.204 \\
\hline \multirow[t]{2}{*}{85.7657} & 17.468 & 47.7697 & 15.0 & \\
\hline & 52.9 & & & 14.208 \\
\hline \multirow[t]{2}{*}{85.7657} & 17.472 & 49.6431 & 15.0 & \\
\hline & 52.9 & & & 14.212 \\
\hline \multirow[t]{2}{*}{85.1832} & 17.476 & 50.5819 & 15.0 & \\
\hline & 52.9 & & 76 & 14.216 \\
\hline \multirow[t]{2}{*}{85.1832} & 17.48 & 49.6431 & 15.0 & \\
\hline & 53.8 & & 76 & 14.22 \\
\hline \multirow[t]{2}{*}{84.6011} & 17.484 & 49.6431 & 15.1 & \\
\hline & 55.6 & & 39 & 14.224 \\
\hline 85.7657 & 17.4 & 056 & & \\
\hline & 52.9 & & 92 & 14.228 \\
\hline 85.7657 & 17.492 & & & \\
\hline & 53.8 & & 27 & 14.232 \\
\hline 85.7657 & 17.4 & & & \\
\hline & 54.7 & & 27 & 14.236 \\
\hline 85.1832 & 17.5 & & & \\
\hline & 53.85 & & 071 & 14.24 \\
\hline 11 & 17.5 & & & \\
\hline & 54.7 & & 76 & 14.244 \\
\hline 332 & 17.508 & & & \\
\hline & 54.7 & & 92 & 14.248 \\
\hline 011 & 17.512 & 56 & & \\
\hline & & & & 14.252 \\
\hline 85.1832 & 17.5 & 7056 & & \\
\hline & 55.6 & & & 14.256 \\
\hline 84.0194 & 17.52 & 49. & & \\
\hline & & & & 14.26 \\
\hline 84.6011 & 17.5 & 19 & 15. & \\
\hline & 56.5 & 8 & 39 & 14.264 \\
\hline 83.4381 & 17.528 & 50.5819 & 15.1 & \\
\hline & 56.5 & & 92 & 14.268 \\
\hline 84.6011 & 17.532 & 056 & 15.1 & \\
\hline & 55. & & 71 & 14.272 \\
\hline 85.1832 & 17.536 & 47.7697 & 15.1 & \\
\hline & 55.6 & 14 & 27 & 14.276 \\
\hline 84.0194 & & 48.7056 & & \\
\hline & 55.6 & & 27 & 14.28 \\
\hline 83.4381 & & & & \\
\hline & 53.8 & & 38.5327 & 4.284 \\
\hline 83.4381 & & & & \\
\hline & 56.5711 & 14.232 & 40. & 4.288 \\
\hline 82.8573 & & 49.6431 & & \\
\hline & 55.6651 & & 38.5 & 4.29 \\
\hline 82.8573 & 17.556 & 50.5819 & 15.1 & \\
\hline & 56.5711 & & 38.5327 & 14.2 \\
\hline 82.8573 & 17.56 & 49.6431 & 15.176 & \\
\hline & 56.5711 & & 38.5327 & 14.3 \\
\hline 82.8573 & 17.564 & 50.5819 & 15.18 & \\
\hline & 55.6651 & & 476 & 14.3 \\
\hline 82.8573 & 17.568 & 50.5819 & 15.184 & \\
\hline & 56.5711 & & 38.5327 & 14.308 \\
\hline $82.85 / 3$ & 17.572 & 49.6431 & 15.188 & \\
\hline & 55.6651 & 14.256 & 39.4192 & 14.312 \\
\hline 2.8573 & 17.576 & 50.5819 & 15.192 & \\
\hline
\end{tabular}

\begin{tabular}{|c|c|c|c|}
\hline 64.7042 & 16.28 & 59.0794 & 16.06 \\
\hline 64.7042 & 16.284 & 59.0794 & 16.064 \\
\hline 65.5975 & 16.288 & 62.0319 & 16.068 \\
\hline 65.5975 & 16.292 & 62.0319 & 16.072 \\
\hline 66.4919 & 16.296 & 62.0319 & 16.076 \\
\hline 66.4919 & 16.3 & 59.0794 & 16.08 \\
\hline 67.3874 & 16.304 & 56.1398 & 16.084 \\
\hline 66.4919 & 16.308 & 58.0981 & 16.088 \\
\hline 63.8121 & 16.312 & 59.0794 & 16.092 \\
\hline 65.5975 & 16.316 & 59.0794 & 16.096 \\
\hline 64.7042 & 16.32 & 59.0794 & 16.1 \\
\hline 65.5975 & 16.324 & 61.0463 & 16.104 \\
\hline 66.4919 & 16.328 & 62.0319 & 16.108 \\
\hline 66.4919 & 16.332 & 61.0463 & 16.112 \\
\hline 68.284 & 16.336 & 58.0981 & 16.116 \\
\hline 67.3874 & 16.34 & 58.0981 & 16.12 \\
\hline 67.3874 & 16.344 & 58.0981 & 16.124 \\
\hline 66.4919 & 16.348 & 58.0981 & 16.128 \\
\hline 66.4919 & 16.352 & 58.0981 & 16.132 \\
\hline 67.3874 & 16.356 & 59.0794 & 16.136 \\
\hline 65.5975 & 16.36 & 64.0074 & 16.14 \\
\hline 62.9211 & 16.364 & 62.0319 & 16.144 \\
\hline 65.5975 & 16.368 & 62.0319 & 16.148 \\
\hline 65.5975 & 16.372 & 61.0463 & 16.152 \\
\hline 65.5975 & 16.376 & 58.0981 & 16.156 \\
\hline 65.5975 & 16.38 & 58.0981 & 16.16 \\
\hline 67.3874 & 16.384 & 58.0981 & 16.164 \\
\hline 68.284 & 16.388 & 58.0981 & 16.168 \\
\hline 65.5975 & 16.392 & 59.0794 & 16.172 \\
\hline 65.5975 & 16.396 & 62.0319 & 16.176 \\
\hline 65.5975 & 16.4 & 62.0319 & 16.18 \\
\hline 66.4919 & 16.404 & 64.0074 & 16.184 \\
\hline 65.5975 & 16.408 & 62.0319 & 16.188 \\
\hline 65.5975 & 16.412 & 61.0463 & 16.192 \\
\hline 66.4919 & 16.416 & 58.0981 & 16.196 \\
\hline
\end{tabular}




\begin{tabular}{|c|c|c|c|c|}
\hline & 5.6651 & 14.26 & 37.6476 & 14.316 \\
\hline \multirow[t]{2}{*}{82.2769} & 17.58 & 48.7056 & 15.196 & \\
\hline & 55.6651 & 14.264 & 38.5327 & 14.32 \\
\hline \multirow[t]{2}{*}{81.6969} & 17.584 & 48.7056 & 15.2 & \\
\hline & 56.5711 & 14.268 & 37.6476 & 14.324 \\
\hline \multirow[t]{2}{*}{81.6969} & 17.588 & 48.7056 & 15.204 & \\
\hline & 56.5711 & 14.272 & 38.5327 & 14.328 \\
\hline \multirow[t]{2}{*}{81.1173} & 17.592 & 49.6431 & 15.208 & \\
\hline & 56.5711 & 14.276 & 37.6476 & 14.332 \\
\hline \multirow[t]{2}{*}{80.5382} & 17.596 & 49.6431 & 15.212 & \\
\hline & 55.6651 & 14.28 & 38.5327 & 14.336 \\
\hline \multirow[t]{2}{*}{81.6969} & 17.6 & 49.6431 & 15.216 & \\
\hline & 56.57 & 14.284 & 39.4192 & 14.34 \\
\hline \multirow[t]{2}{*}{81.1173} & 17.604 & 49.6431 & 15.22 & \\
\hline & 55.6651 & 14.288 & 38.5327 & 14.344 \\
\hline \multirow[t]{2}{*}{81.6969} & 17.608 & 48.7056 & 15.224 & \\
\hline & 56.5711 & 14.292 & 37.6476 & 14.348 \\
\hline \multirow[t]{2}{*}{81.1173} & 17.612 & 49.6431 & 15.228 & \\
\hline & 55.6 & 4.296 & 476 & 14.352 \\
\hline \multirow[t]{2}{*}{81.1173} & 17.616 & 48.7056 & 15.232 & \\
\hline & & & 37.6476 & 14.356 \\
\hline \multirow[t]{2}{*}{80.5382} & 17.62 & 49.6431 & 15.236 & \\
\hline & & & 476 & 14.36 \\
\hline \multirow[t]{2}{*}{81.6969} & 17.6 & 49.6431 & 15.24 & \\
\hline & 56.5 & 8 & 38.5327 & 14.364 \\
\hline 80.5382 & 17.6 & 48.7056 & 15.244 & \\
\hline & 55.6 & 2 & 37.6 & 14.368 \\
\hline 80.5382 & 17.6 & 51.5221 & & \\
\hline & 55.6 & 16 & 37.6476 & 14.372 \\
\hline 80.5382 & 17.6 & 50.5 & 15.252 & \\
\hline & 56.5711 & 14. & 39.4192 & 14.376 \\
\hline 80.5382 & 17.64 & 50.5 & 15.256 & \\
\hline & 55.6 & 4 & 192 & 14.38 \\
\hline 5969 & 17.6 & 31 & & \\
\hline & 56.5 & 14.3 & 40.3071 & 14.384 \\
\hline 80.5382 & 17.6 & 31 & & \\
\hline & 55.6 & 2 & 971 & 14.388 \\
\hline 382 & 17.6 & 31 & 15.268 & \\
\hline & & & & 14.392 \\
\hline 81.6969 & 17.6 & 50.5819 & 15. & \\
\hline & & & & 14.396 \\
\hline 81.1173 & 17.66 & 49.6 & 15. & \\
\hline & 56.571 & & 327 & 14.4 \\
\hline 81.1173 & 17.6 & 49.6431 & 15.28 & \\
\hline & 55.6 & 14.348 & 071 & 14.404 \\
\hline 5382 & 17.668 & 50.5819 & 15.284 & \\
\hline & 55.6 & 143 & 176 & 14.408 \\
\hline 80.5382 & 17.672 & 51.5221 & 15.288 & \\
\hline & 56.5 & 6 & 476 & 14.412 \\
\hline 80.5382 & 17.676 & 50.5819 & 15.292 & \\
\hline & 55.6 & 14.36 & 36.7638 & 14.416 \\
\hline 80.5382 & & & 15.296 & \\
\hline & 54.7603 & & 37.6476 & 14.42 \\
\hline 80.5382 & & & & \\
\hline & 53.8 & 14.368 & 37.6476 & 14.424 \\
\hline 79.9594 & & 49.6431 & & \\
\hline & 55.6651 & 14.372 & 36.7638 & 14.428 \\
\hline 80.5382 & 17.692 & 50.5819 & 15.308 & \\
\hline & 55.6651 & & 35.8814 & 14.43 \\
\hline 80.5382 & 17.696 & 49.6431 & 15.312 & \\
\hline & 54.7603 & & 37.6476 & 14.4 \\
\hline 80.5382 & 17.7 & 48.7056 & 15.316 & \\
\hline & 55.66 & & 37.6476 & 14.44 \\
\hline 00.5502 & 17.704 & 50.5819 & 15.32 & \\
\hline & 54.7603 & & 39.4192 & 14.44 \\
\hline 00.5502 & 17.708 & 50.5819 & 15.324 & \\
\hline & 55.6651 & 14.392 & 37.6476 & 14.448 \\
\hline $11 / 3$ & 17.712 & 51.5221 & 15.328 & \\
\hline & 55.6651 & 14.396 & 37.6476 & 14.452 \\
\hline 382 & 17.716 & 49.6431 & 15.332 & \\
\hline
\end{tabular}

\begin{tabular}{|c|c|c|c|}
\hline 66.4919 & 16.42 & 58.0981 & 16.2 \\
\hline 68.284 & 16.424 & 58.0981 & 16.204 \\
\hline 63.8121 & 16.428 & 61.0463 & 16.208 \\
\hline 64.7042 & 16.432 & 59.0794 & 16.212 \\
\hline 64.7042 & 16.436 & 62.0319 & 16.216 \\
\hline 66.4919 & 16.44 & 62.0319 & 16.22 \\
\hline 63.8121 & 16.444 & 62.0319 & 16.224 \\
\hline 64.7042 & 16.448 & 62.0319 & 16.228 \\
\hline 64.7042 & 16.452 & 63.0189 & 16.232 \\
\hline 65.5975 & 16.456 & 62.0319 & 16.236 \\
\hline 66.4919 & 16.46 & 62.0319 & 16.24 \\
\hline 65.5975 & 16.464 & 64.0074 & 16.244 \\
\hline 65.5975 & 16.468 & 63.0189 & 16.248 \\
\hline 64.7042 & 16.472 & 64.9972 & 16.252 \\
\hline 64.7042 & 16.476 & 64.9972 & 16.256 \\
\hline 66.4919 & 16.48 & 65.9884 & 16.26 \\
\hline 65.5975 & 16.484 & 64.9972 & 16.264 \\
\hline 64.7042 & 16.488 & 65.9884 & 16.268 \\
\hline 66.4919 & 16.492 & 64.9972 & 16.272 \\
\hline 67.3874 & 16.496 & 64.9972 & 16.276 \\
\hline 65.5975 & 16.5 & 65.9884 & 16.28 \\
\hline 65.5975 & 16.504 & 65.9884 & 16.284 \\
\hline 67.3874 & 16.508 & 66.9811 & 16.288 \\
\hline 69.1818 & 16.512 & 68.9705 & 16.292 \\
\hline 67.3874 & 16.516 & 66.9811 & 16.296 \\
\hline 67.3874 & 16.52 & 66.9811 & 16.3 \\
\hline 69.1818 & 16.524 & 67.9751 & 16.304 \\
\hline 69.1818 & 16.528 & 64.9972 & 16.308 \\
\hline 68.284 & 16.532 & 64.9972 & 16.312 \\
\hline 69.1818 & 16.536 & 64.9972 & 16.316 \\
\hline 65.5975 & 16.54 & 64.9972 & 16.32 \\
\hline 69.1818 & 16.544 & 65.9884 & 16.324 \\
\hline 68.284 & 16.548 & 66.9811 & 16.328 \\
\hline 66.4919 & 16.552 & 66.9811 & 16.332 \\
\hline 67.3874 & 16.556 & 66.9811 & 16.336 \\
\hline
\end{tabular}




\begin{tabular}{|c|c|c|c|c|}
\hline & 56.5711 & 14.4 & 38.5327 & 14.456 \\
\hline \multirow[t]{2}{*}{80.5382} & 17.72 & 50.5819 & 15.336 & \\
\hline & 55.6651 & 14.404 & 38.5327 & 14.46 \\
\hline \multirow[t]{2}{*}{80.5382} & 17.724 & 49.6431 & 15.34 & \\
\hline & 55.6651 & 14.408 & 36.7638 & 14.464 \\
\hline \multirow[t]{2}{*}{81.1173} & 17.728 & 50.5819 & 15.344 & \\
\hline & 56.5711 & 14.412 & 37.6476 & 14.468 \\
\hline \multirow[t]{2}{*}{80.5382} & 17.732 & 49.6431 & 15.348 & \\
\hline & 54.7603 & 14.416 & 37.6476 & 14.472 \\
\hline \multirow[t]{2}{*}{80.5382} & 17.736 & 51.5221 & 15.352 & \\
\hline & 54.7603 & 14.42 & 37.6476 & 14.476 \\
\hline \multirow[t]{2}{*}{80.5382} & 17.74 & 50.5819 & 15.356 & \\
\hline & 54.7603 & 14.424 & 36.7638 & 14.48 \\
\hline \multirow[t]{2}{*}{80.5382} & 17.744 & 49.6431 & 15.36 & \\
\hline & 55.6651 & 14.428 & 40.3071 & 14.484 \\
\hline \multirow[t]{2}{*}{80.5382} & 17.748 & 50.5819 & 15.364 & \\
\hline & 55.6651 & 14.432 & 38.5327 & 14.488 \\
\hline \multirow[t]{2}{*}{81.1173} & 17.752 & 50.5819 & 15.368 & \\
\hline & 56.5711 & 14.436 & 38.5327 & 14.492 \\
\hline \multirow[t]{2}{*}{81.1173} & 17.756 & 50.5819 & 15.372 & \\
\hline & 55.6651 & 14.44 & 38.5327 & 14.496 \\
\hline \multirow[t]{2}{*}{82.2769} & 17.76 & 50.5819 & 15.376 & \\
\hline & 55.6651 & 14.444 & 37.6476 & 14.5 \\
\hline \multirow[t]{2}{*}{82.2769} & 17.764 & 49.6431 & 15.38 & \\
\hline & 57.4784 & 14.448 & 38.5327 & 14.504 \\
\hline 82.2769 & 17.768 & 51.5221 & 15.384 & \\
\hline & 57.4784 & 14.452 & 38.5327 & 14.508 \\
\hline 80.5382 & 17.772 & 50.5819 & 15.388 & \\
\hline & 56.5711 & 14.456 & 36.7638 & 14.512 \\
\hline 81.6969 & 17.776 & 51.5221 & 15.392 & \\
\hline & 55.6651 & 14.46 & 38.5327 & 14.516 \\
\hline 81.1173 & 17.78 & 50.5819 & 15.396 & \\
\hline & 56.5711 & 14.464 & 37.6476 & 14.52 \\
\hline 81.1173 & 17.784 & 51.5221 & 15.4 & \\
\hline & 55.6651 & 14.468 & 38.5327 & 14.524 \\
\hline 80.5382 & 17.788 & 50.5819 & 15.404 & \\
\hline & 56.5711 & 14.472 & 37.6476 & 14.528 \\
\hline 81.6969 & 17.792 & 52.4637 & 15.408 & \\
\hline & 56.5711 & 14.476 & 37.6476 & 14.532 \\
\hline 81.6969 & 17.796 & 51.5221 & 15.412 & \\
\hline & 56.5711 & 14.48 & 38.5327 & 14.536 \\
\hline 81.6969 & 17.8 & 51.5221 & 15.416 & \\
\hline & 56.5711 & 14.484 & 38.5327 & 14.54 \\
\hline 81.1173 & 17.804 & 50.5819 & 15.42 & \\
\hline & 57.4784 & 14.488 & 38.5327 & 14.544 \\
\hline 81.1173 & 17.808 & 51.5221 & 15.424 & \\
\hline & 57.4784 & 14.492 & 38.5327 & 14.548 \\
\hline 80.5382 & 17.812 & 52.4637 & 15.428 & \\
\hline & 56.5711 & 14.496 & 38.5327 & 14.552 \\
\hline 81.6969 & 17.816 & 52.4637 & 15.432 & \\
\hline & 60.2078 & 14.5 & 37.6476 & 14.556 \\
\hline 80.5382 & 17.82 & 54.3511 & 15.436 & \\
\hline & 56.5711 & 14.504 & 37.6476 & 14.56 \\
\hline 80.5382 & 17.824 & 51.5221 & 15.44 & \\
\hline & 57.4784 & 14.508 & 38.5327 & 14.564 \\
\hline 81.1173 & 17.828 & 50.5819 & 15.444 & \\
\hline & 56.5711 & 14.512 & 38.5327 & 14.568 \\
\hline 80.5382 & 17.832 & 50.5819 & 15.448 & \\
\hline & 56.5711 & 14.516 & 37.6476 & 14.572 \\
\hline 81.1173 & 17.836 & 50.5819 & 15.452 & \\
\hline & 56.5711 & 14.52 & 37.6476 & 14.576 \\
\hline 81.1173 & 17.84 & 51.5221 & 15.456 & \\
\hline & 56.5711 & 14.524 & 37.6476 & 14.58 \\
\hline 81.6969 & 17.844 & 50.5819 & 15.46 & \\
\hline & 56.5711 & 14.528 & 39.4192 & 14.584 \\
\hline 81.1173 & 17.848 & 51.5221 & 15.464 & \\
\hline & 59.2967 & 14.532 & 39.4192 & 14.588 \\
\hline 80.5382 & 17.852 & 52.4637 & 15.468 & \\
\hline & 58.387 & 14.536 & 39.4192 & 14.592 \\
\hline 80.5382 & 17.856 & 51.5221 & 15.472 & \\
\hline
\end{tabular}

\begin{tabular}{|c|c|c|c|}
\hline 65.5975 & 16.56 & 67.9751 & 16.34 \\
\hline 67.3874 & 16.564 & 65.9884 & 16.344 \\
\hline 68.284 & 16.568 & 64.9972 & 16.348 \\
\hline 67.3874 & 16.572 & 65.9884 & 16.352 \\
\hline 68.284 & 16.576 & 65.9884 & 16.356 \\
\hline 68.284 & 16.58 & 66.9811 & 16.36 \\
\hline 68.284 & 16.584 & 67.9751 & 16.364 \\
\hline 67.3874 & 16.588 & 67.9751 & 16.368 \\
\hline 67.3874 & 16.592 & 67.9751 & 16.372 \\
\hline 68.284 & 16.596 & 66.9811 & 16.376 \\
\hline 68.284 & 16.6 & 66.9811 & 16.38 \\
\hline 68.284 & 16.604 & 64.9972 & 16.384 \\
\hline 69.1818 & 16.608 & 62.0319 & 16.388 \\
\hline 67.3874 & 16.612 & 63.0189 & 16.392 \\
\hline 69.1818 & 16.616 & 65.9884 & 16.396 \\
\hline 70.0806 & 16.62 & 64.9972 & 16.4 \\
\hline 68.284 & 16.624 & 67.9751 & 16.404 \\
\hline 68.284 & 16.628 & 66.9811 & 16.408 \\
\hline 68.284 & 16.632 & 66.9811 & 16.412 \\
\hline 68.284 & 16.636 & 65.9884 & 16.416 \\
\hline 69.1818 & 16.64 & 64.9972 & 16.42 \\
\hline 66.4919 & 16.644 & 63.0189 & 16.424 \\
\hline 70.9807 & 16.648 & 63.0189 & 16.428 \\
\hline 70.0806 & 16.652 & 64.0074 & 16.432 \\
\hline 68.284 & 16.656 & 65.9884 & 16.436 \\
\hline 70.0806 & 16.66 & 65.9884 & 16.44 \\
\hline 69.1818 & 16.664 & 66.9811 & 16.444 \\
\hline 68.284 & 16.668 & 64.9972 & 16.448 \\
\hline 69.1818 & 16.672 & 66.9811 & 16.452 \\
\hline 68.284 & 16.676 & 64.9972 & 16.456 \\
\hline 69.1818 & 16.68 & 64.0074 & 16.46 \\
\hline 69.1818 & 16.684 & 64.9972 & 16.464 \\
\hline 69.1818 & 16.688 & 63.0189 & 16.468 \\
\hline 69.1818 & 16.692 & 65.9884 & 16.472 \\
\hline 69.1818 & 16.696 & 64.9972 & 16.476 \\
\hline
\end{tabular}




\begin{tabular}{|c|c|c|c|c|}
\hline & 58.387 & 14.54 & 39.4192 & 14.596 \\
\hline \multirow[t]{2}{*}{80.5382} & 17.86 & 52.4637 & 15.476 & \\
\hline & 58.387 & 14.544 & 38.5327 & 14.6 \\
\hline \multirow[t]{2}{*}{80.5382} & 17.864 & 51.5221 & 15.48 & \\
\hline & 59.2967 & 14.548 & 38.5327 & 14.604 \\
\hline \multirow[t]{2}{*}{80.5382} & 17.868 & 50.5819 & 15.484 & \\
\hline & 57.4784 & 14.552 & 40.3071 & 14.608 \\
\hline \multirow[t]{2}{*}{80.5382} & 17.872 & 54.3511 & 15.488 & \\
\hline & 58.387 & 14.556 & 38.5327 & 14.612 \\
\hline \multirow[t]{2}{*}{79.9594} & 17.876 & 51.5221 & 15.492 & \\
\hline & 60.2078 & 14.56 & 39.4192 & 14.616 \\
\hline \multirow[t]{2}{*}{79.9594} & 17.88 & 52.4637 & 15.496 & \\
\hline & 59.2967 & 14.564 & 40.3071 & 14.62 \\
\hline \multirow[t]{2}{*}{80.5382} & 17.884 & 54.3511 & 15.5 & \\
\hline & 58.387 & 14.568 & 41.1963 & 14.624 \\
\hline \multirow[t]{2}{*}{80.5382} & 17.888 & 51.5221 & 15.504 & \\
\hline & 59.2967 & 14.572 & 38.5327 & 14.628 \\
\hline \multirow[t]{2}{*}{80.5382} & 17.892 & 53.4067 & 15.508 & \\
\hline & 58.387 & 14.576 & 38.5327 & 14.632 \\
\hline \multirow[t]{2}{*}{79.3811} & 17.896 & 53.4067 & 15.512 & \\
\hline & 57.4784 & 14.58 & 38.5327 & 14.636 \\
\hline \multirow[t]{2}{*}{79.9594} & 17.9 & 52.4637 & 15.516 & \\
\hline & 59.2 & 14.5 & 38.5327 & 14.64 \\
\hline \multirow[t]{2}{*}{78.2259} & 17.904 & 52.4637 & 15.52 & \\
\hline & 57.4784 & 14.588 & 39.4192 & 14.644 \\
\hline 78.2259 & 17.908 & 53.4067 & 15.524 & \\
\hline & 60.2078 & 14.592 & 39. & 14.648 \\
\hline 79.9594 & 17.912 & 53.4067 & 15.528 & \\
\hline & 60.2078 & 14.596 & 39.4192 & 14.652 \\
\hline 78.2259 & 17.916 & 53.4067 & 15.532 & \\
\hline & 59.2967 & 14.6 & 40.3071 & 14.656 \\
\hline 78.8033 & 17.92 & 53.4067 & 15.536 & \\
\hline & 60.2078 & 14.604 & 39.4192 & 14.66 \\
\hline 78.8033 & 17.924 & 53.4 & 15.54 & \\
\hline & 58.387 & 14.608 & 40.3071 & 14.664 \\
\hline 78.2259 & 17.928 & 52.4637 & 15.544 & \\
\hline & 60.2078 & 14.6 & 39. & 14.668 \\
\hline 77.0723 & 17.932 & 52.4637 & 15.548 & \\
\hline & 61.12 & & & 14.672 \\
\hline 77.6489 & 17.936 & 53.4067 & 15 & \\
\hline & 61.12 & & & 14.676 \\
\hline 78.2259 & 17.94 & 53.4067 & 15.556 & \\
\hline & 60.2078 & 14.624 & 39.4192 & 14.68 \\
\hline 77.6489 & 17.944 & 52.4637 & 15.56 & \\
\hline & 60.2078 & 14.628 & 40.3071 & 14.684 \\
\hline 76.4962 & 17.948 & 53.4067 & 15.564 & \\
\hline & 60.2078 & 14.632 & 38.5327 & 14.688 \\
\hline 77.6489 & 17.952 & 54.3511 & 15.568 & \\
\hline & 61.12 & 14.6 & 40.3071 & 14.692 \\
\hline 77.0723 & 17.956 & 54.3511 & 15.572 & \\
\hline & 60.2078 & 14.64 & 40.3071 & 14.696 \\
\hline 75.9205 & 17.96 & 54.3511 & & \\
\hline & 61.12 & 14.644 & 40.3071 & 14.7 \\
\hline 78.2259 & 17.964 & 54.3511 & 15.58 & \\
\hline & 61.12 & 14.648 & 40.3071 & 14.704 \\
\hline 76.4962 & 17.968 & 54.3511 & 15.584 & \\
\hline & 62.0335 & 14.652 & 40.3071 & 4.708 \\
\hline 76.4962 & 17.972 & 54.3511 & 15.588 & \\
\hline & 62.0335 & 14.656 & 41.1963 & 4.712 \\
\hline 75.3452 & 17.976 & 54.3511 & 15.592 & \\
\hline & 60.2078 & 14.66 & 41.1963 & 14.716 \\
\hline 76.4962 & 17.98 & 54.3511 & 15.596 & \\
\hline & 61.12 & 14.664 & 40.3071 & 14.72 \\
\hline 75.9205 & 17.984 & 54.3511 & 15.6 & \\
\hline & 61.12 & 14.668 & 40.3071 & 14.72 \\
\hline 75.9205 & 17.988 & 53.4067 & 15.604 & \\
\hline & 60.2078 & 14.672 & 41.1963 & 14.728 \\
\hline 75.9205 & 17.992 & 54.3511 & 15.608 & \\
\hline & 60.2078 & 14.676 & 40.3071 & 14.732 \\
\hline 5.9205 & 17.996 & 54.3511 & 15.612 & \\
\hline
\end{tabular}

\begin{tabular}{|c|c|c|c|}
\hline 68.284 & 16.7 & 65.9884 & 16.48 \\
\hline 69.1818 & 16.704 & 64.9972 & 16.484 \\
\hline 68.284 & 16.708 & 64.9972 & 16.488 \\
\hline 65.5975 & 16.712 & 64.0074 & 16.492 \\
\hline 67.3874 & 16.716 & 65.9884 & 16.496 \\
\hline 69.1818 & 16.72 & 63.0189 & 16.5 \\
\hline 69.1818 & 16.724 & 65.9884 & 16.504 \\
\hline 68.284 & 16.728 & 64.0074 & 16.508 \\
\hline 70.0806 & 16.732 & 64.0074 & 16.512 \\
\hline 69.1818 & 16.736 & 64.9972 & 16.516 \\
\hline 70.0806 & 16.74 & 65.9884 & 16.52 \\
\hline 69.1818 & 16.744 & 66.9811 & 16.524 \\
\hline 69.1818 & 16.748 & 66.9811 & 16.528 \\
\hline 71.8818 & 16.752 & 63.0189 & 16.532 \\
\hline 69.1818 & 16.756 & 64.9972 & 16.536 \\
\hline 69.1818 & 16.76 & 65.9884 & 16.54 \\
\hline 69.1818 & 16.764 & 65.9884 & 16.544 \\
\hline 69.1818 & 16.768 & 64.9972 & 16.548 \\
\hline 69.1818 & 16.772 & 66.9811 & 16.552 \\
\hline 70.0806 & 16.776 & 67.9751 & 16.556 \\
\hline 69.1818 & 16.78 & 67.9751 & 16.56 \\
\hline 69.1818 & 16.784 & 66.9811 & 16.564 \\
\hline 69.1818 & 16.788 & 66.9811 & 16.568 \\
\hline 69.1818 & 16.792 & 65.9884 & 16.572 \\
\hline 70.0806 & 16.796 & 65.9884 & 16.576 \\
\hline 70.0806 & 16.8 & 65.9884 & 16.58 \\
\hline 70.0806 & 16.804 & 65.9884 & 16.584 \\
\hline 70.9807 & 16.808 & 65.9884 & 16.588 \\
\hline 68.284 & 16.812 & 65.9884 & 16.592 \\
\hline 67.3874 & 16.816 & 67.9751 & 16.596 \\
\hline 70.9807 & 16.82 & 69.9673 & 16.6 \\
\hline 68.284 & 16.824 & 69.9673 & 16.604 \\
\hline 69.1818 & 16.828 & 66.9811 & 16.608 \\
\hline 70.0806 & 16.832 & 67.9751 & 16.612 \\
\hline 70.0806 & 16.836 & 66.9811 & 16.616 \\
\hline
\end{tabular}




\begin{tabular}{|c|c|c|c|c|}
\hline & 60.2078 & 14.68 & 41.1963 & 14.736 \\
\hline \multirow[t]{2}{*}{75.9205} & 18 & 54.3511 & 15.616 & \\
\hline & 59.2967 & 14.684 & 38.5327 & 14.74 \\
\hline \multirow[t]{2}{*}{75.3452} & 18.004 & 54.3511 & 15.62 & \\
\hline & 60.2078 & 14.688 & 40.3071 & 14.744 \\
\hline \multirow[t]{2}{*}{75.3452} & 18.008 & 54.3511 & 15.624 & \\
\hline & 60.2078 & 14.692 & 41.1963 & 14.748 \\
\hline \multirow[t]{2}{*}{75.3452} & 18.012 & 54.3511 & 15.628 & \\
\hline & 61.12 & 14.696 & 40.3071 & 14.752 \\
\hline \multirow[t]{2}{*}{74.7704} & 18.016 & 54.3511 & 15.632 & \\
\hline & 61.12 & 14.7 & 41.1963 & 14.756 \\
\hline \multirow[t]{2}{*}{75.3452} & 18.02 & 55.2969 & 15.636 & \\
\hline & 62.9481 & 14.704 & 40.3071 & 14.76 \\
\hline \multirow[t]{2}{*}{74.196} & 18.024 & 55.2969 & 15.64 & \\
\hline & 60.2078 & 14.708 & 41.1963 & 14.764 \\
\hline \multirow[t]{2}{*}{75.3452} & 18.028 & 55.2969 & 15.644 & \\
\hline & 61.12 & 14.712 & 40.3071 & 14.768 \\
\hline \multirow[t]{2}{*}{73.6221} & 18.032 & 54.3511 & 15.648 & \\
\hline & 61.12 & 14.716 & 42. & 14.772 \\
\hline \multirow[t]{2}{*}{73.6221} & 18.036 & 54.3511 & 15.652 & \\
\hline & 61.12 & 14.72 & 41.1963 & 14.776 \\
\hline \multirow[t]{2}{*}{73.0486} & 18.04 & 54.3511 & 15.656 & \\
\hline & 61.12 & 14.724 & 41.1963 & 14.78 \\
\hline \multirow[t]{2}{*}{73.0486} & 18.044 & 54.3511 & 15.66 & \\
\hline & 61.12 & 14.728 & 963 & 14.784 \\
\hline 71.3308 & 18.048 & 55.2969 & 15.664 & \\
\hline & 60.2078 & 14.732 & 41. & 14.788 \\
\hline 71.9029 & 18.052 & 55.2969 & 15.668 & \\
\hline & 61.12 & 14.736 & 41.1963 & 14.792 \\
\hline 71.9029 & 18.056 & 55.2969 & 15.672 & \\
\hline & 61.12 & 14.74 & 41.1963 & 14.796 \\
\hline 71.3308 & 18.06 & 56.244 & 15.676 & \\
\hline & 61.12 & 14.7 & 42.9788 & 14.8 \\
\hline 71.3308 & 18.064 & 54.3 & & \\
\hline & 60.2078 & 14.7 & 41.1963 & 14.804 \\
\hline 71.3308 & 18.068 & 54.3511 & & \\
\hline & 60.2078 & 14.7 & 42.0869 & 14.808 \\
\hline 71.3308 & 18.072 & 55.2969 & 15.688 & \\
\hline & 62.0335 & 14.756 & 963 & 14.812 \\
\hline 3308 & 18.076 & 55.2969 & 15 & \\
\hline & 60.2078 & 14.76 & 963 & 14.816 \\
\hline 308 & 18.08 & 55.2969 & 15 & \\
\hline & 61.12 & 14.76 & 869 & 14.82 \\
\hline 308 & 18.084 & 57.1925 & 15.7 & \\
\hline & 62.0335 & 14.768 & 42.0869 & 14.824 \\
\hline 70.7591 & 18.088 & 56.244 & 15.704 & \\
\hline & 61.12 & 14.772 & 42.0869 & 14.828 \\
\hline 69.0466 & 18.092 & 56.244 & & \\
\hline & 60.2078 & 14.7 & 369 & 14.832 \\
\hline 69.617 & 18.096 & 57.1925 & 15.712 & \\
\hline & 62.0335 & 14.78 & 44.7666 & 14.836 \\
\hline 67.9072 & 18.1 & 56.244 & & \\
\hline & 62.9481 & 14.784 & 42.0869 & 14.84 \\
\hline 67.9072 & 18.104 & 56.244 & 15.72 & \\
\hline & 62.0335 & 14.788 & 42.9788 & 14.844 \\
\hline 68.4767 & 18.108 & 56.244 & 15.724 & \\
\hline & 61.12 & 14.792 & 42.9788 & 14.848 \\
\hline 66.7696 & 18.112 & 54.3511 & & \\
\hline & 60.2078 & 14.796 & & 14.852 \\
\hline 68.4767 & 18.116 & 54.3511 & 15.732 & \\
\hline & 62.9481 & 14.8 & 43.872 & 14.85 \\
\hline 66.7696 & 18.12 & 55.2969 & 15.736 & \\
\hline & 63.8641 & 14.804 & 42.0869 & 14.86 \\
\hline 67.3382 & 18.124 & 54.3511 & 15.74 & \\
\hline & 61.12 & 14.808 & 42.9788 & 14.86 \\
\hline 67.3382 & 18.128 & 55.2969 & 15.744 & \\
\hline & 63.8641 & 14.812 & 42.9788 & 14.868 \\
\hline 1090 & 18.132 & 56.244 & 15.748 & \\
\hline & 62.0335 & 14.816 & 43.872 & 14.872 \\
\hline 7696 & 18.136 & 57.1925 & 15.752 & \\
\hline
\end{tabular}

\begin{tabular}{|c|c|c|c|}
\hline 70.0806 & 16.84 & 66.9811 & 16.62 \\
\hline 69.1818 & 16.844 & 65.9884 & 16.624 \\
\hline 69.1818 & 16.848 & 65.9884 & 16.628 \\
\hline 69.1818 & 16.852 & 65.9884 & 16.632 \\
\hline 69.1818 & 16.856 & 66.9811 & 16.636 \\
\hline 70.0806 & 16.86 & 66.9811 & 16.64 \\
\hline 70.9807 & 16.864 & 68.9705 & 16.644 \\
\hline 72.7839 & 16.868 & 67.9751 & 16.648 \\
\hline 69.1818 & 16.872 & 69.9673 & 16.652 \\
\hline 70.0806 & 16.876 & 67.9751 & 16.656 \\
\hline 70.9807 & 16.88 & 68.9705 & 16.66 \\
\hline 69.1818 & 16.884 & 68.9705 & 16.664 \\
\hline 70.0806 & 16.888 & 69.9673 & 16.668 \\
\hline 70.0806 & 16.892 & 69.9673 & 16.672 \\
\hline 70.0806 & 16.896 & 68.9705 & 16.676 \\
\hline 69.1818 & 16.9 & 69.9673 & 16.68 \\
\hline 69.1818 & 16.904 & 69.9673 & 16.684 \\
\hline 70.9807 & 16.908 & 69.9673 & 16.688 \\
\hline 70.0806 & 16.912 & 68.9705 & 16.692 \\
\hline 71.8818 & 16.916 & 68.9705 & 16.696 \\
\hline 71.8818 & 16.92 & 68.9705 & 16.7 \\
\hline 70.0806 & 16.924 & 68.9705 & 16.704 \\
\hline 72.7839 & 16.928 & 69.9673 & 16.708 \\
\hline 70.9807 & 16.932 & 69.9673 & 16.712 \\
\hline 71.8818 & 16.936 & 69.9673 & 16.716 \\
\hline 71.8818 & 16.94 & 68.9705 & 16.72 \\
\hline 70.0806 & 16.944 & 69.9673 & 16.724 \\
\hline 70.9807 & 16.948 & 69.9673 & 16.728 \\
\hline 72.7839 & 16.952 & 70.9654 & 16.732 \\
\hline 74.5917 & 16.956 & 69.9673 & 16.736 \\
\hline 76.4037 & 16.96 & 68.9705 & 16.74 \\
\hline 75.4971 & 16.964 & 68.9705 & 16.744 \\
\hline 76.4037 & 16.968 & 70.9654 & 16.748 \\
\hline 71.8818 & 16.972 & 69.9673 & 16.752 \\
\hline 69.1818 & 16.976 & 69.9673 & 16.756 \\
\hline
\end{tabular}




\begin{tabular}{|c|c|c|c|c|}
\hline & 62.0335 & 14.82 & 42.9788 & 4.876 \\
\hline \multirow[t]{2}{*}{66.7696} & 18.14 & 56.244 & 15.756 & \\
\hline & 62.0335 & 14.824 & 43.872 & 14.88 \\
\hline \multirow[t]{2}{*}{66.7696} & 18.144 & 54.3511 & 15.76 & \\
\hline & 62.0335 & 14.828 & 43.872 & 4.884 \\
\hline \multirow[t]{2}{*}{66.7696} & 18.148 & 57.1925 & 15.764 & \\
\hline & 62.9481 & 14.832 & 43.872 & 4.888 \\
\hline \multirow[t]{2}{*}{66.7696} & 18.152 & 57.1925 & 15.768 & \\
\hline & 63.8641 & 14.836 & 44.7666 & 4.892 \\
\hline \multirow[t]{2}{*}{66.7696} & 18.156 & 57.1925 & 15.772 & \\
\hline & 62.9481 & 14.84 & 44.7666 & 14.896 \\
\hline \multirow[t]{2}{*}{66.2015} & 18.16 & 58.1424 & 15.776 & \\
\hline & 62.9481 & 4.844 & 43.872 & 14.9 \\
\hline \multirow[t]{2}{*}{66.7696} & 18.164 & 57.1925 & 15.78 & \\
\hline & 62.9481 & & 43.872 & 14.904 \\
\hline \multirow[t]{2}{*}{66.2015} & 18.168 & 56.2 & 15. & \\
\hline & 63 & & & 4.908 \\
\hline \multirow[t]{2}{*}{65.6338} & 18.172 & 57.1925 & 15. & \\
\hline & & & & 14.912 \\
\hline \multirow[t]{2}{*}{66.2015} & 18.1 & 59.0936 & 15. & \\
\hline & 63. & & 42. & 4.916 \\
\hline \multirow{2}{*}{66.7696} & 18.18 & & 15. & \\
\hline & 63. & & 42.9788 & 4.92 \\
\hline \multirow[t]{2}{*}{65.0667} & 18.1 & & 15. & \\
\hline & 62.9481 & 14. & 43. & 14.924 \\
\hline 65.6338 & 18.188 & 424 & 15. & \\
\hline & 62.9481 & 14. & 44.7666 & 4.928 \\
\hline 65.0667 & 18.192 & 24 & 15.808 & \\
\hline & 63.8 & 14. & 44. & 14.932 \\
\hline 64.4999 & 18.196 & 24 & & \\
\hline & 63. & 14 & 44. & 14.936 \\
\hline 64. & 18.2 & 24 & 15. & \\
\hline & 63.86 & & 44. & 14.94 \\
\hline 99 & 18. & & 15.82 & \\
\hline & & & & 14.944 \\
\hline 64.4999 & 18.2 & 25 & 15. & \\
\hline & & & & 48 \\
\hline 63.3678 & 18.212 & 24 & 15. & \\
\hline & 41 & & 666 & 14.952 \\
\hline 63.3678 & 18.216 & 57. & 15. & \\
\hline & 63.8 & 14. & 25 & 14.956 \\
\hline 64.4999 & 18.22 & 58 & 15. & \\
\hline & 63. & 4 & 25 & 14.96 \\
\hline 63.3678 & 18.2 & 59.0 & & \\
\hline & 64. & 8 & 45 & 14.964 \\
\hline 64.4999 & 18.228 & 24 & & \\
\hline & 63. & 2 & 45. & 14.968 \\
\hline 63.3678 & & & & \\
\hline & 63.8641 & & 44.7666 & 14.972 \\
\hline 63.3678 & & 25 & & \\
\hline & 64.7811 & & 45. & 4.976 \\
\hline 63.9337 & 18.24 & 24 & 15. & \\
\hline & 63. & & 44. & 4.98 \\
\hline 63.3678 & 18.244 & 24 & 15. & \\
\hline & & & 44 & 4.984 \\
\hline 62.2376 & 18.248 & 25 & 15. & \\
\hline & & & 44. & 4.988 \\
\hline 62.2376 & 18.252 & 57. & 15.8 & \\
\hline & & & & 4.992 \\
\hline 62.2376 & 18.256 & 36 & 15. & \\
\hline & 65.6995 & & 598 & 14.996 \\
\hline 62.2376 & 18.26 & 59.0936 & 15.876 & \\
\hline & 66.619 & & 45.6625 & 15 \\
\hline 62.2376 & 18.264 & 58.1424 & 15.88 & \\
\hline & & & & 15.004 \\
\hline 62.2376 & 18.268 & 58.1424 & 15.884 & \\
\hline & 64.7811 & 14.952 & 45.6625 & 15.008 \\
\hline 62.2376 & 18.272 & 58.1424 & 15.888 & \\
\hline & 64.7811 & 14.956 & 45.6625 & 15.012 \\
\hline 61.1092 & 18.276 & 59.0936 & 15.892 & \\
\hline
\end{tabular}

\begin{tabular}{|c|c|c|c|}
\hline 69.1818 & 16.98 & 68.9705 & 16.76 \\
\hline 71.8818 & 16.984 & 69.9673 & 16.764 \\
\hline 70.9807 & 16.988 & 69.9673 & 16.768 \\
\hline 72.7839 & 16.992 & 67.9751 & 16.772 \\
\hline 72.7839 & 16.996 & 66.9811 & 16.776 \\
\hline 70.9807 & 17 & 68.9705 & 16.78 \\
\hline 74.5917 & 17.004 & 68.9705 & 16.784 \\
\hline 72.7839 & 17.008 & 69.9673 & 16.788 \\
\hline 75.4971 & 17.012 & 69.9673 & 16.792 \\
\hline 75.4971 & 17.016 & 69.9673 & 16.796 \\
\hline 72.7839 & 17.02 & 68.9705 & 16.8 \\
\hline 74.5917 & 17.024 & 69.9673 & 16.804 \\
\hline 70.9807 & 17.028 & 66.9811 & 16.808 \\
\hline 73.6872 & 17.032 & 66.9811 & 16.812 \\
\hline 76.4037 & 17.036 & 68.9705 & 16.816 \\
\hline 78.2201 & 17.04 & 67.9751 & 16.82 \\
\hline 76.4037 & 17.044 & 69.9673 & 16.824 \\
\hline 77.3114 & 17.048 & 67.9751 & 16.828 \\
\hline 74.5917 & 17.052 & 69.9673 & 16.832 \\
\hline 70.0806 & 17.056 & 67.9751 & 16.836 \\
\hline 70.9807 & 17.06 & 67.9751 & 16.84 \\
\hline 70.0806 & 17.064 & 66.9811 & 16.844 \\
\hline 71.8818 & 17.068 & 65.9884 & 16.848 \\
\hline 73.6872 & 17.072 & 66.9811 & 16.852 \\
\hline 73.6872 & 17.076 & 66.9811 & 16.856 \\
\hline 76.4037 & 17.08 & 68.9705 & 16.86 \\
\hline 74.5917 & 17.084 & 69.9673 & 16.864 \\
\hline 74.5917 & 17.088 & 68.9705 & 16.868 \\
\hline 73.6872 & 17.092 & 69.9673 & 16.872 \\
\hline 74.5917 & 17.096 & 68.9705 & 16.876 \\
\hline 75.4971 & 17.1 & 66.9811 & 16.88 \\
\hline 75.4971 & 17.104 & 66.9811 & 16.884 \\
\hline 74.5917 & 17.108 & 67.9751 & 16.888 \\
\hline 75.4971 & 17.112 & 69.9673 & 16.892 \\
\hline 72.7839 & 17.116 & 69.9673 & 16.896 \\
\hline
\end{tabular}




\begin{tabular}{|c|c|c|c|c|}
\hline & 65.6995 & 14.96 & 45.6625 & 5.016 \\
\hline \multirow[t]{2}{*}{61.6732} & 18.28 & 58.1424 & 15.896 & \\
\hline & 65.6995 & 14.964 & 46.5598 & 15.02 \\
\hline \multirow[t]{2}{*}{60.5457} & 18.284 & 59.0936 & 15.9 & \\
\hline & 64.7811 & 14.968 & 46.5598 & 15.024 \\
\hline \multirow[t]{2}{*}{59.9827} & 18.288 & 58.1424 & 15.904 & \\
\hline & 64.7811 & 14.972 & 46.5598 & 5.028 \\
\hline \multirow[t]{2}{*}{59.9827} & 18.292 & 58.1424 & 15.908 & \\
\hline & 63.8641 & 14.976 & 45.6625 & 5.032 \\
\hline \multirow[t]{2}{*}{59.9827} & 18.296 & 58.1424 & 15.912 & \\
\hline & 64.7811 & 14.98 & 47.4583 & 5.036 \\
\hline \multirow[t]{2}{*}{60.5457} & 18.3 & 59.0936 & 15.916 & \\
\hline & 64.7811 & 14.984 & 45.6625 & 15.04 \\
\hline \multirow[t]{2}{*}{58.2964} & 18.304 & 59.0936 & 15.92 & \\
\hline & 65.6995 & 14.988 & 47.4583 & 15.044 \\
\hline \multirow[t]{2}{*}{58.2964} & 18.308 & 59.0936 & 15.924 & \\
\hline & 64.7811 & 14.992 & 47.4583 & 15.048 \\
\hline \multirow[t]{2}{*}{57.7353} & 18.312 & 58.1424 & 15.928 & \\
\hline & 65.6995 & 14.996 & 48.3582 & 15.052 \\
\hline \multirow[t]{2}{*}{57.7353} & 18.316 & 58.1424 & 15.932 & \\
\hline & 63.8 & 15 & 46.5598 & 15.056 \\
\hline \multirow[t]{2}{*}{58.858} & 18.32 & 58.1424 & 15.936 & \\
\hline & 64.7811 & & 47.4583 & 15.06 \\
\hline \multirow{2}{*}{57.7353} & 18.324 & 59.0936 & 15.94 & \\
\hline & 64.7811 & & 47.4583 & 15.064 \\
\hline 57.1746 & 18.328 & 59.0936 & 15. & \\
\hline & 63.8641 & 15. & 46. & 15.068 \\
\hline 56.6144 & 18.332 & 59.0 & & \\
\hline & 66.619 & 15.016 & 48.3582 & 15.072 \\
\hline 55.4955 & 18.336 & 58.1424 & 15.952 & \\
\hline & 64.7811 & 15.02 & 47.4583 & 5.076 \\
\hline 55.4955 & 18.34 & 59.0936 & 15.956 & \\
\hline & 66.619 & 15 & 47.4583 & 15.08 \\
\hline 955 & 18.344 & 36 & 15.96 & \\
\hline & 65.6995 & 15 & 48.3582 & 15.084 \\
\hline 55.4955 & 18.348 & 62 & & \\
\hline & 65.6995 & 15 & 582 & 15.088 \\
\hline 3784 & 18.352 & 59. & 15.968 & \\
\hline & & & & 15.092 \\
\hline 3784 & 18.356 & 60.0462 & 15. & \\
\hline & 67.5397 & & & 15.096 \\
\hline 53.2633 & 18.36 & 58. & 15 & \\
\hline & 63.8641 & 15.0 & & 15.1 \\
\hline 3.8206 & 18.364 & 60. & 15.98 & \\
\hline & 64.7811 & 15 & 47.4583 & 15.104 \\
\hline 53.2633 & 18.368 & 58.1424 & 15.984 & \\
\hline & 64.7811 & 15 & 47.4583 & 15.108 \\
\hline 53.2633 & 18.372 & 58.1424 & 15.988 & \\
\hline & 64.7811 & 15 & 582 & 15.112 \\
\hline 53.2633 & 18.376 & 60.0462 & 15.992 & \\
\hline & 64.7811 & 15 & 47.4583 & 15.116 \\
\hline 53.8206 & 18.38 & 58.1424 & 15.996 & \\
\hline & 64.7811 & & 48.3582 & 15.12 \\
\hline 52.7064 & 18.384 & 58.1424 & 16 & \\
\hline & 64.7811 & 15. & 46.5 & 15.124 \\
\hline 52.7064 & 18.388 & 58.1424 & & \\
\hline & 65.6995 & & 48. & 15.128 \\
\hline 52.1501 & 18.392 & 56.244 & 16.008 & \\
\hline & 64.7811 & & 47.4583 & 5.132 \\
\hline 51.0389 & 18.396 & 53.4067 & 16.012 & \\
\hline & 63.8641 & 15.08 & 48.3582 & 15.136 \\
\hline 51.0389 & 18.4 & 45.9019 & 16.016 & \\
\hline & 64.7811 & 15.084 & 47.4583 & 15.1 \\
\hline 51.0389 & 18.404 & 41.2577 & 16.02 & \\
\hline & 66.619 & 15.088 & 48.3582 & 15.14 \\
\hline 51.0389 & 18.408 & 39.4102 & 16.024 & \\
\hline & 66.619 & & 45.6625 & 15.148 \\
\hline .0389 & 18.412 & 43.1111 & 16.028 & \\
\hline & 65.6995 & 15.096 & 48.3582 & 15.152 \\
\hline .0389 & 18.416 & 44.9702 & 16.032 & \\
\hline
\end{tabular}

\begin{tabular}{|c|c|c|c|}
\hline 72.7839 & 17.12 & 69.9673 & 16.9 \\
\hline 73.6872 & 17.124 & 69.9673 & 16.904 \\
\hline 73.6872 & 17.128 & 69.9673 & 16.908 \\
\hline 70.9807 & 17.132 & 68.9705 & 16.912 \\
\hline 70.9807 & 17.136 & 67.9751 & 16.916 \\
\hline 70.9807 & 17.14 & 67.9751 & 16.92 \\
\hline 72.7839 & 17.144 & 66.9811 & 16.924 \\
\hline 71.8818 & 17.148 & 68.9705 & 16.928 \\
\hline 73.6872 & 17.152 & 70.9654 & 16.932 \\
\hline 73.6872 & 17.156 & 69.9673 & 16.936 \\
\hline 70.9807 & 17.16 & 69.9673 & 16.94 \\
\hline 72.7839 & 17.164 & 68.9705 & 16.944 \\
\hline 71.8818 & 17.168 & 69.9673 & 16.948 \\
\hline 72.7839 & 17.172 & 69.9673 & 16.952 \\
\hline 70.9807 & 17.176 & 72.9659 & 16.956 \\
\hline 70.9807 & 17.18 & 73.9681 & 16.96 \\
\hline 70.9807 & 17.184 & 73.9681 & 16.964 \\
\hline 70.9807 & 17.188 & 73.9681 & 16.968 \\
\hline 70.9807 & 17.192 & 71.965 & 16.972 \\
\hline 70.0806 & 17.196 & 69.9673 & 16.976 \\
\hline 71.8818 & 17.2 & 68.9705 & 16.98 \\
\hline 70.0806 & 17.204 & 66.9811 & 16.984 \\
\hline 69.1818 & 17.208 & 69.9673 & 16.988 \\
\hline 69.1818 & 17.212 & 71.965 & 16.992 \\
\hline 70.0806 & 17.216 & 73.9681 & 16.996 \\
\hline 68.284 & 17.22 & 74.9717 & 17 \\
\hline 70.0806 & 17.224 & 74.9717 & 17.004 \\
\hline 70.9807 & 17.228 & 73.9681 & 17.008 \\
\hline 73.6872 & 17.232 & 73.9681 & 17.012 \\
\hline 72.7839 & 17.236 & 70.9654 & 17.016 \\
\hline 70.9807 & 17.24 & 69.9673 & 17.02 \\
\hline 71.8818 & 17.244 & 71.965 & 17.024 \\
\hline 69.1818 & 17.248 & 74.9717 & 17.028 \\
\hline 68.284 & 17.252 & 75.9766 & 17.032 \\
\hline 68.284 & 17.256 & 77.9904 & 17.036 \\
\hline
\end{tabular}




\begin{tabular}{|c|c|c|c|c|}
\hline & 66.619 & 15.1 & 47.4583 & 15.156 \\
\hline \multirow[t]{2}{*}{50.4839} & 18.42 & 49.6431 & 16.036 & \\
\hline & 65.6995 & 15.104 & 48.3582 & 15.16 \\
\hline \multirow[t]{2}{*}{49.9296} & 18.424 & 50.5819 & 16.04 & \\
\hline & 64.7811 & 15.108 & 47.4583 & 15.164 \\
\hline \multirow[t]{2}{*}{48.2693} & 18.428 & 50.5819 & 16.044 & \\
\hline & 65.6995 & 15.112 & 48.3582 & 5.168 \\
\hline \multirow[t]{2}{*}{47.1649} & 18.432 & 47.7697 & 16.048 & \\
\hline & 64.7811 & 15.116 & 47.4583 & 15.172 \\
\hline \multirow[t]{2}{*}{46.6135} & 18.436 & 44.0399 & 16.052 & \\
\hline & 64.7811 & 15.12 & 46.5598 & 15.176 \\
\hline \multirow[t]{2}{*}{46.6135} & 18.44 & 42.1837 & 16.056 & \\
\hline & 64.7811 & 15.124 & 48.3582 & 15.18 \\
\hline \multirow[t]{2}{*}{46.6135} & 18.444 & 41.2577 & 16.06 & \\
\hline & 65.6995 & 15.128 & 582 & 15.184 \\
\hline \multirow[t]{2}{*}{46.6135} & 18.448 & 40.3332 & 16.064 & \\
\hline & 64.7 & 15.132 & 82 & 15.188 \\
\hline \multirow[t]{2}{*}{45.512} & 18.4 & 44.0399 & 16.068 & \\
\hline & 63.8 & & 93 & 15.192 \\
\hline \multirow[t]{2}{*}{45.512} & 18.456 & 46.8351 & 16.072 & \\
\hline & & & 83 & 15.196 \\
\hline \multirow[t]{2}{*}{44.4126} & 18.46 & 49.6431 & 16.0 & \\
\hline & & & & 15.2 \\
\hline \multirow[t]{2}{*}{43.8637} & 18.4 & 51.5221 & 16. & \\
\hline & & & 48 & 15.204 \\
\hline 43.8637 & 18.4 & 49.6431 & & \\
\hline & $64{ }^{2}$ & 15 & 82 & 15.208 \\
\hline 44.4126 & 18.472 & 50.5819 & & \\
\hline & 65.6 & 15. & 83 & 15.212 \\
\hline 43.3152 & 18.4 & 44.0 & & \\
\hline & 63.864 & & 593 & 15.216 \\
\hline 44.4126 & 18.48 & & & \\
\hline & 63.8 & 15. & 49.2593 & 15.22 \\
\hline 26 & 184 & 44.0 & 16.1 & \\
\hline & 65.6 & 15. & 2593 & 15.224 \\
\hline 44.4126 & & $47 .^{\circ}$ & & \\
\hline & & 15.1 & & 5.228 \\
\hline 44.4126 & 184 & 48.7 & 16. & \\
\hline & & & & 5.232 \\
\hline 42.2198 & 18.4 & 48.7056 & 16 & \\
\hline & & & & 5.236 \\
\hline 42.7673 & 18.5 & 50.5 & 16 & \\
\hline & & & & 15.24 \\
\hline 41.1264 & 18.5 & 51.5 & 16.12 & \\
\hline & 64.7 & 15. & 593 & 15.244 \\
\hline 40.0351 & 18.508 & 48.7 & 16.124 & \\
\hline & 65. & 15.1 & 593 & 15.248 \\
\hline 40.0351 & 18.512 & 46.8 & & \\
\hline & 63 & & 593 & 15.252 \\
\hline 40.0351 & 18.516 & 49.6431 & & \\
\hline & 64.7 & 15.2 & 593 & 15.256 \\
\hline 40.5806 & 18.52 & & & \\
\hline & 64.7 & 15.2 & 51.0656 & 15.26 \\
\hline 40.0351 & 18.524 & 48.7056 & & \\
\hline & 65.6 & 15.2 & 618 & 15.264 \\
\hline 39.4902 & & 52.4637 & & \\
\hline & 63.8641 & 15.212 & 50.1 & 5.268 \\
\hline 40.0351 & 18.532 & 53.4067 & 16.1 & \\
\hline & & & 56 & 5.272 \\
\hline 40.0351 & 18.536 & 54.3511 & 16.1 & \\
\hline & & & 49.2593 & 15.2 \\
\hline 40.0351 & 18.54 & 54.3511 & 16.156 & \\
\hline & & & 49.2593 & 15.28 \\
\hline 38.402 & 18.544 & 55.2969 & 16.16 & \\
\hline & & & 50.1618 & 15.2 \\
\hline 37.8586 & 18.548 & 54.3511 & 16.164 & \\
\hline & & 15.232 & & 15.288 \\
\hline 38.402 & 18.552 & 53.4067 & 16.168 & \\
\hline & 64.7811 & 15.236 & 51.0656 & 15.292 \\
\hline .8586 & 18.556 & 51.5221 & 16.172 & \\
\hline
\end{tabular}

\begin{tabular}{|c|c|c|c|}
\hline 70.0806 & 17.26 & 77.9904 & 17.04 \\
\hline 70.9807 & 17.264 & 77.9904 & 17.044 \\
\hline 70.0806 & 17.268 & 77.9904 & 17.048 \\
\hline 72.7839 & 17.272 & 74.9717 & 17.052 \\
\hline 73.6872 & 17.276 & 72.9659 & 17.056 \\
\hline 72.7839 & 17.28 & 71.965 & 17.06 \\
\hline 72.7839 & 17.284 & 73.9681 & 17.064 \\
\hline 70.9807 & 17.288 & 74.9717 & 17.068 \\
\hline 70.9807 & 17.292 & 75.9766 & 17.072 \\
\hline 70.9807 & 17.296 & 77.9904 & 17.076 \\
\hline 72.7839 & 17.3 & 77.9904 & 17.08 \\
\hline 73.6872 & 17.304 & 76.9828 & 17.084 \\
\hline 74.5917 & 17.308 & 74.9717 & 17.088 \\
\hline 75.4971 & 17.312 & 74.9717 & 17.092 \\
\hline 76.4037 & 17.316 & 73.9681 & 17.096 \\
\hline 73.6872 & 17.32 & 73.9681 & 17.1 \\
\hline 74.5917 & 17.324 & 73.9681 & 17.104 \\
\hline 74.5917 & 17.328 & 75.9766 & 17.108 \\
\hline 73.6872 & 17.332 & 77.9904 & 17.112 \\
\hline 74.5917 & 17.336 & 77.9904 & 17.116 \\
\hline 73.6872 & 17.34 & 76.9828 & 17.12 \\
\hline 75.4971 & 17.344 & 73.9681 & 17.124 \\
\hline 76.4037 & 17.348 & 73.9681 & 17.128 \\
\hline 80.0408 & 17.352 & 72.9659 & 17.132 \\
\hline 80.0408 & 17.356 & 71.965 & 17.136 \\
\hline 77.3114 & 17.36 & 72.9659 & 17.14 \\
\hline 76.4037 & 17.364 & 73.9681 & 17.144 \\
\hline 75.4971 & 17.368 & 74.9717 & 17.148 \\
\hline 74.5917 & 17.372 & 75.9766 & 17.152 \\
\hline 74.5917 & 17.376 & 73.9681 & 17.156 \\
\hline 76.4037 & 17.38 & 75.9766 & 17.16 \\
\hline 77.3114 & 17.384 & 73.9681 & 17.164 \\
\hline 78.2201 & 17.388 & 72.9659 & 17.168 \\
\hline 78.2201 & 17.392 & 70.9654 & 17.172 \\
\hline 78.2201 & 17.396 & 71.965 & 17.176 \\
\hline
\end{tabular}




\begin{tabular}{|c|c|c|c|c|}
\hline & 65.6995 & 15.24 & 50.1618 & 5.296 \\
\hline \multirow[t]{2}{*}{37.8586} & 18.56 & 54.3511 & 16.176 & \\
\hline & 65.6995 & 15.244 & 49.2593 & 15.3 \\
\hline \multirow[t]{2}{*}{37.8586} & 18.564 & 54.3511 & 16.18 & \\
\hline & 64.7811 & 15.248 & 50.1618 & 15.304 \\
\hline \multirow[t]{2}{*}{38.402} & 18.568 & 58.1424 & 16.184 & \\
\hline & 67.5397 & 15.252 & 50.1618 & 5.308 \\
\hline \multirow[t]{2}{*}{37.8586} & 18.572 & 58.1424 & 16.188 & \\
\hline & 66.619 & 15.256 & 48.3582 & 15.312 \\
\hline \multirow[t]{2}{*}{37.8586} & 18.576 & 60.0462 & 16.192 & \\
\hline & 66.619 & 15.26 & 49.2593 & 15.316 \\
\hline \multirow[t]{2}{*}{37.8586} & 18.58 & 62.9119 & 16.196 & \\
\hline & 65.6995 & 15.264 & 50.1618 & 15.32 \\
\hline \multirow[t]{2}{*}{37.8586} & 18.584 & 61.0001 & 16.2 & \\
\hline & 66.619 & 15.268 & 50.1618 & 15.324 \\
\hline \multirow[t]{2}{*}{36.7734} & 18.588 & 60.0462 & 16.204 & \\
\hline & 65.6995 & 15.272 & 518 & 15.328 \\
\hline \multirow[t]{2}{*}{36.2316} & 18.592 & 56.244 & 16.208 & \\
\hline & 67. & 15.276 & 618 & 15.332 \\
\hline \multirow[t]{2}{*}{36.2316} & 18.596 & 58.1424 & 16. & \\
\hline & 66.619 & 15.28 & 593 & 5.336 \\
\hline \multirow[t]{2}{*}{35.6903} & 18.6 & 58.1424 & & \\
\hline & 67.5397 & 15.284 & 48.3582 & 15.34 \\
\hline \multirow[t]{2}{*}{35.6903} & 18.604 & 61.0001 & 16.22 & \\
\hline & 67.5397 & 15.288 & 49.2593 & 15.344 \\
\hline 35.6903 & 18.608 & 60.0462 & 16.224 & \\
\hline & 67.5397 & 15.292 & 50.1618 & 15.348 \\
\hline 5.6903 & 18.612 & 61.9553 & & \\
\hline & 67.5397 & 15.296 & 618 & 15.352 \\
\hline 496 & 18.616 & 61.9553 & & \\
\hline & 67.5397 & 15.3 & 618 & 15.356 \\
\hline 903 & 18.62 & 61.95 & & \\
\hline & 67.5397 & 15.304 & 656 & 15.36 \\
\hline 6903 & 18.624 & 61.9 & 16 & \\
\hline & 67.5397 & 15.30 & & 15.364 \\
\hline 6903 & 18.628 & 61.0 & 16 & \\
\hline & 67.5397 & & & 15.368 \\
\hline 1496 & 18.632 & 60.0462 & 16. & \\
\hline & 67.5 & 15.316 & 707 & 15.372 \\
\hline 6903 & 18.636 & 58.1424 & 16. & \\
\hline & 67.5397 & 15.32 & 618 & 15.376 \\
\hline 6903 & 18.64 & 58.1424 & 16.256 & \\
\hline & 66.619 & 15.324 & 707 & 15.38 \\
\hline 6903 & 18.644 & 57.1925 & 16.26 & \\
\hline & 66. & 15. & 656 & 15.384 \\
\hline 35.1496 & & 58.1424 & 16.264 & \\
\hline & 67.5397 & 15.3 & 656 & 15.388 \\
\hline 35.6903 & 18.652 & 57.1925 & & \\
\hline & 67.5397 & 15.336 & 51.9707 & 5.392 \\
\hline 34.0696 & 18.656 & 58.1424 & & \\
\hline & 65.6995 & 15.34 & 707 & 15.396 \\
\hline 35.1496 & 18.66 & 59.0936 & 16. & \\
\hline & 66.619 & 15.34 & 656 & 15.4 \\
\hline 34.6093 & 18.664 & 59.0936 & 16.28 & \\
\hline & 67.5397 & 15.348 & 51.0656 & 15.404 \\
\hline 35.6903 & 18.668 & 61.9553 & 16.284 & \\
\hline & 67.5397 & 15.352 & 51.0656 & 15.408 \\
\hline 35.6903 & 18.672 & 62.9119 & 16.288 & \\
\hline & 68.4616 & 15.356 & 51.0656 & 15.412 \\
\hline 34.6093 & 18.676 & 62.9119 & 16.292 & \\
\hline & 67.5397 & 15.36 & 51.0656 & 15.416 \\
\hline 30.1470 & 18.68 & 61.9553 & 16.296 & \\
\hline & 67.5397 & & 51.0656 & 15.42 \\
\hline 0696 & 18.684 & 61.0001 & 16.3 & \\
\hline & 67.5397 & & & 15.424 \\
\hline 6093 & 18.688 & 61.9553 & 16.304 & \\
\hline & 67.5397 & 15.372 & 51.9707 & 15.428 \\
\hline 6093 & 18.692 & 59.0936 & 16.308 & \\
\hline & 66.619 & 15.376 & 51.9707 & 15.432 \\
\hline 1496 & 18.696 & 61.9553 & 16.312 & \\
\hline
\end{tabular}

\begin{tabular}{|c|c|c|c|}
\hline 78.2201 & 17.4 & 71.965 & 17.18 \\
\hline 78.2201 & 17.404 & 71.965 & 17.184 \\
\hline 76.4037 & 17.408 & 73.9681 & 17.188 \\
\hline 77.3114 & 17.412 & 72.9659 & 17.192 \\
\hline 75.4971 & 17.416 & 70.9654 & 17.196 \\
\hline 78.2201 & 17.42 & 70.9654 & 17.2 \\
\hline 79.1299 & 17.424 & 68.9705 & 17.204 \\
\hline 78.2201 & 17.428 & 67.9751 & 17.208 \\
\hline 80.0408 & 17.432 & 67.9751 & 17.212 \\
\hline 79.1299 & 17.436 & 69.9673 & 17.216 \\
\hline 78.2201 & 17.44 & 69.9673 & 17.22 \\
\hline 77.3114 & 17.444 & 69.9673 & 17.224 \\
\hline 77.3114 & 17.448 & 69.9673 & 17.228 \\
\hline 75.4971 & 17.452 & 69.9673 & 17.232 \\
\hline 75.4971 & 17.456 & 68.9705 & 17.236 \\
\hline 75.4971 & 17.46 & 67.9751 & 17.24 \\
\hline 76.4037 & 17.464 & 66.9811 & 17.244 \\
\hline 78.2201 & 17.468 & 65.9884 & 17.248 \\
\hline 78.2201 & 17.472 & 67.9751 & 17.252 \\
\hline 76.4037 & 17.476 & 67.9751 & 17.256 \\
\hline 78.2201 & 17.48 & 68.9705 & 17.26 \\
\hline 77.3114 & 17.484 & 66.9811 & 17.264 \\
\hline 76.4037 & 17.488 & 68.9705 & 17.268 \\
\hline 76.4037 & 17.492 & 67.9751 & 17.272 \\
\hline 76.4037 & 17.496 & 67.9751 & 17.276 \\
\hline 76.4037 & 17.5 & 67.9751 & 17.28 \\
\hline 77.3114 & 17.504 & 65.9884 & 17.284 \\
\hline 78.2201 & 17.508 & 66.9811 & 17.288 \\
\hline 78.2201 & 17.512 & 66.9811 & 17.292 \\
\hline 78.2201 & 17.516 & 68.9705 & 17.296 \\
\hline 76.4037 & 17.52 & 65.9884 & 17.3 \\
\hline 75.4971 & 17.524 & 69.9673 & 17.304 \\
\hline 76.4037 & 17.528 & 66.9811 & 17.308 \\
\hline 77.3114 & 17.532 & 67.9751 & 17.312 \\
\hline 76.4037 & 17.536 & 68.9705 & 17.316 \\
\hline
\end{tabular}




\begin{tabular}{|c|c|c|c|c|}
\hline & 68.4616 & 15.38 & 51.9707 & 5.436 \\
\hline \multirow[t]{2}{*}{35.6903} & 18.7 & 61.0001 & 16.316 & \\
\hline & 67.5397 & 15.384 & 51.9707 & 15.44 \\
\hline \multirow[t]{2}{*}{35.1496} & 18.704 & 61.9553 & 16.32 & \\
\hline & 66.619 & 15.388 & 51.9707 & 15.444 \\
\hline \multirow[t]{2}{*}{35.1496} & 18.708 & 61.9553 & 16.324 & \\
\hline & 65.6995 & 15.392 & 51.9707 & 15.448 \\
\hline \multirow[t]{2}{*}{35.1496} & 18.712 & 61.0001 & 16.328 & \\
\hline & 67.5397 & 15.396 & 51.9707 & 15.452 \\
\hline \multirow[t]{2}{*}{34.6093} & 18.716 & 58.1424 & 16.332 & \\
\hline & 66.619 & 15.4 & 51.9707 & 15.456 \\
\hline \multirow[t]{2}{*}{35.6903} & 18.72 & 60.0462 & 16.336 & \\
\hline & 67.5397 & 15.404 & 51.0656 & 15.46 \\
\hline \multirow[t]{2}{*}{34.6093} & 18.724 & 58.1424 & 16.34 & \\
\hline & 67.5397 & 15.408 & 51.9707 & 15.464 \\
\hline \multirow[t]{2}{*}{35.1496} & 18.728 & 59.0936 & 16.344 & \\
\hline & 67.5397 & 15.412 & 556 & 15.468 \\
\hline \multirow[t]{2}{*}{35.1496} & 18.732 & 59.0936 & 16.348 & \\
\hline & 65.6995 & 15.416 & 56 & 15.472 \\
\hline \multirow[t]{2}{*}{35.1496} & 18.736 & 61.0001 & 16. & \\
\hline & 67.5397 & 15.42 & 707 & 15.476 \\
\hline \multirow[t]{2}{*}{35.1496} & 18.74 & 61.9553 & & \\
\hline & 67.5397 & 15.424 & 51.5 & 15.48 \\
\hline \multirow[t]{2}{*}{35.1496} & 18.744 & 61.0001 & 16.36 & \\
\hline & 67.5397 & 15.428 & 52.877 & 15.484 \\
\hline 34.6093 & 18.748 & 58.1424 & 16.364 & \\
\hline & 68.4616 & 15.432 & 51.9707 & 15.488 \\
\hline 5.6903 & 18.752 & 58.1424 & & \\
\hline & 68.4616 & 15.436 & 847 & 15.492 \\
\hline 1496 & 18.756 & 57.1925 & & \\
\hline & 69.3847 & 15.44 & 707 & 15.496 \\
\hline 903 & 18.76 & 57. & & \\
\hline & 67.5397 & 15.444 & & 15.5 \\
\hline 6903 & 18.764 & 58. & 16 & \\
\hline & 67.5397 & 15.448 & & 5.504 \\
\hline 6093 & 18.768 & 59.0 & 16 & \\
\hline & 68.4616 & & & 15.508 \\
\hline 1496 & 18.772 & 59.0936 & 16 & \\
\hline & 70.3089 & 15.456 & 347 & 15.512 \\
\hline 6903 & 18.776 & 58.1424 & 16 & \\
\hline & 67.5397 & 15.46 & 52 & 15.516 \\
\hline 6093 & 18.78 & 58.1424 & 16 & \\
\hline & 67.5397 & 15.464 & 847 & 15.52 \\
\hline 35.6903 & 18.784 & 60.0462 & 16.4 & \\
\hline & 68.4616 & 15. & 707 & 15.524 \\
\hline 34.6093 & 18.788 & 58.1424 & & \\
\hline & 67.5397 & 15. & 52. & 15.528 \\
\hline 35.6903 & 18.792 & 60.0462 & & \\
\hline & 68.4616 & 15.476 & 53.7847 & 15532 \\
\hline 35.1496 & 18.796 & 59.0936 & & \\
\hline & 69.3847 & 15.48 & 52 & 15.536 \\
\hline 35.6903 & 18.8 & 57.1925 & 16. & \\
\hline & 67.5397 & 15.484 & 347 & 15.54 \\
\hline 35.6903 & 18.804 & 57.1925 & 16.42 & \\
\hline & 67.5397 & 15.488 & & 5.54 \\
\hline 35.1496 & 18.808 & 58.1424 & 16. & \\
\hline & 68.4616 & 15.492 & 54.6936 & 5.5 \\
\hline 35.1496 & 18.812 & 58.1424 & 16.428 & \\
\hline & 69.3847 & 15.496 & 53.7847 & 0.2 \\
\hline 35.6903 & 18.816 & 58.1424 & 16.432 & \\
\hline & 70.3089 & 15.5 & 53.7847 & 15.556 \\
\hline 35.6903 & 18.82 & 60.0462 & 16.436 & \\
\hline & 68.4616 & & 53.7847 & 15.56 \\
\hline 903 & 18.824 & 59.0936 & 16.44 & \\
\hline & 68.4616 & 15.508 & & 15.564 \\
\hline 496 & 18.828 & 58.1424 & 16.444 & \\
\hline & 69.3847 & 15.512 & 52.877 & 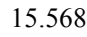 \\
\hline 6903 & 18.832 & 57.1925 & & \\
\hline & 67.5397 & 15.516 & 53.7847 & 15 \\
\hline .6093 & 18.836 & 57.1925 & 16.452 & \\
\hline
\end{tabular}

\begin{tabular}{|c|c|c|c|}
\hline 77.3114 & 17.54 & 69.9673 & 17.32 \\
\hline 78.2201 & 17.544 & 68.9705 & 17.324 \\
\hline 77.3114 & 17.548 & 69.9673 & 17.328 \\
\hline 76.4037 & 17.552 & 70.9654 & 17.332 \\
\hline 78.2201 & 17.556 & 71.965 & 17.336 \\
\hline 77.3114 & 17.56 & 71.965 & 17.34 \\
\hline 76.4037 & 17.564 & 69.9673 & 17.344 \\
\hline 75.4971 & 17.568 & 69.9673 & 17.348 \\
\hline 76.4037 & 17.572 & 70.9654 & 17.352 \\
\hline 76.4037 & 17.576 & 70.9654 & 17.356 \\
\hline 77.3114 & 17.58 & 69.9673 & 17.36 \\
\hline 77.3114 & 17.584 & 70.9654 & 17.364 \\
\hline 78.2201 & 17.588 & 73.9681 & 17.368 \\
\hline 77.3114 & 17.592 & 75.9766 & 17.372 \\
\hline 80.0408 & 17.596 & 75.9766 & 17.376 \\
\hline 77.3114 & 17.6 & 74.9717 & 17.38 \\
\hline 78.2201 & 17.604 & 74.9717 & 17.384 \\
\hline 79.1299 & 17.608 & 74.9717 & 17.388 \\
\hline 78.2201 & 17.612 & 75.9766 & 17.392 \\
\hline 78.2201 & 17.616 & 75.9766 & 17.396 \\
\hline 78.2201 & 17.62 & 75.9766 & 17.4 \\
\hline 79.1299 & 17.624 & 76.9828 & 17.404 \\
\hline 79.1299 & 17.628 & 76.9828 & 17.408 \\
\hline 80.0408 & 17.632 & 77.9904 & 17.412 \\
\hline 80.9527 & 17.636 & 77.9904 & 17.416 \\
\hline 80.0408 & 17.64 & 77.9904 & 17.42 \\
\hline 79.1299 & 17.644 & 77.9904 & 17.424 \\
\hline 79.1299 & 17.648 & 76.9828 & 17.428 \\
\hline 80.0408 & 17.652 & 75.9766 & 17.432 \\
\hline 81.8657 & 17.656 & 76.9828 & 17.436 \\
\hline 81.8657 & 17.66 & 76.9828 & 17.44 \\
\hline 81.8657 & 17.664 & 77.9904 & 17.444 \\
\hline 81.8657 & 17.668 & 77.9904 & 17.448 \\
\hline 80.0408 & 17.672 & 77.9904 & 17.452 \\
\hline 80.9527 & 17.676 & 78.9993 & 17.456 \\
\hline
\end{tabular}




\begin{tabular}{|c|c|c|c|c|}
\hline & 68.4616 & 15.52 & 52.877 & 15.576 \\
\hline \multirow[t]{2}{*}{35.1496} & 18.84 & 55.2969 & 16.456 & \\
\hline & 67.5397 & 15.524 & 53.7847 & 15.58 \\
\hline \multirow[t]{2}{*}{35.6903} & 18.844 & 57.1925 & 16.46 & \\
\hline & 67.5397 & 15.528 & 55.6038 & 15.584 \\
\hline \multirow[t]{2}{*}{35.6903} & 18.848 & 56.244 & 16.464 & \\
\hline & 68.4616 & 15.532 & 54.6936 & 15.588 \\
\hline \multirow[t]{2}{*}{35.6903} & 18.852 & 56.244 & 16.468 & \\
\hline & 67.5397 & 15.536 & 53.7847 & 15.592 \\
\hline \multirow[t]{2}{*}{35.6903} & 18.856 & 57.1925 & 16.472 & \\
\hline & 68.4616 & 15.54 & 54.6936 & 15.596 \\
\hline \multirow[t]{2}{*}{35.6903} & 18.86 & 58.1424 & 16.476 & \\
\hline & 68.4616 & 15.544 & 55.6038 & 15.6 \\
\hline \multirow[t]{2}{*}{35.6903} & 18.864 & 58.1424 & 16.48 & \\
\hline & 70.3089 & 15.548 & 54.6936 & 15.604 \\
\hline \multirow[t]{2}{*}{35.6903} & 18.868 & 58.1424 & 16.484 & \\
\hline & 71.2344 & 15.552 & 936 & 15.608 \\
\hline \multirow[t]{2}{*}{35.6903} & 18.872 & 58.1424 & 16.488 & \\
\hline & 68. & & 38 & 15.612 \\
\hline \multirow[t]{2}{*}{35.6903} & 18.876 & 57.1925 & 16. & \\
\hline & 69.3847 & 15.56 & 038 & 15.616 \\
\hline \multirow[t]{2}{*}{35.6903} & 18.88 & 57.1925 & 16. & \\
\hline & 69.3847 & 15.564 & 038 & 15.62 \\
\hline \multirow[t]{2}{*}{35.6903} & 18.884 & 58.1424 & 16 & \\
\hline & 70.3089 & 15.568 & 55.6038 & 15.624 \\
\hline 36.2316 & 18.888 & 59.0936 & & \\
\hline & 69.3847 & 15.572 & 54.6936 & 15.628 \\
\hline 5.6903 & 18.892 & 59.0936 & & \\
\hline & 69.3847 & 15.576 & 038 & 15.632 \\
\hline 903 & 18.896 & 62 & & \\
\hline & 68.4616 & 15.58 & 936 & 15.636 \\
\hline 6 & 18.9 & 61.0001 & & \\
\hline & 68.4616 & & & 15.64 \\
\hline 6903 & 18.9 & 59. & 16 & \\
\hline & 68.4616 & & & 15.644 \\
\hline 6903 & 18.908 & 59.0 & 16 & \\
\hline & 67.5 & & & 15.648 \\
\hline 6903 & 18.912 & 60.0462 & 16. & \\
\hline & 68.4616 & 15.596 & 038 & 15.652 \\
\hline 7734 & 18.916 & 61.0001 & 16 & \\
\hline & 70.3 & 15.6 & 153 & 15.656 \\
\hline 7.3158 & 18.92 & 60.0462 & 16. & \\
\hline & 70. & 15.604 & 936 & 15.66 \\
\hline 35.6903 & 18.924 & 61.9553 & & \\
\hline & 70.3089 & 15. & 38 & 15.664 \\
\hline 35.6903 & 18.928 & 61.9553 & & \\
\hline & 71.2344 & 15.612 & 153 & 15.668 \\
\hline 36.7734 & 18.932 & 62.9119 & & \\
\hline & 69.3847 & 15.616 & 54.6936 & 15.672 \\
\hline 35.6903 & 18.936 & 61.9553 & & \\
\hline & 68.4616 & 15.62 & 53 & 15.676 \\
\hline 36.7734 & 18.94 & 61.0001 & 16 & \\
\hline & 71.2344 & 15.624 & 038 & 15.68 \\
\hline 36.2316 & 18.944 & 62.9119 & 16.56 & \\
\hline & 69.3847 & 15.628 & & 15.684 \\
\hline 35.6903 & 18.948 & 61.0001 & 16.564 & \\
\hline & 70.3089 & 15.632 & 54.6936 & 15.688 \\
\hline 37.8586 & 18.952 & 61.9 & 16.568 & \\
\hline & 70.3089 & & & 15.69 \\
\hline 37.3158 & 18.956 & 61.9553 & 16.572 & \\
\hline & 71.2344 & 15.64 & 55.6038 & 15.696 \\
\hline 37.3158 & 18.96 & 61.9553 & 16.576 & \\
\hline & 69.3847 & 15.644 & 54.6936 & 15.7 \\
\hline 7734 & 18.964 & 63.8699 & 16.58 & \\
\hline & 70.3089 & & & 15.704 \\
\hline 73 & 18.968 & 63.8699 & 16.584 & \\
\hline & 70.3089 & 15.652 & 55.6038 & 15.708 \\
\hline 3158 & 18.972 & 62.9119 & & \\
\hline & 70.3089 & 15.656 & 55.6038 & 15.712 \\
\hline 7.8586 & 18.976 & 63.8699 & 16.592 & \\
\hline
\end{tabular}

\begin{tabular}{|c|c|c|c|}
\hline 80.9527 & 17.68 & 78.9993 & 17.46 \\
\hline 80.0408 & 17.684 & 77.9904 & 17.464 \\
\hline 81.8657 & 17.688 & 77.9904 & 17.468 \\
\hline 82.7798 & 17.692 & 77.9904 & 17.472 \\
\hline 85.5282 & 17.696 & 77.9904 & 17.476 \\
\hline 86.4464 & 17.7 & 77.9904 & 17.48 \\
\hline 83.6949 & 17.704 & 77.9904 & 17.484 \\
\hline 86.4464 & 17.708 & 78.9993 & 17.488 \\
\hline 88.286 & 17.712 & 81.021 & 17.492 \\
\hline 84.611 & 17.716 & 80.0095 & 17.496 \\
\hline 86.4464 & 17.72 & 81.021 & 17.5 \\
\hline 86.4464 & 17.724 & 78.9993 & 17.504 \\
\hline 84.611 & 17.728 & 78.9993 & 17.508 \\
\hline 82.7798 & 17.732 & 77.9904 & 17.512 \\
\hline 84.611 & 17.736 & 77.9904 & 17.516 \\
\hline 82.7798 & 17.74 & 76.9828 & 17.52 \\
\hline 82.7798 & 17.744 & 78.9993 & 17.524 \\
\hline 80.9527 & 17.748 & 77.9904 & 17.528 \\
\hline 81.8657 & 17.752 & 77.9904 & 17.532 \\
\hline 82.7798 & 17.756 & 77.9904 & 17.536 \\
\hline 82.7798 & 17.76 & 77.9904 & 17.54 \\
\hline 82.7798 & 17.764 & 77.9904 & 17.544 \\
\hline 81.8657 & 17.768 & 75.9766 & 17.548 \\
\hline 83.6949 & 17.772 & 77.9904 & 17.552 \\
\hline 83.6949 & 17.776 & 76.9828 & 17.556 \\
\hline 82.7798 & 17.78 & 76.9828 & 17.56 \\
\hline 82.7798 & 17.784 & 76.9828 & 17.564 \\
\hline 83.6949 & 17.788 & 77.9904 & 17.568 \\
\hline 82.7798 & 17.792 & 74.9717 & 17.572 \\
\hline 81.8657 & 17.796 & 76.9828 & 17.576 \\
\hline 84.611 & 17.8 & 74.9717 & 17.58 \\
\hline 81.8657 & 17.804 & 73.9681 & 17.584 \\
\hline 81.8657 & 17.808 & 73.9681 & 17.588 \\
\hline 82.7798 & 17.812 & 74.9717 & 17.592 \\
\hline 81.8657 & 17.816 & 74.9717 & 17.596 \\
\hline
\end{tabular}




\begin{tabular}{|c|c|c|c|c|}
\hline & 70.3089 & 15.66 & 55.6038 & 15.716 \\
\hline \multirow[t]{2}{*}{36.7734} & 18.98 & 63.8699 & 16.596 & \\
\hline & 70.3089 & 15.664 & 56.5153 & 15.72 \\
\hline \multirow[t]{2}{*}{36.7734} & 18.984 & 63.8699 & 16.6 & \\
\hline & 70.3089 & 15.668 & 56.5153 & 15.724 \\
\hline \multirow[t]{2}{*}{37.3158} & 18.988 & 63.8699 & 16.604 & \\
\hline & 69.3847 & 15.672 & 56.5153 & 15.728 \\
\hline \multirow[t]{2}{*}{36.7734} & 18.992 & 64.8291 & 16.608 & \\
\hline & 70.3089 & 15.676 & 55.6038 & 15.732 \\
\hline \multirow[t]{2}{*}{36.2316} & 18.996 & 64.8291 & 16.612 & \\
\hline & 70.3089 & 15.68 & 56.5153 & 5.736 \\
\hline \multirow[t]{2}{*}{36.7734} & 19 & 66.7515 & 16.616 & \\
\hline & 70.3089 & 15.684 & 56.5153 & 15.74 \\
\hline \multirow[t]{2}{*}{37.8586} & 19.004 & 66.7515 & 16.62 & \\
\hline & 71.2344 & 15.688 & 153 & 15.744 \\
\hline \multirow[t]{2}{*}{37.8586} & 19.008 & 67.7147 & 16.624 & \\
\hline & 72.1 & 15.6 & 38 & 15.748 \\
\hline \multirow[t]{2}{*}{37.8586} & 19.012 & 69.645 & 16.628 & \\
\hline & 71.2 & & & 15.752 \\
\hline \multirow[t]{2}{*}{36.7734} & 19.016 & 67.7147 & 16.632 & \\
\hline & 71.2344 & 15.7 & & 15.756 \\
\hline \multirow[t]{2}{*}{37.8586} & 19.02 & 68.6792 & 16.6 & \\
\hline & 71.2344 & & & 15.76 \\
\hline \multirow[t]{2}{*}{37.8586} & 19.024 & 66.7515 & 16.64 & \\
\hline & 71.2 & $15 .^{\circ}$ & & 15.7 \\
\hline 36.2316 & 19.028 & 68.6792 & 16.644 & \\
\hline & 72. & 15. & 53 & 15.768 \\
\hline 36.7734 & 19.032 & 67.7147 & & \\
\hline & 72. & 15.716 & 56 & 72 \\
\hline 37.8586 & & & & \\
\hline & 71. & 15.72 & 56 & 6 \\
\hline 37.8586 & & 67.7147 & & \\
\hline & 71.2344 & 15.724 & & 57 \\
\hline 37.8586 & 19.044 & 67.7147 & & \\
\hline & 71.2344 & 15.728 & 57. & 5.784 \\
\hline 37.8586 & 19.048 & 67.7147 & & \\
\hline & 72.1609 & 15.732 & 53 & 5.7 \\
\hline 158 & 19.052 & 65.7897 & 16. & \\
\hline & & & & 2 \\
\hline 3158 & 19.0 & 897 & 16 & \\
\hline & & & & 6 \\
\hline 37.8586 & 19.06 & 66.7515 & 16 & \\
\hline & & & & 10 \\
\hline 37.8586 & 19.064 & 66.7515 & 16 & \\
\hline & & & & 15.804 \\
\hline 158 & 10068 & 65.7897 & 16. & \\
\hline & 72. & 15.7 & 73 & 15.808 \\
\hline 36.7734 & 19.072 & 66.7515 & & \\
\hline & 72. & 15. & 38 & 15.812 \\
\hline 37.3158 & 19.076 & 65.7897 & 16.692 & \\
\hline & 71.2344 & 15.76 & 56. & 15.816 \\
\hline 37.3158 & 19.08 & 66.7515 & & \\
\hline & 72.1609 & 15.764 & 59.2573 & 15.82 \\
\hline 37.3158 & & 67.7147 & 16.7 & \\
\hline & 72.1609 & 15.768 & 153 & 15.824 \\
\hline 37.3158 & & 65.7897 & & \\
\hline & 72.1609 & 15.772 & 56 & 8 \\
\hline 37.3158 & & & & \\
\hline & 72.1 & 15.776 & & 2 \\
\hline 37.8586 & 19.096 & 66.7515 & 16.7 & \\
\hline & 71.2344 & & & 15.836 \\
\hline 37.8586 & 19.1 & 65.7897 & 16.7 & \\
\hline & 71.2344 & & 59.2573 & 15.8 \\
\hline 37.8586 & 19.104 & 65.7897 & 16.72 & \\
\hline & 72.1609 & & & 58.820 \\
\hline 37.8586 & 19.108 & 66.7515 & 16.724 & \\
\hline & 72.1609 & 15.792 & 58.342 & 58 \\
\hline 8586 & 19.112 & 65.7897 & 16.728 & \\
\hline & 72.1609 & 15.796 & 58.342 & 15.852 \\
\hline 3158 & 19.116 & 65.7897 & 16.732 & \\
\hline
\end{tabular}

\begin{tabular}{|c|c|c|c|}
\hline 81.8657 & 17.82 & 73.9681 & 17.6 \\
\hline 80.0408 & 17.824 & 73.9681 & 17.604 \\
\hline 79.1299 & 17.828 & 74.9717 & 17.608 \\
\hline 80.0408 & 17.832 & 73.9681 & 17.612 \\
\hline 80.0408 & 17.836 & 73.9681 & 17.616 \\
\hline 80.0408 & 17.84 & 73.9681 & 17.62 \\
\hline 80.0408 & 17.844 & 74.9717 & 17.624 \\
\hline 80.9527 & 17.848 & 73.9681 & 17.628 \\
\hline 80.9527 & 17.852 & 73.9681 & 17.632 \\
\hline 80.0408 & 17.856 & 74.9717 & 17.636 \\
\hline 80.0408 & 17.86 & 75.9766 & 17.64 \\
\hline 82.7798 & 17.864 & 74.9717 & 17.644 \\
\hline 82.7798 & 17.868 & 73.9681 & 17.648 \\
\hline 81.8657 & 17.872 & 74.9717 & 17.652 \\
\hline 82.7798 & 17.876 & 73.9681 & 17.656 \\
\hline 79.1299 & 17.88 & 74.9717 & 17.66 \\
\hline 79.1299 & 17.884 & 74.9717 & 17.664 \\
\hline 80.0408 & 17.888 & 73.9681 & 17.668 \\
\hline 80.9527 & 17.892 & 74.9717 & 17.672 \\
\hline 80.0408 & 17.896 & 74.9717 & 17.676 \\
\hline 81.8657 & 17.9 & 74.9717 & 17.68 \\
\hline 80.0408 & 17.904 & 75.9766 & 17.684 \\
\hline 81.8657 & 17.908 & 77.9904 & 17.688 \\
\hline 80.9527 & 17.912 & 75.9766 & 17.692 \\
\hline 81.8657 & 17.916 & 76.9828 & 17.696 \\
\hline 80.0408 & 17.92 & 73.9681 & 17.7 \\
\hline 81.8657 & 17.924 & 75.9766 & 17.704 \\
\hline 81.8657 & 17.928 & 75.9766 & 17.708 \\
\hline 80.9527 & 17.932 & 75.9766 & 17.712 \\
\hline 81.8657 & 17.936 & 75.9766 & 17.716 \\
\hline 83.6949 & 17.94 & 77.9904 & 17.72 \\
\hline 83.6949 & 17.944 & 76.9828 & 17.724 \\
\hline 82.7798 & 17.948 & 78.9993 & 17.728 \\
\hline 83.6949 & 17.952 & 77.9904 & 17.732 \\
\hline 81.8657 & 17.956 & 81.021 & 17.736 \\
\hline
\end{tabular}




\begin{tabular}{|c|c|c|c|c|}
\hline & 72.1609 & 15.8 & 58.342 & 15.856 \\
\hline \multirow[t]{2}{*}{37.8586} & 19.12 & 66.7515 & 16.736 & \\
\hline & 72.1609 & 15.804 & 57.428 & 15.86 \\
\hline \multirow[t]{2}{*}{37.8586} & 19.124 & 66.7515 & 16.74 & \\
\hline & 73.0887 & 15.808 & 58.342 & 15.864 \\
\hline \multirow[t]{2}{*}{37.8586} & 19.128 & 67.7147 & 16.744 & \\
\hline & 73.0887 & 15.812 & 59.2573 & 15.868 \\
\hline \multirow[t]{2}{*}{37.8586} & 19.132 & 68.6792 & 16.748 & \\
\hline & 71.2344 & 15.816 & 58.342 & 15.872 \\
\hline \multirow[t]{2}{*}{37.3158} & 19.136 & 70.612 & 16.752 & \\
\hline & 74.0176 & 15.82 & 58.342 & 5.876 \\
\hline \multirow[t]{2}{*}{37.8586} & 19.14 & 73.5209 & 16.756 & \\
\hline & 74.0176 & 15.824 & 57.428 & 15.88 \\
\hline \multirow[t]{2}{*}{37.8586} & 19.144 & 72.55 & 16.76 & \\
\hline & 71.2344 & 15.828 & 57.428 & 15.884 \\
\hline \multirow[t]{2}{*}{37.3158} & 19.148 & 70.6 & 16.764 & \\
\hline & 73.0 & 15.8 & & 15.888 \\
\hline \multirow[t]{2}{*}{37.3158} & 19.152 & 70.612 & 16.768 & \\
\hline & 73.0887 & & 59.2573 & 15.892 \\
\hline \multirow[t]{2}{*}{37.3158} & 19.156 & 68.6792 & 16.772 & \\
\hline & 72.1609 & & 573 & 15.896 \\
\hline \multirow[t]{2}{*}{37.3158} & 19.16 & 65.7897 & 16.776 & \\
\hline & 73.0 & & & 15.9 \\
\hline \multirow[t]{2}{*}{36.7734} & 19.164 & 65.7897 & 16.78 & \\
\hline & 73.0 & & 59.2573 & 15.904 \\
\hline 37.3158 & 19.168 & 66.7 & & \\
\hline & 73.0 & 15. & & 5.908 \\
\hline 36.2316 & 19.172 & & & \\
\hline & 74.0176 & 15 & 573 & .912 \\
\hline 36.7734 & 19.176 & & & \\
\hline & 72.1 & 15 & & 916 \\
\hline 36.7734 & 19.18 & & & \\
\hline & 72.1609 & 15. & & 5.92 \\
\hline 3158 & 19.184 & & 16.8 & \\
\hline & 73.0887 & 15. & 2573 & 15.924 \\
\hline 734 & 19.188 & & & \\
\hline & 72.1609 & & 738 & 5.928 \\
\hline 158 & 19.192 & 97 & 16.808 & \\
\hline & 72.1609 & & & 932 \\
\hline 7734 & 19.1 & 65. & 16 & \\
\hline & 73.0887 & & & 936 \\
\hline 37.3158 & 19.2 & 63.8 & 16 & \\
\hline & & & & 15.94 \\
\hline 37.3158 & 19.204 & 91 & 16.82 & \\
\hline & 71.2 & & & 15.944 \\
\hline 158 & 19.208 & 91 & 16.824 & \\
\hline & 74.0 & 15 & 58 & 15.948 \\
\hline 36.2316 & 19.212 & 66.7515 & 16.828 & \\
\hline & 74.0 & 15 & 573 & 15.952 \\
\hline 36.2316 & 19.216 & 68.6792 & 16.832 & \\
\hline & 73.0887 & 15.9 & 58 & 15.956 \\
\hline 37.3158 & 19.22 & 69.645 & & \\
\hline & 72.1609 & 15.9 & 58 & 15.96 \\
\hline 36.7734 & 19.224 & 69.645 & & \\
\hline & 73.0887 & 15.9 & & 15.964 \\
\hline 36.7734 & 19.228 & 66.7 & & \\
\hline & 73.0887 & 15.912 & 59.2573 & .968 \\
\hline 36.7734 & 19.232 & & & \\
\hline & 73.0887 & & 59.2573 & 5.972 \\
\hline 36.7734 & 19.236 & 63.8699 & 16.852 & \\
\hline & 74.0176 & & 59.2573 & 15.976 \\
\hline 37.3158 & 19.24 & 64.8291 & 16.856 & \\
\hline & 72.1609 & & 60.1738 & 15.9 \\
\hline 37.3158 & 19.244 & 65.7897 & 16.86 & \\
\hline & 73.0887 & & 59.2573 & 5.98 \\
\hline 37.3158 & 19.248 & 66.7515 & 16.864 & \\
\hline & 73.0887 & 15.932 & 59.2573 & 15.98 \\
\hline 1734 & 19.252 & 69.645 & 16.868 & \\
\hline & 73.0887 & 15.936 & 59.2573 & 15.99 \\
\hline 2316 & 19.256 & 70.612 & 16.872 & \\
\hline
\end{tabular}

\begin{tabular}{|c|c|c|c|}
\hline 82.7798 & 17.96 & 77.9904 & 17.74 \\
\hline 81.8657 & 17.964 & 78.9993 & 17.744 \\
\hline 80.9527 & 17.968 & 80.0095 & 17.748 \\
\hline 81.8657 & 17.972 & 80.0095 & 17.752 \\
\hline 82.7798 & 17.976 & 78.9993 & 17.756 \\
\hline 83.6949 & 17.98 & 82.0338 & 17.76 \\
\hline 81.8657 & 17.984 & 80.0095 & 17.764 \\
\hline 80.0408 & 17.988 & 81.021 & 17.768 \\
\hline 82.7798 & 17.992 & 81.021 & 17.772 \\
\hline 83.6949 & 17.996 & 77.9904 & 17.776 \\
\hline 83.6949 & 18 & 81.021 & 17.78 \\
\hline 83.6949 & 18.004 & 81.021 & 17.784 \\
\hline 82.7798 & 18.008 & 82.0338 & 17.788 \\
\hline 81.8657 & 18.012 & 82.0338 & 17.792 \\
\hline 80.9527 & 18.016 & 82.0338 & 17.796 \\
\hline 82.7798 & 18.02 & 83.048 & 17.8 \\
\hline 82.7798 & 18.024 & 85.08 & 17.804 \\
\hline 82.7798 & 18.028 & 84.0634 & 17.808 \\
\hline 85.5282 & 18.032 & 83.048 & 17.812 \\
\hline 85.5282 & 18.036 & 84.0634 & 17.816 \\
\hline 83.6949 & 18.04 & 83.048 & 17.82 \\
\hline 83.6949 & 18.044 & 84.0634 & 17.824 \\
\hline 85.5282 & 18.048 & 83.048 & 17.828 \\
\hline 86.4464 & 18.052 & 82.0338 & 17.832 \\
\hline 86.4464 & 18.056 & 84.0634 & 17.836 \\
\hline 85.5282 & 18.06 & 84.0634 & 17.84 \\
\hline 82.7798 & 18.064 & 83.048 & 17.844 \\
\hline 81.8657 & 18.068 & 82.0338 & 17.848 \\
\hline 84.611 & 18.072 & 82.0338 & 17.852 \\
\hline 83.6949 & 18.076 & 82.0338 & 17.856 \\
\hline 83.6949 & 18.08 & 82.0338 & 17.86 \\
\hline 83.6949 & 18.084 & 82.0338 & 17.864 \\
\hline 86.4464 & 18.088 & 84.0634 & 17.868 \\
\hline 85.5282 & 18.092 & 82.0338 & 17.872 \\
\hline 86.4464 & 18.096 & 82.0338 & 17.876 \\
\hline
\end{tabular}




\begin{tabular}{|c|c|c|c|c|}
\hline & 73.0887 & 15.94 & 58.342 & 15.996 \\
\hline \multirow[t]{2}{*}{37.3158} & 19.26 & 71.5804 & 16.876 & \\
\hline & 73.0887 & 15.944 & 59.2573 & 16 \\
\hline \multirow[t]{2}{*}{36.2316} & 19.264 & 70.612 & 16.88 & \\
\hline & 4.0176 & 15.948 & 56.5153 & 16.004 \\
\hline \multirow[t]{2}{*}{36.2316} & 19.268 & 69.645 & 16.884 & \\
\hline & 74.9477 & 15.952 & 57.428 & 16.008 \\
\hline \multirow[t]{2}{*}{37.3158} & 19.272 & 68.6792 & 16.888 & \\
\hline & 74.9477 & 15.956 & 56.5153 & 16.012 \\
\hline \multirow[t]{2}{*}{37.3158} & 19.276 & 65.7897 & 16.892 & \\
\hline & 74.0176 & 15.96 & 56.5153 & 16.016 \\
\hline \multirow[t]{2}{*}{37.3158} & 19.28 & 65.7897 & 16.896 & \\
\hline & 74.0176 & 15.964 & 57.428 & 16.02 \\
\hline \multirow[t]{2}{*}{37.3158} & 19.284 & 66.7515 & 16.9 & \\
\hline & 73.0887 & 15.968 & 58.342 & 16.024 \\
\hline \multirow[t]{2}{*}{37.8586} & 19.288 & 67.7147 & 16.904 & \\
\hline & 74.0176 & 15.972 & 57.428 & 16.028 \\
\hline \multirow[t]{2}{*}{36.7734} & 19.292 & 69.645 & 16.908 & \\
\hline & 74.9477 & 15.976 & 54.6936 & 16.032 \\
\hline \multirow[t]{2}{*}{37.8586} & 19.296 & 69.645 & 16.912 & \\
\hline & 74.9477 & 15.98 & 54.6936 & 16.036 \\
\hline \multirow[t]{2}{*}{37.8586} & 19.3 & 69.645 & 16.916 & \\
\hline & 73.0887 & 15. & 936 & 16.04 \\
\hline \multirow[t]{2}{*}{37.8586} & 19.304 & 69.645 & 16.92 & \\
\hline & 73.0887 & 15. & 936 & 16.044 \\
\hline 37.3158 & 19.308 & 66.7515 & 16.924 & \\
\hline & 74.0176 & 15.992 & 936 & 16.048 \\
\hline 37.8586 & 19.312 & 66.7515 & 16.928 & \\
\hline & 71.2344 & 15.996 & 52.877 & 16.052 \\
\hline 37.8586 & 19.316 & 71.5804 & & \\
\hline & 72.1609 & 16 & 52. & 16.056 \\
\hline 37.3158 & 19.32 & 72.55 & & \\
\hline & 66.619 & 16.004 & 53.7847 & 16.06 \\
\hline 37.3158 & 19.324 & 72.55 & 16.94 & \\
\hline & 52.9545 & 16.008 & 54.6936 & 16.064 \\
\hline 37.3158 & 19.328 & 72.55 & 16.944 & \\
\hline & 20.5032 & 16.012 & 56.5153 & 16.068 \\
\hline 37.8586 & 19.332 & 70.612 & 16.948 & \\
\hline & & & & 16.072 \\
\hline 37.8586 & 19.336 & 68.6792 & 16.952 & \\
\hline & & & & 16.076 \\
\hline 37.8586 & 19.34 & 68.6792 & 16.956 & \\
\hline & & & & 16.08 \\
\hline 37.8586 & 19.344 & 71.5804 & 16.96 & \\
\hline & & & 55.6038 & 16.084 \\
\hline 37.8586 & 19.348 & 75.4665 & 16.964 & \\
\hline & & & 54.6936 & 16.088 \\
\hline 38.402 & 19.352 & 81.3332 & 16.968 & \\
\hline & & & 56.5153 & 16.092 \\
\hline 37.8586 & 19.356 & 82.3153 & 16.972 & \\
\hline & & & 56.5153 & 16.096 \\
\hline 37.8586 & 19.36 & 81.3332 & 16.976 & \\
\hline & & & 56.5153 & 16.1 \\
\hline 37.8586 & 19.364 & 75.4665 & 16.98 & \\
\hline & & & 55.6038 & 16.104 \\
\hline 37.8586 & 19.368 & 71.5804 & 16.984 & \\
\hline & & & & 6.108 \\
\hline 37.8586 & 19.372 & 69.645 & & \\
\hline & & & & 16.112 \\
\hline 38.402 & 19.376 & 69.645 & 16.992 & \\
\hline & & & 55.6038 & 16.116 \\
\hline 38.402 & 19.38 & 74.493 & 16.996 & \\
\hline & & & 55.6038 & 16.12 \\
\hline 38.402 & 19.384 & 80.3523 & 17 & \\
\hline & & & & 16.124 \\
\hline 37.8586 & 19.388 & 87.2439 & 17.004 & \\
\hline & & & & 16.128 \\
\hline 38.402 & 19.392 & 88.2332 & 17.008 & \\
\hline & & & 55.6038 & 16.132 \\
\hline 402 & 9.396 & 87.2439 & 17.012 & \\
\hline
\end{tabular}

\begin{tabular}{|c|c|c|c|}
\hline 84.611 & 18.1 & 82.0338 & 17.88 \\
\hline 87.3657 & 18.104 & 83.048 & 17.884 \\
\hline 87.3657 & 18.108 & 82.0338 & 17.888 \\
\hline 88.286 & 18.112 & 83.048 & 17.892 \\
\hline 86.4464 & 18.116 & 82.0338 & 17.896 \\
\hline 85.5282 & 18.12 & 81.021 & 17.9 \\
\hline 84.611 & 18.124 & 82.0338 & 17.904 \\
\hline 86.4464 & 18.128 & 80.0095 & 17.908 \\
\hline 85.5282 & 18.132 & 77.9904 & 17.912 \\
\hline 85.5282 & 18.136 & 82.0338 & 17.916 \\
\hline 84.611 & 18.14 & 82.0338 & 17.92 \\
\hline 85.5282 & 18.144 & 80.0095 & 17.924 \\
\hline 85.5282 & 18.148 & 81.021 & 17.928 \\
\hline 84.611 & 18.152 & 81.021 & 17.932 \\
\hline 83.6949 & 18.156 & 78.9993 & 17.936 \\
\hline 87.3657 & 18.16 & 77.9904 & 17.94 \\
\hline 84.611 & 18.164 & 77.9904 & 17.944 \\
\hline 86.4464 & 18.168 & 78.9993 & 17.948 \\
\hline 87.3657 & 18.172 & 78.9993 & 17.952 \\
\hline 86.4464 & 18.176 & 77.9904 & 17.956 \\
\hline 87.3657 & 18.18 & 77.9904 & 17.96 \\
\hline 86.4464 & 18.184 & 77.9904 & 17.964 \\
\hline 84.611 & 18.188 & 77.9904 & 17.968 \\
\hline 86.4464 & 18.192 & 77.9904 & 17.972 \\
\hline 84.611 & 18.196 & 77.9904 & 17.976 \\
\hline 86.4464 & 18.2 & 77.9904 & 17.98 \\
\hline 85.5282 & 18.204 & 77.9904 & 17.984 \\
\hline 85.5282 & 18.208 & 77.9904 & 17.988 \\
\hline 87.3657 & 18.212 & 77.9904 & 17.992 \\
\hline 88.286 & 18.216 & 77.9904 & 17.996 \\
\hline 87.3657 & 18.22 & 78.9993 & 18 \\
\hline 88.286 & 18.224 & 77.9904 & 18.004 \\
\hline 87.3657 & 18.228 & 77.9904 & 18.008 \\
\hline 87.3657 & 18.232 & 78.9993 & 18.012 \\
\hline 87.3657 & 18.236 & 77.9904 & 18.016 \\
\hline
\end{tabular}




\begin{tabular}{|c|c|c|c|c|}
\hline \multirow{3}{*}{38.402} & & & 60.1 & 16.1 \\
\hline & 9.4 & 6.2557 & 17.016 & \\
\hline & & & 62.0105 & 16.14 \\
\hline \multirow[t]{2}{*}{38.9458} & 19.404 & 80.3523 & 17.02 & \\
\hline & & & 22 & 16.144 \\
\hline \multirow[t]{2}{*}{39.4902} & 19.408 & 76.4411 & 17.024 & \\
\hline & & & 64.7747 & 16.148 \\
\hline \multirow[t]{2}{*}{38.402} & 19.412 & 76.4411 & 17.028 & \\
\hline & & & 63.8522 & 16.152 \\
\hline \multirow[t]{2}{*}{37.8586} & 19.416 & 76.4411 & 17.032 & \\
\hline & & & 522 & 16.156 \\
\hline \multirow{2}{*}{37.8586} & 19.42 & 76.4411 & 17. & \\
\hline & & & & 16.16 \\
\hline \multirow[t]{2}{*}{38.402} & 19.424 & 80.3523 & 17. & \\
\hline & & & 573 & 16.164 \\
\hline \multirow[t]{2}{*}{38.402} & 19.428 & 84.2831 & & \\
\hline & & & 73 & 16.168 \\
\hline \multirow[t]{2}{*}{39.4902} & 19.432 & 85.2688 & 17. & \\
\hline & & & & 16.172 \\
\hline \multirow[t]{2}{*}{38.402} & 19.436 & 85.2688 & & \\
\hline & & & & 16.1 \\
\hline \multirow[t]{2}{*}{37.8586} & 19.44 & 83.2986 & 17 & \\
\hline & & & & 16.18 \\
\hline \multirow[t]{2}{*}{38.402} & 19.444 & 78.3942 & 17 & \\
\hline & & & & 16.184 \\
\hline 37.8586 & 19.448 & 72.55 & & \\
\hline & & & & 16.188 \\
\hline 38.9458 & 19.452 & 70.612 & & \\
\hline & & & & 16.192 \\
\hline 8.402 & 19.45 & 2.55 & 17 & \\
\hline & & & & 16.196 \\
\hline 38.9458 & 19.46 & 74.493 & 6 & \\
\hline & & & & 16.2 \\
\hline 38.9458 & 19.464 & 79.3727 & & \\
\hline & & & & 16.204 \\
\hline 38.402 & 19.468 & 30.3523 & & \\
\hline & & & & 08 \\
\hline 38.402 & 19.472 & 82.3153 & 1 & \\
\hline & & & & 16.212 \\
\hline 39.4902 & 19.476 & 81.3332 & & \\
\hline & & & & 16 \\
\hline 39.4902 & 19.48 & 76.4411 & 17. & \\
\hline & & & & 16.22 \\
\hline 39.4902 & 19.484 & 75.4 & & \\
\hline & & & & 16.224 \\
\hline 37.8586 & 19.488 & 72.55 & 17 & \\
\hline & & & & 16.228 \\
\hline 38.402 & 19.492 & 71.5804 & & \\
\hline & & & & 16.232 \\
\hline 38.9458 & 19.496 & 9.0 & & \\
\hline & & & & 16.236 \\
\hline 38.9458 & 19.5 & 9.6 & & \\
\hline & & & & 16.24 \\
\hline 38.9458 & 19.504 & 70.612 & 17.12 & \\
\hline & & & & 44 \\
\hline 38.9458 & 19.508 & 70.612 & 17. & \\
\hline & & & & 16. \\
\hline 39.4902 & 19.512 & 71.5804 & 17. & \\
\hline & & & & 16.252 \\
\hline 39.4902 & 19.516 & 69.645 & & \\
\hline & & & & 16.256 \\
\hline 39.4902 & 19.52 & 67.7147 & 17.136 & \\
\hline & & & & 16.26 \\
\hline 38.945 & 19.5 & 66.7515 & 17.14 & \\
\hline & & & & 16.264 \\
\hline 39.4902 & 19.528 & 64.8291 & 17.144 & \\
\hline & & & & 16.268 \\
\hline 40.0351 & 19.532 & 65.7897 & 17.1 & \\
\hline & & & & \\
\hline .035 & 19.536 & 4.82 & 17.152 & \\
\hline
\end{tabular}

\begin{tabular}{|c|c|c|c|}
\hline 7.3657 & 18.24 & 77.9904 & 18.02 \\
\hline 7.3657 & 18.244 & 77.9904 & 18.024 \\
\hline 8.286 & 18.248 & 77.9904 & 18.028 \\
\hline 9.2073 & 18.252 & 77.9904 & 18.032 \\
\hline 9.2073 & 18.256 & 78.9993 & 18.036 \\
\hline 9.2073 & 18.26 & 77.9904 & 18.04 \\
\hline 0.1296 & 18.264 & 30.0095 & 18.044 \\
\hline 1.0529 & 18.268 & 80.0095 & 18.048 \\
\hline 88.286 & 18.272 & 78.9993 & 18.052 \\
\hline 90.1296 & 18.276 & 81.021 & 18.056 \\
\hline 1.0529 & 18.28 & 80.0095 & 18.06 \\
\hline 89.2073 & 18.284 & 78.9993 & 18.064 \\
\hline 89.2073 & 18.288 & 80.0095 & 18.068 \\
\hline 0.1296 & 18.292 & 82.0338 & 18.072 \\
\hline 0.1296 & 18.296 & 82.0338 & 18.076 \\
\hline 1.0529 & 18.3 & 82.0338 & 18.08 \\
\hline 89.2073 & 18.304 & 81.021 & 18.084 \\
\hline 89.2073 & 18.308 & 82.0338 & 18.088 \\
\hline 0.1296 & 18.312 & 80.0095 & 18.092 \\
\hline 1.9773 & 18.316 & 77.9904 & 18.096 \\
\hline 1.9773 & 18.32 & 82.0338 & 18.1 \\
\hline 91.0529 & 18.324 & 82.0338 & 18.104 \\
\hline 1.9773 & 18.328 & 82.0338 & 18.108 \\
\hline 89.2073 & 18.332 & 81.021 & 18.112 \\
\hline 91.0529 & 18.336 & 84.0634 & 18.116 \\
\hline 91.9773 & 18.34 & 84.0634 & 18.12 \\
\hline 91.0529 & 18.344 & 86.098 & 18.124 \\
\hline 90.1296 & 18.348 & 86.098 & 18.128 \\
\hline 92.9026 & 18.352 & 36.098 & 18.132 \\
\hline 91.9773 & 18.356 & 37.1172 & 18.136 \\
\hline 91.0529 & 18.36 & 86.098 & 18.1 \\
\hline 92.9026 & 18.364 & 86.098 & 18.144 \\
\hline 91.9773 & 18.368 & 87.1172 & 18.148 \\
\hline 91.0529 & 18.372 & 88.1378 & 18.15 \\
\hline 91.97 & 18.37 & 86.098 & 18.15 \\
\hline
\end{tabular}




\begin{tabular}{|c|c|c|c|c|}
\hline \multirow{3}{*}{39.4902} & & & 70.3 & 16.276 \\
\hline & 9.54 & 5.7897 & 17.156 & \\
\hline & & & 68.4776 & 16.28 \\
\hline \multirow[t]{2}{*}{39.4902} & 19.544 & 64.8291 & 17.16 & \\
\hline & & & 67.5501 & 16.284 \\
\hline \multirow{2}{*}{38.9458} & 19.548 & 65.7897 & 17. & \\
\hline & & & 67.5501 & 6.288 \\
\hline \multirow[t]{2}{*}{38.9458} & 19.552 & 65.7897 & 17.168 & \\
\hline & & & 67.5501 & 16.292 \\
\hline \multirow[t]{2}{*}{39.4902} & 19.556 & 65.7897 & 17.172 & \\
\hline & & & 62 & 16.296 \\
\hline \multirow[t]{2}{*}{39.4902} & 19.56 & 65.7897 & 17. & \\
\hline & & & & 16.3 \\
\hline \multirow[t]{2}{*}{38.9458} & 19.564 & 4.8291 & & \\
\hline & & & 996 & 6.304 \\
\hline \multirow[t]{2}{*}{40.0351} & 19.568 & 61.0001 & 17. & \\
\hline & & & 501 & 16.308 \\
\hline \multirow[t]{2}{*}{39.4902} & 19.572 & 61.0001 & & \\
\hline & & & & 16.312 \\
\hline \multirow[t]{2}{*}{38.9458} & 19.576 & 59.0936 & & \\
\hline & & & & 16. \\
\hline \multirow[t]{2}{*}{38.9458} & 19.58 & 61.0001 & 17 & \\
\hline & & & 62.0105 & 16.32 \\
\hline \multirow[t]{2}{*}{39.4902} & 19.584 & 61.9553 & 17 & \\
\hline & & & 38 & 16.324 \\
\hline 39.4902 & 19.588 & 62.9119 & & \\
\hline & & & & 6.328 \\
\hline 38.9458 & 19.592 & 62.9119 & & \\
\hline & & & & 16.332 \\
\hline 38.402 & 19.596 & 63.8699 & 17 & \\
\hline & & & & 36 \\
\hline 39.4902 & 19.6 & 65.7897 & & \\
\hline & & & & 16.34 \\
\hline 39.4902 & 19.604 & 63.8699 & & \\
\hline & & & & 16 \\
\hline 40.0351 & 19.608 & 63.8699 & 17 & \\
\hline & & & & 6.348 \\
\hline 40.0351 & 19.612 & 2.9119 & 8 & \\
\hline & & & & 52 \\
\hline 40.0351 & 19.616 & 61.9553 & & \\
\hline & & & & 16.356 \\
\hline 38.402 & 19.62 & 62.9119 & 17 & \\
\hline & & & & 16.36 \\
\hline 40.0351 & 19.624 & 63.8699 & & \\
\hline & & & & 64 \\
\hline 40.0351 & 19.628 & 64.8291 & 1 & \\
\hline & & & & 16.368 \\
\hline 40.0351 & 19.632 & 65.7897 & & \\
\hline & & & & 72 \\
\hline 40.0351 & 19.636 & 67.7147 & 17. & \\
\hline & & & & 6.376 \\
\hline 40.0351 & 19.64 & 67.7147 & & \\
\hline & & & & 16.38 \\
\hline 40.5806 & 19.644 & 68.6792 & & \\
\hline & & & & 16.384 \\
\hline 40.0351 & 19.648 & 67.7147 & 17. & \\
\hline & & & & 16.388 \\
\hline 40.0351 & 19.652 & 5.7897 & 1 & \\
\hline & & & & 16.392 \\
\hline 41.1264 & 19.656 & 67.7147 & 17. & \\
\hline & & & & 16.396 \\
\hline 40.0351 & 19.66 & 66.7515 & 17.276 & \\
\hline & & & & 16.4 \\
\hline 41.1264 & 19.664 & 69.645 & 17.2 & \\
\hline & & & & 16.404 \\
\hline 40.0351 & 19.668 & 71.5804 & 17.284 & \\
\hline & & & 58.342 & 16.408 \\
\hline 40.0351 & 19.672 & 73.5209 & 17.2 & \\
\hline & & & & \\
\hline 40.58 & 19.6 & 4.49 & 17.292 & \\
\hline
\end{tabular}

\begin{tabular}{|c|c|c|c|}
\hline .9773 & 18.38 & 86.098 & 18.16 \\
\hline 91.0529 & 18.384 & 86.098 & 18.164 \\
\hline 90.1296 & 18.388 & 38.1378 & 18.168 \\
\hline 3.829 & 18.392 & 88.1378 & 18.172 \\
\hline 1.9773 & 18.396 & 89.1595 & 18.176 \\
\hline 5.6847 & 18.4 & 88.1378 & 18.18 \\
\hline 1.9773 & 18.404 & 89.1595 & 18.184 \\
\hline 2.9026 & 18.408 & 90.1825 & 18.188 \\
\hline 90.1296 & 18.412 & 90.1825 & 18.192 \\
\hline 3.829 & 18.416 & 88.1378 & 18.196 \\
\hline 1.9773 & 18.42 & 87.1172 & 18.2 \\
\hline 92.9026 & 18.424 & 90.1825 & 18.204 \\
\hline 92.9026 & 18.428 & 89.1595 & 18.208 \\
\hline 91.9773 & 18.432 & 90.1825 & 18.212 \\
\hline 1.9773 & 18.436 & 88.1378 & 18.216 \\
\hline 91.0529 & 18.44 & 90.1825 & 18.22 \\
\hline 91.9773 & 18.444 & 89.1595 & 18.224 \\
\hline 1.9773 & 18.448 & 89.1595 & 18.228 \\
\hline 91.0529 & 18.452 & 90.1825 & 18.232 \\
\hline 92.9026 & 18.456 & 87.1172 & 18.236 \\
\hline 2.9026 & 18.46 & 89.1595 & 18.24 \\
\hline 93.829 & 18.464 & 90.1825 & 18.244 \\
\hline 91.9773 & 18.468 & 90.1825 & 18.248 \\
\hline 91.0529 & 18.472 & 88.1378 & 18.252 \\
\hline 91.0529 & 18.476 & 90.1825 & 18.256 \\
\hline 91.0529 & 18.48 & 0.1825 & 18.26 \\
\hline 93.829 & 18.484 & 90.1825 & 18.264 \\
\hline 92.9026 & 18.488 & 88.1378 & 18.268 \\
\hline 92.9026 & 18.492 & 87.1172 & 18.272 \\
\hline 91.9773 & 18.496 & 8.1378 & 18.276 \\
\hline 93.829 & 18.5 & 87.1172 & 18.28 \\
\hline 92.9026 & 18.504 & 86.098 & 18.284 \\
\hline 91.9773 & 18.508 & 87.1172 & 18.288 \\
\hline 94.756 & 18.512 & 89.1595 & 18.29 \\
\hline $91.9^{7}$ & 18.5 & 87.1172 & 18.29 \\
\hline
\end{tabular}




\begin{tabular}{|c|c|c|c|c|}
\hline & & & 62.0105 & 16.416 \\
\hline \multirow[t]{2}{*}{40.0351} & 19.68 & 75.4665 & 17.296 & \\
\hline & & & 62.0105 & 16.42 \\
\hline \multirow[t]{2}{*}{40.0351} & 19.684 & 73.5209 & 17.3 & \\
\hline & & & 62.0105 & 16.424 \\
\hline \multirow{2}{*}{40.0351} & 19.688 & 74.493 & 17.304 & \\
\hline & & & 61.0915 & 16.428 \\
\hline \multirow[t]{2}{*}{40.5806} & 19.692 & 73.5209 & 17.308 & \\
\hline & & & 60.1738 & 16.432 \\
\hline \multirow[t]{2}{*}{41.1264} & 19.696 & 74.493 & 17.312 & \\
\hline & & & 58.342 & 16.436 \\
\hline \multirow[t]{2}{*}{41.1264} & 19.7 & 74.493 & 17.316 & \\
\hline & & & 58.342 & 16.44 \\
\hline \multirow[t]{2}{*}{41.6729} & 19.704 & 76.4411 & 17.32 & \\
\hline & & & 58.342 & 16.444 \\
\hline \multirow[t]{2}{*}{40.0351} & 19.708 & 76.4411 & 17.324 & \\
\hline & & & 59.2573 & 16.448 \\
\hline \multirow[t]{2}{*}{40.5806} & 19.712 & 77.4171 & 17.328 & \\
\hline & & & 62.0105 & 16.452 \\
\hline \multirow[t]{2}{*}{40.0351} & 19.716 & 78.3942 & 17.332 & \\
\hline & & & 65.6987 & 16.456 \\
\hline \multirow[t]{2}{*}{41.1264} & 19.72 & 78.3942 & 17.336 & \\
\hline & & & 64.7747 & 16.46 \\
\hline \multirow[t]{2}{*}{41.1264} & 19.724 & 79.3727 & 17.34 & \\
\hline & & & 63.8522 & 16.464 \\
\hline 41.1264 & 19.728 & 77.4171 & 17.344 & \\
\hline & & & 62. & 16.468 \\
\hline 41.6729 & 19.732 & 79.3727 & 17.348 & \\
\hline & & & 62.0105 & 16.472 \\
\hline 41.6729 & 19.736 & 80.3523 & 17.352 & \\
\hline & & & 61. & 6.476 \\
\hline 41.1264 & 19.74 & 81.3332 & 17.356 & \\
\hline & & & 62.0105 & 16.48 \\
\hline 42.2198 & 19.744 & 81.3332 & 17.36 & \\
\hline & & & 63.8522 & 16.484 \\
\hline 41.6729 & 19.748 & 83.2986 & 17.364 & \\
\hline & & & 65.6 & 16.488 \\
\hline 42.2198 & 19.752 & 85.2688 & 17.368 & \\
\hline & & & 65.6987 & 16.492 \\
\hline 42.2198 & 19.756 & 85.2688 & 17.3 & \\
\hline & & & 66.6237 & 16.496 \\
\hline 42.2198 & 19.76 & 84.2831 & 17.376 & \\
\hline 42.7673 & 10 & 1 & $\begin{array}{l}65.6987 \\
17.38\end{array}$ & 16.5 \\
\hline 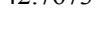 & & & 65.6 & 04 \\
\hline 42.2198 & 19.768 & 82.3153 & 17.384 & \\
\hline & & & 62.9307 & 16.508 \\
\hline 41.6729 & 19.772 & 81.3332 & 17.388 & \\
\hline & & & 61.0915 & 16.512 \\
\hline 42.2198 & 19.776 & 81.3332 & 17.392 & \\
\hline & & & 62.9307 & 16.516 \\
\hline 42.7673 & 19.78 & 82.3153 & 17.396 & \\
\hline & & & 62.9307 & 16.52 \\
\hline 43.3152 & 19.784 & 84.2831 & 17.4 & \\
\hline & & & 65.6987 & 16.524 \\
\hline 42.2198 & 19.788 & 84.2831 & 17.404 & \\
\hline & & & 65.65 & 16.528 \\
\hline 42.7673 & 19.792 & 85.2688 & 17.408 & \\
\hline & & & 67.5501 & 16.532 \\
\hline 43.3152 & 19.796 & 85.2688 & 17.412 & \\
\hline & & & 67.5501 & 16.536 \\
\hline 43.3152 & 19.8 & 84.2831 & 17.416 & \\
\hline & & & 68.4776 & 16.54 \\
\hline 42.7673 & 19.804 & 83.2986 & 17.42 & \\
\hline & & & 67.5501 & 16.544 \\
\hline 42.2198 & 19.808 & 85.2688 & 17.424 & \\
\hline & & & 67.5501 & 16.548 \\
\hline 42.2198 & 19.812 & 83.2986 & 17.428 & \\
\hline & & & 66.6237 & 52 \\
\hline 42.7673 & 19.816 & 81.3332 & 17.432 & \\
\hline
\end{tabular}

\begin{tabular}{|c|c|c|c|}
\hline 5.6847 & 18.52 & 87.1172 & 18.3 \\
\hline 94.7563 & 18.524 & 86.098 & 18.304 \\
\hline 92.9026 & 18.528 & 87.1172 & 18.308 \\
\hline 3.829 & 18.532 & 87.1172 & 18.312 \\
\hline 93.829 & 18.536 & 87.1172 & 18.316 \\
\hline 92.9026 & 18.54 & 85.08 & 18.32 \\
\hline 95.6847 & 18.544 & 86.098 & 18.324 \\
\hline 93.829 & 18.548 & 89.1595 & 18.328 \\
\hline 95.6847 & 18.552 & 87.1172 & 18.332 \\
\hline 93.829 & 18.556 & 89.1595 & 18.336 \\
\hline 94.7563 & 18.56 & 86.098 & 18.34 \\
\hline 95.6847 & 18.564 & 86.098 & 18.344 \\
\hline 97.5443 & 18.568 & 86.098 & 18.348 \\
\hline 95.6847 & 18.572 & 86.098 & 18.352 \\
\hline 96.614 & 18.576 & 86.098 & 18.356 \\
\hline 94.7563 & 18.58 & 86.098 & 18.36 \\
\hline 95.6847 & 18.584 & 88.1378 & 18.364 \\
\hline 94.7563 & 18.588 & 88.1378 & 18.368 \\
\hline 94.7563 & 18.592 & 88.1378 & 18.372 \\
\hline 92.9026 & 18.596 & 87.1172 & 18.376 \\
\hline 94.7563 & 18.6 & 87.1172 & 18.38 \\
\hline 96.614 & 18.604 & 88.1378 & 18.384 \\
\hline 95.6847 & 18.608 & 87.1172 & 18.388 \\
\hline 95.6847 & 18.612 & 88.1378 & 18.392 \\
\hline 98.4756 & 18.616 & 88.1378 & 18.396 \\
\hline 95.6847 & 18.62 & 90.1825 & 18.4 \\
\hline 93.829 & 18.624 & 90.1825 & 18.404 \\
\hline 96.614 & 18.628 & 89.1595 & 18.408 \\
\hline 97.5443 & 18.632 & 90.1825 & 18.412 \\
\hline 95.6847 & 18.636 & 89.1595 & 18.416 \\
\hline 98.4756 & 18.64 & 89.1595 & 18.42 \\
\hline 96.614 & 18.644 & 89.1595 & 18.424 \\
\hline 97.5443 & 18.648 & 90.1825 & 18.428 \\
\hline 97.5443 & 18.652 & 90.1825 & 18.43 \\
\hline 8.0 & 18.6 & 9.18 & 10 \\
\hline
\end{tabular}




\begin{tabular}{|c|c|c|c|c|}
\hline \multirow[b]{2}{*}{42.7673} & & & 67.5 & 16.556 \\
\hline & 9.82 & 80.3523 & $\begin{array}{l}17.436 \\
67.5501\end{array}$ & \\
\hline \multirow[t]{2}{*}{43.8637} & 19.824 & 78.3942 & 17.44 & \\
\hline & & & 68.4776 & 16.564 \\
\hline \multirow[t]{2}{*}{43.3152} & 19.828 & 81.3332 & 17. & \\
\hline & & & & 16.568 \\
\hline \multirow[t]{2}{*}{42.7673} & 19.832 & 81.3332 & 17.448 & \\
\hline & & & 71.2673 & 16.572 \\
\hline \multirow[t]{2}{*}{42.2198} & 19.836 & 81.3332 & 17. & \\
\hline & & & 71.2 & 16.576 \\
\hline \multirow[t]{2}{*}{43.8637} & 19.84 & 79.3727 & & \\
\hline & & & & 16.58 \\
\hline \multirow[t]{2}{*}{42.7673} & 19.844 & 79.3727 & 17. & \\
\hline & & & 72. & 6.584 \\
\hline \multirow[t]{2}{*}{42.7673} & 19.848 & 77.4171 & 17. & \\
\hline & & & & 16.588 \\
\hline \multirow[t]{2}{*}{43.8637} & 19.852 & 76.4411 & & \\
\hline & & & & 16.592 \\
\hline \multirow{2}{*}{43.8637} & 19.856 & 76.4411 & & \\
\hline & & & & 96 \\
\hline \multirow[t]{2}{*}{42.2198} & 19.86 & 77.4171 & 17 & \\
\hline & & & & 6.6 \\
\hline \multirow[t]{2}{*}{43.3152} & 19.864 & 77.4171 & 17 & \\
\hline & & & & 16.604 \\
\hline 44.4126 & 19.868 & 76.4411 & & \\
\hline & & & & 10.000 \\
\hline 43.8637 & 19.872 & 77.4171 & & \\
\hline & & & & 16.612 \\
\hline 43.3152 & 19.876 & 75.4665 & & \\
\hline & & & & 16.616 \\
\hline 43.8637 & 19.88 & 74.493 & 17. & \\
\hline & & & & 16.62 \\
\hline 44.4126 & 19.884 & 76.4411 & & \\
\hline & & & & 6.624 \\
\hline 43.8637 & 19.888 & 76.4411 & & \\
\hline & & & & 6.628 \\
\hline 43.3152 & 19.892 & 75.46 & 17 & \\
\hline & & & & 16.632 \\
\hline 42.2198 & 19.896 & 75.4665 & & \\
\hline & & & & 6.636 \\
\hline 42.7673 & 19.9 & 76.4411 & 17. & \\
\hline & & & & 16.64 \\
\hline 42.2198 & 19.904 & 75.4665 & 17 & \\
\hline & & & & 6.644 \\
\hline 43.3152 & 19.908 & 74.493 & & \\
\hline & & & & 16.648 \\
\hline 43.3152 & 19.912 & 74.493 & & \\
\hline & & & & 6.652 \\
\hline 43.8637 & 19.916 & 73.5209 & & \\
\hline & & & & 5.656 \\
\hline 43.8637 & 19.92 & 73.5209 & & \\
\hline & & & & 16.66 \\
\hline 43.3152 & 19.924 & 73.5209 & & \\
\hline & & & & 6.664 \\
\hline 43.3152 & 19.928 & 74.49 & 17. & \\
\hline & & & & 16.668 \\
\hline 43.3152 & 19.932 & 74.49 & 17. & \\
\hline & & & & 16.672 \\
\hline 43.8637 & 19.936 & 74.493 & & \\
\hline & & & & 16.676 \\
\hline 43.8637 & 19.94 & 74.493 & 17.5 & \\
\hline & & & & 16. \\
\hline 44.41 & 19.94 & 75.4665 & 17.5 & \\
\hline & & & & 16.684 \\
\hline 43.8637 & 19.948 & 75.4665 & 17.564 & \\
\hline & & & & 16.688 \\
\hline 44.412 & 19.952 & 74.493 & 17.5 & \\
\hline & & & & \\
\hline .86 & 19.956 & 74.49 & 17.572 & \\
\hline
\end{tabular}

\begin{tabular}{|c|c|c|c|}
\hline 96.614 & 18.66 & 90.1825 & 18.44 \\
\hline 98.4756 & 18.664 & 90.1825 & 18.444 \\
\hline 97.5443 & 8.668 & 9.1595 & 8.448 \\
\hline 97.5443 & 18.672 & 90.1825 & 18.452 \\
\hline 8.4756 & 18.676 & 90.1825 & 18.456 \\
\hline 98.4756 & 18.68 & 90.1825 & 18.46 \\
\hline 98.4756 & 18.684 & 91.2068 & 18.464 \\
\hline 98.4756 & 18.688 & 91.2068 & 18.468 \\
\hline 98.4756 & 18.692 & 90.1825 & 18.472 \\
\hline 98.4756 & 18.696 & 91.2068 & 18.476 \\
\hline 99.4079 & 18.7 & 91.2068 & 18.48 \\
\hline 99.4079 & 18.704 & 90.1825 & 18.484 \\
\hline 96.614 & 18.708 & 91.2068 & 18.488 \\
\hline 98.4756 & 18.712 & 91.2068 & 18.492 \\
\hline 97.5443 & 18.716 & 93.259 & 18.496 \\
\hline 99.4079 & 18.72 & 91.2068 & 18.5 \\
\hline 99.4079 & 18.724 & 94.287 & 18.504 \\
\hline 98.4756 & 18.728 & 92.2323 & 18.508 \\
\hline 99.4079 & 18.732 & 93.259 & 18.512 \\
\hline 98.4756 & 18.736 & 92.2323 & 18.516 \\
\hline 96.614 & 18.74 & 92.2323 & 18.52 \\
\hline 98.4756 & 18.744 & 93.259 & 18.524 \\
\hline 96.614 & 18.748 & 3.259 & 18.528 \\
\hline 96.614 & 18.752 & 4.287 & 18.532 \\
\hline 97.5443 & 18.756 & 91.2068 & 18.536 \\
\hline 96.614 & 18.76 & 94.287 & 18.5 \\
\hline 97.5443 & 18.764 & 3.259 & 18.544 \\
\hline 99.4079 & 18.768 & 94.287 & 18.548 \\
\hline 97.5443 & 18.772 & 94.287 & 18.552 \\
\hline 96.614 & 18.776 & 93.259 & 18.556 \\
\hline 96.614 & 18.78 & 93.259 & 18.56 \\
\hline 97.544 & 18.784 & 93.259 & 18.564 \\
\hline 96.614 & 18.788 & 94.287 & 18.568 \\
\hline 97.5443 & 18.792 & 94.287 & 18.572 \\
\hline 866 & 18.79 & 95.316 & 18 \\
\hline
\end{tabular}




\begin{tabular}{|c|c|c|c|c|}
\hline \multirow{3}{*}{44.4126} & & & 70.3362 & 16.696 \\
\hline & 19.96 & 74.493 & 17.576 & \\
\hline & & & 68.4776 & 16.7 \\
\hline \multirow[t]{2}{*}{44.4126} & 19.964 & 73.5209 & 17.58 & \\
\hline & & & 68.4776 & 16.704 \\
\hline \multirow[t]{2}{*}{43.3152} & 19.968 & 74.493 & 17.584 & \\
\hline & & & 68.4776 & 16.708 \\
\hline \multirow[t]{2}{*}{43.8637} & 19.972 & 74.493 & 17.588 & \\
\hline & & & 69.4063 & 16.712 \\
\hline \multirow[t]{2}{*}{43.3152} & 19.976 & 77.4171 & 17.592 & \\
\hline & & & 68.4776 & 16.716 \\
\hline \multirow[t]{2}{*}{43.3152} & 19.98 & 77.4171 & 17.596 & \\
\hline & & & 70.3362 & 16.72 \\
\hline \multirow[t]{2}{*}{44.4126} & 19.984 & 77.4171 & 17.6 & \\
\hline & & & 69.4063 & 16.724 \\
\hline \multirow[t]{2}{*}{43.3152} & 19.988 & 78.3942 & 17.604 & \\
\hline & & & 71.2673 & 16.728 \\
\hline \multirow[t]{2}{*}{43.3152} & 19.992 & 78.3942 & 17.608 & \\
\hline & & & 70.3362 & 16.732 \\
\hline \multirow[t]{2}{*}{43.8637} & 19.996 & 79.3727 & 17.612 & \\
\hline & & & 67.5501 & 16.736 \\
\hline \multirow[t]{2}{*}{42.7673} & 20 & 77.4171 & 17.616 & \\
\hline & & & 67.5501 & 16.74 \\
\hline \multirow[t]{2}{*}{43.3152} & 20.004 & 77.4171 & 17.62 & \\
\hline & & & 67.5501 & 16.744 \\
\hline 43.8637 & 20.008 & 80.3523 & 17.624 & \\
\hline & & & 66.6237 & 16.748 \\
\hline 43.8637 & 20.012 & 78.3942 & 17.628 & \\
\hline & & & 66.6237 & 16.752 \\
\hline 44.4126 & 20.016 & 83.2986 & 17.632 & \\
\hline & & & 67.5501 & 16.756 \\
\hline 44.4126 & 20.02 & 82.3153 & 17.636 & \\
\hline & & & 69.4063 & 16.76 \\
\hline 44.4126 & 20.024 & 84.2831 & 17.64 & \\
\hline & & & 66.6237 & 16.764 \\
\hline 44.4126 & 20.028 & 84.2831 & 17.644 & \\
\hline & & & 67.5501 & 16.768 \\
\hline 43.8637 & 20.032 & 84.2831 & 17.648 & \\
\hline & & & 66.6237 & 16.772 \\
\hline 44.4126 & 20.036 & 83.2986 & 17.652 & \\
\hline & & & 67.5501 & 16.776 \\
\hline 44.9621 & 20.04 & 84.2831 & 17.656 & \\
\hline & & & 66.6237 & 16.78 \\
\hline 44.4126 & 20.044 & 84.2831 & 17.66 & \\
\hline & & & 67.5501 & 16.784 \\
\hline 43.3152 & 20.048 & 85.2688 & 17.664 & \\
\hline & & & 67.5501 & 16.788 \\
\hline 44.4126 & 20.052 & 85.2688 & 17.668 & \\
\hline & & & 66.6237 & 16.792 \\
\hline 44.4126 & 20.056 & 86.2557 & 17.672 & \\
\hline & & & 68.4776 & 16.796 \\
\hline 45.512 & 20.06 & 87.2439 & 17.676 & \\
\hline & & & 68.4776 & 16.8 \\
\hline 44.9621 & 20.064 & 87.2439 & 17.68 & \\
\hline & & & 66.6237 & 16.804 \\
\hline 45.512 & 20.068 & 88.2332 & 17.684 & \\
\hline & & & 66.6237 & 16.808 \\
\hline 44.4126 & 20.072 & 87.2439 & 17.688 & \\
\hline & & & 67.5501 & 16.812 \\
\hline 44.9621 & 20.076 & 92.2022 & 17.692 & \\
\hline & & & 66.6237 & 16.816 \\
\hline 45.512 & 20.08 & 95.1911 & 17.696 & \\
\hline & & & 67.5501 & 16.82 \\
\hline 43.8637 & 20.084 & 97.1894 & 17.7 & \\
\hline & & & 68.4776 & 16.824 \\
\hline 44.9621 & 20.088 & 100.195 & 17.704 & \\
\hline & & & 68.4776 & 16.828 \\
\hline 44.4126 & 20.092 & 102.205 & 17.708 & \\
\hline & & & 68.4776 & 16.832 \\
\hline 44.9621 & 20.096 & 106.237 & 17.712 & \\
\hline
\end{tabular}

\begin{tabular}{|c|c|c|c|}
\hline 97.5443 & 18.8 & 94.287 & 18.58 \\
\hline 96.614 & 18.804 & 94.287 & 18.584 \\
\hline 96.614 & 18.808 & 95.3162 & 18.588 \\
\hline 97.5443 & 18.812 & 94.287 & 18.592 \\
\hline 97.5443 & 18.816 & 94.287 & 18.596 \\
\hline 97.5443 & 18.82 & 94.287 & 18.6 \\
\hline 96.614 & 18.824 & 94.287 & 18.604 \\
\hline 97.5443 & 18.828 & 94.287 & 18.608 \\
\hline 96.614 & 18.832 & 94.287 & 18.612 \\
\hline 95.6847 & 18.836 & 95.3162 & 18.616 \\
\hline 97.5443 & 18.84 & 93.259 & 18.62 \\
\hline 96.614 & 18.844 & 95.3162 & 18.624 \\
\hline 96.614 & 18.848 & 95.3162 & 18.628 \\
\hline 97.5443 & 18.852 & 94.287 & 18.632 \\
\hline 99.4079 & 18.856 & 93.259 & 18.636 \\
\hline 98.4756 & 18.86 & 94.287 & 18.64 \\
\hline 100.341 & 18.864 & 94.287 & 18.644 \\
\hline 99.4079 & 18.868 & 93.259 & 18.648 \\
\hline 98.4756 & 18.872 & 93.259 & 18.652 \\
\hline 98.4756 & 18.876 & 94.287 & 18.656 \\
\hline 98.4756 & 18.88 & 93.259 & 18.66 \\
\hline 100.341 & 18.884 & 93.259 & 18.664 \\
\hline 98.4756 & 18.888 & 92.2323 & 18.668 \\
\hline 99.4079 & 18.892 & 92.2323 & 18.672 \\
\hline 98.4756 & 18.896 & 94.287 & 18.676 \\
\hline 100.341 & 18.9 & 94.287 & 18.68 \\
\hline 101.275 & 18.904 & 91.2068 & 18.684 \\
\hline 99.4079 & 18.908 & 93.259 & 18.688 \\
\hline 99.4079 & 18.912 & 93.259 & 18.692 \\
\hline 102.21 & 18.916 & 93.259 & 18.696 \\
\hline 101.275 & 18.92 & 94.287 & 18.7 \\
\hline 101.275 & 18.924 & 92.2323 & 18.704 \\
\hline 101.275 & 18.928 & 91.2068 & 18.708 \\
\hline 102.21 & 18.932 & 94.287 & 18.712 \\
\hline 100.341 & 18.936 & 91.2068 & 18.716 \\
\hline
\end{tabular}




\begin{tabular}{|c|c|c|c|c|}
\hline & & & 69.4063 & 16.836 \\
\hline \multirow[t]{2}{*}{46.6135} & 20.1 & 108.26 & 17.716 & \\
\hline & & & 69.4063 & 16.84 \\
\hline \multirow[t]{2}{*}{46.0625} & 20.104 & 105.228 & 17.72 & \\
\hline & & & 69.4063 & 16.844 \\
\hline \multirow[t]{2}{*}{46.6135} & 20.108 & 105.228 & 17.724 & \\
\hline & & & 67.5501 & 16.848 \\
\hline \multirow[t]{2}{*}{44.9621} & 20.112 & 99.1923 & 17.728 & \\
\hline & & & 67.5501 & 16.852 \\
\hline \multirow[t]{2}{*}{45.512} & 20.116 & 97.1894 & 17.732 & \\
\hline & & & 69.4063 & 16.856 \\
\hline \multirow[t]{2}{*}{45.512} & 20.12 & 93.1973 & 17.736 & \\
\hline & & & 69.4063 & 16.86 \\
\hline \multirow[t]{2}{*}{44.4126} & 20.124 & 90.2153 & 17.74 & \\
\hline & & & 69.4063 & 16.864 \\
\hline \multirow[t]{2}{*}{45.512} & 20.128 & 88.2332 & 17.744 & \\
\hline & & & 70.3362 & 16.868 \\
\hline \multirow[t]{2}{*}{46.0625} & 20.132 & 89.2237 & 17.748 & \\
\hline & & & 70.3362 & 16.872 \\
\hline \multirow[t]{2}{*}{46.0625} & 20.136 & 89.2237 & 17.752 & \\
\hline & & & 71.2673 & 16.876 \\
\hline \multirow[t]{2}{*}{44.9621} & 20.14 & 91.2081 & 17.756 & \\
\hline & & & 72.1996 & 16.88 \\
\hline \multirow[t]{2}{*}{45.512} & 20.144 & 90.2153 & 17.76 & \\
\hline & & & 70.3362 & 16.884 \\
\hline 46.0625 & 20.148 & 94.1936 & 17.764 & \\
\hline & & & 69.4063 & 16.888 \\
\hline 46.0625 & 20.152 & 93.1973 & 17.768 & \\
\hline & & & 72.1996 & 16.892 \\
\hline 44.9621 & 20.156 & 93.1973 & 17.772 & \\
\hline & & & 72.1996 & 16.896 \\
\hline 46.6135 & 20.16 & 93.1973 & 17.776 & \\
\hline & & & 72.1996 & 16.9 \\
\hline 46.6135 & 20.164 & 92.2022 & 17.78 & \\
\hline & & & 74.0676 & 16.904 \\
\hline 46.6135 & 20.168 & 90.2153 & 17.784 & \\
\hline & & & 74.0676 & 16.908 \\
\hline 46.0625 & 20.172 & 87.2439 & 17.788 & \\
\hline & & & 75.0034 & 16.912 \\
\hline 46.6135 & 20.176 & 87.2439 & 17.792 & \\
\hline & & & 73.133 & 16.916 \\
\hline 46.6135 & 20.18 & 87.2439 & 17.796 & \\
\hline & & & 73.133 & 16.92 \\
\hline 46.6135 & 20.184 & 88.2332 & 17.8 & \\
\hline & & & 72.1996 & 16.924 \\
\hline 46.6135 & 20.188 & 89.2237 & 17.804 & \\
\hline & & & 75.0034 & 16.928 \\
\hline 46.6135 & 20.192 & 88.2332 & 17.808 & \\
\hline & & & 74.0676 & 16.932 \\
\hline 46.6135 & 20.196 & 86.2557 & 17.812 & \\
\hline & & & 74.0676 & 16.936 \\
\hline 46.6135 & 20.2 & 85.2688 & 17.816 & \\
\hline & & & 75.9404 & 16.94 \\
\hline 47.1649 & 20.204 & 84.2831 & 17.82 & \\
\hline & & & 75.9404 & 16.944 \\
\hline 46.6135 & 20.208 & 82.3153 & 17.824 & \\
\hline & & & 79.6998 & 16.948 \\
\hline 47.1649 & 20.212 & 80.3523 & 17.828 & \\
\hline & & & 79.6998 & 16.952 \\
\hline 46.6135 & 20.216 & 79.3727 & 17.832 & \\
\hline & & & 81.5863 & 16.956 \\
\hline 46.6135 & 20.22 & 79.3727 & 17.836 & \\
\hline & & & 81.5863 & 16.96 \\
\hline 46.6135 & 20.224 & 78.3942 & 17.84 & \\
\hline & & & 79.6998 & 16.964 \\
\hline 46.6135 & 20.228 & 78.3942 & 17.844 & \\
\hline & & & 79.6998 & 16.968 \\
\hline 46.6135 & 20.232 & 80.3523 & 17.848 & \\
\hline & & & 82.5313 & 16.972 \\
\hline 46.6135 & 20.236 & 83.2986 & 17.852 & \\
\hline
\end{tabular}

\begin{tabular}{|c|c|c|c|}
\hline 102.21 & 18.94 & 93.259 & 18.72 \\
\hline 103.146 & 18.944 & 92.2323 & 18.724 \\
\hline 105.022 & 18.948 & 94.287 & 18.728 \\
\hline 102.21 & 18.952 & 94.287 & 18.732 \\
\hline 103.146 & 18.956 & 93.259 & 18.736 \\
\hline 103.146 & 18.96 & 93.259 & 18.74 \\
\hline 105.961 & 18.964 & 94.287 & 18.744 \\
\hline 105.022 & 18.968 & 93.259 & 18.748 \\
\hline 104.084 & 18.972 & 92.2323 & 18.752 \\
\hline 104.084 & 18.976 & 93.259 & 18.756 \\
\hline 104.084 & 18.98 & 92.2323 & 18.76 \\
\hline 102.21 & 18.984 & 93.259 & 18.764 \\
\hline 105.022 & 18.988 & 93.259 & 18.768 \\
\hline 104.084 & 18.992 & 93.259 & 18.772 \\
\hline 105.961 & 18.996 & 94.287 & 18.776 \\
\hline 106.9 & 19 & 96.3466 & 18.78 \\
\hline 105.961 & 19.004 & 95.3162 & 18.784 \\
\hline 105.961 & 19.008 & 98.411 & 18.788 \\
\hline 105.022 & 19.012 & 99.445 & 18.792 \\
\hline 106.9 & 19.016 & 100.48 & 18.796 \\
\hline 105.022 & 19.02 & 102.554 & 18.8 \\
\hline 104.084 & 19.024 & 103.593 & 18.804 \\
\hline 105.022 & 19.028 & 105.674 & 18.808 \\
\hline 105.961 & 19.032 & 106.716 & 18.812 \\
\hline 105.022 & 19.036 & 106.716 & 18.816 \\
\hline 103.146 & 19.04 & 107.76 & 18.82 \\
\hline 105.022 & 19.044 & 109.85 & 18.824 \\
\hline 103.146 & 19.048 & 110.896 & 18.828 \\
\hline 105.022 & 19.052 & 110.896 & 18.832 \\
\hline 105.022 & 19.056 & 114.044 & 18.836 \\
\hline 105.961 & 19.06 & 116.147 & 18.84 \\
\hline 105.961 & 19.064 & 118.255 & 18.844 \\
\hline 104.084 & 19.068 & 119.311 & 18.848 \\
\hline 105.022 & 19.072 & 119.311 & 18.852 \\
\hline 104.084 & 19.076 & 119.311 & 18.856 \\
\hline
\end{tabular}




\begin{tabular}{|c|c|c|c|c|}
\hline \multirow{3}{*}{46.6135} & & & 83.4774 & 16.976 \\
\hline & 20.24 & 82.3153 & 17.856 & \\
\hline & & & 89.1773 & 16.98 \\
\hline \multirow[t]{2}{*}{46.6135} & 20.244 & 81.3332 & 17.86 & \\
\hline & & & 88.2245 & 16.984 \\
\hline \multirow[t]{2}{*}{46.6135} & 20.248 & 80.3523 & 17.864 & \\
\hline & & & 90.1311 & 16.988 \\
\hline \multirow[t]{2}{*}{46.6135} & 20.252 & 78.3942 & 17.868 & \\
\hline & & & 91.086 & 16.992 \\
\hline \multirow[t]{2}{*}{46.6135} & 20.256 & 78.3942 & 17.872 & \\
\hline & & & 91.086 & 16.996 \\
\hline \multirow[t]{2}{*}{47.7168} & 20.26 & 75.4665 & 17.876 & \\
\hline & & & 90.1311 & 17 \\
\hline \multirow[t]{2}{*}{47.1649} & 20.264 & 76.4411 & 17.88 & \\
\hline & & & 91.086 & 17.004 \\
\hline \multirow[t]{2}{*}{47.7168} & 20.268 & 75.4665 & 17.884 & \\
\hline & & & 89.1773 & 17.008 \\
\hline \multirow[t]{2}{*}{46.6135} & 20.272 & 74.493 & 17.888 & \\
\hline & & & 90.1311 & 17.012 \\
\hline \multirow[t]{2}{*}{47.7168} & 20.276 & 76.4411 & 17.892 & \\
\hline & & & 89.1773 & 17.016 \\
\hline \multirow[t]{2}{*}{46.6135} & 20.28 & 76.4411 & 17.896 & \\
\hline & & & 88.2245 & 17.02 \\
\hline \multirow[t]{2}{*}{46.6135} & 20.284 & 76.4411 & 17.9 & \\
\hline & & & 89.1773 & 17.024 \\
\hline 47.1649 & 20.288 & 77.4171 & 17.904 & \\
\hline & & & 89.1773 & 17.028 \\
\hline 47.1649 & 20.292 & 77.4171 & 17.908 & \\
\hline & & & 89.1773 & 17.032 \\
\hline 47.1649 & 20.296 & 75.4665 & 17.912 & \\
\hline & & & 91.086 & 17.036 \\
\hline 47.1649 & 20.3 & 74.493 & 17.916 & \\
\hline & & & 90.1311 & 17.04 \\
\hline 46.6135 & 20.304 & 76.4411 & 17.92 & \\
\hline & & & 89.1773 & 17.044 \\
\hline 47.7168 & 20.308 & 76.4411 & 17.924 & \\
\hline & & & 89.1773 & 17.048 \\
\hline 46.6135 & 20.312 & 77.4171 & 17.928 & \\
\hline & & & 88.2245 & 17.052 \\
\hline 47.1649 & 20.316 & 78.3942 & 17.932 & \\
\hline & & & 85.3729 & 17.056 \\
\hline 46.6135 & 20.32 & 79.3727 & 17.936 & \\
\hline & & & 82.5313 & 17.06 \\
\hline 46.6135 & 20.324 & 79.3727 & 17.94 & \\
\hline & & & 84.4246 & 17.064 \\
\hline 48.2693 & 20.328 & 80.3523 & 17.944 & \\
\hline & & & 86.3224 & 17.068 \\
\hline 47.1649 & 20.332 & 79.3727 & 17.948 & \\
\hline & & & 90.1311 & 17.072 \\
\hline 47.7168 & 20.336 & 79.3727 & 17.952 & \\
\hline & & & 90.1311 & 17.076 \\
\hline 47.7168 & 20.34 & 79.3727 & 17.956 & \\
\hline & & & 88.2245 & 17.08 \\
\hline 49.3756 & 20.344 & 80.3523 & 17.96 & \\
\hline & & & 86.3224 & 17.084 \\
\hline 48.8222 & 20.348 & 82.3153 & 17.964 & \\
\hline & & & 80.6425 & 17.088 \\
\hline 47.7168 & 20.352 & 80.3523 & 17.968 & \\
\hline & & & 74.0676 & 17.092 \\
\hline 47.1649 & 20.356 & 80.3523 & 17.972 & \\
\hline & & & 71.2673 & 17.096 \\
\hline 47.1649 & 20.36 & 81.3332 & 17.976 & \\
\hline & & & 70.3362 & 17.1 \\
\hline 47.1649 & 20.364 & 79.3727 & 17.98 & \\
\hline & & & 70.3362 & 17.104 \\
\hline 46.6135 & 20.368 & 80.3523 & 17.984 & \\
\hline & & & 75.9404 & 17.108 \\
\hline 47.1649 & 20.372 & 78.3942 & 17.988 & \\
\hline & & & 79.6998 & 17.112 \\
\hline 48.2693 & 20.376 & 78.3942 & 17.992 & \\
\hline
\end{tabular}

\begin{tabular}{|c|c|c|c|}
\hline 105.022 & 19.08 & 119.311 & 18.86 \\
\hline 105.022 & 19.084 & 119.311 & 18.864 \\
\hline 105.022 & 19.088 & 119.311 & 18.868 \\
\hline 105.022 & 19.092 & 120.367 & 18.872 \\
\hline 104.084 & 19.096 & 120.367 & 18.876 \\
\hline 104.084 & 19.1 & 118.255 & 18.88 \\
\hline 104.084 & 19.104 & 120.367 & 18.884 \\
\hline 105.961 & 19.108 & 119.311 & 18.888 \\
\hline 105.022 & 19.112 & 120.367 & 18.892 \\
\hline 105.022 & 19.116 & 120.367 & 18.896 \\
\hline 102.21 & 19.12 & 120.367 & 18.9 \\
\hline 103.146 & 19.124 & 121.425 & 18.904 \\
\hline 102.21 & 19.128 & 122.484 & 18.908 \\
\hline 104.084 & 19.132 & 123.544 & 18.912 \\
\hline 104.084 & 19.136 & 123.544 & 18.916 \\
\hline 105.961 & 19.14 & 122.484 & 18.92 \\
\hline 105.022 & 19.144 & 123.544 & 18.924 \\
\hline 105.022 & 19.148 & 122.484 & 18.928 \\
\hline 105.022 & 19.152 & 122.484 & 18.932 \\
\hline 105.022 & 19.156 & 122.484 & 18.936 \\
\hline 106.9 & 19.16 & 123.544 & 18.94 \\
\hline 107.841 & 19.164 & 123.544 & 18.944 \\
\hline 105.961 & 19.168 & 123.544 & 18.948 \\
\hline 105.961 & 19.172 & 123.544 & 18.952 \\
\hline 104.084 & 19.176 & 123.544 & 18.956 \\
\hline 103.146 & 19.18 & 123.544 & 18.96 \\
\hline 103.146 & 19.184 & 123.544 & 18.964 \\
\hline 103.146 & 19.188 & 123.544 & 18.968 \\
\hline 104.084 & 19.192 & 123.544 & 18.972 \\
\hline 103.146 & 19.196 & 125.667 & 18.976 \\
\hline 104.084 & 19.2 & 124.605 & 18.98 \\
\hline 105.022 & 19.204 & 123.544 & 18.984 \\
\hline 105.961 & 19.208 & 123.544 & 18.988 \\
\hline 104.084 & 19.212 & 123.544 & 18.992 \\
\hline 105.022 & 19.216 & 124.605 & 18.996 \\
\hline
\end{tabular}




\begin{tabular}{|c|c|c|c|c|}
\hline & & & 81.5863 & 17.116 \\
\hline \multirow[t]{2}{*}{47.7168} & 20.38 & 77.4171 & 17.996 & \\
\hline & & & 78.7582 & 17.12 \\
\hline \multirow[t]{2}{*}{47.1649} & 20.384 & 77.4171 & 18 & \\
\hline & & & 74.0676 & 17.124 \\
\hline \multirow[t]{2}{*}{47.1649} & 20.388 & 77.4171 & 18.004 & \\
\hline & & & 68.4776 & 17.128 \\
\hline \multirow[t]{2}{*}{48.8222} & 20.392 & 77.4171 & 18.008 & \\
\hline & & & 66.6237 & 17.132 \\
\hline \multirow[t]{2}{*}{48.8222} & 20.396 & 79.3727 & 18.012 & \\
\hline & & & 65.6987 & 17.136 \\
\hline \multirow[t]{2}{*}{48.2693} & 20.4 & 80.3523 & 18.016 & \\
\hline & & & 65.6987 & 17.14 \\
\hline \multirow[t]{2}{*}{47.7168} & 20.404 & 80.3523 & 18.02 & \\
\hline & & & 67.5501 & 17.144 \\
\hline \multirow[t]{2}{*}{49.3756} & 20.408 & 82.3153 & 18.024 & \\
\hline & & & 71.2673 & 17.148 \\
\hline \multirow[t]{2}{*}{49.3756} & 20.412 & 85.2688 & 18.028 & \\
\hline & & & & 17.152 \\
\hline \multirow[t]{2}{*}{49.3756} & 20.416 & 85.2688 & 18.032 & \\
\hline & & & 73 & 17.156 \\
\hline \multirow[t]{2}{*}{49.9296} & 20.42 & 85.2688 & 18.036 & \\
\hline & & & 362 & 17.16 \\
\hline \multirow[t]{2}{*}{50.4839} & 20.424 & 85.2688 & & \\
\hline & & & 237 & 17.164 \\
\hline 47.7168 & 20.428 & 84.2831 & & \\
\hline & & & 307 & 17.168 \\
\hline 49.3756 & 20.432 & 86.2557 & 18.048 & \\
\hline & & & 57.428 & 17.172 \\
\hline 51.0389 & 20.436 & 85.2688 & 18.052 & \\
\hline & & & 54.6936 & 17.176 \\
\hline 50.4839 & 20.44 & 86.2557 & 18.056 & \\
\hline & & & 55.6038 & 17.18 \\
\hline 50.4839 & 20.444 & 88.2332 & 18.06 & \\
\hline & & & 59 & 17.184 \\
\hline 51.0389 & 20.448 & 89.2237 & 18.064 & \\
\hline & & & & 17.188 \\
\hline 49.9296 & 20.452 & 86.2557 & 18.068 & \\
\hline & & & 747 & 17.192 \\
\hline 51.0389 & 20.456 & 88.2332 & 18.072 & \\
\hline & & & 67 & 17.196 \\
\hline 50.4839 & 20.46 & 85.2688 & 18.076 & \\
\hline & & & 67.5501 & 17.2 \\
\hline 51.0389 & 20.464 & 88.2332 & 18.08 & \\
\hline & & & 64.7747 & 17.204 \\
\hline 51.0389 & 20.468 & 88.2332 & 18.084 & \\
\hline & & & & 17.208 \\
\hline 51.0389 & 20.472 & 90.2153 & 18. & \\
\hline & & & 57.428 & 17.212 \\
\hline 51.0389 & 20.476 & 92.2022 & 18.092 & \\
\hline & & & 56 & 17.216 \\
\hline 50.4839 & 20.48 & 94.1936 & & \\
\hline & & & & 17.22 \\
\hline 51.0389 & 20.484 & 95.1911 & 18.1 & \\
\hline & & & & 17.224 \\
\hline 51.0389 & 20.488 & 98.1903 & & \\
\hline & & & & 17.228 \\
\hline 51.0389 & 20.492 & 96.1897 & & \\
\hline & & & 69.4063 & 17.232 \\
\hline 51.0389 & 20.496 & 93.1973 & 18.112 & \\
\hline & & & 70.3362 & 17.236 \\
\hline 51.0389 & 20.5 & 93.1973 & 18.116 & \\
\hline & & & 69.4063 & 17.24 \\
\hline 51.0389 & 20.504 & 92.2022 & 18.12 & \\
\hline & & & & 17.244 \\
\hline 51.0389 & 20.508 & 89.2237 & 18.124 & \\
\hline & & & 63.8522 & 17.248 \\
\hline 51.0389 & 20.512 & 89.2237 & 18.128 & \\
\hline & & & 63.8522 & 17.252 \\
\hline 51.0389 & 20.516 & 88.2332 & 18.132 & \\
\hline
\end{tabular}

\begin{tabular}{|c|c|c|c|}
\hline 107.841 & 19.22 & 123.544 & 19 \\
\hline 105.022 & 19.224 & 123.544 & 19.004 \\
\hline 106.9 & 19.228 & 123.544 & 19.008 \\
\hline 105.961 & 19.232 & 123.544 & 19.012 \\
\hline 107.841 & 19.236 & 124.605 & 19.016 \\
\hline 105.961 & 19.24 & 122.484 & 19.02 \\
\hline 106.9 & 19.244 & 123.544 & 19.024 \\
\hline 107.841 & 19.248 & 123.544 & 19.028 \\
\hline 106.9 & 19.252 & 123.544 & 19.032 \\
\hline 107.841 & 19.256 & 123.544 & 19.036 \\
\hline 107.841 & 19.26 & 123.544 & 19.04 \\
\hline 106.9 & 19.264 & 123.544 & 19.044 \\
\hline 107.841 & 19.268 & 123.544 & 19.048 \\
\hline 107.841 & 19.272 & 123.544 & 19.052 \\
\hline 109.726 & 19.276 & 123.544 & 19.056 \\
\hline 108.783 & 19.28 & 124.605 & 19.06 \\
\hline 108.783 & 19.284 & 123.544 & 19.064 \\
\hline 107.841 & 19.288 & 124.605 & 19.068 \\
\hline 110.669 & 19.292 & 123.544 & 19.072 \\
\hline 107.841 & 19.296 & 123.544 & 19.076 \\
\hline 109.726 & 19.3 & 124.605 & 19.08 \\
\hline 108.783 & 19.304 & 123.544 & 19.084 \\
\hline 109.726 & 19.308 & 123.544 & 19.088 \\
\hline 108.783 & 19.312 & 124.605 & 19.092 \\
\hline 109.726 & 19.316 & 124.605 & 19.096 \\
\hline 110.669 & 19.32 & 125.667 & 19.1 \\
\hline 110.669 & 19.324 & 125.667 & 19.104 \\
\hline 109.726 & 19.328 & 124.605 & 19.108 \\
\hline 110.669 & 19.332 & 124.605 & 19.112 \\
\hline 110.669 & 19.336 & 123.544 & 19.116 \\
\hline 110.669 & 19.34 & 123.544 & 19.12 \\
\hline 109.726 & 19.344 & 123.544 & 19.124 \\
\hline 110.669 & 19.348 & 125.667 & 19.128 \\
\hline 110.669 & 19.352 & 124.605 & 19.132 \\
\hline 110.669 & 19.356 & 126.73 & 19.136 \\
\hline
\end{tabular}




\begin{tabular}{|c|c|c|c|c|}
\hline \multirow{3}{*}{89} & \multirow{3}{*}{20.52} & \multirow[b]{2}{*}{88.2332} & 64.7747 & \multirow[t]{2}{*}{17.256} \\
\hline & & & 18.136 & \\
\hline & & & 66.6237 & 17.26 \\
\hline \multirow[t]{2}{*}{51.0389} & 20.524 & 88.2332 & 18.14 & \\
\hline & & & 70.3362 & 17.264 \\
\hline \multirow{2}{*}{51.0389} & 20.528 & 89.2237 & 18.144 & \\
\hline & & & 75.0034 & 17.268 \\
\hline \multirow[t]{2}{*}{51.0389} & 20.532 & 88.2332 & 18.148 & \\
\hline & & & 76.8785 & 17.272 \\
\hline \multirow[t]{2}{*}{51.0389} & 20.536 & 89.2237 & 18.152 & \\
\hline & & & 75.9404 & 17.276 \\
\hline \multirow[t]{2}{*}{51.0389} & 20.54 & 88.2332 & 18.156 & \\
\hline & & & 75.0034 & 17.28 \\
\hline \multirow[t]{2}{*}{51.0389} & 20.544 & 87.2439 & 18.16 & \\
\hline & & & 72.1996 & 17.284 \\
\hline \multirow[t]{2}{*}{51.0389} & 20.548 & 88.2332 & 18.164 & \\
\hline & & & 69.4063 & 17.288 \\
\hline \multirow[t]{2}{*}{51.0389} & 20.552 & 89.2237 & 18.168 & \\
\hline & & & 69.4063 & 17.292 \\
\hline 51.5942 & 20.556 & 88.2332 & 18.172 & \\
\hline & & & 70.3362 & 17.296 \\
\hline 51.0389 & 20.56 & 89.2237 & 18.176 & \\
\hline & & & 74.0676 & 17.3 \\
\hline 51.0389 & 20.564 & 90.2153 & 18.18 & \\
\hline & & & 79.6998 & 17.304 \\
\hline 51.0389 & 20.568 & 88.2332 & 18.184 & \\
\hline & & & 84.4246 & 17.308 \\
\hline 51.0389 & 20.572 & 86.2557 & 18.188 & \\
\hline & & & 86.3224 & 17.312 \\
\hline 51.0389 & 20.576 & 86.2557 & 18.192 & \\
\hline & & & 86.3224 & 17.316 \\
\hline 51.0389 & 20.58 & 86.2557 & 18.196 & \\
\hline & & & 85.3729 & 17.32 \\
\hline 51.5942 & 20.584 & 85.2688 & 18.2 & \\
\hline & & & 82.5313 & 17.324 \\
\hline 51.5942 & 20.588 & 85.2688 & 18.204 & \\
\hline & & & 81.5863 & 17.328 \\
\hline 52.1501 & 20.592 & 86.2557 & 18.208 & \\
\hline & & & 80.6425 & 17.332 \\
\hline 51.5942 & 20.596 & 86.2557 & 18.212 & \\
\hline & & & 83.4774 & 17.336 \\
\hline 51.5942 & 20.6 & 88.2332 & 18.216 & \\
\hline & & & 85.3729 & 17.34 \\
\hline 51.0389 & 20.604 & 88.2332 & 18.22 & \\
\hline & & & 88.2245 & 17.344 \\
\hline 51.0389 & 20.608 & 87.2439 & 18.224 & \\
\hline & & & 93.9572 & 17.348 \\
\hline 51.0389 & 20.612 & 88.2332 & 18.228 & \\
\hline & & & 94.9164 & 17.352 \\
\hline 51.0389 & 20.616 & 86.2557 & 18.232 & \\
\hline & & & 93.9572 & 17.356 \\
\hline 51.0389 & 20.62 & 85.2688 & 18.236 & \\
\hline & & & 92.042 & 17.36 \\
\hline 52.1501 & 20.624 & 85.2688 & 18.24 & \\
\hline & & & 90.1311 & 17.364 \\
\hline 52.1501 & 20.628 & 84.2831 & 18.244 & \\
\hline & & & 88.2245 & 17.368 \\
\hline 52.7064 & 20.632 & 85.2688 & 18.248 & \\
\hline & & & 87.2729 & 17.372 \\
\hline 51.5942 & 20.636 & 87.2439 & 18.252 & \\
\hline & & & 88.2245 & 17.376 \\
\hline 51.5942 & 20.64 & 88.2332 & 18.256 & \\
\hline & & & 92.042 & 17.38 \\
\hline 51.0389 & 20.644 & 89.2237 & 18.26 & \\
\hline & & & 92.042 & 17.384 \\
\hline 52.1501 & 20.648 & 89.2237 & 18.264 & \\
\hline & & & 94.9164 & 17.388 \\
\hline 52.7064 & 20.652 & 89.2237 & 18.268 & \\
\hline & & & 94.9164 & 17.392 \\
\hline 51.5942 & 20.656 & 89.2237 & 18.272 & \\
\hline
\end{tabular}

\begin{tabular}{|c|c|c|c|}
\hline 111.614 & 19.36 & 127.794 & 19.14 \\
\hline 110.669 & 19.364 & 128.859 & 19.144 \\
\hline 110.669 & 19.368 & 126.73 & 19.148 \\
\hline 111.614 & 19.372 & 125.667 & 19.152 \\
\hline 108.783 & 19.376 & 124.605 & 19.156 \\
\hline 110.669 & 19.38 & 124.605 & 19.16 \\
\hline 109.726 & 19.384 & 125.667 & 19.164 \\
\hline 110.669 & 19.388 & 126.73 & 19.168 \\
\hline 109.726 & 19.392 & 128.859 & 19.172 \\
\hline 112.559 & 19.396 & 127.794 & 19.176 \\
\hline 110.669 & 19.4 & 127.794 & 19.18 \\
\hline 110.669 & 19.404 & 128.859 & 19.184 \\
\hline 111.614 & 19.408 & 127.794 & 19.188 \\
\hline 112.559 & 19.412 & 127.794 & 19.192 \\
\hline 112.559 & 19.416 & 128.859 & 19.196 \\
\hline 112.559 & 19.42 & 126.73 & 19.2 \\
\hline 111.614 & 19.424 & 125.667 & 19.204 \\
\hline 111.614 & 19.428 & 127.794 & 19.208 \\
\hline 112.559 & 19.432 & 128.859 & 19.212 \\
\hline 111.614 & 19.436 & 128.859 & 19.216 \\
\hline 111.614 & 19.44 & 128.859 & 19.22 \\
\hline 111.614 & 19.444 & 127.794 & 19.224 \\
\hline 111.614 & 19.448 & 127.794 & 19.228 \\
\hline 111.614 & 19.452 & 129.925 & 19.232 \\
\hline 111.614 & 19.456 & 129.925 & 19.236 \\
\hline 110.669 & 19.46 & 130.992 & 19.24 \\
\hline 109.726 & 19.464 & 129.925 & 19.244 \\
\hline 110.669 & 19.468 & 130.992 & 19.248 \\
\hline 110.669 & 19.472 & 128.859 & 19.252 \\
\hline 110.669 & 19.476 & 128.859 & 19.256 \\
\hline 109.726 & 19.48 & 130.992 & 19.26 \\
\hline 111.614 & 19.484 & 130.992 & 19.264 \\
\hline 109.726 & 19.488 & 132.06 & 19.268 \\
\hline 109.726 & 19.492 & 129.925 & 19.27 \\
\hline 110. & 19 & 130.992 & 19. \\
\hline
\end{tabular}




\begin{tabular}{|c|c|c|c|c|}
\hline \multirow{3}{*}{.5942} & \multirow{3}{*}{20.66} & \multirow{3}{*}{88.2332} & 95.8767 & \multirow[t]{2}{*}{17.396} \\
\hline & & & 18.276 & \\
\hline & & & 90.1311 & 17.4 \\
\hline \multirow[t]{2}{*}{51.0389} & 20.664 & 87.2439 & 18.28 & \\
\hline & & & 90.1311 & 17.404 \\
\hline \multirow[t]{2}{*}{51.0389} & 20.668 & 85.2688 & 18.284 & \\
\hline & & & 89.1773 & 17.408 \\
\hline \multirow[t]{2}{*}{51.0389} & 20.672 & 85.2688 & 18.288 & \\
\hline & & & 89.1773 & 17.412 \\
\hline \multirow[t]{2}{*}{51.0389} & 20.676 & 85.2688 & 18.292 & \\
\hline & & & 89.1773 & 17.416 \\
\hline \multirow[t]{2}{*}{51.5942} & 20.68 & 85.2688 & 18.296 & \\
\hline & & & 91.086 & 17.42 \\
\hline \multirow[t]{2}{*}{52.7064} & 20.684 & 85.2688 & 18.3 & \\
\hline & & & 92.042 & 17.424 \\
\hline \multirow[t]{2}{*}{52.7064} & 20.688 & 86.2557 & 18.304 & \\
\hline & & & 93.9572 & 17.428 \\
\hline \multirow[t]{2}{*}{52.7064} & 20.692 & 89.2237 & 18.308 & \\
\hline & & & 93.9572 & 17.432 \\
\hline 53.2633 & 20.696 & 88.2332 & 18.312 & \\
\hline & & & 91.086 & 17.436 \\
\hline 52.7064 & 20.7 & 88.2332 & 18.316 & \\
\hline & & & 89.1773 & 17.44 \\
\hline 53.2633 & 20.704 & 88.2332 & 18.32 & \\
\hline & & & 88.2245 & 17.444 \\
\hline 53.2633 & 20.708 & 87.2439 & 18.324 & \\
\hline & & & 84.4246 & 17.448 \\
\hline 53.2633 & 20.712 & 88.2332 & 18.328 & \\
\hline & & & 87.2729 & 17.452 \\
\hline 52.7064 & 20.716 & 89.2237 & 18.332 & \\
\hline & & & 85.3729 & 17.456 \\
\hline 52.7064 & 20.72 & 89.2237 & 18.336 & \\
\hline & & & 86.3224 & 17.46 \\
\hline 52.1501 & 20.724 & 89.2237 & 18.34 & \\
\hline & & & 87.2729 & 17.464 \\
\hline 52.1501 & 20.728 & 89.2237 & 18.344 & \\
\hline & & & 87.2729 & 17.468 \\
\hline 52.1501 & 20.732 & 90.2153 & 18.348 & \\
\hline & & & 86.3224 & 17.472 \\
\hline 53.2633 & 20.736 & 89.2237 & 18.352 & \\
\hline & & & 84. & 17.476 \\
\hline 52.7064 & 20.74 & 90.2153 & 18.356 & \\
\hline & & & 81.5863 & 17.48 \\
\hline 52.7064 & 20.744 & 91.2081 & 18.36 & \\
\hline & & & 79.6998 & 17.484 \\
\hline 53.2633 & 20.748 & 91.2081 & 18.364 & \\
\hline & & & 78.7582 & 17.488 \\
\hline 53.2633 & 20.752 & 91.2081 & 18.368 & \\
\hline & & & 80.6425 & 17.492 \\
\hline 53.2633 & 20.756 & 90.2153 & 18.372 & \\
\hline & & & 79.6998 & 17.496 \\
\hline 53.8206 & 20.76 & 91.2081 & 18.376 & \\
\hline & & & 79.6998 & 17.5 \\
\hline 53.2633 & 20.764 & 92.2022 & 18.38 & \\
\hline & & & 81.5863 & 17.504 \\
\hline 53.2633 & 20.768 & 94.1936 & 18.384 & \\
\hline & & & 81.5863 & 17.508 \\
\hline 52.7064 & 20.772 & 95.1911 & 18.388 & \\
\hline & & & 80.6425 & 17.512 \\
\hline 53.2633 & 20.776 & 96.1897 & 18.392 & \\
\hline & & & 78.7582 & 17.516 \\
\hline 53.2633 & 20.78 & 96.1897 & 18.396 & \\
\hline & & & 76.8785 & 17.52 \\
\hline 53.2633 & 20.784 & 95.1911 & 18.4 & \\
\hline & & & 75.9404 & 17.524 \\
\hline 53.2633 & 20.788 & 98.1903 & 18.404 & \\
\hline & & & 75.9404 & 17.528 \\
\hline 53.2633 & 20.792 & 97.1894 & 18.408 & \\
\hline & & & 75.0034 & 17.532 \\
\hline 53.2633 & 20.796 & 96.1897 & 18.412 & \\
\hline
\end{tabular}

\begin{tabular}{|c|c|c|c|}
\hline 2.559 & 19.5 & 130.992 & 19.28 \\
\hline 111.614 & 19.504 & 127.794 & 19.284 \\
\hline 14.453 & 19.508 & 26.73 & 19.288 \\
\hline 12.559 & 19.512 & 128.859 & 19.292 \\
\hline 10.669 & 19.516 & 128.859 & 19.296 \\
\hline 111.614 & 19.52 & 129.925 & 19.3 \\
\hline 112.559 & 19.524 & 128.859 & 19.304 \\
\hline 10.669 & 19.528 & 128.859 & 19.308 \\
\hline 109.726 & 19.532 & 128.859 & 19.312 \\
\hline 110.669 & 19.536 & 127.794 & 19.316 \\
\hline 109.726 & 19.54 & 127.794 & 19.32 \\
\hline 109.726 & 19.544 & 128.859 & 19.324 \\
\hline 110.669 & 19.548 & 128.859 & 19.328 \\
\hline 110.669 & 19.552 & 130.992 & 19.332 \\
\hline 111.614 & 19.556 & 129.925 & 19.336 \\
\hline 112.559 & 19.56 & 129.925 & 19.34 \\
\hline 112.559 & 19.564 & 128.859 & 19.344 \\
\hline 110.669 & 19.568 & 128.859 & 19.348 \\
\hline 109.726 & 19.572 & 130.992 & 19.352 \\
\hline 111.614 & 19.576 & 130.992 & 19.356 \\
\hline 111.614 & 19.58 & 132.06 & 19.36 \\
\hline 111.614 & 19.584 & 130.992 & 19.364 \\
\hline 113.505 & 19.588 & 128.859 & 19.368 \\
\hline 114.453 & 19.592 & 128.859 & 19.372 \\
\hline 112.559 & 19.596 & 129.925 & 19.376 \\
\hline 112.55 & 19.6 & 129.925 & 19.38 \\
\hline 113.505 & 19.604 & 132.06 & 19.384 \\
\hline 115.401 & 19.608 & 130.992 & 19.388 \\
\hline 115.401 & 19.612 & 130.992 & 19.392 \\
\hline 117.3 & 19.616 & 132.06 & 19.396 \\
\hline 115.401 & 19.62 & 132.06 & 19. \\
\hline 110.66 & 19.624 & 132.06 & 19.404 \\
\hline 114.45 & 19.628 & 130.992 & 19.408 \\
\hline 112.55 & 19.632 & 129.925 & 19.41 \\
\hline 115.4 & 19.6 & 132.06 & 19.41 \\
\hline
\end{tabular}




\begin{tabular}{|c|c|c|c|c|}
\hline & \multirow{3}{*}{20.8} & \multirow[b]{2}{*}{94.1936} & 76.87 & \multirow[t]{2}{*}{17.536} \\
\hline & & & 18.416 & \\
\hline & & & 75.9404 & 17.54 \\
\hline \multirow[t]{2}{*}{54.3784} & 20.804 & 94.1936 & 18.42 & \\
\hline & & & 77. & 17.544 \\
\hline \multirow[t]{2}{*}{53.8206} & 20.808 & 95.1911 & 18.4 & \\
\hline & & & 75.9 & 17.548 \\
\hline \multirow[t]{2}{*}{53.8206} & 20.812 & 94.1936 & 18.428 & \\
\hline & & & 76.8 & 17.552 \\
\hline \multirow[t]{2}{*}{53.2633} & 20.816 & 96.1897 & 18.4 & \\
\hline & & & & 17.556 \\
\hline \multirow[t]{2}{*}{53.2633} & 20.82 & 95.1911 & 18. & \\
\hline & & & & 17.56 \\
\hline \multirow[t]{2}{*}{53.2633} & 20.824 & 96.1897 & 18. & \\
\hline & & & 75. & 7.564 \\
\hline \multirow[t]{2}{*}{53.2633} & 20.828 & 96.1897 & 18. & \\
\hline & & & 75. & 17.568 \\
\hline \multirow[t]{2}{*}{52.7064} & 20.832 & 99.1923 & & \\
\hline & & & & 17.572 \\
\hline 53.2633 & 20.836 & 98.1903 & & \\
\hline & & & & טדו. \\
\hline 53.2633 & 20.84 & 97.1894 & 18. & \\
\hline & & & & 17.58 \\
\hline 53.8206 & 20.844 & 96.1897 & 18. & \\
\hline & & & & 17.584 \\
\hline 54.3784 & 20.848 & 97.1894 & 18. & \\
\hline & & & & 17.588 \\
\hline 54.3784 & 20.852 & 97.1894 & & \\
\hline & & & & 7.592 \\
\hline 54.9367 & 20.856 & 97.1894 & 18. & \\
\hline & & & & 17.596 \\
\hline 53.2633 & 20.86 & 99.1923 & 18. & \\
\hline & & & & 17.6 \\
\hline 53.8206 & 20.864 & 97.1894 & 18 & \\
\hline & & & & 7.604 \\
\hline 54.9367 & 20.868 & 98.1903 & & \\
\hline & & & & 7.608 \\
\hline 54.9367 & 20.872 & 97.18 & 18 & \\
\hline & & & & 17.612 \\
\hline 53.8206 & 20.876 & 97.1894 & & \\
\hline & & & & 7.616 \\
\hline 54.3784 & 20.88 & 96.1897 & 18. & \\
\hline & & & & 7.62 \\
\hline 54.9367 & 20.88 & 96.1897 & 18 & \\
\hline & & & & 17.624 \\
\hline 55.4955 & 20.888 & 96.1897 & 18 & \\
\hline & & & & 17.628 \\
\hline 54.9367 & 20.892 & 97.1894 & & \\
\hline & & & & 7.632 \\
\hline 54.9367 & 20.896 & 97.1894 & 18. & \\
\hline & & & & 7.636 \\
\hline 55.4955 & 20.9 & 96.1897 & 18 & \\
\hline & & & & 17.64 \\
\hline 54.3784 & 20.904 & 97.1894 & & \\
\hline & & & & 7.644 \\
\hline 54.3784 & 20.908 & 97.1894 & 18.5 & \\
\hline & & & & 17.648 \\
\hline 55.4955 & 20.912 & 97.1894 & 18.5 & \\
\hline & & & & 17.652 \\
\hline 55.4955 & 20.916 & 97.1894 & & \\
\hline & & & & 17.656 \\
\hline 55.4955 & 20.92 & 97.1894 & 18.5 & \\
\hline & & & & 17.66 \\
\hline 55.4955 & 20.92 & 98.1903 & 18. & \\
\hline & & & & 17.664 \\
\hline 55.4955 & 20.928 & 97.1894 & 18.544 & \\
\hline & & & & 17.668 \\
\hline 55.4955 & 20.932 & 97.1894 & 18.5 & \\
\hline & & & & \\
\hline 56.05 & 20.9 & 95.1911 & 18.552 & \\
\hline
\end{tabular}

\begin{tabular}{|c|c|c|c|}
\hline 13.505 & 19.64 & 128.859 & 19.42 \\
\hline 115.401 & 19.644 & 130.992 & 19.424 \\
\hline 115.401 & 19.648 & 129.925 & 19.428 \\
\hline 114.453 & 19.652 & 128.859 & 19.432 \\
\hline 113.505 & 19.656 & 132.06 & 19.436 \\
\hline 117.3 & 19.66 & 132.06 & 19.44 \\
\hline 119.202 & 19.664 & 132.06 & 19.444 \\
\hline 123.017 & 19.668 & 130.992 & 19.448 \\
\hline 125.888 & 19.672 & 133.129 & 19.452 \\
\hline 126.846 & 19.676 & 130.992 & 19.456 \\
\hline 129.727 & 19.68 & 130.992 & 19.46 \\
\hline 135.51 & 19.684 & 129.925 & 19.464 \\
\hline 138.413 & 19.688 & 130.992 & 19.468 \\
\hline 140.351 & 19.692 & 129.925 & 19.472 \\
\hline 139.382 & 19.696 & 133.129 & 19.476 \\
\hline 144.238 & 19.7 & 130.992 & 19.48 \\
\hline 144.238 & 19.704 & 134.199 & 19.484 \\
\hline 142.293 & 19.708 & 132.06 & 19.488 \\
\hline 140.351 & 19.712 & 132.06 & 19.492 \\
\hline 143.266 & 19.716 & 133.129 & 19.496 \\
\hline 142.293 & 19.72 & 132.06 & 19.5 \\
\hline 141.322 & 19.724 & 134.199 & 19.504 \\
\hline 141.322 & 19.728 & 134.199 & 19.508 \\
\hline 140.351 & 19.732 & 134.199 & 19.512 \\
\hline 141.322 & 19.736 & 134.199 & 19.516 \\
\hline 142.293 & 19.74 & 134.199 & 19.52 \\
\hline 142.293 & 19.744 & 134.199 & 19.524 \\
\hline 141.322 & 19.748 & 134.199 & 19.528 \\
\hline 143.266 & 19.752 & 135.27 & 19.532 \\
\hline 143.266 & 19.756 & 135.27 & 19.536 \\
\hline 142.293 & 19.76 & 134.199 & 19.54 \\
\hline 141.322 & 19.764 & 133.129 & 19.544 \\
\hline 142.293 & 19.768 & 133.129 & 19.548 \\
\hline 141.32 & 19.772 & 136.342 & 19.5 \\
\hline 139 & 19.7 & 133.129 & 19.556 \\
\hline
\end{tabular}




\begin{tabular}{|c|c|c|c|c|}
\hline 55.4955 & 0.94 & .1911 & $\begin{array}{l}90.1311 \\
18.556 \\
90.1311\end{array}$ & 17. \\
\hline 56.0547 & 20.944 & 95.1911 & 18.56 & \\
\hline 55.4955 & 20.948 & 98.1903 & 6 & 17.684 \\
\hline \multirow[t]{2}{*}{55.4955} & 20.952 & 99.1923 & & 17.688 \\
\hline & & & 93.5 & 17.692 \\
\hline \multirow[t]{2}{*}{55.4955} & 20.956 & 97.1894 & 18.5 & \\
\hline & & & 93. & 17.696 \\
\hline \multirow[t]{2}{*}{56.0547} & 20.96 & 96.1897 & 18. & \\
\hline & 20.964 & 96.1897 & & 17.7 \\
\hline 55.4955 & & & & 7.704 \\
\hline \multirow[t]{2}{*}{56.0547} & 20.968 & 96.1897 & & \\
\hline & & & & 17.708 \\
\hline \multirow{2}{*}{56.0547} & 20.972 & 95.1911 & & \\
\hline & & & & 17.712 \\
\hline \multirow{2}{*}{56.0547} & 20.976 & 94.1936 & & \\
\hline & & & & 7.71 \\
\hline \multirow[t]{2}{*}{57.1746} & 20.98 & 94.1936 & 18. & \\
\hline & & & & 7.72 \\
\hline \multirow[t]{2}{*}{57.1746} & 20.984 & 92.2022 & 18 & \\
\hline & & & & 17.724 \\
\hline \multirow[t]{2}{*}{57.1746} & 20.988 & 93.1973 & & \\
\hline & & & & 17.728 \\
\hline \multirow[t]{2}{*}{57.1746} & 20.992 & 94.1936 & & \\
\hline & & & & 7.732 \\
\hline 56.6144 & 20.996 & 93.1973 & 18. & \\
\hline & & & & 17.736 \\
\hline 57.7353 & 21 & 95.1911 & 18. & \\
\hline & & & & 17.74 \\
\hline 57.7353 & 21.004 & 93.1973 & & \\
\hline & & & & 7.744 \\
\hline 55.4955 & 21.008 & 94.1936 & & \\
\hline & & & & 7.748 \\
\hline 57.1746 & 21.012 & 97.1894 & & \\
\hline & & & & 17.752 \\
\hline 57.7353 & 21.016 & 96.1897 & & \\
\hline & & & & 7.756 \\
\hline 56.6144 & 21.02 & 95.1911 & 18. & \\
\hline & & & & 7.76 \\
\hline 56.0547 & 21.02 & 95.1911 & 18 & \\
\hline & & & & 7.764 \\
\hline 57.1746 & 21.028 & 94.1936 & & \\
\hline & & & & 7.768 \\
\hline 57.1746 & 21.032 & 4.1936 & & \\
\hline & & & & 7.772 \\
\hline 56.6144 & 21.036 & 93.1973 & 18. & \\
\hline & & & & 7.776 \\
\hline 57.735 & 21.04 & 95.1911 & & \\
\hline & & & & 17.78 \\
\hline 57.7353 & 21.044 & 4.1936 & & \\
\hline & & & & 7.784 \\
\hline 57.7353 & 21.04 & 94.1936 & 18. & \\
\hline & & & & 17.788 \\
\hline 57.735 & 21.052 & 95.1911 & & \\
\hline & & & & 17.792 \\
\hline 57.7353 & 21.056 & 94.1936 & & \\
\hline & & & & 17.796 \\
\hline 57.7353 & 21.06 & 95.1911 & 18.67 & \\
\hline & & & & 17.8 \\
\hline 58.2964 & 21.06 & 96.1897 & 18. & \\
\hline & & & 92.9 & 17.804 \\
\hline 57.7353 & 21.068 & 93.1973 & 18.684 & \\
\hline & & & 92.9991 & 17.808 \\
\hline 57.735 & 21.072 & 94.193 & 18.6 & \\
\hline & & & & \\
\hline 57.1746 & 21.0 & $4.1 S$ & 18.692 & \\
\hline
\end{tabular}

\begin{tabular}{|c|c|c|c|}
\hline 40.351 & 19.78 & 134.199 & 19.56 \\
\hline 41.322 & 19.784 & 133.129 & 19.564 \\
\hline 140.351 & 19.788 & 135.27 & 19.568 \\
\hline 40.351 & 19.792 & 133.129 & 19.572 \\
\hline 140.351 & 19.796 & 135.27 & 19.576 \\
\hline 139.382 & 19.8 & 32.06 & 19.58 \\
\hline 139.382 & 19.804 & 136.342 & 19.584 \\
\hline 138.413 & 19.808 & 133.129 & 19.588 \\
\hline 138.413 & 19.812 & 135.27 & 19.592 \\
\hline 138.413 & 19.816 & 134.199 & 19.596 \\
\hline 137.444 & 19.82 & 134.199 & 19.6 \\
\hline 137.444 & 19.824 & 134.199 & 19.604 \\
\hline 137.444 & 19.828 & 135.27 & 19.608 \\
\hline 137.444 & 19.832 & 135.27 & 19.612 \\
\hline 138.413 & 19.836 & 134.199 & 19.616 \\
\hline 139.382 & 19.84 & 134.199 & 19.62 \\
\hline 138.413 & 19.844 & 135.27 & 19.624 \\
\hline 140.351 & 19.848 & 136.342 & 19.628 \\
\hline 141.322 & 19.852 & 136.342 & 19.632 \\
\hline 139.382 & 19.856 & 135.27 & 19.636 \\
\hline 138.413 & 19.86 & 133.129 & 19.64 \\
\hline 139.382 & 19.864 & 134.199 & 19.644 \\
\hline 140.351 & 19.868 & 133.129 & 19.648 \\
\hline 139.382 & 19.872 & 134.199 & 19.652 \\
\hline 139.382 & 19.876 & 134.199 & 19.656 \\
\hline 138.413 & 19.8 & 35.27 & 19.66 \\
\hline 138.413 & 19.884 & 35.27 & 19.664 \\
\hline 137.444 & 19.888 & 134.199 & 19.668 \\
\hline 135.51 & 19.892 & 132.06 & 19.672 \\
\hline 138.413 & 19.896 & 35.27 & 19.676 \\
\hline 137.444 & 19.9 & 134.199 & 19.68 \\
\hline 138.413 & 19.904 & 135.27 & 19.684 \\
\hline 139.382 & 19.908 & 136.342 & 19.688 \\
\hline 138.413 & 19.912 & 137.415 & 19.69 \\
\hline 138.4 & 19.916 & 137.415 & 19.6 \\
\hline
\end{tabular}




\begin{tabular}{|c|c|c|c|c|}
\hline & & & 93.9572 & 17.816 \\
\hline \multirow[t]{2}{*}{57.7353} & 21.08 & 93.1973 & 18.696 & \\
\hline & & & 92.9991 & 17.82 \\
\hline \multirow[t]{2}{*}{58.2964} & 21.084 & 95.1911 & 18.7 & \\
\hline & & & 91.086 & 7.824 \\
\hline \multirow[t]{2}{*}{57.1746} & 21.088 & 96.1897 & 18.704 & \\
\hline & & & 89.1773 & 17.828 \\
\hline \multirow[t]{2}{*}{57.7353} & 21.092 & 96.1897 & 18.708 & \\
\hline & & & 89.1773 & 17.832 \\
\hline \multirow[t]{2}{*}{58.2964} & 21.096 & 97.1894 & 18.712 & \\
\hline & & & 89.1773 & 17.836 \\
\hline \multirow[t]{2}{*}{57.7353} & 21.1 & 97.1894 & 18.716 & \\
\hline & & & 89.1773 & 17.84 \\
\hline \multirow[t]{2}{*}{58.858} & 21.104 & 97.1894 & 18.72 & \\
\hline & & & 90.1311 & 17.844 \\
\hline \multirow[t]{2}{*}{59.9827} & 21.108 & 98.1903 & 18.724 & \\
\hline & & & 90.1311 & 17.848 \\
\hline \multirow[t]{2}{*}{58.2964} & 21.112 & 97.1894 & 18.728 & \\
\hline & & & 88.2245 & 17.852 \\
\hline \multirow[t]{2}{*}{59.4201} & 21.116 & 96.1897 & 18.732 & \\
\hline & & & 86.3224 & 17.856 \\
\hline \multirow[t]{2}{*}{59.4201} & 21.12 & 96.1897 & 18.736 & \\
\hline & & & 84.4246 & 17.86 \\
\hline \multirow[t]{2}{*}{59.4201} & 21.124 & 97.1894 & 18.74 & \\
\hline & & & 83.4774 & 17.864 \\
\hline 58.2964 & 21.128 & 96.1897 & 18.744 & \\
\hline & & & 81.5863 & 17.868 \\
\hline 58.858 & 21.132 & 97.1894 & 18.748 & \\
\hline & & & 80.6425 & 17.872 \\
\hline 57.7353 & 21.136 & 98.1903 & 18.752 & \\
\hline & & & 80.6425 & 17.876 \\
\hline 58.2964 & 21.14 & 97.1894 & 18.756 & \\
\hline & & & 82.5313 & 17.88 \\
\hline 58.2964 & 21.144 & 99.1923 & 18.76 & \\
\hline & & & 81.5863 & 17.884 \\
\hline 58.2964 & 21.148 & 99.1923 & 18.764 & \\
\hline & & & 82.5313 & 17.888 \\
\hline 59.4201 & 21.152 & 99.1923 & 18.768 & \\
\hline & & & 81.5863 & 17.892 \\
\hline 58.2964 & 21.156 & 99.1923 & 18.772 & \\
\hline & & & 78.7582 & 17.896 \\
\hline 59.4201 & 21.16 & 99.1923 & 18.776 & \\
\hline & & & 81.5863 & 17.9 \\
\hline 59.9827 & 21.164 & 99.1923 & 18.78 & \\
\hline & & & 81.5863 & 17.904 \\
\hline 59.9827 & 21.168 & 98.1903 & 18.784 & \\
\hline & & & 81.5863 & 17.908 \\
\hline 59.4201 & 21.172 & 98.1903 & 18.788 & \\
\hline & & & 81.5863 & 17.912 \\
\hline 59.4201 & 21.176 & 99.1923 & 18.792 & \\
\hline & & & 81.5863 & 17.916 \\
\hline 59.4201 & 21.18 & 101.2 & 18.796 & \\
\hline & & & 84.4246 & 17.92 \\
\hline 58.858 & 21.184 & 99.1923 & 18.8 & \\
\hline & & & 85.37 & 17.924 \\
\hline 59.4201 & 21.188 & 100.195 & 18.804 & \\
\hline & & & 83.4774 & 17.928 \\
\hline 58.2964 & 21.192 & 99.1923 & 18.808 & \\
\hline & & & 82.5313 & 17.932 \\
\hline 59.9827 & 21.196 & 101.2 & 18.812 & \\
\hline & & & 81.5863 & 17.936 \\
\hline 58.858 & 21.2 & 100.195 & 18.816 & \\
\hline & & & 80.6425 & 17.94 \\
\hline 58.858 & 21.204 & 101.2 & 18.82 & \\
\hline & & & 79.6998 & 17.944 \\
\hline 60.5457 & 21.208 & 100.195 & 18.824 & \\
\hline & & & 80.6425 & 17.948 \\
\hline 59.9827 & 21.212 & 100.195 & 18.828 & \\
\hline & & & 80.6425 & 17.952 \\
\hline 59.9827 & 21.216 & 101.2 & 18.832 & \\
\hline
\end{tabular}

\begin{tabular}{|c|c|c|c|}
\hline 136.477 & 19.92 & 135.27 & 19.7 \\
\hline 138.413 & 19.924 & 135.27 & 19.704 \\
\hline 136.477 & 19.928 & 136.342 & 19.708 \\
\hline 138.413 & 19.932 & 136.342 & 19.712 \\
\hline 138.413 & 19.936 & 134.199 & 19.716 \\
\hline 139.382 & 19.94 & 136.342 & 19.72 \\
\hline 139.382 & 19.944 & 136.342 & 19.724 \\
\hline 139.382 & 19.948 & 136.342 & 19.728 \\
\hline 140.351 & 19.952 & 136.342 & 19.732 \\
\hline 139.382 & 19.956 & 136.342 & 19.736 \\
\hline 141.322 & 19.96 & 136.342 & 19.74 \\
\hline 138.413 & 19.964 & 136.342 & 19.744 \\
\hline 140.351 & 19.968 & 136.342 & 19.748 \\
\hline 139.382 & 19.972 & 135.27 & 19.752 \\
\hline 139.382 & 19.976 & 138.489 & 19.756 \\
\hline 140.351 & 19.98 & 138.489 & 19.76 \\
\hline 141.322 & 19.984 & 136.342 & 19.764 \\
\hline 142.293 & 19.988 & 136.342 & 19.768 \\
\hline 141.322 & 19.992 & 136.342 & 19.772 \\
\hline 141.322 & 19.996 & 136.342 & 19.776 \\
\hline 142.293 & 20 & 139.564 & 19.78 \\
\hline 142.293 & 20.004 & 138.489 & 19.784 \\
\hline 143.266 & 20.008 & 139.564 & 19.788 \\
\hline 143.266 & 20.012 & 140.64 & 19.792 \\
\hline 143.266 & 20.016 & 138.489 & 19.796 \\
\hline 142.293 & 20.02 & 138.489 & 19.8 \\
\hline 142.293 & 20.024 & 138.489 & 19.804 \\
\hline 143.266 & 20.028 & 139.564 & 19.808 \\
\hline 144.238 & 20.032 & 140.64 & 19.812 \\
\hline 145.212 & 20.036 & 139.564 & 19.816 \\
\hline 143.266 & 20.04 & 140.64 & 19.82 \\
\hline 140.351 & 20.044 & 140.64 & 19.824 \\
\hline 143.266 & 20.048 & 140.64 & 19.828 \\
\hline 143.266 & 20.052 & 140.64 & 19.832 \\
\hline 144.238 & 20.056 & 141.717 & 19.836 \\
\hline
\end{tabular}




\begin{tabular}{|c|c|c|c|c|}
\hline & & & 82.5313 & 7.956 \\
\hline \multirow[t]{2}{*}{60.5457} & 1.22 & 01.2 & 18.836 & \\
\hline & & & 82.5313 & 17.96 \\
\hline \multirow[t]{2}{*}{61.6732} & 21.224 & 101.2 & 18.84 & \\
\hline & & & 83.4774 & 17.964 \\
\hline \multirow[t]{2}{*}{60.5457} & 21.228 & 103.211 & 18.844 & \\
\hline & & & 84.4246 & 17.968 \\
\hline \multirow[t]{2}{*}{59.9827} & 21.232 & 101.2 & 18.848 & \\
\hline & & & 83. & 17.972 \\
\hline \multirow[t]{2}{*}{60.5457} & 21.236 & 101.2 & 18.8 & \\
\hline & & & 46 & 17.976 \\
\hline \multirow[t]{2}{*}{59.9827} & 21.24 & 101.2 & & \\
\hline & & & & 17.98 \\
\hline \multirow[t]{2}{*}{59.9827} & 21.244 & 100.195 & 18.86 & \\
\hline & & & 246 & 17.984 \\
\hline \multirow[t]{2}{*}{60.5457} & 21.248 & 101.2 & & \\
\hline & & & 46 & 17.988 \\
\hline \multirow[t]{2}{*}{61.1092} & 21.252 & 100.195 & & \\
\hline & & & & 17.992 \\
\hline \multirow[t]{2}{*}{59.9827} & 21.256 & 102.205 & & \\
\hline & & & & 17.996 \\
\hline \multirow[t]{2}{*}{60.5457} & 21.26 & 102.205 & 18. & \\
\hline & & & & 18 \\
\hline \multirow[t]{2}{*}{60.5457} & 21.264 & 101.2 & 18 & \\
\hline & & & & 18.004 \\
\hline 62.2376 & 21.268 & 101.2 & & \\
\hline & & & & 18.008 \\
\hline 59.9827 & 21.272 & 101.2 & & \\
\hline & & & & 18.012 \\
\hline 62.2376 & 21.276 & 101.2 & & \\
\hline & & & & 18.016 \\
\hline 61.6732 & 21.28 & 102.205 & & \\
\hline & & & & 18.02 \\
\hline 61.6732 & 21.284 & 101.2 & & \\
\hline & & & & 18.024 \\
\hline 59.9827 & 21.288 & 103.211 & & \\
\hline & & & & 8.028 \\
\hline 61.1092 & 21.292 & 101.2 & 18 & \\
\hline & & & & 18.032 \\
\hline 61.6732 & 21.296 & 102.205 & & \\
\hline & & & & 18.036 \\
\hline 60.5457 & 21.3 & 102.205 & 18. & \\
\hline & & & & 8.04 \\
\hline 61.6732 & 21.304 & 103.211 & 18 & \\
\hline & & & & 18.044 \\
\hline 62.2376 & 21.308 & 102.205 & & \\
\hline & & & & 8.048 \\
\hline 61.6732 & 21.312 & 102.205 & 18 & \\
\hline & & & & 8.052 \\
\hline 62.2376 & 21.316 & 101 & 18. & \\
\hline & & & & 18.056 \\
\hline 62.2376 & 21.32 & 102.205 & & \\
\hline & & & & 18.06 \\
\hline 62.2376 & 21.324 & 102.205 & 18. & \\
\hline & & & & 18.064 \\
\hline 62.2376 & 21.328 & 102.205 & 18. & \\
\hline & & & & 18.068 \\
\hline 62.2376 & 21.332 & 102.205 & 18. & \\
\hline & & & & 18.072 \\
\hline 62.2376 & 21.336 & 105.228 & & \\
\hline & & & & 18.076 \\
\hline 61.6732 & 21.34 & 105.228 & 18.9 & \\
\hline & & & & 18.08 \\
\hline 62.8025 & 21.34 & 105.228 & 18. & \\
\hline & & & 99.7281 & 18.084 \\
\hline 62.2376 & 21.348 & 105.228 & 18.964 & \\
\hline & & & 99.7 & 18.088 \\
\hline 62.2376 & 21.352 & 105.228 & 18.90 & \\
\hline & & & & 18.092 \\
\hline 62.2376 & 21.35 & 105.228 & 18.972 & \\
\hline
\end{tabular}

\begin{tabular}{|c|c|c|c|}
\hline 144.238 & 20.06 & 139.564 & 19.84 \\
\hline 44.238 & 20.064 & 140.64 & 19.844 \\
\hline 144.238 & 0.068 & 41.717 & 19.848 \\
\hline 144.238 & 20.072 & 140.64 & 19.852 \\
\hline 145.212 & 20.076 & 140.64 & 19.856 \\
\hline 145.212 & 20.08 & 140.64 & 19.86 \\
\hline 146.186 & 20.084 & 141.717 & 19.864 \\
\hline 144.238 & 20.088 & 142.795 & 19.868 \\
\hline 145.212 & 20.092 & 140.64 & 19.872 \\
\hline 144.238 & 20.096 & 140.64 & 19.876 \\
\hline 146.186 & 20.1 & 140.64 & 19.88 \\
\hline 146.186 & 20.104 & 141.717 & 19.884 \\
\hline 147.162 & 20.108 & 141.717 & 19.888 \\
\hline 145.212 & 20.112 & 141.717 & 19.892 \\
\hline 146.186 & 20.116 & 140.64 & 19.896 \\
\hline 146.186 & 20.12 & 140.64 & 19.9 \\
\hline 144.238 & 20.124 & 140.64 & 19.904 \\
\hline 148.137 & 20.128 & 140.64 & 19.908 \\
\hline 148.137 & 20.132 & 140.64 & 19.912 \\
\hline 147.162 & 20.136 & 140.64 & 19.916 \\
\hline 150.092 & 20.14 & 141.717 & 19.92 \\
\hline 148.137 & 20.144 & 140.64 & 19.924 \\
\hline 147.162 & 20.148 & 141.717 & 19.928 \\
\hline 148.137 & 20.152 & 141.717 & 19.932 \\
\hline 148.137 & 20.156 & 141.717 & 19.936 \\
\hline 147.162 & 20.16 & 141.717 & 19.9 \\
\hline 148.137 & 20.164 & 140.64 & 19.944 \\
\hline 149.114 & 20.168 & 142.795 & 19.948 \\
\hline 149.114 & 20.172 & 140.64 & 19.952 \\
\hline 148.137 & 20.176 & 140.64 & 19.956 \\
\hline 148.137 & 20.18 & 140.64 & 19.9 \\
\hline 148.137 & 20.184 & 140.64 & 19.964 \\
\hline 149.114 & 20.188 & 140.64 & 19.968 \\
\hline 148.137 & 20.192 & 140.64 & 19.97 \\
\hline 47. & 20.196 & 140.6 & \\
\hline
\end{tabular}




\begin{tabular}{|c|c|c|c|c|}
\hline \multirow[b]{2}{*}{62.2376} & & & 97.8 & .0 \\
\hline & 1.36 & 105.228 & 18.976 & \\
\hline \multirow[t]{2}{*}{62.2376} & 21.364 & 105.228 & 18.98 & \\
\hline & & & 99.7281 & 18.104 \\
\hline \multirow[t]{2}{*}{63.3678} & 21.368 & 106.237 & 18.9 & \\
\hline & & & 98.7 & 18.108 \\
\hline \multirow[t]{2}{*}{62.8025} & 21.372 & 105.228 & 18.9 & \\
\hline & & & 99.7 & 18.112 \\
\hline \multirow[t]{2}{*}{63.9337} & 21.376 & 106.237 & 18. & \\
\hline & & & 10 & 18.116 \\
\hline \multirow[t]{2}{*}{63.9337} & 21.38 & 107.248 & 18. & \\
\hline & & & 102 & 18.12 \\
\hline \multirow[t]{2}{*}{63.9337} & 21.384 & 106.237 & 19 & \\
\hline & & & 102 & 18.124 \\
\hline \multirow[t]{2}{*}{64.4999} & 21.388 & 106.237 & & \\
\hline & & & & 18.128 \\
\hline \multirow[t]{2}{*}{64.4999} & 21.392 & 108.26 & & \\
\hline & & & & 18.132 \\
\hline \multirow[t]{2}{*}{63.3678} & 21.396 & 109.273 & & \\
\hline & & & & 8.13 \\
\hline \multirow[t]{2}{*}{64.4999} & 21.4 & 108.26 & 19. & \\
\hline & & & & 18.14 \\
\hline \multirow[t]{2}{*}{63.9337} & 21.404 & 110.287 & 19 & \\
\hline & & & & 18.144 \\
\hline 64.4999 & 21.408 & 109.273 & & \\
\hline & & & & 18.148 \\
\hline 64.4999 & 21.412 & 106.237 & & \\
\hline & & & & 18.152 \\
\hline 63.9337 & 21.416 & 107.248 & & \\
\hline & & & & 18.156 \\
\hline 64.4999 & 21.42 & 105.228 & 19. & \\
\hline & & & & 18.16 \\
\hline 64.4999 & 21.424 & 105.228 & 19. & \\
\hline & & & & 18.164 \\
\hline 63.9337 & 21.428 & 104.219 & & \\
\hline & & & & 18.168 \\
\hline 64.4999 & 21.4 & 105.228 & 19. & \\
\hline & & & & 18.172 \\
\hline 63.9337 & 21.436 & 103.211 & & \\
\hline & & & & 18.176 \\
\hline 64.4999 & 21.44 & 105.228 & 19. & \\
\hline & & & & 18.18 \\
\hline 64.4999 & 21.444 & 105.228 & & \\
\hline & & & & 18.184 \\
\hline 64.4999 & 21.448 & 105.228 & 19 & \\
\hline & & & & 18.188 \\
\hline 64.4999 & 21.452 & 108.26 & & \\
\hline & & & & 18.192 \\
\hline 65.0667 & 21.456 & 107.248 & 19. & \\
\hline & & & & 18.196 \\
\hline 65.0667 & 21.46 & 105.228 & & \\
\hline & & & & 18.2 \\
\hline 64.4999 & 21.464 & 103.211 & & \\
\hline & & & & 8.204 \\
\hline 65.6338 & 21.468 & 104.219 & & \\
\hline & & & & 18.208 \\
\hline 65.6338 & 21.47 & 104.219 & 19. & \\
\hline & & & & 18.212 \\
\hline 64.4999 & 21.476 & 104.219 & & \\
\hline & & & & 18.216 \\
\hline 65.0667 & 21.48 & 105.2 & 19.0 & \\
\hline & & & & 18.2 \\
\hline 65.6338 & 21.48 & 105.228 & 19 & \\
\hline & & & & 18.224 \\
\hline 66.7696 & 21.488 & 105.228 & 19.104 & \\
\hline & & & 93.9 & 18.228 \\
\hline 65.6338 & 21.492 & 105.22 & 19.1 & \\
\hline & & & & 18.23 \\
\hline \% & 21.4 & 104.2 & 19.112 & \\
\hline
\end{tabular}

\begin{tabular}{|c|c|c|c|}
\hline 146.186 & 20.2 & 140.64 & 19.98 \\
\hline 147.162 & 20.204 & 40.64 & 19.984 \\
\hline 47.162 & 0.208 & 40.64 & 19.988 \\
\hline 148.137 & 20.212 & 140.64 & 19.992 \\
\hline 149.114 & 20.216 & 140.64 & 19.996 \\
\hline 148.137 & 20.22 & 140.64 & 20 \\
\hline 147.162 & 20.224 & 138.489 & 20.004 \\
\hline 147.162 & 20.228 & 140.64 & 20.008 \\
\hline 147.162 & 20.232 & 140.64 & 20.012 \\
\hline 148.137 & 20.236 & 140.64 & 20.016 \\
\hline 146.186 & 20.24 & 140.64 & 20.02 \\
\hline 147.162 & 20.244 & 140.64 & 20.024 \\
\hline 148.137 & 20.248 & 140.64 & 20.028 \\
\hline 147.162 & 20.252 & 139.564 & 20.032 \\
\hline 147.162 & 20.256 & 140.64 & 20.036 \\
\hline 146.186 & 20.26 & 140.64 & 20.04 \\
\hline 148.137 & 20.264 & 139.564 & 20.044 \\
\hline 148.137 & 20.268 & 140.64 & 20.048 \\
\hline 148.137 & 20.272 & 139.564 & 20.052 \\
\hline 148.137 & 20.276 & 140.64 & 20.056 \\
\hline 146.186 & 20.28 & 140.64 & 20.06 \\
\hline 146.186 & 20.284 & 139.564 & 20.064 \\
\hline 147.162 & 20.288 & 140.64 & 20.068 \\
\hline 149.114 & 20.292 & 140.64 & 20.072 \\
\hline 147.162 & 20.296 & 140.64 & 20.076 \\
\hline 148.137 & 20.3 & 139.564 & 20.08 \\
\hline 149.114 & 20.304 & 140.64 & 20.084 \\
\hline 149.114 & 20.308 & 140.64 & 20.088 \\
\hline 147.162 & 20.312 & 140.64 & 20.092 \\
\hline 147.162 & 20.316 & 140.64 & 20.096 \\
\hline 151.07 & 20.32 & 140.64 & 20.1 \\
\hline 148.137 & 20.324 & 140.64 & 20.104 \\
\hline 146.186 & 20.32 & 140.64 & 20.108 \\
\hline 146.186 & 20.332 & 140.64 & 20.112 \\
\hline 146. & 20.3 & 140.64 & 20.11 \\
\hline
\end{tabular}




\begin{tabular}{|c|c|c|c|c|}
\hline \multirow{3}{*}{65.0667} & & & 95.8767 & 18.236 \\
\hline & 1.5 & 105.228 & 19.116 & \\
\hline & & & 93.9572 & 18.24 \\
\hline \multirow[t]{2}{*}{66.2015} & 21.504 & 105.228 & 19.12 & \\
\hline & & & 94.9164 & 18.244 \\
\hline \multirow[t]{2}{*}{65.6338} & 21.508 & 106.237 & 19.1 & \\
\hline & & & 94.9 & 18.248 \\
\hline \multirow[t]{2}{*}{66.7696} & 21.512 & 105.228 & 19.128 & \\
\hline & & & 93.9 & 18.252 \\
\hline \multirow[t]{2}{*}{65.6338} & 21.516 & 107.248 & 19.1 & \\
\hline & & & 93. & 18.256 \\
\hline \multirow[t]{2}{*}{66.2015} & 21.52 & 105.228 & 19. & \\
\hline & & & & 18.26 \\
\hline \multirow[t]{2}{*}{66.2015} & 21.524 & 107.248 & & \\
\hline & & & & .264 \\
\hline \multirow[t]{2}{*}{66.2015} & 21.528 & 108.26 & 19. & \\
\hline & & & & 18.268 \\
\hline \multirow[t]{2}{*}{65.0667} & 21.532 & 107.248 & & \\
\hline & & & & 18.272 \\
\hline \multirow{2}{*}{66.7696} & 21.536 & 108.26 & & \\
\hline & & & & 10 \\
\hline \multirow[t]{2}{*}{66.2015} & 21.54 & 108.26 & 19. & \\
\hline & & & & 8.28 \\
\hline \multirow[t]{2}{*}{66.2015} & 21.544 & 109.273 & 19. & \\
\hline & & & & 18.284 \\
\hline 66.7696 & 21.548 & 107.248 & 19. & \\
\hline & & & & 18.288 \\
\hline 66.7696 & 21.552 & 106.237 & & \\
\hline & & & & 3.292 \\
\hline 66.7696 & 21.556 & 106.237 & 19. & \\
\hline & & & & 18.296 \\
\hline 66.7696 & 21.56 & 105.228 & 19. & \\
\hline & & & & 18.3 \\
\hline 66.7696 & 21.564 & 105.228 & 19 & \\
\hline & & & & 8.304 \\
\hline 66.7696 & 21.568 & 105.228 & & \\
\hline & & & & 18.308 \\
\hline 66.7696 & $21.5^{\circ}$ & 106.237 & 19. & \\
\hline & & & & 18.312 \\
\hline 66.7696 & 21.576 & 105.228 & & \\
\hline & & & & 8.316 \\
\hline 66.7696 & 21.58 & 106.237 & 19. & \\
\hline & & & & 8.32 \\
\hline 66.7696 & 21.584 & 108.26 & & \\
\hline & & & & 18.324 \\
\hline 66.7696 & 21.588 & 109.273 & & \\
\hline & & & & 8.328 \\
\hline 66.7696 & 21.592 & 109.273 & & \\
\hline & & & & 8.332 \\
\hline 66.7696 & 21.596 & 109.273 & & \\
\hline & & & & 18.336 \\
\hline 66.7696 & 21.6 & 108.26 & & \\
\hline & & & & 18.34 \\
\hline 66.7696 & 21.604 & 109.273 & 19 & \\
\hline & & & & 8.344 \\
\hline 66.7696 & 21.608 & 109.273 & 19. & \\
\hline & & & & 18.348 \\
\hline 67.3382 & 21.612 & 105.228 & & \\
\hline & & & & 18.352 \\
\hline 67.3382 & 21.616 & 107.248 & & \\
\hline & & & & 8.356 \\
\hline 66.7696 & 21.62 & 110.287 & & \\
\hline & & & & 18. \\
\hline 66.2015 & 21.624 & 109.273 & 19. & \\
\hline & & & & 18.364 \\
\hline 66.7696 & 21.628 & 110.287 & 19.244 & \\
\hline & & & & 18.368 \\
\hline 66.7696 & 21.63 & 110.287 & 19.248 & \\
\hline & & & & \\
\hline 5.76 & 21.6 & 107.2 & 19.252 & \\
\hline
\end{tabular}

\begin{tabular}{|c|c|c|c|}
\hline 148.137 & 20.34 & 140.64 & 20.12 \\
\hline 149.114 & 20.344 & 139.564 & 20.124 \\
\hline 150.092 & 20.348 & 140.64 & 20.128 \\
\hline 149.114 & 20.352 & 139.564 & 20.132 \\
\hline 146.186 & 20.356 & 140.64 & 20.136 \\
\hline 149.114 & 20.36 & 140.64 & 20.14 \\
\hline 148.137 & 20.364 & 140.64 & 20.144 \\
\hline 148.137 & 20.368 & 140.64 & 20.148 \\
\hline 150.092 & 20.372 & 140.64 & 20.152 \\
\hline 149.114 & 20.376 & 140.64 & 20.156 \\
\hline 148.137 & 20.38 & 140.64 & 20.16 \\
\hline 147.162 & 20.384 & 140.64 & 20.164 \\
\hline 150.092 & 20.388 & 140.64 & 20.168 \\
\hline 149.114 & 20.392 & 140.64 & 20.172 \\
\hline 151.07 & 20.396 & 140.64 & 20.176 \\
\hline 151.07 & 20.4 & 140.64 & 20.18 \\
\hline 149.114 & 20.404 & 142.795 & 20.184 \\
\hline 152.048 & 20.408 & 140.64 & 20.188 \\
\hline 153.028 & 20.412 & 140.64 & 20.192 \\
\hline 151.07 & 20.416 & 141.717 & 20.196 \\
\hline 149.114 & 20.42 & 142.795 & 20.2 \\
\hline 151.07 & 20.424 & 141.717 & 20.204 \\
\hline 151.07 & 20.428 & 141.717 & 20.208 \\
\hline 152.048 & 20.432 & 142.795 & 20.212 \\
\hline 151.07 & 20.436 & 143.873 & 20.216 \\
\hline 152.048 & 20.44 & 142.795 & 20.22 \\
\hline 153.028 & 20.444 & 141.717 & 20.224 \\
\hline 153.028 & 20.448 & 143.873 & 20.228 \\
\hline 151.07 & 20.452 & 143.873 & 20.232 \\
\hline 152.048 & 20.456 & 142.795 & 20.236 \\
\hline 153.028 & 20.46 & 141.717 & 20.24 \\
\hline 151.07 & 20.464 & 142.795 & 20.244 \\
\hline 153.028 & 20.468 & 140.64 & 20.248 \\
\hline 151.07 & 20.472 & 141.717 & 20.252 \\
\hline $151.0^{\circ}$ & 20.476 & 141.717 & 20.256 \\
\hline
\end{tabular}




\begin{tabular}{|c|c|c|c|c|}
\hline & & & 100.694 & 18.376 \\
\hline \multirow[t]{2}{*}{67.3382} & 1.64 & 09.273 & 19.256 & \\
\hline & & & 100.694 & 18.38 \\
\hline \multirow{2}{*}{66.7696} & 21.644 & 109.273 & 19.26 & \\
\hline & & & 100.694 & 18.384 \\
\hline \multirow[t]{2}{*}{66.7696} & 21.648 & 109.273 & 19.264 & \\
\hline & & & 100.694 & 18.388 \\
\hline \multirow[t]{2}{*}{66.7696} & 21.652 & 108.26 & 19.268 & \\
\hline & & & 37 & 18.392 \\
\hline \multirow[t]{2}{*}{66.7696} & 21.656 & 107.248 & 19.272 & \\
\hline & & & 99 & 18.396 \\
\hline \multirow[t]{2}{*}{66.7696} & 21.66 & 109.273 & & \\
\hline & & & & 18.4 \\
\hline \multirow[t]{2}{*}{67.3382} & 21.664 & 106.237 & 19. & \\
\hline & & & 694 & 18.404 \\
\hline \multirow[t]{2}{*}{67.3382} & 21.668 & 108.26 & & \\
\hline & & & & 18.408 \\
\hline \multirow[t]{2}{*}{67.3382} & 21.672 & 109.273 & & \\
\hline & & & & 18.412 \\
\hline \multirow[t]{2}{*}{66.7696} & 21.676 & 109.273 & 19 & \\
\hline & & & & 18.416 \\
\hline \multirow[t]{2}{*}{66.7696} & 21.68 & 108.26 & 19. & \\
\hline & & & 66 & 18.42 \\
\hline \multirow[t]{2}{*}{66.7696} & 21.684 & 106.237 & 19 & \\
\hline & & & & 18.424 \\
\hline 67.3382 & 21.688 & 107.248 & & \\
\hline & & & & 18.428 \\
\hline 67.9072 & 21.692 & 107.248 & 19. & \\
\hline & & & & 18.432 \\
\hline 67.9072 & 21.696 & 109.273 & 19 & \\
\hline & & & & 18.436 \\
\hline 67.3382 & 21.7 & 108.26 & & \\
\hline & & & & 18.44 \\
\hline 69.0466 & 21.704 & 110.287 & & \\
\hline & & & & 18.444 \\
\hline 68.4767 & 21.708 & 109.273 & 19 & \\
\hline & & & & 8.448 \\
\hline 69.0466 & 21.712 & 108.26 & $1 \mathrm{c}$ & \\
\hline & & & & 18.452 \\
\hline 68.4767 & 21.716 & 109.273 & & \\
\hline & & & & 18.456 \\
\hline 67.9072 & 21.72 & 109.273 & 19. & \\
\hline & & & & 18.46 \\
\hline 68.4767 & 21.724 & 109.273 & 1 & \\
\hline & & & & 18.464 \\
\hline 68.4767 & 21.728 & 109.273 & & \\
\hline & & & & 8.468 \\
\hline 69.0466 & 21.732 & 109.273 & 19. & \\
\hline & & & & 18.472 \\
\hline 69.0466 & 21.736 & 109.273 & 1 & \\
\hline & & & & 18.476 \\
\hline 69.0466 & 21.74 & 109.273 & & \\
\hline & & & & 18.48 \\
\hline 69.0466 & 21.744 & 108.26 & 19. & \\
\hline & & & & 18.484 \\
\hline 69.0466 & 21.748 & 108.26 & 19. & \\
\hline & & & & 18.488 \\
\hline 68.4767 & 21.752 & 110.287 & 19.3 & \\
\hline & & & & 18.492 \\
\hline 69.617 & 21.756 & 111.302 & & \\
\hline & & & & 18.496 \\
\hline 69.617 & 21.76 & 111.302 & 19.376 & \\
\hline & & & & 18.5 \\
\hline 70.759 & 21.76 & 112.318 & 19.3 & \\
\hline & & & 108 & 18.504 \\
\hline 70.1878 & 21.768 & 111.302 & 19. & \\
\hline & & & & 18.508 \\
\hline 69.617 & 21.77 & 108.2 & & \\
\hline & & & & 18.512 \\
\hline 69.6 & 21.77 & 109.2 & 19.392 & \\
\hline
\end{tabular}

\begin{tabular}{|c|c|c|c|}
\hline 152.048 & 20.48 & 142.795 & 20.26 \\
\hline 51.07 & 20.484 & 141.717 & 20.264 \\
\hline 2.048 & 0.488 & 41.717 & 20.268 \\
\hline 52.048 & 20.492 & 141.717 & 20.272 \\
\hline 54.008 & 20.496 & 142.795 & 20.276 \\
\hline 154.989 & 20.5 & 141.717 & 20.28 \\
\hline 156.953 & 20.504 & 140.64 & 20.284 \\
\hline 158.92 & 20.508 & 142.795 & 20.288 \\
\hline 161.876 & 20.512 & 141.717 & 20.292 \\
\hline 162.862 & 20.516 & 141.717 & 20.296 \\
\hline 164.837 & 20.52 & 141.717 & 20.3 \\
\hline 163.849 & 20.524 & 142.795 & 20.304 \\
\hline 162.862 & 20.528 & 141.717 & 20.308 \\
\hline 160.89 & 20.532 & 141.717 & 20.312 \\
\hline 160.89 & 20.536 & 142.795 & 20.316 \\
\hline 158.92 & 20.54 & 143.873 & 20.32 \\
\hline 159.905 & 20.544 & 140.64 & 20.324 \\
\hline 159.905 & 20.548 & 140.64 & 20.328 \\
\hline 158.92 & 20.552 & 140.64 & 20.332 \\
\hline 159.905 & 20.556 & 41.717 & 20.336 \\
\hline 157.936 & 20.56 & 141.717 & 20.34 \\
\hline 59.905 & 20.564 & 140.64 & 20.344 \\
\hline 159.905 & 20.568 & 41.717 & 20.348 \\
\hline 159.905 & 20.572 & 140.64 & 20.352 \\
\hline 159.905 & 20.576 & 141.717 & 20.356 \\
\hline 157.936 & 20.58 & 141.717 & 20.36 \\
\hline 158.92 & 20.584 & 141.717 & 20.364 \\
\hline 157.936 & 20.588 & 143.873 & 20.368 \\
\hline 156.953 & 20.592 & 143.873 & 20.372 \\
\hline 155.971 & 20.596 & 142.795 & 20.376 \\
\hline 156.953 & 20.6 & 143.873 & 20.38 \\
\hline 157.936 & 20.604 & 144.953 & 20.384 \\
\hline 157.936 & 20.608 & 144.953 & 20.388 \\
\hline 158.92 & 20.612 & 142.795 & 20.39 \\
\hline 158 & 20.616 & 143.87 & 20 \\
\hline
\end{tabular}




\begin{tabular}{|c|c|c|c|c|}
\hline & & & 105.536 & 18.516 \\
\hline \multirow[t]{2}{*}{70.1878} & 21.78 & 108.26 & 19.396 & \\
\hline & & & 103.596 & 18.52 \\
\hline \multirow[t]{2}{*}{69.617} & 21.784 & 110.287 & 19.4 & \\
\hline & & & 104.566 & 18.524 \\
\hline \multirow[t]{2}{*}{70.1878} & 21.788 & 110.287 & 19.404 & \\
\hline & & & 104.566 & 18.528 \\
\hline \multirow[t]{2}{*}{70.7591} & 21.792 & 111.302 & 19.408 & \\
\hline & & & 106.508 & 18.532 \\
\hline \multirow[t]{2}{*}{69.617} & 21.796 & 111.302 & 19.412 & \\
\hline & & & 106.508 & 18.536 \\
\hline \multirow[t]{2}{*}{71.3308} & 21.8 & 113.335 & 19.416 & \\
\hline & & & 104.566 & 18.54 \\
\hline \multirow[t]{2}{*}{71.3308} & 21.804 & 112.318 & 19.42 & \\
\hline & & & 103.596 & 18.544 \\
\hline \multirow[t]{2}{*}{71.3308} & 21.808 & 113.335 & 19.424 & \\
\hline & & & 104.566 & 18.548 \\
\hline \multirow[t]{2}{*}{71.3308} & 21.812 & 113.335 & 19.428 & \\
\hline & & & 103.596 & 18.552 \\
\hline \multirow[t]{2}{*}{71.3308} & 21.816 & 113.335 & 19.432 & \\
\hline & & & 102.628 & 18.556 \\
\hline \multirow[t]{2}{*}{70.7591} & 21.82 & 111.302 & 19.436 & \\
\hline & & & 103.596 & 18.56 \\
\hline \multirow[t]{2}{*}{70.7591} & 21.824 & 109.273 & 19.44 & \\
\hline & & & 103.596 & 18.564 \\
\hline 70.7591 & 21.828 & 109.273 & 19.444 & \\
\hline & & & 103.596 & 18.568 \\
\hline 70.1878 & 21.832 & 110.287 & 19.448 & \\
\hline & & & 596 & 18.572 \\
\hline 69.0466 & 21.836 & 111.302 & & \\
\hline & & & 566 & 18.576 \\
\hline 67.3382 & 21.84 & 112.318 & & \\
\hline & & & 103.596 & 18.58 \\
\hline 66.2015 & 21.844 & 112.318 & 19.46 & \\
\hline & & & 103.596 & 18.584 \\
\hline 65.6338 & 21.848 & 113.335 & 19.464 & \\
\hline & & & 103.596 & 18.588 \\
\hline 64.4999 & 21.852 & 110.287 & 19.468 & \\
\hline & & & 103.596 & 18.592 \\
\hline 64.4999 & 21.856 & 110.287 & 19.472 & \\
\hline & & & & 18.596 \\
\hline 64.4999 & 21.86 & 112.318 & 19.476 & \\
\hline & & & 102.628 & 18.6 \\
\hline 66.7696 & 21.864 & 112.318 & 19.48 & \\
\hline & & & & 18.604 \\
\hline 66.7696 & 21.868 & 113.335 & 19.484 & \\
\hline & & & 103.596 & 18.608 \\
\hline 67.3382 & 21.872 & 112.318 & 19.488 & \\
\hline & & & 104.566 & 18.612 \\
\hline 67.9072 & 21.876 & 111.302 & 19.492 & \\
\hline & & & 103.596 & 18.616 \\
\hline 69.0466 & 21.88 & 110.287 & 19.496 & \\
\hline & & & 103.596 & 18.62 \\
\hline 69.0466 & 21.884 & 109.273 & 19.5 & \\
\hline & & & 100.694 & 18.624 \\
\hline 69.0466 & 21.888 & 111.302 & 19.504 & \\
\hline & & & & 18.628 \\
\hline 69.0466 & 21.892 & 113.335 & 19 & \\
\hline & & & 99.7281 & 18.632 \\
\hline 68.4767 & 21.896 & 111.302 & 19.512 & \\
\hline & & & 99.7281 & 18.636 \\
\hline 69.617 & 21.9 & 112.318 & 19.516 & \\
\hline & & & 100.694 & 18.64 \\
\hline 69.0466 & 21.904 & 112.318 & 19.52 & \\
\hline & & & 100.694 & 18.644 \\
\hline 68.4767 & 21.908 & 111.302 & 19.524 & \\
\hline & & & 100.694 & 18.648 \\
\hline 69.0466 & 21.912 & 111.302 & 19.528 & \\
\hline & & & 101.66 & 18.652 \\
\hline 69.046 & 21.916 & 111.302 & 19.532 & \\
\hline
\end{tabular}

\begin{tabular}{|c|c|c|c|}
\hline 160.89 & 20.62 & 143.873 & 20.4 \\
\hline 159.905 & 20.624 & 143.873 & 20.404 \\
\hline 158.92 & 20.628 & 144.953 & 20.408 \\
\hline 157.936 & 20.632 & 144.953 & 20.412 \\
\hline 157.936 & 20.636 & 144.953 & 20.416 \\
\hline 156.953 & 20.64 & 144.953 & 20.42 \\
\hline 154.989 & 20.644 & 143.873 & 20.424 \\
\hline 154.008 & 20.648 & 144.953 & 20.428 \\
\hline 154.989 & 20.652 & 144.953 & 20.432 \\
\hline 154.989 & 20.656 & 143.873 & 20.436 \\
\hline 155.971 & 20.66 & 144.953 & 20.44 \\
\hline 159.905 & 20.664 & 144.953 & 20.444 \\
\hline 160.89 & 20.668 & 144.953 & 20.448 \\
\hline 160.89 & 20.672 & 143.873 & 20.452 \\
\hline 160.89 & 20.676 & 146.034 & 20.456 \\
\hline 156.953 & 20.68 & 144.953 & 20.46 \\
\hline 158.92 & 20.684 & 144.953 & 20.464 \\
\hline 155.971 & 20.688 & 144.953 & 20.468 \\
\hline 154.008 & 20.692 & 144.953 & 20.472 \\
\hline 154.008 & 20.696 & 144.953 & 20.476 \\
\hline 154.989 & 20.7 & 144.953 & 20.48 \\
\hline 156.953 & 20.704 & 146.034 & 20.484 \\
\hline 158.92 & 20.708 & 144.953 & 20.488 \\
\hline 158.92 & 20.712 & 146.034 & 20.492 \\
\hline 159.905 & 20.716 & 147.115 & 20.496 \\
\hline 162.862 & 20.72 & 148.198 & 20.5 \\
\hline 163.849 & 20.724 & 144.953 & 20.504 \\
\hline 162.862 & 20.728 & 141.717 & 20.508 \\
\hline 161.876 & 20.732 & 136.342 & 20.512 \\
\hline 155.971 & 20.736 & 128.859 & 20.516 \\
\hline 152.048 & 20.74 & 124.605 & 20.52 \\
\hline 145.212 & 20.744 & 123.544 & 20.524 \\
\hline 141.322 & 20.748 & 123.544 & 20.528 \\
\hline 142.293 & 20.752 & 126.73 & 20.532 \\
\hline 143.266 & 20.756 & 130.992 & 20.536 \\
\hline
\end{tabular}




\begin{tabular}{|c|c|c|c|c|}
\hline & & & 100.694 & .656 \\
\hline \multirow[t]{2}{*}{69.617} & 1.92 & 13.335 & 19.536 & \\
\hline & & & 101.66 & 18.66 \\
\hline \multirow[t]{2}{*}{70.7591} & 21.924 & 111.302 & 19.54 & \\
\hline & & & 101.66 & 8.664 \\
\hline \multirow[t]{2}{*}{71.3308} & 21.928 & 112.318 & 19.544 & \\
\hline & & & 100.694 & 18.668 \\
\hline \multirow[t]{2}{*}{71.3308} & 21.932 & 111.302 & 19.548 & \\
\hline & & & 100.694 & 18.672 \\
\hline \multirow[t]{2}{*}{70.1878} & 21.936 & 111.302 & 19.552 & \\
\hline & & & 101.66 & 18.676 \\
\hline \multirow{2}{*}{67.3382} & 21.94 & 114.354 & & \\
\hline & & & & 18.68 \\
\hline \multirow[t]{2}{*}{66.7696} & 21.944 & 113.335 & 19.56 & \\
\hline & & & 101.66 & 18.684 \\
\hline \multirow[t]{2}{*}{66.2015} & 21.948 & 113.335 & 19.564 & \\
\hline & & & 102.628 & 18.688 \\
\hline \multirow[t]{2}{*}{65.0667} & 21.952 & 114.354 & 19 & \\
\hline & & & & 18.692 \\
\hline \multirow[t]{2}{*}{66.2015} & 21.956 & 114.354 & & \\
\hline & & & & 18.696 \\
\hline \multirow[t]{2}{*}{66.7696} & 21.96 & 115.373 & 19. & \\
\hline & & & 28 & 18.7 \\
\hline \multirow[t]{2}{*}{67.3382} & 21.964 & 113.335 & 19. & \\
\hline & & & & 18.704 \\
\hline \multirow[t]{2}{*}{67.3382} & 21.968 & 112.318 & & \\
\hline & & & & 18.708 \\
\hline 67.9072 & 21.972 & 113.335 & 19. & \\
\hline & & & & 18.712 \\
\hline 67.9072 & 21.976 & 115.373 & 19 & \\
\hline & & & & 18.716 \\
\hline 67.3382 & 21.98 & 114.354 & 6 & \\
\hline & & & & 18.72 \\
\hline 66.7696 & 21.984 & 113.335 & & \\
\hline & & & & 18.724 \\
\hline 66.7696 & 21.988 & 113.335 & 19 & \\
\hline & & & & 18.728 \\
\hline 66.1 & 21 & 113.335 & & \\
\hline 66.7696 & 21.996 & 114.354 & & \\
\hline & & & & 18.736 \\
\hline 66.7696 & 22 & 113.335 & 19. & \\
\hline & & & & 18.74 \\
\hline 66.7696 & 22.004 & 114.354 & & \\
\hline & & & & 18.744 \\
\hline 68.4767 & 22.008 & 115.373 & & \\
\hline & & & & 8.748 \\
\hline 69.617 & 22.012 & 113.335 & 19 & \\
\hline & & & & 18.752 \\
\hline 70.1878 & 22.016 & 115.373 & 19 & \\
\hline & & & & 8.756 \\
\hline 70.7591 & 22.02 & 115.373 & 1 & \\
\hline & & & & 18.76 \\
\hline 70.7591 & 22.024 & 115.373 & 19. & \\
\hline & & & & 18.764 \\
\hline 71.3308 & 22.028 & 116.393 & 19. & \\
\hline & & & & 18.768 \\
\hline 72.4756 & 22.032 & 117.415 & 19.6 & \\
\hline & & & & 18.772 \\
\hline 71.9029 & 22.036 & 117.415 & & \\
\hline & & & & 76 \\
\hline 71.9029 & 22.04 & 116.393 & 19.6 & \\
\hline & & & & 18.78 \\
\hline 70.75 & 22.04 & 117.415 & 19. & \\
\hline & & & 107.48 & 18.784 \\
\hline 71.3308 & 22.048 & 116.393 & 19.6 & \\
\hline & & & & 18.788 \\
\hline 71.33 & 22.0 & 116.393 & $\begin{array}{l}19.668 \\
106.508\end{array}$ & 187 \\
\hline 70.7591 & 22.056 & 116.393 & 19.672 & \\
\hline
\end{tabular}

\begin{tabular}{|c|c|c|c|}
\hline 143.266 & 20.76 & 134.199 & 20.54 \\
\hline 147.162 & 20.764 & 135.27 & 20.544 \\
\hline 45.212 & 20.768 & 136.342 & 20.548 \\
\hline 147.162 & 20.772 & 139.564 & 20.552 \\
\hline 45.212 & 20.776 & 137.415 & 20.556 \\
\hline 144.238 & 20.78 & 138.489 & 20.56 \\
\hline 144.238 & 20.784 & 139.564 & 20.564 \\
\hline 144.238 & 20.788 & 140.64 & 20.568 \\
\hline 146.186 & 20.792 & 140.64 & 20.572 \\
\hline 148.137 & 20.796 & 140.64 & 20.576 \\
\hline 148.137 & 20.8 & 140.64 & 20.58 \\
\hline 150.092 & 20.804 & 140.64 & 20.584 \\
\hline 153.028 & 20.808 & 140.64 & 20.588 \\
\hline 152.048 & 20.812 & 140.64 & 20.592 \\
\hline 153.028 & 20.816 & 142.795 & 20.596 \\
\hline 153.028 & 20.82 & 141.717 & 20.6 \\
\hline 150.092 & 20.824 & 142.795 & 20.604 \\
\hline 149.114 & 20.828 & 140.64 & 20.608 \\
\hline 147.162 & 20.832 & 140.64 & 20.612 \\
\hline 148.137 & 20.836 & 141.717 & 20.616 \\
\hline 148.137 & 20.84 & 140.64 & 20.62 \\
\hline 149.114 & 20.844 & 144.953 & 20.624 \\
\hline 151.07 & 20.848 & 144.953 & 20.628 \\
\hline 154.989 & 20.852 & 144.953 & 20.632 \\
\hline 154.989 & 20.856 & 141.717 & 20.636 \\
\hline 154.008 & 20.86 & 143.873 & 20.64 \\
\hline 154.989 & 20.864 & 143.873 & 20.644 \\
\hline 152.048 & 20.868 & 143.873 & 20.648 \\
\hline 151.07 & 20.872 & 146.034 & 20.652 \\
\hline 150.092 & 20.876 & 144.953 & 20.656 \\
\hline 151.07 & 20.88 & 146.034 & 20.66 \\
\hline 153.028 & 20.884 & 146.034 & 20.664 \\
\hline 154.008 & 20.888 & 144.953 & 20.668 \\
\hline 155.971 & 20.892 & 144.953 & 20.672 \\
\hline 55.9 & 20.896 & 143.873 & 20. \\
\hline
\end{tabular}




\begin{tabular}{|c|c|c|c|c|}
\hline & & & 106.508 & 18.796 \\
\hline \multirow[t]{2}{*}{71.3308} & 22.06 & 114.354 & 19.676 & \\
\hline & & & 107.48 & 18.8 \\
\hline \multirow[t]{2}{*}{71.3308} & 22.064 & 114.354 & 19.68 & \\
\hline & & & 107.48 & 18.804 \\
\hline \multirow{2}{*}{71.3308} & 22.068 & 113.335 & 19.684 & \\
\hline & & & 108.454 & 18.808 \\
\hline \multirow[t]{2}{*}{71.9029} & 22.072 & 112.318 & 19.688 & \\
\hline & & & 107.48 & 18.812 \\
\hline \multirow[t]{2}{*}{72.4756} & 22.076 & 114.354 & 19.692 & \\
\hline & & & 108.454 & 18.816 \\
\hline \multirow[t]{2}{*}{71.3308} & 22.08 & 116.393 & 19.696 & \\
\hline & & & 108.454 & 18.82 \\
\hline \multirow[t]{2}{*}{69.617} & 22.084 & 114.354 & 19.7 & \\
\hline & & & 109.429 & 18.824 \\
\hline \multirow[t]{45}{*}{47.1649} & 22.088 & 117.415 & 19.704 & \\
\hline & & & 109.429 & 18.828 \\
\hline & 110.073 & 19.100 & 108.454 & 18.832 \\
\hline & 117.415 & 19.712 & & \\
\hline & & & 109.429 & 18.836 \\
\hline & 117.415 & 19.716 & & \\
\hline & 116.393 & 19.72 & 110.404 & 18.84 \\
\hline & & & 109.429 & 18.844 \\
\hline & 117.415 & 19.724 & & \\
\hline & & & 108.454 & 18.848 \\
\hline & 117.415 & 19.728 & & \\
\hline & & & 111.381 & 18.852 \\
\hline & $11 / .413$ & 19.132 & 110.404 & 18.856 \\
\hline & 117.415 & 19.736 & & \\
\hline & & & 110.404 & 18.86 \\
\hline & 118.437 & 19.74 & & \\
\hline & 117 & 1972 & 109.429 & 18.864 \\
\hline & Heno & 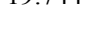 & 111.381 & 18.868 \\
\hline & 117.415 & 19.748 & & \\
\hline & 104 & & 111.381 & 18.872 \\
\hline & 117.70 & 19.172 & 112.358 & 18.876 \\
\hline & 118.437 & 19.756 & & \\
\hline & & & 111.381 & 18.88 \\
\hline & 119.46 & 19.76 & & \\
\hline & & & 111.381 & 18.884 \\
\hline & 118.437 & 19.764 & 4 & \\
\hline & 117.415 & 19.768 & דיד. & 10.000 \\
\hline & & & 111.381 & 18.892 \\
\hline & 118.437 & 19.772 & & \\
\hline & & & 112.358 & 18.896 \\
\hline & 119.46 & 19.776 & & \\
\hline & 11946 & $197-3$ & 111.381 & 18.9 \\
\hline & 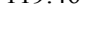 & 15.10 & 110.404 & 18.904 \\
\hline & 118.437 & 19.784 & & \\
\hline & & & 112.358 & 18.908 \\
\hline & 118.437 & 19.788 & & \\
\hline & 1184 & & 112.358 & 18.912 \\
\hline & 110.751 & 10.18 & 111.381 & 18.916 \\
\hline & 119.46 & 19.796 & & \\
\hline & & & 112.358 & 18.92 \\
\hline & 117.415 & 19.8 & & \\
\hline & & & 111.381 & 18.924 \\
\hline & 117.70 & 17.00 & 114.316 & 18.928 \\
\hline & 118.437 & 19.808 & & \\
\hline & 1194 & 198 & 112.358 & 18.932 \\
\hline
\end{tabular}

\begin{tabular}{|c|c|c|c|}
\hline 158.92 & 20.9 & 144.953 & 20.68 \\
\hline 157.936 & 20.904 & 147.115 & 20.684 \\
\hline 157.936 & 20.908 & 147.115 & 20.688 \\
\hline 158.92 & 20.912 & 147.115 & 20.692 \\
\hline 155.971 & 20.916 & 148.198 & 20.696 \\
\hline 155.971 & 20.92 & 150.365 & 20.7 \\
\hline 156.953 & 20.924 & 147.115 & 20.704 \\
\hline 156.953 & 20.928 & 144.953 & 20.708 \\
\hline 155.971 & 20.932 & 144.953 & 20.712 \\
\hline 158.92 & 20.936 & 146.034 & 20.716 \\
\hline 157.936 & 20.94 & 144.953 & 20.72 \\
\hline 162.862 & 20.944 & 144.953 & 20.724 \\
\hline 162.862 & 20.948 & 141.717 & 20.728 \\
\hline 161.876 & 20.952 & 138.489 & 20.732 \\
\hline 163.849 & 20.956 & 138.489 & 20.736 \\
\hline 162.862 & 20.96 & 141.717 & 20.74 \\
\hline 160.89 & 20.964 & 150.365 & 20.744 \\
\hline 159.905 & 20.968 & 159.071 & 20.748 \\
\hline 161.876 & 20.972 & 168.93 & 20.752 \\
\hline 162.862 & 20.976 & 175.538 & 20.756 \\
\hline 159.905 & 20.98 & 176.642 & 20.76 \\
\hline 161.876 & 20.984 & 177.747 & 20.764 \\
\hline 159.905 & 20.988 & 176.642 & 20.768 \\
\hline 164.837 & 20.992 & 175.538 & 20.772 \\
\hline 163.849 & 20.996 & 172.23 & 20.776 \\
\hline 163.849 & 21 & 171.129 & 20.78 \\
\hline 162.862 & 21.004 & 171.129 & 20.784 \\
\hline 164.837 & 21.008 & 171.129 & 20.788 \\
\hline 163.849 & 21.012 & 168.93 & 20.792 \\
\hline 159.905 & 21.016 & 166.733 & 20.796 \\
\hline 161.876 & 21.02 & 163.444 & 20.8 \\
\hline 161.876 & 21.024 & 163.444 & 20.804 \\
\hline 161.876 & 21.028 & 161.256 & 20.808 \\
\hline 161.87 & 21.032 & 161.256 & 20.8 \\
\hline 162.86 & 21.036 & 159.071 & 20.816 \\
\hline
\end{tabular}




\begin{tabular}{|c|c|c|c|c|c|c|c|}
\hline & & 111.381 & 18.936 & 161.876 & 21.04 & 157.98 & 20.82 \\
\hline 119.46 & 19.816 & 110.404 & 18.94 & 165.826 & 21.044 & 157.98 & 20.824 \\
\hline 117.415 & 19.82 & & & & & & \\
\hline & & 111.381 & 18.944 & 162.862 & 21.048 & 157.98 & 20.828 \\
\hline 117.415 & 19.824 & & & & & & \\
\hline & & 112.358 & 18.948 & 161.876 & 21.052 & 157.98 & 20.832 \\
\hline 118.437 & 19.828 & & & & & & \\
\hline & & 110.404 & 18.952 & 162.862 & 21.056 & 159.071 & 20.836 \\
\hline 110.451 & 17.052 & 112.358 & 18.956 & 161.876 & 21.06 & 159.071 & 20.84 \\
\hline 119.46 & 19.836 & & & & & & \\
\hline 120485 & 1984 & 111.381 & 18.96 & 158.92 & 21.064 & 161.256 & 20.844 \\
\hline 120.700 & & 112.358 & 18.964 & 158.92 & 21.068 & 160.163 & 20.848 \\
\hline 117.415 & 19.844 & & & & & & \\
\hline 11046 & 108 & 113.337 & 18.968 & 161.876 & 21.072 & 157.98 & 20.852 \\
\hline 117.40 & 19.040 & 113.337 & 18.972 & 160.89 & 21.076 & 157.98 & 20.856 \\
\hline 120.485 & 19.852 & & & & & & \\
\hline & & 112.358 & 18.976 & 161.876 & 21.08 & 157.98 & 20.86 \\
\hline 119.46 & 19.856 & & & & & & \\
\hline 120.485 & 19.86 & 109.429 & 18.98 & 162.862 & 21.084 & 156.889 & 20.864 \\
\hline & & 111.381 & 18.984 & 160.89 & 21.088 & 156.889 & 20.868 \\
\hline 120.485 & 19.864 & & & & & & \\
\hline & & 112.358 & 18.988 & 161.876 & 21.092 & 157.98 & 20.872 \\
\hline 121.51 & 19.868 & & & & & & \\
\hline & & 112.358 & 18.992 & 160.89 & 21.096 & 156.889 & 20.876 \\
\hline 120.485 & 19.872 & 112358 & 18996 & 150905 & 211 & 15708 & 2088 \\
\hline 121.51 & 19.876 & 112.050 & 10.990 & 159.900 & 21.1 & 151.90 & 20.80 \\
\hline & & 112.358 & 19 & 157.936 & 21.104 & 157.98 & 20.884 \\
\hline 120.485 & 19.88 & & & & & & \\
\hline & & 113.337 & 19.004 & 156.953 & 21.108 & 157.98 & 20.888 \\
\hline 121.51 & 19.884 & & & & & & \\
\hline 11946 & 19888 & 111.381 & 19.008 & 158.92 & 21.112 & 156.889 & 20.892 \\
\hline 11 . & & 112.358 & 19.012 & 158.92 & 21.116 & 155.8 & 20.896 \\
\hline 120.485 & 19.892 & & & & & & \\
\hline & & 112.358 & 19.016 & 158.92 & 21.12 & 155.8 & 20.9 \\
\hline 121.51 & 19.896 & & & & & & \\
\hline 121.51 & 19.9 & 110.404 & 19.02 & 157.936 & 21.124 & 153.623 & 20.904 \\
\hline 11946 & 19904 & 111.381 & 19.024 & 159.905 & 21.128 & 153.623 & 20.908 \\
\hline 117.40 & & 113.337 & 19.028 & 157.936 & 21.132 & 155.8 & 20.912 \\
\hline 121.51 & 19.908 & & & & & & \\
\hline 122536 & 100 & 112.358 & 19.032 & 158.92 & 21.136 & 154.711 & 20.916 \\
\hline 122.550 & 19.912 & 112.358 & 19.036 & 156.953 & 21.14 & 154.711 & 20.92 \\
\hline 121.51 & 19.916 & & & & & & \\
\hline & & 112.358 & 19.04 & 158.92 & 21.144 & 154.711 & 20.924 \\
\hline 121.51 & 19.92 & & & & & & \\
\hline & 1 & 111.381 & 19.044 & 156.953 & 21.148 & 155.8 & 20.928 \\
\hline 122.550 & & 110.404 & 19.048 & 155.971 & 21.152 & 154.711 & 20.932 \\
\hline 121.51 & 19.928 & 111.381 & 19.052 & 155.971 & 21.156 & 154.711 & 20.936 \\
\hline 120.485 & 19.932 & & & & & & \\
\hline & & 112.358 & 19.056 & 155.971 & 21.16 & 154.711 & 20.94 \\
\hline 120.485 & 19.936 & & & & & & \\
\hline 11946 & 100 & 112.358 & 19.06 & 154.008 & 21.164 & 153.623 & 20.944 \\
\hline 119.40 & 19.94 & 111.381 & 19.064 & 155.971 & 21.168 & 151.45 & 20.948 \\
\hline 121.51 & 19.944 & & & & & & \\
\hline & & 111.381 & 19.068 & 154.989 & 21.172 & 153.623 & 20.952 \\
\hline 121.51 & 19.948 & & & & & & \\
\hline 121.51 & 19.952 & 110.404 & 19.072 & 155.971 & 21.176 & 153.623 & 20.956 \\
\hline
\end{tabular}




\begin{tabular}{|c|c|c|c|}
\hline & & 10.404 & 9.07 \\
\hline 4 & 19.9 & 11.381 & 19.08 \\
\hline & & 12.358 & 19.084 \\
\hline 3.56 & 1 & 12.358 & 19.088 \\
\hline 2.536 & 19.972 & 11.381 & 19.092 \\
\hline 23.5 & 19.9 & 12.358 & 19.096 \\
\hline 21.51 & 19.9 & 13.337 & 19.1 \\
\hline 21.51 & 19.984 & 15.296 & 19.104 \\
\hline 21.5 & 19 & 113.337 & 19.108 \\
\hline 23.564 & 19.992 & & \\
\hline 23.564 & 19.9 & 16.278 & 19.116 \\
\hline 123.564 & 20 & 115.296 & 19.12 \\
\hline 122.536 & 20.00 & 78 & 19.124 \\
\hline 121.51 & 20.00 & 13.337 & 19.128 \\
\hline 21.51 & 20.01 & 13.337 & 19.132 \\
\hline 121.51 & 20.0 & 112.358 & 19.136 \\
\hline 120.485 & 20.0 & 37 & 9.14 \\
\hline 123.564 & 20.024 & 13.337 & 19.144 \\
\hline 122.536 & 20.0 & 14.316 & 19.148 \\
\hline 122.536 & 20.03 & 3.337 & 9.152 \\
\hline 124.592 & 20.036 & 16.278 & 19.156 \\
\hline 122.536 & 20.04 & 15.296 & 19.16 \\
\hline 125.621 & 20.0 & 14.316 & 19.164 \\
\hline 124.592 & 20.048 & 37 & 9.168 \\
\hline 122.536 & 20.052 & 96 & 9.172 \\
\hline 122.536 & 20.0 & 6.278 & 19.176 \\
\hline 122.536 & 20.06 & 96 & 9.18 \\
\hline 121.51 & 20.064 & 5.296 & 19.184 \\
\hline 121.51 & 20.0 & 96 & 188 \\
\hline 123.564 & 20.07 & 19.227 & 19.192 \\
\hline 122.536 & 20.07 & 43 & 9.196 \\
\hline 123.564 & 20.08 & 27 & 19. \\
\hline 124.592 & 20.08 & 8.243 & 9. \\
\hline 124.592 & 20.088 & 20.213 & 19.208 \\
\hline 25. & 0.09 & 117.26 & 212 \\
\hline
\end{tabular}

\begin{tabular}{|c|c|c|c|}
\hline 154.989 & 21.18 & 153.623 & 20.96 \\
\hline 56.953 & 21.184 & 153.623 & 20.964 \\
\hline 154.989 & 21.188 & 52.536 & 20.968 \\
\hline 154.989 & 21.192 & 151.45 & 20.972 \\
\hline 154.008 & 21.196 & 152.536 & 20.976 \\
\hline 155.971 & 21.2 & 150.365 & 20.98 \\
\hline 154.008 & 21.204 & 150.365 & 20.984 \\
\hline 154.989 & 21.208 & 149.281 & 20.988 \\
\hline 154.989 & 21.212 & 151.45 & 20.992 \\
\hline 155.971 & 21.216 & 152.536 & 20.996 \\
\hline 154.989 & 21.22 & 153.623 & 21 \\
\hline 154.008 & 21.224 & 150.365 & 21.004 \\
\hline 154.008 & 21.228 & 152.536 & 21.008 \\
\hline 154.008 & 21.232 & 152.536 & 21.012 \\
\hline 153.028 & 21.236 & 150.365 & 21.016 \\
\hline 153.028 & 21.24 & 153.623 & 21.02 \\
\hline 152.048 & 21.244 & 153.623 & 21.024 \\
\hline 153.028 & 21.248 & 153.623 & 21.028 \\
\hline 152.048 & 21.252 & 154.711 & 21.032 \\
\hline 152.048 & 21.256 & 153.623 & 21.036 \\
\hline 152.048 & 21.26 & 152.536 & 21.04 \\
\hline 154.008 & 21.264 & 153.623 & 21.044 \\
\hline 153.028 & 21.268 & 153.623 & 21.048 \\
\hline 152.048 & 21.272 & 153.623 & 21.052 \\
\hline 152.048 & 21.276 & 154.711 & 21.056 \\
\hline 153.028 & 21.28 & 153.623 & 21.06 \\
\hline 154.008 & 21.284 & 155.8 & 21.064 \\
\hline 153.028 & 21.288 & 55.8 & 21.068 \\
\hline 154.008 & 21.292 & 157.98 & 21.072 \\
\hline 153.028 & 21.296 & 156.889 & 21.076 \\
\hline 152.048 & 21.3 & 157.98 & 21.08 \\
\hline 154.008 & 21.304 & 157.98 & 21.084 \\
\hline 151.07 & 21.308 & 157.98 & 21.088 \\
\hline 151.07 & 21.312 & 156.889 & 21.092 \\
\hline 153. & 21.3 & 101 & 21 \\
\hline
\end{tabular}




\begin{tabular}{|c|c|c|c|c|c|c|c|}
\hline & & 116.278 & 19.216 & 153.028 & 21.32 & 157.98 & 21.1 \\
\hline 123.564 & 20.096 & & & & & & \\
\hline 121.51 & 20.1 & 115.296 & 19.22 & 154.008 & 21.324 & 157.98 & 21.104 \\
\hline & & 114.316 & 19.224 & 152.048 & 21.328 & 157.98 & 21.108 \\
\hline 123.564 & 20.104 & & & & & & \\
\hline & & 115.296 & 19.228 & 151.07 & 21.332 & 157.98 & 21.112 \\
\hline 124.592 & 20.108 & & & & & & \\
\hline & & 116.278 & 19.232 & 155.971 & 21.336 & 159.071 & 21.116 \\
\hline 123.564 & 20.112 & & & & & & \\
\hline 126652 & 20116 & 116.278 & 19.236 & 154.008 & 21.34 & 156.889 & 21.12 \\
\hline 120.052 & 20.110 & 117.26 & 19.24 & 156.953 & 21.344 & 159.071 & 21.124 \\
\hline 125.621 & 20.12 & & & & & & \\
\hline & & 117.26 & 19.244 & 156.953 & 21.348 & 157.98 & 21.128 \\
\hline 125.621 & 20.124 & & & & & & \\
\hline & & 118.243 & 19.248 & 156.953 & 21.352 & 157.98 & 21.132 \\
\hline 126.652 & 20.128 & 116.278 & & 156053 & 21356 & 159071 & 21136 \\
\hline 124.592 & 20.132 & 110.278 & 19.252 & 156.953 & 21.356 & 159.071 & 21.150 \\
\hline & & 117.26 & 19.256 & 156.953 & 21.36 & 157.98 & 21.14 \\
\hline 126.652 & 20.136 & & & & & & \\
\hline & & 117.26 & 19.26 & 156.953 & 21.364 & 159.071 & 21.144 \\
\hline 122.536 & 20.14 & 116.278 & 19264 & 155971 & 21368 & 160163 & 21148 \\
\hline 123.564 & 20.144 & 110.210 & 19.204 & 153.911 & 21.500 & 100.105 & 21.140 \\
\hline & & 117.26 & 19.268 & 156.953 & 21.372 & 160.163 & 21.152 \\
\hline 125.621 & 20.148 & & & & & & \\
\hline & & 118.243 & 19.272 & 156.953 & 21.376 & 161.256 & 21.156 \\
\hline 124.592 & 20.152 & & & & & & \\
\hline 124.592 & 20156 & 119.227 & 19.276 & 157.936 & 21.38 & 160.163 & 21.16 \\
\hline & & 119.227 & 19.28 & 155.971 & 21.384 & 160.163 & 21.164 \\
\hline 125.621 & 20.16 & & & & & & \\
\hline & & 118.243 & 19.284 & 156.953 & 21.388 & 160.163 & 21.168 \\
\hline 125.621 & 20.164 & & & & & & \\
\hline 126652 & 20168 & 116.278 & 19.288 & 157.936 & 21.392 & 157.98 & 21.172 \\
\hline & & 118.243 & 19.292 & 157.936 & 21.396 & 161.256 & 21.176 \\
\hline 126.652 & 20.172 & & & & & & \\
\hline & & 119.227 & 19.296 & 158.92 & 21.4 & 161.256 & 21.18 \\
\hline 126.652 & 20.176 & & & & & & \\
\hline 128715 & 2018 & 117.26 & 19.3 & 155.971 & 21.404 & 161.256 & 21.184 \\
\hline 120.115 & 20.10 & 119.227 & 19.304 & 155.971 & 21.408 & 161.256 & 21.188 \\
\hline 126.652 & 20.184 & & & & & & \\
\hline & & 117.26 & 19.308 & 154.989 & 21.412 & 162.35 & 21.192 \\
\hline 126.652 & 20.188 & & & & & & \\
\hline 126652 & 102 & 117.26 & 19.312 & 156.953 & 21.416 & 161.256 & 21.196 \\
\hline 120.052 & 20.172 & 117.26 & 19.316 & 157.936 & 21.42 & 160.163 & 21.2 \\
\hline 125.621 & 20.196 & & & & & & \\
\hline & & 117.26 & 19.32 & 157.936 & 21.424 & 159.071 & 21.204 \\
\hline 125.621 & 20.2 & & & & & & \\
\hline & & 116.278 & 19.324 & 157.936 & 21.428 & 161.256 & 21.208 \\
\hline 125.621 & 20.204 & 116.278 & 19.328 & 157.936 & 21.432 & 160.163 & 21.212 \\
\hline 124.592 & 20.208 & & & & & & \\
\hline & & 114.316 & 19.332 & 158.92 & 21.436 & 161.256 & 21.216 \\
\hline 124.592 & 20.212 & & & & & & \\
\hline & & 115.296 & 19.336 & 158.92 & 21.44 & 162.35 & 21.22 \\
\hline 125.621 & 20.216 & & $10 ? 0$ & 1580 & 21.44 & 161256 & 21224 \\
\hline 126.652 & 20.22 & 115.290 & 19.04 & 158.92 & 21.444 & 101.250 & 21.224 \\
\hline 127683 & 20224 & 114.316 & 19.344 & 159.905 & 21.448 & 161.256 & 21.228 \\
\hline & & 117.26 & 19.348 & 154.989 & 21.452 & 162.35 & 21.232 \\
\hline 126.652 & 20.228 & & & & & & \\
\hline 125.621 & 20.232 & 117.26 & 19.352 & 155.971 & 21.456 & 162.35 & 21.236 \\
\hline
\end{tabular}




\begin{tabular}{|c|c|c|c|c|c|c|c|}
\hline & & 117.26 & 19.356 & 156.953 & 21.46 & 161.256 & 21.24 \\
\hline 126.652 & 20.236 & & & & & & \\
\hline 125621 & 2024 & 117.26 & 19.36 & 155.971 & 21.464 & 162.35 & 21.244 \\
\hline 125.021 & & 118.243 & 19.364 & 156.953 & 21.468 & 163.444 & 21.248 \\
\hline 125.621 & 20.244 & & & & & & \\
\hline & & 118.243 & 19.368 & 156.953 & 21.472 & 162.35 & 21.252 \\
\hline 126.652 & 20.248 & & & & & & \\
\hline & & 118.243 & 19.372 & 156.953 & 21.476 & 162.35 & 21.256 \\
\hline 126.652 & 20.252 & & & & & & \\
\hline 126652 & 20256 & 116.278 & 19.376 & 159.905 & 21.48 & 165.636 & 21.26 \\
\hline 120.052 & 20.250 & 117.26 & 19.38 & 158.92 & 21.484 & 165.636 & 21.264 \\
\hline 127.683 & 20.26 & & & & & & \\
\hline & & 118.243 & 19.384 & 159.905 & 21.488 & 165.636 & 21.268 \\
\hline 127.683 & 20.264 & & & & & & \\
\hline & & 119.227 & 19.388 & 159.905 & 21.492 & 163.444 & 21.272 \\
\hline 126.652 & 20.268 & 11726 & 19392 & 156053 & 21.496 & 163444 & 21276 \\
\hline 128.715 & 20.272 & 117.20 & 19.392 & 150.953 & 21.490 & 105.444 & 21.210 \\
\hline & & 119.227 & 19.396 & 156.953 & 21.5 & 163.444 & 21.28 \\
\hline 126.652 & 20.276 & & & & & & \\
\hline & & 119.227 & 19.4 & 157.936 & 21.504 & 164.54 & 21.284 \\
\hline 126.652 & 20.28 & 118.243 & 19,404 & 156953 & 21508 & 163.444 & 21.288 \\
\hline 126.652 & 20.284 & $110.2+3$ & 17.707 & 10. & 21.000 & 105.444 & 21.200 \\
\hline & & 116.278 & 19.408 & 156.953 & 21.512 & 162.35 & 21.292 \\
\hline 127.683 & 20.288 & & & & & & \\
\hline & & 117.26 & 19.412 & 157.936 & 21.516 & 165.636 & 21.296 \\
\hline 128.715 & 20.292 & 116278 & 19416 & & 2152 & 16454 & 213 \\
\hline 128.715 & 20.296 & 110.210 & 19.410 & $15 / .936$ & 21.52 & 164.24 & 21.3 \\
\hline & & 115.296 & 19.42 & 157.936 & 21.524 & 167.831 & 21.304 \\
\hline 129.748 & 20.3 & & & & & & \\
\hline & & 113.337 & 19.424 & 156.953 & 21.528 & 166.733 & 21.308 \\
\hline 128.715 & 20.304 & & & & & & \\
\hline 127.683 & 20.308 & 115.296 & 19.428 & 160.89 & 21.532 & 167.831 & 21.312 \\
\hline & & 116.278 & 19.432 & 158.92 & 21.536 & 168.93 & 21.316 \\
\hline 128.715 & 20.312 & & & & & & \\
\hline & & 116.278 & 19.436 & 159.905 & 21.54 & 167.831 & 21.32 \\
\hline 128.715 & 20.316 & 119.227 & 19.44 & 159.905 & 21.544 & 168.93 & 21.324 \\
\hline 127.683 & 20.32 & & & & & & \\
\hline & & 119.227 & 19.444 & 158.92 & 21.548 & 167.831 & 21.328 \\
\hline 128.715 & 20.324 & & & & & & \\
\hline & & 118.243 & 19.448 & 159.905 & 21.552 & 167.831 & 21.332 \\
\hline 127.683 & 20.328 & & & & & & \\
\hline 128715 & 20332 & 117.26 & 19.452 & 159.905 & 21.556 & 170.029 & 21.336 \\
\hline 120.110 & 20.052 & 117.26 & 19.456 & 159.905 & 21.56 & 164.54 & 21.34 \\
\hline 127.683 & 20.336 & & & & & & \\
\hline & & 119.227 & 19.46 & 156.953 & 21.564 & 165.636 & 21.344 \\
\hline 129.748 & 20.34 & & & & & & \\
\hline 128.715 & 20.344 & 118.243 & 19.464 & 159.905 & 21.568 & 163.444 & 21.348 \\
\hline 120748 & 20348 & 118.243 & 19.468 & 155.971 & 21.572 & 164.54 & 21.352 \\
\hline 129.140 & 20.070 & 119.227 & 19.472 & 158.92 & 21.576 & 163.444 & 21.356 \\
\hline 129.748 & 20.352 & & & & & & \\
\hline & & 120.213 & 19.476 & 159.905 & 21.58 & 164.54 & 21.36 \\
\hline 129.748 & 20.356 & & & & & & \\
\hline 128715 & 2036 & 118.243 & 19.48 & 157.936 & 21.584 & 167.831 & 21.364 \\
\hline 129748 & $\begin{array}{l}20.50 \\
20364\end{array}$ & 118.243 & 19.484 & 158.92 & 21.588 & 164.54 & 21.368 \\
\hline & & 119.227 & 19.488 & 159.905 & 21.592 & 165.636 & 21.372 \\
\hline 128.715 & 20.368 & & & & & & \\
\hline 128.715 & 20.372 & 117.26 & 19.492 & 157.936 & 21.596 & 165.636 & 21.376 \\
\hline
\end{tabular}




\begin{tabular}{|c|c|c|c|c|c|c|c|}
\hline & & 118.243 & 19.496 & 158.92 & 21.6 & 164.54 & 21.38 \\
\hline 129.748 & 20.376 & 116.278 & 19.5 & 158.92 & 21.604 & 167.831 & 21.384 \\
\hline 129.748 & 20.38 & 114.316 & 19.504 & 156.953 & 21.608 & 165.636 & 21.388 \\
\hline 130.782 & 20.384 & & & & & & \\
\hline 130.782 & 20.388 & 113.337 & 19.508 & 156.953 & 21.612 & 166.733 & 21.392 \\
\hline 129.748 & 20.392 & 116.278 & 19.512 & 155.971 & 21.616 & 166.733 & 21.396 \\
\hline 129.748 & 20.396 & 115.296 & 19.516 & 156.953 & 21.62 & 168.93 & 21.4 \\
\hline 129.748 & 20.4 & 115.296 & 19.52 & 158.92 & 21.624 & 170.029 & 21.404 \\
\hline 128.715 & 20.404 & 117.26 & 19.524 & 159.905 & 21.628 & 167.831 & 21.408 \\
\hline 129.748 & 20.408 & 117.26 & 19.528 & 159.905 & 21.632 & 168.93 & 21.412 \\
\hline 128.715 & 20.412 & 117.26 & 19.532 & 160.89 & 21.636 & 167.831 & 21.416 \\
\hline 129.748 & 20.416 & 119.227 & 19.536 & 159.905 & 21.64 & 170.029 & 21.42 \\
\hline 130.782 & 20.42 & 117.26 & 19.54 & 159.905 & 21.644 & 171.129 & 21.424 \\
\hline 130.782 & 20.424 & 117.26 & 19.544 & 159.905 & 21.648 & 170.029 & 21.428 \\
\hline 129.748 & 20.428 & 119.227 & 19.548 & 157.936 & 21.652 & 170.029 & 21.432 \\
\hline 129.748 & 20.432 & 118.243 & 19.552 & 159.905 & 21.656 & 170.029 & 21.436 \\
\hline 130.782 & 20.436 & 118.243 & 19.556 & 159.905 & 21.66 & 170.029 & 21.44 \\
\hline 131.818 & 20.44 & 118.243 & 19.56 & 159.905 & 21.664 & 172.23 & 21.444 \\
\hline 130.782 & 20.444 & 119.227 & 19.564 & 159.905 & 21.668 & 172.23 & 21.448 \\
\hline 129.748 & 20.448 & 120.213 & 19.568 & 161.876 & 21.672 & 173.332 & 21.452 \\
\hline 131.818 & 20.452 & 118.243 & 19.572 & 159.905 & 21.676 & 174.434 & 21.456 \\
\hline 131.818 & 20.456 & 117.26 & 19.576 & 159.905 & 21.68 & 173.332 & 21.46 \\
\hline 130.782 & 20.46 & 119.227 & 19.58 & 161.876 & 21.684 & 173.332 & 21.464 \\
\hline 131.818 & 20.464 & 118.243 & 19.584 & 161.876 & 21.688 & 174.434 & 21.468 \\
\hline 131.818 & 20.468 & 117.26 & 19.588 & 158.92 & 21.692 & 173.332 & 21.472 \\
\hline 130.782 & 20.472 & 118.243 & 19.592 & 160.89 & 21.696 & 173.332 & 21.476 \\
\hline 132.854 & 20.476 & 118.243 & 19.596 & 158.92 & 21.7 & 173.332 & 21.48 \\
\hline 131.818 & 20.48 & 119.227 & 19.6 & 158.92 & 21.704 & 174.434 & 21.484 \\
\hline 132.854 & 20.484 & 117.26 & 19.604 & 158.92 & 21.708 & 175.538 & 21.488 \\
\hline 130.782 & 20.488 & 118.243 & 19.608 & 159.905 & 21.712 & 175.538 & 21.492 \\
\hline 131.818 & 20.492 & 118.243 & 19.612 & 161.876 & 21.716 & 174.434 & 21.496 \\
\hline 133.891 & 20.496 & 117.26 & 19.616 & 161.876 & 21.72 & 174.434 & 21.5 \\
\hline 133.891 & 20.5 & 118.243 & 19.62 & 160.89 & 21.724 & 175.538 & 21.504 \\
\hline 137.007 & 20.504 & 120.213 & 19.624 & 163.849 & 21.728 & 174.434 & 21.508 \\
\hline 138.048 & 20.508 & 120.213 & 19.628 & 161.876 & 21.732 & 175.538 & 21.512 \\
\hline 138.048 & 20.512 & 120.213 & 19.632 & 162.862 & 21.736 & 174.434 & 21.516 \\
\hline
\end{tabular}




\begin{tabular}{|c|c|c|c|c|c|c|c|}
\hline & & 120.213 & 19.636 & 162.862 & 21.74 & 175.538 & 21.52 \\
\hline 138.048 & 20.516 & קיר ח20 & 1064 & 161076 & 2174 & 0 & 21524 \\
\hline 138.048 & 20.52 & 120.213 & 19.04 & $101.8 / 0$ & 21.744 & 175.538 & 21.524 \\
\hline & & 120.213 & 19.644 & 162.862 & 21.748 & 175.538 & 21.528 \\
\hline 138.048 & 20.524 & & & & & & \\
\hline & & 119.227 & 19.648 & 162.862 & 21.752 & 175.538 & 21.532 \\
\hline 138.048 & 20.528 & & & & & & \\
\hline 138048 & & 119.227 & 19.652 & 162.862 & 21.756 & 175.538 & 21.536 \\
\hline 130080 & 20536 & 120.213 & 19.656 & 163.849 & 21.76 & 175.538 & 21.54 \\
\hline 139.089 & 20.536 & 119.227 & 19.66 & 161.876 & 21.764 & 174.434 & 21.544 \\
\hline 139.089 & 20.54 & & & & & & \\
\hline & & 119.227 & 19.664 & 162.862 & 21.768 & 175.538 & 21.548 \\
\hline 139.089 & 20.544 & 118243 & 10.668 & 163810 & $?$ & 175538 & 21552 \\
\hline 140.132 & 20.548 & 10.2 & 1.000 & 105.047 & 21.112 & 175.530 & 21.032 \\
\hline 138048 & 20552 & 119.227 & 19.672 & 163.849 & 21.776 & 175.538 & 21.556 \\
\hline & & 120.213 & 19.676 & 164.837 & 21.78 & 175.538 & 21.56 \\
\hline 139.089 & 20.556 & 120213 & & $16-2>$ & 21 & 28 & 21564 \\
\hline 139.089 & 20.56 & $120.21 J$ & 19.08 & 102.002 & 21.184 & $1 / 5.538$ & 21.504 \\
\hline 138048 & 20564 & 119.227 & 19.684 & 162.862 & 21.788 & 175.538 & 21.568 \\
\hline 138.048 & 20.568 & 120.213 & 19.688 & 162.862 & 21.792 & 174.434 & 21.572 \\
\hline 134.928 & 20.572 & 120.213 & 19.692 & 163.849 & 21.796 & 175.538 & 21.576 \\
\hline 135.967 & 20.576 & 119.227 & 19.696 & 161.876 & 21.8 & 175.538 & 21.58 \\
\hline 139.089 & 20.58 & 119.227 & 19.7 & 161.876 & 21.804 & 175.538 & 21.584 \\
\hline 141.175 & 20.584 & 120.213 & 19.704 & 162.862 & 21.808 & 175.538 & 21.588 \\
\hline 142.219 & 20.588 & 120.213 & 19.708 & 162.862 & 21.812 & 175.538 & 21.592 \\
\hline 138.048 & 20.592 & 121.199 & 19.712 & 163.849 & 21.816 & 175.538 & 21.596 \\
\hline 138.048 & 20.596 & 123.174 & 19.716 & 164.837 & 21.82 & 175.538 & 21.6 \\
\hline 134.928 & 20.6 & 123.174 & 19.72 & 164.837 & 21.824 & 175.538 & 21.604 \\
\hline 133.891 & 20.604 & 122.186 & 19.724 & 165.826 & 21.828 & 175.538 & 21.608 \\
\hline 132.854 & 20.608 & 122.186 & 19.728 & 164.837 & 21.832 & 175.538 & 21.612 \\
\hline 134.928 & 20.612 & 121.199 & 19.732 & 163.849 & 21.836 & 175.538 & 21.616 \\
\hline 139.089 & 20.616 & 123.174 & 19.736 & 163.849 & 21.84 & 175.538 & 21.62 \\
\hline 142.219 & 20.62 & 122.186 & 19.74 & 162.862 & 21.844 & 175.538 & 21.624 \\
\hline 144.31 & 20.624 & 120.213 & 19.744 & 162.862 & 21.848 & 175.538 & 21.628 \\
\hline 139.089 & 20.628 & 120.213 & 19.748 & 164.837 & 21.852 & 175.538 & 21.632 \\
\hline 138.048 & 20.632 & 121.199 & 19.752 & 163.849 & 21.856 & 175.538 & 21.636 \\
\hline 135.967 & 20.636 & 120.213 & 19.756 & 163.849 & 21.86 & 175.538 & 21.64 \\
\hline 135.967 & 20.64 & 121.199 & 19.76 & 164.837 & 21.864 & 175.538 & 21.644 \\
\hline 133.891 & 20.644 & 121.199 & 19.764 & 165.826 & 21.868 & 175.538 & 21.648 \\
\hline 133.891 & 20.648 & 121.199 & 19.768 & 163.849 & 21.872 & 176.642 & 21.652 \\
\hline 135.967 & 20.652 & 122.186 & 19.772 & 161.876 & 21.876 & 175.538 & 21.656 \\
\hline
\end{tabular}




\begin{tabular}{|c|c|c|c|}
\hline & & 19.227 & 9.776 \\
\hline & 20.66 & 20.213 & 19.78 \\
\hline & & 20.213 & 19.784 \\
\hline 7.00 & 20.6 & 21.199 & 19.788 \\
\hline 7.007 & 20.672 & 20.213 & 19.792 \\
\hline 8.0 & 20.6 & 24.162 & 19.796 \\
\hline 8.048 & 20.6 & 24.162 & 19.8 \\
\hline 37.007 & 20.684 & 24.162 & 19.804 \\
\hline 4.9 & 20.6 & 23.174 & 19.808 \\
\hline 34.928 & 20.692 & & \\
\hline 34.928 & 20.6 & 62 & 19.816 \\
\hline 3.891 & 20 & 24. & 19.82 \\
\hline 137.007 & 20.70 & 2 & 19.820 \\
\hline 137.007 & 20.708 & 24.162 & 19.832 \\
\hline 37.007 & 20.712 & 44 & 19.836 \\
\hline 135.967 & 20.72 & 74 & 19.84 \\
\hline 140.132 & 20.72 & 23.174 & 19.844 \\
\hline 1.175 & 20.7 & 24.162 & 19.848 \\
\hline 139.089 & 20.7 & 36 & 19.852 \\
\hline 137.007 & 20.736 & 24.162 & 19.856 \\
\hline 132.854 & 20.7 & 23.174 & 19.86 \\
\hline 27.683 & 20 & & 98 \\
\hline 124.592 & 20.7 & 22.186 & 19.872 \\
\hline 123.564 & 20.752 & 9 & 19.876 \\
\hline 124.592 & 20.7 & 3.174 & 19.88 \\
\hline 125.62 & 20. & 122.186 & 19.884 \\
\hline 129.748 & 20.764 & 24.162 & 19.888 \\
\hline 130.782 & 20.7 & 2 & 19.892 \\
\hline 129.74 & 20.7 & 124.162 & 19.896 \\
\hline 129.748 & 20.7 & 24.162 & 19.9 \\
\hline 128.715 & 20.7 & 126.143 & 19.904 \\
\hline 127.683 & 20.78 & & \\
\hline 127.683 & 20.788 & 124.162 & 19.908 \\
\hline 27.68 & 20.79 & 125.152 & 12 \\
\hline
\end{tabular}

\begin{tabular}{|c|c|c|c|}
\hline 163.849 & 21.88 & 175.538 & 21.66 \\
\hline 164.837 & 21.884 & 175.538 & 21.664 \\
\hline 65.826 & 21.888 & 75.538 & 21.668 \\
\hline 164.837 & 21.892 & 75.538 & 21.672 \\
\hline 163.849 & 21.896 & 175.538 & 21.676 \\
\hline 164.837 & 21.9 & 175.538 & 21.68 \\
\hline 165.826 & 21.904 & 175.538 & 21.684 \\
\hline 165.826 & 21.908 & 175.538 & 21.688 \\
\hline 165.826 & 21.912 & 175.538 & 21.692 \\
\hline 163.849 & 21.916 & 175.538 & 21.696 \\
\hline 163.849 & 21.92 & 175.538 & 21.7 \\
\hline 163.849 & 21.924 & 175.538 & 21.704 \\
\hline 164.837 & 21.928 & 175.538 & 21.708 \\
\hline 166.815 & 21.932 & 175.538 & 21.712 \\
\hline 164.837 & 21.936 & 175.538 & 21.716 \\
\hline 163.849 & 21.94 & 175.538 & 21.72 \\
\hline 165.826 & 21.944 & 175.538 & 21.724 \\
\hline 164.837 & 21.948 & 175.538 & 21.728 \\
\hline 163.849 & 21.952 & 175.538 & 21.732 \\
\hline 165.826 & 21.956 & 176.642 & 21.736 \\
\hline 165.826 & 21.96 & 175.538 & 21.74 \\
\hline 165.826 & 21.964 & 175.538 & 21.744 \\
\hline 165.826 & 21.968 & 175.538 & 21.748 \\
\hline 164.837 & 21.972 & 177.747 & 21.752 \\
\hline 165.826 & 21.976 & 175.538 & 21.756 \\
\hline 165.826 & 21.98 & 77.747 & 21.76 \\
\hline 165.826 & 21.98 & 175.538 & 21.764 \\
\hline 165.826 & 21.988 & 176.642 & 21.768 \\
\hline 166.815 & 21.992 & 175.538 & 21.772 \\
\hline 164.837 & 21.996 & 177.747 & 21.776 \\
\hline 165.826 & 22 & 176.642 & 21.7 \\
\hline 166.815 & 22.004 & 176.642 & 21.784 \\
\hline 165.82 & 22.008 & 177.747 & 21.788 \\
\hline 165.826 & 22.012 & 179.958 & 21.79 \\
\hline 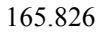 & 22.016 & 178.852 & 21. \\
\hline
\end{tabular}




\begin{tabular}{|c|c|c|c|c|c|c|c|}
\hline & & 124.162 & 19.916 & 166.815 & 22.02 & 178.852 & 21.8 \\
\hline 127.683 & 20.796 & & & & & & \\
\hline 129.748 & 20.8 & 125.152 & 19.92 & 164.837 & 22.024 & 178.852 & 21.804 \\
\hline & & 125.152 & 19.924 & 163.849 & 22.028 & 176.642 & 21.808 \\
\hline 132.854 & 20.804 & & & & & & \\
\hline & & 124.162 & 19.928 & 168.795 & 22.032 & 178.852 & 21.812 \\
\hline 133.891 & 20.808 & & & & & & \\
\hline & & 126.143 & 19.932 & 164.837 & 22.036 & 178.852 & 21.816 \\
\hline 133.891 & 20.812 & & & & & & \\
\hline 133891 & 20816 & 126.143 & 19.936 & 165.826 & 22.04 & 179.958 & 21.82 \\
\hline 153.091 & 20.010 & 124.162 & 19.94 & 165.826 & 22.044 & 181.065 & 21.824 \\
\hline 130.782 & 20.82 & & & & & & \\
\hline & & 125.152 & 19.944 & 166.815 & 22.048 & 179.958 & 21.828 \\
\hline 130.782 & 20.824 & & & & & & \\
\hline & & 125.152 & 19.948 & 166.815 & 22.052 & 179.958 & 21.832 \\
\hline 129.748 & 20.828 & 127.134 & 19.952 & 166.815 & 22.056 & 179.958 & 21.836 \\
\hline 129.748 & 20.832 & & & & & & \\
\hline & & 124.162 & 19.956 & 166.815 & 22.06 & 179.958 & 21.84 \\
\hline 132.854 & 20.836 & & & & & & \\
\hline & & 125.152 & 19.96 & 167.805 & 22.064 & 179.958 & 21.844 \\
\hline 133.891 & 20.84 & 125.152 & 19.964 & 168795 & 22.068 & 179.958 & 21.848 \\
\hline 135.967 & 20.844 & 120.152 & - & (10.1) & 22.000 & 117.950 & 21.040 \\
\hline & & 125.152 & 19.968 & 167.805 & 22.072 & 178.852 & 21.852 \\
\hline 135.967 & 20.848 & & & & & & \\
\hline & & 127.134 & 19.972 & 167.805 & 22.076 & 178.852 & 21.856 \\
\hline 134.928 & 20.852 & & & & & & \\
\hline 134.928 & 20.856 & 128.127 & 19.976 & 167.805 & 22.08 & 178.852 & 21.86 \\
\hline & & 128.127 & 19.98 & 167.805 & 22.084 & 181.065 & 21.864 \\
\hline 133.891 & 20.86 & & & & & & \\
\hline & & 125.152 & 19.984 & 167.805 & 22.088 & 179.958 & 21.868 \\
\hline 134.928 & 20.864 & & & & & & \\
\hline 134.928 & 20.868 & 127.134 & 19.988 & 168.795 & 22.092 & 178.852 & 21.872 \\
\hline & & 125.152 & 19.992 & 170.778 & 22.096 & 182.173 & 21.876 \\
\hline 135.967 & 20.872 & & & & & & \\
\hline & & 128.127 & 19.996 & 169.786 & 22.1 & 183.282 & 21.88 \\
\hline 137.007 & 20.876 & & & & & & \\
\hline & & 128.127 & 20 & 170.778 & 22.104 & 183.282 & 21.884 \\
\hline 135.967 & 20.88 & 128.127 & 20.004 & 167.805 & 22.108 & 182.173 & 21.888 \\
\hline 137.007 & 20.884 & & & & & & \\
\hline & & 127.134 & 20.008 & 167.805 & 22.112 & 183.282 & 21.892 \\
\hline 138.048 & 20.888 & & & & & & \\
\hline 137007 & 20802 & 127.134 & 20.012 & 167.805 & 22.116 & 184.391 & 21.896 \\
\hline 157.001 & 20.072 & 127.134 & 20.016 & 169.786 & 22.12 & 183.282 & 21.9 \\
\hline 138.048 & 20.896 & & & & & & \\
\hline & & 126.143 & 20.02 & 170.778 & 22.124 & 184.391 & 21.904 \\
\hline 140.132 & 20.9 & & & & & & \\
\hline & & 126.143 & 20.024 & 170.778 & 22.128 & 185.501 & 21.908 \\
\hline 141.175 & 20.904 & 127.134 & 20.028 & 170.778 & 22.132 & 184.391 & 21.912 \\
\hline 142.219 & 20.908 & & & & & & \\
\hline & & 126.143 & 20.032 & 172.763 & 22.136 & 184.391 & 21.916 \\
\hline 142.219 & 20.912 & & & & & & \\
\hline & & 128.127 & 20.036 & 172.763 & 22.14 & 185.501 & 21.92 \\
\hline 142.219 & 20.916 & & & & & & \\
\hline 140.132 & 20.92 & 128.127 & 20.04 & 172.763 & 22.144 & 185.501 & 21.924 \\
\hline 140.132 & 20.924 & 127.134 & 20.044 & 171.77 & 22.148 & 183.282 & 21.928 \\
\hline & & 127.134 & 20.048 & 174.751 & 22.152 & 184.391 & 21.932 \\
\hline 138.048 & 20.928 & & & & & & \\
\hline & 20.932 & 128.127 & 20.052 & 172.763 & 22.156 & 183.282 & 21.936 \\
\hline
\end{tabular}




\begin{tabular}{|c|c|c|c|}
\hline & & 28.127 & 0.056 \\
\hline 0.1 & 0.93 & 27.134 & 20.06 \\
\hline 40.132 & 20.94 & & \\
\hline 2.219 & 20.944 & & 0.0 \\
\hline 2.219 & 2 & 28.127 & 20.068 \\
\hline & & 28.127 & 20.072 \\
\hline 6.4 & 20.952 & 129.12 & 20.076 \\
\hline 46.405 & 20.956 & 29.12 & 0.08 \\
\hline 46.405 & 20.96 & & .00 \\
\hline 45.357 & 20.964 & 9.12 & 20.088 \\
\hline 46.405 & 20.968 & 29.12 & 20.092 \\
\hline 145.357 & 20.972 & & \\
\hline 45.357 & 20.976 & 29.12 & 20.096 \\
\hline 145.357 & 20.98 & 30.114 & 20.1 \\
\hline 146.405 & 20.9 & 8.127 & 20.104 \\
\hline 146.405 & 20.988 & 31.109 & 20.108 \\
\hline 150.605 & 20.992 & 28.127 & 20.112 \\
\hline 151.657 & 20.9 & 28.127 & 20.116 \\
\hline 150.605 & 21 & 28.127 & 20.12 \\
\hline 151.657 & 21.004 & 28.127 & 20.124 \\
\hline 48.503 & 21.0 & 29.12 & 20.128 \\
\hline 147.454 & 21.01 & 8.127 & 0.132 \\
\hline 146.405 & 21.016 & 28.127 & 20.136 \\
\hline 146.405 & 21.02 & 28.127 & 20.14 \\
\hline 147.454 & 21.0 & 29.12 & 20.144 \\
\hline 148.503 & 21.028 & 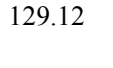 & 20.148 \\
\hline 148.503 & 21.032 & 28.127 & 20.152 \\
\hline 149.553 & 21.03 & 30.114 & 20.156 \\
\hline 149.553 & 21.04 & 129.12 & 20.16 \\
\hline 149.553 & 21.044 & 7 & 0.164 \\
\hline 149.553 & 21.04 & 8.127 & 20.168 \\
\hline 149.5 & 21.05 & 9.12 & 20.172 \\
\hline 146.405 & 21.056 & 4 & 0.176 \\
\hline 146.405 & 21.06 & 4 & 20.18 \\
\hline 6.4 & 21 & 131.109 & 20.184 \\
\hline 145.35 & 21.068 & 129.12 & 20.188 \\
\hline 46. & 21.0 & 129.12 & 19 \\
\hline
\end{tabular}

\begin{tabular}{|c|c|c|c|}
\hline 173.757 & 22.16 & 183.282 & 21.94 \\
\hline 73.757 & 22.164 & 184.391 & 21.944 \\
\hline 172.763 & 22.168 & 182.173 & 21.948 \\
\hline 171.77 & 22.172 & 184.391 & 21.952 \\
\hline 171.77 & 22.176 & 184.391 & 21.956 \\
\hline 172.763 & 22.18 & 184.391 & 21.96 \\
\hline 173.757 & 22.184 & 184.391 & 21.964 \\
\hline 174.751 & 22.188 & 183.282 & 21.968 \\
\hline 174.751 & 22.192 & 185.501 & 21.972 \\
\hline 172.763 & 22.196 & 186.611 & 21.976 \\
\hline 172.763 & 22.2 & 185.501 & 21.98 \\
\hline 171.77 & 22.204 & 186.611 & 21.984 \\
\hline 171.77 & 22.208 & 187.722 & 21.988 \\
\hline 173.757 & 22.212 & 186.611 & 21.992 \\
\hline 176.741 & 22.216 & 184.391 & 21.996 \\
\hline 170.778 & 22.22 & 184.391 & 22 \\
\hline 172.763 & 22.224 & 184.391 & 22.004 \\
\hline 169.786 & 22.228 & 186.611 & 22.008 \\
\hline 167.805 & 22.232 & 185.501 & 22.012 \\
\hline 165.826 & 22.236 & 185.501 & 22.016 \\
\hline 164.837 & 22.24 & 183.282 & 22.02 \\
\hline 163.849 & 22.244 & 185.501 & 22.024 \\
\hline 163.849 & 22.248 & 185.501 & 22.028 \\
\hline 164.837 & 22.252 & 184.391 & 22.032 \\
\hline 163.849 & 22.256 & 183.282 & 22.036 \\
\hline 164.837 & 22.26 & 184.391 & 22.04 \\
\hline 164.837 & 22.264 & 184.391 & 22.044 \\
\hline 163.849 & 22.268 & 184.391 & 22.048 \\
\hline 165.826 & 22.272 & 185.501 & 22.052 \\
\hline 163.849 & 22.276 & 184.391 & 22.056 \\
\hline 160.89 & 22.28 & 184.391 & 22.06 \\
\hline 150.092 & 22.2 & 187.722 & 22.064 \\
\hline 125.888 & 22.2 & 186.611 & 22.0 \\
\hline 96.614 & 22.292 & 186.611 & 22.072 \\
\hline 70. & 22 & 185.501 & 22. \\
\hline
\end{tabular}




\begin{tabular}{|c|c|c|c|c|c|c|c|}
\hline & & 129.12 & 20.196 & 44.4833 & 22.3 & 186.611 & 22.08 \\
\hline 146.405 & 21.076 & & & & & & \\
\hline 148 & 21 & 129.12 & 20.2 & 23.2522 & 22.304 & 186.611 & 22.084 \\
\hline 170.000 & 21.00 & 129.12 & 20.204 & & & 188.834 & 22.088 \\
\hline 147.454 & 21.084 & & & & & & \\
\hline & & 128.127 & 20.208 & & & 184.391 & 22.092 \\
\hline 147.454 & 21.088 & & & & & & \\
\hline & & 128.127 & 20.212 & & & 184.391 & 22.096 \\
\hline 146.405 & 21.092 & & & & & & \\
\hline & & 130.114 & 20.216 & & & 184.391 & 22.1 \\
\hline 144.31 & 21.096 & 128.127 & 20.22 & & & 184.391 & 22.104 \\
\hline 144.31 & 21.1 & 120.121 & 20.22 & & & (10) & 25.101 \\
\hline & & 128.127 & 20.224 & & & 184.391 & 22.108 \\
\hline 144.31 & 21.104 & & & & & & \\
\hline & & 130.114 & 20.228 & & & 184.391 & 22.112 \\
\hline 146.405 & 21.108 & & & & & & \\
\hline 145.357 & 21.112 & 129.12 & 20.232 & & & 183.282 & 22.116 \\
\hline & & 130.114 & 20.236 & & & 184.391 & 22.12 \\
\hline 145.357 & 21.116 & & & & & & \\
\hline & & 129.12 & 20.24 & & & 184.391 & 22.124 \\
\hline 146.405 & 21.12 & & & & & & \\
\hline 145357 & 21124 & 129.12 & 20.244 & & & 184.391 & 22.128 \\
\hline - 17.50 & 21.124 & 128.127 & 20.248 & & & 183.282 & 22.132 \\
\hline 144.31 & 21.128 & & & & & & \\
\hline & & 130.114 & 20.252 & & & 183.282 & 22.136 \\
\hline 143.264 & 21.132 & & & & & & \\
\hline & & 130.114 & 20.256 & & & 183.282 & 22.14 \\
\hline 143.264 & 21.136 & & & & & & \\
\hline 143.264 & 21.14 & 128.127 & 20.26 & & & 184.391 & 22.144 \\
\hline 175.204 & & 129.12 & 20.264 & & & 184.391 & 22.148 \\
\hline 141.175 & 21.144 & & & & & & \\
\hline & & 128.127 & 20.268 & & & 185.501 & 22.152 \\
\hline 144.31 & 21.148 & & & & & & \\
\hline 142.219 & 21.152 & 128.127 & 20.272 & & & 184.391 & 22.156 \\
\hline & & 131.109 & 20.276 & & & 183.282 & 22.16 \\
\hline 145.357 & 21.156 & & & & & & \\
\hline & & 129.12 & 20.28 & & & 187.722 & 22.164 \\
\hline 144.31 & 21.16 & & & & & & \\
\hline 142.219 & 21.164 & 129.12 & 20.284 & & & 184.391 & 22.168 \\
\hline 10.213 & 21001 & 130.114 & 20.288 & & & 184.391 & 22.172 \\
\hline 142.219 & 21.168 & & & & & & \\
\hline & & 130.114 & 20.292 & & & 183.282 & 22.176 \\
\hline 142.219 & 21.172 & & & & & & \\
\hline 141175 & & 128.127 & 20.296 & & & 183.282 & 22.18 \\
\hline $141.1 / 5$ & $21.1 / 6$ & 129.12 & 20.3 & & & 184.391 & 22.184 \\
\hline 141.175 & 21.18 & & & & & & \\
\hline & & 130.114 & 20.304 & & & 183.282 & 22.188 \\
\hline 141.175 & 21.184 & & & & & & \\
\hline & & 129.12 & 20.308 & & & 184.391 & 22.192 \\
\hline 143.264 & 21.188 & 128.127 & 20.312 & & & 185.501 & 22.196 \\
\hline 142.219 & 21.192 & & & & & & \\
\hline & & 130.114 & 20.316 & & & 183.282 & 22.2 \\
\hline 143.264 & 21.196 & & & & & & \\
\hline 142,21 & & 129.12 & 20.32 & & & 185.501 & 22.204 \\
\hline 142.219 & 21.2 & 128.127 & 20.324 & & & 185.501 & 22.208 \\
\hline 143.264 & 21.204 & & & & & & \\
\hline & & 129.12 & 20.328 & & & 184.391 & 22.212 \\
\hline 142.219 & 21.208 & & & & & & \\
\hline 140.132 & 21.212 & 129.12 & 20.332 & & & 185.501 & 22.216 \\
\hline
\end{tabular}




\begin{tabular}{|c|c|c|c|c|c|}
\hline & & 131.109 & 20.336 & 185.501 & 22.22 \\
\hline 140.132 & 21.210 & 129.12 & 20.34 & 188.834 & 22.224 \\
\hline 141.175 & 21.22 & & & & \\
\hline & & 129.12 & 20.344 & 188.834 & 22.228 \\
\hline 142.219 & 21.224 & & & & \\
\hline & & 128.127 & 20.348 & 191.06 & 22.232 \\
\hline 142.219 & 21.228 & & & & \\
\hline 141.175 & 21.232 & 130.114 & 20.352 & 191.06 & 22.236 \\
\hline & & 129.12 & 20.356 & 191.06 & 22.24 \\
\hline 142.219 & 21.236 & & & & \\
\hline 141.175 & 21.24 & 130.114 & 20.36 & 193.289 & 22.244 \\
\hline & & 129.12 & 20.364 & 193.289 & 22.248 \\
\hline 141.175 & 21.244 & 129.12 & 20.368 & 193.289 & 22.252 \\
\hline 141.175 & 21.248 & & & & \\
\hline & & 127.134 & 20.372 & 193.289 & 22.256 \\
\hline $141.1 / 5$ & 21.252 & 129.12 & 20.376 & 193.289 & 22.26 \\
\hline 143.264 & 21.256 & & & & \\
\hline & & 129.12 & 20.38 & 192.174 & 22.264 \\
\hline 142.219 & 21.26 & & & & \\
\hline & & 129.12 & 20.384 & 193.289 & 22.268 \\
\hline 145.357 & 21.264 & & & & \\
\hline & & 129.12 & 20.388 & 193.289 & 22.272 \\
\hline 144.31 & 21.268 & 12912 & & 103280 & 22.276 \\
\hline 142.219 & 21.272 & 129.12 & 20.392 & 193.289 & 22.210 \\
\hline & & 131.109 & 20.396 & 191.06 & 22.28 \\
\hline 143.264 & 21.276 & & & & \\
\hline & & 129.12 & 20.4 & 193.289 & 22.284 \\
\hline 145.357 & 21.28 & & & & \\
\hline 143.264 & 21284 & 128.127 & 20.404 & 193.289 & 22.288 \\
\hline 143.204 & 21.204 & 129.12 & 20.408 & 197.754 & 22.292 \\
\hline 144.31 & 21.288 & & & & \\
\hline & & 130.114 & 20.412 & 204.47 & 22.296 \\
\hline 144.31 & 21.292 & & & & \\
\hline 143264 & 21296 & 130.114 & 20.416 & 208.959 & 22.3 \\
\hline 143.204 & 21.290 & 130.114 & 20.42 & 211.208 & 22.304 \\
\hline 144.31 & 21.3 & & & & \\
\hline & & 130.114 & 20.424 & 212.332 & 22.308 \\
\hline 143.264 & 21.304 & & & & \\
\hline & & 130.114 & 20.428 & 216.838 & 22.312 \\
\hline 144.31 & 21.308 & 130.114 & 20.432 & 219.093 & 22.316 \\
\hline 146.405 & 21.312 & & & & \\
\hline & & 132.105 & 20.436 & 222.481 & 22.32 \\
\hline 146.405 & 21.316 & & & & \\
\hline & & 130.114 & 20.44 & 224.742 & 22.324 \\
\hline 144.31 & 21.32 & 130.114 & 20.444 & 224.742 & 22.328 \\
\hline 142.219 & 21.324 & & & & \\
\hline & & 132.105 & 20.448 & 230.402 & 22.332 \\
\hline 143.264 & 21.328 & & & & \\
\hline & & 132.105 & 20.452 & 229.269 & 22.336 \\
\hline 145.357 & 21.332 & 132105 & 20.156 & 23040? & 2334 \\
\hline 146.405 & 21.336 & 152.100 & 20.450 & 250.402 & 22.54 \\
\hline & & 132.105 & 20.46 & 231.535 & 22.344 \\
\hline 147.454 & 21.34 & & & & \\
\hline & & 132.105 & 20.464 & 236.074 & 22.348 \\
\hline 148.503 & 21.344 & & & & \\
\hline 149553 & 21348 & 130.114 & 20.468 & 237.209 & 22.352 \\
\hline (19.0. & & 130.114 & 20.472 & 238.345 & 22.356 \\
\hline 151.657 & 21.352 & & & & \\
\hline
\end{tabular}




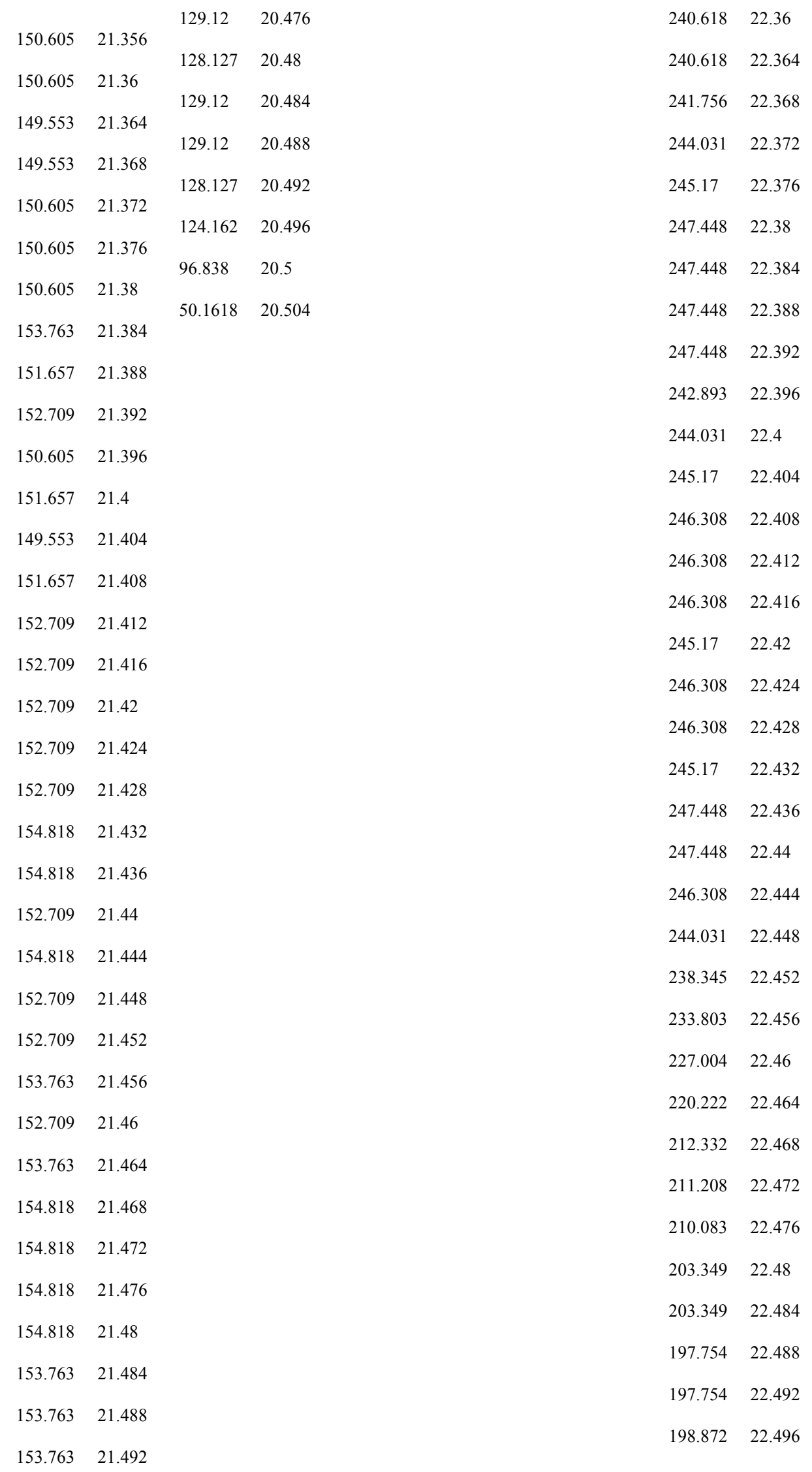




\begin{tabular}{|c|c|}
\hline 153.763 & 21.496 \\
\hline 154.818 & 21.5 \\
\hline 153.763 & 21.504 \\
\hline 152.709 & 21.508 \\
\hline 152.709 & 21.512 \\
\hline 152.709 & 21.516 \\
\hline 152.709 & 21.52 \\
\hline 151.657 & 21.524 \\
\hline 153.763 & 21.528 \\
\hline 153.763 & 21.532 \\
\hline 154.818 & 21.536 \\
\hline 154.818 & 21.54 \\
\hline 154.818 & 21.544 \\
\hline 154.818 & 21.548 \\
\hline 151.657 & 21.552 \\
\hline 153.763 & 21.556 \\
\hline 152.709 & 21.56 \\
\hline 152.709 & 21.564 \\
\hline 153.763 & 21.568 \\
\hline 153.763 & 21.572 \\
\hline 153.763 & 21.576 \\
\hline 154.818 & 21.58 \\
\hline 154.818 & 21.584 \\
\hline 154.818 & 21.588 \\
\hline 155.873 & 21.592 \\
\hline 154.818 & 21.596 \\
\hline 154.818 & 21.6 \\
\hline 154.818 & 21.604 \\
\hline 154.818 & 21.608 \\
\hline 154.818 & 21.612 \\
\hline 153.763 & 21.616 \\
\hline 154.818 & 21.62 \\
\hline 155.873 & 21.624 \\
\hline 155.873 & 21.628 \\
\hline 154.818 & 21. \\
\hline
\end{tabular}

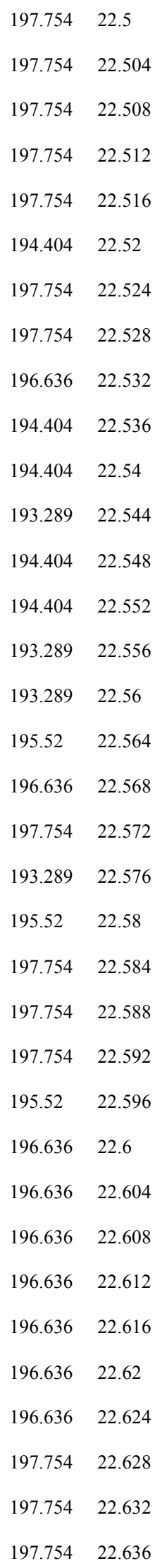




\begin{tabular}{|c|c|}
\hline 154.818 & 21.636 \\
\hline 154.818 & 21.64 \\
\hline 154.818 & 21.644 \\
\hline 154.818 & 21.648 \\
\hline 156.929 & 21.652 \\
\hline 156.929 & 21.656 \\
\hline 155.873 & 21.66 \\
\hline 155.873 & 21.664 \\
\hline 157.986 & 21.668 \\
\hline 157.986 & 21.672 \\
\hline 157.986 & 21.676 \\
\hline 155.873 & 21.68 \\
\hline 157.986 & 21.684 \\
\hline 155.873 & 21.688 \\
\hline 156.929 & 21.692 \\
\hline 154.818 & 21.696 \\
\hline 159.044 & 21.7 \\
\hline 159.044 & 21.704 \\
\hline 157.986 & 21.708 \\
\hline 159.044 & 21.712 \\
\hline 156.929 & 21.716 \\
\hline 160.102 & 21.72 \\
\hline 159.044 & 21.724 \\
\hline 160.102 & 21.728 \\
\hline 159.044 & 21.732 \\
\hline 159.044 & 21.736 \\
\hline 160.102 & 21.74 \\
\hline 154.818 & 21.744 \\
\hline 121.51 & 21.748 \\
\hline 50.5819 & 21.752 \\
\hline
\end{tabular}

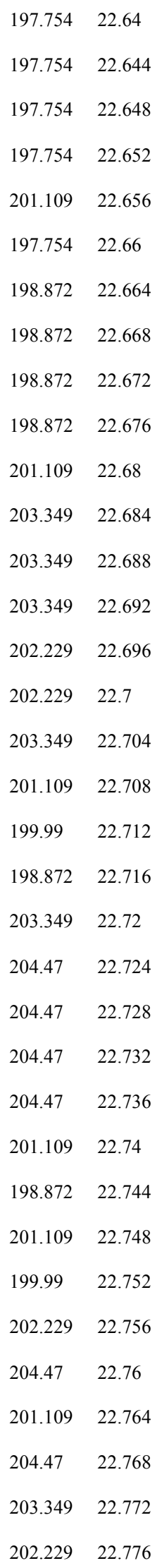




\begin{tabular}{|c|c|}
\hline 204.47 & 22.78 \\
\hline 201.109 & 22.784 \\
\hline 202.229 & 22.788 \\
\hline 203.349 & 22.792 \\
\hline 201.109 & 22.796 \\
\hline 202.229 & 22.8 \\
\hline 201.109 & 22.804 \\
\hline 202.229 & 22.808 \\
\hline 202.229 & 22.812 \\
\hline 202.229 & 22.816 \\
\hline 203.349 & 22.82 \\
\hline 203.349 & 22.824 \\
\hline 203.349 & 22.828 \\
\hline 202.229 & 22.832 \\
\hline 202.229 & 22.836 \\
\hline 201.109 & 22.84 \\
\hline 197.754 & 22.844 \\
\hline 201.109 & 22.848 \\
\hline 199.99 & 22.852 \\
\hline 202.229 & 22.856 \\
\hline 201.109 & 22.86 \\
\hline 201.109 & 22.864 \\
\hline 203.349 & 22.868 \\
\hline 203.349 & 22.872 \\
\hline 203.349 & 22.876 \\
\hline 202.229 & 22.88 \\
\hline 202.229 & 22.884 \\
\hline 201.109 & 22.888 \\
\hline 203.349 & 22.892 \\
\hline 198.872 & 22.896 \\
\hline 202.229 & 22.9 \\
\hline 198.872 & 22.904 \\
\hline 202.229 & 22.908 \\
\hline 201.109 & 22.912 \\
\hline 202.229 & 22.916 \\
\hline
\end{tabular}




\begin{tabular}{|c|c|}
\hline 201.109 & 22.92 \\
\hline 204.47 & 22.924 \\
\hline 202.229 & 22.928 \\
\hline 199.99 & 22.932 \\
\hline 201.109 & 22.936 \\
\hline 199.99 & 22.94 \\
\hline 201.109 & 22.944 \\
\hline 201.109 & 22.948 \\
\hline 202.229 & 22.952 \\
\hline 203.349 & 22.956 \\
\hline 203.349 & 22.96 \\
\hline 205.591 & 22.964 \\
\hline 205.591 & 22.968 \\
\hline 205.591 & 22.972 \\
\hline 206.713 & 22.976 \\
\hline 205.591 & 22.98 \\
\hline 204.47 & 22.984 \\
\hline 205.591 & 22.988 \\
\hline 207.836 & 22.992 \\
\hline 207.836 & 22.996 \\
\hline 206.713 & 23 \\
\hline 207.836 & 23.004 \\
\hline 210.083 & 23.008 \\
\hline 206.713 & 23.012 \\
\hline 208.959 & 23.016 \\
\hline 210.083 & 23.02 \\
\hline 208.959 & 23.024 \\
\hline 210.083 & 23.028 \\
\hline 211.208 & 23.032 \\
\hline 210.083 & 23.036 \\
\hline 211.208 & 23.04 \\
\hline 211.208 & 23.044 \\
\hline 211.208 & 23.048 \\
\hline 210.083 & 23.052 \\
\hline 211.208 & 23.056 \\
\hline
\end{tabular}




\begin{tabular}{ll}
211.208 & 23.06 \\
212.332 & 23.064 \\
212.332 & 23.068 \\
211.208 & 23.072 \\
211.208 & 23.076 \\
212.332 & 23.08 \\
212.332 & 23.084 \\
211.208 & 23.088 \\
211.208 & 23.092 \\
212.332 & 23.096 \\
212.332 & 23.1 \\
\hline 213.458 & 23.104 \\
211.208 & 23.108 \\
213.53 \\
213.584 & 23.112 \\
213.43
\end{tabular}




\begin{tabular}{|c|c|}
\hline 214.584 & 23.2 \\
\hline 215.711 & 23.204 \\
\hline 214.584 & 23.208 \\
\hline 214.584 & 23.212 \\
\hline 215.711 & 23.216 \\
\hline 215.711 & 23.22 \\
\hline 215.711 & 23.224 \\
\hline 214.584 & 23.228 \\
\hline 212.332 & 23.232 \\
\hline 214.584 & 23.236 \\
\hline 214.584 & 23.24 \\
\hline 212.332 & 23.244 \\
\hline 215.711 & 23.248 \\
\hline 215.711 & 23.252 \\
\hline 215.711 & 23.256 \\
\hline 215.711 & 23.26 \\
\hline 214.584 & 23.264 \\
\hline 212.332 & 23.268 \\
\hline 215.711 & 23.272 \\
\hline 215.711 & 23.276 \\
\hline 214.584 & 23.28 \\
\hline 214.584 & 23.284 \\
\hline 215.711 & 23.288 \\
\hline 213.458 & 23.292 \\
\hline 214.584 & 23.296 \\
\hline 215.711 & 23.3 \\
\hline 215.711 & 23.304 \\
\hline 215.711 & 23.308 \\
\hline 217.965 & 23.312 \\
\hline 215.711 & 23.316 \\
\hline 217.965 & 23.32 \\
\hline 215.711 & 23.324 \\
\hline 215.711 & 23.328 \\
\hline 215.711 & 23.332 \\
\hline 215.711 & 23.336 \\
\hline
\end{tabular}




\begin{tabular}{|c|c|}
\hline 216.838 & 23.34 \\
\hline 219.093 & 23.344 \\
\hline 217.965 & 23.348 \\
\hline 219.093 & 23.352 \\
\hline 220.222 & 23.356 \\
\hline 219.093 & 23.36 \\
\hline 219.093 & 23.364 \\
\hline 219.093 & 23.368 \\
\hline 221.351 & 23.372 \\
\hline 223.611 & 23.376 \\
\hline 222.481 & 23.38 \\
\hline 224.742 & 23.384 \\
\hline 224.742 & 23.388 \\
\hline 224.742 & 23.392 \\
\hline 224.742 & 23.396 \\
\hline 224.742 & 23.4 \\
\hline 221.351 & 23.404 \\
\hline 224.742 & 23.408 \\
\hline 223.611 & 23.412 \\
\hline 223.611 & 23.416 \\
\hline 224.742 & 23.42 \\
\hline 224.742 & 23.424 \\
\hline 224.742 & 23.428 \\
\hline 224.742 & 23.432 \\
\hline 224.742 & 23.436 \\
\hline 225.873 & 23.44 \\
\hline 225.873 & 23.444 \\
\hline 225.873 & 23.448 \\
\hline 224.742 & 23.452 \\
\hline 228.136 & 23.456 \\
\hline 228.136 & 23.46 \\
\hline 227.004 & 23.464 \\
\hline 225.873 & 23.468 \\
\hline 225.873 & 23.472 \\
\hline 228.136 & 23.476 \\
\hline
\end{tabular}




\begin{tabular}{|c|c|}
\hline 225.873 & 23.48 \\
\hline 227.004 & 23.484 \\
\hline 228.136 & 23.488 \\
\hline 225.873 & 23.492 \\
\hline 228.136 & 23.496 \\
\hline 229.269 & 23.5 \\
\hline 228.136 & 23.504 \\
\hline 228.136 & 23.508 \\
\hline 229.269 & 23.512 \\
\hline 228.136 & 23.516 \\
\hline 228.136 & 23.52 \\
\hline 228.136 & 23.524 \\
\hline 228.136 & 23.528 \\
\hline 229.269 & 23.532 \\
\hline 229.269 & 23.536 \\
\hline 229.269 & 23.54 \\
\hline 228.136 & 23.544 \\
\hline 225.873 & 23.548 \\
\hline 227.004 & 23.552 \\
\hline 227.004 & 23.556 \\
\hline 224.742 & 23.56 \\
\hline 217.965 & 23.564 \\
\hline 213.458 & 23.568 \\
\hline 214.584 & 23.572 \\
\hline 217.965 & 23.576 \\
\hline 225.873 & 23.58 \\
\hline 231.535 & 23.584 \\
\hline 234.938 & 23.588 \\
\hline 236.074 & 23.592 \\
\hline 233.803 & 23.596 \\
\hline 230.402 & 23.6 \\
\hline 227.004 & 23.604 \\
\hline 227.004 & 23.608 \\
\hline 225.873 & 23.612 \\
\hline 228.136 & 23.616 \\
\hline
\end{tabular}




\begin{tabular}{|c|c|}
\hline 228.136 & 23.62 \\
\hline 229.269 & 23.624 \\
\hline 228.136 & 23.628 \\
\hline 228.136 & 23.632 \\
\hline 229.269 & 23.636 \\
\hline 227.004 & 23.64 \\
\hline 224.742 & 23.644 \\
\hline 223.611 & 23.648 \\
\hline 222.481 & 23.652 \\
\hline 224.742 & 23.656 \\
\hline 224.742 & 23.66 \\
\hline 227.004 & 23.664 \\
\hline 230.402 & 23.668 \\
\hline 229.269 & 23.672 \\
\hline 229.269 & 23.676 \\
\hline 229.269 & 23.68 \\
\hline 228.136 & 23.684 \\
\hline 228.136 & 23.688 \\
\hline 229.269 & 23.692 \\
\hline 230.402 & 23.696 \\
\hline 227.004 & 23.7 \\
\hline 229.269 & 23.704 \\
\hline 229.269 & 23.708 \\
\hline 229.269 & 23.712 \\
\hline 229.269 & 23.716 \\
\hline 229.269 & 23.72 \\
\hline 230.402 & 23.724 \\
\hline 233.803 & 23.728 \\
\hline 233.803 & 23.732 \\
\hline 232.669 & 23.736 \\
\hline 234.938 & 23.74 \\
\hline 234.938 & 23.744 \\
\hline 233.803 & 23.748 \\
\hline 233.803 & 23.752 \\
\hline 233.803 & 23.75 \\
\hline
\end{tabular}




\begin{tabular}{|c|c|}
\hline 234.938 & 23.76 \\
\hline 238.345 & 23.764 \\
\hline 239.482 & 23.768 \\
\hline 238.345 & 23.772 \\
\hline 238.345 & 23.776 \\
\hline 240.618 & 23.78 \\
\hline 238.345 & 23.784 \\
\hline 239.482 & 23.788 \\
\hline 240.618 & 23.792 \\
\hline 241.756 & 23.796 \\
\hline 244.031 & 23.8 \\
\hline 242.893 & 23.804 \\
\hline 242.893 & 23.808 \\
\hline 245.17 & 23.812 \\
\hline 245.17 & 23.816 \\
\hline 244.031 & 23.82 \\
\hline 247.448 & 23.824 \\
\hline 244.031 & 23.828 \\
\hline 245.17 & 23.832 \\
\hline 245.17 & 23.836 \\
\hline 246.308 & 23.84 \\
\hline 246.308 & 23.844 \\
\hline 247.448 & 23.848 \\
\hline 249.727 & 23.852 \\
\hline 249.727 & 23.856 \\
\hline 248.587 & 23.86 \\
\hline 250.867 & 23.864 \\
\hline 248.587 & 23.868 \\
\hline 248.587 & 23.872 \\
\hline 247.448 & 23.876 \\
\hline 249.727 & 23.88 \\
\hline 250.867 & 23.884 \\
\hline 252.008 & 23.888 \\
\hline 250.867 & 23.892 \\
\hline 252.008 & 23.896 \\
\hline
\end{tabular}




\begin{tabular}{|c|c|}
\hline 252.008 & 23.9 \\
\hline 252.008 & 23.904 \\
\hline 252.008 & 23.908 \\
\hline 252.008 & 23.912 \\
\hline 252.008 & 23.916 \\
\hline 250.867 & 23.92 \\
\hline 252.008 & 23.924 \\
\hline 252.008 & 23.928 \\
\hline 253.148 & 23.932 \\
\hline 250.867 & 23.936 \\
\hline 250.867 & 23.94 \\
\hline 252.008 & 23.944 \\
\hline 252.008 & 23.948 \\
\hline 253.148 & 23.952 \\
\hline 252.008 & 23.956 \\
\hline 252.008 & 23.96 \\
\hline 252.008 & 23.964 \\
\hline 253.148 & 23.968 \\
\hline 253.148 & 23.972 \\
\hline 252.008 & 23.976 \\
\hline 250.867 & 23.98 \\
\hline 252.008 & 23.984 \\
\hline 250.867 & 23.988 \\
\hline 252.008 & 23.992 \\
\hline 252.008 & 23.996 \\
\hline 252.008 & 24 \\
\hline 252.008 & 24.004 \\
\hline 252.008 & 24.008 \\
\hline 253.148 & 24.012 \\
\hline 253.148 & 24.016 \\
\hline 253.148 & 24.02 \\
\hline 250.867 & 24.024 \\
\hline 252.008 & 24.028 \\
\hline 250.867 & 24.032 \\
\hline 250.867 & 24.03 \\
\hline
\end{tabular}




\begin{tabular}{|c|c|}
\hline 252.008 & 24.04 \\
\hline 252.008 & 24.044 \\
\hline 252.008 & 24.048 \\
\hline 252.008 & 24.052 \\
\hline 254.29 & 24.056 \\
\hline 252.008 & 24.06 \\
\hline 255.431 & 24.064 \\
\hline 253.148 & 24.068 \\
\hline 253.148 & 24.072 \\
\hline 252.008 & 24.076 \\
\hline 252.008 & 24.08 \\
\hline 253.148 & 24.084 \\
\hline 254.29 & 24.088 \\
\hline 255.431 & 24.092 \\
\hline 254.29 & 24.096 \\
\hline 253.148 & 24.1 \\
\hline 253.148 & 24.104 \\
\hline 254.29 & 24.108 \\
\hline 255.431 & 24.112 \\
\hline 256.573 & 24.116 \\
\hline 256.573 & 24.12 \\
\hline 255.431 & 24.124 \\
\hline 255.431 & 24.128 \\
\hline 255.431 & 24.132 \\
\hline 254.29 & 24.136 \\
\hline 254.29 & 24.14 \\
\hline 254.29 & 24.144 \\
\hline 256.573 & 24.148 \\
\hline 256.573 & 24.152 \\
\hline 256.573 & 24.156 \\
\hline 257.715 & 24.16 \\
\hline 258.858 & 24.164 \\
\hline 260 & 24.168 \\
\hline 258.858 & 24.172 \\
\hline 258.85 & 24.176 \\
\hline
\end{tabular}




\begin{tabular}{|c|c|}
\hline 257.715 & 24.18 \\
\hline 258.858 & 24.184 \\
\hline 261.143 & 24.188 \\
\hline 261.143 & 24.192 \\
\hline 263.43 & 24.196 \\
\hline 262.287 & 24.2 \\
\hline 262.287 & 24.204 \\
\hline 263.43 & 24.208 \\
\hline 262.287 & 24.212 \\
\hline 263.43 & 24.216 \\
\hline 263.43 & 24.22 \\
\hline 264.574 & 24.224 \\
\hline 263.43 & 24.228 \\
\hline 264.574 & 24.232 \\
\hline 263.43 & 24.236 \\
\hline 265.718 & 24.24 \\
\hline 265.718 & 24.244 \\
\hline 265.718 & 24.248 \\
\hline 266.863 & 24.252 \\
\hline 266.863 & 24.256 \\
\hline 268.007 & 24.26 \\
\hline 266.863 & 24.264 \\
\hline 265.718 & 24.268 \\
\hline 268.007 & 24.272 \\
\hline 266.863 & 24.276 \\
\hline 268.007 & 24.28 \\
\hline 268.007 & 24.284 \\
\hline 269.152 & 24.288 \\
\hline 271.443 & 24.292 \\
\hline 270.297 & 24.296 \\
\hline 271.443 & 24.3 \\
\hline 271.443 & 24.304 \\
\hline 271.443 & 24.308 \\
\hline 271.443 & 24.312 \\
\hline 273.734 & 24.316 \\
\hline
\end{tabular}




\begin{tabular}{|c|c|}
\hline 272.588 & 24.32 \\
\hline 274.88 & 24.324 \\
\hline 276.027 & 24.328 \\
\hline 274.88 & 24.332 \\
\hline 276.027 & 24.336 \\
\hline 278.32 & 24.34 \\
\hline 277.173 & 24.344 \\
\hline 278.32 & 24.348 \\
\hline 279.467 & 24.352 \\
\hline 281.761 & 24.356 \\
\hline 279.467 & 24.36 \\
\hline 280.614 & 24.364 \\
\hline 281.761 & 24.368 \\
\hline 280.614 & 24.372 \\
\hline 279.467 & 24.376 \\
\hline 282.908 & 24.38 \\
\hline 284.056 & 24.384 \\
\hline 284.056 & 24.388 \\
\hline 284.056 & 24.392 \\
\hline 286.352 & 24.396 \\
\hline 284.056 & 24.4 \\
\hline 285.204 & 24.404 \\
\hline 286.352 & 24.408 \\
\hline 287.5 & 24.412 \\
\hline 287.5 & 24.416 \\
\hline 288.648 & 24.42 \\
\hline 289.796 & 24.424 \\
\hline 289.796 & 24.428 \\
\hline 289.796 & 24.432 \\
\hline 294.391 & 24.436 \\
\hline 293.242 & 24.44 \\
\hline 295.54 & 24.444 \\
\hline 302.435 & 24.448 \\
\hline 316.232 & 24.452 \\
\hline 341.514 & 24.456 \\
\hline
\end{tabular}




\begin{tabular}{|c|c|}
\hline 366.726 & 24.46 \\
\hline 392.938 & 24.464 \\
\hline 412.172 & 24.468 \\
\hline 425.659 & 24.472 \\
\hline 433.487 & 24.476 \\
\hline 441.284 & 24.48 \\
\hline 444.615 & 24.484 \\
\hline 445.724 & 24.488 \\
\hline 439.06 & 24.492 \\
\hline 435.718 & 24.496 \\
\hline 427.899 & 24.5 \\
\hline 418.926 & 24.504 \\
\hline 415.551 & 24.508 \\
\hline 408.788 & 24.512 \\
\hline 403.138 & 24.516 \\
\hline 399.742 & 24.52 \\
\hline 397.476 & 24.524 \\
\hline 394.073 & 24.528 \\
\hline 392.938 & 24.532 \\
\hline 388.393 & 24.536 \\
\hline 386.118 & 24.54 \\
\hline 380.425 & 24.544 \\
\hline 380.425 & 24.548 \\
\hline 372.44 & 24.552 \\
\hline 369.012 & 24.556 \\
\hline 362.15 & 24.56 \\
\hline 352.987 & 24.564 \\
\hline 339.218 & 24.568 \\
\hline 325.429 & 24.572 \\
\hline 312.782 & 24.576 \\
\hline 296.689 & 24.58 \\
\hline 282.908 & 24.584 \\
\hline 265.718 & 24.588 \\
\hline 253.148 & 24.592 \\
\hline 233.803 & 24.596 \\
\hline
\end{tabular}




\begin{tabular}{ll}
217.965 & 24.6 \\
202.229 & 24.604 \\
189.947 & 24.608 \\
179.958 & 24.612 \\
176.642 & 24.616 \\
174.434 & 24.62 \\
167.831 & 24.624 \\
\hline 160.163 & 24.628 \\
\hline 152.536 & 24.632 \\
\hline 146.034 & 24.636 \\
\hline 140.64 & 24.64 \\
\hline 140.64 & 24.644 \\
\hline 140.64 & 24.648 \\
\hline 138.489 & 24.652 \\
\hline 139.564 & 24.656 \\
\hline 134.199 & 24.66 \\
\hline 127.794 & 24.664 \\
123.544 & 24.668 \\
\hline 122.484 & 24.672 \\
119.311 & 24.676 \\
117.201 & 24.68 \\
111.944 & 24.684 \\
111.944 & 24.688 \\
\hline 110.896 & 24.692 \\
112.993 & 24.696 \\
\hline
\end{tabular}


Appendix C - Gauge data for experiment 4535 .

\begin{tabular}{|c|c|c|c|c|c|c|c|c|c|c|c|}
\hline \multirow[t]{2}{*}{0.538458} & 10 & 1.05199 & 10 & 2.11574 & 10 & 2.59721 & 10 & 0.458308 & 10 & 0.499752 & 10 \\
\hline & 1.85468 & 10 & 1.65629 & 10 & & & & & & & \\
\hline \multirow[t]{2}{*}{0.538458} & 10.004 & 1.39652 & 10.004 & 0.638699 & 10.004 & 0.257073 & 10.004 & 4.54137 & 10.004 & 2.09605 & 10.004 \\
\hline & 0.234906 & 10.004 & 1.11461 & 10.004 & & & & & & & \\
\hline \multirow[t]{2}{*}{0.283072} & 10.008 & 3.39167 & 10.008 & 4.1951 & 10.008 & 0.257073 & 10.008 & 1.96417 & 10.008 & 3.00547 & 10.008 \\
\hline & 2.0745 & 10.008 & 0.62663 & 10.008 & & & & & & & \\
\hline \multirow[t]{2}{*}{0.538458} & 10.012 & 3.39167 & 10.012 & 0.638699 & 10.012 & 0.257073 & 10.012 & 1.96417 & 10.012 & 2.09605 & 10.012 \\
\hline & 0.234906 & 10.012 & 0.62663 & 10.012 & & & & & & & \\
\hline \multirow[t]{2}{*}{0.538458} & 10.016 & 1.05199 & 10.016 & 0.638699 & 10.016 & 2.59721 & 10.016 & 1.96417 & 10.016 & 3.00547 & 10.016 \\
\hline & 0.234906 & 10.016 & 1.11461 & 10.016 & & & & & & & \\
\hline \multirow[t]{2}{*}{0.283072} & 10.02 & 2.08764 & 10.02 & 1.96842 & 10.02 & 0.257073 & 10.02 & 0.458308 & 10.02 & 0.499752 & 10.02 \\
\hline & 0.234906 & 10.02 & 1.65629 & 10.02 & & & & & & & \\
\hline \multirow[t]{2}{*}{0.283072} & 10.024 & 1.05199 & 10.024 & 0.638699 & 10.024 & 0.257073 & 10.024 & 0.458308 & 10.024 & 1.65098 & 10.024 \\
\hline & 2.41693 & 10.024 & 0.62663 & 10.024 & & & & & & & \\
\hline \multirow[t]{2}{*}{0.538458} & 10.028 & 1.05199 & 10.028 & 0.638699 & 10.028 & 0.257073 & 10.028 & 0.458308 & 10.028 & 4.8466 & 10.028 \\
\hline & 0.234906 & 10.028 & 1.65629 & 10.028 & & & & & & & \\
\hline \multirow[t]{2}{*}{0.283072} & 10.032 & 1.05199 & 10.032 & 2.11574 & 10.032 & 0.257073 & 10.032 & 2.43745 & 10.032 & 1.65098 & 10.032 \\
\hline & 0.234906 & 10.032 & 1.11461 & 10.032 & & & & & & & \\
\hline \multirow[t]{2}{*}{0.538458} & 10.036 & 3.39167 & 10.036 & 0.638699 & 10.036 & 2.59721 & 10.036 & 1.96417 & 10.036 & 0.499752 & 10.036 \\
\hline & 0.234906 & 10.036 & 2.052 & 10.036 & & & & & & & \\
\hline 0.283072 & 10.04 & 2.08764 & 10.04 & 0.638699 & 10.04 & 2.59721 & 10.04 & 0.458308 & 10.04 & 1.65098 & 10.04 \\
\hline & 1.85468 & 10.04 & 1.65629 & 10.04 & & & & & & & \\
\hline 0.283072 & 10.044 & 1.05199 & 10.044 & 0.638699 & 10.044 & 0.257073 & 10.044 & 2.01015 & 10.044 & 3.00547 & 10.044 \\
\hline & 1.85468 & 10.044 & 0.62663 & 10.044 & & & & & & & \\
\hline 0.538458 & 10.048 & 1.39652 & 10.048 & 0.638699 & 10.048 & 0.257073 & 10.048 & 2.43745 & 10.048 & 1.65098 & 10.048 \\
\hline & 2.41693 & 10.048 & 0.62663 & 10.048 & & & & & & & \\
\hline 0.538458 & 10.052 & 5.12714 & 10.052 & 0.638699 & 10.052 & 0.257073 & 10.052 & 2.43745 & 10.052 & 3.00547 & 10.052 \\
\hline & 0.234906 & 10.052 & 1.11461 & 10.052 & & & & & & & \\
\hline 0.283072 & 10.056 & 1.05199 & 10.056 & 2.11574 & 10.056 & 2.59721 & 10.056 & 1.96417 & 10.056 & 4.8466 & 10.056 \\
\hline & 2.41693 & 10.056 & 1.11461 & 10.056 & & & & & & & \\
\hline 0.538458 & 10.06 & 3.39167 & 10.06 & 0.638699 & 10.06 & 0.257073 & 10.06 & 0.458308 & 10.06 & 3.00547 & 10.06 \\
\hline & 1.85468 & 10.06 & 1.11461 & 10.06 & & & & & & & \\
\hline 0.283072 & 10.064 & 3.39167 & 10.064 & 0.638699 & 10.064 & 0.257073 & 10.064 & 2.43745 & 10.064 & 1.78639 & 10.064 \\
\hline & 0.234906 & 10.064 & 1.11461 & 10.064 & & & & & & & \\
\hline 0.538458 & 10.068 & 3.39167 & 10.068 & 0.638699 & 10.068 & 0.257073 & 10.068 & 4.54137 & 10.068 & 0.499752 & 10.068 \\
\hline & 2.0745 & 10.068 & 1.11461 & 10.068 & & & & & & & \\
\hline 0.538458 & 10.072 & 3.39167 & 10.072 & 0.638699 & 10.072 & 2.59721 & 10.072 & 4.54137 & 10.072 & 3.00547 & 10.072 \\
\hline & 0.234906 & 10.072 & 1.11461 & 10.072 & & & & & & & \\
\hline 0.538458 & 10.076 & 1.05199 & 10.076 & 4.1951 & 10.076 & 0.257073 & 10.076 & 4.54137 & 10.076 & 0.499752 & 10.076 \\
\hline & 1.85468 & 10.076 & 1.11461 & 10.076 & & & & & & & \\
\hline 0.283072 & 10.08 & 2.08764 & 10.08 & 0.638699 & 10.08 & 0.257073 & 10.08 & 6.11771 & 10.08 & 1.65098 & 10.08 \\
\hline & 1.85468 & 10.08 & 1.11461 & 10.08 & & & & & & & \\
\hline 0.283072 & 10.084 & 1.05199 & 10.084 & 2.11574 & 10.084 & 0.257073 & 10.084 & 0.458308 & 10.084 & 0.499752 & 10.084 \\
\hline & 1.85468 & 10.084 & 1.11461 & 10.084 & & & & & & & \\
\hline 0.283072 & 10.088 & 1.39652 & 10.088 & 0.638699 & 10.088 & 2.59721 & 10.088 & 2.43745 & 10.088 & 0.499752 & 10.088 \\
\hline & 0.234906 & 10.088 & 1.11461 & 10.088 & & & & & & & \\
\hline 1.30685 & 10.092 & 1.05199 & 10.092 & 0.638699 & 10.092 & 2.59721 & 10.092 & 0.458308 & 10.092 & 1.65098 & 10.092 \\
\hline & 0.234906 & 10.092 & 0.62663 & 10.092 & & & & & & & \\
\hline 0.538458 & 10.096 & 1.39652 & 10.096 & 2.11574 & 10.096 & 0.257073 & 10.096 & 2.43745 & 10.096 & 3.00547 & 10.096 \\
\hline & 0.234906 & 10.096 & 2.62938 & 10.096 & & & & & & & \\
\hline 0.538458 & 10.1 & 1.39652 & 10.1 & 0.638699 & 10.1 & 0.257073 & 10.1 & 0.458308 & 10.1 & 1.65098 & 10.1 \\
\hline & 1.85468 & 10.1 & 1.11461 & 10.1 & & & & & & & \\
\hline 0.538458 & 10.104 & 1.39652 & 10.104 & 2.11574 & 10.104 & 0.257073 & 10.104 & 1.96417 & 10.104 & 3.00547 & 10.104 \\
\hline & 2.41693 & 10.104 & 0.62663 & 10.104 & & & & & & & \\
\hline 0.283072 & 10.108 & 2.08764 & 10.108 & 0.638699 & 10.108 & 0.257073 & 10.108 & 2.43745 & 10.108 & 3.00547 & 10.108 \\
\hline & 2.41693 & 10.108 & 0.62663 & 10.108 & & & & & & & \\
\hline 0.538458 & 10.112 & 1.39652 & 10.112 & 2.11574 & 10.112 & 0.257073 & 10.112 & 1.96417 & 10.112 & 0.499752 & 10.112 \\
\hline & 1.85468 & 10.112 & 1.11461 & 10.112 & & & & & & & \\
\hline 0.283072 & 10.116 & 1.39652 & 10.116 & 0.638699 & 10.116 & 0.257073 & 10.116 & 1.96417 & 10.116 & 1.65098 & 10.116 \\
\hline & 0.234906 & 10.116 & 1.11461 & 10.116 & & & & & & & \\
\hline 0.538458 & 10.12 & 2.08764 & 10.12 & 0.638699 & 10.12 & 0.257073 & 10.12 & 1.96417 & 10.12 & 1.65098 & 10.12 \\
\hline & 0.234906 & 10.12 & 0.62663 & 10.12 & & & & & & & \\
\hline 0.942169 & 10.124 & 1.39652 & 10.124 & 0.638699 & 10.124 & 0.257073 & 10.124 & 4.54137 & 10.124 & 0.499752 & 10.124 \\
\hline & 2.41693 & 10.124 & 1.11461 & 10.124 & & & & & & & \\
\hline 0.538458 & 10.128 & 3.39167 & 10.128 & 2.11574 & 10.128 & 0.257073 & 10.128 & 0.458308 & 10.128 & 1.65098 & 10.128 \\
\hline & 0.234906 & 10.128 & 2.62938 & 10.128 & & & & & & & \\
\hline
\end{tabular}




\begin{tabular}{|c|c|c|c|c|c|c|c|c|c|c|c|}
\hline 0.538458 & $\begin{array}{l}10.132 \\
2.41693\end{array}$ & $\begin{array}{l}1.05199 \\
10.132\end{array}$ & $\begin{array}{l}10.132 \\
1.11461\end{array}$ & $\begin{array}{l}1.96842 \\
10.132\end{array}$ & 10.132 & 0.257073 & 10.132 & 1.96417 & 10.132 & 1.65098 & 10.132 \\
\hline 0.283072 & 10.136 & 1.05199 & 10.136 & 0.638699 & 10.136 & 0.257073 & 10.136 & 1.96417 & 10.136 & 0.499752 & 10.136 \\
\hline \multirow[t]{2}{*}{0.538458} & $\begin{array}{l}2.41693 \\
10.14\end{array}$ & $\begin{array}{l}10.136 \\
1.05199\end{array}$ & $\begin{array}{l}1.11461 \\
10.14\end{array}$ & $\begin{array}{l}10.136 \\
0.638699 \\
10.14\end{array}$ & 10.14 & 0.257073 & 10.14 & 0.458308 & 10.14 & 2.09605 & 10.14 \\
\hline & $\begin{array}{l}2.41693 \\
10.144\end{array}$ & $\begin{array}{l}10.14 \\
2.08764\end{array}$ & $\begin{array}{l}1.11461 \\
10.144\end{array}$ & $\begin{array}{l}10.14 \\
0.638699\end{array}$ & & & & & & & \\
\hline 0.538458 & 2.41693 & 10.144 & 0.62663 & 10.144 & & & & & & & \\
\hline \multirow[t]{2}{*}{0.942169} & 10.148 & 1.39652 & 10.148 & 0.638699 & 10.148 & 0.257073 & 10.148 & 2.43745 & 10.148 & 1.65098 & 10.148 \\
\hline & 0.234906 & 10.148 & 0.62663 & 10.148 & & & & & & & \\
\hline \multirow[t]{2}{*}{0.283072} & 10.152 & 1.05199 & 10.152 & 0.638699 & 10.152 & 0.257073 & 10.152 & 4.54137 & 10.152 & 2.09605 & 10.152 \\
\hline & 1.85468 & 10.152 & 61 & 10.152 & & & & & & & \\
\hline \multirow[t]{2}{*}{0.538458} & 10.156 & 1.05199 & 10 & 0.63 & 10.156 & 0.257073 & 10.156 & 0.458308 & 10.156 & 1.65098 & 10.156 \\
\hline & 0.234906 & .156 & 61 & 10.1 & & & & & & & \\
\hline \multirow[t]{2}{*}{0.283072} & 10.16 & 3.39167 & 10.16 & 1.96842 & 10.16 & 0.257073 & 10.16 & 2.01015 & 10.16 & 3.00547 & 10.16 \\
\hline & 2.41693 & 10.16 & & 10.16 & & & & & & & \\
\hline \multirow[t]{2}{*}{0.538458} & 10.164 & 1.39652 & 10.1 & 0.638699 & 10.164 & 0.257073 & 10.164 & 0.458308 & 10.164 & 3.00547 & 10.164 \\
\hline & 0.234906 & 10164 & & 10.164 & & & & & & & \\
\hline \multirow[t]{2}{*}{0.942169} & 10.16 & 52 & & 0.6 & 10.168 & 0.257073 & 10.168 & 458308 & 10.1 & .00547 & 10.168 \\
\hline & 0.234906 & 10.168 & & 10. & & & & & & & \\
\hline 0.283072 & 10.172 & 1.05 & 10. & 2.11 & 10.172 & 0.257073 & 10.172 & .43745 & 10.172 & 1.65098 & 10.172 \\
\hline & 2.41 & 1 & & 10. & & & & & & & \\
\hline 0.283072 & 10.176 & 1.05 & 10. & 2.1 & 10.176 & 0.257073 & 10.176 & 2.43745 & 10.176 & 1.65098 & 10.176 \\
\hline & 0.234906 & 10176 & & 10.176 & & & & & & & \\
\hline 0.283072 & 10.18 & & 10. & & 10.18 & 0.257073 & 10.18 & 0.458308 & 10.18 & 4.8466 & 10.18 \\
\hline & 0.2 & 1 & & & & & & & & & \\
\hline 0.942169 & 10.184 & 52 & 10 & 2.1 & 10.184 & 0.257073 & 10.184 & 2.43745 & 10.184 & 3.00547 & 10.184 \\
\hline & & & & & & & & & & & \\
\hline 0.283072 & 10.188 & 1.39 & 10. & 1.9 & 10.188 & 0.257073 & 10.188 & 1.96417 & 10.188 & 2.09605 & 10.188 \\
\hline & 0.234906 & 1 & & 10. & & & & & & & \\
\hline 0.538458 & 10.192 & 52 & & & 10.192 & 1.91927 & 10.192 & 2.43745 & 10.192 & 0.499752 & 10.192 \\
\hline & 0.2 & & & & & & & & & & \\
\hline 0.942169 & 10.196 & & & & 10.196 & 0.257073 & 10.196 & 17 & 10.1 & 499752 & 10.196 \\
\hline & & & & & & & & & & & \\
\hline 0.942169 & 10.2 & 1.396 & 10 & 0.638 & 10.2 & .257073 & 10.2 & 0.458308 & 10.2 & 3.00547 & 10.2 \\
\hline & 1.854 & 10.2 & & 102 & & & & & & & \\
\hline 0.283072 & 10.204 & 4 & 1 & 8699 & 10.204 & 0.257073 & 10.204 & 1.96417 & 10.204 & 3.00547 & 10.204 \\
\hline & 0.23 & 1 & & 10. & & & & & & & \\
\hline 0.538458 & 10.2 & & & & 10.208 & 1.91927 & 10.208 & 2.01015 & 10.208 & 3.00547 & 10.208 \\
\hline & 0. & & & & & & & & & & \\
\hline 1.30685 & 10.21 & 14 & 1 & 2.1 & 10.212 & 257073 & 10.212 & 17 & 10.212 & .499752 & 10.212 \\
\hline & 0.23 & & & & & & & & & & \\
\hline 2.00895 & 10.216 & 67 & 1 & 2.1 & 10.216 & .257073 & 10.216 & .458308 & 10.216 & 3.00547 & 10.216 \\
\hline & 2.41 & & & $10-2$ & & & & & & & \\
\hline 0.538458 & 10.22 & 99 & 1 & 4. & 10.22 & 0.257073 & 10.22 & 0.458308 & 10.22 & 1.65098 & 10.22 \\
\hline & & & & & & & & & & & \\
\hline 0.283072 & 10.22 & & & 2.1 & 10.224 & 2.59721 & 10.224 & 0.458308 & 10.224 & 3.00547 & 10.224 \\
\hline & & & & & & & & & & & \\
\hline 0.538458 & 10.2 & & & 1.9 & 10.228 & 0.257073 & 10.228 & 1.96417 & 10.228 & 0.499752 & 10.228 \\
\hline & 0.234906 & & & 10.2 & & & & & & & \\
\hline 0.283072 & 10.232 & 1.05199 & & 2.11574 & 10.232 & 2.59721 & 10.232 & 1.96417 & 10.232 & 3.00547 & 10.232 \\
\hline & & & & & & & & & & & \\
\hline 0.538458 & 10.2 & & & 99 & 10.236 & 0.257073 & 10.236 & 2.01015 & 10.23 & 1.65098 & 10.236 \\
\hline & & & & & & & & & & & \\
\hline 0.283072 & 10.24 & & & 0.638699 & 10.24 & 0.257073 & 10.24 & 0.458308 & 10.24 & 3.00547 & 10.24 \\
\hline & & & & & & & & & & & \\
\hline 0.283072 & 10.244 & 9652 & & 2.11574 & 10.244 & 0.257073 & 10.244 & 0.458308 & 10.244 & 0.499752 & 10.244 \\
\hline & 0.2349 & & & & & & & & & & \\
\hline 0.942169 & 10.248 & & & 4.1951 & 10.248 & 2.59721 & 10.248 & 458308 & 10. & 499752 & 10.2 \\
\hline & & & & & & & & & & & \\
\hline 0.538458 & 10.252 & 52 & 1 & 0.6 & 10.252 & 6.19748 & 10.2 & 2.43745 & 10.2 & 3.00547 & 10.252 \\
\hline & & & & & & & & & & & \\
\hline 0.283072 & 10.256 & 1.05199 & 1 & 0.638699 & 10.256 & 0.257073 & 10.256 & 1.96417 & 10.256 & 3.00547 & 10.256 \\
\hline & 4906 & & & & & & & & & & \\
\hline 0.283072 & 10.26 & 5199 & & 4.1951 & 10.26 & 0.257073 & 10.26 & 1.96417 & 10.26 & 3.00547 & 10.26 \\
\hline & & & & & & & & & & & \\
\hline 0.942169 & 10.264 & 3.39167 & & 0.638699 & 10.26 & 0.257073 & 10.2 & 458308 & 10.2 & 499752 & 02 \\
\hline & & & & & & & & & & & \\
\hline 0.283072 & 10.268 & 1.39652 & 10.268 & 0.638699 & 10.268 & 0.257073 & 10.268 & 0.458308 & 10.268 & 0.499752 & 10.268 \\
\hline & 2.41693 & 10.268 & 1.11461 & 10.268 & & & & & & & \\
\hline
\end{tabular}




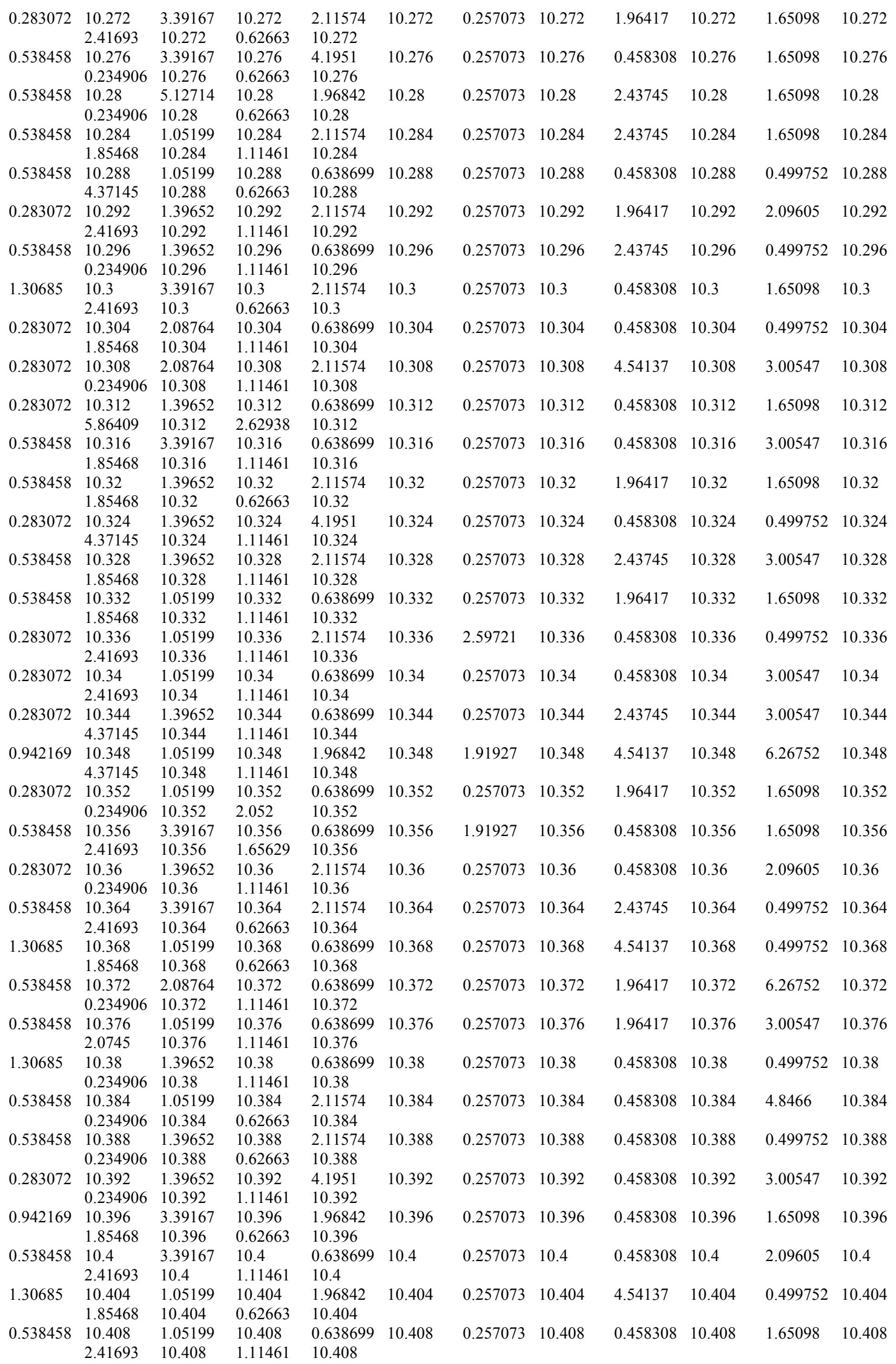




\begin{tabular}{|c|c|c|c|c|c|c|c|c|c|c|c|}
\hline 0.538458 & $\begin{array}{l}10.412 \\
0.234906\end{array}$ & $\begin{array}{l}1.39652 \\
10.412\end{array}$ & $\begin{array}{l}10.412 \\
1.11461\end{array}$ & $\begin{array}{l}2.11574 \\
10.412\end{array}$ & 10.412 & 0.257073 & 10.412 & 1.96417 & 10.412 & 0.499752 & 10.412 \\
\hline \multirow[t]{2}{*}{0.942169} & 10.416 & 1.05199 & 10.416 & 0.638699 & 10.416 & 1.91927 & 10.416 & 96417 & 10.416 & 6.26752 & 10.416 \\
\hline & 0.234906 & 10.416 & 0.62663 & 10.416 & & & & & & & \\
\hline \multirow[t]{2}{*}{0.538458} & 10.42 & 1.05199 & 10.42 & 2.11574 & 10.42 & 2.59721 & 10.42 & 1.96417 & 10.42 & 0.499752 & 10.42 \\
\hline & 0.234906 & 0.42 & 0.62663 & 10.42 & & & & & & & \\
\hline \multirow[t]{2}{*}{0.538458} & 10.424 & 5.12714 & 10.424 & 2.11574 & 10.424 & 0.257073 & 10.424 & 0.458308 & 10.424 & 4.8466 & 10.424 \\
\hline & 4.37145 & 10.424 & 2.62938 & 10.424 & & & & & & & \\
\hline \multirow[t]{2}{*}{0.538458} & 10.428 & 1.05199 & 10.428 & 2.11574 & 10.428 & 0.257073 & 10.428 & 2.43745 & 10.428 & 4.8466 & 10.428 \\
\hline & 1.85468 & 10.428 & 1.11461 & 10.428 & & & & & & & \\
\hline \multirow[t]{2}{*}{0.283072} & 10.432 & 1.05199 & 10.432 & 2.11574 & 10.432 & 0.257073 & 10.432 & 0.458308 & 10.432 & 1.65098 & 10.432 \\
\hline & 0.234906 & 10.432 & 1.65629 & 10.432 & & & & & & & \\
\hline \multirow[t]{2}{*}{0.538458} & 10.436 & 1.39 & & 0.638699 & 10.436 & 0.257073 & 10.436 & 0.458308 & 10.436 & 1.65098 & 10.436 \\
\hline & 4.37 & 10.4 & & 10. & & & & & & & \\
\hline \multirow[t]{2}{*}{0.942169} & 10.44 & 5.12714 & 10.44 & 4.1951 & 10.44 & 0.257073 & 10.44 & .458308 & 10.44 & .499752 & 10.44 \\
\hline & 2.41693 & 1 & & 10.44 & & & & & & & \\
\hline \multirow[t]{2}{*}{0.283072} & 10.444 & 1.39652 & 10.444 & 2.11574 & 10.444 & 0.257073 & 10.444 & 1.96417 & 10.444 & 3.00547 & 10.444 \\
\hline & 2.41693 & 10.444 & & 10.444 & & & & & & & \\
\hline 0.283072 & 10.4 & 99 & & & 10.448 & 2.59721 & 10.448 & 2.43745 & 10.448 & 4.8466 & 10.448 \\
\hline & 2.41693 & 1 & & 10. & & & & & & & \\
\hline 0.942169 & 10. & 7 & 1 & 0.6 & 10.452 & 0.257073 & 10.452 & .458308 & 10.452 & 3.00547 & 0.452 \\
\hline & 2.41 & & & & & & & & & & \\
\hline 0.538458 & 10.45 & 7 & 10. & 699 & 10.456 & .257073 & 10.456 & 0.458308 & 10.456 & 3.00547 & 0.456 \\
\hline & 0.23 & 1 & & & & & & & & & \\
\hline 0.283072 & 10.46 & 3.39167 & 1 & 1.9 & 10.46 & 0.257073 & 10.46 & 2.43745 & 10.46 & 1.65098 & 10.46 \\
\hline & 1.85468 & 1 & & 10.46 & & & & & & & \\
\hline 0.283072 & 10.4 & & & 699 & 10.464 & 0.257073 & 10.464 & 1.96417 & 10.464 & 1.65098 & 10.70 \\
\hline & 0.23 & & & & & & & & & & \\
\hline 0.942169 & 10.468 & & & 699 & 10.468 & 0.257073 & 10.468 & 2.43745 & 10.468 & 0.499752 & 10.468 \\
\hline & 0.23 & & & & & & & & & & \\
\hline 0.538458 & 10.472 & 1.39 & 1 & 2.1 & 10.472 & 0.257073 & 10.472 & 2.43745 & 10.472 & 1.65098 & 10.472 \\
\hline & 0.23 & 10.47 & & 10.472 & & & & & & & \\
\hline 0.538458 & 10.476 & 2 & & 699 & 10.476 & 0.257073 & 10.476 & 2.43745 & 10.476 & 4.8466 & 10.476 \\
\hline & & & & & & & & & & & \\
\hline 0.942169 & 10.4 & & & & 10.48 & .257073 & 10.48 & 43745 & 10.48 & 3.00547 & 0.48 \\
\hline & & & & & & & & & & & \\
\hline 0.283072 & 10.4 & & 1 & 699 & 10.484 & 0.257073 & 10.484 & 1.96417 & 10.484 & 0.499752 & 10.484 \\
\hline & 0.23 & 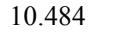 & & 10484 & & & & & & & \\
\hline 0.942169 & 10.488 & 67 & 1 & 699 & 10.488 & 0.257073 & 10.488 & 1.96417 & 10.488 & 0.499752 & 10.488 \\
\hline & & & & & & & & & & & \\
\hline 0.283072 & 10.4 & & & 699 & 10.492 & 2.59721 & 10.492 & 1.96417 & 10.492 & 1.65098 & 10.492 \\
\hline & 0.23 & & & & & & & & & & \\
\hline 0.538458 & 10.49 & & 1 & 699 & 10.496 & 59721 & 10.4 & 45 & 10. & 1.65098 & 0.496 \\
\hline & 0.23 & & & & & & & & & & \\
\hline 0.538458 & 10.5 & 5 & & 699 & 10.5 & 0.257073 & 10.5 & 1.96417 & 10.5 & 1.65098 & 10.5 \\
\hline & $2.416 \mathrm{~S}$ & & & & & & & & & & \\
\hline 0.538458 & 10.5 & 2 & 1 & 1 & 10.504 & 2.59721 & 10.504 & 0.458308 & 10.504 & 1.65098 & 0.504 \\
\hline & 0. & & & & & & & & & & \\
\hline 0.538458 & 10.5 & 7 & 1 & 699 & 10.508 & 0.257073 & 10.508 & 0.458308 & 10.508 & 1.65098 & 10.508 \\
\hline & & & & & & & & & & & \\
\hline 0.538458 & 10.51 & & & & 10.512 & 0.257073 & 10.512 & 1.96417 & 10.512 & 1.78639 & 10.512 \\
\hline & & & & & & & & & & & \\
\hline 2.00895 & 10.51 & 9167 & 10.516 & 1.96842 & 10.516 & 0.257073 & 10.516 & 0.458308 & 10.516 & .8466 & 10.516 \\
\hline & & & & & & & & & & & \\
\hline 0.538458 & 10.52 & 1 & & 699 & 10.52 & 2.59721 & 10.52 & 0.458308 & 10.52 & 0.499752 & 10.52 \\
\hline & & & & & & & & & & & \\
\hline 0.538458 & 10.52 & & 1 & 1.96842 & 10.524 & 0.257073 & 10.524 & 0.458308 & 10.524 & 0.499752 & 10.524 \\
\hline & & & & & & & & & & & \\
\hline 0.538458 & 10.528 & 9652 & 1 & 0.638699 & 10.528 & 0.257073 & 10.528 & 1.96417 & 10.528 & 0.499752 & 10.52 \\
\hline & & & & & & & & & & & \\
\hline 0.538458 & 10.53 & & & & 10.53 & .257073 & 10.53 & .458308 & 10.53 & 4.846 & 20 \\
\hline & & & & & & & & & & & \\
\hline 0.283072 & 10.5 & 1 & 1 & 2.1 & 10.536 & 0.257073 & 10.536 & 0.458308 & 10.536 & 0.499752 & 10.536 \\
\hline & & & & & & & & & & & \\
\hline 0.283072 & 10.54 & 1 & & 2.11574 & 10.54 & 0.257073 & 10.54 & 1.96417 & 10.54 & 1.65098 & 10.54 \\
\hline & 1.8 & & 0 & 10. & & & & & & & \\
\hline 0.538458 & 10.544 & 5.12714 & 10.544 & 2.11574 & 10.544 & 0.257073 & 10.544 & 0.458308 & 10.544 & 1.65098 & 10.5 \\
\hline & & & & & & & & & & & \\
\hline 1 & 10.54 & & 10.5 & 0.638699 & 10.548 & 0.257073 & 10.548 & 1.96417 & 10.548 & 3.00547 & 10.548 \\
\hline & 2.41 & & & & & & & & & & \\
\hline
\end{tabular}




\begin{tabular}{|c|c|c|c|c|c|c|c|c|c|c|c|}
\hline 0.283072 & 10.552 & $\begin{array}{l}3.39167 \\
10.552\end{array}$ & 10.552 & $\begin{array}{l}1.96842 \\
10.552\end{array}$ & 10.552 & 0.257073 & 10.552 & 2.01015 & 10.552 & 2.09605 & 10.552 \\
\hline \multirow[t]{2}{*}{0.283072} & 10.556 & 1.39652 & 10.556 & 4.1951 & 10.556 & 0.257073 & 10.556 & 4.54137 & 10.556 & 0.499752 & 10.556 \\
\hline & 2.41693 & 10.556 & 0.62663 & 10.556 & & & & & & & \\
\hline \multirow[t]{2}{*}{0.538458} & 10.56 & 1.39652 & 10.56 & 0.638699 & 10.56 & 0.257073 & 10.56 & 0.458308 & 10.56 & 0.499752 & 10.56 \\
\hline & 0.234906 & .56 & 1.11461 & 10.56 & & & & & & & \\
\hline \multirow[t]{2}{*}{0.283072} & 10.564 & 1.05199 & 10.564 & 2.11574 & 10.564 & 0.257073 & 10.564 & 0.458308 & 10.564 & 3.00547 & 10.564 \\
\hline & 0.234906 & 10.564 & 1.11461 & 10.564 & & & & & & & \\
\hline \multirow[t]{2}{*}{0.538458} & 10.568 & 1.05199 & 10.568 & 0.638699 & 10.568 & 0.257073 & 10.568 & 0.458308 & 10.568 & 2.09605 & 10.568 \\
\hline & 1.85468 & 10.568 & 0.62663 & 10.568 & & & & & & & \\
\hline \multirow[t]{2}{*}{0.283072} & 10.572 & 1.05199 & 10.572 & 2.11574 & 10.572 & 0.257073 & 10.572 & 4.54137 & 10.572 & 1.65098 & 10.572 \\
\hline & 1.85468 & 10.572 & 1.65629 & 10.572 & & & & & & & \\
\hline \multirow[t]{2}{*}{0.538458} & 10.57 & 199 & 10. & 0.638699 & 10.576 & 0.257073 & 10.576 & 6.11771 & 10.576 & 3.00547 & 10.576 \\
\hline & 2.41693 & 10.576 & 61 & 10.576 & & & & & & & \\
\hline \multirow[t]{2}{*}{0.283072} & 10.58 & 1.05199 & 10.58 & 0.638699 & 10.58 & 2.59721 & 10.58 & 2.43745 & 10.58 & 4.8466 & 10.58 \\
\hline & 4.37145 & 10.58 & & 10.58 & & & & & & & \\
\hline \multirow[t]{2}{*}{0.538458} & 10.584 & 1.05199 & 10.5 & 1.9 & 10.584 & 2.59721 & 10.584 & 4.54137 & 10.584 & 1.65098 & 10.584 \\
\hline & 0.234906 & 1 & & 10. & & & & & & & \\
\hline 0.283072 & 10.5 & 52 & 10 & 1.9 & 10.588 & 0.257073 & 10.588 & 96417 & 10.588 & .00547 & 10.588 \\
\hline & 0.234906 & 8 & & 10. & & & & & & & \\
\hline 0.538458 & 10.592 & 3.39167 & 10. & 2.1 & 10.592 & 0.257073 & 10.592 & 0.458308 & 10.592 & 3.00547 & 10.592 \\
\hline & 0.234906 & 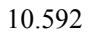 & & & & & & & & & \\
\hline 0.283072 & 10.596 & 1.39652 & 10. & 2.11574 & 10.596 & 0.257073 & 10.596 & 2.43745 & 10.596 & 3.00547 & 10.596 \\
\hline & 0.234906 & 15 & & 10.596 & & & & & & & \\
\hline 0.283072 & 10.6 & & & 99 & 10.6 & 0.257073 & 10.6 & 0.458308 & 10.6 & 0.499752 & 10.6 \\
\hline & 2.4 & & & & & & & & & & \\
\hline 0.283072 & 10.60 & 5 & 10 & 4.1 & 10.604 & 0.257073 & 10.604 & 0.458308 & 10.604 & 1.65098 & 10.604 \\
\hline & 0.234906 & 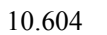 & & & & & & & & & \\
\hline 0.283072 & 10.608 & 1.05 & 10. & 0.638699 & 10.608 & 0.257073 & 10.608 & 0.458308 & 10.608 & 1.65098 & 10.608 \\
\hline & 2.41693 & 1 & 63 & 10608 & & & & & & & \\
\hline 0.538458 & 10.61 & 54 & 1 & 699 & 10.612 & 2.59721 & 10.612 & 1.96417 & 10.612 & 1.65098 & 10.612 \\
\hline & 5.8 & & & 10 & & & & & & & \\
\hline 0.538458 & 10.6 & & & 699 & 10.616 & 0.257073 & 10.616 & 2.01015 & 10.616 & .78639 & 10.616 \\
\hline & 0.23 & & & & & & & & & & \\
\hline 0.538458 & 10.62 & 3.39167 & 10. & 2.11574 & 10.62 & 0.257073 & 10.62 & 2.43745 & 10.62 & .499752 & 10.62 \\
\hline & 2.4169 & 1 & & 10.62 & & & & & & & \\
\hline 0.538458 & 10.62 & 7 & 1 & 8699 & 10.624 & 0.257073 & 10.624 & 0.458308 & 10.624 & 2.09605 & 10.624 \\
\hline & 2.41 & & & 10 & & & & & & & \\
\hline 0.538458 & 10.62 & & & & 10.628 & 0.257073 & 10.628 & 0.458308 & 10.628 & 0.499752 & 10.628 \\
\hline & 0.2 & & & & & & & & & & \\
\hline 1.30685 & 10.632 & 1.05 & 1 & 2.1 & 10.632 & 0.257073 & 10.632 & 0.458308 & 10.632 & 0.499752 & 10.632 \\
\hline & 2. & & & & & & & & & & \\
\hline 0.538458 & 10.63 & 9 & 1 & 0.638699 & 10.636 & 2.59721 & 10.636 & 2.01015 & 10.636 & .00547 & 10.636 \\
\hline & 2.41693 & & & 106 & & & & & & & \\
\hline 1.30685 & 10.64 & 67 & 10 & 574 & 10.64 & 0.257073 & 10.64 & 0.458308 & 10.64 & 1.65098 & 10.64 \\
\hline & & & & & & & & & & & \\
\hline 1.30685 & 10.6 & & 4 & 2.1 & 10.644 & 0.257073 & 10.644 & 2.43745 & 10.644 & 0.499752 & 10.644 \\
\hline & 06 & & & & & & & & & & \\
\hline 0.538458 & 10.64 & & 1 & 0.638699 & 10.648 & 0.257073 & 10.648 & 2.43745 & 10.648 & 0.499752 & 10.648 \\
\hline & 1.85 & & & & & & & & & & \\
\hline 2.00895 & 10.652 & 64 & 10. & 2.11574 & 10.652 & 59721 & 10.652 & 458308 & 10.652 & .499752 & 10.652 \\
\hline & & & & & & & & & & & \\
\hline 0.942169 & 10. & & & & 10.656 & 0.257073 & 10.656 & 458308 & 10.6 & 00547 & 0.656 \\
\hline & & & & & & & & & & & \\
\hline 0.942169 & 10.66 & 714 & 1 & 1.96842 & 10.66 & 2.59721 & 10.66 & 0.458308 & 10.66 & 0.499752 & 10.66 \\
\hline & & & & & & & & & & & \\
\hline 0.538458 & 10.664 & 5199 & 10.6 & 0.638699 & 10.664 & 0.257073 & 10.664 & 0.458308 & 10.664 & 1.65098 & 10.664 \\
\hline & 0.234906 & & & & & & & & & & \\
\hline 0.538458 & 10.668 & 652 & & 2.11574 & 10.668 & 0.257073 & 10.668 & 2.43745 & 10.668 & 499752 & 10.668 \\
\hline & & & & & & & & & & & \\
\hline 0.538458 & 10.672 & 1.05 & 1 & 2.1 & 10.672 & 0.257073 & 10.672 & 2.43745 & 10.67 & .09605 & 0.672 \\
\hline & & & & & & & & & & & \\
\hline 0.538458 & 10.676 & 1.05199 & 1 & 0.638699 & 10.676 & 0.257073 & 10.676 & 2.43745 & 10.676 & 0.499752 & 10.676 \\
\hline & 0.234906 & & & & & & & & & & \\
\hline 0.538458 & 10.68 & 2.08764 & & 2.11574 & 10.68 & 0.257073 & 10.68 & 4.54137 & 10.68 & 0.499752 & 10.68 \\
\hline & 0.23490 & & & & & & & & & & \\
\hline 0.538458 & 10.684 & 1.39652 & & 0.638699 & 10.684 & 0.257073 & 10.68 & 1.96417 & 10.68 & .499752 & 10 \\
\hline & & & & & & & & & & & \\
\hline 0.942169 & 10.688 & 3.39167 & 10.688 & 0.638699 & 10.688 & 0.257073 & 10.688 & 1.96417 & 10.688 & 3.00547 & 10.688 \\
\hline & 0.234906 & 10.688 & 0.62663 & 10.688 & & & & & & & \\
\hline
\end{tabular}




\begin{tabular}{|c|c|c|c|c|c|c|c|c|c|c|c|}
\hline 0.538458 & $\begin{array}{l}10.692 \\
1.85468\end{array}$ & $\begin{array}{l}5.12714 \\
10.692\end{array}$ & $\begin{array}{l}10.692 \\
0.62663\end{array}$ & $\begin{array}{l}2.11574 \\
10.692\end{array}$ & 10.692 & 0.257073 & 10.692 & 0.458308 & 10.692 & 3.00547 & 0.692 \\
\hline \multirow[t]{2}{*}{0.942169} & 10.696 & 1.39652 & 10.696 & 2.11574 & 10.696 & 0.257073 & 10.696 & 0.458308 & 10.696 & 3.00547 & 10.696 \\
\hline & 1.85468 & 10.696 & 0.62663 & 10.696 & & & & & & & \\
\hline \multirow[t]{2}{*}{0.942169} & 10.7 & 3.39167 & 10.7 & 4.1951 & 10.7 & 0.257073 & 10.7 & 4.54137 & 10.7 & 1.65098 & 10.7 \\
\hline & 0.234906 & 0.7 & 0.62663 & 10.7 & & & & & & & \\
\hline \multirow[t]{2}{*}{0.283072} & 10.704 & 1.05199 & 10.704 & 0.638699 & 10.704 & 0.257073 & 10.704 & 1.96417 & 10.704 & 1.65098 & 10.704 \\
\hline & 1.85468 & 10.704 & 2.62938 & 10.704 & & & & & & & \\
\hline \multirow[t]{2}{*}{0.283072} & 10.708 & 3.39167 & 10.708 & 2.11574 & 10.708 & 0.257073 & 10.708 & 4.54137 & 10.708 & 1.65098 & 10.708 \\
\hline & 0.234906 & 10.708 & 1.65629 & 10.708 & & & & & & & \\
\hline \multirow[t]{2}{*}{0.942169} & 10.712 & 1.05199 & 10.712 & 2.11574 & 10.712 & 0.257073 & 10.712 & 2.01015 & 10.712 & 1.65098 & 10.712 \\
\hline & 2.41693 & 10.712 & 1.65629 & 10.712 & & & & & & & \\
\hline \multirow[t]{2}{*}{0.538458} & 10.716 & 99 & 10.716 & 0.638699 & 10.716 & 0.257073 & 10.716 & 0.458308 & 10.716 & 0.499752 & 10.716 \\
\hline & 1.85468 & 10.716 & 0.62663 & 10.716 & & & & & & & \\
\hline \multirow[t]{2}{*}{0.942169} & 10.72 & 1.05199 & 10.72 & 0.638699 & 10.72 & 0.257073 & 10.72 & 2.43745 & 10.72 & 3.00547 & 10.72 \\
\hline & 1.85468 & 10.72 & 1.11461 & 10.72 & & & & & & & \\
\hline \multirow[t]{2}{*}{0.538458} & 10.724 & 1.3 & 10.724 & 4.1 & 10.724 & 0.257073 & 10.724 & 0.458308 & 10.724 & 0.499752 & 10.724 \\
\hline & 2.41693 & & 0.62663 & 10. & & & & & & & \\
\hline 0.538458 & 10.728 & 2 & 10.728 & 2.1 & 10.728 & 0.257073 & 10.728 & 458308 & 10.72 & .8466 & 10.728 \\
\hline & 0.234906 & 10.7 & 1.65629 & 10. & & & & & & & \\
\hline 0.283072 & 10.732 & 3.39 & 10.732 & 0.6 & 10.732 & 0.257073 & 10.732 & 0.458308 & 10.732 & .499752 & 10.732 \\
\hline & 0.234906 & 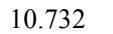 & 1.11461 & & & & & & & & \\
\hline 0.538458 & 10.736 & 3.39 & 10.736 & 3699 & 10.736 & 0.257073 & 10.736 & 1.96417 & 10.736 & 3.00547 & 10.736 \\
\hline & 2.41693 & 1 & 1.11461 & 10736 & & & & & & & \\
\hline 0.942169 & 10.74 & & 10.74 & & 10.74 & 0.257073 & 10.74 & 1.96417 & 10.74 & 1.65098 & 10.74 \\
\hline & 0.234906 & & & & & & & & & & \\
\hline 0.942169 & 10.744 & 52 & 10.744 & 0.6 & 10.744 & 0.257073 & 10.744 & 0.458308 & 10.744 & 0.499752 & 10.744 \\
\hline & 0.234906 & & & & & & & & & & \\
\hline 0.283072 & 10.748 & 1.05199 & 10.748 & 0.638699 & 10.748 & 2.59721 & 10.748 & 0.458308 & 10.748 & 0.499752 & 10.748 \\
\hline & 0.234906 & 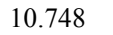 & 461 & 107 & & & & & & & \\
\hline 0.283072 & 10.752 & 9 & .752 & & 10.752 & 0.257073 & 10.752 & 0.458308 & 10.752 & 0.499752 & 10.752 \\
\hline & 0.23 & & & & & & & & & & \\
\hline 0.283072 & 10.756 & & 6 & & 10.756 & 0.257073 & 10.756 & 1.96417 & 10.756 & .499752 & 10.75 \\
\hline & 0.234906 & & & & & & & & & & \\
\hline 0.283072 & 10.76 & 1.05199 & 10.76 & 4. & 10.76 & 0.257073 & 10.76 & 1.96417 & 10.76 & .499752 & 10.76 \\
\hline & 2.0745 & & 1 & 107 & & & & & & & \\
\hline 0.283072 & 10.764 & 2 & 64 & 74 & 10.764 & 0.257073 & 10.764 & 2.43745 & 10.764 & 2.09605 & 10.764 \\
\hline & 4.37145 & 1 & 63 & 10 & & & & & & & \\
\hline 0.283072 & 10.76 & & & & 10.768 & 0.257073 & 10.768 & 0.458308 & 10.768 & 1.65098 & 10.768 \\
\hline & 0.2 & & & & & & & & & & \\
\hline 0.283072 & 10.772 & 1.0 & 10.772 & 2.11 & 10.772 & 0.257073 & 10.772 & 2.43745 & 10.772 & 1.65098 & 10.772 \\
\hline & 2.4169 & & & & & & & & & & \\
\hline 0.283072 & 10.776 & 1 & 1 & 01 & 10.776 & 2.59721 & 10.776 & 458308 & 10.776 & 0.499752 & 10.776 \\
\hline & 2.41693 & & 61 & 107 & & & & & & & \\
\hline 0.538458 & 10.78 & 3 & 10.78 & 74 & 10.78 & 4.65092 & 10.78 & 1.96417 & 10.78 & 4.8466 & 10.78 \\
\hline & 4. & & & & & & & & & & \\
\hline 0.538458 & 10.784 & & 1 & 74 & 10.784 & 0.257073 & 10.784 & 0.458308 & 10.784 & 1.65098 & 10.784 \\
\hline & 0.234906 & & & & & & & & & & \\
\hline 0.538458 & 10.788 & 54 & & 2.11 & 10.788 & 0.257073 & 10.788 & 1.96417 & 10.788 & 0.499752 & 10.788 \\
\hline & 0.23490 & & & & & & & & & & \\
\hline 1.30685 & 10.792 & 105 & 10.792 & 0.638699 & 10.792 & .257073 & 10.792 & .96417 & 10.792 & .499752 & 10.792 \\
\hline & & & & & & & & & & & \\
\hline 0.283072 & 10. & & & & 10.796 & 257073 & 10.7 & 8308 & 10.7 & 499752 & 10.796 \\
\hline & 2 & & & & & & & & & & \\
\hline 0.283072 & 10.8 & 5199 & & 4.1951 & 10.8 & 0.257073 & 10.8 & 2.43745 & 10.8 & .499752 & 10.8 \\
\hline & 2. & & & & & & & & & & \\
\hline 0.538458 & 10.804 & 1 & 10.804 & 2.11574 & 10.804 & 0.257073 & 10.804 & 0.458308 & 10.804 & 3.00547 & 10.804 \\
\hline & 0.23490 & & & & & & & & & & \\
\hline 0.283072 & 10.808 & & & 2.11574 & 10.808 & 2.59721 & 10.808 & 0.458308 & 10.808 & .00547 & 10.808 \\
\hline & & & & & & & & & & & \\
\hline 0.283072 & 10.812 & 1.05199 & 1 & 2.11574 & 10.812 & 0.257073 & 10.812 & 1.96417 & 10.81 & 0.499752 & 10.812 \\
\hline & & & & & & & & & & & \\
\hline 2.00895 & 10.816 & 2.08764 & 816 & 2.11574 & 10.816 & 0.257073 & 10.816 & 0.458308 & 10.816 & 1.65098 & 10.816 \\
\hline & 0.234906 & & & & & & & & & & \\
\hline 0.538458 & 10.82 & 5199 & & 2.11574 & 10.82 & 2.59721 & 10.82 & 0.458308 & 10.82 & 4.8466 & 10.82 \\
\hline & & & & & & & & & & & \\
\hline 0.538458 & 10.824 & 1.05199 & & 0.638699 & 10.82 & 2.59721 & 10.82 & 96417 & 10.8 & 499752 & 10 \\
\hline & & & & & & & & & & & \\
\hline 0.283072 & 10.828 & 1.39652 & 10.828 & 0.638699 & 10.828 & 2.59721 & 10.828 & 1.96417 & 10.828 & 0.499752 & 10.828 \\
\hline & 0.234906 & 10.828 & 1.11461 & 10.828 & & & & & & & \\
\hline
\end{tabular}




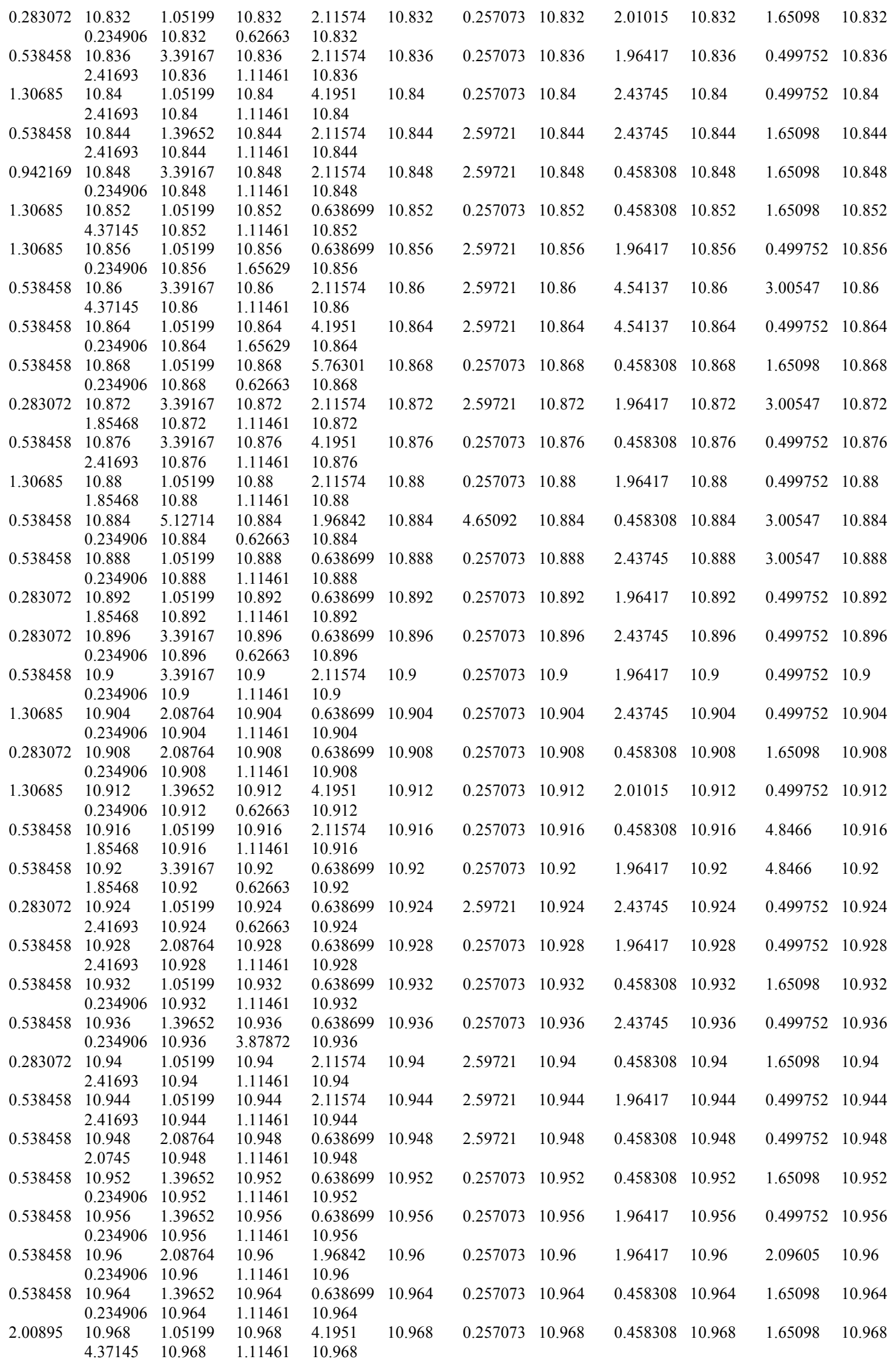




\begin{tabular}{|c|c|c|c|c|c|c|c|c|c|c|c|}
\hline 2.00895 & $\begin{array}{l}10.972 \\
4.37145\end{array}$ & $\begin{array}{l}1.39652 \\
10.972\end{array}$ & $\begin{array}{l}10.972 \\
1.65629\end{array}$ & $\begin{array}{l}0.638699 \\
10.972\end{array}$ & 10.972 & 0.257073 & 10.972 & 1.96417 & 10.972 & 0.499752 & 10.972 \\
\hline \multirow[t]{2}{*}{1.30685} & 10.976 & 3.39167 & 10.976 & 0.638699 & 10.976 & 257073 & 10.976 & 2.43745 & 10.976 & .09605 & 10.976 \\
\hline & 2.41693 & 10.976 & 1.11461 & 10.976 & & & & & & & \\
\hline \multirow[t]{2}{*}{0.283072} & 10.98 & 1.05199 & 10.98 & 4.1951 & 10.98 & .257073 & 10.98 & 2.43745 & 10.98 & 1.65098 & 10.98 \\
\hline & 1.85468 & 10.98 & 1.11461 & 10.98 & & & & & & & \\
\hline \multirow[t]{2}{*}{0.538458} & 10.984 & 1.39652 & 10.984 & 0.638699 & 10.984 & 0.257073 & 10.984 & 0.458308 & 10.984 & 3.00547 & 10.984 \\
\hline & 4.37145 & 10.984 & 2.62938 & 10.984 & & & & & & & \\
\hline \multirow[t]{2}{*}{2.65138} & 10.988 & 1.39652 & 0.988 & 0.638699 & 10.988 & 2.59721 & 10.988 & 4.54137 & 10.988 & 3.00547 & 10.988 \\
\hline & 0.234906 & 10.988 & 2.62938 & 10.988 & & & & & & & \\
\hline \multirow[t]{2}{*}{0.538458} & 10.992 & 1.39652 & 10.992 & 1.96842 & 10.992 & 2.59721 & 10.992 & 2.01015 & 10.992 & 1.65098 & 10.992 \\
\hline & 1.85468 & 10.992 & 1.11461 & 10.992 & & & & & & & \\
\hline \multirow[t]{2}{*}{0.283072} & 10.996 & 1.05 & 10.9 & & 10.996 & 2.59721 & 10.996 & 4.54137 & 10.996 & 0.499752 & 10.996 \\
\hline & 1.85468 & 1 & 61 & 10 & & & & & & & \\
\hline \multirow[t]{2}{*}{1.30685} & 11 & 1.05 & 11 & 4.1951 & 11 & 2.59721 & 11 & 1.96417 & 11 & 1.78639 & 11 \\
\hline & 0.234906 & 1 & 61 & 11 & & & & & & & \\
\hline \multirow[t]{2}{*}{0.538458} & 11.004 & 1.39652 & 11.004 & 4.1951 & 11.004 & 4.65092 & 11.004 & 0.458308 & 11.004 & 0.499752 & 11.004 \\
\hline & 0.234906 & 110 & 0.62663 & 11.0 & & & & & & & \\
\hline 0.283072 & 11.008 & 99 & & & 11.008 & 0.257073 & 11.008 & 2.43745 & 11.008 & 1.65098 & 11.008 \\
\hline & 2.41693 & 1 & & 11. & & & & & & & \\
\hline 0.283072 & 11.012 & 67 & 1 & 0.6 & 11.012 & 0.257073 & 11.012 & .54137 & 11.012 & 0.499752 & 11.012 \\
\hline & 4.37145 & 1 & & 1 . & & & & & & & \\
\hline 0.283072 & 11.016 & 99 & 6 & 0.6 & 11.016 & .257073 & 11.016 & 2.43745 & 11.016 & 1.65098 & 1.016 \\
\hline & 0.234906 & 110 & & 11. & & & & & & & \\
\hline 0.942169 & 11.02 & 2.08 & 1 & 0.638699 & 11.02 & 0.257073 & 11.02 & 2.01015 & 11.02 & 1.65098 & 11.02 \\
\hline & 1.85468 & 11. & & 11.02 & & & & & & & \\
\hline 0.538458 & 11.024 & 52 & & 0.6 & 11.024 & 0.257073 & 11.024 & 2.43745 & 11.024 & 2.09605 & 11.02 \\
\hline & 0.234906 & & & & & & & & & & \\
\hline 0.283072 & 11.028 & 64 & 11 & 0.6 & 11.028 & 0.257073 & 11.028 & 2.43745 & 11.028 & 499752 & 11.028 \\
\hline & 0.234906 & 1 & & & & & & & & & \\
\hline 0.283072 & 11.032 & 1.39 & 1 & 4.1 & 11.032 & 0.257073 & 11.032 & 0.458308 & 11.032 & 0.499752 & 11.032 \\
\hline & 0.234906 & 10 & 63 & 11 & & & & & & & \\
\hline 0.283072 & 11.036 & 9 & & & 11.036 & 0.257073 & 11.036 & 0.458308 & 11.036 & 3.00547 & 11.036 \\
\hline & 0. & & & & & & & & & & \\
\hline 0.283072 & 11.04 & 57 & 1 & 699 & 11.04 & 2.59721 & 11.04 & 1.96417 & 11.04 & 0.499752 & 11.04 \\
\hline & 0 . & & & & & & & & & & \\
\hline 0.283072 & 11.044 & 7 & 1 & 8699 & 11.044 & 0.257073 & 11.044 & 0.458308 & 11.044 & 0.499752 & 11.044 \\
\hline & 0.234906 & 0 & 72 & 11044 & & & & & & & \\
\hline 0.942169 & 11.048 & 57 & 1 & 699 & 11.048 & 0.257073 & 11.048 & 1.96417 & 11.048 & 3.00547 & 11.048 \\
\hline & & & & & & & & & & & \\
\hline 0.538458 & 11.0 & & & 99 & 11.052 & 0.257073 & 11.052 & 1.96417 & 11.052 & 1.65098 & 11.05 \\
\hline & 0.2 & & & & & & & & & & \\
\hline 0.942169 & 11.056 & 2 & 6 & 0.63 & 11.056 & 257073 & 11.056 & 17 & 11.056 & .00547 & 1.056 \\
\hline & 1.85 & & & & & & & & & & \\
\hline 0.538458 & 11.06 & 2 & 1 & 2.11574 & 11.06 & 2.59721 & 11.06 & 1.96417 & 11.06 & 0.499752 & 11.06 \\
\hline & 0.234906 & 0 & & 118 & & & & & & & \\
\hline 0.283072 & 11.064 & 9 & 1 & 74 & 11.064 & .59721 & 11.064 & 0.458308 & 11.064 & . 499752 & 11.064 \\
\hline & & & & & & & & & & & \\
\hline 0.283072 & 11.068 & 9 & 1 & 4. & 11.068 & 0.257073 & 11.068 & 4.54137 & 11.068 & 2.09605 & 11.068 \\
\hline & & & & & & & & & & & \\
\hline 0.942169 & 11.07 & & & 74 & 11.072 & 2.59721 & 11.072 & 2.43 & 11.072 & 0.499752 & 11.072 \\
\hline & 0.23 & & & & & & & & & & \\
\hline 0.942169 & 11.076 & 14 & 1 & 4 & 11.076 & 59721 & 11.076 & 2.43745 & 11.076 & 3.00547 & 11.076 \\
\hline & & & & & & & & & & & \\
\hline 0.942169 & 11.08 & 3 & & 4.1 & 11.08 & .257073 & 11.08 & 4.54137 & 11.08 & 3.00547 & 11.08 \\
\hline & & & & & & & & & & & \\
\hline 0.283072 & 11.084 & 2 & 1 & 0.638699 & 11.084 & 0.257073 & 11.084 & 2.43745 & 11.084 & 4.8466 & 11.084 \\
\hline & 0.23 & & & & & & & & & & \\
\hline 0.283072 & 11.088 & 39652 & 1 & 4.1951 & 11.088 & 1.91927 & 11.088 & 2.43745 & 11.088 & 0.499752 & 11.088 \\
\hline & 0.2 & & & & & & & & & & \\
\hline 0.538458 & 11.092 & & & 0.6 & 11.092 & 59721 & 11.09 & 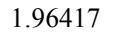 & 11.09 & .84 & 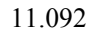 \\
\hline & & & & & & & & & & & \\
\hline 0.283072 & 11.096 & 1 & 1 & 4.1 & 11.096 & 0.257073 & 11.096 & 4.54137 & 11.096 & 3.00547 & 11.096 \\
\hline & & & & & & & & & & & \\
\hline 0.283072 & 11.1 & 1 & & 0.638699 & 11.1 & 0.257073 & 11.1 & 1.96417 & 11.1 & 0.499752 & 11.1 \\
\hline & 0.234906 & & 1 & & & & & & & & \\
\hline 0.538458 & 11.104 & 5.12714 & & 4.1951 & 11.104 & 0.257073 & 11.104 & 0.458308 & 11.104 & 1.65098 & 11.104 \\
\hline & & & & & & & & & & & \\
\hline . & 11.108 & & 11.108 & 2.11574 & 11.108 & 0.257073 & 11.108 & 0.458308 & 11.108 & 1.65098 & 11.108 \\
\hline & 2.41693 & 11 & 11461 & 1110 & & & & & & & \\
\hline
\end{tabular}




\begin{tabular}{|c|c|c|c|c|c|c|c|c|c|c|c|}
\hline 0.283072 & $\begin{array}{l}11.112 \\
2.41693\end{array}$ & $\begin{array}{l}3.39167 \\
11.112\end{array}$ & $\begin{array}{l}11.112 \\
1.11461\end{array}$ & $\begin{array}{l}0.638699 \\
11.112\end{array}$ & 11.112 & 0.257073 & 11.112 & 2.43745 & 11.112 & 0.499752 & 11.112 \\
\hline \multirow[t]{2}{*}{0.283072} & 11.116 & 1.39652 & 11.116 & 0.638699 & 11.116 & 0.257073 & 11.116 & 0.458308 & 11.116 & 0.499752 & 11.116 \\
\hline & 2.41693 & 11.116 & 1.11461 & 11.116 & & & & & & & \\
\hline \multirow[t]{2}{*}{0.538458} & 11.12 & 1.05199 & 11.12 & 0.638699 & 11.12 & 0.257073 & 11.12 & 2.43745 & 11.12 & 3.00547 & 11.12 \\
\hline & 2.41693 & 11.12 & 0.62663 & 11.12 & & & & & & & \\
\hline \multirow[t]{2}{*}{0.283072} & 11.124 & 3.39167 & 11.124 & 0.638699 & 11.124 & 0.257073 & 11.124 & 1.96417 & 11.124 & 1.65098 & 11.124 \\
\hline & 2.41693 & 11.124 & 1.65629 & 11.124 & & & & & & & \\
\hline \multirow[t]{2}{*}{0.538458} & 11.128 & 2.08764 & 11.128 & 2.11574 & 11.128 & 0.257073 & 11.128 & 2.01015 & 11.128 & 0.499752 & 11.128 \\
\hline & 4.37145 & 11.128 & 0.62 & 11.128 & & & & & & & \\
\hline \multirow[t]{2}{*}{0.538458} & 11.132 & 5.12714 & 11. & 0.638699 & 11.132 & 0.257073 & 11.132 & 1.96417 & 11.132 & 3.00547 & 11.132 \\
\hline & 0.234906 & 11.132 & 0.62663 & 11.132 & & & & & & & \\
\hline \multirow[t]{2}{*}{0.538458} & 11.136 & 1.05 & & 0.638699 & 11.136 & 0.257073 & 11.136 & 1.96417 & 11.136 & 3.00547 & 11.136 \\
\hline & 0.234906 & 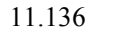 & & 11.1 & & & & & & & \\
\hline \multirow[t]{2}{*}{0.942169} & 11.14 & 5.12714 & 11.14 & 1.96842 & 11.14 & 0.257073 & 11.14 & .43745 & 11.14 & 4.8466 & 1.14 \\
\hline & 0.234906 & 14 & & 11.14 & & & & & & & \\
\hline \multirow[t]{2}{*}{0.283072} & 11.144 & 3.39167 & 11.1 & 0.638699 & 11.144 & 0.257073 & 11.144 & 0.458308 & 11.144 & 3.00547 & 11.144 \\
\hline & 1.85468 & 11.144 & & 11.144 & & & & & & & \\
\hline 0.538458 & 11.1 & & & 699 & 11.148 & 0.257073 & 11.148 & 0.458308 & 11.148 & 4.8466 & 11.148 \\
\hline & 2.41693 & & & 11. & & & & & & & \\
\hline .283072 & 11.15 & 52 & & 0.6 & 11.152 & 0.257073 & 11.152 & .54137 & 11.152 & 3.00547 & 1.152 \\
\hline & 0.23 & 1 & & & & & & & & & \\
\hline 0.538458 & 11.156 & 7 & 1 & 1.9 & 11.15 & .257073 & 11.1 & .43745 & 11.156 & 3.00547 & 11.156 \\
\hline & 0.23 & 1 & & 11. & & & & & & & \\
\hline 0.538458 & 11.16 & 52 & 1 & 2.1 & 11.16 & 0.257073 & 11.16 & 0.458308 & 11.16 & 0.499752 & 11.16 \\
\hline & 0.234906 & 11 & & 11. & & & & & & & \\
\hline 0.283072 & 11.16 & & & & 11.164 & 0.257073 & 11.164 & 0.458308 & 11.1 & 52 & 11.164 \\
\hline & 2.41 & & & & & & & & & & \\
\hline 0.538458 & 11.168 & 1.3 & & 0.6 & 11.168 & 0.257073 & 11.168 & 2.43745 & 11.168 & 0.499752 & 11.168 \\
\hline & 2.4 & & & & & & & & & & \\
\hline 1.30685 & 11.17 & 99 & & & 11.172 & 0.257073 & 11.172 & 2.43745 & 11.172 & 0.499752 & 11.172 \\
\hline & 0.23 & 1 & & 11 & & & & & & & \\
\hline 0.538458 & 11.176 & & & 699 & 11.176 & 0.257073 & 11.176 & 0.458308 & 11.176 & 1.65098 & 11.176 \\
\hline & & & & & & & & & & & \\
\hline 1.30685 & 11.1 & & 1 & 2.1 & 11.18 & 0.257073 & 11.18 & 1.96417 & 11.18 & 4.8466 & 11.18 \\
\hline & & & & & & & & & & & \\
\hline 0.538458 & 11.1 & & & & 11.184 & 0.257073 & 11.184 & 0.458308 & 11.184 & 3.00547 & 11.184 \\
\hline & 0.23 & 1 & & & & & & & & & \\
\hline 0.283072 & 11.1 & 19 & & 74 & 11.188 & 0.257073 & 11.188 & 0.458308 & 11.188 & 0.499752 & 11.188 \\
\hline & & & & & & & & & & & \\
\hline 0.538458 & 11. & & & & 11.192 & 0.257073 & 11.192 & 745 & 11.192 & .00547 & 11.192 \\
\hline & 0.23 & & & & & & & & & & \\
\hline 0.283072 & 11.196 & & & 599 & 11.196 & 073 & 11.1 & 08 & 11. & .00547 & 11.196 \\
\hline & 0.23 & & & & & & & & & & \\
\hline 0.283072 & 11.2 & 5199 & & 2.11574 & 11.2 & 0.257073 & 11.2 & 0.458308 & 11.2 & 0.499752 & 11.2 \\
\hline & 0.234906 & & & 111 & & & & & & & \\
\hline 0.283072 & 11.2 & 9652 & 1 & 99 & 11.204 & 2.59721 & 11.204 & 2.01015 & 11.204 & .00547 & 11.204 \\
\hline & & & & & & & & & & & \\
\hline 0.538458 & 11.2 & 9 & 1 & 99 & 11.208 & 0.257073 & 11.208 & 1.96417 & 11.208 & 0.499752 & 11.2 \\
\hline & & & & & & & & & & & \\
\hline 0.942169 & 11.21 & & & 74 & 11.212 & 0.257073 & 11.21 & 1.96417 & 11.212 & 0.499752 & 11.212 \\
\hline & & & & & & & & & & & \\
\hline 0.283072 & 11.216 & 57 & & 8699 & 11.216 & 2.59721 & 11.216 & 0.458308 & 11.216 & 3.00547 & 11.216 \\
\hline & & & & & & & & & & & \\
\hline 0.538458 & 11.22 & 9 & & 2. & 11.22 & 2.59721 & 11.2 & 0.458308 & 11.2 & 3.00547 & 11.22 \\
\hline & & & & & & & & & & & \\
\hline 0.283072 & 11.22 & 1 & & 2.11574 & 11.224 & 2.59721 & 11.224 & 2.01015 & 11.224 & 0.499752 & 11.2 \\
\hline & & & & & & & & & & & \\
\hline 0.942169 & 11.228 & 1.05199 & & 2.11574 & 11.228 & 0.257073 & 11.228 & 0.458308 & 11.228 & 0.499752 & 11.22 \\
\hline & & & & & & & & & & & \\
\hline 0.942169 & 11.232 & & & 2.11574 & 11.23 & 2.59721 & 11.23 & .458308 & 11.23 & 1.65098 & 11. \\
\hline & & & & & & & & & & & \\
\hline 0.538458 & 11.236 & 1 & 1 & 0.6 & 11.236 & 0.257073 & 11.236 & 2.43745 & 11.236 & 1.65098 & 11.236 \\
\hline & & & & & & & & & & & \\
\hline 0.283072 & 11.24 & 652 & 11.2 & 0.638699 & 11.24 & 0.257073 & 11.24 & 1.96417 & 11.24 & 1.78639 & 11.24 \\
\hline & 2.41693 & & & & & & & & & & \\
\hline 1.41445 & 11.24 & 9167 & & 4.1951 & 11.244 & 0.257073 & 11.244 & 1.96417 & 11.244 & 1.65098 & 11.2 \\
\hline & & & & & & & & & & & \\
\hline 年 & & 764 & & 0.638699 & 11.248 & 0.257073 & 11.248 & 0.458308 & 11.248 & 3.00547 & - \\
\hline & 1. & & & & & & & & & & \\
\hline
\end{tabular}




\begin{tabular}{|c|c|c|c|c|c|c|c|c|c|c|c|}
\hline 0.283072 & $\begin{array}{l}11.252 \\
0.234906\end{array}$ & $\begin{array}{l}1.39652 \\
11.252\end{array}$ & $\begin{array}{l}11.252 \\
0.62663\end{array}$ & $\begin{array}{l}2.11574 \\
11.252\end{array}$ & 11.252 & 0.257073 & 11.252 & 1.96417 & 11.252 & 0.499752 & 11.252 \\
\hline 0.283072 & 11.256 & 1.39652 & 11.256 & 1.96842 & 11.256 & 0.257073 & 11.256 & 4.54137 & 11.256 & 3.00547 & 11.256 \\
\hline \multirow[t]{2}{*}{0.538458} & $\begin{array}{l}1.85468 \\
11.26\end{array}$ & $\begin{array}{l}11.256 \\
1.05199\end{array}$ & $\begin{array}{l}1.11461 \\
11.26\end{array}$ & $\begin{array}{l}11.256 \\
0.638699\end{array}$ & 11.26 & 0.257073 & 11.26 & 0.458308 & 11.26 & 1.65098 & 11.26 \\
\hline & 2.0745 & 11.26 & 1.11461 & 11.26 & & & & & & & \\
\hline \multirow[t]{2}{*}{0.283072} & 11.264 & 2.08764 & 1.264 & 0.638699 & 11.264 & 0.257073 & 11.264 & 0.458308 & 11.264 & 3.00547 & 11.264 \\
\hline & 0.234906 & 11.264 & 1.11461 & 1.264 & & & & & & & \\
\hline \multirow[t]{2}{*}{0.538458} & 11.268 & 1.39652 & 11.268 & 1.96842 & 11.268 & 0.257073 & 11.268 & 0.458308 & 11.268 & 0.499752 & 11.268 \\
\hline & 4.37145 & 11.268 & 0.62663 & 11.268 & & & & & & & \\
\hline \multirow[t]{2}{*}{0.283072} & 11.272 & 2.08764 & 11.272 & 0.638699 & 11.272 & 2.59721 & 11.272 & 1.96417 & 11.272 & 1.65098 & 11.272 \\
\hline & 2.41693 & 11.272 & 61 & 11.272 & & & & & & & \\
\hline \multirow[t]{2}{*}{0.283072} & 11.276 & 3.39167 & 11.276 & 0.63 & 11.276 & 0.257073 & 11.276 & 1.96417 & 11.276 & .499752 & 11.276 \\
\hline & 2.41693 & 11.276 & 461 & 6 & & & & & & & \\
\hline \multirow[t]{2}{*}{0.283072} & 11.28 & 1.39652 & 11.28 & 11574 & 11.28 & 1.91927 & 11.28 & 4.54137 & 11.28 & 3.00547 & 11.28 \\
\hline & 2.41693 & 11.28 & 0.62663 & 11.28 & & & & & & & \\
\hline \multirow[t]{2}{*}{0.538458} & 11.284 & 1.39652 & & & 11.284 & 0.257073 & 11.284 & 0.458308 & 11.284 & 1.65098 & 11.284 \\
\hline & 0.234906 & 11284 & & 11 & & & & & & & \\
\hline 0.538458 & 11.28 & 1.05199 & & 0.6 & 11.288 & 0.257073 & 11.288 & .54137 & 11.2 & . 499752 & 11.288 \\
\hline & 4.37145 & 11.288 & & 11 & & & & & & & \\
\hline 0.942169 & 11.292 & 1.05 & 1 & 0.6 & 11.292 & 0.257073 & 11.292 & .458308 & 11.292 & 0.499752 & 11.292 \\
\hline & 2.41 & 1 & & & & & & & & & \\
\hline 0.942169 & 11.296 & 5.12714 & 6 & 2.1 & 11.296 & 0.257073 & 11.296 & 2.43745 & 11.296 & 1.65098 & 11.296 \\
\hline & 0.234906 & 11296 & & 11296 & & & & & & & \\
\hline 0.283072 & 11.3 & 7 & & 99 & 11.3 & 0.257073 & 11.3 & 2.43745 & 11.3 & 3.00547 & 11.3 \\
\hline & 2.4 & & & & & & & & & & \\
\hline 0.942169 & 11.30 & 99 & & 2.1 & 11.304 & 0.257073 & 11.304 & 0.458308 & 11.304 & 3.00547 & 1.304 \\
\hline & 0.23 & 1 & & & & & & & & & \\
\hline 0.942169 & 11.308 & 3.39167 & 1 & 3699 & 11.308 & 0.257073 & 11.308 & 4.54137 & 11.308 & 6.26752 & 11.308 \\
\hline & 1.85 & 11.308 & & 11308 & & & & & & & \\
\hline 0.283072 & 11.31 & 99 & & 699 & 11.312 & 0.257073 & 11.312 & 1.96417 & 11.312 & 1.65098 & 11.312 \\
\hline & 0.2 & & & & & & & & & & \\
\hline 0.942169 & 11.3 & 52 & & 2.1 & 11.316 & 0.257073 & 11.316 & 0.458308 & 11.316 & .09605 & 1.316 \\
\hline & 0.2 & & & & & & & & & & \\
\hline 0.538458 & 11.32 & 3.39167 & 1 & 0.638699 & 11.32 & .257073 & 11.32 & .96417 & 11.32 & .8466 & 1.32 \\
\hline & 4.3714 & 11.32 & & 112 & & & & & & & \\
\hline 0.283072 & 11.32 & 99 & 4 & & 11.324 & 0.257073 & 11.324 & 2.43745 & 11.324 & 0.499752 & 11.324 \\
\hline & 0.23 & 1 & & & & & & & & & \\
\hline 0.283072 & 11. & & & & 11.328 & 0.257073 & 11.328 & .458308 & 11.328 & .00547 & 11.328 \\
\hline & & & & & & & & & & & \\
\hline 0.538458 & 11.33 & 99 & 1 & 2.1 & 11.332 & 257073 & 11.332 & 58308 & 11.332 & 752 & 11.332 \\
\hline & 0.23 & 1 & & & & & & & & & \\
\hline 0.283072 & 11.336 & 99 & 1 & 4.1 & 11.336 & 0.257073 & 11.336 & .458308 & 11.336 & 1.65098 & 11.336 \\
\hline & 0.234 & 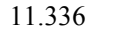 & & 11 & & & & & & & \\
\hline 1.30685 & 11.34 & 52 & & 574 & 11.34 & 0.257073 & 11.34 & 0.458308 & 11.34 & 1.65098 & 11.34 \\
\hline & & & & & & & & & & & \\
\hline 0.283072 & 11.344 & 99 & & 699 & 11.344 & 2.59721 & 11.344 & 2.43745 & 11.344 & 0.499752 & 11.3 \\
\hline & & & & & & & & & & & \\
\hline 0.538458 & 11.3 & & & 8699 & 11.348 & 0.257073 & 11.348 & 1.96417 & 11.348 & 2.09605 & 11.348 \\
\hline & 0.23 & & & & & & & & & & \\
\hline 0.538458 & 11.352 & 3.39167 & & 0.638699 & 11.352 & 0.257073 & 11.352 & 0.458308 & 11.352 & 0.499752 & 11.352 \\
\hline & & & & & & & & & & & \\
\hline 0.283072 & 11 & & & 99 & 11.356 & 0.257073 & 11.356 & 0.458308 & 11.356 & .00547 & 11.356 \\
\hline & & & & & & & & & & & \\
\hline 0.283072 & 11.36 & 1 & & 8699 & 11.36 & 0.257073 & 11.36 & 2.43745 & 11.36 & 3.00547 & 11.36 \\
\hline & 0.23490 & & & & & & & & & & \\
\hline 0.538458 & 11.364 & 5199 & & 0.638699 & 11.364 & 0.257073 & 11.364 & 2.43745 & 11.364 & 4.8466 & 11.364 \\
\hline & 0.234 & & & & & & & & & & \\
\hline 0.538458 & 11.368 & 714 & & 0.638699 & 11.368 & 257073 & 11.368 & 458308 & 11.368 & .499752 & 11 \\
\hline & & & & & & & & & & & \\
\hline 0.942169 & 11.372 & 52 & 1 & 699 & 11.372 & 0.257073 & 11.372 & 1.96417 & 11.37 & 1.65098 & 1.372 \\
\hline & & & & & & & & & & & \\
\hline 0.538458 & 11.376 & 2.08764 & 1 & 0.638699 & 11.376 & 0.257073 & 11.376 & 0.458308 & 11.376 & 1.65098 & 11.376 \\
\hline & 0.234906 & & & & & & & & & & \\
\hline 0.283072 & 11.38 & 1.05199 & & 0.638699 & 11.38 & 0.257073 & 11.38 & 1.96417 & 11.38 & .00547 & 11.38 \\
\hline & & & & & & & & & & & \\
\hline 0.538458 & 11.384 & 1.39652 & & 0.638699 & 11.38 & 0.257073 & 11.38 & 1.96417 & 11.38 & 499752 & 11.3 \\
\hline & & & & & & & & & & & \\
\hline 0.538458 & 11.388 & 3.39167 & 11.388 & 0.638699 & 11.388 & 0.257073 & 11.388 & 1.96417 & 11.388 & 0.499752 & 11.388 \\
\hline & 2.41693 & 11.388 & 1.11461 & 11.388 & & & & & & & \\
\hline
\end{tabular}




\begin{tabular}{|c|c|c|c|c|c|c|c|c|c|c|c|}
\hline 0.538458 & $\begin{array}{l}11.392 \\
0.234906\end{array}$ & $\begin{array}{l}1.05199 \\
11.392\end{array}$ & $\begin{array}{l}11.392 \\
1.11461\end{array}$ & $\begin{array}{l}0.638699 \\
11.392\end{array}$ & 11.392 & 0.257073 & 11.392 & 2.43745 & 11.392 & 0.499752 & 11.392 \\
\hline \multirow[t]{2}{*}{0.283072} & 11.396 & 1.05199 & 11.396 & 0.638699 & 11.396 & 2.59721 & 11.396 & 01015 & 11.396 & .00547 & 1.396 \\
\hline & 2.0745 & 11.396 & 1.11461 & 11.396 & & & & & & & \\
\hline \multirow[t]{2}{*}{0.283072} & 11.4 & 1.39652 & 11.4 & 2.11574 & 11.4 & 2.59721 & 11.4 & 0.458308 & 11.4 & 4.8466 & 11.4 \\
\hline & 2.41693 & 11.4 & 2.62938 & 11.4 & & & & & & & \\
\hline \multirow[t]{2}{*}{0.538458} & 11.404 & 1.05199 & 11.404 & 2.11574 & 11.404 & 0.257073 & 11.404 & 0.458308 & 11.404 & 0.499752 & 11.404 \\
\hline & 2.41693 & 11.404 & 11461 & 11.404 & & & & & & & \\
\hline \multirow[t]{2}{*}{0.283072} & 11.408 & 5.12714 & 1.408 & 4.1951 & 11.408 & 4.65092 & 11.408 & 0.458308 & 11.408 & 4.8466 & 11.408 \\
\hline & 1.85468 & 11.408 & 1.11461 & 11.408 & & & & & & & \\
\hline \multirow[t]{2}{*}{1.30685} & 11.412 & 1.05199 & 11.412 & 2.11574 & 11.412 & 0.257073 & 11.412 & 2.43745 & 11.412 & 3.00547 & 11.412 \\
\hline & 0.234906 & 11.412 & 1.11461 & 11.412 & & & & & & & \\
\hline \multirow[t]{2}{*}{0.538458} & 11.416 & 1.05 & & 0.638699 & 11.416 & 1.91927 & 11.416 & 1.96417 & 11.416 & 1.65098 & 11.416 \\
\hline & 0.234906 & 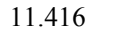 & & 11. & & & & & & & \\
\hline \multirow[t]{2}{*}{0.283072} & 11.42 & 5.12714 & 1.42 & 1.96842 & 11.42 & 0.257073 & 11.42 & .458308 & 11.42 & 0.499752 & 11.42 \\
\hline & 4.37145 & 11.42 & & 11.42 & & & & & & & \\
\hline \multirow[t]{2}{*}{0.942169} & 11.424 & 1.39652 & 11.4 & 0.638699 & 11.424 & 2.59721 & 11.424 & 0.458308 & 11.424 & 1.65098 & 11.424 \\
\hline & 2.41693 & 11.4 & & 11.424 & & & & & & & \\
\hline 0.538458 & 11.428 & & & & 11.428 & 0.257073 & 11.428 & 2.43745 & 11.428 & 0.499752 & 11.428 \\
\hline & 2.41693 & 11. & & 11. & & & & & & & \\
\hline 283072 & 11.432 & & & 2.1 & 11.432 & 0.257073 & 11.432 & .96417 & 11.432 & 4.8466 & 1.432 \\
\hline & 2.41693 & & & & & & & & & & \\
\hline 0.283072 & 11.436 & 7 & & 1.9 & 11.436 & .257073 & 11.436 & .43745 & 11.436 & 5.26752 & 11.436 \\
\hline & 2.41693 & & & 11. & & & & & & & \\
\hline 0.283072 & 11.44 & 99 & 4 & 0.638699 & 11.44 & 2.59721 & 11.44 & 0.458308 & 11.44 & 3.00547 & 11.44 \\
\hline & 0.234906 & 114 & & 11.44 & & & & & & & \\
\hline 0.538458 & 11.4 & & & & 11.444 & 0.257073 & 11.444 & .01015 & 11.4 & .00547 & 11.4 \\
\hline & 2.4 & & & & & & & & & & \\
\hline 0.538458 & 11.448 & & 1 & 0.6 & 11.448 & 0.257073 & 11.448 & 0.458308 & 11.448 & 4.8466 & 11.448 \\
\hline & 0.234906 & & & & & & & & & & \\
\hline 0.942169 & 11.452 & 1.3 & & & 11.452 & 0.257073 & 11.452 & 0.458308 & 11.452 & 3.00547 & 11.452 \\
\hline & 4.37145 & 11. & & 11 & & & & & & & \\
\hline 0.283072 & 11.456 & & & & 11.456 & 0.257073 & 11.456 & 0.458308 & 11.456 & 3.00547 & 11.456 \\
\hline & & & & & & & & & & & \\
\hline 0.942169 & 11.46 & & & 4.1 & 11.46 & .257073 & 11.46 & 43745 & 11.46 & 1.65098 & 11.46 \\
\hline & & & & & & & & & & & \\
\hline 0.538458 & 11.46 & & & 699 & 11.464 & 0.257073 & 11.464 & 0.458308 & 11.464 & 1.65098 & 11.464 \\
\hline & 2.41693 & & & & & & & & & & \\
\hline 0.942169 & 11.46 & 1 & & 4. & 11.468 & 0.257073 & 11.468 & .458308 & 11.468 & 1.65098 & 11.468 \\
\hline & & & & & & & & & & & \\
\hline 0.283072 & 11.472 & & & & 11.47 & 0.257073 & 11.472 & 0.458308 & 11.472 & 0.499752 & 11.472 \\
\hline & & & & & & & & & & & \\
\hline 0.538458 & 11.476 & & & 0.6 & 11.476 & 73 & 11.4 & 417 & 11. & .8466 & 1.476 \\
\hline & 0.23 & & & & & & & & & & \\
\hline 0.942169 & 11.48 & 1 & & 8699 & 11.48 & 0.257073 & 11.48 & 1.96417 & 11.48 & 3.00547 & 11.48 \\
\hline & 2.41693 & 11.4 & & & & & & & & & \\
\hline 0.283072 & 11.4 & 9 & & 74 & 11.484 & 1.91927 & 11.484 & 2.43745 & 11.484 & .65098 & 11.484 \\
\hline & & & & & & & & & & & \\
\hline 0.283072 & 11.4 & 9 & 1 & 2. & 11.488 & 0.257073 & 11.488 & 0.458308 & 11.488 & 4.8466 & 11.488 \\
\hline & & & & & & & & & & & \\
\hline 0.283072 & 11.49 & & & & 11.492 & 1.91927 & 11.4 & .458308 & 11.4 & 5.26752 & 11.492 \\
\hline & & & & & & & & & & & \\
\hline 0.538458 & 11.496 & & & 4.1 & 11.496 & 0.257073 & 11.496 & 2.43745 & 11.496 & 1.65098 & 11.496 \\
\hline & & & & & & & & & & & \\
\hline 0.538458 & 11.5 & 2 & & 599 & 11.5 & 2.59721 & 11.5 & .458308 & 11.5 & 3.00547 & 11.5 \\
\hline & & & & & & & & & & & \\
\hline 0.283072 & 11.50 & & & 99 & 11.504 & 0.257073 & 11.504 & 0.458308 & 11.504 & 0.499752 & 11.504 \\
\hline & & & & & & & & & & & \\
\hline 0.538458 & 11.508 & 9167 & & 0.638699 & 11.508 & 4.65092 & 11.508 & .458308 & 11.508 & 8466 & 11.508 \\
\hline & & & & & & & & & & & \\
\hline 1.30685 & 11.512 & & & 0.638699 & 11.51 & 0.257073 & 11.512 & 54137 & 11.5 & .00547 & 11.512 \\
\hline & & & & & & & & & & & \\
\hline 0.283072 & 11.516 & 99 & & 2.1 & 11.516 & 2.59721 & 11.516 & 1.96417 & 11.516 & 0.499752 & 11.516 \\
\hline & & & & & & & & & & & \\
\hline 0.538458 & 11.52 & 167 & & 3699 & 11.52 & 0.257073 & 11.52 & 2.01015 & 11.52 & 4.8466 & 11.52 \\
\hline & 1.85468 & & & 11.52 & & & & & & & \\
\hline 0.538458 & 11.524 & 1.39652 & & 0.638699 & 11.524 & 2.59721 & 11.524 & 1.96417 & 11.524 & 0.499752 & 11.524 \\
\hline & & & & & & & & & & & \\
\hline 1. & 11.52 & & 11.528 & 0.638699 & 11.528 & 0.257073 & 11.528 & 4.54137 & 11.528 & 0.499752 & 11.52 \\
\hline & 2.41 & & & & & & & & & & \\
\hline
\end{tabular}




\begin{tabular}{|c|c|c|c|c|c|c|c|c|c|c|c|}
\hline 0.283072 & $\begin{array}{l}11.532 \\
2.41693\end{array}$ & $\begin{array}{l}5.12714 \\
11.532\end{array}$ & $\begin{array}{l}11.532 \\
1.11461\end{array}$ & $\begin{array}{l}0.638699 \\
11.532\end{array}$ & 11.532 & 0.257073 & 11.532 & 0.458308 & 11.532 & 0.499752 & 11.532 \\
\hline \multirow[t]{2}{*}{0.538458} & 11.536 & 3.39167 & 11.536 & 0.638699 & 11.536 & 0.257073 & 11.536 & 0.458308 & 11.536 & 1.65098 & 11.536 \\
\hline & 0.234906 & 11.536 & 0.62663 & 11.536 & & & & & & & \\
\hline \multirow[t]{2}{*}{1.30685} & 11.54 & 1.05199 & 11.54 & 0.638699 & 11.54 & 0.257073 & 11.54 & 0.458308 & 11.54 & 1.65098 & 11.54 \\
\hline & 1.85468 & 11.54 & 1.11461 & 11.54 & & & & & & & \\
\hline \multirow[t]{2}{*}{0.538458} & 11.544 & 3.39167 & 11.544 & 0.638699 & 11.544 & 2.59721 & 11.544 & 1.96417 & 11.544 & 0.499752 & 11.544 \\
\hline & 2.41693 & 11.544 & 1.11461 & 11.544 & & & & & & & \\
\hline \multirow[t]{2}{*}{0.538458} & 11.548 & 2.08764 & 11.548 & 0.638699 & 11.548 & 2.59721 & 11.548 & 0.458308 & 11.548 & 3.00547 & 11.548 \\
\hline & 5.86409 & 11.548 & 0.62663 & 11.548 & & & & & & & \\
\hline \multirow[t]{2}{*}{0.538458} & 11.552 & 1.05199 & 11.552 & 2.11574 & 11.552 & 0.257073 & 11.552 & 0.458308 & 11.552 & 0.499752 & 11.552 \\
\hline & 2.41693 & 11.552 & 1.11461 & 11.552 & & & & & & & \\
\hline \multirow[t]{2}{*}{1.30685} & 11.556 & & & 2.11574 & 11.556 & 0.257073 & 11.556 & 4.54137 & 11.556 & 4.8466 & 1.556 \\
\hline & 2.41693 & 11.556 & 1.11461 & 11.556 & & & & & & & \\
\hline \multirow[t]{2}{*}{0.538458} & 11.56 & 1.05199 & 11.56 & 0.638699 & 11.56 & 0.257073 & 11.56 & 2.01015 & 11.56 & 0.499752 & 11.56 \\
\hline & 2.41693 & 11.56 & 1.11461 & 1156 & & & & & & & \\
\hline \multirow[t]{2}{*}{0.283072} & 11.564 & 1.39652 & 11.564 & 2.1 & 11.564 & 0.257073 & 11.564 & 0.458308 & 11.564 & 3.00547 & 11.564 \\
\hline & 2.41693 & 11.5 & & 115 & & & & & & & \\
\hline 0.538458 & 11.568 & 1.3 & 11. & & 11.568 & 0.257073 & 11.568 & 0.458308 & 11.568 & 0.499752 & 11.568 \\
\hline & 1.85468 & 11. & & 11. & & & & & & & \\
\hline 0.283072 & 11.572 & 1.39 & 11. & 0.6 & 11.572 & 2.59721 & 11.572 & .458308 & 11.572 & 1.65098 & 1.572 \\
\hline & 0.234906 & 1 & & & & & & & & & \\
\hline 0.283072 & 11.576 & 3.39167 & 11. & 0.638699 & 11.576 & 0.257073 & 11.576 & 0.458308 & 11.576 & 1.65098 & 11.576 \\
\hline & 2.41693 & 11.576 & & 11.576 & & & & & & & \\
\hline 0.538458 & 11.58 & & & & 11.58 & 2.59721 & 11.58 & 0.458308 & 11.58 & 3.00547 & 11.58 \\
\hline & 1.8 & & & 11 & & & & & & & \\
\hline 0.283072 & 11.584 & 3.3 & 11. & 699 & 11.584 & 2.59721 & 11.584 & 2.43745 & 11.584 & 3.00547 & 11.584 \\
\hline & 4.37145 & & & & & & & & & & \\
\hline 0.538458 & 11.588 & 1.05199 & 11. & 2.11574 & 11.588 & 2.59721 & 11.588 & 2.43745 & 11.588 & 3.00547 & 11.588 \\
\hline & 0.234906 & 115 & 61 & 11588 & & & & & & & \\
\hline 0.538458 & 11.592 & 1.0 & & & 11.592 & 2.59721 & 11.592 & 1.96417 & 11.592 & 0.499752 & 11.592 \\
\hline & 2.4 & & & 11 & & & & & & & \\
\hline 0.538458 & 11.5 & & & 2.1 & 11.596 & 0.257073 & 11.596 & 2.43745 & 11.596 & 0.499752 & 11.596 \\
\hline & 0.23 & & & & & & & & & & \\
\hline 0.538458 & 11.6 & 1.05199 & & 2.11574 & 11.6 & 4.65 & 11.6 & 15 & 11.6 & .00547 & 1.6 \\
\hline & 0.234 & & 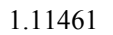 & 11.6 & & & & & & & \\
\hline 0.283072 & 11.604 & 3.39167 & 1 & 0.638699 & 11.604 & 2.59721 & 11.604 & 1.96417 & 11.604 & 3.00547 & 11.604 \\
\hline & 0.23 & 1 & & 11 & & & & & & & \\
\hline 0.283072 & 11.6 & & & 699 & 11.608 & 6.19748 & 11.608 & 1.96417 & 11.608 & 1.65098 & 11.608 \\
\hline & 2.4 & & & & & & & & & & \\
\hline 0.283072 & 11.612 & 1.39652 & 1 & 0.63 & 11.612 & 0.257073 & 11.612 & 1.96417 & 11.612 & 1.65098 & 1.612 \\
\hline & 0.23 & so & & & & & & & & & \\
\hline 0.942169 & 11.616 & 2.08764 & 1 & 2.11574 & 11.616 & 0.257073 & 11.616 & 1.96417 & 11.616 & 1.65098 & 11.616 \\
\hline & 0.234906 & 6 & & 116 & & & & & & & \\
\hline 0.283072 & 11.62 & 99 & 1 & 574 & 11.62 & 0.257073 & 11.62 & 1.96417 & 11.62 & 4.8466 & 11.62 \\
\hline & & & & & & & & & & & \\
\hline 0.283072 & 11.62 & 1.0 & 1 & 699 & 11.624 & 2.59721 & 11.624 & 4.54137 & 11.624 & 1.65098 & 11.624 \\
\hline & & & & & & & & & & & \\
\hline 0.538458 & 11.62 & 2 & 1 & 2.11574 & 11.628 & 0.257073 & 11.628 & 0.458308 & 11.628 & 1.65098 & 11.628 \\
\hline & 0.234906 & & & 110 & & & & & & & \\
\hline 0.538458 & 11.632 & 1.39652 & 11. & 0.638699 & 11.632 & 0.257073 & 11.632 & .458308 & 1.632 & .499752 & 11.632 \\
\hline & & & & & & & & & & & \\
\hline 0.283072 & 11.63 & 4 & & 2.1 & 11.636 & 0.257073 & 11.636 & 1.96417 & 11.636 & 1.65098 & 11.636 \\
\hline & & & & & & & & & & & \\
\hline 0.538458 & 11.64 & 3.3916 & & 0.638699 & 11.64 & 0.257073 & 11.64 & 0.458308 & 11.64 & 3.00547 & 11.64 \\
\hline & 0.23490 & & & & & & & & & & \\
\hline 0.538458 & 11.644 & 2.08764 & 11.644 & 0.638699 & 11.644 & 0.257073 & 11.644 & 2.43745 & 11.644 & 3.00547 & 11.644 \\
\hline & 2.416 & & & & & & & & & & \\
\hline 0.538458 & 11.648 & 3. & & 699 & 11.648 & 0.257073 & 11.648 & 458308 & 11.6 & .00547 & 48 \\
\hline & & & & & & & & & & & \\
\hline 0.538458 & 11.652 & 3.39167 & 2 & 2.11574 & 11.652 & 0.257073 & 11.65 & 1.96417 & 11.652 & 1.78639 & 1.652 \\
\hline & & & & & & & & & & & \\
\hline 0.538458 & 11.656 & 1.39652 & 11.656 & 2.11574 & 11.656 & 2.59721 & 11.656 & 2.01015 & 11.656 & 1.65098 & 11.656 \\
\hline & 2.41693 & 11.656 & 1.1146 & 11.656 & & & & & & & \\
\hline 0.283072 & 11.66 & 1.05199 & 11.66 & 0.638699 & 11.66 & 0.257073 & 11.66 & 4.54137 & 11.66 & 1.65098 & 11.66 \\
\hline & & & & & & & & & & & \\
\hline 0.283072 & 11.664 & 652 & 11.664 & 0.638699 & 11.664 & 2.59721 & 11.664 & 2.43745 & 11.664 & 2.09605 & 11.66 \\
\hline & & & & & & & & & & & \\
\hline 0.538458 & 11.668 & 3.39167 & 11.668 & 2.11574 & 11.668 & 4.65092 & 11.668 & 1.96417 & 11.668 & 6.26752 & 11.668 \\
\hline & 0.234906 & 11.668 & 1.11461 & 11.668 & & & & & & & \\
\hline
\end{tabular}




\begin{tabular}{|c|c|c|c|c|c|c|c|c|c|c|c|}
\hline 0.942169 & $\begin{array}{l}11.672 \\
2.41693\end{array}$ & $\begin{array}{l}3.39167 \\
11.672\end{array}$ & $\begin{array}{l}11.672 \\
1.65629\end{array}$ & $\begin{array}{l}2.11574 \\
11.672\end{array}$ & 11.672 & 0.257073 & 11.672 & 2.43745 & 11.672 & 0.499752 & 11.672 \\
\hline \multirow[t]{2}{*}{0.538458} & 11.676 & 3.39167 & 11.676 & 0.638699 & 11.676 & 4.65092 & 11.676 & 0.458308 & 11.676 & 3.00547 & 11.676 \\
\hline & 2.41693 & 11.676 & 1.11461 & 11.676 & & & & & & & \\
\hline \multirow[t]{2}{*}{0.942169} & 11.68 & 1.39652 & 11.68 & 2.11574 & 11.68 & 0.257073 & 11.68 & 2.43745 & 11.68 & 1.65098 & 11.68 \\
\hline & 4.37145 & 11.68 & 1.11461 & 11.68 & & & & & & & \\
\hline \multirow[t]{2}{*}{0.538458} & 11.684 & 3.39167 & 11.684 & 0.638699 & 11.684 & 0.257073 & 11.684 & 1.96417 & 11.684 & 0.499752 & 11.684 \\
\hline & 2.41693 & 11.684 & 1.11461 & 11.684 & & & & & & & \\
\hline \multirow[t]{2}{*}{0.283072} & 11.688 & 1.05199 & 1.688 & 2.11574 & 11.688 & 0.257073 & 11.688 & 2.43745 & 11.688 & 1.65098 & 11.688 \\
\hline & 5.86409 & 11.688 & 63 & 11.688 & & & & & & & \\
\hline \multirow[t]{2}{*}{0.538458} & 11.692 & 1.39652 & 11.692 & 2.11574 & 11.692 & 2.59721 & 11.692 & 0.458308 & 11.692 & 3.00547 & 11.692 \\
\hline & 0.234906 & 11.692 & 1.11461 & 11.692 & & & & & & & \\
\hline \multirow[t]{2}{*}{0.942169} & 11.696 & 3.39 & & 0.638699 & 11.696 & 0.257073 & 11.696 & 2.43745 & 11.696 & 3.00547 & 11.696 \\
\hline & 2.41693 & 1 & 53 & 11. & & & & & & & \\
\hline \multirow[t]{2}{*}{0.942169} & 11.7 & 3.39167 & 11.7 & 2.11574 & 11.7 & 0.257073 & 11.7 & 0.458308 & 11.7 & 3.00547 & 11.7 \\
\hline & 0.234906 & 11.7 & & 117 & & & & & & & \\
\hline \multirow[t]{2}{*}{0.538458} & 11.704 & 1.39652 & 11.704 & 2.11574 & 11.704 & 0.257073 & 11.704 & 2.43745 & 11.704 & 0.499752 & 11.704 \\
\hline & 1.85468 & 11.704 & 0.62663 & 11.704 & & & & & & & \\
\hline 0.942169 & 11.708 & 3.3 & & 3699 & 11.708 & 0.257073 & 11.708 & 2.43745 & 11.708 & 3.00547 & 11.708 \\
\hline & 2.41693 & 1 & & 11. & & & & & & & \\
\hline 0.538458 & 11.712 & 2 & & 0.6 & 11.712 & 2.59721 & 11.712 & 2.43745 & 11.712 & 1.65098 & 11.712 \\
\hline & 0.23 & 1 & & & & & & & & & \\
\hline 1.41445 & 11.716 & 2 & 6 & 574 & 11.716 & 1.91927 & 11.716 & .96417 & 11.716 & 1.65098 & 11.716 \\
\hline & 5.86409 & 1 & & 11. & & & & & & & \\
\hline 0.942169 & 11.72 & 1.0 & 11 & 0.638699 & 11.72 & 2.59721 & 11.72 & 1.96417 & 11.72 & 1.65098 & 11.72 \\
\hline & 5.86409 & 11. & & 11.72 & & & & & & & \\
\hline 0.942169 & 11.724 & & & 699 & 11.724 & 2.59721 & 11.724 & 1.96417 & 11.724 & .00547 & 11.724 \\
\hline & 906 & & & & & & & & & & \\
\hline 1.41445 & 11.72 & & & 699 & 11.728 & .257073 & 11.728 & 745 & 11.7 & 52 & 11.728 \\
\hline & 4.3 & 1 & & 11 & & & & & & & \\
\hline 0.942169 & 11.732 & 4 & & 574 & 11.732 & 2.59721 & 11.732 & 0.458308 & 11.732 & 0.499752 & 11.732 \\
\hline & 2.41693 & 11 & & 11 & & & & & & & \\
\hline 0.942169 & 11.736 & & & & 11.736 & 0.257073 & 11.736 & 1.96417 & 11.736 & 0.499752 & 11.736 \\
\hline & & & & & & & & & & & \\
\hline 1.7384 & 11.74 & & 1 & 3699 & 11.74 & 2.59721 & 11.74 & 0.458308 & 11.74 & . 499752 & 11.74 \\
\hline & & & & & & & & & & & \\
\hline 2.05747 & 11.74 & 2 & & 574 & 11.744 & 0.257073 & 11.744 & 1.96417 & 11.744 & 0.499752 & 11.744 \\
\hline & 2.41693 & 1 & 61 & 11744 & & & & & & & \\
\hline 1.91101 & 11.748 & 52 & 1 & 699 & 11.748 & 0.257073 & 11.748 & 2.43745 & 11.748 & 2.09605 & 11.748 \\
\hline & & & & & & & & & & & \\
\hline 0.249867 & 11.7 & & & 599 & 11.752 & 0.257073 & 11.752 & .458308 & 11.752 & .00547 & 11.752 \\
\hline & 906 & & & & & & & & & & \\
\hline-2.8711 & 11.756 & 99 & & 1.9 & 11.756 & 257073 & 11.756 & 308 & 11.756 & .00547 & 11.756 \\
\hline & 0.23 & & & & & & & & & & \\
\hline-5.98593 & 11.76 & 9167 & 1 & 1.96842 & 11.76 & 0.257073 & 11.76 & 0.458308 & 11.76 & 3.00547 & 11.76 \\
\hline & 2.4169 & 1 & & 1176 & & & & & & & \\
\hline-9.29232 & 11.76 & 9 & & 8699 & 11.764 & 0.257073 & 11.764 & 1.96417 & 11.764 & . 499752 & 11.764 \\
\hline & & & & & & & & & & & \\
\hline-12.1348 & 11.768 & 9 & & 4.19 & 11.768 & 1.91927 & 11.768 & .43745 & 11.7 & .09605 & 11.768 \\
\hline & & & & & & & & & & & \\
\hline-13.8735 & 11.77 & & & & 11.772 & 0.257073 & 11.772 & 0.458308 & 11.772 & 0.499752 & 11.772 \\
\hline & & & & & & & & & & & \\
\hline-13.6566 & 11.776 & 67 & 1 & 01 & 11.776 & 0.257073 & 11.776 & 1.96417 & 11.776 & 8466 & 11.776 \\
\hline & & & & & & & & & & & \\
\hline-12.3526 & 11.78 & 1 & & 74 & 11.78 & 4.65092 & 11.78 & .458308 & 11.78 & 3.00547 & 11.78 \\
\hline & & & & & & & & & & & \\
\hline-10.3881 & 11.784 & 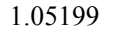 & 1 & 2.11574 & 11.784 & 0.257073 & 11.784 & 1.96417 & 11.784 & 1.65098 & 11.784 \\
\hline & 0.23 & & & & & & & & & & \\
\hline-9.95015 & 11.788 & 5.12714 & 1 & 0.638699 & 11.788 & 4.65092 & 11.788 & 0.458308 & 11.788 & 4.8466 & 11.788 \\
\hline & & & & & & & & & & & \\
\hline-9.73099 & 11.792 & & & & 11.79 & 0.257073 & 11.79 & 1.47 & $11.7 \mathrm{~s}$ & . 499752 & 11.7 \\
\hline & & & & & & & & & & & \\
\hline-10.8255 & 11.796 & 1.05199 & 1 & 1.9 & 11.796 & 0.257073 & 11.796 & 0.458308 & 11.796 & 1.65098 & 11.796 \\
\hline & 0.2 & & & & & & & & & & \\
\hline-11.2624 & 11.8 & 1.39652 & & 0.638699 & 11.8 & 0.257073 & 11.8 & 2.01015 & 11.8 & 0.499752 & 11.8 \\
\hline & 5.86409 & & & & & & & & & & \\
\hline-7.09131 & 11.804 & 1.05199 & 11.804 & 0.638699 & 11.804 & 0.257073 & 11.804 & 2.43745 & 11.804 & 1.65098 & 11.804 \\
\hline & & & & & & & & & & & \\
\hline 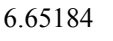 & & & & 2.11574 & 11.808 & 2.59721 & 11.808 & 0.458308 & 11.808 & 0.499752 & 11.808 \\
\hline & 4.37145 & & 31 & 1808 & & & & & & & \\
\hline
\end{tabular}




\begin{tabular}{|c|c|c|c|c|c|c|c|c|c|c|c|}
\hline 0.9149 & $\begin{array}{l}11.812 \\
0.234906\end{array}$ & $\begin{array}{l}1.39652 \\
11.812\end{array}$ & $\begin{array}{l}11.812 \\
2.62938\end{array}$ & $\begin{array}{l}2.11574 \\
11.812\end{array}$ & 11.812 & 2.59721 & 1.812 & 0.458308 & 11.812 & .499752 & 11.812 \\
\hline 54.6311 & $\begin{array}{l}11.816 \\
0.234906\end{array}$ & $\begin{array}{l}5.12714 \\
11.816\end{array}$ & $\begin{array}{l}11.816 \\
1.11461\end{array}$ & $\begin{array}{l}4.1951 \\
11.816\end{array}$ & 11.816 & 2.59721 & 11.816 & .11771 & 11.816 & 0.499752 & 11.816 \\
\hline 73.5908 & $\begin{array}{l}11.82 \\
2.41693\end{array}$ & $\begin{array}{l}3.39167 \\
11.82\end{array}$ & $\begin{array}{l}11.82 \\
1.86553\end{array}$ & $\begin{array}{l}8.09208 \\
11.82\end{array}$ & 11.82 & 4.65092 & 11.82 & 2.43745 & 11.82 & 2.09605 & 11.82 \\
\hline 85.2072 & $\begin{array}{l}11.824 \\
4.37145\end{array}$ & $\begin{array}{l}6.48356 \\
11.824\end{array}$ & $\begin{array}{l}11.824 \\
2.07023\end{array}$ & $\begin{array}{l}5.76301 \\
11.824\end{array}$ & 11.824 & 0.257073 & 11.824 & 0.458308 & 11.824 & 3.00547 & 11.824 \\
\hline 90.9483 & $\begin{array}{l}11.828 \\
0.234906\end{array}$ & $\begin{array}{l}9.53289 \\
11.828\end{array}$ & $\begin{array}{l}11.828 \\
0.62663\end{array}$ & $\begin{array}{l}2.11574 \\
11.828\end{array}$ & 11.828 & 0.257073 & 11.828 & 1.96417 & 11.828 & 3.00547 & 11.828 \\
\hline 93.0457 & & & & & 11.83 & 4.65092 & 11.832 & 0.458308 & 11.832 & .00547 & 1.832 \\
\hline 93.3083 & $\begin{array}{l}11.836 \\
2.41693\end{array}$ & $\begin{array}{l}7.61275 \\
11.836\end{array}$ & & $\begin{array}{l}0.638699 \\
11.836\end{array}$ & 11.836 & .257073 & 11.836 & .458308 & 11.836 & 1.65098 & 11.836 \\
\hline 92.7833 & $\begin{array}{l}11.84 \\
4.37145\end{array}$ & $\begin{array}{l}5.12714 \\
11.84\end{array}$ & $\begin{array}{l}11.84 \\
5.82038\end{array}$ & $\begin{array}{l}1.96842 \\
11.84\end{array}$ & 11.84 & 2.59721 & 11.84 & 4.54137 & 11.84 & 0.499752 & 11.84 \\
\hline 92.5209 & & & & & 11.844 & 2.59721 & 11.844 & 4.54137 & 11.844 & .09605 & 11.844 \\
\hline 91.9964 & & & & $\begin{array}{l}699 \\
8\end{array}$ & 11.8 & .257073 & 11.848 & 45 & 11.848 & 05 & 1.848 \\
\hline 92.7833 & $\begin{array}{l}11.852 \\
0.234906\end{array}$ & $\begin{array}{l}0.591617 \\
11.852\end{array}$ & & & 11.85 & .257073 & 11.852 & .96417 & 11.852 & .09605 & 1.852 \\
\hline 93.8336 & & & & & 11.856 & 1.91927 & 11.856 & 0.458308 & 11.856 & 0.499752 & 11.856 \\
\hline 94.6223 & & & & & 11.86 & 2.03425 & 11.86 & 1.96417 & 11.86 & .499752 & 11.86 \\
\hline 95.1484 & & & & $\begin{array}{l}699 \\
4\end{array}$ & 11.8 & 27 & 4 & 08 & 4 & 6 & 64 \\
\hline 94.8853 & & & & & 11.868 & 0.257073 & 11.868 & 2.01015 & 11.868 & 3.00547 & 11.868 \\
\hline 93.8336 & & & & & 11.872 & 2.59721 & 11.872 & 1.96417 & 11.872 & 3.00547 & 11.872 \\
\hline 92.2586 & $\begin{array}{l}11.876 \\
0.234906\end{array}$ & & & 699 & 11.87 & 7.44 & 1.876 & 17 & 11.876 & 547 & 11.876 \\
\hline 91.734 & $\begin{array}{l}11 \\
2 .\end{array}$ & & & & 1188 & 610740 & 1100 & .96417 & 11.88 & .00547 & 11.88 \\
\hline 90.9483 & $\begin{array}{l}11 . \\
0.2\end{array}$ & & & & 11.884 & 6.19748 & 11.884 & 0.458308 & 11.884 & 3.00547 & 11.884 \\
\hline 89.9015 & & & & & 11.888 & 2.59721 & 11.888 & 0.458308 & 11.888 & .499752 & 11.888 \\
\hline 89.1172 & & & & & 1 & 73 & 2 & 5 & 2 & 66 & 92 \\
\hline 87.811 & & & & & 11.896 & 1.91927 & 11.896 & .43745 & 11.896 & 1.65098 & 11.896 \\
\hline 87.8118 & 11.9 & $\begin{array}{l}2 \\
1\end{array}$ & & 574 & 11.9 & .91927 & 119 & 01015 & 1 & 65098 & 11.9 \\
\hline 87.8118 & & & & & 11.904 & 0.257073 & .904 & 458308 & 11.904 & .499752 & 11.904 \\
\hline 88.85 & $\begin{array}{l}11.90 \\
2.416\end{array}$ & $\begin{array}{l}2 \\
1\end{array}$ & $\begin{array}{l}8 \\
63\end{array}$ & $\begin{array}{l}8699 \\
08\end{array}$ & 11.908 & 1.91927 & 11.908 & 1.96417 & 11.908 & 3.00547 & 11.908 \\
\hline 88.856 & & & & & 11.91 & 0.257073 & 11.912 & 0.458308 & 11.912 & 3.00547 & 11.912 \\
\hline 88.3338 & & & & & 11.916 & 557073 & .916 & 308 & 11.916 & 00547 & 1.916 \\
\hline 87.551 & $\begin{array}{l}11.92 \\
1.854\end{array}$ & $\begin{array}{l}8 \\
1\end{array}$ & & $\begin{array}{l}4 . \\
11\end{array}$ & 11.92 & 257073 & 11.9 & 0.458308 & 11.92 & 499752 & 11.92 \\
\hline 87.029 & $\begin{array}{l}11.924 \\
2.41693\end{array}$ & & & & 1100 & 73 & 1.924 & 7 & 10 & .00547 & 11.924 \\
\hline 87.811 & $\begin{array}{l}11.928 \\
0.234906\end{array}$ & & & & 11.928 & 73 & 8 & 165 & 11.928 & 52 & 28 \\
\hline 88.3338 & & & & & 2 & 257073 & .932 & 43745 & 11.932 & .00547 & 11.932 \\
\hline 89.117 & $\begin{array}{l}11.936 \\
0.234906\end{array}$ & $\begin{array}{l}6.48356 \\
11.936\end{array}$ & & $\begin{array}{l}4.1951 \\
11.936\end{array}$ & 11.936 & 4.65092 & 11.936 & 0.458308 & 11.936 & 1.65098 & 11.936 \\
\hline 90.68 & $\begin{array}{l}11.94 \\
0.234906\end{array}$ & $\begin{array}{l}5.12714 \\
11.94\end{array}$ & 11.94 & $\begin{array}{l}0.638699 \\
11.94\end{array}$ & 11. & 4.65092 & 1 & 308 & 11.94 & 99752 & 11.94 \\
\hline 91.4722 & & & & & & 2.59721 & 11.944 & 6.11771 & 11.944 & 52 & 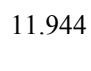 \\
\hline 5209 & $\begin{array}{l}11.948 \\
0.234906\end{array}$ & $\begin{array}{l}3.39167 \\
11.948\end{array}$ & $\begin{array}{l}11.948 \\
1.11461\end{array}$ & $\begin{array}{l}2.11574 \\
11.948\end{array}$ & 11.948 & 2.59721 & 11.948 & 1.96417 & 11.948 & 1.65098 & 11.948 \\
\hline
\end{tabular}




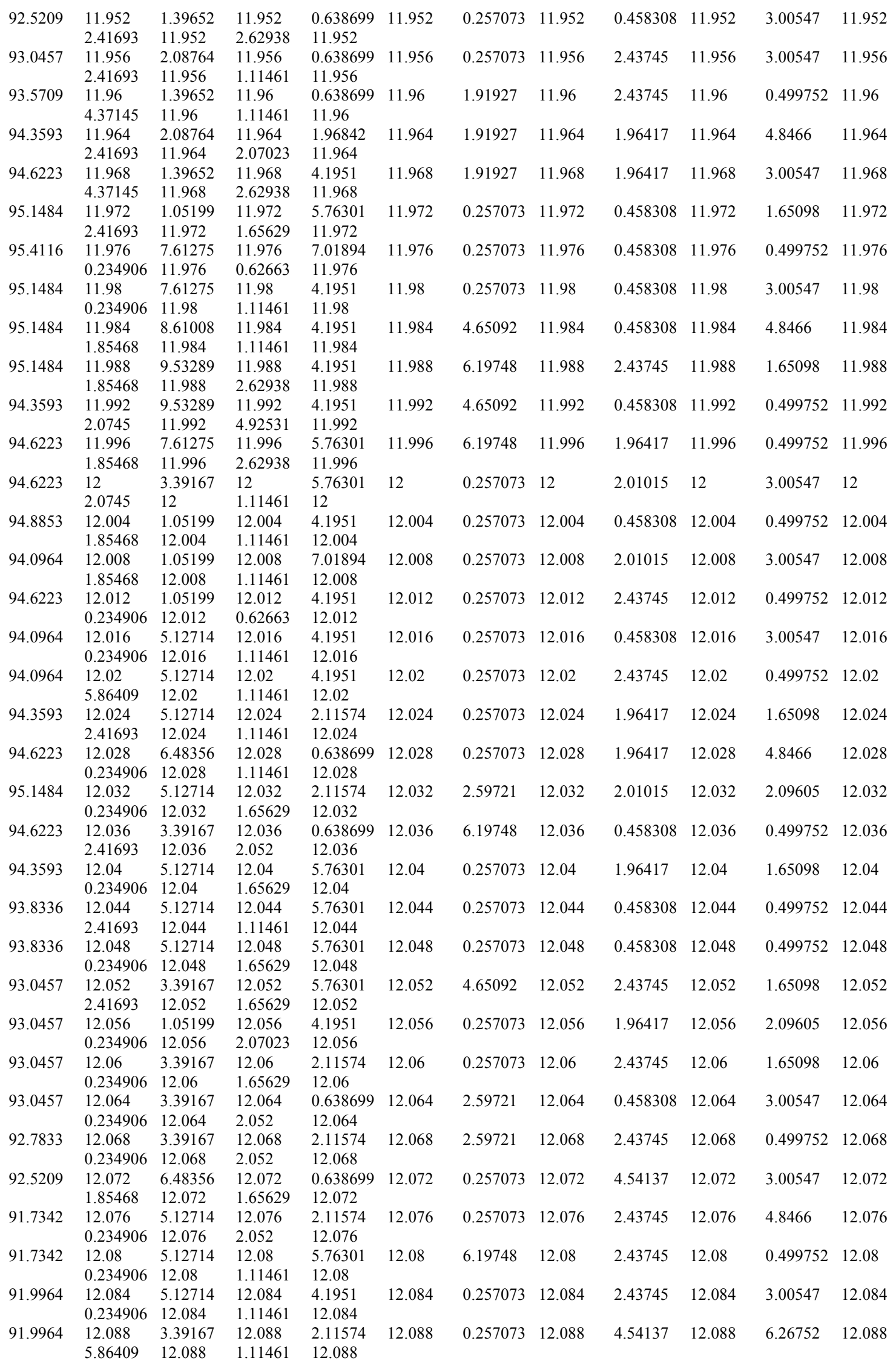




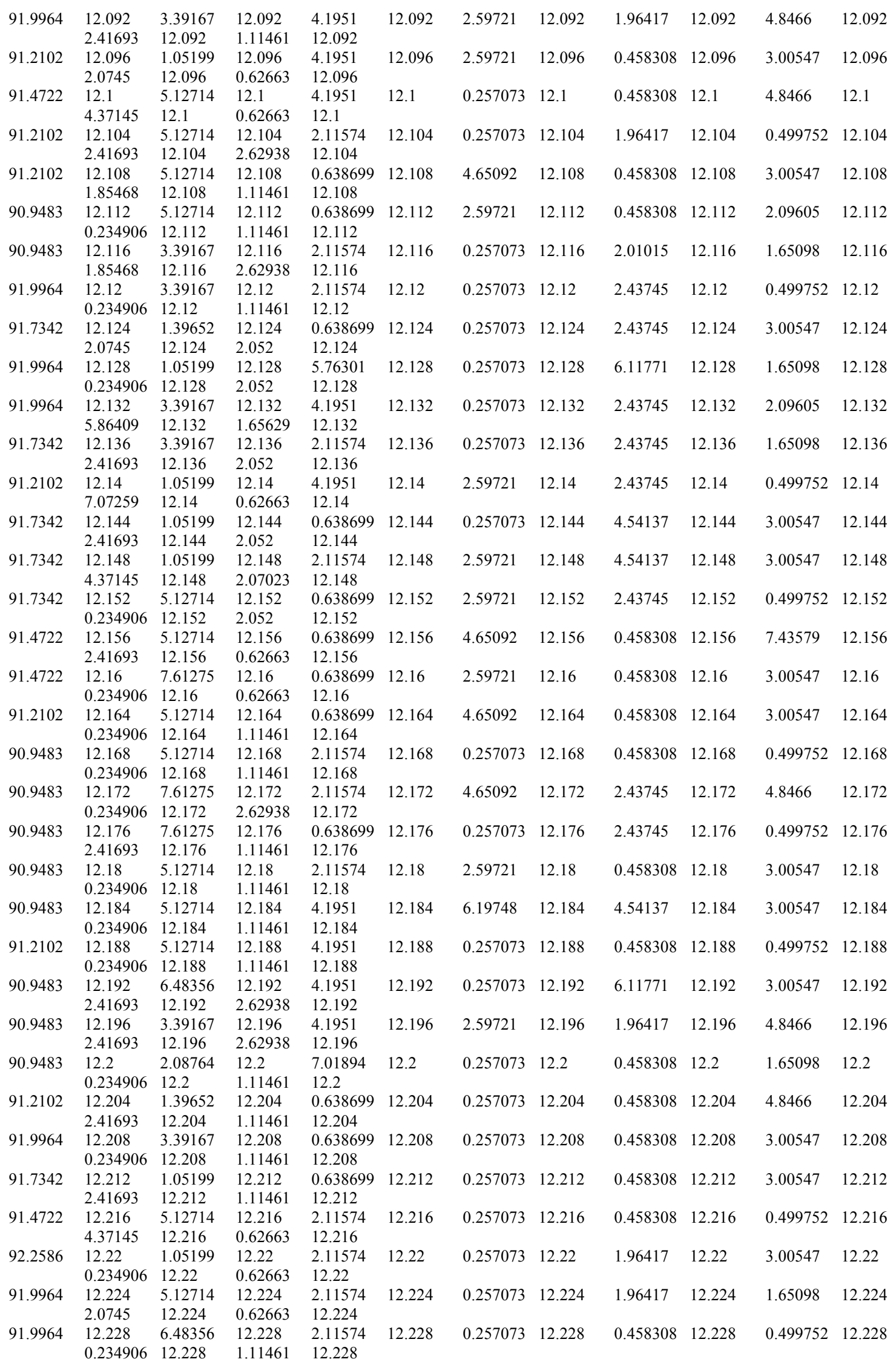




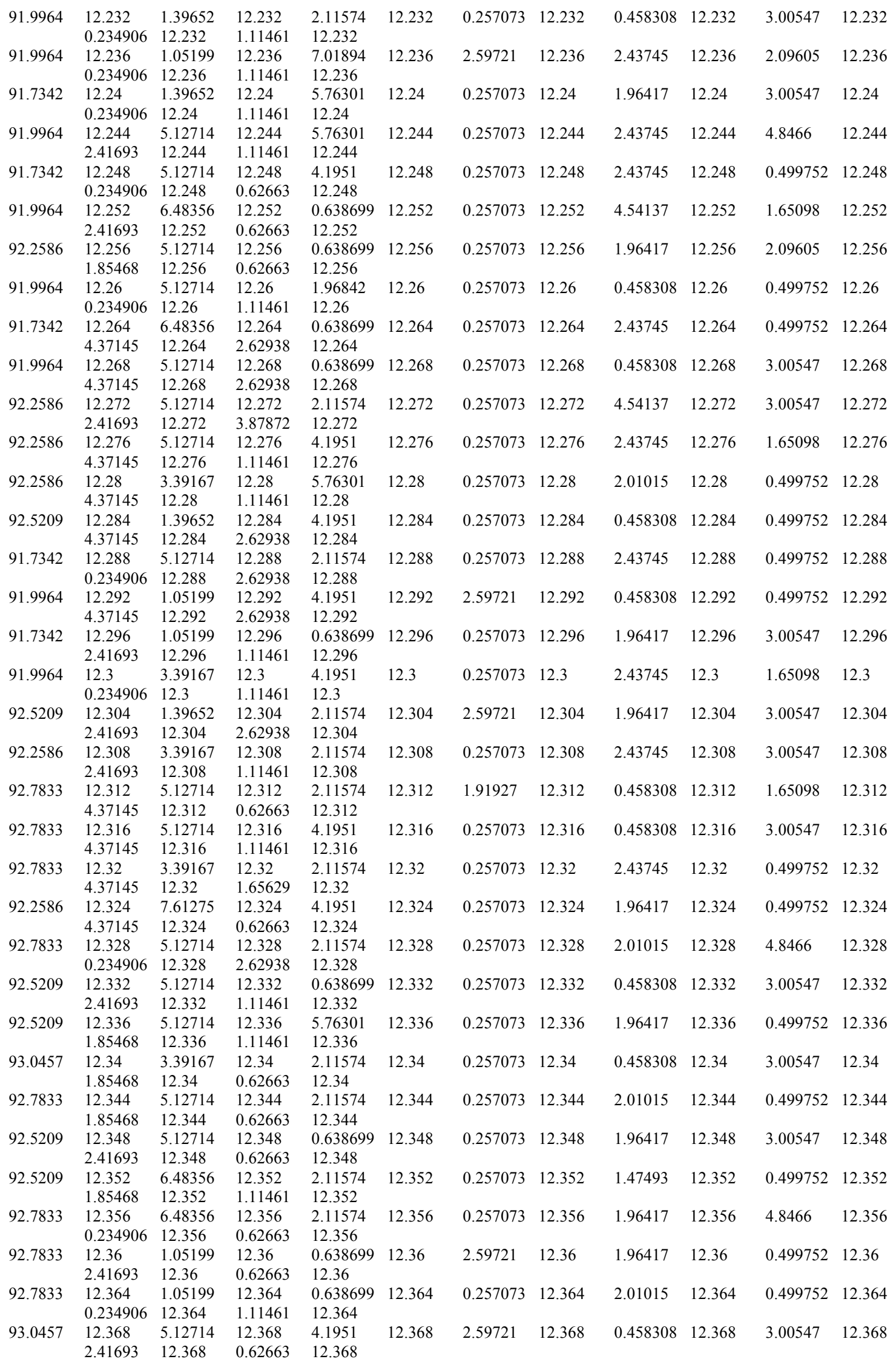




\begin{tabular}{|c|c|c|c|c|c|c|c|c|c|c|c|}
\hline 93.0457 & $\begin{array}{l}12.372 \\
1.85468\end{array}$ & $\begin{array}{l}3.39167 \\
12.372\end{array}$ & $\begin{array}{l}12.372 \\
0.62663\end{array}$ & $\begin{array}{l}0.638699 \\
12.372\end{array}$ & 12.372 & 0.257073 & 12.372 & 0.458308 & 12.372 & 3.00547 & 12.372 \\
\hline \multirow[t]{2}{*}{93.0457} & 12.376 & 5.12714 & 12.376 & 2.11574 & 12.376 & 0.257073 & 12.376 & 0.458308 & 12.376 & 0.499752 & 12.376 \\
\hline & 0.234906 & 12.376 & 1.11461 & 12.376 & & & & & & & \\
\hline \multirow[t]{2}{*}{92.7833} & 12.38 & 5.12714 & 12.38 & 4.1951 & 12.38 & 0.257073 & 12.38 & 4.54137 & 12.38 & 0.499752 & 12.38 \\
\hline & 0.234906 & 12.38 & 1.65629 & 12.38 & & & & & & & \\
\hline \multirow[t]{2}{*}{93.0457} & 12.384 & 6.48356 & 12.384 & 4.1951 & 12.384 & 0.257073 & 12.384 & 2.43745 & 12.384 & 1.65098 & 12.384 \\
\hline & 2.41693 & 12.384 & 1.65629 & 12.384 & & & & & & & \\
\hline \multirow[t]{2}{*}{93.0457} & 12.388 & 5.12714 & 12.388 & 4.1951 & 12.388 & 0.257073 & 12.388 & 0.458308 & 12.388 & 1.65098 & 12.388 \\
\hline & 0.234906 & 12.388 & 1.65629 & 12.388 & & & & & & & \\
\hline \multirow[t]{2}{*}{92.7833} & 12.392 & 6.48356 & 12.392 & 4.1951 & 12.392 & 0.257073 & 12.392 & 0.458308 & 12.392 & 3.00547 & 12.392 \\
\hline & 4.37145 & 12.392 & 1.11461 & 12.392 & & & & & & & \\
\hline \multirow[t]{2}{*}{93.3083} & 12.396 & 6.48 & 12.396 & & 12.396 & 0.257073 & 12.396 & 0.458308 & 12.396 & 3.00547 & 12.396 \\
\hline & 2.41693 & 12.3 & 61 & 12. & & & & & & & \\
\hline \multirow[t]{2}{*}{93.0457} & 12.4 & 5.12714 & 12.4 & 2.11574 & 12.4 & 0.257073 & 12.4 & 2.43745 & 12.4 & 3.00547 & 12.4 \\
\hline & 5.86409 & 12.4 & 1.65629 & 124 & & & & & & & \\
\hline \multirow[t]{2}{*}{93.3083} & 12.404 & 5.12714 & 12.404 & 0.638699 & 12.404 & 0.257073 & 12.404 & 0.458308 & 12.404 & 1.78639 & 12.404 \\
\hline & 0.234906 & 124 & 0.62663 & 12.404 & & & & & & & \\
\hline 93.5709 & 12.408 & 57 & 12.4 & & 12.408 & 0.257073 & 12.408 & 4.54137 & 12.408 & 4.8466 & 12.408 \\
\hline & 4.3 & 12. & 2 & 12 & & & & & & & \\
\hline 93.3083 & 12.412 & 6 & 12.412 & 2.1 & 12.412 & 4.65092 & 12.412 & 4.54137 & 12.412 & 1.65098 & 12.412 \\
\hline & 4.37145 & 1 & 2.052 & & & & & & & & \\
\hline 93.0457 & 12.416 & 7 & 12.416 & 2.11 & 12.416 & .257073 & 12.416 & .458308 & 12.416 & 2.09605 & 12.416 \\
\hline & 2.41 & 12. & 0.62 & 12. & & & & & & & \\
\hline 93.3083 & 12.42 & 5.12714 & 12.42 & 4.1951 & 12.42 & 0.257073 & 12.42 & 0.458308 & 12.42 & 3.00547 & 12.42 \\
\hline & 2.41693 & 12.4 & 2.052 & 12.42 & & & & & & & \\
\hline 93.0457 & 12. & & 4 & 4.1 & 12.424 & 0.257073 & 12.424 & 1.96417 & 12.424 & 3.00547 & 12.4 \\
\hline & & & & & & & & & & & \\
\hline 93.3083 & 12.4 & & 1 & 4.1 & 12.428 & 0.257073 & 12.428 & 1.96417 & 12.428 & .00547 & 12.4 \\
\hline & 2. & & 63 & & & & & & & & \\
\hline 93.5709 & 12.432 & 2.08 & 12.432 & 2.1 & 12.432 & 0.257073 & 12.432 & 1.96417 & 12.432 & 4.8466 & 12.432 \\
\hline & 1.8 & 12. & 2.052 & 12. & & & & & & & \\
\hline 93.0457 & 12.436 & 4 & 12. & & 12.436 & 0.257073 & 12.436 & 0.458308 & 12.436 & 0.499752 & 12.436 \\
\hline & & & & & & & & & & & \\
\hline 93.0457 & 12.44 & 4 & 4 & 699 & 12.44 & 0.257073 & 12.44 & 1.96417 & 12.44 & 3.00547 & 12.44 \\
\hline & & & & & & & & & & & \\
\hline 93.0457 & 12.4 & 4 & 1 & 0.638699 & 12.444 & 2.59721 & 12.444 & 1.96417 & 12.444 & 1.65098 & 12.444 \\
\hline & 1.85 & 12.4 & 63 & 12444 & & & & & & & \\
\hline 93.0457 & 12.4 & 3 & 1 & 74 & 12.448 & 2.59721 & 12.448 & 2.43745 & 12.448 & 0.499752 & 12.448 \\
\hline & & & & & & & & & & & \\
\hline 93.0457 & 12. & & & & 12.452 & 4.65092 & 12.452 & 2.43745 & 12.452 & 3.00547 & 12.452 \\
\hline & & & & & & & & & & & \\
\hline 93.3083 & 12.45 & & 56 & 0.6 & 12.456 & 073 & 12.456 & 308 & 12.456 & 47 & 12.456 \\
\hline & & & & & & & & & & & \\
\hline 93.0457 & 12.46 & 1.05199 & 1 & 2.11574 & 12.46 & 0.257073 & 12.46 & 1.96417 & 12.46 & 0.499752 & 12.46 \\
\hline & 0.234906 & 12.46 & & 1246 & & & & & & & \\
\hline 93.3083 & 12.4 & 2 & 1 & 0.638699 & 12.464 & 2.59721 & 12.464 & 0.458308 & 12.464 & 1.65098 & 12.464 \\
\hline & & & & & & & & & & & \\
\hline 92.7833 & 12. & 9 & 1 & 2. & 12.468 & 0.257073 & 12.468 & 2.43745 & 12.468 & 4.8466 & 12.4 \\
\hline & & & & & & & & & & & \\
\hline 93.3083 & 12.47 & & 12.472 & 2.1 & 12.472 & 0.257073 & 12.47 & 0.458308 & 12.472 & 0.499752 & 12.472 \\
\hline & & & & & & & & & & & \\
\hline 93.0457 & 12.476 & 64 & 1 & 4. & 12.476 & 0.257073 & 12.476 & 458308 & 12.476 & . 499752 & 12.476 \\
\hline & & & & & & & & & & & \\
\hline 92.7833 & 12. & & 1 & 4.1 & 12.48 & 0.257073 & 12.48 & .458308 & 12.48 & 1.65098 & 12.48 \\
\hline & & & & & & & & & & & \\
\hline 93.0457 & 12.4 & 1 & 1 & 0.638699 & 12.484 & 0.257073 & 12.484 & 0.458308 & 12.484 & 1.65098 & 12.4 \\
\hline & & & & & & & & & & & \\
\hline 93.0457 & 12.488 & 1.05199 & 12.488 & 2.11574 & 12.488 & 0.257073 & 12.488 & 1.96417 & 12.488 & 4.8466 & 12.48 \\
\hline & & & & & & & & & & & \\
\hline 93.0457 & 12.49 & & & & 12.49 & 0.257073 & 12.49 & .96417 & 12.4 & 3.00547 & 12.4 \\
\hline & & & & & & & & & & & \\
\hline 93.0457 & 12.496 & 4 & 1 & 4.1 & 12.496 & 0.257073 & 12.496 & 2.43745 & 12.496 & 0.499752 & 12.496 \\
\hline & & & & & & & & & & & \\
\hline 93.0457 & 12.5 & 1.0 & & 2.11574 & 12.5 & 0.257073 & 12.5 & 2.43745 & 12.5 & 1.65098 & 12.5 \\
\hline & & & & 12. & & & & & & & \\
\hline 92.7833 & 12.504 & 1.05199 & 12.504 & 4.1951 & 12.504 & 0.257073 & 12.504 & 0.458308 & 12.504 & 1.65098 & 12.504 \\
\hline & & & & & & & & & & & \\
\hline . & & 1.05199 & 12.508 & 0.638699 & 12.508 & 0.257073 & 12.508 & 2.43745 & 12.508 & 1.65098 & Fon \\
\hline & 1.83408 & & 33 & & & & & & & & \\
\hline
\end{tabular}




\begin{tabular}{|c|c|c|c|c|c|c|c|c|c|c|c|}
\hline 92.7833 & $\begin{array}{l}12.512 \\
0.234906\end{array}$ & $\begin{array}{l}1.05199 \\
12.512\end{array}$ & $\begin{array}{l}12.512 \\
1.11461\end{array}$ & $\begin{array}{l}0.638699 \\
12.512\end{array}$ & 12.512 & 0.257073 & 12.512 & 2.43745 & 12.512 & 2.09605 & 12.512 \\
\hline \multirow[t]{2}{*}{93.0457} & 12.516 & 1.05199 & 12.516 & 2.11574 & 12.516 & 2.59721 & 12.516 & 2.43745 & 12.516 & 1.65098 & 12.516 \\
\hline & 0.234906 & 12.516 & 0.62663 & 12.516 & & & & & & & \\
\hline \multirow[t]{2}{*}{93.3083} & 12.52 & 2.08764 & 12.52 & 2.11574 & 12.52 & 2.59721 & 12.52 & 0.458308 & 12.52 & 1.65098 & 12.52 \\
\hline & 2.41693 & 12.52 & 0.62663 & 12.52 & & & & & & & \\
\hline \multirow[t]{2}{*}{93.0457} & 12.524 & 2.08764 & 12.524 & 0.638699 & 12.524 & 2.59721 & 12.524 & 2.43745 & 12.524 & 6.26752 & 12.524 \\
\hline & 1.85468 & 12.524 & 1.11461 & 12.524 & & & & & & & \\
\hline \multirow[t]{2}{*}{93.0457} & 12.528 & 2.08764 & 12.528 & 2.11574 & 12.528 & 0.257073 & 12.528 & 1.96417 & 12.528 & 0.499752 & 12.528 \\
\hline & 2.41693 & 12.528 & 1.11461 & 12.528 & & & & & & & \\
\hline \multirow[t]{2}{*}{92.7833} & 12.532 & 2.08764 & 12.532 & 2.11574 & 12.532 & 2.59721 & 12.532 & 2.43745 & 12.532 & 3.00547 & 12.532 \\
\hline & 2.41693 & 12.532 & 0.62663 & 12.532 & & & & & & & \\
\hline \multirow[t]{2}{*}{93.0457} & 12.536 & 1.05199 & 12.536 & 0.638699 & 12.536 & 2.59721 & 12.536 & 0.458308 & 12.536 & 3.00547 & 12.536 \\
\hline & 2.41693 & 12.536 & 1.11461 & 12.536 & & & & & & & \\
\hline \multirow[t]{2}{*}{93.0457} & 12.54 & 1.05199 & 12.54 & 2.11574 & 12.54 & 0.257073 & 12.54 & 2.43745 & 12.54 & 3.00547 & 12.54 \\
\hline & 4.37145 & 12.54 & 0.62663 & 12.54 & & & & & & & \\
\hline \multirow[t]{2}{*}{93.0457} & 12.544 & 1.39652 & 12.544 & 0.638699 & 12.544 & 0.257073 & 12.544 & 0.458308 & 12.544 & 3.00547 & 12.544 \\
\hline & 4.37145 & 12.544 & 1.65629 & 12.544 & & & & & & & \\
\hline 93.0457 & 12.548 & 1.39652 & 12.548 & 2.11574 & 12.548 & 2.59721 & 12.548 & 4.54137 & 12.548 & 4.8466 & 12.548 \\
\hline & 0.234906 & 12.548 & 0.62663 & 12.548 & & & & & & & \\
\hline 93.0457 & 12.552 & 1.05199 & 12.552 & 0.638699 & 12.552 & 2.59721 & 12.552 & 2.43745 & 12.552 & 3.00547 & 12.552 \\
\hline & 2.41693 & 12.552 & 0.62663 & 12.552 & & & & & & & \\
\hline 92.7833 & 12.556 & 1.39652 & 12.556 & 4.1951 & 12.556 & 0.257073 & 12.556 & 2.01015 & 12.556 & 1.65098 & 12.556 \\
\hline & 4.37145 & 12.556 & 1.11461 & 12.556 & & & & & & & \\
\hline 93.0457 & 12.56 & 1.39652 & 12.56 & 2.11574 & 12.56 & 0.257073 & 12.56 & 2.43745 & 12.56 & 4.8466 & 12.56 \\
\hline & 2.41693 & 12.56 & 1.11461 & 12.56 & & & & & & & \\
\hline 93.0457 & 12.564 & 1.05199 & 12.564 & 2.11574 & 12.564 & 0.257073 & 12.564 & 1.96417 & 12.564 & 1.65098 & 12.564 \\
\hline & 0.234906 & 12.564 & 1.11461 & 12.564 & & & & & & & \\
\hline 93.0457 & 12.568 & 3.39167 & 12.568 & 4.1951 & 12.568 & 0.257073 & 12.568 & 2.43745 & 12.568 & 3.00547 & 12.568 \\
\hline & 2.41693 & 12.568 & 1.11461 & 12.568 & & & & & & & \\
\hline 92.5209 & 12.572 & 1.39652 & 12.572 & 5.76301 & 12.572 & 0.257073 & 12.572 & 2.43745 & 12.572 & 3.00547 & 12.572 \\
\hline & 0.234906 & 12.572 & 2.62938 & 12.572 & & & & & & & \\
\hline 92.7833 & 12.576 & 3.39167 & 12.576 & 5.76301 & 12.576 & 0.257073 & 12.576 & 1.96417 & 12.576 & 0.499752 & 12.576 \\
\hline & 0.234906 & 12.576 & 1.11461 & 12.576 & & & & & & & \\
\hline 92.7833 & 12.58 & 1.05199 & 12.58 & 4.1951 & 12.58 & 2.59721 & 12.58 & 1.96417 & 12.58 & 0.499752 & 12.58 \\
\hline & 0.234906 & 12.58 & 0.6 & 12.58 & & & & & & & \\
\hline 93.0457 & 12.584 & 5.12714 & 12.584 & 5.76301 & 12.584 & 0.257073 & 12.584 & 1.96417 & 12.584 & 0.499752 & 12.584 \\
\hline & 2.41693 & 12.584 & 1.11461 & 12.584 & & & & & & & \\
\hline 93.0457 & 12.588 & 1.05199 & 12.588 & 4.1951 & 12.588 & 2.59721 & 12.588 & 0.458308 & 12.588 & 3.00547 & 12.588 \\
\hline & 2.41693 & 12.588 & 1.11461 & 12.588 & & & & & & & \\
\hline 93.0457 & 12.592 & 5.12714 & 12.592 & 4.1951 & 12.592 & 0.257073 & 12.592 & 1.96417 & 12.592 & 3.00547 & 12.592 \\
\hline & 0.234906 & 12.5 & 1.1 & 12. & & & & & & & \\
\hline 93.0457 & 12.596 & 5.12714 & 12.596 & 0.638699 & 12.596 & 0.257073 & 12.596 & 2.43745 & 12.596 & 1.65098 & 12.596 \\
\hline & 0.234906 & 12.596 & 1.11461 & 12.596 & & & & & & & \\
\hline 93.0457 & 12.6 & 6.48356 & 12.6 & 2.11574 & 12.6 & 2.59721 & 12.6 & 2.43745 & 12.6 & 4.8466 & 12.6 \\
\hline & 0.234906 & 12.6 & 3.87872 & 12.6 & & & & & & & \\
\hline 93.0457 & 12.604 & 5.12714 & 12.604 & 4.1951 & 12.604 & 0.257073 & 12.604 & 1.96417 & 12.604 & 0.499752 & 12.604 \\
\hline & 1.85468 & 12.604 & 3.87872 & 12.604 & & & & & & & \\
\hline 93.0457 & 12.608 & 8.61008 & 12.608 & 2.11574 & 12.608 & 0.257073 & 12.608 & 1.96417 & 12.608 & 0.499752 & 12.608 \\
\hline & 0.234906 & 12.608 & 372 & 12.60 & & & & & & & \\
\hline 93.0457 & 12.612 & 8.61008 & 12.612 & 0.638699 & 12.612 & 0.257073 & 12.612 & 2.01015 & 12.612 & 6.26752 & 12.612 \\
\hline & 0.234906 & 12.612 & 1.11461 & 12. & & & & & & & \\
\hline 93.3083 & 12.616 & 7.61275 & 12.616 & 0.638699 & 12.616 & 2.59721 & 12.616 & 0.458308 & 12.616 & 0.499752 & 12.616 \\
\hline & 0.234906 & 12.616 & 1.11461 & 12.616 & & & & & & & \\
\hline 93.0457 & 12.62 & 8.61008 & 12.62 & 0.638699 & 12.62 & 0.257073 & 12.62 & 1.96417 & 12.62 & 1.65098 & 12.62 \\
\hline & 0.234906 & 12.6 & & 12.62 & & & & & & & \\
\hline 93.3083 & 12.624 & 9.53289 & 12.624 & 0.638699 & 12.624 & 0.257073 & 12.624 & 0.458308 & 12.624 & 0.499752 & 12.624 \\
\hline & 0.234906 & 12.624 & 1.11461 & 12.6 & & & & & & & \\
\hline 93.3083 & 12.628 & 7.61275 & 12.628 & 2.11574 & 12.628 & 0.257073 & 12.628 & 2.43745 & 12.628 & 3.00547 & 12.628 \\
\hline & 2.41693 & 12.628 & 1.11461 & 12.628 & & & & & & & \\
\hline 93.8336 & 12.632 & 9.53289 & 12.632 & 2.11574 & 12.632 & 0.257073 & 12.632 & 2.43745 & 12.632 & 4.8466 & 12.632 \\
\hline & 1.85468 & & & & & & & & & & \\
\hline 93.5709 & 12.636 & 9.53289 & 12.636 & 4.1951 & 12.636 & 2.59721 & 12.636 & 2.43745 & 12.636 & 3.00547 & 12.636 \\
\hline & 0.234906 & 12.636 & 1.11461 & 12.636 & & & & & & & \\
\hline 93.5709 & 12.64 & 7.61275 & 12.64 & 4.1951 & 12.64 & 2.59721 & 12.64 & 0.458308 & 12.64 & 0.499752 & 12.64 \\
\hline & 2.0745 & 12.64 & 2.62938 & 12.64 & & & & & & & \\
\hline 94.0964 & 12.644 & 9.53289 & 12.644 & 2.11574 & 12.644 & 2.59721 & 12.644 & 4.54137 & 12.644 & 3.00547 & 12.644 \\
\hline & 2.41693 & 12.644 & 2.62938 & 12.644 & & & & & & & \\
\hline 93.5709 & 12.648 & 9.53289 & 12.648 & 2.11574 & 12.648 & 2.59721 & 12.648 & 7.38555 & 12.648 & 1.65098 & 12.648 \\
\hline & 2.41693 & 12.648 & 1.11461 & 12.648 & & & & & & & \\
\hline
\end{tabular}




\begin{tabular}{|c|c|c|c|c|c|c|c|c|c|c|c|}
\hline 93.8336 & $\begin{array}{l}12.652 \\
0.234906\end{array}$ & $\begin{array}{l}9.53289 \\
12.652\end{array}$ & $\begin{array}{l}12.652 \\
0.62663\end{array}$ & $\begin{array}{l}4.1951 \\
12.652\end{array}$ & 12.652 & 2.59721 & 12.652 & 2.43745 & 12.652 & 4.8466 & 12.652 \\
\hline \multirow[t]{2}{*}{94.0964} & 12.656 & 9.53289 & 12.656 & 2.11574 & 12.656 & 2.59721 & 12.656 & 0.458308 & 12.656 & 0.499752 & 12.656 \\
\hline & 41693 & 12.656 & 1.11461 & 2.656 & & & & & & & \\
\hline \multirow[t]{2}{*}{94.0964} & 12.66 & 7.61275 & 12.66 & 4.1951 & 12.66 & 0.257073 & 12.66 & 1.96417 & 12.66 & 4.8466 & 12.66 \\
\hline & 0.234906 & 12.66 & 3.87872 & 12.66 & & & & & & & \\
\hline \multirow[t]{2}{*}{94.0964} & 12.664 & 6.48356 & 12.664 & 0.638699 & 12.664 & 0.257073 & 12.664 & 2.01015 & 12.664 & 3.00547 & 12.664 \\
\hline & 4.37145 & 12.664 & 0.62663 & 12.664 & & & & & & & \\
\hline \multirow[t]{2}{*}{94.0964} & 12.668 & 9.53289 & 2.668 & 0.638699 & 12.668 & 0.257073 & 12.668 & 2.43745 & 12.668 & 4.8466 & 12.668 \\
\hline & 0.234906 & 12.668 & 1.11461 & 12.668 & & & & & & & \\
\hline \multirow[t]{2}{*}{93.5709} & 12.672 & 7.61275 & 12.672 & 5.76301 & 12.672 & 0.257073 & 12.672 & 0.458308 & 12.672 & 0.499752 & 12.672 \\
\hline & 4.37145 & 12.672 & 2.62938 & 12.672 & & & & & & & \\
\hline \multirow[t]{2}{*}{93.8336} & 12.676 & 8.61008 & 126 & 0.638699 & 12.676 & 2.59721 & 12.676 & 4.54137 & 12.676 & 4.8466 & 12.676 \\
\hline & 0.23 & 12.676 & 51 & 12.676 & & & & & & & \\
\hline \multirow[t]{2}{*}{94.0964} & 12.68 & 8.61008 & 2.68 & 0.638699 & 12.68 & 0.257073 & 12.68 & 0.458308 & 12.68 & 3.00547 & 12.68 \\
\hline & 2.41 & 12.68 & 1 & 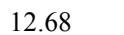 & & & & & & & \\
\hline \multirow[t]{2}{*}{93.8336} & 12.68 & 11.2741 & 12.6 & 2.11574 & 12.684 & 2.59721 & 12.684 & 1.96417 & 12.684 & 0.499752 & 12.684 \\
\hline & 5.86 & 12.684 & 1.1 & 12684 & & & & & & & \\
\hline 94.0964 & 12.6 & 16.3 & 1 & & 12.688 & 0.257073 & 12.688 & 1.96417 & 12.688 & 0.499752 & 12.688 \\
\hline & 2.41 & 12.6 & 61 & 12 & & & & & & & \\
\hline 94.0964 & 12. & 18 & 1 & 2.1 & 12.692 & 0.257073 & 12.692 & 2.01015 & 12.692 & 3.00547 & 12.692 \\
\hline & 1.8 & 1 & & & & & & & & & \\
\hline 93.8336 & 12.6 & 35.1 & 12. & 574 & 12.696 & .257073 & 12.696 & .43745 & 12.696 & 1.65098 & 12.696 \\
\hline & 0.234906 & 12.696 & 0.62 & 12 & & & & & & & \\
\hline 94.0964 & 12.7 & 47.5155 & 12.7 & 0.638699 & 12.7 & 0.257073 & 12.7 & 2.43745 & 12.7 & 3.00547 & 12.7 \\
\hline & 1.85 & 12.7 & 0.62663 & 127 & & & & & & & \\
\hline 94.0964 & 12.7 & 266 & & 574 & 12.704 & 0.257073 & 12.704 & 1.96417 & 12.704 & .09605 & 12.70 \\
\hline & 0.2 & & & & & & & & & & \\
\hline 94.3593 & 12.7 & 47 & 1 & & 12.708 & 1.91927 & 12.708 & 1.96417 & 12.708 & 1.65098 & 12.708 \\
\hline & & 1 & & & & & & & & & \\
\hline 94.0964 & 12.7 & 82.2439 & 1 & 0.638699 & 12.712 & 0.257073 & 12.712 & 1.96417 & 12.712 & 3.00547 & 12.712 \\
\hline & 0.2 & 12712 & 1.1 & 12712 & & & & & & & \\
\hline 94.6223 & 12.7 & 7 & 12.7 & 0.638699 & 12.716 & 2.59721 & 12.716 & 0.458308 & 12.716 & 1.78639 & 12.716 \\
\hline & & & & & & & & & & & \\
\hline 94.3593 & 12.7 & 72 & 1 & & 12.72 & 0.257073 & 12.72 & 1.96417 & 12.72 & 1.65098 & 12.72 \\
\hline & & & & & & & & & & & \\
\hline 94.8853 & 12.7 & .0 & 1 & & 12.724 & 4.65092 & 12.724 & 1.96417 & 12.724 & 0.499752 & 12.724 \\
\hline & 0.234906 & 12.724 & 61 & 12724 & & & & & & & \\
\hline 95.1484 & 12.7 & 24 & 1 & 74 & 12.728 & 2.59721 & 12.728 & 4.54137 & 12.728 & 1.65098 & 12.728 \\
\hline & & & & & & & & & & & \\
\hline 94.8853 & 12. & 8 & & 699 & 12.732 & 2.59721 & 12.732 & 1.96417 & 12.732 & .09605 & 12.732 \\
\hline & & & & & & & & & & & \\
\hline 94.8853 & 12.73 & 7672 & 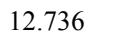 & 74 & 12.736 & 59721 & 12.736 & 15 & 12.736 & .499752 & 12.736 \\
\hline & 4.37 & & & & & & & & & & \\
\hline 94.6223 & 12.74 & 89.7672 & 1 & 2.11574 & 12.74 & 0.257073 & 12.74 & 0.458308 & 12.74 & 1.65098 & 12.74 \\
\hline & 2.4169 & 12.74 & & 1274 & & & & & & & \\
\hline 94.8853 & 12 & 4 & & 699 & 12.744 & 0.257073 & 12.744 & 0.458308 & 12.744 & .499752 & 12.744 \\
\hline & & & & & & & & & & & \\
\hline 94.3593 & 12. & 88 & 1 & 74 & 12.748 & 0.257073 & 12.748 & 0.458308 & 12.748 & 1.65098 & 12.748 \\
\hline & & & & & & & & & & & \\
\hline 95.1484 & 12.7 & 041 & 1 & 0.638699 & 12.752 & 0.257073 & 12.752 & 2.43745 & 12.752 & 0.499752 & 12.752 \\
\hline & & & & & & & & & & & \\
\hline 94.6223 & 12.7 & 41 & 1 & 699 & 12.756 & 59721 & 12.756 & 43745 & 12.756 & .00547 & 12.756 \\
\hline & & & & & & & & & & & \\
\hline 95.1484 & 12. & 45 & & 74 & 12.76 & 257073 & 12.76 & 2.43745 & 12.76 & .499752 & 12.76 \\
\hline & & & & & & & & & & & \\
\hline 95.1484 & 12.7 & 95.4545 & 1 & 4.1951 & 12.764 & 0.257073 & 12.764 & 1.96417 & 12.764 & 0.499752 & 12.764 \\
\hline & & & & & & & & & & & \\
\hline 95.1484 & 12.76 & 97.3587 & 12.7 & 2.11574 & 12.768 & 0.257073 & 12.768 & 2.43745 & 12.768 & 3.00547 & 12.768 \\
\hline & & & & & & & & & & & \\
\hline 95.1484 & 12.77 & 98.3 & & 0.6 & 12.77 & .257073 & 12.77 & 10 & $12.7^{\prime}$ & .65098 & 12.772 \\
\hline & & & & & & & & & & & \\
\hline 95.1484 & 12.776 & 98.3123 & 12.7 & 2.11574 & 12.776 & 2.59721 & 12.776 & 2.43745 & 12.776 & 2.09605 & 12.776 \\
\hline & & & & & & & & & & & \\
\hline 95.1484 & 12.78 & 99.2669 & 12.78 & 2.11574 & 12.78 & 0.257073 & 12.78 & 1.96417 & 12.78 & 0.499752 & 12.78 \\
\hline & & & & & & & & & & & \\
\hline 95.1484 & 12.784 & 98.3123 & & & 12.78 & 0.257073 & 12.78 & 1.9641 & 12.784 & 3.00547 & 12.1 \\
\hline & & & & & & & & & & & \\
\hline 0.711 & 12.788 & 98.3123 & 12.788 & 2.11574 & 12.788 & 0.257073 & 12.788 & 2.43745 & 12.788 & 3.00547 & 12.788 \\
\hline & 0.234906 & 88 & 29 & & & & & & & & \\
\hline
\end{tabular}




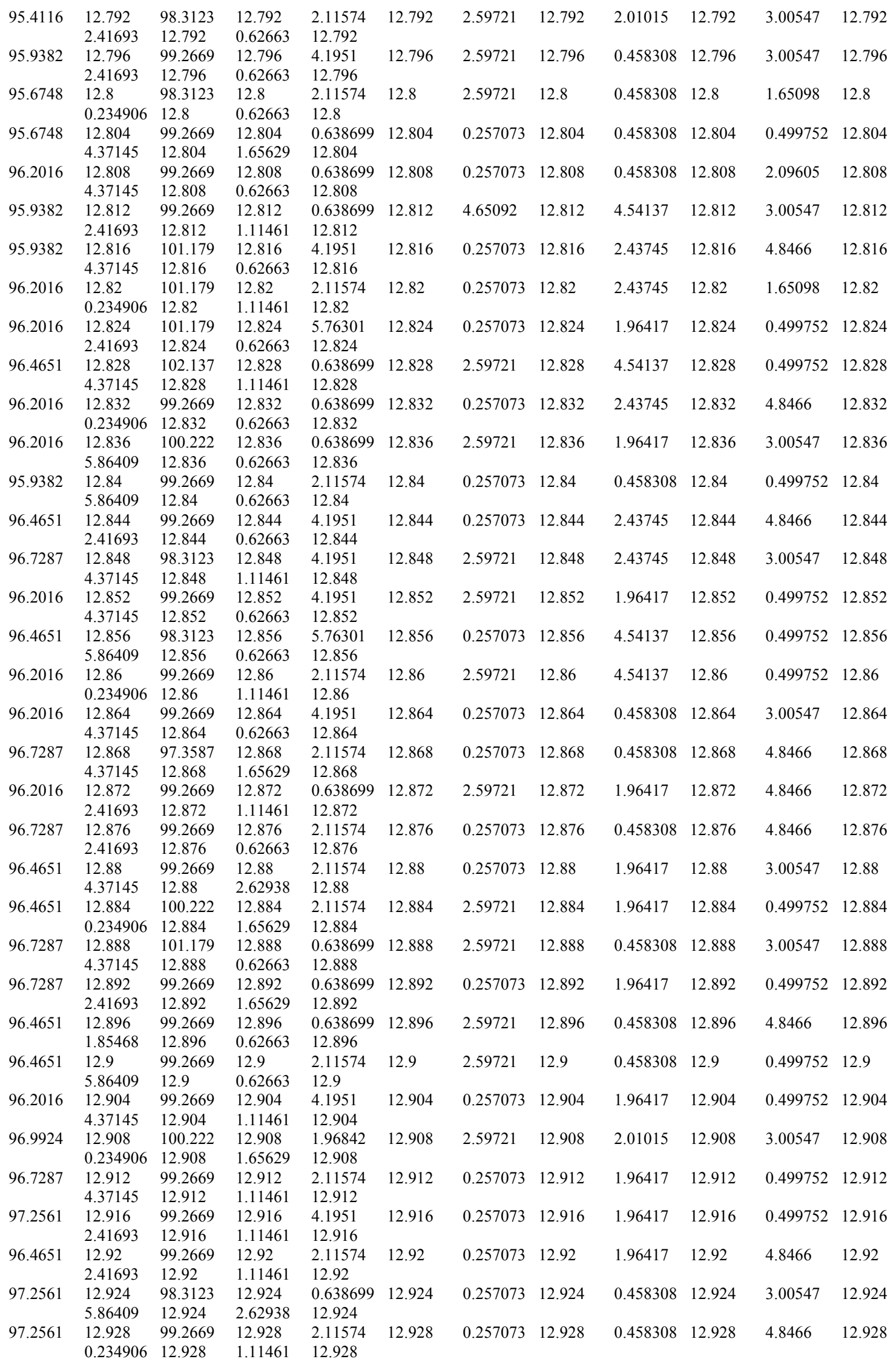




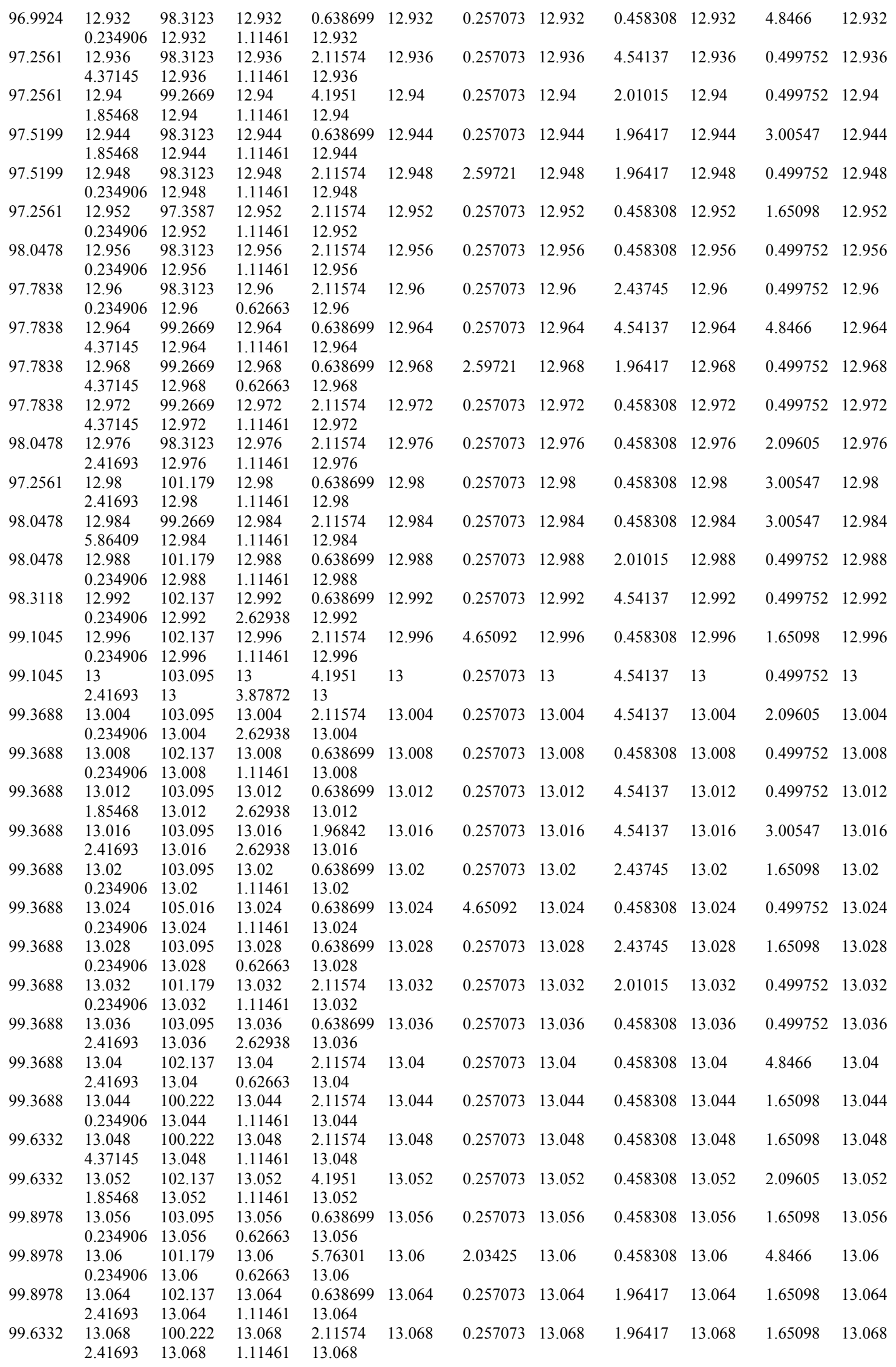




\begin{tabular}{|c|c|c|c|c|c|c|c|c|c|c|c|}
\hline 100.162 & $\begin{array}{l}13.072 \\
0.234906\end{array}$ & $\begin{array}{l}99.2669 \\
13.072\end{array}$ & $\begin{array}{l}13.072 \\
1.11461\end{array}$ & $\begin{array}{l}0.638699 \\
13.072\end{array}$ & 13.072 & 2.59721 & 13.072 & 1.96417 & 13.072 & 0.499752 & 13.072 \\
\hline \multirow[t]{2}{*}{100.162} & 13.076 & 100.222 & 13.076 & 2.11574 & 13.076 & 2.59721 & 13.076 & 0.458308 & 13.076 & 499752 & 13.076 \\
\hline & 0.234906 & 13.076 & 0.62663 & 13.076 & & & & & & & \\
\hline \multirow[t]{2}{*}{100.162} & 13.08 & 99.2669 & 3.08 & 1.96842 & 13.08 & .257073 & 13.08 & 1.96417 & 13.08 & 0.499752 & 13.08 \\
\hline & 2.41693 & 13.08 & 2.62938 & 13.08 & & & & & & & \\
\hline \multirow[t]{2}{*}{100.162} & 13.084 & 100.222 & 13.084 & 0.638699 & 13.084 & 0.257073 & 13.084 & 2.43745 & 13.084 & 0.499752 & 13.084 \\
\hline & 2.41693 & 13.084 & 0.62663 & 13.084 & & & & & & & \\
\hline \multirow[t]{2}{*}{100.427} & 13.088 & 100.222 & 3.088 & 0.638699 & 13.088 & 0.257073 & 13.088 & 1.96417 & 13.088 & 1.65098 & 13.088 \\
\hline & 2.41693 & 13.088 & 0.62663 & 13.088 & & & & & & & \\
\hline \multirow[t]{2}{*}{100.427} & 13.092 & 101.179 & 13.092 & 2.11574 & 13.092 & 0.257073 & 13.092 & 2.43745 & 13.092 & 0.499752 & 13.092 \\
\hline & 0.234906 & 13.092 & 0.62663 & 13.092 & & & & & & & \\
\hline \multirow[t]{2}{*}{100.427} & 13.096 & 100. & & & 13.096 & 0.257073 & 13.096 & 2.43745 & 13.096 & 4.8466 & 13.096 \\
\hline & 1.85 & 13.0 & & 13 & & & & & & & \\
\hline \multirow[t]{2}{*}{100.957} & 13.1 & 100.222 & 13.1 & 2.11574 & 13.1 & 2.59721 & 13.1 & .458308 & 13.1 & 1.65098 & 13.1 \\
\hline & 2.41693 & 13.1 & 1461 & 131 & & & & & & & \\
\hline \multirow[t]{2}{*}{100.427} & 13.104 & 101.179 & 13.104 & 2.11574 & 13.104 & 2.59721 & 13.104 & 2.43745 & 13.104 & 0.499752 & 13.104 \\
\hline & 2.41693 & 13.104 & 61 & 13.104 & & & & & & & \\
\hline 100.957 & 13.1 & 22 & & 699 & 13.108 & 2.59721 & 13.108 & 0.458308 & 13.108 & 1.65098 & 13.108 \\
\hline & 0.23 & 13.108 & & 13 & & & & & & & \\
\hline 101.222 & 13. & 100 & 1 & 4.1 & 13.112 & 2.59721 & 13.112 & 0.458308 & 13.112 & 1.65098 & 13.112 \\
\hline & & 13.1 & & & & & & & & & \\
\hline 101.487 & 13.1 & 100.222 & 6 & 2.1 & 13.116 & 1.91927 & 13.116 & .458308 & 13.116 & 1.65098 & 13.116 \\
\hline & 4.3 & 13.116 & & 13 & & & & & & & \\
\hline 100.957 & 13.12 & 99.2669 & 1 & 4.1951 & 13.12 & 0.257073 & 13.12 & 2.43745 & 13.12 & 0.499752 & 13.12 \\
\hline & 2.41693 & 13.12 & & 13.12 & & & & & & & \\
\hline 101.222 & 13. & 99.2669 & 1 & & 13.124 & 0.257073 & 13.124 & 1.96417 & 13.124 & . 499752 & 13.124 \\
\hline & & 13.124 & & & & & & & & & \\
\hline 101.487 & 13. & 100.222 & 1 & 0.6 & 13.128 & 0.257073 & 13.128 & 0.458308 & 13.128 & 3.00547 & 13.128 \\
\hline & & 13.128 & & & & & & & & & \\
\hline 101.487 & 13.1 & 98.3123 & 1 & 699 & 13.132 & 2.59721 & 13.132 & 2.43745 & 13.132 & 0.499752 & 13.132 \\
\hline & 2.4 & 13.132 & 61 & 13 & & & & & & & \\
\hline 101.752 & 13.1 & 101.179 & 6 & 74 & 13.136 & 2.59721 & 13.136 & 2.43745 & 13.136 & 3.00547 & 13.136 \\
\hline & & & & & & & & & & & \\
\hline 102.017 & 13. & 99. & & 4.1 & 13.14 & 0.257073 & 13.14 & .43745 & 13.14 & 4.8466 & 13.14 \\
\hline & & & & & & & & & & & \\
\hline 102.282 & 13.1 & & & & 13.144 & .257073 & 13.144 & 2.01015 & 13.144 & 0.499752 & 13.144 \\
\hline & 2.41 & 13.144 & & 13 & & & & & & & \\
\hline 102.017 & 13 & 22 & 1 & 99 & 13.148 & 2.59721 & 13.148 & 1.96417 & 13.148 & 3.00547 & 13.148 \\
\hline & & & & & & & & & & & \\
\hline 101.752 & 13 & & & & 13.152 & 0.257073 & 13.152 & 1.96417 & 13.152 & 0.499752 & 13.152 \\
\hline & & & & & & & & & & & \\
\hline 102.017 & 13.15 & 100.222 & 6 & 2.1 & 13.156 & 59721 & 13.156 & 15 & 13.156 & 0.499752 & 13.156 \\
\hline & & & & & & & & & & & \\
\hline 101.487 & 13.16 & .179 & 1 & 4.1 & 13.16 & 0.257073 & 13.16 & 2.43745 & 13.16 & 1.65098 & 13.16 \\
\hline & 2. & 13.16 & & 13 & & & & & & & \\
\hline 101.752 & 13 & 2 & 1 & 1 & 13.164 & 2.59721 & 13.164 & 2.01015 & 13.164 & .00547 & 13.164 \\
\hline & & & & & & & & & & & \\
\hline 101.752 & 13. & 9 & & 4. & 13.168 & 0.257073 & 13.168 & 1.96417 & 13.16 & 1.65098 & 13.168 \\
\hline & & & & & & & & & & & \\
\hline 102.282 & 13.1 & & & 2.11 & 13.172 & 0.257073 & 13.172 & 2.43745 & 13.172 & 3.00547 & 13.172 \\
\hline & & & & & & & & & & & \\
\hline 102.547 & 13.17 & 101.179 & 13.176 & 0.638699 & 13.176 & 0.257073 & 13.176 & 1.96417 & 13.176 & 6.26752 & 13.176 \\
\hline & & & & & & & & & & & \\
\hline 102.282 & 13. & 1 & & 2. & 13.18 & 0.257073 & 13.18 & 0.458308 & 13.18 & 2.09605 & 13.18 \\
\hline & & & & & & & & & & & \\
\hline 102.282 & 13.18 & 1 & 1 & 0.638699 & 13.184 & 0.257073 & 13.184 & 0.458308 & 13.184 & 1.65098 & 13.184 \\
\hline & & & & & & & & & & & \\
\hline 102.547 & 13.18 & 3.095 & 1 & 2.11574 & 13.188 & 0.257073 & 13.188 & 2.01015 & 13.188 & 1.65098 & 13.188 \\
\hline & & & & & & & & & & & \\
\hline 102.547 & 13.19 & 101.179 & & 2.11574 & 13.19 & 0.257073 & 13.19 & . & 13.19 & 3.00547 & 13.192 \\
\hline & & & & & & & & & & & \\
\hline 102.547 & 13.1 & 1 & 1 & 699 & 13.196 & 0.257073 & 13.196 & 4.54137 & 13.196 & 1.65098 & 13.196 \\
\hline & & & & & & & & & & & \\
\hline 102.547 & 13.2 & 100.2 & & 2.11574 & 13.2 & 2.59721 & 13.2 & 0.458308 & 13.2 & 3.00547 & 13.2 \\
\hline & & & & 13 & & & & & & & \\
\hline 102.547 & 13.20 & 101.179 & 1 & 2.11574 & 13.204 & 0.257073 & 13.204 & 0.458308 & 13.204 & 3.00547 & 13.204 \\
\hline & & & & & & & & & & & \\
\hline 03.07 & 13.20 & 102.137 & & 2.11574 & 13.208 & 0.257073 & 13.208 & 1.96417 & 13.208 & 0.499752 & 13.20 \\
\hline & 4.37145 & & & & & & & & & & \\
\hline
\end{tabular}




\begin{tabular}{|c|c|c|c|c|c|c|c|c|c|c|c|}
\hline 103.078 & $\begin{array}{l}13.212 \\
0.234906\end{array}$ & $\begin{array}{l}101.179 \\
13.212\end{array}$ & $\begin{array}{l}13.212 \\
0.62663\end{array}$ & $\begin{array}{l}0.638699 \\
13.212\end{array}$ & 13.212 & 0.257073 & 13.212 & 2.43745 & 13.212 & 1.65098 & 13.212 \\
\hline \multirow[t]{2}{*}{103.078} & 13.216 & 101.179 & 13.216 & 0.638699 & 13.216 & 0.257073 & 13.216 & 1.96417 & 13.216 & 0.499752 & 13.216 \\
\hline & 5.86409 & 13.216 & 1.11461 & 3.216 & & & & & & & \\
\hline \multirow[t]{2}{*}{102.813} & 13.22 & 104.055 & 3.22 & 0.638699 & 13.22 & 0.257073 & 13.22 & 0.458308 & 13.22 & 3.00547 & 13.22 \\
\hline & 0.234906 & 13.22 & 1.11461 & 13.22 & & & & & & & \\
\hline \multirow[t]{2}{*}{103.609} & 13.224 & 103.095 & 13.224 & 2.11574 & 13.224 & 0.257073 & 13.224 & 4.54137 & 13.224 & 3.00547 & 13.224 \\
\hline & 0.234906 & 3.224 & 1.11461 & 13.224 & & & & & & & \\
\hline \multirow[t]{2}{*}{103.344} & 13.228 & 102.137 & 3.228 & 0.638699 & 13.228 & 0.257073 & 13.228 & 0.458308 & 13.228 & 4.8466 & 13.228 \\
\hline & 0.234906 & .228 & 1.11461 & 13.228 & & & & & & & \\
\hline \multirow[t]{2}{*}{103.875} & 13.232 & 102. & 13.232 & 0.638699 & 13.232 & 0.257073 & 13.232 & 1.96417 & 13.232 & 2.09605 & 13.232 \\
\hline & 0.234906 & 13.232 & 1.11461 & 13.232 & & & & & & & \\
\hline \multirow[t]{2}{*}{103.344} & 13.236 & 55 & & 0.638699 & 13.236 & 2.59721 & 13.236 & 0.458308 & 13.236 & 0.499752 & 13.236 \\
\hline & 0.23 & .2 & & 13. & & & & & & & \\
\hline \multirow[t]{2}{*}{103.609} & 13.24 & 103.095 & 13.24 & 2.11574 & 13.24 & 2.59721 & 13.24 & 0.458308 & 13.24 & 0.499752 & 13.24 \\
\hline & 2.41693 & 13.24 & 63 & 1224 & & & & & & & \\
\hline \multirow[t]{2}{*}{103.609} & 13.244 & 103.095 & 13.2 & 0.638699 & 13.244 & 2.59721 & 13.244 & 0.458308 & 13.244 & 4.8466 & 13.244 \\
\hline & 2.41693 & 13.244 & 1.11 & 13.244 & & & & & & & \\
\hline 103.609 & 13.2 & 5 & & 699 & 13.248 & 0.257073 & 13.248 & 0.458308 & 13.248 & 1.65098 & 13.248 \\
\hline & 2.41693 & 13.2 & 51 & 13.248 & & & & & & & \\
\hline 103.344 & 13.2 & 7 & 1 & 2.1 & 13.252 & 2.59721 & 13.252 & 4.54137 & 13.252 & 1.65098 & 13.2 \\
\hline & 0.23 & 1 & & 13 & & & & & & & \\
\hline 103.609 & 13.256 & 102 & 1 & 0.6 & 13.256 & 0.257073 & 13.256 & 2.43745 & 13.256 & 3.00547 & 13.256 \\
\hline & 4.37 & 1 & 0.6 & 13 & & & & & & & \\
\hline 103.875 & 13.26 & 104.055 & 1 & 0.638699 & 13.26 & 2.59721 & 13.26 & 0.458308 & 13.26 & 0.499752 & 13.26 \\
\hline & 0.234906 & 1326 & & 13.26 & & & & & & & \\
\hline 104.141 & 13.2 & 37 & & & 13.264 & 0.257073 & 13.264 & 0.458308 & 13.264 & 0.499752 & 13.264 \\
\hline & & & & & & & & & & & \\
\hline 104.406 & 13.2 & 7 & 1 & 0.6 & 13.268 & 0.257073 & 13.268 & 0.458308 & 13.268 & 1.65098 & 13.268 \\
\hline & & & & & & & & & & & \\
\hline 104.141 & 13.272 & 102 & 1 & 3699 & 13.272 & 0.257073 & 13.272 & 1.96417 & 13.272 & 3.00547 & 13.272 \\
\hline & $4.3^{7}$ & 132 & & 13 & & & & & & & \\
\hline 104.406 & 13.27 & 103 & 13. & 699 & 13.276 & 0.257073 & 13.276 & 0.458308 & 13.276 & 3.00547 & 13.276 \\
\hline & & & & & & & & & & & \\
\hline 104.672 & 13.28 & 5 & 1 & 699 & 13.28 & 0.257073 & 13.28 & 0.458308 & 13.28 & 4.8466 & 13.28 \\
\hline & & & & & & & & & & & \\
\hline 104.672 & 13.2 & 95 & 1 & & 13.284 & 0.257073 & 13.284 & 2.43745 & 13.284 & 0.499752 & 13 \\
\hline & 0.23 & 1 & 29 & 122 & & & & & & & \\
\hline 104.672 & 13.2 & 5 & 1 & 99 & 13.288 & 0.257073 & 13.288 & 1.96417 & 13.288 & 1.65098 & 13.288 \\
\hline & & & & & & & & & & & \\
\hline 104.672 & 13 & & & & 13.292 & 0.257073 & 13.292 & 1.96417 & 13.292 & .09605 & 13.292 \\
\hline & & & & & & & & & & & \\
\hline 104.672 & 13.29 & 7 & 1 & 699 & 13.296 & 257073 & 13.296 & 1.96417 & 13.296 & .499752 & 13.296 \\
\hline & 0.23 & & & & & & & & & & \\
\hline 104.672 & 13.3 & 1.179 & & 0.638699 & 13.3 & 0.257073 & 13.3 & 2.43745 & 13.3 & 0.499752 & 13.3 \\
\hline & 0.2 & & & & & & & & & & \\
\hline 104.672 & 13. & 5 & 1 & 74 & 13.304 & 0.257073 & 13.304 & 0.458308 & 13.304 & 1.65098 & 3.304 \\
\hline & & & & & & & & & & & \\
\hline 105.204 & 13. & 37 & 1 & 74 & 13.308 & 0.257073 & 13.308 & 2.43745 & 13.308 & 3.00547 & 13.308 \\
\hline & & & & & & & & & & & \\
\hline 105.204 & 13.3 & & & 1.96 & 13.312 & .257073 & 13.312 & 2.01015 & 13.312 & 6.26752 & 13.312 \\
\hline & & & & & & & & & & & \\
\hline 105.204 & 13.31 & 37 & 13.3 & 699 & 13.316 & 0.257073 & 13.316 & 1.96417 & 13.316 & 0.499752 & 13.316 \\
\hline & & & & & & & & & & & \\
\hline 105.471 & 13. & 1 & 1 & 699 & 13.32 & 0.257073 & 13.3 & .458308 & 13.3 & 0.499752 & 13.32 \\
\hline & & & & & & & & & & & \\
\hline 105.471 & 13.3 & 1 & 1 & 2.11574 & 13.324 & 0.257073 & 13.324 & 2.01015 & 13.324 & 2.09605 & 13.324 \\
\hline & & & & & & & & & & & \\
\hline 105.737 & 13.32 & 103.095 & 13.3 & 0.638699 & 13.328 & 2.59721 & 13.328 & 0.458308 & 13.328 & 1.65098 & 13.328 \\
\hline & & & & & & & & & & & \\
\hline 105.737 & 13.3 & 10 & & 2.11 & 13.3 & 0.257073 & 13.33 & 458308 & 13.3 & 26752 & 13.332 \\
\hline & & & & & & & & & & & \\
\hline 105.737 & 13.3 & 1 & 1 & 0.638699 & 13.336 & 0.257073 & 13.336 & 0.458308 & 13.336 & 0.499752 & 13.336 \\
\hline & & & & & & & & & & & \\
\hline 106.003 & 13.34 & 103.095 & 13.3 & 2.11574 & 13.34 & 2.59721 & 13.34 & 4.54137 & 13.34 & 0.499752 & 13.34 \\
\hline & & & & & & & & & & & \\
\hline 105.737 & 13.344 & 102.137 & & 2.11574 & 13.344 & 0.257073 & 13.344 & 2.43745 & 13.344 & 1.65098 & 0. \\
\hline & & & & & & & & & & & \\
\hline . & & & 13.348 & 2.11574 & 13.348 & 0.257073 & 13.348 & 0.458308 & 13.348 & 0.499752 & 13.3 \\
\hline & 4.37145 & & 38 & & & & & & & & \\
\hline
\end{tabular}




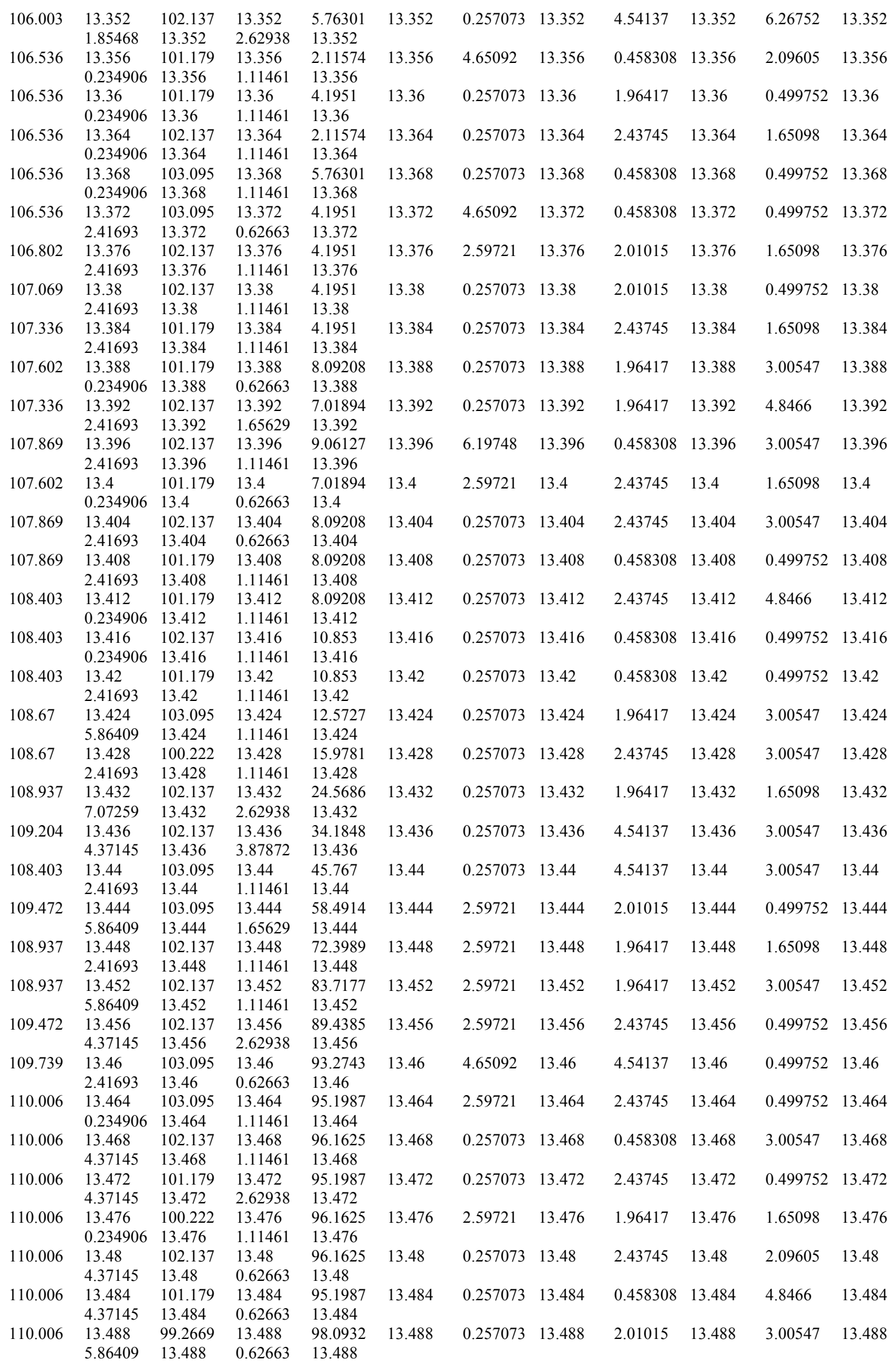




\begin{tabular}{|c|c|c|c|c|c|c|c|c|c|c|c|}
\hline 110.006 & $\begin{array}{l}13.492 \\
0.234906\end{array}$ & $\begin{array}{l}99.2669 \\
13.492\end{array}$ & $\begin{array}{l}13.492 \\
0.62663\end{array}$ & $\begin{array}{l}99.0602 \\
13.492\end{array}$ & 13.492 & 0.257073 & 13.492 & 2.43745 & 13.492 & 0.499752 & 13.492 \\
\hline \multirow[t]{2}{*}{110.006} & 13.496 & 100.222 & 13.496 & 101.967 & 13.496 & 0.257073 & 13.496 & 0.458308 & 13.496 & 0.499752 & 13.496 \\
\hline & .37145 & 13.496 & 1.11461 & 13.496 & & & & & & & \\
\hline \multirow[t]{2}{*}{110.274} & 13.5 & 100.222 & 13.5 & 100.997 & 13.5 & 0.257073 & 13.5 & 4.54137 & 13.5 & 3.00547 & 13.5 \\
\hline & 0.234906 & 13.5 & 1.11461 & 13.5 & & & & & & & \\
\hline \multirow[t]{2}{*}{110.274} & 13.504 & 102.137 & 13.504 & 101.967 & 13.504 & 0.257073 & 13.504 & 0.458308 & 13.504 & 4.8466 & 13.504 \\
\hline & 0.234906 & 3.504 & 2.62938 & 13.504 & & & & & & & \\
\hline \multirow[t]{2}{*}{110.541} & 13.508 & 100.222 & 13.508 & 101.967 & 13.508 & 0.257073 & 13.508 & 0.458308 & 13.508 & 0.499752 & 13.508 \\
\hline & 2.41693 & 13.508 & 0.62663 & 13.508 & & & & & & & \\
\hline \multirow[t]{2}{*}{110.274} & 13.512 & 100.222 & 13.512 & 102.938 & 13.512 & 0.257073 & 13.512 & 1.96417 & 13.512 & 0.499752 & 13.512 \\
\hline & 2.41693 & 13.512 & 0.62663 & 13.512 & & & & & & & \\
\hline \multirow[t]{2}{*}{110.541} & 13.516 & 99.2669 & 13.516 & 105.858 & 13.516 & 0.257073 & 13.516 & 2.43745 & 13.516 & 3.00547 & 13.516 \\
\hline & 4.37 & 13.5 & & 13.516 & & & & & & & \\
\hline \multirow[t]{2}{*}{110.809} & 13.52 & 100.222 & 13 & 106.833 & 13.52 & 0.257073 & 13.52 & 1.96417 & 13.52 & 0.499752 & 13.52 \\
\hline & 2.41 & 13.52 & 63 & 13. & & & & & & & \\
\hline \multirow[t]{2}{*}{110.809} & 13.524 & 99.2669 & 13.524 & 109.764 & 13.524 & 0.257073 & 13.524 & 1.96417 & 13.524 & 1.78639 & 13.524 \\
\hline & 2. & 13.524 & 63 & 13.524 & & & & & & & \\
\hline 110.541 & 13.5 & 99.2 & 8 & 109.764 & 13.528 & 0.257073 & 13.528 & 1.96417 & 13.528 & 3.00547 & 13.528 \\
\hline & 1.85468 & 13.528 & 1.11461 & 13.528 & & & & & & & \\
\hline 111.077 & & 100. & & & 13.532 & 1.91927 & 13.532 & 0.458308 & 13.532 & 1.65098 & 13.532 \\
\hline & 2. & 1 & 2 & & & & & & & & \\
\hline 111.077 & 13.53 & 101.179 & 13. & 108 & 13.536 & 2.59721 & 13.536 & 2.01015 & 13.536 & 0.499752 & 13.536 \\
\hline & 2.41693 & 13.536 & 61 & & & & & & & & \\
\hline 111.077 & 13.54 & 99.2669 & 54 & 110.744 & 13.54 & 4.65092 & 13.54 & 0.458308 & 13.54 & 0.499752 & 13.54 \\
\hline & 2.41693 & 13.54 & 1.11461 & 13.54 & & & & & & & \\
\hline 111.077 & 13.5 & 100.222 & 13.5 & 110 & 13.544 & 4.65092 & 13.544 & 2.43745 & 13.544 & 3.00547 & 13.544 \\
\hline & 0.2 & 1 & & 13 & & & & & & & \\
\hline 111.077 & 13. & 59 & & 11 & 13.548 & 0.257073 & 13.548 & 1.96417 & 13.548 & 1.65098 & 13.548 \\
\hline & 0.2 & 1 & & & & & & & & & \\
\hline 110.809 & 13.5 & 3123 & 1 & 109 & 13.5 & 2.59721 & 13.552 & 0.458308 & 13.552 & 4.8466 & 13.552 \\
\hline & 4. & 1 & 61 & 13 & & & & & & & \\
\hline 111.344 & 13.5 & 99.2669 & 13. & 110.744 & 13.556 & 2.59721 & 13.556 & 1.96417 & 13.556 & 0.499752 & 13.556 \\
\hline & & & & & & & & & & & \\
\hline 111.612 & 13 & 69 & & & 13.56 & 2.59721 & 13.56 & 0.458308 & 13.56 & .26752 & 13.56 \\
\hline & & 1 & & & & & & & & & \\
\hline 111.344 & 13. & 99.2669 & 1 & 44 & 13.564 & 0.257073 & 13.564 & 4.54137 & 13.564 & 3.00547 & 13.564 \\
\hline & & 1 & & & & & & & & & \\
\hline 111.077 & 13 & 669 & 1 & & 13.568 & 73 & 13.568 & 308 & 13.568 & .8466 & 13.568 \\
\hline & 0. & 1 & & 13 & & & & & & & \\
\hline 111.88 & 13 & 9 & & & 13.572 & 0.257073 & 13.572 & 4.54137 & 13.572 & 4.8466 & 13.572 \\
\hline & & & & & & & & & & & \\
\hline 111.88 & 13. & 99.2 & 1 & 11 & 13.576 & 0.257073 & 13.576 & 1.96417 & 13.576 & 1.65098 & 13.576 \\
\hline & & & & & & & & & & & \\
\hline 111.344 & 13.5 & 312 & & 109.764 & 13.58 & 2.59721 & 13.58 & 1.96417 & 13.58 & 1.65098 & 13.58 \\
\hline & & 1 & & 1250 & & & & & & & \\
\hline 111.612 & 13.58 & 99.2669 & 1 & 109.764 & 13.584 & 0.257073 & 13.584 & 0.458308 & 13.584 & 3.00547 & 13.584 \\
\hline & & & & & & & & & & & \\
\hline 111.612 & & & & & 13.588 & 0.257073 & 13.588 & 1.96417 & 13.588 & 0.499752 & 13.588 \\
\hline & & & & & & & & & & & \\
\hline 111.88 & 13.59 & 99.2669 & 2 & 109.764 & 13.592 & 0.257073 & 13.592 & 2.43745 & 13.592 & 4.8466 & 13.592 \\
\hline & & & & & & & & & & & \\
\hline 112.148 & 13.5 & 3587 & 6 & 109.764 & 13.596 & 2.59721 & 13.596 & 0.458308 & 13.596 & 1.65098 & 13.596 \\
\hline & & & & & & & & & & & \\
\hline 111.88 & 13. & 98.3123 & & 111.724 & 13.6 & 0.257073 & 13.6 & 2.43745 & 13.6 & 1.65098 & 13.6 \\
\hline & & & & & & & & & & & \\
\hline 112.148 & 13.604 & 96.4061 & 4 & 110.744 & 13.604 & 0.257073 & 13.604 & 2.01015 & 13.604 & 0.499752 & 13.604 \\
\hline & & & & & & & & & & & \\
\hline 112.148 & 13.60 & 2669 & 1 & 110.744 & 13.608 & 2.59721 & 13.608 & 1.96417 & 13.608 & 0.499752 & 13.608 \\
\hline & & & & & & & & & & & \\
\hline 112.148 & 13.6 & 97.3587 & & 111.724 & 13.612 & 0.257073 & 13.612 & 0.458308 & 13.612 & 4.8466 & 13.612 \\
\hline & & & & & & & & & & & \\
\hline 112.148 & 13.6 & 87 & & & 13.616 & 257073 & 13.616 & 1.96417 & 13.616 & 499752 & 13.616 \\
\hline & & & & & & & & & & & \\
\hline 112.148 & 13.62 & 96.4061 & 13.62 & 112.705 & 13.62 & 0.257073 & 13.62 & 0.458308 & 13.62 & 0.499752 & 13.62 \\
\hline & & 13.62 & & 13.62 & & & & & & & \\
\hline 112.416 & 13.624 & 98.3123 & 13.624 & 111.724 & 13.624 & 0.257073 & 13.624 & 4.54137 & 13.624 & 1.65098 & 13.624 \\
\hline & & & & & & & & & & & \\
\hline 2.68 & 13.62 & 95.4545 & 13.628 & 111.724 & 13.628 & 0.257073 & 13.628 & 0.458308 & 13.628 & 0.499752 & 13.6 \\
\hline & .234906 & 28 & 1.11461 & & & & & & & & \\
\hline
\end{tabular}




\begin{tabular}{|c|c|c|c|c|c|c|c|c|c|c|c|}
\hline 112.952 & $\begin{array}{l}13.632 \\
0.234906\end{array}$ & $\begin{array}{l}96.4061 \\
13.632\end{array}$ & $\begin{array}{l}13.632 \\
1.65629\end{array}$ & $\begin{array}{l}112.705 \\
13.632\end{array}$ & 13.632 & 4.65092 & 13.632 & 2.43745 & 13.632 & 0.499752 & 13.632 \\
\hline \multirow[t]{2}{*}{112.416} & 13.636 & 95.4545 & 13.636 & 111.724 & 13.636 & 0.257073 & 13.636 & 2.43745 & 13.636 & 3.00547 & 13.636 \\
\hline & .41693 & 13.636 & 1.11461 & 13.636 & & & & & & & \\
\hline \multirow[t]{2}{*}{112.684} & 13.64 & 96.4061 & 13.64 & 113.687 & 13.64 & 0.257073 & 13.64 & 0.458308 & 13.64 & 0.499752 & 13.64 \\
\hline & 2.41693 & 13.64 & 0.62663 & 13.64 & & & & & & & \\
\hline \multirow[t]{2}{*}{113.221} & 13.644 & 94.5041 & 13.644 & 113.687 & 13.644 & 2.59721 & 13.644 & 2.43745 & 13.644 & 2.09605 & 13.644 \\
\hline & 0.234906 & 13.644 & 0.62663 & 13.644 & & & & & & & \\
\hline \multirow[t]{2}{*}{112.952} & 13.648 & 96.4061 & 3.648 & 114.67 & 13.648 & 0.257073 & 13.648 & 1.96417 & 13.648 & 3.00547 & 13.648 \\
\hline & 2.41693 & 13.648 & 1.65629 & 13.648 & & & & & & & \\
\hline \multirow[t]{2}{*}{113.221} & 13.652 & 96.4061 & 13.652 & 113.687 & 13.652 & 0.257073 & 13.652 & 1.96417 & 13.652 & 1.65098 & 13.652 \\
\hline & 4.37145 & 13.652 & 1.11461 & 13.652 & & & & & & & \\
\hline \multirow[t]{2}{*}{113.221} & 13.65 & 96.4061 & 13.6 & 111.724 & 13.656 & 0.257073 & 13.656 & 2.43745 & 13.656 & 3.00547 & 13.656 \\
\hline & $5.8 \mathrm{c}$ & 13.6 & & 13.656 & & & & & & & \\
\hline \multirow[t]{2}{*}{113.757} & 13.66 & 96.4 & 6 & 112.705 & 13.66 & 0.257073 & 13.66 & 1.96417 & 13.66 & 3.00547 & 13.66 \\
\hline & 4.37145 & 13.6 & 2 & 13.66 & & & & & & & \\
\hline \multirow[t]{2}{*}{113.757} & 13.664 & 95.4545 & 13.664 & 113.687 & 13.664 & 0.257073 & 13.664 & 0.458308 & 13.664 & 0.499752 & 13.664 \\
\hline & 0.23 & 1 & & 13 & & & & & & & \\
\hline 114.026 & 13.668 & 1 & 13. & 687 & 13.668 & 0.257073 & 13.668 & 0.458308 & 13.668 & 0.499752 & 13.668 \\
\hline & 0.234906 & 13.668 & 63 & 13.668 & & & & & & & \\
\hline 113.757 & 13.6 & 1 & & & 13.672 & 4.65092 & 13.672 & 0.458308 & 13.672 & 1.65098 & 13.672 \\
\hline & 1.8 & 1 & & 13 & & & & & & & \\
\hline 113.757 & 13.676 & 94.5041 & 13. & 113.687 & 13.676 & 0.257073 & 13.676 & 0.458308 & 13.676 & 1.65098 & 13.676 \\
\hline & 0.234906 & 13.6 & & 13. & & & & & & & \\
\hline 114.295 & 13.68 & .4 & 1 & 113.687 & 13.68 & 2.59721 & 13.68 & 1.96417 & 13.68 & 0.499752 & 13.68 \\
\hline & 0.234906 & 13.68 & 1.11461 & 13,68 & & & & & & & \\
\hline 114.026 & 13.6 & 95.4 & 13. & 11 & 13.684 & 2.59721 & 13.684 & 0.458308 & 13.684 & 2.09605 & 13.684 \\
\hline & $7 .($ & & & & & & & & & & \\
\hline 114.026 & 13.6 & 93.5 & 1 & 113 & 13.688 & 0.257073 & 13.688 & 1.96417 & 13.688 & 1.65098 & 13.6 \\
\hline & 7.0 & 13.6 & & & & & & & & & \\
\hline 114.563 & 13.692 & 93.5 & 1 & 113.687 & 13.692 & 0.257073 & 13.692 & 4.54137 & 13.692 & 0.499752 & 13.692 \\
\hline & 2.4 & 13. & 29 & 13 & & & & & & & \\
\hline 114.563 & 13.69 & 95.4 & 13. & 114.67 & 13.696 & 0.257073 & 13.696 & 2.43745 & 13.696 & 3.00547 & 13.696 \\
\hline & & & & & & & & & & & \\
\hline 114.832 & 13 & 9 & & 705 & 13.7 & 1.91927 & 13.7 & 0.458308 & 13.7 & 3.00547 & 13.7 \\
\hline & & 1 & & & & & & & & & \\
\hline 114.563 & 13.7 & & & 57 & 13.704 & 0.257073 & 13.704 & 1.96417 & 13.704 & 3.00547 & 13.704 \\
\hline & & 1 & & & & & & & & & \\
\hline 114.563 & 13.7 & 9 & 1 & 54 & 13.708 & 0.257073 & 13.708 & 2.43745 & 13.708 & 0.499752 & 13.708 \\
\hline & & & & & & & & & & & \\
\hline 114.563 & 13. & 9 & & & 13.712 & .59721 & 13.712 & 1.96417 & 13.712 & 0.499752 & 13.712 \\
\hline & & 1 & & & & & & & & & \\
\hline 115.101 & 13.71 & 91.6 & 1 & 39 & 13.716 & 2.59721 & 13.716 & 1.96417 & 13.716 & 1.65098 & 13.716 \\
\hline & & & & & & & & & & & \\
\hline 115.37 & 13.7 & 93.5546 & 1 & 117.625 & 13.72 & 6.19748 & 13.72 & 458308 & 13.72 & 1.65098 & 13.72 \\
\hline & & 13.7 & & 1277 & & & & & & & \\
\hline 115.37 & 13 & 92.6062 & 1 & 118.612 & 13.724 & 0.257073 & 13.724 & 1.96417 & 13.724 & 4.8466 & 13.724 \\
\hline & & & & & & & & & & & \\
\hline 115.37 & 13 & 9 & 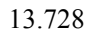 & 11 & 13.728 & 2.59721 & 13.728 & 1.96417 & 13.728 & 3.00547 & 13.728 \\
\hline & & & & & & & & & & & \\
\hline 115.638 & 13.7 & 9 & & 512 & 13.73 & 2.59721 & 13.732 & 45 & 13.73 & 1.65098 & 13.732 \\
\hline & & & & & & & & & & & \\
\hline 116.177 & 13.73 & 93.5546 & 1 & 120.588 & 13.736 & 0.257073 & 13.736 & 0.458308 & 13.736 & 0.499752 & 13.736 \\
\hline & & & & & & & & & & & \\
\hline 116.446 & 13.74 & 9 & & & 13.74 & 59721 & 13.7 & 458308 & 13.74 & .09605 & 13.74 \\
\hline & & & & & & & & & & & \\
\hline 116.446 & 13.74 & 2 & 1 & 120.588 & 13.744 & 0.257073 & 13.74 & .458308 & 13.744 & 1.65098 & 13.744 \\
\hline & & & & & & & & & & & \\
\hline 116.446 & 13.748 & 93.5546 & 13.7 & 121.578 & 13.748 & 0.257073 & 13.748 & 0.458308 & 13.748 & 0.499752 & 13.748 \\
\hline & & & & & & & & & & & \\
\hline 116.715 & & & & 122.569 & 13.752 & 0.257073 & 13.752 & 08 & 13.7 & .00547 & 13.752 \\
\hline & & & & & & & & & & & \\
\hline 116.715 & 13.756 & 9 & 1 & 578 & 13.756 & 0.257073 & 13.756 & 2.43745 & 13.756 & 0.499752 & 13.756 \\
\hline & & & & & & & & & & & \\
\hline 116.715 & 13.76 & 91.6588 & 13.7 & 120.588 & 13.76 & 2.59721 & 13.76 & 2.01015 & 13.76 & 3.00547 & 13.76 \\
\hline & 2.41693 & 13.76 & & 13.76 & & & & & & & \\
\hline 116.446 & 13.764 & 92.6062 & 13.764 & 121.578 & 13.764 & 0.257073 & 13.764 & 2.01015 & 13.764 & 1.65098 & 13.764 \\
\hline & & & & & & & & & & & \\
\hline 16.446 & & 91.6588 & 13.7 & 121.578 & 13.768 & 0.257073 & 13.768 & 0.458308 & 13.768 & 4.8466 & 13.768 \\
\hline & 4.37145 & 1 & 63 & & & & & & & & \\
\hline
\end{tabular}




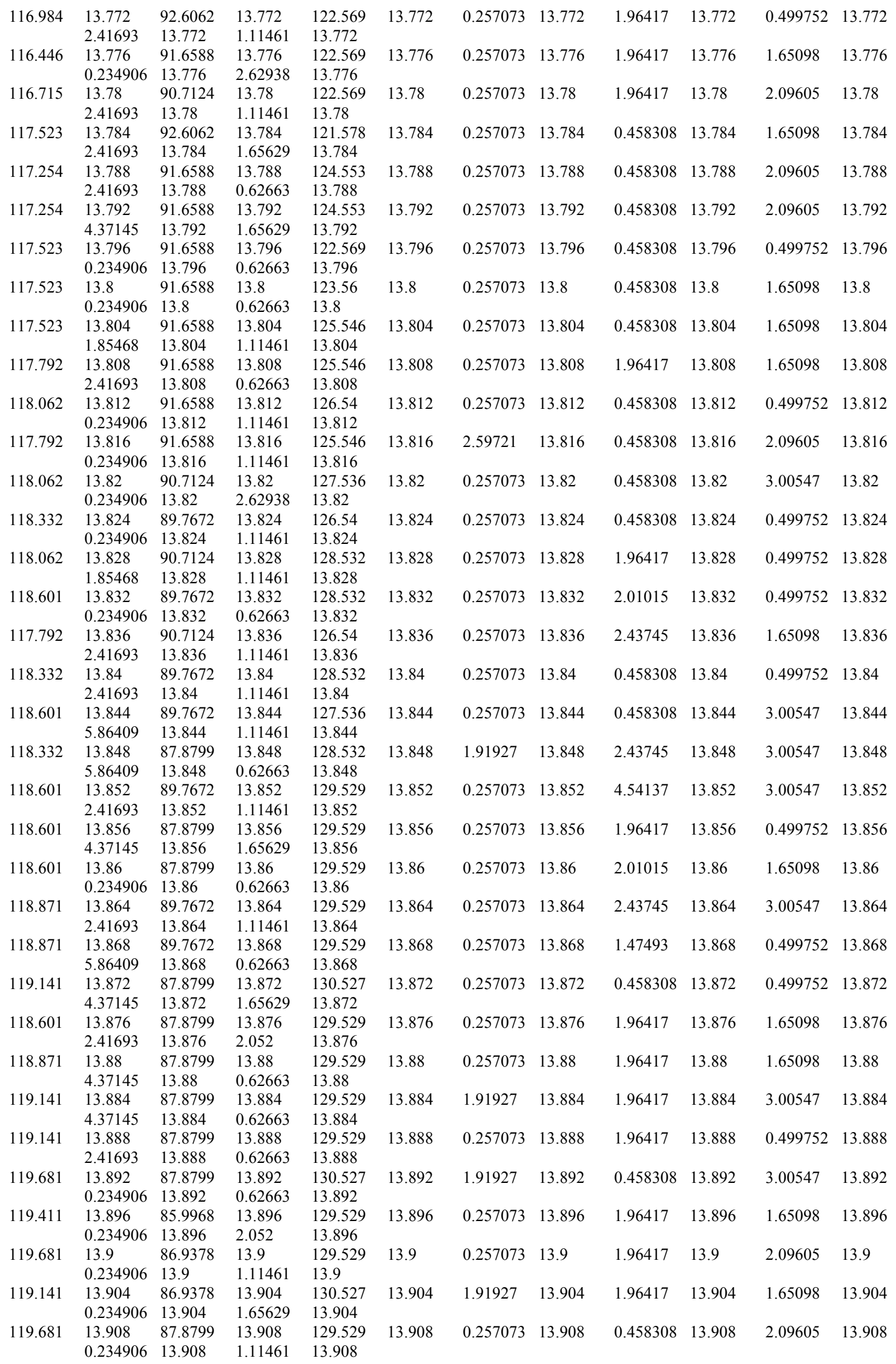




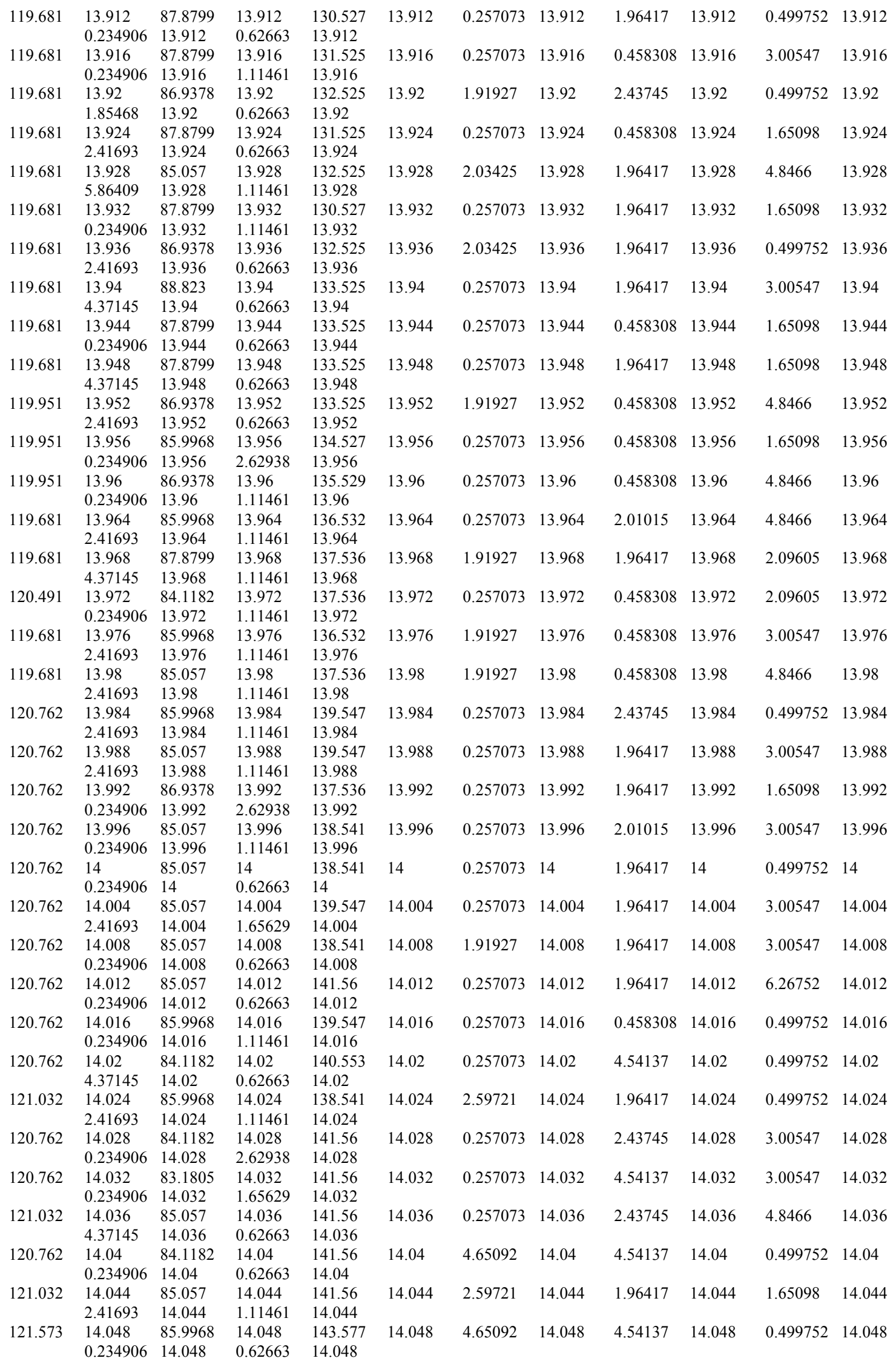




\begin{tabular}{|c|c|c|c|c|c|c|c|c|c|c|c|}
\hline 120.762 & $\begin{array}{l}14.052 \\
2.41693\end{array}$ & $\begin{array}{l}84.1182 \\
14.052\end{array}$ & $\begin{array}{l}14.052 \\
0.62663\end{array}$ & $\begin{array}{l}142.568 \\
14.052\end{array}$ & 14.052 & 4.65092 & 14.052 & 1.96417 & 14.052 & 3.00547 & 14.052 \\
\hline \multirow[t]{2}{*}{120.762} & 14.056 & 85.057 & 14.056 & 145.598 & 14.056 & 4.65092 & 14.056 & 4.54137 & 14.056 & 0.499752 & 14.056 \\
\hline & 2.41693 & 14.056 & 1.11461 & 14.056 & & & & & & & \\
\hline \multirow[t]{2}{*}{120.762} & 14.06 & 85.057 & 14.06 & 143.577 & 14.06 & 8.53183 & 14.06 & 0.458308 & 14.06 & 4.8466 & 14.06 \\
\hline & 2.41693 & 14.06 & 1.11461 & 14.06 & & & & & & & \\
\hline \multirow[t]{2}{*}{121.302} & 14.064 & 85.057 & 14.064 & 145.598 & 14.064 & 6.19748 & 14.064 & 1.96417 & 14.064 & 4.8466 & 14.064 \\
\hline & 2.41693 & 14.064 & 1.11461 & 14.064 & & & & & & & \\
\hline \multirow[t]{2}{*}{121.032} & 14.068 & 83.1805 & 4.068 & 144.587 & 14.068 & 7.44777 & 14.068 & 0.458308 & 14.068 & 3.00547 & 14.068 \\
\hline & 2.41693 & 14.068 & 1.11461 & 14.068 & & & & & & & \\
\hline \multirow[t]{2}{*}{121.032} & 14.072 & 84.1182 & 14.072 & 145.598 & 14.072 & 8.53183 & 14.072 & 1.96417 & 14.072 & 3.00547 & 14.072 \\
\hline & 2.41693 & 14.072 & 1.11461 & 14.072 & & & & & & & \\
\hline \multirow[t]{2}{*}{121.032} & 14.076 & 85.9968 & 14.0 & 145.598 & 14.076 & 8.53183 & 14.076 & 1.96417 & 14.076 & 3.00547 & 14.076 \\
\hline & 0.234906 & 14.076 & 1.6 & 14.076 & & & & & & & \\
\hline \multirow[t]{2}{*}{120.762} & 14.08 & 84.1182 & 14.08 & 145.598 & 14.08 & 9.52542 & 14.08 & 0.458308 & 14.08 & 4.8466 & 14.08 \\
\hline & 2.41693 & 14.08 & & 14.08 & & & & & & & \\
\hline \multirow[t]{2}{*}{121.302} & 14.084 & 84.1182 & 14. & 146.609 & 14.084 & 11.3923 & 14.084 & 1.96417 & 14.084 & 3.00547 & 14.084 \\
\hline & 2.41693 & 14.084 & & 14.084 & & & & & & & \\
\hline 121.573 & 14.088 & 84.1182 & 14. & 145.598 & 14.088 & 11.3923 & 14.088 & 1.96417 & 14.088 & 0.499752 & 14.088 \\
\hline & 4.37145 & 14.088 & & 14.088 & & & & & & & \\
\hline 121.302 & 14.0 & 05 & 14 & 146 & 14.092 & 10.4712 & 14.092 & 2.43745 & 14.092 & 4.8466 & 14.092 \\
\hline & 906 & 1 & & 14. & & & & & & & \\
\hline 121.843 & 14.096 & 5.0 & 1 & 146 & 14.09 & 1.3923 & 14.09 & 458308 & 14.096 & .09605 & 4.096 \\
\hline & 0.234906 & 14.0 & & 14. & & & & & & & \\
\hline 122.655 & 14.1 & 83.1805 & 1 & 147.621 & 14.1 & 12.3014 & 14.1 & 0.458308 & 14.1 & 0.499752 & 14.1 \\
\hline & 0.234906 & 14.1 & 461 & 14.1 & & & & & & & \\
\hline 122.655 & 14.1 & 84.1182 & 14 & 09 & 14.104 & 15.9107 & 14.104 & 0.458308 & 14.104 & 3.00547 & 14.104 \\
\hline & & & & & & & & & & & \\
\hline 123.468 & 14.108 & 83.1 & 1 & 147 & 14.108 & 23.1826 & 14.108 & 2.43745 & 14.108 & 3.00547 & 14.108 \\
\hline & & 1 & & & & & & & & & \\
\hline 123.468 & 14.1 & 81.3 & 1 & 148.634 & 14.112 & 36.1575 & 14.112 & 0.458308 & 14.112 & 0.499752 & 14.112 \\
\hline & 2.4 & 14.1 & 0.6 & 14.112 & & & & & & & \\
\hline 123.468 & 14.116 & 81.3084 & 14. & 145.598 & 14.116 & 52.3213 & 14.116 & 0.458308 & 14.116 & 1.65098 & 14.116 \\
\hline & & & & & & & & & & & \\
\hline 122.926 & 14.12 & 34 & & 148.634 & 14.12 & 72.8657 & 14.12 & 0.458308 & 14.12 & 3.00547 & 14.12 \\
\hline & & & & & & & & & & & \\
\hline 122.926 & 14.1 & 4 & & 48 & 14.12 & 97.0592 & 14.124 & 0.458308 & 14.124 & 0.499752 & 14.124 \\
\hline & 1.85 & 1 & & 14.124 & & & & & & & \\
\hline 123.739 & 14.128 & 81.3084 & 1 & 148.634 & 14.128 & 112.532 & 14.128 & 4.54137 & 14.128 & 3.00547 & 14.128 \\
\hline & 0.2 & & & & & & & & & & \\
\hline 123.739 & 14 & & & & 14.132 & 128.251 & 14.132 & 2.43745 & 14.132 & 0.499752 & 14.132 \\
\hline & & & & & & & & & & & \\
\hline 124.553 & 14.136 & 8 & & 48 & 14.13 & 136.728 & 14.1 & 1.96417 & 14.136 & 3.00547 & 14.136 \\
\hline & & & & & & & & & & & \\
\hline 124.553 & 14.14 & 81.3084 & 1 & 150.663 & 14.14 & 142.058 & 14.14 & 4.54137 & 14.14 & 3.00547 & 14.14 \\
\hline & 2.4169 & 1 & & 1414 & & & & & & & \\
\hline 124.281 & 14. & $8 \quad-$ & & 149.648 & 14.144 & 146.338 & 14.1 & 1.96417 & 14.144 & 0.499752 & 14.144 \\
\hline & & & & & & & & & & & \\
\hline 124.553 & 14.1 & 5 & & 15 & 14.14 & 147.41 & 14.148 & 2.01015 & 14.1 & 1.65098 & 14.148 \\
\hline & & & & & & & & & & & \\
\hline 124.01 & 14.15 & & & 63 & 14.152 & 151.709 & 14.152 & 0.458308 & 14.152 & 1.65098 & 14.152 \\
\hline & & & & & & & & & & & \\
\hline 124.01 & 14 & 82 & & 152.694 & 14.156 & 154.942 & 14.156 & 458308 & 14.156 & .499752 & 14.156 \\
\hline & & & & & & & & & & & \\
\hline 123.739 & 14 & 8 & & 94 & 14.16 & 159.264 & 14.1 & 1.96417 & 14.16 & 3.00547 & 14.16 \\
\hline & & & & & & & & & & & \\
\hline 124.281 & 14.164 & & & 152.694 & 14.164 & 162.515 & 14.164 & 2.01015 & 14.164 & 1.65098 & 14.164 \\
\hline & & & & & & & & & & & \\
\hline 124.824 & 14.168 & 82.2439 & 14.16 & 154.729 & 14.168 & 166.86 & 14.168 & 1.96417 & 14.168 & 0.499752 & 14.168 \\
\hline & & & & & & & & & & & \\
\hline 125.367 & & & & & $14.1^{7}$ & 170.12 & 14.17 & .458308 & $14.1^{\top}$ & 84 & 14.172 \\
\hline & & & & & & & & & & & \\
\hline 125.367 & 14.1 & 8 & & 153 & 14.176 & 173.402 & 14.176 & 1.96417 & 14.176 & 0.499752 & 14.176 \\
\hline & & & & & & & & & & & \\
\hline 124.824 & 14.18 & 81.3084 & 14.18 & 153.711 & 14.18 & 177.778 & 14.18 & 0.458308 & 14.18 & 4.8466 & 14.18 \\
\hline & 4.37145 & & & 14.1 & & & & & & & \\
\hline 125.095 & 14.184 & 82.2439 & & 153.711 & 14.184 & 182.166 & 14.184 & 2.01015 & 14.184 & 0.499752 & 14.184 \\
\hline & & & & & & & & & & & \\
\hline 24.824 & 14.188 & 81.3084 & & 153.711 & 14.188 & 185.465 & 14.188 & 0.458308 & 14.188 & 3.00547 & 14.188 \\
\hline & 2.41693 & 1 & 61 & & & & & & & & \\
\hline
\end{tabular}




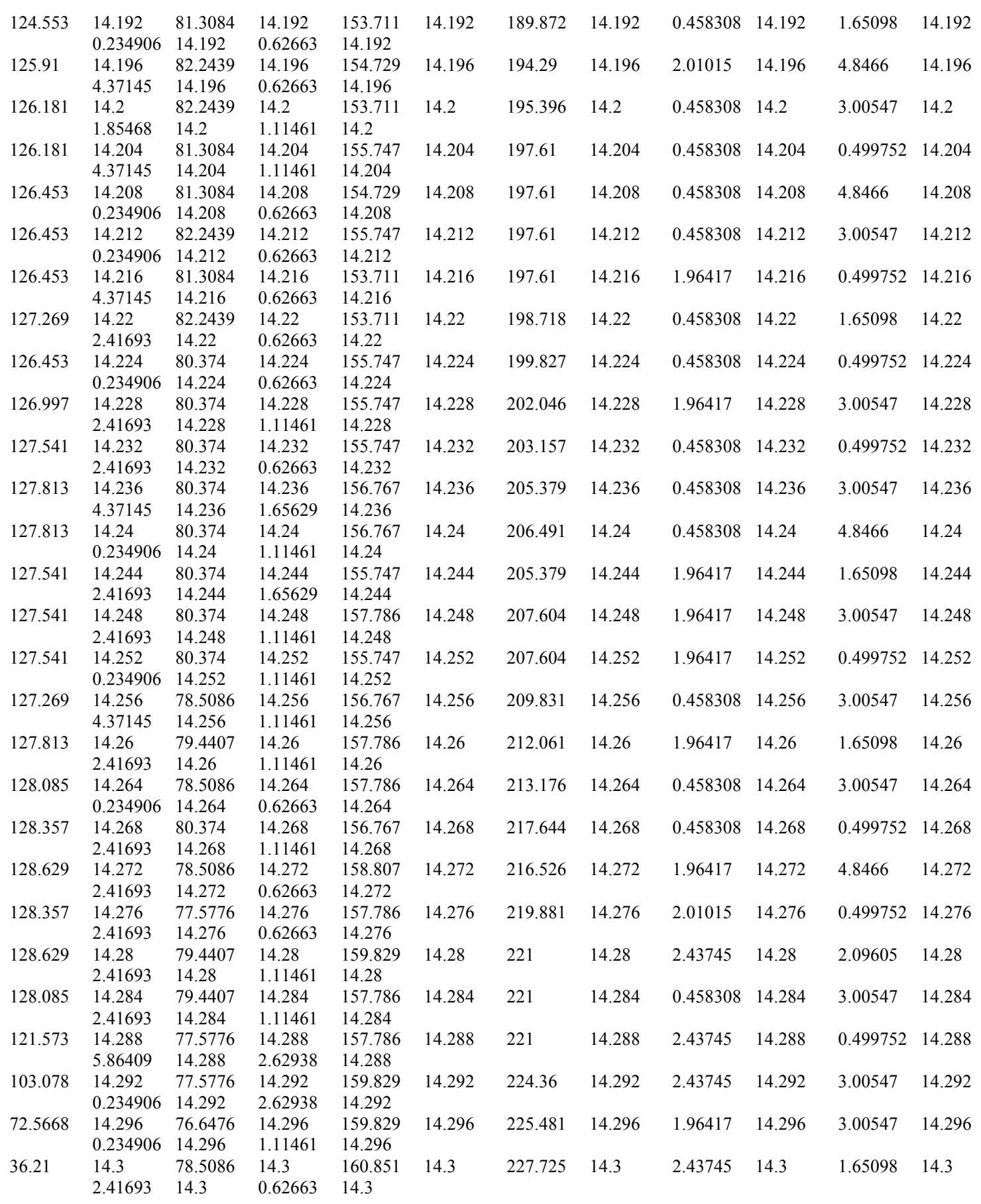


Appendix D - Gauge data for experiment 2S-47.

\begin{tabular}{|c|c|c|c|c|c|c|c|c|c|c|}
\hline-0.1000 & 0.0000 & 0.0093 & 0.0199 & 0.0103 & 0.0111 & 0.0116 & 0.0000 & 0.0126 & -0.0129 & 0.0135 \\
\hline-0.0960 & 0.0000 & 0.0093 & 0.0000 & 0.0206 & 0.0000 & 0.0232 & 0.0127 & 0.0126 & 0.0000 & 0.0000 \\
\hline-0.0920 & 0.0000 & 0.0093 & 0.0099 & 0.0103 & 0.0000 & 0.0000 & 0.0000 & 0.0251 & 0.0000 & 0.0135 \\
\hline-0.0880 & 0.0000 & 0.0187 & 0.0000 & 0.0000 & 0.0223 & 0.0116 & 0.0381 & 0.0000 & -0.0129 & 0.0000 \\
\hline-0.0840 & 0.0080 & 0.0187 & 0.0099 & 0.0103 & 0.0111 & 0.0000 & 0.0127 & 0.0126 & -0.0129 & 0.0000 \\
\hline-0.0800 & 0.0000 & 0.0093 & 0.0099 & 0.0103 & 0.0111 & 0.0000 & 0.0000 & 0.0251 & 0.0000 & 0.0000 \\
\hline-0.0760 & 0.0080 & 0.0093 & 0.0099 & 0.0103 & 0.0000 & 0.0000 & 0.0127 & 0.0126 & 0.0000 & 0.0135 \\
\hline-0.0720 & 0.0000 & 0.0187 & 0.0099 & 0.0000 & 0.0111 & 0.0116 & 0.0127 & 0.0126 & 0.0000 & 0.0135 \\
\hline-0.0680 & 0.0160 & 0.0093 & 0.0099 & 0.0000 & 0.0000 & 0.0116 & 0.0254 & 0.0126 & -0.0129 & 0.0135 \\
\hline-0.0640 & 0.0000 & 0.0000 & 0.0000 & -0.0103 & 0.0000 & 0.0116 & 0.0127 & 0.0126 & -0.0129 & 0.0135 \\
\hline-0.0600 & 0.0000 & 0.0093 & 0.0000 & 0.0103 & 0.0223 & 0.0116 & 0.0127 & 0.0126 & 0.0000 & 0.0000 \\
\hline-0.0560 & 0.0000 & 0.0093 & 0.0099 & 0.0000 & 0.0000 & 0.0116 & 0.0127 & 0.0126 & -0.0259 & 0.0135 \\
\hline-0.0520 & 0.0000 & 0.0093 & 0.0099 & 0.0000 & 0.0111 & 0.0116 & 0.0127 & 0.0126 & -0.0129 & 0.0000 \\
\hline-0.0480 & 0.0080 & 0.0093 & 0.0099 & 0.0206 & 0.0111 & 0.0116 & 0.0127 & 0.0126 & 0.0129 & 0.0000 \\
\hline-0.0440 & 0.0080 & 0.0093 & 0.0199 & 0.0206 & 0.0111 & 0.0116 & 0.0127 & 0.0126 & 0.0000 & 0.0000 \\
\hline-0.0400 & 0.0000 & 0.0093 & 0.0099 & 0.0103 & 0.0000 & 0.0000 & 0.0127 & 0.0126 & 0.0000 & -0.0135 \\
\hline-0.0360 & 0.0080 & 0.0187 & 0.0199 & 0.0206 & 0.0000 & 0.0116 & 0.0000 & 0.0126 & -0.0129 & 0.0000 \\
\hline-0.0320 & 0.0080 & 0.0187 & 0.0099 & 0.0103 & 0.0111 & 0.0116 & 0.0127 & 0.0251 & 0.0000 & 0.0000 \\
\hline-0.0280 & 0.0000 & 0.0000 & 0.0099 & 0.0103 & 0.0111 & 0.0116 & 0.0127 & 0.0126 & 0.0000 & -0.0135 \\
\hline-0.0240 & 0.0000 & 0.0093 & 0.0099 & 0.0000 & 0.0000 & 0.0116 & 0.0254 & 0.0126 & 0.0129 & 0.0000 \\
\hline-0.0200 & 0.0160 & 0.0093 & 0.0000 & 0.0000 & 0.0111 & 0.0000 & 0.0127 & 0.0126 & 0.0000 & 0.0000 \\
\hline-0.0160 & 0.0479 & 0.0093 & 0.0199 & 0.0206 & 111 & 0.0000 & 0.0127 & 0.0251 & 0.0129 & 0.0000 \\
\hline-0.0120 & -0.0400 & 0.0187 & 0.0099 & 0.0206 & 0.0000 & 0.0000 & 0.0000 & 0.0126 & 0.0000 & 0.0000 \\
\hline-0.0080 & -0.0240 & 0.0187 & 0.0199 & 0.0206 & & & 0.0127 & & 0.0000 & 0.0000 \\
\hline-0.0040 & 0.0559 & 0.0187 & -0.0099 & 0.0206 & -0.0111 & -0.0116 & 0.0000 & 0.0126 & 0.0000 & -0.0135 \\
\hline 0.0000 & 1.1344 & 0.0280 & -0.0099 & -0.0103 & -0.0111 & 0.0000 & 0.0000 & & 0.0000 & 0.0404 \\
\hline 0.0040 & 1.5262 & 0.0187 & 0.0000 & 0.0000 & 0.0000 & 0.0000 & 0.0000 & 0.0126 & 0.0000 & -0.0135 \\
\hline 0.0080 & 1.3822 & 0.0093 & & 0.0206 & -0.0111 & & 0.0000 & & & 0.0135 \\
\hline 0.0120 & 1.4147 & 0.0187 & 0.0199 & 0.0103 & 0.0111 & 0.0116 & -0.0127 & 0.0251 & 0.0000 & -0.0135 \\
\hline 0.0160 & 1.4227 & 0.0187 & 0.0099 & 0.0103 & 0.0111 & 0.0116 & 0.0254 & 0.0126 & 0.0129 & 0.0135 \\
\hline 0.0200 & 1.4383 & 0.0280 & 0.0099 & 0.0206 & 0.0111 & 0.0116 & 0.0127 & 0.0126 & 0.0000 & 0.0000 \\
\hline 0.0240 & 1.4228 & 0.0187 & -0.0099 & 0.0103 & 0.0111 & 0.0116 & 0.0127 & 0.0251 & 0.0000 & 0.0000 \\
\hline 0.0280 & 1.4305 & 0.0000 & 0.0099 & 0.0000 & 0.0000 & 0.0116 & -0.0127 & 0.0377 & 0.0000 & 0.0000 \\
\hline 0.0320 & 1.4308 & 0.0093 & 0.0099 & 0.0000 & 0.0000 & 0.0116 & 0.0000 & 0.0126 & 0.0000 & -0.0135 \\
\hline 0.0360 & & 0.0093 & 0.0099 & 0.0103 & -0.0111 & & 0.0000 & 0.0126 & 0.0000 & 0.0000 \\
\hline 0.0400 & 1.4381 & 0.0093 & 0.0099 & 0.0103 & 0.0000 & 16 & 0.0000 & 0.0126 & 0.0000 & -0.0135 \\
\hline & & & & & & & & & & 0.0000 \\
\hline 0.0480 & 1.4301 & 0.0093 & 0.0199 & 0.0000 & 0.0111 & 0.0116 & 0.0127 & 0.0251 & 0.0129 & 0.0000 \\
\hline 0.0520 & & & & & & & & & & 0.0135 \\
\hline 0.0560 & 1.4308 & 0.0187 & 0.0000 & 0.0103 & 0.0111 & 0.0000 & 0.0254 & 0.0126 & 0.0000 & 0.0000 \\
\hline & & & & & & & & & & 0.0000 \\
\hline 0.0640 & 1.4381 & 0.0187 & 0.0099 & 0.0103 & 0.0000 & 0.0232 & 0.0127 & 0.0251 & 0.0129 & 0.0000 \\
\hline 0.0680 & 1.4143 & 0.0093 & 0.0000 & 0.0103 & 0.0111 & 0.0000 & 0.0127 & 0.0126 & -0.0129 & -0.0135 \\
\hline 0.0720 & 1.4386 & 0.0000 & -0.0099 & 0.0103 & 0.0000 & 0.0116 & 0.0127 & 0.0126 & 0.0000 & 0.0135 \\
\hline 0.0760 & 1.4306 & 0.0093 & 0.0099 & 0.0103 & 0.0000 & 0.0116 & 0.0127 & 0.0126 & 0.0129 & 0.0000 \\
\hline 0.0800 & 1.4303 & 0.0093 & 0.0099 & 0.0103 & 0.0000 & 0.0116 & 0.0000 & 0.0251 & -0.0129 & 0.0000 \\
\hline 0.0840 & 1.4383 & 0.0187 & 0.0099 & 0.0206 & 0.0111 & 0.0116 & 0.0127 & 0.0000 & 0.0000 & 0.0000 \\
\hline 0.0880 & 1.4305 & 0.0093 & 0.0000 & 0.0000 & 0.0111 & 0.0000 & 0.0127 & 0.0126 & -0.0129 & 0.0000 \\
\hline 0.0920 & 1.4225 & 0.0093 & 0.0099 & 0.0103 & 0.0111 & 0.0116 & 0.0254 & 0.0251 & 0.0129 & 0.0135 \\
\hline 0.0960 & 1.4225 & 0.0093 & 0.0000 & 0.0103 & 0.0000 & 0.0000 & 0.0254 & 0.0126 & 0.0000 & 0.0000 \\
\hline 0.1000 & 1.4225 & 0.0187 & 0.0099 & 0.0000 & -0.0111 & 0.0000 & 0.0254 & 0.0126 & 0.0000 & 0.0000 \\
\hline 0.1040 & 1.4225 & 0.0093 & 0.0000 & & & 0.0116 & 0.0000 & 0.0126 & 0.0129 & 0.0000 \\
\hline 0.1080 & 1.4385 & 0.0093 & 0.0099 & 0.0000 & 0.0111 & 0.0116 & 0.0127 & 0.0000 & 0.0000 & 0.0000 \\
\hline 0.1120 & 1.4225 & 0.0187 & 0.0199 & & 0.0000 & 0.0000 & 0.0000 & 0.0126 & 0.0129 & 0.0000 \\
\hline 0.1160 & 1.4385 & 0.0187 & 0.0000 & 0.0103 & 0.0000 & 0.0116 & 0.0127 & 0.0126 & -0.0129 & 0.0000 \\
\hline 0.1200 & 1.4385 & 0.0093 & 0.0000 & 0.0103 & & & 0.0127 & 0.0126 & -0.0129 & 0.0000 \\
\hline 0.1240 & 1.4305 & 0.0093 & 0.0099 & 0.0000 & 0.0000 & 0.0116 & 0.0000 & 0.0126 & 0.0000 & -0.0135 \\
\hline 0.1280 & 1.4225 & 0.0093 & 0.0099 & 0.0103 & 0.0111 & 0.0116 & 0.0000 & 0.0126 & 0.0000 & 0.0000 \\
\hline 0.1320 & 1.4305 & 0.0093 & -0.0099 & 0.0103 & 0.0000 & 0.0116 & 0.0254 & 0.0126 & 0.0000 & 0.0000 \\
\hline 0.1360 & 1.4145 & 0.0093 & 0.0000 & 0.0103 & 0.0111 & 0.0000 & 0.0254 & 0.0126 & -0.0129 & -0.0135 \\
\hline 0.1400 & 1.4305 & 0.0187 & 0.0199 & 0.0206 & 0.0223 & 0.0116 & 0.0127 & 0.0126 & -0.0129 & 0.0135 \\
\hline 0.1440 & 1.4225 & 0.0187 & 0.0099 & 0.0103 & 0.0000 & 0.0000 & 0.0127 & 0.0126 & 0.0000 & 0.0000 \\
\hline 0.1480 & 1.4145 & 0.0093 & 0.0199 & 0.0103 & 0.0000 & 0.0116 & 0.0127 & 0.0126 & 0.0000 & -0.0135 \\
\hline 0.1520 & 1.4225 & 0.0093 & 0.0099 & 0.0000 & 0.0000 & 0.0116 & 0.0254 & 0.0251 & -0.0129 & 0.0000 \\
\hline 0.1560 & 1.4225 & 0.0093 & 0.0199 & 0.0103 & 0.0111 & 0.0116 & 0.0000 & 0.0126 & 0.0129 & 0.0000 \\
\hline 0.1600 & 1.4225 & 0.0000 & 0.0099 & 0.0206 & 0.0111 & 0.0000 & 0.0254 & 0.0000 & 0.0000 & -0.0135 \\
\hline
\end{tabular}




\begin{tabular}{|c|c|c|c|c|c|c|c|c|c|c|}
\hline 0.1640 & 1.4145 & 0.0187 & 0.0099 & 0.0206 & -0.0111 & 0.0116 & 0.0127 & 0.0126 & 0.0129 & 0.0000 \\
\hline 0.1680 & 1.4145 & 0.0187 & 0.0099 & 0.0103 & 0.0111 & 0.0116 & 0.0127 & 0.0126 & 0.0000 & 0.0000 \\
\hline 0.1720 & 1.4145 & 0093 & 0.0099 & 0.0206 & 0000 & 0.0000 & 0.0127 & 0.0251 & 0.0000 & .0000 \\
\hline 0.1760 & 1.4145 & .0093 & .0000 & 0.0103 & 0111 & 0.0116 & 0.0127 & 0.0251 & 0.0129 & .0000 \\
\hline 0.1800 & 1.4145 & 0093 & .0000 & .0103 & .0111 & .0116 & .0127 & 0.0126 & 0.0129 & .0000 \\
\hline 0.1840 & 1.4065 & 0187 & .0099 & .0103 & & & & & .0000 & 0.0000 \\
\hline 0.1880 & 1.4225 & .0187 & .0199 & 0.0000 & .0000 & & & 0126 & 0.0129 & 0.0000 \\
\hline 0.1920 & 1.4145 & .0093 & .0199 & 0103 & & & 0.0254 & 0.0377 & .0000 & 0.0000 \\
\hline 0.1960 & 14065 & 0187 & 0.0099 & 0206 & & & & 0126 & 0000 & .0000 \\
\hline 0.2000 & 4145 & 0840 & -0.0596 & -0.0206 & .0446 & 0.0232 & & & & 0000 \\
\hline 0.2040 & 1.4065 & 6255 & -0.1292 & -0.1032 & 0.0780 & -0.0463 & -0.0127 & .0377 & 0000 & .0000 \\
\hline 0.2080 & 1.4145 & & -0.0497 & -0.0516 & 0.0446 & -0.0347 & & & .0259 & 0.0269 \\
\hline 0.2120 & 1.4145 & 1.4099 & -0.0199 & & & & & & & 0.0000 \\
\hline 0.2160 & 1.3665 & 1.4379 & 0.0099 & -0.0103 & & & & 126 & 0.0388 & -0.0135 \\
\hline 0.2200 & 1.3905 & & .0099 & & & & & & 0.0129 & 0.0000 \\
\hline 0.2240 & & & & & & & & & & .0135 \\
\hline 0.2280 & 1.41 & & 0000 & & & & & & & .0135 \\
\hline 0.2320 & 1.3985 & 1.4472 & .0099 & & 00 & & & & & 0000 \\
\hline 0.2360 & 1.4065 & & .0099 & & & & & & & 0.0135 \\
\hline 0.2400 & 1.4065 & 1.4472 & 0.0099 & & & & & & & 0.0000 \\
\hline 0.2440 & 1.4065 & & .0099 & & & & & & & 0.0135 \\
\hline 0.2480 & & & & & & & & & & 0.0000 \\
\hline 0.2520 & & & & & & & & & & 0000 \\
\hline 0.2560 & 5 & & 9 & & & & & & & 0000 \\
\hline 0.2600 & & & & & & & & & & 0000 \\
\hline 0.2640 & 138 & & 99 & & & & & & & .0135 \\
\hline 0.2680 & 5 & & .0099 & & & & & & & -0.0135 \\
\hline 0.2720 & 3905 & & . 0099 & & & & & & & 0.0000 \\
\hline & & & & & & & & & & 0.0000 \\
\hline 0.2800 & & & & & & & & & & 135 \\
\hline 0.2840 & & & & & & & & & & 000 \\
\hline 0.2880 & & & & & & & & & & 135 \\
\hline 0.2920 & 101205 & & .0199 & 6 & & & & & & 0.0000 \\
\hline 0.2960 & 5 & 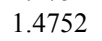 & 99 & 0 & & & & & & 000 \\
\hline 0.3000 & 13825 & & 0199 & & & & & & & .0135 \\
\hline & & & & & & & & & & \\
\hline & & & & & & & & & & 000 \\
\hline 0.3120 & 1.3905 & & 99 & & & & & & & .0000 \\
\hline 0.3160 & & & & & & & & & & 0.0000 \\
\hline & & & & & & & & & & \\
\hline 0.3240 & 3905 & 15033 & 0199 & & & & & & & 0.0000 \\
\hline 0.3280 & & & & & & & & & & 0.0000 \\
\hline & & & & & & & & & & 000 \\
\hline 0 . & & & & & & & & & & 35 \\
\hline 0.3400 & & & & & & & & & & 135 \\
\hline & & & & & & & & & & 135 \\
\hline 0.3480 & & & & .1136 & & & 31 & & & 0.0000 \\
\hline 0.3520 & & & 3813 & -0.0723 & -0.0446 & & & & & -0.0269 \\
\hline & & & & & & & & & & -0.0269 \\
\hline & & & & & & & & & & -0.0135 \\
\hline 0 & & & & & & & & & & 00 \\
\hline 0.3 & & & & & & & & & & 135 \\
\hline 0.3720 & & & & & & & & & & \\
\hline 0.3760 & & & & & & & & & & 0.0135 \\
\hline 0.3800 & & & & & & & & & & 0.0135 \\
\hline & & & & & & & & & & \\
\hline & & & & & & & & & & -0.0135 \\
\hline 0.3920 & & & & & & & & & 0 & -0.0135 \\
\hline & & & & & & & & & & -0.0135 \\
\hline 0.4000 & & & & & & & & & & 0.0000 \\
\hline 0.4040 & & & 204 & & & & & & 00 & 0.0135 \\
\hline 0.4080 & 1.3665 & & & & & & & & & 135 \\
\hline 0.4120 & & & & & & & & & & 0.0000 \\
\hline 0.4160 & & & & & & & & & & 0.0000 \\
\hline & & & & & & & & & & 0.0000 \\
\hline & & & & & & & & & & 0.0135 \\
\hline & & & & & & & & & & 0.0000 \\
\hline 0.4320 & 1.3506 & & & 0.0103 & & 0.0000 & 0.0254 & 26 & 0.0000 & 0.0135 \\
\hline 0.4360 & 1.3585 & 1.4939 & 1.5402 & 0.0103 & 0.0000 & 0.0116 & 0.0254 & 0.0251 & -0.0129 & 0.0000 \\
\hline 0.4400 & 1.3585 & 1.4846 & 1.5402 & 0.0103 & 0.0111 & 0.0116 & 0.0000 & 0.0251 & 0.0000 & 0.0000 \\
\hline
\end{tabular}




\begin{tabular}{|c|c|c|c|c|c|c|c|c|c|c|}
\hline 0.4440 & 1.3506 & 1.4846 & 1.5601 & 0.0000 & 0.0000 & 0.0116 & 0.0127 & 0.0251 & -0.0129 & 0.0135 \\
\hline 0.4480 & 1.3506 & 1.4939 & 1.5502 & -0.0103 & 0.0000 & -0.0116 & -0.0127 & 0.0126 & -0.0129 & 0.0000 \\
\hline 0.4520 & 1.3426 & 1.4939 & 1.5601 & 0.0103 & 0.0111 & 0.0000 & 0.0127 & 0.0251 & 0.0000 & 0.0000 \\
\hline 0.4560 & 1.3506 & 1.4846 & 1.5402 & .0103 & .0000 & 0.0116 & 0.0127 & .0251 & .0129 & 0.0000 \\
\hline 0.4600 & 1.3506 & 1.4846 & .5601 & .0000 & .0000 & .0000 & 0.0254 & 0.0251 & 0.0129 & 0.0135 \\
\hline 0.4640 & 1.3506 & 1.4846 & .5502 & .0103 & .0000 & .0116 & & & 0.0129 & -0.0135 \\
\hline 0.4680 & 1.3506 & 1.4846 & .5701 & 0.0103 & .0000 & & & 0.0251 & & -0.0135 \\
\hline 0.4720 & 1.3585 & 1.4846 & .5701 & .0103 & .0000 & & & 0.0251 & .0000 & 0.0000 \\
\hline 0.4760 & 1.3426 & 1.4846 & .5701 & 0103 & 0000 & 232 & & & 0000 & .0000 \\
\hline 0.4800 & 3426 & 1.4939 & 5701 & 3 & 0000 & & & & & 0000 \\
\hline 0.4840 & 1.3426 & 1.4752 & 1.5402 & 1342 & .0446 & .0232 & & 126 & 0000 & 0.0135 \\
\hline 0.4880 & 1.3346 & .4379 & .4707 & 9601 & .1115 & & & & & 0.0269 \\
\hline 0.4920 & 1.3426 & 1.4006 & & & & & & & & -0.0404 \\
\hline 0.4960 & 1.3346 & 1.4192 & .5303 & & 0.0223 & & & -0.0126 & 0.0259 & -0.0269 \\
\hline 0.5000 & 1.3346 & 1.4566 & .5601 & & .0000 & & & & & 0.0000 \\
\hline 0.5040 & & & & & & & & & & -0.0135 \\
\hline 0.5080 & 3266 & 19 & & & .0111 & & & & & 000 \\
\hline 0.5120 & 1.3266 & .4659 & 01 & & .0111 & & & & & 135 \\
\hline 0.5160 & 3266 & 4752 & & & .0000 & & & & & \\
\hline 0.5200 & 1.3186 & 1.4659 & & & & & & & & 0.0000 \\
\hline 0.5240 & 3266 & & & & .0111 & & & & & -0.0135 \\
\hline & & & & & & & & & & 000 \\
\hline 0.5320 & 66 & & & & & & & & & 000 \\
\hline 0.5360 & 46 & & & & 00 & & & & & 000 \\
\hline 0.5400 & 3106 & 4566 & 01 & & & & & & & 0000 \\
\hline 0.5440 & 3266 & .4472 & & & 0 & & & & & 0000 \\
\hline 0.5480 & 1.3106 & 14659 & & & & & & & & -0.0135 \\
\hline 0.5520 & 3186 & & & & & & & & & 0.0135 \\
\hline & & & & & & & & & & \\
\hline 0.5 & & & & & & & & & & 135 \\
\hline 0.5640 & & & & & & & & & & 135 \\
\hline 0.5680 & & & & & & & & & & \\
\hline 0.5720 & 13186 & 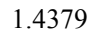 & & & 1 & & & & & 000 \\
\hline 0.5760 & 3106 & 9 & 12 & 11 & 00 & & & & & 00 \\
\hline 0.5800 & 3106 & & & & & & & & & 0135 \\
\hline & & & & & & & & & & \\
\hline & & & & & & & & & & \\
\hline 0.5920 & 1.3106 & & & & .0000 & & & & & -0.0135 \\
\hline 0.5960 & & & & & & & & & & \\
\hline & & & & & & & & & & 269 \\
\hline 0.6040 & 1.3026 & 1.4286 & .5701 & & 0.0111 & & & & & -0.0135 \\
\hline 0.6080 & & & & & & & & & & 0.0135 \\
\hline & & & & & & & & & & \\
\hline 0.6 & 46 & & & & & & & & & 35 \\
\hline 0.6200 & 46 & & & & & & & & & 0.0000 \\
\hline 0.62 & & & & & & & & & & 0.0135 \\
\hline 0.6280 & & & & & & & & & & -0.0269 \\
\hline 0.6320 & 1.3026 & & & & 1.6386 & -0.0 & & & & -0.0135 \\
\hline & & & & & & & & & & \\
\hline 0.6 & & & & & & & & & & 000 \\
\hline 0.6440 & & & & & & & & & & 135 \\
\hline 0.6480 & & & & & & & & & & -0.0269 \\
\hline 0.6520 & & & & & & & & & & -0.0135 \\
\hline 0.6560 & 1.2866 & & & & & -0.0116 & & & & -0.0135 \\
\hline 0.6600 & & & & & & & & & & 0.0000 \\
\hline & & & & & & & & & & 0.0135 \\
\hline 0.6680 & & & & & & & & & & \\
\hline 0.6720 & & & & & & & & & & 0.0135 \\
\hline & & & & & & & & & 00 & -0.0135 \\
\hline 0.6800 & & & & & & & & & & 0.0135 \\
\hline 0.6840 & 1.2946 & 12 & & & 1.7389 & & & & -0.0129 & 0.0135 \\
\hline 0.6880 & 1.2866 & & & & & & & & 00 & -0.0135 \\
\hline 0.6920 & & & & & & & & & & 0.0000 \\
\hline 0.6960 & & & & & & & & & & 0.0000 \\
\hline & & & & & & & & & & 0.0000 \\
\hline & & & & & & & & & & 0.0000 \\
\hline & & & & & & & & & & 0.0000 \\
\hline 0.7120 & 1.2866 & & 1.5 & 1 & 1.7612 & 16 & & 77 & 0.0259 & -0.0135 \\
\hline 0.7160 & 1.2786 & 1.3632 & 1.4806 & 1.6414 & 1.7724 & 0.0116 & 0.0127 & 0.0251 & 0.0000 & -0.0135 \\
\hline 0.7200 & 1.2786 & 1.3632 & 1.5005 & 1.6414 & 1.7724 & 0.0000 & 0.0127 & 0.0000 & 0.0000 & 0.0269 \\
\hline
\end{tabular}




\begin{tabular}{|c|c|c|c|c|c|c|c|c|c|c|}
\hline 0.7240 & 1.2866 & 1.3632 & 1.4906 & 1.6414 & 1.7724 & 0.0116 & 0.0000 & 0.0126 & -0.0129 & 0.0135 \\
\hline 0.7280 & 1.2866 & 1.3725 & 1.4806 & 1.6414 & 1.7724 & 0.0116 & 0.0000 & 0.0126 & -0.0129 & 0.0000 \\
\hline 0.7320 & 1.2786 & 1.3725 & 1.4707 & 1.6311 & 1.7835 & 0.0116 & 0.0000 & 0.0126 & 0.0129 & 0.0000 \\
\hline 0.7360 & 1.2786 & 1.3632 & 1.4806 & 1.6311 & 1.7835 & 0.0116 & 0.0254 & 0.0251 & 0.0129 & 0.0000 \\
\hline 0.7400 & 1.2786 & 1.3632 & 1.4806 & 1.6311 & 1.7724 & 0.0000 & 0.0127 & 0.0126 & 0.0000 & 0.0000 \\
\hline 0.7440 & 1.2786 & 1.3539 & 1.4707 & 1.6311 & 1.7724 & 0.0232 & 0.0000 & 0.0251 & -0.0129 & 0.0000 \\
\hline 0.7480 & 1.2786 & 1.3539 & 1.4806 & 1.6208 & 1.7835 & 0.0116 & 0.0127 & 0.0126 & 0.0129 & -0.0135 \\
\hline 0.7520 & 1.2786 & 1.3539 & 1.4707 & 1.6208 & 1.7835 & 0.0116 & 0.0254 & 0.0251 & 0.0000 & 0.0000 \\
\hline 0.7560 & 1.2627 & 1.3539 & 1.4409 & 1.6105 & 1.7055 & 0.7412 & -0.0127 & -0.0251 & 0.0000 & 0.0000 \\
\hline 0.7600 & 1.2786 & 1.3259 & 1.4111 & 1.5279 & 1.6832 & 1.5982 & -0.0890 & -0.0879 & -0.0129 & -0.0135 \\
\hline 0.7640 & 1.2786 & 1.2979 & 1.4210 & 1.5588 & 1.7278 & 1.7603 & -0.0636 & -0.0377 & -0.0388 & -0.0538 \\
\hline 0.7680 & 1.2627 & 1.3072 & 1.4309 & 1.5795 & 1.7501 & 1.7603 & -0.0254 & -0.0126 & -0.0517 & -0.0269 \\
\hline 0.7720 & 1.2627 & 1.3352 & 1.4309 & 1.5898 & 1.7612 & 1.7834 & 0.0000 & 0.0000 & -0.0259 & 0.0000 \\
\hline 0.7760 & 1.2627 & 1.3352 & 1.4409 & 1.5898 & 1.7612 & 1.8182 & -0.0127 & 0.0000 & 0.0000 & -0.0135 \\
\hline 0.7800 & 1.2627 & 1.3259 & 1.4508 & 1.5898 & 1.7612 & 1.8413 & 0.0000 & 0.0126 & -0.0129 & -0.0269 \\
\hline 0.7840 & 1.2627 & 1.3259 & 1.4409 & 1.5898 & 1.7612 & 1.8645 & 0.0000 & 0.0126 & -0.0129 & -0.013 \\
\hline 0.7880 & 1.2707 & 1.3352 & 1.4409 & 1.5898 & 1.7612 & 1.8877 & 0.0000 & 0.0126 & -0.0129 & 0.0000 \\
\hline 0.7920 & 1.2707 & 1.3259 & 1.4309 & 1.5898 & 1.7501 & 1.8645 & 0.0127 & 0.0126 & -0.0129 & 0.0000 \\
\hline 0.7960 & 1.2707 & 1.3352 & 1.4409 & 1.6001 & 1.7501 & 1.8877 & 0.0000 & 0.0126 & 0.0000 & 0.0135 \\
\hline 0.8000 & 1.2627 & 1.3259 & 1.4309 & 1.5898 & 1.7501 & 1.8993 & 0.0127 & 0.0126 & 0.0000 & -0.0135 \\
\hline 0.8040 & 1.2707 & 1.3165 & 1.4409 & 1.5692 & 1.7389 & 1.9108 & 0.0000 & 0.0126 & -0.0129 & -0.013 \\
\hline 0.8080 & 1.2467 & 1.3259 & 1.4210 & 1.5692 & 1.7389 & & 0.0127 & 0.0126 & -0.0129 & 0.0000 \\
\hline 0.8120 & 1.2547 & 1.3165 & 1.4111 & 1.5692 & 1.7389 & 1.8993 & 0.0000 & 0.0126 & 0.0000 & -0.0135 \\
\hline 0.8160 & 1.2547 & 1.3165 & 1.4309 & 1.5692 & 1.7278 & 1.9108 & 0.0000 & 0.0126 & 0.0000 & -0.0135 \\
\hline 0.8200 & 1.2627 & 1.3072 & 1.4309 & 1.5588 & 1.7278 & 1.8993 & 0.0000 & 0.0000 & 0.0000 & -0.0135 \\
\hline 0.8240 & 1.2547 & 1.3165 & 1.4210 & 1.5485 & 1.7278 & 1.9108 & 0.0000 & 0.0126 & 0.0000 & 0.0135 \\
\hline 0.8280 & 1.2627 & 1.3259 & 1.4111 & 1.5588 & 1.7166 & 1.9108 & 0.0127 & 0.0126 & 0.0000 & 0.0135 \\
\hline 0.8320 & 1.2627 & 1.3072 & 1.3912 & 1.5485 & 1.7166 & 1.8993 & 0.0000 & 0.0126 & 0.0000 & -0.0135 \\
\hline 0.8360 & 1.2547 & 1.3072 & 1.4111 & 1.5485 & 1.6943 & 1.8993 & 0.0000 & 0.0251 & 0.0129 & 0.0000 \\
\hline 0.8400 & 1.2547 & 1.3165 & 1.4011 & 1.5382 & 1.7055 & 1.8993 & 0.0127 & 0.0000 & 0.0129 & 0.0000 \\
\hline 0.8440 & 1.2467 & 1.3072 & 1.4111 & 1.5485 & 1.6609 & 1.9108 & 0.0254 & 0.0126 & -0.0129 & -0.0135 \\
\hline 0.8480 & 1.2467 & 1.2979 & 1.4011 & 1.5382 & 1.6609 & 1.8993 & 0.0127 & 377 & 0.0000 & 0.0000 \\
\hline 0.8520 & 1.2627 & 1.2979 & & 1.5279 & & & & & 0.0000 & 0.0135 \\
\hline 0.8560 & 1.2547 & 1.3072 & 1.3912 & 1.5175 & 1.6720 & 1.8877 & 0.0000 & 0.0126 & -0.0129 & 0.0000 \\
\hline 0.8600 & 1.2707 & 1.2979 & 1.3912 & & & & 0.0127 & 0.0000 & 0.0000 & 0.0000 \\
\hline 0.8640 & 1.2547 & 1.2885 & 1.3912 & 1.5279 & 1.6609 & 1.8761 & 0.0254 & 0.0126 & 0.0000 & 0.0135 \\
\hline 0.8680 & 1.2547 & 1.2885 & 1.3912 & & & & & & & \\
\hline 0.8720 & 1.2547 & 1.2979 & 1.3912 & 1.5175 & 1.6497 & 1.8645 & 0.0127 & 0.0126 & 0.0000 & -0.0135 \\
\hline 0.8760 & 1.2467 & 1.2979 & 1.3813 & 1.5072 & 1.6609 & 1.8529 & 0.0127 & 0.0251 & 0.0000 & 0.0000 \\
\hline 0.8800 & 1.2467 & 1.2885 & 1.3713 & 1.4762 & 1.5940 & 1.7603 & 0.1398 & -0.0251 & 0.0000 & 0.0135 \\
\hline 0.8840 & 1.2467 & 1.2885 & 1.3415 & 1.4453 & 1.5717 & 1.7487 & 1.3220 & -0.1130 & -0.0259 & -0.0135 \\
\hline 0.8880 & 1.2547 & 1.2605 & 1.3415 & 1.4453 & 1.5940 & 1.7950 & 1.9576 & -0.0377 & -0.1552 & -0.0404 \\
\hline 0.8920 & 1.2467 & 1.2792 & 1.3515 & 1.4762 & 1.6163 & 1.8066 & 2.0593 & 0.0000 & -0.0388 & -0.0135 \\
\hline 0.8960 & 1.2627 & 1.2792 & 1.3614 & 1.4762 & 1.6163 & 1.8066 & 2.0593 & 0.0000 & 0.0000 & 0.0000 \\
\hline 0.9000 & 1.2467 & 1.2885 & 1.3614 & 1.4659 & 1.6163 & 1.7950 & 2.0848 & 0.0000 & -0.0129 & -0.0135 \\
\hline 0.9040 & 1.2467 & 1.2885 & & & & & & & -0.0129 & 0.0000 \\
\hline 0.9080 & 1.2467 & 1.2792 & 1.3515 & 1.4762 & 1.6052 & 1.8066 & 2.1102 & 0.0126 & -0.0129 & 0.0135 \\
\hline 0.9120 & 1.2387 & 1.2699 & & & & & & & -0.0129 & 0.0000 \\
\hline 0.9160 & 1.2387 & 1.2792 & 1.3515 & 1.4659 & 1.6052 & 1.7834 & 2.0975 & 0.0251 & -0.0129 & 0.0000 \\
\hline 0.9200 & 1.2467 & 1.2792 & 1.3316 & 1.4659 & 1.5940 & & & & 0.0000 & -0.0269 \\
\hline 0.9240 & 1.2387 & 1.2792 & 1.3415 & 1.4659 & 1.5940 & 1.7603 & 2.0466 & 0.0251 & 0.0000 & 0.0000 \\
\hline 0.9280 & 1.2387 & 1.2792 & 1.3316 & 1.4556 & 1.5829 & 1.7487 & 2.0466 & 0.0251 & -0.0129 & 0.0135 \\
\hline 0.9320 & 1.2387 & 1.2699 & 1.3316 & 1.4556 & 1.5829 & 1.7487 & 2.0212 & 0.0126 & -0.0129 & 0.0000 \\
\hline 0.9360 & 1.2307 & 1.2605 & 1.3316 & 1.4453 & 1.5829 & 1.7603 & 2.0085 & 0.0251 & 0.0000 & 0.0135 \\
\hline 0.9400 & 1.2227 & 1.2605 & 1.3316 & 1.4453 & 1.5717 & 1.7371 & 2.0085 & 0.0126 & 0.0000 & 0.0000 \\
\hline 0.9440 & 1.2307 & 1.2699 & 1.3316 & 1.4349 & 1.5717 & 1.7255 & 1.9958 & 0.0126 & -0.0129 & -0.0135 \\
\hline 0.9480 & 1.2307 & 1.2699 & 1.3217 & 1.4349 & 1.5606 & 1.7255 & 1.9704 & 0.0126 & 0.0000 & 0.0000 \\
\hline 0.9520 & 1.2307 & 1.2605 & 1.3316 & 1.4349 & 1.5606 & 1.7255 & 1.9576 & 0.0251 & 0.0000 & -0.0135 \\
\hline 0.9560 & 1.2307 & 1.2699 & 1.3316 & 1.4349 & 1.5606 & 1.7255 & 1.9449 & 0.0126 & -0.0129 & 0.0000 \\
\hline 0.9600 & 1.2307 & 1.2418 & 1.3217 & 1.4349 & 1.5494 & 1.7140 & 1.9322 & 0.0251 & 0.0000 & 0.0000 \\
\hline 0.9640 & 1.2227 & 1.2699 & 1.3217 & 1.4040 & 1.5383 & 1.7024 & 1.9322 & 0.0251 & -0.0259 & \\
\hline 0.9680 & 1.2227 & 1.2699 & 1.3117 & 1.4040 & 1.5494 & 1.7024 & 1.9068 & 0.0251 & -0.0129 & -0.013 \\
\hline 0.9720 & 1.2227 & 1.2605 & 1.3117 & 1.4040 & 1.5383 & 1.6908 & 1.8941 & 0.0251 & 0.0129 & -0.013 \\
\hline 0.9760 & 1.2227 & 1.2512 & 1.3117 & 1.4143 & 1.5383 & 1.6676 & 1.8941 & 0.0126 & -0.0129 & 0.0000 \\
\hline 0.9800 & 1.2147 & 1.2605 & 1.3117 & 1.4143 & 1.5383 & 1.6561 & 1.8941 & 0.0126 & -0.0129 & 0.0135 \\
\hline 0.9840 & 1.2067 & 1.2605 & 1.3018 & 1.3937 & 1.5160 & 1.6561 & 1.8814 & 0.0126 & 0.0129 & 0.0135 \\
\hline 0.9880 & 1.2227 & 1.2512 & 1.3018 & 1.3937 & 1.5048 & 1.6213 & 1.8432 & 0.2135 & 0.0129 & 0.0135 \\
\hline 0.9920 & 1.2227 & 1.2325 & 1.2819 & 1.3420 & 1.4602 & 1.5634 & 1.7543 & 1.8085 & -0.0129 & -0.0135 \\
\hline 0.9960 & 1.1987 & 1.2325 & 1.2720 & 1.3627 & 1.4714 & 1.6097 & 1.7543 & 2.2356 & -0.1423 & -0.080 \\
\hline 1.0000 & 1.1987 & 1.2138 & 1.2720 & 1.3524 & 1.4825 & 1.6445 & 1.8051 & 2.2356 & -0.0647 & -0.053 \\
\hline
\end{tabular}




\begin{tabular}{|c|c|c|c|c|c|c|c|c|c|c|}
\hline 1.0040 & 1.1987 & 1.2325 & 1.2819 & 1.3937 & 1.4937 & 1.6329 & 1.8178 & 2.1728 & 0.0000 & -0.0135 \\
\hline 1.0080 & 1.1987 & 1.2325 & 1.2819 & 1.3730 & 1.4825 & 1.6329 & 1.8178 & 2.1225 & 0.0129 & 0.0135 \\
\hline 1.0120 & 1.1987 & 1.2418 & 1.2918 & 1.3833 & 1.4825 & 1.6329 & 1.8178 & 2.1100 & -0.0129 & 0.0135 \\
\hline 1.0160 & 1.1987 & 1.2418 & 1.2819 & 1.3833 & 1.4825 & 1.6097 & 1.8051 & 2.0848 & -0.0129 & 0.0135 \\
\hline 1.0200 & 1.1987 & 1.2418 & 1.2720 & 1.3730 & 1.4825 & 1.6213 & 1.7924 & 2.0597 & 0.0000 & 0.0000 \\
\hline 1.0240 & 1.1987 & 1.2325 & 1.2819 & 1.3833 & 1.4825 & 1.6213 & 1.7924 & 2.0220 & 0.0000 & 0.0000 \\
\hline 1.0280 & 1.1987 & 1.2325 & 1.2918 & 1.3524 & 1.4825 & 1.6097 & 1.7924 & 2.0095 & -0.0129 & -0.0135 \\
\hline 1.0320 & 1.1908 & 1.2325 & 1.2720 & 1.3627 & 1.4825 & 1.6213 & 1.7924 & 1.9844 & 0.0129 & 0.0000 \\
\hline 1.0360 & 1.2147 & 1.2232 & 1.2720 & 1.3627 & .4714 & 1.5982 & 1.7670 & 1.9718 & 0.0000 & 0.0000 \\
\hline 1.0400 & 1.1987 & 1.2232 & 1.2 & 1.3627 & 1.4714 & 1.6097 & & & 29 & 0.0135 \\
\hline 1.0440 & 1.2067 & 1.2232 & 1.2819 & 1.3627 & 1.4379 & 1.5866 & 1.7543 & 216 & 0.0000 & 0.0135 \\
\hline 1.0480 & 1.1987 & 1.2232 & .2620 & 1.3420 & 1.4491 & 1.5982 & 1.7415 & & 0.0000 & 0.0135 \\
\hline 1.0520 & 1.1987 & 1.2232 & .2620 & 1.3420 & 1.4491 & 1.5866 & 1.7415 & & & 0.0000 \\
\hline 1.0560 & 1.1908 & 1.2232 & 1.2521 & 1.3420 & 1.4379 & 1.5866 & & & 0.0129 & 0.0000 \\
\hline 1.0600 & 1.1987 & 1.2232 & & & & & & & & 0.0000 \\
\hline 1.0640 & & & & & & & & & & .0135 \\
\hline 1.0680 & 1.1908 & 1.2232 & & 7 & 1.4268 & & & & & 0135 \\
\hline 1.0720 & 1.1828 & 1.2232 & 1.2422 & 17 & .4268 & & 1.7161 & & & 0.0000 \\
\hline 1.0760 & 1.1828 & 1.2138 & & & & & & & & -0.0135 \\
\hline 1.0800 & 1.1908 & 1.2138 & 1.2521 & 1.3214 & & & & & & 0.0000 \\
\hline 1.0840 & 1.1908 & 1.2045 & & & & & & & & 0.0135 \\
\hline & & & & & & & & & & -0.0269 \\
\hline 1.0920 & 1.1 & 1.2 & & & & & & & & 000 \\
\hline 1.0960 & 1.1828 & 1.2045 & 23 & & 12 & & & & & -0.0539 \\
\hline 1.1000 & 1.1828 & 1.2045 & .2223 & 3008 & 1.3823 & 1.5171 & 1.6 & & & -0.0942 \\
\hline 1.1040 & 1.1748 & 1.1952 & & 3111 & 1.3935 & 1.5287 & 1. & & & -0.0269 \\
\hline 1.1080 & 1.1668 & 1.1952 & 1.2422 & .3111 & 1.3935 & 1.5287 & & & & 0.0135 \\
\hline 1.1120 & 1.1588 & 1.2045 & & & 1.4045 & 1.5287 & & & & 0.0135 \\
\hline & & & & & & & & & & 0.0000 \\
\hline 0 & 1.1 & & & & & & & & & 000 \\
\hline 1.1240 & 1.15 & 1.2 & & & & & & & & -0.0135 \\
\hline 1.1280 & 1.1588 & 1.1952 & 22 & 111 & 1.3823 & 87 & 1.6 & & & 0.0135 \\
\hline 1.1320 & 1.1668 & 1.1952 & & & 1.4045 & & 16 & & & 0.0000 \\
\hline 1.1360 & 1.1668 & 1.2045 & 22 & 04 & 1.3823 & 55 & 1.6 & & & 0.0135 \\
\hline 1.1400 & 1.1748 & 15 & & & 1.3823 & 1.5055 & & & & 0.0000 \\
\hline & & & & & & & & & & \\
\hline 1.1 & 1 & & & & & & & & & 135 \\
\hline 1.1520 & 1.1588 & 1.1952 & & & 1.3600 & & & & & 0.0000 \\
\hline 11560 & & & & & & & & & & 0.0000 \\
\hline & & & & & & & & & & -0.0135 \\
\hline 1.1640 & 1.1668 & 1.1858 & 1.2322 & 1.2904 & 1.3712 & 1.4823 & 1.6017 & & 1.9016 & -0.0135 \\
\hline 1.1680 & & & & & & & & & & -0.0135 \\
\hline & & & & & & & & & & 00 \\
\hline 1.1760 & 1 & & & & & & & & & 00 \\
\hline 1.1800 & 1.1588 & 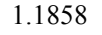 & & & & & 90 & & & 0.0135 \\
\hline 1.1840 & 1.1588 & & & & & & & & & 0.0000 \\
\hline & 1.15 & & 23 & 2 & 1.36 & & & & & -0.0135 \\
\hline 1.1920 & 1.1508 & 1.1858 & & & 1.3489 & 1.4592 & & & & 0.0000 \\
\hline & & & & & & & & & & -0.0269 \\
\hline 1 ? & & & & & & & & & & 0.2020 \\
\hline O & & & & & & & & & & 1.4541 \\
\hline & 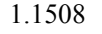 & & & & & & & & & 1.9792 \\
\hline & & & & & & & & & & 1.9792 \\
\hline 1.2160 & 1.1508 & 1.1672 & 1.2024 & 1.2699 & 1.3377 & 1.4244 & & & 111 & 1.9119 \\
\hline 1.2200 & & & & & & & & & & 1.8984 \\
\hline & & & & & & & & & & 1.8715 \\
\hline 1 & & & & & & & & & & 1.8446 \\
\hline 1.2320 & 0 & & & & & & & & 23 & 1.8311 \\
\hline & & 1.1672 & & & & & & & & 1.8042 \\
\hline 1.2400 & 1.1 & 1.1 & 1.2 & 1.2595 & 1.3377 & & & & & 1.7907 \\
\hline 1.2440 & 1.1268 & 1.1672 & 1.2024 & 1.2595 & 1.3266 & 1.4244 & 1.5000 & 1.6327 & 1.7593 & 1.7907 \\
\hline 1.2480 & 1.1428 & & & & 1.3266 & 1.4244 & 1.5000 & & 64 & 1.7638 \\
\hline 1.2520 & 1.1348 & & & & & & & & & 1.7638 \\
\hline 1.2560 & & & & & & & & & & 1.7503 \\
\hline & & & & & & & & & 7464 & 1.7234 \\
\hline & & 1 & & 2595 & 1.3266 & 1.4129 & 1.4873 & 76 & 335 & 1.7234 \\
\hline & & & & & & & & & & 1.7099 \\
\hline 1.2720 & 1.1268 & 1.1578 & 1.2024 & 1.2699 & 1.3377 & 1.3897 & & 1.5950 & 1.7076 & 1.7099 \\
\hline 1.2760 & 1.1268 & 1.1672 & 1.2024 & 1.2595 & 1.3377 & 1.4013 & 1.4746 & 1.5950 & 1.7205 & 1.7099 \\
\hline 1.2800 & 1.1268 & 1.1672 & 1.1726 & 1.2699 & 1.3377 & 1.4013 & 1.4873 & 1.5824 & 1.7076 & 1.6965 \\
\hline
\end{tabular}




\begin{tabular}{|c|c|c|c|c|c|c|c|c|c|c|}
\hline 1.2840 & 1.1188 & 1.1672 & 1.1925 & 1.2492 & 1.3154 & 1.3781 & 1.4619 & 1.5824 & 1.6947 & 1.7099 \\
\hline 1.2880 & 1.1188 & 1.1672 & 1.1925 & 1.2492 & 1.3266 & 1.3781 & 1.4746 & 1.5824 & 1.6947 & 1.7099 \\
\hline 1.2920 & 1.1188 & 1.1672 & 1.1825 & 1.2595 & 1.3154 & 1.3897 & 1.4619 & 1.5699 & 1.6947 & 1.6830 \\
\hline 1.2960 & 1.1188 & 1.1578 & 1.2024 & 1.2595 & 1.3043 & 1.3781 & 1.4619 & 1.5824 & 1.6817 & 1.6965 \\
\hline 1.3000 & 1.1188 & 1.1578 & 1.1925 & 1.2595 & 1.3043 & 1.3781 & 1.4619 & 1.5573 & 1.6817 & 1.6830 \\
\hline 1.3040 & 1.1188 & 1.1485 & 1.1925 & 1.2389 & 1.3154 & 1.3781 & 1.4619 & 1.5573 & 1.6558 & 1.6830 \\
\hline 1.3080 & 1.1188 & 1.1765 & 1.2024 & 1.2595 & 1.3154 & 1.3781 & 1.4492 & 1.5699 & 1.6558 & 1.6830 \\
\hline 1.3120 & 1.1108 & 1.1485 & 1.2024 & 1.2595 & 1.3154 & 1.3781 & 1.4619 & 1.5824 & 1.6688 & 1.6561 \\
\hline 1.3160 & 1.1108 & 1.1672 & 1.2024 & 1.2595 & 1.3043 & 1.3781 & 1.4365 & 1.5573 & 1.6429 & 1.6561 \\
\hline 1.3200 & 1.1188 & 1.1672 & 1.1925 & 1.2492 & 1.3043 & 1.3665 & 1.4492 & 1.5573 & 1.6170 & 1.6561 \\
\hline 1.3240 & 1.1268 & 1.1672 & 1.2024 & 1.2595 & 1.3043 & 1.3550 & 1.4492 & 1.5322 & 1.6170 & 1.6426 \\
\hline 1.3280 & 1.1188 & 1.1672 & 1.2124 & 1.2595 & 1.3043 & 1.3550 & 1.4365 & 1.5448 & 1.6558 & 1.6561 \\
\hline 1.3320 & 1.1188 & 1.1578 & 1.1925 & 1.2595 & 1.2931 & 1.3665 & 1.4365 & 1.5322 & 1.6558 & 1.6291 \\
\hline 1.3360 & 1.1188 & 1.1485 & 1.1925 & 1.2595 & 1.2931 & 1.3550 & 1.4365 & 1.5322 & 1.6300 & 1.6426 \\
\hline 1.3400 & 1.1348 & 1.1485 & 1.2024 & 1.2595 & 1.2931 & 1.3550 & 1.4237 & 1.5322 & 1.6170 & 1.6291 \\
\hline 1.3440 & 1.1267 & 1.1578 & 1.1925 & 1.2595 & 1.2931 & 1.3665 & 1.4365 & 1.5322 & 1.6300 & 1.6291 \\
\hline 1.3480 & 1.1187 & 1.1578 & 1.2124 & 1.2492 & 1.2931 & 1.3434 & 1.4237 & 1.5322 & 1.6170 & 1.6291 \\
\hline 1.3520 & 1.1267 & 1.1485 & 1.1925 & 1.2698 & 1.2820 & 1.3550 & 1.4110 & 1.5322 & 1.6300 & 1.6157 \\
\hline 1.3560 & 1.1347 & 1.1485 & 1.2124 & 1.2698 & 1.2931 & 1.3318 & 1.4237 & 1.5196 & 1.6041 & 1.6157 \\
\hline 1.3600 & 1.1347 & 1.1578 & 1.2124 & 1.2698 & 1.2931 & 1.3318 & 1.4110 & 1.5322 & 1.5912 & 1.6022 \\
\hline 1.3640 & 1.1347 & 1.1578 & 1.2124 & 1.2594 & 1.2820 & 1.3434 & 1.4110 & 1.5196 & 1.6041 & 1.5887 \\
\hline 1.3680 & 1.1347 & 1.1578 & 1.1925 & 1.2491 & 1.2820 & 1.3550 & 1.4110 & 1.5196 & 1.6170 & 1.6022 \\
\hline 1.3720 & 1.1428 & 1.1578 & 1.2124 & 1.2491 & 1.2931 & 1.3318 & 1.4110 & 1.4945 & 1.6170 & 1.6022 \\
\hline 1.3760 & 1.1268 & 1.1485 & 1.2024 & 1.2594 & 1.2820 & 1.3318 & 1.4110 & 1.4945 & 1.6041 & 1.6022 \\
\hline 1.3800 & 1.1428 & 1.1485 & 1.2124 & 1.2594 & 1.2819 & 1.3434 & 1.4110 & 1.5071 & 1.6041 & 1.6022 \\
\hline 1.3840 & 1.1348 & 1.1672 & 1.1925 & 1.2594 & 1.2707 & 1.3318 & 1.3983 & 1.5071 & 1.6041 & 1.6022 \\
\hline 1.3880 & 1.1428 & 1.1485 & 1.2024 & 1.2698 & 1.2819 & 1.3434 & 1.3983 & 1.4945 & 1.6041 & 1.6022 \\
\hline 1.3920 & 1.1588 & 1.1578 & 1.2024 & 1.2594 & 1.2707 & 1.3434 & 1.3983 & 1.4820 & 1.5912 & 1.5753 \\
\hline 1.3960 & 1.1508 & 1.1672 & 1.2124 & 1.2698 & 1.2707 & 1.3202 & 1.3983 & 1.4820 & 1.5912 & 1.5887 \\
\hline 1.4000 & 1.1508 & 1.1578 & 1.2 & 1.2 & 1.2 & 1.3 & 1.3 & 1.4945 & 1.5 & 1.6022 \\
\hline 1.4040 & 1.1508 & 1.1672 & 1.1 & 1.2698 & 1.2596 & 1.3318 & 1.3856 & 1.4820 & 1.5 & 1.5618 \\
\hline 1.4080 & 1.1503 & 1.1391 & 1.1925 & 1.2594 & 1.2819 & 1.3202 & 1.3856 & 1.4820 & 1.5524 & 1.5618 \\
\hline 1.4120 & 1.1507 & 1.1485 & 1.2124 & 1.2594 & 1.2596 & 1.3318 & 1.3856 & 1.4694 & 1.5782 & 1.5483 \\
\hline 1.4160 & 1.1503 & 1.1578 & 1.2024 & 1.2698 & 1.2596 & 1.3202 & 1.3729 & 1.4694 & 1.5912 & 1.5349 \\
\hline 1.4200 & 1.1589 & 1.1578 & 1.2024 & 1.2698 & 1.2707 & 1.3318 & 1.3729 & 1.4820 & 1.5782 & 1.5214 \\
\hline 1.4240 & 1.1583 & 1.1578 & 1.2024 & 1.2698 & 1.2707 & 1.3202 & 1.3729 & 1.4694 & 1.5912 & 1.5214 \\
\hline 1.4280 & 1.1587 & 1.1 & 1.2 & 1.2 & 1.2 & 1.3 & 29 & 68 & 1. & 1.5214 \\
\hline 1.4320 & 1.1509 & 1.1672 & 1.2124 & 1.2801 & 1.2707 & 1.3318 & 1.3856 & 1.4568 & 1.5912 & 1.5483 \\
\hline 1.4360 & 1.1583 & 1.1672 & 1.1925 & 1.2698 & 1.2819 & 1.3202 & 1.3729 & 1.4568 & 1.5912 & 1.5214 \\
\hline 1.4400 & 1.1505 & 1.1485 & 1.1925 & 1.2594 & 1.2819 & 1.3318 & 1.3602 & 1.4568 & 1.5782 & 1.5214 \\
\hline 1.4440 & 1.1509 & 1.1672 & 1.2124 & 1.2594 & 1.2819 & 1.3318 & 1.3602 & 1.4443 & 1.5782 & 1.5079 \\
\hline 1.4480 & 1.1431 & 1.1578 & 1.2124 & 1.2594 & 1.2597 & 1.3202 & 1.3729 & 1.4192 & 1.5782 & 1.5214 \\
\hline 1.4520 & 1.1587 & 1.1578 & 1.2024 & 1.2797 & 1.2708 & 1.3318 & 1.3602 & 1.4443 & .5653 & 1.5079 \\
\hline 1.4560 & 1.1510 & 1.1578 & 1.2024 & 1.2801 & 1.2708 & 1.3318 & 1.3475 & 1.4568 & 1.5 & 1.5079 \\
\hline 1.4600 & 1.1432 & 1.1485 & 1.2024 & 1.2797 & 1.2708 & 1.3318 & 1.3602 & 1.4317 & 1.5782 & 1.5079 \\
\hline 1.4640 & 1.1425 & 1.1578 & 1.2024 & 1.2698 & 1.2820 & 1.3434 & 1.3602 & 1.4192 & 1.5912 & 1.4945 \\
\hline 1.4680 & 1.1429 & 1.1672 & 1.2124 & 1.2694 & 1.2597 & 1.3318 & 1.3475 & 1.4192 & 1.5912 & 1.4810 \\
\hline 1.4720 & 1.1351 & 1.1578 & 1.2024 & 1.2591 & 1.2820 & 1.3086 & 1.3729 & 1.4317 & 1.5912 & 1.4810 \\
\hline 1.4760 & 1.1352 & 1.1672 & 1.2124 & 1.2801 & 1.2820 & 1.3202 & 1.3602 & 1.4192 & 1.5912 & 1.4541 \\
\hline 1.4800 & 1.1427 & 1.1578 & 1.2024 & 1.2591 & 1.2820 & 1.3318 & 1.3475 & 1.4192 & 1.5782 & 1.4675 \\
\hline 1.4840 & 1.1509 & 1.1672 & 1.2223 & 1.2694 & 1.2820 & 1.3318 & 1.3475 & 1.4192 & 1.6041 & 1.4541 \\
\hline 1.4880 & 1.1429 & 1.1578 & 1.2124 & 1.2698 & 1.2708 & 1.3434 & 1.3475 & 1.4317 & 1.5912 & 1.4272 \\
\hline 1.4920 & 1.1429 & 1.1578 & 1.2024 & 1.2804 & 1.2708 & 1.3318 & 1.3475 & 1.4066 & 1.6170 & 1.4406 \\
\hline 1.4960 & 1.1269 & 1.1578 & 1.1925 & 1.2698 & 1.2931 & 1.3202 & 1.3348 & 1.4066 & 1.6170 & 1.4406 \\
\hline 1.5000 & 1.1349 & 1.1578 & 1.2223 & 1.2804 & 1.2820 & 1.3202 & 1.3348 & 1.4192 & 1.6041 & 1.4272 \\
\hline
\end{tabular}


Appendix E - Gauge data for experiment 2S-50.

\begin{tabular}{|c|c|c|c|c|c|c|c|c|c|c|c|}
\hline 480 & $9 e-3$ & $46 e-3$ & 000 & 0000 & 99 & 00 & 08 & 09 & 0.0000 & 0.0118 & $6.8330 \mathrm{e}-3$ \\
\hline-0.2440 & 0.0000 & 0.0159 & 0.0000 & 0.0000 & 0.0000 & 0.0103 & -0.0108 & 0.0109 & 0.0115 & 0.0118 & -0.0129 \\
\hline-0.2400 & $-6.5169 \mathrm{e}-3$ & $37.9446 \mathrm{e}-3$ & 0.0000 & 0.0096 & 0.0000 & -0.0103 & .0000 & 0.0109 & 0.0000 & .0000 & $6.0724 \mathrm{e}-3$ \\
\hline .2360 & 0.0000 & 0.0159 & $-9.3023 e-3$ & 30.0192 & .0000 & .0000 & .0000 & .0109 & .0000 & 0.0118 & .0102 \\
\hline .2320 & $-6.5214 \mathrm{e}-3$ & 30.0000 & 0.0000 & 0.0096 & 0099 & .0103 & .0108 & .0109 & .0000 & .0118 & .0307 \\
\hline .2280 & $-6.5169 \mathrm{e}-3$ & 30.0000 & 0.0000 & -0.0096 & 0198 & 0103 & 0000 & .0109 & .0000 & & 0231 \\
\hline 0.2240 & 0.0000 & 0.0000 & $9.3023 e-3$ & 0.0000 & 0.0099 & & & & -0.0115 & .0118 & .0292 \\
\hline & .0000 & 0.0000 & $9.3008 \mathrm{e}-3$ & 0.0000 & 0.0099 & -0.0103 & .0000 & 09 & & 118 & \\
\hline .2160 & .0000 & 0.0159 & $9.3008 \mathrm{e}-3$ & 0.0000 & .0000 & & & & & & \\
\hline .2120 & 0.0000 & 0.01 & -9.3008 & 30.0096 & 0.0099 & 3 & & & 15 & & \\
\hline 2080 & $-6.5169 \mathrm{e}-3$ & 30.0159 & 0.0000 & 0.0096 & 00 & & & & & & De-3 \\
\hline .2040 & $6.5169 \mathrm{e}-3$ & 0.0000 & $9.3023 e-3$ & 0.0000 & -0.0099 & 103 & & & & & \\
\hline .2000 & $-6.5214 \mathrm{e}-3$ & $37.9473 \mathrm{e}-3$ & 0.0186 & 0.0192 & 0.0000 & 0103 & & & & & e-3 \\
\hline & -6.51 & 30.0000 & & & & & & & & & $3 e-3$ \\
\hline 0.1920 & -0.0130 & 0.0000 & 0.0000 & 0.0000 & 099 & & & & & 118 & $77 e-3$ \\
\hline & $-6.5169 \mathrm{e}-3$ & $37.9473 e-3$ & & & & & & & & & \\
\hline & .0 & 7.94 & 0.0000 & 0.0000 & & & & & & & \\
\hline & 0000 & $7.9473 e-3$ & $-9.3008 \mathrm{e}-3$ & $3-0.0096$ & & & & & & & \\
\hline & $9 e-3$ & $7.9446 e-3$ & 0.0000 & & & & & & & & \\
\hline .1720 & 00 & 7.94 & $3 e-3$ & & & & & & & & \\
\hline & 0 & 0.0000 & & & & & & & & & e-3 \\
\hline & .0000 & $7.9446 \mathrm{e}-3$ & 0.0000 & & & & & & & & 3e-3 \\
\hline & -6.5 & 30.0000 & 9.3 & & & & & & & & \\
\hline & -6.5 & 30 & & & & & & & & & \\
\hline & 00 & 0.0000 & 9.3 & 0.0000 & & & & & & & \\
\hline & & & & & & & & & & & \\
\hline-0 & -651 & $37.9473 e-3$ & 0.0 & & & & & & & & \\
\hline 1400 & 00000 & $7.9473 e-3$ & 0.0000 & & & & & & & & \\
\hline & -6.5 & 30.0 & $3 e-3$ & & & & & & & & \\
\hline & 0.0 & $6 e-3$ & & & & & & & & & \\
\hline & -6.5 & $30 .(1-x$ & $3 e-3$ & & & & & & & & \\
\hline & & & & & & & & & & & \\
\hline & -6.5 & & 9.3 & & & & & & & & \\
\hline & & & & & & & & & & & \\
\hline 0 & $-0 .($ & 7.9 & $-9.3023 e-3$ & 30.0 & & & & & & & \\
\hline & -6. & & & & & & & & & & \\
\hline & -6 & $e-3$ & $e-3$ & & & & & & & & \\
\hline & 65 & & & & & & & & & & \\
\hline & & & & & & & & & & & \\
\hline & & & & & & & & & & & \\
\hline & & & & & & & & & & & \\
\hline & & & & & & & & & & & \\
\hline & & $37.9446 \mathrm{e}-3$ & & & & & & & & & \\
\hline & & & & & & & & & & & \\
\hline & -6. & 6e-3 & & & & & & & & & \\
\hline & & & & & & & & & & & \\
\hline & & & & & & & & & & & \\
\hline-0.0600 & 0.0000 & 7.9446e-3 & $-9.3008 e-3$ & & & & & & & & $3 e-3$ \\
\hline & & & & & & & & & & & \\
\hline & & 30. & -3 & & & & & & & & \\
\hline & & $6 e-3$ & & & & & & & & & \\
\hline & & 7.9 & & & & & & & & & $\alpha$ \\
\hline & & & 9.3 & & & & & & & & \\
\hline$-0 .(1 \quad r y$ & 6. & $7.9446 \mathrm{e}-3$ & & & & & & & & & $8 \mathrm{e}-3$ \\
\hline & & 0.0000 & $23 e-3$ & & 099 & & & & & & \\
\hline & & & & & & & & & & & \\
\hline & & & & & & & & & & & \\
\hline & & & $3 e-3$ & & & & & & & & \\
\hline & & -7.94 & & & & & & & & & \\
\hline & & & & & & & & & & & -2 \\
\hline$-8.0000 \mathrm{e}$ & & 0.00 & 0000 & 0.0000 & & & & & & & \\
\hline$-4.0000 \mathrm{e}$ & 0.2933 & -0.015 & $9.3023 e-3$ & -0.0096 & 0.0099 & & & & & & 0.0192 \\
\hline-6.3063 & & 0 & תרקט & $9.3023 \mathrm{e}-$ & 0.0096 & 0.0000 & 0000 & -0.0108 & 0.0109 & 0.0000 & -0.0118 \\
\hline & & & & & & & & & & & \\
\hline & & & & & & & & & & & \\
\hline & & & & & & & & & & & \\
\hline & & 0.0000 & 00 & 0.0000 & 0.0099 & 0.0000 & -0.0108 & 0.0109 & 0.0000 & .0000 & 0.0193 \\
\hline
\end{tabular}




\begin{tabular}{|c|c|c|c|c|c|c|c|c|c|c|c|}
\hline 0.0160 & 1.2709 & $7.9554 \mathrm{e}-3$ & $9.3023 \mathrm{e}-3$ & 0.0000 & 0.0099 & -0.0103 & -0.0108 & 0.0000 & -0.0115 & -0.0236 & 0.0176 \\
\hline 0.0200 & 1.2579 & $7.9464 \mathrm{e}-3$ & 0.0000 & 0.0000 & 0.0099 & -0.0103 & 0.0108 & 0.0109 & 0.0115 & -0.0118 & 0.0285 \\
\hline 0.0240 & 1.2383 & $7.9464 \mathrm{e}-3$ & 0.0186 & 0.0000 & 0.0000 & -0.0103 & 0.0108 & 0.0000 & -0.0115 & 0.0000 & 0.0132 \\
\hline 0.0280 & 1.2318 & $7.9464 \mathrm{e}-3$ & 0.0000 & 0.0000 & 0.0099 & 0.0000 & -0.0108 & 0.0000 & 0.0000 & 0.0000 & 0.0121 \\
\hline 0.0320 & 1.2448 & 0.0000 & 0.0000 & 0.0000 & 0.0000 & 0.0000 & 0.0108 & 0.0109 & -0.0115 & -0.0118 & 0.0142 \\
\hline 0.0360 & 1.2579 & $7.9464 \mathrm{e}-3$ & 0.0000 & 0.0000 & 0.0099 & 0.0103 & 0.0000 & 0.0109 & 0.0115 & -0.0118 & 0.0370 \\
\hline 0.0400 & 1.2579 & 0.0000 & $9.3023 e-3$ & 0.0000 & 0.0099 & 0.0103 & 0.0108 & 0.0109 & 0.0000 & -0.0118 & $7.8765 e-3$ \\
\hline 0.0440 & 1.2513 & $7.9464 \mathrm{e}-3$ & 0.0000 & 0.0096 & 0.0099 & 0.0000 & 0.0216 & 0.0218 & 0.0000 & 0.0000 & 0.0265 \\
\hline 0.0480 & 1.2513 & 0.0000 & 0.0000 & 0.0096 & 0.0000 & 0.0000 & -0.0108 & 0.0109 & 0.0000 & 0.0000 & $4.3641 \mathrm{e}-3$ \\
\hline 0.0520 & 1.2579 & $7.9464 \mathrm{e}-3$ & 0.0000 & 0.0096 & 0.0000 & -0.0103 & 0.0000 & 0.0000 & 0.0000 & -0.0118 & $2.7581 \mathrm{e}-3$ \\
\hline 0.0560 & 1.2579 & $7.9464 \mathrm{e}-3$ & $9.3023 e-3$ & 0.0096 & 0.0000 & -0.0103 & 0.0323 & 0.0109 & 0.0000 & 0.0000 & 0.0162 \\
\hline 0.0600 & 1.2579 & $-7.9446 e-3$ & 0.0000 & 0.0000 & 0.0000 & 0.0000 & 0.0000 & 0.0109 & -0.0115 & 0.0118 & $1.8025 \mathrm{e}-3$ \\
\hline 0.0640 & 1.2513 & $7.9446 \mathrm{e}-3$ & $9.3023 e-3$ & 0.0000 & 0.0099 & -0.0103 & 0.0000 & 0.0000 & -0.0230 & -0.0118 & $6.5470 \mathrm{e}-3$ \\
\hline 0.0680 & 1.2579 & $7.9473 e-3$ & $9.3023 e-3$ & 0.0000 & 0.0000 & 0.0000 & 0.0108 & 0.0109 & 0.0000 & -0.0118 & $7.8765 \mathrm{e}-3$ \\
\hline 0.0720 & 1.2513 & 0.0159 & 0.0000 & 0.0096 & 0.0099 & 0.0000 & 0.0108 & 0.0109 & -0.0115 & -0.0118 & -0.0105 \\
\hline 0.0760 & 1.2513 & $7.9446 \mathrm{e}-3$ & 0.0000 & 0.0000 & 0.0000 & & & 0.0109 & 0.0115 & 0.0000 & 0.0150 \\
\hline 0.0800 & 1.2513 & $7.9446 e-3$ & $9.3023 e-3$ & 0.0192 & 0.0000 & -0.0103 & 0.0000 & 0.0109 & 0.0000 & -0.0236 & 0.0170 \\
\hline 0.0840 & 1.2448 & $7.9446 \mathrm{e}-3$ & 0.0000 & 0.0000 & 0.0099 & 0.0000 & 0.0108 & 0.0109 & 0.0000 & 0.0000 & 0.0164 \\
\hline 0.0880 & 1.2579 & 0.0000 & $9.3023 e-3$ & 0.0000 & 0.0000 & -0.0103 & 0.0000 & 0.0109 & 0.0 & 0.0118 & 0.0222 \\
\hline 0.0920 & 1.2513 & 0.0000 & 0.0000 & 0.0000 & 0.0000 & -0.0103 & 0.0216 & 0.0218 & -0.0115 & 0.0000 & 0.0306 \\
\hline 0.0960 & 1.2448 & $7.9446 \mathrm{e}-3$ & 0.0000 & 0.0000 & 0.0000 & 0.0000 & 0.0000 & 0.0109 & 0.0115 & 0.0000 & 0.0240 \\
\hline 0.1000 & 1.2513 & $7.9446 \mathrm{e}-3$ & 0.0000 & 0.0000 & 0.0000 & 0.0103 & 0.0108 & 0.0109 & 0.0000 & -0.0118 & 0.0190 \\
\hline 0.1040 & 1.2644 & $7.9446 \mathrm{e}-3$ & $9.3023 e-3$ & 0.0000 & -0.0099 & 0.0000 & 0.0000 & 0.0000 & -0.0115 & -0.0118 & 0.0201 \\
\hline 0.1080 & 1.2513 & $7.9446 e-3$ & 0.0000 & 0.0096 & 0.0000 & 0.0000 & 0.0108 & 0.0109 & 0.0000 & 0.0000 & $4.0797 \mathrm{e}-3$ \\
\hline 0.1120 & 1.2513 & $7.9446 \mathrm{e}-3$ & 0.0000 & 0.0096 & 0.0000 & 0.0000 & 0.0000 & 0.0000 & -0.0115 & -0.0118 & $-3.4165 e-3$ \\
\hline 0.1160 & 1.2644 & $7.9446 \mathrm{e}-3$ & $9.3023 e-3$ & 0.0000 & 0.0000 & 0.0 & 0.0 & 0.0109 & 0. & -0.0118 & $-3.4165 \mathrm{e}-3$ \\
\hline 0.1200 & 1.2513 & 0.0000 & $9.3023 \mathrm{e}-3$ & 0.0000 & 0.0099 & 0.0000 & 0.0108 & 0.0109 & 115 & -0.0118 & 0.0252 \\
\hline 0.1240 & 1.2513 & $7.9446 \mathrm{e}-3$ & 0.0000 & 0.0096 & 0.0000 & 0.0 & 0.0108 & 0.0000 & 0.0000 & 0.0000 & 0.0167 \\
\hline 0.1280 & 1.2579 & 0.0000 & $9.3023 e-3$ & 0.0096 & 0.0000 & 0.0 & 0.0 & 0.0109 & 0.0 & -0.0118 & $2.0883 \mathrm{e}-3$ \\
\hline 0.1320 & 1.2513 & 0.0159 & 0.0000 & 0.0096 & -0.0099 & 0.0000 & 0.0000 & 0.0000 & 0.0 & -0.0118 & 0.0197 \\
\hline 0.1360 & 1.2513 & $7.9446 \mathrm{e}-3$ & 0.0000 & 0.0096 & 0.0000 & 0.0000 & 0.0108 & 0.0109 & 0.0000 & 0.0000 & 0.0207 \\
\hline 0.1400 & 1.2709 & $7.9446 \mathrm{e}-3$ & $9.3008 \mathrm{e}-3$ & 0.0000 & 0.0099 & 0.0000 & 0.0216 & 0.0109 & 15 & 0.0000 & 0.0168 \\
\hline 0.1440 & 1.2513 & $7.9446 \mathrm{e}-3$ & 0.0000 & 0.0000 & -0.0099 & 0.0 & 0.0 & 09 & 0. & 0.0 & 07 \\
\hline 0.1480 & 1.2579 & $7.9446 \mathrm{e}-3$ & $9.3023 e-3$ & 0.0000 & 0.0099 & 0.0000 & 0.0000 & 0.0218 & 0.0000 & 0.0000 & $7.4003 e-3$ \\
\hline 0.1520 & 1.2644 & $7.9446 \mathrm{e}-3$ & 0.0000 & 0.0000 & 0.0099 & 0.0103 & 0.0216 & 0.0109 & 0.0000 & 0.0118 & 0.0119 \\
\hline 0.1560 & 1.2644 & $7.9446 \mathrm{e}-3$ & 0.0000 & -0.0096 & 0.0099 & 0.0 & 0.0108 & 0.0109 & 0.0000 & 0.0000 & 0.0235 \\
\hline 0.1600 & 1.2579 & $7.9446 e-3$ & $-9.3023 e-3$ & 0.0000 & 0.0099 & 0.0000 & 0.0000 & 0.0109 & -0.0115 & -0.0118 & 0.0239 \\
\hline 0.1640 & 1.2579 & $7.9446 \mathrm{e}-3$ & 0.0000 & 0.0096 & 0.0000 & 0.0000 & 0.0000 & 0.0218 & -0.0115 & -0.0118 & 0.0235 \\
\hline 0.1680 & 1.2513 & 0.0000 & $9.3023 e-3$ & 0.0096 & 0.0099 & & & 0.0109 & & 0.0000 & $5.6937 \mathrm{e}-3$ \\
\hline 0.1720 & 1.2579 & $7.9464 \mathrm{e}-3$ & $-9.3008 e-3$ & -0.0096 & 0.0000 & 0. & 0. & 18 & 0 & 00 & -0.0167 \\
\hline 0.1760 & 1.2513 & 0.0000 & 0.0186 & 0.0000 & 0.0099 & 0.0000 & -0.0108 & 0.0000 & -0.0115 & 0.0000 & 0.0297 \\
\hline 0.1800 & 1.2709 & $7.9464 \mathrm{e}-3$ & $9.3023 e-3$ & 0.0000 & 0.0000 & 0.0000 & 0.0108 & 0.0109 & 0.0115 & 0.0000 & $6.0740 \mathrm{e}-3$ \\
\hline 0.1840 & 1.2513 & 0.0159 & 0.0000 & 0.0000 & 0.0000 & 0.0000 & 0.0108 & 0.0109 & 0.0000 & 0.0000 & 0.0140 \\
\hline 0.1880 & 1.2579 & $7.9446 \mathrm{e}-3$ & $-9.3008 e-3$ & 0.0096 & 0.0000 & -0.0103 & 0.0000 & 0.0109 & 0.0000 & 0.0000 & $9.7743 e-3$ \\
\hline 0.1920 & 1.2513 & 0.0000 & $9.3023 e-3$ & 0.0096 & -0.0099 & 0.0000 & -0.0108 & 0.0109 & -0.0115 & -0.0118 & 0.0140 \\
\hline 0.1960 & 1.2579 & $7.9464 \mathrm{e}-3$ & $9.3023 e-3$ & 0.0000 & 0.0099 & 0.0103 & 0.0000 & 0.0109 & 0.0000 & 0.0000 & $-7.4957 \mathrm{e}-3$ \\
\hline 0.2000 & 1.2579 & $4 e-3$ & 9.30 & 96 & 00 & -0 & 0. & 00 & 15 & 0.0000 & $2.6639 \mathrm{e}-3$ \\
\hline 0.2040 & 1.2513 & 0.0000 & $9.3023 e-3$ & 0.0096 & 0.0000 & 0.0000 & 0.0108 & 0.0109 & 0.0000 & 0.0236 & 0.0138 \\
\hline 0.2080 & 1.2513 & 0.0000 & $9.3023 e-3$ & 0.0096 & -0.0099 & 0.0000 & 0.0000 & 0.0000 & 0.0115 & 0.0000 & $-2.2772 \mathrm{e}-3$ \\
\hline 0.2120 & 1.2513 & 0.0000 & 0.0000 & 0.0096 & 0.0099 & 0.0000 & -0.0108 & 0.0109 & 0.0000 & 0.0000 & 0.0207 \\
\hline 0.2160 & 1.2513 & $-7.9464 \mathrm{e}-3$ & 0.0000 & 0.0096 & 0.0099 & -0.0103 & 0.0108 & 0.0000 & 0.0000 & 0.0000 & 0.0305 \\
\hline 0.2200 & 1.2644 & 0.0000 & 0.0000 & 0.0000 & -0.0099 & 0.0000 & 0.0108 & 0.0109 & -0.0115 & -0.0118 & -0.0108 \\
\hline 0.2240 & 1.2448 & $7.9464 \mathrm{e}-3$ & 0.0000 & 0.0096 & 0.0099 & 0.0000 & -0.0 & 09 & 15 & -0.0118 & $5.7880 \mathrm{e}-3$ \\
\hline 0.2280 & 1.2448 & $7.9464 \mathrm{e}-3$ & 0.0000 & 0.0000 & -0.0099 & 0.0103 & -0.0108 & 0.0109 & 0.0000 & 0.0000 & $-8.7305 e-3$ \\
\hline 0.2320 & 1.2448 & $7.9464 \mathrm{e}-3$ & $9.3023 e-3$ & 0.0000 & 0.0000 & 0.0000 & 0.0108 & -0.0109 & -0.0115 & 0.0000 & 0.0178 \\
\hline 0.2360 & 1.2513 & $7.9464 \mathrm{e}-3$ & $9.3008 \mathrm{e}-3$ & 0.0000 & 0.0000 & 0.0000 & 0.0108 & 0.0109 & 0.0000 & 0.0000 & $8.7283 e-3$ \\
\hline 0.2400 & 1.2318 & $7.9446 \mathrm{e}-3$ & 0.0000 & 0.0000 & 0.0000 & 0.0000 & 0.0108 & 0.0109 & 0.0000 & 0.0000 & $-2.9419 \mathrm{e}-3$ \\
\hline 0.2440 & 1.2448 & $7.9446 \mathrm{e}-3$ & $9.3023 e-3$ & 0.0000 & 0.0099 & 0.0000 & 0.0108 & 0.0109 & -0.0115 & 0.0118 & -0.0257 \\
\hline 0.2480 & 1.2579 & 0.0159 & $9.3023 e-3$ & 0.0000 & 0.0099 & 0.0000 & 0.0108 & 0.0218 & -0.0115 & 0.0000 & 0.0134 \\
\hline 0.2520 & 1.2513 & 0.0159 & 0.0000 & 0.0000 & 0.0000 & 0.0103 & -0.0108 & 0.0109 & -0.0115 & 0.0000 & $7.4962 \mathrm{e}-3$ \\
\hline 0.2560 & 1.2513 & $7.9464 \mathrm{e}-3$ & 0.0000 & 0.0000 & 0.0099 & 0.0000 & 0.0000 & 0.0000 & 0.0000 & 0.0000 & 0.0344 \\
\hline 0.2600 & 1.2579 & 0.0000 & $9.3023 e-3$ & 0.0096 & 0.0000 & 0.0000 & 0.0000 & 0.0000 & 0.0115 & 0.0000 & 0.0173 \\
\hline 0.2640 & 1.2448 & 0.0000 & $9.3023 e-3$ & 0.0000 & 0.0000 & 0.0000 & -0.0108 & 0.0218 & -0.0115 & 0.0000 & $1.9847 \mathrm{e}-3$ \\
\hline 0.2680 & 1.2579 & $7.9446 \mathrm{e}-3$ & 0.0000 & 0.0000 & 0.0000 & 0.0000 & 0.0108 & 0.0109 & 0.0000 & 0.0000 & 0.0151 \\
\hline 0.2720 & 1.2579 & $7.9446 \mathrm{e}-3$ & $9.3023 \mathrm{e}-3$ & 0.0192 & 0.0099 & 0.0000 & 0.0108 & 0.0000 & 0.0000 & 0.0118 & 0.0227 \\
\hline 0.2760 & 1.2448 & $7.9464 \mathrm{e}-3$ & 0.0000 & 0.0096 & 0.0000 & 0.0000 & 0.0000 & 0.0109 & 0.0000 & -0.0118 & $-9.3946 e-3$ \\
\hline 0.2800 & 1.2513 & $7.9446 \mathrm{e}-3$ & 0.0000 & 0.0096 & 0.0099 & -0.0103 & 0.0108 & 0.0109 & 0.0000 & 0.0000 & 0.0330 \\
\hline 0.2840 & & $7.9446 \mathrm{e}-3$ & 0.0000 & 0.0000 & 0.0000 & & 0.0000 & 0.0109 & 0.0000 & 0.0000 & 0.0214 \\
\hline 0.2880 & 1.2513 & 0.0000 & $9.3023 \mathrm{e}-3$ & 0.0000 & 0.0099 & 0.0000 & 0.0000 & 0.0218 & 0.0000 & 0.0000 & -0.0140 \\
\hline 0.2920 & 1.2579 & $7.9464 \mathrm{e}-3$ & 0.0000 & 0.0000 & 0.0000 & 0.0000 & 0.0000 & 0.0109 & -0.0115 & 0.0000 & $-5.9701 \mathrm{e}-3$ \\
\hline
\end{tabular}




\begin{tabular}{|c|c|c|c|c|c|c|c|c|c|c|c|}
\hline 0.2960 & 1.2513 & 0.0000 & $9.3008 \mathrm{e}-3$ & 0.0000 & 0.0099 & -0.0103 & 0.0000 & 0.0000 & 0.0000 & 0.0000 & $8.1610 \mathrm{e}-3$ \\
\hline 0.3000 & 1.2579 & $7.9464 \mathrm{e}-3$ & 0.0000 & 0.0192 & 0.0000 & -0.0206 & 0.0108 & 0.0000 & 0.0000 & -0.0118 & $-9.5832 \mathrm{e}-3$ \\
\hline 0.3040 & 1.2513 & 0.0159 & $9.3023 e-3$ & 0.0192 & 0.0000 & 0.0000 & 0.0108 & 0.0109 & 0.0115 & 0.0000 & $6.4511 \mathrm{e}-3$ \\
\hline 0.3080 & 1.2579 & $-7.9464 \mathrm{e}-3$ & 0.0186 & 0.0096 & 0.0000 & 0.0000 & 0.0108 & 0.0109 & 0.0000 & -0.0118 & $9.8724 \mathrm{e}-3$ \\
\hline 0.3120 & 1.2513 & 0.0000 & $9.3023 e-3$ & 0.0096 & 0.0099 & 0.0103 & 0.0108 & 0.0109 & 0.0000 & -0.0118 & 0.0237 \\
\hline 0.3160 & 1.2579 & $7.9464 \mathrm{e}-3$ & $9.3023 e-3$ & 0.0000 & 0.0000 & -0.0103 & 0.0108 & 0.0109 & -0.0115 & 0.0000 & $-3.1305 e-3$ \\
\hline 0.3200 & 1.2448 & 0.0000 & 0.0186 & 0.0000 & 0.0000 & 0.0000 & 0.0000 & -0.0109 & 0.0000 & 0.0000 & 0.0205 \\
\hline 0.3240 & 1.2579 & $7.9464 \mathrm{e}-3$ & 0.0000 & 0.0000 & 0.0000 & 0.0000 & 0.0108 & 0.0109 & -0.0115 & 0.0118 & $-1.9016 \mathrm{e}-4$ \\
\hline 0.3280 & 1.2644 & 0.0000 & $9.3023 \mathrm{e}-3$ & 0.0000 & -0.0099 & 0.0103 & 0.0108 & 0.0109 & -0.0115 & -0.0118 & 0.0135 \\
\hline 0.3320 & 1.2644 & $7.9464 \mathrm{e}-3$ & $9.3023 e-3$ & 0.0000 & -0.0099 & 0.0000 & 0.0216 & 0.0218 & 0.0000 & -0.0118 & $4.8419 \mathrm{e}-3$ \\
\hline 0.3360 & 1.2709 & $7.9464 \mathrm{e}-3$ & 0.0000 & 0.0192 & 0.0000 & 0.0000 & 0.0000 & 0.0109 & 0.0000 & 0.0000 & 0.0165 \\
\hline 0.3400 & 1.2513 & 0.0159 & 0.0000 & 0.0096 & 0.0000 & 0.0000 & 0.0000 & 0.0000 & 0.0000 & 0.0000 & 0.0279 \\
\hline 0.3440 & 1.2579 & 0.0000 & 0.0000 & 0.0096 & 0.0099 & -0.0103 & 0.0000 & 0.0000 & 0.0000 & 0.0000 & $43 e-3$ \\
\hline 0.3480 & 1.2579 & $7.9464 \mathrm{e}-3$ & 0.0000 & 0.0192 & 0.0000 & -0.0103 & 0.0000 & 0.0109 & -0.0115 & -0.0118 & 0.0360 \\
\hline 0.3520 & 1.2579 & 0.0000 & 0.0000 & 0.0096 & 0.0000 & -0.0103 & 0.0108 & 0.0109 & -0.0230 & 0.0000 & 0.0287 \\
\hline 0.3560 & 1.2579 & $7.9464 \mathrm{e}-3$ & 0.0000 & 0.0096 & 0.0099 & -0.0103 & 0.0108 & 0.0000 & 0.0000 & 0.0000 & \\
\hline 0.3600 & 1.2513 & 0.0000 & 0.0000 & 0.0096 & 0.0099 & 0.0000 & 0.0108 & 0.0109 & -0.0115 & 0.0000 & $6.9273 e-3$ \\
\hline 0.3640 & 1.2644 & $7.9464 \mathrm{e}-3$ & 0.0186 & 0.0096 & 0.0099 & 0.0000 & -0.0108 & 0.0109 & 0.0115 & -0.0236 & -0.0103 \\
\hline 0.3680 & 1.2644 & $7.9464 \mathrm{e}-3$ & $-9.3023 e-3$ & 0.0000 & -0.0099 & -0.0103 & 0.0108 & 0.0109 & 0.0000 & 0.0000 & $92 \mathrm{e}-3$ \\
\hline 0.3720 & 1.2644 & $7.9464 \mathrm{e}-3$ & $9.3023 e-3$ & 0.0096 & 0.0198 & -0.0103 & 0.0108 & 0.0000 & 0.0000 & 0.0000 & -0.0115 \\
\hline 0.3760 & 1.2644 & 0.0000 & $9.3023 e-3$ & 0.0000 & 0.0099 & 0.0103 & 0.0000 & 0.0000 & 0.0000 & 0.0000 & 32 \\
\hline 0.3800 & 1.2644 & 0.0000 & $9.3023 e-3$ & -0.0096 & 0.0099 & -0.0103 & 0.0216 & 0.0109 & 0.0115 & 0.0118 & 156 \\
\hline 0.3840 & 1.2644 & 0.0000 & $9.3023 e-3$ & 0.0096 & 0.0000 & 0.0103 & 0.0108 & 0.0109 & -0.0115 & -0.0118 & $-1.6131 \mathrm{e}-3$ \\
\hline 0.3880 & 1.2644 & 0.0000 & 0.0000 & 0.0000 & 0.0000 & -0.0103 & 0.0216 & 0.0218 & 0.0115 & 0.0000 & $2.2771 \mathrm{e}-3$ \\
\hline 0.3920 & 1.2644 & $7.9464 \mathrm{e}-3$ & $9.3023 e-3$ & 0.0000 & -0.0099 & 0.0000 & 0.0108 & 0.0109 & -0.0115 & -0.0118 & 19 \\
\hline 0.3960 & 1.2709 & $7.9464 \mathrm{e}-3$ & 0.0000 & -0.0096 & 0.0099 & 03 & 0.0216 & 0.0218 & -0.0115 & 0.0000 & \\
\hline 0.4000 & 1.2644 & $7.9464 \mathrm{e}-3$ & 0.0186 & 0.0000 & 0.0000 & 0.0000 & 0.0000 & 0.0109 & 0.0000 & 0.0000 & 0.0244 \\
\hline 0.4040 & 1.2644 & $7.9464 \mathrm{e}-3$ & $-9.3023 e-3$ & 0.0000 & 0.0099 & 0.0000 & 0.0000 & 0.0109 & 0.0000 & -0.0118 & $73 e-3$ \\
\hline 0.4080 & 1.2709 & 0.0000 & 0.0000 & 0.0096 & 0.0000 & -0.0103 & -0.0108 & 0.0109 & 0.0115 & -0.0118 & 0.0441 \\
\hline 0.4120 & 1.2774 & 0.0000 & $9.3008 \mathrm{e}-3$ & 0.0000 & 0.0000 & 0.0103 & 0.0108 & 0.0109 & 0.0000 & 0.0000 & $-8.5397 \mathrm{e}-3$ \\
\hline 0.4160 & 1.2579 & 0.0000 & 0.0000 & 0.0192 & 0.0198 & 0.0000 & 0.0000 & 0.0109 & -0.0115 & 0.0000 & $5.5988 \mathrm{e}-3$ \\
\hline 0.4200 & 1.2579 & $7.9464 \mathrm{e}-3$ & 0.0000 & 0.0000 & 0.0198 & -0.0103 & 0.0216 & 0.0109 & 0.0000 & $0 .($ & $54 \mathrm{e}-3$ \\
\hline 0.4240 & 1.2644 & 0.0000 & 0.0000 & 0.0000 & 0.0099 & 0.0000 & 0.0000 & 0.0000 & -0.0115 & 0.0000 & 0.0 \\
\hline 0.4280 & 1.2513 & 0.0159 & $9.3008 \mathrm{e}-3$ & 0.0096 & 0.0099 & 0.0000 & 0.0108 & 0.0218 & 0.0000 & 0.0000 & $-5.7889 e-3$ \\
\hline 0.4320 & 1.2644 & $7.9464 \mathrm{e}-3$ & $9.3023 e-3$ & 0.0096 & 0.0000 & -0.0103 & 0.0000 & 0.0218 & 0.0000 & -0.0118 & 0.0116 \\
\hline 0.4360 & 1.2709 & 0.0000 & $9.3023 e-3$ & 0.0000 & 0.0099 & 0.0103 & 0.0108 & 0.0109 & -0.0115 & 0.0000 & $5.8822 \mathrm{e}-3$ \\
\hline 0.4400 & 1.2644 & 0.0000 & 0.0186 & -0.0096 & 0.0000 & -0.0103 & 0.0108 & 0.0000 & 0.0000 & 0.0000 & $-6.4511 e-3$ \\
\hline 0.4440 & 1.2579 & 0.0000 & 0.0000 & 0.0000 & -0.0099 & 0.0000 & 0.0216 & 0.0109 & -0.0115 & -0.0118 & $3.7010 \mathrm{e}-3$ \\
\hline 0.4480 & 1.2579 & 0 & 0.0 & 96 & 00 & & & 00 & & 18 & \\
\hline 0.4520 & 1.2513 & 0.0000 & $9.3023 e-3$ & 0.0096 & 0.0099 & 0.0103 & 0.0108 & 0.0109 & 0.0000 & 0.0118 & $968 \mathrm{e}-3$ \\
\hline 0.4560 & 1.2513 & 0.0000 & $9.3023 e-3$ & 0.0000 & 0.0000 & 0.0000 & -0.0108 & 0.0109 & 0.0000 & -0.0118 & 23 \\
\hline 0.4600 & 1.2579 & 0.0000 & $9.3023 e-3$ & 0.0096 & -0.0099 & 0.0000 & 0.0216 & 0.0000 & -0.0115 & -0.0236 & 0.0121 \\
\hline 0.4640 & 1.2513 & $7.9464 \mathrm{e}-3$ & 0.0000 & 0.0192 & 0.0099 & 0.0000 & 0.0108 & 0.0000 & 0.0000 & -0.0118 & $5.6937 \mathrm{e}-3$ \\
\hline 0.4680 & 1.2448 & $7.9464 \mathrm{e}-3$ & $9.3023 e-3$ & 0.0096 & 0.0000 & -0.0103 & -0.0108 & 0.0218 & -0.0115 & 0.0000 & 0.0137 \\
\hline 0.4720 & 1.2579 & $7.9446 \mathrm{e}-3$ & $-9.3023 e-3$ & -0.0096 & 0.0000 & 0.0103 & 0.0000 & 0.0109 & -0.0115 & 0.0000 & 0.0102 \\
\hline 0.4760 & 1.2513 & 7.94 & $9.3023 e-3$ & 0.0000 & 00 & 3 & 0. & 18 & 0. & 00 & \\
\hline 0.4800 & 1.2579 & $7.9464 \mathrm{e}-3$ & 0.0000 & 0.0000 & 0.0000 & 0.0000 & 0.0108 & 0.0000 & -0.0115 & 0.0118 & $21 \mathrm{e}-3$ \\
\hline 0.4840 & 1.2513 & 0.0000 & $9.3008 \mathrm{e}-3$ & 0.0000 & -0.0099 & 0.0000 & 0.0108 & 0.0000 & -0.0115 & 0.0000 & 0.0237 \\
\hline 0.4880 & 1.2448 & 0.0000 & $9.3008 \mathrm{e}-3$ & 0.0000 & 0.0198 & 0.0000 & 0.0000 & 0.0000 & -0.0115 & 0.0000 & 0.0266 \\
\hline 0.4920 & 1.2513 & 0.0000 & 0.0000 & 0.0000 & 0.0000 & 0.0000 & 0.0000 & 0.0218 & 0.0000 & -0.0118 & 0.0228 \\
\hline 0.4960 & 1.2513 & 0.0000 & $9.3023 e-3$ & 0.0192 & 0.0099 & 0.0000 & 0.0000 & 0.0109 & -0.0115 & -0.0118 & 0.0178 \\
\hline 0.5000 & 1.2513 & $7.9464 \mathrm{e}-3$ & $9.3023 e-3$ & 0.0096 & 0.0000 & & & 0.0218 & 0.0000 & 0.0000 & \\
\hline 0.5040 & 1.2513 & $7.9464 \mathrm{e}-3$ & 0.0000 & 0.0096 & 00 & 0 & 0. & 09 & 0. & 00 & $8 e-3$ \\
\hline 0.5080 & 1.2513 & $7.9464 \mathrm{e}-3$ & 0.0000 & 0.0192 & 0099 & 0.0103 & 0.0 & 0.0109 & 0.0000 & 0.0118 & 0.0192 \\
\hline 0.5120 & 1.2318 & 0.0000 & 0.0000 & 0.0096 & 0.0099 & 0.0000 & 0.0000 & 0.0109 & 0.0000 & 0.0118 & $4.7461 \mathrm{e}-4$ \\
\hline 0.5160 & 1.2448 & $-7.9464 \mathrm{e}-3$ & 0.0000 & 0.0000 & 0.0099 & 0.0103 & 0.0000 & 0.0000 & 0.0000 & 0.0000 & $7.9692 \mathrm{e}-3$ \\
\hline 0.5200 & 1.2513 & 0.0000 & 0.0186 & 0.0096 & -0.0099 & 0.0000 & 0.0216 & 0.0109 & 0.0000 & -0.0118 & $-6.3573 e-3$ \\
\hline 0.5240 & 1.2448 & $7.9464 \mathrm{e}-3$ & 0.0000 & 0.0096 & 0.0000 & 0.0000 & 0.0108 & 0.0109 & 0.0000 & 0.0000 & -0.0123 \\
\hline 0.5280 & 1.2448 & $7.9554 \mathrm{e}-3$ & 0.0000 & 0.0096 & 0.0000 & 0.0103 & 0.0000 & 0.0109 & -0.0115 & -0.0118 & 0.0203 \\
\hline 0.5320 & 1.2513 & 0.0000 & 0.0000 & 0.0096 & -0.0099 & 0.0000 & 0.0000 & 0.0218 & -0.0115 & 0.0000 & -0.0131 \\
\hline 0.5360 & 1.2383 & 0.0000 & $9.3023 \mathrm{e}-3$ & 0.0000 & 0.0099 & 0.0000 & 0.0108 & 0.0109 & 0.0000 & 0.0000 & $5.3134 \mathrm{e}-3$ \\
\hline 0.5400 & 1.2513 & $7.9554 \mathrm{e}-3$ & 0.0000 & 0.0000 & 0.0099 & -0.0103 & & 0.0109 & 0.0000 & 0.0000 & $6.9267 \mathrm{e}-3$ \\
\hline 0.5440 & 1.2383 & 0.0000 & 0.0000 & 0.0096 & 0.0099 & 0.0103 & 0.0216 & 0.0218 & 0.0000 & -0.0118 & 0.0134 \\
\hline 0.5480 & 1.2383 & 0.0000 & 0.0000 & 0.0000 & -0.0099 & 0.0103 & 0.0000 & 0.0109 & 0.0000 & -0.0118 & $-7.4962 \mathrm{e}-3$ \\
\hline 0.5520 & 1.2318 & $7.9554 \mathrm{e}-3$ & $9.3023 e-3$ & 0.0096 & 0.0099 & 0.0000 & 0.0108 & 0.0000 & -0.0115 & 0.0000 & 0.0327 \\
\hline 0.5560 & 1.2383 & 0.0000 & $9.3008 \mathrm{e}-3$ & 0.0000 & 0.0099 & 0.0103 & 0.0000 & 0.0327 & 0.0115 & -0.0118 & $6.8314 \mathrm{e}-3$ \\
\hline 0.5600 & 1.2383 & $7.9464 \mathrm{e}-3$ & 0.0000 & 0.0096 & 0.0000 & 0.0000 & -0.0108 & 0.0218 & -0.0115 & -0.0118 & 0.0224 \\
\hline 0.5640 & 1.2318 & $7.9554 \mathrm{e}-3$ & $-9.3023 e-3$ & 0.0000 & 0.0000 & 0.0103 & 0.0108 & 0.0109 & 0.0000 & 0.0118 & 0.0165 \\
\hline 0.5680 & 1.2188 & 0.0000 & 0.0186 & 0.0000 & 0.0000 & 0.0000 & 0.0108 & 0.0109 & -0.0115 & 0.0118 & 0.0414 \\
\hline 0.5720 & 1.2318 & $7.9464 \mathrm{e}-3$ & $9.3008 \mathrm{e}-3$ & 0.0000 & 0.0099 & 0.0000 & 0.0108 & 0.0109 & -0.0115 & 0.0000 & 0.0205 \\
\hline
\end{tabular}




\begin{tabular}{|c|c|c|c|c|c|c|c|c|c|c|c|}
\hline 0.5760 & 1.2122 & $7.9464 \mathrm{e}-3$ & $9.3023 e-3$ & 0.0096 & 0.0198 & 0.0103 & 0.0000 & 0.0109 & 0.0000 & 0.0000 & -0.0187 \\
\hline 0.5800 & 1.2122 & 0.0159 & 0.0000 & 0.0000 & 0.0000 & 0.0000 & 0.0108 & 0.0109 & 0.0230 & -0.0118 & $-3.4117 \mathrm{e}-3$ \\
\hline 0.5840 & 1.2187 & 0.0000 & 0.0000 & -0.0096 & 0.0000 & 0.0000 & 0.0108 & 0.0000 & -0.0115 & 0.0000 & 0.0169 \\
\hline 0.5880 & 1.2187 & $7.9103 e-3$ & $9.3023 e-3$ & 0.0000 & -0.0099 & 0.0000 & 0.0000 & 0.0109 & -0.0115 & -0.0118 & 0.0250 \\
\hline 0.5920 & 1.2187 & 0.0159 & $-9.3023 e-3$ & 0.0096 & 0.0000 & 0.0103 & 0.0108 & 0.0109 & 0.0000 & 0.0000 & $9.5848 \mathrm{e}-3$ \\
\hline 0.5960 & 1.2187 & 0.0319 & $9.3023 e-3$ & 0.0000 & 0.0000 & 0.0000 & 0.0108 & 0.0109 & 0.0000 & -0.0118 & $-2.8467 \mathrm{e}-3$ \\
\hline 0.6000 & 1.2187 & 0.0557 & 0.0186 & 0.0096 & 0.0000 & 0.0103 & 0.0108 & 0.0109 & 0.0000 & -0.0118 & $1.4227 \mathrm{e}-3$ \\
\hline 0.6040 & 1.2122 & 0.2782 & $9.3023 e-3$ & 0.0000 & 0.0000 & -0.0103 & 0.0000 & 0.0109 & 0.0000 & 0.0000 & $-2.1828 \mathrm{e}-3$ \\
\hline 0.6080 & 1.1991 & 0.5722 & 0.0000 & 0.0096 & -0.0099 & 0.0000 & -0.0108 & 0.0109 & -0.0230 & 0.0000 & 0.0222 \\
\hline 0.6120 & 1.1926 & 0.8345 & $9.3023 e-3$ & 0.0096 & 0.0000 & 0.0103 & 0.0108 & 0.0109 & 0.0000 & 0.0000 & 0.0107 \\
\hline 0.6160 & 1.1991 & 1.0967 & 0.0000 & 0.0096 & 0.0000 & 0.0103 & 0.0000 & 0.0109 & 0.0000 & -0.0118 & 0.0234 \\
\hline 0.6200 & 1.2056 & 1.2875 & $9.3023 e-3$ & 0.0000 & 0.0000 & 0.0000 & 0.0108 & 0.0109 & 0.0000 & -0.0118 & $1.5181 \mathrm{e}-3$ \\
\hline 0.6240 & 1.2187 & 1.3034 & 0.0000 & 0.0000 & -0.0099 & 0.0000 & 0.0108 & 0.0109 & -0.0115 & -0.0118 & $9.2988 \mathrm{e}-3$ \\
\hline 0.6280 & 1.2252 & 1.2875 & $9.3023 e-3$ & 0.0096 & 0.0198 & 0.0000 & 0.0108 & 0.0218 & -0.0115 & 0.0000 & $-7.3072 \mathrm{e}-3$ \\
\hline 0.6320 & 1.2187 & 1.2796 & 0.0186 & 0.0000 & 0.0000 & -0.0103 & 0.0000 & 0.0109 & -0.0115 & -0.0118 & $-1.8041 \mathrm{e}-3$ \\
\hline 0.6360 & 1.2187 & 1.2875 & 0.0000 & 0.0096 & 0.0000 & 0.0103 & 0.0108 & 0.0218 & 0.0000 & 0.0000 & 0.0314 \\
\hline 0.6400 & 1.2187 & 1.2875 & 0.0186 & 0.0000 & 0.0099 & 0.0103 & -0.0108 & 0.0109 & 0.0000 & 0.0000 & 0.0155 \\
\hline 0.6440 & 1.2122 & 1.2875 & $-9.3008 e-3$ & 0.0096 & 0.0000 & 0.0000 & 0.0108 & 0.0218 & 0.0115 & 0.0118 & $151 \mathrm{e}-3$ \\
\hline 0.6480 & 1.2122 & 1.2875 & 0.0000 & 0.0000 & 0.0000 & 0.0000 & 0.0108 & 0.0218 & 0.0000 & 0.0000 & $8.2553 \mathrm{e}-3$ \\
\hline 0.6520 & 1.2122 & 1.3034 & 0.0000 & 0.0000 & 0.0000 & 0.0000 & 0.0216 & 0.0218 & -0.0115 & -0.0118 & 0.0107 \\
\hline 0.6560 & 1.2057 & 1.2954 & 0.0000 & 0.0192 & 0.0198 & -0.0103 & 0.0000 & 218 & 230 & -0.0118 & \\
\hline 0.6600 & 1.2057 & 1.3034 & 0.0000 & 0.0000 & 0.0000 & 0.0000 & -0.0108 & 0.0109 & 0.0000 & -0.0118 & \\
\hline 0.6640 & 1.2057 & 1.3034 & 0.0000 & 0.0000 & 0.0000 & -0.0103 & 0.0000 & 0.0109 & 0.0000 & -0.0118 & 84 \\
\hline 0.6680 & 1.2057 & 1.2954 & $9.3023 e-3$ & 0.0096 & 0.0099 & 0.0000 & 0.0108 & 0.0109 & 0.0000 & -0.0118 & $673 e-3$ \\
\hline 0.6720 & 1.2057 & 1.3034 & 0.0000 & 0.0096 & 0.0099 & -0.0103 & 0.0000 & 0.0109 & -0.0115 & -0.0118 & 0.0112 \\
\hline 0.6760 & 1.2122 & 1.3113 & 0.0000 & 0.0096 & 0.0099 & 0.0000 & 0.0108 & 0.0218 & 0.0000 & 0.0000 & $8.6340 \mathrm{e}-3$ \\
\hline 0.6800 & 1.2057 & 1.3113 & 0.0000 & 0.0192 & 0.0000 & 0.0000 & 0.0000 & 0.0109 & 0.0000 & -0.0118 & 05 \\
\hline 0.6840 & 1.2057 & 1.3193 & $9.3008 \mathrm{e}-3$ & 0.0096 & 0.0099 & 03 & 0.0000 & 0.0218 & 15 & -0.0236 & \\
\hline 0.6880 & 1.2057 & 1.3193 & 0.0000 & -0.0096 & 0.0000 & -0.0103 & 0.0000 & 0.0109 & 0.0000 & -0.0118 & $26 e-3$ \\
\hline 0.6920 & 1.2122 & 1.3113 & 0.0000 & 0.0000 & 0.0000 & 0.0 & 0.0108 & 0.0000 & 00 & 0.0000 & \\
\hline 0.6960 & 1.2122 & 1.3113 & $9.3023 e-3$ & 0.0000 & 0.0000 & -0.0103 & 0.0000 & 0.0000 & 00 & 000 & \\
\hline 0.7000 & 1.2057 & 1.3193 & 0.0000 & 0.0096 & 0.0099 & 0.0000 & 0.0108 & 0.0000 & -0.0115 & 0.0118 & $-4.4600 \mathrm{e}-3$ \\
\hline 0.7040 & 1.1992 & 1.3193 & 0.0000 & 0.0192 & 0.0099 & 0.0000 & 0.0108 & 0.0218 & -0.0115 & -0.0118 & $8.2553 \mathrm{e}-3$ \\
\hline 0.7080 & 1.1992 & 132 & 0.0000 & 0.0096 & 0.0099 & 0.0103 & 0.0000 & 0.0109 & 115 & 0.0000 & 276 \\
\hline 0.7120 & 1.1992 & 1.3 & 0.0 & 0.0096 & 0.0000 & 00 & -0.0108 & 09 & -0 & 00 & 0. \\
\hline 0.7160 & 1.1927 & 1.3193 & 0.0000 & 0.0096 & 0.0000 & -0.0206 & 0.0108 & 0.0109 & -0.0115 & 0.0000 & 0.0154 \\
\hline 0.7200 & 1.1927 & 1.3272 & $9.3023 e-3$ & 0.0000 & 0.0000 & 0.0000 & 0.0000 & 0.0109 & 0.0000 & -0.0118 & -0.0156 \\
\hline 0.7240 & 1.1992 & 1.3431 & $9.3023 e-3$ & 0.0000 & -0.0099 & 0.0000 & 0.0000 & 0.0109 & 0.0000 & -0.0118 & -0.0237 \\
\hline 0.7280 & 1.1992 & 1.3352 & 0.0000 & 0.0096 & 0.0000 & 0.0000 & 0.0108 & 0.0218 & -0.0115 & -0.0118 & 0.0101 \\
\hline 0.7320 & 1.1927 & 1.3352 & 0.0000 & 0.0000 & 0.0099 & -0.0103 & 0.0216 & 0.0109 & -0.0115 & 0.0000 & $4.6486 \mathrm{e}-3$ \\
\hline 0.7360 & 1.1992 & 1.3 & $9.3023 e-3$ & 0.0096 & 0.0 & 0.0 & 0. & 09 & 00 & 00 & $08 \mathrm{e}-3$ \\
\hline 0.7400 & 1.1992 & 1.3 & 0.0000 & 0.0000 & 0.0099 & -0.0103 & 0.0000 & 0.0109 & -0. & 0.0000 & $6.4524 \mathrm{e}-3$ \\
\hline 0.7440 & 1.1927 & 1.3272 & 0.0000 & 0.0000 & 0.0000 & -0.0103 & 0.0000 & 0.0109 & 0.0000 & 0.0000 & -0.0118 \\
\hline 0.7480 & 1.1862 & 1.3431 & 0.0186 & 0.0000 & 0.0000 & & 0.0108 & 0.0109 & 00 & 0.0118 & $-4.7445 \mathrm{e}-3$ \\
\hline 0.7520 & 1.1862 & 1.3511 & $9.3023 e-3$ & 0.0096 & 0.0000 & 0.0000 & 0.0108 & 0.0109 & 0.0115 & 0.0118 & 0.0184 \\
\hline 0.7560 & 1.1927 & 1.3431 & 0.0000 & 0.0000 & 0.0000 & 0.0103 & 0.0000 & 0.0109 & -0.0115 & 0.0000 & 0.0203 \\
\hline 0.7600 & 1.1797 & 1.3431 & 0.0000 & 0.0096 & 0.0099 & 0.0103 & 0.0000 & 0.0109 & 0.0000 & -0.0118 & 0.0252 \\
\hline 0.7640 & 1.1797 & 1.3 & 0.0000 & 0.0096 & 0.0000 & & 0.0108 & 18 & 00 & 0.0118 & -0.0327 \\
\hline 0.7680 & 1.1862 & 128 & 0.0000 & 0.0096 & 0 & 00 & 0.0 & 99 & 08 & 236 & 0.0 \\
\hline 0.7720 & 1.1862 & 1.3511 & $-9.3023 e-3$ & 0.0096 & 0.0198 & 0.0103 & 0.0000 & 0.0109 & -0.0115 & 0.0000 & 0.0 \\
\hline 0.7760 & 1.1797 & 1.3590 & 0.0000 & 0.0000 & 0.0000 & 0.0103 & 0.0108 & 0.0109 & 0.0000 & -0.0118 & 0.0362 \\
\hline 0.7800 & 1.1797 & 1.3670 & $-9.3008 e-3$ & 0.0000 & 0.0099 & 0.0000 & 0.0108 & 0.0109 & 0.0115 & 0.0000 & $8.2553 \mathrm{e}-3$ \\
\hline 0.7840 & 1.1732 & 1.3670 & 0.0000 & 0.0096 & 0.0000 & 0.0000 & 0.0108 & 0.0218 & -0.0115 & -0.0118 & $-1.5181 \mathrm{e}-3$ \\
\hline 0.7880 & 1.1927 & 1.3749 & $9.3023 e-3$ & -0.0096 & 0.0000 & 0.0103 & 0.0216 & 0.0000 & 0.0115 & -0.0118 & 0.0181 \\
\hline 0.7920 & 1.1732 & 1.3670 & 00000 & 0.0000 & 0.0000 & 0.0000 & 0.0108 & 0.0000 & 0.0000 & 0.0000 & 0.0179 \\
\hline 0.7960 & 1.1797 & 1.3749 & $9.3023 e-3$ & 0.0000 & 0.0000 & 0.0000 & 0.0108 & 0.0109 & -0.0115 & -0.0118 & 0.0206 \\
\hline 0.8000 & 1.1732 & 1.3670 & $-9.3023 e-3$ & 0.0096 & -0.0099 & 0.0000 & -0.0108 & 0.0109 & 0.0000 & 0.0118 & $-9.5880 e-5$ \\
\hline 0.8040 & 1.1797 & 1.3749 & 0.0000 & 0.0000 & -0.0099 & 0.0000 & 0.0000 & 0.0109 & -0.0115 & 0.0000 & 0.0186 \\
\hline 0.8080 & 1.1732 & 1.3670 & 0.0186 & 0.0096 & 0.0000 & 0.0000 & 0.0108 & 0.0109 & 0.0115 & 0.0000 & -0.0100 \\
\hline 0.8120 & 1.1797 & 1.3670 & 0.0000 & 0.0000 & 0.0000 & 0.0000 & 0.0216 & 0.0109 & 0.0000 & 0.0000 & $-6.0676 \mathrm{e}-3$ \\
\hline 0.8160 & 1.1732 & 1.3749 & 0.0000 & 0.0000 & 0.0000 & 0.0103 & 0.0000 & 0.0109 & 0.0115 & -0.0118 & $-9.7686 e-3$ \\
\hline 0.8200 & 1.1732 & 1.3829 & $9.3023 e-3$ & 0.0000 & 0.0000 & 0.0103 & -0.0108 & 0.0109 & 0.0000 & 0.0000 & $-6.2626 \mathrm{e}-3$ \\
\hline 0.8240 & 1.1601 & 1.3829 & $9.3023 e-3$ & 0.0096 & 0.0000 & -0.0103 & -0.0108 & 0.0000 & -0.0115 & 0.0000 & 0.0230 \\
\hline 0.8280 & 1.1601 & 1.3829 & 0.0651 & 0.0000 & -0.0297 & -0.0206 & -0.0108 & -0.0109 & -0.0230 & & $-3.8899 \mathrm{e}-3$ \\
\hline 0.8320 & 1.1732 & 1.3908 & 0.3069 & -0.0288 & -0.0496 & -0.0310 & -0.0323 & -0.0109 & -0.0115 & 0.0000 & $7.9708 \mathrm{e}-3$ \\
\hline 0.8360 & 1.1732 & 1.3749 & 0.5673 & -0.0385 & -0.0595 & -0.0516 & -0.0323 & -0.0218 & -0.0230 & -0.0236 & 0.0109 \\
\hline 0.8400 & 1.1666 & 1.3670 & 0.9116 & -0.0673 & -0.0496 & -0.0413 & -0.0323 & -0.0218 & -0.0345 & -0.0236 & $-2.5632 \mathrm{e}-3$ \\
\hline 0.8440 & 1.1666 & 1.3590 & 1.1906 & -0.0673 & -0.0198 & -0.0413 & -0.0108 & 0.0000 & -0.0345 & -0.0118 & 0.0115 \\
\hline 0.8480 & 1.1666 & 1.3670 & 1.2743 & -0.0385 & 0.0000 & -0.0103 & 0.0000 & 0.0000 & 0.0000 & -0.0236 & $1.8025 \mathrm{e}-3$ \\
\hline 0.8520 & 1.1601 & 1.3749 & 1.3115 & -0.0096 & 0.0000 & -0.0103 & 0.0000 & 0.0109 & -0.0115 & 0.0000 & $6.7276 \mathrm{e}-3$ \\
\hline
\end{tabular}




\begin{tabular}{|c|c|c|c|c|c|c|c|c|c|c|c|}
\hline 0.8560 & 1.1601 & 1.3829 & 1.3208 & 0.0000 & 0.0000 & 0.0000 & 0.0108 & 0.0109 & -0.0115 & -0.0118 & 0.0130 \\
\hline 0.8600 & 1.1601 & 1.3829 & 1.3115 & 0.0000 & -0.0099 & -0.0103 & 0.0000 & 0.0109 & -0.0115 & -0.0118 & -0.0136 \\
\hline 0.8640 & 1.1471 & 1.3829 & 1.3208 & -0.0096 & 0.0000 & 0.0000 & 0.0000 & 0.0109 & -0.0115 & 0.0118 & 0.0259 \\
\hline 0.8680 & 1.1601 & 1.3829 & 1.3115 & 0.0000 & 0.0099 & 0.0000 & 0.0216 & -0.0109 & 0.0000 & -0.0236 & $9.0137 \mathrm{e}-3$ \\
\hline 0.8720 & 1.1536 & 1.3829 & 1.3208 & 0.0000 & 0.0000 & -0.0103 & 0.0000 & 0.0000 & 0.0000 & 0.0000 & 0.0518 \\
\hline 0.8760 & 1.1536 & 1.3908 & 1.3208 & 0.0096 & 0.0000 & 0.0000 & 0.0108 & 0.0109 & 0.0115 & 0.0118 & $-6.6477 \mathrm{e}-3$ \\
\hline 0.8800 & 1.1471 & 1.3829 & 1.3301 & 0.0000 & 0.0000 & -0.0103 & 0.0000 & 0.0109 & 0.0000 & -0.0118 & $2.7518 \mathrm{e}-3$ \\
\hline 0.8840 & 1.1471 & 1.3987 & 1.3301 & 0.0000 & 0.0000 & 0.0000 & 0.0108 & 0.0109 & -0.0115 & 0.0118 & 0.0154 \\
\hline 0.8880 & 1.1536 & 1.3908 & 1.3301 & 0.0000 & 0.0000 & 0.0000 & 0.0108 & 0.0109 & 0.0000 & 0.0000 & $6.1683 \mathrm{e}-3$ \\
\hline 0.8920 & 1.1406 & 1.3908 & 1.3301 & 0.0000 & 0.0099 & 0.0000 & 0.0108 & 0.0109 & 0.0000 & -0.0118 & $4.6502 \mathrm{e}-3$ \\
\hline 0.8960 & 1.1341 & 1.3908 & 1.3301 & 0.0000 & 0.0000 & 0.0000 & 0.0108 & 0.0000 & -0.0115 & -0.0236 & -0.0137 \\
\hline 0.9000 & 1.1406 & 1.3908 & 1.3301 & 0.0096 & 0.0000 & 0.0000 & 0.0108 & 0.0218 & 0.0000 & 0.0000 & $7.8765 e-3$ \\
\hline 0.9040 & 1.1341 & 1.3829 & 1.3487 & -0.0096 & 0.0000 & -0.0103 & 0.0000 & 0.0000 & 0.0000 & -0.0118 & 0.0133 \\
\hline 0.9080 & 1.1406 & 1.3909 & 1.3394 & 0.0000 & 0.0099 & -0.0103 & 0.0000 & 0.0218 & 0.0000 & 0.0000 & 0.0283 \\
\hline 0.9120 & 1.1406 & 1.3829 & 1.3487 & 0.0096 & 0.0000 & 0.0000 & 0.0000 & 0.0109 & 000 & 0.0000 & $-6.3584 \mathrm{e}-3$ \\
\hline 0.9160 & 1.1275 & 1.3909 & 1.3487 & 0.0096 & 0.0000 & -0.0103 & 0.0000 & 0.0109 & -0.0115 & 0.0000 & 253 \\
\hline 0.9200 & 1.1275 & 1.3829 & 1.3580 & 0.0000 & 0.0000 & -0.0103 & 0.0108 & 0.0109 & 0.0000 & -0.0118 & 0.0214 \\
\hline 0.9240 & 1.1341 & 1.3909 & 1.3580 & -0.0096 & 0.0000 & -0.0103 & 0.0216 & 0.0000 & -0.0115 & -0.0118 & 0.0226 \\
\hline 0.9280 & 1.1341 & 1.3988 & 1.3580 & 0.0096 & 0.0000 & 0.0000 & 0.0108 & 0.0000 & 0.0000 & 0.0118 & $-1.3279 \mathrm{e}-3$ \\
\hline 0.9320 & 1.1209 & 1.3829 & 1.3673 & 0.0000 & 0.0099 & -0.0103 & 0.0000 & 0.0109 & 0.0000 & -0.0118 & 0.0315 \\
\hline 0.9360 & 1.1209 & 1.3909 & 1.3673 & 0.0000 & 0.0000 & 0.0000 & 0.0000 & 0.0109 & & -0.0118 & -0.0378 \\
\hline 0.9400 & 1.1274 & 1.3909 & 1.3673 & 0.0096 & 0.0000 & 0.0000 & 0.0108 & 0.0218 & -0.0115 & 118 & $48 \mathrm{e}-3$ \\
\hline 0.9440 & 1.1144 & 1.3988 & 1.3673 & 0.0000 & 0.0099 & 0.0000 & 0.0000 & 0.0000 & 0.0115 & 0.0 & $387 e-3$ \\
\hline 0.9480 & 1.1209 & 1.3988 & 1.3766 & 0.0000 & 0.0000 & 0.0000 & 0.0000 & 0.0109 & 0.0115 & -0.0118 & -0.0124 \\
\hline 0.9520 & 1.1144 & 1.3829 & 1.3766 & 0.0000 & 0.0099 & 0.0103 & 0.0000 & -0.0109 & -0.0230 & 0.0000 & $7.6867 \mathrm{e}-3$ \\
\hline 0.9560 & 1.1144 & 1.3988 & 1.3766 & 0.0000 & 0.0099 & -0.0103 & 0.0000 & 0.0218 & 0.0000 & -0.0118 & -0.0137 \\
\hline 0.9600 & 1.1079 & 1.3988 & 1.3766 & 0.0000 & 0.0000 & 0.0103 & 0.0000 & 0.0109 & -0.0230 & -0.0118 & 0.0165 \\
\hline 0.9640 & 1.0949 & 1.4068 & 1.3766 & 0.0000 & 0.0000 & 0.0000 & 0.0108 & 0.0000 & & -0.0118 & $19 e-3$ \\
\hline 0.9680 & 1.0949 & 1.3988 & 1.3859 & 0.0000 & 0.0099 & -0 & 0.0108 & 0.0109 & & 0.0 & $200 e-3$ \\
\hline 0.9720 & 1.1079 & 1.3909 & 1.3766 & 0.0096 & 0.0000 & 0.0000 & 0.0216 & 0.0109 & 0.0000 & -0.0118 & -0.0149 \\
\hline 0.9760 & 1.0949 & 1.4068 & 1.3859 & 0.0192 & -0.0099 & -0.0206 & 0.0000 & 0.0000 & & -0 & $72 e-3$ \\
\hline 0.9800 & 1.1014 & 1.3988 & 1.3766 & 0.0192 & -0.0396 & -0.0103 & -0.0216 & -0.0109 & 0.0 & -0.0118 & -0.0343 \\
\hline 0.9840 & 1.1014 & 1.3909 & 1.3580 & 0.1635 & -0.0496 & -0.0516 & -0.0323 & -0.0218 & -0.0230 & -0.0236 & 0.0183 \\
\hline 0.9880 & 1.0949 & 1.3988 & 1.3301 & 0.4520 & -0.0694 & -0.0619 & -0.0323 & -0.0327 & -0.0115 & -0.0236 & 0.0251 \\
\hline 0.9920 & 1.1014 & 1.3988 & 1.3208 & 0.8078 & -0.0595 & -0.0619 & -0.0215 & -0.0218 & 45 & 236 & 114 \\
\hline 0.9960 & 1.1014 & 1.3 & 1.3 & 59 & -0.0297 & -0 & 08 & 00 & 5 & -0 & \\
\hline 1.0000 & 1.1014 & 1.3909 & 1.3673 & 1.2789 & -0.0099 & -0.0206 & 0.0000 & 0.0000 & -0.0115 & -0.0118 & $-2.8476 \mathrm{e}-3$ \\
\hline 1.0040 & 1.1209 & 1.4147 & 1.3859 & 1.3077 & -0.0099 & 0.0000 & 0.0108 & 0.0109 & -0.0115 & -0.0236 & $-9.3946 e-3$ \\
\hline 1.0080 & 1.1014 & 1.3988 & 1.3952 & 1.3366 & 0.0000 & 0.0000 & 0.0000 & 0.0000 & 0.0000 & 0.0118 & -0.0316 \\
\hline 1.0120 & 1.1209 & 1.3909 & 1.3952 & 1.3366 & 0.0099 & 0.0000 & 0.0108 & 0.0109 & -0.0115 & -0.0118 & -0.0196 \\
\hline 1.0160 & 1.1014 & 1.3988 & 1.4045 & 1.3462 & 0.0000 & -0.0103 & 0.0000 & 0.0109 & 0.0000 & 0.0000 & $-5.1232 \mathrm{e}-3$ \\
\hline 1.0200 & 1.1079 & 1.3988 & 1.4045 & 1.3366 & 0.0000 & 0.0000 & 0.0216 & 0.0109 & 115 & 18 & $3.9854 \mathrm{e}-3$ \\
\hline 1.0240 & 1.1014 & 1.3988 & 1.4138 & 62 & 0.0000 & -0 & -0.0 & 09 & 0. & $0 .($ & 25 \\
\hline 1.0280 & 1.0949 & 1.4068 & 1.4045 & 1.3558 & 0.0000 & 0.0000 & -0.0108 & 0.0109 & 0.0000 & -0.0118 & 0.0136 \\
\hline 1.0320 & 1.0818 & 1.4068 & 1.4138 & 1.3654 & -0.0099 & -0.0103 & 0.0000 & 0.0000 & -0.0115 & -0.0118 & 0.0221 \\
\hline 1.0360 & 1.0818 & 1.4068 & 1.4045 & 1.3654 & 0.0099 & 0.0103 & 0.0216 & 0.0109 & -0.0115 & 0.0000 & 0.0217 \\
\hline 1.0400 & 1.0688 & 1.3988 & 1.4138 & 1.3751 & 0.0000 & -0.0103 & 0.0000 & 0.0109 & 0.0000 & 0.0000 & -0.0112 \\
\hline 1.0440 & 1.0623 & 1.4068 & 1.4045 & 1.3751 & 0.0000 & 0.0103 & 0.0108 & 0.0218 & -0.0115 & -0.0118 & $-5.3134 \mathrm{e}-3$ \\
\hline 1.0480 & 1.0558 & 1.4068 & 1.4045 & 51 & 0.0000 & -0 . & 00 & 09 & 15 & 118 & -0.0141 \\
\hline 1.0520 & 1.0558 & 1.3988 & 1.4045 & 1.3751 & 0.0000 & 0.0103 & 0.0000 & 0.0000 & 0.0115 & 0.0000 & 0.0193 \\
\hline 1.0560 & 1.0493 & 1.3988 & 1.4138 & 1.3751 & -0.0099 & -0.0103 & 0.0000 & 0.0109 & -0.0115 & 0.0000 & 0.0330 \\
\hline 1.0600 & 1.0427 & 1.4147 & 1.4138 & 1.3847 & 0.0198 & 0.0000 & 0.0108 & 0.0000 & 0.0000 & 0.0118 & 0.0222 \\
\hline 1.0640 & 1.0297 & 1.3988 & 1.4138 & 1.3943 & 0.0000 & 0.0103 & 0.0216 & 0.0109 & 0.0000 & -0.0118 & $-7.2119 \mathrm{e}-3$ \\
\hline 1.0680 & 1.0297 & 1.4068 & 1.4045 & 1.4039 & 0.0099 & 0.0000 & 0.0108 & 0.0218 & 0.0000 & -0.0118 & 0.0334 \\
\hline 1.0720 & 1.0297 & 1.3987 & 1.4045 & 1.4039 & 0.0000 & -0.0103 & 0.0108 & 0.0000 & 0.0000 & -0.0118 & $7.8754 \mathrm{e}-3$ \\
\hline 1.0760 & 1.0167 & 1.4068 & 1.4138 & 1.4039 & -0.0099 & 0.0000 & 0.0000 & 0.0109 & 0.0000 & 0.0000 & -0.0150 \\
\hline 1.0800 & 1.0102 & 1.4067 & 1.4138 & 1.4039 & 0.0000 & -0.0103 & 0.0108 & -0.0109 & 0.0000 & 0.0000 & $1.4238 \mathrm{e}-3$ \\
\hline 1.0840 & 1.0167 & 1.4067 & 1.4231 & 1.4135 & 0.0099 & 0.0000 & 0.0000 & 0.0109 & 0.0000 & -0.0118 & 0.0194 \\
\hline 1.0880 & 1.0036 & 1.3908 & 1.4138 & 1.4135 & 0.0000 & 0.0000 & 0.0108 & 0.0000 & -0.0230 & 0.0000 & 0.0377 \\
\hline 1.0920 & 1.0036 & 1.4067 & 1.4138 & 1.4135 & 0.0000 & -0.0103 & 0.0108 & 0.0109 & 0.0000 & 0.0000 & 0.0309 \\
\hline 1.0960 & 0.9905 & 1.4067 & 1.4324 & 1.4135 & 0.0000 & -0.0103 & 0.0000 & 0.0109 & -0.0115 & -0.0118 & $6.9257 \mathrm{e}-3$ \\
\hline 1.1000 & 0.9970 & 1.4067 & 1.4324 & 1.4135 & -0.0099 & 0.0000 & 0.0000 & 0.0109 & -0.0115 & -0.0118 & -0.0138 \\
\hline 1.1040 & 0.9970 & 1.3987 & 1.4138 & 1.4135 & -0.0099 & 0.0000 & 0.0108 & 0.0109 & 0.0000 & -0.0236 & 0.0122 \\
\hline 1.1080 & 0.9905 & 1.3908 & 1.4231 & 1.4231 & 0.0099 & 0.0000 & 0.0000 & 0.0109 & 0.0000 & 0.0000 & $1.7083 \mathrm{e}-3$ \\
\hline 1.1120 & 0.9905 & & 1.4417 & 1.4231 & 0.0000 & 0.0000 & 0.0000 & 0.0109 & -0.0115 & 0.0000 & $5.7880 \mathrm{e}-3$ \\
\hline 1.1160 & 0.9905 & 1.3987 & 1.4324 & 1.4231 & 0.0000 & 0.0000 & 0.0108 & 0.0109 & -0.0115 & -0.0118 & 0.0149 \\
\hline 1.1200 & 0.9775 & 1.3987 & 1.4324 & 1.4231 & 0.0099 & -0.0103 & 0.0000 & 0.0109 & -0.0115 & -0.0118 & 0.0331 \\
\hline 1.1240 & 0.9775 & 1.3987 & 1.4324 & 1.4328 & 0.0198 & -0.0103 & 0.0000 & 0.0000 & 0.0000 & -0.0118 & $9.1087 \mathrm{e}-3$ \\
\hline 1.1280 & 0.9645 & 1.3908 & 1.4510 & 1.4328 & 0.1982 & -0.0310 & -0.0216 & 0.0000 & -0.0230 & 0.0118 & 0.0217 \\
\hline 1.1320 & 0.9645 & 1.4146 & 1.4324 & 1.4231 & 0.4857 & -0.0413 & -0.0216 & 0.0000 & -0.0230 & -0.0118 & $5.5035 \mathrm{e}-3$ \\
\hline
\end{tabular}




\begin{tabular}{|c|c|c|c|c|c|c|c|c|c|c|c|}
\hline 1.1360 & 0.9645 & 1.3908 & 1.4231 & 1.4039 & 0.8128 & -0.0516 & -0.0539 & -0.0218 & -0.0230 & -0.0236 & 0.0169 \\
\hline 1.1400 & 0.9710 & 1.3829 & 1.3952 & 1.3943 & 1.1498 & -0.0619 & -0.0431 & -0.0218 & -0.0345 & -0.0236 & $9.6788 \mathrm{e}-3$ \\
\hline 1.1440 & 0.9645 & 1.3829 & 1.3859 & 1.3751 & 1.3381 & -0.0516 & -0.0323 & -0.0218 & -0.0230 & -0.0118 & -0.0239 \\
\hline 1.1480 & 0.9579 & 1.3987 & 1.4045 & 1.3943 & 1.3778 & -0.0103 & -0.0108 & -0.0109 & -0.0115 & -0.0118 & -0.0365 \\
\hline 1.1520 & 0.9449 & 1.3829 & 1.4324 & 1.4135 & 1.3778 & -0.0206 & -0.0108 & 0.0000 & 0.0000 & 0.0000 & -0.0200 \\
\hline 1.1560 & 0.9449 & 1.3908 & 1.4324 & 1.4424 & 1.3976 & -0.0103 & 0.0000 & 0.0109 & 0.0000 & 0.0000 & -0.0224 \\
\hline 1.1600 & 0.9384 & 1.3908 & 1.4417 & 1.4424 & 1.3877 & 0.0000 & -0.0216 & 0.0109 & -0.0230 & 0.0000 & 0.0111 \\
\hline 1.1640 & 0.9384 & 1.3908 & 1.4417 & 1.4520 & 1.3976 & 0.0000 & 0.0108 & 0.0109 & 0.0000 & -0.0118 & $3.4149 \mathrm{e}-3$ \\
\hline 1.1680 & 0.9384 & 1.3829 & 1.4510 & 1.4520 & 1.3877 & -0.0206 & 0.0108 & 0.0109 & 0.0115 & 0.0000 & -0.0153 \\
\hline 1.1720 & 0.9384 & 1.3908 & 1.4417 & 1.4520 & 1.4075 & 0.0000 & 0.0000 & 0.0000 & 0.0000 & -0.0236 & 0.0379 \\
\hline 1.1760 & 0.9319 & 1.3829 & 1.4510 & 1.4520 & 1.4174 & 0.0000 & 0.0000 & 0.0000 & 0.0000 & 0.0000 & 0.0236 \\
\hline 1.1800 & 0.9254 & 1.3749 & 1.4510 & 1.4520 & 1.4174 & 0.0000 & 0.0108 & 0.0000 & -0.0115 & 0.0000 & 0.0222 \\
\hline 1.1840 & 0.9319 & 1.3749 & 1.4603 & 1.4616 & 1.4273 & 0.0000 & 0.0000 & 0.0109 & 0.0000 & 0.0000 & 0.0130 \\
\hline 1.1880 & 0.9188 & 1.3829 & 1.4603 & 1.4616 & 1.4174 & 0.0000 & 0.0000 & 0.0109 & 0.0000 & 0.0118 & $2.1813 e-3$ \\
\hline 1.1920 & 0.9254 & 1.3829 & 1.4603 & 1.4616 & 1.4273 & -0.0103 & 0.0108 & 0.0109 & -0.0115 & 0.0118 & -0.0203 \\
\hline 1.1960 & 0.9319 & 1.3829 & 1.4510 & 1.4616 & 1.4372 & -0.0103 & 0.0108 & 0.0109 & 0.0000 & 0.0000 & 0.0204 \\
\hline 1.2000 & 0.9254 & 1.3749 & 1.4510 & 1.4712 & 1.4273 & -0.0103 & 0.0000 & 0.0000 & 0.0115 & -0.0118 & 0.0158 \\
\hline 1.2040 & 0.9384 & 1.3670 & 1.4603 & 1.4712 & 1.4372 & -0.0103 & 0.0000 & 0.0109 & 0.0000 & -0.0118 & $6.2626 \mathrm{e}-3$ \\
\hline 1.2080 & 0.9384 & 1.3670 & 1.4603 & 1.4712 & 1.4472 & 0.0000 & 0.0108 & 0.0109 & -0.0115 & 0.0000 & 223 \\
\hline 1.2120 & 0.9384 & 1.3749 & 1.4603 & 1.4712 & 1.4472 & 0.0000 & 0.0108 & 0.0218 & -0.0115 & 0.0000 & $-3.7873 e-4$ \\
\hline 1.2160 & 0.9384 & 1.3670 & 1.4603 & 1.4712 & 1.4571 & 0.0000 & 0.0000 & 0.0109 & -0.0115 & 0.0000 & -0.0266 \\
\hline 1.2200 & 0.9384 & 1.3749 & 1.4603 & 1.4712 & 1.4571 & -0.0103 & 0.0216 & 0.0109 & 0.0000 & 0.0000 & $1.6140 \mathrm{e}-3$ \\
\hline 1.2240 & 0.9384 & 1.3590 & 1.4603 & 1.4712 & 1.4571 & 0.0000 & 0.0108 & 0.0109 & -0.0230 & -0.0118 & -0.0115 \\
\hline 1.2280 & 0.9449 & 1.3590 & 1.4603 & 1.4712 & 1.4571 & 0.0000 & 0.0000 & 0.0000 & 0.0115 & 0.0000 & $-6.7292 \mathrm{e}-3$ \\
\hline 1.2320 & 0.9319 & 1.3590 & 1.4510 & 1.4616 & 1.4571 & -0.0103 & 0.0000 & 0.0109 & 115 & 0.0000 & \\
\hline 1.2360 & 0.9254 & 1.3670 & 1.4510 & 1.4712 & 1.4571 & 0.0000 & 0.0000 & 0.0109 & 00 & -0.0118 & $97 e-3$ \\
\hline 1.2400 & 0.9188 & 1.3590 & 1.4510 & 1.4808 & 1.4769 & 0.0000 & -0.0108 & 0.0109 & -0.0115 & 0.0000 & 0.0269 \\
\hline 1.2440 & 0.9188 & 1.3590 & 1.4417 & 1.4808 & 1.4769 & -0.0103 & 0.0000 & 0.0000 & -0.0115 & 0.0000 & 0.0107 \\
\hline 1.2480 & 0.9384 & 1.3431 & 1.4510 & 1.4905 & 1.4769 & 0.0000 & 0.0000 & -0.0109 & 0.0000 & 0.0000 & $9.7750 \mathrm{e}-3$ \\
\hline 1.2520 & 0.9319 & 1.3590 & 1.4510 & 1.4808 & 1.4769 & 0.0000 & 0.0108 & 0.0109 & -0.0115 & 0.0000 & 0.0247 \\
\hline 1.2560 & 0.9254 & 1.3511 & 1.4603 & 1.4808 & 1.4670 & 0.0000 & 0.0108 & 0.0109 & -0.0115 & 0.0000 & 0.0124 \\
\hline 1.2600 & 0.9123 & 1.3590 & 1.4603 & 1.4808 & 1.4868 & 0 & 0.0 & 0.0109 & 0.0 & 0.0 & $54 \mathrm{e}-3$ \\
\hline 1.2640 & 0.8928 & 1.3511 & 1.4603 & 1.4905 & 1.4868 & 00 & 0.0108 & 0.0000 & -0.0 & 0.0000 & -0 \\
\hline 1.2680 & 0.8863 & 1.3511 & 1.4603 & 1.4905 & 1.4967 & -0.0103 & 0.0000 & 0.0109 & -0.0115 & 0.0000 & -0.0208 \\
\hline 1.2720 & 0.8798 & 1.3511 & 1.4603 & 1.4905 & 1.4967 & -0.0103 & -0.0108 & 0.0109 & 0.0000 & 0.0000 & $2.5632 \mathrm{e}-3$ \\
\hline 1.2760 & 0.8602 & 1.3590 & 1.4510 & 1.4905 & 1.4769 & 0.1341 & -0.0108 & 0.0000 & 0.0000 & -0.0118 & 0.0423 \\
\hline 1.2800 & 0.8537 & 1.3511 & 1.4510 & 1.4905 & 1.4571 & 0.4435 & -0.0323 & -0.0218 & -0.0115 & -0.0118 & $-9.1086 \mathrm{e}-3$ \\
\hline 1.2840 & 0.8472 & 1.3590 & 1.4510 & 1.4616 & 1.4372 & 0.8045 & -0.0539 & -0.0436 & -0.0115 & -0.0236 & $-7.0215 \mathrm{e}-3$ \\
\hline 1.2880 & 0.8537 & 1.3 & 1.4 & 1. & 72 & & -0 & 27 & -0 & 173 & $13 e-3$ \\
\hline 1.2920 & 0.8667 & 1.3431 & 1.4231 & 1.4424 & 1.4571 & 1.3719 & -0.0108 & -0.0218 & -0.0345 & -0.0355 & -0.0117 \\
\hline 1.2960 & 0.8472 & 1.3511 & 1.4138 & 1.4616 & 1.4967 & 1.4028 & -0.0108 & -0.0218 & 0.0000 & -0.0355 & -0.0176 \\
\hline 1.3000 & 0.8732 & 1.3431 & 1.4417 & 1.4712 & 1.4967 & 1.4234 & 0.0000 & 0.0109 & -0.0115 & -0.0236 & $-9.4921 \mathrm{e}-4$ \\
\hline 1.3040 & 0.8602 & 1.3431 & 1.4417 & 1.4905 & 1.5165 & 1.4337 & 0.0000 & 0.0000 & 0.0000 & -0.0118 & 0.0138 \\
\hline 1.3080 & 0.8146 & 1.3431 & 1.4417 & 1.4905 & 1.5066 & 1.4441 & 0.0108 & 0.0218 & -0.0115 & 0.0118 & -0.0160 \\
\hline 1.3120 & 0.7885 & 1.3431 & 1.4603 & 1.4905 & 1.5264 & 1.4544 & -0.0108 & 0.0000 & -0.0115 & 0.0118 & $-7.1175 e-3$ \\
\hline 1.3160 & 0.7755 & 1.3 & 03 & 1. & 64 & 17 & 0. & 09 & 5 & 18 & $72 \mathrm{e}-3$ \\
\hline 1.3200 & 0.7625 & 1.3431 & 1.4417 & 1.5097 & 1.5165 & 1.4647 & -0.0108 & 0.0109 & -0.0115 & -0.0118 & -0.0114 \\
\hline 1.3240 & 0.7364 & 1.3352 & 1.4510 & 1.5001 & 1.5165 & 1.4750 & 0.0000 & 0.0109 & 0.0000 & 0.0000 & -0.0263 \\
\hline 1.3280 & 0.6843 & 1.3352 & 1.4603 & 1.5001 & 1.5165 & 1.4750 & 0.0000 & 0.0218 & -0.0115 & -0.0236 & $-7.4003 e-3$ \\
\hline 1.3320 & 0.6712 & 1.3193 & 1.4510 & 1.5001 & 1.5165 & 1.4853 & -0.0108 & 0.0109 & 0.0000 & 0.0000 & $-6.6317 e-4$ \\
\hline 1.3360 & 0.6908 & 1.3193 & 1.4510 & 1.5097 & 1.5165 & 1.4956 & 0.0000 & 0.0109 & 0.0000 & 0.0118 & $2.9418 \mathrm{e}-3$ \\
\hline 1.3400 & 0.6908 & 1.3272 & 1.4510 & 1.4905 & 1.5364 & 1.4853 & -0.0108 & 0.0109 & 0.0115 & 0.0236 & 0.0313 \\
\hline 1.3440 & 0.6973 & 1.3113 & 1.4510 & 1.5001 & 1.5364 & 1.4956 & 0.0000 & 0.0109 & -0.0115 & 0.0118 & $-5.5978 \mathrm{e}-3$ \\
\hline 1.3480 & 0.7038 & 1.3272 & 1.4510 & 1.5001 & 1.5264 & 1.5059 & 0.0000 & 0.0109 & -0.0115 & 0.0118 & $9.6791 \mathrm{e}-3$ \\
\hline 1.3520 & 0.7299 & 1.3113 & 1.4510 & 1.5097 & 1.5364 & 1.4956 & 0.0216 & 0.0109 & 0.0000 & 0.0000 & $5.7896 \mathrm{e}-3$ \\
\hline 1.3560 & 0.7234 & 1.3113 & 1.4417 & 1.5097 & 1.5364 & 1.5163 & 0.0000 & 0.0109 & 0.0000 & -0.0118 & 0.0121 \\
\hline 1.3600 & 0.7103 & 1.3113 & 1.4510 & 1.5097 & 1.5264 & 1.5163 & 0.0000 & 0.0000 & 0.0000 & 0.0000 & 0.0380 \\
\hline 1.3640 & 0.7169 & 1.3034 & 1.4417 & 1.5001 & 1.5364 & 1.5163 & 0.0000 & 0.0109 & 0.0000 & -0.0118 & 0.0150 \\
\hline 1.3680 & 0.7038 & 1.2954 & 1.4417 & 1.5097 & 1.5264 & 1.5266 & 0.0000 & -0.0109 & 0.0000 & -0.0118 & $-7.5921 \mathrm{e}-3$ \\
\hline 1.3720 & 0.7169 & 1.2954 & 1.4417 & 1.4905 & 1.5364 & 1.5266 & 0.0000 & 0.0109 & -0.0115 & 0.0118 & $-1.3279 \mathrm{e}-3$ \\
\hline 1.3760 & 0.7234 & 1.2954 & 1.4510 & 1.5193 & 1.5364 & 1.5163 & 0.0000 & 0.0000 & 0.0000 & -0.0118 & $1.5181 \mathrm{e}-3$ \\
\hline 1.3800 & 0.7299 & 1.2875 & 1.4417 & 1.5097 & 1.5364 & 1.5369 & -0.0216 & 0.0109 & 0.0115 & 0.0000 & $9.4889 \mathrm{e}-3$ \\
\hline 1.3840 & 0.7364 & 1.2875 & 1.4510 & 1.5097 & 1.5264 & 1.5369 & 0.0000 & 0.0109 & 0.0000 & 0.0000 & $-8.6404 \mathrm{e}-3$ \\
\hline 1.3880 & 0.7364 & 1.2875 & 1.4417 & 1.5001 & 1.5364 & 1.5472 & 0.0216 & 0.0218 & 0.0115 & 0.0000 & $-1.2337 \mathrm{e}-3$ \\
\hline 1.3920 & 0.7429 & 1.2796 & 1.4417 & 1.5001 & 1.5364 & 1.5369 & 0.0000 & 0.0000 & 0.0000 & -0.0236 & $-1.9016 \mathrm{e}-4$ \\
\hline 1.3960 & 0.7559 & 1.2716 & 1.4417 & 1.5097 & 1.5562 & 1.5575 & 0.0000 & 0.0000 & 0.0000 & -0.0118 & -0.0239 \\
\hline 1.4000 & 0.7559 & 1.2637 & 1.4417 & 1.5097 & 1.5463 & 1.5575 & 0.0000 & 0.0109 & 0.0000 & 0.0000 & $5.4076 \mathrm{e}-3$ \\
\hline 1.4040 & 0.7559 & 1.2716 & 1.4324 & 1.5001 & 1.5463 & 1.5472 & 0.0216 & 0.0000 & 0.0000 & 0.0000 & -0.0168 \\
\hline 1.4080 & 0.7559 & 1.2557 & 1.4324 & 1.5097 & 1.5463 & 1.5575 & 0.0108 & 0.0109 & 0.0000 & 0.0000 & -0.0212 \\
\hline 1.4120 & 0.7625 & 1.2637 & 1.4324 & 1.5097 & 1.5562 & 1.5678 & 0.0108 & 0.0109 & 0.0000 & 0.0118 & $-1.6140 \mathrm{e}-3$ \\
\hline
\end{tabular}




\begin{tabular}{|c|c|c|c|c|c|c|c|c|c|c|c|}
\hline 1.4160 & 0.7690 & 1.2557 & 1.4324 & 1.5097 & 1.5562 & 1.5781 & 0.0108 & 0.0000 & 0.0000 & 0.0118 & -0.0213 \\
\hline 1.4200 & 0.7625 & 1.2478 & 1.4324 & 1.5097 & 1.5562 & 1.5575 & 0.0970 & 0.0000 & -0.0115 & 0.0000 & $-1.9016 \mathrm{e}-4$ \\
\hline 1.4240 & 0.7625 & 1.2398 & 1.4324 & 1.5097 & 1.5264 & 1.5369 & 0.4525 & -0.0218 & -0.0460 & 0.0000 & 0.0112 \\
\hline 1.4280 & 0.7625 & 1.2557 & 1.4138 & 1.5097 & 1.5165 & 1.5266 & 0.8513 & -0.0327 & -0.0460 & -0.0355 & 0.0223 \\
\hline 1.4320 & 0.7559 & 1.2239 & 1.4138 & 1.4905 & 1.4967 & 1.5266 & 1.2824 & -0.0545 & -0.0690 & -0.0236 & 0.0131 \\
\hline 1.4360 & 0.7559 & 1.2239 & 1.4045 & 1.4712 & 1.5165 & 1.5472 & 1.4440 & -0.0327 & -0.0575 & -0.0236 & -0.0135 \\
\hline 1.4400 & 0.7429 & 1.2239 & 1.3952 & 1.4712 & 1.5364 & 1.5678 & 1.4978 & -0.0218 & -0.0345 & -0.0118 & -0.0268 \\
\hline 1.4440 & 0.7494 & 1.2239 & 1.4138 & 1.4808 & 1.5562 & 1.5885 & 1.5086 & -0.0109 & -0.0115 & -0.0118 & $-3.7010 e-3$ \\
\hline 1.4480 & 0.7429 & 1.2239 & 1.4231 & 1.4905 & 1.5463 & 1.5781 & 1.5086 & 0.0000 & -0.0115 & 0.0000 & -0.0141 \\
\hline 1.4520 & 0.7364 & 1.2319 & 1.4138 & 1.4905 & 1.5562 & 1.5988 & 1.5194 & 0.0000 & 0.0000 & -0.0118 & 0.0147 \\
\hline 1.4560 & 0.7429 & 1.2239 & 1.4231 & 1.5097 & 1.5562 & 1.5885 & 1.5302 & 0.0000 & 0.0000 & -0.0118 & $2.1813 \mathrm{e}-3$ \\
\hline 1.4600 & 0.7559 & 1.2239 & 1.4231 & 1.5001 & 1.5562 & 1.5885 & 1.5409 & -0.0109 & 0.0000 & -0.0118 & -0.0118 \\
\hline 1.4640 & 0.7559 & 1.2160 & 1.4231 & 1.4905 & 1.5661 & 1.5885 & 1.5625 & 0.0109 & 0.0000 & 0.0000 & $3.9854 \mathrm{e}-3$ \\
\hline 1.4680 & 0.7559 & 1.2239 & 1.4045 & 1.5001 & 1.5463 & 1.5988 & 1.5733 & 0.0109 & -0.0115 & 0.0000 & $7.1175 e-3$ \\
\hline 1.4720 & 0.7559 & 1.2239 & 1.4138 & 1.4905 & 1.5661 & 1.5988 & 1.5733 & 0.0000 & 0.0000 & 0.0000 & 0.0192 \\
\hline 1.4760 & 0.7559 & 1.2319 & 1.4231 & 1.4808 & 1.5562 & 1.6091 & 1.5733 & 0.0109 & -0.0115 & 0.0000 & $1.2335 \mathrm{e}-3$ \\
\hline 1.4800 & 0.7625 & 1.2239 & 1.4231 & 1.4905 & 1.5562 & 1.5988 & 1.5840 & 0.0109 & -0.0230 & 0.0000 & 0.0169 \\
\hline 1.4840 & 0.7690 & 1.2239 & 1.4045 & 1.4905 & 1.5562 & 1.5988 & 1.5948 & 0.0218 & -0.0115 & 0.0000 & $6.7365 e-3$ \\
\hline 1.4880 & 0.7625 & 1.2160 & 1.4138 & 1.4905 & 1.5463 & 1.5988 & 1.6056 & 0.0109 & -0.0115 & 0.0000 & $-6.9265 e-3$ \\
\hline 1.4920 & 0.7755 & 1.2239 & 1.3952 & 1.5001 & 1.5562 & 1.6091 & 1.5948 & 0.0000 & 0.0000 & -0.0118 & $8.4454 \mathrm{e}-3$ \\
\hline 1.4960 & 0.7755 & 1.2160 & 1.4138 & 1.4905 & 1.5562 & 1.6194 & 1.6164 & 0.0109 & 0.0000 & -0.0118 & -0.0185 \\
\hline 1.5000 & 0.7690 & 1.2080 & 1.4138 & 1.4905 & 1.5562 & 1.6091 & 1.6056 & 0.0218 & 0.0000 & 0.0236 & 0.0205 \\
\hline 1.5040 & 0.7755 & 1.2080 & 1.3952 & 1.4905 & 1.5562 & 1.6091 & 1.6164 & 0.0109 & 0.0000 & 0.0000 & $64 e-3$ \\
\hline 1.5080 & 0.7755 & 1.2080 & 1.3952 & 1.4905 & 1.5562 & 1.6091 & 1.6164 & 0.0109 & -0.0115 & 0.0000 & -0.0128 \\
\hline 1.5120 & 0.7755 & 1.2080 & 1.4045 & 1.4905 & 1.5562 & 1.6091 & 1.6271 & 0.0109 & -0.0115 & -0.0118 & $9.4921 \mathrm{e}-3$ \\
\hline 1.5160 & 0.7820 & 1.2160 & 1.3952 & 1.4808 & 1.5562 & 1.6091 & 1.6271 & 0.0109 & 0.0000 & -0.0118 & $7.5905 e-4$ \\
\hline 1.5200 & 0.7690 & 1.2001 & 1.3952 & 1.4905 & 1.5562 & 1.5988 & 1.6271 & 0.0000 & 0.0000 & 0.0000 & 0.0258 \\
\hline 1.5240 & 0.7625 & 1.2001 & 1.3952 & 1.4905 & 1.5562 & 1.5988 & 1.6379 & 0.0000 & 0.0000 & -0.0118 & 0.0285 \\
\hline 1.5280 & 0.7559 & 1.2080 & 1.3952 & 1.4905 & 1.5562 & 1.5988 & 1.6487 & 0.0109 & -0.0115 & -0.0118 & 0.0170 \\
\hline 1.5320 & 0.7559 & 1.2001 & 1.3952 & 1.4808 & 1.5562 & 1.5988 & 1.6 & 0.0109 & 15 & $0 .($ & $88 \mathrm{e}-3$ \\
\hline 1.5360 & 0.7559 & 1.1921 & 1.3952 & 1.4808 & 1.5562 & 1.6091 & 1.6271 & 0.0109 & -0.0115 & -0.0118 & 0.0165 \\
\hline 1.5400 & 0.7559 & 1.1921 & 1.3859 & 1.4712 & 1.5463 & 1.6091 & 1.6702 & 0.0109 & 0.0000 & -0.0118 & 54 \\
\hline 1.5440 & 0.7494 & 1.1921 & 1.3859 & 1.4808 & 1.5463 & 1.6091 & 1.6702 & 0.0109 & -0.0115 & 0.0000 & $6.0730 \mathrm{e}-3$ \\
\hline 1.5480 & 0.7429 & 1.1921 & 1.3859 & 1.4712 & 1.5562 & 1.6194 & 1.6702 & 0.0109 & 0.0000 & 0.0000 & $5.7800 \mathrm{e}-3$ \\
\hline 1.5520 & 0.7429 & 1.1921 & 1.3859 & 1.4712 & 1.5463 & 1.5988 & 1.6595 & 0.0109 & 0.0000 & 0.0000 & $-7.5905 e-3$ \\
\hline 1.5560 & 0.7429 & 1.1921 & 1.3859 & 1.4712 & 1.5364 & 1.6091 & 1.6702 & 0.0000 & & -0.0118 & $-9.6823 e-3$ \\
\hline 1.5600 & 0.7429 & 1.1842 & 1.3766 & 1.4616 & 1.5463 & 1.6 & 1. & 18 & $0 .($ & $0 .($ & $91 \mathrm{e}-3$ \\
\hline 1.5640 & 0.7299 & 1.1762 & 1.3859 & 1.4712 & 1.5463 & 1.5885 & 1.6271 & 0.2942 & -0.0345 & -0.0118 & -0.0174 \\
\hline 1.5680 & 0.7299 & 1.1683 & 1.3766 & 1.4616 & 1.5264 & 1.5781 & 1.6164 & 0.7954 & -0.0575 & -0.0591 & $3.4165 \mathrm{e}-3$ \\
\hline 1.5720 & 0.7234 & 1.1604 & 1.3487 & 1.4424 & 1.5066 & 1.5575 & 1.6164 & 1.3511 & -0.0690 & -0.0828 & $9.4282 \mathrm{e}-5$ \\
\hline 1.5760 & 0.7169 & 1.1604 & 1.3487 & 1.4231 & 1.5165 & 1.5885 & 1.6595 & 1.5690 & -0.0460 & -0.0591 & $-3.3206 e-3$ \\
\hline 1.5800 & 0.7299 & 1.1762 & 1.3487 & 1.4328 & 1.5264 & 1.5988 & 1.6810 & 1.6126 & -0.0230 & -0.0118 & $4.7443 \mathrm{e}-4$ \\
\hline 1.5840 & 0.7103 & 1.1 & 1.3 & 1.4328 & 1.5364 & 1.5 & 1. & 26 & 15 & 18 & 0.0 \\
\hline 1.5880 & 0.7234 & 1.1604 & 1.3580 & 1.4424 & 1.5364 & 1.5988 & 1.6 & 1.6453 & 0.0000 & -0.0236 & $-2.5632 e-3$ \\
\hline 1.5920 & 0.7234 & 1.1524 & 1.3580 & 1.4520 & 1.5463 & 1.5988 & 1.6810 & 1.6453 & 0.0 & 0.0000 & 0.0150 \\
\hline 1.5960 & 0.7038 & 1.1524 & 1.3580 & 1.4328 & 1.5264 & 1.5988 & 1.6810 & 1.6562 & 0.0000 & 0.0000 & $2.5616 \mathrm{e}-3$ \\
\hline 1.6000 & 0.7038 & 1.1445 & 1.3580 & 1.4424 & 1.5364 & 1.5988 & 1.6810 & 1.6562 & 0.0000 & 0.0000 & -0.0148 \\
\hline 1.6040 & 0.7169 & 1.1445 & 1.3673 & 1.4424 & 1.5364 & 1.6091 & 1.6918 & 1.6889 & 0.0000 & -0.0118 & $1.8025 \mathrm{e}-3$ \\
\hline 1.6080 & 0.7103 & 1.1445 & 1.3580 & 1.4520 & 1.5364 & 1.5988 & & & -0.0230 & -0.0118 & $6.1619 \mathrm{e}-3$ \\
\hline 1.6120 & 0.7169 & 1.1524 & 1.3 & 1.4424 & 1.5165 & 1.5 & 1. & 89 & 0. & 0. & 329 \\
\hline 1.6160 & 0.7103 & 1.1445 & 1.3487 & 1.4424 & 1.5165 & 1.5988 & 1.6918 & 1.7107 & -0.0115 & -0.0118 & $5.0289 \mathrm{e}-3$ \\
\hline 1.6200 & 0.7234 & 1.1524 & 1.3394 & 1.4328 & 1.5364 & 1.5988 & 1.6 & 1.7325 & 0.0000 & -0.0118 & 0.0155 \\
\hline 1.6240 & 0.7169 & 1.1604 & 1.3487 & 1.4328 & 1.5264 & 1.5988 & 1.7026 & 1.7216 & 0.0000 & 0.0000 & $-3.1313 e-3$ \\
\hline 1.6280 & 0.7103 & 1.1683 & 1.3394 & 1.4231 & 1.5264 & 1.5988 & 1.7026 & 1.7216 & -0.0115 & 0.0118 & $-7.4019 e-3$ \\
\hline 1.6320 & 0.7169 & 1.1524 & 1.3394 & 1.4231 & 1.5165 & 1.5988 & 1.6918 & 1.7325 & 0.0000 & 0.0000 & 0.0112 \\
\hline 1.6360 & 0.7299 & 1.1604 & 1.3394 & 1.4328 & 1.5264 & 1.5988 & 1.6918 & 1.7543 & 0.0115 & -0.0236 & $-2.1819 e-3$ \\
\hline 1.6400 & 0.7103 & 1.1524 & 1.3394 & 1.4231 & 1.4967 & 1.5988 & 1.6810 & 1.7543 & 0.0000 & -0.0118 & 0.0154 \\
\hline 1.6440 & 0.7103 & 1.1524 & 1.3394 & 1.4231 & 1.5066 & 1.5988 & 1.6918 & 1.7434 & 0.0115 & -0.0118 & -0.0103 \\
\hline 1.6480 & 0.7169 & 1.1604 & 1.3208 & 1.4135 & 1.5066 & 1.5988 & 1.6918 & 1.7652 & 0.0000 & -0.0118 & $-9.5832 e-3$ \\
\hline 1.6520 & 0.7169 & 1.1604 & 1.3301 & 1.4039 & 1.5066 & 1.5988 & 1.6918 & 1.7652 & 0.0000 & -0.0118 & $9.0138 \mathrm{e}-3$ \\
\hline 1.6560 & 0.7169 & 1.1604 & 1.3301 & 1.4135 & 1.5165 & 1.5781 & 1.6918 & 1.7543 & 0.0000 & 0.0000 & $-5.2191 e-3$ \\
\hline 1.6600 & 0.7234 & 1.1683 & 1.3301 & 1.4135 & 1.5066 & 1.5988 & 1.6918 & 1.7652 & 0.0115 & 0.0000 & 0.0167 \\
\hline 1.6640 & 0.7103 & 1.1524 & 1.3394 & 1.4135 & 1.5066 & 1.5885 & 1.6702 & 1.7760 & -0.0115 & 0.0000 & 0.0142 \\
\hline 1.6680 & 0.7103 & 1.1524 & 1.3208 & 1.4231 & 1.4967 & 1.5885 & 1.6702 & 1.7869 & 0.0000 & 0.0000 & -0.0250 \\
\hline 1.6720 & 0.7103 & 1.1524 & 1.3301 & 1.4039 & 1.4868 & 1.5885 & 1.6810 & 1.7760 & 0.0000 & -0.0118 & $5.4081 \mathrm{e}-3$ \\
\hline 1.6760 & 0.7104 & 1.1524 & 1.3115 & 1.4039 & 1.4868 & 1.5781 & 1.6810 & 1.7760 & -0.0115 & -0.0118 & -0.0133 \\
\hline 1.6800 & 0.7104 & 1.1524 & 1.3208 & 1.4135 & 1.4868 & 1.5885 & 1.6702 & 1.7869 & 0.0000 & 0.0000 & 0.0179 \\
\hline 1.6840 & 0.7104 & 1.1604 & 1.3115 & 1.4039 & 1.4868 & 1.5781 & 1.6702 & 1.7869 & -0.0115 & -0.0118 & $1.4238 \mathrm{e}-3$ \\
\hline 1.6880 & 0.7104 & 1.1605 & 1.3115 & 1.3943 & 1.4967 & 1.5678 & 1.6810 & 1.7869 & -0.0115 & 0.0118 & -0.0114 \\
\hline 1.6920 & 0.7104 & 1.1607 & 1.3115 & 1.3943 & 1.4868 & 1.5678 & 1.6702 & 1.7978 & -0.0115 & 0.0236 & 0.0111 \\
\hline
\end{tabular}




\begin{tabular}{|c|c|c|c|c|c|c|c|c|c|c|c|}
\hline 1.6960 & 0.7169 & 1.1528 & 1.3115 & 1.3943 & 1.4769 & 1.5781 & 1.6702 & 1.8087 & 0.0000 & -0.0118 & $5.6937 \mathrm{e}-3$ \\
\hline 1.7000 & 0.7235 & 1.1679 & 1.3115 & 1.3847 & 1.4670 & 1.5575 & 1.6487 & 1.7543 & 0.2415 & -0.0355 & -0.0142 \\
\hline 1.7040 & 0.7169 & 1.1607 & 1.3022 & 1.3943 & 1.4571 & 1.5472 & 1.6164 & 1.7216 & 0.9661 & -0.0709 & 0.0178 \\
\hline 1.7080 & 0.7235 & 1.1607 & 1.3022 & 1.3751 & 1.4472 & 1.5369 & 1.6271 & 1.7325 & 1.6216 & -0.0591 & -0.0139 \\
\hline 1.7120 & 0.7235 & 1.1607 & 1.2929 & 1.3751 & 1.4472 & 1.5472 & 1.6487 & 1.7652 & 1.7596 & -0.0473 & -0.0587 \\
\hline 1.7160 & 0.7365 & 1.1600 & 1.2929 & 1.3654 & 1.4670 & 1.5575 & 1.6595 & 1.7760 & 1.7826 & 0.0000 & -0.0739 \\
\hline 1.7200 & 0.7365 & 1.1441 & 1.3022 & 1.3654 & 1.4571 & 1.5678 & 1.6595 & 1.7760 & 1.7711 & 0.0000 & -0.0195 \\
\hline 1.7240 & 0.7365 & 1.1605 & 1.2929 & 1.3654 & 1.4571 & 1.5678 & 1.6595 & 1.7869 & 1.8056 & -0.0118 & -0.0374 \\
\hline 1.7280 & 0.7365 & 1.1607 & 1.3022 & 1.3654 & 1.4571 & 1.5575 & 1.6595 & 1.7869 & 1.8286 & 0.0000 & 0.0175 \\
\hline 1.7320 & 0.7300 & 1.1520 & 1.2929 & 1.3654 & 1.4571 & 1.5472 & 1.6595 & 1.7760 & 1.8401 & -0.0118 & $9.5848 \mathrm{e}-3$ \\
\hline 1.7360 & 0.7430 & 1.1604 & 1.2929 & 1.3654 & 1.4571 & 1.5575 & 1.6487 & 1.7869 & 1.8401 & -0.0118 & 0.0250 \\
\hline 1.7400 & 0.7560 & 1.1605 & 1.3022 & 1.3654 & 1.4571 & 1.5472 & 1.6379 & 1.7760 & 1.8516 & -0.0118 & -0.0248 \\
\hline 1.7440 & 0.7495 & 1.1687 & 1.2929 & 1.3654 & 1.4372 & 1.5369 & 1.6487 & 1.7652 & 1.8861 & -0.0118 & $2.0870 \mathrm{e}-3$ \\
\hline 1.7480 & 0.7430 & 1.1840 & 1.2743 & 1.3654 & 1.4472 & 1.5472 & 1.6595 & 1.7869 & 1.8861 & -0.0236 & $3.1321 \mathrm{e}-3$ \\
\hline 1.7520 & 0.7495 & 1.1764 & 1.2929 & 1.3654 & 1.4472 & 1.5369 & 1.6487 & 1.7652 & 1.8861 & -0.0118 & 0.0148 \\
\hline 1.7560 & 0.7625 & 1.1766 & 1.2836 & 1.3558 & 1.4372 & 1.5266 & 1.6487 & 1.7652 & 1.8861 & 0.0000 & $7.5905 e-4$ \\
\hline 1.7600 & 0.7495 & 1.1840 & 1.2743 & 1.3558 & 1.4372 & 1.5369 & 1.6271 & 1.7760 & 1.8976 & -0.0236 & 0.0237 \\
\hline 1.7640 & 0.7625 & 1.1763 & 1.2743 & 1.3558 & 1.4372 & 1.5369 & 1.6271 & 1.7652 & 1.8976 & 0.0000 & $5.7049 \mathrm{e}-3$ \\
\hline 1.7680 & 0.7560 & 1.1686 & 1.2836 & 1.3558 & 1.4273 & 1.5472 & 1.6271 & 1.7543 & 1.8861 & -0.0118 & -0.0183 \\
\hline 1.7720 & 0.7690 & 1.1686 & 1.2836 & 1.3558 & 1.4273 & 1.5266 & 1.6164 & 1.7652 & 1.9091 & -0.0236 & -0.0113 \\
\hline 1.7760 & 0.7690 & 1.1765 & 1.2743 & 1.3366 & 1.4273 & 1.5266 & 1.6379 & 1.7543 & 1.8976 & 0.0118 & $6.6381 \mathrm{e}-4$ \\
\hline 1.7800 & 0.7755 & 1.1762 & 1.2743 & 1.3462 & 1.4174 & 1.5163 & 1.6271 & 1.7543 & 1.9091 & 0.0000 & $8.9200 \mathrm{e}-3$ \\
\hline 1.7840 & 0.7821 & 1.1680 & 1.2743 & 1.3462 & 1.4174 & 1.5059 & 1.6164 & 1.7543 & 1.9206 & 0.0118 & 0.0110 \\
\hline 1.7880 & 0.7821 & 1.1838 & 1.2743 & 1.3366 & 1.4174 & 1.5059 & 1.6164 & 1.7434 & 1.9091 & 0.0000 & $9.3941 \mathrm{e}-3$ \\
\hline 1.7920 & 0.7755 & 1.1683 & 1.2650 & 1.3270 & 1.4174 & 1.5059 & 1.6164 & 1.7434 & 1.9091 & 0.0000 & -0.0195 \\
\hline 1.7960 & 0.7951 & 1.1761 & 1.2650 & 1.3174 & 1.4075 & 1.5059 & 1.6164 & 1.7325 & 1.9091 & -0.0118 & $-2.2776 \mathrm{e}-3$ \\
\hline 1.8000 & 0.7886 & 1.1685 & 1.2557 & 1.3366 & 1.4174 & 1.4956 & 1.6164 & 1.7216 & 1.8861 & -0.0236 & $7.1159 e-3$ \\
\hline 1.8040 & 0.7886 & 1.1685 & 1.2557 & 1.3366 & 1.4075 & 1.5163 & 1.6164 & 1.7107 & 1.8861 & 0.0000 & $5.2186 \mathrm{e}-3$ \\
\hline 1.8080 & 0.7886 & 1.1679 & 1.2557 & 1.3270 & 1.3976 & 1.4956 & 1.6164 & 1.6998 & 1.8976 & -0.0118 & $9.1086 \mathrm{e}-3$ \\
\hline 1.8120 & 0.8082 & 1.1759 & 1.2557 & 1.3366 & 1.4075 & 1.5059 & 1.6056 & 1.6 & 1.8746 & -0.0118 & 0.0390 \\
\hline 1.8160 & 0.7951 & 1.1685 & 1.2557 & 1.3270 & 1.4075 & 1.4956 & 1.5948 & 1.6998 & 1.8631 & -0.0118 & $-9.2992 \mathrm{e}-3$ \\
\hline 1.8200 & 0.8082 & 1.1685 & 1.2557 & 1.3270 & 1.3976 & 1.4956 & 1.5948 & 1.7216 & 1.8861 & 0.0000 & $7.2121 \mathrm{e}-3$ \\
\hline 1.8240 & 0.8147 & 1.1602 & 1.2464 & 1.3174 & 1.3976 & 1.5059 & 1.5948 & 1.6998 & 1.8631 & 0.0237 & $-5.9778 \mathrm{e}-3$ \\
\hline 1.8280 & 0.8212 & 1.1600 & 1.2557 & 1.3174 & 1.3877 & 1.4544 & 1.5733 & 1.6344 & 1.8056 & 0.7923 & $8.0651 \mathrm{e}-3$ \\
\hline 1.8320 & 0.8277 & 1.1607 & 1.2464 & 1.3077 & 1.3679 & 1.4544 & 1.5517 & 1.6562 & 1.7941 & 1.9159 & -0.0136 \\
\hline 1.8360 & 0.8407 & 1.1679 & 1.2185 & 1.2885 & 1.3877 & 1.4647 & 1.5733 & 1.6780 & 1.8171 & 2.1406 & -0 \\
\hline 1.8400 & 0.8342 & 1.1602 & 1.2371 & 1.2789 & 1.3877 & 1.4647 & 1.5840 & 1.6998 & 1.8 & 2.1 & 713 \\
\hline 1.8440 & 0.8473 & 1.1604 & 1.2371 & 1.3077 & 1.3778 & 1.4750 & 1.5625 & 1.6998 & 1.8286 & 2.1169 & -0.0219 \\
\hline 1.8480 & 0.8407 & 1.1526 & 1.2464 & 1.3077 & 1.3877 & 1.4853 & 1.5733 & 1.6780 & 1.8171 & 2.1288 & $-9.7734 \mathrm{e}-3$ \\
\hline 1.8520 & 0.8538 & 1.1607 & 1.2371 & 1.2981 & 1.3778 & 1.4750 & 1.5625 & 1.6780 & 1.8171 & 2.1406 & $-4.7301 \mathrm{e}-4$ \\
\hline 1.8560 & 0.8407 & 1.1600 & 1.2371 & 1.3077 & 1.3976 & 1.4544 & 1.5733 & 1.6671 & 1.8171 & 2.1051 & -0.0237 \\
\hline 1.8600 & 0.8473 & 1.1762 & 1.2278 & 1.2789 & 1.3778 & 1.4647 & 1.5733 & 1.6780 & 1.8171 & 2.0933 & -0.0146 \\
\hline 1.8640 & 0.8407 & 1.1 & 1.2 & 1.3 & 1.3679 & 1.4647 & 1.5 & 1. & 1. & 2. & 0. \\
\hline 1.8680 & 0.8603 & 1.1685 & 1.2185 & 1.2885 & 1.3679 & 1.4647 & 1.5733 & 1.6780 & 1.8056 & 2.0814 & $8.2553 \mathrm{e}-3$ \\
\hline 1.8720 & 0.8538 & 1.1685 & 1.2278 & 1.2981 & 1.3579 & 1.4544 & 1.5517 & 1.6671 & 1.7941 & 2.0341 & -0.0141 \\
\hline 1.8760 & 0.8603 & 1.1685 & 1.2185 & 1.2885 & 1.3579 & 1.4544 & 1.5517 & 1.6671 & 1.7826 & 2.0105 & -0.0183 \\
\hline 1.8800 & 0.8603 & 1.1685 & 1.2185 & 1.2789 & 1.3679 & 1.4441 & 1.5517 & 1.6671 & 1.7826 & 2.0105 & $-6.6477 \mathrm{e}-4$ \\
\hline 1.8840 & 0.8603 & 1.1685 & 1.2185 & 1.2885 & 1.3480 & 1.4337 & 1.5409 & 1.6671 & 1.7711 & 1.9987 & $9.0143 \mathrm{e}-3$ \\
\hline 1.8880 & 0.8603 & 1.1683 & 1.2092 & 1.2789 & 1.3579 & 1.4337 & 1.5409 & 1.6562 & 1.7826 & 1.9750 & $-1.9927 \mathrm{e}-3$ \\
\hline 1.8920 & 0.8603 & 1.1604 & 1.2092 & 1.2693 & 1.3579 & 1.4441 & 1.5409 & 71 & 1.7 & 13 & 0.0241 \\
\hline 1.8960 & 0.8603 & 1.1604 & 1.2185 & 1.2789 & 1.3480 & 1.4337 & 1.5302 & 1.6453 & 1.7481 & 1.9632 & $-2.5618 \mathrm{e}-3$ \\
\hline 1.9000 & 0.8668 & 1.1764 & 1.1999 & 1.2693 & 1.3381 & 1.4234 & 1.5302 & 1.6562 & 1.7596 & 1.9277 & -0.0255 \\
\hline 1.9040 & 0.8603 & 1.1604 & 1.2092 & 1.2693 & 1.3579 & 1.4234 & 1.5194 & 1.6453 & 1.7481 & 1.9277 & $-6.4527 e-3$ \\
\hline 1.9080 & 0.8668 & 1.1522 & 1.2092 & 1.2693 & 1.3480 & 1.4234 & 1.5302 & 1.6453 & 1.7481 & 1.9277 & 0.0198 \\
\hline 1.9120 & 0.8668 & 1.1604 & 1.2092 & 1.2693 & 1.3381 & 1.4234 & 1.5194 & 1.6235 & 1.7366 & 1.9040 & $7.3029 e-3$ \\
\hline 1.9160 & 0.8668 & 1.1605 & 1.2092 & 1.2597 & 1.3381 & 1.4131 & 1.5302 & 1.6344 & 1.7366 & 1.9040 & $5.8966 \mathrm{e}-3$ \\
\hline 1.9200 & 0.8538 & 1.1687 & 1.1999 & 1.2597 & 1.3381 & 1.4234 & 1.5086 & 1.6235 & 1.7251 & 1.8922 & 0.0255 \\
\hline 1.9240 & 0.8538 & 1.1607 & 1.1999 & 1.2597 & 1.3282 & 1.3925 & 1.5086 & 1.6126 & 1.7136 & 1.8922 & $9.8708 \mathrm{e}-3$ \\
\hline 1.9280 & 0.8538 & 1.1602 & 1.1906 & 1.2597 & 1.3282 & 1.4028 & 1.5194 & 1.6017 & 1.7251 & 1.8686 & -0.0171 \\
\hline 1.9320 & 0.8538 & 1.1687 & 1.1906 & 1.2404 & 1.3282 & 1.4028 & 1.5086 & 1.6235 & 1.7136 & 1.8686 & 0.0196 \\
\hline 1.9360 & 0.8538 & 1.1766 & 1.1813 & 1.2404 & 1.3084 & 1.3925 & 1.4655 & 1.5908 & 1.6446 & 1.8212 & $-6.5358 \mathrm{e}-3$ \\
\hline 1.9400 & 0.8473 & 1.1599 & 1.1813 & 1.2597 & 1.2985 & 1.3822 & 1.4655 & 1.5908 & 1.6216 & 1.8094 & $8.2553 \mathrm{e}-3$ \\
\hline 1.9440 & 0.8473 & 1.1603 & 1.1720 & 1.2405 & 1.2985 & 1.3925 & 1.4871 & 1.5690 & 1.6561 & 1.8331 & 0.6884 \\
\hline 1.9480 & 0.8473 & 1.1679 & 1.1720 & 1.2404 & 1.3084 & 1.3822 & 1.4871 & 1.6017 & 1.6906 & 1.8331 & 2.1261 \\
\hline 1.9520 & 0.8407 & 1.1762 & 1.1720 & 1.2308 & 1.3084 & 1.3822 & 1.4763 & 1.5908 & 1.6791 & 1.8212 & 2.3793 \\
\hline 1.9560 & 0.8472 & 1.1845 & 1.1627 & 1.2404 & 1.2985 & 1.3719 & 1.4871 & 1.5799 & 1.6791 & 1.8212 & 2.3011 \\
\hline 1.9600 & 0.8407 & 1.1760 & 1.1720 & 1.2404 & 1.2886 & 1.3822 & 1.4871 & 1.5799 & 1.6791 & 1.8212 & 2.2303 \\
\hline 1.9640 & 0.8472 & 1.1843 & 1.1627 & 1.2309 & 1.2985 & 1.3822 & 1.4655 & 1.5690 & 1.6791 & 1.8212 & 2.2239 \\
\hline 1.9680 & 0.8472 & 1.1840 & 1.1720 & 1.2213 & 1.2886 & 1.3719 & 1.4763 & 1.5690 & 1.6906 & 1.8094 & 2.1669 \\
\hline 1.9720 & 0.8537 & 1.2004 & 1.1720 & 1.2213 & 1.2886 & 1.3719 & 1.4763 & 1.5799 & 1.6676 & 1.8094 & 2.1287 \\
\hline
\end{tabular}




\begin{tabular}{|c|c|c|c|c|c|c|c|c|c|c|c|}
\hline 1.9760 & 0.8472 & 1.1997 & 1.1627 & 1.2309 & 1.2985 & 1.3719 & 1.4655 & 1.5690 & 1.6676 & 1.8094 & 2.0841 \\
\hline 1.9800 & 0.8472 & 1.1921 & 1.1534 & 1.2213 & 1.2886 & 1.3615 & 1.4763 & 1.5799 & 1.6561 & 1.7976 & 2.0724 \\
\hline 1.9840 & 0.8472 & 1.1997 & 1.1720 & 1.2117 & 1.2985 & 1.3615 & 1.4548 & 1.5690 & 1.6676 & 1.7739 & 2.0581 \\
\hline 1.9880 & 0.8537 & 1.1998 & 1.1534 & 1.2117 & 1.2786 & 1.3615 & 1.4548 & 1.5581 & 1.6561 & 1.7739 & 2.0435 \\
\hline 1.9920 & 0.8407 & 1.2004 & 1.1627 & 1.2213 & 1.2786 & 1.3615 & 1.4440 & 1.5581 & 1.6561 & 1.7739 & 2.0299 \\
\hline 1.9960 & 0.8541 & 1.1997 & 1.1534 & 1.2213 & 1.2786 & 1.3615 & 1.4548 & 1.5581 & 1.6446 & 1.7739 & 2.0128 \\
\hline 2.0000 & 0.8539 & 1.1917 & 1.1441 & 1.2020 & 1.2786 & 1.3409 & 1.4440 & 1.5472 & 1.6561 & 1.7739 & 1.9961 \\
\hline 2.0040 & 0.8535 & 1.1921 & 1.1441 & 1.2020 & 1.2687 & 1.3512 & 1.4440 & 1.5472 & 1.6446 & 1.7621 & 1.9836 \\
\hline 2.0080 & 0.8535 & 1.1921 & 1.1534 & 1.2020 & 1.2588 & 1.3409 & 1.4440 & 1.5581 & 1.6331 & 1.7621 & 1.9727 \\
\hline 2.0120 & 0.8534 & 1.2002 & 1.1441 & 1.2020 & 1.2687 & 1.3409 & 1.4332 & 1.5472 & 1.6216 & 1.7739 & 1.9339 \\
\hline 2.0160 & 0.8604 & 1.1922 & 1.1441 & 1.1828 & 1.2588 & 1.3512 & 1.4224 & 1.5363 & 1.6331 & 1.7503 & 1.9385 \\
\hline 2.0200 & 0.8667 & 1.1924 & 1.1441 & 1.1828 & 1.2588 & 1.3409 & 1.4332 & 1.5363 & 1.6331 & 1.7503 & 1.9390 \\
\hline 2.0240 & 0.8600 & 1.1924 & 1.1441 & 1.2020 & 1.2588 & 1.3409 & 1.4224 & 1.5363 & 1.6331 & 1.7503 & 1.9309 \\
\hline 2.0280 & 0.8736 & 1.1924 & 1.1441 & 1.1924 & 1.2588 & 1.3409 & 1.4332 & 1.5363 & 1.6101 & 1.7385 & 1.9229 \\
\hline 2.0320 & 0.8598 & 1.1843 & 1.1348 & 1.1828 & 1.2489 & 1.3306 & 1.4224 & 1.5363 & 1.6216 & 1.7385 & 1.8919 \\
\hline 2.0360 & 0.8736 & 1.1841 & 1.1348 & 1.1924 & 1.2489 & 1.3306 & 1.4224 & 1.5254 & 1.6216 & 1.7030 & 1.9117 \\
\hline 2.0400 & 0.8664 & 1.1839 & 1.1348 & 1.1828 & 1.2489 & 1.3203 & 1.4224 & 1.5254 & 1.6101 & 1.7148 & 1.9221 \\
\hline 2.0440 & 0.8664 & 1.1758 & 1.1441 & 1.1828 & 1.2489 & 1.3306 & 1.4224 & 1.5254 & 1.6216 & 1.7030 & 1.8668 \\
\hline 2.0480 & 0.8535 & 1.1604 & 1.1348 & 1.1924 & 1.2390 & 1.3203 & 1.4117 & 1.5145 & 1.6216 & 1.7030 & 1.9011 \\
\hline 2.0520 & 0.8537 & 1.1604 & 1.1162 & 1.1636 & 1.2390 & 1.3306 & 1.4117 & 1.5145 & 1.6101 & 1.7148 & 1.8882 \\
\hline 2.0560 & 0.8472 & 1.1525 & 1.1255 & 1.1732 & 1.2192 & 1.3100 & 1.4009 & 1.5145 & 1.6101 & 1.6911 & 1.8663 \\
\hline 2.0600 & 0.8665 & 1.1446 & 1.1255 & 1.1732 & 1.2390 & 1.3100 & 1.4009 & 1.5145 & 1.6216 & 1.6 & 1.8775 \\
\hline 2.0640 & 0.8535 & 1.1287 & 1.1162 & 1.1732 & 1.2291 & 1.3100 & 1.4009 & 1.5254 & 1.5986 & 1.6793 & 1.8637 \\
\hline 2.0680 & 0.8533 & 1.1368 & 1.1162 & 1.1636 & 1.2291 & 1.3100 & 1.4009 & 1.4928 & 1.6101 & 1.6793 & 1.8681 \\
\hline 2.0720 & 0.8539 & 1.1281 & 1.1162 & 1.1636 & 1.2390 & 1.2997 & 1.4009 & 1.4819 & 1.6101 & 1.6911 & 1.8687 \\
\hline 2.0760 & 0.8472 & 1.1285 & 1.1162 & 1.1540 & 1.2291 & 1.3100 & 1.3793 & 1.5036 & 1.5986 & 1.6675 & 1.8391 \\
\hline 2.0800 & 0.8470 & 1.1122 & 1.1162 & 1.1636 & 1.2192 & 1.2997 & 1.3793 & 1.5036 & 1.6216 & 1.6793 & 1.8188 \\
\hline 2.0840 & 0.8468 & 1.1124 & 1.1069 & 1.1540 & 1.2093 & 1.3100 & 1.3901 & 1.4928 & 1.5986 & & 1.8546 \\
\hline 2.0880 & 0.8340 & 1.1126 & 1.1 & 1.1 & 1.2192 & 1.2893 & 1.3901 & 1.4710 & 1.6 & 1. & 1.8128 \\
\hline 2.0920 & 0.8475 & 1.1046 & 1.1069 & 1.1636 & 1.2192 & 1.2893 & 1.3901 & 1.4928 & 1.5871 & 1. & 1.8291 \\
\hline 2.0960 & 0.8475 & 1.0967 & 1.1162 & 1.1444 & 1.2093 & 1.2790 & 1.3793 & 1.4819 & 1.5871 & 1.6675 & 1.8387 \\
\hline 2.1000 & 0.8468 & 1.0887 & 1.1069 & 1.1540 & 1.1994 & 1.2893 & 1.3686 & 1.4710 & 1.5756 & 1.6675 & 1.8343 \\
\hline 2.1040 & 0.8403 & 1.0887 & 1.0883 & .1347 & 1.2291 & 1.2997 & 1.3793 & 1.4819 & 1.5756 & 1.6675 & 1.7883 \\
\hline 2.1080 & 0.8539 & 1.0967 & 1.1069 & 1.1347 & 1.2093 & 1.2790 & 1.3793 & 1.4819 & 1.5756 & 1.6 & 1.7997 \\
\hline 2.1120 & 0.8410 & 1.0887 & 1.0976 & 1.1444 & 1.1994 & 1.2790 & 1.3578 & 1.4710 & 1.5641 & & 1.7795 \\
\hline 2.1160 & 0.8475 & 1.0967 & 1.0 & 1. & 1.2093 & 1.2 & 1.3686 & 1.4 & 1.5 & & 1.8077 \\
\hline 2.1200 & 0.8410 & 1.0965 & 1.1162 & 1.1444 & 1.1994 & 1.2790 & 1.3686 & 1.4710 & 1.5641 & 1.6 & 1.7990 \\
\hline 2.1240 & 0.8410 & 1.0965 & 1.0976 & 1.1444 & 1.1994 & 1.2893 & 1.3686 & 1.4710 & 1.5641 & 1.6438 & 1.7984 \\
\hline 2.1280 & 0.8273 & 1.0965 & 1.0883 & 1.1251 & 1.1994 & 1.2790 & 1.3686 & 1.4710 & 1.5641 & 1.6438 & 1.7862 \\
\hline 2.1320 & 0.8475 & 1.0884 & 1.1069 & 1.1347 & 1.1994 & 1.2790 & 1.3686 & 1.4492 & 1.5526 & 1.6320 & 1.7727 \\
\hline 2.1360 & 0.8340 & 1.0963 & 1.0883 & 1.1251 & 1.1894 & 1.2584 & 1.3470 & 1.4492 & 1.5526 & 1.6320 & 1.7492 \\
\hline 2.1400 & 0.8340 & 1.0884 & 1.0883 & 1.1347 & 1.1894 & 1.2584 & 1.3470 & 1.4492 & 26 & & 1.7722 \\
\hline 2.1440 & 0.8209 & 1.0884 & 1.0 & 1. & 1.1894 & 1.2584 & 1.3470 & 1. & 1.5 & 1. & 1.8006 \\
\hline 2.1480 & 0.8273 & 1.0812 & 1.0883 & 1.1347 & 1.1894 & 1.2790 & 1.3578 & 1.4710 & 1.5526 & 1.6202 & 1.7629 \\
\hline 2.1520 & 0.8273 & 1.0732 & 1.0883 & 1.1347 & 1.1795 & 1.2687 & 1.3470 & 1.4492 & 1.5411 & 1.6202 & 1.7621 \\
\hline 2.1560 & 0.8209 & 1.0732 & 1.0883 & 1.1347 & 1.1795 & 1.2584 & 1.3578 & 1.4601 & 1.5296 & 1.6084 & 1.7709 \\
\hline 2.1600 & 0.8273 & 1.0651 & 1.0790 & 1.1059 & 1.1795 & 1.2584 & 1.3470 & 1.4492 & 1.5296 & 1.6202 & 1.7514 \\
\hline 2.1640 & 0.8209 & 1.0651 & 1.0790 & 1.1155 & 1.1795 & 1.2584 & 1.3470 & 1.4383 & 1.5296 & 1.6202 & 1.7190 \\
\hline 2.1680 & 0.8144 & 1.0488 & & 1.1 & 1.1696 & 1.2 & 1.3470 & 1. & 1.5 & & 1.7592 \\
\hline 2.1720 & 0.8081 & 1.0566 & 1.0790 & 1.1155 & 1.1696 & 1.2687 & 1.3470 & 1.4492 & 1.5296 & 1.6 & 1.7172 \\
\hline 2.1760 & 0.7952 & 1.0335 & 1.0790 & 1.1155 & 1.1696 & 1.2481 & 1.3470 & 1.4492 & 1.5066 & 1.5965 & 1.6849 \\
\hline 2.1800 & 0.8017 & 1.0335 & 1.0604 & 1.1155 & 1.1597 & 1.2481 & 1.3362 & 1.4274 & 1.5181 & 1.5965 & 1.7022 \\
\hline 2.1840 & 0.7954 & 1.0335 & 1.0790 & 1.1155 & 1.1597 & 1.2481 & 1.3362 & 1.4383 & 1.5181 & 1.5965 & 1.6891 \\
\hline 2.1880 & 0.8019 & 1.0096 & 1.0790 & 1.1347 & 1.1696 & 1.2481 & 1.3362 & 1.4274 & 1.5066 & 1.5847 & 1.7332 \\
\hline 2.1920 & 0.7947 & 1.0095 & 1.0604 & 1.1155 & 1.1696 & 1.2584 & 1.3362 & 1.4383 & 1.5181 & 1.5847 & 1.7060 \\
\hline 2.1960 & 0.8084 & 1.0015 & 1.0697 & 1.0963 & 1.1597 & 1.2481 & 1.3362 & 1.4274 & 1.5066 & 1.5729 & 1.7071 \\
\hline 2.2000 & 0.7947 & 0.9936 & 1.0697 & 1.0963 & 1.1597 & 1.2378 & 1.3362 & 1.4274 & 1.4951 & 1.5610 & 1.7023 \\
\hline 2.2040 & 0.7883 & 1.0017 & 1.0697 & 1.0963 & 1.1597 & 1.2481 & 1.3361 & 1.4274 & 1.4951 & 1.5847 & 1.6950 \\
\hline 2.2080 & 0.7753 & 0.9854 & 1.0697 & 1.1059 & 1.1597 & 1.2378 & 1.3361 & 1.4274 & 1.4951 & 1.5729 & 1.7110 \\
\hline 2.2120 & 0.7889 & 0.9936 & 1.0418 & 1.0963 & 1.1498 & 1.2481 & 1.3361 & 1.4274 & 1.5066 & 1.5610 & 1.7030 \\
\hline 2.2160 & 0.7887 & 0.9775 & 1.0418 & 1.1059 & 1.1498 & 1.2481 & 1.3361 & 1.4165 & 1.4951 & 1.5729 & 1.6937 \\
\hline 2.2200 & 0.7883 & 0.9856 & 1.0604 & 1.0963 & 1.1597 & 1.2378 & 1.3361 & 1.4274 & 1.4836 & 1.5492 & 1.6840 \\
\hline 2.2240 & 0.7818 & 0.9938 & 1.0604 & 1.0963 & 1.1399 & 1.2378 & 1.3361 & 1.4056 & 1.4836 & 1.5610 & 1.6837 \\
\hline 2.2280 & 0.7688 & 0.9858 & 1.0511 & 1.1059 & 1.1498 & 1.2378 & 1.3254 & 1.4274 & 1.4836 & 1.5610 & 1.6974 \\
\hline 2.2320 & 0.7751 & 0.9771 & 1.0604 & 1.0963 & 1.1498 & 1.2275 & 1.3146 & 1.4165 & 1.4721 & 1.5610 & 1.6896 \\
\hline 2.2360 & 0.7688 & 0.9771 & 1.0418 & 1.0770 & 1.1498 & 1.2275 & 1.3254 & 1.4165 & 1.4721 & 1.5610 & 1.6672 \\
\hline 2.2400 & 0.7625 & 0.9853 & 1.0604 & 1.0963 & 1.1399 & 1.2378 & 1.3254 & 1.4056 & 1.4606 & 1.5492 & 1.6662 \\
\hline 2.2440 & 0.7625 & 0.9771 & 1.0511 & 1.0867 & 1.1300 & 1.2378 & 1.3146 & 1.4056 & 1.4721 & 1.5374 & 1.6646 \\
\hline 2.2480 & 0.7625 & 0.9853 & 1.0418 & 1.0867 & 1.1300 & 1.2275 & 1.3146 & 1.3947 & 1.4721 & 1.5374 & 1.6646 \\
\hline 2.2520 & 0.7626 & 0.9853 & 1.0511 & 1.0770 & 1.1399 & 1.2275 & 1.3146 & 1.4056 & 1.4721 & 1.5256 & 1.6718 \\
\hline
\end{tabular}




\begin{tabular}{|c|c|c|c|c|c|c|c|c|c|c|c|}
\hline 2.2560 & 0.7433 & 1.0254 & 1.0418 & 1.0770 & 1.1300 & 1.2171 & 1.3146 & 1.4056 & 1.4606 & 1.5256 & 1.6654 \\
\hline .2600 & 0.7362 & 1.0172 & 1.0418 & 1.0770 & 1.1300 & 1.2171 & 1.3146 & 1.4056 & 1.4606 & 1.5374 & 1.6357 \\
\hline 2640 & 0.7299 & 0.9934 & 1.0511 & 1.0770 & 1.1300 & 1.2068 & 1.2930 & 1.3838 & 1.4491 & 1.5374 & 1.6621 \\
\hline 2680 & .7301 & 1.0015 & 1.0139 & 1.0578 & 1.1300 & 1.2171 & 1.3038 & 1.3838 & 1.4491 & 1.5256 & 1.6558 \\
\hline 2720 & 0.7172 & 1.0015 & 1.0325 & 1.0674 & 1.1300 & 1.2171 & 1.3038 & 1.3838 & 1.4491 & 1.5137 & 6461 \\
\hline .2760 & 0.7237 & 1.0015 & 1.0325 & 1.0770 & 1.1201 & 1.2171 & 1.3038 & 1.3729 & 1.4376 & 1.5256 & .6670 \\
\hline 2800 & 0.7102 & 1.0015 & 1.0325 & 1.0867 & 1.1201 & 1.2171 & 1.3038 & 1.3838 & 1.4261 & 1.5256 & 1.6424 \\
\hline 2840 & .7102 & 1.0015 & 1.0325 & 1.0770 & 1.1300 & 1.2275 & 1.3038 & 1.3729 & 1.4146 & 1.5137 & 1.6280 \\
\hline 2880 & 0.7102 & 0.9936 & 1.0325 & 1.0674 & 1.1300 & 1.2068 & 2823 & 1.3620 & 1.4146 & 1.5137 & 6309 \\
\hline 2920 & 0.7038 & 0.9936 & 1.0232 & 1.0578 & 1.1300 & 1.2171 & 1.2930 & 1.3838 & 1.4031 & 1.5137 & 1.6183 \\
\hline 2960 & .7038 & 0.9936 & 1.0325 & 1.0770 & 1.1101 & 1.1965 & 1.3038 & 1.3729 & 1.3916 & 1.5137 & 1.6095 \\
\hline 3000 & .6843 & 0.9856 & 1.0232 & 1.0674 & 1.1201 & 1.2171 & & 1.3838 & 1.4031 & 1.4901 & 1.6255 \\
\hline 2.3040 & .6843 & 0.9856 & & 1.0482 & & & & & & & 1.6017 \\
\hline .3080 & 0.6843 & 0.9856 & 1.0 & 1.0674 & 1.1 & 65 & & 1.3620 & & & 6328 \\
\hline 3120 & 0.6843 & 0.9856 & 1.0139 & 1.0770 & 1.1101 & 068 & & 1.3620 & 1.4031 & 783 & .6191 \\
\hline 3160 & 0.6846 & 0.9938 & 1.0139 & 1.0674 & 1.1101 & 2171 & 2930 & 1.3511 & 1.4146 & 901 & 6186 \\
\hline 3200 & 0.6712 & 0.9856 & 1.0232 & 1.0578 & 1.1101 & 1.1862 & 1.2823 & 1.3402 & 1.4031 & 1.4783 & .5767 \\
\hline 3240 & .6712 & 0.9938 & 1.0232 & 1.0386 & 1.1101 & 2068 & 2715 & 1.3511 & 1.4261 & 4664 & .5919 \\
\hline 2.3280 & 0.6647 & 0.9938 & & 1.0482 & & & & & & & 5937 \\
\hline 2.3320 & 0.6582 & 0.9 & & 1.0 & & & & & & & 5723 \\
\hline 2.3360 & 6582 & 0.9938 & & 1.0 & & & & & & & 306 \\
\hline 3400 & 0.6517 & 0.9938 & 1.0139 & 1.0578 & 1.1101 & & & 1.3620 & & & .5873 \\
\hline 3440 & .6517 & 0.9858 & 1.0139 & 1.0578 & 1.1002 & 965 & 1.2823 & 1.3402 & 1.3801 & 1.4664 & 1.5819 \\
\hline 3480 & 6387 & 0.9858 & 1.0046 & 1.0386 & & & 607 & 1.3402 & 1.3916 & & .6023 \\
\hline 2.3520 & .6321 & 0.9938 & 1.0 & 1.0482 & $1 .($ & & & 1.3 & 916 & & .5843 \\
\hline 2.3560 & 0.6321 & 0.9936 & & & & & & & & & \\
\hline 2.3600 & 0.6321 & & & & & & & & & & \\
\hline 2.3640 & 0.6256 & 0.9936 & & 1.0 & & & & & & & 632 \\
\hline 3680 & .6126 & 0.9853 & 1.0 & 1.0386 & & & & 93 & 01 & & 1.5558 \\
\hline 3720 & 6191 & 0.9771 & 0.95 & 1.0386 & 03 & 59 & 07 & 1.3293 & 1.3916 & 46 & 1.5806 \\
\hline 3760 & 6061 & 0.9771 & & & & & & & & & 395 \\
\hline 2.3800 & .6126 & 0.9851 & 0. & 1.0290 & 4 & & & 1.3184 & & & 5275 \\
\hline 2.3840 & .6061 & & & & & & & & & & \\
\hline 2.3880 & 61 & & & & & & & & & & 392 \\
\hline 2.3920 & 5996 & 0.9699 & 0 & 1.0193 & & & & 1.2966 & & & 1.5310 \\
\hline 3960 & 5996 & 0.9851 & 0 & 1.01 & & & & 1.3075 & & & 1.5593 \\
\hline 4000 & 5930 & 858 & & & & & & & & & $50 ?$ \\
\hline 2.4040 & 5930 & 0.9696 & 1.0 & 1.0193 & & & 84 & 1.2857 & & & 1.5467 \\
\hline 2.4080 & .6126 & 0.9694 & & & & & & & & & .5406 \\
\hline 2.4120 & 5930 & 0.9773 & & & & & & & & & \\
\hline 2.4160 & 5 & 2 & & & & & & & & & 179 \\
\hline 2.4200 & 5865 & 0.9620 & 1.9 & 1.00 & & & 84 & 1.2748 & 56 & & 1.5144 \\
\hline 2.4240 & 5800 & 0.9538 & 2.06 & 1.0001 & & & 176 & 1.2748 & 41 & 55 & 1.4914 \\
\hline .4280 & 5865 & 0.9618 & & & & & & & & & .5167 \\
\hline 2.4320 & 5800 & 0.9697 & 2.2413 & 1.0 & & & & 1.2639 & 26 & & 1.5323 \\
\hline 2.4360 & & & & & & & & & & & .5208 \\
\hline 2.4400 & & & & & & & & & & & 5232 \\
\hline 2.4440 & 0.5865 & 0.9612 & & & & & & & & & 1.5227 \\
\hline 2.4480 & 5735 & 20 & 2 & 1.9 & & & & 1.2 & 26 & & 1.4847 \\
\hline .4520 & 5800 & 0.9540 & 5296 & 2.1347 & 1.0606 & & 58 & 1.2530 & 1.3111 & 36 & 1.4976 \\
\hline 4560 & 5735 & 0.9540 & 2.5575 & 2.2599 & & & & 530 & 111 & & 1.468 \\
\hline 2.4600 & 0.5800 & 0.9620 & 2.59 & 2.3560 & & & & 1.2530 & 1.2996 & & 1.4849 \\
\hline 2.4640 & & & & & & & & & & & 4679 \\
\hline 2.4680 & .5800 & & & & & & & & & & 1.477 \\
\hline 2.4720 & .5735 & & & 2.5483 & & & & 1.2312 & 1.2766 & 600 & 1.4587 \\
\hline 2.4760 & 5865 & 0.9618 & & 2.6155 & & & 1.1745 & 1.2312 & 1.2881 & 1.3482 & 1.452 \\
\hline & 5735 & 0.9376 & 2.7435 & 2.6835 & 2.4585 & & 45 & 1.2312 & 81 & 182 & $1455^{3}$ \\
\hline 2.4840 & .5865 & 0.9457 & 2.7714 & 2.7307 & 2.5577 & 1.1965 & 1.1422 & 1.2312 & 1.2996 & 1.3718 & 1.4494 \\
\hline 2.4880 & 0.5865 & 0.9455 & 2.7993 & 2.7788 & 2.6267 & 1.5369 & 1.1422 & 1.1877 & 1.2766 & 1.3482 & 1.4641 \\
\hline 2.4920 & 0.5930 & 0.9535 & 2.7993 & 2.7987 & 2.6957 & 1.8876 & 1.1206 & 1.1986 & 1.2766 & 1.3245 & 1.437 \\
\hline 4960 & 0.5930 & 0.9454 & 2.8272 & 2.8269 & 2.7851 & 2. & & 377 & 1.2651 & 1.3245 & 1.466 \\
\hline 2.5000 & 0.5865 & 0.9300 & 2.8458 & 2.8650 & 2.8444 & 2.3312 & 1.1422 & 1.1877 & 1.2536 & 1.3363 & 1.452 \\
\hline
\end{tabular}


Appendix F - Gauge data for experiment 2S-54.

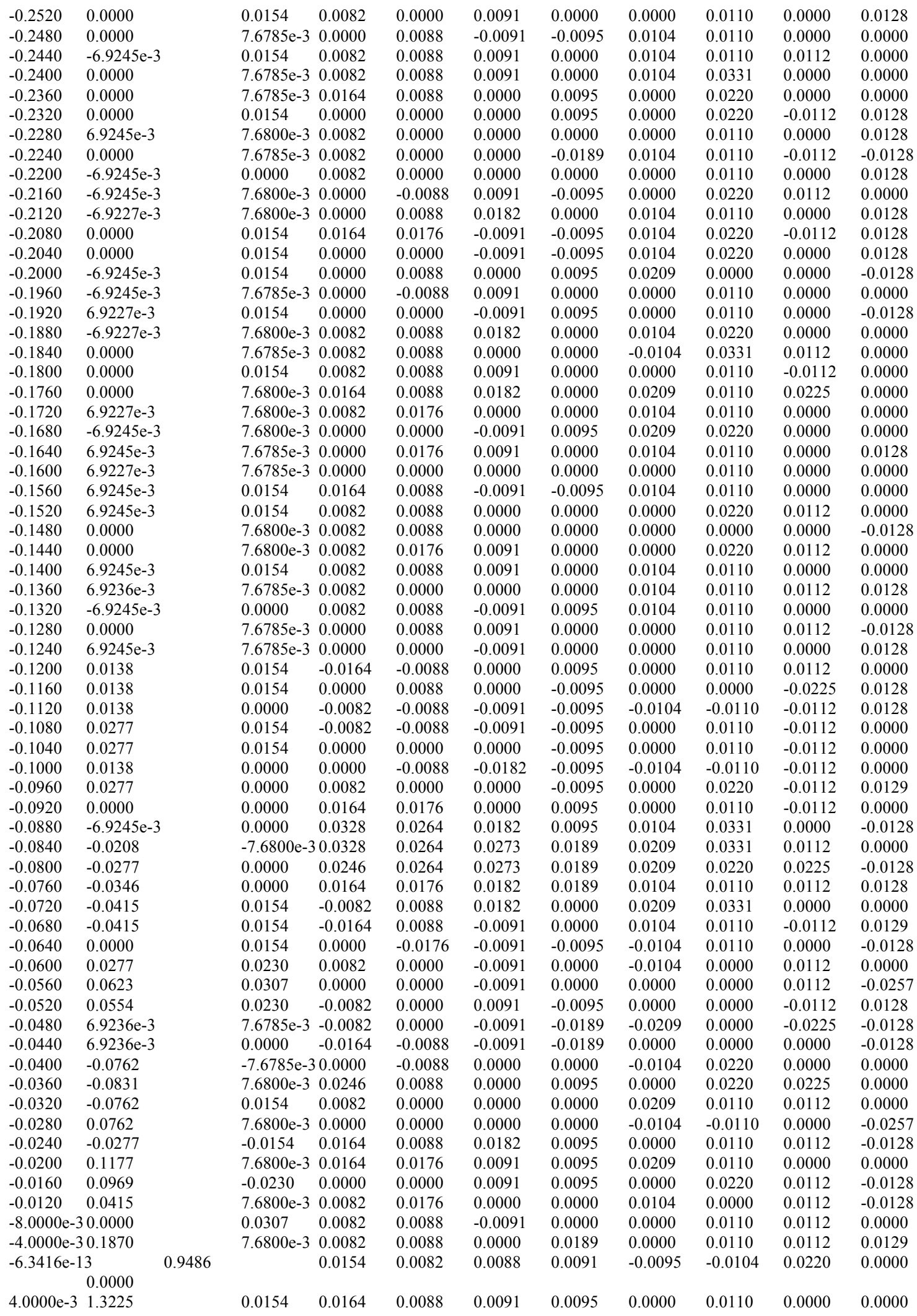




\begin{tabular}{|c|c|}
\hline & \\
\hline 0.0120 & 197 \\
\hline .0160 & 239 \\
\hline 0200 & 2463 \\
\hline 0240 & \\
\hline 0280 & 287 \\
\hline 0320 & 267 \\
\hline 0360 & 25 \\
\hline .0400 & 1.274 \\
\hline .0440 & דיר \\
\hline .0480 & .20 \\
\hline .0520 & 1.260 \\
\hline 560 & \\
\hline 0600 & \\
\hline 0640 & 1.301 \\
\hline 0680 & .266 \\
\hline .0720 & 1268 \\
\hline 0760 & .28 \\
\hline 9800 & 281 \\
\hline 0840 & 260 \\
\hline 0880 & \\
\hline 0920 & \\
\hline 0960 & .294 \\
\hline 1000 & 6 \\
\hline 1040 & .26 \\
\hline 1080 & 267 \\
\hline 120 & 28 \\
\hline 1160 & 274 \\
\hline 1200 & .274 \\
\hline 1240 & .281 \\
\hline & \\
\hline 20 & 260 \\
\hline 360 & 274 \\
\hline 400 & 267 \\
\hline 1440 & $1 / 2$ \\
\hline 1480 & 12 \\
\hline & 127 \\
\hline 0 & 281 \\
\hline 0 & 281 \\
\hline 640 & 172 \\
\hline 1680 & 272 \\
\hline 0.1720 & .274 \\
\hline 1760 & 1.27 \\
\hline 1800 & 20. \\
\hline 10 & \\
\hline 0 & 1.274 \\
\hline 1920 & 272 \\
\hline $10 \Omega$ & .27 \\
\hline קO & $1.26^{\circ}$ \\
\hline 2040 & \\
\hline 2080 & 120. \\
\hline 20 & 2 \\
\hline 0 & 12 \\
\hline 2200 & $1.26^{7}$ \\
\hline 2240 & $1.26 \mathrm{C}$ \\
\hline 0.2280 & 1.23 \\
\hline 0.2520 & 1.23 \\
\hline 002 & 126 \\
\hline 2400 & 12 \\
\hline 2440 & 12 \\
\hline 2480 & 10 \\
\hline 2520 & 166 \\
\hline 0.2560 & 1.20 \\
\hline 0.2600 & 1.26 \\
\hline 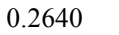 & \\
\hline 0.2680 & \\
\hline (270 & \\
\hline 77 & \\
\hline 0.2800 & $1.26^{\circ}$ \\
\hline 0.2840 & 1.2 \\
\hline
\end{tabular}

\begin{tabular}{|c|c|c|c|c|c|c|c|c|}
\hline 0.0230 & 0.0082 & 0.0088 & 0.0091 & 0.0095 & 0.0209 & 0.0220 & 0.0112 & 0.0000 \\
\hline 0.0154 & 0.0082 & 0.0088 & 0.0000 & 0.0000 & 0.0000 & 0.0000 & -0.0112 & 0.0128 \\
\hline $7.6785 \mathrm{e}-3$ & 0.0164 & 0.0088 & 0.0091 & -0.0095 & 0.0313 & 0.0220 & 0.0000 & 0.0128 \\
\hline 0.0000 & 0.0000 & 0176 & 0182 & 0.0000 & .0104 & 0.0110 & 0.0000 & 0128 \\
\hline $7.6785 e-3$ & 0.0000 & 0088 & 0.0091 & 0.0000 & 0209 & .0000 & .0000 & 0000 \\
\hline $7.6785 \mathrm{e}-3$ & 0.0082 & -0.0088 & 0.0091 & -0.0095 & 0000 & .0000 & 000 & .012 \\
\hline $7.6785 \mathrm{e}-3$ & -0.0082 & .0088 & .0091 & 0.0095 & 0104 & 0220 & 0112 & .0129 \\
\hline $7.6800 \mathrm{e}-3$ & 0.0082 & 0.0088 & .0000 & .0095 & .0104 & 0110 & 112 & 0000 \\
\hline 0.0154 & 0.0000 & .0088 & .0000 & 0.0000 & .0104 & .0110 & 000 & 0000 \\
\hline $7.6785 e-3$ & 0.0082 & .0000 & .0000 & 0.0000 & .0104 & .0110 & 000 & 0128 \\
\hline $7.6785 \mathrm{e}-3$ & 0.0164 & .0088 & .0091 & 0.0000 & 0104 & .0110 & 0.0000 & 0128 \\
\hline 0.0000 & 0.0082 & 0.0000 & .0000 & & & & & 128 \\
\hline $7.6785 e-3$ & 0.0000 & .0000 & 0091 & 0000 & & & & 128 \\
\hline $7.6785 \mathrm{e}-3$ & -0.0082 & 0.0088 & .0000 & 0000 & 000 & 10 & & 0129 \\
\hline $7.6785 e-3$ & 0.0000 & 0.0000 & 0.0000 & -0.0095 & 000 & 0110 & & 0257 \\
\hline $7.6800 \mathrm{e}-3$ & 0.0164 & 0.0176 & 0.0091 & 0.0095 & .0104 & 0110 & & .0000 \\
\hline $7.6785 e-3$ & 0.0082 & 0.0088 & -0.0091 & 0.0095 & 0000 & 20 & 00 & 0000 \\
\hline $7.6785 \mathrm{e}-3$ & 0.0082 & 0.0000 & .0000 & 0.0000 & .0104 & & & 0000 \\
\hline 0.0000 & 0.0082 & 38 & 1 & 95 & & & & 000 \\
\hline 0.0000 & 0.0000 & 0 & 91 & 00 & 104 & 00 & & 000 \\
\hline 0.0000 & 0.0082 & 0088 & 0000 & 00 & & 000 & & 000 \\
\hline 0.0154 & 0.0082 & 00 & .0000 & & & 110 & & .0000 \\
\hline $.6800 \mathrm{e}-3$ & 0.0000 & .0000 & .0000 & & & & & 0128 \\
\hline 7.68 & 0.0082 & & & 95 & & & & 000 \\
\hline e-3 & 0.0082 & & & & & & & \\
\hline 0.00 & 0.0082 & & & & & & & 128 \\
\hline $7.6785 \mathrm{e}-3$ & 0.0082 & 88 & 1 & 995 & & 10 & & 000 \\
\hline $7.6785 e-3$ & 0.0082 & 0000 & .0000 & 0.0000 & 00 & 10 & 12 & 0128 \\
\hline 0.0154 & 0.0082 & & 91 & & & & & 0000 \\
\hline $7.6785 e-3$ & 0.0082 & 38 & 0 & & & & & -0.0257 \\
\hline $7.6800 \mathrm{e}-3$ & 0.0082 & & & & & & & \\
\hline 0.00 & -0.0082 & & & & & & & 00 \\
\hline 7.67 & 0.0000 & & & & & & & 00 \\
\hline $7.6785 \mathrm{e}-3$ & 0.0082 & & 1 & 0 & 104 & & & 128 \\
\hline 0.0154 & 0.0000 & 00 & 91 & .0095 & & & & 000 \\
\hline & & & & & & & & 128 \\
\hline 0.0154 & 0.0082 & & & & 00 & & 112 & 0128 \\
\hline 7.67 & 0.0000 & & & & & & & \\
\hline & 0.0 & & & & & & & \\
\hline 0.00 & 0.0082 & & & & & & & 000 \\
\hline $7.6785 \mathrm{e}-3$ & 0.0082 & 0000 & 0 & 95 & & 00 & & .0128 \\
\hline & 0.0082 & & & & & & & \\
\hline 7.67 & -0.0246 & 0088 & & 89 & & 10 & & -0.0128 \\
\hline $7.6785 e-3$ & -0.0492 & & & & & & & -0.0128 \\
\hline & -0.0492 & & & & & & & -0.0128 \\
\hline & -0.0 & & & & & & & \\
\hline $7.6800 \mathrm{e}-3$ & -0.0410 & & & 79 & 209 & 00 & & 128 \\
\hline 0.0000 & -0.0164 & .0176 & 82 & -0.0095 & 104 & 000 & & -0.0128 \\
\hline & -0.0082 & & & & & & & 000 \\
\hline 0.1843 & 0.0000 & 000 & & -0.0095 & & & & -0.0128 \\
\hline & & & & & & & & \\
\hline & & & & & & & & \\
\hline 0.9 & 00 & & & & & & & \\
\hline 1.1596 & 0.0082 & & 0 & 95 & 00 & 10 & & 128 \\
\hline & & & & & & & & 000 \\
\hline 1.2364 & 0.0082 & 0.0000 & 0 & & 000 & & & .0000 \\
\hline 1.2518 & 0.0082 & & & & & & & 000 \\
\hline 1.2441 & & & & & & & & 0000 \\
\hline & & & & & & & & \\
\hline & & & & & & & & \\
\hline & & & & & & & & DQ0 \\
\hline 1.25 & 0.0082 & & & 995 & & & & 128 \\
\hline 1.2594 & 0.0000 & 0.0000 & -0.0091 & 0.0000 & 0.0000 & 0.0220 & 0.0000 & 0.0128 \\
\hline 1.2671 & 0.0000 & & & 000 & 000 & 0.0000 & 0000 & .0128 \\
\hline & 0.0000 & & & & & & & \\
\hline & 0.0000 & & & -0.0095 & 0.0000 & & & -0.012 \\
\hline & & & & & & & & \\
\hline & & & & & & & & .0000 \\
\hline 1.2825 & 0.0082 & 0.0088 & 0.0000 & 0.0095 & 0.0104 & 0.0110 & 0.0000 & 0.0128 \\
\hline 1.2748 & 0.0082 & 0.0088 & 0.0000 & 0.0000 & 0.0000 & 0.0110 & 0.0000 & 0.0000 \\
\hline
\end{tabular}




\begin{tabular}{|c|c|c|c|c|c|c|c|c|c|c|}
\hline 0.2880 & 1.2740 & 1.2748 & 0.0000 & 0.0000 & 0.0091 & -0.0095 & 0.0000 & 0.0110 & 0.0000 & 0.0000 \\
\hline 0.2920 & 1.2810 & 1.2825 & 0.0000 & 0.0000 & 0.0091 & -0.0095 & 0.0104 & 0.0220 & 0.0000 & 0.0128 \\
\hline 0.2960 & 1.2879 & 1.2825 & 0.0082 & 0.0088 & 0.0091 & 0.0000 & 0.0000 & 0.0110 & 0.0000 & 0.0000 \\
\hline 0.3000 & 1.2602 & 1.2748 & 0.0082 & 0.0088 & 0.0091 & 0.0000 & 0.0000 & 0.0000 & 0.0112 & -0.0128 \\
\hline 0.3040 & 1.2671 & 1.2902 & 0.0164 & 0.0088 & 0.0091 & 0.0095 & 0.0000 & 0.0110 & 0.0112 & 0.0000 \\
\hline 0.3080 & 1.2671 & 1.2748 & 0.0656 & 0.0088 & 0.0000 & 0.0000 & 0.0000 & 0.0000 & -0.0112 & 0.0000 \\
\hline 0.3120 & 1.2671 & 1.2825 & 0.3444 & -0.0352 & -0.0273 & -0.0284 & -0.0313 & -0.0110 & 0.0000 & 0.0128 \\
\hline 0.3160 & 1.2671 & 1.2825 & 0.7052 & -0.0791 & -0.0547 & -0.0379 & -0.0313 & -0.0110 & -0.0112 & -0.0257 \\
\hline 0.3200 & 1.2671 & 1.3055 & 0.9347 & -0.0527 & -0.0273 & -0.0284 & -0.0209 & -0.0110 & -0.0337 & -0.0128 \\
\hline 0.3240 & 1.2533 & 1.2902 & 1.1479 & -0.0440 & -0.0273 & -0.0284 & -0.0209 & -0.0110 & -0.0225 & -0.0128 \\
\hline 0.3280 & 1.2671 & 1.2902 & 1.2217 & -0.0176 & -0.0182 & -0.0379 & -0.0104 & -0.0110 & -0.0225 & -0.0129 \\
\hline 0.3320 & 1.2740 & 1.2825 & 1.2463 & 0.0000 & -0.0091 & -0.0189 & -0.0104 & -0.0110 & 0.0000 & -0.0128 \\
\hline 0.3360 & 1.2671 & 1.2825 & 1.2627 & -0.0088 & 0.0000 & 0.0000 & -0.0104 & 0.0220 & 0.0112 & -0.0128 \\
\hline 0.3400 & 1.2671 & 1.2441 & 1.2627 & 0.0000 & 0.0091 & 0.0095 & -0.0104 & -0.0110 & 0.0000 & 0.0000 \\
\hline 0.3440 & 1.2671 & 1.2364 & 1.2545 & 0.0000 & 0.0000 & 0.0000 & 0.0104 & 0.0110 & -0.0112 & 0.0128 \\
\hline 0.3480 & 1.2671 & 1.2441 & 1.2627 & -0.0088 & 0.0000 & -0.0095 & 0.0000 & 0.0000 & 0.0000 & 0.0128 \\
\hline 0.3520 & 1.2671 & 1.2518 & 1.2709 & 0.0000 & -0.0091 & -0.0095 & 0.0000 & 0.0110 & -0.0112 & -0.0128 \\
\hline 0.3560 & 1.2671 & 1.2671 & 1.2709 & 0.0000 & 0.0000 & 0.0095 & 0.0104 & 0.0110 & -0.0112 & -0.0257 \\
\hline 0.3600 & 1.2740 & 1.2825 & 1.2709 & 0.0000 & 0.0091 & 0.0095 & 0.0104 & 0.0110 & 0.0112 & -0.0128 \\
\hline 0.3640 & 1.2740 & 1.2902 & 1.2791 & 0.0176 & 0.0000 & 0.0095 & 0.0000 & 0.0110 & 0.0000 & 0.0000 \\
\hline 0.3680 & 1.2671 & 1.2902 & 1.2873 & 0.0000 & 0.0000 & 0.0095 & -0.0104 & 0.0110 & 0.0000 & 0.0000 \\
\hline 0.3720 & 1.2602 & 1.2902 & 1.2791 & 0.0088 & 0.0000 & 0.0000 & 0.0104 & 0.0110 & 0.0000 & 0.0000 \\
\hline 0.3760 & 1.2671 & 1.2902 & 1.2791 & 0.0000 & 0.0091 & 0.0095 & 0.0104 & 0.0000 & 0.0112 & 0.0000 \\
\hline 0.3800 & 1.2671 & 1.2902 & 1.2873 & 0.0088 & 0.0091 & 0.0000 & 0.0104 & 0.0000 & 0.0225 & -0.0128 \\
\hline 0.3840 & 1.2671 & 1.2978 & 1.2873 & 0.0000 & 0.0000 & -0.0095 & 0.0104 & 0.0110 & 0.0000 & 0.0000 \\
\hline 0.3880 & 1.2740 & 1.2902 & 1.2955 & 0.0088 & -0.0091 & 0.0000 & 0.0000 & 0.0220 & -0.0112 & 0.0000 \\
\hline 0.3920 & 1.2671 & 1.2902 & 1.2955 & 0.0000 & 0.0091 & -0.0095 & 0.0104 & 0.0331 & 0.0000 & -0.0128 \\
\hline 0.3960 & 1.2671 & 1.2902 & 1.2791 & 0.0176 & 0.0000 & -0.0095 & 0.0000 & 0.0000 & 0.0000 & 0.0000 \\
\hline 0.4000 & 1.2671 & 1.2978 & 1.2955 & 0.0000 & 0.0000 & 0.0000 & 0.0104 & 0.0110 & 0.0000 & -0.0128 \\
\hline 0.4040 & 1.2602 & 1.2978 & 1.2955 & 0.0088 & 0.0091 & 0.0000 & 0.0104 & 0.0220 & 0.0000 & 0.0128 \\
\hline 0.4080 & 1.2602 & 1.2978 & 1.2955 & 0.0088 & 0.0091 & 0.0095 & 0.0104 & 0.0220 & 0.0112 & 0.0128 \\
\hline 0.4120 & 1.2602 & 1.2902 & 1.2955 & 0.0088 & 0.0000 & 0.0000 & -0.0104 & 0.0220 & 0.0000 & 0.0000 \\
\hline 0.4160 & 1.2671 & 1.2902 & 1.2873 & 0.0088 & 0.0000 & -0.0095 & 0.0104 & 0.0110 & -0.0112 & 0.0000 \\
\hline 0.4200 & 1.2740 & 1.2902 & 1.2873 & 0.0000 & 0.0182 & 0.0000 & 0.0104 & 0.0000 & 0.0225 & -0.0128 \\
\hline 0.4240 & 1.2740 & 1.2978 & 1.2955 & 0.0000 & 0.0000 & 0.0000 & -0.0104 & 0.0110 & -0.0112 & -0.0128 \\
\hline 0.4280 & 1.2671 & 1.2902 & 1.2955 & 0.0000 & 0.0091 & 0.0095 & 0.0000 & 0.0110 & -0.0112 & -0.0128 \\
\hline 0.4320 & 1.2602 & 1.2978 & 1.2873 & 0.0088 & 0.0091 & 0.0000 & 0.0000 & 0.0000 & 0.0112 & 0.0257 \\
\hline 0.4360 & 1.2602 & 1.2825 & 1.3037 & 0.0000 & 0.0000 & 0.0000 & 0.0000 & 0.0220 & 0.0112 & 0.0128 \\
\hline 0.4400 & 1.2602 & 1.2902 & 1.2873 & 0.0176 & -0.0091 & 0.0000 & 0.0104 & 0.0220 & 0.0112 & 0.0000 \\
\hline 0.4440 & 1.2533 & 1.2978 & 1.2791 & 0.1846 & -0.0182 & -0.0379 & -0.0104 & -0.0220 & 0.0000 & -0.0128 \\
\hline 0.4480 & 1.2671 & 1.2978 & 1.2463 & 0.5801 & -0.0638 & -0.0568 & -0.0209 & -0.0110 & -0.0337 & -0.0128 \\
\hline 0.4520 & 1.2602 & 1.2902 & 1.2545 & 0.9229 & -0.0547 & -0.0568 & -0.0313 & -0.0220 & -0.0337 & -0.0257 \\
\hline 0.4560 & 1.2602 & 1.2902 & 1.2627 & 1.1338 & -0.0455 & -0.0379 & -0.0209 & -0.0110 & -0.0225 & 0.0000 \\
\hline 0.4600 & 1.2602 & 1.2902 & 1.2791 & 1.2481 & -0.0091 & -0.0284 & -0.0209 & -0.0110 & -0.0112 & 0.0000 \\
\hline 0.4640 & 1.2602 & 1.2978 & 1.2873 & 1.2657 & 0.0000 & -0.0189 & -0.0104 & 0.0110 & -0.0112 & 0.0000 \\
\hline 0.4680 & 1.2533 & 1.2902 & 1.3037 & 1.2745 & 0.0091 & -0.0095 & -0.0104 & 0.0000 & -0.0112 & 0.0000 \\
\hline 0.4720 & 1.2671 & 1.2825 & 1.3119 & 1.2657 & 0.0091 & 0.0000 & 0.0104 & 0.0110 & 0.0000 & -0.0128 \\
\hline 0.4760 & 1.2602 & 1.2518 & 1.3119 & 1.2657 & 0.0000 & 0.0095 & 0.0000 & 0.0000 & 0.0112 & 0.0129 \\
\hline 0.4800 & 1.2533 & 1.2518 & 1.2955 & 1.2657 & 0.0000 & 0.0000 & 0.0000 & 0.0110 & 0.0112 & -0.0128 \\
\hline 0.4840 & 1.2533 & 1.2518 & 1.3119 & 1.2745 & 0.0000 & -0.0095 & 0.0104 & 0.0110 & 0.0000 & -0.0128 \\
\hline 0.4880 & 1.2602 & 1.2902 & 1.3037 & 1.2745 & 0.0000 & 0.0000 & 0.0104 & 0.0000 & 0.0000 & 0.0000 \\
\hline 0.4920 & 1.2671 & 1.2902 & 1.3037 & 1.3008 & 0.0000 & 0.0000 & -0.0104 & 0.0110 & 0.0000 & 0.0128 \\
\hline 0.4960 & 1.2671 & 1.2978 & 1.3201 & 1.2833 & 0.0000 & -0.0095 & 0.0104 & 0.0220 & 0.0112 & 0.0000 \\
\hline 0.5000 & 1.2533 & 1.2978 & 1.3037 & 1.2920 & 0.0000 & 0.0000 & -0.0104 & 0.0110 & 0.0000 & 0.0000 \\
\hline 0.5040 & 1.2533 & 1.2978 & 1.3037 & 1.2920 & 0.0000 & -0.0095 & -0.0104 & 0.0110 & 0.0000 & -0.0128 \\
\hline 0.5080 & 1.2533 & 1.2978 & 1.3119 & 1.2920 & 0.0000 & 0.0095 & 0.0104 & 0.0000 & 0.0000 & 0.0128 \\
\hline 0.5120 & 1.2533 & 1.3055 & 1.3119 & 1.3008 & 0.0000 & 0.0000 & 0.0000 & 0.0110 & -0.0112 & 0.0000 \\
\hline 0.5160 & 1.2533 & 1.2978 & 1.3119 & 1.2920 & 0.0091 & 0.0000 & 0.0209 & 0.0110 & 0.0000 & 0.0128 \\
\hline 0.5200 & 1.2602 & 1.2978 & 1.3037 & 1.3008 & 0.0000 & 0.0000 & 0.0000 & 0.0220 & 0.0000 & -0.0128 \\
\hline 0.5240 & 1.2463 & 1.2978 & 1.3201 & 1.3008 & 0.0000 & 0.0095 & 0.0104 & 0.0110 & -0.0112 & 0.0000 \\
\hline 0.5280 & 1.2602 & 1.2978 & 1.3119 & 1.2920 & 0.0091 & 0.0000 & 0.0104 & 0.0000 & 0.0000 & 0.0000 \\
\hline 0.5320 & 1.2463 & 1.2825 & 1.3119 & 1.3096 & 0.0091 & 0.0095 & 0.0104 & 0.0110 & 0.0112 & 0.0000 \\
\hline 0.5360 & 1.2533 & 1.2978 & 1.3201 & 1.3008 & 0.0000 & 0.0000 & 0.0000 & 0.0110 & 0.0112 & 0.0128 \\
\hline 0.5400 & 1.2533 & 1.2978 & 1.3119 & 1.3008 & 0.0000 & 0.0095 & -0.0104 & 0.0000 & 0.0112 & 0.0000 \\
\hline 0.5440 & 1.2394 & 1.3055 & 1.3037 & 1.3008 & 0.0091 & 0.0095 & 0.0000 & 0.0000 & 0.0000 & 0.0128 \\
\hline 0.5480 & 1.2463 & 1.2978 & 1.3119 & 1.3096 & 0.0000 & 0.0095 & 0.0104 & 0.0110 & 0.0112 & 0.0000 \\
\hline 0.5520 & 1.2463 & 1.3055 & 1.3037 & 1.3184 & 0.0091 & 0.0000 & 0.0104 & 0.0220 & 0.0112 & 0.0000 \\
\hline 0.5560 & 1.2533 & 1.2978 & 1.3037 & 1.3096 & 0.0091 & 0.0095 & 0.0000 & 0.0110 & 0.0112 & -0.0128 \\
\hline 0.5600 & 1.2533 & 1.3055 & 1.3037 & 1.3008 & 0.0091 & 0.0095 & 0.0104 & 0.0000 & 0.0112 & 0.0000 \\
\hline 0.5640 & 1.2463 & 1.2978 & 1.3201 & 1.3184 & 0.0000 & 0.0000 & -0.0209 & 0.0220 & -0.0112 & 0.0000 \\
\hline
\end{tabular}




\begin{tabular}{|c|c|c|c|c|c|c|c|c|c|c|}
\hline 0.5680 & 1.2463 & 1.3055 & 1.3119 & 1.3008 & -0.0091 & 0.0095 & 0.0104 & 0.0000 & 0.0112 & 0.0000 \\
\hline 0.5720 & 1.2325 & 1.2978 & 1.3201 & 1.3096 & 0.0091 & 0.0000 & -0.0104 & 0.0110 & 0.0112 & 0.0000 \\
\hline 0.5760 & 1.2394 & 1.2978 & 1.2955 & 1.3008 & 0.1548 & -0.0284 & -0.0209 & 0.0000 & -0.0112 & 0.0000 \\
\hline 0.5800 & 1.2463 & 1.3055 & 1.2955 & 1.2833 & 0.5740 & -0.0568 & -0.0418 & -0.0220 & -0.0112 & -0.0128 \\
\hline 0.5840 & 1.2463 & 1.2978 & 1.2791 & 1.2657 & 0.9748 & -0.0473 & -0.0418 & -0.0220 & -0.0337 & -0.0129 \\
\hline 0.5880 & 1.2394 & 1.2978 & 1.2873 & 1.2745 & 1.1752 & -0.0379 & -0.0313 & -0.0110 & -0.0337 & -0.0129 \\
\hline 0.5920 & 1.2463 & 1.3055 & 1.2955 & 1.2920 & 1.2572 & -0.0284 & -0.0209 & -0.0110 & -0.0225 & 0.0000 \\
\hline 0.5960 & 1.2463 & 1.3055 & 1.3037 & 1.3096 & 1.2846 & -0.0189 & 0.0000 & 0.0110 & -0.0112 & 0.0000 \\
\hline 0.6000 & 1.2463 & 1.3055 & 1.3201 & 1.3096 & 1.2755 & -0.0095 & -0.0104 & 0.0220 & 0.0000 & -0.0128 \\
\hline 0.6040 & 1.2463 & 1.2978 & 1.3283 & 1.3184 & 1.2846 & 0.0000 & -0.0104 & 0.0110 & 0.0000 & -0.0257 \\
\hline 0.6080 & 1.2394 & 1.2748 & 1.3283 & 1.3272 & 1.2846 & 0.0095 & -0.0104 & 0.0220 & -0.0112 & 0.0000 \\
\hline 0.6120 & 1.2463 & 1.2748 & 1.3365 & 1.3096 & 1.2937 & 0.0000 & 0.0000 & 0.0110 & -0.0112 & 0.0000 \\
\hline 0.6160 & 1.2463 & 1.2902 & 1.3283 & 1.3096 & 1.2846 & 0.0000 & 0.0000 & 0.0220 & 0.0112 & -0.0128 \\
\hline 0.6200 & 1.2394 & 1.2902 & 1.3283 & 1.3184 & 1.3119 & -0.0095 & -0.0104 & 0.0110 & 0.0000 & -0.0128 \\
\hline 0.6240 & 1.2463 & 1.2978 & 1.3365 & 1.3184 & 1.3119 & -0.0095 & -0.0104 & 0.0220 & -0.0112 & 0.0000 \\
\hline 0.6280 & 1.2325 & 1.2978 & 1.3201 & 1.3096 & 1.3119 & 0.0095 & 0.0000 & 0.0110 & -0.0112 & 0.0128 \\
\hline 0.6320 & 1.2394 & 1.3055 & 1.3283 & 1.3272 & 1.3119 & 0.0000 & 0.0104 & 0.0110 & 0.0000 & -0.0128 \\
\hline 0.6360 & 1.2394 & 1.2978 & 1.3365 & 1.3184 & 1.3028 & 0.0000 & -0.0104 & 0.0110 & 0.0000 & -0.0128 \\
\hline 0.6400 & 1.2463 & 1.3055 & 1.3201 & 1.3184 & 1.3210 & 0.0095 & -0.0104 & 0.0000 & 0.0000 & 0.0000 \\
\hline 0.6440 & 1.2325 & 1.3055 & 1.3283 & 1.3272 & 1.3210 & 0.0095 & 0.0000 & 0.0110 & 0.0000 & 0.0128 \\
\hline 0.6480 & 1.2325 & 1.3055 & 1.3283 & 1.3272 & 1.3210 & 0.0000 & 0.0000 & 0.0000 & -0.0112 & 0.0128 \\
\hline 0.6520 & 1.2394 & 1.3055 & 1.3447 & 1.3272 & 1.3210 & -0.0095 & -0.0104 & 0.0110 & -0.0112 & 0.0000 \\
\hline 0.6560 & 1.2394 & 1.3055 & 1.3447 & 1.3184 & 1.3210 & 0.0000 & -0.0104 & 0.0331 & -0.0112 & -0.0128 \\
\hline 0.6600 & 1.2256 & 1.2978 & 1.3365 & 1.3272 & 1.3210 & 0.0095 & 0.0000 & 0.0220 & 0.0112 & -0.0128 \\
\hline 0.6640 & 1.2325 & 1.3055 & 1.3447 & 1.3360 & 1.3210 & 0.0000 & 0.0104 & 0.0000 & 0.0112 & 0.0000 \\
\hline 0.6680 & 1.2325 & 1.3055 & 1.3447 & 1.3272 & 1.3210 & 0.0000 & 0.0104 & 0.0220 & 0.0000 & -0.0128 \\
\hline 0.6720 & 1.2256 & 1.3055 & 1.3447 & 1.3272 & 1.3210 & 0.0095 & 0.0104 & 0.0220 & 0.0000 & -0.0128 \\
\hline 0.6760 & 1.2186 & 1.3055 & 1.3283 & 1.3184 & 1.3301 & 0.0000 & 0.0000 & 0.0110 & 0.0000 & 0.0000 \\
\hline 0.6800 & 1.2186 & 1.3055 & 1.3365 & 1.3184 & 1.3301 & 0.0000 & 0.0104 & 0.0220 & 0.0000 & -0.0128 \\
\hline 0.6840 & 1.2256 & 1.2978 & 1.3365 & 1.3272 & 1.3301 & 0.0000 & 0.0000 & 0.0110 & 0.0112 & 0.0000 \\
\hline 0.6880 & 1.2256 & 1.2978 & 1.3365 & 1.3272 & 1.3301 & 0.0000 & 0.0000 & 0.0220 & 0.0000 & -0.0128 \\
\hline 0.6920 & 1.2256 & 1.3055 & 1.3365 & 1.3272 & 1.3392 & 0.0000 & 0.0000 & 0.0220 & 0.0000 & 0.0000 \\
\hline 0.6960 & 1.2256 & 1.3055 & 1.3283 & 1.3272 & 1.3301 & -0.0095 & 0.0104 & 0.0000 & 0.0000 & 0.0128 \\
\hline 0.7000 & 1.2117 & 1.3132 & 1.3365 & 1.3360 & 1.3301 & -0.0095 & 0.0104 & 0.0000 & 0.0000 & 0.0000 \\
\hline 0.7040 & 1.2186 & 1.3055 & 1.3283 & 1.3360 & 1.3301 & 0.0000 & 0.0104 & 0.0220 & 0.0000 & -0.0128 \\
\hline 0.7080 & 1.2117 & 1.3055 & 1.3365 & 1.3360 & 1.3301 & 0.0852 & -0.0104 & 0.0000 & 0.0000 & -0.0128 \\
\hline 0.7120 & 1.2256 & 1.2978 & 1.3201 & 1.3272 & 1.3028 & 0.4639 & -0.0313 & -0.0331 & -0.0112 & -0.0128 \\
\hline 0.7160 & 1.2048 & 1.3132 & 1.3119 & 1.2920 & 1.2937 & 0.9184 & -0.0522 & -0.0331 & -0.0337 & -0.0257 \\
\hline 0.7200 & 1.2048 & 1.3132 & 1.3037 & 1.3096 & 1.3028 & 1.1835 & -0.0313 & -0.0110 & -0.0225 & -0.0385 \\
\hline 0.7240 & 1.2117 & 1.2978 & 1.3119 & 1.3184 & 1.3210 & 1.2876 & -0.0104 & 0.0000 & 0.0000 & -0.0128 \\
\hline 0.7280 & 1.2117 & 1.2978 & 1.3283 & 1.3360 & 1.3392 & 1.2781 & 0.0000 & 0.0110 & -0.0112 & 0.0000 \\
\hline 0.7320 & 1.2117 & 1.3055 & 1.3447 & 1.3448 & 1.3392 & 1.2876 & 0.0000 & 0.0000 & 0.0000 & -0.0128 \\
\hline 0.7360 & 1.2048 & 1.2978 & 1.3365 & 1.3448 & 1.3574 & 1.2971 & 0.0000 & 0.0110 & 0.0000 & 0.0128 \\
\hline 0.7400 & 1.2117 & 1.2902 & 1.3447 & 1.3536 & 1.3483 & 1.3065 & 0.0104 & -0.0110 & 0.0000 & 0.0129 \\
\hline 0.7440 & 1.2048 & 1.2825 & 1.3283 & 1.3448 & 1.3574 & 1.2971 & 0.0000 & 0.0000 & 0.0000 & -0.0128 \\
\hline 0.7480 & 1.2048 & 1.2748 & 1.3365 & 1.3448 & 1.3483 & 1.3065 & -0.0104 & 0.0000 & 0.0112 & 0.0000 \\
\hline 0.7520 & 1.2048 & 1.2902 & 1.3365 & 1.3536 & 1.3483 & 1.3160 & 0.0104 & 0.0220 & 0.0000 & -0.0128 \\
\hline 0.7560 & 1.2117 & 1.2902 & 1.3447 & 1.3448 & 1.3574 & 1.3160 & 0.0209 & 0.0110 & 0.0112 & 0.0000 \\
\hline 0.7600 & 1.2048 & 1.2978 & 1.3447 & 1.3448 & 1.3574 & 1.3160 & 0.0000 & 0.0110 & 0.0000 & 0.0000 \\
\hline 0.7640 & 1.1979 & 1.2978 & 1.3447 & 1.3448 & 1.3574 & 1.3255 & 0.0209 & -0.0110 & 0.0000 & 0.0000 \\
\hline 0.7680 & 1.2048 & 1.3055 & 1.3365 & 1.3536 & 1.3574 & 1.3255 & -0.0104 & 0.0110 & 0.0000 & 0.0000 \\
\hline 0.7720 & 1.1979 & 1.2978 & 1.3447 & 1.3624 & 1.3574 & 1.3349 & 0.0000 & 0.0000 & -0.0112 & 0.0128 \\
\hline 0.7760 & 1.2048 & 1.3055 & 1.3447 & 1.3536 & 1.3574 & 1.3349 & 0.0000 & 0.0220 & -0.0112 & -0.0128 \\
\hline 0.7800 & 1.1979 & 1.3055 & 1.3447 & 1.3536 & 1.3574 & 1.3444 & -0.0104 & 0.0000 & 0.0000 & 0.0000 \\
\hline 0.7840 & 1.1910 & 1.3055 & 1.3447 & 1.3624 & 1.3666 & 1.3539 & 0.0000 & 0.0220 & 0.0112 & 0.0000 \\
\hline 0.7880 & 1.2048 & 1.2978 & 1.3447 & 1.3624 & 1.3574 & 1.3349 & 0.0209 & 0.0000 & -0.0112 & -0.0128 \\
\hline 0.7920 & 1.2048 & 1.3055 & 1.3447 & 1.3448 & 1.3574 & 1.3444 & 0.0104 & 0.0220 & 0.0112 & 0.0000 \\
\hline 0.7960 & 1.1910 & 1.3055 & 1.3447 & 1.3536 & 1.3757 & 1.3444 & 0.0104 & 0.0110 & 0.0225 & 0.0257 \\
\hline 0.8000 & 1.2048 & 1.2978 & 1.3365 & 1.3536 & 1.3574 & 1.3444 & 0.0104 & 0.0000 & 0.0112 & 0.0000 \\
\hline 0.8040 & 1.2048 & 1.2978 & 1.3447 & 1.3536 & 1.3666 & 1.3444 & 0.0000 & 0.0220 & 0.0112 & 0.0000 \\
\hline 0.8080 & 1.1979 & 1.2978 & 1.3447 & 1.3624 & 1.3666 & 1.3539 & 0.0000 & 0.0331 & 0.0225 & 0.0000 \\
\hline 0.8120 & 1.2048 & 1.2978 & 1.3447 & 1.3712 & 1.3574 & 1.3444 & 0.0104 & 0.0220 & 0.0000 & 0.0129 \\
\hline 0.8160 & 1.2048 & 1.2978 & 1.3447 & 1.3799 & 1.3574 & 1.3539 & 0.0104 & 0.0110 & 0.0000 & 0.0000 \\
\hline 0.8200 & 1.1910 & 1.2902 & 1.3365 & 1.3624 & 1.3574 & 1.3633 & -0.0104 & 0.0110 & 0.0000 & 0.0000 \\
\hline 0.8240 & 1.1910 & 1.2978 & 1.3447 & 1.3624 & 1.3666 & 1.3539 & 0.0104 & 0.0000 & 0.0000 & 0.0000 \\
\hline 0.8280 & 1.1910 & 1.2902 & 1.3447 & 1.3712 & 1.3666 & 1.3633 & 0.0000 & 0.0000 & 0.0000 & 0.0128 \\
\hline 0.8320 & 1.1910 & 1.2978 & 1.3447 & 1.3624 & 1.3574 & 1.3539 & 0.0104 & 0.0110 & -0.0112 & 0.0128 \\
\hline 0.8360 & 1.1840 & 1.2978 & 1.3447 & 1.3624 & 1.3757 & 1.3633 & 0.0104 & 0.0000 & 0.0000 & 0.0000 \\
\hline 0.8400 & 1.1979 & 1.2978 & 1.3283 & 1.3624 & 1.3666 & 1.3444 & 0.1253 & -0.0110 & -0.0112 & -0.0128 \\
\hline 0.8440 & 1.1979 & 1.2978 & 1.3283 & 1.3624 & 1.3392 & 1.3255 & 0.5430 & -0.0441 & -0.0337 & -0.0128 \\
\hline
\end{tabular}




\begin{tabular}{|c|c|c|c|c|c|c|c|c|c|c|}
\hline 0.8480 & 1.1910 & 1.2902 & 1.3201 & 1.3448 & 1.3301 & 1.3160 & 1.0025 & -0.0441 & -0.0562 & -0.0257 \\
\hline 0.8520 & 1.1979 & 1.2902 & 1.3201 & 1.3536 & 1.3483 & 1.3349 & 1.2532 & -0.0220 & -0.0449 & -0.0128 \\
\hline 0.8560 & 1.1840 & 1.2902 & 1.3365 & 1.3448 & 1.3483 & 1.3444 & 1.3158 & 0.0000 & -0.0112 & 0.0000 \\
\hline 0.8600 & 1.1840 & 1.2902 & 1.3365 & 1.3536 & 1.3757 & 1.3633 & 1.3054 & 0.0000 & -0.0112 & 0.0128 \\
\hline 0.8640 & 1.1910 & 1.2902 & 1.3365 & 1.3624 & 1.3757 & 1.3728 & 1.3158 & 0.0110 & -0.0112 & 0.0000 \\
\hline 0.8680 & 1.1840 & 1.2902 & 1.3365 & 1.3712 & 1.3757 & 1.3728 & 1.3367 & 0.0220 & 0.0000 & 0.0000 \\
\hline 0.8720 & 1.1840 & 1.2825 & 1.3447 & 1.3624 & 1.3848 & 1.3823 & 1.3367 & 0.0110 & 0.0112 & -0.0128 \\
\hline 0.8760 & 1.1840 & 1.2748 & 1.3447 & 1.3624 & 1.3848 & 1.3728 & 1.3367 & 0.0000 & 0.0000 & -0.0128 \\
\hline 0.8800 & 1.1910 & 1.2748 & 1.3447 & 1.3624 & 1.3757 & 1.3728 & 1.3471 & 0.0110 & -0.0112 & 0.0128 \\
\hline 0.8840 & 1.1840 & 1.2671 & 1.3365 & 1.3712 & 1.3757 & 1.3728 & 1.3576 & 0.0110 & 0.0112 & 0.0000 \\
\hline 0.8880 & 1.1840 & 1.2825 & 1.3447 & 1.3712 & 1.3848 & 1.3823 & 1.3680 & 0.0110 & 0.0000 & 0.0000 \\
\hline 0.8920 & 1.1771 & 1.2902 & 1.3365 & 1.3712 & 1.3757 & 1.3823 & 1.3576 & 0.0110 & 0.0000 & 0.0128 \\
\hline 0.8960 & 1.1771 & 1.2902 & 1.3365 & 1.3624 & 1.3848 & 1.3823 & 1.3576 & 0.0220 & -0.0112 & -0.0128 \\
\hline 0.9000 & 1.1910 & 1.2902 & 1.3365 & 1.3712 & 1.3939 & 1.3823 & 1.3680 & 0.0110 & 0.0112 & 0.0000 \\
\hline 0.9040 & 1.1910 & 1.2748 & 1.3365 & 1.3712 & 1.4030 & 1.3823 & 1.3785 & 0.0110 & 0.0000 & 0.0000 \\
\hline 0.9080 & 1.1771 & 1.2902 & 1.3447 & 1.3799 & 1.3848 & 1.3823 & 1.3785 & 0.0110 & 0.0000 & 0.0128 \\
\hline 0.9120 & 1.1702 & 1.2902 & 1.3447 & 1.3712 & 1.4030 & 1.3823 & 1.3785 & 0.0000 & 0.0112 & 0.0000 \\
\hline 0.9160 & 1.1840 & 1.2748 & 1.3447 & 1.3799 & 1.3939 & 1.4012 & 1.3785 & 0.0000 & 0.0000 & 0.0128 \\
\hline 0.9200 & 1.1840 & 1.2902 & 1.3365 & 1.3712 & 1.3848 & 1.3917 & 1.3785 & 0.0110 & 0.0112 & 0.0000 \\
\hline 0.9240 & 1.1771 & 1.2825 & 1.3283 & 1.3712 & 1.3939 & 1.3917 & 1.3785 & 0.0000 & 0.0112 & -0.0128 \\
\hline 0.9280 & 1.1771 & 1.2902 & 1.3283 & 1.3712 & 1.4030 & 1.3917 & 1.3785 & 0.0220 & 0.0000 & 0.0000 \\
\hline 0.9320 & 1.1702 & 1.2825 & 1.3365 & 1.3799 & 1.4030 & 1.4012 & 1.3785 & 0.0110 & 0.0112 & 0.0129 \\
\hline 0.9360 & 1.1702 & 1.2902 & 1.3201 & 1.3624 & 1.4030 & 1.4107 & 1.3889 & 0.0110 & 0.0112 & 0.0128 \\
\hline 0.9400 & 1.1702 & 1.2902 & 1.3283 & 1.3712 & 1.4030 & 1.4012 & 1.3889 & 0.0110 & 0.0000 & 0.0128 \\
\hline 0.9440 & 1.1702 & 1.2825 & 1.3365 & 1.3624 & 1.4121 & 1.3917 & 1.3993 & 0.0220 & 0.0000 & 0.0000 \\
\hline 0.9480 & 1.1702 & 1.2748 & 1.3283 & 1.3887 & 1.4030 & 1.3823 & 1.3993 & 0.0110 & 0.0000 & 0.0128 \\
\hline 0.9520 & 1.1702 & 1.2748 & 1.3283 & 1.3712 & 1.3848 & 1.3917 & 1.3993 & 0.0000 & -0.0112 & 0.0128 \\
\hline 0.9560 & 1.1563 & 1.2825 & 1.3283 & 1.3712 & 1.3939 & 1.3917 & 1.3993 & 0.0110 & 0.0112 & 0.0000 \\
\hline 0.9600 & 1.1563 & 1.2671 & 1.3283 & 1.3624 & 1.4030 & 1.3917 & 1.3993 & 0.0110 & 0.0000 & 0.0000 \\
\hline 0.9640 & 1.1702 & 1.2671 & 1.3365 & 1.3624 & 1.4030 & 1.4107 & 1.4098 & 0.0110 & 0.0000 & 0.0000 \\
\hline 0.9680 & 1.1702 & 1.2748 & 1.3201 & 1.3712 & 1.4121 & 1.3917 & 1.3993 & 0.1102 & 0.0112 & 0.0000 \\
\hline 0.9720 & 1.1563 & 1.2825 & 1.3365 & 1.3624 & 1.3939 & 1.3823 & 1.3785 & 0.5180 & -0.0112 & -0.0128 \\
\hline 0.9760 & 1.1563 & 1.2748 & 1.3283 & 1.3624 & 1.3757 & 1.3728 & 1.3785 & 1.0468 & -0.0449 & -0.0257 \\
\hline 0.9800 & 1.1563 & 1.2671 & 1.3119 & 1.3624 & 1.3848 & 1.3823 & 1.3785 & 1.3002 & -0.0449 & -0.0385 \\
\hline 0.9840 & 1.1563 & 1.2671 & 1.3119 & 1.3624 & 1.3848 & 1.4012 & 1.3889 & 1.3443 & -0.0225 & -0.0257 \\
\hline 0.9880 & 1.1494 & 1.2748 & 1.3283 & 1.3624 & 1.3848 & 1.4107 & 1.4098 & 1.3443 & 0.0000 & 0.0000 \\
\hline 0.9920 & 1.1494 & 1.2825 & 1.3283 & 1.3712 & 1.4121 & 1.4107 & 1.3993 & 1.3553 & 0.0000 & 0.0000 \\
\hline 0.9960 & 1.1563 & 1.2671 & 1.3283 & 1.3799 & 1.4030 & 1.4201 & 1.4098 & 1.3553 & -0.0112 & 0.0128 \\
\hline 1.0000 & 1.1563 & 1.2671 & 1.3283 & 1.3712 & 1.4121 & 1.4107 & 1.4098 & 1.3663 & 0.0000 & 0.0128 \\
\hline 1.0040 & 1.1563 & 1.2671 & 1.3283 & 1.3799 & 1.4030 & 1.4107 & 1.4098 & 1.3773 & 0.0000 & 0.0128 \\
\hline 1.0080 & 1.1494 & 1.2594 & 1.3283 & 1.3712 & 1.4121 & 1.4201 & 1.4307 & 1.3663 & 0.0000 & 0.0000 \\
\hline 1.0120 & 1.1494 & 1.2594 & 1.3201 & 1.3799 & 1.4030 & 1.4201 & 1.4411 & 1.3773 & 0.0112 & 0.0000 \\
\hline 1.0160 & 1.1425 & 1.2594 & 1.3283 & 1.3712 & 1.4030 & 1.4201 & 1.4307 & 1.3773 & 0.0000 & -0.0129 \\
\hline 1.0200 & 1.1356 & 1.2594 & 1.3365 & 1.3624 & 1.4121 & 1.4107 & 1.4307 & 1.3773 & 0.0112 & 0.0000 \\
\hline 1.0240 & 1.1494 & 1.2594 & 1.3283 & 1.3799 & 1.4121 & 1.4107 & 1.4307 & 1.3885 & 0.0112 & 0.0000 \\
\hline 1.0280 & 1.1356 & 1.2671 & 1.3283 & 1.3712 & 1.4121 & 1.4201 & 1.4307 & 1.3885 & -0.0112 & 0.0000 \\
\hline 1.0320 & 1.1425 & 1.2748 & 1.3119 & 1.3799 & 1.4212 & 1.4201 & 1.4411 & 1.4105 & 0.0000 & 0.0000 \\
\hline 1.0360 & 1.1356 & 1.2594 & 1.3119 & 1.3799 & 1.4121 & 1.4296 & 1.4411 & 1.4105 & 0.0000 & 0.0000 \\
\hline 1.0400 & 1.1286 & 1.2518 & 1.3201 & 1.3799 & 1.4212 & 1.4201 & 1.4411 & 1.4105 & -0.0112 & -0.0128 \\
\hline 1.0440 & 1.1356 & 1.2594 & 1.3201 & 1.3712 & 1.4121 & 1.4201 & 1.4411 & 1.3995 & 0.0000 & -0.0129 \\
\hline 1.0480 & 1.1356 & 1.2594 & 1.3201 & 1.3712 & 1.4121 & 1.4296 & 1.4516 & 1.4215 & 0.0112 & -0.0129 \\
\hline 1.0520 & 1.1425 & 1.2594 & 1.3119 & 1.3624 & 1.4212 & 1.4296 & 1.4411 & 1.4105 & 0.0000 & 0.0000 \\
\hline 1.0560 & 1.1425 & 1.2594 & 1.3201 & 1.3712 & 1.4121 & 1.4296 & 1.4516 & 1.4215 & -0.0112 & 0.0000 \\
\hline 1.0600 & 1.1356 & 1.2594 & 1.3119 & 1.3712 & 1.4121 & 1.4391 & 1.4516 & 1.4215 & 0.0000 & 0.0128 \\
\hline 1.0640 & 1.1425 & 1.2518 & 1.3119 & 1.3624 & 1.4121 & 1.4296 & 1.4516 & 1.4325 & -0.0112 & 0.0000 \\
\hline 1.0680 & 1.1286 & 1.2671 & 1.3201 & 1.3624 & 1.4212 & 1.4391 & 1.4516 & 1.4215 & 0.0112 & -0.0128 \\
\hline 1.0720 & 1.1356 & 1.2594 & 1.3037 & 1.3624 & 1.4121 & 1.4296 & 1.4516 & 1.4325 & 0.0000 & 0.0128 \\
\hline 1.0760 & 1.1356 & 1.2441 & 1.3037 & 1.3624 & 1.4121 & 1.4391 & 1.4516 & 1.4325 & 0.0000 & 0.0128 \\
\hline 1.0800 & 1.1217 & 1.2518 & 1.3201 & 1.3712 & 1.4030 & 1.4485 & 1.4307 & 1.4215 & 0.0000 & 0.0000 \\
\hline 1.0840 & 1.1217 & 1.2518 & 1.3119 & 1.3712 & 1.4121 & 1.4391 & 1.4307 & 1.4436 & 0.0000 & 0.0000 \\
\hline 1.0880 & 1.1286 & 1.2518 & 1.3119 & 1.3712 & 1.4121 & 1.4296 & 1.4411 & 1.4436 & -0.0112 & 0.0000 \\
\hline 1.0920 & 1.1079 & 1.2518 & 1.3201 & 1.3624 & 1.4121 & 1.4391 & 1.4620 & 1.4436 & 0.0112 & 0.0000 \\
\hline 1.0960 & 1.1148 & 1.2518 & 1.3119 & 1.3624 & 1.4212 & 1.4296 & 1.4516 & 1.4436 & -0.0112 & -0.0129 \\
\hline 1.1000 & 1.1217 & 1.2518 & 1.3119 & 1.3624 & 1.4121 & 1.4296 & 1.4411 & 1.4105 & 0.1236 & -0.0257 \\
\hline 1.1040 & 1.1148 & 1.2518 & 1.3037 & 1.3624 & 1.4121 & 1.4296 & 1.4202 & 1.4105 & 0.6065 & -0.0514 \\
\hline 1.1080 & 1.1148 & 1.2441 & 1.2955 & 1.3536 & 1.4030 & 1.4201 & 1.4202 & 1.4215 & 1.1344 & -0.0128 \\
\hline 1.1120 & 1.1079 & 1.2518 & 1.2955 & 1.3536 & 1.4030 & 1.4391 & 1.4307 & 1.4436 & 1.3366 & -0.0129 \\
\hline 1.1160 & 1.1079 & 1.2364 & 1.3037 & 1.3624 & 1.4121 & 1.4296 & 1.4620 & 1.4546 & 1.3703 & -0.0257 \\
\hline 1.1200 & 1.1148 & 1.2518 & 1.2955 & 1.3536 & 1.4121 & 1.4296 & 1.4724 & 1.4546 & 1.3591 & -0.0129 \\
\hline 1.1240 & 1.1079 & 1.2441 & 1.2955 & 1.3712 & 1.4121 & 1.4485 & 1.4724 & 1.4546 & 1.3815 & -0.0129 \\
\hline
\end{tabular}




\begin{tabular}{|c|c|c|c|c|c|c|c|c|c|c|}
\hline 1.1280 & 1.1009 & 1.2364 & 1.2955 & 1.3536 & 1.4121 & 1.4485 & 1.4724 & 1.4546 & 1.3815 & 0.0000 \\
\hline 1.1320 & 1.1009 & 1.2364 & 1.2955 & 1.3536 & 1.4121 & 1.4485 & 1.4724 & 1.4656 & 1.3928 & -0.0128 \\
\hline 1.1360 & 1.1079 & 1.2364 & 1.3037 & 1.3624 & 1.4121 & 1.4391 & 1.4724 & 1.4766 & 1.3928 & 0.0000 \\
\hline 1.1400 & 1.1009 & 1.2287 & 1.3037 & 1.3536 & 1.4030 & 1.4391 & 1.4724 & 1.4656 & 1.3815 & 0.0000 \\
\hline 1.1440 & 1.1009 & 1.2441 & 1.2955 & 1.3536 & 1.4030 & 1.4485 & 1.4829 & 1.4656 & 1.4152 & 0.0000 \\
\hline 1.1480 & 1.1009 & 1.2364 & 1.3037 & 1.3536 & 1.4121 & 1.4485 & 1.4724 & 1.4766 & 1.4377 & 0.0000 \\
\hline 1.1520 & 1.0940 & 1.2364 & 1.2873 & 1.3448 & 1.4121 & 1.4485 & 1.4620 & 1.4656 & 1.4265 & 0.0000 \\
\hline 1.1560 & 1.0871 & 1.2364 & 1.2873 & 1.3536 & 1.4212 & 1.4580 & 1.4724 & 1.4766 & 1.4377 & 0.0000 \\
\hline 1.1600 & 1.0940 & 1.2441 & 1.2955 & 1.3536 & 1.4212 & 1.4391 & 1.4724 & 1.4766 & 1.4265 & -0.0129 \\
\hline 1.1640 & 1.0940 & 1.2441 & 1.2955 & 1.3536 & 1.4212 & 1.4485 & 1.4724 & 1.4766 & 1.4489 & 0.0000 \\
\hline 1.1680 & 1.0940 & 1.2364 & 1.3037 & 1.3624 & 1.4121 & 1.4391 & 1.4829 & 1.4766 & 1.4377 & -0.0128 \\
\hline 1.1720 & 1.0871 & 1.2364 & 1.2955 & 1.3536 & 1.4030 & 1.4391 & 1.4829 & 1.4766 & 1.4489 & -0.0128 \\
\hline 1.1760 & 1.0871 & 1.2364 & 1.2955 & 1.3536 & 1.4121 & 1.4580 & 1.4829 & 1.4876 & 1.4489 & 0.0128 \\
\hline 1.1800 & 1.0940 & 1.2287 & 1.2955 & 1.3448 & 1.4030 & 1.4580 & 1.4829 & 1.4766 & 1.4489 & 0.0128 \\
\hline 1.1840 & 1.0940 & 1.2364 & 1.2791 & 1.3360 & 1.4030 & 1.4485 & 1.4829 & 1.4876 & 1.4602 & 0.0000 \\
\hline 1.1880 & 1.0802 & 1.2287 & 1.2955 & 1.3448 & 1.4121 & 1.4485 & 1.4829 & 1.4876 & 1.4602 & 0.0000 \\
\hline 1.1920 & 1.0871 & 1.2364 & 1.2873 & 1.3448 & 1.3939 & 1.4485 & 1.4933 & 1.4766 & 1.4602 & 0.0000 \\
\hline 1.1960 & 1.0802 & 1.2287 & 1.2873 & 1.3536 & 1.4121 & 1.4485 & 1.4724 & 1.4986 & 1.4602 & -0.0128 \\
\hline 1.2000 & 1.0871 & 1.2364 & 1.2873 & 1.3536 & 1.4030 & 1.4391 & 1.4829 & 1.5097 & 1.4714 & -0.0128 \\
\hline 1.2040 & 1.0802 & 1.2287 & 1.2955 & 1.3536 & 1.4121 & 1.4580 & 1.4829 & 1.4766 & 1.4714 & 0.0000 \\
\hline 1.2080 & 1.0732 & 1.2287 & 1.2873 & 1.3448 & 1.4121 & 1.4485 & 1.4829 & 1.4766 & 1.4826 & 0.0000 \\
\hline 1.2120 & 1.0663 & 1.2287 & 1.2955 & 1.3448 & 1.4121 & 1.4485 & 1.4933 & 1.4766 & 1.4826 & 0.0000 \\
\hline 1.2160 & 1.0663 & 1.2210 & 1.2791 & 1.3448 & 1.4030 & 1.4485 & 1.4933 & 1.4876 & 1.4714 & 0.0000 \\
\hline 1.2200 & 1.0594 & 1.2210 & 1.2791 & 1.3360 & 1.3939 & 1.4485 & 1.4933 & 1.4876 & 1.4826 & -0.0129 \\
\hline 1.2240 & 1.0663 & 1.2210 & 1.2873 & 1.3448 & 1.4030 & 1.4485 & 1.4933 & 1.4876 & 1.4939 & 0.0385 \\
\hline 1.2280 & 1.0663 & 1.2287 & 1.2791 & 1.3536 & 1.3939 & 1.4391 & 1.4933 & 1.4766 & 1.4714 & 0.3340 \\
\hline 1.2320 & 1.0663 & 1.2210 & 1.2791 & 1.3360 & 1.3939 & 1.4485 & 1.4829 & 1.4876 & 1.4714 & 0.9122 \\
\hline 1.2360 & 1.0594 & 1.2210 & 1.2791 & 1.3360 & 1.3939 & 1.4201 & 1.4829 & 1.4876 & 1.4602 & 1.2976 \\
\hline 1.2400 & 1.0594 & 1.2287 & 1.2791 & 1.3448 & 1.3848 & 1.4485 & 1.4933 & 1.4986 & 1.4939 & 1.4004 \\
\hline 1.2440 & 1.0525 & 1.2134 & 1.2709 & 1.3360 & 1.3939 & 1.4485 & 1.4933 & 1.4986 & 1.4939 & 1.4133 \\
\hline 1.2480 & 1.0525 & 1.2210 & 1.2709 & 1.3360 & 1.3939 & 1.4391 & 1.4829 & 1.5317 & 1.4939 & 1.4004 \\
\hline 1.2520 & 1.0525 & 1.2057 & 1.2791 & 1.3272 & 1.4030 & 1.4485 & 1.4829 & 1.5097 & 1.5051 & 1.4518 \\
\hline 1.2560 & 1.0594 & 1.2134 & 1.2709 & 1.3360 & 1.3939 & 1.4485 & 1.4829 & 1.5207 & 1.5276 & 1.4261 \\
\hline 1.2600 & 1.0456 & 1.2134 & 1.2791 & 1.3360 & 1.3939 & 1.4485 & 1.4829 & 1.5097 & 1.5051 & 1.4261 \\
\hline 1.2640 & 1.0525 & 1.2057 & 1.2709 & 1.3360 & 1.3848 & 1.4391 & 1.4933 & 1.5097 & 1.5051 & 1.4647 \\
\hline 1.2680 & 1.0456 & 1.1980 & 1.2791 & 1.3360 & 1.3939 & 1.4485 & 1.5038 & 1.5207 & 1.5163 & 1.4647 \\
\hline 1.2720 & 1.0386 & 1.2057 & 1.2627 & 1.3360 & 1.3939 & 1.4485 & 1.5038 & 1.5207 & 1.5163 & 1.4775 \\
\hline 1.2760 & 1.0456 & 1.2057 & 1.2709 & 1.3272 & 1.3939 & 1.4580 & 1.4933 & 1.5207 & 1.5163 & 1.4904 \\
\hline 1.2800 & 1.0456 & 1.1980 & 1.2709 & 1.3272 & 1.3939 & 1.4296 & 1.5038 & 1.5207 & 1.5163 & 1.4775 \\
\hline 1.2840 & 1.0456 & 1.2134 & 1.2627 & 1.3184 & 1.3848 & 1.4485 & 1.5038 & 1.5207 & 1.5276 & 1.4904 \\
\hline 1.2880 & 1.0386 & 1.2057 & 1.2709 & 1.3272 & 1.4030 & 1.4391 & 1.4933 & 1.5207 & 1.5276 & 1.4904 \\
\hline 1.2920 & 1.0386 & 1.2057 & 1.2627 & 1.3272 & 1.3848 & 1.4485 & 1.4724 & 1.5317 & 1.5388 & 1.4904 \\
\hline 1.2960 & 1.0525 & 1.2134 & 1.2627 & 1.3184 & 1.3939 & 1.4391 & 1.4829 & 1.5207 & 1.5276 & 1.4904 \\
\hline 1.3000 & 1.0456 & 1.1980 & 1.2627 & 1.3184 & 1.3757 & 1.4391 & 1.4933 & 1.5207 & 1.5276 & 1.5032 \\
\hline 1.3040 & 1.0386 & 1.1980 & 1.2627 & 1.3184 & 1.3848 & 1.4391 & 1.4933 & 1.5317 & 1.5388 & 1.5032 \\
\hline 1.3080 & 1.0248 & 1.1980 & 1.2463 & 1.3272 & 1.3757 & 1.4391 & 1.4933 & 1.5317 & 1.5388 & 1.5289 \\
\hline 1.3120 & 1.0317 & 1.1980 & 1.2627 & 1.3184 & 1.3757 & 1.4485 & 1.4933 & 1.5317 & 1.5388 & 1.5161 \\
\hline 1.3160 & 1.0317 & 1.2057 & 1.2627 & 1.3272 & 1.3848 & 1.4485 & 1.4933 & 1.5317 & 1.5388 & 1.5032 \\
\hline 1.3200 & 1.0317 & 1.2134 & 1.2627 & 1.3272 & 1.3939 & 1.4391 & 1.5038 & 1.5317 & 1.5500 & 1.5289 \\
\hline 1.3240 & 1.0179 & 1.1980 & 1.2545 & 1.3096 & 1.3757 & 1.4296 & 1.4933 & 1.5427 & 1.5500 & 1.5289 \\
\hline 1.3280 & 1.0248 & 1.1980 & 1.2545 & 1.3184 & 1.3757 & 1.4296 & 1.4933 & 1.5317 & 1.5276 & 1.5289 \\
\hline 1.3320 & 1.0248 & 1.1826 & 1.2463 & 1.3096 & 1.3757 & 1.4296 & 1.4933 & 1.5317 & 1.5276 & 1.5546 \\
\hline 1.3360 & 1.0248 & 1.1903 & 1.2463 & 1.3096 & 1.3757 & 1.4296 & 1.5038 & 1.5317 & 1.5388 & 1.5418 \\
\hline 1.3400 & 1.0179 & 1.1903 & 1.2545 & 1.3184 & 1.3666 & 1.4391 & 1.4933 & 1.5317 & 1.5388 & 1.5418 \\
\hline 1.3440 & 1.0179 & 1.1903 & 1.2463 & 1.3184 & 1.3757 & 1.4201 & 1.4933 & 1.5207 & 1.5276 & 1.5418 \\
\hline 1.3480 & 1.0179 & 1.1903 & 1.2545 & 1.3096 & 1.3757 & 1.4391 & 1.4829 & 1.5427 & 1.5276 & 1.5546 \\
\hline 1.3520 & 1.0040 & 1.1980 & 1.2545 & 1.3008 & 1.3757 & 1.4201 & 1.4933 & 1.5427 & 1.5500 & 1.5418 \\
\hline 1.3560 & 1.0317 & 1.1903 & 1.2545 & 1.3184 & 1.3757 & 1.4201 & 1.4829 & 1.5427 & 1.5500 & 1.5418 \\
\hline 1.3600 & 1.0109 & 1.1826 & 1.2463 & 1.3096 & 1.3757 & 1.4201 & 1.4933 & 1.5427 & 1.5500 & 1.5546 \\
\hline 1.3640 & 1.0040 & 1.1826 & 1.2463 & 1.3008 & 1.3757 & 1.4296 & 1.5038 & 1.5317 & 1.5276 & 1.5418 \\
\hline 1.3680 & 1.0040 & 1.1903 & 1.2381 & 1.3096 & 1.3574 & 1.4296 & 1.4933 & 1.5427 & 1.5500 & 1.5675 \\
\hline 1.3720 & 0.9832 & 1.1903 & 1.2381 & 1.2920 & 1.3666 & 1.4296 & 1.4724 & 1.5207 & 1.5612 & 1.5803 \\
\hline 1.3760 & 0.9902 & 1.1903 & 1.2299 & 1.2920 & 1.3666 & 1.4201 & 1.4829 & 1.5427 & 1.5500 & 1.5675 \\
\hline 1.3800 & 0.9832 & 1.1903 & 1.2381 & 1.3008 & 1.3666 & 1.4296 & 1.4933 & 1.5207 & 1.5500 & 1.5675 \\
\hline 1.3840 & 0.9832 & 1.1826 & 1.2299 & 1.3008 & 1.3666 & 1.4107 & 1.4933 & 1.5207 & 1.5612 & 1.5675 \\
\hline 1.3880 & 0.9832 & 1.1826 & 1.2299 & 1.2920 & 1.3666 & 1.4201 & 1.4829 & 1.5317 & 1.5725 & 1.5675 \\
\hline 1.3920 & 0.9763 & 1.1903 & 1.2299 & 1.2920 & 1.3666 & 1.4296 & 1.4829 & 1.5317 & 1.5725 & 1.5675 \\
\hline 1.3960 & 0.9763 & 1.1826 & 1.2299 & 1.3008 & 1.3666 & 1.4296 & 1.4829 & 1.5427 & 1.5500 & 1.5803 \\
\hline 1.4000 & 0.9625 & 1.1826 & 1.2299 & 1.2920 & 1.3666 & 1.4201 & 1.4829 & 1.5317 & 1.5612 & 1.5932 \\
\hline 1.4040 & 0.9625 & 1.1750 & 1.2299 & 1.2920 & 1.3574 & 1.4201 & 1.4829 & 1.5427 & 1.5725 & 1.5803 \\
\hline
\end{tabular}




\begin{tabular}{|c|c|c|c|c|c|c|c|c|c|c|}
\hline 1.4080 & 0.9555 & 1.1673 & 1.2299 & 1.2920 & 1.3574 & 1.4201 & 1.4829 & 1.5207 & 1.5612 & 1.5675 \\
\hline 1.4120 & 0.9486 & 1.1750 & 1.2299 & 1.2833 & 1.3574 & 1.4107 & 1.4829 & 1.5207 & 1.5725 & 1.5932 \\
\hline 1.4160 & 0.9486 & 1.1750 & 1.2299 & 1.2833 & 1.3574 & 1.4107 & 1.4829 & 1.5537 & 1.5725 & 1.5932 \\
\hline 1.4200 & 0.9486 & 1.1750 & 1.2381 & 1.2920 & 1.3574 & 1.4107 & 1.4724 & 1.5317 & 1.5612 & 1.5803 \\
\hline 1.4240 & 0.9486 & 1.1750 & 1.2299 & 1.2920 & 1.3483 & 1.4012 & 1.4829 & 1.5317 & 1.5612 & 1.5932 \\
\hline 1.4280 & 0.9417 & 1.1750 & 1.2217 & 1.2745 & 1.3574 & 1.4107 & 1.4724 & 1.5317 & 1.5837 & 1.5932 \\
\hline 1.4320 & 0.9417 & 1.1750 & 1.2299 & 1.2745 & 1.3483 & 1.4012 & 1.4829 & 1.5317 & 1.5837 & 1.5932 \\
\hline 1.4360 & 0.9279 & 1.1750 & 1.2299 & 1.2833 & 1.3483 & 1.4107 & 1.4724 & 1.5317 & 1.5837 & 1.5932 \\
\hline 1.4400 & 0.9348 & 1.1673 & 1.2299 & 1.2833 & 1.3574 & 1.4107 & 1.4829 & 1.5427 & 1.5725 & 1.6060 \\
\hline 1.4440 & 0.9209 & 1.1673 & 1.2299 & 1.2833 & 1.3392 & 1.4107 & 1.4724 & 1.5317 & 1.5725 & 1.6060 \\
\hline 1.4480 & 0.9209 & 1.1596 & 1.2135 & 1.2745 & 1.3483 & 1.4012 & 1.4724 & 1.5207 & 1.5725 & 1.5803 \\
\hline 1.4520 & 0.9071 & 1.1673 & 1.2135 & 1.2833 & 1.3392 & 1.4012 & 1.4724 & 1.5317 & 1.5725 & 1.6060 \\
\hline 1.4560 & 0.9209 & 1.1596 & 1.2135 & 1.2745 & 1.3392 & 1.4012 & 1.4724 & 1.5207 & 1.5612 & 1.6188 \\
\hline 1.4600 & 0.9140 & 1.1673 & 1.2299 & 1.2745 & 1.3392 & 1.4107 & 1.4724 & 1.5317 & 1.5725 & 1.5803 \\
\hline 1.4640 & 0.9140 & 1.1673 & 1.2053 & 1.2745 & 1.3392 & 1.3917 & 1.4620 & 1.5207 & 1.5837 & 1.5803 \\
\hline 1.4680 & 0.9140 & 1.1596 & 1.2135 & 1.2657 & 1.3392 & 1.3917 & 1.4724 & 1.5097 & 1.5725 & 1.5932 \\
\hline 1.4720 & 0.9140 & 1.1673 & 1.2135 & 1.2833 & 1.3392 & 1.3917 & 1.4620 & 1.5317 & 1.5725 & 1.6060 \\
\hline 1.4760 & 0.9140 & 1.1596 & 1.2135 & 1.2745 & 1.3301 & 1.4012 & 1.4829 & 1.5207 & 1.5949 & 1.6060 \\
\hline 1.4800 & 0.9071 & 1.1596 & 1.2135 & 1.2745 & 1.3301 & 1.3917 & 1.4724 & 1.5207 & 1.5837 & 1.5932 \\
\hline 1.4840 & 0.9140 & 1.1596 & 1.2135 & 1.2745 & 1.3392 & 1.3917 & 1.4620 & 1.5317 & 1.5837 & 1.6188 \\
\hline 1.4880 & 0.9209 & 1.1673 & 1.2135 & 1.2657 & 1.3210 & 1.3917 & 1.4724 & 1.5207 & 1.5725 & 1.6188 \\
\hline 1.4920 & 0.9209 & 1.1596 & 1.2053 & 1.2657 & 1.3210 & 1.3917 & 1.4516 & 1.5207 & 1.5612 & 1.6060 \\
\hline 1.4960 & 0.9140 & 1.1519 & 1.2053 & 1.2569 & 1.3210 & 1.3823 & 1.4620 & 1.5317 & 1.5837 & 1.5932 \\
\hline 1.5000 & 0.9209 & 1.1596 & 1.2053 & 1.2569 & 1.3301 & 1.3917 & 1.4620 & 1.5207 & 1.5725 & 1.6060 \\
\hline 1.5040 & 0.9209 & 1.1596 & 1.2053 & 1.2569 & 1.3210 & 1.3917 & 1.4620 & 1.5207 & 1.5725 & 1.6060 \\
\hline 1.5080 & 0.9140 & 1.1596 & 1.1971 & 1.2569 & 1.3210 & 1.3823 & 1.4516 & 1.5097 & 1.5725 & 1.6188 \\
\hline 1.5120 & 0.9140 & 1.1673 & 1.1971 & 1.2569 & 1.3210 & 1.3728 & 1.4516 & 1.5097 & 1.5837 & 1.6317 \\
\hline 1.5160 & 0.9071 & 1.1519 & 1.1971 & 1.2481 & 1.3119 & 1.3823 & 1.4411 & 1.4986 & 1.5725 & 1.6317 \\
\hline 1.5200 & 0.9071 & 1.1596 & 1.1971 & 1.2481 & 1.3119 & 1.3728 & 1.4516 & 1.5207 & 1.5725 & 1.6060 \\
\hline 1.5240 & 0.9279 & 1.1519 & 1.1971 & 1.2569 & 1.3210 & 1.3728 & 1.4411 & 1.5097 & 1.5725 & 1.6060 \\
\hline 1.5280 & 0.9209 & 1.1596 & 1.1889 & 1.2481 & 1.3028 & 1.3728 & 1.4516 & 1.5207 & 1.5612 & 1.6188 \\
\hline 1.5320 & 0.9140 & 1.1596 & 1.1971 & 1.2481 & 1.3119 & 1.3728 & 1.4411 & 1.5097 & 1.5612 & 1.6317 \\
\hline 1.5360 & 0.9209 & 1.1442 & 1.1971 & 1.2393 & 1.3119 & 1.3728 & 1.4411 & 1.5207 & 1.5612 & 1.6060 \\
\hline 1.5400 & 0.9209 & 1.1442 & 1.1889 & 1.2393 & 1.3119 & 1.3633 & 1.4411 & 1.5097 & 1.5725 & 1.6060 \\
\hline 1.5440 & 0.9209 & 1.1519 & 1.1889 & 1.2481 & 1.3119 & 1.3633 & 1.4307 & 1.5097 & 1.5612 & 1.6060 \\
\hline 1.5480 & 0.9140 & 1.1442 & 1.1889 & 1.2569 & 1.3119 & 1.3728 & 1.4411 & 1.5097 & 1.5612 & 1.6317 \\
\hline 1.5520 & 0.9071 & 1.1366 & 1.1889 & 1.2393 & 1.3119 & 1.3633 & 1.4411 & 1.5097 & 1.5612 & 1.6317 \\
\hline 1.5560 & 0.8932 & 1.1519 & 1.1971 & 1.2481 & 1.3119 & 1.3633 & 1.4202 & 1.4876 & 1.5725 & 1.6188 \\
\hline 1.5600 & 0.8863 & 1.1442 & 1.1807 & 1.2393 & 1.3028 & 1.3728 & 1.4307 & 1.5097 & 1.5612 & 1.6188 \\
\hline 1.5640 & 0.8794 & 1.1442 & 1.1807 & 1.2393 & 1.3028 & 1.3633 & 1.4307 & 1.4986 & 1.5612 & 1.6188 \\
\hline 1.5680 & 0.8863 & 1.1442 & 1.1807 & 1.2393 & 1.3028 & 1.3539 & 1.4202 & 1.4986 & 1.5500 & 1.6317 \\
\hline 1.5720 & 0.8725 & 1.1289 & 1.1807 & 1.2393 & 1.3028 & 1.3539 & 1.4202 & 1.4986 & 1.5612 & 1.6317 \\
\hline 1.5760 & 0.8725 & 1.1442 & 1.1807 & 1.2393 & 1.2937 & 1.3633 & 1.4202 & 1.4876 & 1.5500 & 1.6188 \\
\hline 1.5800 & 0.8586 & 1.1442 & 1.1807 & 1.2393 & 1.3028 & 1.3539 & 1.4202 & 1.4876 & 1.5388 & 1.6188 \\
\hline 1.5840 & 0.8655 & 1.1366 & 1.1807 & 1.2305 & 1.2937 & 1.3444 & 1.4098 & 1.4876 & 1.5388 & 1.6188 \\
\hline 1.5880 & 0.8517 & 1.1366 & 1.1807 & 1.2217 & 1.2937 & 1.3444 & 1.4098 & 1.4986 & 1.5388 & 1.6188 \\
\hline 1.5920 & 0.8586 & 1.1366 & 1.1807 & 1.2217 & 1.2755 & 1.3444 & 1.4098 & 1.4876 & 1.5500 & 1.6188 \\
\hline 1.5960 & 0.8517 & 1.1289 & 1.1807 & 1.2217 & 1.2846 & 1.3444 & 1.4202 & 1.4986 & 1.5500 & 1.6317 \\
\hline 1.6000 & 0.8586 & 1.1442 & 1.1807 & 1.2129 & 1.2846 & 1.3349 & 1.4098 & 1.4766 & 1.5388 & 1.6188 \\
\hline 1.6040 & 0.8586 & 1.1289 & 1.1725 & 1.2305 & 1.2937 & 1.3539 & 1.4098 & 1.4876 & 1.5388 & 1.6188 \\
\hline 1.6080 & 0.8586 & 1.1289 & 1.1643 & 1.2217 & 1.2755 & 1.3444 & 1.4202 & 1.4766 & 1.5388 & 1.6060 \\
\hline 1.6120 & 0.8586 & 1.1289 & 1.1725 & 1.2217 & 1.2846 & 1.3160 & 1.4098 & 1.4766 & 1.5388 & 1.6060 \\
\hline 1.6160 & 0.8517 & 1.1289 & 1.1561 & 1.2217 & 1.2755 & 1.3349 & 1.3993 & 1.4876 & 1.5276 & 1.6060 \\
\hline 1.6200 & 0.8517 & 1.1212 & 1.1643 & 1.2217 & 1.3028 & 1.3444 & 1.3993 & 1.4876 & 1.5500 & 1.5932 \\
\hline 1.6240 & 0.8517 & 1.1366 & 1.1725 & 1.2217 & 1.2846 & 1.3444 & 1.4098 & 1.4876 & 1.5388 & 1.6060 \\
\hline 1.6280 & 0.8517 & 1.1289 & 1.1725 & 1.2217 & 1.2846 & 1.3349 & 1.4098 & 1.4656 & 1.5388 & 1.6060 \\
\hline 1.6320 & 0.8448 & 1.1289 & 1.1725 & 1.2129 & 1.2572 & 1.3255 & 1.3889 & 1.4876 & 1.5276 & 1.6060 \\
\hline 1.6360 & 0.8448 & 1.1289 & 1.1643 & 1.2129 & 1.2663 & 1.3349 & 1.3889 & 1.4766 & 1.5388 & 1.6060 \\
\hline 1.6400 & 0.8517 & 1.1289 & 1.1643 & 1.2129 & 1.2663 & 1.3255 & 1.3993 & 1.4656 & 1.5388 & 1.6060 \\
\hline 1.6440 & 0.8448 & 1.1212 & 1.1643 & 1.2217 & 1.2663 & 1.3255 & 1.3889 & 1.4656 & 1.5388 & 1.5803 \\
\hline 1.6480 & 0.8378 & 1.1212 & 1.1561 & 1.2041 & 1.2481 & 1.3160 & 1.3889 & 1.4546 & 1.5276 & 1.6060 \\
\hline 1.6520 & 0.8309 & 1.1212 & 1.1561 & 1.1954 & 1.2663 & 1.3160 & 1.3889 & 1.4546 & 1.5163 & 1.5932 \\
\hline 1.6560 & 0.8378 & 1.1289 & 1.1561 & 1.1954 & 1.2572 & 1.3255 & 1.3889 & 1.4766 & 1.5051 & 1.5932 \\
\hline 1.6600 & 0.8309 & 1.1212 & 1.1479 & 1.1954 & 1.2663 & 1.3160 & 1.3785 & 1.4546 & 1.5051 & 1.5675 \\
\hline 1.6640 & 0.8378 & 1.1289 & 1.1479 & 1.2041 & 1.2481 & 1.3160 & 1.3785 & 1.4546 & 1.5163 & 1.6060 \\
\hline 1.6680 & 0.8378 & 1.1212 & 1.1561 & 1.1866 & 1.2572 & 1.3065 & 1.3785 & 1.4325 & 1.5163 & 1.5932 \\
\hline 1.6720 & 0.8517 & 1.1212 & 1.1561 & 1.1954 & 1.2572 & 1.3065 & 1.3785 & 1.4546 & 1.5163 & 1.5932 \\
\hline 1.6760 & 0.8517 & 1.1212 & 1.1479 & 1.2041 & 1.2390 & 1.3065 & 1.3785 & 1.4546 & 1.5051 & 1.5803 \\
\hline 1.6800 & 0.8517 & 1.1212 & 1.1479 & 1.1954 & 1.2572 & 1.2971 & 1.3680 & 1.4436 & 1.5051 & 1.5932 \\
\hline 1.6840 & 0.8586 & 1.1135 & 1.1561 & 1.1866 & 1.2572 & 1.2971 & 1.3785 & 1.4325 & 1.4939 & 1.5803 \\
\hline
\end{tabular}




\begin{tabular}{|c|c|c|c|c|c|c|c|c|c|c|}
\hline 1.6880 & 0.8517 & 1.1212 & 1.1479 & 1.1778 & 1.2481 & 1.2971 & 1.3785 & 1.4546 & 1.5051 & 1.5803 \\
\hline 1.6920 & 0.8517 & 1.1058 & 1.1643 & 1.1866 & 1.2572 & 1.3065 & 1.3785 & 1.4325 & 1.5051 & 1.5675 \\
\hline 1.6960 & 0.8586 & 1.1135 & 1.1479 & 1.1778 & 1.2572 & 1.2971 & 1.3680 & 1.4436 & 1.4939 & 1.5546 \\
\hline 1.7000 & 0.8517 & 1.1135 & 1.1479 & 1.1954 & 1.2481 & 1.2971 & 1.3785 & 1.4325 & 1.4939 & 1.5803 \\
\hline 1.7040 & 0.8448 & 1.1135 & 1.1479 & 1.1778 & 1.2481 & 1.2971 & 1.3576 & 1.4215 & 1.4939 & 1.5418 \\
\hline 1.7080 & 0.8448 & 1.1135 & 1.1479 & 1.1866 & 1.2390 & 1.2876 & 1.3471 & 1.4436 & 1.4939 & 1.5546 \\
\hline 1.7120 & 0.8448 & 1.1135 & 1.1479 & 1.1778 & 1.2481 & 1.2876 & 1.3471 & 1.4215 & 1.4826 & 1.5675 \\
\hline 1.7160 & 0.8378 & 1.1058 & 1.1479 & 1.1690 & 1.2390 & 1.2876 & 1.3576 & 1.4215 & 1.4939 & 1.5546 \\
\hline 1.7200 & 0.8448 & 1.1135 & 1.1397 & 1.1778 & 1.2299 & 1.2876 & 1.3576 & 1.4215 & 1.4826 & 1.5546 \\
\hline 1.7240 & 0.8448 & 1.1135 & 1.1397 & 1.1602 & 1.2299 & 1.2971 & 1.3471 & 1.4325 & 1.4714 & 1.5546 \\
\hline 1.7280 & 0.8378 & 1.1135 & 1.1397 & 1.1778 & 1.2208 & 1.2781 & 1.3471 & 1.4215 & 1.4714 & 1.5418 \\
\hline 1.7320 & 0.8448 & 1.1135 & 1.1397 & 1.1602 & 1.2390 & 1.2876 & 1.3576 & 1.4105 & 1.4714 & 1.5546 \\
\hline 1.7360 & 0.8378 & 1.1212 & 1.1397 & 1.1690 & 1.2208 & 1.2781 & 1.3471 & 1.4105 & 1.4826 & 1.5546 \\
\hline 1.7400 & 0.8309 & 1.0982 & 1.1397 & 1.1778 & 1.2299 & 1.2687 & 1.3262 & 1.4215 & 1.4714 & 1.5418 \\
\hline 1.7440 & 0.8378 & 1.0982 & 1.1397 & 1.1778 & 1.2299 & 1.2781 & 1.3471 & 1.3994 & 1.4714 & 1.5418 \\
\hline 1.7480 & 0.8378 & 1.1058 & 1.1397 & 1.1690 & 1.2299 & 1.2781 & 1.3367 & 1.4105 & 1.4714 & 1.5546 \\
\hline 1.7520 & 0.8309 & 1.1058 & 1.1397 & 1.1690 & 1.2299 & 1.2876 & 1.3367 & 1.4215 & 1.4714 & 1.5161 \\
\hline 1.7560 & 0.8309 & 1.1058 & 1.1233 & 1.1602 & 1.2299 & 1.2687 & 1.3367 & 1.3994 & 1.4826 & 1.5418 \\
\hline 1.7600 & 0.8378 & 1.1135 & 1.1315 & 1.1602 & 1.2299 & 1.2687 & 1.3471 & 1.4215 & 1.4602 & 1.5418 \\
\hline 1.7640 & 0.8309 & 1.0982 & 1.1315 & 1.1690 & 1.2117 & 1.2592 & 1.3367 & 1.4105 & 1.4602 & 1.5418 \\
\hline 1.7680 & 0.8448 & 1.0982 & 1.1315 & 1.1602 & 1.2208 & 1.2687 & 1.3367 & 1.4105 & 1.4602 & 1.5289 \\
\hline 1.7720 & 0.8309 & 1.1058 & 1.1397 & 1.1602 & 1.2299 & 1.2781 & 1.3471 & 1.3994 & 1.4602 & 1.5418 \\
\hline 1.7760 & 0.8378 & 1.0982 & 1.1233 & 1.1602 & 1.2026 & 1.2592 & 1.3367 & 1.4104 & 1.4602 & 1.5289 \\
\hline 1.7800 & 0.8309 & 1.1058 & 1.1233 & 1.1690 & 1.2208 & 1.2687 & 1.3367 & 1.3883 & 1.4489 & 1.5161 \\
\hline 1.7840 & 0.8378 & 1.1135 & 1.1233 & 1.1602 & 1.2026 & 1.2687 & 1.3262 & 1.3883 & 1.4377 & 1.5161 \\
\hline 1.7880 & 0.8309 & 1.1058 & 1.1233 & 1.1602 & 1.2117 & 1.2592 & 1.3262 & 1.3773 & 1.4377 & 1.5289 \\
\hline 1.7920 & 0.8309 & 1.0982 & 1.1233 & 1.1602 & 1.2117 & 1.2497 & 1.3158 & 1.3663 & 1.4489 & 1.5161 \\
\hline 1.7960 & 0.8309 & 1.0982 & 1.1151 & 1.1514 & 1.2026 & 1.2687 & 1.3262 & 1.3883 & 1.4377 & 1.5161 \\
\hline 1.8000 & 0.8378 & 1.0905 & 1.1233 & 1.1514 & 1.2026 & 1.2497 & 1.3158 & 1.3663 & 1.4377 & 1.5032 \\
\hline 1.8040 & 0.8309 & 1.0982 & 1.1151 & 1.1426 & 1.1935 & 1.2497 & 1.3158 & 1.3773 & 1.4265 & 1.5161 \\
\hline 1.8080 & 0.8378 & 1.0905 & 1.1233 & 1.1514 & 1.1935 & 1.2497 & 1.3054 & 1.3773 & 1.4377 & 1.5161 \\
\hline 1.8120 & 0.8171 & 1.0982 & 1.1151 & 1.1426 & 1.2026 & 1.2403 & 1.3054 & 1.3663 & 1.4377 & 1.5032 \\
\hline 1.8160 & 0.8171 & 1.0982 & 1.1151 & 1.1426 & 1.1935 & 1.2403 & 1.3158 & 1.3773 & 1.4377 & 1.5032 \\
\hline 1.8200 & 0.8240 & 1.0905 & 1.1151 & 1.1514 & 1.1935 & 1.2497 & 1.3054 & 1.3553 & 1.4489 & 1.5032 \\
\hline 1.8240 & 0.8309 & 1.0982 & 1.1069 & 1.1514 & 1.1935 & 1.2403 & 1.3054 & 1.3553 & 1.4377 & 1.5032 \\
\hline 1.8280 & 0.8309 & 1.0982 & 1.1151 & 1.1602 & 1.2026 & 1.2497 & 1.3054 & 1.3553 & 1.4152 & 1.5032 \\
\hline 1.8320 & 0.8378 & 1.0982 & 1.1151 & 1.1426 & 1.2026 & 1.2403 & 1.3158 & 1.3443 & 1.4265 & 1.4647 \\
\hline 1.8360 & 0.8309 & 1.0905 & 1.1233 & 1.1338 & 1.1935 & 1.2308 & 1.3158 & 1.3663 & 1.4265 & 1.4775 \\
\hline 1.8400 & 0.8378 & 1.0905 & 1.1069 & 1.1426 & 1.2026 & 1.2403 & 1.2845 & 1.3553 & 1.4152 & 1.4904 \\
\hline 1.8440 & 0.8378 & 1.0905 & 1.1069 & 1.1426 & 1.1752 & 1.2308 & 1.2740 & 1.3443 & 1.4040 & 1.4775 \\
\hline 1.8480 & 0.8378 & 1.0905 & 1.1151 & 1.1514 & 1.1844 & 1.2213 & 1.2845 & 1.3443 & 1.3928 & 1.4904 \\
\hline 1.8520 & 0.8448 & 1.1058 & 1.1069 & 1.1338 & 1.1844 & 1.2403 & 1.2845 & 1.3443 & 1.4040 & 1.4775 \\
\hline 1.8560 & 0.8378 & 1.1058 & 1.1069 & 1.1250 & 1.1752 & 1.2213 & 1.2845 & 1.3553 & 1.4040 & 1.4775 \\
\hline 1.8600 & 0.8447 & 1.0905 & 1.1069 & 1.1338 & 1.1752 & 1.2308 & 1.2845 & 1.3333 & 1.4040 & 1.4647 \\
\hline 1.8640 & 0.8585 & 1.0828 & 1.0987 & 1.1338 & 1.1752 & 1.2308 & 1.2845 & 1.3443 & 1.4040 & 1.4647 \\
\hline 1.8680 & 0.8585 & 1.0828 & 1.1069 & 1.1338 & 1.1844 & 1.2213 & 1.2740 & 1.3333 & 1.4040 & 1.4775 \\
\hline 1.8720 & 0.8585 & 1.0828 & 1.1069 & 1.1338 & 1.1752 & 1.2119 & 1.2740 & 1.3443 & 1.4040 & 1.4647 \\
\hline 1.8760 & 0.8585 & 1.0905 & 1.1151 & 1.1250 & 1.1752 & 1.2119 & 1.2845 & 1.3333 & 1.4040 & 1.4518 \\
\hline 1.8800 & 0.8655 & 1.0828 & 1.1151 & 1.1250 & 1.1844 & 1.2119 & 1.2845 & 1.3222 & 1.4040 & 1.4518 \\
\hline 1.8840 & 0.8655 & 1.0828 & 1.1069 & 1.1162 & 1.1844 & 1.2213 & 1.2636 & 1.3222 & 1.3928 & 1.4518 \\
\hline 1.8880 & 0.8725 & 1.0905 & 1.0905 & 1.1250 & 1.1661 & 1.2213 & 1.2636 & 1.3333 & 1.3815 & 1.4647 \\
\hline 1.8920 & 0.8585 & 1.0751 & 1.0905 & 1.1162 & 1.1661 & 1.2213 & 1.2845 & 1.3333 & 1.3928 & 1.4647 \\
\hline 1.8960 & 0.8725 & 1.0905 & 1.0987 & 1.1162 & 1.1661 & 1.2119 & 1.2636 & 1.3333 & 1.3815 & 1.4390 \\
\hline 1.9000 & 0.8655 & 1.0674 & 1.0987 & 1.1162 & 1.1661 & 1.2024 & 1.2636 & 1.3222 & 1.3703 & 1.4390 \\
\hline 1.9040 & 0.8725 & 1.0828 & 1.0987 & 1.1162 & 1.1661 & 1.2024 & 1.2636 & 1.3222 & 1.3703 & 1.4390 \\
\hline 1.9080 & 0.8655 & 1.0828 & 1.0905 & 1.1250 & 1.1570 & 1.2024 & 1.2636 & 1.3112 & 1.3703 & 1.4390 \\
\hline 1.9120 & 0.8725 & 1.0828 & 1.0905 & 1.1075 & 1.1570 & 1.1929 & 1.2636 & 1.3112 & 1.3815 & 1.4261 \\
\hline 1.9160 & 0.8725 & 1.0828 & 1.0987 & 1.1075 & 1.1479 & 1.2024 & 1.2532 & 1.3002 & 1.3703 & 1.4390 \\
\hline 1.9200 & 0.8655 & 1.0751 & 1.0823 & 1.1075 & 1.1570 & 1.1929 & 1.2532 & 1.3112 & 1.3703 & 1.4261 \\
\hline 1.9240 & 0.8585 & 1.0828 & 1.0905 & 1.1162 & 1.1661 & 1.1929 & 1.2532 & 1.3112 & 1.3815 & 1.4133 \\
\hline 1.9280 & 0.8655 & 1.0751 & 1.0823 & 1.1075 & 1.1661 & 1.1929 & 1.2532 & 1.3112 & 1.3703 & 1.4261 \\
\hline 1.9320 & 0.8585 & 1.0751 & 1.0905 & 1.0987 & 1.1479 & 1.1929 & 1.2427 & 1.3002 & 1.3928 & 1.4261 \\
\hline 1.9360 & 0.8586 & 1.0751 & 1.0905 & 1.0987 & 1.1570 & 1.1929 & 1.2532 & 1.3222 & 1.3815 & 1.4261 \\
\hline 1.9400 & 0.8586 & 1.0751 & 1.0905 & 1.0987 & 1.1570 & 1.2024 & 1.2532 & 1.3002 & 1.3703 & 1.4261 \\
\hline 1.9440 & 0.8586 & 1.0751 & 1.0659 & 1.0811 & 1.1388 & 1.1740 & 1.2532 & 1.2782 & 1.3703 & 1.4004 \\
\hline 1.9480 & 0.8516 & 1.0674 & 1.0495 & 1.0723 & 1.1206 & 1.1740 & 1.2218 & 1.2892 & 1.3591 & 1.4004 \\
\hline 1.9520 & 0.8516 & 1.0751 & 1.0495 & 1.0723 & 1.1115 & 1.1645 & 1.2218 & 1.2782 & 1.3478 & 1.3876 \\
\hline 1.9560 & 0.8516 & 1.0598 & 1.0495 & 1.0723 & 1.1206 & 1.1740 & 1.2114 & 1.2782 & 1.3591 & 1.3876 \\
\hline 1.9600 & 0.8378 & 1.0674 & 1.0659 & 1.0723 & 1.1206 & 1.1740 & 1.2218 & 1.3112 & 1.3366 & 1.3876 \\
\hline 1.9640 & 0.8378 & 1.0751 & 1.0577 & 1.0811 & 1.1206 & 1.1645 & 1.2218 & 1.2892 & 1.3591 & 1.3876 \\
\hline
\end{tabular}




$\begin{array}{lllllllllll}1.9680 & 0.8378 & 1.0828 & 1.0577 & 1.0811 & 1.1206 & 1.1551 & 1.2218 & 1.2892 & 1.3478 & 1.4004 \\ 1.9720 & 0.8447 & 1.1519 & 1.1561 & 1.0547 & 1.1115 & 1.1456 & 1.2009 & 1.2892 & 1.3478 & 1.3876 \\ 1.9760 & 0.8309 & 1.3286 & 1.3447 & 1.0283 & 1.0750 & 1.1456 & 1.2009 & 1.2671 & 1.3478 & 1.3747 \\ 1.9800 & 0.8309 & 1.4591 & 1.4841 & 1.0196 & 1.0750 & 1.1361 & 1.1905 & 1.2892 & 1.3366 & 1.3619 \\ 1.9840 & 0.8239 & 1.5744 & 1.5826 & 1.0283 & 1.0750 & 1.1267 & 1.2009 & 1.2892 & 1.3591 & 1.3747 \\ 1.9880 & 0.8309 & 1.6589 & 1.6564 & 1.0371 & 1.1024 & 1.1551 & 1.2009 & 1.2892 & 1.3478 & 1.3490 \\ 1.9920 & 0.8309 & 1.7203 & 1.7138 & 1.0371 & 1.0932 & 1.1456 & 1.2114 & 1.2892 & 1.3366 & 1.3619 \\ 1.9960 & 0.8378 & 1.7894 & 1.8122 & 1.0371 & 1.0932 & 1.1361 & 1.2009 & 1.2892 & 1.3366 & 1.3619 \\ 2.0000 & 0.8378 & 1.9046 & 1.9352 & 1.1162 & 1.0659 & 1.1267 & 1.2114 & 1.2782 & 1.3254 & 1.3490\end{array}$


Appendix G - Gauge data for experiment 2S-64.

\begin{tabular}{|c|c|c|c|c|c|c|c|c|c|c|}
\hline-0.1000 & 0.0125 & $4.0847 \mathrm{e}-3$ & -0.0077 & -0.0210 & -0.0132 & 0.0027 & 0.0107 & -0.0155 & 0.0104 & $-7.0138 \mathrm{e}-4$ \\
\hline-0.0980 & 0.0144 & -0.0169 & -0.0139 & -0.0022 & -0.0170 & 0.0035 & -0.0074 & -0.0138 & -0.0010 & -0.0132 \\
\hline-0.0960 & 0.0110 & $-3.6589 \mathrm{e}-3$ & 0.0165 & 0.0013 & -0.0024 & 0.0016 & -0.0203 & 0.0084 & -0.0122 & -0.0104 \\
\hline-0.0940 & $-6.6266 e-3$ & $-1.7015 e-3$ & -0.0075 & -0.0076 & 0.0054 & 0.0132 & 0.0220 & -0.0162 & -0.0067 & -0.0361 \\
\hline-0.0920 & $3.9900 \mathrm{e}-3$ & $7.9138 \mathrm{e}-3$ & 0.0195 & -0.0121 & -0.0138 & -0.0028 & -0.0055 & -0.0028 & -0.0215 & -0.0297 \\
\hline-0.0900 & $2.4934 \mathrm{e}-3$ & $-6.8464 e-4$ & -0.0264 & -0.0041 & 0.0142 & -0.0348 & -0.0038 & -0.0090 & -0.0319 & $6.0171 \mathrm{e}-3$ \\
\hline-0.0880 & 0.0105 & $-1.8717 \mathrm{e}-3$ & -0.0115 & 0.0007 & -0.0120 & 0.0068 & -0.0351 & 0.0005 & 0.0153 & $-8.1261 \mathrm{e}-3$ \\
\hline-0.0860 & 0.0218 & $7.5762 \mathrm{e}-3$ & -0.0073 & -0.0173 & -0.0179 & -0.0064 & 0.0005 & -0.0259 & -0.0169 & $4.0125 \mathrm{e}-3$ \\
\hline-0.0840 & $4.2043 \mathrm{e}-3$ & 0.0132 & -0.0053 & -0.0274 & -0.0149 & -0.0060 & 0.0158 & 0.0100 & 0.0163 & $5.5228 \mathrm{e}-3$ \\
\hline-0.0820 & 0.0139 & -0.0233 & -0.0117 & -0.0375 & -0.0307 & -0.0101 & -0.0403 & -0.0185 & -0.0001 & $7.6238 \mathrm{e}-3$ \\
\hline-0.0800 & 0.0276 & 0.0107 & -0.0231 & -0.0099 & -0.0200 & 0.0069 & -0.0107 & 0.0095 & -0.0128 & $-2.9083 e-3$ \\
\hline-0.0780 & $-5.3443 e-3$ & $7.7434 \mathrm{e}-3$ & 0.0025 & -0.0080 & -0.0231 & -0.0214 & 0.0081 & -0.0023 & 0.0082 & -0.0250 \\
\hline-0.0760 & $-2.9211 \mathrm{e}-3$ & $3.1486 \mathrm{e}-3$ & -0.0244 & -0.0112 & -0.0015 & -0.0145 & 0.0003 & 0.0144 & -0.0005 & -0.0392 \\
\hline-0.0740 & 0.0105 & 0.0192 & -0.0122 & -0.0109 & -0.0025 & -0.0006 & 0.0193 & -0.0068 & -0.0035 & -0.0187 \\
\hline-0.0720 & 0.0108 & $-5.1893 e-3$ & -0.0184 & -0.0430 & -0.0098 & 0.0183 & 0.0212 & -0.0090 & 0.0123 & $2.5070 \mathrm{e}-3$ \\
\hline-0.0700 & 0.0107 & $7.4883 e-3$ & -0.0101 & 0.0054 & -0.0216 & 0.0207 & -0.0146 & -0.0035 & 0.0260 & 0.0129 \\
\hline-0.0680 & $-3.9900 \mathrm{e}-3$ & $-5.9128 \mathrm{e}-4$ & -0.0097 & -0.0084 & -0.0055 & 0.0158 & -0.0245 & -0.0029 & -0.0001 & -0.0102 \\
\hline-0.0660 & 0.0189 & $5.1060 \mathrm{e}-3$ & -0.0143 & -0.0149 & 0.0037 & -0.0002 & 0.0014 & 0.0113 & -0.0088 & 0.0158 \\
\hline-0.0640 & $-4.6320 \mathrm{e}-3$ & -0.0113 & 0.0117 & -0.0142 & -0.0076 & -0.0074 & 0.0025 & -0.0330 & -0.0310 & -0.0275 \\
\hline-0.0620 & $-8.5454 \mathrm{e}-4$ & $-3.9140 \mathrm{e}-3$ & -0.0140 & -0.0085 & -0.0296 & 0.0245 & -0.0188 & -0.0054 & -0.0273 & 0.0171 \\
\hline-0.0600 & 0.0143 & -0.0195 & 0.0084 & 0.0150 & -0.0035 & -0.0081 & 0.0004 & -0.0150 & 0.0099 & $2.0063 e-3$ \\
\hline-0.0580 & 0.0131 & 0.0113 & -0.0258 & -0.0169 & -0.0148 & -0.0322 & 0.0013 & -0.0165 & 0.0004 & $-7.6238 \mathrm{e}-3$ \\
\hline-0.0560 & 0.0112 & $-9.5344 \mathrm{e}-3$ & 0.0052 & -0.0091 & -0.0269 & 0.0193 & -0.0115 & -0.0033 & -0.0429 & -0.0188 \\
\hline-0.0540 & $7.6964 \mathrm{e}-3$ & $-5.7860 e-3$ & -0.0059 & -0.0243 & -0.0487 & 0.0047 & 0.0098 & -0.0033 & 0.0230 & $-4.9145 e-3$ \\
\hline-0.0520 & 0.0185 & -0.0218 & -0.0087 & -0.0265 & -0.0229 & -0.0272 & 0.0112 & 0.0047 & -0.0174 & 0.0198 \\
\hline-0.0500 & 0.0239 & $1.6167 \mathrm{e}-3$ & 0.0019 & -0.0084 & -0.0158 & -0.0055 & 0.0255 & -0.0164 & -0.0235 & -0.0151 \\
\hline-0.0480 & $9.0489 \mathrm{e}-3$ & $-5.1037 \mathrm{e}-4$ & -0.0133 & -0.0236 & 0.0076 & -0.0283 & -0.0079 & -0.0149 & 0.0114 & $4.1120 \mathrm{e}-3$ \\
\hline-0.0460 & $8.4041 \mathrm{e}-3$ & 0.0191 & -0.0131 & -0.0162 & -0.0192 & -0.0050 & -0.0286 & -0.0178 & -0.0051 & 0.0331 \\
\hline-0.0440 & $5.2723 \mathrm{e}-3$ & $-4.3405 e-3$ & -0.0152 & -0.0117 & -0.0247 & 0.0218 & 0.0149 & 0.0098 & -0.0300 & $1.4044 \mathrm{e}-3$ \\
\hline-0.0420 & 0.0177 & $4.0845 \mathrm{e}-3$ & -0.0163 & -0.0064 & 0.0001 & 0.0098 & 0.0023 & -0.0054 & -0.0394 & 0.0210 \\
\hline-0.0400 & 0.0231 & $-6.8075 e-4$ & -0.0171 & -0.0128 & -0.0066 & 0.0180 & -0.0053 & 0.0101 & 0.0143 & $7.1246 \mathrm{e}-3$ \\
\hline-0.0380 & $-9.9408 \mathrm{e}-4$ & $8.5580 \mathrm{e}-4$ & -0.0110 & 0.0018 & -0.0125 & -0.0053 & 0.0219 & 0.0101 & 0.0134 & -0.0153 \\
\hline-0.0360 & $7.9800 \mathrm{e}-3$ & $-5.9568 \mathrm{e}-3$ & -0.0070 & -0.0194 & -0.0081 & -0.0092 & -0.0133 & -0.0021 & 0.0065 & 0.0122 \\
\hline-0.0340 & $-7.2686 \mathrm{e}-3$ & -0.0124 & -0.0232 & -0.0253 & -0.0188 & 0.0104 & 0.0338 & 0.0393 & 0.0194 & $-7.4231 e-3$ \\
\hline-0.0320 & $-8.5546 \mathrm{e}-4$ & $6.5523 \mathrm{e}-3$ & -0.0154 & -0.0166 & -0.0130 & -0.0113 & -0.0030 & 0.0163 & 0.0045 & -0.0293 \\
\hline-0.0300 & 0.0100 & $-5.6156 e-3$ & 0.0133 & -0.0092 & -0.0163 & -0.0077 & 0.0123 & 0.0006 & -0.0115 & $-6.2198 \mathrm{e}-3$ \\
\hline-0.0280 & 0.0336 & $6.2123 e-3$ & -0.0001 & -0.0093 & -0.0082 & 0.0115 & -0.0202 & -0.0068 & -0.0066 & -0.0356 \\
\hline-0.0260 & $7.6936 \mathrm{e}-3$ & $-1.1063 e-3$ & 0.0035 & 0.0080 & -0.0216 & 0.0177 & -0.0216 & 0.0193 & -0.0170 & -0.0116 \\
\hline-0.0240 & $9.5472 \mathrm{e}-3$ & -0.0124 & -0.0226 & 0.0253 & -0.0047 & -0.0105 & 0.0077 & -0.0263 & -0.0019 & $-4.7144 \mathrm{e}-3$ \\
\hline-0.0220 & $-8.9066 \mathrm{e}-3$ & -0.0103 & 0.0030 & -0.0215 & -0.0101 & 0.0002 & -0.0016 & 0.0119 & -0.0294 & -0.0169 \\
\hline-0.0200 & $-9.6191 \mathrm{e}-3$ & 0.0128 & 0.0039 & 0.0020 & 0.0080 & -0.0198 & -0.0355 & 0.0035 & -0.0063 & -0.0111 \\
\hline-0.0180 & 0.0165 & 0.0123 & 0.0186 & -0.0123 & -0.0129 & -0.0061 & 0.0000 & -0.0265 & 0.0065 & -0.0193 \\
\hline-0.0160 & 0.0222 & $1.1062 \mathrm{e}-3$ & -0.0081 & 0.0237 & -0.0065 & 0.0076 & -0.0175 & -0.0091 & 0.0033 & $-5.1167 e-3$ \\
\hline-0.0140 & $-8.6959 \mathrm{e}-3$ & $8.0842 \mathrm{e}-3$ & 0.0215 & 0.0050 & -0.0201 & -0.0171 & 0.0010 & -0.0168 & 0.0264 & $-3.0174 \mathrm{e}-4$ \\
\hline-0.0120 & $7.4811 \mathrm{e}-3$ & $8.5930 \mathrm{e}-3$ & -0.0173 & -0.0070 & -0.0014 & 0.0075 & -0.0323 & 0.0281 & 0.0089 & -0.0138 \\
\hline-0.0100 & 0.0142 & $-8.5946 \mathrm{e}-3$ & 0.0134 & -0.0076 & -0.0065 & 0.0092 & -0.0086 & 0.0042 & -0.0064 & 0.0128 \\
\hline \multicolumn{2}{|c|}{$-8.0000 \mathrm{e}-30.0227$} & $8.5922 \mathrm{e}-3$ & 0.0174 & 0.0109 & -0.0082 & 0.0055 & 0.0074 & -0.0046 & -0.0057 & $-8.0250 \mathrm{e}-3$ \\
\hline \multicolumn{2}{|c|}{$-6.0000 e-3-5.8432 e-3$} & $8.5907 \mathrm{e}-3$ & 0.0064 & 0.0026 & -0.0006 & -0.0022 & 0.0062 & -0.0078 & -0.0004 & $-1.4044 \mathrm{e}-3$ \\
\hline \multicolumn{2}{|c|}{$-4.0000 e-30.0353$} & $9.3593 \mathrm{e}-4$ & -0.0059 & -0.0094 & 0.0015 & 0.0056 & -0.0096 & -0.0026 & 0.0018 & $-1.9067 e-3$ \\
\hline \multicolumn{2}{|c|}{$-2.0000 \mathrm{e}-30.1398$} & $-3.9989 e-3$ & 30.0065 & 0.0043 & -0.0280 & 0.0134 & -0.0197 & 0.0043 & 0.0189 & -0.0139 \\
\hline $1.6159 \mathrm{e}-12$ & 0.2877 & & $-3.3999 e-3$ & $3-0.0111$ & -0.0021 & -0.0018 & 0.0045 & -0.0177 & -0.0129 & 0.0252 \\
\hline \multicolumn{11}{|c|}{$-1.8056 \mathrm{e}-3$} \\
\hline $2.0000 \mathrm{e}-3$ & 0.3708 & -0.0146 & -0.0217 & 0.0092 & -0.0188 & 0.0116 & -0.0336 & -0.0208 & 0.0036 & -0.0311 \\
\hline $4.0000 \mathrm{e}-3$ & 0.4619 & $9.5337 \mathrm{e}-3$ & -0.0182 & 0.0013 & 0.0001 & -0.0184 & -0.0245 & -0.0191 & 0.0103 & -0.0118 \\
\hline $6.0000 \mathrm{e}-3$ & 0.5744 & -0.0152 & -0.0105 & -0.0068 & -0.0480 & -0.0087 & 0.0077 & 0.0081 & 0.0041 & -0.0151 \\
\hline $8.0000 \mathrm{e}-3$ & 0.6508 & 0.0211 & -0.0038 & -0.0060 & -0.0207 & 0.0017 & -0.0279 & -0.0122 & 0.0155 & $6.0027 \mathrm{e}-4$ \\
\hline 0.0100 & 0.7205 & $9.0173 \mathrm{e}-3$ & 0.0171 & -0.0024 & -0.0009 & -0.0104 & -0.0227 & -0.0020 & 0.0350 & -0.0201 \\
\hline 0.0120 & 0.8192 & -0.0334 & 0.0152 & 0.0090 & -0.0075 & -0.0074 & 0.0109 & -0.0246 & 0.0290 & $2.1074 \mathrm{e}-3$ \\
\hline 0.0140 & 0.9544 & $5.2748 \mathrm{e}-3$ & -0.0015 & -0.0163 & -0.0205 & 0.0007 & -0.0149 & 0.0051 & -0.0054 & $2.7092 \mathrm{e}-3$ \\
\hline 0.0160 & 1.0423 & 0.0183 & 0.0020 & -0.0185 & -0.0029 & 0.0049 & -0.0186 & 0.0052 & 0.0255 & $2.2072 \mathrm{e}-3$ \\
\hline 0.0180 & 1.1669 & -0.0112 & 0.0022 & 0.0111 & 0.0006 & -0.0163 & 0.0064 & 0.0031 & 0.0266 & $1.9067 \mathrm{e}-3$ \\
\hline 0.0200 & 1.3061 & $-8.6794 \mathrm{e}-3$ & -0.0069 & 0.0107 & -0.0106 & 0.0063 & -0.0211 & -0.0054 & -0.0151 & -0.0102 \\
\hline 0.0220 & 1.4466 & $-1.7871 \mathrm{e}-3$ & 30.0036 & -0.0086 & -0.0067 & -0.0060 & -0.0225 & -0.0242 & 0.0067 & -0.0258 \\
\hline 0.0240 & 1.4548 & $2.5518 \mathrm{e}-4$ & -0.0078 & 0.0050 & 0.0134 & -0.0052 & -0.0057 & -0.0225 & -0.0249 & $9.4294 \mathrm{e}-3$ \\
\hline 0.0260 & 1.4835 & $3.9126 \mathrm{e}-3$ & -0.0025 & 0.0050 & -0.0264 & -0.0280 & -0.0149 & 0.0015 & -0.0023 & -0.0138 \\
\hline 0.0280 & 1.4833 & -0.0149 & -0.0120 & -0.0049 & -0.0248 & 0.0094 & 0.0180 & -0.0074 & 0.0039 & $7.1217 \mathrm{e}-3$ \\
\hline
\end{tabular}




\begin{tabular}{|c|c|c|c|c|c|c|c|c|c|c|}
\hline 0.0300 & 1.4944 & 0.0294 & -0.0006 & -0.0010 & -0.0021 & -0.0133 & 0.0200 & -0.0129 & 0.0041 & $-5.4169 e-3$ \\
\hline 0.0320 & 1.4704 & 0.0177 & -0.0192 & 0.0045 & -0.0090 & -0.0262 & -0.0202 & -0.0135 & -0.0151 & $7.5239 e-3$ \\
\hline 0.0340 & 1.4816 & -0.0105 & 0.0098 & -0.0088 & -0.0107 & 0.0007 & -0.0179 & -0.0125 & -0.0110 & 0.0256 \\
\hline 0.0360 & 1.4788 & 0.0197 & 0.0262 & -0.0004 & -0.0154 & 0.0039 & -0.0321 & -0.0140 & -0.0050 & $-4.5136 \mathrm{e}-3$ \\
\hline 0.0380 & 1.5084 & $1.2759 \mathrm{e}-3$ & -0.0082 & -0.0161 & -0.0190 & -0.0094 & 0.0125 & -0.0036 & -0.0077 & 0.0186 \\
\hline 0.0400 & 1.4911 & $8.1698 \mathrm{e}-3$ & 0.0081 & 0.0145 & -0.0099 & -0.0017 & -0.0114 & -0.0174 & 0.0189 & -0.0170 \\
\hline 0.0420 & 1.4941 & 0.0365 & 0.0068 & -0.0235 & -0.0134 & -0.0037 & -0.0007 & 0.0121 & 0.0118 & $-5.4169 \mathrm{e}-3$ \\
\hline 0.0440 & 1.5013 & $4.5070 \mathrm{e}-3$ & 0.0106 & -0.0212 & -0.0026 & 0.0011 & -0.0268 & 0.0016 & 0.0094 & 0.0160 \\
\hline 0.0460 & 1.4898 & $3.3182 \mathrm{e}-3$ & 0.0025 & 0.0031 & -0.0113 & 0.0035 & 0.0125 & 0.0218 & 0.0319 & -0.0142 \\
\hline 0.0480 & 1.5061 & $-7.2331 e-3$ & -0.0061 & 0.0062 & 0.0112 & -0.0049 & 0.0003 & 0.0070 & -0.0120 & 0.0164 \\
\hline 0.0500 & 1.5095 & 0.0263 & 0.0320 & -0.0041 & -0.0207 & -0.0062 & 0.0210 & 0.0023 & 0.0074 & $-2.1074 \mathrm{e}-3$ \\
\hline 0.0520 & 1.5028 & $1.8750 \mathrm{e}-3$ & 0.0058 & -0.0099 & -0.0022 & 0.0162 & -0.0150 & -0.0095 & -0.0065 & -0.0275 \\
\hline 0.0540 & 1.5172 & $3.4037 \mathrm{e}-3$ & 0.0119 & -0.0165 & -0.0215 & 0.0244 & -0.0075 & 0.0052 & 0.0145 & 0.0182 \\
\hline 0.0560 & 1.5075 & 7.9986e-3 & -0.0006 & 0.0074 & -0.0126 & -0.0173 & -0.0043 & -0.0203 & 0.0152 & $3.0097 \mathrm{e}-3$ \\
\hline 0.0580 & 1.4831 & $-3.0653 e-3$ & 0.0095 & 0.0129 & -0.0141 & 0.0093 & -0.0023 & 0.0255 & 0.0109 & -0.0199 \\
\hline 0.0600 & 1.4787 & $-8.5098 \mathrm{e}-3$ & -0.0116 & -0.0087 & 0.0160 & -0.0088 & -0.0062 & 0.0128 & 0.0116 & $3.2100 \mathrm{e}-3$ \\
\hline 0.0620 & 1.4869 & 0.0180 & -0.0044 & -0.0062 & -0.0170 & 0.0050 & -0.0126 & 0.0083 & -0.0221 & -0.0366 \\
\hline 0.0640 & 1.4856 & -0.0134 & 0.0061 & -0.0014 & -0.0119 & -0.0116 & -0.0050 & -0.0062 & 0.0075 & -0.0213 \\
\hline 0.0660 & 1.5295 & $-1.3614 \mathrm{e}-3$ & -0.0005 & -0.0036 & 0.0086 & 0.0130 & -0.0062 & 0.0066 & -0.0032 & -0.0184 \\
\hline 0.0680 & 1.5344 & 0.0146 & 0.0031 & 0.0168 & -0.0426 & -0.0124 & -0.0044 & -0.0082 & 0.0168 & $-3.3095 \mathrm{e}-3$ \\
\hline 0.0700 & 1.4945 & 0.0117 & -0.0170 & 0.0043 & -0.0019 & -0.0105 & 0.0176 & 0.0121 & 0.0066 & 0.0119 \\
\hline 0.0720 & 1.5059 & $7.3988 \mathrm{e}-3$ & -0.0065 & 0.0152 & -0.0277 & -0.0060 & -0.0066 & -0.0060 & -0.0203 & $8.0250 \mathrm{e}-4$ \\
\hline 0.0740 & 1.5132 & $-2.0423 e-3$ & -0.0037 & 0.0097 & 0.0129 & -0.0067 & 0.0022 & -0.0360 & 0.0049 & -0.0259 \\
\hline 0.0760 & 1.4973 & $-6.3850 e-3$ & -0.0026 & -0.0043 & -0.0311 & 0.0225 & -0.0119 & -0.0303 & 0.0023 & -0.0102 \\
\hline 0.0780 & 1.5120 & 0.0256 & -0.0065 & 0.0316 & -0.0150 & -0.0180 & -0.0003 & 0.0007 & -0.0091 & $9.4294 \mathrm{e}-3$ \\
\hline 0.0800 & 1.4952 & $5.9595 \mathrm{e}-4$ & -0.0221 & 0.0160 & 0.0064 & 0.0416 & 0.0082 & 0.0301 & 0.0075 & -0.0202 \\
\hline 0.0820 & 1.4951 & 0.0204 & 0.0095 & 0.0222 & -0.0268 & 0.0313 & -0.0116 & 0.0024 & 0.0065 & -0.0234 \\
\hline 0.0840 & 1.5038 & $3.0638 \mathrm{e}-3$ & 0.0066 & -0.0060 & -0.0174 & -0.0101 & 0.0343 & -0.0204 & 0.0037 & 0.0256 \\
\hline 0.0860 & 1.4842 & 0.0134 & 0.0106 & -0.0356 & -0.0156 & -0.0080 & -0.0018 & -0.0176 & 0.0002 & $-3.8116 \mathrm{e}-3$ \\
\hline 0.0880 & 1.5139 & 0.0151 & -0.0120 & -0.0084 & -0.0058 & -0.0230 & -0.0048 & -0.0193 & 0.0100 & -0.0101 \\
\hline 0.0900 & 1.5099 & $-6.2123 e-3$ & 0.0125 & -0.0088 & -0.0084 & 0.0132 & -0.0124 & -0.0015 & -0.0006 & -0.0184 \\
\hline 0.0920 & 1.5064 & $-1.1911 \mathrm{e}-3$ & -0.0084 & -0.0217 & -0.0090 & -0.0401 & -0.0249 & 0.0118 & -0.0056 & 0.0256 \\
\hline 0.0940 & 1.5164 & $7.5738 \mathrm{e}-3$ & -0.0032 & 0.0157 & 0.0080 & 0.0274 & 0.0015 & -0.0121 & -0.0029 & -0.0254 \\
\hline 0.0960 & 1.4908 & $-6.7219 \mathrm{e}-3$ & 0.0096 & 0.0058 & 0.0238 & 0.0005 & -0.0021 & 0.0147 & 0.0183 & $6.6206 \mathrm{e}-3$ \\
\hline 0.0980 & 1.5105 & $-7.9986 \mathrm{e}-3$ & 0.0071 & 0.0057 & 0.0006 & 0.0045 & -0.0184 & 0.0053 & -0.0090 & $-5.3170 \mathrm{e}-3$ \\
\hline 0.1000 & 1.4924 & -0.0183 & 0.0099 & 0.0120 & 0.0011 & 0.0080 & 0.0100 & 0.0076 & 0.0097 & $-9.0201 \mathrm{e}-4$ \\
\hline 0.1020 & 1.5138 & -0.0146 & -0.0133 & 0.0105 & -0.0092 & -0.0165 & 0.0177 & -0.0077 & -0.0216 & -0.0150 \\
\hline 0.1040 & 1.4978 & 0.0126 & -0.0017 & -0.0148 & 0.0034 & -0.0377 & -0.0393 & 0.0095 & -0.0186 & -0.0430 \\
\hline 0.1060 & 1.5005 & $-5.3610 \mathrm{e}-3$ & -0.0174 & 0.0023 & -0.0074 & -0.0021 & 0.0029 & 0.0122 & -0.0094 & $-5.7170 \mathrm{e}-3$ \\
\hline 0.1080 & 1.5357 & 0.0136 & 0.0088 & 0.0183 & -0.0019 & -0.0353 & -0.0007 & -0.0119 & 0.0006 & $-1.4039 \mathrm{e}-3$ \\
\hline 0.1100 & 1.5087 & $-1.7023 e-3$ & 0.0005 & 0.0023 & -0.0032 & 0.0070 & 0.0017 & 0.0041 & -0.0147 & $-8.7280 \mathrm{e}-3$ \\
\hline 0.1120 & 1.5238 & $5.3610 \mathrm{e}-3$ & -0.0027 & 0.0096 & 0.0200 & -0.0132 & -0.0023 & -0.0055 & -0.0017 & $-5.9192 \mathrm{e}-3$ \\
\hline 0.1140 & 1.5172 & 0.0204 & 0.0314 & 0.0093 & -0.0085 & 0.0114 & -0.0166 & -0.0003 & -0.0020 & -0.0108 \\
\hline 0.1160 & 1.5153 & $2.5518 \mathrm{e}-4$ & -0.0020 & 0.0044 & -0.0232 & 0.0006 & -0.0117 & 0.0044 & 0.0081 & -0.0252 \\
\hline 0.1180 & 1.5109 & $4.0829 \mathrm{e}-3$ & -0.0134 & -0.0309 & -0.0212 & 0.0044 & -0.0027 & 0.0065 & -0.0265 & -0.0127 \\
\hline 0.1200 & 1.4978 & 0.0134 & 0.0114 & -0.0167 & 0.0156 & 0.0086 & 0.0123 & 0.0138 & -0.0040 & $8.9270 \mathrm{e}-3$ \\
\hline 0.1220 & 1.4994 & -0.0106 & -0.0085 & 0.0078 & -0.0327 & -0.0264 & 0.0082 & -0.0021 & -0.0206 & $-4.9158 \mathrm{e}-3$ \\
\hline 0.1240 & 1.5048 & $4.4229 \mathrm{e}-3$ & 0.0020 & 0.0338 & 0.0094 & 0.0057 & 0.0110 & 0.0045 & -0.0133 & $5.5169 \mathrm{e}-3$ \\
\hline 0.1260 & 1.4929 & $6.6363 \mathrm{e}-3$ & 0.0068 & 0.0105 & -0.0170 & 0.0228 & -0.0018 & -0.0098 & 0.0240 & $1.2037 \mathrm{e}-3$ \\
\hline 0.1280 & 1.5088 & $8.9353 \mathrm{e}-3$ & -0.0114 & 0.0088 & -0.0170 & -0.0013 & -0.0441 & -0.0133 & -0.0088 & 0.0169 \\
\hline 0.1300 & 1.5086 & $4.4284 \mathrm{e}-3$ & 0.0136 & 0.0101 & 0.0145 & -0.0073 & -0.0105 & -0.0143 & 0.0164 & -0.0205 \\
\hline 0.1320 & 1.5156 & $8.0873 e-3$ & -0.0176 & -0.0015 & -0.0044 & -0.0031 & -0.0075 & 0.0006 & 0.0016 & 0.0192 \\
\hline 0.1340 & 1.4980 & 0.0112 & 0.0031 & -0.0216 & -0.0113 & -0.0016 & 0.0052 & -0.0008 & 0.0055 & $-5.1163 e-3$ \\
\hline 0.1360 & 1.4941 & $-4.4253 e-3$ & -0.0274 & -0.0090 & -0.0043 & 0.0112 & 0.0272 & 0.0055 & 0.0398 & $-5.6175 e-3$ \\
\hline 0.1380 & 1.4913 & $-7.8290 \mathrm{e}-3$ & 0.0062 & 0.0164 & -0.0142 & 0.0015 & -0.0031 & -0.0030 & 0.0204 & $-4.6141 \mathrm{e}-3$ \\
\hline 0.1400 & 1.5078 & $3.8278 \mathrm{e}-3$ & 0.0160 & -0.0004 & -0.0124 & -0.0178 & -0.0331 & -0.0135 & -0.0194 & $6.0184 \mathrm{e}-3$ \\
\hline 0.1420 & 1.5003 & 0.0155 & -0.0070 & -0.0361 & -0.0031 & 0.0180 & -0.0121 & -0.0022 & -0.0070 & -0.0303 \\
\hline 0.1440 & 1.5205 & 0.0128 & -0.0261 & -0.0170 & 0.0015 & -0.0081 & 0.0037 & -0.0264 & 0.0030 & $7.0138 \mathrm{e}-4$ \\
\hline 0.1460 & 1.5111 & -0.0208 & -0.0168 & -0.0365 & -0.0165 & 0.0028 & -0.0353 & 0.0132 & -0.0072 & $5.4169 \mathrm{e}-3$ \\
\hline 0.1480 & 1.4987 & $4.4276 \mathrm{e}-3$ & 0.0106 & 0.0178 & 0.0112 & 0.0003 & -0.0066 & 0.0071 & 0.0007 & $-3.0094 \mathrm{e}-3$ \\
\hline 0.1500 & 1.4929 & $-1.9551 \mathrm{e}-3$ & -0.0095 & -0.0007 & 0.0037 & -0.0262 & 0.0221 & -0.0021 & -0.0374 & -0.0162 \\
\hline 0.1520 & 1.4962 & $-9.0162 e-3$ & 0.0050 & 0.0132 & -0.0094 & 0.0081 & -0.0217 & -0.0256 & -0.0122 & $-8.9286 \mathrm{e}-3$ \\
\hline 0.1540 & 1.4902 & $-7.9138 \mathrm{e}-3$ & 0.0188 & 0.0040 & 0.0006 & -0.0027 & -0.0037 & 0.0086 & -0.0201 & -0.0276 \\
\hline 0.1560 & 1.4917 & $8.4242 \mathrm{e}-3$ & -0.0152 & -0.0066 & 0.0032 & 0.0118 & -0.0159 & -0.0045 & -0.0094 & -0.0293 \\
\hline 0.1580 & 1.5101 & $-5.1037 e-3$ & 0.0141 & 0.0047 & -0.0111 & -0.0208 & -0.0156 & -0.0219 & -0.0345 & -0.0108 \\
\hline 0.1600 & 1.4961 & $8.3409 \mathrm{e}-3$ & -0.0126 & -0.0059 & -0.0088 & -0.0037 & -0.0125 & -0.0046 & 0.0161 & $-8.9334 \mathrm{e}-3$ \\
\hline 0.1620 & 1.4992 & $-9.1026 e-3$ & -0.0014 & 0.0089 & 0.0118 & 0.0247 & 0.0018 & -0.0044 & 0.0025 & -0.0116 \\
\hline 0.1640 & 1.4934 & $7.1478 \mathrm{e}-3$ & 0.0017 & 0.0097 & 0.0053 & 0.0208 & -0.0121 & 0.0095 & -0.0001 & $2.9086 \mathrm{e}-3$ \\
\hline 0.1660 & 1.5001 & $7.7434 \mathrm{e}-3$ & 0.0029 & 0.0166 & -0.0206 & 0.0337 & 0.0021 & -0.0145 & 0.0003 & $8.7264 \mathrm{e}-3$ \\
\hline 0.1680 & 1.5127 & 0.0120 & -0.0159 & -0.0237 & 0.0017 & -0.0331 & -0.0141 & -0.0335 & -0.0269 & -0.0215 \\
\hline
\end{tabular}




\begin{tabular}{|c|c|}
\hline 0.1700 & 1.4967 \\
\hline 0.1720 & 1.5175 \\
\hline 0.1740 & 1.4856 \\
\hline 0.1760 & 1.4914 \\
\hline 0.1780 & 1.4907 \\
\hline 0.1800 & 1.4942 \\
\hline 0.1820 & 1.4904 \\
\hline 0.1840 & 1.4793 \\
\hline 0.1860 & 1.4990 \\
\hline 0.1880 & 1.4909 \\
\hline 0.1900 & 1.4921 \\
\hline 0.1920 & 1.4814 \\
\hline 0.1940 & 1.4802 \\
\hline 0.1960 & 1.4739 \\
\hline 0.1980 & 1.4746 \\
\hline 0.2000 & 1.4778 \\
\hline 0.2020 & 1.4919 \\
\hline 0.2040 & 1.4790 \\
\hline 0.2060 & 1.4918 \\
\hline 0.2080 & 1.4824 \\
\hline 0.2100 & 1.4875 \\
\hline 0.2120 & 1.4842 \\
\hline 0.2140 & 1.4886 \\
\hline 0.2160 & 1.4889 \\
\hline 0.2180 & 1.4898 \\
\hline 0.2200 & 1.5102 \\
\hline 0.2220 & 1.5227 \\
\hline 0.2240 & 1.4615 \\
\hline 0.2260 & 1.4793 \\
\hline 0.2280 & 1.4754 \\
\hline 0.2300 & 1.5032 \\
\hline 0.2320 & 1.4535 \\
\hline 0.2340 & 1.4624 \\
\hline 0.2360 & 1.4837 \\
\hline 0.2380 & 1.4763 \\
\hline 0.2400 & 1.4826 \\
\hline 0.2420 & 1.4650 \\
\hline 0.2440 & 1.4476 \\
\hline 0.2460 & 1.4632 \\
\hline 0.2480 & 1.4649 \\
\hline 0.2500 & 1.4569 \\
\hline 0.2520 & 1.4748 \\
\hline 0.2540 & 1.4893 \\
\hline 0.2560 & 1.4741 \\
\hline 0.2580 & 1.4770 \\
\hline 0.2600 & 1.4792 \\
\hline 0.2620 & 1.4742 \\
\hline 0.2640 & 1.4652 \\
\hline 0.2660 & 1.4771 \\
\hline 0.2680 & 1.4967 \\
\hline 0.2700 & 1.4539 \\
\hline 0.2720 & 1.4725 \\
\hline 0.2740 & 1.4826 \\
\hline 0.2760 & 1.4820 \\
\hline 0.2780 & 1.4577 \\
\hline 0.2800 & 1.4641 \\
\hline 0.2820 & 1.4651 \\
\hline 0.2840 & 1.4559 \\
\hline 0.2860 & 1.4731 \\
\hline 0.2880 & 1.4919 \\
\hline 0.2900 & 1.4621 \\
\hline 0.2920 & 1.4688 \\
\hline 0.2940 & 1.4769 \\
\hline 0.2960 & 1.4651 \\
\hline 0.2980 & 1.4908 \\
\hline 0.3000 & 1.4603 \\
\hline 0.3020 & 1.4723 \\
\hline 0.3040 & 1.4564 \\
\hline 0.3060 & 1.4592 \\
\hline 0.3080 & 1.4545 \\
\hline
\end{tabular}

\begin{tabular}{|c|c|c|c|c|c|c|c|c|}
\hline 44 & 037 & -0.0090 & -0.0130 & -0.0085 & 0.0027 & -0.0152 & -0.0043 & -0.0118 \\
\hline-0.0241 & 0.0251 & 0.0055 & 0.0048 & -0.0082 & 0.0157 & -0.0041 & -0.0286 & -0.0312 \\
\hline $8.6770 \mathrm{e}-3$ & -0.0082 & .0089 & 0.0018 & 0.0013 & -0.0046 & 0.0113 & -0.0009 & $.3263 \mathrm{e}-3$ \\
\hline 0.0114 & -0.0058 & .0131 & 0.0183 & .0035 & -0.0025 & 884 & -0.0069 & -0.0184 \\
\hline$-9.3601 e-3$ & -0.0167 & 0089 & 00179 & 0228 & 0.0234 & -0.0206 & 0.0004 & .0183 \\
\hline 0.0185 & -0.0069 & 0.0157 & -0.0002 & 0060 & 0029 & -0.0 & 0.0116 & 0214 \\
\hline $4.9325 \mathrm{e}-3$ & 0.0055 & 0.0207 & -0.0216 & .0028 & 0192 & -0.0090 & .0011 & 254 \\
\hline-0.0212 & -0.0082 & 0025 & 0.0130 & .0104 & & & 0203 & \\
\hline 0.0101 & -0.0099 & .0109 & .0159 & 0072 & 0003 & 032 & 0.0188 & \\
\hline $1278 \mathrm{e}-3$ & -0.0179 & 0023 & & & & & & $54 \mathrm{e}-3$ \\
\hline $.7219 e-3$ & -0.0060 & -0.0149 & & & & & -0.0084 & \\
\hline $7.1498 \mathrm{e}-3$ & & 0.0350 & & & & & & 10 \\
\hline 0.02 & -0.0412 & .0343 & & & & & & \\
\hline 0.0628 & -0.0457 & 52 & & 246 & 329 & & & $6 e-3$ \\
\hline 0.1373 & -0.0300 & -0.0172 & & 443 & 165 & & 0108 & \\
\hline & -0.0368 & -0.0478 & & & 0136 & & -0.0190 & \\
\hline 2998 & -0.0 & -0.0390 & & & & & 245 & $81 e-3$ \\
\hline & & -0.0311 & & & & & & \\
\hline 43 & & 77 & & & & & & \\
\hline 0.55 & -0.0 & -0.0 & & & & & & \\
\hline 0.6036 & -0.0697 & -0.0675 & & & 529 & & & 4 \\
\hline & -0.0603 & 47 & & & & & & \\
\hline & & -0.0856 & & & & & & \\
\hline 6 & & -0.0 & & & & & & \\
\hline & & & & & & & & $5 e-3$ \\
\hline & & & & & & & & \\
\hline 17 & -0.0 & -0.0 & & & & & & $6 e-3$ \\
\hline 168 & -0.0347 & -0.0453 & & & & & 139 & \\
\hline & & & & & & & & -3 \\
\hline 1 & -0.0 & 55 & & & & & & \\
\hline & & & & & & & & \\
\hline & & & & & & & & \\
\hline & & & & & & & & \\
\hline 1.51 & -0.0 & 55 & & & 202 & & & $e-$ \\
\hline & -0.0123 & & & & & & & \\
\hline & & 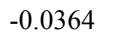 & & & & & & 4 \\
\hline 1.5334 & $-0 .($ & 1 & & & & & 96 & \\
\hline & & & & & & & & e-3 \\
\hline & & & & & & & & \\
\hline U & -0.0 & 2 & & & & & & \\
\hline & -0.0 & 76 & & & & & & \\
\hline & & & & & & & & \\
\hline 1.5429 & & 9 & & & & & & \\
\hline & & & & & & & & $9 e-3$ \\
\hline & & & & & & & & \\
\hline & & & & & & & & \\
\hline 6 & -( & 2 & & & & & & $4 e-4$ \\
\hline & -0.0 & 2 & & & & & & \\
\hline & & -0.0 & & & & & & e-3 \\
\hline 1.5688 & -0.0454 & -0.0447 & & & & & 137 & $1 e-3$ \\
\hline & & & & & & & & \\
\hline & & & & & & & & \\
\hline & -( & & & & & & & -3 \\
\hline 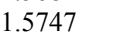 & 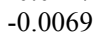 & & 3 & & & & & $7 e-3$ \\
\hline & & & & & & & & \\
\hline 1.5686 & -0.00 & 65 & & & 374 & & 105 & \\
\hline & & & & & & & & $08 \mathrm{e}-3$ \\
\hline & & & & & & & & \\
\hline & & & & & & & & 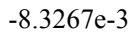 \\
\hline & & & & & & & & \\
\hline & & & & & & & 62 & $e-3$ \\
\hline & & -0.0 & & & & & & \\
\hline 1.6058 & -0.0178 & -0.0176 & -0.0257 & 24 & 0.0102 & -0.0018 & 211 & 43 \\
\hline 1.5801 & -0.0104 & 0.00 & -0 . & & 092 & 12 & 0.0009 & \\
\hline & & & & & & & & \\
\hline & & & & & & & 0050 & \\
\hline & & & & & & & & \\
\hline & & & & & & & & \\
\hline 1.5778 & -0.0233 & 0.0055 & 0.0043 & 0.0119 & -0.0115 & -0.0379 & 0.0074 & $-2.4075 e-3$ \\
\hline 1.5949 & 0.0089 & 0.0001 & -0.0271 & -0.0149 & 0.0258 & 0.0303 & -0.0127 & -0.0353 \\
\hline
\end{tabular}




\begin{tabular}{|c|c|c|c|c|c|c|c|c|c|c|}
\hline 0.3100 & 1.4594 & 1.5887 & -0.0018 & -0.0155 & -0.0022 & 0.0046 & -0.0033 & 0.0224 & -0.0087 & -0.0116 \\
\hline 0.3120 & 1.4515 & 1.5762 & 0.0117 & 0.0128 & -0.0332 & -0.0323 & 0.0252 & 0.0030 & 0.0104 & 0.0152 \\
\hline 0.3140 & 1.4372 & 1.5926 & -0.0107 & -0.0118 & -0.0008 & -0.0032 & 0.0346 & -0.0080 & -0.0240 & $2.6081 \mathrm{e}-3$ \\
\hline 0.3160 & 1.4472 & 1.6042 & -0.0284 & -0.0033 & 0.0067 & 0.0255 & 0.0050 & -0.0207 & 0.0162 & $-2.9083 e-3$ \\
\hline 0.3180 & 1.4644 & 1.6042 & -0.0056 & -0.0254 & -0.0227 & -0.0071 & -0.0027 & -0.0284 & 0.0074 & 0.0000 \\
\hline 0.3200 & 1.4596 & 1.5957 & -0.0034 & -0.0350 & -0.0147 & -0.0005 & -0.0032 & -0.0250 & -0.0017 & 0.0107 \\
\hline 0.3220 & 1.4347 & 1.5949 & -0.0158 & -0.0048 & -0.0144 & -0.0170 & 0.0089 & 0.0111 & -0.0231 & 0.0187 \\
\hline 0.3240 & 1.4612 & 1.5988 & -0.0011 & -0.0186 & -0.0206 & -0.0168 & -0.0032 & -0.0182 & 0.0091 & $7.2228 \mathrm{e}-3$ \\
\hline 0.3260 & 1.4446 & 1.5996 & -0.0071 & 0.0242 & -0.0016 & -0.0233 & -0.0516 & 0.0081 & -0.0183 & $-6.8216 \mathrm{e}-3$ \\
\hline 0.3280 & 1.4453 & 1.6035 & 0.0632 & -0.0075 & -0.0155 & 0.0052 & -0.0118 & 0.0125 & -0.0201 & $-3.7112 \mathrm{e}-3$ \\
\hline 0.3300 & 1.4433 & 1.6136 & 0.1472 & -0.0427 & -0.0081 & -0.0071 & -0.0014 & -0.0414 & 0.0067 & -0.0220 \\
\hline 0.3320 & 1.4431 & 1.5965 & 0.2343 & -0.0625 & -0.0522 & 0.0080 & 0.0098 & -0.0161 & -0.0161 & -0.0371 \\
\hline 0.3340 & 1.4388 & 1.5895 & 0.3206 & -0.0384 & -0.0173 & -0.0302 & -0.0291 & -0.0205 & -0.0204 & -0.0123 \\
\hline 0.3360 & 1.4554 & 1.5933 & 0.3668 & -0.0449 & -0.0200 & -0.0173 & -0.0291 & -0.0306 & -0.0253 & -0.0257 \\
\hline 0.3380 & 1.4406 & 1.5832 & 0.4703 & -0.0507 & -0.0661 & -0.0245 & -0.0239 & -0.0166 & 0.0015 & -0.0204 \\
\hline 0.3400 & 1.4639 & 1.5739 & 0.5480 & -0.0816 & -0.0351 & -0.0270 & -0.0234 & -0.0135 & -0.0115 & $-5.7170 \mathrm{e}-3$ \\
\hline 0.3420 & 1.4307 & 1.5708 & 0.6267 & -0.0546 & -0.0382 & -0.0523 & -0.0289 & -0.0243 & -0.0220 & 0.0106 \\
\hline 0.3440 & 1.4479 & 1.5692 & 0.7663 & -0.0650 & -0.0608 & -0.0245 & -0.0077 & -0.0058 & -0.0226 & -0.0160 \\
\hline 0.3460 & 1.4275 & 1.5539 & 0.8647 & -0.0450 & -0.0509 & -0.0245 & -0.0250 & -0.0092 & 0.0033 & -0.0210 \\
\hline 0.3480 & 1.4447 & 1.5747 & 0.9611 & -0.0660 & -0.0494 & -0.0348 & -0.0111 & -0.0295 & -0.0429 & -0.0126 \\
\hline 0.3500 & 1.4548 & 1.5481 & 1.0577 & -0.0636 & -0.0798 & -0.0194 & -0.0495 & -0.0295 & -0.0242 & $3.2100 \mathrm{e}-3$ \\
\hline 0.3520 & 1.4521 & 1.5603 & 1.1677 & -0.0536 & -0.0855 & -0.0003 & -0.0347 & -0.0377 & -0.0252 & $3.3106 \mathrm{e}-3$ \\
\hline 0.3540 & 1.4421 & 1.5460 & 1.2674 & -0.0619 & -0.0402 & -0.0246 & -0.0451 & -0.0402 & -0.0262 & $-8.0250 \mathrm{e}-3$ \\
\hline 0.3560 & 1.4183 & 1.5630 & 1.3348 & -0.0819 & -0.0554 & -0.0453 & -0.0174 & -0.0027 & -0.0131 & $-6.2191 \mathrm{e}-3$ \\
\hline 0.3580 & 1.4281 & 1.5599 & 1.4203 & -0.0937 & -0.0412 & -0.0399 & -0.0198 & 0.0003 & -0.0068 & -0.0227 \\
\hline 0.3600 & 1.4481 & 1.5546 & 1.4993 & -0.0349 & -0.0450 & -0.0146 & -0.0539 & -0.0155 & -0.0105 & $-9.8306 \mathrm{e}-3$ \\
\hline 0.3620 & 1.4339 & 1.5552 & 1.5463 & -0.0246 & -0.0528 & -0.0202 & -0.0155 & -0.0313 & -0.0041 & $7.8241 \mathrm{e}-3$ \\
\hline 0.3640 & 1.4478 & 1.5627 & 1.5910 & -0.0472 & -0.0729 & -0.0138 & -0.0317 & -0.0460 & -0.0198 & -0.0266 \\
\hline 0.3660 & 1.4397 & 1.5700 & 1.6060 & -0.0050 & -0.0437 & -0.0212 & -0.0292 & -0.0195 & -0.0191 & -0.0216 \\
\hline 0.3680 & 1.4319 & 1.5716 & 1.6116 & -0.0251 & -0.0215 & -0.0093 & -0.0350 & -0.0484 & 0.0167 & $-8.1248 \mathrm{e}-3$ \\
\hline 0.3700 & 1.4450 & 1.6035 & 1.6259 & -0.0159 & -0.0547 & -0.0154 & -0.0061 & -0.0148 & -0.0334 & $4.0125 \mathrm{e}-3$ \\
\hline 0.3720 & 1.4270 & 1.5770 & 1.6274 & 0.0118 & -0.0120 & -0.0192 & -0.0009 & -0.0164 & -0.0099 & -0.0118 \\
\hline 0.3740 & 1.4428 & 1.5840 & 1.6339 & -0.0260 & -0.0311 & 0.0056 & -0.0318 & -0.0241 & 0.0114 & 0.0168 \\
\hline 0.3760 & 1.4411 & 1.5957 & 1.6399 & -0.0018 & -0.0121 & 0.0039 & 0.0099 & 0.0123 & -0.0244 & -0.0227 \\
\hline 0.3780 & 1.4429 & 1.5848 & 1.6631 & -0.0257 & -0.0023 & 0.0029 & -0.0137 & -0.0263 & 0.0082 & $4.6144 \mathrm{e}-3$ \\
\hline 0.3800 & 1.4286 & 1.6392 & 1.6640 & 0.0032 & -0.0414 & 0.0271 & -0.0025 & 0.0089 & -0.0005 & -0.0299 \\
\hline 0.3820 & 1.4366 & 1.6229 & 1.6483 & -0.0147 & -0.0126 & 0.0059 & -0.0018 & -0.0069 & -0.0083 & -0.0169 \\
\hline 0.3840 & 1.4139 & 1.6089 & 1.6737 & -0.0015 & -0.0280 & -0.0155 & 0.0103 & -0.0114 & -0.0216 & 0.0129 \\
\hline 0.3860 & 1.4210 & 1.5957 & 1.6753 & -0.0018 & -0.0260 & -0.0249 & 0.0159 & -0.0051 & -0.0226 & -0.0157 \\
\hline 0.3880 & 1.4503 & 1.6066 & 1.6861 & 0.0017 & -0.0216 & -0.0176 & -0.0365 & -0.0058 & -0.0012 & $-8.0250 \mathrm{e}-3$ \\
\hline 0.3900 & 1.4373 & 1.5871 & 1.6894 & -0.0377 & -0.0148 & 0.0014 & 0.0013 & -0.0025 & -0.0056 & 0.0229 \\
\hline 0.3920 & 1.4136 & 1.6097 & 1.6588 & -0.0246 & 0.0111 & -0.0386 & -0.0025 & 0.0090 & -0.0154 & $9.6303 e-3$ \\
\hline 0.3940 & 1.4321 & 1.5980 & 1.6886 & -0.0141 & 0.0178 & 0.0080 & -0.0353 & -0.0264 & -0.0056 & -0.0173 \\
\hline 0.3960 & 1.4338 & 1.6027 & 1.6850 & 0.0117 & 0.0203 & 0.0050 & -0.0303 & -0.0083 & -0.0214 & $5.7181 \mathrm{e}-3$ \\
\hline 0.3980 & 1.4293 & 1.5786 & 1.6977 & 0.0074 & -0.0306 & 0.0196 & 0.0086 & -0.0150 & 0.0303 & $-8.3267 \mathrm{e}-3$ \\
\hline 0.4000 & 1.4197 & 1.5996 & 1.6952 & 0.0007 & -0.0233 & -0.0486 & -0.0124 & 0.0035 & -0.0143 & $2.7092 \mathrm{e}-3$ \\
\hline 0.4020 & 1.4338 & 1.5693 & 1.6778 & -0.0164 & -0.0329 & -0.0028 & 0.0084 & 0.0070 & -0.0206 & $-3.5105 e-3$ \\
\hline 0.4040 & 1.4189 & 1.5949 & 1.7060 & 0.0098 & -0.0293 & -0.0082 & -0.0224 & 0.0231 & -0.0193 & -0.0203 \\
\hline 0.4060 & 1.4169 & 1.5918 & 1.6903 & 0.0055 & 0.0002 & 0.0158 & -0.0029 & -0.0244 & 0.0107 & $-5.8181 \mathrm{e}-3$ \\
\hline 0.4080 & 1.4122 & 1.5863 & 1.6927 & 0.0062 & -0.0166 & -0.0059 & -0.0209 & -0.0069 & 0.0177 & 0.0220 \\
\hline 0.4100 & 1.4270 & 1.6112 & 1.7069 & -0.0207 & 0.0224 & -0.0196 & 0.0223 & -0.0090 & -0.0038 & $4.7152 \mathrm{e}-3$ \\
\hline 0.4120 & 1.4112 & 1.5902 & 1.6903 & 0.0080 & -0.0061 & -0.0089 & 0.0070 & -0.0159 & 0.0199 & $4.8150 \mathrm{e}-3$ \\
\hline 0.4140 & 1.4354 & 1.5988 & 1.7019 & -0.0067 & 0.0093 & -0.0260 & 0.0018 & -0.0293 & -0.0136 & -0.0289 \\
\hline 0.4160 & 1.4251 & 1.6035 & 1.7177 & -0.0069 & -0.0051 & -0.0069 & 0.0007 & 0.0113 & 0.0051 & $6.0187 \mathrm{e}-4$ \\
\hline 0.4180 & 1.4181 & 1.5988 & 1.7243 & -0.0037 & -0.0123 & -0.0136 & 0.0001 & 0.0100 & -0.0128 & $-2.9099 \mathrm{e}-3$ \\
\hline 0.4200 & 1.4355 & 1.6011 & 1.7152 & -0.0166 & -0.0196 & 0.0168 & -0.0076 & 0.0210 & -0.0123 & -0.0292 \\
\hline 0.4220 & 1.4209 & 1.5832 & 1.7094 & 0.0127 & -0.0121 & -0.0021 & -0.0249 & 0.0161 & 0.0116 & -0.0244 \\
\hline 0.4240 & 1.4023 & 1.5723 & 1.6927 & 0.0055 & 0.0075 & -0.0248 & -0.0160 & 0.0032 & -0.0063 & 0.0301 \\
\hline 0.4260 & 1.4048 & 1.5910 & 1.7119 & -0.0011 & -0.0116 & 0.0018 & -0.0055 & -0.0026 & -0.0016 & 0.0204 \\
\hline 0.4280 & 1.4300 & 1.5770 & 1.7285 & 0.0078 & 0.0019 & 0.0113 & 0.0001 & -0.0083 & -0.0205 & -0.0114 \\
\hline 0.4300 & 1.4221 & 1.5762 & 1.7285 & 0.0036 & 0.0094 & -0.0122 & -0.0103 & 0.0186 & 0.0116 & $7.3236 \mathrm{e}-3$ \\
\hline 0.4320 & 1.4093 & 1.5980 & 1.7077 & -0.0143 & 0.0276 & -0.0122 & 0.0128 & 0.0008 & -0.0301 & 0.0147 \\
\hline 0.4340 & 1.4330 & 1.5635 & 1.7077 & -0.0079 & -0.0335 & 0.0111 & 0.0078 & -0.0091 & 0.0018 & $8.4263 e-3$ \\
\hline 0.4360 & 1.3946 & 1.5879 & 1.7285 & 0.0084 & -0.0088 & -0.0228 & 0.0002 & -0.0165 & 0.0114 & -0.0172 \\
\hline 0.4380 & 1.4150 & 1.5933 & 1.7094 & 0.0226 & 0.0088 & 0.0184 & -0.0283 & -0.0002 & -0.0216 & -0.0142 \\
\hline 0.4400 & 1.4080 & 1.5887 & 1.7476 & 0.0117 & -0.0290 & -0.0155 & 0.0025 & -0.0261 & 0.0070 & -0.0455 \\
\hline 0.4420 & 1.4012 & 1.5696 & 1.6969 & -0.0279 & -0.0357 & -0.0025 & -0.0303 & -0.0072 & -0.0070 & $3.1089 e-3$ \\
\hline 0.4440 & 1.4017 & 1.5607 & 1.7035 & 0.0174 & 0.0016 & -0.0007 & -0.0075 & 0.0039 & -0.0046 & 0.0137 \\
\hline 0.4460 & 1.4402 & 1.5762 & 1.7534 & 0.0056 & -0.0175 & -0.0003 & 0.0037 & -0.0054 & -0.0090 & $-4.8150 \mathrm{e}-3$ \\
\hline 0.4480 & 1.4291 & 1.5622 & 1.7260 & -0.0098 & 0.0001 & 0.0279 & -0.0159 & 0.0133 & -0.0045 & 0.0127 \\
\hline
\end{tabular}




\begin{tabular}{|c|c|c|c|c|c|c|c|c|c|c|}
\hline 0.4500 & 1.3954 & 1.5708 & 1.7185 & 0.0245 & -0.0536 & 0.0186 & -0.0036 & -0.0013 & 0.0252 & -0.0195 \\
\hline 0.4520 & 1.4140 & 1.5570 & 1.6994 & 0.0198 & 0.0251 & -0.0083 & 0.0001 & -0.0022 & 0.0143 & -0.0114 \\
\hline 0.4540 & 1.4038 & 1.5786 & 1.7493 & -0.0019 & -0.0033 & 0.0050 & 0.0011 & 0.0029 & 0.0057 & 0.0118 \\
\hline 0.4560 & 1.4176 & 1.5630 & 1.7276 & 0.0068 & 0.0325 & -0.0180 & 0.0015 & 0.0099 & -0.0254 & 0.0201 \\
\hline 0.4580 & 1.3901 & 1.5513 & 1.7310 & 0.0006 & -0.0128 & 0.0118 & 0.0193 & -0.0023 & -0.0335 & $-3.6113 e-3$ \\
\hline 0.4600 & 1.3892 & 1.5778 & 1.6944 & -0.0056 & -0.0142 & -0.0096 & 0.0003 & -0.0015 & -0.0183 & -0.0217 \\
\hline 0.4620 & 1.4016 & 1.5458 & 1.7019 & 0.0170 & 0.0059 & 0.0049 & -0.0203 & -0.0238 & 0.0005 & $4.0125 \mathrm{e}-4$ \\
\hline 0.4640 & 1.4006 & 1.5537 & 1.7285 & 0.0999 & -0.0271 & 0.0145 & -0.0210 & -0.0223 & -0.0070 & $1.7061 \mathrm{e}-3$ \\
\hline 0.4660 & 1.3928 & 1.5545 & 1.6774 & 0.1670 & -0.0127 & -0.0151 & -0.0140 & -0.0088 & -0.0136 & $4.5133 \mathrm{e}-3$ \\
\hline 0.4680 & 1.3984 & 1.5520 & 1.6944 & 0.3004 & -0.0538 & -0.0004 & 0.0086 & 0.0062 & 0.0143 & $-1.3033 e-3$ \\
\hline 0.4700 & 1.4008 & 1.5430 & 1.6853 & 0.3961 & -0.0331 & -0.0204 & -0.0066 & -0.0029 & -0.0258 & $-8.1245 e-3$ \\
\hline 0.4720 & 1.3971 & 1.5435 & 1.6861 & 0.5038 & -0.0543 & -0.0475 & -0.0302 & -0.0332 & -0.0391 & -0.0185 \\
\hline 0.4740 & 1.4116 & 1.5302 & 1.6919 & 0.6237 & -0.0554 & -0.0682 & -0.0355 & -0.0403 & -0.0074 & -0.0318 \\
\hline 0.4760 & 1.4083 & 1.5314 & 1.6756 & 0.7658 & -0.0500 & -0.0403 & -0.0118 & -0.0150 & -0.0193 & -0.0248 \\
\hline 0.4780 & 1.3774 & 1.5177 & 1.6844 & 0.8818 & -0.0563 & -0.0495 & -0.0464 & -0.0474 & -0.0156 & $1.3049 \mathrm{e}-3$ \\
\hline 0.4800 & 1.3987 & 1.5327 & 1.6465 & 0.9927 & -0.0404 & -0.0465 & -0.0133 & -0.0257 & -0.0166 & -0.0186 \\
\hline 0.4820 & 1.4058 & 1.4955 & 1.6669 & 1.1105 & -0.0409 & -0.0685 & -0.0190 & -0.0389 & -0.0393 & -0.0235 \\
\hline 0.4840 & 1.3822 & 1.4991 & 1.6986 & 1.2682 & -0.0574 & -0.0507 & -0.0513 & -0.0355 & -0.0386 & -0.0171 \\
\hline 0.4860 & 1.4194 & 1.4847 & 1.6441 & 1.3740 & -0.0647 & -0.0384 & -0.0633 & -0.0358 & -0.0088 & -0.0530 \\
\hline 0.4880 & 1.4059 & 1.4905 & 1.6735 & 1.4816 & -0.0545 & -0.0607 & -0.0402 & -0.0170 & -0.0416 & $5.6175 \mathrm{e}-3$ \\
\hline 0.4900 & 1.3988 & 1.4956 & 1.6637 & 1.5490 & -0.0548 & -0.0456 & -0.0257 & -0.0290 & -0.0380 & -0.0298 \\
\hline 0.4920 & 1.3669 & 1.4759 & 1.6851 & 1.6508 & -0.0418 & -0.0406 & -0.0204 & -0.0198 & -0.0214 & -0.0104 \\
\hline 0.4940 & 1.3913 & 1.4966 & 1.6686 & 1.6768 & -0.0666 & -0.0497 & -0.0252 & -0.0623 & -0.0215 & -0.0409 \\
\hline 0.4960 & 1.3997 & 1.4943 & 1.6811 & 1.7352 & -0.0667 & -0.0456 & -0.0531 & -0.0468 & -0.0197 & -0.0163 \\
\hline 0.4980 & 1.4013 & 1.5005 & 1.6894 & 1.7522 & -0.0237 & -0.0255 & -0.0462 & -0.0130 & -0.0114 & $-8.5262 \mathrm{e}-3$ \\
\hline 0.5000 & 1.4141 & 1.5140 & 1.6911 & 1.7629 & -0.0177 & -0.0050 & -0.0079 & -0.0166 & -0.0075 & -0.0184 \\
\hline 0.5020 & 1.3896 & 1.5065 & 1.7052 & 1.7780 & 0.0113 & -0.0443 & -0.0275 & -0.0302 & -0.0049 & $9.7295 e-3$ \\
\hline 0.5040 & 1.4052 & 1.4778 & 1.6919 & 1.7747 & -0.0124 & -0.0195 & -0.0211 & -0.0464 & -0.0167 & $-1.0031 \mathrm{e}-3$ \\
\hline 0.5060 & 1.3856 & 1.5144 & 1.7060 & 1.8147 & 0.0129 & 0.0021 & -0.0306 & -0.0285 & -0.0116 & -0.0118 \\
\hline 0.5080 & 1.3976 & 1.5033 & 1.6954 & 1.7916 & -0.0164 & -0.0030 & -0.0172 & -0.0108 & -0.0225 & -0.0361 \\
\hline 0.5100 & 1.4099 & 1.5222 & 1.7044 & 1.7942 & -0.0229 & -0.0322 & -0.0249 & 0.0090 & -0.0333 & -0.0103 \\
\hline 0.5120 & 1.3767 & 1.5213 & 1.6878 & 1.8112 & -0.0121 & -0.0064 & 0.0055 & -0.0219 & -0.0142 & 0.0331 \\
\hline 0.5140 & 1.4058 & 1.5173 & 1.6919 & 1.8512 & -0.0130 & -0.0292 & -0.0228 & -0.0140 & -0.0073 & -0.0392 \\
\hline 0.5160 & 1.3794 & 1.5029 & 1.6944 & 1.8201 & -0.0041 & 0.0155 & -0.0257 & -0.0279 & -0.0192 & $-7.9242 \mathrm{e}-3$ \\
\hline 0.5180 & 1.3776 & 1.5330 & 1.6969 & 1.7969 & 0.0081 & -0.0046 & -0.0240 & -0.0125 & -0.0025 & -0.0139 \\
\hline 0.5200 & 1.3994 & 1.5320 & 1.6816 & 1.8334 & -0.0205 & 0.0043 & -0.0011 & -0.0071 & 0.0048 & -0.0131 \\
\hline 0.5220 & 1.3768 & 1.5182 & 1.6906 & 1.7977 & 0.0044 & -0.0033 & -0.0002 & 0.0043 & 0.0123 & -0.0119 \\
\hline 0.5240 & 1.3948 & 1.5044 & 1.6691 & 1.8352 & 0.0082 & -0.0202 & 0.0121 & -0.0046 & -0.0077 & $-4.8086 e-3$ \\
\hline 0.5260 & 1.3934 & 1.5122 & 1.6687 & 1.8227 & 0.0244 & 0.0049 & 0.0013 & 0.0018 & 0.0209 & -0.0161 \\
\hline 0.5280 & 1.3756 & 1.5168 & 1.6830 & 1.8441 & 0.0053 & -0.0069 & -0.0100 & 0.0303 & -0.0339 & 0.0184 \\
\hline 0.5300 & 1.3891 & 1.5142 & 1.6853 & 1.8307 & -0.0136 & 0.0292 & -0.0239 & 0.0088 & 0.0065 & -0.0243 \\
\hline 0.5320 & 1.3828 & 1.4920 & 1.6703 & 1.8548 & -0.0067 & 0.0346 & -0.0102 & -0.0114 & -0.0189 & 0.0187 \\
\hline 0.5340 & 1.3880 & 1.5023 & 1.6787 & 1.8476 & 0.0012 & 0.0161 & 0.0020 & 0.0029 & -0.0117 & $9.5289 \mathrm{e}-3$ \\
\hline 0.5360 & 1.3963 & 1.5029 & 1.6879 & 1.8743 & -0.0127 & -0.0201 & -0.0038 & 0.0027 & 0.0073 & $-3.5117 e-3$ \\
\hline 0.5380 & 1.3830 & 1.5222 & 1.6443 & 1.8583 & -0.0053 & -0.0133 & -0.0084 & -0.0209 & -0.0233 & $-4.2128 \mathrm{e}-3$ \\
\hline 0.5400 & 1.3957 & 1.5056 & 1.6546 & 1.8227 & -0.0156 & -0.0014 & -0.0003 & 0.0136 & 0.0181 & 0.0113 \\
\hline 0.5420 & 1.3823 & 1.4970 & 1.7027 & 1.8450 & -0.0218 & 0.0396 & 0.0053 & 0.0010 & -0.0027 & -0.0135 \\
\hline 0.5440 & 1.3915 & 1.4983 & 1.6861 & 1.8717 & 0.0299 & -0.0251 & -0.0079 & -0.0018 & 0.0021 & -0.0117 \\
\hline 0.5460 & 1.3633 & 1.5165 & 1.6506 & 1.8619 & 0.0075 & -0.0216 & 0.0086 & -0.0064 & -0.0068 & $3.4103 e-3$ \\
\hline 0.5480 & 1.3911 & 1.4992 & 1.6615 & 1.8227 & -0.0331 & 0.0057 & -0.0029 & -0.0158 & -0.0074 & -0.0193 \\
\hline 0.5500 & 1.3962 & 1.4913 & 1.6869 & 1.8476 & 0.0221 & -0.0021 & 0.0298 & 0.0154 & 0.0031 & $6.9219 \mathrm{e}-3$ \\
\hline 0.5520 & 1.3820 & 1.4819 & 1.6579 & 1.8307 & -0.0027 & -0.0032 & -0.0082 & 0.0094 & -0.0063 & $-6.0187 e-4$ \\
\hline 0.5540 & 1.3765 & 1.5014 & 1.6778 & 1.8289 & 0.0029 & -0.0050 & -0.0082 & 0.0024 & -0.0117 & 0.0101 \\
\hline 0.5560 & 1.3702 & 1.4948 & 1.6240 & 1.8441 & 0.0152 & -0.0072 & -0.0049 & -0.0039 & 0.0185 & $-2.5086 e-3$ \\
\hline 0.5580 & 1.3755 & 1.4677 & 1.6581 & 1.8272 & 0.0089 & -0.0278 & -0.0140 & -0.0221 & -0.0285 & $2.9094 \mathrm{e}-3$ \\
\hline 0.5600 & 1.3682 & 1.4742 & 1.6234 & 1.8672 & 0.0308 & -0.0198 & -0.0022 & 0.0145 & 0.0035 & 0.0161 \\
\hline 0.5620 & 1.3825 & 1.4864 & 1.6397 & 1.8325 & 0.0071 & 0.0162 & 0.0016 & -0.0076 & 0.0032 & $-4.8150 e-3$ \\
\hline 0.5640 & 1.3828 & 1.4695 & 1.6289 & 1.8361 & -0.0017 & 0.0020 & 0.0155 & 0.0102 & -0.0071 & $1.4044 \mathrm{e}-3$ \\
\hline 0.5660 & 1.3684 & 1.5034 & 1.6342 & 1.8316 & -0.0015 & 0.0065 & -0.0020 & 0.0064 & 0.0277 & 0.0137 \\
\hline 0.5680 & 1.3799 & 1.4675 & 1.6498 & 1.8476 & -0.0106 & 0.0151 & 0.0026 & -0.0035 & 0.0007 & $-1.9116 \mathrm{e}-3$ \\
\hline 0.5700 & 1.3643 & 1.4881 & 1.6337 & 1.8485 & 0.0169 & -0.0090 & 0.0245 & -0.0118 & -0.0089 & $9.0201 \mathrm{e}-4$ \\
\hline 0.5720 & 1.3822 & 1.4728 & 1.6248 & 1.8654 & 0.0093 & 0.0059 & -0.0020 & -0.0173 & -0.0100 & -0.0196 \\
\hline 0.5740 & 1.3571 & 1.4738 & 1.6229 & 1.8298 & 0.0037 & 0.0167 & -0.0200 & 0.0158 & 0.0053 & -0.0123 \\
\hline 0.5760 & 1.3860 & 1.4628 & 1.6177 & 1.8227 & -0.0108 & -0.0105 & 0.0001 & -0.0068 & -0.0022 & $-6.0188 e-3$ \\
\hline 0.5780 & 1.3681 & 1.4657 & 1.6116 & 1.8450 & 0.0074 & -0.0186 & -0.0052 & 0.0125 & 0.0042 & $3.8114 \mathrm{e}-3$ \\
\hline 0.5800 & 1.3952 & 1.4883 & 1.5934 & 1.8218 & -0.0031 & 0.0342 & -0.0074 & -0.0115 & -0.0174 & $-7.7233 e-3$ \\
\hline 0.5820 & 1.3449 & 1.4980 & 1.6019 & 1.8138 & 0.0106 & -0.0190 & -0.0217 & 0.0086 & -0.0116 & $6.1187 \mathrm{e}-3$ \\
\hline 0.5840 & 1.3568 & 1.4460 & 1.6168 & 1.8165 & 0.0149 & 0.0179 & 0.0108 & -0.0047 & -0.0343 & $1.1034 \mathrm{e}-3$ \\
\hline 0.5860 & 1.3835 & 1.4578 & 1.6096 & 1.8120 & -0.0050 & 0.0033 & 0.0277 & -0.0252 & -0.0013 & $-5.3170 e-3$ \\
\hline 0.5880 & 1.3915 & 1.4524 & 1.5924 & 1.8192 & -0.0196 & -0.0116 & 0.0028 & 0.0203 & -0.0189 & $4.0125 \mathrm{e}-3$ \\
\hline
\end{tabular}




\begin{tabular}{|c|c|c|c|c|c|c|c|c|c|c|}
\hline 0.5900 & 1.3723 & 1.4520 & 1.5722 & 1.8005 & 0.0783 & -0.0055 & 0.0046 & 0.0103 & 0.0036 & $-9.0281 \mathrm{e}-3$ \\
\hline 0.5920 & 1.3732 & 1.4369 & 1.5739 & 1.7752 & 0.2006 & -0.0209 & -0.0025 & -0.0002 & -0.0029 & -0.0447 \\
\hline 0.5940 & 1.3955 & 1.4662 & 1.5483 & 1.7862 & 0.3138 & -0.0309 & -0.0233 & -0.0076 & -0.0224 & -0.0287 \\
\hline 0.5960 & 1.3781 & 1.4633 & 1.5571 & 1.7642 & 0.4386 & -0.0097 & -0.0500 & -0.0063 & -0.0029 & $-6.6202 \mathrm{e}-3$ \\
\hline 0.5980 & 1.3758 & 1.4426 & 1.5338 & 1.7553 & 0.6584 & -0.0715 & -0.0532 & -0.0126 & -0.0526 & $-4.2131 \mathrm{e}-3$ \\
\hline 0.6000 & 1.3747 & 1.4265 & 1.5331 & 1.7323 & 0.7750 & -0.0369 & -0.0547 & -0.0393 & -0.0423 & $-2.6081 \mathrm{e}-3$ \\
\hline 0.6020 & 1.3811 & 1.4118 & 1.5626 & 1.7513 & 0.9357 & -0.0573 & -0.0572 & -0.0361 & -0.0300 & -0.0411 \\
\hline 0.6040 & 1.3742 & 1.4100 & 1.5324 & 1.7465 & 1.0724 & -0.0461 & -0.0622 & -0.0276 & -0.0258 & -0.0225 \\
\hline 0.6060 & 1.3928 & 1.4194 & 1.5525 & 1.7222 & 1.2068 & -0.0714 & -0.0716 & -0.0367 & -0.0482 & -0.0147 \\
\hline 0.6080 & 1.3738 & 1.4055 & 1.5397 & 1.7424 & 1.3918 & -0.0852 & -0.0574 & -0.0482 & -0.0371 & -0.0167 \\
\hline 0.6100 & 1.3872 & 1.4163 & 1.5463 & 1.7109 & 1.5165 & -0.0470 & -0.0818 & -0.0390 & -0.0646 & -0.0167 \\
\hline 0.6120 & 1.3863 & 1.4128 & 1.4946 & 1.7275 & 1.6891 & -0.0674 & -0.0363 & -0.0349 & -0.0508 & 0.0289 \\
\hline 0.6140 & 1.3699 & 1.4089 & 1.5063 & 1.7197 & 1.8048 & -0.0517 & -0.0512 & -0.0456 & -0.0663 & -0.0302 \\
\hline 0.6160 & 1.3611 & 1.3889 & 1.5443 & 1.7222 & 1.8940 & -0.0562 & -0.0832 & -0.0007 & -0.0545 & -0.0390 \\
\hline 0.6180 & 1.3658 & 1.3972 & 1.5267 & 1.7412 & 1.9418 & -0.0450 & -0.0595 & -0.0471 & -0.0329 & $-4.2131 \mathrm{e}-3$ \\
\hline 0.6200 & 1.3587 & 1.4234 & 1.5399 & 1.7289 & 1.9640 & -0.0512 & -0.0436 & -0.0399 & -0.0346 & $1.4044 \mathrm{e}-3$ \\
\hline 0.6220 & 1.3641 & 1.3950 & 1.5427 & 1.7274 & 2.0014 & -0.0225 & -0.0373 & -0.0113 & -0.0276 & $-9.0345 \mathrm{e}-3$ \\
\hline 0.6240 & 1.3658 & 1.3837 & 1.5628 & 1.7565 & 2.0244 & -0.0241 & -0.0026 & -0.0319 & -0.0169 & -0.0208 \\
\hline 0.6260 & 1.3533 & 1.4047 & 1.5291 & 1.7344 & 2.0369 & -0.0208 & -0.0111 & -0.0280 & -0.0051 & 0.0185 \\
\hline 0.6280 & 1.3607 & 1.4163 & 1.5554 & 1.7540 & 2.0149 & -0.0263 & 0.0029 & -0.0209 & -0.0268 & $-9.9317 \mathrm{e}-3$ \\
\hline 0.6300 & 1.3795 & 1.4279 & 1.5401 & 1.7320 & 2.0149 & -0.0107 & -0.0011 & -0.0233 & -0.0359 & $-1.4044 \mathrm{e}-3$ \\
\hline 0.6320 & 1.3739 & 1.4362 & 1.5618 & 1.7191 & 2.0312 & 0.0101 & 0.0129 & -0.0048 & -0.0062 & -0.0174 \\
\hline 0.6340 & 1.3510 & 1.4135 & 1.5499 & 1.7074 & 2.0264 & -0.0242 & -0.0229 & 0.0119 & 0.0056 & -0.0276 \\
\hline 0.6360 & 1.3769 & 1.4156 & 1.5340 & 1.7233 & 2.0350 & -0.0066 & 0.0249 & -0.0116 & 0.0001 & $1.2038 \mathrm{e}-3$ \\
\hline 0.6380 & 1.3921 & 1.4358 & 1.5330 & 1.7002 & 2.0043 & -0.0015 & 0.0176 & -0.0131 & 0.0012 & -0.0125 \\
\hline 0.6400 & 1.3516 & 1.4173 & 1.4931 & 1.7121 & 2.0168 & -0.0209 & 0.0097 & -0.0032 & 0.0144 & 0.0118 \\
\hline 0.6420 & 1.3649 & 1.4130 & 1.5425 & 1.7393 & 2.0101 & 0.0097 & 0.0193 & -0.0155 & 0.0125 & 0.0537 \\
\hline 0.6440 & 1.3727 & 1.4134 & 1.5155 & 1.7163 & 1.9909 & 0.0084 & -0.0044 & -0.0281 & 0.0042 & $-2.8088 \mathrm{e}-3$ \\
\hline 0.6460 & 1.3669 & 1.4070 & 1.5397 & 1.7082 & 2.0149 & -0.0180 & 0.0108 & -0.0074 & -0.0014 & $-2.2066 \mathrm{e}-3$ \\
\hline 0.6480 & 1.3809 & 1.4223 & 1.5530 & 1.7135 & 2.0034 & 0.0286 & -0.0014 & 0.0126 & -0.0020 & -0.0257 \\
\hline 0.6500 & 1.3786 & 1.3960 & 1.5316 & 1.6942 & 1.9861 & -0.0005 & 0.0294 & -0.0183 & 0.0128 & $3.8119 \mathrm{e}-3$ \\
\hline 0.6520 & 1.3603 & 1.4210 & 1.5132 & 1.6809 & 2.0187 & 0.0228 & 0.0149 & -0.0027 & 0.0024 & -0.0258 \\
\hline 0.6540 & 1.3852 & 1.3935 & 1.5492 & 1.6882 & 1.9525 & 0.0098 & -0.0105 & 0.0147 & -0.0010 & $-6.5206 \mathrm{e}-3$ \\
\hline 0.6560 & 1.3794 & 1.3928 & 1.5134 & 1.6971 & 1.9660 & 0.0040 & 0.0019 & 0.0129 & 0.0046 & $-6.3202 \mathrm{e}-3$ \\
\hline 0.6580 & 1.3758 & 1.4059 & 1.5251 & 1.7042 & 1.9698 & -0.0030 & 0.0001 & 0.0095 & 0.0067 & 0.0240 \\
\hline 0.6600 & 1.3819 & 1.4097 & 1.5062 & 1.6850 & 1.9755 & -0.0043 & 0.0077 & 0.0076 & 0.0146 & 0.0207 \\
\hline 0.6620 & 1.3632 & 1.4231 & 1.5322 & 1.6810 & 1.9536 & -0.0092 & 0.0243 & 0.0168 & -0.0016 & $5.9189 \mathrm{e}-3$ \\
\hline 0.6640 & 1.3768 & 1.4018 & 1.5100 & 1.6632 & 1.9551 & 0.0077 & 0.0382 & -0.0015 & -0.0007 & 0.0219 \\
\hline 0.6660 & 1.4063 & 1.3904 & 1.5080 & 1.7054 & 1.9285 & 0.0206 & -0.0005 & -0.0007 & -0.0005 & 0.0128 \\
\hline 0.6680 & 1.3868 & 1.4041 & 1.5096 & 1.6825 & 1.9650 & 0.0110 & 0.0102 & -0.0241 & 0.0134 & $1.3033 e-3$ \\
\hline 0.6700 & 1.3742 & 1.4086 & 1.5065 & 1.6770 & 1.9362 & -0.0050 & 0.0123 & -0.0126 & -0.0003 & $-6.8212 \mathrm{e}-3$ \\
\hline 0.6720 & 1.3746 & 1.3843 & 1.4992 & 1.6670 & 1.9099 & -0.0138 & -0.0058 & -0.0218 & 0.0091 & $-9.5289 \mathrm{e}-3$ \\
\hline 0.6740 & 1.3883 & 1.3920 & 1.5103 & 1.6836 & 1.9192 & -0.0143 & 0.0097 & 0.0152 & -0.0123 & $6.6206 e-3$ \\
\hline 0.6760 & 1.3788 & 1.3897 & 1.5120 & 1.6940 & 1.9110 & 0.0423 & 0.0222 & 0.0114 & 0.0036 & $-4.4137 \mathrm{e}-3$ \\
\hline 0.6780 & 1.3607 & 1.3933 & 1.4911 & 1.6531 & 1.9009 & 0.0209 & 0.0169 & 0.0005 & -0.0029 & $2.1074 \mathrm{e}-3$ \\
\hline 0.6800 & 1.3751 & 1.3878 & 1.5040 & 1.6746 & 1.9109 & -0.0137 & 0.0171 & -0.0222 & 0.0036 & $-9.6300 e-5$ \\
\hline 0.6820 & 1.4006 & 1.3908 & 1.4892 & 1.6726 & 1.8967 & 0.0153 & -0.0281 & 0.0008 & 0.0141 & -0.0167 \\
\hline 0.6840 & 1.3949 & 1.3911 & 1.5005 & 1.6625 & 1.8688 & 0.0154 & 0.0116 & -0.0204 & 0.0085 & -0.0249 \\
\hline 0.6860 & 1.3903 & 1.4075 & 1.4955 & 1.6772 & 1.8538 & 0.0121 & 0.0070 & 0.0038 & -0.0093 & $2.8084 \mathrm{e}-3$ \\
\hline 0.6880 & 1.4003 & 1.4029 & 1.4910 & 1.6590 & 1.8585 & 0.0154 & -0.0155 & -0.0180 & 0.0069 & $-1.0031 \mathrm{e}-3$ \\
\hline 0.6900 & 1.3797 & 1.3839 & 1.4837 & 1.6478 & 1.8493 & -0.0239 & -0.0003 & 0.0084 & -0.0088 & -0.0131 \\
\hline 0.6920 & 1.3811 & 1.3636 & 1.4709 & 1.6443 & 1.8634 & 0.0097 & 0.0139 & -0.0248 & -0.0063 & 0.0158 \\
\hline 0.6940 & 1.3959 & 1.3801 & 1.4887 & 1.6450 & 1.8466 & 0.0122 & 0.0063 & 0.0011 & -0.0035 & $8.0253 e-3$ \\
\hline 0.6960 & 1.3743 & 1.3651 & 1.5024 & 1.6203 & 1.8511 & -0.0333 & -0.0092 & 0.0106 & -0.0149 & 0.0222 \\
\hline 0.6980 & 1.3902 & 1.3918 & 1.4786 & 1.6089 & 1.8643 & -0.0024 & 0.0111 & -0.0086 & 0.0096 & $3.5113 \mathrm{e}-3$ \\
\hline 0.7000 & 1.3697 & 1.3974 & 1.4661 & 1.6407 & 1.8227 & 0.0938 & 0.0129 & -0.0221 & 0.0093 & -0.0218 \\
\hline 0.7020 & 1.3721 & 1.3887 & 1.4616 & 1.5927 & 1.8187 & 0.2156 & 0.0101 & -0.0321 & -0.0185 & $-7.0219 \mathrm{e}-4$ \\
\hline 0.7040 & 1.3718 & 1.3827 & 1.4502 & 1.5652 & 1.8091 & 0.3800 & -0.0223 & -0.0330 & -0.0174 & -0.0524 \\
\hline 0.7060 & 1.3675 & 1.3505 & 1.4523 & 1.5845 & 1.7858 & 0.5436 & -0.0185 & -0.0190 & -0.0336 & -0.0441 \\
\hline 0.7080 & 1.3938 & 1.3435 & 1.4405 & 1.5615 & 1.7697 & 0.7407 & -0.0460 & -0.0563 & -0.0294 & -0.0221 \\
\hline 0.7100 & 1.3448 & 1.3742 & 1.4335 & 1.5801 & 1.7699 & 0.9552 & -0.0340 & -0.0723 & -0.0252 & -0.0275 \\
\hline 0.7120 & 1.4038 & 1.3439 & 1.4192 & 1.5676 & 1.7526 & 1.1355 & -0.0433 & -0.0642 & -0.0315 & -0.0453 \\
\hline 0.7140 & 1.3731 & 1.3282 & 1.4244 & 1.5391 & 1.7437 & 1.3280 & -0.0511 & -0.0650 & -0.0307 & -0.0543 \\
\hline 0.7160 & 1.3629 & 1.3256 & 1.4106 & 1.5606 & 1.7459 & 1.4734 & -0.0793 & -0.0660 & -0.0416 & -0.0398 \\
\hline 0.7180 & 1.3521 & 1.3410 & 1.4440 & 1.5388 & 1.7468 & 1.6368 & -0.0730 & -0.0617 & -0.0526 & -0.0688 \\
\hline 0.7200 & 1.3628 & 1.3343 & 1.4383 & 1.5248 & 1.7426 & 1.7759 & -0.0944 & -0.0685 & -0.0572 & -0.0380 \\
\hline 0.7220 & 1.3822 & 1.3471 & 1.4151 & 1.5572 & 1.7706 & 1.8894 & -0.0466 & -0.0630 & -0.0326 & -0.0455 \\
\hline 0.7240 & 1.3823 & 1.3487 & 1.4393 & 1.5470 & 1.7255 & 1.9929 & -0.0475 & -0.0789 & -0.0615 & -0.0349 \\
\hline 0.7260 & 1.3840 & 1.3193 & 1.4196 & 1.5580 & 1.7255 & 2.0598 & -0.0445 & -0.0497 & -0.0039 & -0.0144 \\
\hline 0.7280 & 1.3688 & 1.3377 & 1.4422 & 1.5663 & 1.7294 & 2.0868 & -0.0337 & -0.0752 & -0.0314 & -0.0210 \\
\hline
\end{tabular}




\begin{tabular}{|c|c|c|c|c|c|c|c|c|c|c|}
\hline 0.7300 & 1.3798 & 1.3126 & 1.4165 & 1.5499 & 1.7648 & 2.1255 & $1.1744 \mathrm{e}-3$ & -0.0119 & -0.0254 & $-2.8087 \mathrm{e}-3$ \\
\hline 0.7320 & 1.3602 & 1.3522 & 1.4324 & 1.5648 & 1.7510 & 2.0959 & -0.0298 & $-6.8956 \mathrm{e}-4$ & $4-0.0181$ & $-8.4263 \mathrm{e}-3$ \\
\hline 0.7340 & 1.3685 & 1.3592 & 1.4330 & 1.5510 & 1.7586 & 2.0897 & -0.0292 & -0.0230 & -0.0018 & $6.5211 \mathrm{e}-3$ \\
\hline 0.7360 & 1.3768 & 1.3284 & 1.4059 & 1.5686 & 1.7690 & 2.0730 & $6.7217 \mathrm{e}-3$ & $-3.1550 \mathrm{e}-3$ & $3-0.0062$ & -0.0100 \\
\hline 0.7380 & 1.3724 & 1.3325 & 1.4514 & 1.5670 & 1.7412 & 2.0326 & $-9.3874 \mathrm{e}-3$ & $33.8453 \mathrm{e}-3$ & -0.0218 & $4.3142 \mathrm{e}-3$ \\
\hline 0.7400 & 1.3699 & 1.3596 & 1.4425 & 1.5554 & 1.7594 & 2.0362 & 0.0342 & -0.0177 & 0.0074 & 0.0204 \\
\hline 0.7420 & 1.3746 & 1.3588 & 1.4285 & 1.5286 & 1.7703 & 2.0393 & -0.0219 & $2.7613 e-3$ & -0.0057 & 0.0132 \\
\hline 0.7440 & 1.3747 & 1.3202 & 1.4036 & 1.5386 & 1.7346 & 2.0124 & 0.0110 & $7.0003 \mathrm{e}-3$ & -0.0015 & $-4.6144 \mathrm{e}-3$ \\
\hline 0.7460 & 1.4056 & 1.3336 & 1.4547 & 1.5482 & 1.7262 & 2.0175 & $8.0013 \mathrm{e}-3$ & -0.0186 & -0.0141 & 0.0155 \\
\hline 0.7480 & 1.3645 & 1.3375 & 1.4338 & 1.5640 & 1.7299 & 2.0125 & $6.4069 \mathrm{e}-4$ & $-6.2053 e-3$ & 30.0283 & $-3.3095 \mathrm{e}-3$ \\
\hline 0.7500 & 1.3863 & 1.3434 & 1.4258 & 1.5286 & 1.7312 & 1.9828 & -0.0160 & 0.0174 & 0.0074 & -0.0196 \\
\hline 0.7520 & 1.3671 & 1.3397 & 1.4048 & 1.5518 & 1.7143 & 1.9639 & $5.0152 \mathrm{e}-3$ & -0.0225 & 0.0252 & 0.0202 \\
\hline 0.7540 & 1.3687 & 1.3693 & 1.4039 & 1.5495 & 1.7673 & 1.9960 & -0.0139 & $-3.2538 \mathrm{e}-3$ & 30.0019 & -0.0123 \\
\hline 0.7560 & 1.3620 & 1.3337 & 1.4271 & 1.5278 & 1.6954 & 1.9886 & 0.0462 & 0.0366 & -0.0164 & $-4.4134 \mathrm{e}-3$ \\
\hline 0.7580 & 1.3778 & 1.3571 & 1.3838 & 1.5498 & 1.7299 & 1.9615 & 0.0162 & $-1.6759 \mathrm{e}-3$ & $3-0.0112$ & -0.0307 \\
\hline 0.7600 & 1.3602 & 1.3295 & 1.4120 & 1.5454 & 1.7031 & 1.9373 & $-7.4687 \mathrm{e}-4$ & $4-0.0151$ & -0.0041 & -0.0209 \\
\hline 0.7620 & 1.3858 & 1.3333 & 1.4368 & 1.5272 & 1.6881 & 1.9442 & $7.0328 \mathrm{e}-3$ & $8.6760 \mathrm{e}-3$ & -0.0248 & $5.3126 \mathrm{e}-3$ \\
\hline 0.7640 & 1.3732 & 1.3304 & 1.4080 & 1.5104 & 1.6865 & 1.9404 & 0.0126 & $-1.5775 \mathrm{e}-3$ & 30.0108 & -0.0168 \\
\hline 0.7660 & 1.3761 & 1.3382 & 1.4192 & 1.5175 & 1.6801 & 1.9187 & 0.0288 & 0.0155 & 0.0152 & $-7.0299 \mathrm{e}-4$ \\
\hline 0.7680 & 1.3789 & 1.3424 & 1.3959 & 1.5535 & 1.6919 & 1.9290 & $7.2559 \mathrm{e}-3$ & $-6.0134 \mathrm{e}-3$ & $3-0.0105$ & 0.0178 \\
\hline 0.7700 & 1.3543 & 1.3509 & 1.3943 & 1.5388 & 1.6890 & 1.9204 & -0.0134 & $5.9163 \mathrm{e}-3$ & 0.0176 & -0.0246 \\
\hline 0.7720 & 1.3784 & 1.3429 & 1.4003 & 1.5603 & 1.6801 & 1.8897 & $-4.4808 \mathrm{e}-3$ & $3-0.0222$ & -0.0066 & -0.0106 \\
\hline 0.7740 & 1.3661 & 1.3357 & 1.4026 & 1.5336 & 1.6888 & 1.9115 & 0.0109 & -0.0246 & 0.0140 & $4.5149 \mathrm{e}-3$ \\
\hline 0.7760 & 1.3685 & 1.3553 & 1.3864 & 1.5170 & 1.6779 & 1.8894 & $-5.6513 e-3$ & 30.0145 & 0.0021 & 0.0119 \\
\hline 0.7780 & 1.3774 & 1.3484 & 1.4142 & 1.5181 & 1.6369 & 1.9031 & -0.0160 & $-2.7605 e-3$ & 30.0056 & $4.9156 \mathrm{e}-3$ \\
\hline 0.7800 & 1.3636 & 1.3232 & 1.3932 & 1.5224 & 1.6607 & 1.8768 & $-8.0024 \mathrm{e}-3$ & $33.4503 e-3$ & 0.0256 & $6.3189 \mathrm{e}-3$ \\
\hline 0.7820 & 1.3812 & 1.3439 & 1.4140 & 1.5358 & 1.6531 & 1.8857 & $-7.5677 \mathrm{e}-3$ & $3-0.0195$ & -0.0092 & 0.0153 \\
\hline 0.7840 & 1.3567 & 1.3374 & 1.4162 & 1.5225 & 1.6706 & 1.8595 & $-2.4547 \mathrm{e}-3$ & 30.0102 & -0.0066 & $-6.0188 \mathrm{e}-3$ \\
\hline 0.7860 & 1.3600 & 1.3494 & 1.4005 & 1.5215 & 1.6660 & 1.8579 & 0.0151 & 0.0108 & 0.0110 & $-2.1074 \mathrm{e}-3$ \\
\hline 0.7880 & 1.3791 & 1.3364 & 1.3908 & 1.5121 & 1.6559 & 1.8809 & $-7.3629 \mathrm{e}-3$ & 30.0157 & 0.0114 & -0.0350 \\
\hline 0.7900 & 1.3499 & 1.3357 & 1.4155 & 1.4874 & 1.6660 & 1.8492 & $-2.3472 \mathrm{e}-3$ & 30.0152 & -0.0248 & $-4.5165 e-3$ \\
\hline 0.7920 & 1.3707 & 1.3403 & 1.3803 & 1.4832 & 1.6723 & 1.8430 & $8.3188 \mathrm{e}-3$ & 0.0216 & -0.0018 & 7.4231e-3 \\
\hline 0.7940 & 1.3541 & 1.3345 & 1.3904 & 1.5117 & 1.6673 & 1.8513 & $-3.8351 \mathrm{e}-3$ & $3-0.0253$ & 0.0063 & $9.5294 \mathrm{e}-3$ \\
\hline 0.7960 & 1.3602 & 1.2984 & 1.3644 & 1.5216 & 1.6451 & 1.8523 & 0.0181 & -0.0187 & 0.0037 & $1.0031 \mathrm{e}-4$ \\
\hline 0.7980 & 1.3474 & 1.2979 & 1.3968 & 1.4859 & 1.6356 & 1.8444 & $8.5350 \mathrm{e}-3$ & 0.0145 & 0.0010 & $-2.0063 \mathrm{e}-4$ \\
\hline 0.8000 & 1.3655 & 1.3127 & 1.4177 & 1.5108 & 1.6267 & 1.8068 & -0.0328 & $5.2259 \mathrm{e}-3$ & 0.0110 & -0.0105 \\
\hline 0.8020 & 1.3596 & 1.3081 & 1.3650 & 1.4819 & 1.6424 & 1.8135 & $-4.1537 \mathrm{e}-3$ & $3-0.0115$ & -0.0333 & 0.0183 \\
\hline 0.8040 & 1.3652 & 1.3115 & 1.3682 & 1.4580 & 1.6132 & 1.8119 & 0.0805 & $-8.6775 \mathrm{e}-3$ & $3-0.0180$ & -0.0172 \\
\hline 0.8060 & 1.3530 & 1.3388 & 1.3620 & 1.4881 & 1.6198 & 1.8010 & 0.1859 & -0.0428 & -0.0727 & $5.2162 \mathrm{e}-3$ \\
\hline 0.8080 & 1.3527 & 1.3108 & 1.3777 & 1.4463 & 1.5923 & 1.7845 & 0.3110 & -0.0367 & -0.0523 & $-2.7076 \mathrm{e}-3$ \\
\hline 0.8100 & 1.3553 & 1.3132 & 1.3586 & 1.4505 & 1.5839 & 1.7548 & 0.5091 & -0.0618 & -0.0591 & -0.0294 \\
\hline 0.8120 & 1.3409 & 1.3016 & 1.3256 & 1.4423 & 1.5991 & 1.7231 & 0.7545 & -0.0713 & -0.0988 & -0.0230 \\
\hline 0.8140 & 1.3422 & 1.3070 & 1.3534 & 1.4486 & 1.5628 & 1.7532 & 0.9886 & -0.0372 & -0.0867 & $4.6149 \mathrm{e}-3$ \\
\hline 0.8160 & 1.3467 & 1.2974 & 1.3401 & 1.4390 & 1.5799 & 1.7532 & 1.1832 & -0.0686 & -0.0758 & -0.0198 \\
\hline 0.8180 & 1.3531 & 1.2765 & 1.3475 & 1.4441 & 1.5644 & 1.7334 & 1.3886 & -0.0499 & -0.0656 & -0.0378 \\
\hline 0.8200 & 1.3395 & 1.2807 & 1.3500 & 1.4486 & 1.5802 & 1.7436 & 1.5404 & -0.0562 & -0.0392 & -0.0174 \\
\hline 0.8220 & 1.3653 & 1.2825 & 1.3828 & 1.4719 & 1.5916 & 1.7626 & 1.7185 & -0.0392 & -0.0910 & $-4.7139 \mathrm{e}-3$ \\
\hline 0.8240 & 1.3311 & 1.2845 & 1.3565 & 1.4310 & 1.5664 & 1.7469 & 1.8725 & -0.0788 & -0.0411 & -0.0110 \\
\hline 0.8260 & 1.3465 & 1.3095 & 1.3469 & 1.4535 & 1.5742 & 1.7453 & 2.0419 & -0.0390 & -0.0426 & -0.0198 \\
\hline 0.8280 & 1.3637 & 1.2781 & 1.3551 & 1.4262 & 1.5923 & 1.7546 & 2.0822 & -0.0251 & -0.0370 & $-7.8244 \mathrm{e}-3$ \\
\hline 0.8300 & 1.3502 & 1.2736 & 1.3532 & 1.4510 & 1.5700 & 1.7693 & 2.1540 & -0.0332 & -0.0177 & -0.0201 \\
\hline 0.8320 & 1.3478 & 1.2817 & 1.3673 & 1.4632 & 1.5750 & 1.7648 & 2.1748 & -0.0117 & 0.0056 & -0.0119 \\
\hline 0.8340 & 1.3563 & 1.2883 & 1.3539 & 1.4594 & 1.5860 & 1.7733 & 2.1884 & $3.4513 \mathrm{e}-3$ & 0.0084 & $-3.3108 \mathrm{e}-3$ \\
\hline 0.8360 & 1.3630 & 1.3068 & 1.4056 & 1.4710 & 1.5681 & 1.7669 & 2.1271 & -0.0158 & -0.0001 & -0.0238 \\
\hline 0.8380 & 1.3501 & 1.2846 & 1.3587 & 1.4666 & 1.5940 & 1.7644 & 2.1136 & -0.0160 & 0.0109 & $-6.5200 \mathrm{e}-3$ \\
\hline 0.8400 & 1.3416 & 1.2986 & 1.3576 & 1.4656 & 1.5948 & 1.7798 & 2.0957 & $-7.4938 \mathrm{e}-3$ & $3-0.0048$ & $8.8270 \mathrm{e}-3$ \\
\hline 0.8420 & 1.3631 & 1.3104 & 1.3735 & 1.4460 & 1.5609 & 1.7587 & 2.0667 & $-5.2251 \mathrm{e}-3$ & $3-0.0070$ & 0.0115 \\
\hline 0.8440 & 1.3654 & 1.3140 & 1.3592 & 1.4583 & 1.5816 & 1.7410 & 2.0594 & $1.6762 \mathrm{e}-3$ & -0.0010 & -0.0136 \\
\hline 0.8460 & 1.3444 & 1.3036 & 1.3545 & 1.4598 & 1.5743 & 1.7619 & 2.0334 & 0.0288 & 0.0139 & -0.0146 \\
\hline 0.8480 & 1.3533 & 1.3407 & 1.3505 & 1.4422 & 1.5716 & 1.7070 & 2.0144 & -0.0204 & 0.0094 & $-7.7233 e-3$ \\
\hline 0.8500 & 1.3398 & 1.2884 & 1.3368 & 1.4753 & 1.5501 & 1.7164 & 2.0374 & $6.5074 \mathrm{e}-3$ & 0.0241 & -0.0367 \\
\hline 0.8520 & 1.3506 & 1.2620 & 1.3458 & 1.4280 & 1.5491 & 1.7481 & 1.9728 & $-5.1267 \mathrm{e}-3$ & 30.0011 & $2.0046 \mathrm{e}-3$ \\
\hline 0.8540 & 1.3518 & 1.3006 & 1.3521 & 1.4526 & 1.5839 & 1.7357 & 1.9501 & $-8.2800 \mathrm{e}-3$ & 30.0079 & -0.0133 \\
\hline 0.8560 & 1.3512 & 1.3015 & 1.3708 & 1.4827 & 1.5650 & 1.7351 & 1.9780 & $5.7181 \mathrm{e}-3$ & 0.0018 & $6.7217 \mathrm{e}-3$ \\
\hline 0.8580 & 1.3512 & 1.3132 & 1.3562 & 1.4599 & 1.5733 & 1.7282 & 1.9501 & $-1.6759 \mathrm{e}-3$ & $3-0.0057$ & $-2.0066 \mathrm{e}-3$ \\
\hline 0.8600 & 1.3485 & 1.2958 & 1.3362 & 1.4657 & 1.5736 & 1.7458 & 1.9781 & 0.0176 & 0.0060 & $1.6050 \mathrm{e}-3$ \\
\hline 0.8620 & 1.3385 & 1.2850 & 1.3273 & 1.4493 & 1.5566 & 1.7191 & 1.9324 & -0.0171 & 0.0010 & -0.0122 \\
\hline 0.8640 & 1.3400 & 1.2902 & 1.3388 & 1.4426 & 1.5350 & 1.7284 & 1.8747 & -0.0191 & -0.0145 & -0.0199 \\
\hline 0.8660 & 1.3384 & 1.2699 & 1.3515 & 1.4384 & 1.5707 & 1.6995 & 1.9139 & $8.5829 \mathrm{e}-3$ & 0.0057 & $-3.1089 \mathrm{e}-3$ \\
\hline 0.8680 & 1.3277 & 1.2737 & 1.3448 & 1.4580 & 1.5738 & 1.7102 & 1.8942 & -0.0116 & -0.0051 & $1.1026 \mathrm{e}-3$ \\
\hline
\end{tabular}




\begin{tabular}{|c|c|c|c|c|c|c|c|c|c|c|}
\hline 0.8700 & 1.3522 & 1.2700 & 1.3511 & 1.4506 & 1.5374 & 1.6874 & 1.8967 & -0.0376 & -0.0182 & -0.0389 \\
\hline 0.8720 & 1.3371 & 1.2786 & 1.3564 & 1.4398 & 1.5445 & 1.6982 & 1.8702 & $1.6785 \mathrm{e}-3$ & 0.0132 & $-6.2210 \mathrm{e}-3$ \\
\hline 0.8740 & 1.3185 & 1.2897 & 1.3468 & 1.4426 & 1.5309 & 1.7024 & 1.8985 & $1.1838 \mathrm{e}-3$ & 0.0062 & $4.7139 \mathrm{e}-3$ \\
\hline 0.8760 & 1.3322 & 1.2845 & 1.3365 & 1.4511 & 1.5383 & 1.6971 & 1.9031 & $6.4092 \mathrm{e}-3$ & 0.0433 & -0.0185 \\
\hline 0.8780 & 1.3185 & 1.3141 & 1.3648 & 1.4372 & 1.5463 & 1.6793 & 1.8889 & -0.0232 & -0.0020 & $4.6144 \mathrm{e}-3$ \\
\hline 0.8800 & 1.3303 & 1.3006 & 1.3403 & 1.4272 & 1.5636 & 1.6682 & 1.8865 & -0.0113 & 0.0067 & $-6.0187 \mathrm{e}-4$ \\
\hline 0.8820 & 1.3507 & 1.2813 & 1.3337 & 1.4234 & 1.5620 & 1.6911 & 1.8488 & -0.0101 & -0.0102 & $-8.0250 \mathrm{e}-4$ \\
\hline 0.8840 & 1.3416 & 1.2824 & 1.3570 & 1.4261 & 1.5235 & 1.6896 & 1.8614 & -0.0119 & -0.0058 & 0.0000 \\
\hline 0.8860 & 1.3510 & 1.2798 & 1.3347 & 1.4342 & 1.5416 & 1.6936 & 1.8555 & $5.7194 \mathrm{e}-3$ & 0.0079 & $-5.9176 \mathrm{e}-3$ \\
\hline 0.8880 & 1.3160 & 1.2675 & 1.3600 & 1.4468 & 1.5403 & 1.6915 & 1.8118 & -0.0168 & 0.0090 & -0.0267 \\
\hline 0.8900 & 1.3186 & 1.2705 & 1.3739 & 1.4724 & 1.5324 & 1.6942 & 1.8481 & -0.0130 & 0.0105 & $-6.5179 \mathrm{e}-3$ \\
\hline 0.8920 & 1.3302 & 1.2797 & 1.3385 & 1.4318 & 1.5474 & 1.6723 & 1.8486 & $-8.3822 \mathrm{e}-$ & 30.0039 & -0.0375 \\
\hline 0.8940 & 1.3387 & 1.2986 & 1.3366 & 1.4572 & 1.5348 & 1.6722 & 1.8474 & $-9.7616 \mathrm{e}-$ & $3-0.0042$ & 0.0205 \\
\hline 0.8960 & 1.3167 & 1.3080 & 1.3164 & 1.4354 & 1.5322 & 1.6757 & 1.8160 & $1.7756 \mathrm{e}-3$ & 0.0111 & $7.0219 \mathrm{e}-3$ \\
\hline 0.8980 & 1.3110 & 1.3080 & 1.3400 & 1.4471 & 1.5142 & 1.6694 & 1.8238 & $8.7797 \mathrm{e}-3$ & 0.0045 & $4.4137 \mathrm{e}-3$ \\
\hline 0.9000 & 1.3032 & 1.2797 & 1.3380 & 1.4486 & 1.5071 & 1.6798 & 1.8238 & -0.0100 & 0.0062 & $5.0076 \mathrm{e}-4$ \\
\hline 0.9020 & 1.3179 & 1.2817 & 1.3505 & 1.4637 & 1.5178 & 1.6835 & 1.7961 & -0.0236 & 0.0035 & $-1.5039 \mathrm{e}-3$ \\
\hline 0.9040 & 1.3130 & 1.2905 & 1.3384 & 1.4510 & 1.5264 & 1.6712 & 1.8053 & 0.0324 & -0.0206 & -0.0273 \\
\hline 0.9060 & 1.3047 & 1.2804 & 1.3554 & 1.4099 & 1.5199 & 1.6657 & 1.7766 & 0.1027 & -0.0286 & 0.0140 \\
\hline 0.9080 & 1.3003 & 1.2782 & 1.3284 & 1.4184 & 1.5104 & 1.6364 & 1.7728 & 0.2172 & -0.0450 & -0.0396 \\
\hline 0.9100 & 1.3324 & 1.2849 & 1.3304 & 1.4243 & 1.5038 & 1.6461 & 1.7647 & 0.3954 & -0.0256 & -0.0132 \\
\hline 0.9120 & 1.3240 & 1.2809 & 1.3282 & 1.4289 & 1.4831 & 1.6086 & 1.7810 & 0.5642 & -0.0355 & -0.0616 \\
\hline 0.9140 & 1.3151 & 1.2883 & 1.3106 & 1.4160 & 1.4893 & 1.6249 & 1.7698 & 0.8103 & -0.0479 & -0.0573 \\
\hline 0.9160 & 1.3160 & 1.2899 & 1.2926 & 1.4080 & 1.4823 & 1.6037 & 1.7144 & 1.0670 & -0.0900 & -0.0802 \\
\hline 0.9180 & 1.3363 & 1.2667 & 1.2757 & 1.3972 & 1.4666 & 1.6135 & 1.7241 & 1.3308 & -0.0665 & -0.0790 \\
\hline 0.9200 & 1.3178 & 1.2660 & 1.3075 & 1.4039 & 1.4783 & 1.6055 & 1.7240 & 1.5820 & -0.0796 & -0.0597 \\
\hline 0.9220 & 1.3247 & 1.2751 & 1.3296 & 1.4325 & 1.4677 & 1.6017 & 1.7386 & 1.7043 & -0.0338 & -0.0402 \\
\hline 0.9240 & 1.2941 & 1.2694 & 1.3028 & 1.4089 & 1.4735 & 1.5913 & 1.7394 & 1.8257 & -0.0641 & -0.0178 \\
\hline 0.9260 & 1.2973 & 1.2710 & 1.3158 & 1.4026 & 1.4812 & 1.5901 & 1.7409 & 1.9990 & -0.0614 & -0.0370 \\
\hline 0.9280 & 1.3043 & 1.2699 & 1.3230 & 1.3977 & 1.4715 & 1.6277 & 1.7438 & 2.1184 & -0.0434 & -0.0330 \\
\hline 0.9300 & 1.2989 & 1.2902 & 1.3362 & 1.4068 & 1.4840 & 1.6284 & 1.7403 & 2.1416 & -0.0559 & -0.0224 \\
\hline 0.9320 & 1.2994 & 1.2597 & 1.3402 & 1.4051 & 1.4789 & 1.6273 & 1.7573 & 2.1730 & -0.0327 & -0.0293 \\
\hline 0.9340 & 1.3055 & 1.2611 & 1.3214 & 1.4033 & 1.4982 & 1.6325 & 1.7485 & 2.1821 & -0.0401 & -0.0350 \\
\hline 0.9360 & 1.3078 & 1.2862 & 1.3205 & 1.4078 & 1.4838 & 1.6158 & 1.7559 & 2.1406 & 0.0029 & $-8.6269 \mathrm{e}-3$ \\
\hline 0.9380 & 1.2940 & 1.2579 & 1.3177 & 1.4215 & 1.5178 & 1.6061 & 1.7439 & 2.1786 & -0.0003 & $7.0219 \mathrm{e}-3$ \\
\hline 0.9400 & 1.2923 & 1.2522 & 1.3388 & 1.4215 & 1.5121 & 1.6341 & 1.7509 & 2.0945 & 0.0245 & -0.0111 \\
\hline 0.9420 & 1.3039 & 1.2538 & 1.3355 & 1.4092 & 1.5108 & 1.6170 & 1.7512 & 2.0597 & -0.0043 & $8.4259 \mathrm{e}-3$ \\
\hline 0.9440 & 1.2862 & 1.2753 & 1.3452 & 1.4274 & 1.5203 & 1.6383 & 1.7553 & 2.0764 & 0.0259 & $-1.8051 \mathrm{e}-3$ \\
\hline 0.9460 & 1.2836 & 1.2801 & 1.3450 & 1.4365 & 1.4859 & 1.6431 & 1.7655 & 2.0809 & 0.0174 & -0.0257 \\
\hline 0.9480 & 1.3062 & 1.2932 & 1.3200 & 1.4495 & 1.5043 & 1.6111 & 1.7451 & 2.0299 & -0.0340 & $-1.9067 \mathrm{e}-3$ \\
\hline 0.9500 & 1.3140 & 1.2849 & 1.3103 & 1.4417 & 1.4918 & 1.6209 & 1.7209 & 2.0263 & -0.0074 & $-9.2271 \mathrm{e}-3$ \\
\hline 0.9520 & 1.2750 & 1.2894 & 1.3383 & 1.4323 & 1.4799 & 1.6083 & 1.7274 & 1.9904 & 0.0297 & -0.0232 \\
\hline 0.9540 & 1.3028 & 1.2756 & 1.3373 & 1.4287 & 1.4838 & 1.6249 & 1.7155 & 1.9562 & 0.0104 & $1.4044 \mathrm{e}-3$ \\
\hline 0.9560 & 1.2813 & 1.2626 & 1.3346 & 1.4424 & 1.5024 & 1.6153 & 1.7397 & 1.9455 & -0.0100 & $-1.6050 \mathrm{e}-3$ \\
\hline 0.9580 & 1.2921 & 1.2793 & 1.3245 & 1.4331 & 1.5042 & 1.5974 & 1.7327 & 1.9084 & -0.0239 & $1.4045 \mathrm{e}-3$ \\
\hline 0.9600 & 1.2813 & 1.2413 & 1.3354 & 1.4281 & 1.4623 & 1.5955 & 1.7327 & 1.9350 & -0.0068 & $-5.9192 \mathrm{e}-3$ \\
\hline 0.9620 & 1.2852 & 1.2859 & 1.2889 & 1.4099 & 1.4750 & 1.6080 & 1.7226 & 1.9037 & -0.0030 & -0.0306 \\
\hline 0.9640 & 1.2997 & 1.2713 & 1.3219 & 1.4290 & 1.4632 & 1.5936 & 1.7238 & 1.8987 & 0.0080 & -0.0154 \\
\hline 0.9660 & 1.2953 & 1.2749 & 1.3005 & 1.4096 & 1.4899 & 1.6282 & 1.7481 & 1.8983 & -0.0132 & $8.5262 \mathrm{e}-3$ \\
\hline 0.9680 & 1.2939 & 1.2634 & 1.3171 & 1.4113 & 1.4519 & 1.6022 & 1.6985 & 1.8792 & 0.0074 & $4.3126 e-3$ \\
\hline 0.9700 & 1.2651 & 1.2583 & 1.3166 & 1.3972 & 1.5053 & 1.6233 & 1.6751 & 1.8679 & -0.0369 & -0.0192 \\
\hline 0.9720 & 1.2844 & 1.2446 & 1.3024 & 1.4191 & 1.4908 & 1.6076 & 1.7042 & 1.8640 & -0.0119 & 0.0115 \\
\hline 0.9740 & 1.2862 & 1.2558 & 1.3072 & 1.4172 & 1.4504 & 1.5880 & 1.7037 & 1.8827 & -0.0160 & $7.0227 \mathrm{e}-4$ \\
\hline 0.9760 & 1.2704 & 1.2453 & 1.3301 & 1.4229 & 1.4532 & 1.6244 & 1.7048 & 1.8556 & -0.0188 & -0.0131 \\
\hline 0.9780 & 1.2849 & 1.2390 & 1.3291 & 1.4167 & 1.4906 & 1.5978 & 1.6912 & 1.8603 & -0.0272 & -0.0184 \\
\hline 0.9800 & 1.2618 & 1.2832 & 1.3151 & 1.4381 & 1.4623 & 1.6248 & 1.6960 & 1.8526 & -0.0469 & -0.0281 \\
\hline 0.9820 & 1.2750 & 1.2564 & 1.3192 & 1.4351 & 1.4791 & 1.5887 & 1.6723 & 1.8390 & -0.0234 & -0.0247 \\
\hline 0.9840 & 1.2878 & 1.2776 & 1.2993 & 1.4423 & 1.4893 & 1.5793 & 1.7116 & 1.8582 & -0.0301 & -0.0310 \\
\hline 0.9860 & 1.3040 & 1.2528 & 1.3264 & 1.4331 & 1.5060 & 1.6078 & 1.6755 & 1.8381 & -0.0116 & -0.0181 \\
\hline 0.9880 & 1.2690 & 1.2701 & 1.3302 & 1.4230 & 1.4814 & 1.5863 & 1.6837 & 1.8565 & -0.0163 & -0.0112 \\
\hline 0.9900 & 1.2733 & 1.2450 & 1.3152 & 1.4311 & 1.4950 & 1.5951 & 1.6929 & 1.8081 & -0.0270 & $-4.6080 e-3$ \\
\hline 0.9920 & 1.2837 & 1.2606 & 1.3136 & 1.4428 & 1.4636 & 1.5951 & 1.6768 & 1.8442 & -0.0075 & -0.0116 \\
\hline 0.9940 & 1.2603 & 1.2661 & 1.3366 & 1.4701 & 1.4787 & 1.6012 & 1.6882 & 1.8165 & -0.0046 & 0.0211 \\
\hline 0.9960 & 1.2885 & 1.2635 & 1.3388 & 1.4301 & 1.4802 & 1.6197 & 1.7013 & 1.8182 & -0.0160 & -0.0203 \\
\hline 0.9980 & 1.2808 & 1.2709 & 1.3561 & 1.4614 & 1.4768 & 1.6187 & 1.6806 & 1.8063 & -0.0001 & -0.0110 \\
\hline 1.0000 & 1.2704 & 1.2782 & 1.3224 & 1.4496 & 1.4772 & 1.5949 & 1.7137 & 1.8097 & -0.0058 & $-9.5289 \mathrm{e}-3$ \\
\hline 1.0020 & 1.2739 & 1.3029 & 1.3249 & 1.4640 & 1.4514 & 1.5932 & 1.6770 & 1.8182 & -0.0036 & -0.0104 \\
\hline 1.0040 & 1.2939 & 1.2760 & 1.3112 & 1.4509 & 1.4699 & 1.6144 & 1.6686 & 1.7917 & -0.0324 & $-4.0125 \mathrm{e}-4$ \\
\hline 1.0060 & 1.2721 & 1.2736 & 1.3446 & 1.4455 & 1.4317 & 1.6188 & 1.6830 & 1.7830 & 0.0045 & -0.0135 \\
\hline 1.0080 & 1.2546 & 1.2618 & 1.3591 & 1.4336 & 1.4415 & 1.5838 & 1.6704 & 1.7867 & 0.0061 & -0.0408 \\
\hline
\end{tabular}




\begin{tabular}{|c|c|c|c|c|c|c|c|c|c|c|}
\hline 1.0100 & 1.2693 & 1.2326 & 1.3170 & 1.4762 & 1.4441 & 1.5871 & 1.6880 & 1.7885 & 0.0855 & -0.0417 \\
\hline 1.0120 & 1.2628 & 1.2451 & 1.3270 & 1.4477 & 1.4321 & 1.6043 & 1.6857 & 1.7703 & 0.2564 & -0.0832 \\
\hline 1.0140 & 1.2838 & 1.2792 & 1.3219 & 1.4552 & 1.4435 & 1.5867 & 1.6476 & 1.7450 & 0.5124 & -0.0133 \\
\hline 1.0160 & 1.2973 & 1.2467 & 1.3091 & 1.4357 & 1.4388 & 1.6183 & 1.6498 & 1.7176 & 0.7429 & -0.0535 \\
\hline 1.0180 & 1.2655 & 1.2619 & 1.2964 & 1.4267 & 1.4464 & 1.5744 & 1.6470 & 1.7338 & 0.9777 & -0.0638 \\
\hline 1.0200 & 1.2739 & 1.2777 & 1.3250 & 1.4365 & 1.4370 & 1.5841 & 1.6209 & 1.6826 & 1.2042 & -0.0330 \\
\hline 1.0220 & 1.2737 & 1.2625 & 1.3008 & 1.4318 & 1.4187 & 1.5929 & 1.6460 & 1.7286 & 1.4309 & -0.0499 \\
\hline 1.0240 & 1.2537 & 1.2373 & 1.3151 & 1.4157 & 1.4246 & 1.5672 & 1.6404 & 1.7103 & 1.6009 & -0.0740 \\
\hline 1.0260 & 1.2724 & 1.2326 & 1.3187 & 1.4153 & 1.4360 & 1.5862 & 1.6088 & 1.6947 & 1.7546 & -0.0528 \\
\hline 1.0280 & 1.2558 & 1.2352 & 1.3125 & 1.4432 & 1.4794 & 1.6154 & 1.6209 & 1.7045 & 1.9246 & -0.0328 \\
\hline 1.0300 & 1.2802 & 1.2200 & 1.3362 & 1.4367 & 1.4386 & 1.5974 & 1.6240 & 1.7137 & 2.0772 & -0.0480 \\
\hline 1.0320 & 1.2705 & 1.2342 & 1.3112 & 1.4556 & 1.4291 & 1.5917 & 1.5959 & 1.7160 & 2.1484 & -0.0386 \\
\hline 1.0340 & 1.2617 & 1.2474 & 1.3052 & 1.4527 & 1.4450 & 1.5965 & 1.6239 & 1.6895 & 2.1859 & -0.0470 \\
\hline 1.0360 & 1.2406 & 1.2720 & 1.3168 & 1.4464 & 1.4825 & 1.6083 & 1.6600 & 1.7222 & 2.1660 & $-9.3287 e-3$ \\
\hline 1.0380 & 1.2579 & 1.2672 & 1.3104 & 1.4621 & 1.4755 & 1.6362 & 1.6537 & 1.7281 & 2.1608 & $-8.5322 \mathrm{e}-3$ \\
\hline 1.0400 & 1.2806 & 1.2683 & 1.3209 & 1.4468 & 1.4679 & 1.6162 & 1.6458 & 1.7173 & 2.1210 & $2.0064 \mathrm{e}-3$ \\
\hline 1.0420 & 1.2701 & 1.2964 & 1.3215 & 1.4873 & 1.4789 & 1.6448 & 1.6675 & 1.7416 & 2.1091 & $-6.0188 \mathrm{e}-3$ \\
\hline 1.0440 & 1.2681 & 1.2807 & 1.3166 & 1.4621 & 1.4395 & 1.6328 & 1.6801 & 1.7354 & 2.1087 & $3.0094 \mathrm{e}-3$ \\
\hline 1.0460 & 1.2523 & 1.2653 & 1.3478 & 1.4665 & 1.4710 & 1.6554 & 1.6816 & 1.7333 & 2.0662 & -0.0315 \\
\hline 1.0480 & 1.2563 & 1.2750 & 1.3326 & 1.4846 & 1.4614 & 1.6732 & 1.6867 & 1.7227 & 2.0960 & -0.0419 \\
\hline 1.0500 & 1.2730 & 1.2569 & 1.3287 & 1.4879 & 1.5078 & 1.6316 & 1.6481 & 1.7346 & 2.0608 & $-5.1164 e-3$ \\
\hline 1.0520 & 1.2662 & 1.2881 & 1.3326 & 1.4771 & 1.5022 & 1.6617 & 1.6580 & 1.7022 & 2.0454 & $-9.6316 e-3$ \\
\hline 1.0540 & 1.2604 & 1.3111 & 1.3410 & 1.4486 & 1.4760 & 1.6535 & 1.6849 & 1.6810 & 2.0163 & $-6.2194 \mathrm{e}-3$ \\
\hline 1.0560 & 1.2677 & 1.2986 & 1.3267 & 1.4728 & 1.4888 & 1.6739 & 1.6658 & 1.6939 & 2.0444 & -0.0260 \\
\hline 1.0580 & 1.2579 & 1.3055 & 1.3338 & 1.4653 & 1.4804 & 1.6734 & 1.6607 & 1.7236 & 1.9816 & $-5.8186 e-3$ \\
\hline 1.0600 & 1.2650 & 1.3048 & 1.3120 & 1.4502 & 1.4658 & 1.6820 & 1.6532 & 1.7027 & 1.9940 & -0.0246 \\
\hline 1.0620 & 1.2873 & 1.2900 & 1.3071 & 1.4495 & 1.4835 & 1.6711 & 1.6732 & 1.7224 & 1.9952 & -0.0233 \\
\hline 1.0640 & 1.2632 & 1.2643 & 1.3137 & 1.4830 & 1.4584 & 1.6797 & 1.6772 & 1.6753 & 1.9946 & -0.0152 \\
\hline 1.0660 & 1.2684 & 1.2752 & 1.3222 & 1.4513 & 1.5098 & 1.6528 & 1.6710 & 1.6827 & 1.9631 & -0.0204 \\
\hline 1.0680 & 1.2594 & 1.2775 & 1.3303 & 1.4766 & 1.4707 & 1.6711 & 1.6413 & 1.6882 & 1.9539 & -0.0394 \\
\hline 1.0700 & 1.2679 & 1.3074 & 1.3131 & 1.4505 & 1.4653 & 1.6629 & 1.6811 & 1.6980 & 1.9590 & $-2.2069 \mathrm{e}-3$ \\
\hline 1.0720 & 1.2608 & 1.3109 & 1.3294 & 1.4307 & 1.4742 & 1.6776 & 1.6607 & 1.6855 & 1.9542 & $7.3236 \mathrm{e}-3$ \\
\hline 1.0740 & 1.2550 & 1.3200 & 1.3169 & 1.4364 & 1.4525 & 1.6616 & 1.6660 & 1.6474 & 1.9325 & -0.0128 \\
\hline 1.0760 & 1.2690 & 1.2972 & 1.3000 & 1.4608 & 1.4829 & 1.6699 & 1.6478 & 1.6494 & 1.9388 & -0.0191 \\
\hline 1.0780 & 1.2795 & 1.2794 & 1.3259 & 1.4646 & 1.4609 & 1.6756 & 1.6442 & 1.6724 & 1.9248 & -0.0261 \\
\hline 1.0800 & 1.2774 & 1.2915 & 1.3111 & 1.4756 & 1.5065 & 1.6730 & 1.6617 & 1.6804 & 1.8984 & -0.0336 \\
\hline 1.0820 & 1.2745 & 1.2961 & 1.3256 & 1.4896 & 1.4818 & 1.6546 & 1.6929 & 1.6436 & 1.9462 & $2.6097 \mathrm{e}-3$ \\
\hline 1.0840 & 1.2752 & 1.2849 & 1.3256 & 1.4374 & 1.4990 & 1.6677 & 1.6619 & 1.6709 & 1.9159 & -0.0132 \\
\hline 1.0860 & 1.2698 & 1.2905 & 1.3487 & 1.4792 & 1.4729 & 1.6807 & 1.6547 & 1.6814 & 1.9357 & 0.0213 \\
\hline 1.0880 & 1.2661 & 1.3241 & 1.3489 & 1.4752 & 1.4991 & 1.6884 & 1.6675 & 1.6454 & 1.9305 & $5.8181 \mathrm{e}-3$ \\
\hline 1.0900 & 1.2753 & 1.3278 & 1.3252 & 1.4545 & 1.5209 & 1.6928 & 1.6814 & 1.6544 & 1.9006 & -0.0173 \\
\hline 1.0920 & 1.2617 & 1.3193 & 1.3383 & 1.4756 & 1.5057 & 1.6880 & 1.6797 & 1.6554 & 1.9066 & $3.7120 \mathrm{e}-3$ \\
\hline 1.0940 & 1.2640 & 1.3368 & 1.3252 & 1.4883 & 1.4938 & 1.6853 & 1.6762 & 1.6645 & 1.8898 & -0.0423 \\
\hline 1.0960 & 1.2810 & 1.3499 & 1.3392 & 1.4966 & 1.5197 & 1.6686 & 1.6886 & 1.6682 & 1.8894 & $8.9270 \mathrm{e}-3$ \\
\hline 1.0980 & 1.2737 & 1.3560 & 1.3295 & 1.4818 & 1.5344 & 1.7124 & 1.6438 & 1.6339 & 1.8861 & 0.0182 \\
\hline 1.1000 & 1.2743 & 1.3392 & 1.3277 & 1.4794 & 1.5067 & 1.7065 & 1.6697 & 1.6363 & 1.8825 & -0.0219 \\
\hline 1.1020 & 1.2956 & 1.3370 & 1.3229 & 1.4899 & 1.5276 & 1.7092 & 1.6684 & 1.6533 & 1.8713 & $2.2069 \mathrm{e}-3$ \\
\hline 1.1040 & 1.2666 & 1.3212 & 1.3456 & 1.4863 & 1.5394 & 1.7054 & 1.6680 & 1.6285 & 1.8782 & -0.0189 \\
\hline 1.1060 & 1.2555 & 1.3501 & 1.3428 & 1.4681 & 1.5446 & 1.7129 & 1.6638 & 1.6674 & 1.8714 & 0.0147 \\
\hline 1.1080 & 1.2815 & 1.3394 & 1.3299 & 1.4659 & 1.4925 & 1.6842 & 1.6653 & 1.6248 & 1.8665 & 0.0150 \\
\hline 1.1100 & 1.2698 & 1.3301 & 1.3347 & 1.4412 & 1.5264 & 1.7108 & 1.6737 & 1.6183 & 1.8748 & 0.0198 \\
\hline 1.1120 & 1.2894 & 1.3367 & 1.2801 & 1.4810 & 1.4966 & 1.6918 & 1.6481 & 1.6205 & 1.8675 & -0.0170 \\
\hline 1.1140 & 1.2909 & 1.3496 & 1.2914 & 1.4615 & 1.5379 & 1.7046 & 1.6595 & 1.6025 & 1.8834 & $6.6287 \mathrm{e}-3$ \\
\hline 1.1160 & 1.2693 & 1.3095 & 1.2854 & 1.4422 & 1.4765 & 1.6865 & 1.6528 & 1.6112 & 1.8432 & 0.1164 \\
\hline 1.1180 & 1.2835 & 1.3070 & 1.3034 & 1.4684 & 1.4708 & 1.6769 & 1.6568 & 1.5865 & 1.8480 & 0.3096 \\
\hline 1.1200 & 1.2738 & 1.3069 & 1.3015 & 1.4369 & 1.4836 & 1.6739 & 1.6435 & 1.5983 & 1.8216 & 0.5436 \\
\hline 1.1220 & 1.2910 & 1.3248 & 1.2881 & 1.4443 & 1.5316 & 1.7106 & 1.6509 & 1.5748 & 1.8189 & 0.8540 \\
\hline 1.1240 & 1.3015 & 1.3168 & 1.2861 & 1.4310 & 1.4822 & 1.6825 & 1.6547 & 1.5593 & 1.7962 & 1.1630 \\
\hline 1.1260 & 1.2765 & 1.3079 & 1.2910 & 1.4522 & 1.4841 & 1.7161 & 1.6577 & 1.5526 & 1.7980 & 1.4329 \\
\hline 1.1280 & 1.2825 & 1.3188 & 1.3213 & 1.4427 & 1.5199 & 1.6854 & 1.6323 & 1.5234 & 1.7958 & 1.6647 \\
\hline 1.1300 & 1.2869 & 1.3479 & 1.3001 & 1.4357 & 1.4853 & 1.6982 & 1.6394 & 1.5259 & 1.7972 & 1.8596 \\
\hline 1.1320 & 1.2835 & 1.3418 & 1.2746 & 1.4479 & 1.5254 & 1.6826 & 1.6534 & 1.5472 & 1.8091 & 2.0026 \\
\hline 1.1340 & 1.2747 & 1.3290 & 1.2820 & 1.4165 & 1.4914 & 1.7252 & 1.6583 & 1.5322 & 1.8229 & 2.1184 \\
\hline 1.1360 & 1.2776 & 1.3379 & 1.2984 & 1.4412 & 1.4929 & 1.6886 & 1.6296 & 1.5566 & 1.8086 & 2.2358 \\
\hline 1.1380 & 1.3031 & 1.3203 & 1.3271 & 1.4420 & 1.4829 & 1.7297 & 1.6620 & 1.5249 & 1.8281 & 2.2449 \\
\hline 1.1400 & 1.2897 & 1.3228 & 1.2792 & 1.4249 & 1.5183 & 1.7227 & 1.6458 & 1.5410 & 1.8309 & 2.2067 \\
\hline 1.1420 & 1.2940 & 1.3567 & 1.3184 & 1.4688 & 1.5197 & 1.7247 & 1.6487 & 1.5690 & 1.8240 & 2.1902 \\
\hline 1.1440 & 1.2758 & 1.3480 & 1.3106 & 1.4390 & 1.5211 & 1.7448 & 1.6482 & 1.5514 & 1.8295 & 2.1481 \\
\hline 1.1460 & 1.2817 & 1.3568 & 1.3021 & 1.4597 & 1.5590 & 1.7511 & 1.6455 & 1.5345 & 1.8117 & 2.1197 \\
\hline 1.1480 & 1.2798 & 1.3651 & 1.2973 & 1.4641 & 1.5376 & 1.7734 & 1.6366 & 1.5803 & 1.7934 & 2.1120 \\
\hline
\end{tabular}




\begin{tabular}{|c|c|c|c|c|c|c|c|c|c|c|}
\hline 1.1500 & 1.2843 & 1.3480 & 1.2885 & 1.4509 & 1.5454 & 1.7361 & 1.6745 & 1.5509 & 1.8037 & 2.0984 \\
\hline 1.1520 & 1.2997 & 1.3629 & 1.2824 & 1.4376 & 1.5279 & 1.7735 & 1.6420 & 1.5566 & 1.7914 & 2.0632 \\
\hline 1.1540 & 1.3028 & 1.3810 & 1.2948 & 1.4658 & 1.5615 & 1.7950 & 1.6781 & 1.5433 & 1.8021 & 2.0871 \\
\hline 1.1560 & 1.2947 & 1.3729 & 1.2986 & 1.4274 & 1.5132 & 1.7881 & 1.6568 & 1.5872 & 1.7952 & 2.0634 \\
\hline 1.1580 & 1.3092 & 1.3910 & 1.2994 & 1.4568 & 1.5560 & 1.7910 & 1.6781 & 1.5621 & 1.7804 & 2.0427 \\
\hline 1.1600 & 1.2904 & 1.3992 & 1.3095 & 1.4491 & 1.5235 & 1.7834 & 1.6597 & 1.5366 & 1.7595 & 2.0395 \\
\hline 1.1620 & 1.3234 & 1.3733 & 1.2876 & 1.4552 & 1.5381 & 1.7901 & 1.6435 & 1.5447 & 1.7476 & 2.0462 \\
\hline 1.1640 & 1.3111 & 1.3729 & 1.2673 & 1.4535 & 1.5183 & 1.7887 & 1.6617 & 1.5731 & 1.7407 & 2.0061 \\
\hline 1.1660 & 1.3043 & 1.3492 & 1.2707 & 1.4369 & 1.5186 & 1.7589 & 1.6894 & 1.5314 & 1.7296 & 1.9961 \\
\hline 1.1680 & 1.2927 & 1.3354 & 1.2544 & 1.4331 & 1.5144 & 1.7739 & 1.6652 & 1.5311 & 1.7392 & 1.9955 \\
\hline 1.1700 & 1.3032 & 1.3247 & 1.2637 & 1.4269 & 1.5025 & 1.7842 & 1.6649 & 1.5362 & 1.6952 & 1.9472 \\
\hline 1.1720 & 1.2915 & 1.3582 & 1.2674 & 1.4350 & 1.5080 & 1.7848 & 1.6561 & 1.5300 & 1.7089 & 1.9411 \\
\hline 1.1740 & 1.3049 & 1.3427 & 1.2397 & 1.4090 & 1.5033 & 1.7737 & 1.6711 & 1.5293 & 1.6834 & 1.9404 \\
\hline 1.1760 & 1.2966 & 1.3516 & 1.2661 & 1.4049 & 1.5149 & 1.7909 & 1.6536 & 1.5466 & 1.6883 & 1.9355 \\
\hline 1.1780 & 1.3148 & 1.3786 & 1.2649 & 1.3961 & 1.4968 & 1.7914 & 1.6388 & 1.5336 & 1.6509 & 1.9348 \\
\hline 1.1800 & 1.3136 & 1.3577 & 1.2631 & 1.4096 & 1.4558 & 1.7557 & 1.6244 & 1.5345 & 1.6533 & 1.9096 \\
\hline 1.1820 & 1.3100 & 1.3652 & 1.2446 & 1.3990 & 1.4973 & 1.7747 & 1.6546 & 1.5294 & 1.6710 & 1.9053 \\
\hline 1.1840 & 1.3017 & 1.3572 & 1.2780 & 1.4046 & 1.4960 & 1.7949 & 1.6758 & 1.5423 & 1.6728 & 1.9249 \\
\hline 1.1860 & 1.3108 & 1.3537 & 1.2635 & 1.4187 & 1.4926 & 1.7887 & 1.6284 & 1.5502 & 1.6614 & 1.9231 \\
\hline 1.1880 & 1.3238 & 1.3432 & 1.2842 & 1.3949 & 1.5054 & 1.7973 & 1.6205 & 1.5552 & 1.6536 & 1.8823 \\
\hline 1.1900 & 1.3136 & 1.3638 & 1.3058 & 1.4244 & 1.5119 & 1.8199 & 1.6416 & 1.5284 & 1.6635 & 1.8878 \\
\hline 1.1920 & 1.3262 & 1.3628 & 1.2915 & 1.4179 & 1.4993 & 1.8241 & 1.6299 & 1.5280 & 1.6420 & 1.9053 \\
\hline 1.1940 & 1.3135 & 1.3867 & 1.2652 & 1.4037 & 1.4803 & 1.8212 & 1.6415 & 1.5404 & 1.6436 & 1.8918 \\
\hline 1.1960 & 1.2876 & 1.4086 & 1.2929 & 1.4043 & 1.5062 & 1.8268 & 1.6652 & 1.5346 & 1.6488 & 1.8738 \\
\hline 1.1980 & 1.3174 & 1.3877 & 1.3169 & 1.3978 & 1.4982 & 1.8187 & 1.6410 & 1.5487 & 1.6632 & 1.8833 \\
\hline 1.2000 & 1.3055 & 1.3874 & 1.3092 & 1.4303 & 1.4900 & 1.8406 & 1.6818 & 1.5630 & 1.6656 & 1.8616 \\
\hline 1.2020 & 1.3240 & 1.3915 & 1.2751 & 1.4167 & 1.5086 & 1.8216 & 1.6582 & 1.5356 & 1.6604 & 1.8669 \\
\hline 1.2040 & 1.3291 & 1.4030 & 1.2854 & 1.3896 & 1.4974 & 1.8357 & 1.6655 & 1.5203 & 1.6655 & 1.8795 \\
\hline 1.2060 & 1.3167 & 1.3789 & 1.2885 & 1.4196 & 1.5117 & 1.8628 & 1.6985 & 1.5211 & 1.6691 & 1.8446 \\
\hline 1.2080 & 1.3268 & 1.3834 & 1.2857 & 1.3805 & 1.4947 & 1.8443 & 1.6982 & 1.5668 & 1.6727 & 1.8602 \\
\hline 1.2100 & 1.3273 & 1.3939 & 1.3142 & 1.4108 & 1.4809 & 1.8157 & 1.6622 & 1.5566 & 1.6845 & 1.8352 \\
\hline 1.2120 & 1.3108 & 1.4056 & 1.2818 & 1.3878 & 1.4710 & 1.8248 & 1.6781 & 1.5595 & 1.6817 & 1.8560 \\
\hline 1.2140 & 1.3158 & 1.4056 & 1.2796 & 1.4119 & 1.4646 & 1.8490 & 1.6751 & 1.5496 & 1.6896 & 1.8490 \\
\hline 1.2160 & 1.3217 & 1.3950 & 1.2453 & 1.3811 & 1.4799 & 1.8457 & 1.6486 & 1.5544 & 1.7152 & 1.8181 \\
\hline 1.2180 & 1.3347 & 1.3746 & 1.2919 & 1.3926 & 1.4427 & 1.8387 & 1.6517 & 1.5333 & 1.7238 & 1.8406 \\
\hline 1.2200 & 1.3133 & 1.3723 & 1.2810 & 1.3413 & 1.4521 & 1.8243 & 1.6670 & 1.5436 & 1.7187 & 1.8295 \\
\hline 1.2220 & 1.3229 & 1.3743 & 1.2862 & 1.3783 & 1.4720 & 1.8339 & 1.6889 & 1.5487 & 1.7263 & 1.8185 \\
\hline 1.2240 & 1.3287 & 1.4025 & 1.2682 & 1.3864 & 1.4383 & 1.8483 & 1.6604 & 1.5279 & 1.7040 & 1.8297 \\
\hline 1.2260 & 1.3275 & 1.3686 & 1.2515 & 1.3813 & 1.4409 & 1.8586 & 1.6782 & 1.5269 & 1.7541 & 1.8033 \\
\hline 1.2280 & 1.3298 & 1.3730 & 1.2676 & 1.3726 & 1.4238 & 1.8442 & 1.6620 & 1.5322 & 1.7122 & 1.8288 \\
\hline 1.2300 & 1.3371 & 1.3743 & 1.2584 & 1.3444 & 1.4355 & 1.8214 & 1.6451 & 1.5277 & 1.6813 & 1.8030 \\
\hline 1.2320 & 1.3329 & 1.3904 & 1.2893 & 1.3560 & 1.4236 & 1.8475 & 1.6573 & 1.5328 & 1.7091 & 1.8263 \\
\hline 1.2340 & 1.3270 & 1.3793 & 1.2726 & 1.3603 & 1.4233 & 1.8136 & 1.6611 & 1.5555 & 1.7135 & 1.8115 \\
\hline 1.2360 & 1.3166 & 1.3712 & 1.2685 & 1.3615 & 1.4166 & 1.8412 & 1.6436 & 1.5442 & 1.6961 & 1.8028 \\
\hline 1.2380 & 1.3223 & 1.3648 & 1.2995 & 1.3672 & 1.4191 & 1.8651 & 1.6586 & 1.5576 & 1.6722 & 1.7602 \\
\hline 1.2400 & 1.3149 & 1.3715 & 1.2935 & 1.3701 & 1.4280 & 1.8379 & 1.6525 & 1.5428 & 1.6851 & 1.7660 \\
\hline 1.2420 & 1.3206 & 1.3671 & 1.3190 & 1.3548 & 1.4042 & 1.8621 & 1.6661 & 1.5441 & 1.6964 & 1.7840 \\
\hline 1.2440 & 1.3276 & 1.3873 & 1.2687 & 1.3744 & 1.4317 & 1.8265 & 1.6733 & 1.5958 & 1.6772 & 1.7615 \\
\hline 1.2460 & 1.3249 & 1.4195 & 1.3254 & 1.3459 & 1.4021 & 1.8727 & 1.7090 & 1.5404 & 1.6885 & 1.7896 \\
\hline 1.2480 & 1.3460 & 1.4086 & 1.3171 & 1.3487 & 1.4105 & 1.8586 & 1.6957 & 1.5677 & 1.6832 & 1.7941 \\
\hline 1.2500 & 1.3365 & 1.4028 & 1.3131 & 1.3686 & 1.4186 & 1.8686 & 1.6533 & 1.5910 & 1.6952 & 1.7393 \\
\hline 1.2520 & 1.3297 & 1.4093 & 1.3047 & 1.3767 & 1.4138 & 1.8543 & 1.6806 & 1.5805 & 1.6928 & 1.7896 \\
\hline 1.2540 & 1.3354 & 1.4063 & 1.3050 & 1.3660 & 1.4063 & 1.8625 & 1.7068 & 1.5672 & 1.7297 & 1.7655 \\
\hline 1.2560 & 1.3156 & 1.4084 & 1.3269 & 1.3839 & 1.4064 & 1.8229 & 1.7087 & 1.5669 & 1.7112 & 1.7434 \\
\hline 1.2580 & 1.3208 & 1.3968 & 1.3093 & 1.3613 & 1.3851 & 1.8828 & 1.6999 & 1.5642 & 1.6912 & 1.7567 \\
\hline 1.2600 & 1.3383 & 1.4090 & 1.3062 & 1.3438 & 1.3907 & 1.8521 & 1.7075 & 1.5581 & 1.6962 & 1.7478 \\
\hline 1.2620 & 1.3287 & 1.4044 & 1.3372 & 1.3358 & 1.3893 & 1.8517 & 1.7075 & 1.5867 & 1.6406 & 1.7225 \\
\hline 1.2640 & 1.3251 & 1.3980 & 1.2984 & 1.3542 & 1.3814 & 1.8504 & 1.7169 & 1.5648 & 1.7053 & 1.7403 \\
\hline 1.2660 & 1.3304 & 1.3940 & 1.3264 & 1.3282 & 1.3567 & 1.8321 & 1.6963 & 1.5584 & 1.6666 & 1.7286 \\
\hline 1.2680 & 1.3374 & 1.4148 & 1.2974 & 1.3128 & 1.3680 & 1.8326 & 1.7223 & 1.5581 & 1.6806 & 1.7506 \\
\hline 1.2700 & 1.3397 & 1.3735 & 1.2748 & 1.3152 & 1.3343 & 1.8510 & 1.7037 & 1.5776 & 1.6838 & 1.7140 \\
\hline 1.2720 & 1.3140 & 1.3434 & 1.2965 & 1.3261 & 1.3360 & 1.8462 & 1.7005 & 1.5478 & 1.6674 & 1.6869 \\
\hline 1.2740 & 1.3105 & 1.3686 & 1.2931 & 1.3322 & 1.3336 & 1.8309 & 1.6671 & 1.5562 & 1.6639 & 1.6504 \\
\hline 1.2760 & 1.3023 & 1.3712 & 1.2898 & 1.3146 & 1.3253 & 1.8300 & 1.6995 & 1.5852 & 1.6736 & 1.6174 \\
\hline 1.2780 & 1.3178 & 1.3600 & 1.2761 & 1.3230 & 1.3501 & 1.8246 & 1.7113 & 1.5486 & 1.6557 & 1.5602 \\
\hline 1.2800 & 1.2954 & 1.3725 & 1.2757 & 1.3394 & 1.2856 & 1.8299 & 1.7019 & 1.5711 & 1.6454 & 1.5448 \\
\hline 1.2820 & 1.3103 & 1.3620 & 1.2972 & 1.3104 & 1.2960 & 1.8083 & 1.6864 & 1.5563 & 1.6560 & 1.4912 \\
\hline 1.2840 & 1.3123 & 1.3712 & 1.2796 & 1.3115 & 1.3087 & 1.8074 & 1.6858 & 1.5391 & 1.6794 & 1.4915 \\
\hline 1.2860 & 1.3271 & 1.3645 & 1.2689 & 1.3207 & 1.2856 & 1.8260 & 1.6972 & 1.5564 & 1.6787 & 1.4416 \\
\hline 1.2880 & 1.3295 & 1.3500 & 1.2789 & 1.3215 & 1.2594 & 1.7967 & 1.7038 & 1.5442 & 1.6820 & 1.4325 \\
\hline
\end{tabular}




\begin{tabular}{|c|c|c|c|c|c|c|c|c|c|c|}
\hline 1.2900 & 1.3153 & 1.3366 & 1.3034 & 1.3007 & 1.2759 & 1.8108 & 1.6888 & 1.5639 & 1.6324 & 1.4134 \\
\hline 1.2920 & 1.2773 & 1.3487 & 1.2824 & 1.3282 & 1.2931 & 1.7969 & 1.7172 & 1.5414 & 1.6742 & 1.3766 \\
\hline 1.2940 & 1.3105 & 1.3335 & 1.2890 & 1.3144 & 1.2665 & 1.8178 & 1.7324 & 1.5564 & 1.6707 & 1.3490 \\
\hline 1.2960 & 1.3097 & 1.3438 & 1.3028 & 1.3499 & 1.2741 & 1.8149 & 1.7716 & 1.5559 & 1.6487 & 1.3092 \\
\hline 1.2980 & 1.3074 & 1.3666 & 1.2951 & 1.3207 & 1.2540 & 1.7971 & 1.7448 & 1.5379 & 1.6618 & 1.2837 \\
\hline 1.3000 & 1.2866 & 1.3449 & 1.3043 & 1.3071 & 1.2401 & 1.8112 & 1.7475 & 1.5613 & 1.6575 & 1.2704 \\
\hline 1.3020 & 1.3047 & 1.3568 & 1.2818 & 1.3118 & 1.2473 & 1.7812 & 1.7599 & 1.5910 & 1.6609 & 1.2436 \\
\hline 1.3040 & 1.2916 & 1.3309 & 1.2811 & 1.3290 & 1.2219 & 1.8066 & 1.7513 & 1.6032 & 1.6390 & 1.2198 \\
\hline 1.3060 & 1.2709 & 1.3436 & 1.3026 & 1.3202 & 1.2333 & 1.7868 & 1.7677 & 1.5852 & 1.6732 & 1.2265 \\
\hline 1.3080 & 1.2653 & 1.3473 & 1.3013 & 1.3075 & 1.2486 & 1.7839 & 1.7514 & 1.5962 & 1.6732 & 1.2198 \\
\hline 1.3100 & 1.2904 & 1.3386 & 1.3046 & 1.3352 & 1.2248 & 1.8001 & 1.7806 & 1.5846 & 1.6410 & 1.2198 \\
\hline 1.3120 & 1.2828 & 1.3309 & 1.3162 & 1.3312 & 1.2233 & 1.7690 & 1.7629 & 1.5670 & 1.6348 & 1.2198 \\
\hline 1.3140 & 1.2773 & 1.3477 & 1.3101 & 1.3155 & 1.1843 & 1.8163 & 1.7976 & 1.5767 & 1.6510 & 1.2198 \\
\hline 1.3160 & 1.2742 & 1.3333 & 1.2835 & 1.3101 & 1.1734 & 1.7862 & 1.7935 & 1.5858 & 1.6547 & 1.2198 \\
\hline 1.3180 & 1.2608 & 1.3496 & 1.3003 & 1.3103 & 1.1627 & 1.7879 & 1.8091 & 1.5836 & 1.6375 & 1.2198 \\
\hline 1.3200 & 1.2491 & 1.3409 & 1.2713 & 1.3006 & 1.1681 & 1.7777 & 1.7962 & 1.5648 & 1.6506 & 1.2198 \\
\hline 1.3220 & 1.2322 & 1.2969 & 1.2911 & 1.2958 & 1.1666 & 1.7436 & 1.7784 & 1.5809 & 1.6295 & 1.2198 \\
\hline 1.3240 & 1.2264 & 1.2968 & 1.2848 & 1.3459 & 1.1736 & 1.7462 & 1.8059 & 1.6003 & 1.6454 & 1.2198 \\
\hline 1.3260 & 1.2211 & 1.3113 & 1.2709 & 1.3087 & 1.1370 & 1.7478 & 1.7856 & 1.5897 & 1.6092 & 1.2198 \\
\hline 1.3280 & 1.1949 & 1.2751 & 1.2635 & 1.3014 & 1.1264 & 1.7309 & 1.7947 & 1.5685 & 1.6122 & 1.2198 \\
\hline 1.3300 & 1.2132 & 1.2857 & 1.2521 & 1.2792 & 1.1218 & 1.7270 & 1.8198 & 1.5940 & 1.6110 & 1.2198 \\
\hline 1.3320 & 1.1875 & 1.2957 & 1.2514 & 1.2660 & 1.1148 & 1.7100 & 1.8258 & 1.5674 & 1.6250 & 1.2198 \\
\hline 1.3340 & 1.1644 & 1.2926 & 1.2826 & 1.3061 & 1.1087 & 1.6999 & 1.7909 & 1.5882 & 1.5861 & 1.2198 \\
\hline 1.3360 & 1.1644 & 1.2762 & 1.2650 & 1.3130 & 1.1068 & 1.7138 & 1.8071 & 1.5851 & 1.6272 & 1.2198 \\
\hline 1.3380 & 1.1439 & 1.2922 & 1.2814 & 1.2788 & 1.0912 & 1.7492 & 1.8003 & 1.5648 & 1.6105 & 1.2198 \\
\hline 1.3400 & 1.1173 & 1.2626 & 1.2946 & 1.3014 & 1.0970 & 1.6999 & 1.8046 & 1.5711 & 1.6087 & 1.2198 \\
\hline 1.3420 & 1.1121 & 1.2471 & 1.2809 & 1.3093 & 1.0777 & 1.7329 & 1.8125 & 1.5673 & 1.6194 & 1.2198 \\
\hline 1.3440 & 1.1020 & 1.2842 & 1.3038 & 1.3185 & 1.0836 & 1.6945 & 1.8157 & 1.6002 & 1.6095 & 1.2198 \\
\hline 1.3460 & 1.0809 & 1.2450 & 1.2808 & 1.3259 & 1.0999 & 1.7051 & 1.8287 & 1.5820 & 1.6318 & 1.2198 \\
\hline 1.3480 & 1.0826 & 1.2797 & 1.2756 & 1.3361 & 1.1100 & 1.7021 & 1.8234 & 1.5909 & 1.5781 & 1.2198 \\
\hline 1.3500 & 1.0811 & 1.2851 & 1.3233 & 1.3278 & 1.0380 & 1.6722 & 1.8056 & 1.5865 & 1.6187 & 1.2198 \\
\hline 1.3520 & 1.0605 & 1.3110 & 1.2955 & 1.3283 & 1.0892 & 1.7105 & 1.8622 & 1.5839 & 1.6060 & 1.2198 \\
\hline 1.3540 & 1.0464 & 1.2859 & 1.2957 & 1.3401 & 1.0584 & 1.6997 & 1.8836 & 1.6166 & 1.5655 & 1.2198 \\
\hline 1.3560 & 1.0437 & 1.2702 & 1.3142 & 1.3192 & 1.0646 & 1.6983 & 1.8458 & 1.6088 & 1.6278 & 1.2198 \\
\hline 1.3580 & 1.0367 & 1.2672 & 1.2861 & 1.3220 & 1.0642 & 1.6629 & 1.8932 & 1.6078 & 1.5991 & 1.2198 \\
\hline 1.3600 & 1.0214 & 1.2426 & 1.3111 & 1.3232 & 1.0513 & 1.6913 & 1.8846 & 1.6253 & 1.5877 & 1.2198 \\
\hline 1.3620 & 1.0041 & 1.2660 & 1.2760 & 1.3517 & 1.0737 & 1.6831 & 1.8645 & 1.6061 & 1.5814 & 1.2198 \\
\hline 1.3640 & 0.9836 & 1.2282 & 1.2960 & 1.3258 & 1.0578 & 1.6843 & 1.8997 & 1.6059 & 1.5802 & 1.2198 \\
\hline 1.3660 & 0.9894 & 1.2453 & 1.3072 & 1.3285 & 1.0833 & 1.6849 & 1.9040 & 1.5751 & 1.5817 & 1.2198 \\
\hline 1.3680 & 0.9866 & 1.2624 & 1.2842 & 1.3013 & 1.0537 & 1.6959 & 1.8803 & 1.6400 & 1.5851 & 1.2198 \\
\hline 1.3700 & 0.9828 & 1.2677 & 1.2551 & 1.3281 & 1.0325 & 1.6208 & 1.8819 & 1.6113 & 1.5711 & 1.2198 \\
\hline 1.3720 & 0.9577 & 1.2488 & 1.2703 & 1.3433 & 1.0411 & 1.6944 & 1.9069 & 1.5717 & 1.5779 & 1.2198 \\
\hline 1.3740 & 0.9663 & 1.2183 & 1.2730 & 1.3134 & 1.0087 & 1.6795 & 1.9059 & 1.6019 & 1.5612 & 1.2198 \\
\hline 1.3760 & 0.9577 & 1.2096 & 1.2571 & 1.3251 & 1.0197 & 1.6657 & 1.9128 & 1.6150 & 1.5591 & 1.2198 \\
\hline 1.3780 & 0.9578 & 1.2178 & 1.2536 & 1.2980 & 1.0145 & 1.6564 & 1.8919 & 1.5968 & 1.5459 & 1.2198 \\
\hline 1.3800 & 0.9577 & 1.2336 & 1.2685 & 1.3029 & 1.0053 & 1.6659 & 1.9039 & 1.5981 & 1.5453 & 1.2198 \\
\hline 1.3820 & 0.9577 & 1.1921 & 1.2935 & 1.3356 & 1.0062 & 1.6566 & 1.9181 & 1.5935 & 1.5699 & 1.2198 \\
\hline 1.3840 & 0.9577 & 1.2307 & 1.2530 & 1.3054 & 1.0037 & 1.6335 & 1.9220 & 1.6049 & 1.5235 & 1.2198 \\
\hline 1.3860 & 0.9577 & 1.2001 & 1.2601 & 1.3217 & 1.0233 & 1.6264 & 1.8694 & 1.6037 & 1.5546 & 1.2198 \\
\hline 1.3880 & 0.9577 & 1.2162 & 1.2405 & 1.3150 & 0.9781 & 1.6456 & 1.9079 & 1.6131 & 1.5471 & 1.2198 \\
\hline 1.3900 & 0.9577 & 1.2046 & 1.2563 & 1.3095 & 0.9948 & 1.6447 & 1.9098 & 1.6157 & 1.5603 & 1.2198 \\
\hline 1.3920 & 0.9577 & 1.2189 & 1.2514 & 1.3306 & 1.0047 & 1.6510 & 1.8975 & 1.6041 & 1.5334 & 1.2198 \\
\hline 1.3940 & 0.9577 & 1.1907 & 1.2579 & 1.3068 & 0.9622 & 1.6120 & 1.9142 & 1.6258 & 1.5553 & 1.2198 \\
\hline 1.3960 & 0.9577 & 1.2030 & 1.2537 & 1.3278 & 1.0012 & 1.6382 & 1.9188 & 1.6170 & 1.5185 & 1.2198 \\
\hline 1.3980 & 0.9577 & 1.1850 & 1.2917 & 1.3343 & 0.9684 & 1.6438 & 1.8900 & 1.6241 & 1.5286 & 1.2198 \\
\hline 1.4000 & 0.9577 & 1.2049 & 1.2477 & 1.3358 & 0.9980 & 1.6701 & 1.8953 & 1.6181 & 1.5134 & 1.2198 \\
\hline 1.4020 & 0.9577 & 1.2071 & 1.2806 & 1.3273 & 1.0015 & 1.6661 & 1.9326 & 1.6067 & 1.5344 & 1.2198 \\
\hline 1.4040 & 0.9577 & 1.2399 & 1.2758 & 1.3453 & 1.0060 & 1.6442 & 1.9135 & 1.6392 & 1.5354 & 1.2198 \\
\hline 1.4060 & 0.9577 & 1.2099 & 1.2625 & 1.3323 & 1.0025 & 1.6516 & 1.9436 & 1.6319 & 1.5504 & 1.2198 \\
\hline 1.4080 & 0.9577 & 1.2081 & 1.2787 & 1.3404 & 0.9876 & 1.6271 & 1.9153 & 1.6364 & 1.5622 & 1.2198 \\
\hline 1.4100 & 0.9577 & 1.2141 & 1.2429 & 1.3579 & 1.0024 & 1.6098 & 1.9222 & 1.6679 & 1.5276 & 1.2198 \\
\hline 1.4120 & 0.9577 & 1.1847 & 1.2710 & 1.3560 & 0.9836 & 1.6455 & 1.9317 & 1.6594 & 1.5550 & 1.2198 \\
\hline 1.4140 & 0.9577 & 1.1889 & 1.2617 & 1.3157 & 0.9915 & 1.6071 & 1.9286 & 1.6643 & 1.5498 & 1.2198 \\
\hline 1.4160 & 0.9577 & 1.1728 & 1.2898 & 1.3555 & 0.9808 & 1.6520 & 1.9704 & 1.6439 & 1.5331 & 1.2198 \\
\hline 1.4180 & 0.9577 & 1.1823 & 1.2603 & 1.3451 & 0.9843 & 1.6259 & 1.9349 & 1.6486 & 1.5492 & 1.2198 \\
\hline 1.4200 & 0.9577 & 1.1867 & 1.2490 & 1.3411 & 0.9857 & 1.6328 & 1.9376 & 1.6550 & 1.5384 & 1.2198 \\
\hline 1.4220 & 0.9577 & 1.1867 & 1.2710 & 1.3355 & 1.0020 & 1.6168 & 1.9495 & 1.6496 & 1.5250 & 1.2198 \\
\hline 1.4240 & 0.9577 & 1.1872 & 1.2233 & 1.3290 & 0.9813 & 1.6234 & 1.9396 & 1.6809 & 1.5223 & 1.2198 \\
\hline 1.4260 & 0.9577 & 1.1762 & 1.2305 & 1.3436 & 0.9422 & 1.6364 & 1.9162 & 1.6482 & 1.5163 & 1.2198 \\
\hline 1.4280 & 0.9577 & 1.1522 & 1.2339 & 1.3608 & 0.9692 & 1.6089 & 1.9253 & 1.6590 & 1.5329 & 1.2198 \\
\hline
\end{tabular}




\begin{tabular}{|c|c|c|c|c|c|c|c|c|c|c|}
\hline 1.4300 & 0.9577 & 1.1569 & 1.2271 & 1.3168 & 0.9599 & 1.6290 & 1.9335 & 1.6719 & 1.5144 & 1.2198 \\
\hline 1.4320 & 0.9577 & 1.1366 & 1.2283 & 1.3415 & 0.9636 & 1.6126 & 1.9064 & 1.6651 & 1.5228 & 1.2198 \\
\hline 1.4340 & 0.9577 & 1.1349 & 1.2307 & 1.3341 & 0.9415 & 1.5951 & 1.8943 & 1.6357 & 1.5051 & 1.2198 \\
\hline 1.4360 & 0.9577 & 1.1521 & 1.2472 & 1.3303 & 0.9262 & 1.6345 & 1.8968 & 1.6455 & 1.5091 & 1.2198 \\
\hline 1.4380 & 0.9577 & 1.1567 & 1.2089 & 1.3537 & 0.9578 & 1.5933 & 1.9082 & 1.6539 & 1.4983 & 1.2198 \\
\hline 1.4400 & 0.9577 & 1.1519 & 1.2274 & 1.3427 & 0.9254 & 1.6136 & 1.8960 & 1.6408 & 1.4616 & 1.2198 \\
\hline 1.4420 & 0.9577 & 1.1346 & 1.1786 & 1.3162 & 0.9470 & 1.6011 & 1.8725 & 1.6513 & 1.4866 & 1.2198 \\
\hline 1.4440 & 0.9577 & 1.1293 & 1.2148 & 1.3235 & 0.9416 & 1.5864 & 1.9227 & 1.6376 & 1.4881 & 1.2198 \\
\hline 1.4460 & 0.9577 & 1.1298 & 1.2157 & 1.3054 & 0.9407 & 1.6081 & 1.9328 & 1.6538 & 1.5011 & 1.2198 \\
\hline 1.4480 & 0.9577 & 1.1336 & 1.2290 & 1.3390 & 0.9382 & 1.5713 & 1.9100 & 1.6737 & 1.4572 & 1.2198 \\
\hline 1.4500 & 0.9577 & 1.1153 & 1.2404 & 1.3425 & 0.9256 & 1.5927 & 1.8919 & 1.6738 & 1.5019 & 1.2198 \\
\hline 1.4520 & 0.9577 & 1.1043 & 1.2311 & 1.3714 & 0.9470 & 1.5893 & 1.9100 & 1.6610 & 1.4849 & 1.2198 \\
\hline 1.4540 & 0.9577 & 1.1509 & 1.2409 & 1.3552 & 0.9337 & 1.6076 & 1.8794 & 1.6728 & 1.5127 & 1.2198 \\
\hline 1.4560 & 0.9577 & 1.1323 & 1.2186 & 1.3530 & 0.9418 & 1.6227 & 1.9197 & 1.6836 & 1.5086 & 1.2198 \\
\hline 1.4580 & 0.9577 & 1.1310 & 1.2429 & 1.3322 & 0.9167 & 1.6127 & 1.9384 & 1.7032 & 1.4713 & 1.2198 \\
\hline 1.4600 & 0.9577 & 1.1233 & 1.2148 & 1.3570 & 0.9277 & 1.6295 & 1.9239 & 1.6875 & 1.4767 & 1.2198 \\
\hline 1.4620 & 0.9577 & 1.1147 & 1.2143 & 1.3364 & 0.9224 & 1.5909 & 1.9206 & 1.7008 & 1.4858 & 1.2198 \\
\hline 1.4640 & 0.9577 & 1.1130 & 1.2302 & 1.3563 & 0.9112 & 1.5968 & 1.9433 & 1.7163 & 1.5193 & 1.2198 \\
\hline 1.4660 & 0.9577 & 1.1163 & 1.2411 & 1.3621 & 0.9250 & 1.5890 & 1.9141 & 1.7130 & 1.5139 & 1.2198 \\
\hline 1.4680 & 0.9577 & 1.1160 & 1.2416 & 1.3678 & 0.9248 & 1.5861 & 1.9285 & 1.7181 & 1.4853 & 1.2198 \\
\hline 1.4700 & 0.9577 & 1.1115 & 1.2236 & 1.3678 & 0.9355 & 1.6080 & 1.9199 & 1.7130 & 1.5313 & 1.2198 \\
\hline 1.4720 & 0.9577 & 1.1007 & 1.2563 & 1.3374 & 0.9210 & 1.5862 & 1.9307 & 1.7149 & 1.4923 & 1.2198 \\
\hline 1.4740 & 0.9577 & 1.0933 & 1.2495 & 1.3566 & 0.9080 & 1.5647 & 1.9404 & 1.7354 & 1.4952 & 1.2198 \\
\hline 1.4760 & 0.9577 & 1.0978 & 1.1975 & 1.3721 & 0.9028 & 1.5918 & 1.9278 & 1.7221 & 1.4730 & 1.2198 \\
\hline 1.4780 & 0.9577 & 1.0735 & 1.1986 & 1.3815 & 0.8937 & 1.5681 & 1.9252 & 1.7406 & 1.4915 & 1.2198 \\
\hline 1.4800 & 0.9577 & 1.0861 & 1.1870 & 1.3709 & 0.8952 & 1.5801 & 1.9137 & 1.7100 & 1.4528 & 1.2198 \\
\hline 1.4820 & 0.9577 & 1.0694 & 1.2148 & 1.3641 & 0.8784 & 1.5841 & 1.8936 & 1.7173 & 1.4683 & 1.2198 \\
\hline 1.4840 & 0.9577 & 1.0627 & 1.1973 & 1.3326 & 0.8728 & 1.5508 & 1.9064 & 1.7385 & 1.5019 & 1.2198 \\
\hline 1.4860 & 0.9577 & 1.0670 & 1.2271 & 1.3538 & 0.8771 & 1.5525 & 1.9100 & 1.7303 & 1.4760 & 1.2198 \\
\hline 1.4880 & 0.9577 & 1.0627 & 1.2158 & 1.3679 & 0.8712 & 1.5764 & 1.9024 & 1.7524 & 1.4922 & 1.2198 \\
\hline 1.4900 & 0.9577 & 1.0358 & 1.2098 & 1.3773 & 0.8881 & 1.5689 & 1.8906 & 1.7329 & 1.4812 & 1.2198 \\
\hline 1.4920 & 0.9577 & 1.0686 & 1.1963 & 1.3684 & 0.8610 & 1.6013 & 1.8898 & 1.7341 & 1.4880 & 1.2198 \\
\hline 1.4940 & 0.9577 & 1.0672 & 1.1989 & 1.3737 & 0.8558 & 1.5570 & 1.9279 & 1.7133 & 1.4754 & 1.2198 \\
\hline 1.4960 & 0.9577 & 1.1005 & 1.2021 & 1.3737 & 0.8433 & 1.5628 & 1.9015 & 1.7691 & 1.4933 & 1.2198 \\
\hline 1.4980 & 0.9577 & 1.0740 & 1.1952 & 1.3629 & 0.8428 & 1.5643 & 1.8989 & 1.7601 & 1.5166 & 1.2198 \\
\hline 1.5000 & 0.9577 & 1.0513 & 1.1887 & 1.3500 & 0.8314 & 1.5509 & 1.8965 & 1.7732 & 1.4997 & 1.2198 \\
\hline
\end{tabular}


Appendix $\mathrm{H}-$ Gauge data for experiment 2S-68.

\begin{tabular}{|c|c|c|c|c|c|c|c|c|c|c|c|}
\hline-0.1000 & $1.3105 \mathrm{e}-3$ & 0.0159 & 0.0117 & -0.0042 & -0.0154 & 0.0137 & 0.0198 & -0.0169 & -0.0135 & -0.0003 & -0.0128 \\
\hline-0.0980 & 0.0126 & $8.0539 \mathrm{e}-3$ & $-5.5609 \mathrm{e}-3$ & 30.0039 & 0.0121 & 0.0250 & 0.0096 & -0.0225 & 0.0085 & 0.0033 & 0.0000 \\
\hline-0.0960 & 0.0113 & 0.0170 & 0.0105 & -0.0103 & 0.0076 & 0.0113 & -0.0072 & -0.0094 & 0.0034 & 0.0032 & 0.0000 \\
\hline-0.0940 & 0.0165 & $9.6891 \mathrm{e}-3$ & $-1.3714 \mathrm{e}-3$ & 30.0062 & 0.0072 & 0.0158 & -0.0168 & 0.0010 & 0.0203 & 0.0012 & 0.0000 \\
\hline 0.0920 & 0.0147 & $6.4150 \mathrm{e}-3$ & $5.1290 \mathrm{e}-3$ & 0.0170 & -0.0088 & -0.0074 & -0.0146 & -0.0154 & -0.0276 & -0.0186 & 0.0128 \\
\hline-0.0900 & $5.1865 \mathrm{e}-3$ & 0.0179 & $6.7136 \mathrm{e}-3$ & -0.0103 & 0.0179 & 0.0141 & -0.0026 & 0.0304 & -0.0252 & -0.0031 & 0.0000 \\
\hline-0.0880 & 0.0176 & $-4.9877 \mathrm{e}-3$ & $39.0236 \mathrm{e}-3$ & 0.0007 & 0.0050 & 0.0301 & -0.0208 & 0.0158 & 0.0046 & -0.0403 & -0.0128 \\
\hline-0.0860 & 0.0120 & $-8.4059 \mathrm{e}-3$ & $31.4437 \mathrm{e}-3$ & -0.0020 & -0.0014 & 0.0072 & 0.0019 & -0.0061 & -0.0263 & -0.0101 & -0.0128 \\
\hline-0.0840 & $2.2800 \mathrm{e}-4$ & 0.0151 & $6.8581 \mathrm{e}-3$ & 0.0096 & -0.0033 & 0.0138 & 0.0313 & 0.0163 & -0.0026 & -0.0127 & -0.0128 \\
\hline-0.0820 & $1.6416 \mathrm{e}-4$ & 0.0286 & 0.0160 & -0.0079 & 0.0092 & 0.0118 & -0.0069 & -0.0156 & 0.0067 & 0.0146 & 0.0000 \\
\hline-0.0800 & $-7.4054 \mathrm{e}-2$ & $41.2111 \mathrm{e}-3$ & 0.0333 & -0.0001 & 0.0103 & -0.0276 & -0.0105 & 0.0155 & 0.0096 & -0.0145 & 0.0000 \\
\hline 0.0780 & 0.0126 & $-5.7000 e-4$ & $45.5586 e-3$ & 0.0015 & 0.0145 & 0.0011 & -0.0105 & -0.0295 & -0.0207 & -0.0100 & .0257 \\
\hline 0.0760 & $8.7780 \mathrm{e}-3$ & $-3.7766 \mathrm{e}-3$ & $3-0.0281$ & -0.0008 & 0.0040 & -0.0230 & -0.0088 & 0.0228 & 0.0250 & 0.0120 & 0.0000 \\
\hline 0.0740 & $-9.1218 \mathrm{e}-2$ & 40.0133 & $2.4571 \mathrm{e}-3$ & -0.0109 & -0.0074 & 0.0185 & -0.0053 & 0.0070 & -0.0028 & -0.0017 & 0.0000 \\
\hline-0.0720 & 0.0164 & $8.8309 \mathrm{e}-3$ & $-3.2486 e-3$ & $3-0.0133$ & 0.0021 & 0.0226 & -0.0063 & 0.0049 & 0.0055 & 0.0034 & 0.0128 \\
\hline-0.0700 & $6.6704 \mathrm{e}-3$ & -0.0102 & $-9.3832 \mathrm{e}-4$ & $4-0.0047$ & 0.0015 & -0.0157 & -0.0118 & -0.0229 & 0.0052 & 0.0129 & -0.0128 \\
\hline-0.0680 & $9.8029 \mathrm{e}-3$ & 0.0150 & $5.7026 e-3$ & -0.0051 & 0.0032 & 08 & 021 & 15 & 046 & 076 & -0.0257 \\
\hline 0.0660 & 0.0120 & $3.4920 \mathrm{e}-3$ & 0.0173 & 0.0127 & -0.0022 & -0.0226 & & 10 & 19 & 266 & 0.0000 \\
\hline 0.0640 & $6.3840 \mathrm{e}-3$ & $2.3520 \mathrm{e}-3$ & -0.0139 & 0.0029 & 0.0026 & -0.0019 & -0.0057 & 0.0118 & -0.0060 & -0.0047 & -0.0128 \\
\hline 0.0620 & $6.2162 \mathrm{e}-3$ & $5.3443 \mathrm{e}-3$ & 0.0250 & -0.0015 & 0.0123 & -0.0151 & 0.0005 & 03 & 0.0186 & -0.0054 & 0.0000 \\
\hline-0.0600 & -8.60 & $3-1.99$ & & 0.0070 & -0.0031 & & & & & 023 & 0.0128 \\
\hline-0.0580 & $6.2709 \mathrm{e}-3$ & $-5.8432 \mathrm{e}-3$ & $3-0.0118$ & -0.0073 & -0.0014 & -0.0102 & -0.0202 & -0.0071 & -0.0062 & 0.0082 & -0.0128 \\
\hline-0.0560 & 0.0197 & $-1.4255 e-3$ & $36.4968 \mathrm{e}-3$ & 0.0185 & -0.0032 & -0.0054 & 0.0052 & & 0.0136 & 0.0019 & 0.0000 \\
\hline-0.0540 & $-8.7215 \mathrm{e}-3$ & $3-3.13$ & $e-3$ & 0.0046 & -0. & 44 & 970 & 19 & & 111 & 28 \\
\hline 0.0520 & $9.6325 \mathrm{e}-3$ & $8.4086 \mathrm{e}-3$ & 0.0109 & -0.0112 & -0.0103 & -0.0004 & 0. & -0 & 020 & 06 & 128 \\
\hline 0.0500 & 0.0117 & $6.9829 \mathrm{e}-3$ & 0.0232 & -0.0022 & 0.0084 & -0.0009 & -0.0094 & 87 & 207 & -0.0061 & 0.0000 \\
\hline 0.0480 & -0.0188 & -7.0 & & 0.0242 & & & & & & & \\
\hline-0.0460 & 0.0151 & $-2.7077 \mathrm{e}-3$ & 30.0 & -0.0012 & -0.0026 & 0. & -0.0172 & $0 .($ & 61 & 041 & 0.0000 \\
\hline-0.0440 & 0.0173 & $-7.6909 e-3$ & $39.5288 \mathrm{e}-3$ & 0.0067 & 0.0012 & 0.0024 & 0.0175 & -0.0034 & -0.0033 & 0.0018 & .0000 \\
\hline-0.0420 & 0.0105 & -1.14 & $3-2.0936 e-3$ & $3-0.0054$ & 0.0 & & 43 & & & 038 & 0.0000 \\
\hline-0.0400 & 0.0 & 8.5 & 0.0 & 0.0 & -0 & & 40 & & & 04 & 128 \\
\hline 0.0380 & $5 e-3$ & $5.9847 \mathrm{e}-3$ & 2.16 & 0.0149 & 0.0 & & & & 34 & 25 & 00 \\
\hline 0.0360 & 1.3 & $8.1916 \mathrm{e}-3$ & 0.018 & 0.0040 & -0. & & & & & 092 & 00 \\
\hline-0.0340 & $6.2703 \mathrm{e}-3$ & -0.0106 & -0.0100 & 0.0094 & 0.0 & 0. & -0.0128 & $0 .($ & -0.0006 & 0.0024 & 0.0000 \\
\hline-0.0320 & 0.0126 & 0.0126 & $8.3737 \mathrm{e}-3$ & -0.0113 & -0.0042 & -0.0065 & 0.0039 & 0.0 & -0.0090 & -0.0106 & 0.0000 \\
\hline-0.0300 & $4.0484 \mathrm{e}-3$ & -0.0170 & $-6.3524 \mathrm{e}-3$ & 30.0073 & -0.0073 & -0.0131 & -0.0070 & -0.0111 & 0.0148 & 0.0097 & .0000 \\
\hline-0.0280 & $-6.0420 \mathrm{e}-3$ & $33.4917 \mathrm{e}-3$ & $-9.5996 \mathrm{e}-3$ & & 0.0050 & & & & 44 & & 0.0000 \\
\hline 0.0260 & 10 & 0.0 & $e-3$ & -0 & -0 & & & & 58 & 90 & 00 \\
\hline 0.0240 & $-7.7520 \mathrm{e}-3$ & $36.7689 \mathrm{e}-3$ & 0.0162 & 0.0123 & 0.0012 & -0.0061 & 0. & -0 & 70 & -0.0078 & 0.0000 \\
\hline 0.0220 & 0.0215 & $8.9057 \mathrm{e}-3$ & 0.0324 & 0.0079 & -0.0085 & 001 & -0.0179 & -0 & 151 & -0.0238 & 0.0000 \\
\hline-0.0200 & $7.8095 \mathrm{e}-3$ & $-6.6977 \mathrm{e}-3$ & $33.4650 \mathrm{e}-3$ & 0.0062 & 0.0 & -0.0060 & 0.0 & -0.0091 & & -0.0085 & 0.0257 \\
\hline-0.0180 & $4.0467 \mathrm{e}-3$ & -0.0184 & $4.4044 \mathrm{e}-3$ & 0.0050 & 0.0204 & 0.0284 & -0.0009 & 0.0234 & 0.0059 & -0.0016 & 0.0128 \\
\hline-0.0160 & $-9.2896 e-3$ & 30.0184 & $5.7750 \mathrm{e}-3$ & 0.0173 & 0.0102 & -0.0003 & -0.0085 & & 0.0055 & -0.0331 & 0.0000 \\
\hline-0.0140 & $-1.1965 \mathrm{e}-3$ & $3-1.4966 e-3$ & $3-3.3957 \mathrm{e}-3$ & & -0.0107 & & -0.0028 & & 030 & -0.0010 & .0128 \\
\hline 120 & & & & & & & & & & 45 & \\
\hline 0.0100 & $-2.7360 \mathrm{e}-3$ & 32.9 & $7 e-4$ & -0.0074 & 0.0024 & -0.0013 & 0.0 & 0. & $0 .($ & 17 & -0.0128 \\
\hline $3.0000 \mathrm{e}$ & $3 e-4$ & $-4.9868 \mathrm{e}-3$ & 36.0 & -0.0185 & 0.0188 & & 128 & 67 & 06 & 966 & 00 \\
\hline-6.0000 & $34.7844 \mathrm{e}-3$ & $-4.8453 e-3$ & $3-7.9387 e-4$ & $4-0.0062$ & -0.0012 & -0.0063 & 0.0041 & -0.0125 & 0.0061 & -0.0066 & 0.0128 \\
\hline$-4.0000 e-3$ & 30.0319 & $-8.2609 \mathrm{e}-3$ & $3-7.4359 e-3$ & $3-0.0223$ & 0.0076 & 0.0049 & 0.0022 & 0.0246 & 0.0126 & 0.0136 & -0.0128 \\
\hline$-2.0000 \mathrm{e}-3$ & 30.0604 & -0.0187 & $2.7430 \mathrm{e}-3$ & -0.0061 & -0.0096 & & -0.0213 & -0.0129 & 0.0041 & -0.0134 & 0.0257 \\
\hline $1.6708 \mathrm{e}-1$ & & & & & & 00135 & & & 0.0198 & -0.0159 & -0.0285 \\
\hline $00 \mathrm{e}-3$ & 0.6775 & & & & & & & & & & \\
\hline $4.0000 \mathrm{e}-3$ & 0.9907 & -0.0245 & $9.4548 \mathrm{e}-3$ & -0.0027 & -0.0035 & & & 260 & -0.0143 & -0.0315 & -0.0128 \\
\hline $6.0000 \mathrm{e}-3$ & 1.1519 & -0.0138 & -0.0133 & 0.0131 & -0.0164 & 0.0034 & -0.0028 & -0.0068 & 0.0194 & 0.0087 & 0.0000 \\
\hline $8.0000 \mathrm{e}-3$ & 1.2196 & $1.2859 \mathrm{e}-3$ & 0.0158 & 0.0299 & -0.0183 & 0.0022 & -0.0135 & 0.0003 & -0.0271 & -0.0060 & -0.0128 \\
\hline 0.0100 & 1.1860 & 0.0128 & $-7.2911 \mathrm{e}-3$ & $3-0.0187$ & -0.0174 & 0.0200 & 0.0039 & 0.0004 & -0.0028 & 0.0071 & 0.0128 \\
\hline .0120 & 1.1673 & $6.7032 \mathrm{e}-3$ & $-2.5264 \mathrm{e}-3$ & 30.0094 & 0.0301 & -0.0061 & 0.0057 & -0.0136 & & -0.0149 & -0.0128 \\
\hline .0140 & 1.1418 & 0.0168 & $9.6720 \mathrm{e}-3$ & 0.0014 & 0.0134 & -0.0154 & -0.0139 & 0.0111 & -0.0192 & 0.0175 & 0.0128 \\
\hline & 1.1477 & $-1.4957 \mathrm{e}-3$ & $3-5.8473 e-3$ & 30.0150 & 0.0143 & -0.0164 & -0.0176 & -0.0030 & -0.0076 & -0.0103 & -0.0128 \\
\hline 0.0180 & 1.1615 & -0.0152 & -0.0154 & -0.0130 & -0.0010 & 0.0053 & 0.0006 & -0.0094 & -0.0064 & -0.0029 & 0.0000 \\
\hline 0.0200 & 1.1645 & 0.0197 & 0.0000 & -0.0019 & 0.0124 & -0.0017 & -0.0154 & 0.0203 & -0.0095 & -0.0052 & 0.0000 \\
\hline 0.0220 & 1.1607 & $5.2714 \mathrm{e}-3$ & 0.0131 & 0.0123 & -0.0094 & 0.0007 & 0.0019 & -0.0026 & 0.0040 & 0.0004 & 0.0000 \\
\hline 0.0240 & 1.1842 & $-5.6544 \mathrm{e}-4$ & $4-4.8364$ & 30.0111 & -0.0063 & -0.0014 & 0.0121 & 0.0207 & 0.0048 & 0.0005 & -0.0128 \\
\hline 0.0260 & 1.1841 & $-8.5728 \mathrm{e}-4$ & $4-2.8875 e-3$ & 30.0181 & -0.0059 & -0.0056 & 0.0117 & 0.0058 & -0.0189 & 0.0003 & 0.0000 \\
\hline
\end{tabular}




\begin{tabular}{|c|c|c|c|c|c|c|c|c|c|c|c|}
\hline 0280 & 1.1808 & 0.0161 & $1.0834 \mathrm{e}-3$ & 047 & 0.0109 & -0.0234 & 0.0074 & -0.0028 & -0.0137 & 0.0075 & 0.0000 \\
\hline 0300 & 1.1654 & $1.8514 \mathrm{e}-3$ & $1.9493 \mathrm{e}-3$ & 0.0060 & -0.0116 & 0.0045 & -0.0083 & 0.0087 & 0.0101 & 0.0042 & -0.0128 \\
\hline 0320 & 1.1705 & $9.9408 \mathrm{e}-4$ & $-3.9700 \mathrm{e}-$ & 0.0117 & 0.0094 & 0.0026 & -0.0180 & -0.0085 & -0.0010 & 0.0004 & 0.0128 \\
\hline 0340 & 1.1779 & $1.4318 \mathrm{e}-3$ & $1.7325 \mathrm{e}-3$ & -0.0009 & 0.0003 & -0.0062 & 0.0083 & 0.0095 & 0.0089 & 0.0031 & -0.0128 \\
\hline 0360 & 1.1760 & $1432 \mathrm{e}-3$ & 0.0189 & 0.0123 & .0272 & 0.0015 & .0099 & -0.0144 & 0.0056 & .0181 & 000 \\
\hline 0.0380 & 1.1812 & $0 e-3$ & 0.0121 & 0.0101 & 0152 & -0.0168 & -0.0354 & & & -0.0041 & -0.0128 \\
\hline 0.0400 & 1.1797 & $.5486 \mathrm{e}-3$ & $7.5799 \mathrm{e}-3$ & 0.0059 & -0.0042 & -0.0030 & -0.0070 & 0.0229 & -0.0202 & 0.0151 & 0.0000 \\
\hline 0.0420 & 1.1665 & $4.6339 \mathrm{e}-3$ & -0.0121 & 0.0126 & -0.0175 & -0.0153 & 0.0035 & 0.0076 & -0.0199 & .0045 & .0128 \\
\hline 0440 & 1.1852 & -0.0 & -0.0202 & 0.0294 & & -0.0 & & & & .0018 & \\
\hline 460 & 1.1831 & -0.0183 & $-7.3635 e-3$ & 0.0035 & .0036 & 0.0011 & & & & 0152 & 0257 \\
\hline 0480 & 1.1831 & $.3366 e-3$ & 0.0123 & -0.0052 & 064 & 0.0055 & & & & & \\
\hline 0.0500 & 1.1839 & $5.3840 \mathrm{e}-4$ & $-4.0425 e-3$ & -0.0123 & -0.0044 & -0.0086 & & -0.0020 & 0013 & & 128 \\
\hline 0.0520 & 1.1786 & 0.0142 & $1.9489 \mathrm{e}-3$ & & & & & & & & -0.0128 \\
\hline 0.0540 & 1.1792 & .0111 & -0.0136 & -0.0063 & -0.0180 & & & & -0.0084 & -0.0131 & 128 \\
\hline 0.0560 & & & $-5.6295 \mathrm{e}-$ & 0.0074 & & & & & & & \\
\hline 0580 & & & -3.3 & & & & & & & & \\
\hline 6000 & 1.1789 & & & & 07 & & & & & & \\
\hline 0620 & 1.1707 & & -9.3 & & & & & & & & \\
\hline 0640 & 1.1 & & & & & & & & & & \\
\hline 0660 & 1.1865 & & -1.5 & & & & & & & 075 & 128 \\
\hline 0680 & & & -5.7 & $t-0.0014$ & & & & & & & \\
\hline 0700 & & & 9.8 & & & & & & & & \\
\hline 0720 & & -4.9 & -3 & 12 & & & & & & & \\
\hline 0740 & 1.1814 & 64 & 0.01 & -0.0035 & & & & & & & \\
\hline & & & & -0.0201 & & & & & & & \\
\hline 0780 & 1.1842 & & & & & & & & & & \\
\hline 0800 & 1.1934 & -5.7 & -3.9 & -0.0073 & & & & & & & 128 \\
\hline 0820 & & & & & & & & & & & \\
\hline 0 & & & & & & & & & & & \\
\hline 0860 & & -4.7 & -3 & 01 & & & & & & & \\
\hline & & & & & & & & & & & \\
\hline 0900 & 2 & 3 & -4. & 30. & & & & & & & \\
\hline 0920 & 1.1837 & 5771 & 3.175 & -0.0093 & $0 \Omega \Omega$ & 0.0019 & & & & & 0128 \\
\hline 0940 & 1.1739 & $-0,0$ & & 0.0068 & & & & & & 017 & 28 \\
\hline 0 & & & & & & & & & & & \\
\hline 0 & & & & & & & & & & & \\
\hline 1000 & 30 & & 9.4 & & & & & & & & \\
\hline 1020 & 1.1679 & & -0.0110 & -0.0002 & & & & & & & \\
\hline & & & & & & & & & & & \\
\hline 1060 & 1.1903 & 6.17 & 3.03 & 0.0057 & & & & & & & \\
\hline 1080 & & & & & & & & & & & -0.0128 \\
\hline & & & & & & & & & & & \\
\hline 0 & & & & & & & & & & & \\
\hline 1140 & & & -4.2 & 85 & & & & & & & \\
\hline 1160 & & & & & & & & & & & \\
\hline & & & -6.2 & & & & & & & & \\
\hline 1200 & 1.1917 & & 0.0121 & -0.0086 & -0.0088 & & & & & & 0.0000 \\
\hline 1220 & & & & & & & & & & & 0.0128 \\
\hline 0 & & & $-4.0425 c$ & & & & & & & & \\
\hline 0 & & & -2.6 & 22 & & & & & & & \\
\hline 280 & & & & 0. & & & & & & 130 & \\
\hline & & & & & & & & & & & \\
\hline 1320 & 1.18 & int & $-4.4029 e-3$ & 0.0096 & 30 & & & & & -0.0031 & 00 \\
\hline 1340 & & & 0.0141 & -0.0015 & & & & & & & 28 \\
\hline & & & & & & & & & & & \\
\hline & & & & & & & & & & & \\
\hline & & & -6.13 & & & & & & & & \\
\hline & & & & & & & & & & & \\
\hline 170 & & & e-3 & & & & & & & & \\
\hline 1460 & & $.7000 \mathrm{e}-3$ & -0.0152 & 0.0013 & & & & 0.0 & & 151 & 0.0257 \\
\hline 1480 & & $4.2043 \mathrm{e}-3$ & $7.9387 \mathrm{e}-4$ & 0.0043 & 59 & & & & & 130 & -0.0128 \\
\hline & & & & & & & & & & & \\
\hline 520 & & & $5.1251 \mathrm{e}-3$ & -0.0051 & & & & & & & \\
\hline & & & & & & & & & & 076 & \\
\hline & & & & & & & & & & & \\
\hline & & 0.0112 & $4.2589 \mathrm{e}-3$ & 0.0100 & & & & & & 226 & 0.0000 \\
\hline 1600 & & & $4.6199 \mathrm{e}-3$ & -0.0006 & & & & & & 005 & -0.0128 \\
\hline 1620 & 1.1912 & Be-4 & 0.0126 & -0.0096 & -0.0067 & 0. & 0.0092 & -0.0091 & -0.0042 & 0.0068 & 0000 \\
\hline 1640 & 1.1780 & $-3.2796 \mathrm{e}-3$ & $-3.2486 \mathrm{e}$ & 0.0008 & -0.0043 & -0.0059 & 0.0089 & -0.0 & 0.0 & -0.0242 & 0.0128 \\
\hline 0.1660 & 1.2010 & $2.7789 \mathrm{e}-3$ & $-1.8765 \mathrm{e}-3$ & 0.0087 & -0.0224 & 0.0058 & 0.0012 & -0.0158 & -0.0086 & -0.0008 & 0.0128 \\
\hline
\end{tabular}




\begin{tabular}{|c|c|c|c|c|c|c|c|c|c|c|c|}
\hline 1680 & 1.1857 & 0.0219 & $4.7648 \mathrm{e}-3$ & 0.0143 & 0.0015 & 0.0190 & -0.0181 & 0.0262 & 0.0101 & 0.0114 & 0.0000 \\
\hline 0.1700 & 1.1833 & $9.0489 \mathrm{e}-3$ & -0.0169 & -0.0006 & 0.0117 & 0.0098 & -0.0206 & -0.0090 & 0.0248 & 0.0156 & -0.0128 \\
\hline 0.1720 & 1.1843 & $8.9777 \mathrm{e}-3$ & $7.5072 \mathrm{e}-3$ & 0.0079 & -0.0078 & -0.0169 & -0.0263 & -0.0175 & 0.0145 & 0.0027 & 0128 \\
\hline 0.1740 & 1.1788 & .0199 & -0.0111 & 0.0171 & -0.0051 & 0.0268 & 0.0011 & 0.0311 & 0.0015 & -0.0211 & .0000 \\
\hline 0.1760 & 1.1882 & $.9832 \mathrm{e}-3$ & $6.9323 \mathrm{e}-3$ & -0.0029 & -0.0101 & -0.0114 & -0.0009 & 0.0075 & -0.0036 & -0.0146 & -0.0128 \\
\hline 1780 & 1.1845 & $2800 e-3$ & $-8.1551 e-3$ & -0.0028 & -0.0047 & -0.0291 & 0.0001 & 0.0244 & -0.0149 & 0.0019 & .0000 \\
\hline 1800 & 1.1929 & 0.0170 & $-7.3635 e-3$ & -0.0174 & -0.0145 & -0.0059 & -0.0091 & -0.0063 & 0.0177 & 0.0064 & .0128 \\
\hline 0.1820 & 1.1846 & .0137 & 0.0118 & 0.0050 & -0.0084 & -0.0136 & -0.0095 & -0.0006 & -0.0235 & 0.0060 & 0.0128 \\
\hline 0.1840 & 1.1758 & $.9945 \mathrm{e}-3$ & -0.0253 & -0.0015 & -0.0111 & -0.0094 & -0.0062 & -0.0115 & & -0.0182 & 0.0000 \\
\hline 0.1860 & 1.1836 & 0.0182 & -0.0100 & -0.0174 & -0.0170 & -0.0185 & -0.0224 & 0.0146 & -0.0087 & 54 & .0128 \\
\hline 0.1880 & 1.1851 & .0155 & 0.0219 & -0.0022 & & & & & -0.0053 & & 0.0128 \\
\hline 0.1900 & 1.1814 & $-8.9102 \mathrm{e}-3$ & $-2.7432 e-3$ & 0.0076 & 0.0 & & & -0.0011 & & & 0128 \\
\hline 1920 & 1.1838 & $e-3$ & -1.228 & 0.0252 & 3 & & & & & & .0128 \\
\hline 1940 & 1.1943 & 0.0108 & -8.6 & 0.0008 & 68 & & & & 009 & & \\
\hline 1960 & 1.1954 & $.0443 e-3$ & 4.83 & -0.0058 & & & & & 100 & & 000 \\
\hline 0.1980 & 1.1783 & $4.2773 \mathrm{e}-4$ & -7.579 & & & & & & & & -0.0128 \\
\hline 0.2000 & 1.1827 & 0.0125 & -0.0128 & -0.0004 & 0.0072 & 0.0063 & & & -0.0038 & -0.0290 & -0.0128 \\
\hline 0.2020 & & $16 e-4$ & $2.1660 \mathrm{e}-3$ & & & & & & & & \\
\hline 2040 & 7 & & 0.02 & & & & & & & & \\
\hline 2060 & 1.1814 & e-3 & 5.63 & 0.0225 & & & & & & & \\
\hline 80 & 1.1745 & & & 163 & & & & & & & 28 \\
\hline 2100 & $1.1 \mathrm{c}$ & 1 & & -0.0076 & & & & & & & 00 \\
\hline 2120 & 1.1736 & & -6.8 & -0.0014 & 0.0 & & & & & & 257 \\
\hline 2140 & 1.1815 & e-3 & 9.24 & -0.0031 & & & & & & & 0128 \\
\hline 60 & & & -6.7 & & & & & & & & \\
\hline 180 & & & 6.5 & & & & & & & & \\
\hline 2200 & 1.1796 & $e-3$ & -0.0 & -0. & & & & & & & \\
\hline 2220 & & & & & & & & & & & \\
\hline 2240 & 1.1843 & -3 & -0.0 & & & & & & & & \\
\hline 2260 & 1.1738 & $e-3$ & $9.5985 e-3$ & 0.0023 & -0.0017 & & & & 98 & & 000 \\
\hline 2280 & & $e-3$ & -6.49 & -0.0088 & & & & & & & \\
\hline 00 & & & & & & & & & & & \\
\hline 20 & & & -0.0 & 0.0 & & & & & & & \\
\hline 2340 & 1.1594 & 0 & $-5.9190 e-3$ & 65 & & & & & & & \\
\hline 2360 & & 40750 & -1.8 & & & & & & & & \\
\hline 2380 & 1.1488 & 089 & & & -0.01 & & & & & & 00 \\
\hline 2400 & 1.1654 & & & 0.0297 & -0.0 & 0.0007 & & & & & .0128 \\
\hline 2420 & & & & & & & & & & & 128 \\
\hline & & & & & & & & & & & \\
\hline 60 & & & & & & & & & & & \\
\hline 480 & & & & & & & & & & & \\
\hline 2500 & 1.18 & & & & & & & & & & \\
\hline 2520 & & .0226 & -4.26 & & & & & & & & -0.0128 \\
\hline 2540 & 1.1824 & $.5454 \mathrm{e}-3$ & 0.0147 & -0.0056 & & & & & & & 0.0000 \\
\hline 2560 & & & & & & & & & & & 0.0128 \\
\hline & & & & & & & & & & & \\
\hline 500 & & 9 & -5.7 & & & & & & & & \\
\hline 620 & & & & 26 & & & & & & & \\
\hline 2640 & & & & & & & & & & & \\
\hline 0.2660 & 1.1717 & $6.4132 \mathrm{e}-3$ & $-3.0346 e-3$ & -0.0146 & -0.0030 & & & & -0.0007 & -0.0044 & 0.0000 \\
\hline 2680 & & & 0.0 & & & & & & & & 0000 \\
\hline & & & & & & & & & & & \\
\hline & & & & & & & & & & & \\
\hline 2740 & & & $e-3$ & & & & & & & & 00 \\
\hline 60 & & 5 & & & & & & & & & \\
\hline 2780 & 1.1853 & 0.0254 & 0.0100 & -0.0086 & & & & 0.0 & & -0.0026 & -0.0128 \\
\hline 0.2800 & 1.1966 & $3.2020 \mathrm{e}-3$ & $-8.6394 \mathrm{e}-4$ & -0.0258 & -0.0043 & -0.0011 & & -0.0004 & -0.0008 & -0.0041 & 0.0000 \\
\hline 2820 & & & & & & & & & & 120 & 000 \\
\hline & & & & & & & & & & & \\
\hline 2860 & & & 1.6 & & & & & & & & \\
\hline & & e-3 & & & & & & & & & 00 \\
\hline 00 & & & -4 & & & & & & & & \\
\hline 2920 & 1.1972 & $-7.1939 e-3$ & 0.0148 & 0.0160 & -0.0174 & & -0. & -0.0149 & -0.0178 & -0.0103 & 0.0000 \\
\hline 0.2940 & 1.1872 & $4 e-3$ & $4.6955 \mathrm{e}-3$ & 0.012 & 0.0038 & & -0.0057 & -0.0190 & 0.0020 & -0.0166 & 0.0000 \\
\hline 2960 & 1.1911 & $e-3$ & 0.0173 & -0.0069 & -0.0008 & 44 & 50000 & & -0.0165 & -0.0134 & -0.0128 \\
\hline 2980 & 1.1774 & $e-3$ & -2.1714 & & & & & & & & -0.0128 \\
\hline & & & & & & & & & & & \\
\hline & & & & & & & & & & & 0.0000 \\
\hline & 1.19 & 001 & 0.01 & & & & -0.0 & -0.0 & 0.0012 & -0.0127 & -0.0128 \\
\hline 0.3060 & 1.1921 & 0.0183 & 0.0118 & 0.0057 & 0.0066 & 0.0044 & -0.0033 & 0.0254 & 0.0149 & -0.0140 & -0.0128 \\
\hline
\end{tabular}




\begin{tabular}{|c|c|c|c|c|c|c|c|c|c|c|c|}
\hline 3080 & 1.1799 & 0.0108 & $6.7852 \mathrm{e}-3$ & -0.0198 & 0.0082 & -0.0007 & -0.0205 & 0.0004 & -0.0091 & 0.0034 & 0.0000 \\
\hline 0.3100 & 1.1821 & $9.0489 \mathrm{e}-3$ & $4.4752 \mathrm{e}-3$ & -0.0034 & 0.0048 & 0.0030 & 0.0103 & -0.0107 & -0.0015 & 0.0070 & 0.0000 \\
\hline 0.3120 & 1.1906 & $-7.7666 e-3$ & $-7.2911 c$ & 0.0001 & 0.0283 & -0.0051 & 0.0027 & 0.0102 & 0.0118 & 0.0156 & 0.0257 \\
\hline 0.3140 & 1.1877 & $-4.9832 \mathrm{e}-4$ & 0.0125 & -0.0077 & 0.0064 & 0.0010 & -0.0046 & -0.0150 & 0.0037 & -0.0171 & 0.0128 \\
\hline 0.3160 & 1.1765 & $9.6207 \mathrm{e}-3$ & $-1.1550 \mathrm{e}-3$ & -0.0002 & 0.0083 & 0.0000 & 0.0068 & 0.0033 & -0.0121 & -0.0192 & -0.0128 \\
\hline 0.3180 & 1.1921 & $-7.1227 \mathrm{e}-4$ & $1.9489 \mathrm{e}-3$ & 0.0052 & .0099 & 0.0045 & 0.0132 & 0.0022 & 0.0053 & -0.0056 & 0128 \\
\hline 0.3200 & 1.2026 & -0.0153 & $6.9331 \mathrm{e}-3$ & -0.0138 & 0.0079 & 0.0086 & 0.0174 & -0.0070 & -0.0001 & -0.0007 & 0000 \\
\hline 0.3220 & 1.2024 & $-7.3389 e-3$ & 0.0156 & -0.0140 & .0038 & 0.0115 & -0.0157 & 0.0255 & -0.0062 & 0.0153 & .0128 \\
\hline 0.3240 & 1.1862 & 0.0109 & 0.0170 & 0.0237 & -0.0060 & -0.0080 & -0.0062 & 0.0135 & -0.0040 & -0.0023 & 0.0000 \\
\hline 0.3260 & 1.1667 & -0.0135 & 0.0152 & -0.0040 & -0.0127 & 0.0087 & -0.0015 & 0.0094 & -0.0205 & 0.0093 & -0.0128 \\
\hline 0.3280 & 1.1886 & -0.0195 & $-4.3351 c$ & 0.0052 & 0.0079 & 0.0155 & 0.0046 & -0.0081 & -0.0250 & -0.0211 & 0.0000 \\
\hline 0.3300 & 1.1896 & 00163 & $6.4911 \mathrm{e}-4$ & & -0.0078 & 46 & -0.0 & & 0.0 & & 0000 \\
\hline 0.3320 & 1.1728 & $7 e-3$ & 5.34 & -0.0105 & & 0103 & 0 & 0.0019 & & & 0128 \\
\hline 0.3340 & 1.1789 & $9.3343 e-3$ & $-1.9496 \mathrm{e}-3$ & -0.0092 & 0.0128 & 0.0108 & -0.0132 & 136 & & 228 & 0128 \\
\hline 0.3360 & 1.1797 & $-6.2700 \mathrm{e}-3$ & $-8.7010 \mathrm{e}-4$ & $1-0.0021$ & -0.0044 & 0.0202 & 0.0019 & 0.0061 & -0.0113 & 072 & 0257 \\
\hline 0.3380 & 1.1894 & & & & & -0.0208 & & & & -0.0171 & 0.0128 \\
\hline 0.3400 & 1.1770 & -1.56 & 2.7 & 0.0040 & 0.0131 & 0.0155 & -0.0119 & 0.0169 & 0.0054 & -0.0175 & -0.0257 \\
\hline 0.3420 & 1.1717 & & & & & & & & & & 0.0128 \\
\hline 0.3440 & 1.1830 & & & & & & & & & & 128 \\
\hline 0.3460 & 1.1724 & .0156 & 0.0122 & -0.0069 & 0.0012 & 0.0037 & 92 & 004 & & & 000 \\
\hline 0.3480 & 1.1782 & $3 e-3$ & $-5.4131 e-3$ & -0.0088 & -0.0069 & -0.0065 & 250 & 004 & & 138 & 0128 \\
\hline 0.3500 & 1.1886 & -4.9 & & -0.0176 & 0.0145 & 0.0181 & & & & & 000 \\
\hline 0.3520 & 1.1816 & -0.0189 & 4.47 & -0.0188 & & & & & & 142 & .0128 \\
\hline 0.3540 & 1.1643 & & & -0.0188 & -0.0065 & & & & & & 0.0128 \\
\hline 60 & & & & & & & & & & & 128 \\
\hline 0.3580 & 37 & & & & & & & & & & 128 \\
\hline 0.3600 & 1.1733 & 1.1 & $3 e-3$ & -0.0164 & 0014 & & & & & 012 & 28 \\
\hline 0.3620 & 1.1847 & & & & & & & & & & \\
\hline 0.3640 & 1.1820 & -1 & $6 e-3$ & -0.0 & 227 & & & & & & 000 \\
\hline 0.3660 & 1.1758 & 00 & $8.0819 \mathrm{e}-3$ & 0.0118 & 0.0135 & 99 & -0 . & & & 025 & .0128 \\
\hline 3680 & & & -0.0 & & & & & & & & 0.0000 \\
\hline & & & $-8 .(1$ & & & & & & & & 128 \\
\hline 0.3720 & 26 & & & 0.0 & & & & & & & 00 \\
\hline 0.3740 & 1.1736 & & -0.0 & & & & & & & & 28 \\
\hline 0.3760 & 10 & 0 & 0.0 & & 78 & & & & & & \\
\hline 0.3780 & 1.1781 & $6 e-3$ & 0.0230 & & 0.0002 & -0.0127 & -0.0243 & & & & 0.0000 \\
\hline 0.3800 & 1.1876 & $e-3$ & 0.0106 & -0.0002 & -0.0025 & 0.0189 & -0.0064 & 52 & & 20 & 0.0000 \\
\hline 0.3820 & 11627 & & $8.6625 \mathrm{e}-4$ & -0.0015 & & & & & & & 0128 \\
\hline & & & & & & & & & & & \\
\hline 860 & 3 & & 4.4 & & & & & & & & 128 \\
\hline 0.3880 & & & $6.9269 \mathrm{e}-3$ & & & & & & & & \\
\hline 0.3900 & & -2.99 & $7.8660 \mathrm{e}-3$ & -0.0 & & & & & & & \\
\hline 0.3920 & 1.1929 & -0.0165 & 0.0153 & 0.0160 & & & & & & & 0.0000 \\
\hline 0.3940 & 1.1770 & $-6.0562 \mathrm{e}-3$ & -0.0193 & -0.0097 & 0.0008 & & & -0.0083 & & 177 & 0.0128 \\
\hline 0.3960 & & & 0.0 & & & & & & & & -0.0128 \\
\hline & & -9 . & $-2.7420 e-3$ & & & & & & & & 28 \\
\hline 0.4000 & 7 & 0.01 & -4.33 & 46 & 71 & 50 & & 4 & & 86 & 00 \\
\hline 0.4020 & & & e-3 & & & & & & & & 0.0000 \\
\hline 0.4040 & & -0. & -0.0116 & 0.0111 & & & & & & & -0.0128 \\
\hline 0.4060 & 1.1794 & $3.6338 \mathrm{e}-3$ & $-9.7451 e-3$ & 0.0044 & -0.0045 & -0.0107 & 0.0232 & -0.0031 & 0.0046 & 0.0240 & -0.0128 \\
\hline 0.4080 & & & 0.0108 & 0.0008 & 0161 & -0.0027 & & & & 060 & -0.0128 \\
\hline & & & & & & & & & & & \\
\hline & & & $e-3$ & & & & & & & & \\
\hline 4140 & & $9 e-3$ & 0.0118 & -0.0083 & 78 & & 01 & 7 & 56 & 06 & 28 \\
\hline 4160 & & & $1.8742 \mathrm{e}-3$ & & 0028 & & & & & & 128 \\
\hline 0.4180 & & & -0.0142 & 0.0164 & & & -0.0030 & & & -0.0019 & 0.0000 \\
\hline 0.4200 & 1.1700 & $3.6389 \mathrm{e}-3$ & $5.3407 \mathrm{e}-3$ & -0.0018 & 0.0052 & 0.0052 & 0.0052 & 0.0184 & -0.0119 & -0.0107 & -0.0257 \\
\hline 0.4220 & & -0.0170 & 0.0107 & -0.0026 & & & & & & & 0000 \\
\hline & & $-5.0160 \mathrm{e}-4$ & $-4.5479 e$ & -0.0087 & -0.0143 & & & & & & 0.0000 \\
\hline 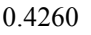 & & 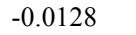 & -0.0176 & 0.0154 & -0.0093 & & 00 & & -0.0 & 228 & -0.0257 \\
\hline & & & $-7.9387 \mathrm{e}-4$ & & 0075 & & & & & -0.0015 & -0.0128 \\
\hline & & & -0.0137 & -0.0 & & & & & & & 0.0000 \\
\hline 0.4320 & 1.1690 & $-9.6189 e-3$ & $4.1144 \mathrm{e}-3$ & -0.0137 & 0.0012 & 0.0071 & 0.0036 & -0.0114 & 0.0107 & 0.0077 & -0.0257 \\
\hline 0.4340 & 1.1752 & 0.0120 & $3.6814 \mathrm{e}-3$ & -0.0149 & 0.0118 & -0.0159 & -0.0194 & 087 & -0.0153 & 0.0018 & 0.0128 \\
\hline 0.4360 & 792 & $9.1173 e-3$ & $3.9701 \mathrm{e}-3$ & -0.0125 & 131 & -0.0241 & 06 & 46 & 223 & 033 & 0.0000 \\
\hline 0.4380 & 1.1804 & $-2.4898 \mathrm{e}-3$ & $2.3100 \mathrm{e}-3$ & 0.0073 & -0.0120 & & 0.0036 & & & -0.0059 & -0.0128 \\
\hline & & & & 80057 & 029 & & & & & & 0.0128 \\
\hline & & & & & & & & & -0.0119 & & \\
\hline & & $2.4934 \mathrm{e}-3$ & $-6.2824 \mathrm{e}-3$ & 0.0199 & -0.0067 & 0.0038 & 0.0130 & 0.02 & -0.0169 & -0.0184 & 0.0128 \\
\hline 0.4460 & 1.1831 & $9.8332 \mathrm{e}-3$ & $-5.0532 \mathrm{e}-3$ & -0.0016 & 0.0027 & 0.0057 & -0.0061 & -0.0010 & -0.0256 & -0.0012 & 0.0000 \\
\hline
\end{tabular}




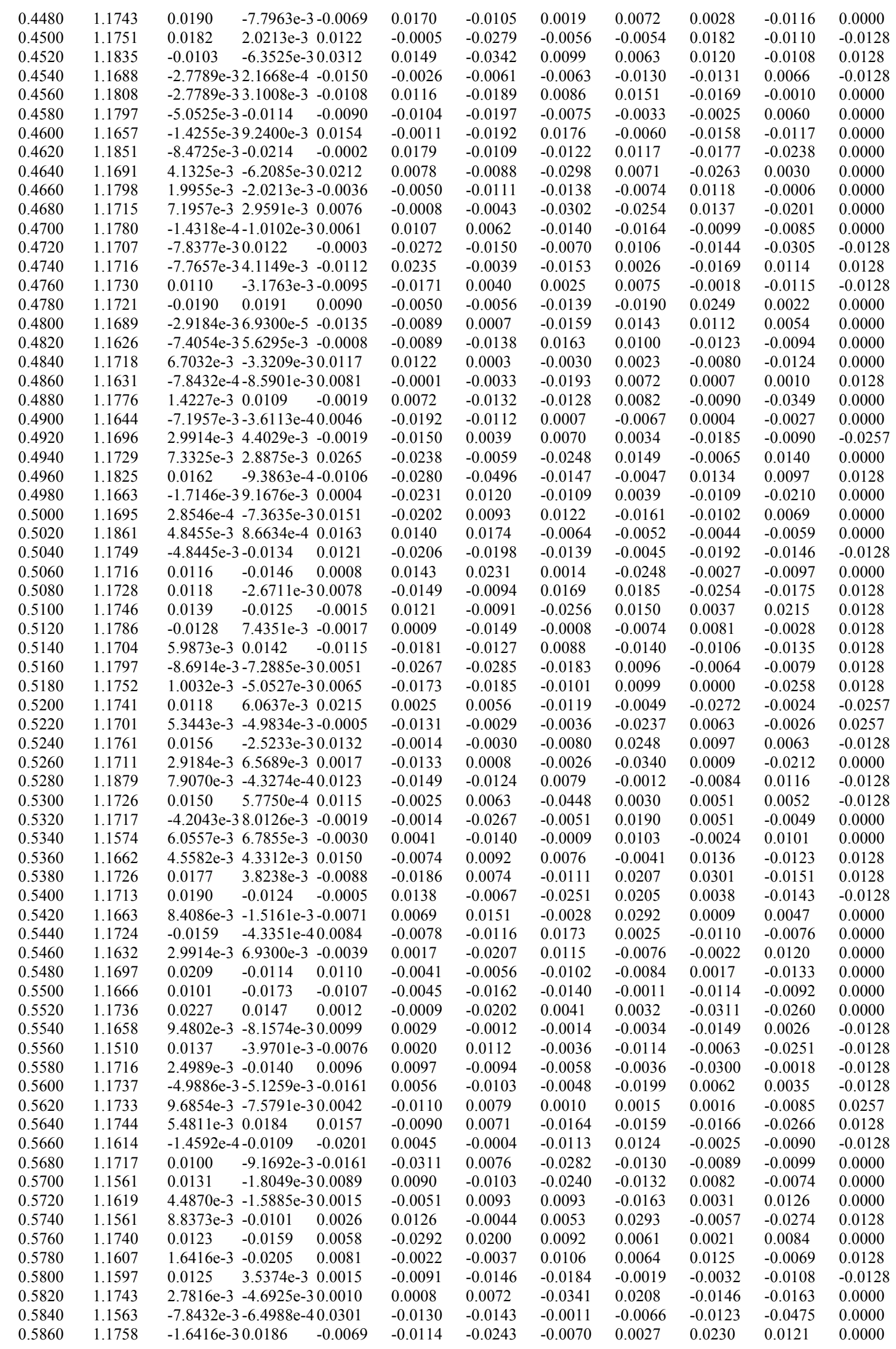




\begin{tabular}{|c|c|c|c|c|c|c|c|c|c|c|c|}
\hline 0.5880 & .1644 & $7.9107 \mathrm{e}-3$ & -0.0111 & -0.0041 & -0.0042 & -0.0029 & -0.0101 & -0.0057 & -0.0271 & 0.0077 & 0.0000 \\
\hline 0.5900 & 1.1675 & $-6.1286 e-3$ & $3.5351 \mathrm{e}-3$ & -0.0009 & 0.0053 & 0.0036 & 0.0087 & 0.0059 & -0.0049 & -0.0261 & 0.0257 \\
\hline 0.5920 & 1.1647 & $6.6941 \mathrm{e}-3$ & $-9.3478 e-4$ & $4-0.0124$ & -0.0002 & 0.0036 & -0.0170 & -0.0187 & -0.0058 & -0.0155 & -0.0128 \\
\hline 0.5940 & 1.1643 & $1.7054 \mathrm{e}-3$ & $4.9811 \mathrm{e}-3$ & 0.0097 & -0.0203 & -0.0313 & -0.0062 & 0.0119 & -0.0155 & -0.0006 & 0.0128 \\
\hline 0.5960 & .1632 & $3357 e-3$ & $-2.3821 \mathrm{e}-3$ & $3-0.0183$ & -0.0254 & 0.0016 & -0.0006 & -0.0057 & -0.0217 & -0.0094 & -0.0128 \\
\hline 0.5980 & .1575 & $3.9216 \mathrm{e}-3$ & $37.4351 \mathrm{e}-3$ & -0.0205 & -0.0120 & 0.0021 & -0.0042 & 0.0043 & -0.0082 & -0.0074 & 0.0000 \\
\hline 0.6000 & .1688 & 0.0142 & $-2.7489 e-3$ & $3-0.0016$ & 0.0018 & -0.0084 & -0.0231 & -0.0203 & 0.0045 & -0.0059 & -0.0128 \\
\hline 0.6020 & 1696 & 0.0273 & $1.9488 \mathrm{e}-3$ & 0.0086 & -0.0069 & -0.0127 & 0.0004 & 0.0156 & 0.0048 & -0.0363 & -0.0128 \\
\hline 0.6040 & .1666 & .0529 & $3.9701 \mathrm{e}-3$ & 0.0252 & -0.0040 & -0.0075 & -0.0176 & -0.0118 & -0.0167 & -0.0229 & 0.0000 \\
\hline 0.6060 & 1225 & .3207 & $-4.3336 \mathrm{e}-3$ & 30.0036 & 0.0034 & 0.0029 & -0.0183 & 0.0036 & -0.0169 & -0.0187 & -0.0128 \\
\hline 0.6080 & .0881 & .7094 & $-4.1144 \mathrm{e}-3$ & $3-0.0099$ & 0.0152 & -0.0126 & -0.0116 & 0.0037 & 0.0054 & -0.0236 & -0.0128 \\
\hline & 1077 & .1099 & $e-3$ & 0.0066 & & & & & & & \\
\hline 0.6120 & 1339 & 1.2384 & $3.0338 \mathrm{e}-3$ & -0.0105 & 0.0022 & -0.0177 & -0.0176 & 0.0108 & 05 & 86 & 0.0000 \\
\hline & 1446 & .3178 & 0.0152 & -0.0109 & 0.0030 & & -0. & & & & 0.0000 \\
\hline 0.6160 & .1635 & 1.3240 & $-9.4556 \mathrm{e}-3$ & $3-0.0136$ & -0.0051 & 0.0118 & -0.0099 & -0.0169 & 31 & & 0.0128 \\
\hline 0.6180 & 1.1532 & 1.2810 & 0.0113 & -0.0153 & -0.0112 & -0.0227 & -0.0246 & 0.0179 & 0.0071 & 157 & -0.0128 \\
\hline 0.6200 & 1.1540 & 1.2349 & 0.0 & -0.0232 & 0.0140 & -0.0102 & -0 . & 0.0 & 0. & 21 & 000 \\
\hline 0.6220 & .1600 & 1.2018 & 0.0166 & -0.0134 & -0.0036 & -0.0021 & 0. & -0.0203 & -0.0086 & -0.0064 & -0.0128 \\
\hline & .1669 & 1.20 & $9 e-3$ & 0.0037 & & 22 & & & & & 128 \\
\hline 0.6260 & 1.1544 & 1.2000 & $6.3525 \mathrm{e}-3$ & 0.0008 & 0.0171 & 0.0078 & -0.0172 & -0.0055 & -0.0113 & -0.0044 & -0.0128 \\
\hline & .1407 & .1980 & $-1.1550 \mathrm{e}-3$ & 0.0161 & -0.0085 & & 103 & & & & .0000 \\
\hline 0.6300 & 1600 & .2202 & 0.0129 & 0.0011 & 0.0168 & 0. & 0.0 & 0.0 & -0 & -0.0063 & 0.0128 \\
\hline & 1592 & 1.2273 & $7.5799 \mathrm{e}-3$ & 0.0221 & -0.0098 & 007 & 21 & 66 & & 026 & \\
\hline 0.6340 & 1535 & 1.2110 & $5.1251 \mathrm{e}-3$ & -0.0066 & -0.0082 & -0.0248 & -0.0001 & 0.00 & 37 & & 0.0000 \\
\hline & & 1.1804 & 2.74 & 0.0052 & & & & & & & \\
\hline 0.6 & .1461 & 1.2168 & -1.8 & 0.0218 & -0.0102 & -0.0093 & 0.0 & -0 . & & & 0.0000 \\
\hline & & 1.2 & 5.9 & -0 . & & & & & & & \\
\hline 0.6 & & 1.21 & & & & & & & -0 & & 0.0000 \\
\hline & 90 & 1.2 & $9 e-4$ & -0.0 & 53 & 97 & & & & & 00 \\
\hline & & & & & & & & & & & -0.0128 \\
\hline 80 & 601 & 1.20 & -3 & 89 & -0 . & 0. & -0 & 71 & & & 00 \\
\hline & & & & & & & & & & & 00 \\
\hline 520 & .1587 & 1.2266 & -2.0 & 30.0160 & -0.0086 & 0. & 77 & -0 . & 91 & -0.0034 & 0.0128 \\
\hline & & 1.2199 & -0.0 & & & & & & & & 00 \\
\hline 60 & 1664 & 1.2296 & 0.0116 & 0.0070 & 0.0 & 028 & 0. & -0 . & & 54 & -0.0128 \\
\hline & .1383 & 1.2104 & $9.7451 \mathrm{e}-3$ & & & -0.0030 & 74 & & & & \\
\hline 00 & 1504 & .24 & 7.36 & 0.0 & 20 & 0. & 0. & 0.0 & -0 & & 128 \\
\hline & 1599 & 1.2340 & 0.01 & -0.0038 & -0.0 & & & & & & 00 \\
\hline & .1495 & 1.2271 & 0.0 & 0.0 & 0.0 & & & & & & 000 \\
\hline & & 1.2 & 7.2 & 0.0 & & & & 84 & & & 000 \\
\hline & .1467 & 1.2204 & -2.3 & -0.0009 & -0.0 & 082 & & & & & 000 \\
\hline & 1388 & .23 & 0.0 & -0.0035 & & & & & & & 28 \\
\hline & & & & & & & & & & & 28 \\
\hline & & .23 & & -0 . & & & & & & & \\
\hline & & & & & & & & & & & \\
\hline & .1711 & .24 & $e-3$ & -0. & 6 & & & & & & 28 \\
\hline & & & & & & & & & & & -0.0128 \\
\hline & .1436 & 1.2320 & -1.8 & -0.0102 & -0.0010 & 52 & & & & & 0.0000 \\
\hline 40 & 1465 & 1.2315 & $6 e-4$ & 0.0089 & -0.0380 & 110 & & & 79 & 61 & 000 \\
\hline & .1725 & 1.2481 & 0.0135 & 0.0034 & -0.0078 & 0.0 & -0 . & 0.0 & 11 & & 0.0128 \\
\hline 80 & .1533 & 1.2257 & $1.7325 \mathrm{e}-3$ & 0.0094 & -0.0017 & 166 & & 50 & 10 & 056 & 257 \\
\hline & & .2203 & 0.0197 & 0.0042 & & 078 & & & & & \\
\hline 20 & 1706 & 1.2462 & 8.95 & 0.0043 & 0.0 & 0.0 & 0. & -0 . & 0. & & 28 \\
\hline & .1461 & .2379 & $6.2047 \mathrm{e}-3$ & -0.0258 & & -0.0036 & & & & & 0.0000 \\
\hline & 1517 & 1.2347 & 7.72 & -0.0150 & 0.0 & 0.0004 & & -0 . & 82 & 45 & 0.0000 \\
\hline & .1568 & 1.2367 & $3.7534 \mathrm{e}-3$ & -0.0183 & & & -0.0045 & & & & -0.0128 \\
\hline & .1521 & 1.2342 & -4.8 & 184 & -0.0082 & -0 . & 0.0022 & 0.0095 & 94 & -0.0094 & 00 \\
\hline 0.7020 & .1631 & 1.2189 & $4.4036 \mathrm{e}-3$ & -0.0186 & 0.0109 & & -3.2458 & $4-0.0163$ & -0.0165 & -0.0014 & 0.0128 \\
\hline & .1657 & 1.2477 & -0.01 & $-6.5585 e-3$ & 30.0116 & 0.0103 & $19 e-3$ & 47 & $-1.4669 \mathrm{e}$ & $3-0.0097$ & 0.0000 \\
\hline & & & & & & & & & & & -0.0128 \\
\hline 0.7080 & .1571 & 1.2249 & 0.0191 & $-8.7180 e-3$ & $3-0.0107$ & 0.0223 & 0.0158 & $4.0646 \mathrm{e}-3$ & $-9.8172 \mathrm{e}$ & $4-0.0026$ & -0.0128 \\
\hline & & 1.23 & 0.01 & & & $3.3884 \mathrm{e}-3$ & $-9.2314 \mathrm{e}-3$ & & 6.8 & & \\
\hline 0.7120 & .1564 & 1.2156 & $-5.7750 e-4$ & -0.0140 & 0.0115 & -0.0323 & $-3.9697 e-3$ & $3-4.4109 e$ & 0.0120 & 0.0004 & 0.0000 \\
\hline 0.7140 & 1.1575 & 1.2275 & $5.5583 \mathrm{e}-3$ & -0.0146 & $1.6563 \mathrm{e}-4$ & $-7.1325 \mathrm{e}-3$ & $32.7543 e-3$ & $-5.3903 e-3$ & 0.0136 & -0.0162 & 0.0128 \\
\hline & .1400 & 1.2189 & 0.0111 & $7.0227 \mathrm{e}-3$ & 0.0130 & -0.0276 & $-4.5372 \mathrm{e}-3$ & $3-3.8884 e-3$ & -9.2302 & 30.0224 & 0.0128 \\
\hline 0.7180 & 1.1669 & 1.2342 & -0.0147 & $6.9461 \mathrm{e}-4$ & -0.0110 & 0.0157 & 0.0137 & 0.0237 & $1.3752 \mathrm{e}-3$ & -0.0092 & 0.0128 \\
\hline & .1417 & 1.2490 & $9.3124 \mathrm{e}-3$ & $-2.9332 \mathrm{e}-3$ & $31.7347 \mathrm{e}-$ & 0.0134 & -0.0151 & $-6.8064 \mathrm{e}$ & -0.0225 & -0.0056 & -0.0128 \\
\hline 0.7220 & 1.1615 & 1.2376 & -0.0162 & -0.0228 & $5.4513 \mathrm{e}-3$ & 0.0265 & -0.0270 & $2.2971 \mathrm{e}-3$ & $5.4994 \mathrm{e}-3$ & -0.0189 & 0.0000 \\
\hline 0.7240 & 1.1590 & 1.2462 & 0.0149 & $1.9266 \mathrm{e}-3$ & -0.0119 & $1.6928 \mathrm{e}-3$ & $-8.5065 e-3$ & 30.0153 & $-5.2052 \mathrm{e}$ & $3-0.0163$ & 0.0000 \\
\hline 0.7260 & 1.1472 & 1.2481 & 0.0204 & $-4.2435 e-3$ & $35.3679 \mathrm{e}-3$ & $-7.3113 e-3$ & $3-0.0182$ & 0.0265 & -0.0218 & -0.0173 & -0.0128 \\
\hline
\end{tabular}




\begin{tabular}{|c|c|c|c|c|c|c|c|c|c|c|c|}
\hline 0.7280 & 1.1413 & 1.2297 & 0.0219 & $2.8566 \mathrm{e}-3$ & -0.0182 & $-7.7564 e-3$ & $3-1.5555 \mathrm{e}-4$ & $4-0.0144$ & -0.0228 & -0.0146 & 0.0128 \\
\hline .7300 & 1.1512 & 1.2454 & 0.0191 & $2.2394 \mathrm{e}-3$ & $1.9814 \mathrm{e}-3$ & $4.3679 \mathrm{e}-3$ & $4.7803 e-3$ & -0.0111 & $-2.8471 \mathrm{e}$ & $3-0.0206$ & 0.0128 \\
\hline 7320 & 1.1448 & 1.2341 & $5.1975 \mathrm{e}-3$ & $-1.5428 \mathrm{e}-3$ & 30.0130 & 0.0175 & $-8.6693 e-3$ & $3-7.1567 e-3$ & $3-4.9023$ & $4-0.0047$ & 0.0000 \\
\hline 7340 & 1.1486 & 1.2402 & $-1.7317 \mathrm{e}-3$ & $3-0.0186$ & $4.9600 \mathrm{e}-4$ & $-8.0264 \mathrm{e}-4$ & $41.3778 \mathrm{e}-3$ & $8.9247 \mathrm{e}-3$ & $4.9149 \mathrm{e}-$ & 0.0048 & 0.0257 \\
\hline 0.7360 & 1.1427 & 1.2436 & 0.0179 & 0.0227 & 0.0197 & 0.0140 & $9.7198 \mathrm{e}-4$ & $-4.8599 \mathrm{e}-3$ & $3-5.3020 e-3$ & 30.0058 & 0.0000 \\
\hline 0.7380 & 1.1488 & 1.2543 & 0.0184 & 0.0307 & -0.0108 & $8.8272 \mathrm{e}-3$ & $-1.4583 \mathrm{e}-3$ & $3-0.0224$ & $5.3020 \mathrm{e}-3$ & 0.0033 & 0.0385 \\
\hline 0.7400 & .1365 & 1.2474 & $5.4116 \mathrm{e}-3$ & 0.0000 & $-6.6894 \mathrm{e}-3$ & $3-0.0106$ & 0.0154 & $1.9435 \mathrm{e}-3$ & -0.0232 & -0.0013 & 0.0128 \\
\hline 7420 & .1526 & 1.2431 & 0.0134 & 0.0140 & $-9.4981 \mathrm{e}-3$ & 30.0304 & $-1.5399 \mathrm{e}-3$ & 30.0118 & $-8.6419 \mathrm{e}-3$ & $3-0.0200$ & 0.0000 \\
\hline 7440 & .1576 & 1.2547 & 0.0128 & $-6.6358 \mathrm{e}-3$ & $39.6628 \mathrm{e}-3$ & -0.0119 & $6.8058 \mathrm{e}-3$ & $-4.6835 e-3$ & $3-6.1868 \mathrm{e}-3$ & $3-0.0052$ & 0.0000 \\
\hline 0.7460 & .1480 & 1.2448 & $3.1763 \mathrm{e}-3$ & $-3.5491 \mathrm{e}-3$ & $35.3679 \mathrm{e}-3$ & $-2.0507 e-3$ & $3-5.9140 e-3$ & $32.6013 \mathrm{e}-4$ & -0.0134 & -0.0053 & -0.0128 \\
\hline .7480 & . 1596 & 1.2506 & 0.0252 & $-4.1660 \mathrm{e}-3$ & $31.3219 \mathrm{e}-3$ & $-2.6742 \mathrm{e}-3$ & $3-7.2915 e-3$ & $3-0.0205$ & $6.4811 \mathrm{e}-3$ & 0.0243 & -0.0128 \\
\hline 0.7500 & 1.1348 & 1.2529 & $2.2375 \mathrm{e}-3$ & $1.1571 \mathrm{e}-3$ & -0.0131 & 0.0257 & -0.0109 & -0.0149 & -0.0102 & -0.0070 & 0.0000 \\
\hline 0.7520 & 1.1430 & 1.2529 & 0.0196 & $-3.3949 \mathrm{e}-3$ & $3-0.0122$ & -0.0163 & $7.7779 \mathrm{e}-3$ & 0.0246 & -0.0259 & -0.0033 & 0.0128 \\
\hline 0.7540 & 1.1264 & 1.2495 & $-8.1574 \mathrm{e}-3$ & $3-1.8514 \mathrm{e}-3$ & $3-0.0164$ & $5.5291 \mathrm{e}-3$ & $8.3447 \mathrm{e}-3$ & $1.8560 \mathrm{e}-3$ & $-7.5621 \mathrm{e}-3$ & $3-0.0056$ & 0.0128 \\
\hline .7560 & .1381 & 1.2316 & -0.0158 & -0.0154 & -0.0266 & 0.0230 & 0.0136 & $5.6550 \mathrm{e}-3$ & $-5.7005 e-3$ & 30.0169 & .0000 \\
\hline 7580 & .1372 & 1.2480 & $-2.2377 \mathrm{e}-3$ & $34.4754 \mathrm{e}-3$ & 0.0149 & $5.0802 \mathrm{e}-3$ & $-8.1799 \mathrm{e}-3$ & $3-6.9805 e-3$ & $33.9218 \mathrm{e}-4$ & -0.0162 & 0.0128 \\
\hline 0.7600 & . 1483 & 1.2445 & $-4.6970 \mathrm{e}-3$ & $3-5.6293 e-3$ & $35.7820 \mathrm{e}-3$ & 0.0148 & $1.2154 \mathrm{e}-3$ & 0.0177 & -0.0215 & 0.0071 & -0.0128 \\
\hline 0.7620 & .1293 & 1.2314 & $4.9811 \mathrm{e}-3$ & 0.0100 & $-9.3307 e-3$ & 30.0106 & $-2.1881 \mathrm{e}-3$ & $7.2452 \mathrm{e}-3$ & $8.4458 \mathrm{e}-3$ & -0.0183 & 0.0257 \\
\hline 0.7640 & 1.1363 & 1.2655 & $5.1952 \mathrm{e}-3$ & 0.0282 & -0.0 & $4.1013 \mathrm{e}-3$ & 0.0167 & $71 e-3$ & $67 e-4$ & 0.0233 & -0.0128 \\
\hline 0.7660 & 1.1491 & 1.2535 & 0.0103 & $1.6978 \mathrm{e}-3$ & $-5.5309 \mathrm{e}-3$ & $32.5011 \mathrm{e}-3$ & $-1.9444 c$ & & & 0.0093 & -0.0128 \\
\hline 0.7680 & .1404 & 1.2406 & 0.0 & 5.01 & 0.0104 & $-2.8530 \mathrm{e}-3$ & 32.18 & $-7.8643 e-3$ & $68 \mathrm{e}-3$ & $30 .($ & 28 \\
\hline 0.7700 & .1398 & 1.2531 & -0.0117 & -0.0205 & 0.0120 & 0.0189 & 7.6966 & -0.0178 & -0.0259 & -0.0201 & .0257 \\
\hline 0.7720 & 1366 & 1.2476 & $4.3120 \mathrm{e}-4$ & $2.4690 \mathrm{e}-3$ & -0.0197 & 0.0161 & -0.0100 & -0.0209 & $1.0810 \mathrm{e}-3$ & -0.0046 & -0.0128 \\
\hline 0.7740 & .1483 & 1.2347 & 0.0147 & 0.0139 & $-7.4339 \mathrm{e}-3$ & $3-6.9556$ & $38.7492 \mathrm{e}-3$ & -0.0102 & $2.9414 \mathrm{e}-3$ & 0.0081 & -0.0128 \\
\hline 0.7760 & 1444 & 1.2611 & $-5.2699 \mathrm{e}-3$ & 30.0101 & -0.0183 & $2.7617 \mathrm{e}-3$ & $-4.8610 \mathrm{e}-3$ & $36.1866 \mathrm{e}-4$ & 0.0201 & -0.0004 & 0.0000 \\
\hline 0.7780 & .1344 & 1.2501 & 0.0159 & -0.0260 & $5.9432 \mathrm{e}-3$ & $9.0944 \mathrm{e}-3$ & 0.0109 & & & 0.0045 & -0.0128 \\
\hline 0.7800 & 96 & 1.2471 & $1 \mathrm{e}-3$ & $-5.2466 \mathrm{e}-3$ & 39.91 & $4.5467 \mathrm{e}-3$ & $4.1314 \mathrm{e}-3$ & & $77 e-3$ & 30.0056 & 28 \\
\hline 0.7820 & 45 & 1.2690 & -4.5 & 32.3 & -6.5 & $3-0$. & 0.0118 & $24 e-3$ & -0 . & 45 & 00 \\
\hline 0.7840 & .1312 & 1.2432 & $-4.5445 \mathrm{e}-3$ & 34.63 & $-5.8639 \mathrm{e}-3$ & $36.8643 \mathrm{e}-3$ & $6.0716 \mathrm{e}-3$ & $-5.4786 e-3$ & $3-0.0122$ & 0.0136 & 0.0128 \\
\hline 0.7860 & .1262 & 1.2597 & 0.0102 & $-8.4127 \mathrm{e}-3$ & $34.0438 \mathrm{e}-3$ & $4.0123 \mathrm{e}-3$ & -0.0156 & $-3.8872 \mathrm{e}-3$ & 30.0106 & -0.0331 & 0.0000 \\
\hline 0.7880 & 1439 & 1.2505 & 0.0105 & 0.0161 & -0.0122 & 0.0155 & $-3.2417 \mathrm{e}-3$ & $3-8.7474 e-3$ & 32.9 & 0.0049 & 0128 \\
\hline 0.7900 & 1.1481 & 1.2599 & $5.0527 \mathrm{e}-4$ & $-8.4106 \mathrm{e}-3$ & $3-0.0265$ & $3.7441 \mathrm{e}-3$ & -3.2427 & 40.0119 & $-2.8485 e-3$ & $3-0.0234$ & 0.0000 \\
\hline 0.7920 & 1.1273 & 1.2454 & $3.6036 \mathrm{e}-4$ & -0.0185 & -0.0112 & 0.0169 & -0.0169 & $4.4177 \mathrm{e}-3$ & & & 0128 \\
\hline 0.7940 & 20 & 1.2 & -1. & $34 .($ & 0.0 & -6 & & 3.1 & & & 00 \\
\hline 0.7960 & 1299 & 1.2332 & 6.20 & 0.0144 & $6.1106 \mathrm{e}-3$ & $6.5999 \mathrm{e}-3$ & 4.04 & $-4.9485 e-3$ & 30.0270 & 0. & -0.0128 \\
\hline 0.7980 & 1610 & 1.2534 & $5.2699 \mathrm{e}-3$ & $-6.0219 \mathrm{e}-3$ & $3-0.0194$ & $-6.6912 \mathrm{e}-3$ & $35.5086 \mathrm{e}-3$ & 0.0107 & $-3.3373 e-3$ & 30.0047 & 0.0128 \\
\hline 0.8000 & 1339 & 2567 & $5.0534 \mathrm{e}-3$ & $1.9291 \mathrm{e}-3$ & 0.0 & $6.2386 \mathrm{e}-4$ & $3.1595 \mathrm{e}-3$ & $-6.1843 e-3$ & $37.1674 \mathrm{e}-3$ & 0.0115 & 000 \\
\hline 0.8020 & 406 & 1.2628 & $-9.8175 e-3$ & $3-0.0113$ & -0.0180 & 0.0247 & 2.998 & -0.0251 & 0.0000 & 0.0109 & 0.0128 \\
\hline 0.8040 & 265 & 1.2719 & 0.0107 & $-4.3207 e-3$ & $3-0.0106$ & $4.0988 \mathrm{e}-3$ & $2.1870 \mathrm{e}-3$ & $5.6550 \mathrm{e}-3$ & -0.0253 & 0.0190 & -0.0128 \\
\hline 0.8060 & & 1.2710 & & $5.0927 \mathrm{e}-3$ & 0.0 & $-1.7784 \mathrm{e}-4$ & & 0.0150 & -0 & -0.0093 & 128 \\
\hline .8080 & 1 & 1.2 & -4.9 & $3-0$. & -0. & -7.6 & $3-2.2690 e-3$ & 22 & te-3 & -0 & 128 \\
\hline 0.8100 & 1435 & 1.2781 & -0.0101 & -0.0187 & $6.9361 \mathrm{e}-3$ & -0.0111 & $-4.2942 \mathrm{e}-3$ & $38.8218 \mathrm{e}-5$ & $6.6777 \mathrm{e}-3$ & -0.0235 & -0.0257 \\
\hline 0.8120 & 1236 & 1.2607 & $-5.4123 e-3$ & $38.8728 \mathrm{e}-3$ & 0.0105 & 0.0120 & $6.1598 \mathrm{e}-3$ & 0.0268 & 0.0157 & 0.0007 & -0.0257 \\
\hline 0.8140 & 1322 & 1.2633 & $5.7981 \mathrm{e}-4$ & -0.0198 & -0.0173 & $2.5854 \mathrm{e}-3$ & $-8.8311 \mathrm{e}-3$ & $3-7.0687$ & $4-3.9280 c$ & 30.0055 & 0.0000 \\
\hline 0.8160 & 1437 & 1.2659 & $-6.3525 e-3$ & $37.0218 \mathrm{e}-3$ & $1.0731 \mathrm{e}-3$ & $2.2291 \mathrm{e}-3$ & $4.0547 \mathrm{e}-4$ & $-1.9442 \mathrm{e}-3$ & 30.0124 & 0.0106 & 0.0128 \\
\hline 0.8180 & .1301 & 1.2694 & $4.3351 \mathrm{e}-4$ & $7.6391 \mathrm{e}-3$ & $3.2215 \mathrm{e}-3$ & -0.0163 & -3.6 & $3-$ & $36.5791 \mathrm{e}-3$ & -0.0118 & 0.0000 \\
\hline 0.8200 & 1364 & 1.2604 & & & -0.0369 & $9.0050 \mathrm{e}-3$ & & & $4 e-4$ & 154 & 0000 \\
\hline 0.8220 & 0 & 1. & & 3.2 & 0.0 & & -4 & $3-1$ & $3-4$ & & 128 \\
\hline .8240 & 1351 & 1.2725 & 0.2 & -9.25 & $4-0.0271$ & -0.0370 & -0.0135 & -0.0194 & $4.6157 \mathrm{e}-3$ & -0.0161 & -0.0128 \\
\hline 0.8260 & .1310 & 1.2588 & 0.3272 & -0.0502 & -0.0288 & -0.0245 & $-9.3983 e-3$ & $3-0.0309$ & 0.0160 & 0.0063 & -0.0128 \\
\hline 0.8280 & 1260 & 2608 & 0.5 & -0.0 & -0.0 & -0.0305 & -0.0397 & -0.0194 & -0.0263 & -0.0354 & 0.0000 \\
\hline 0.8300 & .1219 & 1.2495 & 0.6669 & -0.0812 & -0.0615 & -0.0349 & -0.0441 & -0.0367 & -0.0455 & -0.0288 & 0.0000 \\
\hline 0.8320 & .1185 & 1.2535 & & & -0.0962 & -0.0713 & -0.0465 & -0.0478 & -0.0164 & -0.0375 & -0.0385 \\
\hline 1.8340 & 1323 & 1.2657 & & & -0.0972 & & -0.0346 & -0.0338 & & -0.0359 & -0.0128 \\
\hline .8360 & 1354 & 1.2274 & 1.0 & -0.0 & -0.0 & -0.0718 & -0.0 & -0.0352 & -0.0 & -0.0200 & -0.0128 \\
\hline 0.8380 & 1319 & 1.2624 & 1.1291 & -0.0662 & -0.0667 & -0.0610 & -0.0597 & -0.0399 & -0.0355 & 0.0005 & -0.0128 \\
\hline 0.8400 & 1384 & 1.2452 & 1.1 & -0.0257 & -0.0549 & -0.0364 & -0.0438 & $-8.8366 \mathrm{e}-3$ & $3-0.0447$ & -0.0124 & 0.0000 \\
\hline 0.8420 & .1222 & 1.2545 & 1.1572 & -0.0443 & -0.0479 & -0.0385 & -0.0342 & -0.0400 & -0.0492 & -0.0221 & 0.0000 \\
\hline 0.8440 & 1.1392 & 1.2606 & 1.1937 & -0.0353 & -0.0418 & -0.0590 & -0.0283 & -0.0145 & $-9.6248 \mathrm{e}-$ & $3-0.0192$ & -0.0257 \\
\hline 0.8460 & 1.1267 & 1.2536 & 1.1829 & -0.0177 & -0.0162 & -0.0406 & $-6.8089 e-3$ & $3-0.0268$ & -0.0335 & -0.0302 & -0.0128 \\
\hline 0.8480 & .1273 & 1.2707 & 1.1898 & -0.0124 & -0.0286 & -0.0306 & -0.0193 & $-7.6931 \mathrm{e}-$ & $3-0.0178$ & -0.0062 & -0.0128 \\
\hline .8500 & 1292 & 2408 & 1.2012 & $6.0984 \mathrm{e}-3$ & -0.0155 & -0.0144 & -0.0151 & -0.0328 & 0.0104 & -0.0006 & -0.0128 \\
\hline 0.8520 & 1074 & 1.2543 & 1.2085 & $-7.6539 \mathrm{e}-5$ & $5-0.0139$ & -0.0108 & $2.0253 \mathrm{e}-3$ & $-1.6784 \mathrm{e}-3$ & $33.0445 e-3$ & -0.0083 & -0.0128 \\
\hline 0.8540 & .1277 & 2721 & 1.1806 & $-5.2466 c$ & $3-9.8293$ & $3-0.0290$ & $-5.1850 e-3$ & 30.0133 & $-2.6513 \mathrm{e}$ & $3-0.0244$ & 0.0000 \\
\hline 0.8560 & 1.1292 & 1.2603 & 1.2186 & $-6.1725 e-4$ & $4-0.0197$ & -0.0130 & 0.0108 & $5.9206 \mathrm{e}-3$ & -0.0180 & 0.0123 & 0.0000 \\
\hline 0.8580 & 1.1120 & 1.2502 & 1.1958 & $2.6229 \mathrm{e}-3$ & $-8.4247 \mathrm{e}-3$ & $3-0.0158$ & 0.0115 & -0.0152 & 0.0167 & 0.0041 & 0.0000 \\
\hline .8600 & 1177 & 1.2648 & 1.1853 & $-3.5491 \mathrm{e}-3$ & $36.1952 \mathrm{e}-3$ & $-6.9528 c$ & $37.4540 \mathrm{e}$ & $9.2776 \mathrm{e}-3$ & $4.4189 \mathrm{e}-$ & -0.0180 & 0.0000 \\
\hline 0.8620 & 1296 & 1.2604 & 1.2111 & $1.8517 \mathrm{e}-3$ & -0.0215 & $-4.0123 \mathrm{e}$ & $3-9.7271 e-4$ & $43.9766 \mathrm{e}-3$ & 0.0104 & 0.0064 & 0.0000 \\
\hline 0.8640 & .1206 & 1.2712 & 1.2180 & 0.0129 & -0.0113 & $4.1011 \mathrm{e}-$ & -0.0181 & -0.0102 & $6.1869 \mathrm{e}-3$ & -0.0091 & 0.0000 \\
\hline 0.8660 & 1.1233 & 1.2812 & 1.2151 & $2.0073 \mathrm{e}-3$ & -0.0265 & $-4.0161 \mathrm{e}-3$ & $37.2103 \mathrm{e}-3$ & -0.0106 & -0.0196 & -0.0090 & 0.0000 \\
\hline
\end{tabular}




\begin{tabular}{|c|c|c|c|c|c|c|c|c|c|c|c|}
\hline 0.8680 & 1.1177 & 1.2834 & 2297 & $6.7898 \mathrm{e}-3$ & $2.8069 \mathrm{e}-3$ & -0.0291 & 0.0143 & $3.9766 \mathrm{e}-3$ & -0.0266 & -0.0009 & 0.0257 \\
\hline 0.8700 & 1.1200 & 1.2587 & .2254 & $-8.4868 \mathrm{e}-3$ & $3-4.9600 e-2$ & $4-0.0242$ & $8.9182 \mathrm{e}-4$ & $4.5896 \mathrm{e}-3$ & -0.0325 & -0.0149 & 0.0128 \\
\hline 8720 & 1.1239 & 2682 & 2087 & -0.0143 & -0.0139 & -0.0176 & -0.0262 & -0.0253 & $-6.2850 \mathrm{e}$ & 30.0035 & -0.0128 \\
\hline 8740 & 1.1163 & 2693 & 185 & & -0.0192 & -0.0131 & $-2.8353 \mathrm{e}$ & 1753 & $7502+2$ & 060 & -0.0128 \\
\hline 8760 & 1.1079 & 2758 & 2394 & $3.7809 \mathrm{e}-3$ & -0.0126 & -0.0126 & $8.0886 \mathrm{e}-5$ & 0.0281 & $-3.5359 \mathrm{e}-3$ & 30.0095 & .0128 \\
\hline 0.8780 & 1.1179 & 1.2703 & 2203 & $8.4851 \mathrm{e}-4$ & $-5.3688 \mathrm{e}-3$ & $3-0.0135$ & -0.0116 & 0.0152 & 0.0135 & -0.0008 & 0.0000 \\
\hline 0.8800 & 1.1221 & 1.2749 & 2223 & -0.0130 & -0.0293 & $-7.9380 \mathrm{e}-3$ & $38.7502 \mathrm{e}-3$ & -0.0222 & -0.0233 & 0.0051 & 0128 \\
\hline 0.8820 & 1.1066 & 2709 & 2253 & $-9.4884 \mathrm{e}-3$ & $-5.7882 \mathrm{e}-3$ & $3-0.0134$ & $-3.8078 \mathrm{e}-3$ & $3-6.5428 \mathrm{e}-3$ & $3-0.0100$ & 0.0049 & 0000 \\
\hline 0.8840 & 1.1122 & 2868 & 2341 & -0.0106 & $3.4694 \mathrm{e}-3$ & $-3.1208 \mathrm{e}-3$ & $3-0.0119$ & $-2.2088 \mathrm{e}-3$ & $3-1.3748 e-3$ & $3-0.0331$ & 0000 \\
\hline 8860 & 1085 & 2763 & 2382 & $-8.3353 e-3$ & $3-0.0190$ & -0.0127 & -0.0199 & $7.6003 e-3$ & 0.0397 & -0.0024 & 0128 \\
\hline .8880 & 1.1022 & 2919 & 2425 & & & $-7.2190 \mathrm{e}-3$ & & & $38.0523 \mathrm{e}-3$ & -0.0345 & 0000 \\
\hline 8900 & 1.1291 & .2733 & 2380 & $-7.2539 \mathrm{e}-3$ & $3-2.4738 e$ & $3-6.2367 e-3$ & $3-0.0261$ & -0.0103 & $3.8301 \mathrm{e}-3$ & 0.0021 & 0128 \\
\hline 0.8920 & 1.1064 & & & & & & -9 3174e & & $35.1059 \mathrm{e}$ & 0.0109 & 0128 \\
\hline 0.8940 & 1164 & 2616 & & & 3.7 & $11 \mathrm{e}-3$ & $70 e-3$ & 37.8 & -5.5 & & \\
\hline 0.8960 & 1261 & 2740 & & 97 & -0.0146 & -0.0230 & -0.0203 & -0.0149 & 6.1 & & 000 \\
\hline 0.8980 & & & & & & $-3.3009 \mathrm{e}-3$ & $3-3.9696 e-3$ & & & & 128 \\
\hline 0.9000 & 1.1026 & 1.2 & & $5 e-3$ & $3-8.7545 e-3$ & $3-0.0204$ & -0.0139 & $0 e-3$ & 0.0162 & & 0128 \\
\hline 0.9020 & 1.1175 & .2716 & & & & $-9.8115 \mathrm{e}-3$ & $3-0.0115$ & & $-2.5643 \mathrm{e}-3$ & $3-0.0068$ & 0.0000 \\
\hline 0.9040 & 1.1059 & & & & -0.0127 & $-9.6279 \mathrm{e}-3$ & $3-8.2639 e-3$ & & $4-7.6551 e-3$ & $3-0.0099$ & 128 \\
\hline 0.9060 & & & & -2.0 & $3-3.3866 e-3$ & & & & & & \\
\hline 0.9080 & & & & & & 5.4 & & & & & \\
\hline 0.9100 & 1099 & & & & & & -0 & & 6.6 & & \\
\hline 0.9120 & & & & & & & $59 e-4$ & & & & \\
\hline 0.9140 & 35 & 10 & & $e-3$ & -5.6 & $31.6943 \mathrm{e}-3$ & 4.29 & & & & 28 \\
\hline 0.9160 & 1.1022 & 2924 & & & & $69 \mathrm{e}-2$ & 47.21 & & $-5.2040 \mathrm{e}-3$ & $3-0.0122$ & 257 \\
\hline 0.9180 & 1.0964 & & & & & & & $48 \mathrm{e}-3$ & & & \\
\hline 0 & & & & -2 & & 1. & & & & & \\
\hline 20 & & & & & $3-3$ & $3-0$ & & & & & \\
\hline 0.9240 & 0973 & & & & -0 . & $8 \mathrm{e}-3$ & -5.7 & & & & 257 \\
\hline 0.9260 & & & & & & & & & & & \\
\hline 0.9280 & 7 & 18 & & & $-4.9565 \mathrm{e}-3$ & $3-2.85$ & & -0. & & -0 & 128 \\
\hline 0.9300 & 1.0836 & 24 & 560 & & -1.7 & & $9 e-3$ & $1.8548 \mathrm{e}-3$ & $-2.7499 \mathrm{e}-3$ & 090 & 000 \\
\hline 0.9320 & & & & & & & & & & & \\
\hline & & & & & $3-4$ & $12 \mathrm{e}-3$ & & & & & \\
\hline 360 & 7 & & & & -0 . & -0 & & & & & \\
\hline 0.9380 & & & & & -8 & $3-9$ & & & & & \\
\hline 0.9400 & & & & & & & & & & & \\
\hline 0.9420 & 0885 & & 721 & & & $-6.1511 \mathrm{e}-3$ & & & & & 128 \\
\hline 0.9440 & 0835 & & 600 & & -0.0117 & $-5.8847 \mathrm{e}-3$ & & & -3 & 95 & 0128 \\
\hline 0.9460 & & & & & & & & & & & \\
\hline & & & & & e-3 & & & & & & \\
\hline 00 & & & & & & & -1 & $90 e-3$ & -4 & 86 & \\
\hline 0.9520 & & & & & & 3. & & & & & \\
\hline 0.9540 & & & & & & & & & & & \\
\hline 0.9560 & 1.0953 & & 87 & -2.0 & & -0.0267 & -1.9 & & 273 & & -0.0257 \\
\hline 0.9580 & & & & & & & & & & & 0.0128 \\
\hline 0.9600 & & & & & & & & $4-9$ & & & 128 \\
\hline & & & & 3 & $3-9$ & & & & -3 & & \\
\hline & & & & & & $5 e-3$ & & -2 & & & 00 \\
\hline 0.9660 & & & & & $7.9281 \mathrm{e}-3$ & $4.9043 \mathrm{e}-3$ & $-6.6472 \mathrm{e}-3$ & 30.0166 & $5.7945 \mathrm{e}-3$ & -0.0063 & 128 \\
\hline 0.9680 & & & & $-1.4732 \mathrm{e}-3$ & & -0.0213 & -0.0165 & & -6.4 & & \\
\hline 0.9700 & & & & 4.62 & $-4.2129 \mathrm{e}-3$ & $3-0.0135$ & -0.0100 & $6.1866 \mathrm{e}-4$ & -0.0425 & & -0.0128 \\
\hline & & & & & & $-5.3446 \mathrm{e}-2$ & & & $-5.1034 \mathrm{e}-3$ & & 0.0000 \\
\hline & & & & & & & & $57 e-3$ & & & \\
\hline & & & & & & & $5 e-3$ & 30.0 & & 06 & \\
\hline & & & & & & -3 & $3-0.0$ & -9. & $37 e-3$ & & \\
\hline 0. & & & & & & & $-9.4792 e-3$ & 30.0202 & -0.0186 & & 128 \\
\hline 9820 & & & & & & & -0.0 & & -0. & 33 & 128 \\
\hline 0.9840 & 04 & & & & & & & -0 & -0.0297 & 317 & -0.0128 \\
\hline 0.9860 & & & & & & & & $-9.1013 e-$ & & & -0.0128 \\
\hline & & & & & & & & & & & \\
\hline & & & & & & & & & & & \\
\hline 20 & & & & & & & & & & -0 . & 257 \\
\hline & & & & & & & & & & & \\
\hline & & & & & & & & & -0.0344 & & .0128 \\
\hline 0.9980 & 1.1036 & 1.3022 & 2660 & 1.1859 & -0.0197 & -0.0289 & -0.0169 & -0.0233 & -0.0113 & -0.0299 & -0.0128 \\
\hline > > & & & & & & & $-8.6685 \mathrm{e}$ & & & & \\
\hline & & & & & & 2.0 & -0.0250 & -0 & 504 & 84 & .0128 \\
\hline & & & & & & & & & -0.0289 & -0.0050 & -0.0128 \\
\hline 1.0060 & .0933 & 2844 & 744 & 162 & -0.0125 & -0.0239 & -0.0232 & -5.7430 & -0.0322 & 0.0000 & 0.0128 \\
\hline
\end{tabular}




\begin{tabular}{|c|c|c|c|c|c|c|c|c|c|c|c|}
\hline 1.0080 & 1.1153 & 1.2873 & 1.2883 & 1.2217 & -0.0287 & -0.0123 & -0.0194 & $4.5059 \mathrm{e}-3$ & $-6.2850 \mathrm{e}-3$ & $3-0.0154$ & 0.0128 \\
\hline 1.0100 & 1.1044 & 1.2908 & 1.2734 & 1.2142 & -0.0236 & -0.0165 & -0.0212 & 0.0112 & $-3.9218 \mathrm{e}$ & 0.0349 & 0.0128 \\
\hline 0120 & 1.1140 & 1.2870 & 2936 & 1.2248 & $-5.4525 e-3$ & $3-0.0286$ & $-3.8888 \mathrm{e}-3$ & $3-0.0217$ & $5.5979 \mathrm{e}-3$ & -0.0220 & -0.0128 \\
\hline 0140 & 1.1292 & 2712 & 2804 & 1.2510 & $-2.7214 \mathrm{e}-3$ & $3-4.3679 e-3$ & $38.9120 \mathrm{e}-3$ & -0.0165 & $2.0112 \mathrm{e}-4$ & -0.0352 & 0.0000 \\
\hline 0160 & 1.1105 & & 2867 & .2214 & -0.0138 & -0.0211 & $2.4306 \mathrm{e}-3$ & $2.4746 \mathrm{e}-3$ & -0.0155 & -0.0109 & 0.0000 \\
\hline .0180 & 1.1250 & 2927 & & & $-6.9375 e-3$ & $38.9204 \mathrm{e}-4$ & -0.0154 & $-8.8218 \mathrm{e}-5$ & $56.8745 \mathrm{e}-3$ & -0.0167 & 0.0000 \\
\hline 1.0200 & 1.1285 & 3094 & 2899 & 1.2507 & $9.0919 \mathrm{e}-4$ & -0.0168 & -0.0273 & -0.0144 & $4.9149 \mathrm{e}-4$ & 0.0126 & 0.0128 \\
\hline 1.0220 & 1.1352 & .3067 & 2763 & 1.2345 & -0.0197 & $9.8957 \mathrm{e}-3$ & -0.0161 & $3.5287 \mathrm{e}-4$ & $-3.4371 \mathrm{e}-3$ & & 0.0000 \\
\hline 0240 & 1.1311 & 2935 & & 1 & $-7.4304 e-3$ & $3-2.2253 e-3$ & $853 e-3$ & $3-6.6231 e-3$ & 33.9 & & -0.0128 \\
\hline 260 & 1.1132 & 3027 & 887 & 2545 & 0.0130 & $-5.8011 \mathrm{e}-3$ & $32.9978 \mathrm{e}-3$ & 0.0166 & 1.47 & 020 & 0.0000 \\
\hline 0280 & 1.1124 & 2812 & 2697 & 2640 & $2.6421 \mathrm{e}-3$ & -0.0210 & -0.0236 & -0.0219 & -7.266 & -0.0191 & 0.0128 \\
\hline 0300 & 1.1095 & 3114 & 3003 & .2441 & $-2.3937 \mathrm{e}-3$ & 30.0142 & -0.0285 & $2.2959 \mathrm{e}-3$ & $-1.6701 \mathrm{e}-3$ & $5.0295 \mathrm{e}-3$ & -0.0128 \\
\hline . 0320 & 1.0951 & 2890 & & 1.2654 & & & $-2.0232 \mathrm{e}-3$ & & & -0.0178 & -0.0257 \\
\hline 1.0340 & 1.1088 & 1.2888 & & & & $5.7060 \mathrm{e}-3$ & -0.0335 & $-8.8331 \mathrm{e}$ & & & 0.0000 \\
\hline & & & & & & & & & & & 0.0000 \\
\hline 30 & 1 & 18 & & & & $3 e-3$ & $-8.7503 e-3$ & $3-2.5$ & & $33 e-4$ & 40.0128 \\
\hline 0400 & 1.0795 & 1.2945 & & 1.2726 & $4 e-3$ & $6.7759 \mathrm{e}-3$ & -0.0149 & -7.0 & & 146 & 128 \\
\hline & 1.0791 & 1.2953 & & 1.2631 & $-4.0473 e-3$ & $7 e-3$ & $-8.1871 \mathrm{e}-3$ & 30.0110 & & & \\
\hline & 1.0 & 12 & & & 1e-3 & $2 e-3$ & & & $-6.8738 \mathrm{e}-3$ & & -0.0128 \\
\hline 1.0460 & 1.0900 & 2662 & 2928 & 2776 & & -2.8 & $91 \mathrm{e}-3$ & $-5.4786 e-3$ & 35.70 & $03 e-4$ & -0.0128 \\
\hline & 1.0767 & & & & & & & & & & \\
\hline & 0 & & & & & & & & & & \\
\hline 20 & 76 & & & & & 2.9 & & -9 & & & \\
\hline 0540 & 1.0880 & 30 & & & & -1 . & $3-5$ & $3-6.0972 \mathrm{e}-3$ & $3-0.0104$ & -4 & 128 \\
\hline & 10798 & & & & te-3 & 0.0 & & & $-6.7760 \mathrm{e}-3$ & & \\
\hline 80 & 1.0663 & 74 & & & -9. & & & & & & 0.0000 \\
\hline 1.0600 & 1.0760 & 2933 & & & & & & & & & 30.0128 \\
\hline & & & & & & & & & & & \\
\hline & & & & & & -9 & $9 e-3$ & 2. & & & 128 \\
\hline & & & & & & 30.0 & & & & & \\
\hline & & & & & & & 34. & & & & 0000 \\
\hline 00 & 1.0 & 62 & & 8 & & & & & & $55 e-3$ & $3-0.0128$ \\
\hline 0720 & 10685 & 2925 & 2959 & 2815 & $-5.1979 e-3$ & $3-2.3185 e-3$ & & & & & 0.0128 \\
\hline 0740 & 1.08 & & & & & & & $-5.3044 \mathrm{e}-$ & & $60 e-3$ & 0.0000 \\
\hline & & & & & & & & & & & 000 \\
\hline & & & & & & & & & & & \\
\hline & & & & & & & & & & $e-3$ & \\
\hline & 66 & & & & & & 9.8 & & & $5 e-3$ & -0.0128 \\
\hline & & & & & & & & & & & \\
\hline 1.0860 & 1.0618 & 37 & & & & 5.9 & & 0.0 & 7.0 & & 0.0000 \\
\hline 0880 & & & & & & $46.2424 \mathrm{e}-3$ & & $-7.5110 \mathrm{e}-3$ & & & 0.0000 \\
\hline & & & & & -3 & & $5 e-3$ & & & & \\
\hline & & & & & & & & 1.5 & & & \\
\hline 40 & & & & & & & -8.2 & 30.0191 & $-9.4275 \mathrm{e}-3$ & 92 & \\
\hline & & & & & $8 e-3$ & $27 e-3$ & $0 \mathrm{e}-3$ & $3-0$ & -0.0242 & & 000 \\
\hline & & & & & 0 & 0.0173 & $4.2935 \mathrm{e}-3$ & $-4.0637 \mathrm{e}-3$ & & $4.3250 \mathrm{e}-3$ & 0.0000 \\
\hline 1.1000 & 1.0541 & & & & $-2.2303 e-3$ & $3-5.7948 e-3$ & $3-0.0117$ & -0.0216 & $5.9909 \mathrm{e}-3$ & $-9.4420 \mathrm{e}-3$ & 30.0128 \\
\hline & & & & & & & & $-2.5628 \mathrm{e}$ & & & 0.0128 \\
\hline & & & & & & & & & & $e-4$ & \\
\hline & & & & & & 2.8 & & -0 & -0 . & & \\
\hline 80 & & & & & $8 e-3$ & $3-0.0160$ & -9.8 & $1 e-3$ & 0.0326 & $8.3840 \mathrm{e}-3$ & 00 \\
\hline & & & & & & & -5.4 & $09 e-4$ & $-5.4994 \mathrm{e}-3$ & & \\
\hline 1.1120 & 1.0 & 1.2788 & & & $6.3600 \mathrm{e}-3$ & $5.7060 \mathrm{e}-3$ & 4.6 & 0.0112 & $9.6238 \mathrm{e}-3$ & -0.0177 & -0.0128 \\
\hline 1.1140 & & & & & & & $e-3$ & $34 \mathrm{e}-3$ & $8.8392 \mathrm{e}-3$ & $-3.0005 e-3$ & 30.0128 \\
\hline & & & & & & & & $1.8560 \mathrm{e}-3$ & $4 e-3$ & & 0.0128 \\
\hline & & & & & & & & & & & \\
\hline & & & & & & & -2.0 & $3-0$ & & $0 \mathrm{e}-4$ & 28 \\
\hline & & & & & e-3 & $4 e-3$ & 0.0 & 0.0 & & & 00 \\
\hline & & & & & $0 e-3$ & $3-1.7784 e-4$ & $4-6.4813 e-3$ & $39.7221 \mathrm{e}-4$ & -0.0246 & $5.1129 \mathrm{e}-3$ & 0.0128 \\
\hline 1.1260 & 1.0407 & 3040 & 2932 & 96 & -0.0107 & $8.0264 \mathrm{e}-3$ & $-4.2935 \mathrm{e}-3$ & $34.0648 \mathrm{e}-3$ & -0.0236 & -0.0202 & 0.0128 \\
\hline 1.1280 & & & & & & $36 e-3$ & & -0.0113 & $1.2766 \mathrm{e}-3$ & -0.0183 & 0.0000 \\
\hline & & & & & & & & -7 & $1 \mathrm{e}-3$ & & \\
\hline & & & & & & & & & -0.0275 & $3.3535 \mathrm{e}-3$ & -0.0257 \\
\hline & & & & & & & & & & $-7.4130 \mathrm{e}-3$ & 30.0000 \\
\hline & & & & & & & & & & & -0.0257 \\
\hline & 1.04 & 1.2 & 7 & 3 & & -0.0 & -0.0691 & -0. & -0.0480 & -0.0304 & -0.0257 \\
\hline 400 & 1.0402 & 1.3055 & & & & -0.0629 & & & -0.0184 & -0.0240 & -0.0128 \\
\hline 1420 & 1.0380 & 1.2924 & 01 & 51 & 9 & -0.0722 & -0.0556 & -0.0399 & -0.0327 & -0.0383 & -0.0257 \\
\hline 1440 & 1.0551 & 1.2939 & 710 & 1.2613 & & -0.0 & -0.0 & -0.0483 & -0.0389 & -0.0257 & -0.0385 \\
\hline 1.1460 & 1.0379 & 1.2872 & 1.3024 & 1.2645 & 1.2027 & -0.0374 & -0.0544 & -0.0489 & -0.0586 & -0.0287 & -0.0128 \\
\hline
\end{tabular}




\begin{tabular}{|c|c|c|c|c|c|c|c|c|c|c|c|}
\hline 1.1480 & 1.0534 & 1.3006 & 1.2918 & 1.2768 & 1.2241 & -0.0396 & -0.0449 & -0.0338 & -0.0257 & -0.0188 & -0.0385 \\
\hline 1.1500 & 1.0454 & 1.3012 & 1.2988 & 1.2843 & 1.2179 & -0.0337 & -0.0328 & -0.0262 & -0.0375 & $-3.7955 e-3$ & $3-0.0257$ \\
\hline 1520 & 1.0386 & 1.2859 & 2924 & 1.2795 & 1.2234 & -0.0160 & -0.0160 & -0.0166 & -0.0210 & $7.9425 \mathrm{e}-3$ & -0.0128 \\
\hline 1540 & 1.0581 & .3012 & 3051 & 2935 & 2665 & $4.1902 \mathrm{e}-3$ & -0.0186 & -0.0245 & -0.0138 & $7.9453 e-4$ & 0.000 \\
\hline 1560 & 1.0729 & .2861 & 3151 & 2911 & & $6.7740 \mathrm{e}-3$ & -0.0143 & -0.0172 & -0.0101 & $4.2360 \mathrm{e}-3$ & 0.0000 \\
\hline .1580 & 1.0302 & 2950 & & .3212 & 1.2523 & 0.0239 & $-5.7523 e-3$ & $3-0.0297$ & -0.0251 & $3.5300 \mathrm{e}-4$ & 0.0000 \\
\hline 1.1600 & 1.0389 & 1.2958 & .3169 & 1.3105 & 2633 & $8.9156 \mathrm{e}-3$ & $-2.6703 e-3$ & $3-8.6589 e-$ & $4.7137 \mathrm{e}-3$ & $3-4.6770 e-3$ & 30.0000 \\
\hline 1.1620 & 1.0472 & 1.2808 & .3159 & 1.2924 & 1.2668 & -0.0109 & -0.0307 & -0.0279 & -0.0221 & $-7.5895 e-3$ & 30.0128 \\
\hline 1640 & 1.0584 & 1.28 & & 3055 & 2721 & 0.0 & $-8.2639 \mathrm{e}-3$ & 30.0000 & $-3.8302 \mathrm{e}-3$ & $3-4$ & $3-0.0128$ \\
\hline 1660 & 1.0402 & 1.2820 & 3096 & 3266 & 2577 & -0.0208 & -0.0286 & $-8.7479 \mathrm{e}-3$ & $36.9638 \mathrm{e}-$ & -0.0199 & 0.0128 \\
\hline 1680 & 1.0521 & 2987 & & & 2806 & & $-8.2639 \mathrm{e}-3$ & $3-0.0129$ & $-3.5359 \mathrm{e}-3$ & & 0.0000 \\
\hline 1.1700 & 1.0462 & .2791 & & & 1.2634 & $4.2795 \mathrm{e}-3$ & -0.0179 & $6.8889 \mathrm{e}-3$ & -0.0193 & -0.0324 & -0.0128 \\
\hline 1.1720 & 1.0525 & 2815 & & & & $-4.1939 e-3$ & $3.4833 \mathrm{e}-3$ & -0.0234 & $2.7503 e-3$ & & 0.0000 \\
\hline 1.1740 & 1.0673 & 1.2898 & & & & $08 \mathrm{e}-3$ & $51 \mathrm{e}-3$ & & & & -0.0385 \\
\hline 1.1760 & 1.0351 & & & & & & & -3.0944 & & & 0.0128 \\
\hline 80 & 1.04 & & & & & & $8 e-3$ & $6 e-3$ & -0 & & 000 \\
\hline 1800 & 1.0581 & 59 & & 3301 & 2910 & & 0.0117 & & $93 e-3$ & & -0.0128 \\
\hline & 1.0362 & 20 & & 02 & & $-2.8530 \mathrm{e}-3$ & $3.4024 \mathrm{e}-3$ & & & & 30.0257 \\
\hline & 1.0351 & 60 & & & 1.2953 & $2 e-3$ & & & $-6.3856 e-3$ & & 0.0128 \\
\hline 1.1860 & 1.0312 & 1.2770 & & & 1.2956 & & $-1.2154 \mathrm{e}-3$ & $38.3965 \mathrm{e}-3$ & -0.0102 & -4 & $3-0.0128$ \\
\hline & 1.0341 & & & & & $0 e-3$ & & & 16004 & & -0.0128 \\
\hline & & & & & & & & & & & \\
\hline 20 & 1.0297 & & & & & & $5 e-3$ & 0.0 & -6. & & \\
\hline 40 & 1.0413 & 18 & & 60 & 15 & e-3 & -0.0200 & $-9.7153 e-$ & $4-0.0151$ & & -0.0128 \\
\hline & 1.0362 & & & & & & $-7.6157 \mathrm{e}$ & $39.2776 \mathrm{e}-3$ & & & 28 \\
\hline 1.1980 & 1.0395 & 37 & & 14 & 77 & -7 & & & $3-2$ & $3-0$. & 0.0128 \\
\hline 1.2000 & 1.0273 & & & 3188 & & & $-1.0536 \mathrm{e}-3$ & $3-4.2413 e-3$ & & $0 e-3$ & $3-0.0128$ \\
\hline & 1.0382 & & & & & & & & & & \\
\hline & & & & & & $2+2$ & & & & & \\
\hline 60 & 1 & & & & & 7 & & & -9. & & \\
\hline & 1.04 & & & & & & -0. & -0.0186 & $-1.3752 \mathrm{e}-3$ & & 30.0000 \\
\hline 00 & 1.0321 & & & & & & -1.9 & & & & \\
\hline 1.2120 & 1.0443 & 1.2872 & 0 & 22 & 85 & 0.0 & -9.4 & & & & 0.0000 \\
\hline 2140 & 1.0423 & 75 & & 3232 & 2993 & $-6.5081 \mathrm{e}-3$ & $1.4587 \mathrm{e}-3$ & $06 e-3$ & & & 0.0000 \\
\hline & 1.0 & & & & & & & & & & \\
\hline & & & & & & -2 & e-3 & & & & \\
\hline 200 & 1.0327 & & & & & & $1.6208 \mathrm{e}-3$ & & -0. & $9 \mathrm{e}-3$ & $3-0.0128$ \\
\hline 220 & 1.0439 & & & 91 & & $50 e-3$ & -0.0110 & & $8 e-3$ & & -0.0 \\
\hline 40 & & & & & 72 & & & & & & \\
\hline 1.2260 & 1.0408 & 23 & & 61 & 3070 & & -0. & -7.9 & 7e-3 & 3.7 & 0.0128 \\
\hline 1.2280 & 1.0329 & & & & & $5 e-4$ & & & & & -0.0128 \\
\hline & & & & & & & & & & & \\
\hline & 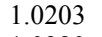 & & & & & & -2 & $3-2$ & $4-0$. & & -0 \\
\hline 340 & 1.0280 & & & & 24 & & -0.0168 & 0.0319 & $-8.3465 \mathrm{e}-3$ & $65 e-3$ & $3-0.0128$ \\
\hline & 1.0234 & & & & & $2 e-3$ & -0.0216 & $-4.4211 \mathrm{e}$ & $3-0.0202$ & -0. & -0.0128 \\
\hline 1.2380 & 1.0276 & 2 & & & 80 & & $-2.0257 \mathrm{e}-3$ & 30.0103 & $9.8172 \mathrm{e}-5$ & 6.70 & -0.0128 \\
\hline 1.2400 & 1.0344 & 2792 & & 3166 & 3393 & 0.0135 & $-1.2112 \mathrm{e}-3$ & $8 \mathrm{e}-3$ & $-4.5179 \mathrm{e}-3$ & $3-5.2$ & $3-0.0128$ \\
\hline 1.2420 & & & & & & & & & & & \\
\hline & & & & & & & & & & & \\
\hline 50 & 6 & & & & & $3 e-3$ & $1 e-3$ & $3-0$. & 0.0 & 0.0125 & -0 \\
\hline 1.2480 & 1.0183 & & & & 26 & & -0.0 & $-7.8638 \mathrm{e}-3$ & $3-0.0100$ & $-3.5300 \mathrm{e}-4$ & 40.0000 \\
\hline & & & & & & & -7.5 & $31.7644 \mathrm{e}-4$ & $+2.9414 \mathrm{e}-$ & & 0.0000 \\
\hline 1.2520 & 1.0284 & 1.2809 & & 351 & 1.3174 & -0.0157 & $5.9147 \mathrm{e}-3$ & -0.0138 & $-7.6602 \mathrm{e}-3$ & $31.7650 \mathrm{e}-3$ & 0.0000 \\
\hline 1.2540 & 1.0348 & & & & & $-9.8066 \mathrm{e}-3$ & & & -0.0163 & & 0.0000 \\
\hline 1.2560 & & & & & & & & $1 \mathrm{e}-4$ & & $1.5010 \mathrm{e}-3$ & -0.0128 \\
\hline & & & & & & & & $2 e-3$ & 0.0 & & \\
\hline & & & & & & & -0. & -0.0113 & $-1.5707 \mathrm{e}-3$ & 34.4 & -0.0128 \\
\hline 20 & & & & & & & $-5.5894 \mathrm{e}$ & $39.1894 \mathrm{e}$ & $9.0353 \mathrm{e}$ & $5 e-3$ & 30.0128 \\
\hline 40 & 29 & & & & 1.3429 & $-1.2487 \mathrm{e}-3$ & $-3.0789 e-3$ & $37.4223 e-3$ & 0.0169 & & -0.0128 \\
\hline 1.2660 & 1.0269 & 1.2696 & 92 & 3653 & 1.3337 & -0.0194 & $-3.9738 \mathrm{e}-3$ & $37.0688 \mathrm{e}-4$ & 0.0205 & 0.0195 & 0.0128 \\
\hline 1.2680 & 1.0232 & & & 3541 & 442 & $4.9037 \mathrm{e}-3$ & $-6.6430 \mathrm{e}-3$ & $3-0.0155$ & -0.0154 & $1.5814 \mathrm{e}-3$ & 0.0000 \\
\hline & & & & & & & & & & & \\
\hline & & & & & & $3 e-3$ & & & 0.0147 & $6.1775 \mathrm{e}-4$ & 0.0000 \\
\hline & & & & & & & & -0.0197 & $-6.2850 e$ & 30.0230 & 0.0128 \\
\hline & & & & & & & & $-7.9509 \mathrm{e}$ & & $8.8250 \mathrm{e}$ & -0.0257 \\
\hline 1.2780 & 1.0164 & 1.27 & 1 & 3617 & 1.3221 & & $-3.1597 \mathrm{e}-3$ & $3-8.5711 \mathrm{e}-3$ & $3-4.8114 \mathrm{e}-3$ & $3-0.0256$ & 0.0000 \\
\hline 1.2800 & 1.0175 & 1.2534 & & 3282 & 403 & & -0.0479 & $8.3943 \mathrm{e}-3$ & -0.0269 & $7.9354 \mathrm{e}-4$ & 0.0000 \\
\hline 1.2820 & 1.0212 & 1.28 & 82 & 1.3363 & 1.3351 & 77 & -0.0276 & -0.0179 & -0.0177 & -0.0360 & 0.0000 \\
\hline 1.2840 & 1.0167 & 1.2617 & 1.2937 & 1.3042 & 1.3115 & & -0.0757 & -0.0532 & -0.0355 & $-8.2080 \mathrm{e}-3$ & 30.0257 \\
\hline 1.2860 & 1.0157 & 1.2757 & 1.3062 & 1.3166 & 1.3035 & 0.6770 & -0.0601 & -0.0634 & -0.0304 & -0.0453 & 0.0000 \\
\hline
\end{tabular}




\begin{tabular}{|c|c|c|c|c|c|c|c|c|c|c|c|}
\hline 1.2880 & 1.0054 & 1.2650 & 1.2881 & 1.2915 & 1.3004 & 0.8712 & -0.0705 & -0.0498 & -0.0350 & -0.0463 & -0.0128 \\
\hline 1.2900 & 1.0274 & 1.2670 & 1.2947 & 1.3040 & 1.2787 & 1.0135 & -0.0643 & -0.0695 & -0.0441 & -0.0172 & -0.0128 \\
\hline 2920 & 1.0206 & 1.2503 & 1.2964 & 1.3108 & 1.2982 & 1.1575 & -0.0702 & -0.0557 & -0.0414 & -0.0470 & -0.0128 \\
\hline 1.2940 & 1.0136 & 1.2680 & 2930 & .3096 & .3021 & 1.2203 & -0.0512 & -0.0570 & -0.0258 & -0.0319 & 0.0000 \\
\hline 1.2960 & 1.0154 & 1.2681 & 3058 & .3184 & .3202 & 1.2633 & -0.0333 & -0.0189 & -0.0381 & -0.0499 & 0.0000 \\
\hline 1.2980 & 1.0083 & 1.2631 & 3047 & 1.3280 & .3379 & 1.2907 & -0.0284 & -0.0229 & $-2.4549 \mathrm{e}-3$ & $3-0.0307$ & 0.0000 \\
\hline 1.3000 & 1.0005 & 1.2659 & .3099 & 1.3255 & .3274 & 1.2855 & -0.0228 & -0.0284 & -0.0268 & -0.0283 & 0.0000 \\
\hline 1.3020 & 0.9937 & 1.2636 & .3024 & 1.3233 & .3477 & 1.2746 & -0.0399 & -0.0208 & -0.0185 & $-6.7960 e-3$ & $3-0.0128$ \\
\hline 1.3040 & 1.0166 & 1.2836 & 3071 & 1.3260 & 3509 & & 75 & -0.0319 & -0.0179 & -0.0162 & -0.0128 \\
\hline 3060 & 1.0182 & 1.2782 & 3252 & 3546 & 3522 & 1.3033 & -0.0160 & $-4.0671 \mathrm{e}-3$ & $3-0.0162$ & -0.0138 & -0.0128 \\
\hline 1.3080 & 1.0075 & 1.2677 & 3105 & 3178 & & & -0.0117 & -0.0149 & $-7.6589 \mathrm{e}-3$ & $3-0.0274$ & -0.0128 \\
\hline 1.3100 & 1.0203 & 2848 & 3172 & 3320 & .3580 & 1.2634 & $-4.6976 \mathrm{e}-3$ & $3-0.0165$ & $-9.7166 e-3$ & $3-0.0200$ & 0.0000 \\
\hline 1.3120 & 1.0119 & 1.2811 & .3125 & 1.3478 & 1.3627 & & & $-1.9442 \mathrm{e}$ & $3-7.7569 e-3$ & $3-6.9710 e$ & 30.0000 \\
\hline 1.3140 & 1.0056 & 1.2686 & 1.3088 & 1.3426 & 1.3450 & & $-8.1871 \mathrm{e}-3$ & $3-1.0609 e-3$ & $3-0.0183$ & $3.8830 \mathrm{e}-3$ & 0.0257 \\
\hline 1.3160 & 1.0066 & & & & & & & & & & -0.0257 \\
\hline 1.3180 & 0.9966 & 1 & & & & & 1e-3 & & & & 0.0000 \\
\hline 200 & 1.0074 & 1.2765 & & 3438 & 1.3714 & 1.3081 & & $-6.7159 \mathrm{e}-3$ & 30.0160 & $-5.8245 \mathrm{e}-3$ & 0.0128 \\
\hline 1.3220 & 0.9999 & 1.2815 & 68 & 539 & 1.3433 & 1.3146 & & 0.0213 & & -3 . & 30.0000 \\
\hline & 1.0001 & 1.2681 & & & & & & & $4.9023 \mathrm{e}-4$ & 0.0192 & 0.0000 \\
\hline 1.3260 & 0.9674 & 1.2701 & & & 1.3672 & & & & -0.0221 & & 0.0000 \\
\hline & 0.9922 & 1.2639 & & & & & & $-4.4222 \mathrm{e}$ & 40.0 & $2.4710 \mathrm{e}-3$ & 0.0128 \\
\hline & 1.00 & & & & 44 & & $6 e-3$ & $-3.8879 \mathrm{e}-3$ & $3-7$ & & \\
\hline 1.3320 & 0.9923 & 1.2 & & & 17 & & $270 e-3$ & 0.0196 & $7 e-4$ & & 28 \\
\hline 3340 & 0.9779 & 1.2841 & & & 1.3756 & 1.3 & $29 \mathrm{e}-3$ & $3-0.0218$ & -0.0144 & $-4.4054 \mathrm{e}$ & +0.0000 \\
\hline 60 & 0.9837 & 1.2757 & 162 & 07 & 3670 & & & -0.0235 & -0.0220 & -0.0192 & \\
\hline 80 & 9742 & & & & & & & $3-0.0154$ & $-4.6157 e-3$ & $3-0$. & 0.0000 \\
\hline 1.3400 & 0.9909 & 1.2772 & 57 & 67 & 1.3680 & & $8.1093 \mathrm{e}-4$ & $-1.5020 \mathrm{e}-3$ & $3-8$. & 276 & 0.0128 \\
\hline & 0.9774 & & & & & & & & & & \\
\hline & 03 & & & & & & & 9.8 & & $5 e-3$ & \\
\hline 60 & 0.9578 & 28 & & & 5 & & $2 e-3$ & -0. & $-9.4276 e-3$ & 30. & \\
\hline 3480 & 0.9587 & 2524 & & & .3783 & & & $-5.3067 e-3$ & $3-0.0116$ & 225 & 0.0000 \\
\hline 3500 & 0.9427 & .2529 & & & 3633 & & & $3-6.1852 \mathrm{e}-3$ & & & -0.0128 \\
\hline 1.3520 & 0.9430 & 1.2649 & 11 & 2 & 1.3747 & 68 & $2.3436 \mathrm{e}-3$ & $-7.1571 \mathrm{e}-3$ & $1 e-3$ & $3-0.0$ & 0.0128 \\
\hline 1.3540 & 0.9446 & 1.2828 & 8 & 9 & 1.3547 & & & $-1.5031 \mathrm{e}-3$ & & $-1.2355 \mathrm{e}-3$ & 30.0000 \\
\hline & 0.9270 & & & & & & & & $9 e-3$ & & \\
\hline & 3 & & & & & & & & & & \\
\hline 3600 & 0.9210 & 1.2 & & & 1.3745 & & $7 e-3$ & 0.0275 & $-7.8512 \mathrm{e}$ & 212 & 28 \\
\hline 3620 & 0.9382 & 1.2605 & & 57 & 1.3971 & 1.3 & -0.0220 & $6.0082 \mathrm{e}-3$ & $-5.4013 e-3$ & 30.0 & -0.0128 \\
\hline & 0.9251 & & & & & & $72 \mathrm{e}-$ & & & 0.0113 & -0.0128 \\
\hline 1.3660 & 0.9171 & 1.2473 & 72 & 05 & 1.3822 & 1.3424 & & 0.0246 & $-7.5616 \mathrm{e}-3$ & $3-9.2655 e-3$ & 30.0000 \\
\hline 1.3680 & 0.9207 & & & & & & & $-7.7753 \mathrm{e}$ & $3-0.0474$ & & 30.0000 \\
\hline & 0.91 & & & & & & & & & & \\
\hline & $x$ & & & & & & $90 e-4$ & $4-5$ & $7 e-3$ & & \\
\hline 740 & 8879 & & & & & & & $6.0086 \mathrm{e}-3$ & -0.0115 & $50 \mathrm{e}-4$ & 40.0128 \\
\hline 3760 & 0.8819 & 69 & & & 67 & & 70e-3 & $2.0324 \mathrm{e}-3$ & -0.0172 & & 0.0000 \\
\hline & & 31 & & & & & & $3.6226 \mathrm{e}-3$ & & & 00 \\
\hline 1.3800 & 0.8794 & 1.2448 & 73 & & 1.3492 & & $3 e-3$ & 0.0183 & 0.0156 & -0.0139 & 0.0000 \\
\hline 1.3820 & & & & & & & & & $8.3528 \mathrm{e}-3$ & $-7.4130 \mathrm{e}$ & 30.0000 \\
\hline 3840 & 0.85 & & & & & & & $18 \mathrm{e}-3$ & $-4.4196 \mathrm{e}-3$ & & \\
\hline 60 & 0.8438 & 2 & & & 00 & & e-3 & -1.7 & 44.2 & 0. & 00 \\
\hline 880 & 8461 & 2536 & & & 21 & & & 0.0 & $-9.8297 e-5$ & $59.7004 e-4$ & 0.0128 \\
\hline & & & & & & & $0 e-3$ & $95 \mathrm{e}-3$ & $-1.5713 \mathrm{e}-3$ & 31 & 0.0128 \\
\hline 1.3920 & 0.8382 & 1.2509 & 43 & 3433 & 1.3845 & & 21 & $4.8601 \mathrm{e}-3$ & -0.0164 & $5.8245 \mathrm{e}-3$ & 0.0000 \\
\hline 1.3940 & 0.8399 & 1.2472 & & & & & $29 e-3$ & -0.0244 & $-5.6953 \mathrm{e}-3$ & $55 \mathrm{e}-$ & 30.0000 \\
\hline & & & & & & & $8 \mathrm{e}-3$ & $3.1804 \mathrm{e}-3$ & $7.4628 \mathrm{e}-3$ & & 0.0128 \\
\hline & & & & & & & & $72 \mathrm{e}-3$ & & te-3 & \\
\hline 00 & 3 & & & & & & & 0.0 & 0.0 & $5 e-3$ & -0.0128 \\
\hline 20 & 8264 & & & & 39 & & -0.0171 & $-3.0043 e-3$ & 30.0100 & -0.0237 & 0.0000 \\
\hline & 8201 & 22 & & & 0 & & $-7.2901 \mathrm{e}$ & $t-3.0039 \mathrm{e}$ & 30.0186 & $-3.5300 \mathrm{e}-3$ & 0.0000 \\
\hline 1.4060 & 0.8159 & 1.2377 & 395 & 631 & 1.4105 & 1.3702 & $8.5071 \mathrm{e}-3$ & -0.0267 & $4.1280 \mathrm{e}-3$ & $-4.6765 e-3$ & 30.0000 \\
\hline 1.4080 & 0.8275 & 1.2499 & & & 1.3633 & & -0.0200 & $8.3946 \mathrm{e}-3$ & $-5.9899 \mathrm{e}-3$ & $3-0.0151$ & -0.0128 \\
\hline & & & & & & & $-2.5114 \mathrm{e}-3$ & 30.0163 & $4.9098 \mathrm{e}-3$ & $55 e-3$ & 0.0128 \\
\hline & 8130 & & & & & & $-5.8333 e-3$ & $3-3.2693 e$ & 30.0109 & -0.0183 & 0.0128 \\
\hline 140 & 8027 & & & & & & $1.6177 \mathrm{e}-2$ & 0.0114 & -0.0128 & $6.1733 \mathrm{e}-$ & -0.0128 \\
\hline & & & & & & & $-7.1294 \mathrm{e}$ & $3-3.0039 e-3$ & $3-1.7673 e-3$ & $3-0.0240$ & -0.0128 \\
\hline & 81 & 1.2395 & & 3702 & 1.4125 & 1.4 & -0.0109 & -0.0107 & $-3.5348 \mathrm{e}-3$ & $3-8.8956 e-5$ & 50.0128 \\
\hline 1.4200 & 0.8021 & 1.2467 & 1.3319 & 3566 & 1.4004 & 1.4097 & -0.0252 & -0.0181 & $-1.6693 e-3$ & $34.4125 \mathrm{e}-3$ & 0.0000 \\
\hline 1.4220 & 0.7989 & 1.2359 & 1.3198 & 1.3821 & 1.3963 & 1.3847 & -0.0203 & $6.8041 \mathrm{e}-3$ & 0.0178 & $4.8573 \mathrm{e}-3$ & 0.0000 \\
\hline 1.4240 & 0.8129 & 1.2433 & 58 & 1.3635 & 1.4015 & 1.4124 & $6.6441 \mathrm{e}-3$ & $-2.2971 \mathrm{e}-$ & $3-0.0127$ & -0.0125 & 0.0000 \\
\hline 1.4260 & 0.8073 & 1.2157 & 1.3101 & 1.3613 & 1.4074 & 1.3818 & 0.0856 & $-7.3334 \mathrm{e}-3$ & $3-0.0204$ & $3.4422 \mathrm{e}-3$ & 0.0000 \\
\hline
\end{tabular}




\begin{tabular}{|c|c|c|c|c|c|c|c|c|c|c|c|}
\hline 1.4280 & 0.8137 & 1.2397 & 1.3111 & 1.3696 & 1.3772 & 1.3857 & 0.2123 & $-7.7756 \mathrm{e}-3$ & $3-0.0398$ & -0.0240 & 0.0000 \\
\hline 1.4300 & 0.8014 & 1.2255 & 1.3148 & 1.3272 & 1.3730 & 1.3654 & 0.3952 & -0.0444 & -0.0261 & -0.0204 & -0.0128 \\
\hline 1.4320 & 0.8001 & 1.2293 & 1.2881 & 1.3518 & 1.3634 & 1.3556 & 0.6352 & -0.0431 & -0.0562 & -0.0335 & -0.0257 \\
\hline 1.4340 & 0.8031 & 1.2387 & .3071 & 1.3384 & .3437 & 1.3664 & 0.8375 & -0.0805 & -0.0660 & -0.0235 & 0.0000 \\
\hline 1.4360 & 0.7902 & 1.2336 & 2879 & .3227 & 1.3254 & 1.3405 & 1.0472 & -0.0923 & -0.0782 & -0.0445 & -0.0257 \\
\hline 1.4380 & 0.8241 & 1.2312 & 2940 & 1.3282 & 1.3325 & 1.3572 & 1.1545 & -0.0954 & -0.1000 & -0.0480 & -0.0257 \\
\hline 1.4400 & 0.8276 & 1.2240 & 1.2953 & 1.3381 & 1.3430 & 1.3707 & 1.2565 & -0.0658 & -0.0449 & -0.0498 & -0.0257 \\
\hline 1.4420 & 0.8154 & 1.2458 & 1.2934 & 1.3613 & 1.3737 & 1.3732 & 1.2972 & -0.0539 & -0.0583 & -0.0492 & -0.0257 \\
\hline 1.4440 & 0.8127 & 1.2366 & 1.2797 & 1.3382 & 1.3541 & & 1.3403 & & -0.0587 & -0.0368 & -0.0128 \\
\hline 1.4460 & 0.8273 & 1.2307 & & 1.3327 & .3500 & & 1.3577 & -0.0 & -0.0284 & $-1.7650 \mathrm{e}-4$ & $4-0.0257$ \\
\hline 1.4480 & 0.8020 & 1.2144 & 2972 & 1.3514 & 1.4161 & 1.3984 & 1.3541 & -0.0134 & -0.0311 & -0.0113 & 0.0000 \\
\hline 1.4500 & 0.8161 & .2229 & & 1.3483 & .3935 & 1.4130 & 1.3369 & $-7.9532 \mathrm{e}-3$ & $3-0.0138$ & -0.0113 & 0.0000 \\
\hline 1.4520 & 0.8089 & 1.2270 & & 1.3508 & 1.3996 & & & & -0.0476 & -0.0216 & -0.0128 \\
\hline 1.4540 & 0.8084 & 1.2098 & 1.3245 & 1.3726 & 1.3932 & 1.4287 & 1.3489 & $1.0597 \mathrm{e}-3$ & $-8.4458 \mathrm{e}$ & $3-5.0281 e-3$ & 30.0000 \\
\hline 1.4560 & 0.8059 & 1.2158 & & 1.3347 & & & & & $32.3574 \mathrm{e}-3$ & -0.0440 & -0.0128 \\
\hline & 0.7985 & & & & & & & & & & \\
\hline 1.4600 & 0.8142 & 1.2344 & & 1.51 & & & & 6.27 & -0.0100 & -0.0168 & 0.0000 \\
\hline 4620 & 0.8108 & 1.2344 & 78 & 1.3542 & 1.4034 & 1.4099 & 1.3602 & $-7.5992 \mathrm{e}-3$ & $3-9.7267 e-3$ & $3-8.9140 \mathrm{e}$ & 0.0257 \\
\hline 1.4640 & 0.8152 & 1.2309 & & & & 1.4036 & & 0.0149 & -0.0143 & 0.0294 & -0.0128 \\
\hline 1.4660 & 0.8119 & 1.2265 & & 1.3584 & & & & $7.4223 e-3$ & $-8.8380 \mathrm{e}-3$ & $3-0.0127$ & -0.0128 \\
\hline 1.4680 & 0.8214 & 1.2196 & & & & & & -1.9 & & $9.8840 \mathrm{e}-3$ & 0.0000 \\
\hline 1.4700 & 0.82 & & & & & & & & & $-2.3835 \mathrm{e}-3$ & 30.0000 \\
\hline 1.4720 & 0.80 & 0 & & 7 & & & & & & $5 e-3$ & 00 \\
\hline 4740 & 0.8175 & 3 & & 4 & 1.4 & & & & -0 & -6 & 28 \\
\hline 1.4760 & 0.8115 & 1.2068 & & 78 & 1.4 & 1.2 & & & -0.0170 & $7.9496 \mathrm{e}-4$ & -0.0128 \\
\hline 4780 & 0.8106 & 1.2240 & & & & & & & $6.7752 \mathrm{e}-3$ & $-3.5304 \mathrm{e}-3$ & 30.0000 \\
\hline 1.4800 & 0.8129 & 1.2126 & & 31 & & & & & $5.3045 \mathrm{e}-3$ & -0.0174 & 0.0000 \\
\hline 1.4820 & 0.8122 & 1.2247 & & & & & & & & $32 e-3$ & 0.0000 \\
\hline & & & & & & & & & $-8.9365 \mathrm{e}-3$ & $17 \mathrm{e}-3$ & \\
\hline 60 & 0 & & & & & & & $23 e-3$ & $9 e-3$ & & 00 \\
\hline 380 & 0.79 & 52 & & & & & & & 5.9 & -6 & 00 \\
\hline 4900 & 0.7998 & 2250 & & 85 & & & & & $13 e-3$ & 35 & 0.0000 \\
\hline 4920 & 0.7900 & & & & 1 & & & 00 & & & 0.0128 \\
\hline 1.4940 & 0.7987 & 1.2284 & 21 & 3 & 138 & 18 & 53 & $4.8600 \mathrm{e}-3$ & -6.5 & & -0.0257 \\
\hline 1.4960 & 0.7934 & 1.2289 & & & 1.3907 & & & $-5.2128 \mathrm{e}$ & 30.0161 & e-3 & -0.0128 \\
\hline & & & & & & & & & & & \\
\hline & 9 & & & & & & & & $72 e-3$ & 35. & 57 \\
\hline 1.5020 & 0.7860 & 2201 & & & 1.4 & & & & -0.0160 & -0 . & -0.0128 \\
\hline & & & & & & & & & & & \\
\hline 1.5060 & 0.7 & 30 & & & & & & & $-7.0706 e-3$ & 20 & -0.0128 \\
\hline 1.5080 & 0.7749 & 1.2087 & 3076 & 3551 & 1.4011 & 1.4094 & 1.4283 & -0.0139 & $3.7371 \mathrm{e}-3$ & 0.0262 & 0.0128 \\
\hline 1.5100 & 0.7796 & & & & & & & & $-4.3203 e-3$ & $32.6475 \mathrm{e}-3$ & 0.0000 \\
\hline & 077 & & & & & & & $2 e-3$ & & & 0.0000 \\
\hline 0 & 2 & & & & & & & e-3 & 0.0255 & -0.0140 & 28 \\
\hline 5160 & 0.7766 & 1.2213 & & & 1.3 & & & -0.0 & $-4.1242 \mathrm{e}-3$ & $3-2.6546 e-$ & +0.0000 \\
\hline 5180 & 0.7897 & & & & & & & & $3.5372 \mathrm{e}-3$ & -0.0158 & -0.0128 \\
\hline 1.5200 & 0.7844 & 1.2098 & & & & & & & 0.0138 & -0.0243 & 0.0000 \\
\hline 1.5220 & 0.7790 & 1.2125 & 3155 & 3672 & 1.4374 & 1.4210 & 1.4315 & 0.0286 & $-6.7765 e-3$ & 30.0164 & -0.0128 \\
\hline & & & & & & & & & & & -0.0128 \\
\hline & 0 & & & & & & & e-3 & $8 \mathrm{e}-3$ & 36 & 28 \\
\hline & 78 & & & & & & & & -0 & $5 e-3$ & 00 \\
\hline 0 & 0.793 & & & & & & & $4 e-3$ & -0. & -0.0195 & -0.0128 \\
\hline 5320 & 0.7723 & 2 & & & & & & & $-5.1072 \mathrm{e}-3$ & $35.2947 \mathrm{e}-3$ & 0.0000 \\
\hline 1.5340 & 0.7882 & 1.1992 & 5 & 3385 & 1.4151 & 1.4463 & 1.4385 & -0.0219 & 0.0134 & -0.0195 & -0.0128 \\
\hline 1.5360 & 0.7871 & & & & & & & $3.5332 \mathrm{e}-4$ & $-3.1425 \mathrm{e}-3$ & $3-0.0243$ & 0.0000 \\
\hline & 0.7982 & & & & & & & & 0.0405 & $-5.7355 e$ & 0.0385 \\
\hline & 0.7718 & & & & & & & $-2.6511 \mathrm{e}$ & $3-1.8654 \mathrm{e}-3$ & & 0.0000 \\
\hline & 7874 & & & & & & & 0.01 & $-3.5347 \mathrm{e}-3$ & $25 \mathrm{e}$ & 0.0128 \\
\hline & 7862 & & & & 2 & & 70 & -0.0 & $-5.8928 \mathrm{e}-3$ & $3-0.0105$ & 0.0000 \\
\hline & 0.7959 & & & & & & & & $2.9464 \mathrm{e}-3$ & -0.0159 & 0.0128 \\
\hline 1.5480 & 0.7807 & 1.2034 & 1.2898 & 1.3525 & 1.4174 & 1.4528 & 1.4466 & -0.0164 & -0.0114 & $3.5300 \mathrm{e}-3$ & 0.0128 \\
\hline 1.5500 & 0.7798 & & & & & & & -0.0155 & -0.0138 & -0.0146 & 0.0000 \\
\hline 1.5520 & 0.7759 & & & & 1.4092 & & & $9.1012 \mathrm{e}-3$ & -0.0169 & $-7.0600 \mathrm{e}$ & 0.0128 \\
\hline & 0.7675 & & & & & & & -0.0358 & $-3.3398 \mathrm{e}$ & $3-9.4431 e$ & 0.0000 \\
\hline & 77 & & & & & & & $-7.9543 \mathrm{e}$ & $45.3033 e-3$ & -0.0113 & 0.0385 \\
\hline & $79^{1}$ & & & & & & & & -0.0179 & -0.0181 & 0.0000 \\
\hline & 07590 & & & & & & & $3.8884 \mathrm{e}-3$ & $-9.1334 \mathrm{e}-3$ & 30.0177 & 0.0128 \\
\hline 1.5620 & 0.7836 & 1.1862 & 1.3127 & 1.3399 & 1.4191 & 1.4566 & 1.4386 & -0.0124 & 0.0233 & $3.0061 \mathrm{e}-3$ & 0.0000 \\
\hline 1.5640 & 0.7861 & 1.1926 & 1.3039 & 1.3496 & 1.4117 & 1.4308 & 1.4493 & $6.8991 \mathrm{e}-3$ & $9.8209 \mathrm{e}-3$ & $-1.8525 \mathrm{e}-3$ & $3-0.0128$ \\
\hline 1.5660 & 0.7855 & 1.2218 & 1.2860 & 1.3581 & 1.4051 & 1.4447 & 1.4365 & -0.0120 & -0.0112 & $-6.0010 e-3$ & 0.0128 \\
\hline
\end{tabular}




\begin{tabular}{|c|c|c|c|c|c|c|c|c|c|c|c|}
\hline 5680 & 0.7659 & 1.1926 & 1.2854 & 1.3462 & 1.4047 & 1.4508 & 1.4465 & 0.0121 & \multicolumn{2}{|c|}{$-9.2314 e-3-0.0355$} & 0.0000 \\
\hline 1.5700 & 0.7648 & 1.2023 & 1.2874 & 1.3463 & 1.4154 & 1.4490 & 1.4483 & 0.0346 & $120-4$ & -0.0263 & 0.0000 \\
\hline 1.5720 & 0.7775 & 1.1978 & 1.2845 & 1.3595 & 1.4129 & 1.4329 & 1.4477 & 0.1234 & $5.9909 \mathrm{e}-3$ & -0.0299 & -0.0257 \\
\hline 1.5740 & 0.7873 & 1.2005 & 2921 & 1.3313 & 1.4030 & 1.4076 & 1.4338 & 0.2748 & -0.0462 & -0.0236 & -0.0128 \\
\hline 1.5760 & 0.7715 & 1.1984 & 1.2793 & 1.3324 & 1.3905 & 1.4107 & 1.4139 & 0.4634 & -0.0734 & -0.0655 & -0.0257 \\
\hline 1.5780 & 0.7786 & 1.1982 & 1.2844 & 1.3534 & 1.3912 & 1.4006 & 1.4042 & 0.6993 & -0.0765 & -0.0692 & -0.0128 \\
\hline 1.5800 & 0.7975 & 1.2176 & 1.2700 & 1.3172 & 1.3788 & 1.3772 & 1.3927 & 0.9361 & -0.0715 & -0.0609 & -0.0257 \\
\hline 1.5820 & 0.7790 & 1.1897 & 1.2862 & 1.3236 & 1.3704 & 1.4101 & 1.4019 & 1.1100 & -0.0849 & -0.0887 & 0.0000 \\
\hline 1.5840 & 0.7760 & 1.1948 & 1.2710 & 1.3134 & 1.3821 & 1.3892 & 1.4031 & 1.2634 & -0.0502 & -0.0640 & -0.0257 \\
\hline 1.5860 & 0.7601 & 1.1973 & 1.2804 & 1.3340 & 1.3693 & & 1.4277 & & & -0.0425 & -0.0257 \\
\hline 1.5880 & 0.7816 & 1.1904 & 2634 & 1.3249 & 1.3909 & 1.4209 & 1.4245 & 1.3933 & -0.0385 & -0.0308 & -0.0257 \\
\hline 1.5900 & 0.7945 & 1.1906 & .2685 & 1.3298 & 1.3733 & 1.4356 & 1.4457 & 206 & 05 & -0.0117 & -0.0257 \\
\hline 1.5920 & 0.7994 & 1.1861 & 1.2564 & 1.3321 & 1.4013 & & 1.4284 & 1.4361 & -0.0104 & $-5.5602 \mathrm{e}$ & 30.0000 \\
\hline 1.5940 & 0.7923 & 1.2117 & 1.2581 & 1.3412 & 1.4218 & 1.4630 & 1.4417 & & -0.0139 & $-6.8835 e-3$ & 30.0000 \\
\hline 1.5960 & 0.7818 & 1.2045 & 1.2663 & 1.3481 & 1.4129 & 1.4622 & 1.4657 & & -0.0121 & 0.0115 & 0.0128 \\
\hline 1.5980 & 0.7964 & & & & & & & & $-7.1694 \mathrm{e}$ & 373240 & 30.0000 \\
\hline 1.6000 & 0.7876 & 1.1996 & & 1.3485 & 1.3927 & 1.4 & & & & & -0.0257 \\
\hline 1.6020 & 0.7962 & 1.2043 & & 1.3461 & 1.3955 & 1.4 & & & & $1.2355 \mathrm{e}-3$ & 0.0128 \\
\hline 1.6040 & 0.8107 & 1.2013 & & & 1.4111 & & & & & -0.0392 & 0.0128 \\
\hline 1.6060 & 0.8010 & 1.1751 & & 1.3 & & & & & & $-6.0010 e-3$ & 0.0128 \\
\hline 1.6080 & 0.8016 & 1.2106 & & & 1.4111 & & & & & -0.0161 & 0.0000 \\
\hline 1.6100 & 0.7923 & & & & & & & & $23 \mathrm{e}-3$ & $3-0.0313$ & 0.0000 \\
\hline & 0.8040 & & & & 1.3 & & & & & & 0000 \\
\hline 1.6140 & 0.8104 & 1.2 & & 1.3 & 1.4 & 1.4 & & & & & 0.0257 \\
\hline 1.6160 & 0.7967 & 1.1954 & & 1.3342 & 1.3973 & 1.4449 & 1. & & & $3.2660 \mathrm{e}-3$ & 0.0128 \\
\hline 1.6180 & 0.8024 & 1.2136 & & & & 1.4 & & & $e-4$ & -0.0326 & -0.0128 \\
\hline 1.6200 & 0.8190 & 1.2089 & 87 & 1.3 & 1.4 & & & & & $-5.4719 \mathrm{e}-3$ & 30.0000 \\
\hline 1.6220 & 0.8131 & 1.1822 & 1.2750 & 1.3538 & 1.3833 & 1.4441 & 1.4709 & & & 0.0102 & -0.0257 \\
\hline 1.6240 & 0.8190 & & & & & & & & & $32 e-3$ & 0.0000 \\
\hline 1.6260 & 0.8058 & & & & & & & & & $0 e-3$ & \\
\hline 1.6280 & 0.8096 & 1.2 & & & 1.4 & & & & & $20 e-3$ & \\
\hline 1.6300 & 0.8073 & 1.1918 & & 379 & 1.3928 & 1.4 & & & & $e-3$ & 0.0128 \\
\hline 1.6320 & 0.8265 & 1.1926 & 68 & & 1.3970 & 60 & & & & & -0.0128 \\
\hline 1.6340 & 0.8467 & 1.2045 & 2777 & 1.3365 & 1.4152 & 1.4476 & & & & 0.0184 & 0.0000 \\
\hline 1.6360 & 0.8289 & 1.1880 & 2745 & 6 & 14 & & & & $-2.1608 \mathrm{e}-3$ & $3-0.0192$ & 0.0000 \\
\hline & & & & & & & & & & $5 e-3$ & 0000 \\
\hline 1.6400 & 0.83 & & & & & & & & & $75 e-3$ & $3-0.0257$ \\
\hline 1.6420 & 0.8306 & 1.1940 & 788 & 1.3325 & 1.3953 & 1.4372 & 1.4627 & 1.4 & -0.0209 & 0.0108 & 0.0128 \\
\hline 1.6440 & 0.8275 & & & & & & & & & $4.0591 \mathrm{e}-3$ & -0.0128 \\
\hline 1.6460 & 0.8110 & 86 & & 88 & 69 & 11 & & & & & 0.0128 \\
\hline 1.6480 & 0.8454 & 1.1684 & 2568 & 1.3308 & 1.3954 & 1.4472 & 1.4872 & & -0.0104 & -0.0258 & -0.0128 \\
\hline 1.6500 & 0.8110 & & & & & & & & & -0.0105 & 0.0128 \\
\hline & 3 & & & & & & & & $6 e-3$ & & 28 \\
\hline 1.6540 & 0.8434 & & & & & & & & -0.0 & $5 e-3$ & 28 \\
\hline 1.6560 & 0.8215 & 1.1821 & 37 & 9 & 1.3 & 56 & & & -0.0122 & -0.0213 & 0.0128 \\
\hline 1.6580 & 0.8336 & 1.1831 & 786 & & 1.3951 & & & & $-4.6160 \mathrm{e}-$ & $3-0.0117$ & -0.0128 \\
\hline 1.6600 & 0.8282 & 1.1607 & 2773 & 1.3454 & 1.4144 & 1.4616 & & & $-5.9846 \mathrm{e}-$ & $3-6.5234 e-3$ & 30.0000 \\
\hline 1.6620 & 0.8437 & 1.1607 & 2483 & 1.3292 & 1.4069 & 1.4313 & 1.4851 & & $-2.6510 \mathrm{e}-3$ & $3-7.1475 e$ & 30.0000 \\
\hline 1.6640 & & & & & & & & & $-3.6327 \mathrm{e}-$ & & -0.0128 \\
\hline 1.6660 & 6 & & & & & & & & & 34. & 0.0128 \\
\hline 6680 & 08408 & 9 & & & & & & & -0. & $0 .($ & 0.0128 \\
\hline 1.6700 & 7 & & & & & & & & 4. & 0.0 & 0.0000 \\
\hline 1.6720 & 0.8390 & 1.1803 & 2738 & 1.3421 & & & & & $3 e-3$ & 7.1461e-3 & 0.0000 \\
\hline 1.6740 & 0.8465 & 1.1839 & 1.2815 & 1.3401 & 1.3715 & & & & $9.4300 \mathrm{e}-3$ & 0.0113 & 0.0000 \\
\hline 1.6760 & 0.8324 & & & & & & & & & -0.0342 & -0.0128 \\
\hline 1.6780 & 0.8284 & & & & & & & & & $-5.6480 e-3$ & -0.0128 \\
\hline 16800 & 09240 & & & & & & & & $7.6551 \mathrm{e}-3$ & $1.0590 \mathrm{e}-3$ & -0.0128 \\
\hline 1.6820 & 8207 & 1.1403 & 21 & 8 & 1.3749 & 1.4 & 1. & 1. & 0.0178 & -0.0152 & 0.0000 \\
\hline 1.6840 & 8331 & 1766 & & & 4034 & & & & $8.7407 e-3$ & $-1.7650 \mathrm{e}-4$ & 0.0128 \\
\hline 1.6860 & 0.8379 & 1.1581 & 1.2681 & 1.3486 & 1.3880 & 1.4 & 1.4779 & 09 & -0.0151 & $-9.0015 e-3$ & 0.0257 \\
\hline 1.6880 & 0.8337 & 1.1731 & 1.2606 & 1.3212 & 1.4019 & 1.4355 & 1.4920 & 1.5105 & $5.9896 \mathrm{e}-3$ & -0.0199 & 0.0128 \\
\hline 1.6900 & 0.8074 & & & & 1.4016 & & & & -0.0240 & -0.0191 & -0.0257 \\
\hline 1.6920 & 0.8147 & & & & & & & & & 0.0131 & 0.0128 \\
\hline 6940 & 8182 & & & & & & & & $2.2626 \mathrm{e}-3$ & $3.2645 \mathrm{e}-3$ & 0.0000 \\
\hline & & & & & & & & & & $5.2950 \mathrm{e}-4$ & -0.0128 \\
\hline & (2) & & & & & & & & $4 e-3$ & $6.2653 e-3$ & 0.0128 \\
\hline 1.7000 & 08017 & 1.1571 & 1.2673 & 1.3259 & 1.3992 & 1.4474 & & & $2.8484 \mathrm{e}-3$ & $-2.1180 \mathrm{e}$ & $3-0.0128$ \\
\hline 1.7020 & 0.8031 & 1.1580 & 1.2593 & 1.3202 & 1.3909 & 1.4397 & 1.4809 & 1.5059 & $5.5006 \mathrm{e}-3$ & -0.0154 & 0.0000 \\
\hline 1.7040 & 0.7967 & 1.1636 & 1.2569 & 1.3303 & 1.3845 & 1.4539 & 1.4865 & 1.5088 & -0.0245 & -0.0125 & 0.0128 \\
\hline 1.7060 & 0.8048 & 1.1581 & 1.2676 & 1.3263 & 1.3702 & 1.4214 & 1.4987 & 1.5436 & 0.0109 & 0.0121 & 0.0257 \\
\hline
\end{tabular}




\begin{tabular}{|c|c|c|c|c|c|c|c|c|c|c|c|}
\hline 1.7080 & 0.8045 & 1.1642 & 1.2572 & 1.3145 & 1.3940 & 1.4361 & 1.5142 & 1.5024 & 0.0183 & 0.0149 & 0.0000 \\
\hline 1.7100 & 0.8056 & 1.1490 & 1.2520 & 1.3210 & 1.3866 & 1.4414 & 1.4881 & 1.5332 & $-9.7166 \mathrm{e}-3$ & $32.2066 \mathrm{e}-3$ & 0.0000 \\
\hline 1.7120 & 0.7807 & 1.1621 & 1.2754 & 1.3198 & 1.3881 & 1.4192 & 1.5041 & 1.5180 & -0.0113 & -0.0159 & 0.0000 \\
\hline 1.7140 & 0.7886 & 1.1613 & 1.2635 & 1.3262 & 1.3937 & 1.4407 & 1.4804 & 1.5050 & 0.0605 & $-4.2360 \mathrm{e}-3$ & 30.0000 \\
\hline 1.7160 & 0.7936 & 1.1677 & 1.2591 & 1.3019 & 1.3911 & 1.4171 & 1.4654 & 1.5043 & 0.2150 & -0.0344 & -0.0257 \\
\hline 1.7180 & 0.7837 & 1.1536 & 1.2461 & 1.3194 & 1.3432 & 1.4321 & 1.4791 & 1.4951 & 0.4511 & -0.0392 & -0.0385 \\
\hline 1.7200 & 0.7925 & 1.1672 & 1.2504 & 1.3189 & 1.3700 & 1.4126 & 1.4259 & 1.4967 & 0.7748 & -0.0726 & -0.0642 \\
\hline 1.7220 & 0.7970 & 1.1635 & 1.2212 & 1.2947 & 1.3744 & 1.4185 & 1.4441 & 1.4747 & 1.0750 & -0.0892 & -0.0642 \\
\hline 1.7240 & 0.7892 & 1.1410 & 1.2305 & 1.2901 & 1.3499 & 1.4206 & & 1.4619 & & -0.0711 & -0.0642 \\
\hline 1.7260 & 0.7811 & 1.1514 & 1.2369 & 1.2869 & 1.3607 & 1.4314 & 1.4570 & 1.5081 & 1.3643 & -0.0446 & -0.0514 \\
\hline 1.7280 & 0.7700 & 1.1606 & 1.2401 & 1.3027 & 1.3748 & 1.4234 & 1.4702 & 1.4994 & 1.4517 & -0.0233 & -0.0385 \\
\hline 1.7300 & 0.7776 & 1.1536 & 1.2450 & 1.3085 & 1.3724 & 1.4350 & 1.4805 & 1.5224 & 1.5000 & -0.0403 & 0.0000 \\
\hline 1.7320 & 0.7770 & 1.1546 & 1.2555 & 1.3027 & 1.3933 & & & & & & -0.0128 \\
\hline 1.7340 & 0.7741 & 1.1778 & 1.2596 & 1.3121 & 1.3813 & & 1.4619 & 1.4823 & & -0.0254 & 0.0128 \\
\hline 1.7360 & 0.7730 & & & & & & & & & $4.2360 \mathrm{e}-3$ & -0.0128 \\
\hline 1.7380 & 0.7712 & 1.1 & .2424 & & 1.4012 & & & & & & 0.0257 \\
\hline 1.7400 & 0.7659 & 1.1458 & 1.2434 & 1.3106 & 1.3939 & 1.4269 & 1.4773 & 1.5266 & & $-6.5302 \mathrm{e}-3$ & 30.0000 \\
\hline 1.7420 & 0.7785 & 1.1342 & 1.2392 & 1.3401 & 1.3763 & 1.4360 & 1.4947 & 1.5213 & 1.5530 & 7.5 & -0.0128 \\
\hline 1.7440 & 0.7864 & 1.1290 & 1.2230 & 1.3060 & 1.3763 & & 1.4 & & & $-3.5300 \mathrm{e}-3$ & 30.0000 \\
\hline 1.7460 & 0.7780 & 1.1432 & 1.2458 & 1.3156 & 1.3848 & & & & & & 0.0128 \\
\hline 1.7480 & 0.7636 & & 1.2450 & & & & & & & $5.1185 \mathrm{e}-3$ & -0.0128 \\
\hline 1.7500 & 0.7 & & & & & & & & & & 0.0000 \\
\hline 1.7520 & 0.7749 & 1.1 & 506 & & 1.3 & & & & & & -0.0128 \\
\hline 1.7540 & 0.7734 & 1.1291 & 399 & 1.3083 & 1.3904 & 1.4 & & 1. & & $00 e-4$ & -0.0128 \\
\hline 1.7560 & 0.7521 & 1.1219 & .2747 & 1.2973 & 1.3805 & 1.4420 & 1.4942 & 1.5419 & 1.5461 & 0.0104 & -0.0128 \\
\hline 1.7580 & 0.7668 & 1.1252 & 2497 & 1.3161 & 1.3789 & 1.4227 & & & & & 0.0000 \\
\hline 1.7600 & 0.7587 & 1.1155 & 1.2437 & 1.2 & 1.3700 & & & & & & -0.0128 \\
\hline 1.7620 & 0.7626 & & & & & & & & & $75 e-3$ & 0.0128 \\
\hline 1.7 & 0.7 & & & & & & & & & & -0.0128 \\
\hline 1.7660 & 0.7730 & 1.1316 & 23 & & 1.3 & & & & & & -0.0128 \\
\hline 1.7680 & 0.7733 & 1.1368 & 2576 & 1.3 & 1.3589 & 1.4 & & & & $e-3$ & $3-0.0128$ \\
\hline 1.7700 & 0.7498 & 1.1300 & 2415 & 1.3078 & 1.3970 & 1.4344 & 1.5045 & & 1.5622 & $-8.7360 \mathrm{e}-3$ & 30.0000 \\
\hline 1.7720 & 0.7749 & .1129 & 2426 & 2915 & 1.3791 & & & 1.5 & & & 0.0000 \\
\hline 1.7740 & 0.7574 & 37 & 198 & 0 & 1.3708 & & & & & e-3 & 0.0000 \\
\hline 1.7760 & 0.7649 & & & & 1.3 & & & & & & 0.0000 \\
\hline 0 & 0 & & & & & & & & & $e-3$ & 0.0000 \\
\hline 1.7800 & 0.7475 & 1.1124 & 523 & & & & & & & & 0.0000 \\
\hline 1.7820 & 0.7450 & 1.1141 & 2509 & 1.2974 & 1.3707 & 1.4037 & 1.4 & 1.5600 & 40 & -0 & 0.0000 \\
\hline 1.7840 & 0.7431 & 1.1158 & 377 & & & & & & & & 0.0000 \\
\hline 1.7860 & 0.7350 & 1.1142 & 2439 & 1.3052 & 1.3589 & 1.4 & 1.4 & 1.5 & & $-9.7950 e-3$ & 30.0000 \\
\hline 1.7880 & 0.7362 & & & & & & & & & & 0.0000 \\
\hline 1.7900 & 0.7416 & & & & & & & & & & 0.0128 \\
\hline 1.7920 & 0.7243 & & 6 & & & & & & & & $3-0.0128$ \\
\hline 1.7940 & 0.7186 & 1.1109 & 458 & & 1.3604 & & & 1. & 79 & $73 e-3$ & 0.0257 \\
\hline 1.7960 & 0.7094 & 1.1155 & 427 & 1.3109 & 1.3711 & 1.4274 & 1.4 & 1.5671 & 929 & 0.0183 & 0.0000 \\
\hline 1.7980 & 0.7032 & & 347 & & & & & & & $-3.9691 \mathrm{e}-3$ & 30.0000 \\
\hline 1.8000 & 0.6944 & 1.1 & 2510 & 68 & 1.3636 & 1.4 & 1.4 & & 1. & -0.0229 & 0.0000 \\
\hline 1.8020 & 0.6997 & & & & & & & & & & 0.0128 \\
\hline 18040 & 0.6927 & & & & & & & & & $53 e-3$ & $3-0.0128$ \\
\hline 1.8060 & 0.6957 & 33 & 03 & 1.2 & 1.3 & & & 1.5 & 25 & $6 e-3$ & 0.0000 \\
\hline 1.8080 & 0.6482 & 1219 & 2376 & & 1.3 & & & 1.5 & & -0 & 0.0128 \\
\hline 1.8100 & 0.6586 & & & & & & & & & $6 e-4$ & 0.0000 \\
\hline 1.8120 & 0.6502 & 1.1165 & 1.2477 & 1.2911 & 1.3796 & & 1.4912 & & & -0.0191 & 0.0000 \\
\hline 1.8140 & 0.6252 & 1.1211 & 2472 & & & & & & & & 0.0128 \\
\hline 1.8160 & & & & & & & & & & $2.1180 \mathrm{e}-3$ & 0.0000 \\
\hline 18180 & 0.6128 & & & & & & & & & & -0.0128 \\
\hline 1.8200 & 0.5976 & 0 & 1 & & 1.3 & & & & & 2.6 & 0.0128 \\
\hline 1.8220 & 0.5766 & 1.1219 & 318 & 82 & 1.3618 & 1.4 & 1. & 70 & 66 & $-5.5605 e-3$ & -0.0128 \\
\hline 1.8240 & 0.5660 & $0 s$ & 470 & 4 & 1.3490 & & & 19 & 80 & $-6.8835 e-3$ & 30.0000 \\
\hline 1.8260 & 0.5714 & 1.1101 & 1.2368 & 1.2840 & 1.3591 & 1.4216 & 1.4848 & 1.5339 & 1.6047 & 0.0145 & -0.0128 \\
\hline 1.8280 & 0.5477 & 1.0839 & 1.2579 & 1.2941 & 1.3616 & & 1.4873 & 54 & 1.6176 & $-8.3887 \mathrm{e}-3$ & 30.0000 \\
\hline 1.8300 & 0.5253 & & & & & & & & & & 0.0257 \\
\hline 1.8320 & 0.5194 & 1.1022 & 1.2265 & 1.2945 & 1.3488 & & & 1.5546 & 1.6254 & $-2.1166 \mathrm{e}-3$ & $3-0.0128$ \\
\hline 8340 & 0.5093 & & 2382 & 1.2780 & 1.3629 & & & 1.5760 & & -0.0200 & 0.0000 \\
\hline & 4969 & & & & & & & & & -0.0119 & 0.0000 \\
\hline 1.8380 & 5034 & 1.1019 & 1.2364 & 1.3114 & 1.3509 & 1.4 & 1.4 & 1.5733 & 1.6056 & -0.0223 & 0.0128 \\
\hline 1.8400 & 0.4717 & 1.0794 & 1.2385 & 1.2941 & 1.3573 & 1.4131 & 1.4950 & 1.5675 & 1.6126 & $-3.7065 e-3$ & 30.0000 \\
\hline 1.8420 & 0.4762 & 1.0852 & 1.2395 & 1.3046 & 1.3633 & 1.4275 & 1.4693 & 1.5448 & 1.6328 & -0.0203 & -0.0128 \\
\hline 1.8440 & 0.4618 & 1.0742 & 1.2432 & 1.2898 & 1.3634 & 1.4251 & 1.4965 & 1.5576 & 1.6376 & $-7.2294 \mathrm{e}$ & 30.0000 \\
\hline 1.8460 & 0.4665 & 1.1067 & 1.2289 & 1.2940 & 1.3421 & 1.4128 & 1.4789 & 1.5566 & 1.6516 & $8.8956 \mathrm{e}-4$ & 0.0000 \\
\hline
\end{tabular}




\begin{tabular}{|c|c|c|c|c|c|c|c|c|c|c|c|}
\hline 1.8480 & 0.4516 & 1.0882 & 1.2334 & 1.2931 & 1.3558 & 1.4052 & 1.4900 & 1.5693 & 1.6125 & $3.1770 \mathrm{e}-3$ & -0.0128 \\
\hline 1.8500 & 0.4592 & 1.0728 & 1.2140 & 1.2876 & 1.3682 & 1.4440 & 1.4815 & 1.5366 & 1.6214 & 0.0340 & 0.0000 \\
\hline 1.8520 & 0.4390 & 1.0892 & 1.2316 & 1.2762 & 1.3465 & 1.4244 & 1.4803 & 1.5411 & 1.6239 & 0.1437 & -0.0257 \\
\hline 1.8540 & 0.4189 & 1.0892 & 1.2178 & 1.2751 & 1.3586 & 1.4202 & 1.4773 & 1.5194 & 1.6066 & 0.3834 & -0.0257 \\
\hline 1.8560 & 0.4181 & 1.0868 & 1.2196 & 1.2817 & 1.3597 & 1.3751 & 1.4601 & 1.5197 & 1.5964 & 0.7772 & -0.0385 \\
\hline 1.8580 & 0.4173 & 1.1024 & 1.2292 & 1.2854 & 1.3477 & 1.3818 & 1.4484 & 1.5071 & 1.5614 & 1.0721 & -0.0385 \\
\hline 1.8600 & 0.3960 & 1.0977 & 1.2284 & 1.2883 & 1.3433 & 1.3757 & 1.4821 & 1.4914 & 1.5811 & 1.3171 & -0.0385 \\
\hline 1.8620 & 0.3985 & 1.0857 & 1.2108 & 1.2598 & 1.3221 & 1.4102 & 1.4577 & 1.5305 & 1.5912 & 1.5003 & -0.0257 \\
\hline 1.8640 & 0.3873 & 1.0858 & 1.2225 & 1.2896 & 1.3447 & 1.4045 & 1.4623 & 1.5209 & 1.5906 & 1.5946 & -0.0257 \\
\hline 1.8660 & 0.3644 & 1.0814 & 1.2223 & 1.2733 & 1.3248 & 1.3937 & 1.4726 & 1.5065 & 1.6117 & & -0.0128 \\
\hline 1.8680 & 0.3659 & 1.0660 & 1.2280 & 1.2810 & 1.3398 & 1.3945 & 1.4670 & 1.5158 & 1.6081 & & -0.0257 \\
\hline 1.8700 & 0.3486 & 1.0852 & .2060 & 1.2622 & 1.3288 & 1.4057 & 1.4977 & 1.5516 & 1.6545 & & 0.0000 \\
\hline 1.8720 & 0.3543 & 1.0991 & 1.2200 & 1.2539 & 1.3460 & 1.3715 & 1.4737 & 1.5557 & 1.6178 & 1.6163 & -0.0128 \\
\hline 1.8740 & 0.3503 & 1.0865 & 1.2392 & 1.2702 & 1.3206 & 1.4081 & 1.4846 & 1.5534 & & & 0.0000 \\
\hline 1.8760 & 0.3506 & 1.0877 & 1.2218 & 1.2505 & 1.3216 & 1.3985 & 1.4921 & 1.5623 & 1.6393 & & 0.0000 \\
\hline 1.8780 & 0.3535 & 1.0550 & & & & & & & & & 0.0257 \\
\hline 1.8800 & 0.3469 & 1.1036 & & 1.2607 & 1.3386 & 1.3965 & & 1.5546 & & & 000 \\
\hline 1.8820 & 0.3186 & 1.0890 & 2325 & 1.2708 & 1.3201 & 1.3905 & 1.4823 & 1.5541 & & & 0.0000 \\
\hline 1.8840 & 0.3239 & 1.0859 & & 1.2776 & 1.3344 & 1.3834 & 1.4709 & 1.5301 & & & -0.0128 \\
\hline 1.8860 & 0.3212 & 1.0863 & 1.2096 & 1.2711 & 1.3490 & & & & & & 0.0000 \\
\hline 1.8880 & 0.3101 & 1.0683 & 1.2170 & 1.2673 & 1.3287 & 1.3915 & & & & & -0.0128 \\
\hline 1.8900 & 0.2938 & 1.0878 & & 1.2687 & 1.3325 & 1.4096 & & & & & 0.0000 \\
\hline 1.8920 & 0.3062 & 1.0616 & & $1.2^{\prime}$ & 1.3 & & & & & & 128 \\
\hline 1.8940 & 0.2901 & 1.0558 & 51 & 1.2523 & 1.3199 & 1.3 & & 1.5 & & & \\
\hline 1.8960 & 0.2876 & 1.0622 & 2063 & 1.2876 & 1.3468 & 1.4128 & 1.4659 & 1.5180 & 1.6430 & & -0.0128 \\
\hline 1.8980 & 0.2792 & 1.0613 & .2144 & 1.2845 & 1.3300 & 1.3789 & 1.4622 & 1.5427 & 1.6459 & & -0.0128 \\
\hline 1.9000 & 0.2826 & 1.0655 & 1.2198 & 1.2710 & 1.3251 & 1.3985 & 1.4684 & 1.5267 & & & 0.0000 \\
\hline 1.9020 & 0.2790 & 1.0545 & 1.2285 & 1.2603 & 1.3591 & 1.4053 & 1.4800 & 1.5440 & & & 0.0128 \\
\hline 1.9040 & 0.2650 & 1.0484 & & & & & & & & & 0.0000 \\
\hline 1.9060 & 0.26 & 1.0 & & & & & & & & & \\
\hline 9080 & 0.2675 & 1.0684 & 82 & 1.2761 & 1.3 & 1.3 & & & & & -0.0128 \\
\hline 1.9100 & 0.2618 & 1.0564 & .2163 & 1.2810 & 1.3002 & 1.4038 & 1.4866 & 1.5400 & & 67 & -0.0128 \\
\hline 1.9120 & 0.2566 & 1.0772 & 2190 & 1.2743 & 1.3463 & 1.4057 & 1.4744 & 1.5374 & & & -0.0128 \\
\hline 1.9140 & 0.2619 & 1.0688 & .2048 & 1.2766 & 1.3464 & 1.3840 & 1.4706 & 1.5 & 1.6 & 1.6 & 0.0128 \\
\hline 1.9160 & 0.2661 & 1.0551 & 1.2140 & 1.2573 & 1.3352 & 1.3911 & 1.4582 & 1.5414 & & & 0.0000 \\
\hline 1.9180 & & & & & & & & & & & \\
\hline 1.9200 & 0.25 & 1.0493 & & 1.2 & & & & & & & 128 \\
\hline 1.9220 & 0.2613 & 1.0748 & 161 & 1.2772 & 1.3383 & 1.3968 & 1.4703 & 1.5 & & 19 & 0.0000 \\
\hline 1.9240 & 0.2716 & 1.0385 & & & & & & & & & -0.0257 \\
\hline 1.9260 & 0.2543 & 1.0428 & 68 & 1.2684 & 1.3287 & 1.3 & 1.4 & & & & -0.0128 \\
\hline 1.9280 & 0.2641 & 1.0442 & 1.2014 & 1.2686 & 1.3428 & 1.3885 & 1.4499 & 1.5250 & 1.6326 & 1.7054 & 0.0128 \\
\hline 1.9300 & 0.2662 & 1.0799 & & & & & & & & & -0.0128 \\
\hline & 0.2 & & & & & & & & & & 00 \\
\hline 9340 & 0.2507 & 1.0677 & 31 & 1.2 & 1.3 & 1.3 & & & & & 0.0000 \\
\hline 1.9360 & 0.2771 & 1.0621 & 87 & 1.2460 & 1.3395 & 1.3665 & 1.4662 & 1.5 & 95 & 1.7089 & -0.0128 \\
\hline 1.9380 & 0.2724 & 1.0459 & 1976 & 1.2313 & & & 1.4417 & & & 1.7311 & 0.0000 \\
\hline 1.9400 & 0.2851 & 1.0526 & 2037 & 1.2533 & 1.3229 & 1.3887 & 1.4646 & 1.5352 & & 1.7191 & 0.0000 \\
\hline 1.9420 & 0.2746 & 1.0280 & 1.1988 & 1.2698 & 1.3105 & 1.3681 & 1.4637 & & 1.6474 & 1.7126 & -0.0257 \\
\hline 1.9440 & & & & & & & & & & & -0.0128 \\
\hline 1.9460 & 0.2740 & & 1 & 1. & & & & & & & 00 \\
\hline 9480 & 0.2689 & .0604 & 2067 & 1.27 & 1.3 & & 1.4 & & & 25 & -0.0128 \\
\hline 1.9500 & 0.2863 & 1.0418 & & & & & & & & & 128 \\
\hline 1.9520 & 0.2870 & 1.0506 & 2157 & 1.2450 & 1.3183 & 1.3798 & 1.4350 & & & 1.7263 & 0.0000 \\
\hline 1.9540 & 0.2706 & 1.0346 & 1.2077 & 1.2403 & 1.3033 & 1.3905 & 1.4598 & 1.5193 & 1.6108 & 1.7270 & 0.0000 \\
\hline 1.9560 & 0.2851 & & & & & & & & & & -0.0128 \\
\hline 1.9580 & 0.2914 & & & 1.2445 & & & & & & & 0.0000 \\
\hline 1.9600 & 0.2712 & 1.0393 & 2070 & 1.2572 & & & & & & 29 & 0.0128 \\
\hline 1.9620 & 0.2896 & 1.0430 & 1995 & 1.2466 & 1.3363 & 1.3743 & 1.4 & 1.5233 & 31 & 1.7375 & 0.0000 \\
\hline 1.9640 & 0.2681 & 1.0359 & 1873 & 2468 & .3189 & 1.3787 & 1.4376 & .5273 & 1.6124 & 1.7332 & 0.0128 \\
\hline 1.9660 & 0.2767 & 1.0415 & 1.2091 & 1.2543 & 1.2954 & 1.3944 & 1.4529 & 1.5252 & 1.6189 & 1.7303 & 0.0128 \\
\hline 1.9680 & 0.2824 & 1.0265 & 1.1967 & 1.2385 & 1.3140 & 1.3834 & 1.4530 & 1.5397 & 1.6210 & 1.7327 & 0.0000 \\
\hline 1.9700 & 0.2982 & 1.0541 & 1.1942 & 1.2352 & 1.3124 & 1.3838 & 1.4634 & 593 & 448 & 1.7084 & 0.0000 \\
\hline 1.9720 & 0.2673 & 1.0375 & & 1.2621 & 1.3160 & & 1.4650 & & 1.6155 & 1.7356 & 0.0000 \\
\hline 1.9740 & 0.2744 & 1.0403 & & 1.2475 & & & 1.4670 & .5309 & 1.6214 & 1.7376 & 0.0000 \\
\hline & 要 & & & & & & & 1.5281 & 1.6253 & 1.7250 & 0.0000 \\
\hline 80 & 0.27 & 1.0347 & & 1.2510 & & & 1.4 & 1.5072 & & 1.7338 & 0.0000 \\
\hline 1.9800 & 0.2740 & 1.0382 & 1.1923 & 1.2412 & 1.3221 & 1.3766 & 1.4417 & 1.5058 & 1.6049 & 1.7449 & 0.0000 \\
\hline 1.9820 & 0.2706 & 1.0275 & 1.1945 & 1.2616 & 1.3189 & 1.3565 & 1.4339 & 1.5062 & 1.6396 & 1.7180 & 0.0257 \\
\hline 1.9840 & 0.2853 & 1.0523 & 1.2010 & 1.2580 & 1.2823 & 1.3877 & 1.4315 & 1.5028 & 1.5978 & 1.7106 & 0.1284 \\
\hline 1.9860 & 0.2874 & 1.0342 & 1.1957 & 1.2500 & 1.2844 & 1.3546 & 1.4238 & 1.5209 & 1.6049 & 1.7071 & 0.3723 \\
\hline
\end{tabular}




\begin{tabular}{|c|c|c|c|c|c|c|c|c|c|c|c|}
\hline 1.9880 & 0.2792 & 1.0448 & 1.1911 & 1.2532 & 1.2917 & 1.3684 & 1.4037 & 1.5115 & 1.5745 & 1.7074 & 0.7319 \\
\hline 1.9900 & 0.2579 & 1.0157 & 1.1846 & 1.2106 & 1.3031 & 1.3688 & 1.4209 & 1.5284 & 1.5484 & 1.6821 & 1.1171 \\
\hline 1.9920 & 0.2669 & 1.0313 & 1.1630 & 1.2410 & 1.2928 & 1.3623 & 1.4035 & 1.5069 & 1.5461 & 1.7037 & 1.4124 \\
\hline 1.9940 & 0.2753 & 1.0234 & 1.1698 & 1.2584 & 1.3056 & 1.3847 & 1.4158 & 1.5073 & 1.5739 & 1.7072 & 1.5665 \\
\hline 1.9960 & 0.2748 & 1.0343 & 1.1702 & 1.2292 & 1.3207 & 1.3683 & 1.4204 & 1.4995 & 1.5558 & 1.7023 & 1.6564 \\
\hline 1.9980 & 0.2522 & 1.0112 & 1.1752 & 1.2373 & 1.3062 & 1.3772 & 1.4360 & 1.5100 & 1.6173 & 1.7145 & 1.7334 \\
\hline 2.0000 & 0.2743 & 1.0450 & 1.1714 & 1.2487 & 1.3145 & 1.3646 & 1.4146 & 1.4838 & 1.5792 & 1.7195 & 1.7334 \\
\hline 2.0020 & 0.2479 & 1.0234 & 1.1761 & 1.2206 & 1.3108 & 1.3528 & 1.4273 & 1.5058 & 1.5819 & 1.7137 & 1.7206 \\
\hline 2.0040 & 0.2467 & 1.0265 & 1.1857 & 1.2426 & 1.3011 & 1.3666 & 1.4410 & 1.4959 & 1.6219 & 1.7315 & 1.7334 \\
\hline 2.0060 & 0.2450 & 1.0312 & 1.1718 & 1.2202 & 1.2915 & 1.3660 & 1.4501 & 1.5054 & 1.5920 & 1.7411 & 1.7077 \\
\hline 2.0080 & 0.2329 & 1.0197 & 1.1698 & 1.2469 & 1.3077 & 1.3632 & 1.4434 & 1.4934 & 1.6189 & 1.7417 & 1.7206 \\
\hline 2.0100 & 0.2426 & 1.0227 & 1.1698 & 1.2403 & 1.2937 & 1.3463 & 1.4469 & 1.4962 & 1.6193 & 1.7156 & 1.7462 \\
\hline 2.0120 & 0.2285 & 1.0282 & 1.1857 & 1.2264 & 1.2806 & 1.3485 & 1.4245 & 1.5038 & 1.6093 & 1.7313 & 1.7334 \\
\hline 2.0140 & 0.2464 & 1.0023 & 1.1722 & 1.2346 & 1.2974 & 1.3517 & 1.4430 & 1.5099 & 1.5992 & 1.7396 & 1.7334 \\
\hline 2.0160 & 0.2424 & 1.0210 & 1.1763 & 1.2434 & 1.2724 & 1.3442 & 1.4202 & 1.4800 & 1.6145 & 1.6999 & 1.7462 \\
\hline 2.0180 & 0.2370 & 1.0091 & 1.1699 & 1.2279 & 1.2836 & 1.3460 & 1.4159 & 1.4786 & 1.6145 & 1.7260 & 1.7462 \\
\hline 2.0200 & 0.2264 & 1.0225 & 1.1739 & 1.2166 & 1.2980 & 1.3394 & 1.4239 & 1.4991 & 1.5998 & 1.7370 & 1.7334 \\
\hline 2.0220 & 0.2177 & 1.0190 & 1.1706 & 1.2538 & 1.2874 & 1.3401 & 1.4326 & 1.5064 & 1.5956 & 1.7126 & 1.7462 \\
\hline 2.0240 & 0.2318 & 1.0064 & 1.1661 & 1.2321 & 1.2993 & 1.3295 & 1.4199 & 1.4842 & 1.5807 & 1.7163 & 1.7462 \\
\hline 2.0260 & 0.2237 & 1.0148 & 1.1682 & 1.2467 & 1.2896 & 1.3337 & 1.4396 & 1.4918 & 1.6142 & 1.7263 & 1.7719 \\
\hline 2.0280 & 0.2155 & 1.0332 & 1.1754 & 1.2292 & 1.2879 & 1.3711 & 1.4167 & 1.4890 & 042 & 1.6769 & 1.7719 \\
\hline 2.0300 & 0.2318 & 1.0222 & 1.1830 & 1.2147 & 1.2862 & 1.3218 & 1.4039 & 1.4809 & 1.5994 & 1.7233 & 1.7848 \\
\hline 2.0320 & 0.2102 & 1.0102 & 1.1851 & 1.2436 & 1.2969 & 1.3525 & 1.4183 & 1.4869 & 1.6098 & 1.7191 & 1.7848 \\
\hline 2.0340 & 0.2246 & 0.9961 & 1.1798 & 1.2331 & 1.2942 & 1.3349 & 1.4062 & 1.4807 & 1.5975 & 1.7157 & 1.7719 \\
\hline 2.0360 & 0.2201 & 1.0255 & 1.1603 & 1.2211 & 1.2749 & 1.3343 & 1.4234 & 1.5213 & 1.5921 & 1.7198 & 1.8104 \\
\hline 2.0380 & 0.2130 & 1.0235 & 1.1749 & 1.2292 & 1.3015 & 1.3324 & 1.3951 & 1.4916 & 1.6069 & 1.6879 & 1.7976 \\
\hline 2.0400 & 0.2049 & 0.9936 & 1.1 & 1.2222 & 1.2950 & 1.3317 & 1.4071 & 64 & & 1.7010 & 1.7976 \\
\hline 2.0420 & 0.2039 & 1.0121 & 1.1655 & 1.2159 & 1.2511 & 1.3219 & 1.4129 & 1.4899 & 695 & 1.7027 & 1.8104 \\
\hline 2.0440 & 0.2023 & 0.9976 & 1.1620 & 1.2134 & 1.2775 & 1.3312 & 1.4075 & 1.4619 & 1.5542 & 1.7225 & 1.7848 \\
\hline 2.0460 & 0.2036 & 0.9908 & 1.1666 & 1.2230 & 1.2952 & 1.3177 & 1.3864 & 1.4941 & 1.6005 & 1.6890 & 1.7848 \\
\hline 2.0480 & 0.1950 & 1.0195 & 1.1667 & 1.2071 & 1.2916 & 1.3162 & 1.4106 & 1.4724 & 1.5861 & 1.7047 & 1.7976 \\
\hline 2.0500 & 0.1853 & 1.0203 & 1.1726 & 1.2138 & 1.2899 & 1.2960 & 1.3787 & 1.5077 & 1.5833 & 1.6777 & 1.8104 \\
\hline 2.0520 & 0.2042 & 1.0053 & 1.1622 & 1.2353 & 1.2737 & 1.3233 & 1.4077 & 1.4580 & 1.5979 & 1.7169 & 1.7976 \\
\hline 2.0540 & 0.1987 & 1.0051 & 1.1 & 1.1923 & 1.2860 & 1.3 & 1.3 & 1.4 & & 65 & 1.7976 \\
\hline 2.0560 & 0.2003 & 0.9938 & 1.1652 & 1.2044 & 1.2653 & 1.3326 & 1.4048 & 1.4901 & 1.5916 & 1.6982 & 1.7976 \\
\hline 2.0580 & 0.2003 & 1.0067 & 1.1673 & 1.2209 & 1.2930 & 1.3446 & 1.3882 & 1.4705 & 1.5867 & 1.6900 & 1.7976 \\
\hline 2.0600 & 0.1903 & 1.0061 & 1.1578 & 1.2291 & 1.2781 & 1.3446 & 1.4042 & 1.4998 & 1.5568 & 1.7026 & 1.7848 \\
\hline 2.0620 & 0.1792 & 0.9867 & 1.1530 & 1.2145 & 1.2878 & 1.3168 & 1.3916 & 1.4566 & 1.5834 & 1.7064 & 1.7976 \\
\hline 2.0640 & 0.1797 & 0.9947 & 1.1648 & 1.2223 & 1.2828 & 1.3041 & 1.4150 & 1.4868 & 1.5927 & 1.6999 & 1.7976 \\
\hline 2.0660 & 0.2010 & 1.0087 & 1.1 & 1.2020 & 1.2733 & 1.3153 & 1.4 & 1.4887 & 1.5627 & 1.6968 & 1.8104 \\
\hline 2.0680 & 0.1810 & 1.0 & 1. & 1.2075 & 1.2784 & 1.3246 & 1.3 & 1.4 & 22 & 90 & 1.8104 \\
\hline 2.0700 & 0.1911 & 1.0017 & 1.1748 & 1.2095 & 1.2839 & 1.3125 & 1.3768 & 1.4765 & 1.5626 & 1.6913 & 1.7976 \\
\hline 2.0720 & 0.1986 & 1.0147 & 1.1491 & 1.2260 & 1.2868 & 1.3344 & 1.4103 & 1.4942 & 1.5494 & 1.6861 & 1.8104 \\
\hline 2.0740 & 0.1872 & 0.9962 & 1.1475 & 1.2238 & 1.2663 & 1.3448 & 1.3983 & 1.4732 & 1.5826 & 1.7101 & 1.8104 \\
\hline 2.0760 & 0.1956 & 0.9954 & 1.1717 & 1.2191 & 1.2779 & 1.3202 & 1.4181 & 1.4622 & 1.5834 & 1.6912 & 1.8104 \\
\hline 2.0780 & 0.1854 & 1.0079 & 1.1642 & 1.2161 & 1.2839 & 1.3195 & 1.3829 & 1.4736 & 1.5506 & 1.6779 & 1.8233 \\
\hline 2.0800 & 0.1634 & 1.0071 & 89 & 1.2025 & 1.2718 & 1.3626 & 1.3940 & 1.4898 & 504 & 531 & 1.7976 \\
\hline 2.0820 & 0.1808 & 0.9890 & 01 & 1.2009 & 1.2814 & 1.3139 & 1.3 & 1. & 67 & 81 & 1.8233 \\
\hline 2.0840 & 0.1792 & 1.0108 & 1.1639 & 1.2045 & 1.2821 & 1.3019 & 1.3873 & 1.4662 & 1.5794 & 1.6752 & 1.7976 \\
\hline 2.0860 & 0.1754 & 1.0122 & 1.1521 & 1.2050 & 1.2564 & 1.2930 & 1.4006 & 1.4332 & 1.5746 & 1.6763 & 1.7976 \\
\hline 2.0880 & 0.1793 & 1.0079 & 1.1500 & 1.2166 & 1.2600 & 1.3074 & 1.4090 & 1.4704 & 1.5828 & 1.6546 & 1.7976 \\
\hline 2.0900 & 0.1722 & 1.0021 & 1.1653 & 1.2412 & 1.2717 & 1.3112 & 1.3740 & 1.4685 & 1.5769 & 1.6710 & 1.8104 \\
\hline 2.0920 & 0.1905 & 1.0191 & 1.1316 & 1.2124 & 1.2736 & 1.3055 & 1.3799 & 1.4696 & 1.5865 & 1.6458 & 1.7719 \\
\hline 2.0940 & 0.1873 & 0.9997 & 1.1458 & 1.2330 & 1.2836 & 1.3214 & 1.3 & 1.4782 & 41 & 542 & 1.7848 \\
\hline 2.0960 & 0.1727 & 0.9955 & 1.1499 & 1.2161 & 1.2622 & 1.3065 & 1.3776 & 1.4613 & 1.5589 & 1.6527 & 1.7848 \\
\hline 2.0980 & 0.1694 & 0.9835 & 1.1520 & 1.2226 & 1.2685 & 1.3213 & 1.3791 & 1.4754 & 1.5169 & 1.6533 & 1.7719 \\
\hline 2.1000 & 0.1913 & 0.9897 & 1.1413 & 1.1906 & 1.2672 & 1.3326 & 1.3789 & 1.4489 & 1.5471 & 1.6354 & 1.7848 \\
\hline 2.1020 & 0.1858 & 0.9738 & 1.1548 & 1.1929 & 1.2690 & 1.3030 & 1.3589 & 1.4528 & 1.5304 & 1.6410 & 1.7976 \\
\hline 2.1040 & 0.1816 & 0.9891 & 1.1546 & 1.1840 & 1.2588 & 1.3176 & 1.3711 & 1.4311 & 1.5461 & 1.6550 & 1.7848 \\
\hline 2.1060 & 0.1717 & 0.9710 & 1.1475 & 1.1838 & 1.2641 & 1.2875 & 1.3590 & 1.4550 & 1.5504 & 1.6340 & 1.7976 \\
\hline 2.1080 & 0.1668 & 0.9694 & 1.1526 & 1.1882 & 1.2529 & 1.3074 & 1.4064 & 1.4504 & 1.5224 & 1.6540 & 1.7719 \\
\hline 2.1100 & 0.1959 & 0.9741 & 1.1586 & 1.2040 & 1.2502 & 1.2921 & 1.3726 & 1.4035 & 1.5303 & 1.6330 & 1.7848 \\
\hline 2.1120 & 0.1880 & 0.9669 & 1.1643 & 1.2064 & 1.2556 & 1.2834 & 1.3764 & 1.4350 & 1.5574 & 1.6057 & 1.7848 \\
\hline 2.1140 & 0.1742 & 0.9808 & 1.1793 & 1.1958 & 1.2278 & 1.3120 & 1.3799 & 1.4296 & 1.5295 & 1.6230 & 1.7848 \\
\hline 2.1160 & 0.1820 & 0.9745 & 1.1471 & 1.2003 & 1.2689 & 1.3225 & 1.3614 & 1.4432 & 1.5426 & 1.6342 & 1.7848 \\
\hline 2.1180 & 0.1746 & 0.9829 & 1.1453 & 1.2011 & 1.2370 & 1.3134 & 1.3562 & 1.4313 & 1.5381 & 1.6663 & 1.7719 \\
\hline 2.1200 & 0.1839 & 0.9536 & 1.1461 & 1.2080 & 1.2522 & 1.2869 & 1.3881 & 1.4406 & 1.5484 & 1.6590 & 1.7591 \\
\hline 2.1220 & 0.1811 & 0.9537 & 1.1539 & 1.1994 & 1.2873 & 1.3080 & 1.3547 & 1.4441 & 1.5300 & 1.6344 & 1.7591 \\
\hline 2.1240 & 0.1718 & 0.9327 & 1.1474 & 1.1936 & 1.2478 & 1.3104 & 1.3578 & 1.4391 & 1.5198 & 1.6196 & 1.7591 \\
\hline 2.1260 & 0.1761 & 0.9342 & 1.1502 & 1.2068 & 1.2663 & 1.3057 & 1.3697 & 1.4264 & 1.5378 & 1.6334 & 1.7591 \\
\hline
\end{tabular}




\begin{tabular}{|c|c|c|c|c|c|c|c|c|c|c|c|}
\hline 2.1280 & 0.1771 & 0.9260 & 1.1460 & 1.2015 & 1.2464 & 1.2749 & 1.3498 & 1.4433 & 1.5242 & 1.6280 & 1.7462 \\
\hline 2.1300 & 0.1742 & 0.9451 & 1.1431 & 1.1994 & 1.2363 & 1.3190 & 1.3530 & 1.4445 & 1.5397 & 1.6265 & 1.7462 \\
\hline 2.1320 & 0.1714 & 0.9097 & 1.1413 & 1.1830 & 1.2363 & 1.2912 & 1.3492 & 1.4169 & 1.5314 & 1.6248 & 1.7719 \\
\hline 2.1340 & 0.1859 & 0.9197 & 1.1552 & 1.2035 & 1.2474 & 1.2892 & 1.3546 & 1.4227 & 1.5105 & 1.6251 & 1.7462 \\
\hline 2.1360 & .1863 & 0.9193 & 1.1331 & 1.1901 & 1.2526 & 1.3163 & 1.3402 & 1.4462 & 1.5148 & 1.6167 & 1.7591 \\
\hline .1380 & 1857 & 0.9163 & 1.1314 & 1.1912 & 1.2445 & 1.3044 & 1.3545 & 1.4247 & 1.5348 & 1.6383 & 1.7462 \\
\hline .1400 & 0.2122 & 0.9154 & 1.1356 & 1.1908 & 1.2428 & 1.3062 & 1.3537 & 1.4256 & 1.5196 & 1.6032 & 1.7462 \\
\hline .1420 & 0.2018 & 0.8884 & 1.1405 & 1.1920 & 1.2527 & 1.2947 & 1.3507 & 1.4188 & 1.4947 & 1.6239 & 1.7462 \\
\hline 2.1440 & .2042 & 0.8903 & 1.1285 & 1.1958 & 1.2469 & 1.2883 & 1.3510 & 1.4113 & 1.5509 & 1.6302 & 1.7591 \\
\hline 2.1460 & .2036 & 0.8865 & 1.1147 & 1.1830 & 1.2715 & 1.3079 & 1.3371 & 1.4335 & 1.5224 & 1.6100 & 1.733 \\
\hline 2.1480 & .2013 & 0.8957 & 1.1277 & 1.1771 & 1.2445 & 1.2937 & 1.3456 & 1.4175 & 1.5020 & 1.6026 & 1.733 \\
\hline 2.1500 & 1976 & 0.8722 & 1.1477 & 1.1905 & 1.2390 & 1.3033 & 1.3622 & 1.4367 & 1.4950 & 1.6114 & 1.733 \\
\hline 1520 & 1968 & 0.8688 & 1.1551 & 1.1964 & 1.2321 & 2823 & 1.3618 & 1.4199 & 1.5200 & 1.6158 & 1.7462 \\
\hline 1540 & 0.1951 & 0.8667 & 1.1583 & 1.1649 & 1.2426 & 1.2712 & 1.3479 & 1.4275 & 1.5146 & 1.6101 & 1.7334 \\
\hline .1560 & 0.1926 & 0.8697 & 1.1518 & 1.2028 & 1.2612 & 1.2876 & 1.3470 & 1.4341 & 1.4784 & 1.6063 & 1.7334 \\
\hline 1580 & 1990 & 0.8677 & 1.1396 & 1.1811 & 1.2429 & 1.2508 & 1.3453 & 1.4229 & 1.4928 & 1.6045 & 1.7206 \\
\hline 2.1600 & .2090 & 0.8432 & 1.1185 & 1.1781 & 1.2449 & 1.2716 & 1.3201 & 1.4171 & 1.4981 & 1.6235 & 1.733 \\
\hline 2.1620 & 2129 & 0.8493 & & & & & & 1.3762 & & 1.5871 & 1720 \\
\hline 2.1640 & .2088 & 0.8626 & 1.1171 & 1.1654 & 1.2258 & & .3304 & 1.4080 & & 1.5953 & 1.7077 \\
\hline 2.1660 & 0.2149 & 0.8459 & 1.1292 & 1.1706 & 1.2374 & 1.2823 & 1.3433 & 1.4109 & 1.4970 & .5826 & 1.7077 \\
\hline 1680 & 2312 & 0.8361 & 1.1334 & 1.1957 & 1.2237 & 1.2678 & 1.3258 & 1.3977 & 1.4727 & 1.5728 & 1.7077 \\
\hline .1700 & .2235 & 0.8507 & 1.1282 & 1.1845 & 1.2228 & 1.2793 & 1.3252 & 1.4098 & 1.5004 & 1.5934 & 1.7077 \\
\hline 2.1720 & 0.2102 & 0.8368 & 1.1388 & 1.1737 & 1.2342 & 1.2945 & 1.3090 & 1.4066 & 1.4909 & 1.5724 & 1.7077 \\
\hline 2.1740 & 0.1996 & 0.8355 & 1.1244 & & 1.2263 & & 1.3340 & 1.3976 & 1.4929 & 1.5940 & 1.69 \\
\hline 2.1760 & 2027 & 0.8 & & & & & & & & & \\
\hline 2.1780 & 2148 & 0.8165 & & 1.1 & 1.2002 & & 23 & 1.4061 & & 1.6077 & 1.694 \\
\hline 2.1800 & .2145 & 0.8209 & 1.1327 & 1.1567 & 1.2358 & 1.2864 & 1.3331 & 1.4037 & 1.4707 & 1.6004 & 1.7077 \\
\hline 2.1820 & 1923 & 0.8324 & 1.1177 & 1.1811 & 1.2274 & 1.2942 & 1.3284 & 1.3931 & 1.4740 & 1.5802 & 1.6949 \\
\hline 2.1840 & 1999 & 0.8213 & 1.1505 & 1.1795 & 1.2294 & 1.2798 & 1.3362 & 1.4083 & 1.4653 & 1.5826 & 1.694 \\
\hline 2.1860 & .2133 & 0.8403 & 1.1482 & 1.1741 & 1.2338 & 1.2716 & 1.3369 & 1.4407 & 1.4732 & 1.5546 & 1.68 \\
\hline 2.1880 & 0.2029 & 0.8150 & & & & & & 1.4056 & & 1.5847 & \\
\hline 2.1900 & 2 & 0.8 & & & & & 94 & 1.4059 & & 1.5 & 1.6949 \\
\hline 2.1920 & 1876 & 0.8 & & & 1.2 & & 38 & 1.3942 & & 1.5831 & 1.7077 \\
\hline .1940 & 1751 & 0.8033 & 1.1301 & 1.1641 & 1.2377 & 984 & 3260 & 1.3968 & 1.4 & 1.5744 & 1.682 \\
\hline 2.1960 & .1873 & 0.7960 & 1.1319 & 1.1666 & 1.2247 & 1.2746 & 1.3101 & 1.3955 & 1.4551 & 1.5967 & 1.682 \\
\hline 2.1980 & 1902 & 0.8110 & & & & & & & & 5502 & 1.682 \\
\hline 2.2000 & 2075 & 0.7960 & 1.1501 & 1.1 & 1.2079 & 658 & 43 & 1.3958 & 1.4723 & 1.5764 & 1.66 \\
\hline 2.2020 & 0.2062 & 0.8050 & & & & & & & & 1.5440 & \\
\hline 2.2040 & & & & & & & & & & & 56 \\
\hline 2.2060 & 2178 & 0.79 & & & 1.2 & & 32 & 40 & 1. & 1.5 & 1.643 \\
\hline 2.2080 & .2169 & 0.8282 & 1.1506 & 1.18 & 1.2407 & 841 & 1.3427 & 1.3666 & 1.4792 & 1.5498 & 1.6692 \\
\hline 2.2100 & 2139 & 0.8077 & 339 & 1.1625 & 1.2308 & 551 & 295 & 1.3858 & 555 & 1.5607 & 1.6564 \\
\hline 2.2120 & 2160 & 0.7996 & 1.1154 & 1.1661 & 1.2256 & 1.2794 & 1.3249 & 1.3734 & 1.4576 & 1.5459 & 1.656 \\
\hline 2.2140 & 2097 & 0.8031 & & 1.1693 & 1.2168 & & 1.3064 & 1.3629 & 1.4479 & 1.5433 & 1.64 \\
\hline 2.2160 & .2002 & & & & & & & & & 1.5442 & \\
\hline 2.2180 & 5 & 0. & & & 39 & & & & & 81 & 1 \\
\hline 2.2200 & 2246 & 0.7781 & 1. & 1.1 & 1.2001 & 55 & 91 & 1.3764 & 68 & 1.5425 & 1.6178 \\
\hline 2.2220 & .2255 & 0.7923 & 1.1118 & 1.1736 & 1.2218 & 551 & 1.2925 & 1.3877 & 1.4336 & 1.5745 & 1.6050 \\
\hline 2.2240 & 2064 & 0.7988 & & & 1.2289 & & 779 & 1.3741 & & .5408 & 1.6307 \\
\hline 2.2260 & 0.2032 & 0.7613 & 1.1375 & 1.1612 & 1.2107 & 1.2761 & 1.3062 & 1.3511 & 1.4259 & 1.5478 & 1.617 \\
\hline 2.2280 & 1998 & 0.7974 & 1.1183 & & 1.2130 & & 1.2747 & 1.3743 & 1.4417 & 1.5231 & 1.60 \\
\hline 2.2300 & & & & & & & & & & & \\
\hline 2.2320 & 0 & & & & & & & 90 & & & 307 \\
\hline 2.2340 & 0.1881 & 0.8144 & 1.1270 & 1.1 & 1.1973 & 17 & 1.3038 & 1.3562 & 1.4258 & 1.5464 & 1.6050 \\
\hline 2.2360 & .2091 & 0.7749 & 1.1220 & 1.1577 & 1.2147 & 1.2500 & 1.3200 & 1.3700 & 1.4638 & 1.5276 & 1.605 \\
\hline 2.2380 & 1912 & 0.7722 & 331 & & 1.2046 & 1.2444 & 1.3102 & 1.3380 & 1.4554 & 1.5436 & 1.617 \\
\hline 2.2400 & 0.1968 & 0.7827 & 1.1306 & 1.1447 & 1.1997 & 1.2616 & 1.3029 & 1.3535 & 1.4449 & 1.5524 & 1.605 \\
\hline 2.2420 & 0.2019 & 0.7778 & & & 1.2086 & & & 1.3762 & 1.4340 & 1.5214 & 1.605 \\
\hline 2.2440 & 2097 & 0.7957 & & & & & & & & 1.5093 & 1.592 \\
\hline 2.2460 & .1997 & & & & & & & & & 1.5274 & 1.617 \\
\hline 2.2480 & .1796 & 0.8012 & 1.1195 & & 1.1898 & 2284 & 1.2995 & 1.3494 & 1.4752 & 1.5186 & 1.579 \\
\hline 2.2500 & 2035 & 0.7738 & & & 1.1801 & & 1.2971 & 1.3443 & 1.4405 & .5070 & 1617 \\
\hline 2.2520 & 0.2116 & 0.7897 & 1.1239 & 1.1538 & 1.1923 & 1.2369 & 1.3122 & 1.3234 & 1.4487 & 1.5083 & 1.592 \\
\hline 2.2540 & 0.2099 & 0.7913 & 1.1127 & 1.1349 & 1.1898 & 1.2520 & 1.2923 & 1.3742 & 1.4511 & 1.5322 & $1.57 \mathrm{c}$ \\
\hline 2.2560 & 0.2240 & 0.7843 & 1.1300 & 1.1655 & 1.2056 & 301 & 1.3014 & 1.3529 & 1.4307 & 1.5127 & 1.55 \\
\hline 2.2580 & 0.2176 & 0.7958 & 1.1240 & 1.1479 & 1.1977 & 1.2392 & 1.2781 & 1.3373 & 1.4217 & 1.5076 & 1.592 \\
\hline 2.2600 & 2065 & 7794 & 91 & & & & & 289 & 208 & .5128 & $1.57 \mathrm{~S}$ \\
\hline & & & & & & & & 1.3517 & 1.4302 & 1.4935 & 1570 \\
\hline & 2195 & 0.7954 & & & 1.1902 & 353 & 1.2706 & 1.3354 & 1.4060 & 1.4890 & 1.579 \\
\hline 2.2660 & 0.2350 & 0.8141 & 1.1059 & 1.1399 & 1.1999 & 1.2186 & 1.2929 & 1.3195 & 1.4205 & 1.4894 & 1.553 \\
\hline
\end{tabular}




\begin{tabular}{|c|c|c|c|c|c|c|c|c|c|c|c|}
\hline 2.2680 & 0.2117 & 0.7842 & 1.0996 & 1.1536 & 1.1892 & 1.2601 & 1.2830 & 1.3523 & 1.4146 & 1.5134 & 1.5665 \\
\hline .2700 & 0.2088 & 0.7821 & 1.1095 & 1.1355 & 1.1938 & 1.2444 & 1.2940 & 1.3660 & 1.3738 & 1.5178 & 1.5536 \\
\hline .2720 & 0.2011 & 0.7926 & 1.1421 & 1.1520 & 1.1997 & 1.2273 & 1.2781 & 1.3271 & 1.3836 & 1.5115 & 1.5536 \\
\hline 2740 & .2022 & 0.7728 & 1.1097 & 1.1583 & 1.1953 & 1.2342 & 1.2851 & 1.3558 & 1.3941 & 1.5011 & 1.5665 \\
\hline .2760 & .1920 & 0.7821 & 1.1100 & 1.1660 & 1.1890 & 1.2402 & 1.2873 & 1.3466 & 1.4115 & 1.4966 & .5536 \\
\hline 2.2780 & 0.2039 & 0.7827 & 1.1028 & 1.1180 & 1.1778 & 1.2288 & 1.2767 & 1.3282 & 1.4021 & 1.4844 & .5665 \\
\hline .2800 & .2055 & 0.7957 & 1.0855 & 1.1357 & 1.1775 & 1.2114 & 1.2922 & 1.2965 & 1.4160 & 1.5019 & 1.5536 \\
\hline 2820 & 2031 & 0.7954 & 1.1039 & 1.1324 & 1.1971 & 1.2228 & 1.2848 & 1.3244 & 1.4159 & 1.5046 & 1.5922 \\
\hline 2840 & 0.2087 & 0.7778 & 1.0860 & 1.1338 & 1.1858 & 1.2115 & 1.3107 & 1.3148 & 1.4141 & 1.4849 & .5536 \\
\hline 2860 & .1929 & 0.8015 & 1.1060 & 1.1342 & 1.1915 & 1.2427 & 1.2961 & 1.3121 & 1.4173 & 1.4994 & .5536 \\
\hline 2880 & .2223 & 0.7759 & 1.1013 & 1.1296 & 1.1629 & 1.2347 & 1.2838 & 1.3437 & 1.4027 & 1.4964 & .5536 \\
\hline 2900 & .2135 & 0.7781 & 1.1038 & 1.1429 & 1.1950 & 1.1902 & 1.2775 & 1.3216 & 1.3914 & 1.4808 & 1.5665 \\
\hline 2.2920 & .1935 & 0.7719 & & & & & 1.2611 & & & & .5665 \\
\hline 2.2940 & 0.2 & 0.79 & 1.0847 & 1.1171 & 1.1618 & 1.2479 & 1.2722 & 1.3339 & & & 1.5665 \\
\hline .2960 & 0.2067 & 0.7664 & 1.0933 & 1.1171 & 1.1 & 1.2435 & 1.2715 & 1.3112 & & 1.5011 & .5280 \\
\hline 2980 & 1986 & 0.7705 & 1.0961 & 1.1176 & 1.1710 & 1.2316 & 1.2762 & 1.3221 & & & 5536 \\
\hline 3000 & .2073 & 0.7731 & 1.0933 & 1.1052 & 1.1786 & 1.2052 & 1.2633 & 1.3048 & 1.3779 & 1.4909 & .5280 \\
\hline 3020 & 1956 & 0.7759 & 1.0852 & 1.1239 & 1.1772 & .2214 & 1.2558 & 1.3191 & 1.3804 & 1.4635 & .5408 \\
\hline 2.3040 & 1995 & 0.7752 & 1.1007 & & & & 1.2414 & & & & 5536 \\
\hline 2.3060 & 0.2085 & 0.7752 & & & & & & & & & 5408 \\
\hline 2.3080 & 1948 & 0.7804 & 1.0 & & & & 89 & & & & 5536 \\
\hline 3100 & 0.1971 & 0.7857 & 1.1012 & 1.1284 & 1.1 & 850 & 1.2573 & 1.2970 & & & .5151 \\
\hline 3120 & .2073 & 0.7666 & 1.0761 & 1.1171 & 1.1822 & 1.2132 & 1.2548 & 1.2829 & 1.3679 & 1.4308 & 1.5280 \\
\hline 3140 & .1882 & 0.7801 & 1.0696 & 1.1168 & 1.1721 & 1.2062 & 1.2662 & 1.3048 & & & .5280 \\
\hline 3160 & 1907 & 0.7738 & 1.0860 & & & 1.2207 & 1.2313 & 1.2967 & & & .5408 \\
\hline 2.3180 & 1970 & 0.7907 & & & & & & & & & 5408 \\
\hline 2.3200 & 8 & 0.78 & & & & & & & & & 280 \\
\hline 2.3220 & 1924 & 0.7633 & 1.0 & & & & 33 & & & & 1.5280 \\
\hline 3240 & .1826 & 0.7656 & 1.0746 & 1.1127 & 1.1 & 055 & 1.2460 & 1.3059 & & & 1.5151 \\
\hline .3260 & 2001 & 0.7731 & 1.0906 & 1.0985 & 1.1754 & 840 & 1.2510 & 1.2899 & 05 & 460 & 1.5280 \\
\hline 3280 & 1877 & 0.7718 & 1.1053 & & & & 1.2528 & 1.2954 & & & .5151 \\
\hline 2.3300 & 1863 & 0.7711 & 1.0881 & & & 37 & 1.2741 & 1.2774 & & & 1.5151 \\
\hline 2.3320 & 1915 & 0.7638 & & & & & & & & & 5151 \\
\hline 2.3340 & 1947 & 0.7747 & & & & & & & & & 1.5151 \\
\hline .3360 & 1794 & 0.7566 & 1.0 & 1.0 & & 10 & 2342 & 1.2785 & & & 1.5280 \\
\hline .3380 & 1950 & 0.7741 & 1.0 & 1.1296 & 1.1 & 910 & 2257 & 1.2927 & & & 1.5023 \\
\hline 3400 & 1868 & 0.7565 & 1.0644 & 1.1036 & 1. & 948 & 2327 & 1.2726 & & 31 & .528 \\
\hline 3420 & 1773 & 0.7511 & & & & & & 1.2871 & & & 1.5151 \\
\hline 2.3440 & 1900 & 0.7638 & 1.0707 & & & & 2361 & 1.2710 & & & .5151 \\
\hline 2.3460 & & 0.7530 & & & & & & & & & \\
\hline 2.3480 & 3 & 74 & & & & & & & & & 1.5151 \\
\hline 2.3500 & 1869 & 0.7425 & 1.0 & & & 96 & 361 & 1.2884 & & & 1.5151 \\
\hline .3520 & 1900 & 0.7376 & 1.0743 & 1.1041 & 1.1 & 955 & 1.2274 & 1.2880 & 17 & 77 & 1.5023 \\
\hline 3540 & 1833 & 0.7515 & 1.0764 & & 29 & & 2230 & 1.2887 & & & 1.5151 \\
\hline 2.3560 & 1862 & 0.7363 & & & & & 1.2037 & 1.2581 & & & 1.5023 \\
\hline 2.3580 & & 0.7376 & & & & & & & & & דיק \\
\hline 2.3600 & & & & & & & & & & & 5023 \\
\hline 2.3620 & 7 & 0.7 & & & & & & 43 & & & 894 \\
\hline .3640 & 1832 & 0.7675 & 0 & & & & 393 & 1.2581 & & & 1.5151 \\
\hline 2.3660 & 1899 & 0.7532 & 1.0604 & 1.1 & 1.1521 & 708 & 1.2431 & 1.2774 & 549 & 123 & 1.4894 \\
\hline 2.3680 & 1783 & 0.7512 & & & & & 2124 & 1.2706 & & & .4894 \\
\hline 2.3700 & 1718 & 0.7594 & & & & & & & & & 1.4894 \\
\hline 2.3720 & & & & & & & & & & & 4766 \\
\hline 2.3740 & .1807 & 0.7494 & & & & & & & & & 1.4894 \\
\hline 2.3760 & .1763 & 0.7435 & & & & & 39 & 1.2762 & & 05 & 1.5151 \\
\hline 2.3780 & .1721 & 0.7363 & 1.0779 & 1.1003 & 1.1 & 787 & 1.2063 & 1.2550 & 52 & 1.4155 & 1.4894 \\
\hline 2.3800 & 1876 & 0.7304 & 1.0626 & 0 & 77 & 796 & 1.1990 & 1.2572 & 66 & 57 & 1.5023 \\
\hline 2.3820 & 1821 & 0.7398 & & & & 748 & 1.2160 & 1.2491 & & & 1.5023 \\
\hline 2.3840 & .1850 & 0.7388 & 1.0573 & 1.0935 & & & 1.2268 & 1.2343 & & 1.4145 & 1.5023 \\
\hline 2.3860 & .1988 & 0.7434 & & & & & & 517 & & 117 & 1.4766 \\
\hline 2.3880 & & 0.7508 & & & & & & & & & 1.476 \\
\hline 2.3900 & 1901 & & & & & & & 1.2733 & & & 1.476 \\
\hline 2.3920 & 1936 & 0.7427 & 1.0500 & 1.0801 & 1.1400 & 440 & 1.2123 & 1.2517 & 709 & 1.4219 & 1.5023 \\
\hline 2.3940 & .1960 & 0.7486 & 1.0579 & & 11131 & 1536 & 1.2099 & 1.2555 & 1.3083 & 1.4171 & 1489 \\
\hline 2.3960 & 1755 & 0.7338 & 1.0608 & & 114 & 1.1622 & 1.1993 & 1.2527 & 35 & 1.4126 & 1.4894 \\
\hline 2.3980 & 0.1746 & 0.7240 & 1.0494 & 1.0999 & 1.1121 & 1.1566 & 1.1853 & 1.2466 & 1.3202 & 1.4001 & 1.5023 \\
\hline 2.4000 & .1768 & 0.7293 & 1.0446 & & & & 1.1801 & 1.2455 & 1.3204 & 1.4014 & 1.489 \\
\hline 2.4020 & 1645 & 0.7276 & $1 .($ & & & & 36 & 1.2449 & 1.2989 & 1.3866 & 1.502 \\
\hline & 686 & 0.7264 & 549 & & & & & 1.2521 & & 1.4188 & 1.476 \\
\hline 2.4060 & 0.1594 & 0.7104 & 1.0466 & 1.0856 & 1.0966 & 1.1412 & 1.2200 & 1.2426 & 1.3097 & 1.4054 & 1.463 \\
\hline
\end{tabular}




\begin{tabular}{|c|c|c|c|c|c|c|c|c|c|c|c|}
\hline 2.4080 & 0.1651 & 0.7365 & 1.0444 & 1.0842 & 1.1083 & 1.1258 & 1.1752 & 1.2199 & 1.3420 & 1.3859 & 1.4766 \\
\hline 2.4100 & 0.1658 & 0.7097 & 1.0468 & 1.0740 & 1.1142 & 1.1393 & 1.1770 & 1.2448 & 1.3061 & 1.4126 & 1.4381 \\
\hline .4120 & 0.1595 & 0.7043 & 1.0349 & 1.0650 & 1.1023 & 1.1275 & 1.1818 & 1.2336 & 1.3173 & 1.3893 & 1.4766 \\
\hline .4140 & 0.1717 & 0.7024 & 1.0400 & 1.0826 & 1.0989 & 1.1262 & 1.1703 & 1.2235 & 1.3099 & 1.4014 & 1.4638 \\
\hline 4160 & 0.1464 & 0.6900 & 1.0399 & 1.0819 & 1.1072 & 1.1451 & 1.1859 & 1.2167 & 1.2917 & 1.3850 & 4638 \\
\hline .4180 & 0.1460 & 0.7036 & 1.0385 & 1.0874 & 1.0852 & 1.1361 & 1.1831 & 1.2362 & 1.2968 & 1.3583 & . 4766 \\
\hline 2.4200 & 0.1579 & 0.6879 & 1.0591 & 1.0683 & 1.1149 & 1.1170 & 2055 & 1.2319 & 1.2960 & 1.3709 & 1.5023 \\
\hline .4220 & 0.1376 & 0.6996 & 1.0430 & 1.0892 & 1.1080 & 1.1395 & 1.1836 & 1.2043 & 1.3242 & 1.3884 & .4638 \\
\hline 4240 & 0.1310 & 0.6998 & 1.0294 & 1.0687 & 1.1151 & 1.1259 & 1957 & 1.2393 & 1.2958 & .3914 & 4766 \\
\hline 4260 & 0.1387 & 0.6986 & 1.0313 & 1.0798 & 1.0920 & 1418 & 1746 & 1.2246 & 1.3098 & 4000 & 4638 \\
\hline 4280 & 0.1255 & 0.6768 & 1.0363 & 1.0697 & 1.1057 & 1.1557 & 1.1700 & 1.2456 & 1.2941 & 1.4023 & 1.4766 \\
\hline 4300 & 1402 & 0.6997 & 1.0199 & 1.0786 & & & 1677 & 1.2123 & 1.3116 & 061 & 4509 \\
\hline 2.4320 & 0.1405 & 0.6606 & 1.0178 & 1.0413 & & 1.1172 & .1681 & & & 186 & .4766 \\
\hline 2.4340 & 0.1413 & 0.6888 & & & & & & & & & 4766 \\
\hline 2.4360 & 0.1483 & 0.6594 & $1 .($ & 1.0 & & & & & & & 4766 \\
\hline 4380 & 1238 & 0.6483 & 0 & 1.0 & & & & & & & 509 \\
\hline .4400 & 0.1067 & 0.6658 & 1.0266 & 1.0755 & 1.1179 & 1.1103 & 1595 & 1.2470 & 951 & 705 & 5023 \\
\hline 4420 & 1263 & 0.6771 & 1.0253 & 1.0538 & & & & & & & .4766 \\
\hline 4440 & 0.1226 & 0.6350 & 1.0162 & 1.0549 & 1.0 & & & 1.2248 & & & .4638 \\
\hline 2.4460 & 0.1176 & 0.6455 & 1.0490 & 1.0497 & & & & & & & 638 \\
\hline 2.4480 & & & & & & & & & & & \\
\hline 2.4500 & 0.1266 & 76 & & & & & & & & & 894 \\
\hline 2.4520 & 0.1223 & 0.6349 & 1.1961 & 1.0431 & 1.0741 & 66 & 24 & 1.1 & 57 & 15 & 1.4509 \\
\hline .4540 & 0.1044 & 0.6144 & 1.2574 & 1.0228 & 1.0738 & 013 & 1455 & 1.1801 & 11 & 36 & 1.4638 \\
\hline 4560 & 1158 & 0.5944 & 1.3164 & & & & & & & & 381 \\
\hline 2.4580 & 0.1200 & 0.6266 & 1.3777 & 1.0446 & 51 & 1.0956 & 52 & 1.2035 & 45 & 75 & 1.4252 \\
\hline 2.4600 & 0.1210 & 0.6181 & & 1.0036 & & & & & & & 81 \\
\hline 2.4620 & 0.1179 & & & & & & & & & & 1.4638 \\
\hline 2.4640 & 4 & 26 & & & & & & & & & 381 \\
\hline 2.4660 & 0.1097 & 0.6059 & 1 & 1.0 & & & 42 & & & & 1.4509 \\
\hline 2.4680 & 0.0953 & 0.6101 & & & & & & & 320 & & 509 \\
\hline 2.4700 & 0951 & 0.6110 & & 0.9915 & 96 & & 1.1294 & & & & 1.3996 \\
\hline 2.4720 & 0.1038 & 0.6120 & 1.8 & 1.0023 & & & 1.1196 & & & & 1.4381 \\
\hline 2.4740 & & & & & & & & & & & 1.4381 \\
\hline 2.4760 & 0.0876 & 0.6165 & & & & & & & & & 1.4252 \\
\hline 478 & 00801 & 06 & & & & & & & & & \\
\hline 2.4800 & 0.0768 & 84 & 1 & 1.1 & 1.0 & & & 1.1 & 1.2522 & 169 & 1.425 \\
\hline 2.4820 & 0.0874 & 0.6503 & 1.9758 & 1.2608 & 1.0279 & 1.0352 & 49 & 1.1578 & 1.2442 & 1.3663 & 1.4252 \\
\hline 4840 & 0.0716 & 0.6411 & & & & & & & & & 14252 \\
\hline 2.4860 & 0.0782 & 0.6393 & 2.0390 & 1.3871 & 1.0040 & 1.0486 & 05 & 1.1434 & 1.2518 & 1.3598 & 1.4252 \\
\hline 2.4880 & 0.0824 & 0.6457 & 2.0782 & 1.4743 & 0.9995 & 238 & 1.0784 & 1.1512 & 469 & 445 & 1.4252 \\
\hline 2.4900 & 0.0779 & 0.6375 & & & & & & & & & 1.4252 \\
\hline 2.4920 & 0.0880 & & & & & & & & 1.2478 & 1.3452 & 1.4252 \\
\hline & & & & & & & & & & 1.3747 & 1.438 \\
\hline & & & & & & 1.0310 & & 1.1628 & 1.2493 & 1.3554 & 1425 \\
\hline & 0.0878 & 0.6530 & 2.1991 & 1.8402 & 0.9990 & 1.0413 & 1.0802 & 1.1620 & 1.2623 & 1.3530 & 1.42 \\
\hline .5000 & 0.0676 & 0.6503 & 2.2199 & 1.9020 & 1.0167 & 1.0423 & 1.1188 & 1.1535 & 1.2669 & 1.3489 & 1.412 \\
\hline
\end{tabular}

